\title{
FINAL REPORT PHASE I
}

\section{VOLUME II OF IV BOOK 3 OF 4}

ASSESSMENT OF UNDERGROUND COAL GASIFICATION IN BITUMINOUS COALS - CATALOG OF BITUMINOUS COALS AND SITE SELECTION

\section{APPENDIX A}

NATIONAL COAL RESOURCE DATA SYSTEM ECOAL, WCOAL AND BMALYT

$$
\text { prepared for the }
$$

U.S. DEPARTMENT OF ENERGY

MORGANTOWN ENERGY TECHNOLOGY CENTER UNDER CONTRACT NO. DE-AC05-80MC 14584

\section{PROCESS DIVISION \\ $\mathrm{OF}$ \\ WILLIAMS BROTHERS ENGINEERING COMPANY \\ A Resource Sciences Company}




\section{DISCLAIMER}

This report was prepared as an account of work sponsored by an agency of the United States Government. Neither the United States Government nor any agency Thereof, nor any of their employees, makes any warranty, express or implied, or assumes any legal liability or responsibility for the accuracy, completeness, or usefulness of any information, apparatus, product, or process disclosed, or represents that its use would not infringe privately owned rights. Reference herein to any specific commercial product, process, or service by trade name, trademark, manufacturer, or otherwise does not necessarily constitute or imply its endorsement, recommendation, or favoring by the United States Government or any agency thereof. The views and opinions of authors expressed herein do not necessarily state or reflect those of the United States Government or any agency thereof. 


\section{DISCLAIMER}

Portions of this document may be illegible in electronic image products. Images are produced from the best available original document. 


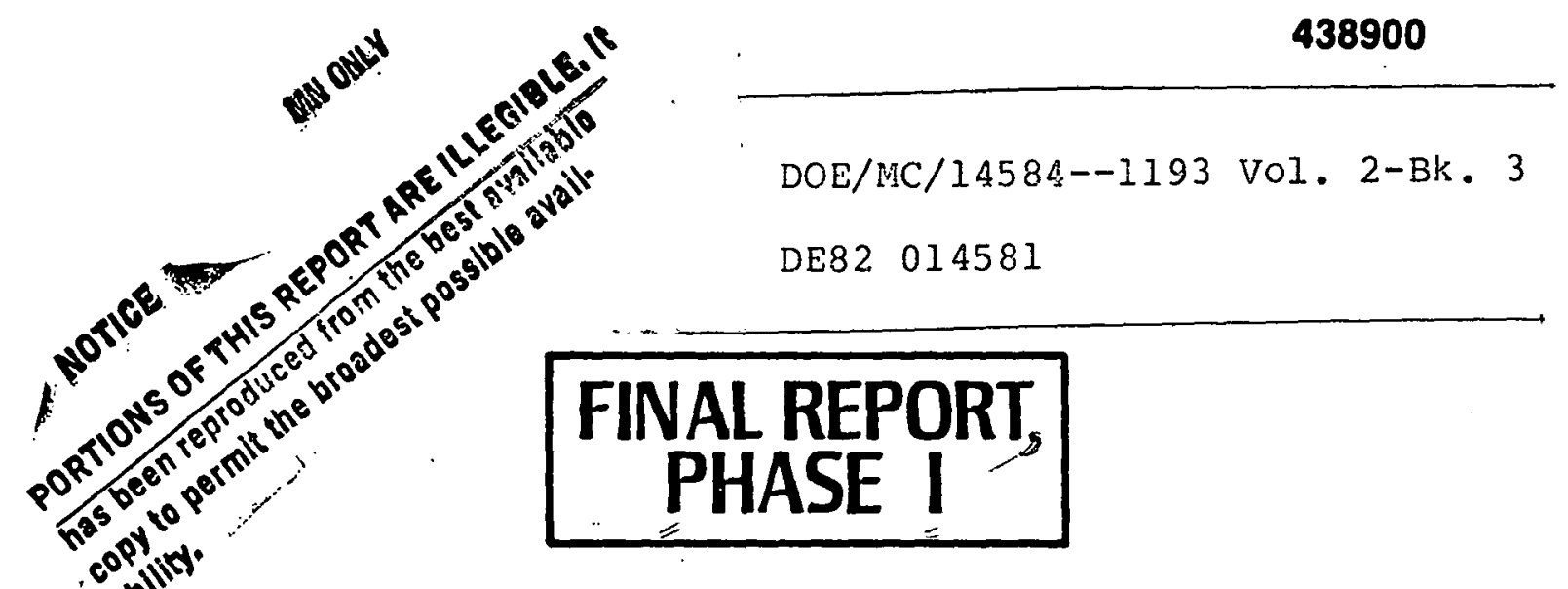

\section{VOLUME II OF IV \\ BOOK 3 OF 4}

$\checkmark$ ASSESSMENT OF UNDERGROUND COAL

GASIFICATION IN BITUMINOUS COALS :

- CATALOG OF BITUMINOUS COALS

AND SITE SELECTION; ${ }^{4}$

$$
\text { APPENDIX } A_{0} 2 H
$$

NATIONAL COAĹ RESOURČE DATA SYSTEM:

ECOAL, WCOAL, AND BMALYT.

$$
\text { prepared for the }
$$

\section{U.S. DEPARTMENT OF ENERGY}

MORGANTOWN ENERGY TECHNOLOGY CENTER

UNDER CONTRACT NO. DE-AC05-80MC 14584

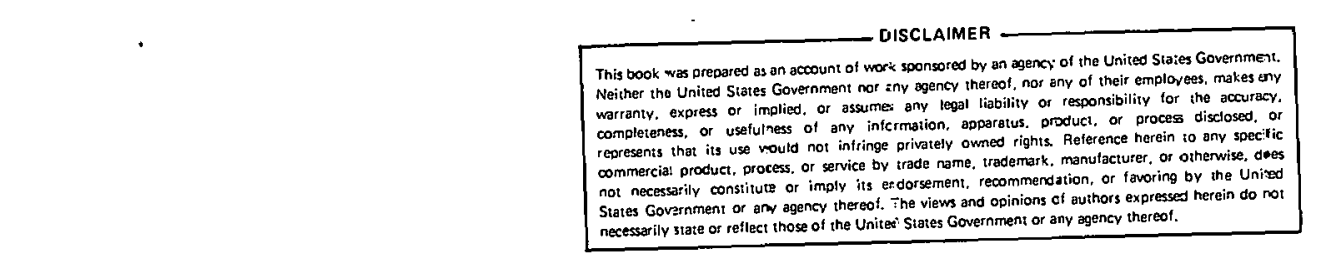

\section{PROCESS DIVISION \\ OF}

\section{WILLIAMS BROTHERS ENGINEERING COMPANY}

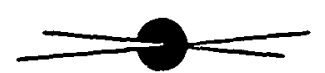

RESOURCE SCIENCES CENTER • TULSA, OKLAHOMA 74177 


\section{DISCLAIMER}

This report was prepared as an account of work sponsored by the United States Government. Neither the United States nor the United States Department of Energy, nor any of their employees, makes any warranty, express or implied, or assumes any legal liability or responsibility for the accuracy, completeness, or usefulness of any information, apparatus, product, or process disclosed, or represents that its use would not infringe privately owned rights. Reference herein to any specific commercial product, process, or service by trade name, mark, manufacturer, or otherwise, does not necessarily constitute or imply its endorsement, recommendation, or favoring by the United States Government or any agency thereof. The views and opinions of authors expressed herein do not necessarily state or reflect those of the United States Government or any agency thereof.

This technical report is being transmitted in advance of DOE review and no further dissemination or publication shall be made of the report without prior approval of the DOE Project/Program Manager. 
Appendix $A$ is a catalog of the bituminous coal in 29 states of the contiguous United States which contain identified bituminous coal resources. 
1 
identified coat resources in olabamo

(In millions of short tons)

\begin{tabular}{|c|c|c|c|c|c|c|c|c|c|c|c|c|}
\hline $\begin{array}{l}\text { rank } \\
\text { coal bed } \\
\text { overburden } \\
=x=x=x=3 x=x=x\end{array}$ & $\begin{array}{r}\text { measur } \\
14-28 \\
=x=x=3 x=20\end{array}$ & $\begin{array}{l}\text { ed and in } \\
28-42 \\
=x=28=\pi x=\end{array}$ & $\begin{array}{l}\text { dicated } \\
\text { Over } 42 \\
=x==x=m=3 \\
\text { bibb }\end{array}$ & $\begin{array}{l}\text { nches) } \\
\text { total } \\
x=0 x=0\end{array}$ & $\begin{aligned} & 14-28 \\
&=x=2 x=2 x=8\end{aligned}$ & $\begin{array}{l}\text { inferred } \\
28-42 \\
=x=2= \pm=2 \\
\text { ounty }\end{array}$ & $\begin{array}{l}\text { (inches) } \\
\text { over } 42 \\
x=x=0 x=0\end{array}$ & $\begin{array}{l}\text { total } \\
=. \cos \end{array}$ & $\begin{array}{c}14-28 \\
=x=2=x x=2\end{array}$ & $\begin{array}{c}\operatorname{cotal}(i \\
28-42 \\
=28=x=21\end{array}$ & $\begin{array}{l}\text { inchess } \\
\text { Over } 42 \\
=x=x=x=x=\end{array}$ & $=\begin{array}{l}\operatorname{total} \\
==2 \operatorname{sen} 2\end{array}$ \\
\hline it & & & & & & & & & & & & \\
\hline 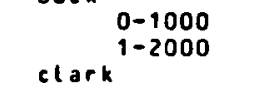 & $\begin{array}{l}0.00 \\
0.00\end{array}$ & $\begin{array}{l}0.00 \\
0.00\end{array}$ & $\begin{array}{l}0.00 \\
0.00\end{array}$ & $\begin{array}{l}0.00 \\
0.00\end{array}$ & $\begin{array}{l}3.00 \\
8.90\end{array}$ & $\begin{array}{l}10.20 \\
19.50\end{array}$ & $\begin{array}{l}2.60 \\
1.30\end{array}$ & $\begin{array}{l}15.80 \\
29.70\end{array}$ & $\begin{array}{l}3.00 \\
8.90\end{array}$ & $\begin{array}{l}10.20 \\
19.50\end{array}$ & $\begin{array}{l}2.60 \\
1.30\end{array}$ & $\begin{array}{l}15.80 \\
29.70\end{array}$ \\
\hline $\begin{array}{r}0-1000 \\
1-2000 \\
\text { gholson }\end{array}$ & $\begin{array}{l}0.00 \\
0.00\end{array}$ & $\begin{array}{l}0.00 \\
0.00\end{array}$ & $\begin{array}{l}0.00 \\
0.00\end{array}$ & $\begin{array}{l}0.00 \\
0.00\end{array}$ & $\begin{array}{l}23.10 \\
39.80\end{array}$ & $\begin{array}{l}21.70 \\
11.40\end{array}$ & $\begin{array}{r}12.10 \\
1.70\end{array}$ & $\begin{array}{l}56.90 \\
52.90\end{array}$ & $\begin{array}{l}23.10 \\
39.80\end{array}$ & $\begin{array}{l}21.70 \\
11.40\end{array}$ & $\begin{array}{r}12.10 \\
1.70\end{array}$ & $\begin{array}{l}56.90 \\
52.90\end{array}$ \\
\hline $\begin{array}{c}\text { gholson } \\
0-1000 \\
1-2000 \\
\text { heleno }\end{array}$ & $\begin{array}{l}0.00 \\
0.00\end{array}$ & $\begin{array}{l}0.00 \\
0.00\end{array}$ & $\begin{array}{l}0.00 \\
0.00\end{array}$ & $\begin{array}{l}0.00 \\
0.00\end{array}$ & $\begin{array}{l}9.30 \\
5.50\end{array}$ & $\begin{array}{l}9.30 \\
7.00\end{array}$ & $\begin{array}{l}1.60 \\
0.00\end{array}$ & $\begin{array}{l}20.20 \\
12.50\end{array}$ & $\begin{array}{l}9.30 \\
5.50\end{array}$ & $\begin{array}{l}9.30 \\
7.00\end{array}$ & $\begin{array}{l}1.60 \\
0.00\end{array}$ & $\begin{array}{l}20.20 \\
12.50\end{array}$ \\
\hline $\begin{array}{r}0-1000 \\
\text { aontevallo }\end{array}$ & 0.00 & 0.00 & 0.00 & 0.00 & 1.80 & 2.80 & 0.00 & 4.60 & 1.80 & 2.80 & 0.00 & 4.60 \\
\hline $\begin{array}{c}0-1000 \\
\text { nunnally }\end{array}$ & 0.00 & 0.00 & 0.00 & 0.00 & 9.80 & 4.60 & 0.00 & 14.40 & 9.80 & 4.60 & 0.00 & 14.40 \\
\hline $\begin{array}{l}0-1000 \\
1-2000 \\
2-3000\end{array}$ & $\begin{array}{l}0.00 \\
0.00 \\
0.00\end{array}$ & $\begin{array}{l}0.00 \\
0.00 \\
0.00\end{array}$ & $\begin{array}{l}0.00 \\
0.00 \\
0.00\end{array}$ & $\begin{array}{l}0.00 \\
0.00 \\
0.00\end{array}$ & $\begin{array}{r}4.10 \\
35.20 \\
29.30\end{array}$ & $\begin{array}{r}19.70 \\
34.20 \\
8.50\end{array}$ & $\begin{array}{l}7.50 \\
2.20 \\
0.00\end{array}$ & $\begin{array}{l}31.30 \\
71.60 \\
37.80\end{array}$ & $\begin{array}{r}4.10 \\
35.20 \\
29.30\end{array}$ & $\begin{array}{r}19.70 \\
34.20 \\
8.50\end{array}$ & $\begin{array}{l}7.50 \\
2.20 \\
0.00\end{array}$ & $\begin{array}{l}31.30 \\
71.60 \\
37.80\end{array}$ \\
\hline $\begin{array}{c}0-1000 \\
1-2000 \\
\text { upper thompson }\end{array}$ & $\begin{array}{l}0.00 \\
0.00\end{array}$ & $\begin{array}{l}0.00 \\
0.00\end{array}$ & $\begin{array}{l}0.00 \\
0.00\end{array}$ & $\begin{array}{l}0.00 \\
0.00\end{array}$ & $\begin{array}{r}3.10 \\
17.90\end{array}$ & $\begin{array}{r}8.60 \\
13.90\end{array}$ & $\begin{array}{l}0.00 \\
0.00\end{array}$ & $\begin{array}{l}19.70 \\
31.80\end{array}$ & $\begin{array}{r}3.10 \\
17.90\end{array}$ & $\begin{array}{r}8.60 \\
13.90\end{array}$ & $\begin{array}{l}0.00 \\
0.00\end{array}$ & $\begin{array}{l}11.70 \\
31.80\end{array}$ \\
\hline $\begin{array}{c}0-1000 \\
1-2000 \\
\text { youngbl ood }\end{array}$ & $\begin{array}{l}0.00 \\
0.00\end{array}$ & $\begin{array}{l}0.00 \\
0.00\end{array}$ & $\begin{array}{l}0.00 \\
0.00\end{array}$ & $\begin{array}{l}0.00 \\
0.00\end{array}$ & $\begin{array}{l}7.80 \\
3.90\end{array}$ & $\begin{array}{r}18.10 \\
0.00\end{array}$ & $\begin{array}{r}03.00 \\
0.00\end{array}$ & $\begin{array}{r}108.90 \\
3.90\end{array}$ & $\begin{array}{l}7.80 \\
3.90\end{array}$ & $\begin{array}{r}18.10 \\
0.00\end{array}$ & $\begin{array}{r}83.00 \\
0.00\end{array}$ & $\begin{array}{r}108.90 \\
3.90\end{array}$ \\
\hline $\begin{array}{r}0-1000 \\
1-2000\end{array}$ & $\begin{array}{r}0.00 \\
0.00 \\
\end{array}$ & $\begin{array}{r}0.00 \\
0.00 \\
-0.0\end{array}$ & $\begin{array}{r}0.00 \\
0.00 \\
\end{array}$ & $\begin{array}{r}0.00 \\
0.00 \\
\hdashline .00\end{array}$ & $\begin{array}{r}37.00 \\
51.00 \\
-5\end{array}$ & $\begin{array}{r}34.30 \\
20.00 \\
-\end{array}$ & $\begin{array}{r}9.00 \\
1.50 \\
\hdashline\end{array}$ & $\begin{array}{r}80.30 \\
79.10 \\
-\end{array}$ & $\begin{array}{r}37.00 \\
51.00 \\
\end{array}$ & $\begin{array}{r}36.30 \\
26.60 \\
-26.0\end{array}$ & $\begin{array}{r}9.00 \\
1.50 \\
-.50\end{array}$ & $\begin{array}{r}80.30 \\
79.10 \\
-2.1 .\end{array}$ \\
\hline county total = & 0.00 & 0.00 & 0.00 & 0.00 & 290.50 & 250.40 & 122.50 & 663.40 & 290.50 & 250.40 & 122.50 & 663.40 \\
\hline & & & blount & & & ounty & & & & & & \\
\hline $\begin{array}{l}\text { it } \\
\text { altoona }\end{array}$ & & & & & & & & & & & & \\
\hline $\begin{array}{r}0-1000 \\
\text { black creek }\end{array}$ & 0.00 & 0.00 & 0.00 & 0.00 & 1.40 & 2.70 & 0.00 & 4.10 & 1.40 & 2.70 & 0.00 & 4.10 \\
\hline $\begin{array}{l}0-1000 \\
\text { swansea }\end{array}$ & 11.90 & 1.80 & 0.00 & 13.70 & 0.00 & 0.00 & 0.00 & 0.00 & 11.90 & 1.80 & 0.00 & 13.70 \\
\hline $\begin{array}{r}0-1000 \\
-\end{array}$ & 0.00 & 0.00 & 0.00 & 0.00 & 1.60 & 2.90 & 0.00 & 4.50 & 1.60 & $\begin{array}{r}2.90 \\
-2 .-1 .\end{array}$ & 0.00 & 4.50 \\
\hline county total $=$ & 11.90 & 1.80 & 0.00 & 13.70 & 3.00 & 5.60 & 0.00 & 8.60 & 14.90 & 7.40 & 0.00 & 23.30 \\
\hline
\end{tabular}




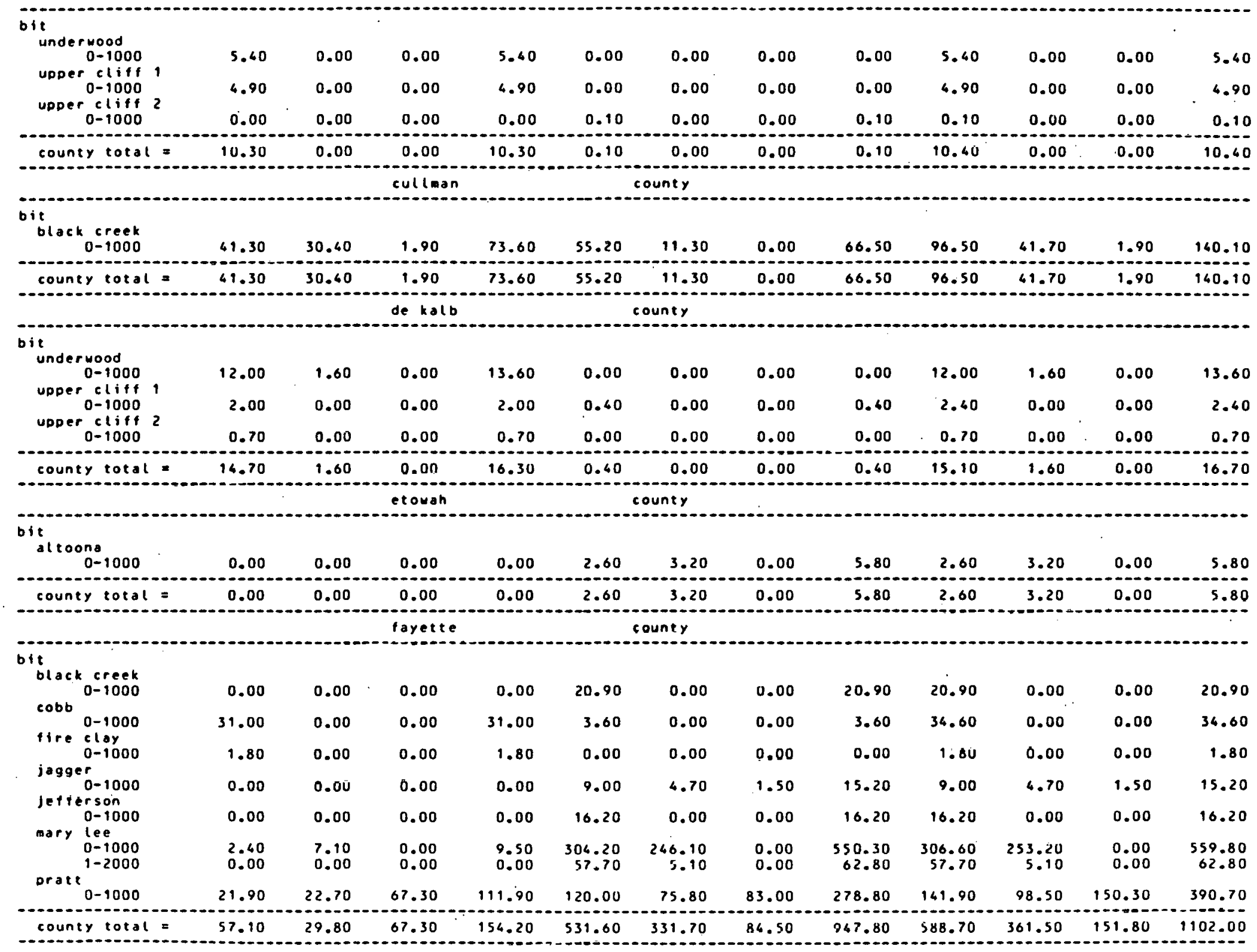


jefferson

county

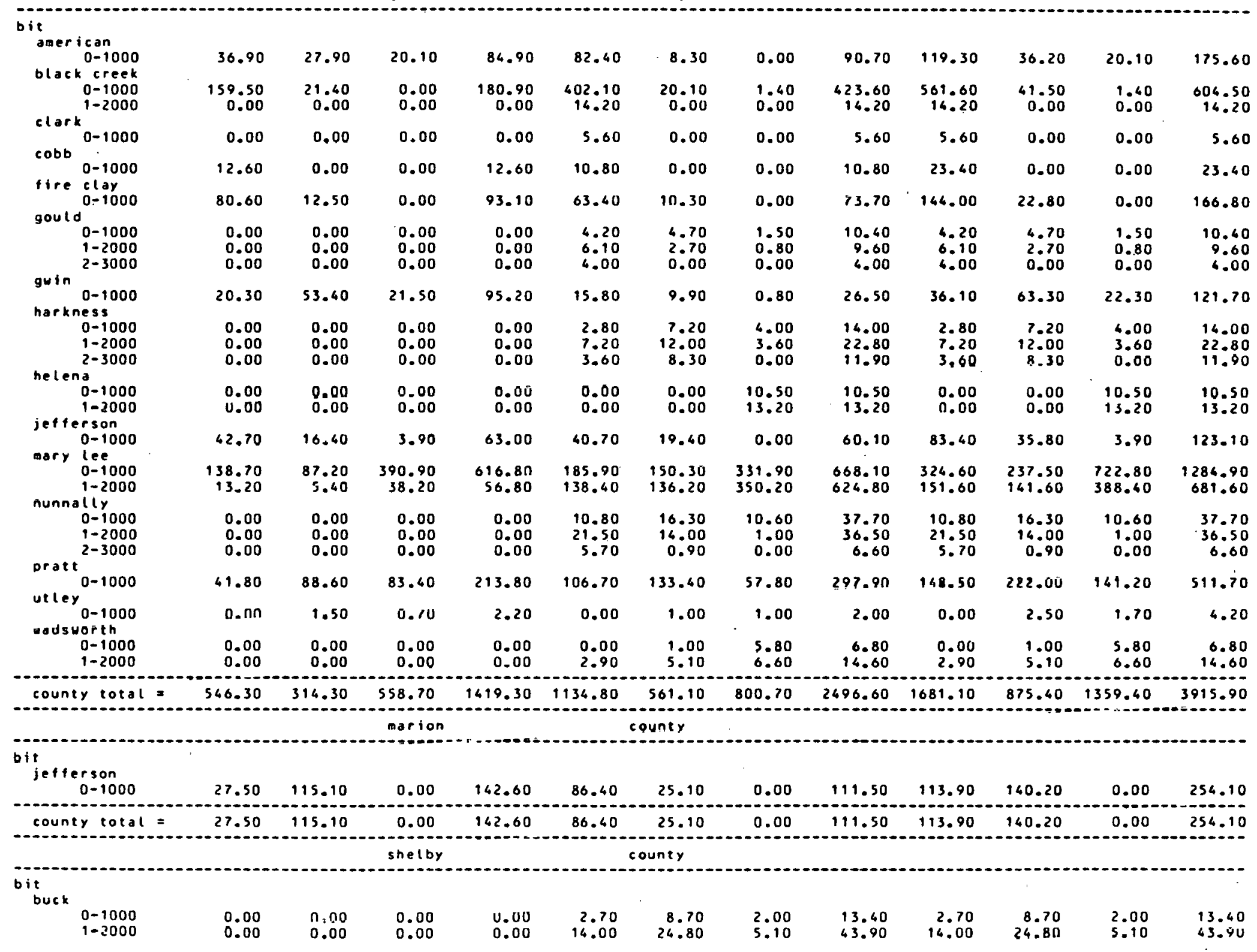




\begin{tabular}{|c|c|c|c|c|c|c|c|c|c|c|c|c|}
\hline $2-3000$ & 0.00 & 0.00 & 0.00 & 0.00 & 4.00 & 2.70 & 0.00 & 6.70 & 4.00 & 2.70 & 0.00 & 6.70 \\
\hline $\begin{array}{r}\text { clark } \\
0-1000 \\
1-2000 \\
2-3000\end{array}$ & $\begin{array}{l}0.00 \\
0.00 \\
0.00\end{array}$ & $\begin{array}{l}0.00 \\
0.00 \\
0.00\end{array}$ & $\begin{array}{l}0.00 \\
0.00 \\
0.00\end{array}$ & $\begin{array}{l}0.00 \\
0.00 \\
0.00\end{array}$ & $\begin{array}{l}20.70 \\
20.30 \\
18.70\end{array}$ & $\begin{array}{l}13.60 \\
50.00 \\
10.10\end{array}$ & $\begin{array}{l}4.90 \\
8.30 \\
0.00\end{array}$ & $\begin{array}{l}39.20 \\
78.60 \\
28.80\end{array}$ & $\begin{array}{l}20.70 \\
20.30 \\
18.70\end{array}$ & $\begin{array}{l}13.60 \\
50.00 \\
10.10\end{array}$ & $\begin{array}{l}4.90 \\
8.30 \\
0.00\end{array}$ & $\begin{array}{l}39.20 \\
78.60 \\
28.80\end{array}$ \\
\hline $\begin{array}{r}\text { ghols son } \\
0-1000 \\
1-2000 \\
2-3000\end{array}$ & $\begin{array}{l}0.00 \\
0.00 \\
0.00\end{array}$ & $\begin{array}{l}0.00 \\
0.00 \\
0.00\end{array}$ & $\begin{array}{l}0.00 \\
0.00 \\
0.00\end{array}$ & $\begin{array}{l}0.00 \\
0.00 \\
0.00\end{array}$ & $\begin{array}{r}17.20 \\
18.60 \\
8.50\end{array}$ & $\begin{array}{l}17.60 \\
44.90 \\
27.50\end{array}$ & $\begin{array}{r}3.10 \\
13.90 \\
6.40\end{array}$ & $\begin{array}{l}37.90 \\
77.40 \\
42.40\end{array}$ & $\begin{array}{r}17.20 \\
18.60 \\
8.50\end{array}$ & $\begin{array}{l}17.60 \\
44.90 \\
27.50\end{array}$ & $\begin{array}{r}3.1 .0 \\
13.90 \\
6.40\end{array}$ & $\begin{array}{l}37.90 \\
77.40 \\
42.40\end{array}$ \\
\hline $\begin{array}{r}\text { gould } \\
0-1000 \\
1-2000 \\
2-3000\end{array}$ & $\begin{array}{l}0.00 \\
0.00 \\
0.00\end{array}$ & $\begin{array}{l}0.00 \\
0.00 \\
0.00\end{array}$ & $\begin{array}{l}0.00 \\
0.00 \\
0.00\end{array}$ & $\begin{array}{l}0.00 \\
0.00 \\
0.00\end{array}$ & $\begin{array}{l}.4 .30 \\
11.10 \\
24.80\end{array}$ & $\begin{array}{l}1.00 \\
3.40 \\
0.00\end{array}$ & $\begin{array}{l}1.20 \\
0.00 \\
0.00\end{array}$ & $\begin{array}{r}6.50 \\
14.50 \\
24.80\end{array}$ & $\begin{array}{r}4.30 \\
11.10 \\
24.80\end{array}$ & $\begin{array}{l}1.00 \\
3.40 \\
0.00\end{array}$ & $\begin{array}{l}1.20 \\
0.00 \\
0.00\end{array}$ & $\begin{array}{r}6.50 \\
14.50 \\
24.80\end{array}$ \\
\hline $\begin{aligned} & \text { hetena } \\
& 0-1000 \\
& 1-2000\end{aligned}$ & $\begin{array}{l}0.00 \\
0.00\end{array}$ & $\begin{array}{l}0.00 \\
0.00\end{array}$ & $\begin{array}{l}0.00 \\
0.00\end{array}$ & $\begin{array}{l}0.00 \\
0.00\end{array}$ & $\begin{array}{l}18.60 \\
26.40\end{array}$ & $\begin{array}{r}18.90 \\
8.80\end{array}$ & $\begin{array}{l}5.00 \\
3.90\end{array}$ & $\begin{array}{l}42.50 \\
39.10\end{array}$ & $\begin{array}{l}18.60 \\
26.40\end{array}$ & $\begin{array}{r}18.90 \\
8.80\end{array}$ & $\begin{array}{l}5.00 \\
3.90\end{array}$ & $\begin{array}{l}42.50 \\
39.10\end{array}$ \\
\hline $\begin{array}{c}\text { lower thompson } \\
0-1000 \\
1-2000\end{array}$ & $\begin{array}{l}0.00 \\
0.00\end{array}$ & $\begin{array}{l}0.00 \\
0.00\end{array}$ & $\begin{array}{l}0.00 \\
0.00\end{array}$ & $\begin{array}{l}0.00 \\
0.00\end{array}$ & $\begin{array}{l}3.40 \\
0.00\end{array}$ & $\begin{array}{r}17.60 \\
2.40\end{array}$ & $\begin{array}{l}9: 10 \\
1.90\end{array}$ & $\begin{array}{r}30.10 \\
4.30\end{array}$ & $\begin{array}{l}3.40 \\
0.00\end{array}$ & $\begin{array}{r}17.60 \\
2.40\end{array}$ & $\begin{array}{l}9.10 \\
1.90\end{array}$ & $\begin{array}{r}30.10 \\
4.30\end{array}$ \\
\hline $\begin{array}{r}\text { are in } \\
0-1000 \\
1-2000 \\
2-3000\end{array}$ & $\begin{array}{l}0.00 \\
0.00 \\
0.00\end{array}$ & $\begin{array}{l}1.20 \\
0.00 \\
0.00\end{array}$ & $\begin{array}{l}3.00 \\
0.00 \\
0.00\end{array}$ & $\begin{array}{l}4.20 \\
0.00 \\
0.00\end{array}$ & $\begin{array}{l}0.00 \\
0.00 \\
0.00\end{array}$ & $\begin{array}{l}0.00 \\
1.00 \\
0.70\end{array}$ & $\begin{array}{l}0.00 \\
2.70 \\
2.00\end{array}$ & $\begin{array}{l}0.00 \\
3.70 \\
2.70\end{array}$ & $\begin{array}{l}0.00 \\
0.00 \\
0.00\end{array}$ & $\begin{array}{l}1.20 \\
1.00 \\
0.70\end{array}$ & $\begin{array}{l}3.00 \\
2.70 \\
.2 .00\end{array}$ & $\begin{array}{l}4.20 \\
3.70 \\
2.70\end{array}$ \\
\hline $\begin{array}{r}\text { montevallo } \\
0-1000 \\
1-2000\end{array}$ & $\begin{array}{l}0.00 \\
0.00\end{array}$ & $\begin{array}{l}0.00 \\
0.00\end{array}$ & $\begin{array}{l}0.00 \\
0.00\end{array}$ & $\begin{array}{l}0.00 \\
0.00\end{array}$ & $\begin{array}{l}8.00 \\
0.00\end{array}$ & $\begin{array}{r}15.80 \\
4.80\end{array}$ & $\begin{array}{r}29.80 \\
3.20\end{array}$ & $\begin{array}{r}53.60 \\
8.00\end{array}$ & $\begin{array}{l}8.00 \\
0.00\end{array}$ & $\begin{array}{r}15.80 \\
4.80\end{array}$ & $\begin{array}{r}29.80 \\
3.20\end{array}$ & $\begin{array}{r}53.60 \\
8.00\end{array}$ \\
\hline $\begin{array}{r}\text { nunnal } 1 y \\
0-1000 \\
1-2000 \\
2-3000\end{array}$ & $\begin{array}{l}0.00 \\
0.00 \\
0.00\end{array}$ & $\begin{array}{l}0.00 \\
0.00 \\
0.00\end{array}$ & $\begin{array}{l}0.00 \\
0.00 \\
0.00\end{array}$ & $\begin{array}{l}0.00 \\
0.00 \\
0.00\end{array}$ & $\begin{array}{l}13.00 \\
25.30 \\
19.20\end{array}$ & $\begin{array}{l}6.30 \\
6.00 \\
2.10\end{array}$ & $\begin{array}{l}1.40 \\
1.80 \\
0.00\end{array}$ & $\begin{array}{l}20.70 \\
33.10 \\
21.30\end{array}$ & $\begin{array}{l}13.00 \\
25.30 \\
19.20\end{array}$ & $\begin{array}{l}6.30 \\
6.00 \\
2.10\end{array}$ & $\begin{array}{l}1.40 \\
1.80 \\
0.00\end{array}$ & $\begin{array}{l}20.70 \\
33.10 \\
21.30\end{array}$ \\
\hline pump $0-1000$ & 0.00 & 0.00 & 0.00 & 0.00 & 5.10 & 0.00 & 0.00 & 3.10 & 5.10 & 0.00 & 0.00 & 5.10 \\
\hline $\begin{array}{c}\text { UDper thomps on } \\
0-1000 \\
1-2000\end{array}$ & $\begin{array}{l}0.00 \\
0.00\end{array}$ & $\begin{array}{l}0.00 \\
0.00\end{array}$ & $\begin{array}{l}0.00 \\
0.00\end{array}$ & $\begin{array}{l}0.00 \\
0.00\end{array}$ & $\begin{array}{r}6.50 \\
11.40\end{array}$ & $\begin{array}{l}7.10 \\
6.20\end{array}$ & $\begin{array}{l}2.00 \\
0.00\end{array}$ & $\begin{array}{l}15.60 \\
17.60\end{array}$ & $\begin{array}{r}6.50 \\
11.40\end{array}$ & $\begin{array}{l}7.10 \\
6.20\end{array}$ & $\begin{array}{l}2.00 \\
0.00\end{array}$ & $\begin{array}{l}15.60 \\
17.60\end{array}$ \\
\hline $\begin{array}{r}\text { wadsworth } \\
0-1000 \\
1-2000 \\
2-3000\end{array}$ & $\begin{array}{l}0.00 \\
0.00 \\
0.00\end{array}$ & $\begin{array}{l}0.00 \\
0.00 \\
0.00\end{array}$ & $\begin{array}{l}0.00 \\
0.00 \\
0.00\end{array}$ & $\begin{array}{l}0.00 \\
0.00 \\
0.00\end{array}$ & $\begin{array}{l}4.30 \\
9.00 \\
5.20\end{array}$ & $\begin{array}{l}7.20 \\
5.20 \\
2.80\end{array}$ & $\begin{array}{l}1.90 \\
0.40 \\
0.00\end{array}$ & $\begin{array}{r}13.40 \\
14.60 \\
8.00\end{array}$ & $\begin{array}{l}4.30 \\
9.00 \\
5.20\end{array}$ & $\begin{array}{l}7.20 \\
5.20 \\
2.80\end{array}$ & $\begin{array}{l}1.90 \\
0.40 \\
0.00\end{array}$ & $\begin{array}{r}13.60 \\
16.60 \\
8.00\end{array}$ \\
\hline $\begin{array}{r}\text { youngblood } \\
0-1000 \\
1-2000 \\
2-3000\end{array}$ & $\begin{array}{l}0.00 \\
0.00 \\
0.00\end{array}$ & $\begin{array}{l}0.00 \\
0.00 \\
0.00\end{array}$ & $\begin{array}{r}0.00 \\
0.00 \\
0.00\end{array}$ & 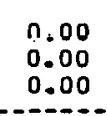 & $\begin{array}{r}8.30 \\
41.60 \\
13.00\end{array}$ & $\begin{array}{l}10.90 \\
27.90 \\
10.40\end{array}$ & $\begin{array}{l}1.40 \\
6.00 \\
0.00\end{array}$ & $\begin{array}{r}20.80 \\
73.50 \\
23.40\end{array}$ & $\begin{array}{r}8.50 \\
49.60 \\
13.00 \\
\end{array}$ & $\begin{array}{l}10.90 \\
27.90 \\
10.60\end{array}$ & $\begin{array}{l}1.60 \\
4.00 \\
0.00\end{array}$ & $\begin{array}{l}20.80 \\
73.50 \\
23.40\end{array}$ \\
\hline county total & 0.00 & 1.20 & 3.00 & 4.20 & 383.40 & 366.40 & 115.40 & 865.20 & 383.40 & 367.60 & 118.40 & 869.40 \\
\hline & & & & & & & & & & & & \\
\hline $\begin{array}{c}\text { broken arrow } \\
0-1000 \\
\text { clark }\end{array}$ & 1.00 & 0.80 & 0.00 & 1.80 & 0.00 & 0.00 & 0.00 & 0.00 & 1.00 & 0.80 & 0.00 & 1.80 \\
\hline $0-1000$ & 0.00 & 0.00 & 0.00 & 0.00 & 5.80 & 0.00 & 0.00 & 5.80 & 5.80 & 0.00 & 0.00 & 5.80 \\
\hline $\begin{array}{c}\text { coat city } \\
0-1000 \\
\text { fairvies }\end{array}$ & 0.60 & 0.20 & 0.00 & 0.80 & 0.00 & 0.00 & 0.50 & 0.50 & 0.60 & 0.20 & 0.50 & 1.30 \\
\hline $\begin{array}{l}0-1000 \\
1-2000\end{array}$ & $\begin{array}{r}13.50 \\
0.00\end{array}$ & $\begin{array}{l}0.60 \\
1.00\end{array}$ & $\begin{array}{l}0.00 \\
0.00\end{array}$ & $\begin{array}{r}14.10 \\
1.00\end{array}$ & $\begin{array}{l}1.90 \\
1.60\end{array}$ & $\begin{array}{l}0.00 \\
0.00\end{array}$ & $\begin{array}{l}0.00 \\
0.00\end{array}$ & $\begin{array}{l}1.90 \\
1.60\end{array}$ & $\begin{array}{r}15.40 \\
1.60\end{array}$ & $\begin{array}{l}0.60 \\
1.00\end{array}$ & $\begin{array}{l}0.00 \\
0.0 n\end{array}$ & $\begin{array}{r}16.00 \\
2.000\end{array}$ \\
\hline $0-1000$ & 1.70 & 0.00 & 0.00 & 1,70 & 0.00 & $0.00 \hat{~}$ & 0.00 & 0.00 & 1.70 & 0.00 & 0.00 & 1.70 \\
\hline
\end{tabular}




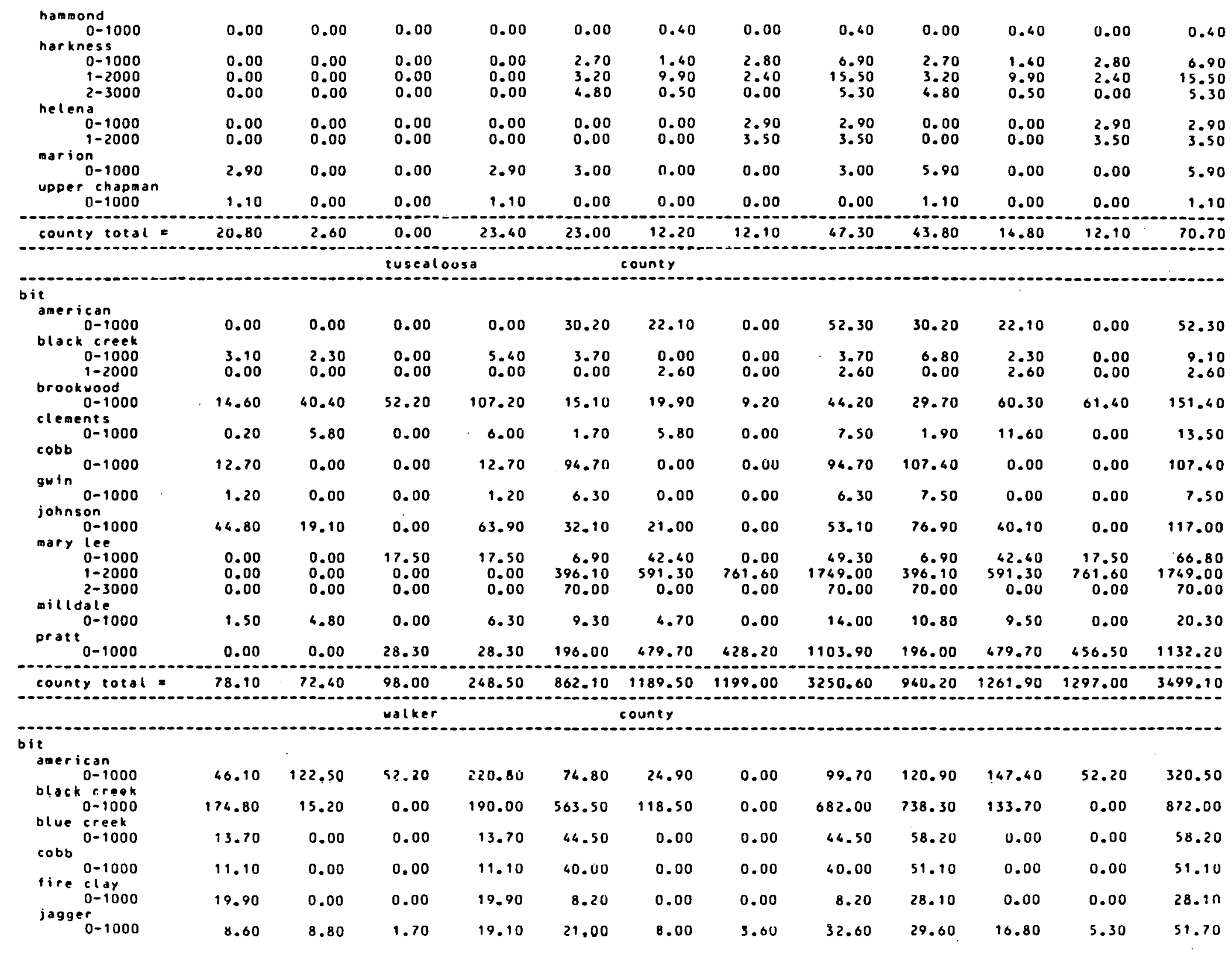




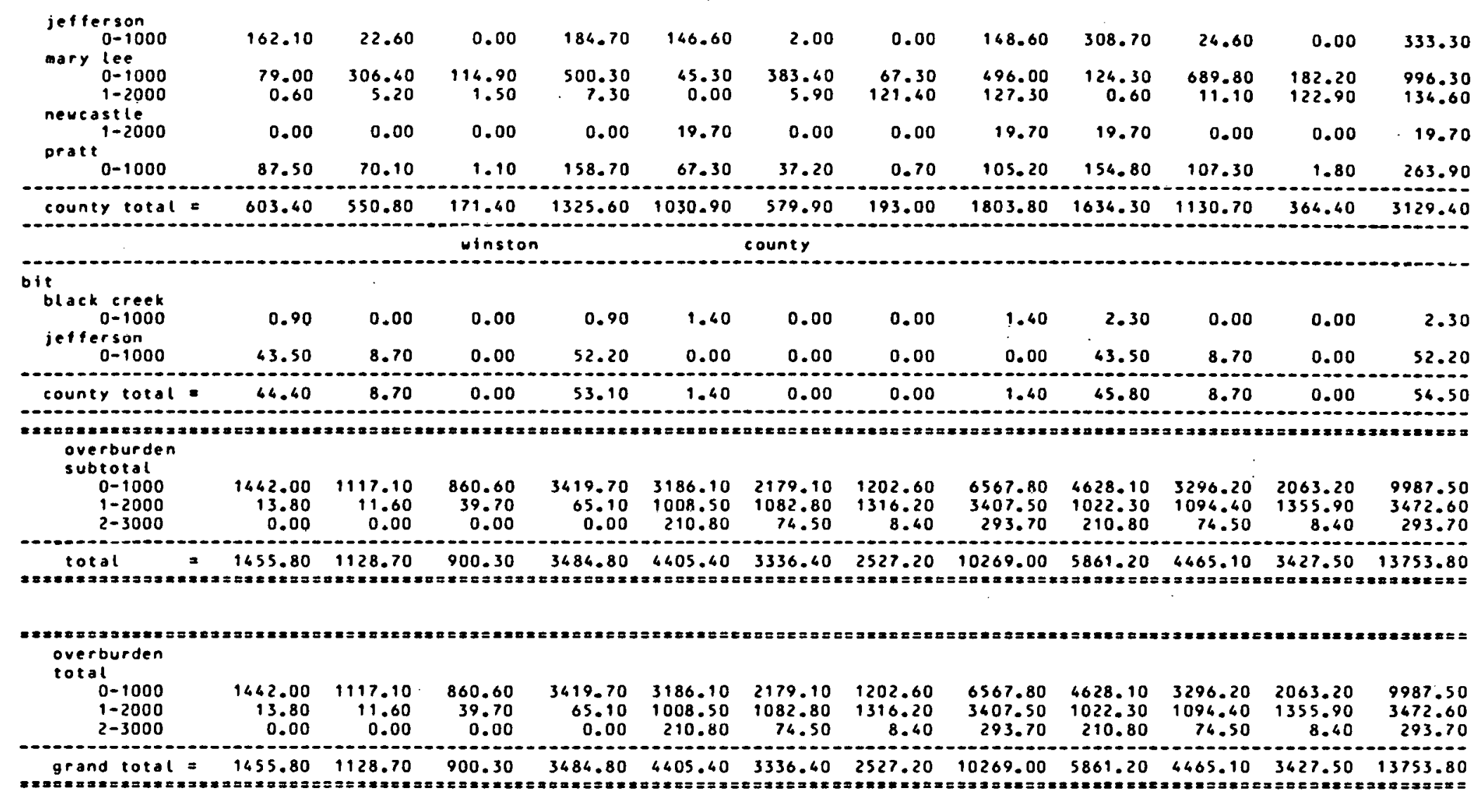

total tonnage of tdentified coal resources in alabama

13753.80

resource figures taken from:

$\begin{array}{lll}\text { source } & \text { year } & \text { base year of resource } \\ \text { usgs bull } 1182 \mathrm{~b} & 1964 & 1958\end{array}$


average analyses of coal in alabama

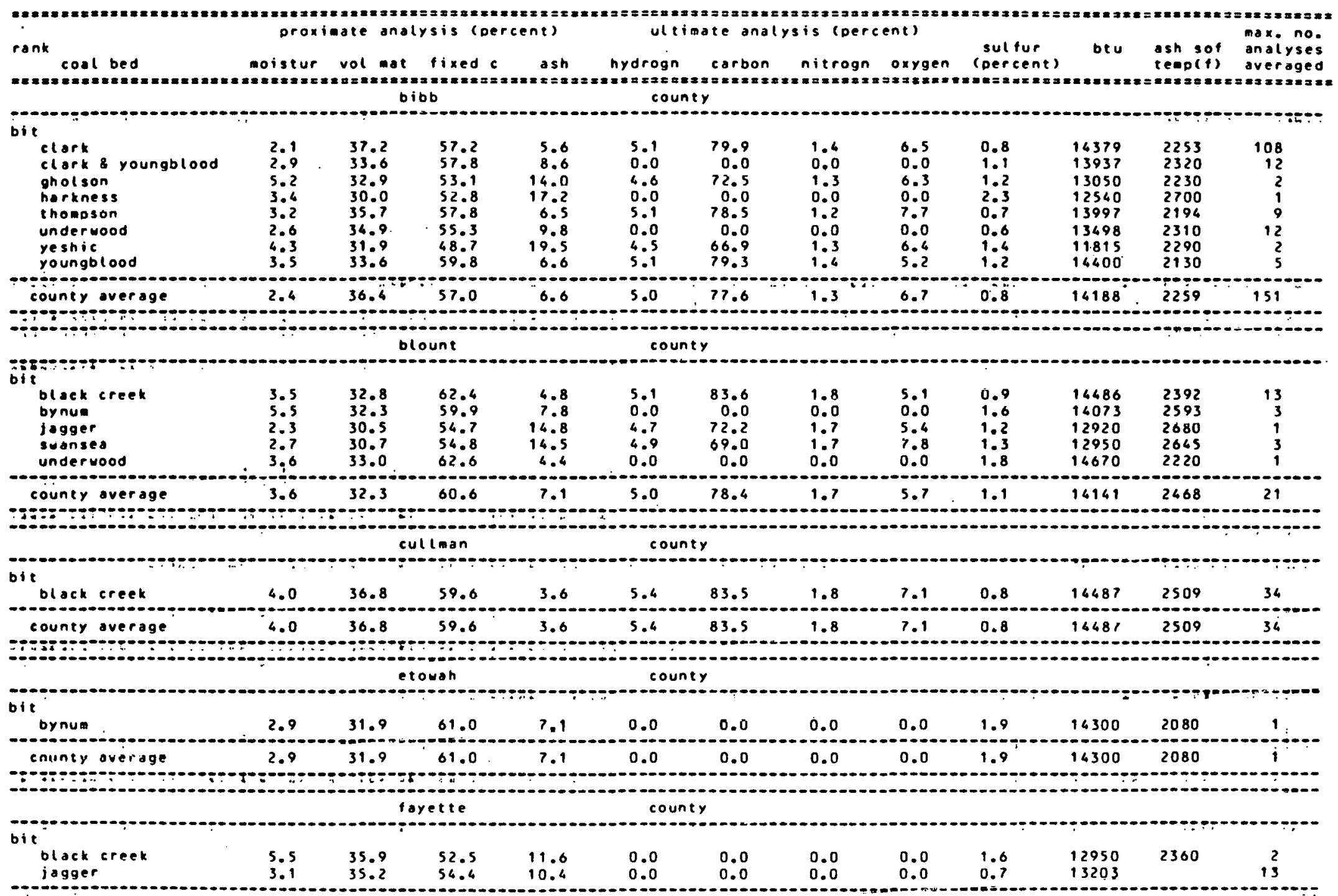




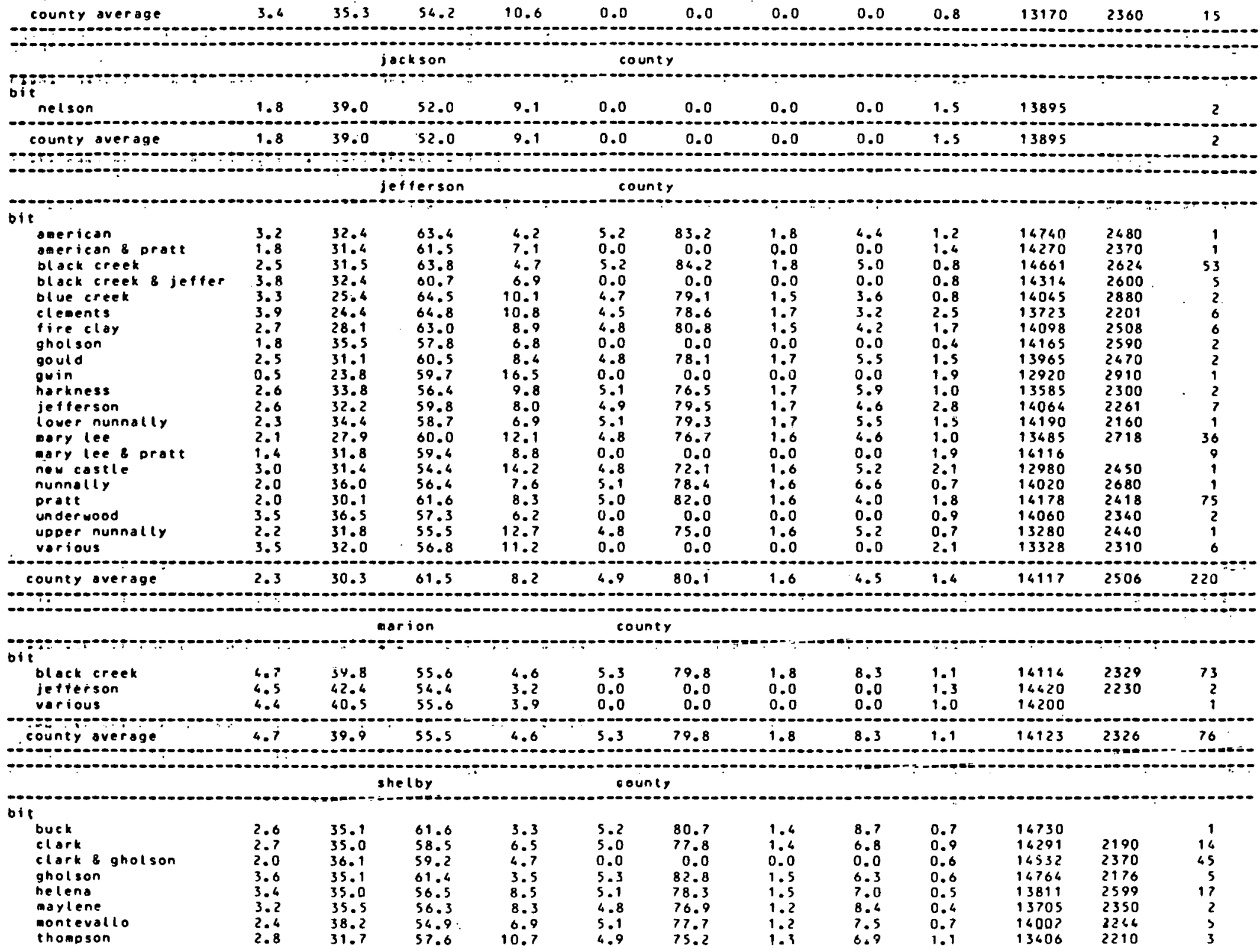




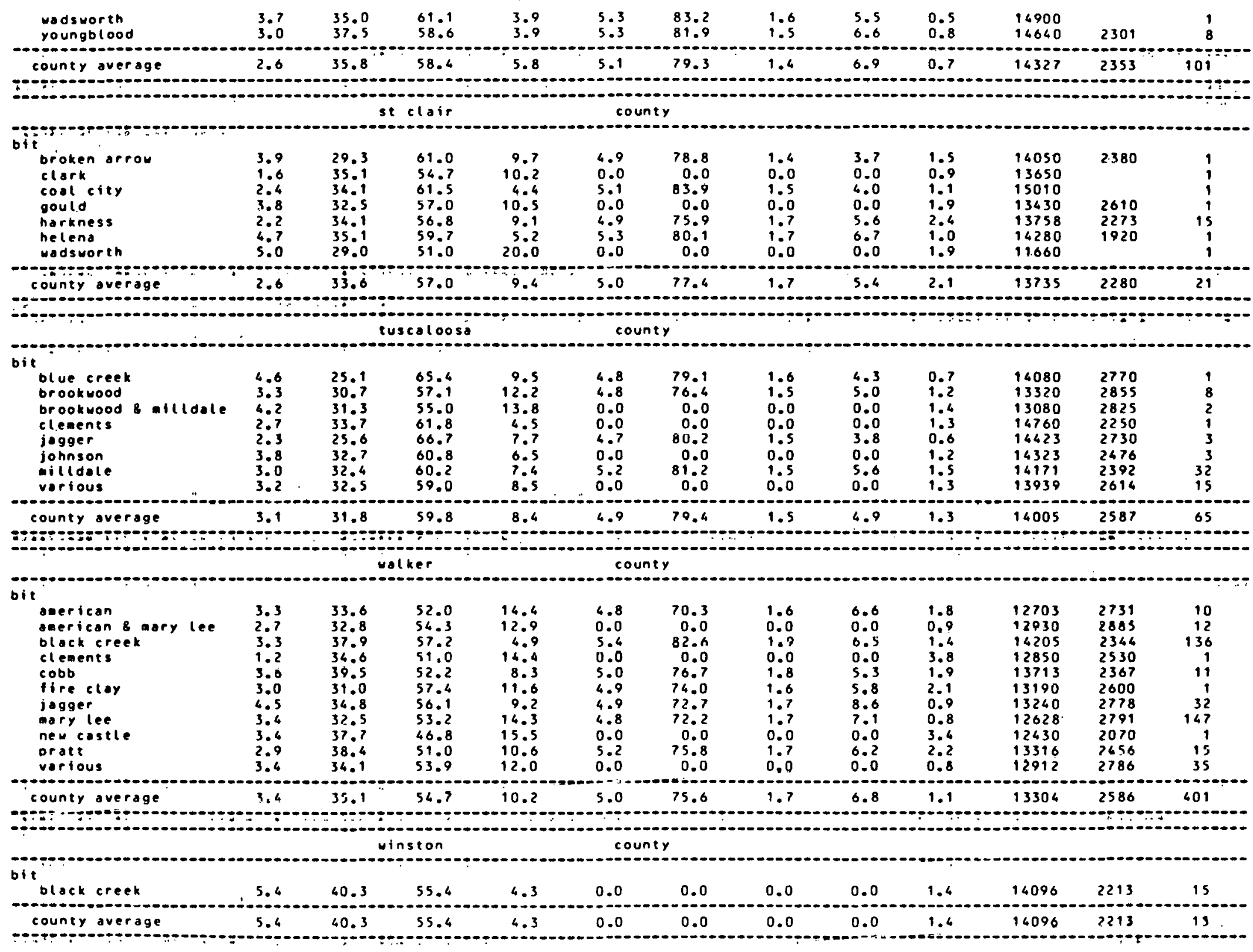




\begin{tabular}{|c|c|c|c|}
\hline \multicolumn{2}{|r|}{ county } & \multicolumn{2}{|r|}{ bed } \\
\hline$a$ l abama & bibb & harkne & ss \\
\hline al acama & blount & swanse & \\
\hline al abama & olount & swanse & \\
\hline al 3030 A & bl nunt & black & creek \\
\hline al doamd & olount & jaguer & \\
\hline al abama & blount & nynuin & \\
\hline al abama & blount & black & creek \\
\hline al acama & blount & black & crepl \\
\hline al abama & olnunt & black & creek \\
\hline ol aban & cullman & black & creak \\
\hline al abama & cullman & black & creek \\
\hline al a oama & cultiman & black & creek \\
\hline al abama & culliman & olack & croek \\
\hline al abama & cutiman & black & creek \\
\hline$a \log a \operatorname{ar}$ & cullman & black & creek \\
\hline al abant & culliman & black & creek \\
\hline al abama & cull man & black & creek \\
\hline al abama & cullman & black & creek \\
\hline al abama & cullman & black & creek \\
\hline a & cullman & black & creek \\
\hline al ahamd & cullman & olack & creek \\
\hline al abama & culiman & black & creek \\
\hline al abama & etnwah & bynum & \\
\hline al abama & jefferson & undery & Innt \\
\hline al abama & jpffercon & variou & \\
\hline 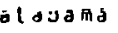 & jefferson & variou & \\
\hline al aband & jefferson & jefter & $\operatorname{sen}$ \\
\hline$a($ a & jefferson & jeffer & son \\
\hline al abama & jefferson & black & creek \\
\hline al abama & jefferson & black & creek \\
\hline al abama & jefterson & mary 1 & lee \\
\hline al abama & jeffersnn & oratt & \\
\hline alabana & jefterson & olack & creek \\
\hline al abama & jefterson & variou & \\
\hline al avama & jefferson & variou & \\
\hline al abana a & jefferson & black & creek \\
\hline al abama & jeftersan & fire $c$ & $=\operatorname{lay}$ \\
\hline al abaina & jefferson & block & creek \\
\hline al abo & jelterson & mary 1 & ee \\
\hline a l a bama & jefferson & black & creek \\
\hline al abama & jefferson & clenen & its \\
\hline$a l a b a m a$ & jeffersan & clemen & nes \\
\hline al abamo & jefferson & clenen & nes \\
\hline alabamt & iefterson & clemen & nes \\
\hline al abama & jefferson & clemen & nes \\
\hline al a a a a a & jefferson & $c l e m e n$ & $n t s$ \\
\hline al abam. & ieffercon & gwin & \\
\hline ol L Gama & marion & black & creek \\
\hline$a(a b \rightarrow n a$ & marion & $h l a c k$ & creek \\
\hline$a($ a b a mu & marion & Dlack & creek \\
\hline
\end{tabular}

\begin{tabular}{|c|c|c|c|c|c|}
\hline \multicolumn{2}{|c|}{ moistur volr } & \multirow{2}{*}{\multicolumn{2}{|c|}{ fixedc ash }} & \multicolumn{2}{|c|}{ sulf } \\
\hline 3.4 & $3 n . n$ & & & 2.3 & 12540 \\
\hline 2.6 & $31 . ?$ & 54.1 & & 1.2 & 12880 \\
\hline $2 . ?$ & $3 ? .2$ & 52.3 & 15.2 & 1.4 & 17780 \\
\hline 2.6 & 37.6 & $6 n .1$ & 7.3 & 0.8 & 14240 \\
\hline 2.3 & 30.5 & 54.7 & 14.8 & 1.2 & 12920 \\
\hline 8.1 & 31.1 & $58 . ?$ & 10.7 & 1.3 & 13540 \\
\hline 4.0 & 33.9 & 62.1 & 4.0 & 1.1 & 16660 \\
\hline 3.8 & 37.8 & 61.8 & 5.4 & 1.1 & 16480 \\
\hline 3.1 & $3 ? .8$ & 64.0 & 3.? & 0.7 & 16940 \\
\hline 4.8 & 34.9 & 56.5 & 3.6 & 1.1 & 13600 \\
\hline 3.2 & 35.6 & 59.0 & 5.6 & 0.9 & 14190 \\
\hline 3.2 & 40.2 & 55.1 & 4.7 & 1.5 & 16260 \\
\hline 3.3 & 40.2 & 55.5 & 4.3 & 1.3 & 14270 \\
\hline $3 . ?$ & 30.0 & 57.5 & 3.5 & 0.9 & 14490 \\
\hline 4.0 & 36.9 & 58.9 & 4.? & 0.7 & 14390 \\
\hline 3.7 & 38.2 & 50.3 & 2.5 & 0.7 & 14670 \\
\hline 3.1 & 39.7 & 58.7 & 1.6 & 0.7 & 14920 \\
\hline 5.7 & 33.6 & 58.2 & 8.2 & 0.8 & 13850 \\
\hline 6.0 & 38.2 & 60.0 & 1.8 & 0.7 & 14800 \\
\hline 5.4 & 34.9 & 00.7 & 4.4 & 0.7 & 14190 \\
\hline$x .0$ & 33.1 & $6 n .9$ & 6.0 & 0.6 & 14020 \\
\hline 9.7 & 32.1 & 63.4 & 3.5 & 0.8 & 14160 \\
\hline$? .9$ & 31.9 & 61.0 & 7.7 & 1.9 & 14300 \\
\hline 4.2 & 34.1 & 57.7 & 3.2 & 0.9 & 13680 \\
\hline 1.9 & 31.3 & 54.8 & 13.9 & 3.7 & 13080 \\
\hline 1.8 & 31.8 & 56.3 & 11.9 & 2.7 & 13420 \\
\hline 6.5 & 31.3 & 56.3 & 12.4 & 3.0 & 13260 \\
\hline 2.6 & 32.4 & 56.8 & 10.8 & 3.0 & 13500 \\
\hline 3.0 & 33.5 & 64.3 & 2.2 & 0.8 & 15090 \\
\hline $5 . ?$ & $? 3.8$ & 60.9 & 6.3 & 0.8 & 14360 \\
\hline 0.8 & 25.7 & 58.5 & 15.8 & 0.7 & 12890 \\
\hline$? .6$ & 28.4 & 61.4 & 10.2 & 2.3 & 13950 \\
\hline 2.8 & $? 0.8$ & 54.5 & 15.7 & 1.2 & 12790 \\
\hline 4.6 & 31.6 & 59.5 & 8.9 & 1.5 & 13600 \\
\hline 5.6 & 34.7 & 58.5 & 6.8 & 1.1 & 13730 \\
\hline 2.7 & 35.8 & 61.6 & $? .6$ & 0.6 & 93040 \\
\hline \}, ? & 39.3 & 57.6 & 11.1 & 2.1 & 13460 \\
\hline 3.5 & $3 n . ?$ & 56.3 & 13.5 & 1.? & 131000 \\
\hline 0.8 & ?. & 57.0 & 16.0 & 1.1 & 12860 \\
\hline 4.6 & $3 ? .2$ & 65.0 & 2.8 & 0.5 & 15020 \\
\hline 4.7 & 73.6 & $6 ? .8$ & 13.6 & 3.8 & 13080 \\
\hline 4. & 25.1 & 66.4 & 8.5 & 1.7 & 14010 \\
\hline 3.? & $? 5 . ?$ & 67.9 & 6.9 & 1.6 & 14560 \\
\hline $3 . ?$ & 23.8 & $6 ? .7$ & 13.5 & 2.1 & 13210 \\
\hline 2.3 & $>3.9$ & $6 \therefore 8$ & 11.6 & 3.9 & 13820 \\
\hline 4.6 & 24.8 & 66.3 & 10.9 & 1.9 & 13660 \\
\hline 0.5 & 23.8 & 50.7 & 16.5 & 1.9 & $1 ? 920$ \\
\hline $0 . ?$ & 36.0 & 611.4 & 3.6 & 0.7 & 14190 \\
\hline & $\therefore$ & $5=$ & 12.9 & 1.3 & 12800 \\
\hline 3 & 35.5 & 54.7 & 0.8 & $1 . \Gamma$ & 13240 \\
\hline
\end{tabular}

\begin{tabular}{|c|c|}
\hline ngilo & isilo \\
\hline 76 & 8.0 \\
\hline 0 & 6.0 \\
\hline 54 & 5.0 \\
\hline 64 & 8.5 \\
\hline 55 & 7.0 \\
\hline 73 & 7.0 \\
\hline 59 & 8.5 \\
\hline 54 & 8.5 \\
\hline 64 & 9.0 \\
\hline 0 & 4.5 \\
\hline 0 & 4.5 \\
\hline$n$ & 4.0 \\
\hline 0 & 5.5 \\
\hline 0 & 5.0 \\
\hline 0 & 5.5 \\
\hline 0 & 5.5 \\
\hline 0 & 6.0 \\
\hline 50 & 7.0 \\
\hline 41 & 6.5 \\
\hline 0 & 1.0 \\
\hline 0 & 3.5 \\
\hline 0 & 1.5 \\
\hline 0 & 8.5 \\
\hline 56 & 6.5 \\
\hline 0 & 7.5 \\
\hline 0 & 7.5 \\
\hline 64 & 8.5 \\
\hline 55 & 8.5 \\
\hline 58 & 8.5 \\
\hline 59 & 8.5 \\
\hline 0 & 9.0 \\
\hline 85 & 7.0 \\
\hline 66 & 7.5 \\
\hline 0 & 8.0 \\
\hline 0 & 1.5 \\
\hline 54 & 8.0 \\
\hline 0 & 6.5 \\
\hline 0 & 7.5 \\
\hline 0 & 8.5 \\
\hline 63 & 9.0 \\
\hline . & 8.0 \\
\hline ** & 8.5 \\
\hline 96 & 9.0 \\
\hline 98 & 8.5 \\
\hline ** & 9.0 \\
\hline 96 & 9.0 \\
\hline 0 & 7.5 \\
\hline 41 & $? .0$ \\
\hline 41 & 3.0 \\
\hline 41 & 1.5 \\
\hline
\end{tabular}




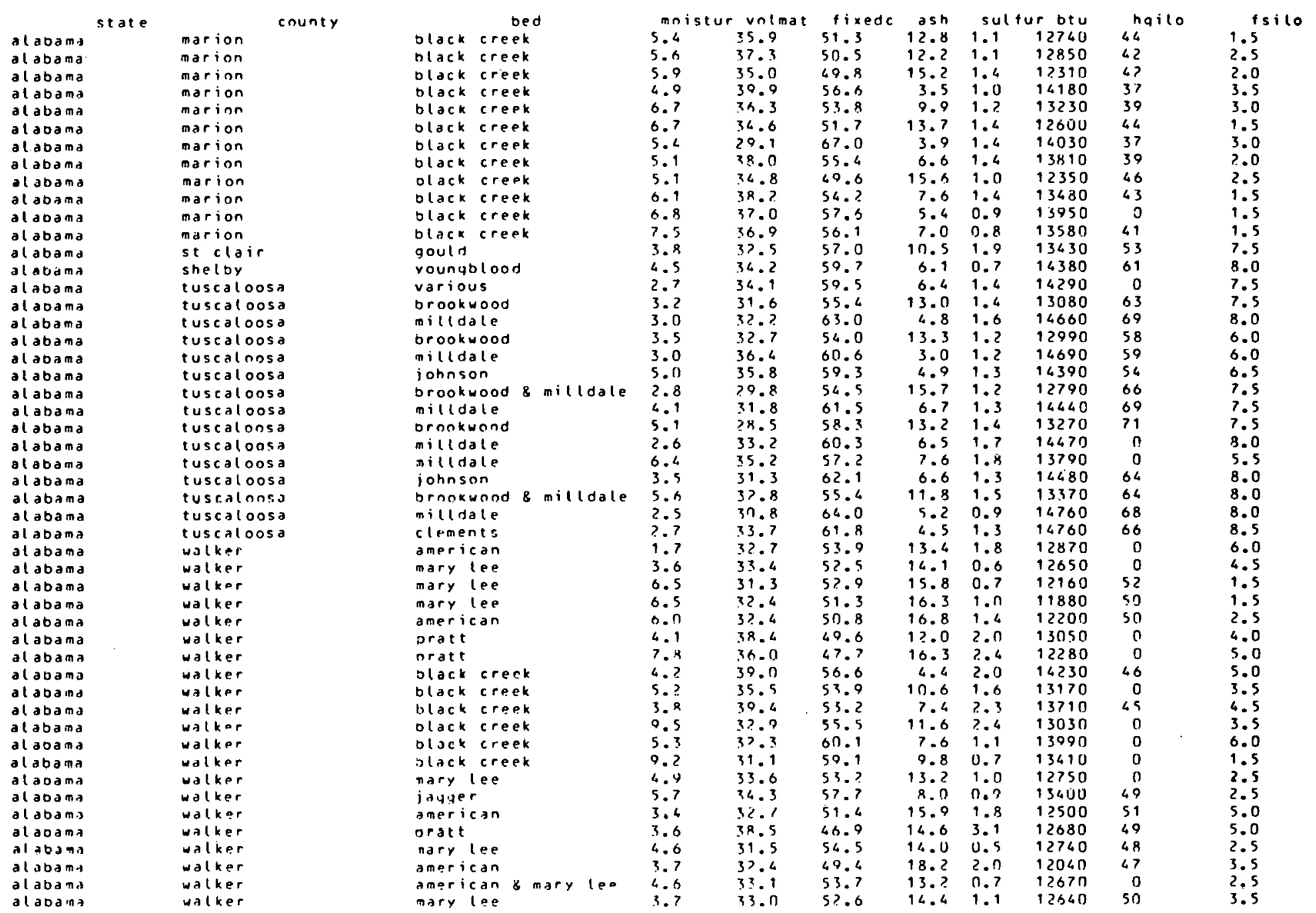




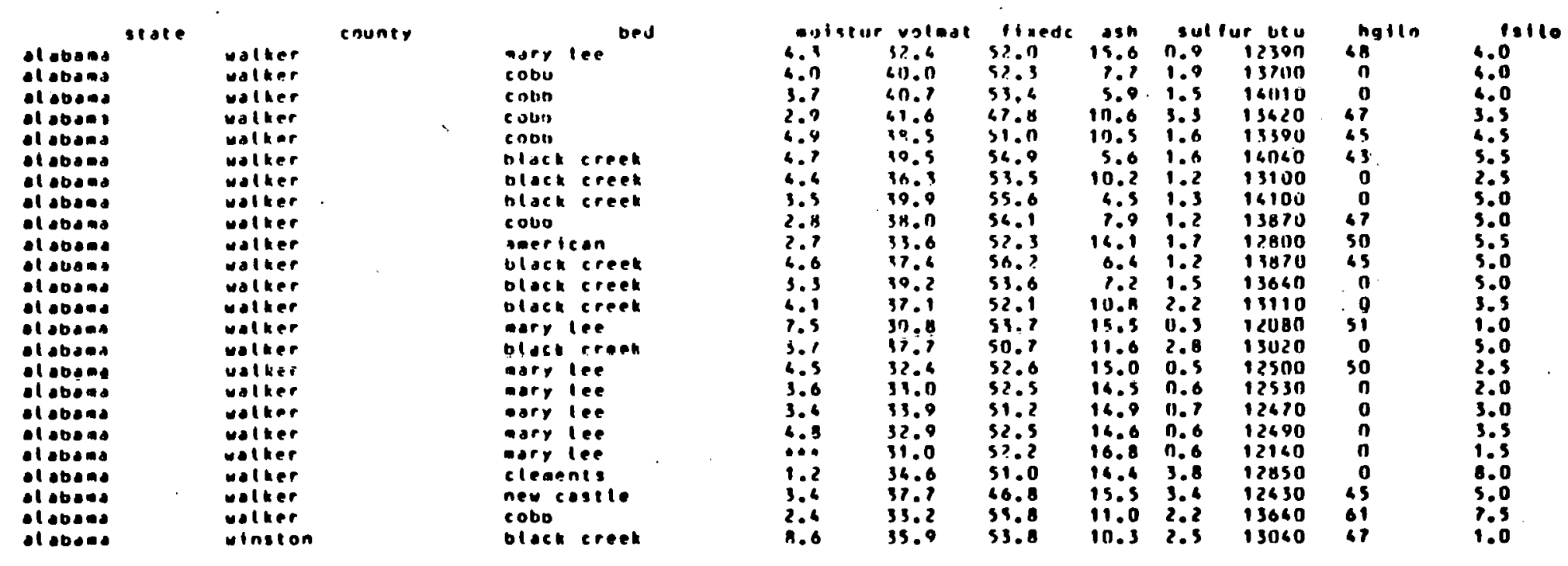



National Coal Resources Data System

U.S. Geological survey

identifled coal resources in arizona

(In aillions of short tons)

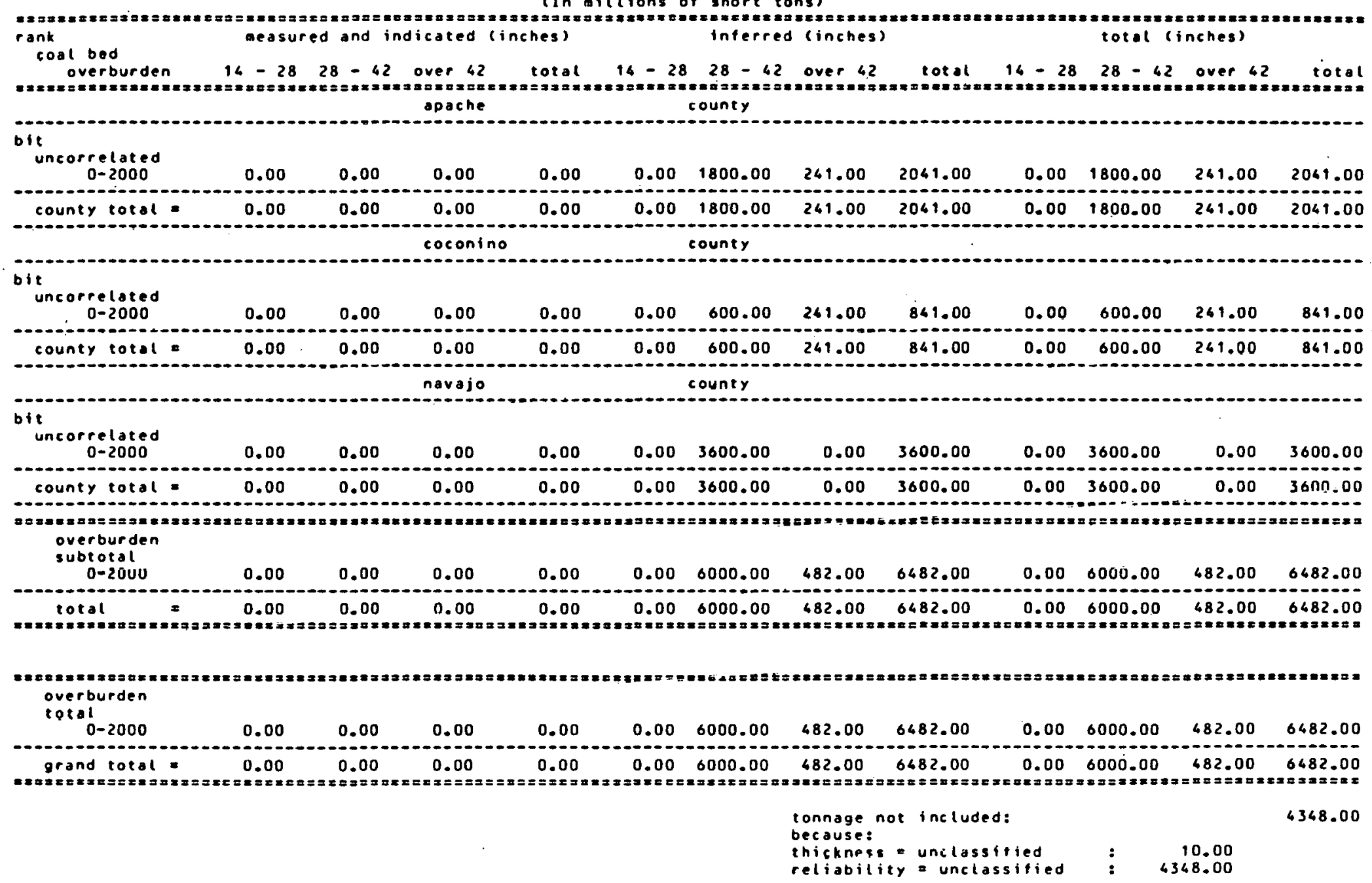


resource tigures taken from:

$\begin{array}{lll}\text { source } & \text { year } & \text { base year of resource } \\ \text { arizona be bull } 182 & 1970 & \text { original } \\ \text { arizona bo bull } 180 & 1969 & \text { original }\end{array}$

records containing unclassiffed thickness and/or reliability

state county

oriz navajo bed

uncorrelated

uncorrelated rank

bit base thickness overburden
year (inches or teet) (teet)

reliability $0-\frac{1000}{20}$

orig $>42$ in orig uncloss $\begin{array}{ll}0-2000 & \text { unclass } \\ \text { uncliass } & \text { unclass }\end{array}$ tonnage

4338.00

338.00
10.00 

National Coal Resources Data System

U.S. Geological survey

identified coal resources in arkansas

(In nillions of stiort tons)

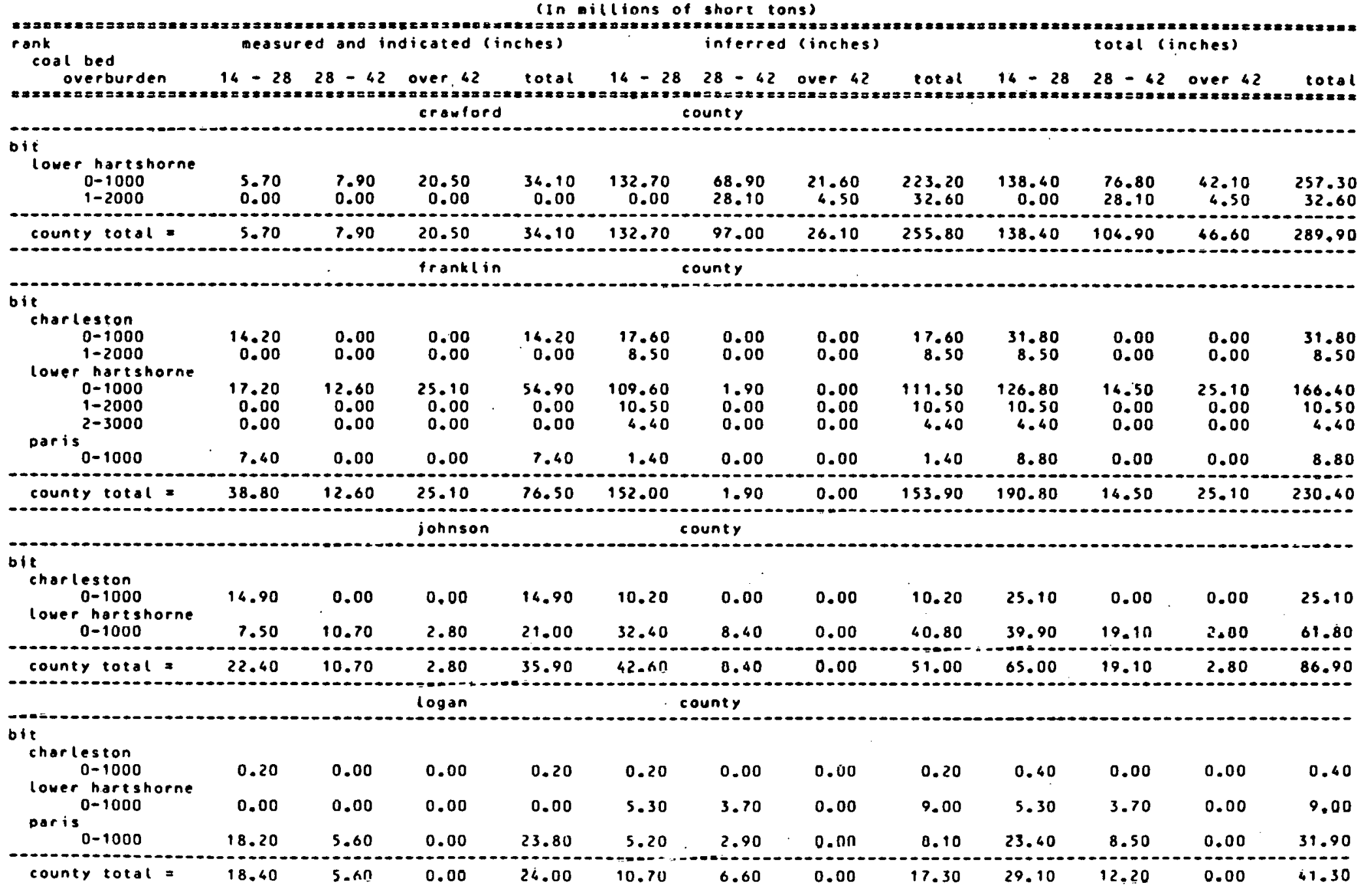


$3 \cot t$

county

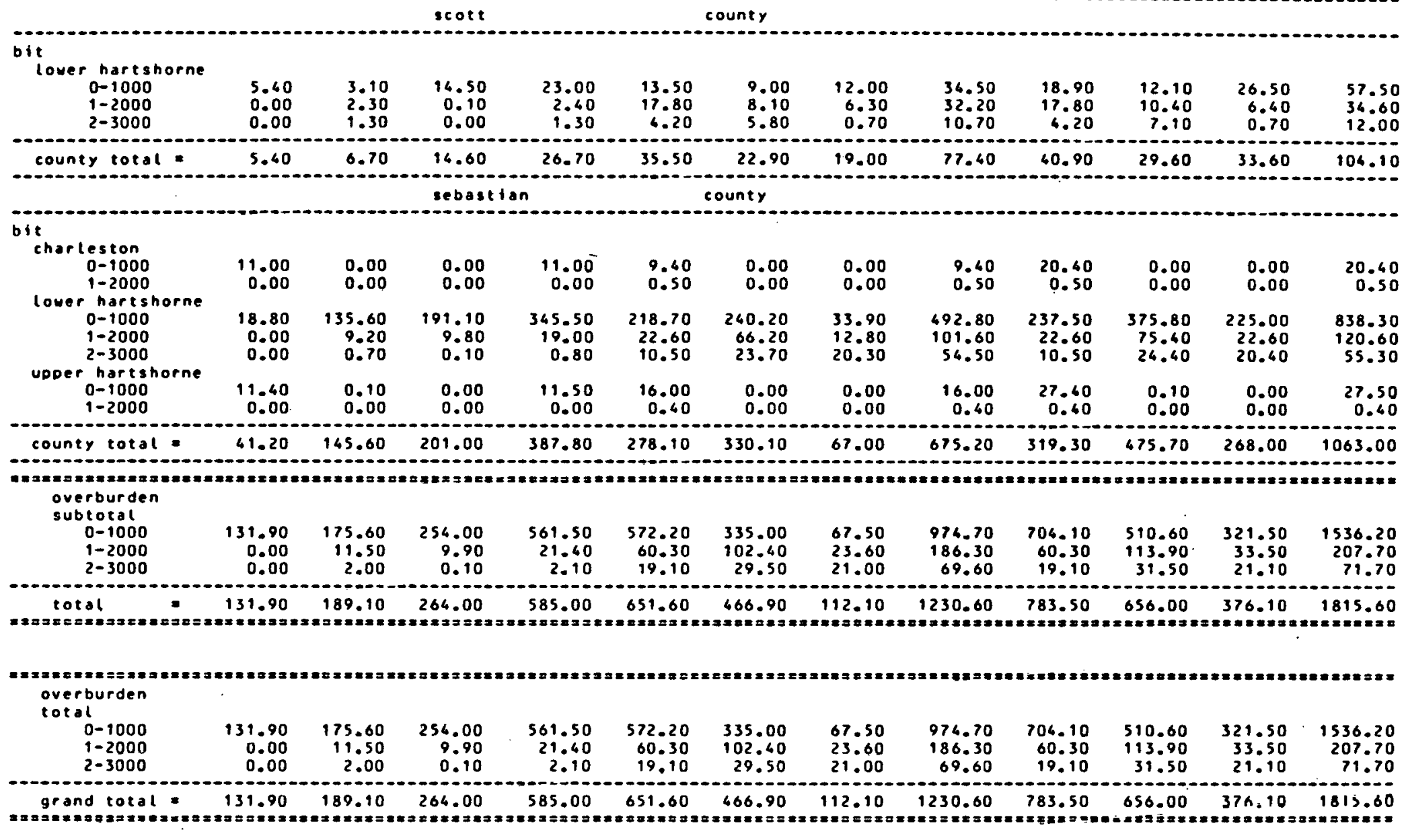

total tonnage of identified coal resources in arkansas

1815.60

resource figures taken from:

source

usgs bull 10720 year

1954 base year of resource

original 
average analyses of coat in arkansas

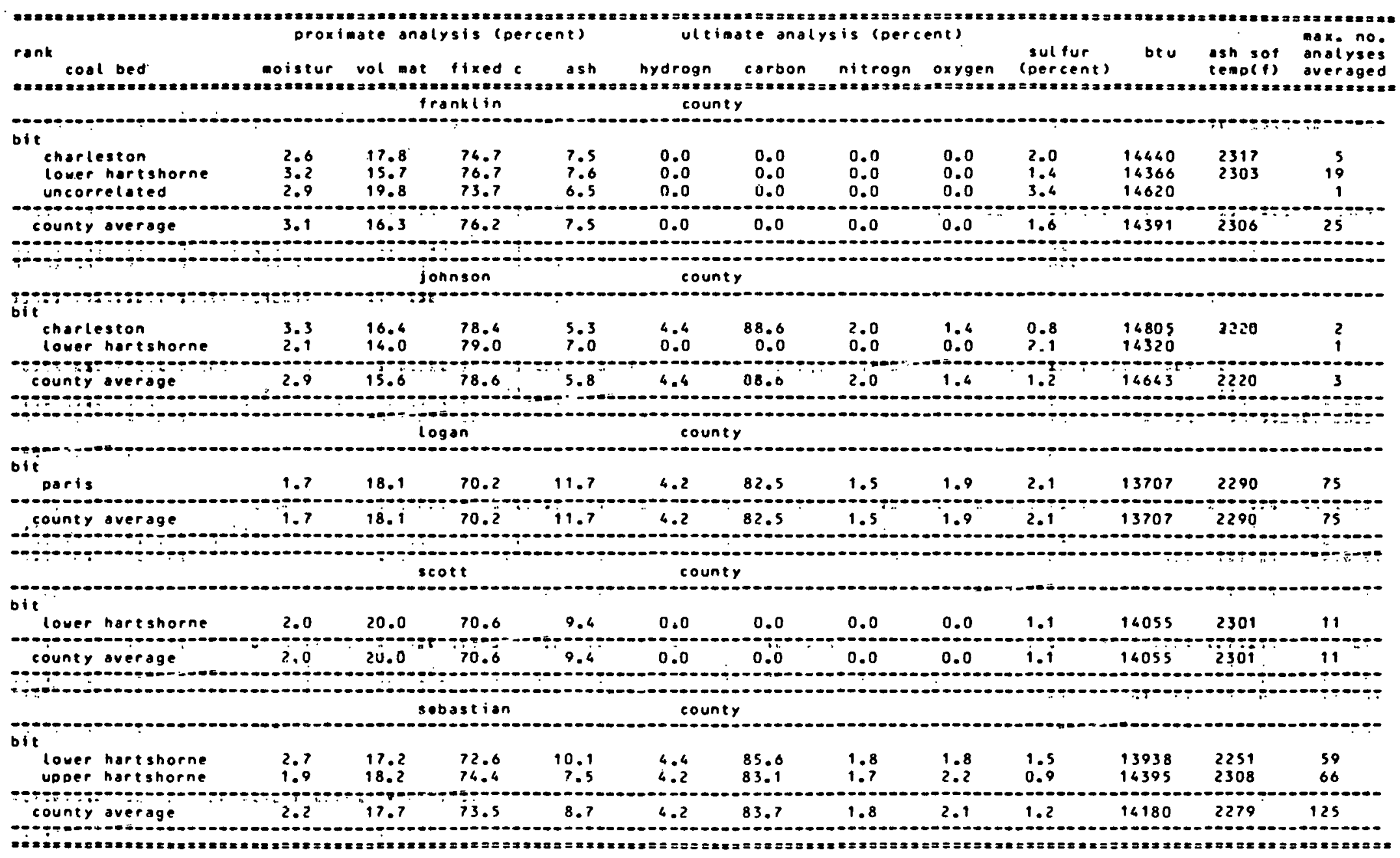


Arkansas

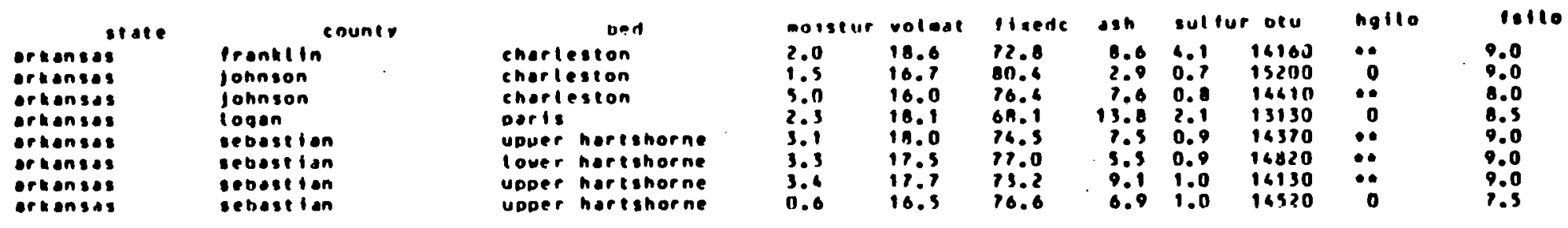




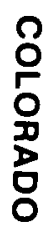


National Coal Resources oata system

U.S. Geological Survey

identified coal resources in colorado

(In millions of short tons)

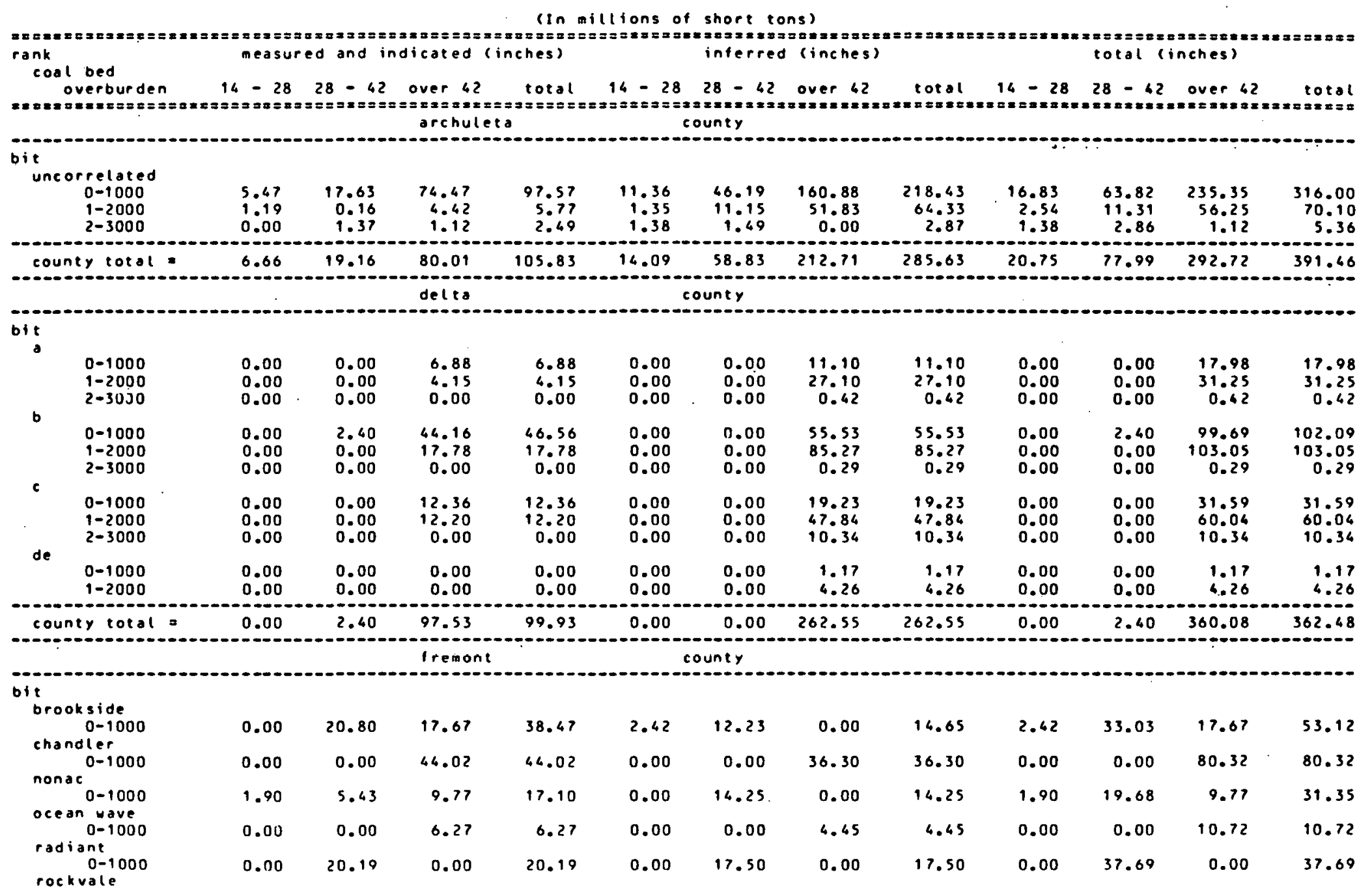




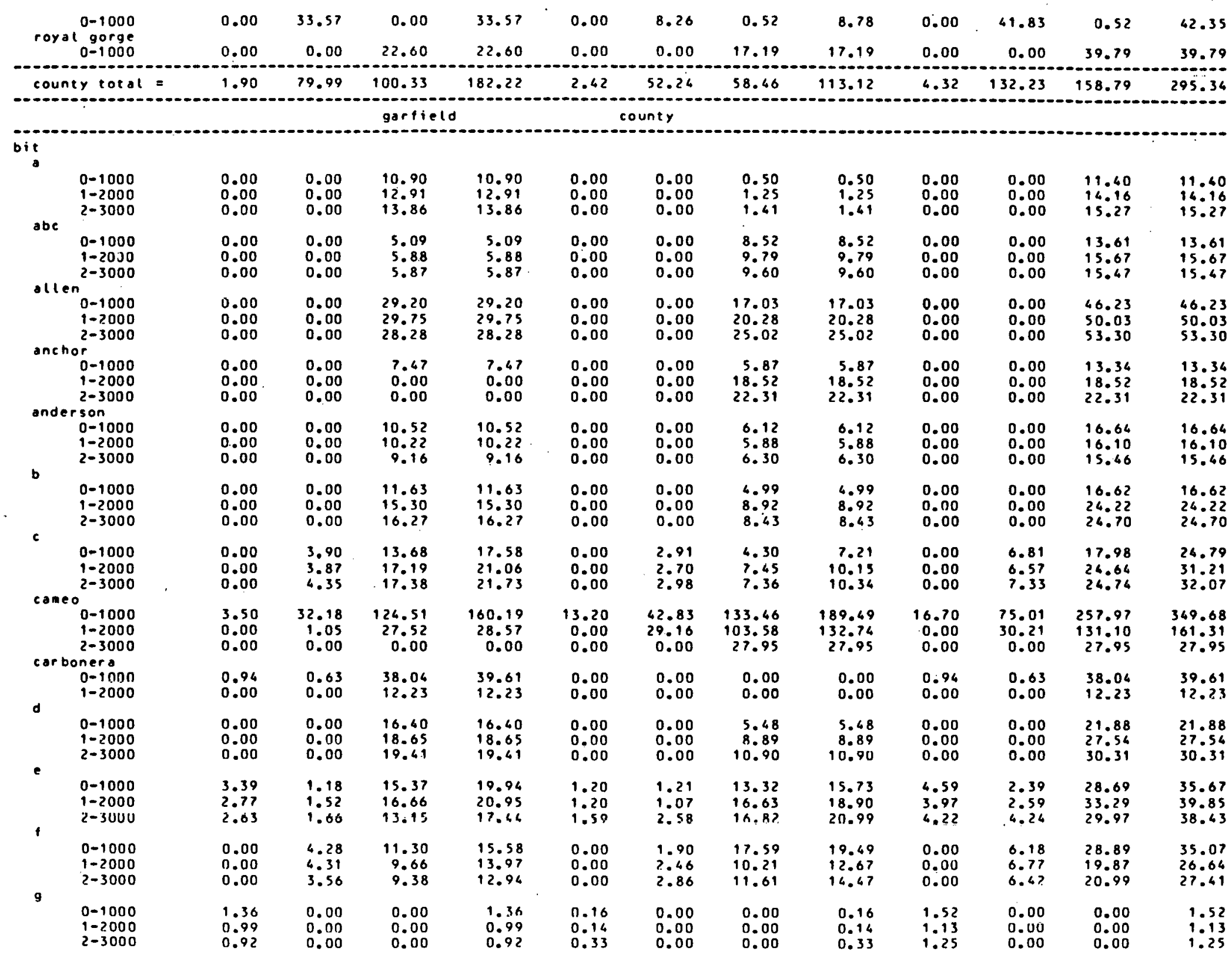




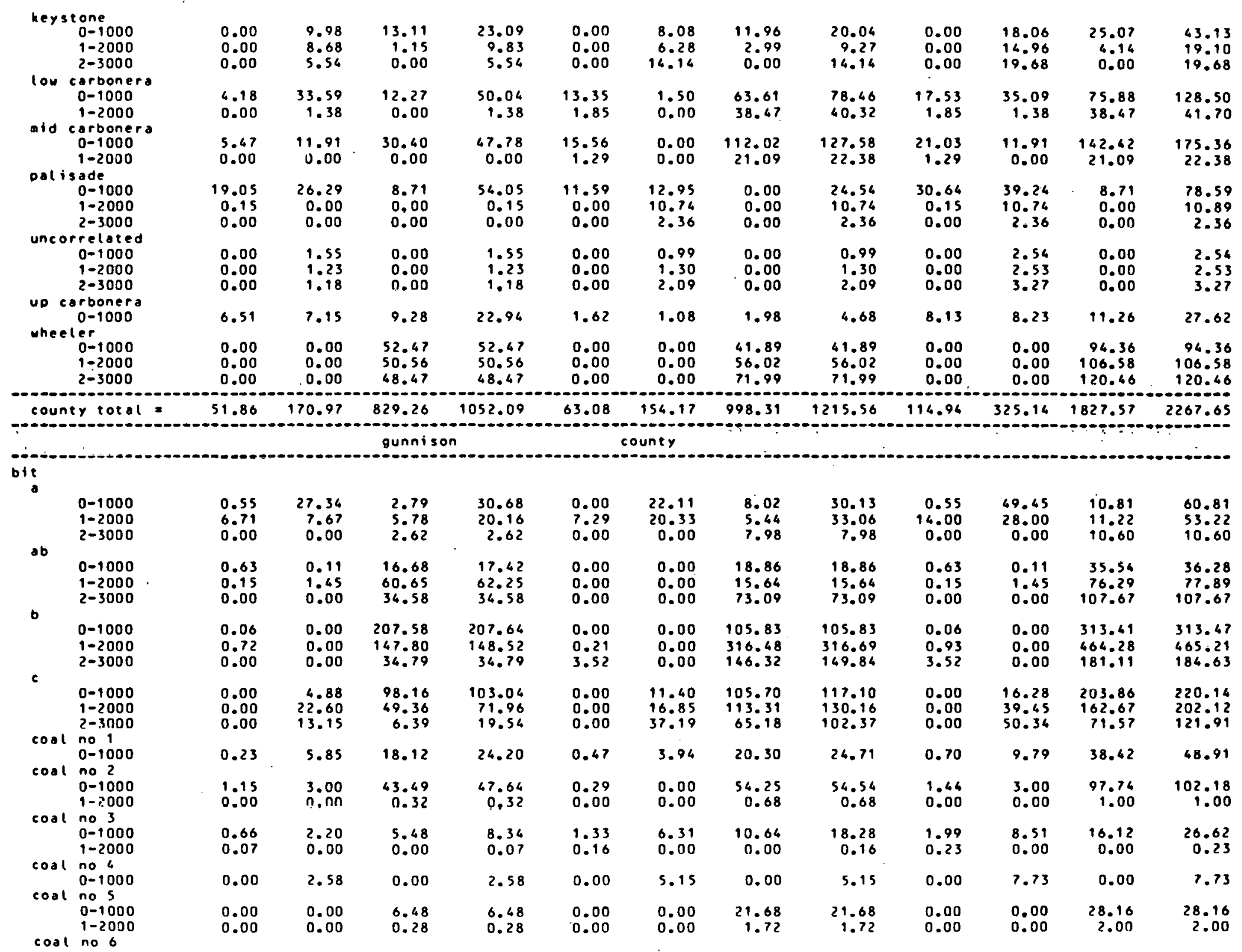




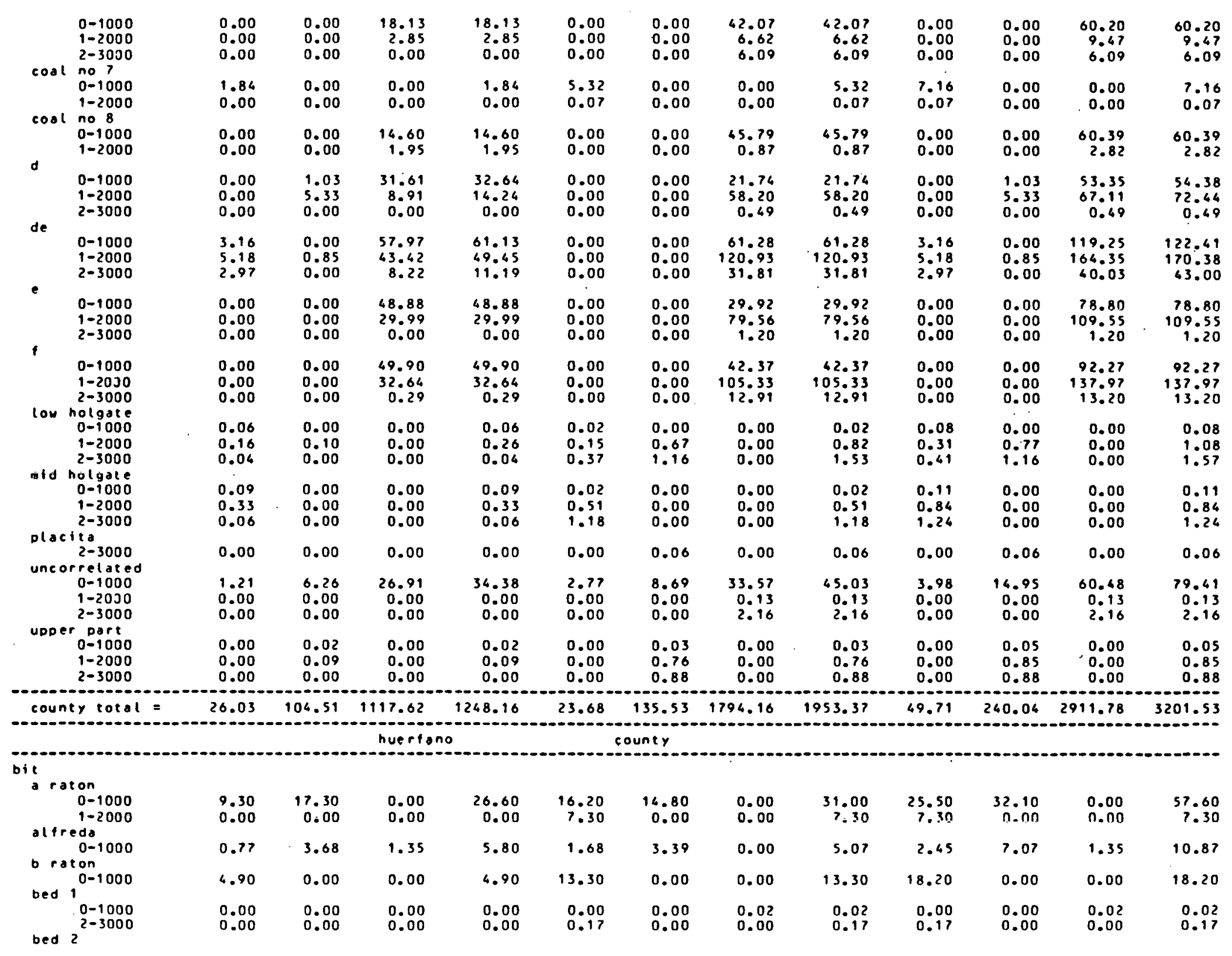




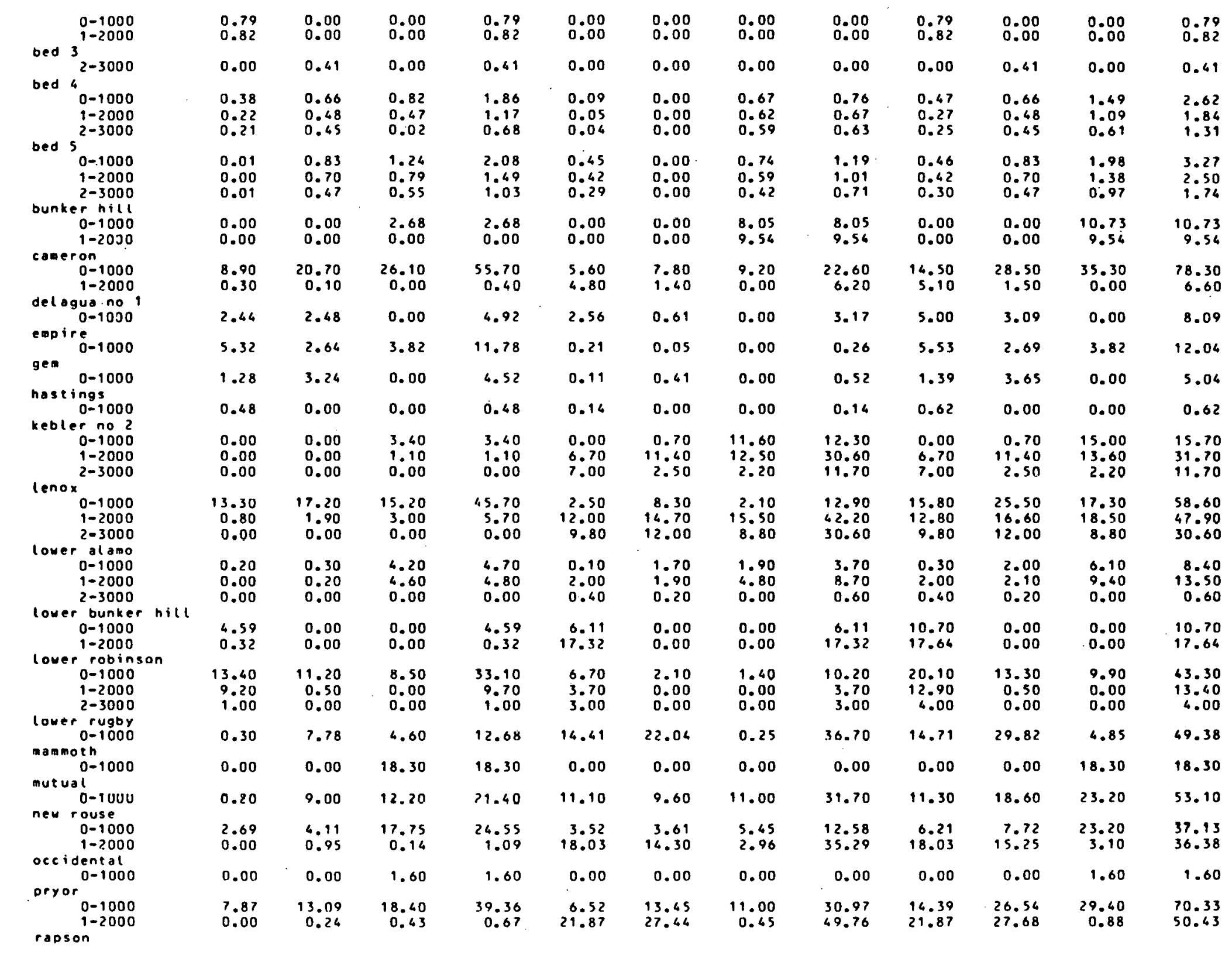




\begin{tabular}{|c|c|c|c|c|c|c|c|c|c|c|c|c|c|}
\hline & $\begin{array}{l}0-1000 \\
1-2000\end{array}$ & $\begin{array}{l}9.64 \\
0.00\end{array}$ & $\begin{array}{l}7.83 \\
0.63\end{array}$ & $\begin{array}{l}5.97 \\
0.68\end{array}$ & $\begin{array}{r}15.24 \\
1.31\end{array}$ & $\begin{array}{l}1.25 \\
3.13\end{array}$ & $\begin{array}{l}15.42 \\
48.46\end{array}$ & $\begin{array}{l}0.66 \\
1.93\end{array}$ & $\begin{array}{l}17.33 \\
53.52\end{array}$ & $\begin{array}{l}2.69 \\
3.13\end{array}$ & $\begin{array}{l}23.25 \\
49.09\end{array}$ & $\begin{array}{l}6.63 \\
2.61\end{array}$ & $\begin{array}{l}32.57 \\
54.83\end{array}$ \\
\hline rea & $\begin{array}{l}0-100.0 \\
1-2000 \\
2-3000\end{array}$ & $\begin{array}{l}8.20 \\
1.20 \\
0.00\end{array}$ & $\begin{array}{l}9.20 \\
0.50 \\
0.00\end{array}$ & $\begin{array}{l}0.00 \\
0.00 \\
0.00\end{array}$ & $\begin{array}{l}17.40 \\
9.70 \\
0.00\end{array}$ & $\begin{array}{l}11.10 \\
18.40 \\
10.50\end{array}$ & $\begin{array}{l}1.30 \\
3.70 \\
0.00\end{array}$ & $\begin{array}{l}0.00 \\
0.00 \\
0.00\end{array}$ & $\begin{array}{l}12.40 \\
22.10 \\
10.50\end{array}$ & $\begin{array}{l}19.30 \\
19.60 \\
10.50\end{array}$ & $\begin{array}{r}1.0 .50 \\
4.20 \\
0.00\end{array}$ & $\begin{array}{l}0.00 \\
0.00 \\
0.00\end{array}$ & $\begin{array}{l}29.80 \\
23.80 \\
10.50\end{array}$ \\
\hline $\begin{array}{l}\text { thoma } \\
\text { unc or }\end{array}$ & $\begin{array}{c}\text { as } \\
0-1000 \\
\text { rrelated }\end{array}$ & 0.46 & 1.29 & 0.00 & 1.75 & 0.86 & 0.00 & 0.00 & 0.86 & 1.32 & 1.29 & 0.00 & 2.61 \\
\hline & $\begin{array}{r}0-1000 \\
1-2000 \\
2-3000 \\
\text { ratamo }\end{array}$ & $\begin{array}{r}21.23 \\
1.03 \\
1.09\end{array}$ & $\begin{array}{l}4.78 \\
0.37 \\
0.76\end{array}$ & $\begin{array}{l}1.13 \\
0.39 \\
0.06\end{array}$ & $\begin{array}{r}27.14 \\
1.79 \\
1.91\end{array}$ & $\begin{array}{r}10.65 \\
2.74 \\
2.70\end{array}$ & $\begin{array}{l}0.58 \\
0.52 \\
0.50\end{array}$ & $\begin{array}{l}0.00 \\
0.00 \\
0.00\end{array}$ & $\begin{array}{r}11.23 \\
3.26 \\
3.20\end{array}$ & $\begin{array}{r}31.88 \\
3.77 \\
3.79\end{array}$ & $\begin{array}{l}5.36 \\
0.89 \\
1.26\end{array}$ & $\begin{array}{l}1.13 \\
0.39 \\
0.06\end{array}$ & $\begin{array}{r}38.37 \\
5.05 \\
5.11\end{array}$ \\
\hline & $\begin{array}{l}0-10 J 0 \\
1-2000\end{array}$ & $\begin{array}{l}0.10 \\
0.20\end{array}$ & $\begin{array}{l}0.60 \\
0.50\end{array}$ & $\begin{array}{l}2.20 \\
0.30\end{array}$ & $\begin{array}{l}2.90 \\
1.00\end{array}$ & $\begin{array}{l}0.30 \\
0.30\end{array}$ & $\begin{array}{l}0.30 \\
0.20\end{array}$ & $\begin{array}{l}0.00 \\
0.00\end{array}$ & $\begin{array}{l}0.60 \\
0.50\end{array}$ & $\begin{array}{l}0.40 \\
0.50\end{array}$ & $\begin{array}{l}0.90 \\
0.70\end{array}$ & $\begin{array}{l}2.20 \\
0.30\end{array}$ & $\begin{array}{l}3.50 \\
1.50\end{array}$ \\
\hline upper & $\begin{array}{l}\text { bunker } \\
0-1000 \\
1-2000 \\
\text { robinson }\end{array}$ & $\begin{array}{l}3.55 \\
0.00\end{array}$ & $\begin{array}{l}2.91 \\
7.10\end{array}$ & $\begin{array}{l}2.77 \\
0.00\end{array}$ & $\begin{array}{l}9.23 \\
7.10\end{array}$ & $\begin{array}{r}3.66 \\
20.06\end{array}$ & $\begin{array}{l}7.84 \\
? .89\end{array}$ & $\begin{array}{l}0.37 \\
0.00\end{array}$ & $\begin{array}{l}11.87 \\
22.95\end{array}$ & $\begin{array}{r}7.21 \\
20.06\end{array}$ & $\begin{array}{r}10.75 \\
9.99\end{array}$ & $\begin{array}{l}3.16 \\
0.00\end{array}$ & $\begin{array}{l}21.10 \\
30.05\end{array}$ \\
\hline & $\begin{array}{l}0-1000 \\
1-2000 \\
2-3000\end{array}$ & $\begin{array}{l}9.70 \\
0.80 \\
0.00\end{array}$ & $\begin{array}{l}7.70 \\
8.80 \\
0.00\end{array}$ & $\begin{array}{l}9.10 \\
3.40 \\
0.00\end{array}$ & $\begin{array}{r}26.50 \\
13.00 \\
0.00\end{array}$ & $\begin{array}{r}7.60 \\
9.00 \\
13.50\end{array}$ & $\begin{array}{l}0.80 \\
0.00 \\
0.00\end{array}$ & $\begin{array}{l}0.00 \\
0.70 \\
0.00\end{array}$ & $\begin{array}{r}8.40 \\
9.70 \\
13.50\end{array}$ & $\begin{array}{r}17.30 \\
9.80 \\
13.50\end{array}$ & $\begin{array}{l}8.50 \\
8.80 \\
0.00\end{array}$ & $\begin{array}{l}9.10 \\
6.10 \\
0.00\end{array}$ & $\begin{array}{l}36.90 \\
22.70 \\
13.50\end{array}$ \\
\hline & en & 0.98 & 3.91 & 0.00 & 4.89 & 5.17 & 0.65 & 0.00 & 5.82 & 6.15 & 4.56 & 0.00 & 10.71 \\
\hline & $\begin{array}{l}0-1000 \\
1-2000 \\
2-3000\end{array}$ & $\begin{array}{l}8.30 \\
2.50 \\
0.00\end{array}$ & $\begin{array}{l}6.90 \\
2.00 \\
0.00\end{array}$ & $\begin{array}{r}10.80 \\
6.40 \\
0.00 \\
\end{array}$ & $\begin{array}{r}26.00 \\
10.90 \\
0.00\end{array}$ & $\begin{array}{l}1.70 \\
0.80 \\
3.40\end{array}$ & $\begin{array}{r}10.60 \\
7.30 \\
2.60 \\
\end{array}$ & $\begin{array}{l}0.70 \\
0.50 \\
0.00\end{array}$ & $\begin{array}{r}13.00 \\
8.60 \\
6.00 \\
\end{array}$ & $\begin{array}{r}10.00 \\
3.30 \\
3.40 \\
\end{array}$ & $\begin{array}{r}17.50 \\
9.30 \\
2.60 \\
\end{array}$ & $\begin{array}{r}11.50 \\
6.90 \\
0.00\end{array}$ & $\begin{array}{r}39.00 \\
19.50 \\
6.00\end{array}$ \\
\hline count & ty total = & 150.78 & 86.39 & 194.46 & 531.63 & 333.01 & 278.06 & 127.21 & 738.28 & 483.79 & 464.45 & 321.67 & 1269.91 \\
\hline \multicolumn{14}{|l|}{ bit } \\
\hline & $\begin{array}{c}\text { onero } \\
0-1000 \\
\text { rrelated }\end{array}$ & 0.00 & 0.00 & 22.54 & 22.54 & 0.00 & 0.00 & 0.00 & 0.00 & 0.00 & 0.00 & 22.54 & 22.54 \\
\hline & $\begin{array}{l}0-1000 \\
1-2000 \\
2-3000\end{array}$ & $\begin{array}{r}17.59 \\
1.31 \\
0.00\end{array}$ & $\begin{array}{l}7.97 \\
2.10 \\
0.00\end{array}$ & $\begin{array}{r}291.74 \\
6.53 \\
0.00\end{array}$ & $\begin{array}{r}317.30 \\
9.94 \\
0.00\end{array}$ & $\begin{array}{r}383.47 \\
13.82 \\
26.10\end{array}$ & $\begin{array}{r}608.35 \\
15.61 \\
32.29\end{array}$ & $\begin{array}{r}1031.97 \\
241.27 \\
389.72\end{array}$ & $\begin{array}{r}2023.79 \\
270.70 \\
448.11\end{array}$ & $\begin{array}{r}401.06 \\
15.13 \\
26.10\end{array}$ & $\begin{array}{r}616.32 \\
17.71 \\
32.29\end{array}$ & $\begin{array}{r}1323.71 \\
247.80 \\
389.72\end{array}$ & $\begin{array}{r}2349.09 \\
280.64 \\
448.11\end{array}$ \\
\hline cour & ey total = & 18.90 & 10.07 & 320.81 & 349.78 & 423.39 & 656.25 & 1662.96 & 2742.60 & 442.29 & 666.32 & 1983.77 & 3092.38 \\
\hline \multicolumn{14}{|l|}{ bit } \\
\hline attre & $\begin{array}{l}0-1000 \\
\text { edd }\end{array}$ & 1.32 & 5.60 & 7.49 & 14.41 & 5.65 & 3.89 & 0.00 & 9.54 & 0.97 & 9.49 & 7.49 & 23.95 \\
\hline oDach & he-1000 & 0.77 & 3.68 & 1.35 & 5.80 & 1.68 & 3.39 & 0.00 & 5.07 & 2.45 & 7.07 & 1.35 & 10.87 \\
\hline$b$ & $\begin{array}{l}0-1000 \\
1-2000\end{array}$ & $\begin{array}{l}7.74 \\
0.01\end{array}$ & $\begin{array}{l}8.59 \\
0.09\end{array}$ & $\begin{array}{r}13.67 \\
0.00\end{array}$ & $\begin{array}{r}30.00 \\
0.10\end{array}$ & $\begin{array}{r}25.05 \\
9.58\end{array}$ & $\begin{array}{r}41.25 \\
7.65\end{array}$ & $\begin{array}{l}3.06 \\
0.00\end{array}$ & $\begin{array}{l}69.36 \\
17.23\end{array}$ & $\begin{array}{r}32.79 \\
9.59\end{array}$ & $\begin{array}{r}44.84 \\
7.74\end{array}$ & $\begin{array}{r}10.73 \\
0.00\end{array}$ & $\begin{array}{l}99.36 \\
17.33\end{array}$ \\
\hline bear & $\begin{array}{l}0-1000 \\
\text { canyon no } 6\end{array}$ & 2.83 & 4.10 & 34.15 & 41.08 & 22.96 & 24.85 & 94.07 & 141.88 & 25.79 & 28.95 & 128.22 & 182.96 \\
\hline bed 1 & $1^{0-1000}$ & 8.36 & 3.63 & 5.98 & 17.97 & 4.61 & 1.75 & 0.26 & 6.62 & 12.97 & 5.38 & 6.26 & 24.59 \\
\hline & $\begin{array}{l}0-1000 \\
1-2000\end{array}$ & $\begin{array}{l}0.05 \\
0.33\end{array}$ & $\begin{array}{l}1.44 \\
1.22\end{array}$ & $\begin{array}{l}0.00 \\
0.00\end{array}$ & $\begin{array}{l}1.49 \\
1.55\end{array}$ & $\begin{array}{l}3.60 \\
1.86\end{array}$ & $\begin{array}{l}4.71 \\
2.73\end{array}$ & $\begin{array}{l}0.02 \\
0.00\end{array}$ & $\begin{array}{l}8.33 \\
4.59\end{array}$ & $\begin{array}{l}3.65 \\
2.19\end{array}$ & $\begin{array}{l}6.15 \\
3.95\end{array}$ & $\begin{array}{l}0.02 \\
0.00\end{array}$ & $\begin{array}{l}9.82 \\
6.14\end{array}$ \\
\hline
\end{tabular}




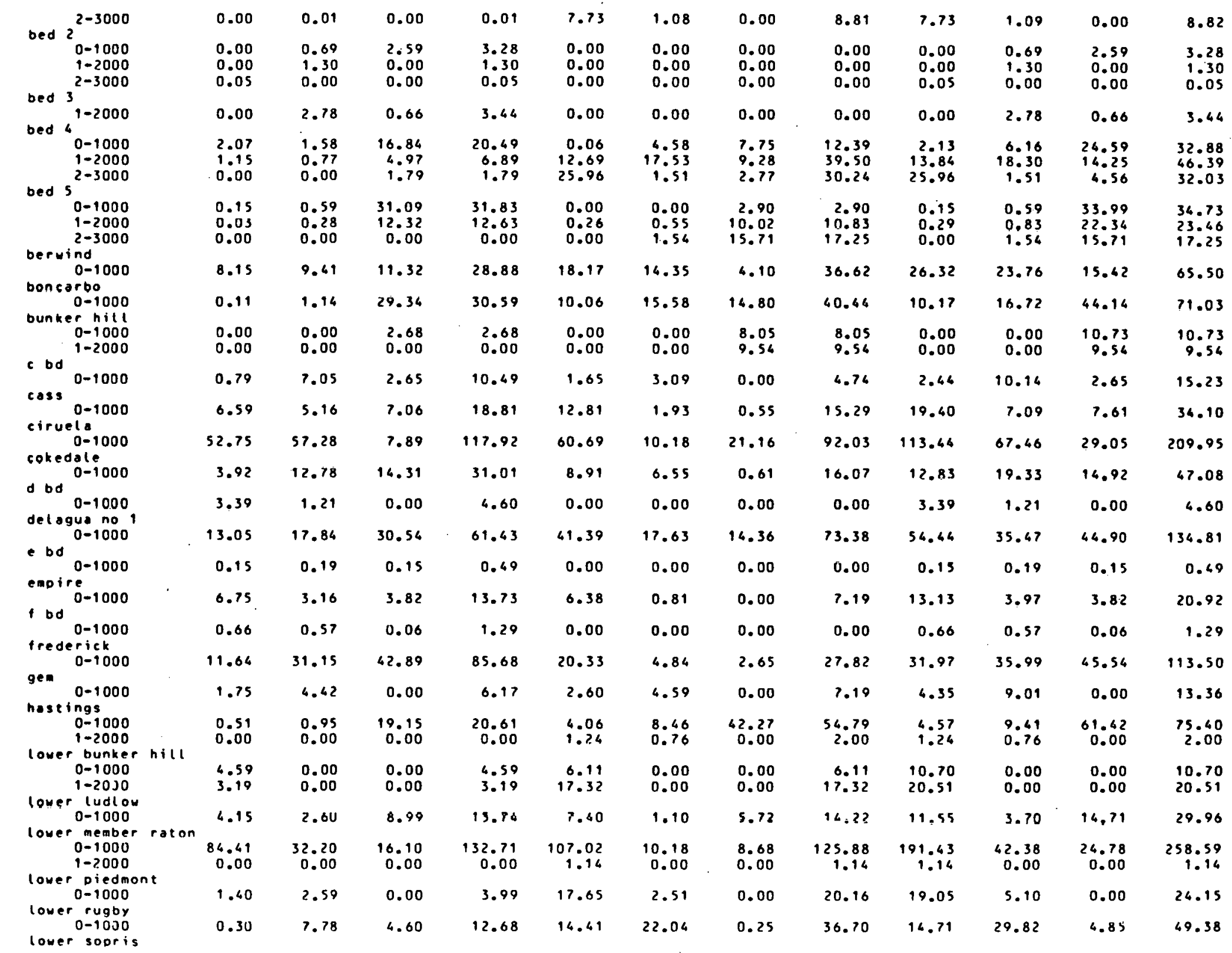




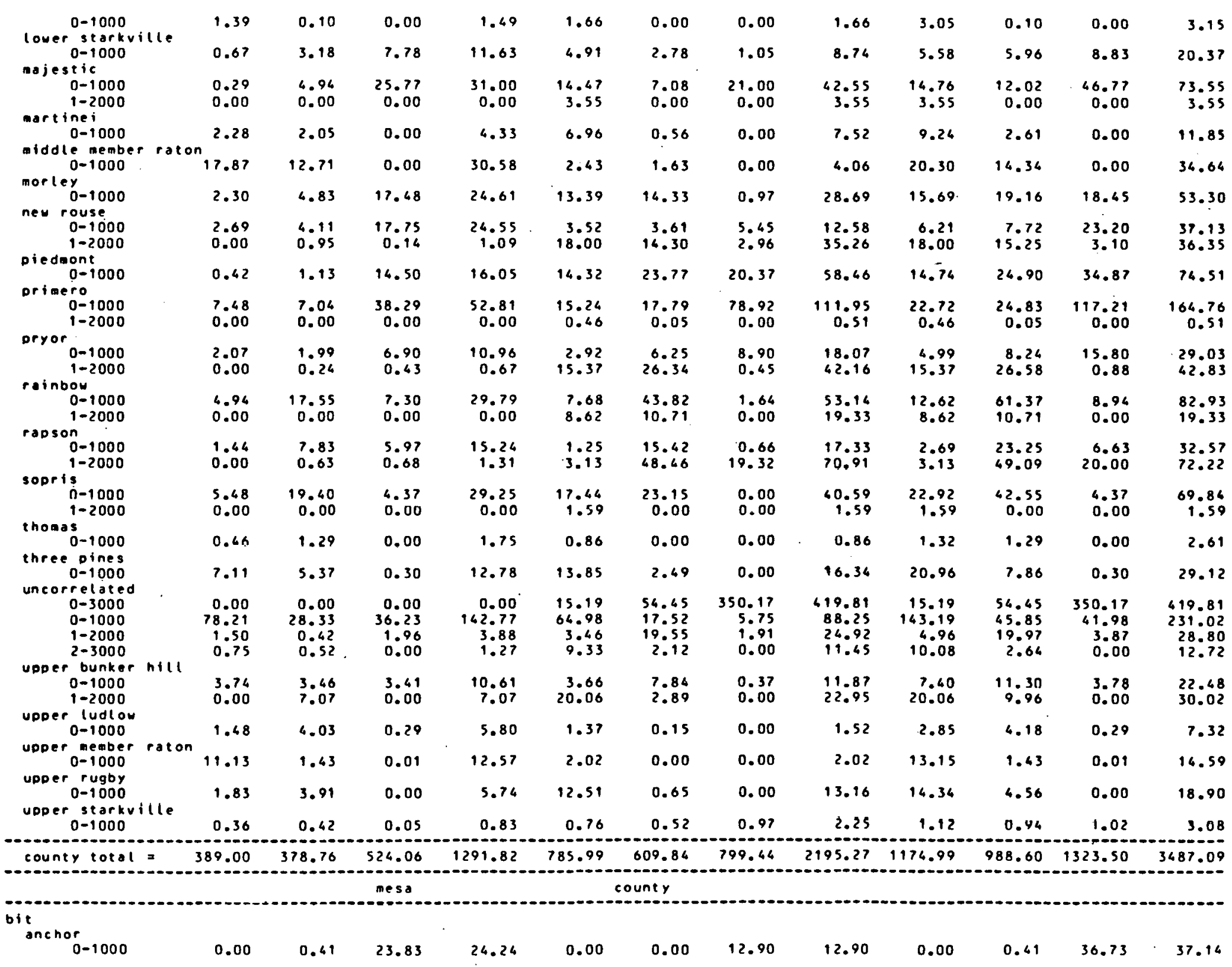




\begin{tabular}{|c|c|c|c|c|c|c|c|c|c|c|c|c|}
\hline $\begin{array}{l}1-2000 \\
2-3000\end{array}$ & $\begin{array}{l}0.00 \\
0.00\end{array}$ & $\begin{array}{l}0.00 \\
0.00\end{array}$ & $\begin{array}{l}0.00 \\
0.00\end{array}$ & $\begin{array}{l}0.00 \\
0.00\end{array}$ & $\begin{array}{l}0.00 \\
0.00\end{array}$ & $\begin{array}{l}0.00 \\
0.00\end{array}$ & $\begin{array}{l}17.85 \\
10.50\end{array}$ & $\begin{array}{l}17.85 \\
10.50\end{array}$ & $\begin{array}{l}0.00 \\
0.00\end{array}$ & $\begin{array}{l}0.00 \\
0.00\end{array}$ & $\begin{array}{l}17.85 \\
10.50\end{array}$ & $\begin{array}{l}17.85 \\
10.50\end{array}$ \\
\hline $\begin{array}{l}0-1000 \\
1-2000 \\
2-3000\end{array}$ & $\begin{array}{l}0.00 \\
0.00 \\
0.00\end{array}$ & $\begin{array}{l}0.00 \\
0.00 \\
0.00\end{array}$ & $\begin{array}{r}104.22 \\
12.63 \\
0.00\end{array}$ & $\begin{array}{r}104.22 \\
12.63 \\
0.00\end{array}$ & $\begin{array}{l}0.00 \\
0.00 \\
0.00\end{array}$ & $\begin{array}{l}0.00 \\
0.00 \\
0.00\end{array}$ & $\begin{array}{r}121.45 \\
129.46 \\
48.91\end{array}$ & $\begin{array}{r}129.45 \\
129.46 \\
48.91\end{array}$ & $\begin{array}{l}0.00 \\
0.00 \\
0.00\end{array}$ & $\begin{array}{l}0.00 \\
0.00 \\
0.00\end{array}$ & $\begin{array}{r}225.67 \\
142.09 \\
48.91\end{array}$ & $\begin{array}{r}225.67 \\
142.09 \\
48.91\end{array}$ \\
\hline $\begin{array}{c}\text { carbonera } \\
0-1000 \\
1-2000\end{array}$ & $\begin{array}{l}0.00 \\
0.00\end{array}$ & $\begin{array}{l}0.00 \\
0.00\end{array}$ & $\begin{array}{r}16.33 \\
3.03\end{array}$ & $\begin{array}{r}16.33 \\
3.03\end{array}$ & $\begin{array}{l}0.00 \\
0.00\end{array}$ & $\begin{array}{l}0.00 \\
0.00\end{array}$ & $\begin{array}{l}0.00 \\
0.00\end{array}$ & $\begin{array}{l}0.00 \\
0.00\end{array}$ & $\begin{array}{l}0.00 \\
0.00\end{array}$ & $\begin{array}{l}0.00 \\
0.00\end{array}$ & $\begin{array}{r}16.33 \\
3.03\end{array}$ & $\begin{array}{r}16.33 \\
3.03\end{array}$ \\
\hline $\begin{array}{r}\text { Dal is ade } \\
0-1000 \\
1-2000 \\
2-3000\end{array}$ & $\begin{array}{l}1.43 \\
0.83 \\
0.00\end{array}$ & $\begin{array}{r}12.54 \\
3.30 \\
0.00\end{array}$ & $\begin{array}{r}72.02 \\
18.06 \\
0.00 \\
\end{array}$ & $\begin{array}{r}85.99 \\
22.19 \\
0.00\end{array}$ & $\begin{array}{l}0.07 \\
0.00 \\
0.00\end{array}$ & $\begin{array}{l}5.61 \\
2.87 \\
0.00\end{array}$ & $\begin{array}{l}176.53 \\
304.30 \\
201.25\end{array}$ & $\begin{array}{l}182.21 \\
307.17 \\
201.25\end{array}$ & $\begin{array}{l}1.50 \\
0.83 \\
0.00\end{array}$ & $\begin{array}{r}18.15 \\
6.17 \\
0.00\end{array}$ & $\begin{array}{l}248.55 \\
322.36 \\
201.25\end{array}$ & $\begin{array}{l}268.20 \\
329.36 \\
201.25\end{array}$ \\
\hline couner iotal = & 2.26 & 16.25 & 250.12 & 268.63 & 0.07 & 8.48 & 1023.15 & 1031.70 & 2.33 & 24.73 & 1273.27 & 1300.33 \\
\hline & & & moffat & & & ty & & & . & & & \\
\hline ? & & & & & & & & & & & & \\
\hline $0-1000$ & 0.00 & 5.88 & 0.00 & 5.88 & 0.00 & 0.00 & 0.00 & 0.00 & 0.00 & 5.88 & 0.00 & 5.88 \\
\hline basuf $^{0-1000}$ & 0.00 & 0.00 & 0.56 & 0.56 & 0.00 & 0.00 & 0.00 & 0.00 & 0.00 & 0.00 & 0.56 & 0.56 \\
\hline $\begin{array}{r}0-1000 \\
1-2000 \\
2-3000 \\
\text { bo group }\end{array}$ & $\begin{array}{l}0.00 \\
0.00 \\
0.00\end{array}$ & $\begin{array}{l}0.00 \\
0.00 \\
0.00\end{array}$ & $\begin{array}{l}30.61 \\
32.74 \\
28.72\end{array}$ & $\begin{array}{l}30.61 \\
32.74 \\
28.72\end{array}$ & $\begin{array}{l}0.00 \\
0.00 \\
0.00\end{array}$ & $\begin{array}{l}0.00 \\
0.00 \\
0.00\end{array}$ & $\begin{array}{l}0.00 \\
0.00 \\
0.00\end{array}$ & $\begin{array}{l}0.00 \\
0.00 \\
0.00\end{array}$ & $\begin{array}{l}0.00 \\
0.00 \\
0.00\end{array}$ & $\begin{array}{l}0.00 \\
0.00 \\
0.00\end{array}$ & $\begin{array}{l}30.61 \\
32.74 \\
28.72\end{array}$ & $\begin{array}{l}30.61 \\
32.74 \\
28.72\end{array}$ \\
\hline $\begin{array}{r}0-1000 \\
1-2000\end{array}$ & $\begin{array}{r}38.90 \\
7.51\end{array}$ & $\begin{array}{r}19.58 \\
2.56\end{array}$ & $\begin{array}{l}78.05 \\
11.09\end{array}$ & $\begin{array}{r}136.49 \\
21.06\end{array}$ & $\begin{array}{l}0.00 \\
0.00\end{array}$ & $\begin{array}{l}0.00 \\
0.00\end{array}$ & $\begin{array}{l}0.00 \\
0.00\end{array}$ & $\begin{array}{l}0.00 \\
0.00\end{array}$ & $\begin{array}{r}38.96 \\
7.51\end{array}$ & $\begin{array}{r}19.48 \\
2.34\end{array}$ & $\begin{array}{l}78.05 \\
11.01\end{array}$ & $\begin{array}{r}136.49 \\
21.06\end{array}$ \\
\hline $\begin{array}{l}0-1000 \\
1-2000\end{array}$ & $\begin{array}{l}3.57 \\
0.00\end{array}$ & $\begin{array}{l}1.79 \\
1.37\end{array}$ & $\begin{array}{l}13.10 \\
17.56\end{array}$ & $\begin{array}{l}18.46 \\
18.93\end{array}$ & $\begin{array}{l}0.00 \\
0.00\end{array}$ & $\begin{array}{l}0.00 \\
0.00\end{array}$ & $\begin{array}{l}0.00 \\
0.00\end{array}$ & $\begin{array}{l}0.00 \\
0.00\end{array}$ & $\begin{array}{l}3.57 \\
0.00\end{array}$ & $\begin{array}{l}1.79 \\
1.37\end{array}$ & $\begin{array}{l}13.10 \\
17.56\end{array}$ & $\begin{array}{l}18.46 \\
18.93\end{array}$ \\
\hline f zone & 0.00 & 0.00 & 8.55 & 8.55 & 0.00 & 0.00 & 0.00 & 0.00 & 0.00 & 0.00 & 8.55 & 8.55 \\
\hline $\begin{array}{r}0-1000 \\
1-2030\end{array}$ & $\begin{array}{l}18.99 \\
22.92\end{array}$ & $\begin{array}{l}1.36 \\
0.00\end{array}$ & $\begin{array}{l}67.84 \\
17.90\end{array}$ & $\begin{array}{l}88.17 \\
40.82\end{array}$ & $\begin{array}{l}0.00 \\
0.00\end{array}$ & $\begin{array}{l}0.00 \\
0.00\end{array}$ & $\begin{array}{l}0.00 \\
0.00\end{array}$ & $\begin{array}{l}0.00 \\
0.00\end{array}$ & $\begin{array}{l}18.99 \\
22.92\end{array}$ & $\begin{array}{l}1.34 \\
0.00\end{array}$ & $\begin{array}{l}67.84 \\
17.90\end{array}$ & $\begin{array}{l}88.17 \\
40.82\end{array}$ \\
\hline $0^{0-1000}$ & 0.10 & 1.67 & 0.87 & 2.64 & 0.06 & 0.00 & 0.00 & 0.06 & 0.16 & 1.67 & 0.87 & 2.70 \\
\hline ff $11^{0-1000}$ & 0.87 & 0.00 & 0.00 & 0.87 & 0.00 & 0.00 & 0.00 & 0.00 & 0.87 & 0.00 & 0.00 & 0.87 \\
\hline if $12^{0-1000}$ & 2.04 & 0.00 & 0.31 & 2.35 & 0.00 & 0.00 & 0.00 & 0.00 & 2.04 & 0.00 & 0.31 & 2.35 \\
\hline ff $13^{0-1000}$ & 0.00 & 0.00 & 8.28 & 8.28 & 0.00 & 0.00 & 0.00 & 0.00 & 0.00 & 0.00 & 8.28 & 8.28 \\
\hline$+14^{0-1000}$ & 0.00 & 1.60 & 0.00 & 1.60 & 0.00 & 0.00 & 0.00 & 0.00 & 0.00 & 1.60 & 0.00 & 1.60 \\
\hline$+15^{0-1000}$ & 0.00 & 0.72 & 0.00 & 0.72 & 0.00 & 0.00 & 0.00 & 0.00 & 0.00 & 0.72 & 0.00 & 0.72 \\
\hline ff $18^{0-1000}$ & 0.66 & 0.00 & 0.24 & 0.90 & 0.17 & 0.00 & 4.61 & 4.78 & 0.85 & 0.00 & 4.05 & 5.68 \\
\hline$+19^{0-1000}$ & 0.00 & 0.00 & 14.85 & 14.85 & 0.00 & 0.00 & 17.86 & 17.86 & 0.00 & 0.00 & 32.71 & 32.71 \\
\hline if $2^{0-1000}$ & 1.85 & 0.00 & 0.00 & 1.85 & 4.16 & 1.63 & 0.00 & 5.79 & 6.01 & 1.63 & 0.00 & 7.64 \\
\hline if $20^{0-1000}$ & 0.70 & 2.18 & 0.00 & 2.88 & 0.00 & 0.23 & 0.00 & 0.23 & 0.70 & 2.41 & 0.00 & 3.11 \\
\hline $0-1000$ & 0.00 & 3.71 & 0.00 & 3.71 & 0.00 & 16.53 & 0.00 & 16.53 & 0.00 & 20.26 & 0.00 & 20.24 \\
\hline
\end{tabular}




\begin{tabular}{|c|c|c|c|c|c|c|c|c|c|c|c|c|c|}
\hline if 21 & $0-1000$ & 0.00 & 0.00 & 2.67 & 2.67 & 0.00 & 2.69 & 7.62 & 10.31 & 0.00 & 2.69 & 10.29 & 12.98 \\
\hline++23 & $3^{0-1000}$ & 0.37 & 0.00 & 0.00 & 0.37 & 0.00 & 0.00 & 0.00 & 0.00. & 0.37 . & 0.00 & 0.00 & 0.37 \\
\hline te 3 & $0-1000$ & 0.99 & 2.55 & 14.98 & 18.52 & 0.12 & 23.58 & 0.00 & 23.70 & $1.11^{\circ}$ & 26.13 & 14.98 & 42.22 \\
\hline $1+4$ & $0-1000$ & 0.51 & 1.25 & 0.00 & 1.76 & 0.00 & 0.33 & 0.00 & 0.33 & 0.51 & 1.58 & 0.00 & 2.09 \\
\hline 45 & $0-1000$ & 0.00 & 0.00 & 0.52 & 0.52 & 0.00 & 0.00 & 0.00 & 0.00 & 0.00 & 0.00 & 0.52 & 0.52 \\
\hline & $0-1000$ & 0.00 & 0.46 & 0.76 & 1.22 & 0.00 & 0.67 & 0.14 & 0.81 & 0.00 & 1.13 & 0.90 & 2.03 \\
\hline 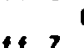 & $0-1000$ & 1.62 & 0.00 & 1.38 & 3.00 & 0.61 & 3.11 & 0.65 & 4.37 & 2.23 & 3.11 & 2.03 & 7.37 \\
\hline $1+9$ & $0-1000$ & 0.69 & 0.00 & 5.39 & 6.08 & 1.00 & 6.19 & 2.48 & 9.67 & 1.69 & 6.19 & 7.87 & 15.75 \\
\hline ft gr & $\begin{array}{l}0-1000 \\
\text { OUD }\end{array}$ & 0.00 & 3.04 & 12.06 & 15.10 & 0.00 & 0.00 & 4.77 & 4.77 & 0.00 & 3.04 & 16.83 & 19.87 \\
\hline goft & $\begin{array}{l}0-1000 \\
1-2000 \\
1\end{array}$ & $\begin{array}{r}90.12 \\
5.27\end{array}$ & $\begin{array}{r}165.64 \\
10.93\end{array}$ & $\begin{array}{r}1167.56 \\
156.01\end{array}$ & $\begin{array}{r}1423.32 \\
172.21\end{array}$ & $\begin{array}{l}0.00 \\
0.00\end{array}$ & $\begin{array}{l}0.00 \\
0.00\end{array}$ & $\begin{array}{l}0.00 \\
0.00\end{array}$ & $\begin{array}{l}0.00 \\
0.00\end{array}$ & $\begin{array}{r}90.12 \\
5.27\end{array}$ & $\begin{array}{r}165.64 \\
10.93\end{array}$ & $\begin{array}{r}1167.56 \\
156.01\end{array}$ & $\begin{array}{r}1423.32 \\
172.21\end{array}$ \\
\hline gott & $\begin{array}{l}0-1000 \\
10\end{array}$ & 0.00 & 1.13 & 0.00 & 1.13 & 0.00 & 0.00 & 0.00 & 0.00 & 0.00 & 1.13 & 0.00 & 1.13 \\
\hline goft & $\begin{array}{l}0-1000 \\
11\end{array}$ & 0.00 & 1.58 & 3.66 & 5.04 & 0.00 & 0.00 & 6.60 & 6.60 & 0.00 & 1.58 & 10.06 & 11.64 \\
\hline goft & $\begin{array}{l}0-1000 \\
12\end{array}$ & 0.00 & 0.00 & 16.52 & 16.52 & 0.00 & 0.00 & 0.00 & 0.00 & 0.00 & 0.00 & 16.52 & 16.52 \\
\hline goft & $\begin{array}{l}0-1000 \\
14\end{array}$ & 0.00 & 0.00 & 1.11 & 1.11 & 0.00 & 0.00 & 0.00 & 0.00 & 0.00 & 0.00 & 1.11 & 1.11 \\
\hline gotf & $\begin{array}{l}0-1000 \\
15\end{array}$ & 0.00 & 0.00 & 4.82 & 4.82 & 0.00 & 0.00 & 0.00 & 0.00 & 0.00 & 0.00 & 4.82 & 4.82 \\
\hline goft 1 & $\begin{array}{l}0-1000 \\
16\end{array}$ & 1.30 & 0.00 & 0.00 & 1.30 & 0.00 & 0.00 & 0.00 & 0.00 & 1.30 & 0.00 & 0.00 & 1.30 \\
\hline goft & $\begin{array}{l}0-1000 \\
17\end{array}$ & 0.00 & 3.06 & 0.00 & 3.06 & 0.00 & 5.48 & 0.00 & 5.48 & 0.00 & 8.54 & 0.00 & 8.54 \\
\hline gof f & $\begin{array}{l}0-1000 \\
18\end{array}$ & 0.00 & 0.00 & 10.39 & 10.39 & 0.00 & 0.00 & 35.21 & 35.21 & 0.00 & 0.00 & 45.60 & 45.60 \\
\hline goft & $\begin{array}{l}0-1000 \\
19\end{array}$ & 0.00 & 1.72 & 0.00 & 1.72 & 0.00 & 0.00 & 0.00 & 0.00 & 0.00 & 1.72 & 0.00 & 1.72 \\
\hline youft? & $z^{0-1000}$ & 0.00 & 0.00 & 10.45 & 10.45 & 0.00 & 0.00 & 37.88 & 37.88 & 0.00 & 0.00 & 48.33 & 48.33 \\
\hline goft & $\begin{array}{l}0-1000 \\
20\end{array}$ & 0.71 & 0.00 & 0.00 & 0.11 & 0.00 & 0.00 & 0.00 & 0.00 & 0.71 & 0.00 & 0.00 & 0.71 \\
\hline $904+$ & $\begin{array}{l}0-1000 \\
22\end{array}$ & 0.00 & 0.00 & 6.63 & 6.63 & 0.00 & 0.00 & 20.43 & 20.43 & 0.00 & 0.00 & 27.06 & 27.06 \\
\hline $90+4$ & $\begin{array}{l}0-1000 \\
3\end{array}$ & 0.00 & 1.06 & 0.12 & 1.18 & 0.00 & 7.00 & 0.00 & 7.00 & 0.00 & 8.06 & 0.12 & 8.18 \\
\hline gote & $\begin{array}{l}0-1000 \\
4\end{array}$ & 0.83 & 0.00 & 0.00 & 0.83 & 0.00 & 0.00 & 0.00 & 0.00 & 0.83 & 0.00 & 0.00 & 0.83 \\
\hline gott & $\begin{array}{l}0-1000 \\
6\end{array}$ & 0.83 & 0.00 & 0.00 & 0.83 & 0.00 & 0,00 & 0.00 & 0.00 & 0.83 & 0.00 & 0.00 & 0.83 \\
\hline got? & 7 & 0.52 & 0.77 & 0.00 & 1.29 & 1.77 & 0.00 & 0.00 & 1.71 & 2.29 & 0.77 & 0.00 & 3.06 \\
\hline gotf 8 & $\begin{array}{l}0-1000 \\
8\end{array}$ & 0.00 & 0.00 & 3.65 & 3.65 & 0.00 & 0.00 & 0.00 & 0.00 & 0.00 & 0.00 & 3.65 & 3.65 \\
\hline $901+8$ & $\begin{array}{l}0-1000 \\
9\end{array}$ & 0.00 & 0.00 & 2.55 & 2.55 & 0.00 & 0.00 & 0.00 & 0.00 & 0.00 & 0.00 & 2.55 & 2.55 \\
\hline h cone & $e^{0-1000}$ & 0.00 & 0.00 & 1.66 & 1.66 & 0.00 & 0.00 & 0.00 & 0.00 & 0.00 & 0.00 & 1.66 & 1.66 \\
\hline
\end{tabular}




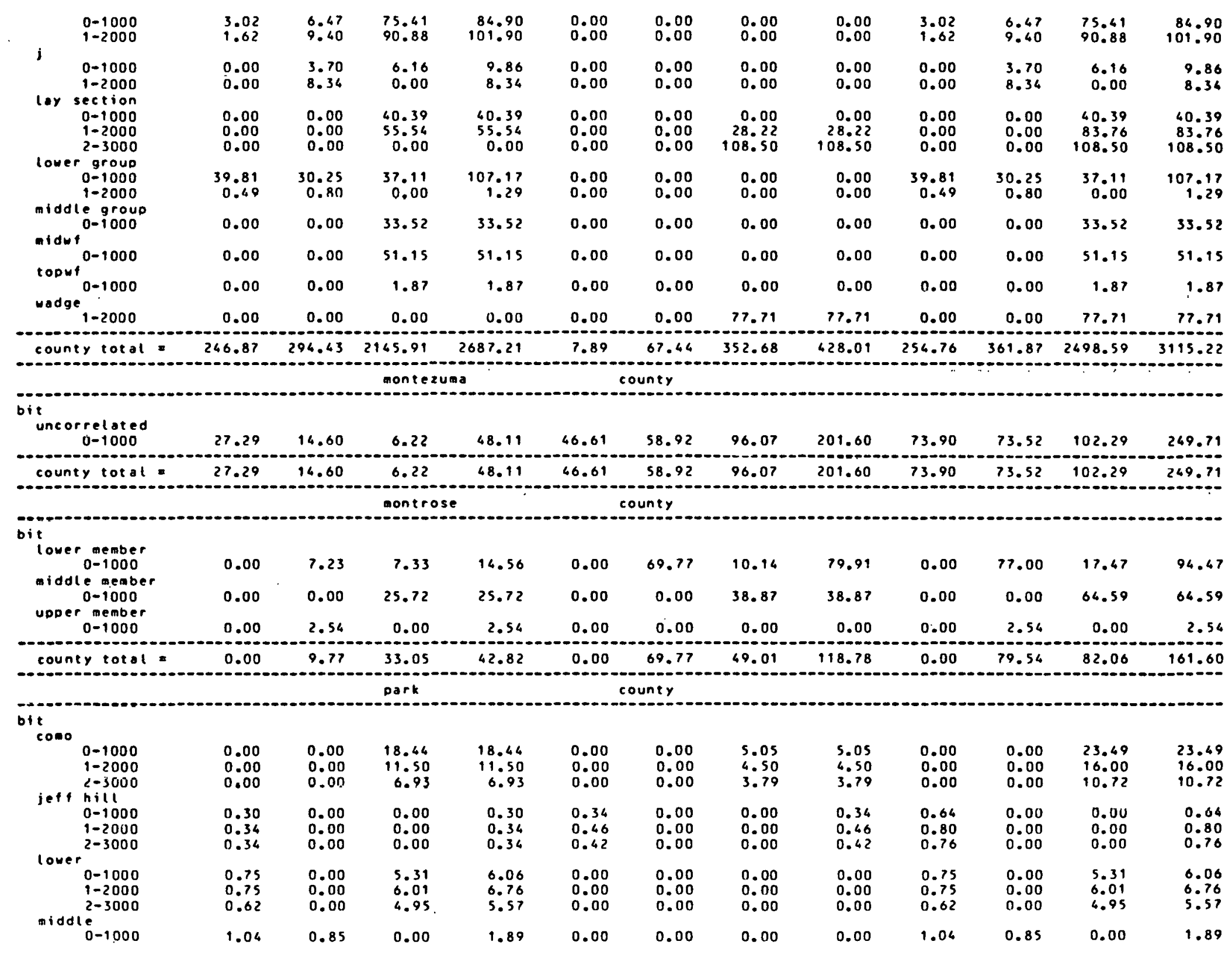




\begin{tabular}{|c|c|c|c|c|c|c|c|c|c|c|c|c|c|}
\hline unoe & $\begin{array}{r}1-2000 \\
2-3000\end{array}$ & $\begin{array}{l}1.04 \\
0.84\end{array}$ & $\begin{array}{l}7.60 \\
7.60\end{array}$ & $\begin{array}{l}0.00 \\
0.00\end{array}$ & $\begin{array}{l}8.64 \\
8.44\end{array}$ & $\begin{array}{l}0.00 \\
0.00\end{array}$ & $\begin{array}{l}0.00 \\
0.00\end{array}$ & $\begin{array}{l}0.00 \\
0.00\end{array}$ & $\begin{array}{l}0.00 \\
0.00\end{array}$ & $\begin{array}{l}9.04 \\
0.84\end{array}$ & $\begin{array}{l}7.60 \\
7.60\end{array}$ & $\begin{array}{l}0.00 \\
0.00\end{array}$ & $\begin{array}{l}8.64 \\
8.44\end{array}$ \\
\hline & $\begin{array}{l}0-1000 \\
1-2000 \\
2-3000\end{array}$ & $\begin{array}{l}0.00 \\
0.00 \\
0.00\end{array}$ & $\begin{array}{l}0.00 \\
0.00 \\
0.00\end{array}$ & $\begin{array}{l}0.71 \\
0.83 \\
0.94\end{array}$ & $\begin{array}{l}0.71 \\
0.83 \\
0.94\end{array}$ & $\begin{array}{l}0.00 \\
0.00 \\
0.00\end{array}$ & $\begin{array}{r}0.00 \\
0.00 \\
0.00\end{array}$ & $\begin{array}{l}0.00 \\
0.00 \\
0.00\end{array}$ & $\begin{array}{l}0.00 \\
0.00 \\
0.00\end{array}$ & $\begin{array}{l}0.00 \\
0.00 \\
0.00\end{array}$ & $\begin{array}{l}0.00 \\
0.00 \\
0.00\end{array}$ & $\begin{array}{l}0.71 \\
0.83 \\
0.94\end{array}$ & $\begin{array}{l}0.71 \\
0.83 \\
0.94\end{array}$ \\
\hline coun & ty total & 6.02 & 16.05 & 55.62 & 77.69 & 1.22 & 0.00 & 13.34 & 14.56 & 7.24 & 16.05 & 68.96 & 92.25 \\
\hline- & - & & & inkin & & & ney & & & & & & \\
\hline \multicolumn{14}{|l|}{ it } \\
\hline & $\begin{array}{l}0-1000 \\
1-2000 \\
2-3000\end{array}$ & $\begin{array}{l}0.00 \\
0.00 \\
0.00\end{array}$ & $\begin{array}{l}0.00 \\
0.00 \\
0.00\end{array}$ & $\begin{array}{l}12.90 \\
14.20 \\
19.60\end{array}$ & $\begin{array}{l}12.90 \\
14.20 \\
19.60\end{array}$ & $\begin{array}{l}0.00 \\
0.00 \\
0.00\end{array}$ & $\begin{array}{l}0.00 \\
0.00 \\
0.00\end{array}$ & $\begin{array}{l}3.68 \\
3.69 \\
6.35\end{array}$ & $\begin{array}{l}3.68 \\
3.49 \\
6.35\end{array}$ & $\begin{array}{l}0.00 \\
0.00 \\
0.00\end{array}$ & $\begin{array}{l}0.00 \\
0.00 \\
0.00\end{array}$ & $\begin{array}{l}16.58 \\
17.69 \\
25.95\end{array}$ & $\begin{array}{l}16.58 \\
17.69 \\
25.95\end{array}$ \\
\hline aller & $\begin{array}{l}0-1000 \\
1--2000 \\
2-3000 \\
n\end{array}$ & $\begin{array}{l}0.00 \\
0.00 \\
0.00\end{array}$ & $\begin{array}{l}0.00 \\
0.00 \\
0.00\end{array}$ & $\begin{array}{l}5.71 \\
4.08 \\
0.00\end{array}$ & $\begin{array}{l}5.71 \\
4.08 \\
0.00\end{array}$ & $\begin{array}{l}0.00 \\
0.00 \\
0.00\end{array}$ & $\begin{array}{l}0.00 \\
0.00 \\
0.00\end{array}$ & $\begin{array}{r}7.36 \\
13.86 \\
6.53\end{array}$ & $\begin{array}{r}7.34 \\
13.86 \\
6.53\end{array}$ & $\begin{array}{l}0.00 \\
0.00 \\
0.00\end{array}$ & $\begin{array}{l}0.00 \\
0.00 \\
0.00\end{array}$ & $\begin{array}{r}13.05 \\
17.94 \\
6.53\end{array}$ & $\begin{array}{r}13.05 \\
17.96 \\
6.53\end{array}$ \\
\hline & $\begin{array}{l}0-1000 \\
1-2000 \\
2-3000 \\
\text { rson }\end{array}$ & $\begin{array}{l}0.00 \\
0.00 \\
0.00\end{array}$ & $\begin{array}{l}0.00 \\
0.00 \\
0.00\end{array}$ & $\begin{array}{l}22.83 \\
16.87 \\
16.69\end{array}$ & $\begin{array}{l}22.83 \\
16.87 \\
16.69\end{array}$ & $\begin{array}{l}0.00 \\
0.00 \\
0.00\end{array}$ & $\begin{array}{l}0.00 \\
0.00 \\
0.00\end{array}$ & $\begin{array}{r}7.64 \\
11.66 \\
10.53\end{array}$ & $\begin{array}{r}7.66 \\
11.66 \\
10.53\end{array}$ & $\begin{array}{l}0.00 \\
0.00 \\
0.00\end{array}$ & $\begin{array}{l}0.00 \\
0.00 \\
0.00\end{array}$ & $\begin{array}{l}30.47 \\
28.53 \\
27.22\end{array}$ & $\begin{array}{l}30.47 \\
28.53 \\
27.22\end{array}$ \\
\hline 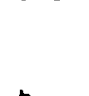 & $\begin{array}{l}0-1000 \\
1-2000 \\
2-3000\end{array}$ & $\begin{array}{l}0.00 \\
0.00 \\
0.00\end{array}$ & $\begin{array}{l}4.89 \\
2.84 \\
2.15\end{array}$ & $\begin{array}{r}11.55 \\
9.30 \\
8.26\end{array}$ & $\begin{array}{l}16.44 \\
12.14 \\
10.41\end{array}$ & $\begin{array}{l}0.00 \\
0.00 \\
0.00\end{array}$ & $\begin{array}{l}4.32 \\
6.82 \\
4.10\end{array}$ & $\begin{array}{l}0.00 \\
0.15 \\
6.54\end{array}$ & $\begin{array}{l}4.32 \\
6.97 \\
8.64\end{array}$ & $\begin{array}{l}0.00 \\
0.00 \\
0.00\end{array}$ & $\begin{array}{l}9.21 \\
9.66 \\
6.25\end{array}$ & $\begin{array}{r}11.55 \\
9.65 \\
12.80\end{array}$ & $\begin{array}{l}20.76 \\
19.11 \\
19.05\end{array}$ \\
\hline$\varepsilon$ & $\begin{array}{l}0-1000 \\
1-2000 \\
2-3000\end{array}$ & $\begin{array}{l}0.00 \\
0.00 \\
0.00\end{array}$ & $\begin{array}{l}1.89 \\
2.45 \\
2.54\end{array}$ & $\begin{array}{l}5.06 \\
4.80 \\
7.85\end{array}$ & $\begin{array}{r}6.95 \\
7.25 \\
10.39\end{array}$ & $\begin{array}{l}0.00 \\
0.00 \\
0.00\end{array}$ & $\begin{array}{l}2.52 \\
2.32 \\
4.25\end{array}$ & $\begin{array}{l}0.00 \\
0.00 \\
0.00\end{array}$ & $\begin{array}{l}2.52 \\
2.32 \\
4.25\end{array}$ & $\begin{array}{l}0.00 \\
0.00 \\
0.00\end{array}$ & $\begin{array}{l}4.61 \\
4.77 \\
6.79\end{array}$ & $\begin{array}{l}5.06 \\
4.80 \\
7.85\end{array}$ & $\begin{array}{r}9.47 \\
9.57 \\
14.64\end{array}$ \\
\hline d & $\begin{array}{l}0-1000 \\
1-2000 \\
2-3000\end{array}$ & $\begin{array}{l}0.00 \\
0.00 \\
0.00\end{array}$ & $\begin{array}{l}0.00 \\
0.00 \\
0.00\end{array}$ & $\begin{array}{l}5.08 \\
4.86 \\
7.63\end{array}$ & $\begin{array}{l}5.08 \\
4.86 \\
7.63\end{array}$ & $\begin{array}{l}0.00 \\
0.00 \\
0.00\end{array}$ & $\begin{array}{l}0.00 \\
0.00 \\
0.00\end{array}$ & $\begin{array}{l}3.02 \\
4.20 \\
4.96\end{array}$ & $\begin{array}{l}3.02 \\
4.20 \\
4.96\end{array}$ & $\begin{array}{l}0.00 \\
0.00 \\
0.00\end{array}$ & $\begin{array}{l}0.00 \\
0.00 \\
0.00\end{array}$ & $\begin{array}{r}8.10 \\
9.06 \\
12.59\end{array}$ & $\begin{array}{r}8.10 \\
9.06 \\
12.59\end{array}$ \\
\hline 0 & $\begin{array}{l}0-1000 \\
1-2000 \\
2-3000\end{array}$ & $\begin{array}{l}0.00 \\
0.00 \\
0.00\end{array}$ & $\begin{array}{l}0.00 \\
0.00 \\
0.00\end{array}$ & $\begin{array}{l}3.42 \\
3.21 \\
5.22\end{array}$ & $\begin{array}{l}3.42 \\
3.21 \\
5.22\end{array}$ & $\begin{array}{l}0.00 \\
0.00 \\
0.00\end{array}$ & $\begin{array}{l}0.00 \\
0.00 \\
0.00\end{array}$ & $\begin{array}{l}0.00 \\
0.00 \\
0.00\end{array}$ & $\begin{array}{l}0.00 \\
0.00 \\
0.00\end{array}$ & $\begin{array}{l}0.00 \\
0.00 \\
0.00\end{array}$ & $\begin{array}{l}0.00 \\
0.00 \\
0.00\end{array}$ & $\begin{array}{l}3.42 \\
3.21 \\
.5 .22\end{array}$ & $\begin{array}{l}3.42 \\
3.21 \\
5.22\end{array}$ \\
\hline 1 & $\begin{array}{l}0-1000 \\
1-2000 \\
2-3000\end{array}$ & $\begin{array}{l}0.44 \\
0.28 \\
0.41\end{array}$ & $\begin{array}{l}3.44 \\
2.43 \\
1.38\end{array}$ & $\begin{array}{l}0.00 \\
0.00 \\
0.00\end{array}$ & $\begin{array}{l}3.88 \\
2.71 \\
1.70\end{array}$ & $\begin{array}{l}0.00 \\
0.00 \\
0.00\end{array}$ & $\begin{array}{l}3.57 \\
3.16 \\
5.60\end{array}$ & $\begin{array}{l}0.00 \\
0.00 \\
0.00\end{array}$ & $\begin{array}{l}3.57 \\
3.16 \\
5.60\end{array}$ & $\begin{array}{l}0.44 \\
0.28 \\
0.41\end{array}$ & $\begin{array}{l}7.01 \\
5.59 \\
0.98\end{array}$ & $\begin{array}{l}0.00 \\
0.00 \\
0.00\end{array}$ & $\begin{array}{l}7.65 \\
5.87 \\
7.39\end{array}$ \\
\hline$g$ & $\begin{array}{l}0-1000 \\
1-2000 \\
2-3000\end{array}$ & $\begin{array}{l}0.00 \\
0.00 \\
0.00\end{array}$ & $\begin{array}{l}4.12 \\
2.86 \\
2.13\end{array}$ & $\begin{array}{l}0.00 \\
0.00 \\
0.00\end{array}$ & $\begin{array}{l}4.12 \\
2.86 \\
2.13\end{array}$ & $\begin{array}{l}0.00 \\
0.00 \\
0.00\end{array}$ & $\begin{array}{l}3.75 \\
3.39 \\
6.24\end{array}$ & $\begin{array}{l}0.00 \\
0.00 \\
0.00\end{array}$ & $\begin{array}{l}3.75 \\
3.39 \\
6.24\end{array}$ & $\begin{array}{l}0.00 \\
0.00 \\
0.00\end{array}$ & $\begin{array}{l}7.87 \\
6.25 \\
8.37\end{array}$ & $\begin{array}{l}0.00 \\
0.00 \\
0.00\end{array}$ & $\begin{array}{l}7.87 \\
6.25 \\
8.37\end{array}$ \\
\hline low & $\begin{array}{r}0-1000 \\
1-2000 \\
2-3030 \\
\text { hol gate }\end{array}$ & $\begin{array}{l}2.24 \\
1.34 \\
1.17\end{array}$ & $\begin{array}{l}0.00 \\
0.00 \\
0.00\end{array}$ & $\begin{array}{l}0.00 \\
0.00 \\
0.00\end{array}$ & $\begin{array}{l}2.24 \\
1.54 \\
1.17\end{array}$ & $\begin{array}{l}2.03 \\
1.85 \\
3.41\end{array}$ & $\begin{array}{l}0.00 \\
0.00 \\
0.00\end{array}$ & $\begin{array}{l}0.00 \\
0.00 \\
0.00\end{array}$ & $\begin{array}{l}2.03 \\
1.85 \\
3.41\end{array}$ & $\begin{array}{l}4.27 \\
3.39 \\
4.38\end{array}$ & $\begin{array}{l}0.00 \\
0.00 \\
0.00\end{array}$ & $\begin{array}{l}0.00 \\
0.00 \\
0.00\end{array}$ & $\begin{array}{l}4.27 \\
3.39 \\
6.58\end{array}$ \\
\hline $\operatorname{mid}$ & $\begin{array}{r}0-1000 \\
1-2000 \\
2-3000 \\
\text { holgate }\end{array}$ & $\begin{array}{l}0.08 \\
0.03 \\
0.00\end{array}$ & $\begin{array}{l}1.45 \\
0.47 \\
0.00\end{array}$ & $\begin{array}{l}0.00 \\
0.00 \\
0.00\end{array}$ & $\begin{array}{l}1.53 \\
0.50 \\
0.00\end{array}$ & $\begin{array}{l}0.14 \\
0.11 \\
0.01\end{array}$ & $\begin{array}{l}0.29 \\
0.59 \\
0.21\end{array}$ & $\begin{array}{l}0.00 \\
0.00 \\
0.00\end{array}$ & $\begin{array}{l}0.43 \\
0.70 \\
0.22\end{array}$ & $\begin{array}{l}0.22 \\
0.14 \\
0.01\end{array}$ & $\begin{array}{l}1.74 \\
1.06 \\
0.21\end{array}$ & $\begin{array}{l}0.00 \\
0.00 \\
0.00\end{array}$ & $\begin{array}{l}1.96 \\
1.20 \\
0.22\end{array}$ \\
\hline & $\begin{array}{l}0-1000 \\
1-2000 \\
2-3000\end{array}$ & $\begin{array}{l}0.61 \\
0.18 \\
0.00\end{array}$ & $\begin{array}{l}0.00 \\
0.00 \\
0.00\end{array}$ & $\begin{array}{l}0.00 \\
0.00 \\
0.00\end{array}$ & $\begin{array}{l}0.61 \\
0.18 \\
0.00\end{array}$ & $\begin{array}{l}0.39 \\
0.44 \\
0.12\end{array}$ & $\begin{array}{l}0.00 \\
0.00 \\
0.00\end{array}$ & $\begin{array}{l}0.00 \\
0.00 \\
0.00\end{array}$ & $\begin{array}{l}0.39 \\
0.44 \\
0.12\end{array}$ & $\begin{array}{l}1.00 \\
0.62 \\
0.12\end{array}$ & $\begin{array}{l}0.00 \\
0.00 \\
0.00\end{array}$ & $\begin{array}{l}0.00 \\
0.00 \\
0.00\end{array}$ & $\begin{array}{l}1.00 \\
0.62 \\
0.12\end{array}$ \\
\hline
\end{tabular}




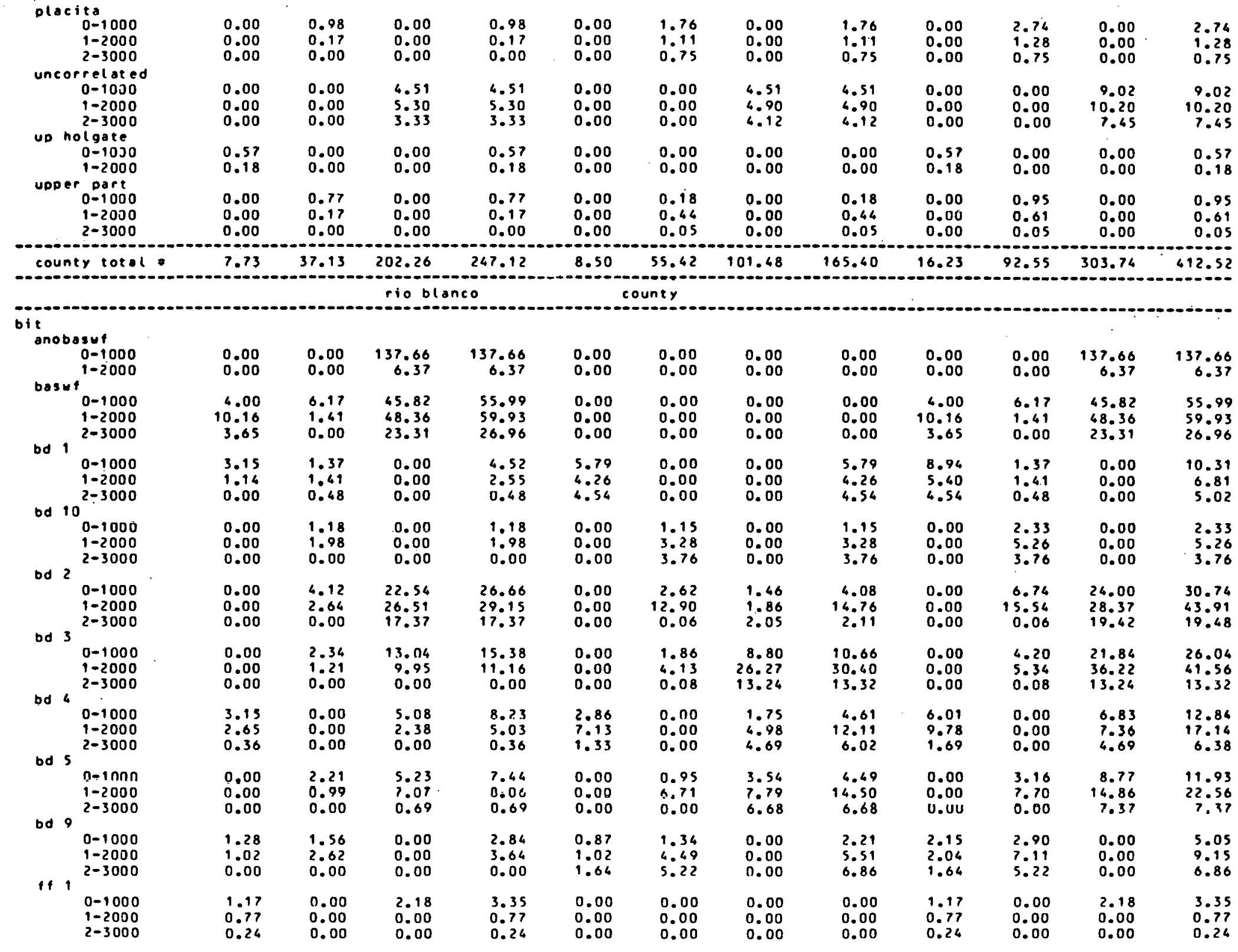




\begin{tabular}{|c|c|c|c|c|c|c|c|c|c|c|c|c|c|}
\hline ff & $\begin{array}{r}0-1000 \\
1-2000 \\
11^{2-3000}\end{array}$ & $\begin{array}{l}1.20 \\
0.90 \\
0.17\end{array}$ & $\begin{array}{l}3.65 \\
2.58 \\
0.45\end{array}$ & $\begin{array}{l}7.31 \\
4.27 \\
0.00\end{array}$ & $\begin{array}{r}12.16 \\
7.75 \\
0.62\end{array}$ & $\begin{array}{l}0.00 \\
0.00 \\
0.31\end{array}$ & $\begin{array}{l}0.00 \\
0.28 \\
0.61\end{array}$ & $\begin{array}{l}0.93 \\
3.20 \\
6.31\end{array}$ & $\begin{array}{l}0.93 \\
3.48 \\
7.23\end{array}$ & $\begin{array}{l}1.20 \\
0.90 \\
0.48\end{array}$ & $\begin{array}{l}3.65 \\
2.86 \\
1.06\end{array}$ & $\begin{array}{l}8.24 \\
7.47 \\
6.31\end{array}$ & $\begin{array}{r}13.09 \\
11.23 \\
7.85\end{array}$ \\
\hline $1 f$ & $\begin{array}{r}0-1000 \\
1-2000 \\
12^{2-3000}\end{array}$ & $\begin{array}{l}3.01 \\
0.00 \\
0.00\end{array}$ & $\begin{array}{l}5.14 \\
3.59 \\
0.31\end{array}$ & $\begin{array}{r}10.53 \\
7.57 \\
1.46\end{array}$ & $\begin{array}{r}18.68 \\
19.16 \\
1.77\end{array}$ & $\begin{array}{l}5.49 \\
0.00 \\
0.00\end{array}$ & $\begin{array}{r}9.48 \\
22.95 \\
8.14\end{array}$ & $\begin{array}{l}9.69 \\
0.14 \\
0.05\end{array}$ & $\begin{array}{r}16.66 \\
23.09 \\
8.19\end{array}$ & $\begin{array}{l}8.50 \\
0.00 \\
0.00\end{array}$ & $\begin{array}{r}14.62 \\
26.54 \\
8.45\end{array}$ & $\begin{array}{r}12.22 \\
7.71 \\
1.51\end{array}$ & $\begin{array}{r}35.34 \\
34.25 \\
9.96\end{array}$ \\
\hline 11 & $\begin{array}{r}0-1000 \\
1-2000 \\
2-3000\end{array}$ & $\begin{array}{l}1.09 \\
1.1 ? \\
0.39\end{array}$ & $\begin{array}{l}1.68 \\
1.32 \\
0.33\end{array}$ & $\begin{array}{r}11.39 \\
0.00 \\
0.00\end{array}$ & $\begin{array}{r}14.08 \\
2.44 \\
0.72\end{array}$ & $\begin{array}{l}0.00 \\
0.00 \\
0.00\end{array}$ & $\begin{array}{l}0.00 \\
0.00 \\
0.00\end{array}$ & $\begin{array}{l}0.00 \\
0.00 \\
0.00\end{array}$ & $\begin{array}{l}0.00 \\
0.00 \\
0.00\end{array}$ & $\begin{array}{l}1.01 \\
1.12 \\
0.39\end{array}$ & $\begin{array}{l}1.68 \\
1.32 \\
0.33\end{array}$ & $\begin{array}{r}11.39 \\
0.00 \\
0.00\end{array}$ & $\begin{array}{r}14.08 \\
2.44 \\
0.72\end{array}$ \\
\hline 11 & $\begin{array}{r}0-1000 \\
1-2000 \\
14^{2-3000}\end{array}$ & $\begin{array}{l}5.46 \\
2.28 \\
0.00\end{array}$ & $\begin{array}{l}0.63 \\
0.02 \\
0.00\end{array}$ & $\begin{array}{l}3.71 \\
2.91 \\
0.73\end{array}$ & $\begin{array}{l}9.80 \\
5.21 \\
0.73\end{array}$ & $\begin{array}{r}5.18 \\
12.50 \\
8.59\end{array}$ & $\begin{array}{l}0.00 \\
0.00 \\
0.00\end{array}$ & $\begin{array}{l}0.00 \\
0.00 \\
0.00\end{array}$ & $\begin{array}{r}5.18 \\
12.50 \\
8.59\end{array}$ & $\begin{array}{r}10.64 \\
14.78 \\
8.59\end{array}$ & $\begin{array}{l}0.63 \\
0.02 \\
0.00\end{array}$ & $\begin{array}{l}3.79 \\
2.91 \\
0.73\end{array}$ & $\begin{array}{r}14.98 \\
17.71 \\
9.32\end{array}$ \\
\hline$f f$ & $\begin{array}{r}0-1000 \\
1-2000 \\
15^{2-3000}\end{array}$ & $\begin{array}{l}2.19 \\
0.00 \\
0.00\end{array}$ & $\begin{array}{l}8.70 \\
2.71 \\
0.40\end{array}$ & $\begin{array}{l}24.17 \\
23.69 \\
12.82\end{array}$ & $\begin{array}{l}35.06 \\
26.40 \\
13.22\end{array}$ & $\begin{array}{l}2.46 \\
0.00 \\
0.00\end{array}$ & $\begin{array}{r}3.19 \\
11.26 \\
7.00\end{array}$ & $\begin{array}{r}3.34 \\
4.41 \\
10.59\end{array}$ & $\begin{array}{r}8.99 \\
15.67 \\
17.59\end{array}$ & $\begin{array}{l}4.65 \\
0.00 \\
0.00\end{array}$ & $\begin{array}{r}11.89 \\
13.97 \\
7.40\end{array}$ & $\begin{array}{l}27.51 \\
28.10 \\
23.41\end{array}$ & $\begin{array}{l}44.05 \\
42.07 \\
30.81\end{array}$ \\
\hline if & $16^{0-1000} \begin{array}{r}1-2000 \\
\end{array}$ & $\begin{array}{l}0.47 \\
0.00\end{array}$ & $\begin{array}{l}2.09 \\
0.00\end{array}$ & $\begin{array}{r}16.32 \\
0.62\end{array}$ & $\begin{array}{r}18.88 \\
0.62\end{array}$ & $\begin{array}{l}0.00 \\
0.00\end{array}$ & $\begin{array}{l}0.00 \\
0.00\end{array}$ & $\begin{array}{l}6.25 \\
0.00\end{array}$ & $\begin{array}{l}6.25 \\
0.00 \\
i\end{array}$ & $\begin{array}{l}0.47 \\
0.00\end{array}$ & $\begin{array}{l}2.09 \\
0.00\end{array}$ & $\begin{array}{r}22.57 \\
0.62\end{array}$ & $\begin{array}{r}25.13 \\
0.62\end{array}$ \\
\hline$f t$ & $17^{0-1000} \begin{array}{r}1-2000 \\
2-3000\end{array}$ & $\begin{array}{l}9.91 \\
1.25 \\
0.00\end{array}$ & $\begin{array}{l}0.00 \\
0.00 \\
0.00\end{array}$ & $\begin{array}{l}6.98 \\
0.00 \\
0.00\end{array}$ & $\begin{array}{l}8.89 \\
1.25 \\
0.00\end{array}$ & $\begin{array}{l}1.41 \\
4.19 \\
0.22\end{array}$ & $\begin{array}{l}0.00 \\
0.00 \\
0.00\end{array}$ & $\begin{array}{l}0.00 \\
0.00 \\
0.00\end{array}$ & $\begin{array}{l}1.41 \\
4.19 \\
0.22\end{array}$ & $\begin{array}{l}3.32 \\
5.44 \\
0.22\end{array}$ & $\begin{array}{l}0.00 \\
0.00 \\
0.00\end{array}$ & $\begin{array}{l}6.98 \\
0.00 \\
0.00\end{array}$ & $\begin{array}{r}10.30 \\
5.64 \\
0.22\end{array}$ \\
\hline 11 & $18^{0-1000}$ & $\begin{array}{l}0.00 \\
0.00\end{array}$ & $\begin{array}{l}0.00 \\
0.00\end{array}$ & $\begin{array}{r}12.91 \\
5.54\end{array}$ & $\begin{array}{r}12.91 \\
5.54\end{array}$ & $\begin{array}{l}0.00 \\
0.00\end{array}$ & $\begin{array}{l}0.00 \\
0.00\end{array}$ & $\begin{array}{l}13.64 \\
40.21\end{array}$ & $\begin{array}{l}13.64 \\
40.21\end{array}$ & $\begin{array}{l}0.00 \\
0.00\end{array}$ & $\begin{array}{l}0.00 \\
0.00\end{array}$ & $\begin{array}{l}26.55 \\
45.75\end{array}$ & $\begin{array}{l}26.55 \\
45.75\end{array}$ \\
\hline$f$ & $\begin{array}{r}0-1000 \\
1-2000 \\
19^{2-3050}\end{array}$ & $\begin{array}{l}3.17 \\
2.35 \\
0.34\end{array}$ & $\begin{array}{l}0.00 \\
0.00 \\
0.00\end{array}$ & $\begin{array}{l}5.48 \\
0.00 \\
0.00\end{array}$ & $\begin{array}{l}8.65 \\
2.35 \\
0.34\end{array}$ & $\begin{array}{l}2.57 \\
6.67 \\
1.49\end{array}$ & $\begin{array}{l}0.00 \\
0.00 \\
0.00\end{array}$ & $\begin{array}{l}0.00 \\
0.00 \\
0.00\end{array}$ & $\begin{array}{l}2.57 \\
6.67 \\
1.49\end{array}$ & $\begin{array}{l}5.74 \\
9.02 \\
1.83\end{array}$ & $\begin{array}{l}0.00 \\
0.00 \\
0.00\end{array}$ & $\begin{array}{l}5.48 \\
0.00 \\
0.00\end{array}$ & $\begin{array}{r}11.22 \\
9.02 \\
1.83\end{array}$ \\
\hline 41 & $2^{0-1000}$ & 1.88 & 2.33 & 0.00 & 4.21 & 0.00 & 2.93 & 0.00 & 2.93 & 1.88 & 5.26 & 0.00 & 7.14 \\
\hline 1 & $\begin{array}{r}0-1000 \\
1-2000 \\
2-3000\end{array}$ & $\begin{array}{l}0.59 \\
0.22 \\
0.00\end{array}$ & $\begin{array}{l}0.00 \\
0.00 \\
0.00\end{array}$ & $\begin{array}{r}53.33 \\
6.64 \\
0.00\end{array}$ & $\begin{array}{r}53.92 \\
6.66 \\
0.00\end{array}$ & $\begin{array}{l}0.00 \\
0.00 \\
0.00\end{array}$ & $\begin{array}{l}0.00 \\
0.00 \\
0.00\end{array}$ & $\begin{array}{r}116.14 \\
241.02 \\
10.03\end{array}$ & $\begin{array}{r}116.14 \\
241.02 \\
10.03\end{array}$ & $\begin{array}{l}0.59 \\
0.22 \\
0.00\end{array}$ & $\begin{array}{l}0.00 \\
0.00 \\
0.00\end{array}$ & $\begin{array}{r}169.67 \\
247.46 \\
10.03\end{array}$ & $\begin{array}{r}170.06 \\
247.68 \\
10.03\end{array}$ \\
\hline 14 & $21^{0-1000} \begin{array}{r}1-2000 \\
\end{array}$ & $\begin{array}{l}1.81 \\
0.00\end{array}$ & $\begin{array}{l}8.68 \\
0.00\end{array}$ & $\begin{array}{r}20.43 \\
1.38\end{array}$ & $\begin{array}{r}30.92 \\
1.38\end{array}$ & $\begin{array}{l}0.00 \\
0.00\end{array}$ & $\begin{array}{l}0.05 \\
0.00\end{array}$ & $\begin{array}{r}13.13 \\
4.60\end{array}$ & $\begin{array}{r}13.18 \\
4.60\end{array}$ & $\begin{array}{l}1.81 \\
0.00\end{array}$ & $\begin{array}{l}8.73 \\
0.00\end{array}$ & $\begin{array}{r}33.56 \\
5.98\end{array}$ & $\begin{array}{r}44.10 \\
5.98\end{array}$ \\
\hline 11 & $22^{0-1000} \begin{array}{c}1-2000 \\
.000\end{array}$ & $\begin{array}{l}0.00 \\
0.00\end{array}$ & $\begin{array}{l}2.18 \\
0.00\end{array}$ & $\begin{array}{l}4.96 \\
3.98\end{array}$ & $\begin{array}{l}7.14 \\
3.98\end{array}$ & $\begin{array}{l}0.00 \\
0.00\end{array}$ & $\begin{array}{l}2.19 \\
0.00\end{array}$ & $\begin{array}{r}1.27 \\
14.15\end{array}$ & $\begin{array}{r}3.46 \\
14.15\end{array}$ & $\begin{array}{l}0.00 \\
0.00\end{array}$ & $\begin{array}{l}4.37 \\
0.00\end{array}$ & $\begin{array}{r}6.23 \\
18.13\end{array}$ & $\begin{array}{l}10.60 \\
18.13\end{array}$ \\
\hline if & $\begin{array}{r}0-1000 \\
1-2000 \\
23\end{array}$ & $\begin{array}{l}2.21 \\
0.06 \\
0.00\end{array}$ & $\begin{array}{l}0.00 \\
0.00 \\
0.00\end{array}$ & $\begin{array}{l}8.17 \\
1.82 \\
0.00\end{array}$ & $\begin{array}{r}10.38 \\
1.88 \\
0.00\end{array}$ & $\begin{array}{l}0.25 \\
0.00 \\
0.00\end{array}$ & $\begin{array}{l}0.37 \\
9.99 \\
2.33\end{array}$ & $\begin{array}{l}7.69 \\
0.94 \\
5.82\end{array}$ & $\begin{array}{l}8.31 \\
2.93 \\
8.15\end{array}$ & $\begin{array}{l}2.46 \\
0.06 \\
0.00\end{array}$ & $\begin{array}{l}0.37 \\
1.99 \\
2.33\end{array}$ & $\begin{array}{r}15.86 \\
2.76 \\
5.82\end{array}$ & $\begin{array}{r}18.69 \\
6.81 \\
8.15\end{array}$ \\
\hline if & $\begin{array}{r}0-1000 \\
1-2000 \\
2-3000\end{array}$ & $\begin{array}{l}1.91 \\
0.00 \\
0.00\end{array}$ & $\begin{array}{r}12.53 \\
0.00 \\
0.00\end{array}$ & $\begin{array}{l}3.15 \\
2.44 \\
0.00\end{array}$ & $\begin{array}{r}17.59 \\
2.44 \\
0.00\end{array}$ & $\begin{array}{l}0.08 \\
0.00 \\
0.00\end{array}$ & $\begin{array}{r}13.61 \\
1.89 \\
0.21\end{array}$ & $\begin{array}{r}1.47 \\
12.22 \\
0.00\end{array}$ & $\begin{array}{r}15.16 \\
14.11 \\
0.21\end{array}$ & $\begin{array}{l}1.44 \\
0.00 \\
0.00\end{array}$ & $\begin{array}{r}26.14 \\
1.89 \\
0.21\end{array}$ & $\begin{array}{r}4.02 \\
14.66 \\
0.00\end{array}$ & $\begin{array}{r}32.75 \\
16.55 \\
0.21\end{array}$ \\
\hline 4 & $\begin{array}{r}0-1000 \\
1-2000 \\
2-3000 \\
3\end{array}$ & $\begin{array}{l}4.07 \\
0.75 \\
0.00\end{array}$ & $\begin{array}{l}3.61 \\
0.00 \\
0.00\end{array}$ & $\begin{array}{l}0.00 \\
0.00 \\
0.00\end{array}$ & $\begin{array}{l}7.48 \\
0.75 \\
0.00\end{array}$ & $\begin{array}{l}2.39 \\
0.93 \\
0.67\end{array}$ & $\begin{array}{l}0.00 \\
4.23 \\
2.83\end{array}$ & $\begin{array}{l}0.00 \\
0.00 \\
0.00\end{array}$ & $\begin{array}{l}2.39 \\
5.16 \\
3.30\end{array}$ & $\begin{array}{l}6.46 \\
1.68 \\
0.47\end{array}$ & $\begin{array}{l}3.61 \\
4.23 \\
2.83\end{array}$ & $\begin{array}{l}0.00 \\
0.00 \\
0.00\end{array}$ & $\begin{array}{l}9.87 \\
5.91 \\
3.30\end{array}$ \\
\hline & $0-1000$ & 0.84 & 0.00 & 29.14 & 29.98 & 0.81 & 0.00 & 36.78 & 37.59 & 1.65 & 0.00 & 65.92 & 67.57 \\
\hline
\end{tabular}




\begin{tabular}{|c|c|c|c|c|c|c|c|c|c|c|c|c|c|}
\hline 186 & $\begin{array}{l}1-2000 \\
2-3000\end{array}$ & $\begin{array}{l}0.73 \\
0.00\end{array}$ & $\begin{array}{l}0.00 \\
0.00\end{array}$ & $\begin{array}{r}21.03 \\
3.29\end{array}$ & $\begin{array}{r}21.76 \\
3.29\end{array}$ & $\begin{array}{l}1.31 \\
0.99\end{array}$ & $\begin{array}{l}0.00 \\
0.00\end{array}$ & $\begin{array}{l}34.47 \\
39.69\end{array}$ & $\begin{array}{l}35.78 \\
40.68\end{array}$ & $\begin{array}{l}2.04 \\
0.99\end{array}$ & $\begin{array}{l}0.00 \\
0.00\end{array}$ & $\begin{array}{l}55.50 \\
42.98\end{array}$ & $\begin{array}{l}57.54 \\
43.97\end{array}$ \\
\hline 5 & $\begin{array}{l}0-1000 \\
1-2000 \\
2-3000\end{array}$ & $\begin{array}{l}0.00 \\
0.00 \\
0.00\end{array}$ & $\begin{array}{l}1.69 \\
0.97 \\
0.00\end{array}$ & $\begin{array}{r}10.10 \\
9.87 \\
1.60\end{array}$ & $\begin{array}{r}11.79 \\
10.84 \\
1.60\end{array}$ & $\begin{array}{l}0.00 \\
0.00 \\
0.00\end{array}$ & $\begin{array}{l}0.00 \\
0.00 \\
0.00\end{array}$ & $\begin{array}{r}7.94 \\
17.45 \\
16.81\end{array}$ & $\begin{array}{r}7.94 \\
17.45 \\
16.81\end{array}$ & $\begin{array}{l}0.00 \\
0.00 \\
0.00\end{array}$ & $\begin{array}{l}1.69 \\
0.97 \\
0.00\end{array}$ & $\begin{array}{l}18.04 \\
27.32 \\
18.41\end{array}$ & $\begin{array}{l}19.73 \\
28.29 \\
18.49\end{array}$ \\
\hline 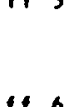 & $\begin{array}{l}0-1000 \\
1-2000 \\
2-3000\end{array}$ & $\begin{array}{l}0.88 \\
0.50 \\
0.00\end{array}$ & $\begin{array}{l}0.00 \\
0.00 \\
0.00\end{array}$ & $\begin{array}{l}3.20 \\
3.71 \\
2.01\end{array}$ & $\begin{array}{l}4.08 \\
4.21 \\
2.01\end{array}$ & $\begin{array}{l}0.00 \\
0.00 \\
0.00\end{array}$ & $\begin{array}{l}0.00 \\
0.00 \\
0.00\end{array}$ & $\begin{array}{l}2.36 \\
2.90 \\
2.36\end{array}$ & $\begin{array}{l}2.36 \\
2.90 \\
2.36\end{array}$ & $\begin{array}{l}0.88 \\
0.50 \\
0.00\end{array}$ & $\begin{array}{l}0.00 \\
0.00 \\
0.00\end{array}$ & $\begin{array}{l}5.56 \\
6.61 \\
4.37\end{array}$ & $\begin{array}{l}6.44 \\
7.11 \\
4.37\end{array}$ \\
\hline$f+7$ & $\begin{array}{l}0-1000 \\
1-2000 \\
2-3000\end{array}$ & $\begin{array}{l}1.15 \\
0.66 \\
0.00\end{array}$ & $\begin{array}{l}2.19 \\
1.70 \\
1.10\end{array}$ & $\begin{array}{l}0.00 \\
0.00 \\
0.00\end{array}$ & $\begin{array}{l}3.34 \\
2.36 \\
1.10\end{array}$ & $\begin{array}{l}0.00 \\
0.00 \\
0.00\end{array}$ & $\begin{array}{l}1.93 \\
2.23 \\
1.76\end{array}$ & $\begin{array}{l}0.00 \\
0.00 \\
0.00\end{array}$ & $\begin{array}{l}1.93 \\
2.23 \\
1.76\end{array}$ & $\begin{array}{l}1.15 \\
0.66 \\
0.00\end{array}$ & $\begin{array}{l}4.12 \\
3.93 \\
2.86\end{array}$ & $\begin{array}{l}0.00 \\
0.00 \\
0.00\end{array}$ & $\begin{array}{l}5.27 \\
4.59 \\
2.86\end{array}$ \\
\hline+8 & $\begin{array}{l}0-1000 \\
1-2000 \\
2-3000\end{array}$ & $\begin{array}{l}2.77 \\
0.75 \\
0.16\end{array}$ & $\begin{array}{l}0.00 \\
0.00 \\
0.00\end{array}$ & $\begin{array}{r}16.91 \\
3.96 \\
1.80\end{array}$ & $\begin{array}{r}19.68 \\
4.71 \\
1.96\end{array}$ & $\begin{array}{l}0.00 \\
0.00 \\
0.00\end{array}$ & $\begin{array}{l}0.29 \\
0.00 \\
0.00\end{array}$ & $\begin{array}{l}20.92 \\
17.34 \\
5.08\end{array}$ & $\begin{array}{r}21.21 \\
17.34 \\
5.08\end{array}$ & $\begin{array}{l}2.77 \\
0.75 \\
0.16\end{array}$ & $\begin{array}{l}0.29 \\
0.00 \\
0.00\end{array}$ & $\begin{array}{r}37.83 \\
21.30 \\
6.88\end{array}$ & $\begin{array}{r}40.89 \\
22.05 \\
7.04\end{array}$ \\
\hline if 9 & $\begin{array}{l}0-1000 \\
1-2000 \\
2-3000\end{array}$ & $\begin{array}{l}0.00 \\
0.00 \\
0.00\end{array}$ & $\begin{array}{l}3.06 \\
1.79 \\
0.00\end{array}$ & $\begin{array}{r}34.18 \\
20.47 \\
6.41\end{array}$ & $\begin{array}{r}37.24 \\
22.26 \\
6.41\end{array}$ & $\begin{array}{l}0.00 \\
0.00 \\
0.00\end{array}$ & $\begin{array}{l}2.56 \\
1.30 \\
1.61\end{array}$ & $\begin{array}{l}15.81 \\
18.90 \\
27.20\end{array}$ & $\begin{array}{l}18.37 \\
20.20 \\
28.81\end{array}$ & $\begin{array}{l}0.00 \\
0.00 \\
0.00\end{array}$ & $\begin{array}{l}5.62 \\
3.09 \\
1.61\end{array}$ & $\begin{array}{l}49.99 \\
39.37 \\
33.61\end{array}$ & $\begin{array}{l}55.61 \\
42.46 \\
35.22\end{array}$ \\
\hline goff & $\begin{array}{l}0-1000 \\
1-2000 \\
2-3000 \\
1\end{array}$ & $\begin{array}{l}2.42 \\
0.52 \\
0.00\end{array}$ & $\begin{array}{l}2.61 \\
3.21 \\
0.69\end{array}$ & $\begin{array}{l}1.70 \\
0.00 \\
0.00\end{array}$ & $\begin{array}{l}6.73 \\
3.73 \\
0.69\end{array}$ & $\begin{array}{l}0.00 \\
0.00 \\
0.00\end{array}$ & $\begin{array}{l}1.13 \\
2.75 \\
2.41\end{array}$ & $\begin{array}{l}0.00 \\
0.00 \\
0.00\end{array}$ & $\begin{array}{l}1.13 \\
2.75 \\
2.41\end{array}$ & $\begin{array}{l}2.42 \\
0.52 \\
0.00\end{array}$ & $\begin{array}{l}3.74 \\
5.96 \\
3.10\end{array}$ & $\begin{array}{l}1.70 \\
0.00 \\
0.00\end{array}$ & $\begin{array}{l}7.86 \\
6.48 \\
3.10\end{array}$ \\
\hline goft & $\begin{array}{l}0-1000 \\
1-2000 \\
i=3000 \\
10\end{array}$ & $\begin{array}{l}0.72 \\
0.63 \\
0.58\end{array}$ & $\begin{array}{l}0.16 \\
0.00 \\
0.00\end{array}$ & $\begin{array}{l}0.00 \\
0.00 \\
0.00\end{array}$ & $\begin{array}{l}0.88 \\
0.63 \\
0.58\end{array}$ & $\begin{array}{l}0.26 \\
0.32 \\
0.40\end{array}$ & $\begin{array}{l}0.00 \\
0.00 \\
0.00\end{array}$ & $\begin{array}{l}0.00 \\
0.00 \\
0.00\end{array}$ & $\begin{array}{l}0.26 \\
0.32 \\
0.40\end{array}$ & $\begin{array}{l}0.98 \\
0.95 \\
0.98\end{array}$ & $\begin{array}{l}0.16 \\
0.00 \\
0.00\end{array}$ & $\begin{array}{l}0.00 \\
0.00 \\
0.00\end{array}$ & $\begin{array}{l}1.14 \\
0.95 \\
0.98\end{array}$ \\
\hline $90 f t$ & $\begin{array}{l}0-1000 \\
1-2000 \\
2-3000 \\
11\end{array}$ & $\begin{array}{l}1.56 \\
9.17 \\
0.27\end{array}$ & $\begin{array}{l}0.00 \\
0.00 \\
0.00\end{array}$ & $\begin{array}{l}3.44 \\
0.00 \\
0.00\end{array}$ & $\begin{array}{l}5.00 \\
1.17 \\
0.27\end{array}$ & $\begin{array}{l}0.56 \\
0.41 \\
3.29\end{array}$ & $\begin{array}{l}0.00 \\
0.00 \\
0.00\end{array}$ & $\begin{array}{l}1.72 \\
0.00 \\
0.00\end{array}$ & $\begin{array}{l}2.28 \\
0.41 \\
3.29\end{array}$ & $\begin{array}{l}2.12 \\
1.58 \\
3.56\end{array}$ & $\begin{array}{l}0.00 \\
0.00 \\
0.00\end{array}$ & $\begin{array}{l}5.16 \\
0.00 \\
0.00\end{array}$ & $\begin{array}{l}7.28 \\
1.58 \\
3.56\end{array}$ \\
\hline $90+1$ & $\begin{array}{l}0-1000 \\
1-2000 \\
2-3000 \\
12\end{array}$ & $\begin{array}{l}1.59 \\
1.47 \\
1.04\end{array}$ & $\begin{array}{l}3.74 \\
2.84 \\
0.00\end{array}$ & $\begin{array}{l}6.07 \\
0.00 \\
0.00\end{array}$ & $\begin{array}{r}11.40 \\
4.31 \\
1.04\end{array}$ & $\begin{array}{l}2.73 \\
1.40 \\
3.46\end{array}$ & $\begin{array}{r}3.75 \\
5.30 \\
10.58\end{array}$ & $\begin{array}{l}5.21 \\
6.59 \\
0.00\end{array}$ & $\begin{array}{l}11.69 \\
13.29 \\
14.04\end{array}$ & $\begin{array}{l}4.32 \\
2.87 \\
4.50\end{array}$ & $\begin{array}{r}7.49 \\
8.14 \\
10.58\end{array}$ & $\begin{array}{r}11.28 \\
6.59 \\
0.00\end{array}$ & $\begin{array}{l}23.09 \\
17.60 \\
15.08\end{array}$ \\
\hline gott & $\begin{array}{l}0-1000 \\
1-2000 \\
2-3000 \\
13\end{array}$ & $\begin{array}{l}0.74 \\
0.32 \\
0.00\end{array}$ & $\begin{array}{l}0.00 \\
0.00 \\
0.00\end{array}$ & $\begin{array}{r}39.97 \\
33.07 \\
1.35\end{array}$ & $\begin{array}{r}40.71 \\
33.39 \\
1.35\end{array}$ & $\begin{array}{l}0.00 \\
0.00 \\
0.00\end{array}$ & $\begin{array}{l}0.00 \\
0.00 \\
0.00\end{array}$ & $\begin{array}{l}43.75 \\
96.85 \\
21.05\end{array}$ & $\begin{array}{l}43.75 \\
96.85 \\
29.05\end{array}$ & $\begin{array}{l}0.74 \\
0.32 \\
0.00\end{array}$ & $\begin{array}{l}0.00 \\
0.00 \\
0.00\end{array}$ & $\begin{array}{r}83.72 \\
129.92 \\
22.40\end{array}$ & $\begin{array}{r}84.46 \\
130.24 \\
22.40\end{array}$ \\
\hline got & $\begin{array}{l}0-1000 \\
1-2000 \\
2-3000 \\
14\end{array}$ & $\begin{array}{l}1.07 \\
0.35 \\
0.00\end{array}$ & $\begin{array}{l}0.79 \\
0.00 \\
0.00\end{array}$ & $\begin{array}{l}0.00 \\
0.00 \\
0.00\end{array}$ & $\begin{array}{l}1.86 \\
0.35 \\
0.00\end{array}$ & $\begin{array}{l}0.72 \\
1.09 \\
2.97\end{array}$ & $\begin{array}{l}0.00 \\
0.00 \\
0.00\end{array}$ & $\begin{array}{l}0.00 \\
0.00 \\
0.00\end{array}$ & $\begin{array}{l}0.72 \\
1.09 \\
2.97\end{array}$ & $\begin{array}{l}1.79 \\
1.44 \\
2.97\end{array}$ & $\begin{array}{l}0.79 \\
0.00 \\
0.00\end{array}$ & $\begin{array}{l}0.00 \\
0.00 \\
0.00\end{array}$ & $\begin{array}{l}2.58 \\
1.66 \\
2.97\end{array}$ \\
\hline gott & $\begin{array}{l}0-1000 \\
15 \\
0-1000\end{array}$ & $\begin{array}{l}0.00 \\
0.49\end{array}$ & $\begin{array}{l}1.02 \\
0.00\end{array}$ & $\begin{array}{l}0.00 \\
u .000\end{array}$ & $\begin{array}{l}1.02 \\
0.49\end{array}$ & $\begin{array}{l}0.00 \\
0.00\end{array}$ & $\begin{array}{l}0.00 \\
0.0 n\end{array}$ & $\begin{array}{l}0.00 \\
0.00\end{array}$ & $\begin{array}{l}0.00 \\
0.00\end{array}$ & $\begin{array}{l}0.00 \\
0.49\end{array}$ & $\begin{array}{l}1.02 \\
0.00\end{array}$ & $\begin{array}{l}0.00 \\
0.00\end{array}$ & $\begin{array}{l}1.02 \\
0.49\end{array}$ \\
\hline $\begin{array}{l}\text { gott } \\
\text { gott } \\
\text { gotf }\end{array}$ & $\begin{array}{l}77 \\
0-1000 \\
19 \\
0-1000 \\
2\end{array}$ & $\begin{array}{l}0.00 \\
0.00\end{array}$ & $\begin{array}{l}0.00 \\
0.00\end{array}$ & $\begin{array}{r}3.69 \\
15.78\end{array}$ & $\begin{array}{r}3.69 \\
15.78\end{array}$ & $\begin{array}{l}0.00 \\
0.00\end{array}$ & $\begin{array}{l}0.00 \\
0.00\end{array}$ & $\begin{array}{r}0.00 \\
80.42\end{array}$ & $\begin{array}{r}0.00 \\
80.42\end{array}$ & $\begin{array}{l}0.00 \\
0.00\end{array}$ & $\begin{array}{l}0.00 \\
0.00\end{array}$ & $\begin{array}{r}3.69 \\
96.20\end{array}$ & $\begin{array}{r}3.69 \\
96.20\end{array}$ \\
\hline $90+4$ & $\begin{array}{l}0-1000 \\
1-2000 \\
2-3000 \\
20\end{array}$ & $\begin{array}{l}5.57 \\
0.50 \\
0.55\end{array}$ & $\begin{array}{l}9.92 \\
0.33 \\
0.00\end{array}$ & $\begin{array}{l}0.00 \\
0.00 \\
0.00\end{array}$ & $\begin{array}{r}15.49 \\
0.83 \\
0.55\end{array}$ & $\begin{array}{l}6.52 \\
2.05 \\
1.77\end{array}$ & $\begin{array}{l}0.00 \\
0.00 \\
0.00\end{array}$ & $\begin{array}{l}0.00 \\
0.00 \\
0.00\end{array}$ & $\begin{array}{l}6.52 \\
2.05 \\
1.77\end{array}$ & $\begin{array}{r}12.09 \\
2.55 \\
2.32\end{array}$ & $\begin{array}{l}9.92 \\
0.33 \\
0.00\end{array}$ & $\begin{array}{l}0.00 \\
0.00 \\
0.00\end{array}$ & $\begin{array}{r}22.01 \\
2.88 \\
2.32\end{array}$ \\
\hline & $0=1000$ & n.hth & 0.00 & 0.00 & 0.66 & 1.74 & 0.00 & 0.00 & 1.74 & 2.40 & 0.00 & 0.00 & 2.40 \\
\hline
\end{tabular}




\begin{tabular}{|c|c|c|c|c|c|c|c|c|c|c|c|c|c|c|}
\hline gott & $\begin{array}{l}21 \\
0-1000 \\
22\end{array}$ & & 1.71 & 0.00 & 0.00 & 1.79 & 4.31 & 0.00 & 0.00 & 4.31 & 6.02 & 0.00 & 0.00 & 6.02 \\
\hline 9044 & $\begin{array}{l}0-1000 \\
3\end{array}$ & & 2.02 & 3.06 & 0.01 & 5.09 & 4.74 & 2.89 & 0.00 & 7.63 & 6.76 & 5.95 & 0.01 & 12.72 \\
\hline gott & ${ }_{4}^{0-1000}$ & & 0.65 & 12.92 & 14.01 & 27.58 & 0.00 & 1.91 & 9.20 & 11.11 & 0.65 & 14.83 & 23.21 & 38.69 \\
\hline goft & $\begin{array}{l}0-1000 \\
1-2000 \\
5\end{array}$ & & $\begin{array}{l}1.88 \\
0.00\end{array}$ & $\begin{array}{l}0.00 \\
0.00\end{array}$ & $\begin{array}{l}0.00 \\
0.00\end{array}$ & $\begin{array}{l}1.88 \\
0.00\end{array}$ & $\begin{array}{l}1.51 \\
1.03\end{array}$ & $\begin{array}{l}0.00 \\
0.00\end{array}$ & $\begin{array}{l}0.00 \\
0.00\end{array}$ & $\begin{array}{l}1.51 \\
1.03\end{array}$ & $\begin{array}{l}3.39 \\
9.03\end{array}$ & $\begin{array}{l}0.00 \\
0.00\end{array}$ & $\begin{array}{l}0.00 \\
0.00\end{array}$ & $\begin{array}{l}3.39 \\
1.03\end{array}$ \\
\hline gotf & $\begin{array}{l}0-1000 \\
6\end{array}$ & & 1.21 & 1.13 & 0.00 & 2.34 & 0.00 & 0.00 & 0,00 & 0.00 & 1.21 & 1.13 & 0.00 & 2.34 \\
\hline gott & $l^{0-1000}$ & & 0.38 & 2.51 & 31.398 & 34.27 & 0.00 & 3.49 & 7.22 & 10.71 & 0.38 & 6.00 & 38.60 & 44.98 \\
\hline gott & $\begin{array}{l}0-1000 \\
1-2000 \\
2=3000 \\
8\end{array}$ & & $\begin{array}{l}2.42 \\
0.32 \\
0.00\end{array}$ & $\begin{array}{l}0.00 \\
0.00 \\
0.00\end{array}$ & $\begin{array}{l}0.00 \\
0.00 \\
0.00\end{array}$ & $\begin{array}{l}2.42 \\
0.32 \\
0.00\end{array}$ & $\begin{array}{l}0.62 \\
1.00 \\
2.09\end{array}$ & $\begin{array}{l}0.00 \\
0.00 \\
0.00\end{array}$ & $\begin{array}{l}0.00 \\
0.00 \\
0.00\end{array}$ & $\begin{array}{l}0.62 \\
1.00 \\
2.09\end{array}$ & $\begin{array}{l}3.04 \\
1.32 \\
2.09\end{array}$ & $\begin{array}{l}0.00 \\
0.00 \\
0.00\end{array}$ & $\begin{array}{l}0.00 \\
0.00 \\
0.00\end{array}$ & $\begin{array}{l}3.06 \\
1.32 \\
2.09\end{array}$ \\
\hline opte & $\begin{array}{l}0-1000 \\
1-2000 \\
2-3000 \\
9\end{array}$ & & $\begin{array}{l}0.00 \\
0.00 \\
0.00\end{array}$ & $\begin{array}{l}1.73 \\
0.00 \\
0.00\end{array}$ & $\begin{array}{l}4.58 \\
3.95 \\
3.82\end{array}$ & $\begin{array}{l}6.31 \\
3.95 \\
3.82\end{array}$ & $\begin{array}{l}0.00 \\
0.00 \\
0.00\end{array}$ & $\begin{array}{l}0.00 \\
0.00 \\
0.00\end{array}$ & $\begin{array}{l}1.84 \\
1.26 \\
7.00\end{array}$ & $\begin{array}{l}1.84 \\
1.24 \\
7.00\end{array}$ & $\begin{array}{l}0.00 \\
0.00 \\
0.00\end{array}$ & $\begin{array}{l}1.73 \\
0.00 \\
0.00\end{array}$ & $\begin{array}{r}6.62 \\
5.19 \\
10.82\end{array}$ & $\begin{array}{r}8.15 \\
5.19 \\
10.82\end{array}$ \\
\hline kerst? & $\begin{array}{l}0-1000 \\
1-2000 \\
2-3000\end{array}$ & & $\begin{array}{l}0.00 \\
0.00 \\
0.00\end{array}$ & $\begin{array}{l}0.00 \\
0.00 \\
0.00\end{array}$ & $\begin{array}{l}4.78 \\
0.83 \\
0.00\end{array}$ & $\begin{array}{l}4.78 \\
0.83 \\
0.00\end{array}$ & $\begin{array}{l}0.00 \\
0.00 \\
0.00\end{array}$ & $\begin{array}{l}0.00 \\
0.00 \\
0.00\end{array}$ & $\begin{array}{l}2.15 \\
2.13 \\
2.93\end{array}$ & $\begin{array}{l}2.15 \\
2.13 \\
2.93\end{array}$ & $\begin{array}{l}0.00 \\
0.00 \\
0.00\end{array}$ & $\begin{array}{l}0.00 \\
0.00 \\
0.00\end{array}$ & $\begin{array}{l}0.93 \\
2.96 \\
2.93\end{array}$ & $\begin{array}{l}6.93 \\
2.96 \\
2.93\end{array}$ \\
\hline Iion & $\begin{array}{l}0-1000 \\
1+2000 \\
2-3000 \\
c\end{array}$ & 1 & $\begin{array}{l}0.00 \\
0.00 \\
0.00\end{array}$ & $\begin{array}{l}0.00 \\
0.00 \\
0.00\end{array}$ & $\begin{array}{l}6.21 \\
6.67 \\
0.00\end{array}$ & $\begin{array}{l}6.21 \\
6.67 \\
0.00\end{array}$ & $\begin{array}{l}0.00 \\
0.00 \\
0.00\end{array}$ & $\begin{array}{l}0.00 \\
0.00 \\
0.00\end{array}$ & $\begin{array}{r}3.22 \\
12.65 \\
20.46\end{array}$ & $\begin{array}{r}3.22 \\
12.65 \\
20.46\end{array}$ & $\begin{array}{l}0.00 \\
0.00 \\
0.00\end{array}$ & $\begin{array}{l}0.00 \\
0.00 \\
0.00\end{array}$ & $\begin{array}{r}9.43 \\
19.32 \\
20.46\end{array}$ & $\begin{array}{r}9.43 \\
19.32 \\
20.46\end{array}$ \\
\hline lion & $\begin{array}{l}0-1000 \\
1-2000 \\
2-3000 \\
\text { canyon }\end{array}$ & 10 & $\begin{array}{l}0.47 \\
0.00 \\
0.00\end{array}$ & $\begin{array}{l}3.55 \\
1.90 \\
0.04\end{array}$ & $\begin{array}{l}0.02 \\
0.00 \\
0.00\end{array}$ & $\begin{array}{l}4.04 \\
1.90 \\
0.04\end{array}$ & $\begin{array}{l}0.00 \\
0.00 \\
0.00\end{array}$ & $\begin{array}{l}0.32 \\
0.00 \\
0.00\end{array}$ & $\begin{array}{l}0.00 \\
0.00 \\
0.00\end{array}$ & $\begin{array}{l}0.32 \\
0.00 \\
0.00\end{array}$ & $\begin{array}{l}0.47 \\
0.00 \\
0.00\end{array}$ & $\begin{array}{l}3.87 \\
1.90 \\
0.04\end{array}$ & $\begin{array}{l}0.02 \\
0.00 \\
0.00\end{array}$ & $\begin{array}{l}4.36 \\
1.90 \\
0.04\end{array}$ \\
\hline lion & $\begin{array}{l}0-1000 \\
1-2000 \\
2-3000 \\
\text { canyon }\end{array}$ & $11^{\circ}$ & $\begin{array}{l}0.00 \\
0.00 \\
0.00\end{array}$ & $\begin{array}{l}0.00 \\
0.00 \\
0.00\end{array}$ & $\begin{array}{l}28.57 \\
17.22 \\
13.57\end{array}$ & $\begin{array}{l}28.57 \\
17.22 \\
13.57\end{array}$ & $\begin{array}{l}0.00 \\
0.00 \\
0.00\end{array}$ & $\begin{array}{l}0.00 \\
0.00 \\
0.00\end{array}$ & $\begin{array}{l}0.00 \\
0.00 \\
0.00\end{array}$ & $\begin{array}{l}0.00 \\
0.00 \\
0.00\end{array}$ & $\begin{array}{l}0.00 \\
0.00 \\
0.00\end{array}$ & $\begin{array}{l}0.00 \\
0.00 \\
0.00\end{array}$ & $\begin{array}{l}28.57 \\
17.22 \\
13.57\end{array}$ & $\begin{array}{l}28.57 \\
17.22 \\
13.57\end{array}$ \\
\hline Ition & $\begin{array}{l}0-1000 \\
1-2000 \\
\text { canyon }\end{array}$ & 12 & $\begin{array}{l}2.25 \\
0.19\end{array}$ & $\begin{array}{l}0.00 \\
0.00\end{array}$ & $\begin{array}{l}0.00 \\
0.00\end{array}$ & $\begin{array}{l}2.25 \\
0.19\end{array}$ & $\begin{array}{l}0.00 \\
0.00\end{array}$ & $\begin{array}{l}0.00 \\
0.00\end{array}$ & $\begin{array}{l}0.00 \\
0.00\end{array}$ & $\begin{array}{l}0.00 \\
0.00\end{array}$ & $\begin{array}{l}2.25 \\
0.19\end{array}$ & $\begin{array}{l}0.00 \\
0.00\end{array}$ & $\begin{array}{l}0.00 \\
0.00\end{array}$ & $\begin{array}{l}2.25 \\
0.19\end{array}$ \\
\hline lion & $\begin{array}{l}0-1000 \\
1-2000 \\
2-3000 \\
\text { canyon }\end{array}$ & 13 & $\begin{array}{l}0.57 \\
0.30 \\
0.10\end{array}$ & $\begin{array}{l}0.86 \\
0.71 \\
0.59\end{array}$ & $\begin{array}{l}1.82 \\
0.39 \\
0.00\end{array}$ & $\begin{array}{l}3.25 \\
1.40 \\
0.69\end{array}$ & $\begin{array}{l}0.64 \\
1.02 \\
0.57\end{array}$ & $\begin{array}{l}1.17 \\
1.61 \\
0.88\end{array}$ & $\begin{array}{l}0.14 \\
1.99 \\
1.07\end{array}$ & $\begin{array}{l}1.95 \\
4.62 \\
2.52\end{array}$ & $\begin{array}{l}1.21 \\
1.32 \\
0.67\end{array}$ & $\begin{array}{l}2.03 \\
2.32 \\
1.47\end{array}$ & $\begin{array}{l}1.96 \\
2.38 \\
1.07\end{array}$ & $\begin{array}{l}5.20 \\
6.02 \\
3.21\end{array}$ \\
\hline Iion & $\begin{array}{l}c a n y 0 n \\
0-1000 \\
1-2000 \\
2-3000 \\
\text { canyon }\end{array}$ & 14 & $\begin{array}{l}0.70 \\
0.19 \\
0.27\end{array}$ & $\begin{array}{l}1.27 \\
0.27 \\
0.00\end{array}$ & $\begin{array}{l}0.00 \\
0.00 \\
0.00\end{array}$ & $\begin{array}{l}1.97 \\
0.46 \\
0.27\end{array}$ & $\begin{array}{l}0.83 \\
1.17 \\
0.14\end{array}$ & $\begin{array}{l}1.24 \\
1.45 \\
0.75\end{array}$ & $\begin{array}{l}0.00 \\
0.00 \\
0.00\end{array}$ & $\begin{array}{l}2.07 \\
2.62 \\
0.89\end{array}$ & $\begin{array}{l}1.53 \\
1.36 \\
0.41\end{array}$ & $\begin{array}{l}2.51 \\
1.72 \\
0.75\end{array}$ & $\begin{array}{l}0.00 \\
0.00 \\
0.00\end{array}$ & $\begin{array}{l}4.04 \\
3.08 \\
1.16\end{array}$ \\
\hline Ifon & $\begin{array}{l}0-1000 \\
1-2000 \\
2-3000 \\
\text { canyon }\end{array}$ & 15 & $\begin{array}{l}1.98 \\
1.43 \\
0.76\end{array}$ & $\begin{array}{l}0.00 \\
0.00 \\
0.00\end{array}$ & $\begin{array}{l}2.84 \\
2.53 \\
1.90\end{array}$ & $\begin{array}{l}4.82 \\
3.96 \\
2.66\end{array}$ & $\begin{array}{l}0.24 \\
0.55 \\
2.28\end{array}$ & $\begin{array}{l}1.80 \\
1.07 \\
0.72\end{array}$ & $\begin{array}{l}0.00 \\
0.0 n \\
0.00\end{array}$ & $\begin{array}{l}2.04 \\
1.62 \\
3.00\end{array}$ & $\begin{array}{l}2.22 \\
1.98 \\
3.04\end{array}$ & $\begin{array}{l}1.80 \\
1.07 \\
0.72\end{array}$ & $\begin{array}{l}2.84 \\
2.53 \\
1.90\end{array}$ & $\begin{array}{l}6.86 \\
5.58 \\
5.60\end{array}$ \\
\hline iron & $\begin{array}{l}\text { canyon } \\
0-1000 \\
1-2000\end{array}$ & 13 & $\begin{array}{l}0.00 \\
0.00\end{array}$ & $\begin{array}{l}0.79 \\
0.36\end{array}$ & $\begin{array}{l}0.00 \\
0.00\end{array}$ & $\begin{array}{l}0.79 \\
0.36\end{array}$ & $\begin{array}{l}0.00 \\
0.00\end{array}$ & $\begin{array}{l}0.00 \\
0.00\end{array}$ & $\begin{array}{l}0.00 \\
0.00\end{array}$ & $\begin{array}{l}0.00 \\
0.00\end{array}$ & $\begin{array}{l}0.00 \\
0.00\end{array}$ & $\begin{array}{l}0.79 \\
0.36\end{array}$ & $\begin{array}{l}0.00 \\
0.00\end{array}$ & $\begin{array}{l}0.79 \\
0.36\end{array}$ \\
\hline lion & $\begin{array}{l}\text { canyon } \\
0-1000 \\
1-2000 \\
2-3000 \\
\text { canyon }\end{array}$ & 17 & $\begin{array}{l}1.03 \\
0.52 \\
0.00\end{array}$ & $\begin{array}{l}0.00 \\
0.00 \\
0.00\end{array}$ & $\begin{array}{l}0.00 \\
0.00 \\
0.00\end{array}$ & $\begin{array}{l}1.03 \\
0.52 \\
0.00\end{array}$ & $\begin{array}{l}0.58 \\
0.67 \\
0.94 \\
-.94\end{array}$ & $\begin{array}{l}0.00 \\
0.00 \\
0.00\end{array}$ & $\begin{array}{l}0.00 \\
0.00 \\
0.00\end{array}$ & $\begin{array}{l}0.58 \\
0.67 \\
0.94\end{array}$ & $\begin{array}{l}1.61 \\
1.19 \\
0.94\end{array}$ & $\begin{array}{l}0.00 \\
0.00 \\
0.00\end{array}$ & $\begin{array}{l}0.00 \\
0.00 \\
0.00\end{array}$ & $\begin{array}{l}1.61 \\
1.19 \\
0.94\end{array}$ \\
\hline
\end{tabular}




\begin{tabular}{|c|c|c|c|c|c|c|c|c|c|c|c|c|c|c|}
\hline & $\begin{array}{l}0-1000 \\
1-2000\end{array}$ & & $\begin{array}{l}0.58 \\
0.27\end{array}$ & $\begin{array}{l}0.00 \\
0.00\end{array}$ & $\begin{array}{l}0.00 \\
0.00\end{array}$ & $\begin{array}{l}0.58 \\
0.27\end{array}$ & $\begin{array}{l}0.00 \\
0.00\end{array}$ & $\begin{array}{l}0.00 \\
0.00\end{array}$ & $\begin{array}{l}0.00 \\
0.00\end{array}$ & $\begin{array}{l}0.00 \\
0.00\end{array}$ & $\begin{array}{l}0.58 \\
0.27\end{array}$ & $\begin{array}{l}0.00 \\
0.00\end{array}$ & $\begin{array}{l}0.00 \\
0.00\end{array}$ & $\begin{array}{l}0.58 \\
0.27\end{array}$ \\
\hline lion & $\begin{array}{l}\text { canyon } \\
0-1000 \\
1-2000 \\
2-30 j 0\end{array}$ & 18 & $\begin{array}{l}0.60 \\
0.27 \\
0.00\end{array}$ & $\begin{array}{l}0.00 \\
0.00 \\
0.00\end{array}$ & $\begin{array}{l}0.00 \\
0.00 \\
0.00\end{array}$ & $\begin{array}{l}0.60 \\
0.27 \\
0.00\end{array}$ & $\begin{array}{l}0.31 \\
0.54 \\
0.74\end{array}$ & $\begin{array}{l}0.00 \\
0.00 \\
0.00\end{array}$ & $\begin{array}{l}0.00 \\
0.00 \\
0.00\end{array}$ & $\begin{array}{l}0.31 \\
0.54 \\
0.74\end{array}$ & $\begin{array}{l}0.91 \\
0.81 \\
0.74\end{array}$ & $\begin{array}{l}0.00 \\
0.00 \\
0.00\end{array}$ & $\begin{array}{l}0.00 \\
0.00 \\
0.00\end{array}$ & $\begin{array}{l}0.91 \\
0.81 \\
0.74\end{array}$ \\
\hline lion & $\begin{array}{l}\text { canyon } \\
0-1000 \\
1-2000 \\
2-3000\end{array}$ & 19 & $\begin{array}{l}1.85 \\
1.60 \\
0.34\end{array}$ & $\begin{array}{l}0.87 \\
0.18 \\
0.00\end{array}$ & $\begin{array}{l}0.00 \\
0.00 \\
0.00\end{array}$ & $\begin{array}{l}2.72 \\
1.78 \\
0.34\end{array}$ & $\begin{array}{l}0.00 \\
0.08 \\
1.50\end{array}$ & $\begin{array}{l}1.09 \\
0.83 \\
0.96\end{array}$ & $\begin{array}{l}0.00 \\
0.00 \\
0.00\end{array}$ & $\begin{array}{l}1.09 \\
0.99 \\
2.46\end{array}$ & $\begin{array}{l}1.85 \\
1.68 \\
1.84\end{array}$ & $\begin{array}{l}1.96 \\
1.01 \\
0.96\end{array}$ & $\begin{array}{l}0.00 \\
0.00 \\
0.00\end{array}$ & $\begin{array}{l}3.81 \\
2.69 \\
2.80\end{array}$ \\
\hline lion & $\begin{array}{l}\text { canyon } \\
0-1000 \\
1-2000 \\
2-3000\end{array}$ & 2 & $\begin{array}{l}0.83 \\
0.65 \\
0.01\end{array}$ & $\begin{array}{l}0.39 \\
0.00 \\
0.00\end{array}$ & $\begin{array}{l}8.50 \\
3.38 \\
2.83\end{array}$ & $\begin{array}{l}9.64 \\
4.03 \\
2.84\end{array}$ & $\begin{array}{l}0.00 \\
0.00 \\
0.00\end{array}$ & $\begin{array}{l}0.00 \\
0.00 \\
0.00\end{array}$ & $\begin{array}{l}6.76 \\
3.61 \\
4.45\end{array}$ & $\begin{array}{l}6.76 \\
3.69 \\
4.45\end{array}$ & $\begin{array}{l}0.83 \\
0.65 \\
0.01\end{array}$ & $\begin{array}{l}0.31 \\
0.00 \\
0.00\end{array}$ & $\begin{array}{r}15.26 \\
6.99 \\
7.28\end{array}$ & $\begin{array}{r}16.40 \\
7.64 \\
7.29\end{array}$ \\
\hline Iion & $\begin{array}{l}\text { conyon } \\
0-1000 \\
1-2000 \\
2-3000\end{array}$ & 20 & $\begin{array}{l}1.37 \\
0.45 \\
0.00\end{array}$ & $\begin{array}{l}0.00 \\
0.00 \\
0.00\end{array}$ & $\begin{array}{l}0.00 \\
0.00 \\
0.00\end{array}$ & $\begin{array}{l}1.37 \\
0.45 \\
0.00\end{array}$ & $\begin{array}{l}0.60 \\
0.90 \\
0.78\end{array}$ & $\begin{array}{l}0.00 \\
0.00 \\
0.00\end{array}$ & $\begin{array}{l}0.00 \\
0.00 \\
0.00\end{array}$ & $\begin{array}{l}0.60 \\
0.90 \\
0.78\end{array}$ & $\begin{array}{l}1.97 \\
1.35 \\
0.78\end{array}$ & $\begin{array}{l}0.00 \\
0.00 \\
0.00\end{array}$ & $\begin{array}{l}0.00 \\
0.00 \\
0.00\end{array}$ & $\begin{array}{l}1.97 \\
1.35 \\
0.78\end{array}$ \\
\hline tron & $\begin{array}{l}\text { canyon } \\
0-1000 \\
1-2000 \\
2-3000 \\
2-3000\end{array}$ & 21 & $\begin{array}{l}0.00 \\
0.00 \\
0.00\end{array}$ & $\begin{array}{l}1.93 \\
1.67 \\
0.35\end{array}$ & $\begin{array}{l}1.48 \\
0.50 \\
0.13\end{array}$ & $\begin{array}{l}3.41 \\
1.97 \\
0.48\end{array}$ & $\begin{array}{l}0.00 \\
0.00 \\
0.00\end{array}$ & $\begin{array}{l}1.60 \\
1.42 \\
2.03\end{array}$ & $\begin{array}{l}1.51 \\
1.26 \\
1.44\end{array}$ & $\begin{array}{l}3.11 \\
2.68 \\
3.47\end{array}$ & $\begin{array}{l}0.00 \\
0.00 \\
0.00\end{array}$ & $\begin{array}{l}3.53 \\
2.89 \\
2.38\end{array}$ & $\begin{array}{l}2.99 \\
1.76 \\
1.57\end{array}$ & $\begin{array}{l}6.52 \\
4.65 \\
3.95\end{array}$ \\
\hline (1) & $\begin{array}{l}0-1000 \\
1-2000 \\
2-3000\end{array}$ & 26 & $\begin{array}{l}0.00 \\
0.00 \\
0.00\end{array}$ & $\begin{array}{l}0.00 \\
0.00 \\
0.00\end{array}$ & $\begin{array}{l}2.70 \\
2.39 \\
0.00\end{array}$ & $\begin{array}{l}2.70 \\
2.39 \\
0.00\end{array}$ & $\begin{array}{l}0.00 \\
0.00 \\
0.00\end{array}$ & $\begin{array}{l}1.33 \\
1.25 \\
9.53\end{array}$ & $\begin{array}{l}1.31 \\
2.79 \\
0.00\end{array}$ & $\begin{array}{l}2.64 \\
4.04 \\
9.53\end{array}$ & $\begin{array}{l}0.00 \\
0.00 \\
0.00\end{array}$ & $\begin{array}{l}1.33 \\
1.25 \\
9.53\end{array}$ & $\begin{array}{l}4.01 \\
5.18 \\
0.00\end{array}$ & $\begin{array}{l}5.34 \\
6.43 \\
9.53\end{array}$ \\
\hline (1) & $\begin{array}{l}0-1000 \\
1-2000 \\
2-3000\end{array}$ & 3 & $\begin{array}{l}1.63 \\
1.88 \\
0.74\end{array}$ & $\begin{array}{l}0.00 \\
0.00 \\
0.00\end{array}$ & $\begin{array}{l}0.00 \\
0.00 \\
0.00\end{array}$ & $\begin{array}{l}1.63 \\
1.88 \\
0.74\end{array}$ & $\begin{array}{l}1.06 \\
1.11 \\
3.89\end{array}$ & $\begin{array}{l}0.00 \\
0.00 \\
0.00\end{array}$ & $\begin{array}{l}0.00 \\
0.00 \\
0.00\end{array}$ & $\begin{array}{l}1.06 \\
1.11 \\
3.89\end{array}$ & $\begin{array}{l}2.69 \\
2.99 \\
4.63\end{array}$ & $\begin{array}{l}0.00 \\
0.00 \\
0.00\end{array}$ & $\begin{array}{l}0.00 \\
0.00 \\
0.00\end{array}$ & $\begin{array}{l}2.69 \\
2.99 \\
4.63\end{array}$ \\
\hline Iton & $\begin{array}{l}\text { cenyon } \\
0-1000 \\
1-2000 \\
2-3000\end{array}$ & 26 & $\begin{array}{l}2.48 \\
1.60 \\
0.00\end{array}$ & $\begin{array}{l}2.31 \\
1.40 \\
1.20\end{array}$ & $\begin{array}{l}0.00 \\
0.00 \\
0.00\end{array}$ & $\begin{array}{l}4.79 \\
3.00 \\
1.20\end{array}$ & $\begin{array}{l}0.00 \\
2.95 \\
5.87\end{array}$ & $\begin{array}{l}4.51 \\
2.14 \\
6.30\end{array}$ & $\begin{array}{l}0.00 \\
0.00 \\
0.00\end{array}$ & $\begin{array}{r}4.51 \\
5.00 \\
12.17\end{array}$ & $\begin{array}{l}2.48 \\
4.55 \\
5.87\end{array}$ & $\begin{array}{l}6.82 \\
3.54 \\
7.50\end{array}$ & $\begin{array}{l}0.00 \\
0.00 \\
0.00\end{array}$ & $\begin{array}{r}9.30 \\
8.09 \\
13.37\end{array}$ \\
\hline lion & $\begin{array}{l}\text { canyon } \\
0-1000 \\
1-2000 \\
2-3000 \\
\text { canyon }\end{array}$ & 3 & $\begin{array}{l}2.97 \\
1.65 \\
0.11\end{array}$ & $\begin{array}{l}0.51 \\
0.00 \\
0.00\end{array}$ & $\begin{array}{l}0.00 \\
0.00 \\
0.00\end{array}$ & $\begin{array}{l}3.48 \\
1.65 \\
0.11\end{array}$ & $\begin{array}{l}1.15 \\
0.66 \\
1.18\end{array}$ & $\begin{array}{l}2.40 \\
0.00 \\
0.00\end{array}$ & $\begin{array}{l}0.00 \\
0.00 \\
0.00\end{array}$ & $\begin{array}{l}3.55 \\
0.66 \\
1.18\end{array}$ & $\begin{array}{l}4.12 \\
2.31 \\
1.29\end{array}$ & $\begin{array}{l}2.91 \\
0.00 \\
0.00\end{array}$ & $\begin{array}{l}0.00 \\
0.00 \\
0.00\end{array}$ & $\begin{array}{l}7.03 \\
2.31 \\
1.29\end{array}$ \\
\hline Cron & $\begin{array}{l}c=1000 \\
0-1000 \\
1-2000 \\
z-3000 \\
\text { canyon }\end{array}$ & 5 & $\begin{array}{l}0.00 \\
0.00 \\
0.00\end{array}$ & $\begin{array}{l}0.00 \\
0.00 \\
0.00\end{array}$ & $\begin{array}{l}7.23 \\
1.98 \\
1.78\end{array}$ & $\begin{array}{l}7.23 \\
1.98 \\
1.78\end{array}$ & $\begin{array}{l}0.00 \\
0.00 \\
0.00\end{array}$ & $\begin{array}{l}0.00 \\
0.00 \\
0.00\end{array}$ & $\begin{array}{l}6.88 \\
2.90 \\
2.95\end{array}$ & $\begin{array}{l}6.88 \\
2.90 \\
2.95\end{array}$ & $\begin{array}{l}0.00 \\
0.00 \\
0.00\end{array}$ & $\begin{array}{l}0.00 \\
0.00 \\
0.00\end{array}$ & $\begin{array}{r}14.11 \\
4.88 \\
4.73\end{array}$ & $\begin{array}{r}14.11 \\
4.88 \\
4.73\end{array}$ \\
\hline lion & $\begin{array}{l}0=1000 \\
1-2000 \\
2-3000 \\
\text { canyon }\end{array}$ & 6 & $\begin{array}{l}1.42 \\
0.36 \\
0.00\end{array}$ & $\begin{array}{l}2.86 \\
1.65 \\
1.22\end{array}$ & $\begin{array}{l}9.62 \\
5.43 \\
4.12\end{array}$ & $\begin{array}{r}13.90 \\
7.42 \\
5.34\end{array}$ & $\begin{array}{l}3.64 \\
3.15 \\
2.34\end{array}$ & $\begin{array}{l}0.00 \\
0.00 \\
0.00\end{array}$ & $\begin{array}{l}5.91 \\
3.58 \\
3.76\end{array}$ & $\begin{array}{l}9.55 \\
6.73 \\
6.10\end{array}$ & $\begin{array}{l}5.06 \\
3.49 \\
2.34\end{array}$ & $\begin{array}{l}2.86 \\
1.65 \\
1.22\end{array}$ & $\begin{array}{r}15.53 \\
9.01 \\
7.88\end{array}$ & $\begin{array}{l}23.45 \\
14.15 \\
11.44\end{array}$ \\
\hline lion & $\begin{array}{l}0-1000 \\
1=2000 \\
2=3000 \\
\text { canyon }\end{array}$ & 7 & $\begin{array}{l}7.23 \\
3.17 \\
0.73\end{array}$ & $\begin{array}{l}0.05 \\
0.02 \\
0.00\end{array}$ & $\begin{array}{l}0.00 \\
0.00 \\
0.00\end{array}$ & $\begin{array}{l}7.2 .8 \\
3.19 \\
0.73\end{array}$ & $\begin{array}{l}1.69 \\
2.71 \\
3.57\end{array}$ & $\begin{array}{l}1.30 \\
0.29 \\
0.89\end{array}$ & $\begin{array}{l}0.00 \\
0.00 \\
0.00\end{array}$ & $\begin{array}{l}2.99 \\
3.00 \\
4.46\end{array}$ & $\begin{array}{l}8.92 \\
5.88 \\
4.30\end{array}$ & $\begin{array}{l}1.35 \\
0.31 \\
0.89\end{array}$ & $\begin{array}{l}0.00 \\
0.00 \\
0.00\end{array}$ & $\begin{array}{r}10.27 \\
6.19 \\
5.19\end{array}$ \\
\hline lion & $\begin{array}{l}0-1000 \\
1-2000 \\
\text { canyon }\end{array}$ & 8 & $\begin{array}{l}1.10 \\
0.07\end{array}$ & $\begin{array}{l}0.00 \\
0.00\end{array}$ & $\begin{array}{l}0.00 \\
0.00\end{array}$ & $\begin{array}{l}1.10 \\
0.07\end{array}$ & $\begin{array}{l}0.00 \\
0.00\end{array}$ & $\begin{array}{l}0.00 \\
0.00\end{array}$ & $\begin{array}{l}0.00 \\
0.00\end{array}$ & $\begin{array}{l}0.00 \\
0.00\end{array}$ & $\begin{array}{l}1.10 \\
0.07\end{array}$ & $\begin{array}{l}0.00 \\
0.00\end{array}$ & $\begin{array}{l}0.00 \\
0.00\end{array}$ & $\begin{array}{l}1.100 \\
0.07\end{array}$ \\
\hline lion & $\begin{array}{l}0-1000 \\
1-2000 \\
2-3000 \\
\text { canyon }\end{array}$ & 9 & $\begin{array}{l}3.34 \\
1.65 \\
1.83\end{array}$ & $\begin{array}{l}0.00 \\
0.00 \\
0.00\end{array}$ & $\begin{array}{l}3.60 \\
2.00 \\
1.15\end{array}$ & $\begin{array}{l}0.94 \\
3.65 \\
2.98\end{array}$ & $\begin{array}{l}0.55 \\
0.26 \\
0.79\end{array}$ & $\begin{array}{l}1.87 \\
1.38 \\
2.29\end{array}$ & $\begin{array}{l}1.71 \\
1.35 \\
2.09\end{array}$ & $\begin{array}{l}4.13 \\
2.99 \\
5.17\end{array}$ & $\begin{array}{l}3.89 \\
1.91 \\
2.62\end{array}$ & $\begin{array}{l}1.87 \\
1.38 \\
2.29\end{array}$ & $\begin{array}{l}5.39 \\
3.35 \\
3.24\end{array}$ & $\begin{array}{r}11.07 \\
6.64 \\
8.15\end{array}$ \\
\hline & $\begin{array}{l}0-1000 \\
1-2000\end{array}$ & & $\begin{array}{l}0.00 \\
0.00\end{array}$ & $\begin{array}{l}3.08 \\
1.50\end{array}$ & $\begin{array}{l}4.40 \\
2.40\end{array}$ & $\begin{array}{l}7.48 \\
3.90\end{array}$ & $\begin{array}{l}0.00 \\
0.00\end{array}$ & $\begin{array}{l}1.75 \\
1.03\end{array}$ & $\begin{array}{l}2.21 \\
1.78\end{array}$ & $\begin{array}{l}3.96 \\
2.81\end{array}$ & $\begin{array}{l}0.00 \\
0.00\end{array}$ & $\begin{array}{l}4.83 \\
2.53\end{array}$ & $\begin{array}{l}6.61 \\
4.18\end{array}$ & $\begin{array}{r}11.44 \\
6.71\end{array}$ \\
\hline
\end{tabular}




\begin{tabular}{|c|c|c|c|c|c|c|c|c|c|c|c|c|c|}
\hline litan & $2-3000$ & 0.00 & 0.79 & 2.17 & 2.96 & 0.00 & 1.05 & 1.59 & 2.64 & 0.00 & 1.86 & 3.76 & 5.60 \\
\hline 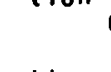 & $\begin{array}{l}0-1000 \\
1-2000\end{array}$ & $\begin{array}{l}0.90 \\
0.12\end{array}$ & $\begin{array}{l}0.00 \\
0.00\end{array}$ & $\begin{array}{l}0.00 \\
0.00\end{array}$ & $\begin{array}{l}0.90 \\
0.12\end{array}$ & $\begin{array}{l}0.00 \\
0.00\end{array}$ & $\begin{array}{l}0.00 \\
0.00\end{array}$ & $\begin{array}{l}0.00 \\
0.00\end{array}$ & $\begin{array}{l}0.00 \\
0.00\end{array}$ & $\begin{array}{l}0.90 \\
0.12\end{array}$ & $\begin{array}{l}0.00 \\
0.00\end{array}$ & $\begin{array}{l}0.00 \\
0.00\end{array}$ & $\begin{array}{l}0.90 \\
0.12\end{array}$ \\
\hline tion & $\begin{array}{l}\text { canyon b } \\
0-1000 \\
1-2000\end{array}$ & $\begin{array}{l}0.00 \\
0.00\end{array}$ & $\begin{array}{l}0.00 \\
0.00\end{array}$ & $\begin{array}{l}3.46 \\
0.53\end{array}$ & $\begin{array}{l}3.46 \\
0.53\end{array}$ & $\begin{array}{l}0.00 \\
0.00\end{array}$ & $\begin{array}{l}0.00 \\
0.00\end{array}$ & $\begin{array}{l}0.00 \\
0.00\end{array}$ & $\begin{array}{l}0.00 \\
0.00\end{array}$ & $\begin{array}{l}0.00 \\
0.00\end{array}$ & $\begin{array}{l}0.00 \\
0.00\end{array}$ & $\begin{array}{l}3.46 \\
0.53\end{array}$ & $\begin{array}{l}3.46 \\
0.53\end{array}$ \\
\hline Iton & $\begin{array}{l}\text { canyon c } \\
0-1000 \\
1-2000 \\
2-3000\end{array}$ & $\begin{array}{l}0.39 \\
0.60 \\
0.27\end{array}$ & $\begin{array}{l}0.00 \\
0.00 \\
0.00\end{array}$ & $\begin{array}{l}0.00 \\
0.00 \\
0.00\end{array}$ & $\begin{array}{l}0.39 \\
0.60 \\
0.27\end{array}$ & $\begin{array}{l}0.00 \\
0.00 \\
0.00\end{array}$ & $\begin{array}{l}0.00 \\
0.00 \\
0.00\end{array}$ & $\begin{array}{l}0.00 \\
0.00 \\
0.00\end{array}$ & $\begin{array}{l}0.00 \\
0.00 \\
0.00\end{array}$ & $\begin{array}{l}0.39 \\
0.60 \\
0.27\end{array}$ & $\begin{array}{l}0.00 \\
0.00 \\
0.00\end{array}$ & $\begin{array}{l}0.00 \\
0.00 \\
0.00\end{array}$ & $\begin{array}{l}0.39 \\
0.60 \\
0.27\end{array}$ \\
\hline Iton & $\begin{array}{l}\text { canyon d } \\
0-1000 \\
1-2000 \\
2-3000 \\
\end{array}$ & $\begin{array}{l}0.00 \\
0.00 \\
0.00\end{array}$ & $\begin{array}{l}0.00 \\
0.00 \\
0.00\end{array}$ & $\begin{array}{l}1.44 \\
1.28 \\
0.40\end{array}$ & $\begin{array}{l}1.44 \\
1.28 \\
0.40\end{array}$ & $\begin{array}{l}0.00 \\
0.00 \\
0.00\end{array}$ & $\begin{array}{l}0.00 \\
0.00 \\
0.00\end{array}$ & $\begin{array}{l}0.00 \\
0.00 \\
0.00\end{array}$ & $\begin{array}{l}0.00 \\
0.00 \\
0.00\end{array}$ & $\begin{array}{l}0.00 \\
0.00 \\
0.00\end{array}$ & $\begin{array}{l}0.00 \\
0.00 \\
0.00\end{array}$ & $\begin{array}{l}1.64 \\
1.28 \\
0.40\end{array}$ & $\begin{array}{l}1.44 \\
1.28 \\
0.40\end{array}$ \\
\hline & $\begin{array}{r}0-1000 \\
1-2000 \\
2-3000\end{array}$ & $\begin{array}{l}0.00 \\
0.00 \\
0.00\end{array}$ & $\begin{array}{l}0.00 \\
0.00 \\
0.00\end{array}$ & $\begin{array}{r}3.97 \\
20.61 \\
22.62\end{array}$ & $\begin{array}{r}3.97 \\
20.61 \\
22.62\end{array}$ & $\begin{array}{l}0.00 \\
0.00 \\
0.00\end{array}$ & $\begin{array}{l}0.00 \\
0.00 \\
0.00\end{array}$ & $\begin{array}{l}0.00 \\
0.00 \\
0.00\end{array}$ & $\begin{array}{l}0.00 \\
0.00 \\
0.00\end{array}$ & $\begin{array}{l}0.00 \\
0.00 \\
0.00\end{array}$ & $\begin{array}{l}0.00 \\
0.00 \\
0.00\end{array}$ & $\begin{array}{r}3.97 \\
20.61 \\
22.62\end{array}$ & $\begin{array}{r}3.97 \\
20.61 \\
22.62\end{array}$ \\
\hline & $\begin{array}{l}0-1000 \\
1-2000 \\
2-3000\end{array}$ & $\begin{array}{l}0.00 \\
0.00 \\
0.00\end{array}$ & $\begin{array}{l}0.00 \\
0.00 \\
0.00\end{array}$ & $\begin{array}{r}181.21 \\
47.27 \\
23.04\end{array}$ & $\begin{array}{r}181.21 \\
47.27 \\
23.04\end{array}$ & $\begin{array}{l}0.00 \\
0.00 \\
0.00\end{array}$ & $\begin{array}{l}0.00 \\
0.00 \\
0.00\end{array}$ & $\begin{array}{l}0.00 \\
0.00 \\
0.00\end{array}$ & $\begin{array}{l}0.00 \\
0.00 \\
0.00\end{array}$ & $\begin{array}{l}0.00 \\
0.00 \\
0.00\end{array}$ & $\begin{array}{l}0.00 \\
0.00 \\
0.00\end{array}$ & $\begin{array}{r}181.21 \\
47.27 \\
23.04\end{array}$ & $\begin{array}{r}181.21 \\
47.27 \\
23.04\end{array}$ \\
\hline cou & yy total & 170.51 & 185.62 & 1429.54 & 1785.67 & 84.39 & 48.23 & 257.03 & 1689.65 & 354.90 & $\begin{array}{r}433.85 \\
-\end{array}$ & 2686.57 & 3675.32 \\
\hline & & & & routt & & & ty & & & & & & \\
\hline it & & & & & & & & & & & & & \\
\hline & $0-1000$ & 0.00 & $0: 00$ & 4.25 & 4.25 & 0.00 & 0.00 & 0.00 & 0.00 & 0.00 & 0.00 & 4.25 & 4.25 \\
\hline bear & $\begin{array}{l}0-1000 \\
\text { river }\end{array}$ & 1.23 & 0.00 & 1.60 & 2.83 & 0.00 & 0.00 & 0.00 & 0.00 & 1.23 & 0.00 & 1.60 & 2.83 \\
\hline & $\begin{array}{l}0-1000 \\
1-2000 \\
0\end{array}$ & $\begin{array}{l}0.00 \\
0.00\end{array}$ & $\begin{array}{l}0.00 \\
0.00\end{array}$ & $\begin{array}{l}76.38 \\
37.48\end{array}$ & $\begin{array}{l}76.38 \\
37.48\end{array}$ & $\begin{array}{l}0.00 \\
0.00\end{array}$ & $\begin{array}{l}0.00 \\
0.00\end{array}$ & $\begin{array}{l}0.00 \\
0.00\end{array}$ & $\begin{array}{l}0.00 \\
0.00\end{array}$ & $\begin{array}{l}0.00 \\
0.00\end{array}$ & $\begin{array}{l}0.00 \\
0.00\end{array}$ & $\begin{array}{l}76.38 \\
37.68\end{array}$ & $\begin{array}{l}76.38 \\
37.48\end{array}$ \\
\hline brook & $1-2000$ & 0.00 & 0.00 & 2.42 & 2.42 & 0.00 & 0.00 & 0.00 & 0.00 & 0.00 & 0.00 & 2.42 & 2.42 \\
\hline 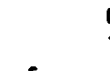 & $\begin{array}{l}0-1000 \\
1-2000\end{array}$ & $\begin{array}{l}0.00 \\
0.00\end{array}$ & $\begin{array}{l}0.94 \\
0.00\end{array}$ & $\begin{array}{l}55.39 \\
45.57\end{array}$ & $\begin{array}{l}56.33 \\
45.57\end{array}$ & $\begin{array}{l}0.00 \\
0.00\end{array}$ & $\begin{array}{l}0.00 \\
0.00\end{array}$ & $\begin{array}{l}0.00 \\
0.00\end{array}$ & $\begin{array}{l}0.00 \\
0.00\end{array}$ & $\begin{array}{l}0.00 \\
0.00\end{array}$ & $\begin{array}{l}0.94 \\
0.00\end{array}$ & $\begin{array}{l}55.39 \\
45.57\end{array}$ & $\begin{array}{l}56.33 \\
45.57\end{array}$ \\
\hline 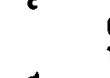 & $\begin{array}{l}0-1000 \\
1-2000\end{array}$ & $\begin{array}{l}0.75 \\
0.00\end{array}$ & $\begin{array}{l}0.00 \\
1.38\end{array}$ & $\begin{array}{l}14.65 \\
12.05\end{array}$ & $\begin{array}{l}15.40 \\
13.43\end{array}$ & $\begin{array}{l}0.00 \\
0.00\end{array}$ & $\begin{array}{l}0.00 \\
0.00\end{array}$ & $\begin{array}{l}0.00 \\
0.00\end{array}$ & $\begin{array}{l}0.00 \\
0.00\end{array}$ & $\begin{array}{l}0.75 \\
0.00\end{array}$ & $\begin{array}{l}0.00 \\
1.38\end{array}$ & $\begin{array}{l}14.09 \\
12.05\end{array}$ & $\begin{array}{l}15.40 \\
13.43\end{array}$ \\
\hline 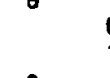 & $\begin{array}{l}0-1000 \\
1-2000\end{array}$ & $\begin{array}{l}0.00 \\
0.00\end{array}$ & $\begin{array}{l}0.83 \\
0.00\end{array}$ & $\begin{array}{r}18.17 \\
9.14\end{array}$ & $\begin{array}{r}19.00 \\
9.16\end{array}$ & $\begin{array}{l}0.00 \\
0.00\end{array}$ & $\begin{array}{l}0.00 \\
0.00\end{array}$ & $\begin{array}{l}0.00 \\
0.00\end{array}$ & $\begin{array}{l}0.00 \\
0.00\end{array}$ & $\begin{array}{l}0.00 \\
0.00\end{array}$ & $\begin{array}{l}0.83 \\
0.00\end{array}$ & $\begin{array}{r}18.17 \\
9.16\end{array}$ & $\begin{array}{r}19.00 \\
9.14\end{array}$ \\
\hline $120 n$ & $e^{0-1000}$ & 0.00 & 0.00 & 2.74 & 2.74 & 0.00 & 0.00 & 0.00 & 0.00 & 0.00 & 0.00 & 2.76 & 2.74 \\
\hline h zon & $\begin{array}{l}0-1000 \\
1-2000 \\
e^{-}\end{array}$ & $\begin{array}{l}17.16 \\
20.96\end{array}$ & $\begin{array}{l}29.40 \\
36.29\end{array}$ & $\begin{array}{l}215.58 \\
176.13\end{array}$ & $\begin{array}{l}262.14 \\
233.38\end{array}$ & $\begin{array}{l}0.0 u \\
0.00\end{array}$ & $\begin{array}{r}0.14 \\
12.13\end{array}$ & $\begin{array}{r}4.68 \\
301.14\end{array}$ & $\begin{array}{r}5.42 \\
313.27\end{array}$ & $\begin{array}{l}17.16 \\
20.96\end{array}$ & $\begin{array}{l}30.14 \\
48.42\end{array}$ & $\begin{array}{l}220.26 \\
477.27\end{array}$ & $\begin{array}{l}267.56 \\
346.09\end{array}$ \\
\hline & $\begin{array}{l}0-1000 \\
1-2000\end{array}$ & $\begin{array}{r}29.74 \\
0.00\end{array}$ & $\begin{array}{l}0.00 \\
0.00\end{array}$ & $\begin{array}{l}148.72 \\
100.30\end{array}$ & $\begin{array}{l}170.46 \\
100.30\end{array}$ & $\begin{array}{l}0.00 \\
0.00\end{array}$ & $\begin{array}{l}0.00 \\
0.00\end{array}$ & $\begin{array}{l}0.00 \\
0.00\end{array}$ & $\begin{array}{l}0.00 \\
0.00\end{array}$ & $\begin{array}{r}21.74 \\
0.00\end{array}$ & $\begin{array}{l}0.00 \\
0.00\end{array}$ & $\begin{array}{l}148.72 \\
100.30\end{array}$ & $\begin{array}{l}170.46 \\
100.30\end{array}$ \\
\hline $\begin{array}{l}\text { J } \\
\text { lenno }\end{array}$ & $0-1000$ & 0.00 & 0.00 & 71.05 & 71.05 & 0.00 & 0.00 & 0.00 & 0.00 & 0.00 & 0.00 & 71.05 & 71.05 \\
\hline & $\begin{array}{l}0-1000 \\
1-2000\end{array}$ & $\begin{array}{r}8.47 \\
12.62\end{array}$ & $\begin{array}{l}50.68 \\
20.53\end{array}$ & $\begin{array}{l}49.09 \\
34.08\end{array}$ & $\begin{array}{r}100.24 \\
67.23\end{array}$ & $\begin{array}{l}0.00 \\
0.00\end{array}$ & $\begin{array}{l}30.98 \\
12.34\end{array}$ & $\begin{array}{l}75.60 \\
93.42\end{array}$ & $\begin{array}{l}106.58 \\
105.76\end{array}$ & $\begin{array}{r}8.47 \\
12.62\end{array}$ & $\begin{array}{l}81.66 \\
32.87\end{array}$ & $\begin{array}{l}116.69 \\
127.50\end{array}$ & $\begin{array}{l}206.82 \\
172.99\end{array}$ \\
\hline
\end{tabular}




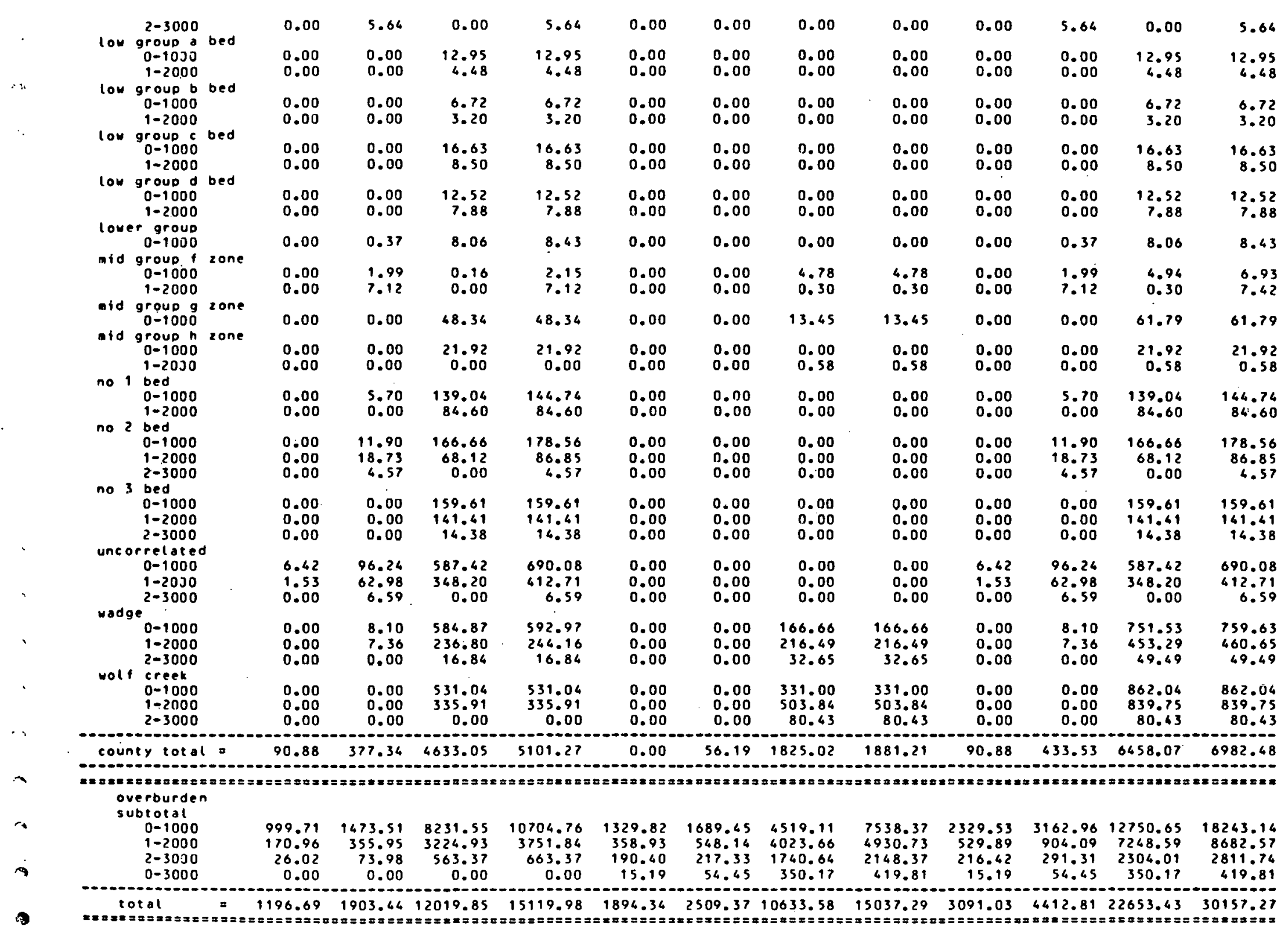




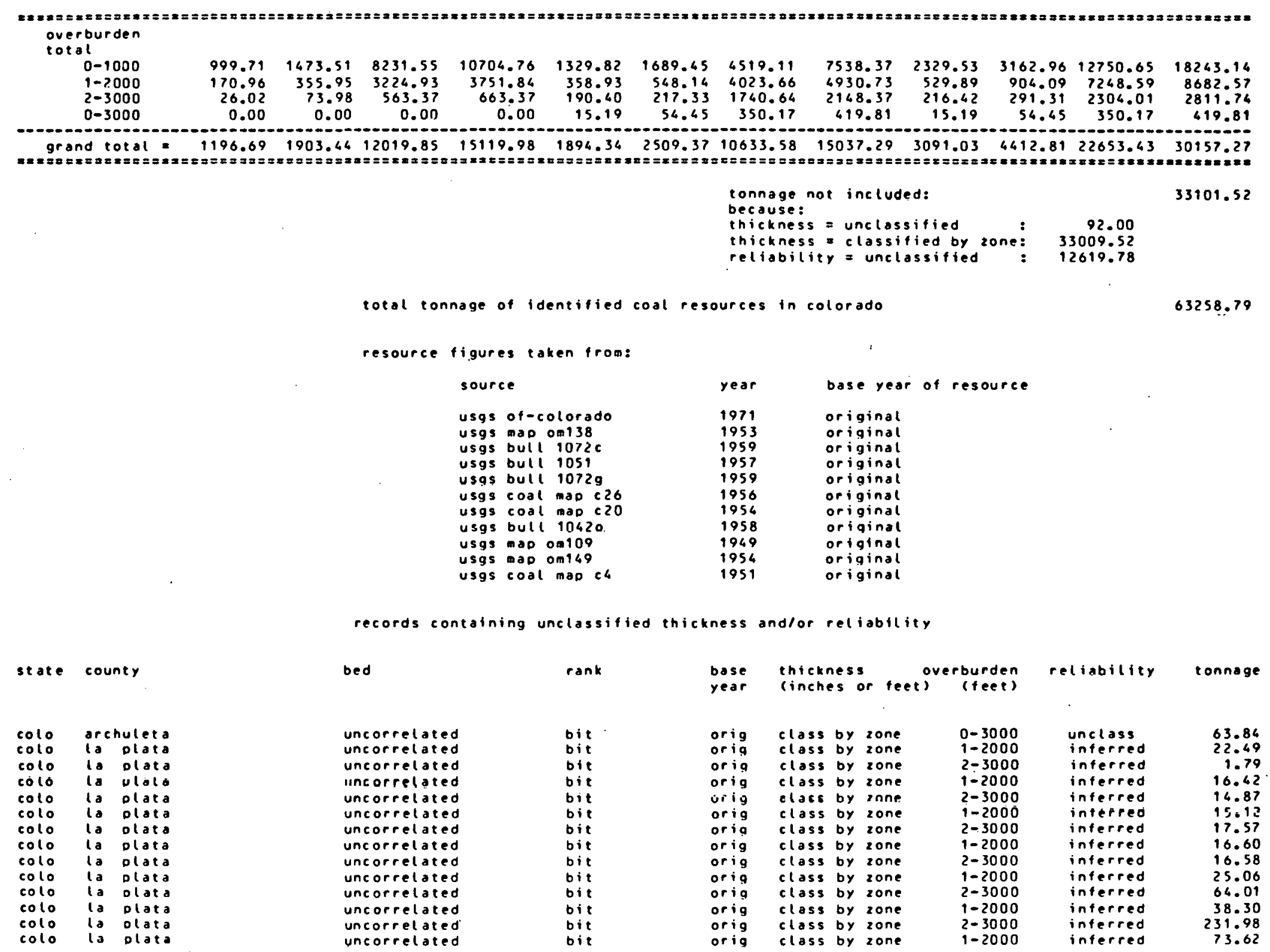




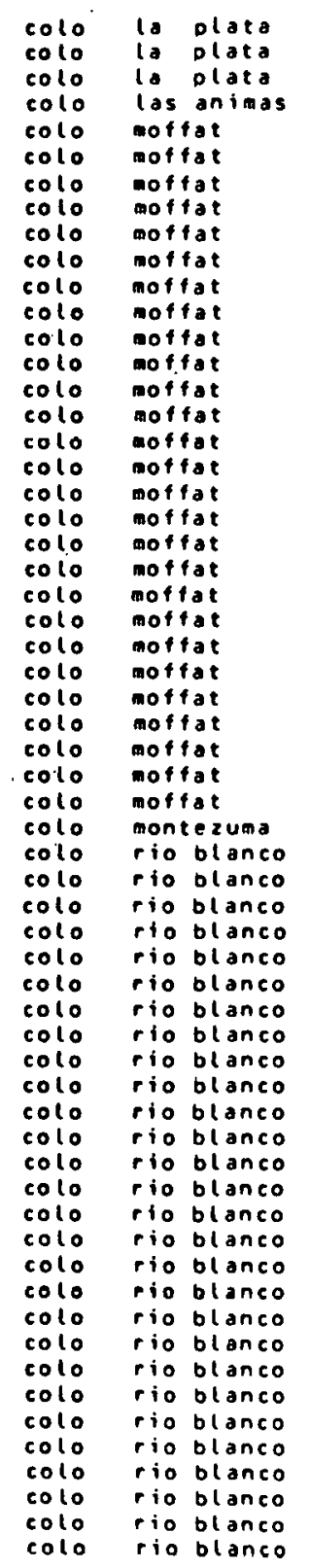

\begin{tabular}{|c|c|}
\hline \\
\hline $\begin{array}{l}\text { uncorrelated } \\
\text { uncorrel ated }\end{array}$ & $\begin{array}{l}\text { bit } \\
\text { bit }\end{array}$ \\
\hline uncorrelated & bit \\
\hline $\begin{array}{l}\text { uncorretoted } \\
\text { uncorrelated }\end{array}$ & $\begin{array}{l}\text { bit } \\
\text { bit }\end{array}$ \\
\hline $\begin{array}{l}\text { uncorrelated } \\
\text { uncorrelated }\end{array}$ & $\begin{array}{l}\text { bit } \\
\text { bit }\end{array}$ \\
\hline uncorrelated & bit \\
\hline uncorrelated & bit \\
\hline uncorrelated & bit \\
\hline uncorrelated & bit \\
\hline uncorrelated & bit \\
\hline unc orrelated & bit \\
\hline uncorrelated & bit \\
\hline unc orrelated & bit \\
\hline uncorrelated & bit \\
\hline uncorrelated & bit \\
\hline uncorrelated & bit \\
\hline uncorrelated & bit \\
\hline uncorrelated & bit \\
\hline unc orrelated & bit \\
\hline uncorrelated & bit \\
\hline uncorrelated & bit \\
\hline uncorrelated & bit \\
\hline uncorrelated & bit \\
\hline uncorrelated & bit \\
\hline unc orrelated & bit \\
\hline uncorrelated & bit \\
\hline uncorrelated & bit \\
\hline uncorrelated & bit \\
\hline uncorrelated & bit \\
\hline uncorrelated & bit \\
\hline unc orrelated & bit \\
\hline uncorrelated & bit \\
\hline uncorrelated & bit \\
\hline uncorrelated & bit \\
\hline unc orrelated & bit \\
\hline uncorrelated & bit \\
\hline uncorrelated & bit \\
\hline uncorrelated & bit \\
\hline uncorrelated & bit \\
\hline uncorrelated & bit \\
\hline unc orrelated & bit \\
\hline nde & bit \\
\hline nde & bit \\
\hline nde & bit \\
\hline uncorrelated & bit \\
\hline uncorrelated & bit \\
\hline uncorrelated & bit \\
\hline uncorrelated & bit \\
\hline unc orrelated & bit \\
\hline uncorrelated & bit \\
\hline uncorrelated & bit \\
\hline uncorrelated & bit \\
\hline uncorrel ated & bit \\
\hline uncorrelated & bit \\
\hline unc orrelated & bit \\
\hline uncorrelated & bit \\
\hline uncorrelated & bit \\
\hline
\end{tabular}

\begin{tabular}{|c|c|c|c|}
\hline $\begin{array}{l}\text { orig } \\
\text { orig } \\
\text { orig } \\
\text { orig }\end{array}$ & $\begin{array}{l}\text { class } \\
\text { class } \\
\text { class } \\
\text { class }\end{array}$ & $\begin{array}{l}\text { by } \\
\text { by } \\
\text { by } \\
\text { by }\end{array}$ & $\begin{array}{l}2 \text { one } \\
\text { zone } \\
\text { zone } \\
\text { zone }\end{array}$ \\
\hline orig & class & by & zone \\
\hline orig & class & by & zone \\
\hline orig & class & by & zone \\
\hline orig & class & by & zone \\
\hline orig & $c t a 3 s$ & by & zone \\
\hline orig & class & by & zone \\
\hline orig & class & by & zone \\
\hline orig & class & by & zone \\
\hline rig & $C \operatorname{loss}$ & by & zone \\
\hline orig & class & by & zone \\
\hline orig & $c \cos 3$ & by & zone \\
\hline orig & closs & by & zone \\
\hline orig & class & by & zone \\
\hline orig & $c \cos 3$ & by & zone \\
\hline orig & ctass & by & zone \\
\hline orig & class & by & zone \\
\hline $\begin{array}{l}\text { orla } \\
\text { orig }\end{array}$ & class. & by & $\begin{array}{l}\text { zone } \\
\text { zone }\end{array}$ \\
\hline orig & class & by & zone \\
\hline orig & class & by & zone \\
\hline orig & class & by & zone \\
\hline orig & class & by & zone \\
\hline orig & class & by & zone \\
\hline orig & closs & by & zone \\
\hline orig & closs & by & zone \\
\hline orig & class & by & zone \\
\hline orig & $\begin{array}{l}\text { class } \\
\text { class }\end{array}$ & by & zone \\
\hline orig & & & zone \\
\hline $\begin{array}{l}\text { Orig } \\
\text { origg }\end{array}$ & unctas & $\begin{array}{l}\text { by } \\
55\end{array}$ & zone \\
\hline orig & class & by & zone \\
\hline orig & class & by & zone \\
\hline orig & class & by & zone \\
\hline orig & class & by & zone \\
\hline orig & class & by & zone \\
\hline rig & class & by & zone \\
\hline orig & class & by & zone \\
\hline orig & class & by & zone \\
\hline orig & class & by & zone \\
\hline rig & closs & by & zone \\
\hline orig & class & by & zone \\
\hline orig & class & by & zone \\
\hline orig & class & by & zone \\
\hline orig & class & by & zone \\
\hline orig & class & by & zone \\
\hline urig & 21633 & by & 20 ne \\
\hline orig & closs & by & zone \\
\hline rig & class & by & zone \\
\hline rig & class & by & zone \\
\hline orig & class & by & zone \\
\hline orig & class & by & 2 or \\
\hline ia & class & by & zone \\
\hline or & class & by & \\
\hline orig & $c$ tass & by & zon \\
\hline orig & class & by & zone \\
\hline
\end{tabular}

$\begin{array}{llr} & \\ 2-3000 & \text { inferred } & 543.15 \\ 2-3000 & \text { inferred } & 430.43 \\ 0-3000 & \text { unclass } & 3292.57 \\ 0-3000 & \text { unclass } & 7988.36 \\ 0-3000 & \text { inferred } & 342.52 \\ 0-3000 & \text { inferred } & 796.05 \\ 0-3000 & \text { inferred } & 27.95 \\ 0-3000 & \text { inferred } & 149.40 \\ 0-3000 & \text { inferred } & 68.54 \\ 0-3000 & \text { inferred } & 962.26 \\ 0-3000 & \text { inferred } & 1295.85 \\ 0-3000 & \text { inferred } & 97.55 \\ \text { unclass } & \text { inferred } & 69.12 \\ \text { unclass } & \text { inferred } & 18.00 \\ \text { unclass } & \text { inferred } & 55.03 \\ \text { unclass } & \text { inferred } & 174.98 \\ \text { unclass } & \text { inferred } & 346.19 \\ \text { uncloss } & \text { inferred } & 254.64 \\ \text { unclass } & \text { inferred } & 563.35 \\ \text { unclass } & \text { inferred } & 808.92 \\ \text { unclass } & \text { inferred } & 10.78 \\ \text { unclass } & \text { inferred } & 570.34 \\ \text { uncloss } & \text { inferred } & 1842.02 \\ \text { unclass } & \text { inferred } & 2222.52 \\ \text { unclass } & \text { inferred } & 1429.54 \\ 0-3000 & \text { inferred } & 11.81 \\ 0-3000 & \text { inferred } & 262.44 \\ 0-3000 & \text { inferred } & 6.16 \\ 0-3000 & \text { inferred } & 18.63 \\ 0-3000 & \text { inferred } & 138.24 \\ 0-3000 & \text { inferred } & 128.99 \\ 0-3000 & \text { unclass } & 1028.20 \\ \text { unclass } & \text { inferred } & 110.08 \\ \text { unclass } & \text { inferred } & 78.13 \\ \text { unclass } & \text { inferred } & 92.00 \\ \text { unclass } & \text { inferred } & 4.56 \\ \text { unclass } & \text { inferred } & 4.56 \\ \text { unclass } & \text { inferred } & 13.83 \\ \text { unclass } & \text { inferred } & 41.48 \\ \text { unclass } & \text { inferred } \\ \text { unclass } & \text { inferred } & 69.20 \\ \text { unclass } & \text { inferred } & 9.20 \\ \text { unclass } & \text { inferred } & 12.31 \\ 0-3000 & \text { inferred } & 20.70 \\ 0-3000 & \text { inferred } & 331.74 \\ 0-3000 & \text { inferred } & 351.35 \\ 0-3000 & \text { inferred } & 442.49 \\ 0-3000 & \text { inferred } & 237.79 \\ 0-3000 & \text { inferred } & 11.44 \\ 0-3000 & \text { inferred } & 113.26 \\ 0-3000 & \text { inferred } & 12.31 \\ 0-30 n 0 & \text { inferred } & 151.64 \\ 0-3000 & \text { inferred } & 102.87 \\ 0-3000 & \text { inferred } & 330.71 \\ 0-3000 & \text { inferred } & 135.59 \\ 0-3000 & \text { inferred } & 161.88 \\ 0-3000 & \text { inferred } & 49.73 \\ 0-3000 & \text { inferred } & 25.86 \\ 0-3000 & \text { inferred } & 62.19 \\ 0-3000 & \text { inferred } & 152.01 \\ 0-3000 & \text { inferred } & 197.10 \\ & & \end{array}$




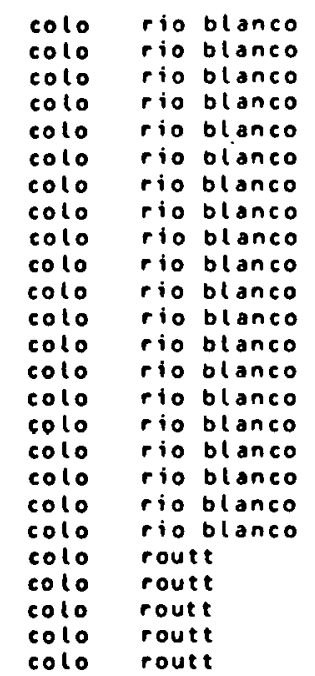

uncorrelated
uncorrelated
uncorrelated
uncorrelated
uncorrelated
uncorrelated
uncorrelated
uncorrelated
uncorrelated
uncorrelated
uncorrelated
uncorrelated
uncorrelated
uncorrelated
uncorrelated
uncorrelated
uncorrelated
uncorrelated
uncorrelated
uncorrelated
uncorrelated
uncorrelated
uncorrelated
uncorrelated
uncorrelated

bit
bit
bit
bit
bit
bit
bit
bit
bit
bit
bit
bit
bit
bit
bit
bit
bit
bit
bit
bit
bit
bit
bit
bit
bit

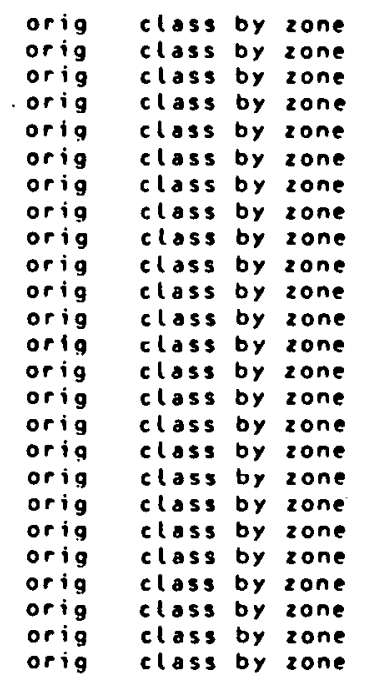

$0-3000$

$0-3000$

$0-3000$

$0-3000$

$0-3000$

$0-3000$

$0-3000$

$0-3000$

0.3000

$0-3000$

$0-3000$
$0-3000$

$0-3000$

$0-3000$

$0-3000$

$0-3000$

$0 \div 3000$

$0-3000$

0-3000

0-3000

unclass

unclass

unclass

unclass
unclass

inferred

interred

inferred

inferred

interred

inferred

inferred

inferred

inferred

inferred

inferred

inferred

inferred

207.36 96.75
190.08 207.36

31.05 10.35
179.64 179.64 196.92 207.36 207.36 44.91 158.94 172.80 183.15 79.47 33.97 419.73 120.96 246.81 13.83 13.83 64.30 98.20 
average analyses of coal in colorado

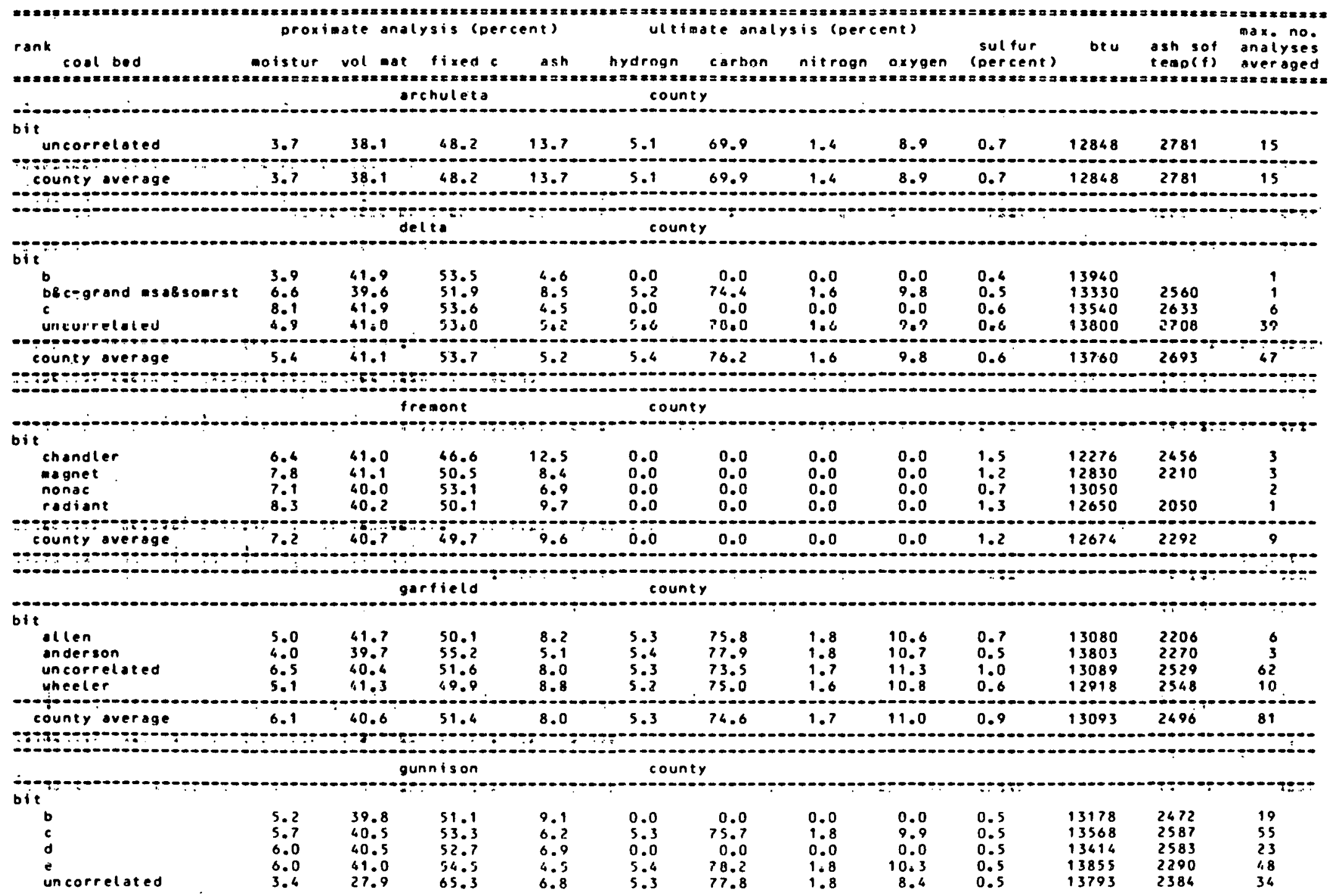




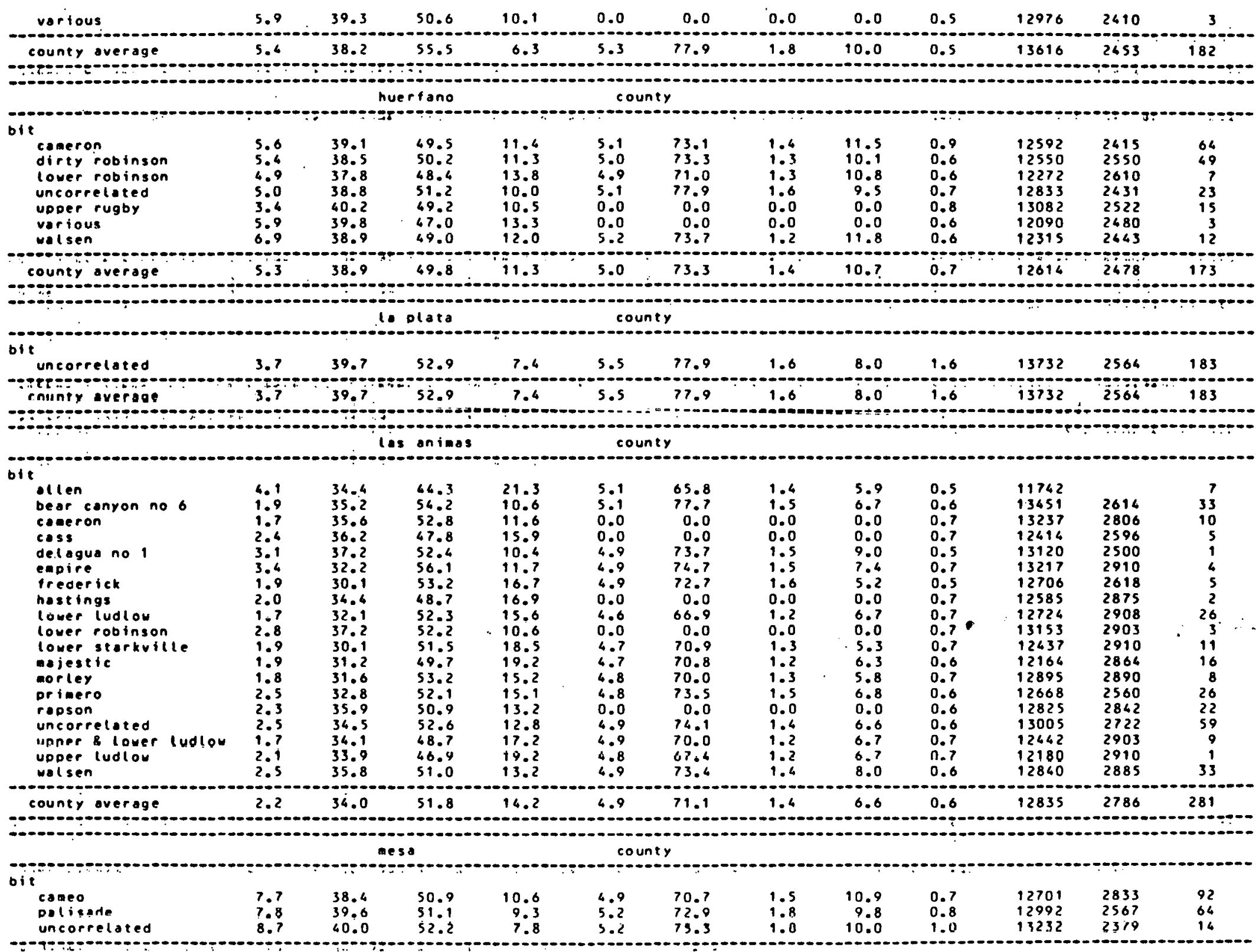




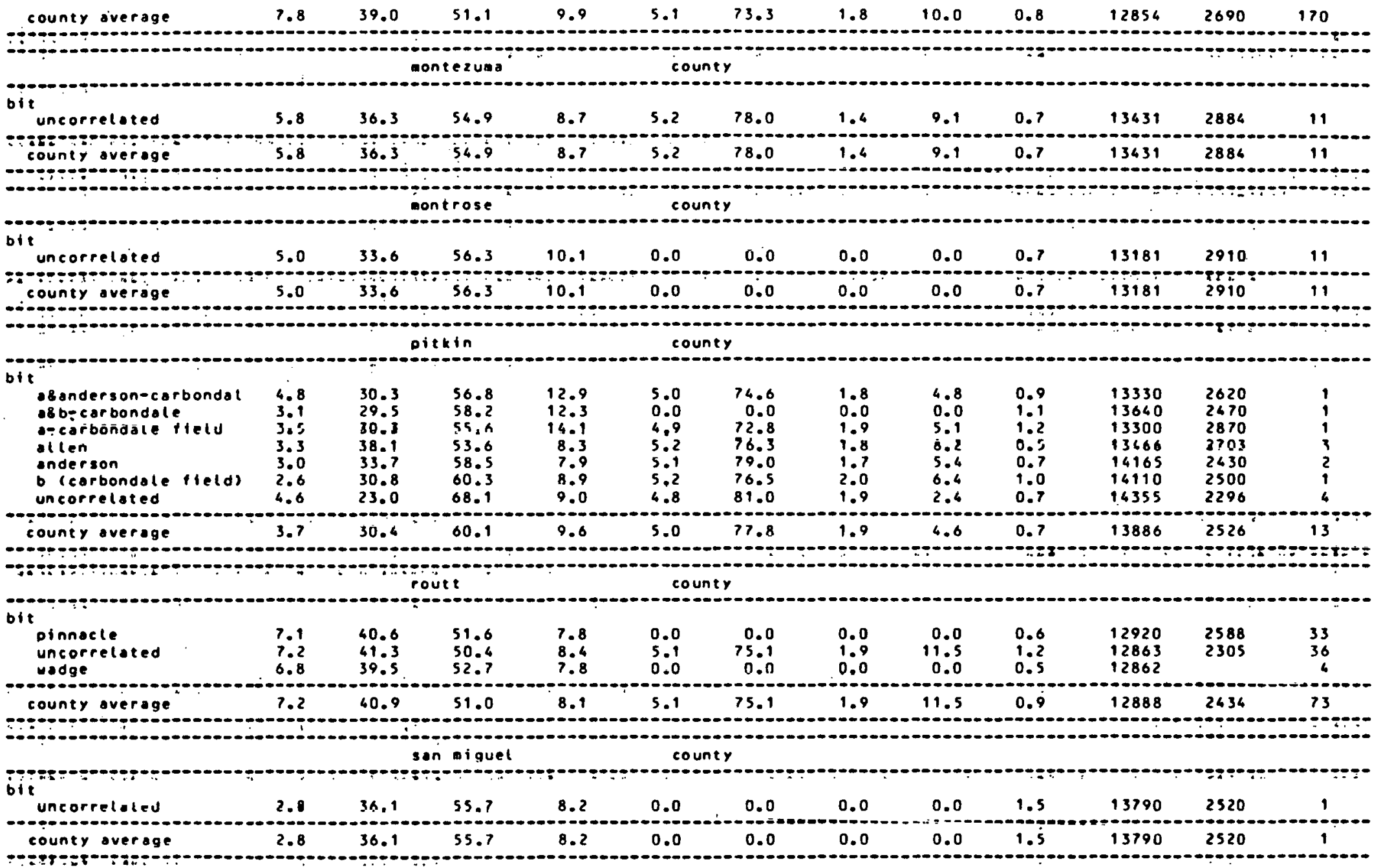

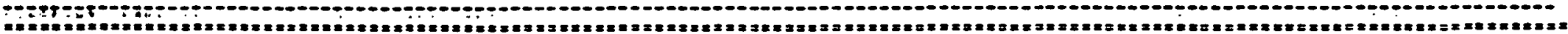


Colorado

\begin{tabular}{|c|c|}
\hline 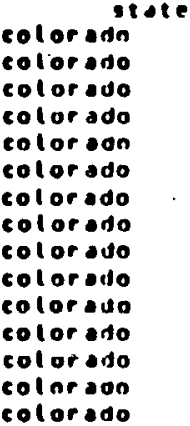 & 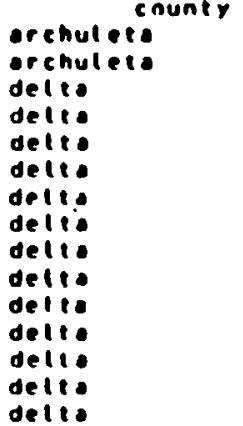 \\
\hline
\end{tabular}

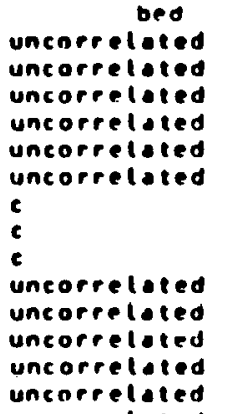

\begin{tabular}{|c|c|c|c|c|c|}
\hline $\begin{array}{l}6.9 \\
4.8 \\
6.0 \\
6.4 \\
6.9 \\
6.8 \\
8.8 \\
7.9 \\
7.4 \\
6.5 \\
4.8 \\
5.3 \\
4.6 \\
3.1\end{array}$ & $\begin{array}{l}4010.1 \\
40.0 \\
30.8 \\
41.8 \\
41.3 \\
40.7 \\
61.8 \\
41.7 \\
61.9 \\
61.3 \\
41.8 \\
41.5 \\
41.2 \\
41.9 \\
40.8 \\
411.7\end{array}$ & $\begin{array}{l}11 x e d c \\
66.2 \\
67.9 \\
53.8 \\
56.8 \\
36.5 \\
56.2 \\
53.7 \\
56.6 \\
53.4 \\
53.3 \\
53.1 \\
53.5 \\
52.7 \\
52.1 \\
53.3\end{array}$ & $\begin{array}{l}134 \\
13.8 \\
12.4 \\
6.8 \\
5.0 \\
4.8 \\
5.2 \\
4.6 \\
3.5 \\
3.3 \\
4.9 \\
3.4 \\
5.3 \\
6.2 \\
7.1\end{array}$ & $\begin{array}{l}20110 \\
0.7 \\
0.0 \\
0.5 \\
0.5 \\
0.5 \\
0.5 \\
0.6 \\
0.6 \\
0.6 \\
0.6 \\
0.5 \\
0.6 \\
0.6 \\
0.6 \\
0.6\end{array}$ & $\begin{array}{l}010 \\
12300 \\
12900 \\
13730 \\
13680 \\
13620 \\
13690 \\
13610 \\
13680 \\
13360 \\
14030 \\
13880 \\
13860 \\
13710 \\
13580 \\
13680\end{array}$ \\
\hline
\end{tabular}

1.110
4.0
4.00
1.0
1.5
1.0
1.0
1.5
1.0
1.5
9.5
3.5
3.0
4.5
3.0
3.5

continued..... 


\begin{tabular}{|c|c|}
\hline & \\
\hline colnradn & delta \\
\hline colorado & gartiel \\
\hline colorado & garfielf \\
\hline colorado & gartiel \\
\hline coloradon & qartiela \\
\hline colorado & qartield \\
\hline colorado & gartield \\
\hline colorado & garfield \\
\hline colorado & qarfield \\
\hline colorado & aarfield \\
\hline coloraco & gartield \\
\hline colorado & gartield \\
\hline colorado & garfield \\
\hline colorado & qartield \\
\hline color a do & gunn is on \\
\hline coloraro & qunnison \\
\hline coloraso & gunnison \\
\hline colorado & gunnison \\
\hline colorado & gunnison \\
\hline colorado & gunnison \\
\hline $\begin{array}{l}\text { colorado } \\
\text { coloradn }\end{array}$ & dunnison \\
\hline colorado & $\begin{array}{l}\text { gunnison } \\
\text { gunnis on }\end{array}$ \\
\hline colorado & gunnison \\
\hline $\begin{array}{l}\text { colorado } \\
\text { coloratan }\end{array}$ & $\begin{array}{l}\text { gunnison } \\
\text { qunnisnnn }\end{array}$ \\
\hline colorado & Junnison \\
\hline colorado & gunnison \\
\hline colorado & gunn is on \\
\hline colorado & gunnison \\
\hline $\begin{array}{l}\text { colorado } \\
\text { colorado }\end{array}$ & $\begin{array}{l}\text { gunnison } \\
\text { gunnison }\end{array}$ \\
\hline colorado & gunnison \\
\hline ronlerads & qunnison \\
\hline colorado & qunnison \\
\hline coloraso & gunnison \\
\hline colorado & gunnison \\
\hline $\begin{array}{l}\text { colorado } \\
\text { colorado }\end{array}$ & $\begin{array}{l}\text { gunnisan } \\
\text { gunnison }\end{array}$ \\
\hline $\begin{array}{l}\text { colorado } \\
\text { colorado }\end{array}$ & $\begin{array}{l}\text { qunnis on } \\
\text { qunnis on }\end{array}$ \\
\hline colorado & gunnison \\
\hline colorado & dunnisnn \\
\hline coloraco & hue, fario \\
\hline cotorado & huertano \\
\hline colorado & huerfano \\
\hline colnrado & huertano \\
\hline colorato & huertano \\
\hline colorado & huerfano \\
\hline colorato & nuerfano \\
\hline colnrado & huerfann \\
\hline
\end{tabular}

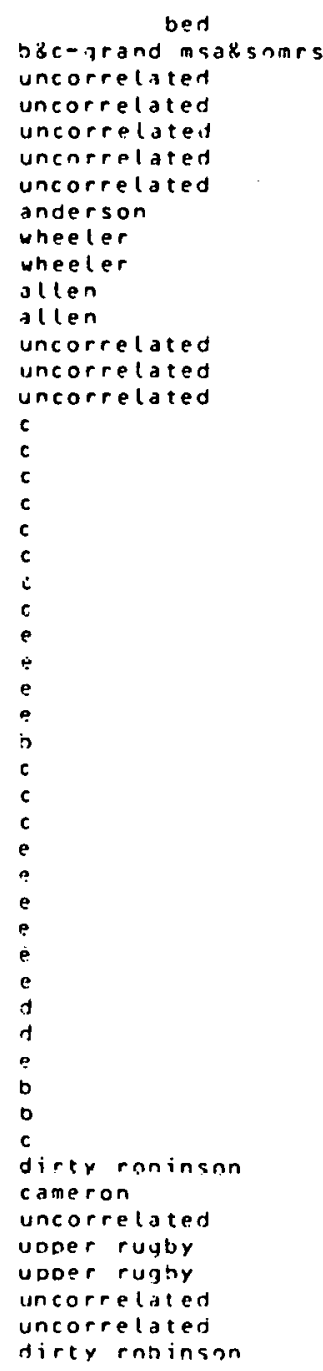

\begin{tabular}{|c|c|c|}
\hline & vol & $f i x \in d c$ \\
\hline 6.6 & 39.6 & 51.9 \\
\hline 5.1 & 61.8 & 53.7 \\
\hline 4.9 & 38.3 & 45.4 \\
\hline 5.2 & 38.6 & 49.3 \\
\hline 4.7 & $41) .0$ & 51.4 \\
\hline 4.4 & 38.3 & 50.9 \\
\hline $4 . ?$ & 38.9 & 54.6 \\
\hline 4.6 & 41.0 & 60.7 \\
\hline 5.3 & $41 . n$ & 49.8 \\
\hline $0 . ?$ & 41.1 & $50 . n$ \\
\hline 4.3 & 49.3 & 50.8 \\
\hline 9.4 & 30.9 & 50.9 \\
\hline 7.5 & 41.8 & 53.1 \\
\hline 5.1 & 41.6 & 49.4 \\
\hline 5.8 & 40.0 & 54.3 \\
\hline 6.1 & 41.5 & 53.4 \\
\hline 5.4 & $4 n: 2$ & $5 ? .94$ \\
\hline 5.7 & 41.5 & $5 \geqslant .1$ \\
\hline 5.9 & $4 \pi .0$ & 59.9 \\
\hline 6.0 & 40.6 & 53.9 \\
\hline A. 3 & $4 \pi . n$ & 53.3 \\
\hline 7.3 & 39.9 & $5>.6$ \\
\hline 0.3 & 41.0 & 53.9 \\
\hline 6.7 & $4 \pi .0$ & 53.4 \\
\hline 6.2 & 40.2 & 56.3 \\
\hline 6.7 & 40.8 & 54.2 \\
\hline 5.1 & 40.1 & $5 ? .7$ \\
\hline 6.6 & 40.0 & 54.7 \\
\hline $\begin{array}{l}0.0 \\
6.1\end{array}$ & 40.5 & 54.5 \\
\hline 7.1 & $4 \pi .4$ & 52.8 \\
\hline 5.0 & 47.0 & 54.5 \\
\hline 6.3 & 40.5 & $55 . ?$ \\
\hline 6.4 & 41.6 & 54.7 \\
\hline 6.8 & 40.9 & 54.7 \\
\hline 6.7) & 40.7 & 54.8 \\
\hline 7.1 & 39.7 & $5 ? .5$ \\
\hline 8.1 & 40.1 & 53.7 \\
\hline 7.5 & 40.8 & 57.7 \\
\hline 6.9 & 67.4 & 54.4 \\
\hline 5.7 & 30.5 & 59.6 \\
\hline 8.2 & 39.5 & 5ก.8 \\
\hline 0.4 & 39.4 & 55.2 \\
\hline 3.6 & $37 ?$ & 48.3 \\
\hline 7.2 & 41.8 & 47.7 \\
\hline 5.6 & 30.2 & 4.7 .1 \\
\hline 4.2 & 40.6 & 50.0 \\
\hline 3.3 & 39.8 & 51.6 \\
\hline 3.6 & 36.0 & 57.6 \\
\hline 4. & 35.4 & $56, ?$ \\
\hline & $? 7,1$ & 472 \\
\hline
\end{tabular}

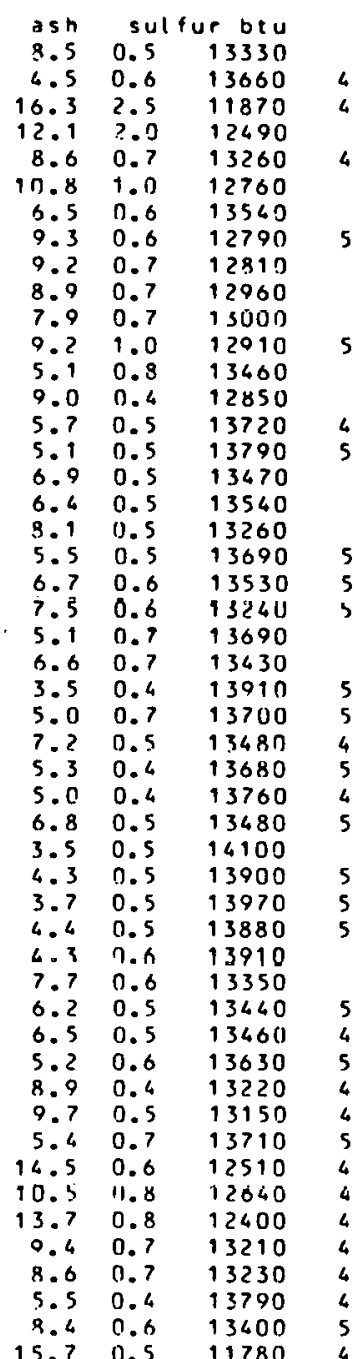

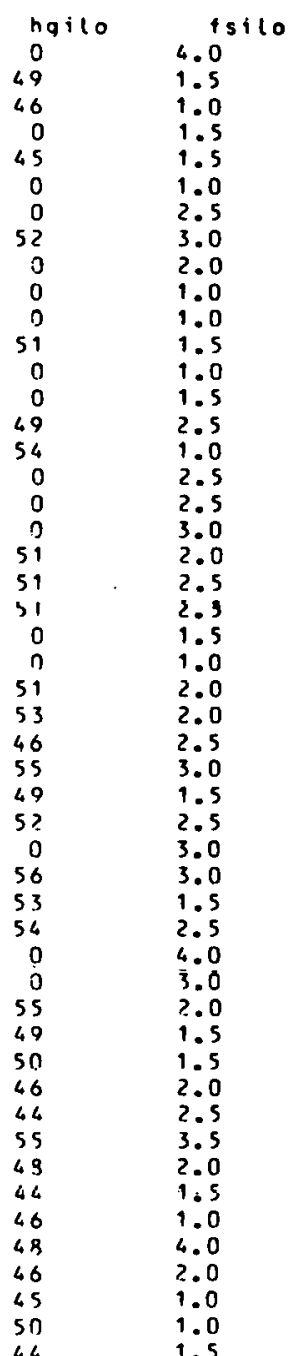




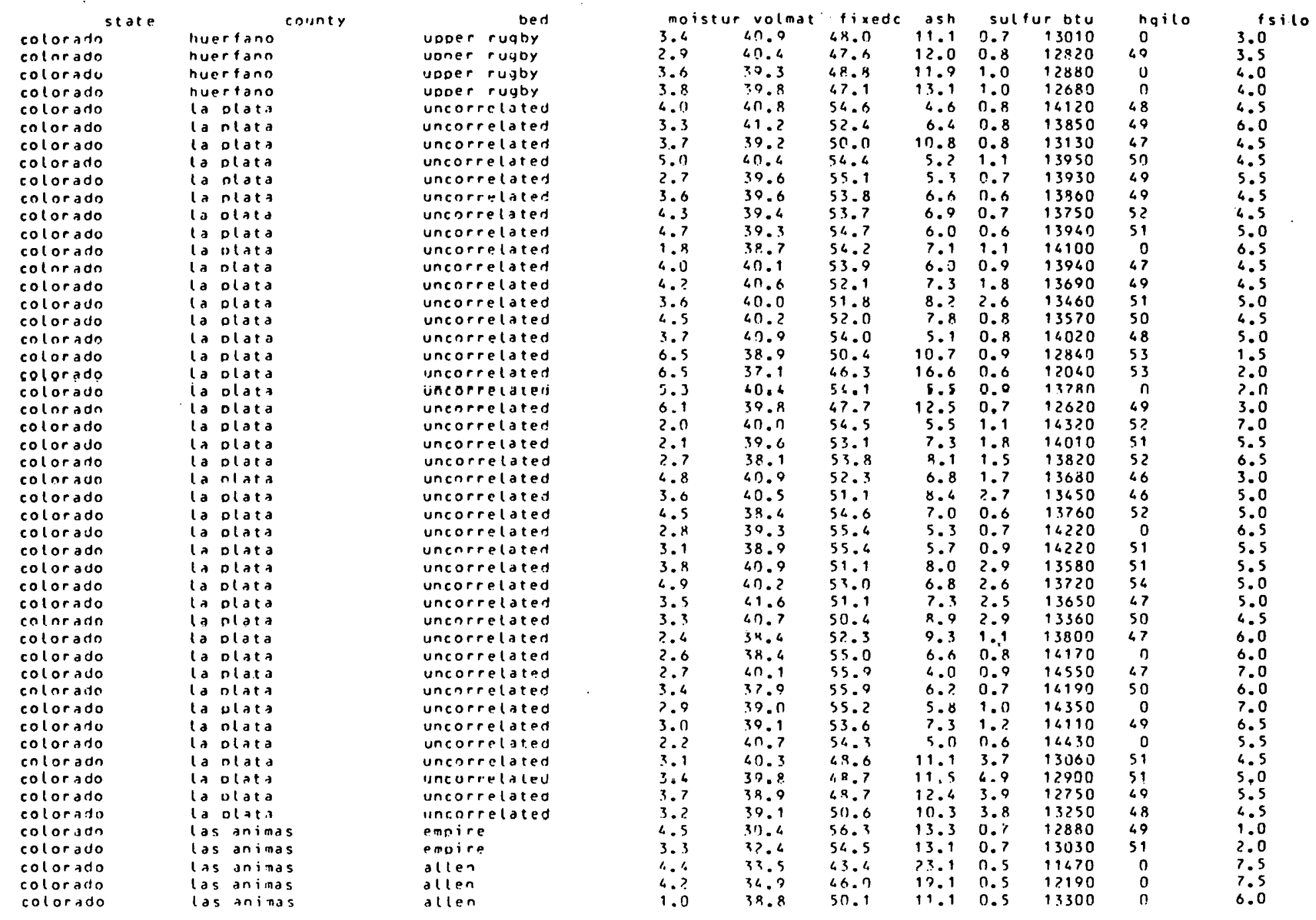




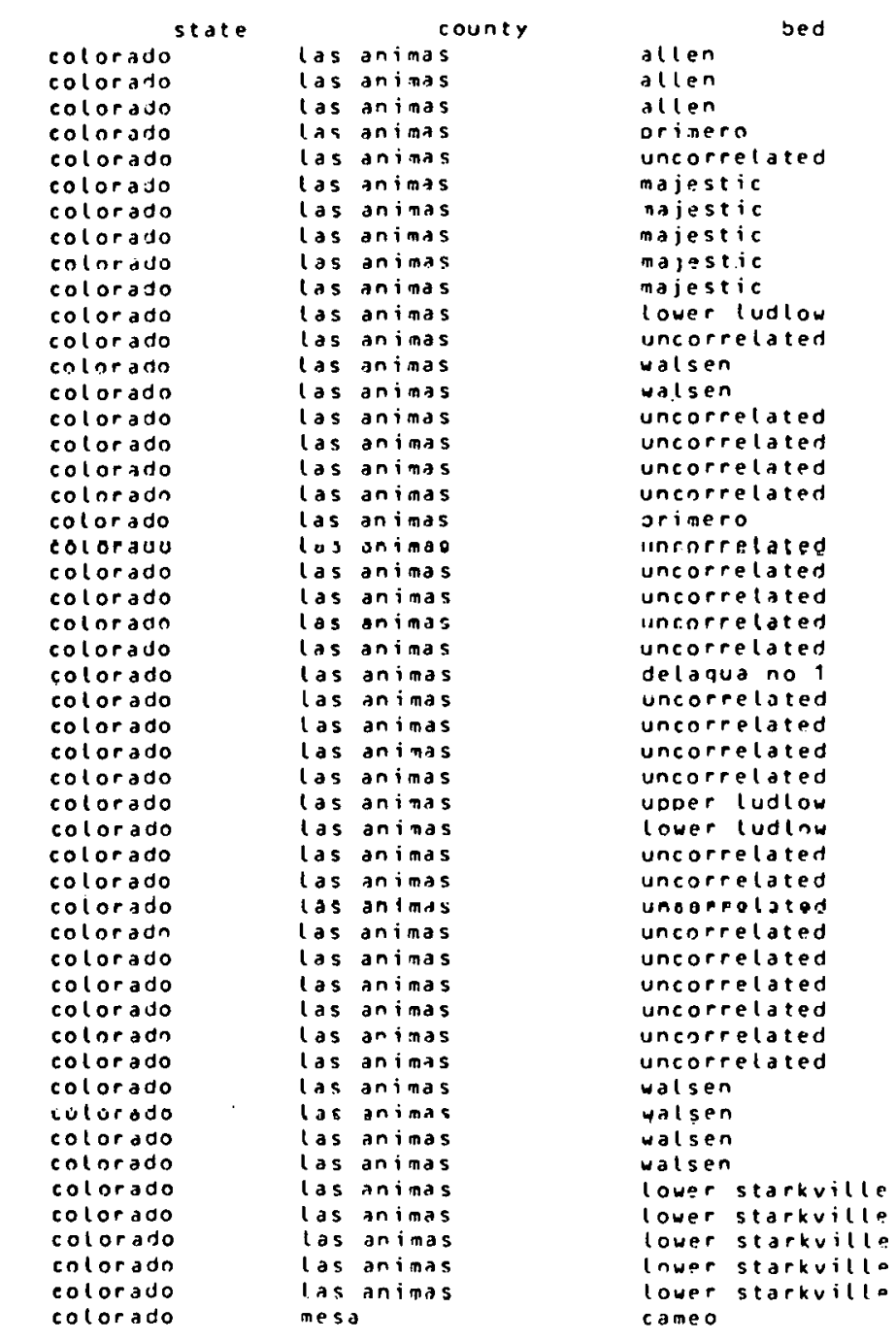

\begin{tabular}{|c|c|c|c|c|c|c|c|}
\hline \multicolumn{2}{|r|}{ t } & \multirow{2}{*}{$\begin{array}{l}f i x e d c \\
43.3\end{array}$} & \multirow{2}{*}{$\begin{array}{l}\text { ash } \\
23.1\end{array}$} & \multicolumn{2}{|c|}{ sult } & 10 & \multirow{2}{*}{ tsilo } \\
\hline 4.1 & 33.6 & & & 0.5 & 11480 & n & \\
\hline 4.6 & 34.0 & 43.5 & & 0.4 & 11580 & $n$ & 7.0 \\
\hline 4.4 & 33.4 & 4?. 5 & 24.1 & 0.5 & 11240 & 0 & 0.5 \\
\hline$? .6$ & 33.7 & 53.1 & $14 . ?$ & 3.7 & 12870 & 59 & $\begin{array}{l}0.3 \\
6.5\end{array}$ \\
\hline 3.6 & 31.5 & 53.0 & 15.5 & 0.6 & 12400 & 55 & 1.0 \\
\hline 2.3 & 30.4 & 47.7 & 21.0 & 0.5 & 11730 & 50 & 3.5 \\
\hline 1.9 & $3 ? .0$ & 49.1 & 18.9 & 0.6 & 12140 & 0 & 5.0 \\
\hline 7.1 & 30.8 & 43.9 & 20.3 & 0.6 & 11950 & 48 & 2.5 \\
\hline 2.2 & 30.7 & 40.7 & 19.6 & 0.5 & 12010 & 0 & 2.0 \\
\hline$? 1$ & 31.4 & $49 ?$ & 10.4 & 0.7 & 12150 & $5 ?$ & 2.0 \\
\hline 2.? & 20.7 & 51.4 & 19.9 & 0.7 & 12060 & 53 & 1.0 \\
\hline 2.5 & 37.2 & $5 n .3$ & 17.5 & 0.6 & 12040 & 47 & 1.0 \\
\hline 2.8 & 33.7 & 54.7 & 11.6 & 0.6 & 13100 & 0 & 1.5 \\
\hline 2.9 & 399 & 55.4 & 11.7 & 0.7 & 13020 & 49 & 1.5 \\
\hline 1.9 & 35.4 & 55.4 & 9.2 & 0.6 & 13880 & 62 & 7.5 \\
\hline 3.1 & 38.1 & 53.6 & 8.3 & 0.8 & 13650 & n & 5.0 \\
\hline$? .5$ & 37.5 & 54.8 & 7.7 & 0.6 & 13770 & 49 & 4.0 \\
\hline 2.7 & 28.5 & 60.7 & 10.8 & 0.8 & 13640 & 80 & 1.0 \\
\hline $2 . ?$ & 33.0 & 51.3 & 15.7 & 0.5 & $1 \geqslant 670$ & 58 & 6.0 \\
\hline$? .6$ & 36.5 & 52.4 & 11.1 & 0.5 & 12880 & 51 & 2.0 \\
\hline 3.5 & 38. & 48.7 & 9.9 & 0,5 & 1 2RSO & $5 ?$ & 4.0 \\
\hline 2.8 & 30.7 & 57.1 & 12.2 & 0.6 & 13190 & 57 & 4.5 \\
\hline 2.1 & 31.7 & 53.5 & 11.8 & 0.6 & 13250 & 0 & 6.0 \\
\hline 3.9 & 30.7 & 54.5 & 14.8 & 0.5 & 12610 & J & 1.5 \\
\hline 3.1 & 37.2 & $5 ? .4$ & 10.4 & 0.5 & 13120 & 0 & 3.5 \\
\hline 2.4 & 35.7 & 5?.? & 12.1 & 0.9 & 13160 & 0 & 4.5 \\
\hline 2.4 & 32.9 & 59.8 & 15.3 & 0.8 & 12860 & 35 & 6.5 \\
\hline 2.1 & 34.7 & 51.4 & 13.9 & 0.7 & 13020 & 55 & 6.0 \\
\hline 2.6 & 34.0 & 51.3 & 14.7 & 0.7 & 12860 & 0 & 0.5 \\
\hline 2.1 & 33.9 & 45.9 & 17.2 & 0.7 & 12180 & 49 & 5.5 \\
\hline$?$ & 30.2 & 50.0 & 19.8 & 0.8 & 11990 & 48 & 4.5 \\
\hline 1.9 & 31.6 & 56.0 & 12.4 & 0.6 & 13350 & 72 & 5.5 \\
\hline 2.4 & 31.7 & 53.6 & 14.7 & 0,5 & $1 \geq 860$ & 75 & 6.5 \\
\hline 1.8 & 30,5 & 50.3 & 19.2 & 0.6 & 12160 & 49 & 4.5 \\
\hline 2.7 & 33.7 & 51.4 & 14.4 & U. 8 & 12700 & 43 & 2.5 \\
\hline 2.5 & 33.1 & 49.0 & 17.9 & 0.7 & 12230 & 5? & 5.5 \\
\hline 2.4 & 35.7 & 49.9 & 14.4 & 0.7 & 12850 & 59 & 6.5 \\
\hline 2.2 & 35.3 & 48.6 & 16.1 & 0.0 & 12530 & 0 & 0.0 \\
\hline 9.8 & 37.0 & $\begin{array}{l}43.0 \\
51.0\end{array}$ & 12.0 & 0.7 & 13130 & 44 & 3.5 \\
\hline 3.9 & 37.2 & 49.0 & 13.8 & 0.7 & $1 \geqslant>30$ & 48 & 3.0 \\
\hline$? .7$ & 34.9 & $\begin{array}{l}48.11 \\
48.7\end{array}$ & 15.0 & 0.6 & 12400 & 44 & $\begin{array}{l}3.0 \\
3.0\end{array}$ \\
\hline 3.2 & 35.0 & 49.0 & 16.0 & 0.6 & 12430 & 46 & 2.0 \\
\hline 3.3 & 39.9 & $\begin{array}{r}49.0 \\
40.2\end{array}$ & 16.0 & 0.7 & $1>530$ & 4? & 2,5 \\
\hline 3.0 & 34.6 & 45.9 & 10.6 & 0.8 & 11810 & 47 & 3.0 \\
\hline 2.1 & 29.7 & $\begin{array}{l}51.5 \\
51.5\end{array}$ & 13.8 & 0.7 & 12380 & 57 & $\begin{array}{l}3.0 \\
7.0\end{array}$ \\
\hline 2.1 & 29.7 & $50 . ?$ & 20.1 & n.s & 12130 & 69 & 5.5 \\
\hline 2.6 & 39.4 & $5 \geqslant .5$ & $1 \mathrm{B.1}$ & 0.9 & 12790 & 0 & 6.5 \\
\hline 2.4 & 31.6 & $5 ? .1$ & 16.3 & 0.8 & 12820 & 66 & 7.0 \\
\hline 9.7 & $3 n .7$ & 52.3 & 17.0 & 0.8 & 12700 & 0 & 6.0 \\
\hline 8.8 & 39.8 & $5 ? .2$ & 9.0 & 0.6 & 12900 & 47 & 1.5 \\
\hline
\end{tabular}




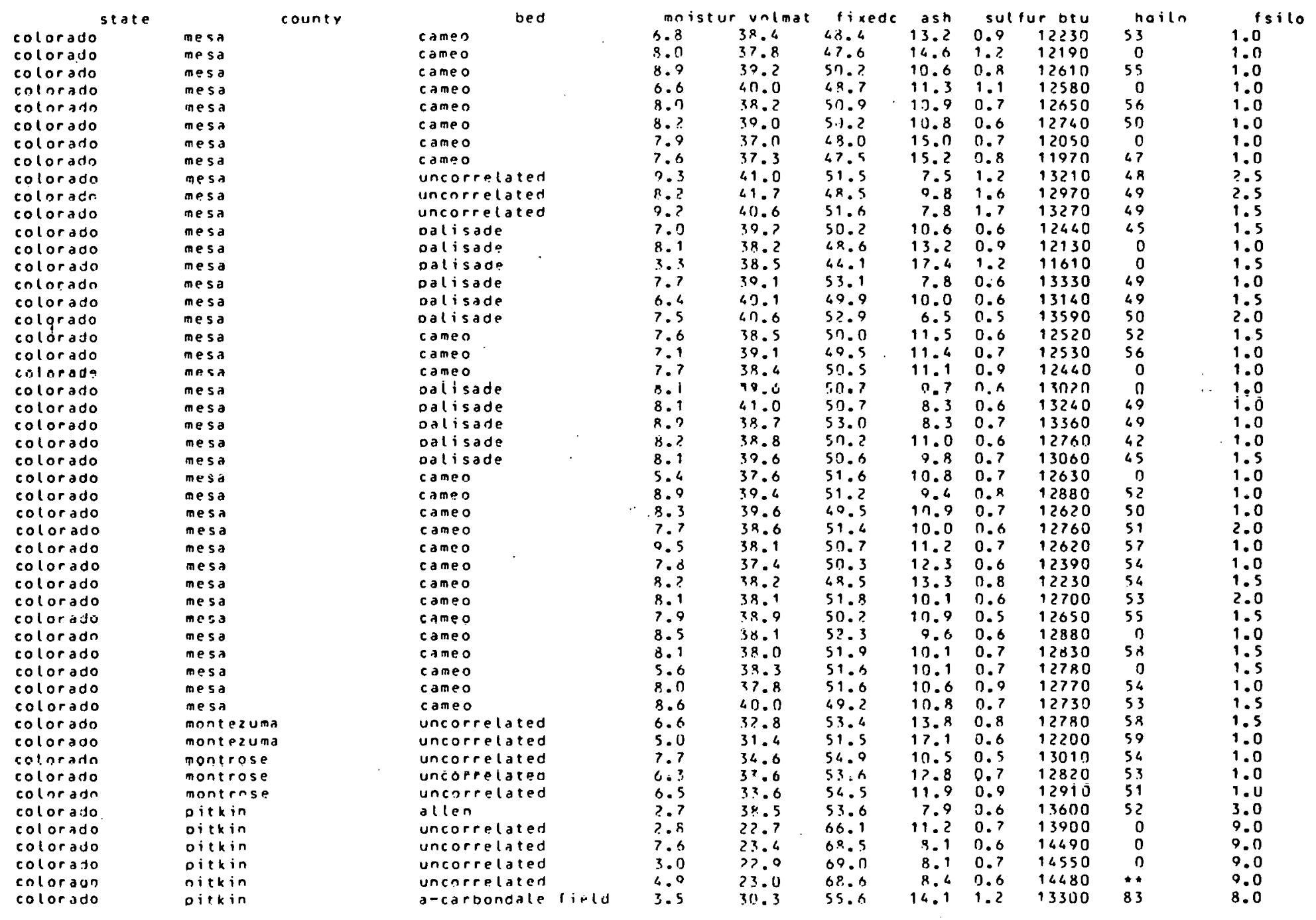


colorado state

colorsao

coloraus

colorado

colorasto

coloran

color $\$ 00$

colorado counter

oichin

ciakto

gitein

oletin

ravit

coute

son aiquel het

U (carunndale lield) abancerson-carbondal ast-cartundate

anterson
onderson

uncerrelaced

oinnocle

uncorpeloced molstur volnat tixedc
2.0

3

3.1 ?0.5 3R.?

12.91 .016110

$\begin{array}{llllll}3.1 & 3.2 & 99.5 & 8.1 & 0.7 & 16100 \\ 2.3 & 3.9 & 57.5 & 1.6 & 0.6 & 14230\end{array}$

$7.241 .3 \quad 41.9 \quad 9.81 .1 \quad 12070$

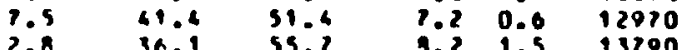

$16.159 .7 \quad 9.2 \quad 1.5 \quad 13790$

$\begin{array}{cc}14+110 & 18110 \\ 69 & 7.0 \\ 11 & 9.0 \\ 86 & 8.0 \\ 71 & 7.0 \\ 64 & 8.0 \\ 11 & 1.5 \\ 50 & 1.5 \\ 0 & 2.0\end{array}$



National Coal Resources Data Systen

U.S. Geological survey

identified coal resources in georgia

\section{(In millions of short tons)}

\begin{tabular}{|c|c|c|c|c|c|c|c|c|c|c|c|c|}
\hline $\begin{array}{l}\text { rank } \\
\text { coal bed } \\
\text { overburden } \\
m=x=0=0 x=a=0\end{array}$ & $\begin{array}{r}\text { aeasur } \\
14=28 \\
=x=2=3 x=0\end{array}$ & $\begin{array}{l}\text { ed and in } \\
\begin{array}{l}28-42 \\
=x=0=8=0\end{array}\end{array}$ & $\begin{array}{l}\text { idicated } \\
\text { Over } 42 \\
=x=a=x=a x \\
\text { dade }\end{array}$ & 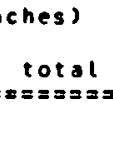 & $=x==x=28$ & $\begin{array}{l}\text { inferred } \\
28-42 \\
=x= \pm=x=2 \\
\text { ounty }\end{array}$ & $\begin{array}{l}\text { (inches) } \\
\text { over } 42 \\
x=x=x=x=x\end{array}$ & $\begin{array}{l}\text { totol } \\
x=2 x=2=\end{array}$ & $\begin{array}{c}14-28 \\
x=x=2 x=x=0\end{array}$ & $\begin{array}{l}\text { total }(i \\
28-42 \\
2 x=x=2=2=\end{array}$ & $\begin{array}{l}\text { nehes) } \\
\text { Over } 42 \\
=x=x=2=x=\end{array}$ & $\begin{array}{l}\operatorname{tat} a l \\
=x=0=\end{array}$ \\
\hline \multicolumn{13}{|l|}{$\begin{array}{l}\text { bit } \\
\text { no } 1\end{array}$} \\
\hline $0-1000$ & 0.50 & 0.00 & 0.00 & 0.50 & 0.00 & 0.00 & 0.00 & 0.00 & 0.50 & 0.00 & 0.00 & 0.50 \\
\hline $0-1000$ & 0.00 & 0.17 & 0.00 & 0.17 & 0.00 & 0.00 & 0.00 & 0.00 & 0.00 & 0.17 & 0.00 & 0.17 \\
\hline $0-1000$ & 3.34 & D.nn & n.nn & 3.34 & 0.00 & 0.00 & 0.00 & 0.00 & 3.34 & 0.00 & 0.00 & 3.34 \\
\hline $0-1000$ & 12.10 & 0.00 & 0.00 & 12.10 & 0.00 & 0.00 & 0.00 & 0.00 & 12.10 & 0.00 & 0.00 & 12.10 \\
\hline $0-1000$ & 0.20 & 0.00 & 0.00 & 0.20 & 0.00 & 0.00 & 0.00 & 0.00 & 0.20 & 0.00 & 0.00 & 0.20 \\
\hline county total & 16.14 & 0.17 & 0.00 & 16.31 & 0.00 & $0 . \mathrm{n}$ & 0.00 & 0.00 & 16.16 & 0.17 & 0.00 & 16.31 \\
\hline \\
\hline${ }_{\text {no }} 3^{0-1000}$ & 0.50 & 0.00 & 0.00 & 0.50 & 0.00 & 0.00 & 0.00 & 0.00 & 0.50 & 0.00 & 0.00 & 0.50 \\
\hline no 4 U-iuvo & 0.00 & 0.33 & 0.00 & 0.33 & $n, n n$ & 0.00 & 0.00 & 0.00 & 0.00 & 0.33 & 0.00 & 0.33 \\
\hline $0-1000$ & 6.66 & 0.00 & 0.00 & 6.66 & 0.00 & 0.00 & 0.00 & 0.00 & 6.66 & 0.00 & 0.00 & 6.66 \\
\hline county total & 7.16 & 0.33 & 0.00 & 7.49 & 0.00 & 0.00 & 0.00 & 0.00 & 7.16 & 0.33 & 0.00 & 7.49 \\
\hline $\begin{array}{c}\text { overburden } \\
\text { subtotal } \\
0-1000\end{array}$ & $23.30^{\circ}$ & 0.50 & 0.00 & 23.80 & u.vu & 0.00 & 0.00 & 0.00 & 23.30 & $0.5 n$ & 0.00 & 23.80 \\
\hline $\begin{array}{l}\operatorname{tot} a l \\
=x=x=x=x=x=0\end{array}$ & $\begin{array}{l}23.30 \\
=20=x=20=\end{array}$ & $\begin{array}{c}0.50 \\
z=2 x=2 x=2\end{array}$ & $\begin{array}{c}0.00 \\
z=x=\geq==x\end{array}$ & $\begin{array}{l}23.80 \\
==== \pm=2\end{array}$ & $\begin{array}{c}0.00 \\
=x=3 x=2 x\end{array}$ & $\begin{array}{c}0.00 \\
x=2=x=2 x\end{array}$ & $\begin{array}{c}0.00 \\
x=2==2 x=\end{array}$ & $\begin{array}{l}0.00 \\
=x=2=\end{array}$ & $\begin{aligned} & 23.30 \\
= & =2 x=2=x\end{aligned}$ & $\begin{array}{l}0.50 \\
=20=2=2\end{array}$ & $=0.00$ & $\begin{array}{l}23.80 \\
x=28=8=\end{array}$ \\
\hline \multicolumn{13}{|l|}{$\begin{array}{l}\text { overburden } \\
\text { total }\end{array}$} \\
\hline $\begin{array}{c}0-1000 \\
0\end{array}$ & .23 .30 & 0.50 & 0.00 & 23.80 & 0.00 & 0.00 & 0.00 & 0.00 & 23.30 & 0.50 & 0.00 & 23.80 \\
\hline$=$ grand total & $=\begin{aligned} & 23.30 \\
= & x===\end{aligned}$ & $\begin{array}{l}0.50 \\
=x=x=2\end{array}$ & $\begin{array}{l}0.00 \\
=x=20\end{array}$ & $\begin{array}{l}23.80 \\
=2=8=x=\end{array}$ & $\begin{array}{l}0.00 \\
x=2 x \leq 2\end{array}$ & 0.00 & 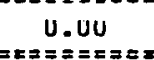 & $\begin{array}{l}0.00 \\
=3=3 x=0 \\
0=0\end{array}$ & $\begin{array}{l}23.30 \\
==* 2 \pi=8\end{array}$ & $\begin{array}{l}0.50 \\
==== \pm\end{array}$ & $\begin{array}{l}n, n n \\
== \pm x=2\end{array}$ & $=23.80$ \\
\hline
\end{tabular}


resource figures taken from:

source

usga coal inu map yeor

1946 base year of resource

1945 
average analyses of coal in georgio

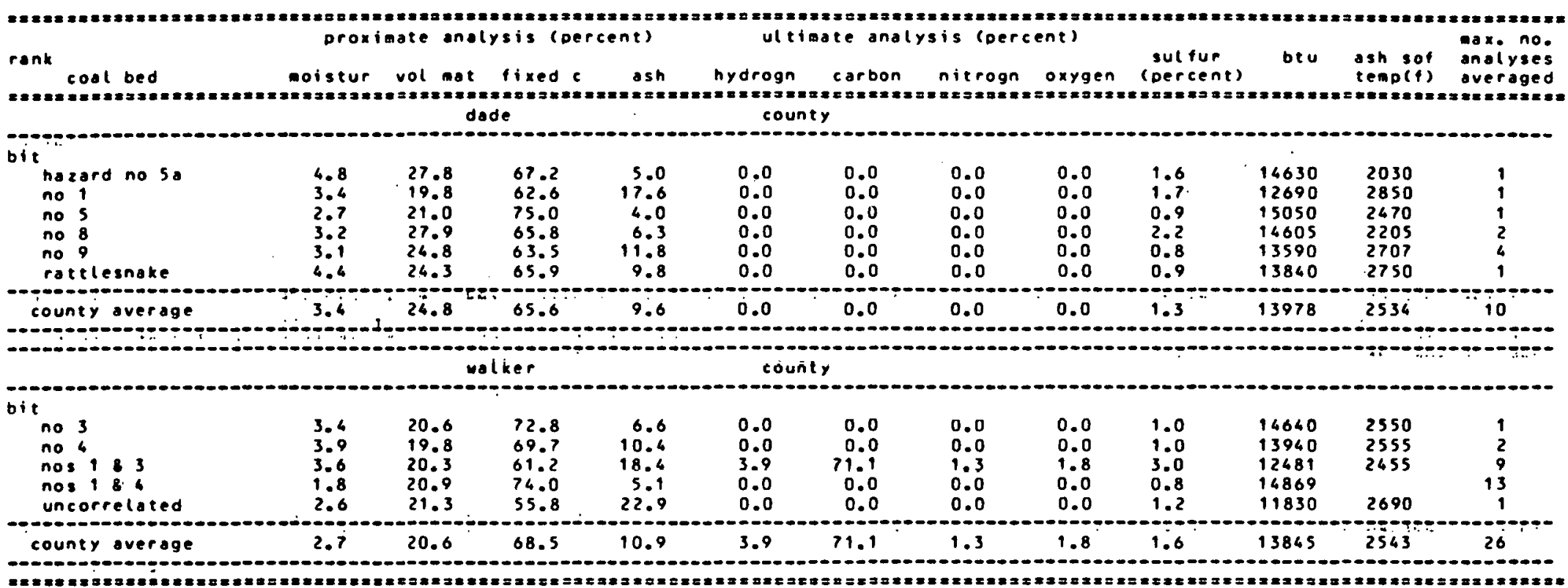



National Coal Resources Data Systed

U.S. Geological survey

identified coal resources in idaho

(In millions of short tons)

all records contain unclassified thickness andor reliability

total tonnages $\quad 9.24$

state county

bed

brown bear

broun bear

progressive

boise

boise

bit
bii
bit
bit
bit
bit

teton

teton

idan teton resource figures taken from:

source

idaho bng Danph 92 base thickness overburden

(inches or feet) (teet)

$0-1000$
$0-3000$
$0-1000$

$0-3000$
$0-1000$

1951 unclass

uncloss

unclass

1951 unclass
1951 unclass

$0-1000$

$0-2000$

$0-1000$

$0-2000$

reliability

tonnage

$\begin{array}{ll}\text { indicatd } & 1.42 \\ \text { inferedd } & 1.62 \\ \text { indicatd } & 2.13 \\ \text { inferred } & 2.13 \\ \text { indicatd } & 1.07 \\ \text { inferred } & 1.07\end{array}$

year base year of resource

$1951 \quad 1951$ 
overoge onalyses of coal in idaho

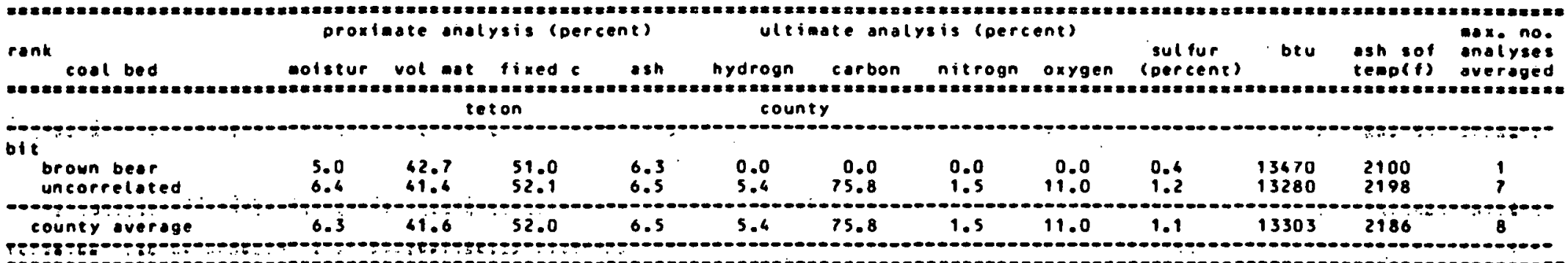

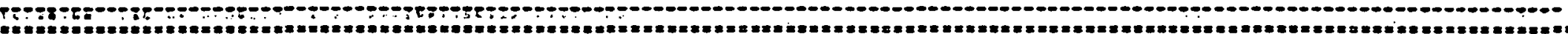


Idaho icte celon county brown bed

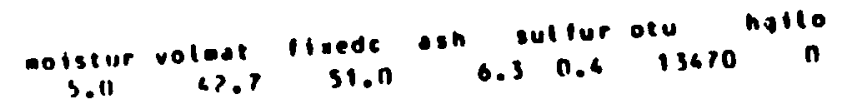

12110

2.5 Idono 
$\bar{E}$
$\frac{\bar{z}}{2}$ 
National Coat Resources data System

U.S. Geological surver

identified coal resources in illinois

(In millions of short tons)

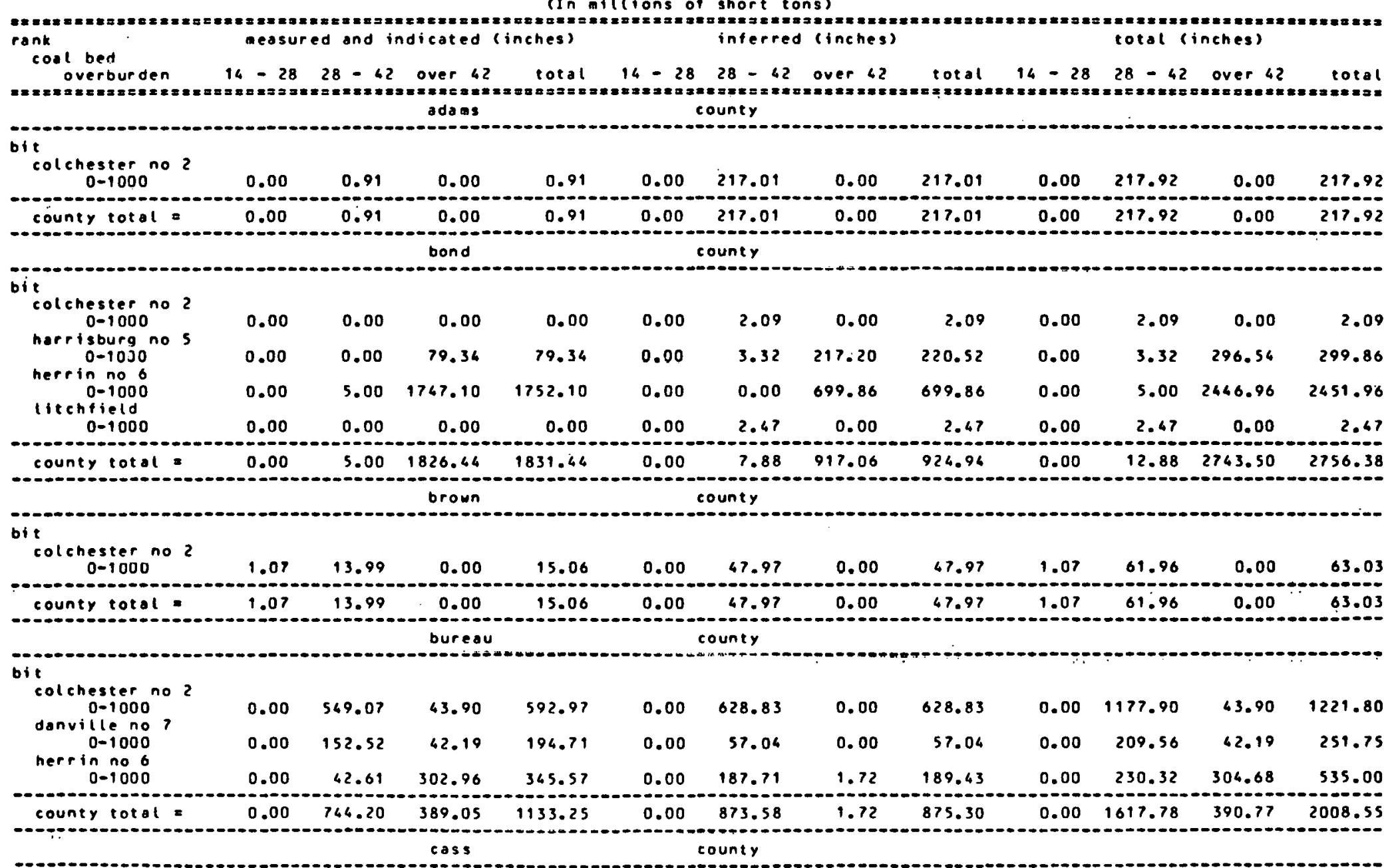

bit 


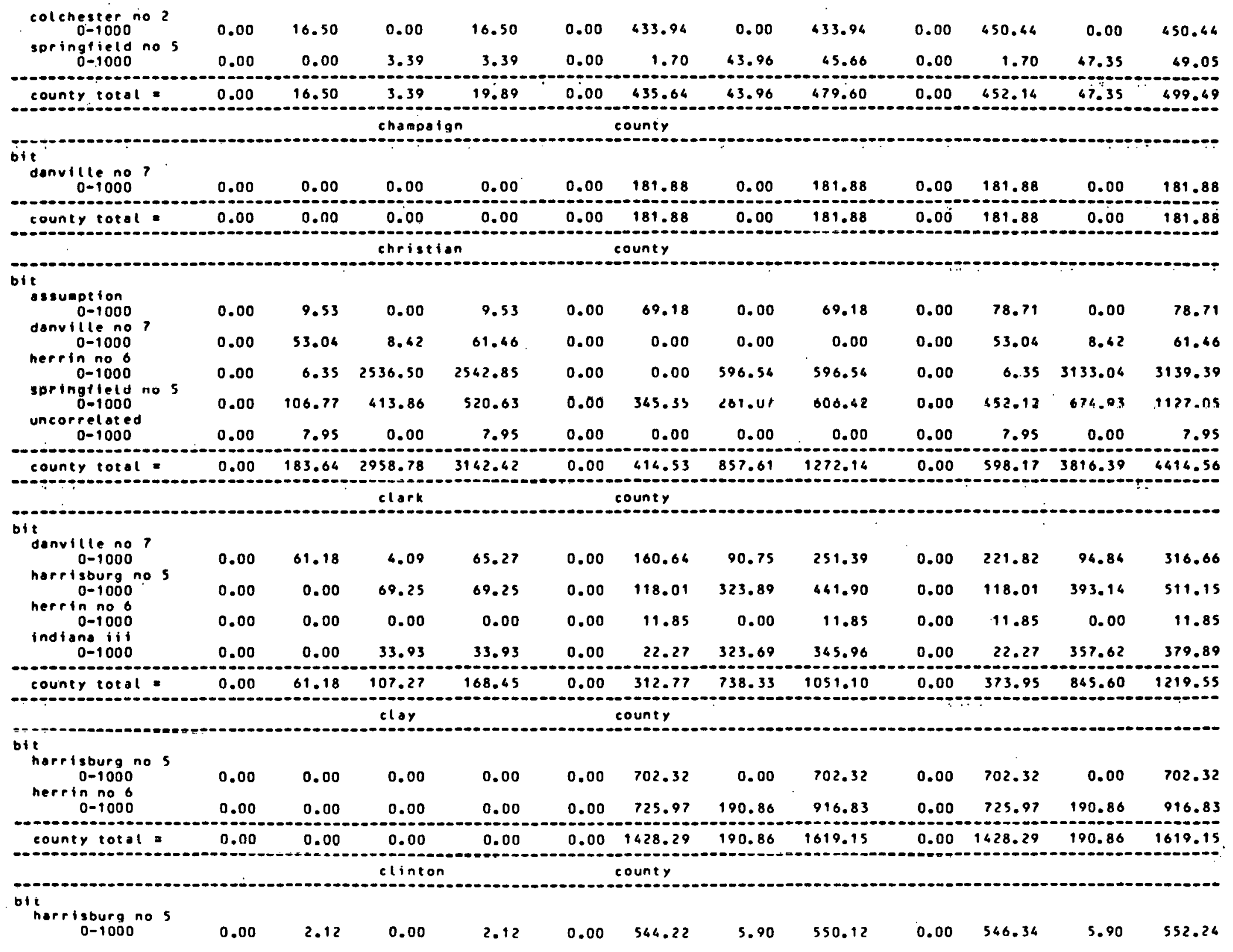




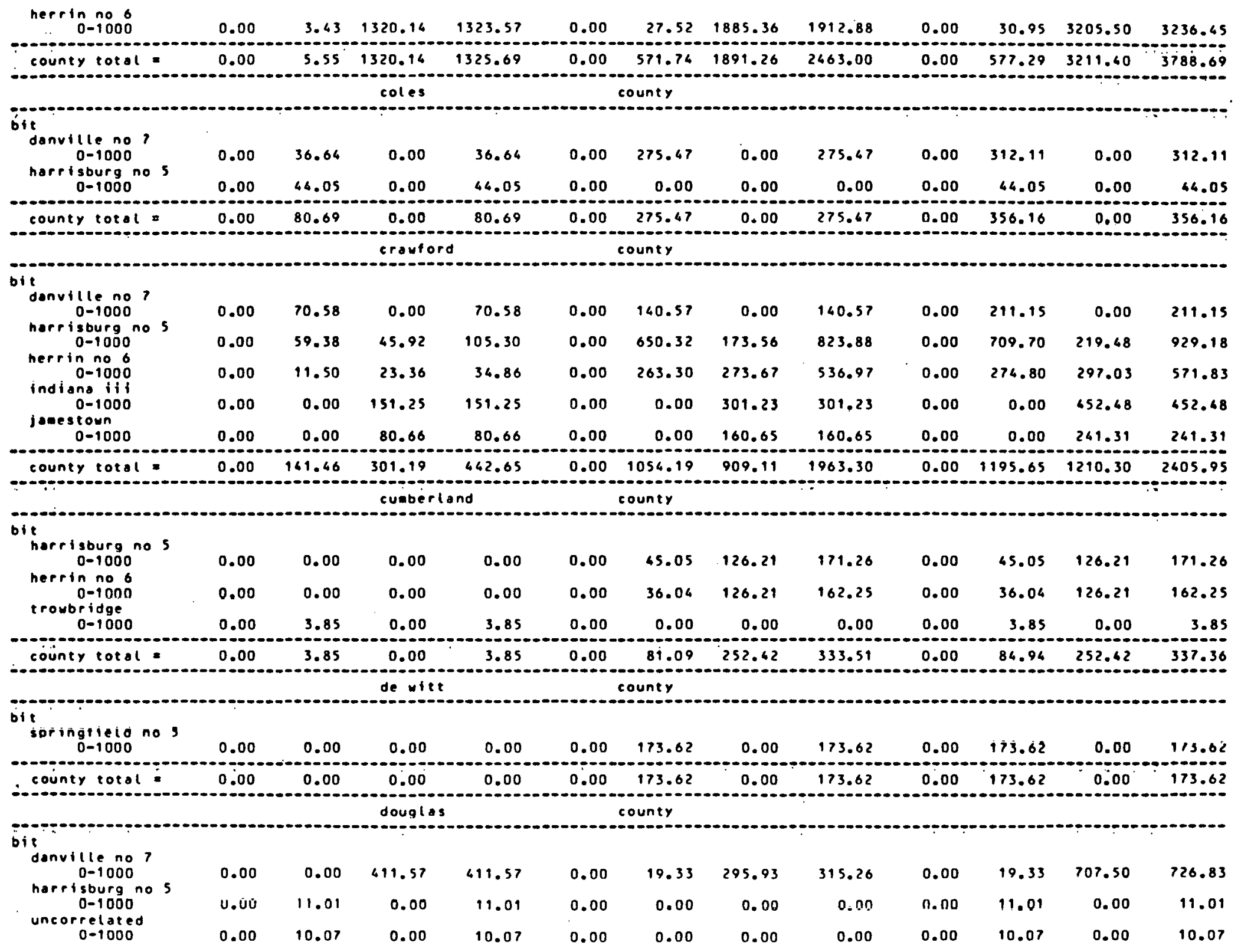




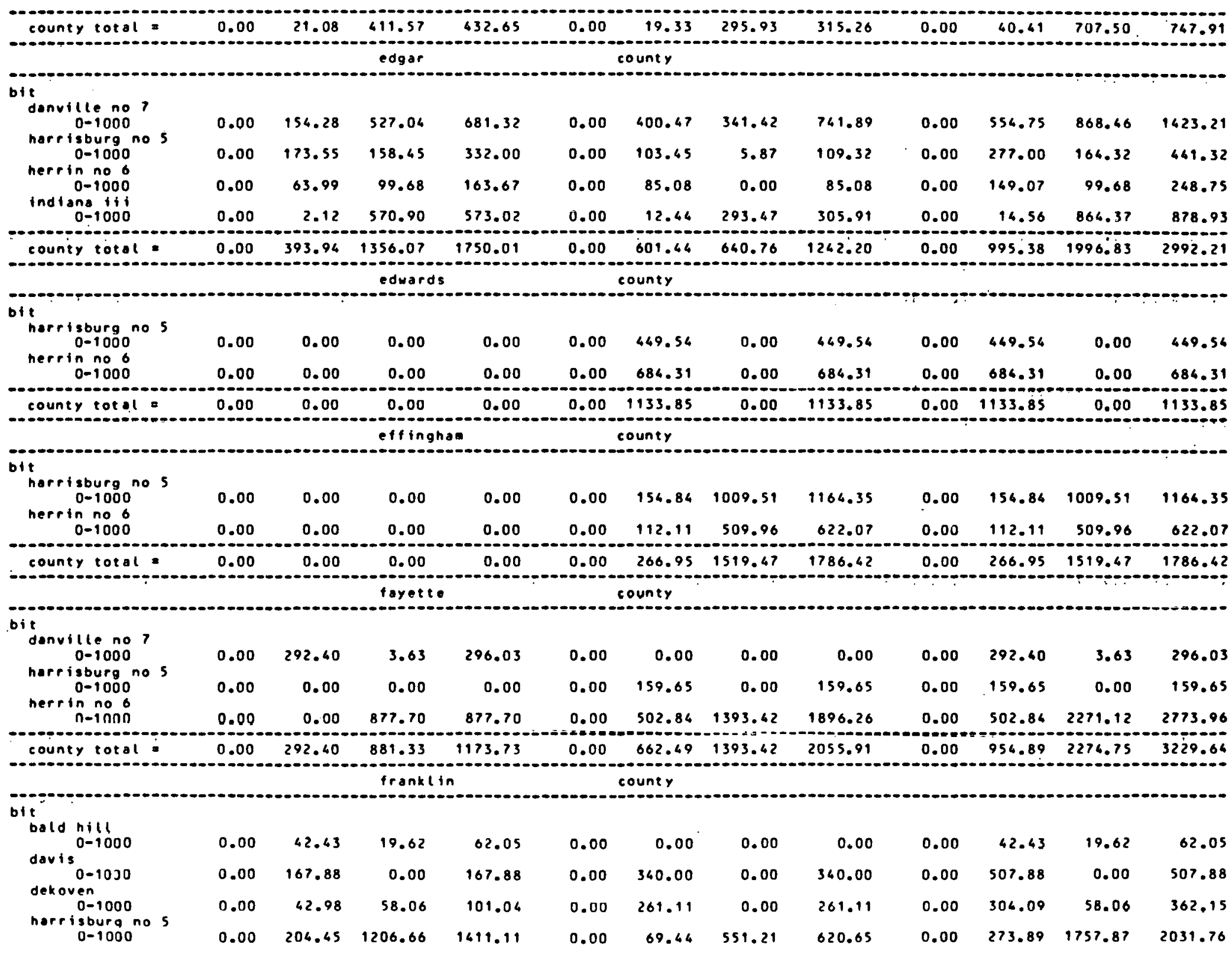




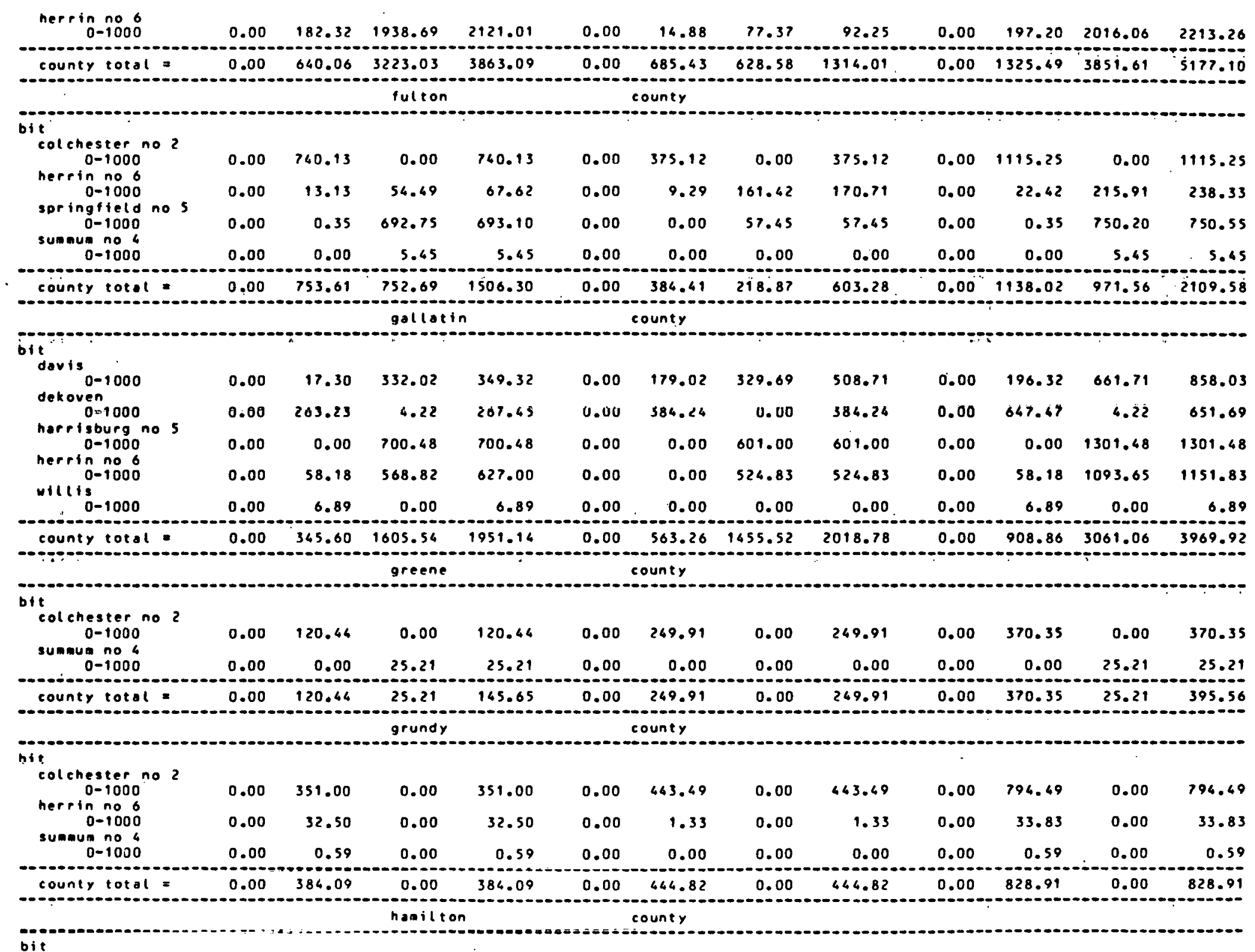




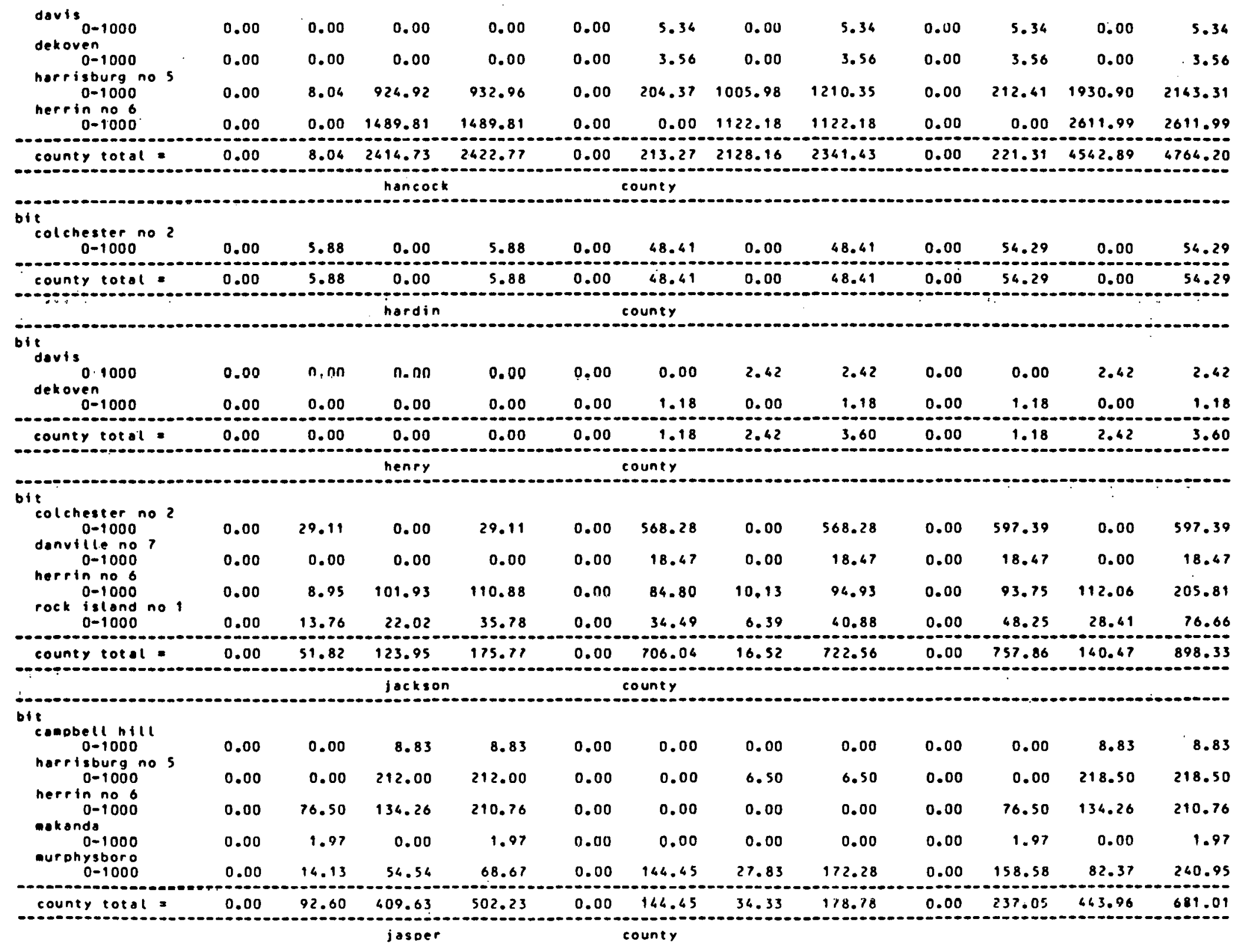




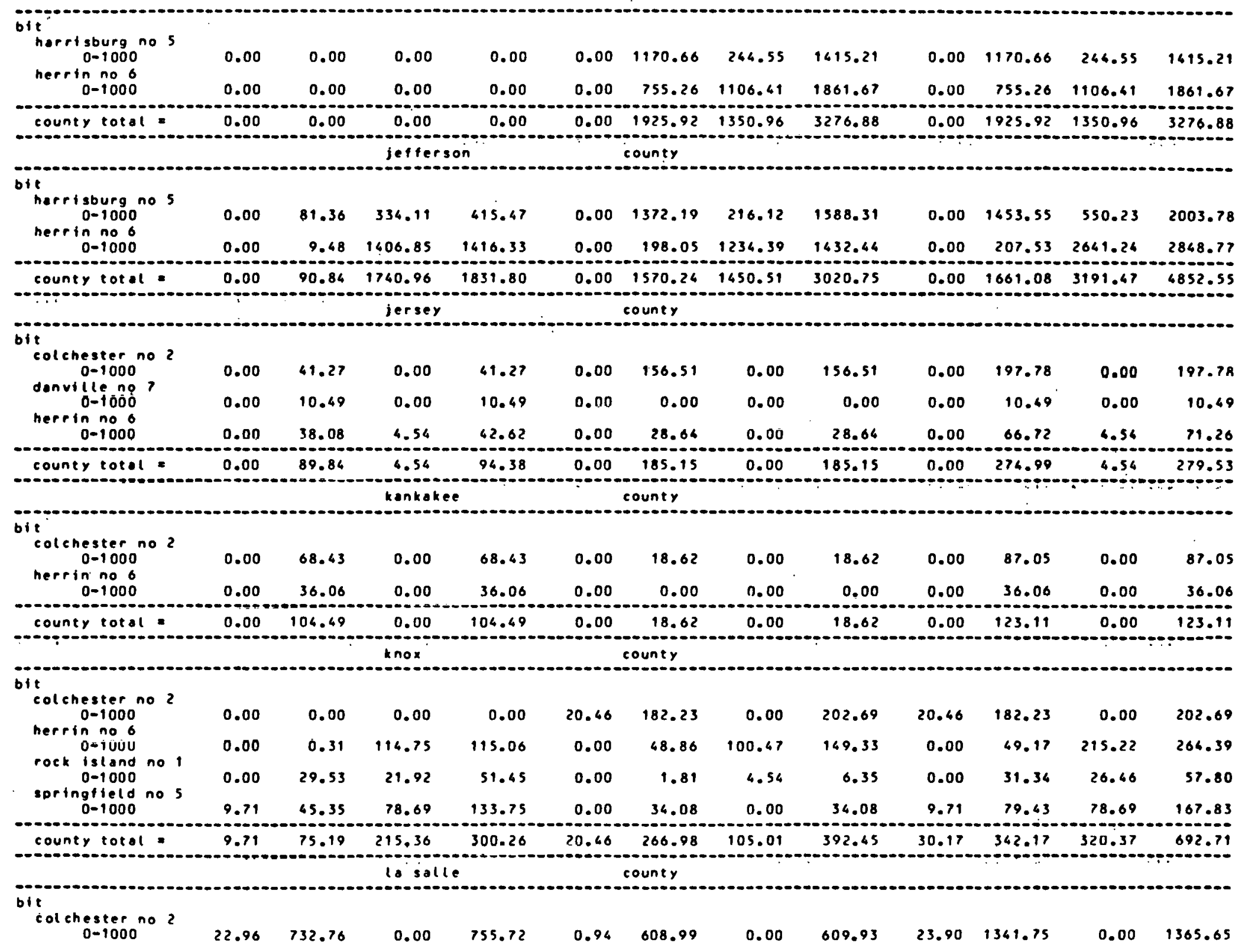




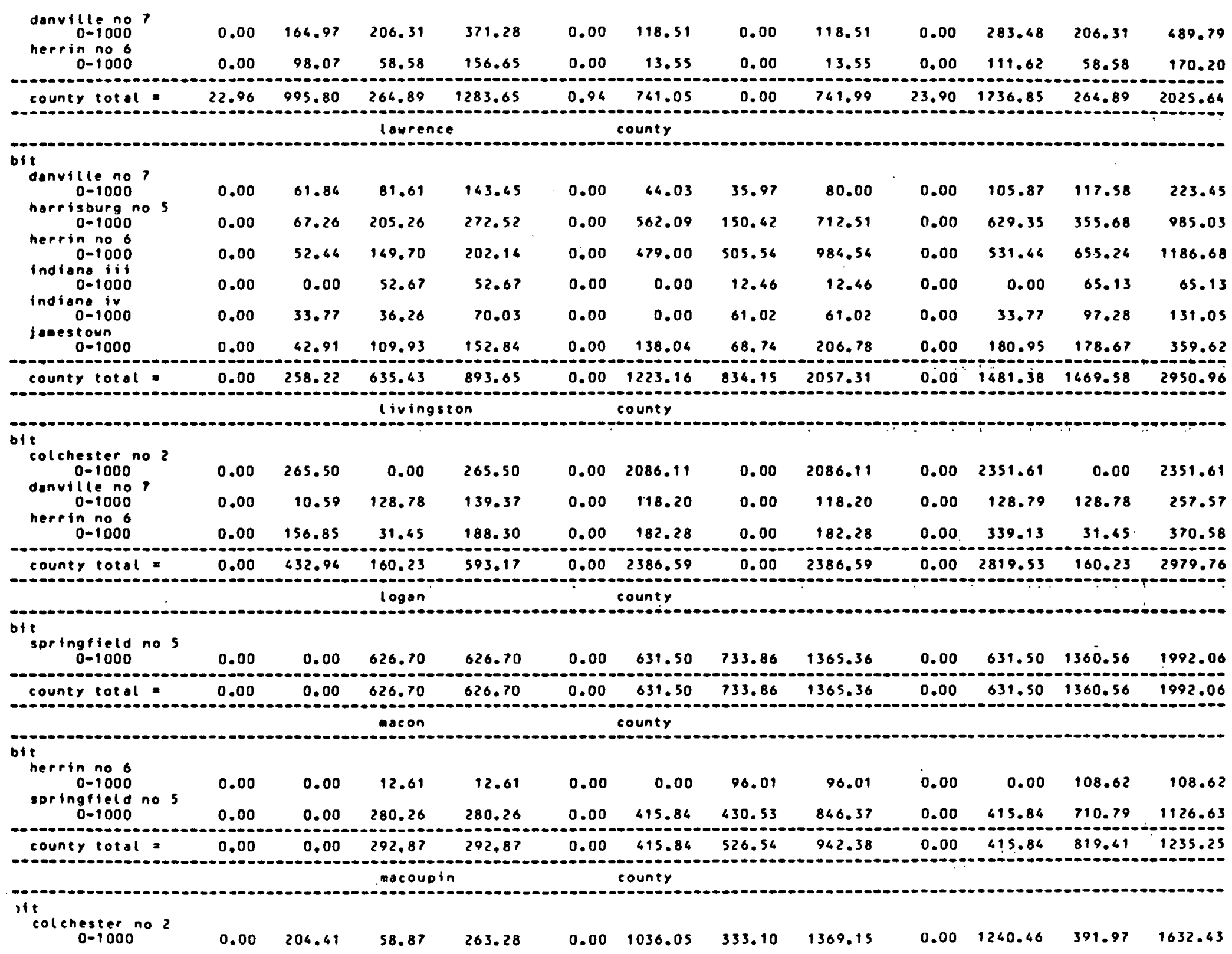




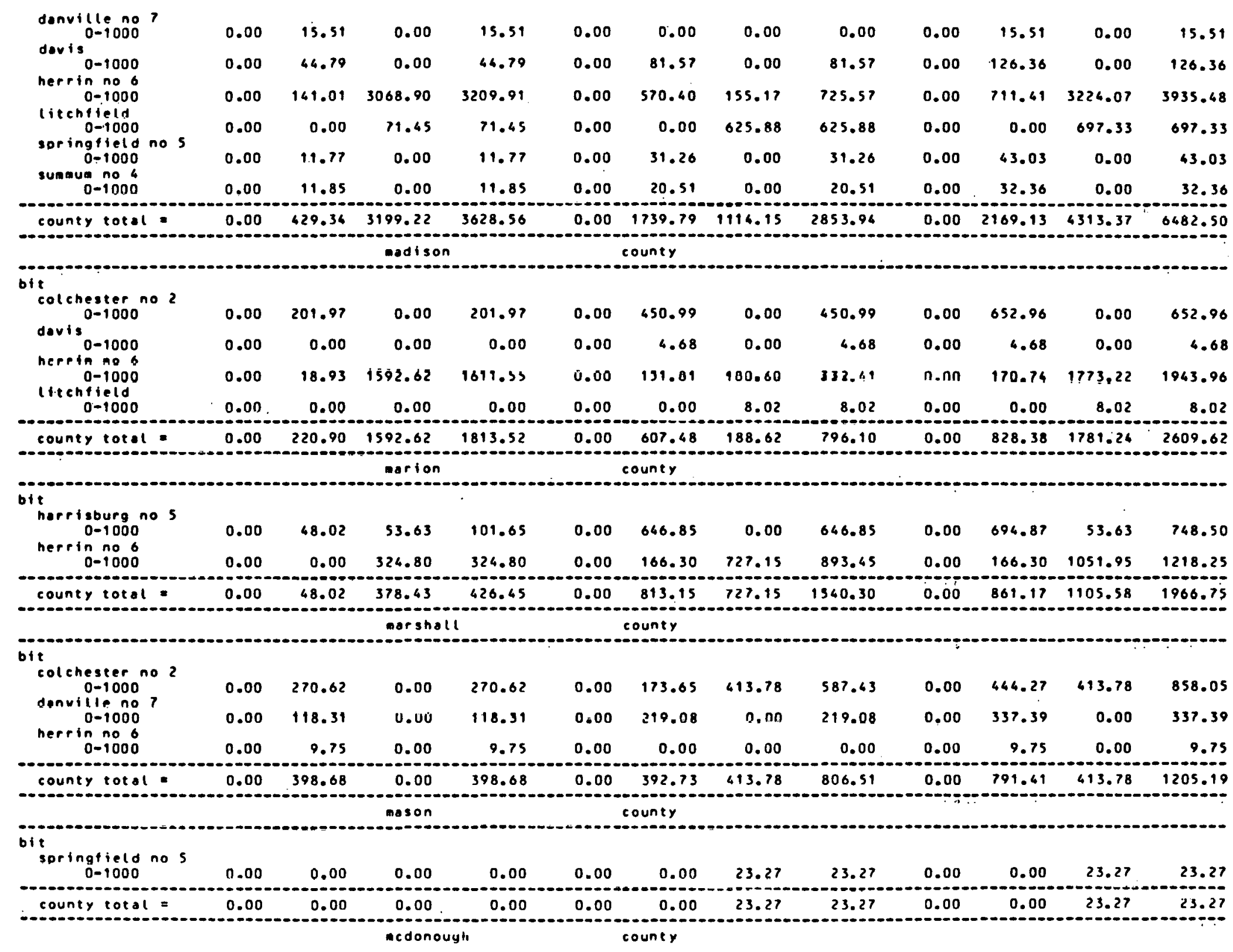




\begin{tabular}{|c|c|c|c|c|c|c|c|c|c|c|c|c|}
\hline $\begin{array}{c}\text { colchester no } 2 \\
0=1000\end{array}$ & 6.31 & 52.49 & 0.00 & 58.80 & 1.11 & 28.01 & 0.00 & 29.12 & 7.42 & 80.50 & 0.00 & 87.92 \\
\hline county total & 6.31 & 52.49 & 0.00 & 58.80 & 1.11 & 28.01 & 0.00 & 29.12 & 7.42 & 80.50 & 0.00 & 87.92 \\
\hline \\
\hline $\begin{array}{c}\text { colchester no } 2 \\
0-1000\end{array}$ & 0.00 & 82.15 & 0.00 & 82.15 & 0.00 & 214.25 & 0.00 & 214.25 & 0.00 & 296.40 & 0.00 & 296.40 \\
\hline $\begin{array}{c}\text { osuring } \\
0-1000\end{array}$ & 0.00 & 123.32 & 108.36 & 231.68 & 0.00 & 371.69 & 0.00 & 371.69 & 0.00 & 495.01 & 108.36 & 603.37 \\
\hline $\begin{array}{c}0-1000 \\
0-100\end{array}$ & 0.00 & 41.88 & 65.24 & 107.12 & 0.00 & 209.22 & 0.00 & 209.22 & 0.00 & 251.10 & 65.24 & 316.34 \\
\hline couney total & 0.00 & 247.35 & 173.60 & 420.95 & 0.00 & 795.16 & 0.00 & 795.16 & 0.00 & 1042.51 & $173.60^{\circ}$ & 1216.11 \\
\hline 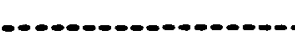 & & & nard & & & unty & & & & & & \\
\hline \\
\hline $\begin{array}{l}0-1000 \\
\text { goringtiold }\end{array}$ & 0.00 & 0.00 & 0.00 & 0.00 & 0.00 & 23.78 & 0.00 & 23.78 & 0.00 & 23.78 & 0.00 & 23.78 \\
\hline $0-1000$ & 0.00 & 0.00 & 1327.67 & 1327.67 & $0 . \overline{0} \overline{0}$ & 38.84 & 501.10 & 334.46 & U.uu & 38.84 & 1828.75 & 1807.01 \\
\hline county total & 0.00 & 0.00 & 1327.67 & 1327.67 & 0.00 & 62.62 & 501.10 & 563.72 & 0.00 & 62.62 & 1828.77 & 1891.39 \\
\hline- & & & & & & & & & & & & \\
\hline \multicolumn{13}{|l|}{ bit } \\
\hline $\begin{array}{l}0-1000 \\
\text { rock island no } 1\end{array}$ & 0.00 & 0.00 & 0.00 & 0.00 & 0.00 & 2.88 & 0.00 & 2.88 & 0.00 & 2.88 & 0.00 & 2.88 \\
\hline $\begin{array}{l}0-1000 \\
0\end{array}$ & 0.00 & 37.57 & 11.57 & 49.14 & 0.00 & 9.81 & 10.31 & 20.12 & 0.00 & 47.38 & 21.88 & 69.26 \\
\hline county total= & 0.00 & 37.57 & 11.57 & 49.16 & 0.00 & 12.69 & 10.31 & 23.00 & 0.00 & 50.26 & 21.88 & 72.16 \\
\hline & & & monroe & & & ouney & & & & & & \\
\hline $\begin{array}{l}\text { bit } \\
\text { harrisburg no } 5 \\
0-1000\end{array}$ & 0.00 & 0.00 & 0.00 & 0.00 & 0.00 & 4.97 & 0.00 & 4.97 & 0.00 & 4.97 & 0.00 & 4.97 \\
\hline couney total = & 0.00 & 0.00 & 0.00 & 0.00 & 0.00 & 4.97 & 0.00 & 4.97 & $0.00^{\circ}$ & 4.97 & 0.00 & 4.97 \\
\hline- & & & mont go & & & buñey & & & & & & \\
\hline \multicolumn{13}{|l|}{ bit } \\
\hline $\begin{array}{c}0-1000 \\
\text { danville no? }\end{array}$ & 0.00 & 146.67 & 5.80 & 152.67 & 0.00 & 325.41 & 80.98 & 406.39 & 0.00 & 472.08 & 86.78 & 558.86 \\
\hline davis $0-1000$ & 0.00 & 24.97 & 0.00 & 24.97 & 0.00 & 0.00 & 0.00 & 0.00 & 0.00 & 24.97 & 0.00 & 24.97 \\
\hline $\begin{array}{r}0-1000 \\
\text { herrin no } 6\end{array}$ & 0.00 & 53.72 & 0.00 & 53.72 & 0.00 & 79.64 & 0.00 & 79.64 & 0.00 & 133.36 & 0.00 & 133.36 \\
\hline $\begin{array}{l}0-1000 \\
\text { litentield }\end{array}$ & $n, n n$ & 13.93 & 3339.57 & 3353.50 & 0.00 & 39.22 & 351.01 & 390.23 & 0.00 & 53.15 & 3690.58 & 3743.73 \\
\hline $0-1000$ & 0.00 & 48.69 & 159.71 & 208.20 & 0.00 & 124.25 & 180.97 & 305.22 & 0.00 & 172.74 & 340.68 & 513.42 \\
\hline
\end{tabular}




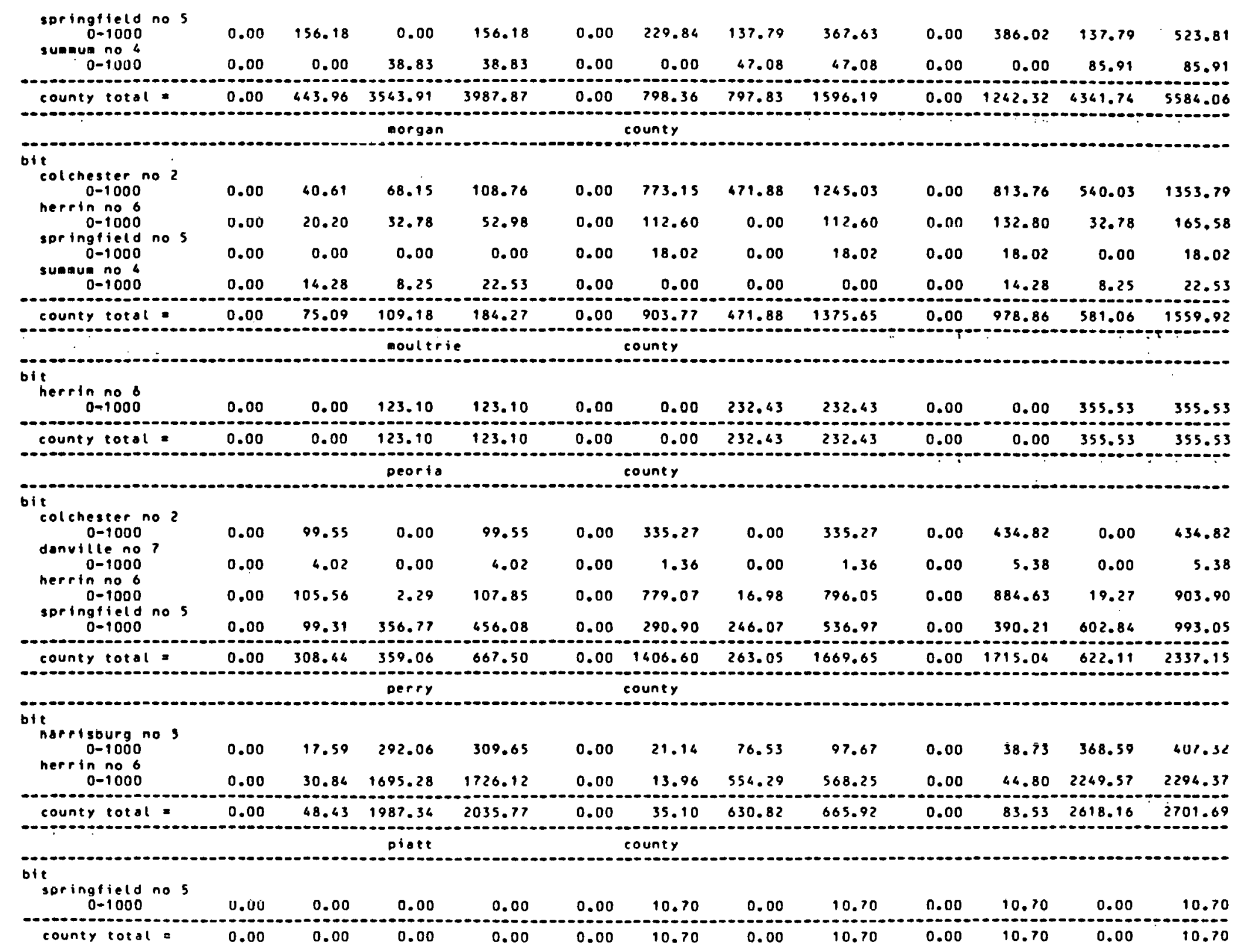




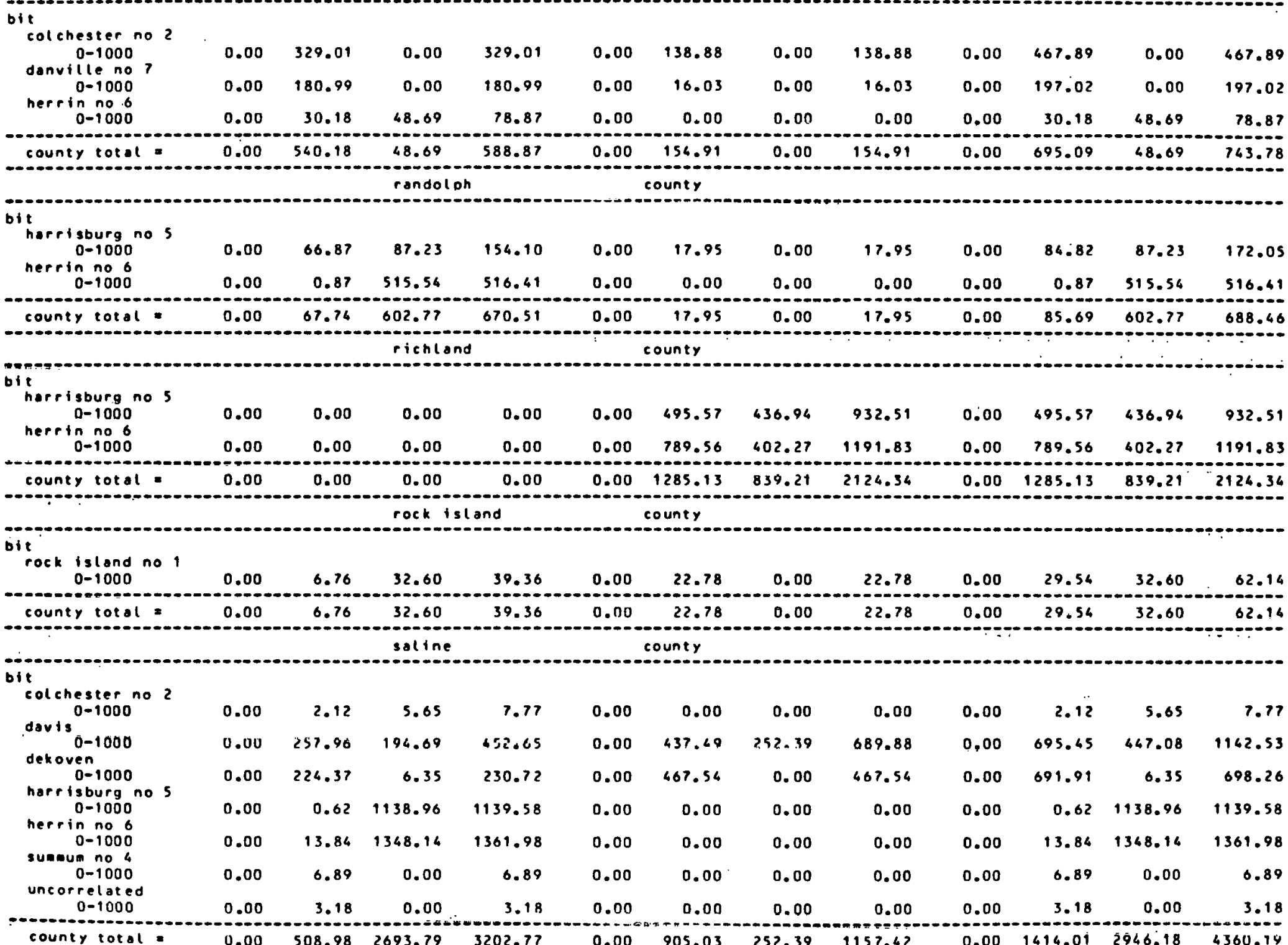




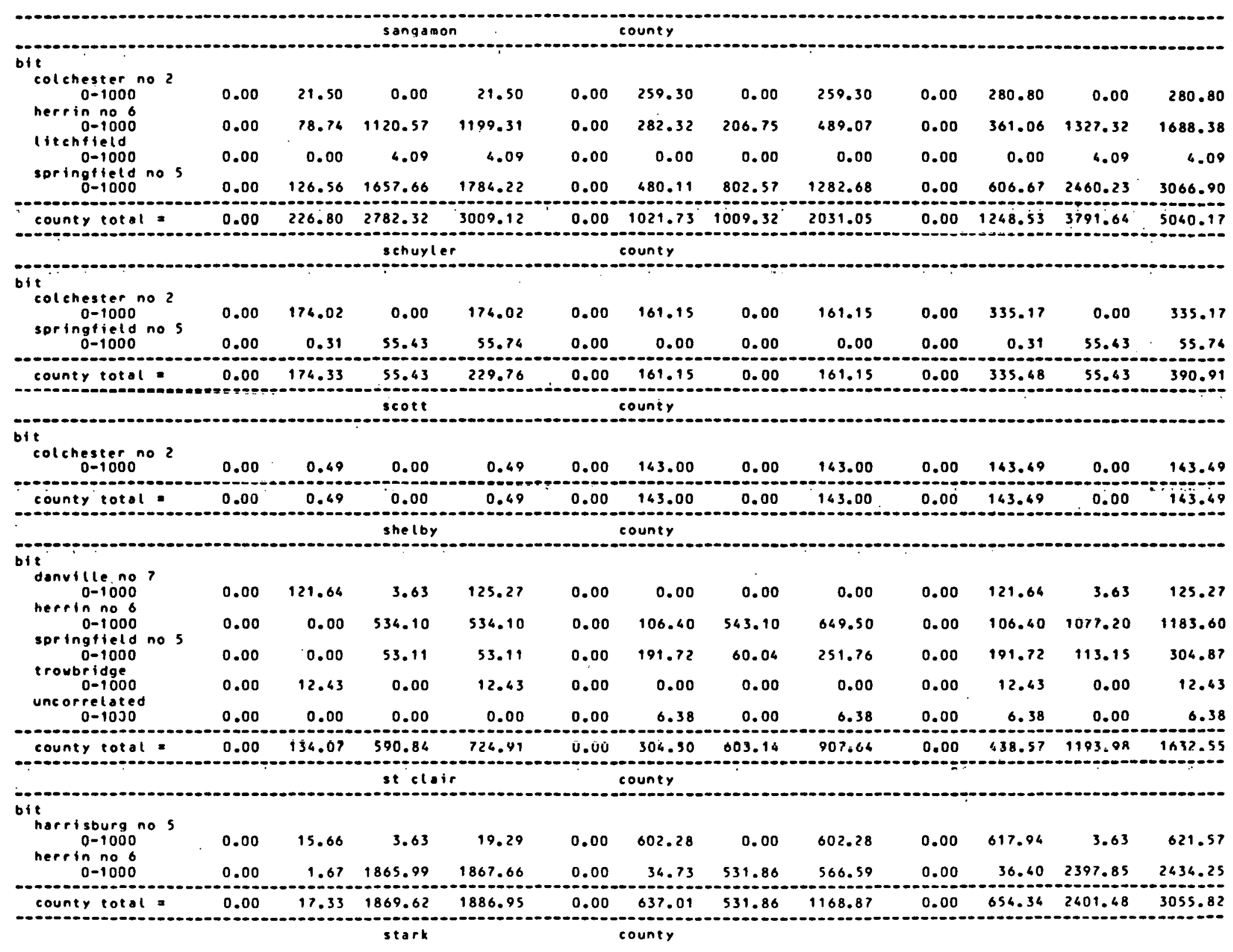




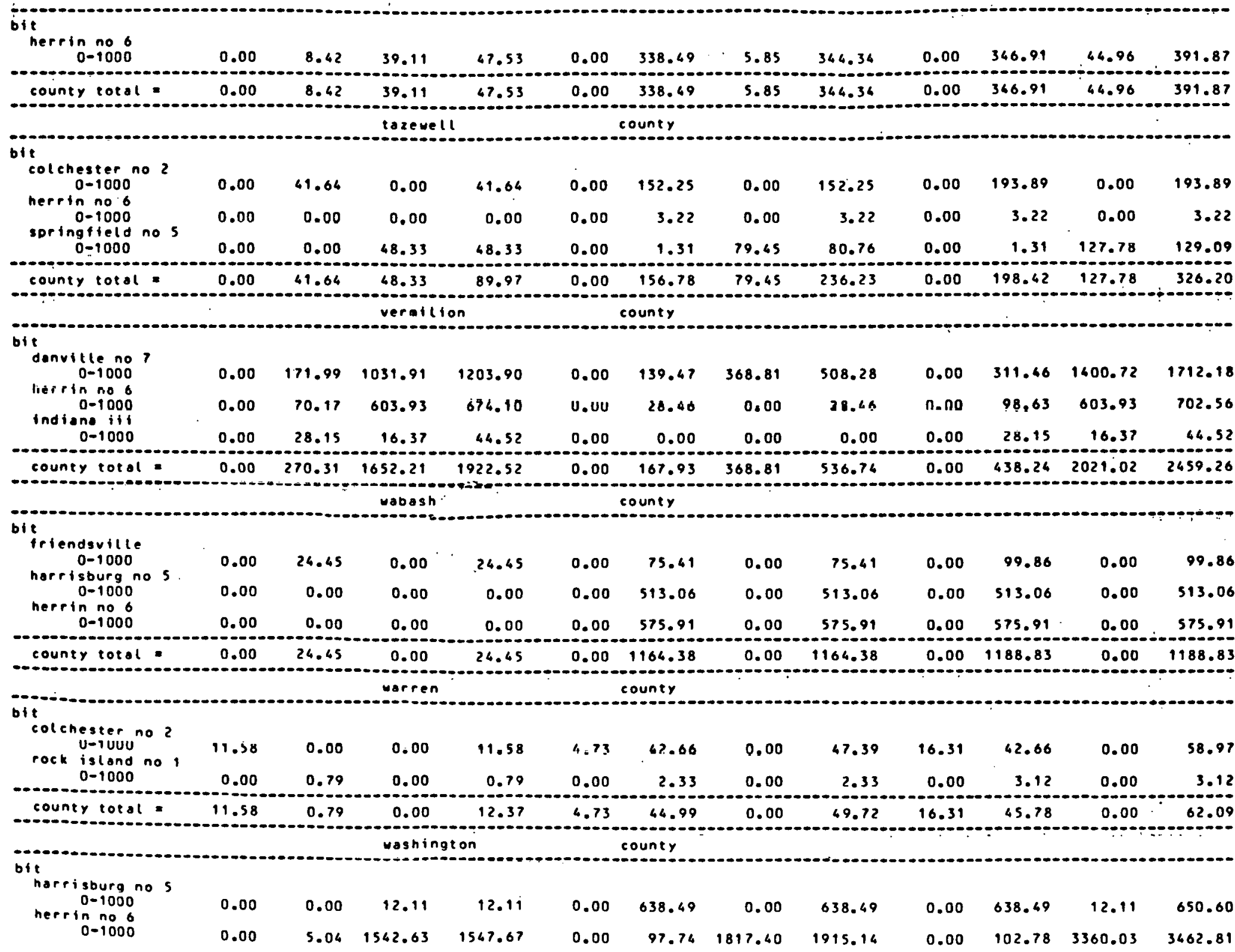




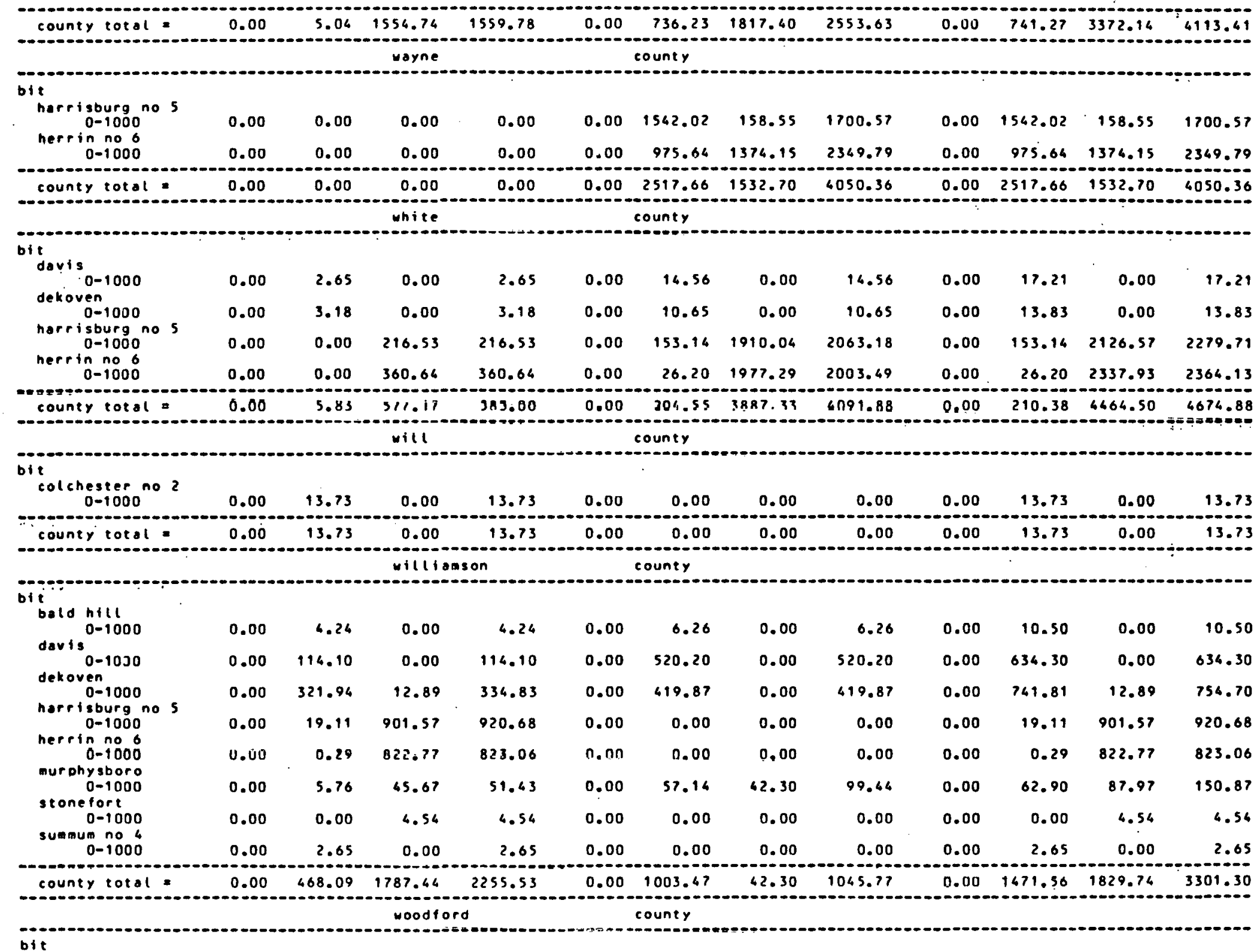




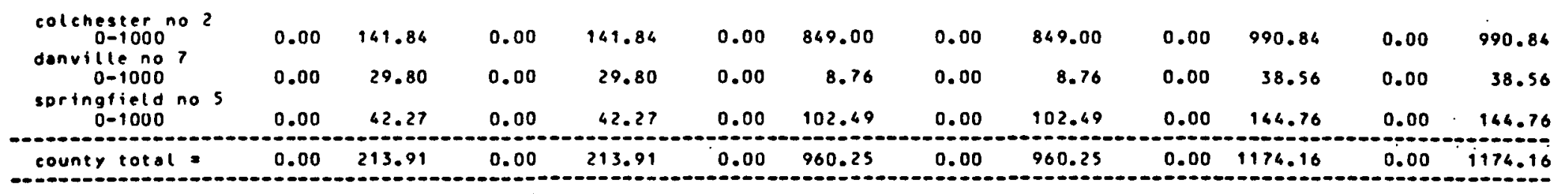
overburden

subtotal

$51.6311453 .0049191 .73 \quad 60696.36 \quad 27.24 \quad 41124.98 \quad 35481.48 \quad 76633.09$

$78.8752577 .98 \quad 84673.21937330 .04$

total $\quad 51.6311453 .0149191 .75 \quad 60696.39 \quad 27.2441125 .0035481 .4976633 .73 \quad 78.8752578 .0184673 .24137330 .12$

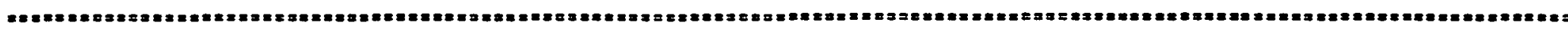
overburden

total

$51.6311453 .0049191 .73 \quad 60696.36$

$27.24 \quad 41124.98 \quad 35489.48 \quad 76633.69$

$78.8752577 .98 \quad 86673.21 \quad 137330.04$

grand total 51.6311453 .0149191 .7560696 .39

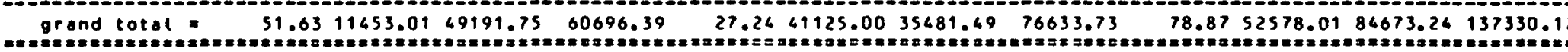
78.8752578 .0184673 .24137330 .12

resource figures taken from:
source
year
base year of resource
' illinots gs bull 78
1952
orfiginal 
average analyses of coal in illinois

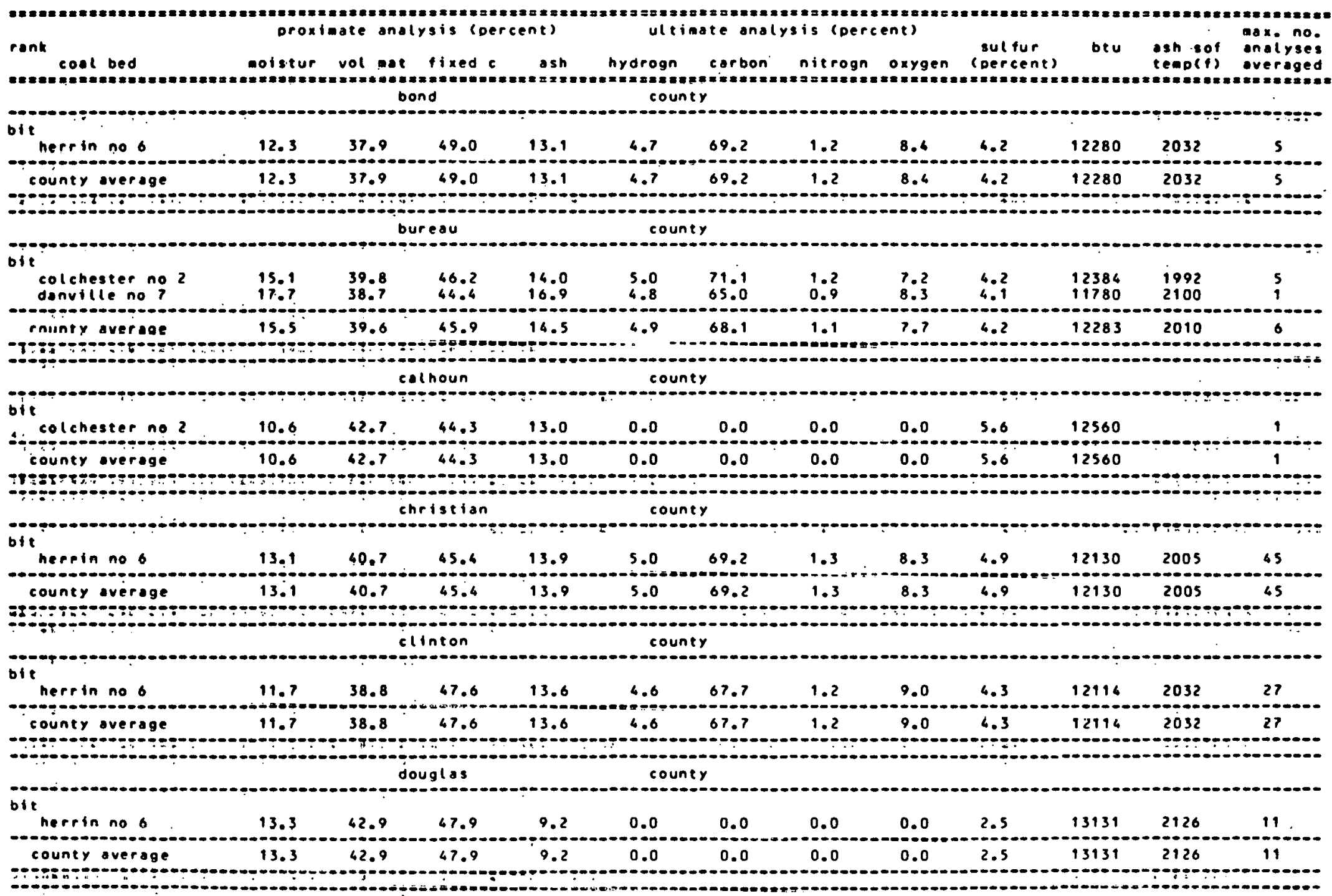


edgar

county

bit nerrin no 6
nounty overage
county overage 14.7 40. gollatin iounty

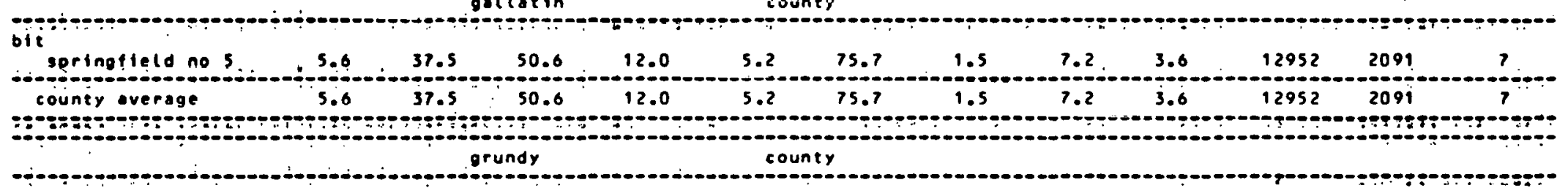

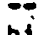

\begin{tabular}{|c|c|c|c|c|c|c|c|c|c|c|c|c|}
\hline $\begin{array}{l}\text { colchester no } \\
\text { denville no }\end{array}$ & $\begin{array}{l}15.1 \\
14.2\end{array}$ & $\begin{array}{l}40.9 \\
41.7\end{array}$ & $\begin{array}{l}48.8 \\
45.6\end{array}$ & $\begin{array}{l}10.2 \\
12.7\end{array}$ & $\begin{array}{l}5.2 \\
0.0\end{array}$ & $\begin{array}{r}73.1 \\
0.0\end{array}$ & $\begin{array}{l}1.2 \\
0.0\end{array}$ & $\begin{array}{l}8.3 \\
0.0\end{array}$ & $\begin{array}{l}3.5 \\
4.7\end{array}$ & $\begin{array}{r}12950 \\
10750\end{array}$ & $\begin{array}{l}1990 \\
2060\end{array}$ & 1 \\
\hline onty sverage & 14.9 & 41.1 & 48.2 & 10.7 & 5.2 & 73.1 & 1.2 & 8.3 & 3.8 & 12510 & 2007 & 5 \\
\hline
\end{tabular}

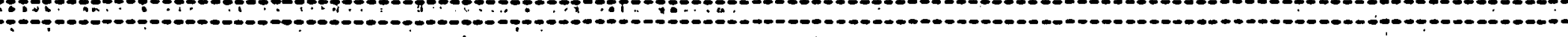
benry county in

colchester no herrin no 6 (a) rock tistand no county overage $15.8 \quad 42.2$ (3) 43.9

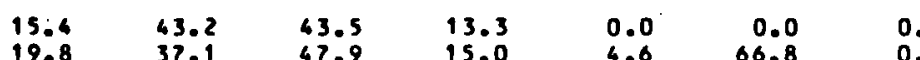

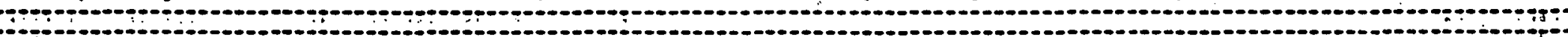

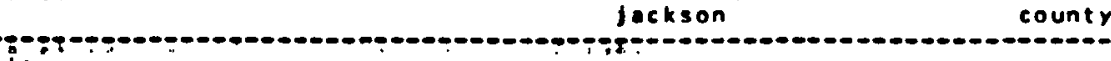

bie

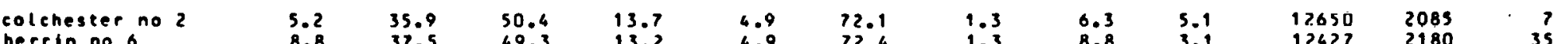
roek island no county average

\begin{tabular}{ll}
5.2 & 35 \\
8.8 & 37 \\
8.9 & 34 \\
\hline 8.2 & 37
\end{tabular}

\begin{tabular}{rrr}
50.4 & 13.7 & 4.9 \\
49.3 & 13.2 & 4.9 \\
56.5 & 8.7 & 5.0 \\
\hline 50.0 & 13.0 & 4.9
\end{tabular}




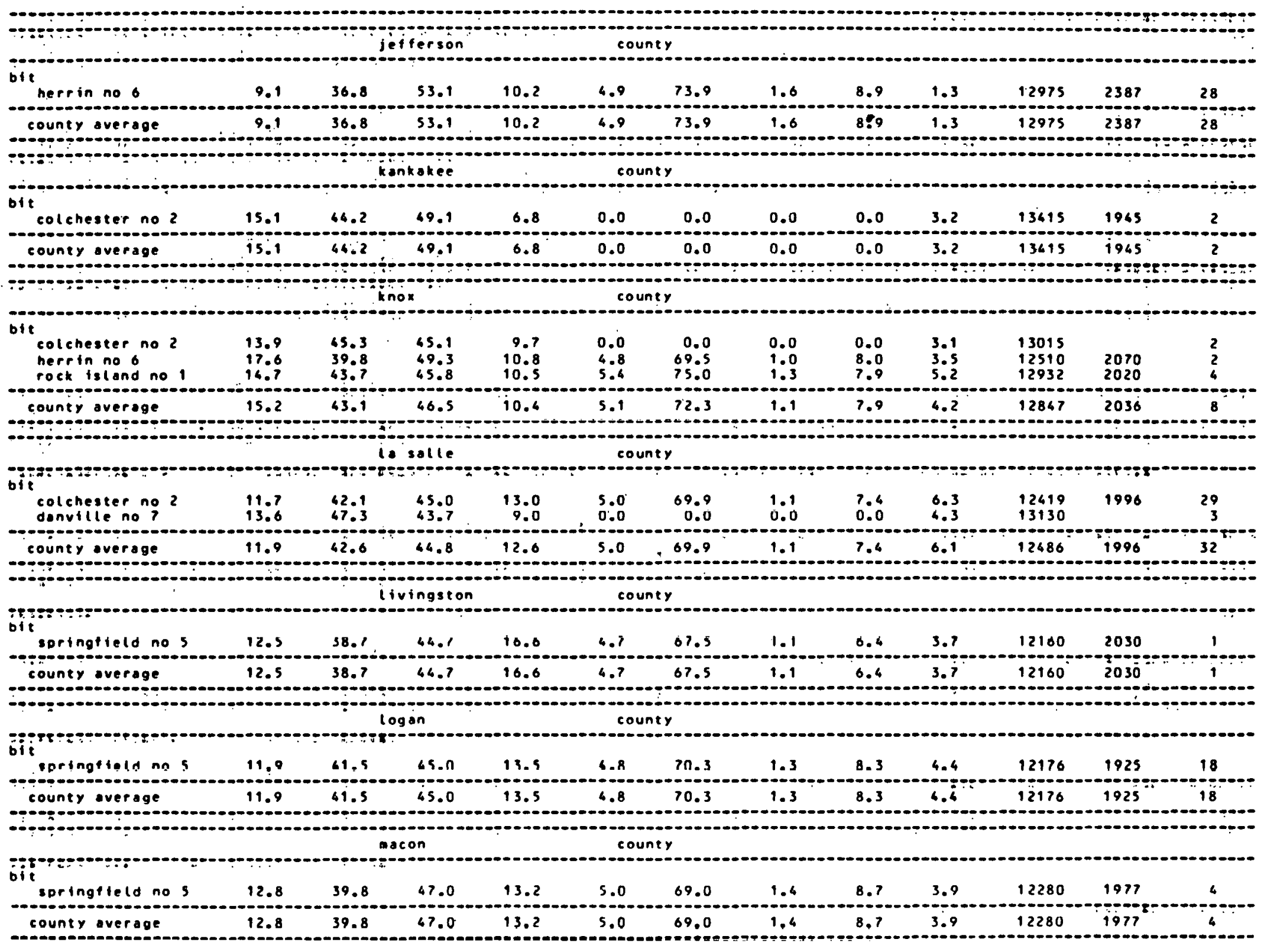




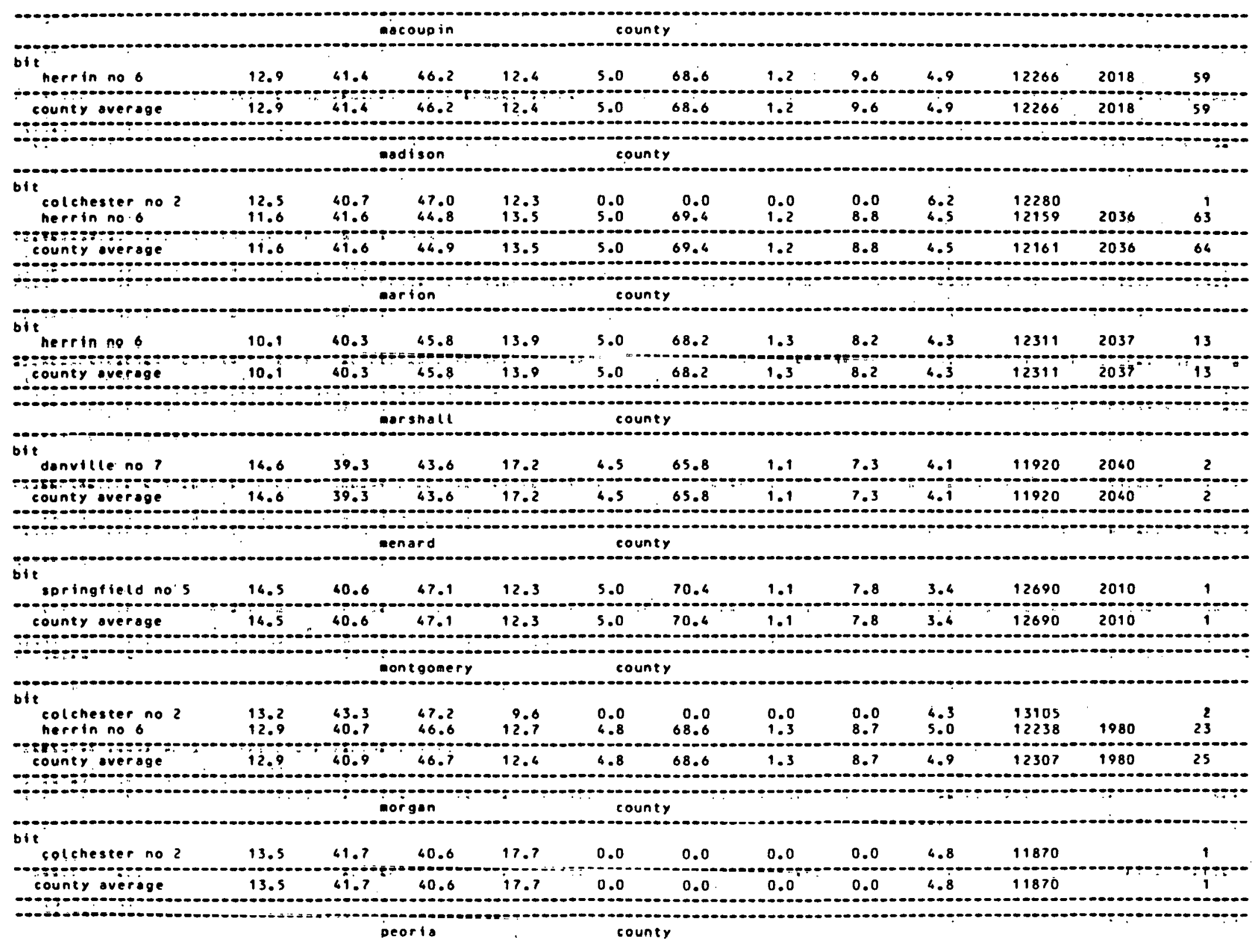




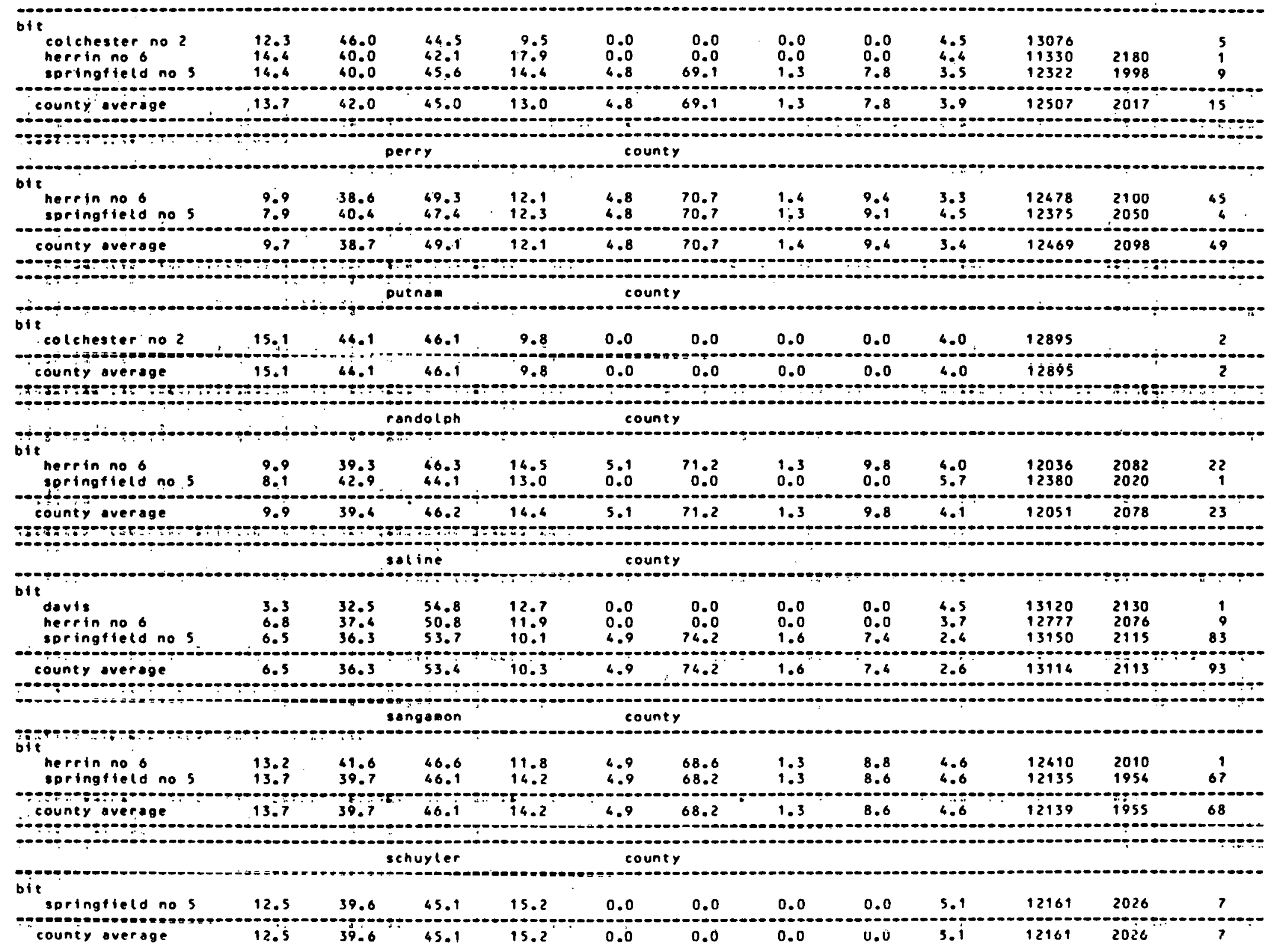




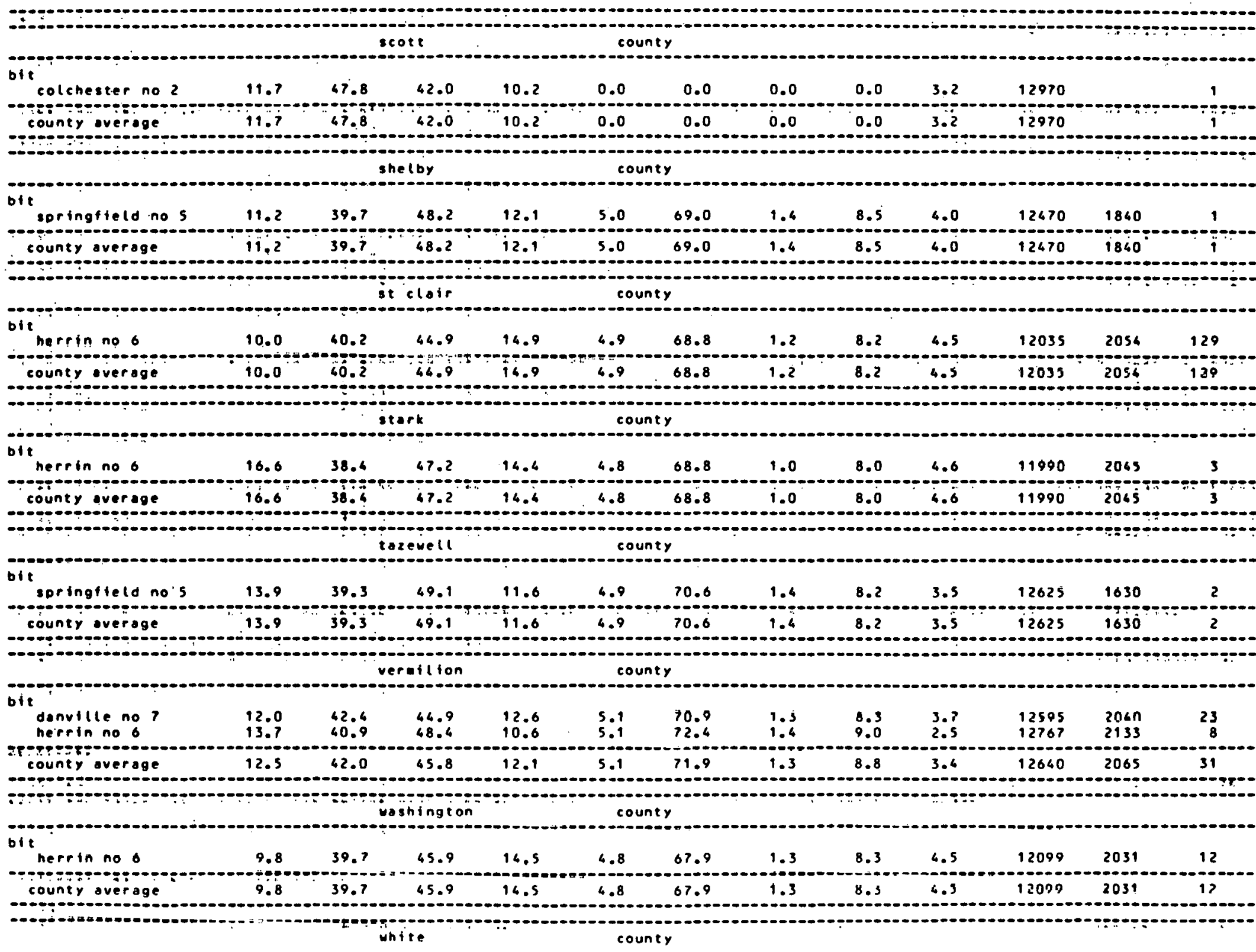




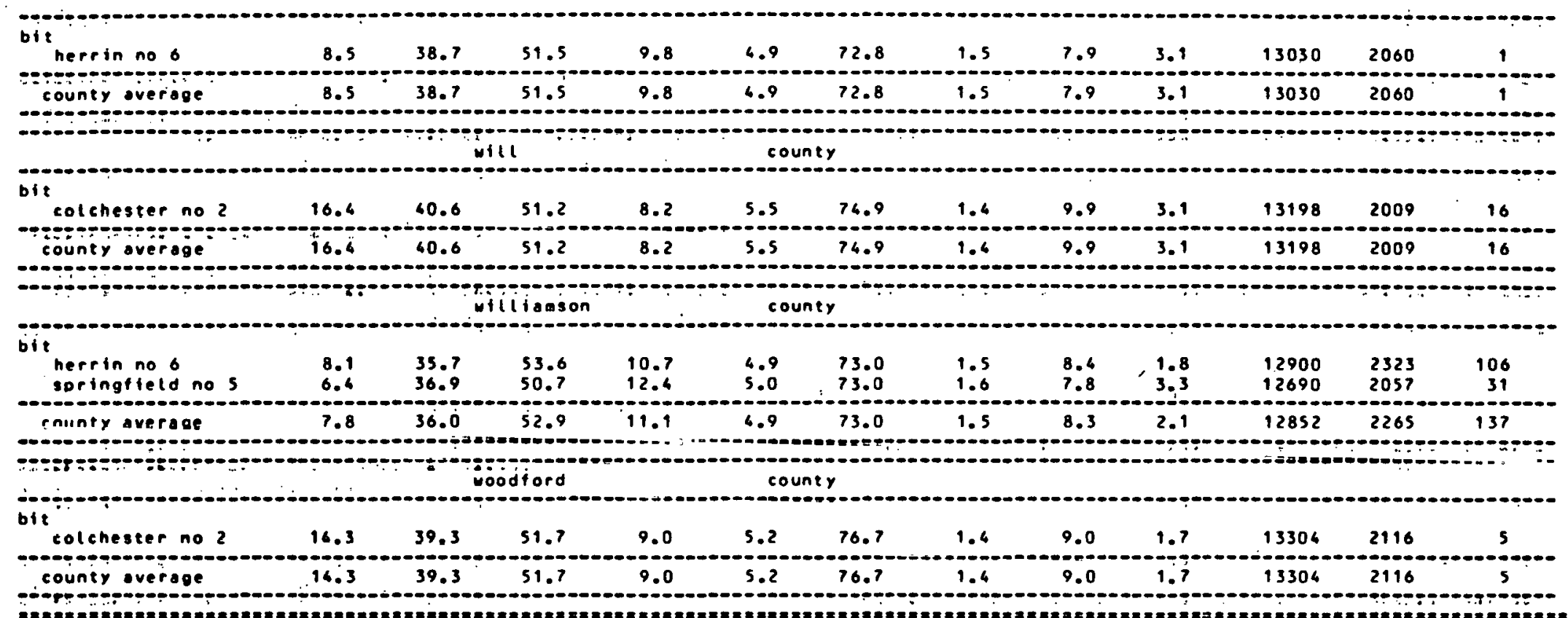




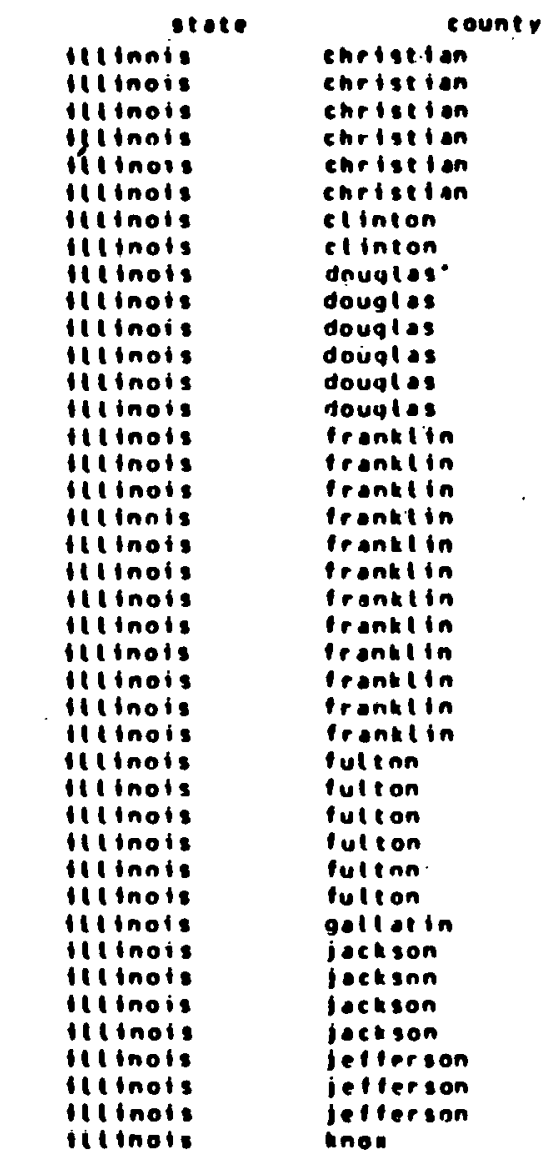

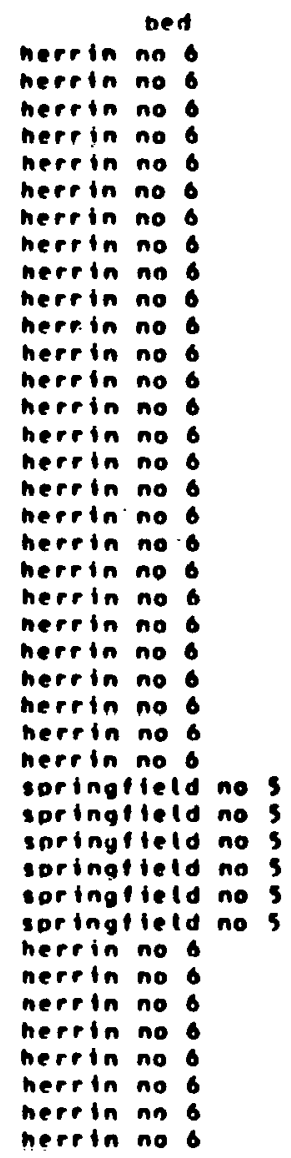

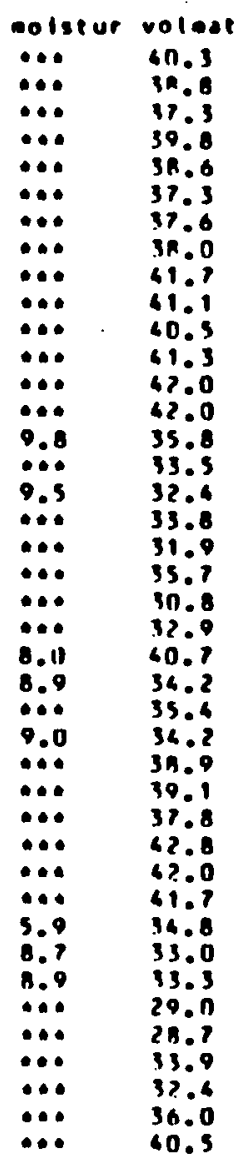

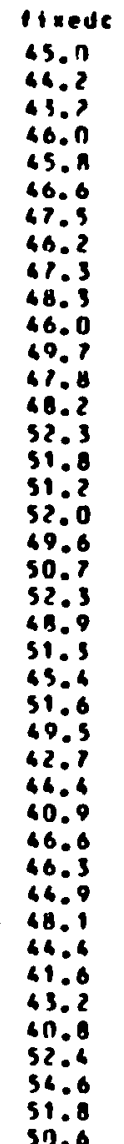

ish sulfur betu

$16.7 \quad 4.8 \quad 11460$

$11.05 .2 \quad 11570$

$19.55 .2 \quad 111110$

$16.24 .5 \quad 12010$

$15.64 .8 \quad 11870$

$16.94 .0 \quad 11800$

$15.84 .1 \quad 11700$

$11.02 .3 \quad 12890$

13.52 .90

13.53 .012400

10.52 .3013260

9.83 .012810

11.93 .212640

$16.7 ? .2 \quad 12660$

$16.41 .6 \quad 11550$

$16.2 \quad 1.6 \quad 12290$

18.51 .511550

$13.61 .8 \quad 12320$

$16.91 .2 \quad 11990$

$8.02 .7 \quad 13310$

$20.64 .0 \quad 11610$

$\begin{array}{lll}16.0 & 1.8 & 12630\end{array}$

18.45 .311290

$16.53 .1 \quad 11750$

$21.32 .4 \quad 10930$

$10.6 \quad 3.3 \quad 12540$

13.63 .9 . 11890

$17.14 .5 \quad 12010$

$22.03 .0 \quad 10870$

23.13 .8 10390

$2 \% 1.4$ 10070

$13.79 .5 \quad 12610$

13.01 .212480

$12.2 \quad 1.2 \quad 12650$

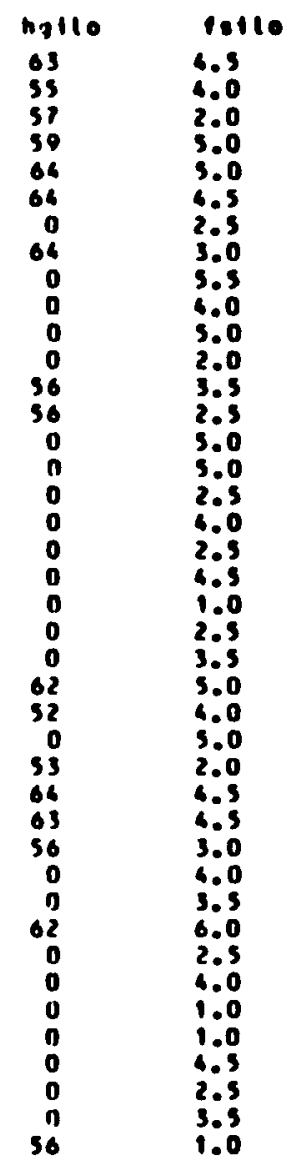

continued...... 


\begin{tabular}{|c|c|c|c|c|c|c|c|c|c|c|c|c|}
\hline & county & \multicolumn{3}{|c|}{ hed } & & \multicolumn{2}{|c|}{ sisodr } & \multicolumn{2}{|c|}{ sulfur otu } & \multicolumn{2}{|r|}{1.} \\
\hline Allinois & logan & soringf & field & d no 5 & $\cdots$ & 41.2 & 47.0 & 16.8 & 4.3 & 19710 & 62 & 3.5 \\
\hline ilinois & macouoin & herrin & no 6 & & 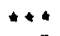 & 39.8 & 43.1 & 17.1 & 5.3 & 11540 & 0 & 2.5 \\
\hline illinois & macouoin & herrin & no 6 & 3 & 4.3 & 42.3 & 44.4 & 13.3 & 5.7 & 11900 & 0 & 4.5 \\
\hline iliinois & macouoin & herrin & no 6 & 6 & $\ldots$ & 47.4 & $47 . n$ & 12.6 & 4.9 & 12230 & 66 & 4.0 \\
\hline illinois & macoupin & herrin & no 6 & 6 & $\ldots$ & 30.8 & 45.8 & 16.4 & 5.0 & 11940 & 65 & 5.5 \\
\hline illinnis & macnunin & nerrin & no 6 & 6 & $\ldots$ & 39.5 & 45.6 & 14.9 & 5.2 & 11760 & 0 & 4.0 \\
\hline illinois & madison & herrin & no 6 & 6 & $\star * *$ & 38.6 & 44.7 & 16.7 & 4.2 & 11570 & 0 & 3.5 \\
\hline illinois & madison & herrin & no 6 & 6 & $\ldots$ & $4 n .0$ & 43.0 & 17.0 & 4.2 & 11580 & 0 & 4.0 \\
\hline Hllinois & madison & herrin & no 6 & 8 & $\ldots$ & 37.6 & 49.1 & 29.3 & 4.7 & 10820 & 0 & 3.0 \\
\hline illinois & madisnn & herrin & no 6 & 6 & $\ldots *$ & 30.8 & 41.4 & 18.8 & 5.1 & 11370 & 58 & 4.5 \\
\hline illinois & marion & nerrin & no 6 & 6 & *\#* & 38.5 & 45.6 & 15.9 & 3.9 & 12090 & 58 & 5.0 \\
\hline illinois & marion & herrin & no 0 & 6 & $\ldots$ & 38.4 & 43.6 & 18.0 & 4.3 & 11580 & 65 & 4.5 \\
\hline ilinois & marion & herrin & no 6 & & 9.7 & 40.2 & 44.7 & 15.1 & 5.0 & 12070 & 64 & 5.0 \\
\hline illinois & montqomery & herrin & no 6 & & $\therefore$ & 41.1 & 47.5 & 11.4 & 4.0 & 12190 & 57 & 3.5 \\
\hline tllinois & montgomery & nerrin & no 6 & 6 & \#* & 37.2 & 47.1 & 15.7 & 4.5 & 11700 & 0 & 3.5 \\
\hline illinois & peoria & herrin & no 0 & 0 & $* *$ & 40.0 & 42.1 & 17.9 & 4.4 & 11330 & 59 & 3.0 \\
\hline illinois & Derry & nerrin & no 6 & & 9.3 & 37.3 & 46.1 & 16.6 & 4.? & 11640 & 0 & 3.5 \\
\hline ilitingis & nerry & herrin & no 6 & 6 & 9.5 & 37.7 & 47.1 & 15.2 & 4.5 & 12030 & 60 & 4.5 \\
\hline illinois & Derry & hero in & nn in & 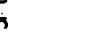 & 3.1 & 30.7 & 47.7 & 13.3 & 3.6 & 12190 & 54 & 3.5 \\
\hline Hllinois & Derry & soringt & icla & d no 5 & 7.2 & 49.8 & 48.0 & 10.2 & 3.9 & 12010 & 61 & 3.0 \\
\hline ilinois & randoloh & soringt & i ield & d no 5 & 8.1 & $4 ? .9$ & 44.1 & 13.0 & 5.7 & 12380 & 0 & 4.0 \\
\hline ilinois & randolon & herrin & no 6 & & $\cdots$ & 38.7 & 44.2 & 17.1 & 4.8 & 11510 & 59 & 4.5 \\
\hline illinois & randoloh & herrin & no 6 & 6 & $\#+$ & 39.6 & 45.7 & 14.7 & 4.1 & 11910 & 60 & 4.0 \\
\hline ilinnois & randalon & nerrin & & 6 & 9.1 & 37.5 & 49.0 & 21.5 & 4.2 & 10790 & 54 & 3.5 \\
\hline fllinois & randolon & herrin & no 6 & & 8.5 & 40.4 & 45.0 & 14.6 & 4.6 & 11850 & 54 & 2.5 \\
\hline illinois & randoloh & nerrin & no 6 & 6 & $\ldots$ & 38.4 & 46.2 & 15.4 & 4.2 & 11890 & 58 & 4.0 \\
\hline illinois & st clair & nerrin & no 6 & 6 & $\star \star *$ & 35.3 & 39.6 & 25.1 & 4.0 & 10090 & 0 & 4.0 \\
\hline illinois & st clair & herrin & no 6 & & 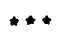 & 30.3 & 43.0 & 17.7 & 4.8 & 11510 & 0 & 5.0 \\
\hline illinois & st clair & nerrin & no 6 & 6 & $\star *$ & 37.6 & 64.1 & 18.3 & 4.5 & 11420 & 61 & 5.0 \\
\hline illinois & st clair & nerrin & no 6 & 8 & $\omega *$ & 36.9 & 44.3 & 18.8 & 5.2 & 11470 & 63 & 4.5 \\
\hline ilinois & st clair & herrin & & & 9.1 & 49.4 & $4 ? .1$ & 16.5 & 6.2 & 11560 & 57 & 4.5 \\
\hline illinois & st clair & herrin & no 6 & 6 & 9.0 & 40.6 & 48.8 & 10.6 & 3.6 & 11820 & 55 & 4.5 \\
\hline illinois & st clair & nerrin & no $n$ & & $+*$ & 35.5 & 41.? & 23.3 & 4.6 & 10520 & 0 & 3.0 \\
\hline illinois & saline & soringt & icla & d no 5 & 8.3 & 33.1 & 50.8 & 16.1 & 2.8 & 12090 & 0 & 5.0 \\
\hline ilinois & saline & davis & & & 3.3 & 32.5 & 54.8 & 12.7 & 4.5 & 13920 & 65 & 8.0 \\
\hline illinois & saline & springf & ielJ & d no 5 & 7.2 & 33.0 & 50.1 & 16.9 & 2.7 & 12070 & 62 & 6.0 \\
\hline illinois & saline & soring $f$ & field & d no 5 & 8.0 & 33.4 & 52.5 & 14.1 & 2.7 & 12530 & 62 & 6.0 \\
\hline ilinois & sanqamon & springt & field & d no 5 & $\because$ & 40.3 & 44.6 & 15.1 & 4.5 & 11840 & 64 & 4.5 \\
\hline Hlinnis & sanaamon & sorinat & ield & d no 5 & $\star *$ & 39.0 & 44.7 & 16.3 & 4.7 & 11760 & 0 & 4.5 \\
\hline flitinois & sangamon & soringt & field & d no 5 & $\ldots$ & 30.4 & 44.7 & 15.9 & 4.6 & 11750 & 67 & 4.0 \\
\hline ilinois & sangamon & soringl & $i \in(, d$ & f no 5 & $\ldots$ & 30.6 & 45.4 & 14.0 & 4.7 & 12150 & 60 & 4.5 \\
\hline ilinois & stark & herrin & no 6 & & 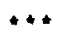 & 37.5 & 44.5 & 18.0 & 4.3 & U & 38 & 3.5 \\
\hline illinois & washingtonn & nerrin & no 6 & & 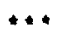 & 40.2 & 45.7 & 14.1 & 4.8 & 12120 & 59 & 4.5 \\
\hline ilinois & will & colches & iter & no? & $\cdots *$ & 36.9 & 47.1 & 16.0 & 3.9 & 11870 & 0 & 3.0 \\
\hline illinois & wili & colches & ster & no 2 & $\ldots$ & 35.3 & 48.5 & $16 . ?$ & 3.7 & 11910 & 0 & 2.0 \\
\hline illinois & williamson & springt & ibla & d no 5 & 7.? & $3 \geq 8$ & 45.2 & 22.0 & 3.0 & 10870 & 0 & 4.0 \\
\hline illinois & williamson & herrin & no 6 & & 7.9 & 37.5 & $5 n .7$ & 11.8 & 3.5 & 12720 & 57 & 3.5 \\
\hline illinois & vilitiomson & nerrin & no $h$ & 3 & 6.9 & 36.5 & 49.8 & 14.7 & 4.2 & 12260 & 58 & 5.0 \\
\hline illinois & williamson & herrin & no $h$ & s & 7.3 & 36.8 & 43.4 & 13.8 & 2.5 & 13490 & 60 & 5.0 \\
\hline illinois & williamson & herrin & no 6 & 6 & $\because$ & 31.7 & 51.0 & 17.3 & 1.1 & 11930 & 0 & 2.5 \\
\hline
\end{tabular}




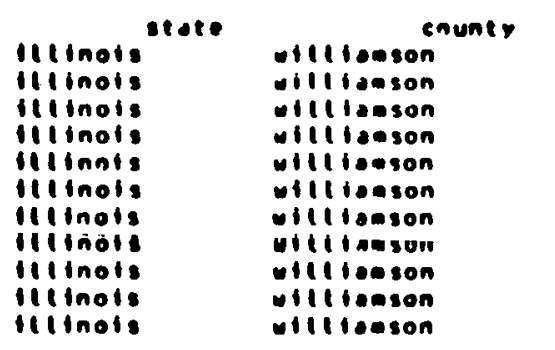

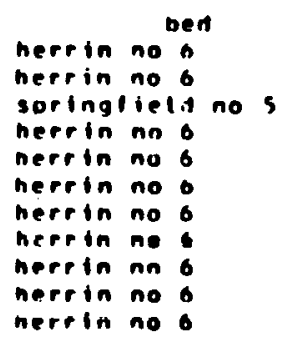

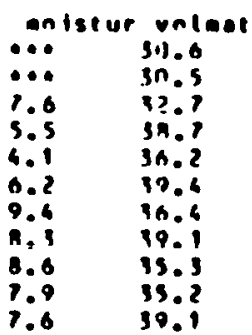

iliede

in sultur oly

iiron

$\begin{array}{llll}51.5 & 18.0 & 1.6 & 11700 \\ 45.3 & 22.0 & 3.5 & 10960\end{array}$

$\begin{array}{llll}6.3 & 22.0 & 3.5 & 10960 \\ 68.9 & 12.8 & 3.6 & 12010\end{array}$

$30.1 \quad 13.73 .1 \quad 12350$

$69.5 \quad 10.8 \quad 3.3 \quad 12830$

69.811 .13 .6

$53.2 \quad 11.5$ i.9 13610

nerelin no 0

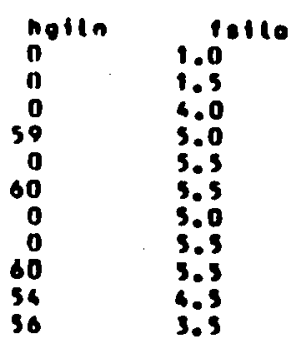


(In millions of short tons)

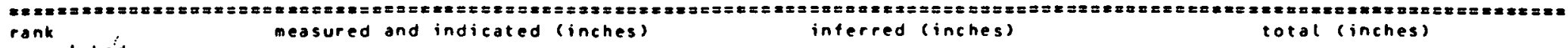
coal bed

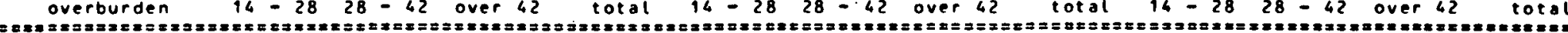
clay county

\begin{tabular}{|c|c|c|c|c|c|c|c|c|c|c|c|c|}
\hline \multicolumn{13}{|l|}{$\begin{array}{l}\text { bit } \\
\text { nde }\end{array}$} \\
\hline $\begin{array}{l}\text { strippable } \\
\text { strip-1000 }\end{array}$ & $\begin{array}{l}3.45 \\
0.02\end{array}$ & $\begin{array}{l}6.94 \\
0.11\end{array}$ & $\begin{array}{r}163.97 \\
81.98\end{array}$ & $\begin{array}{r}174.36 \\
81.31\end{array}$ & $\begin{array}{l}4.37 \\
0.00\end{array}$ & $\begin{array}{r}12.92 \\
0.00\end{array}$ & $\begin{array}{r}39.10 \\
109.48\end{array}$ & $\begin{array}{r}56.39 \\
109.48\end{array}$ & $\begin{array}{l}7.82 \\
0.02\end{array}$ & $\begin{array}{r}19.86 \\
0.11\end{array}$ & $\begin{array}{l}203.07 \\
190.66\end{array}$ & $\begin{array}{l}230.75 \\
190.79\end{array}$ \\
\hline county totol $=$ & 3.67 & 7.05 & 265.15 & 255.67 & 4.37 & 12.92 & 148.58 & 165.87 & 7.84 & 19.97 & 393.73 & 421.54 \\
\hline
\end{tabular}

\begin{tabular}{|c|c|c|c|c|c|c|c|c|c|c|c|c|}
\hline $\begin{array}{l}\text { stripoable } \\
\text { strip-1000 }\end{array}$ & $\begin{array}{r}56.30 \\
133.00\end{array}$ & $\begin{array}{l}114.37 \\
193.82\end{array}$ & $\begin{array}{r}88.63 \\
128.15\end{array}$ & $\begin{array}{l}259.30 \\
454.97\end{array}$ & $\begin{array}{l}8.17 \\
5.94\end{array}$ & $\begin{array}{l}1.41 \\
7.93\end{array}$ & $\begin{array}{r}25.18 \\
106.29\end{array}$ & $\begin{array}{r}34.76 \\
119.36\end{array}$ & $\begin{array}{r}64.47 \\
138.94\end{array}$ & $\begin{array}{l}115.78 \\
200.95\end{array}$ & $\begin{array}{l}113.81 \\
234.64\end{array}$ & $\begin{array}{l}294.06 \\
574.33\end{array}$ \\
\hline county total $=$ & 189.30 & 308.19 & 216.78 & 714.27 & 14.11 & 8.54 & 131.47 & 154.12 & 203.41 & 316.73 & 348.25 & 868. \\
\hline
\end{tabular}

\begin{tabular}{|c|c|c|c|c|c|c|c|c|c|c|c|c|}
\hline strip-1000 & 51.34 & 95.81 & 27.13 & 174.28 & 0.00 & 0.00 & 0.00 & 0.00 & 51.34 & 95.81 & 27.13 & 174.28 \\
\hline ity totat & 51.34 & 95.81 & 27.13 & 176.28 & 0.00 & 0.00 & 0.00 & 0.00 & 51.34 & 95.81 & 27.13 & 174.28 \\
\hline
\end{tabular}
bit

noe

ibson count

$67.68 \quad 211.45 \quad 1192.96 \quad 1472.09 \quad 177.02 \quad 570.272355 .98 \quad 3103.27 \quad 244.70 \quad 781.723548 .94 \quad 4575.36$
green couney

bit

\begin{tabular}{|c|c|c|c|c|c|c|c|c|c|c|c|c|}
\hline $\begin{array}{l}\text { strippable } \\
\text { strio-1000 }\end{array}$ & $\begin{array}{l}8.47 \\
0.00\end{array}$ & $\begin{array}{r}13.14 \\
0.00\end{array}$ & $\begin{array}{l}322.39 \\
251.25\end{array}$ & $\begin{array}{l}364.00 \\
259.25\end{array}$ & $\begin{array}{r}19.94 \\
0.00\end{array}$ & $\begin{array}{r}13.88 \\
0.00\end{array}$ & $\begin{array}{l}106.92 \\
246.14\end{array}$ & $\begin{array}{l}140.74 \\
246.14\end{array}$ & $\begin{array}{r}28.41 \\
0.00\end{array}$ & $\begin{array}{r}27.02 \\
0.00\end{array}$ & $\begin{array}{l}429.31 \\
497.39\end{array}$ & $\begin{array}{r}484.74 \\
497.39\end{array}$ \\
\hline councy total $=$ & 8.47 & 13.14 & 573.64 & 595.25 & 19.96 & 13.88 & 353.06 & 386.88 & 28.41 & 27.02 & 920.10 & 982.13 \\
\hline
\end{tabular}




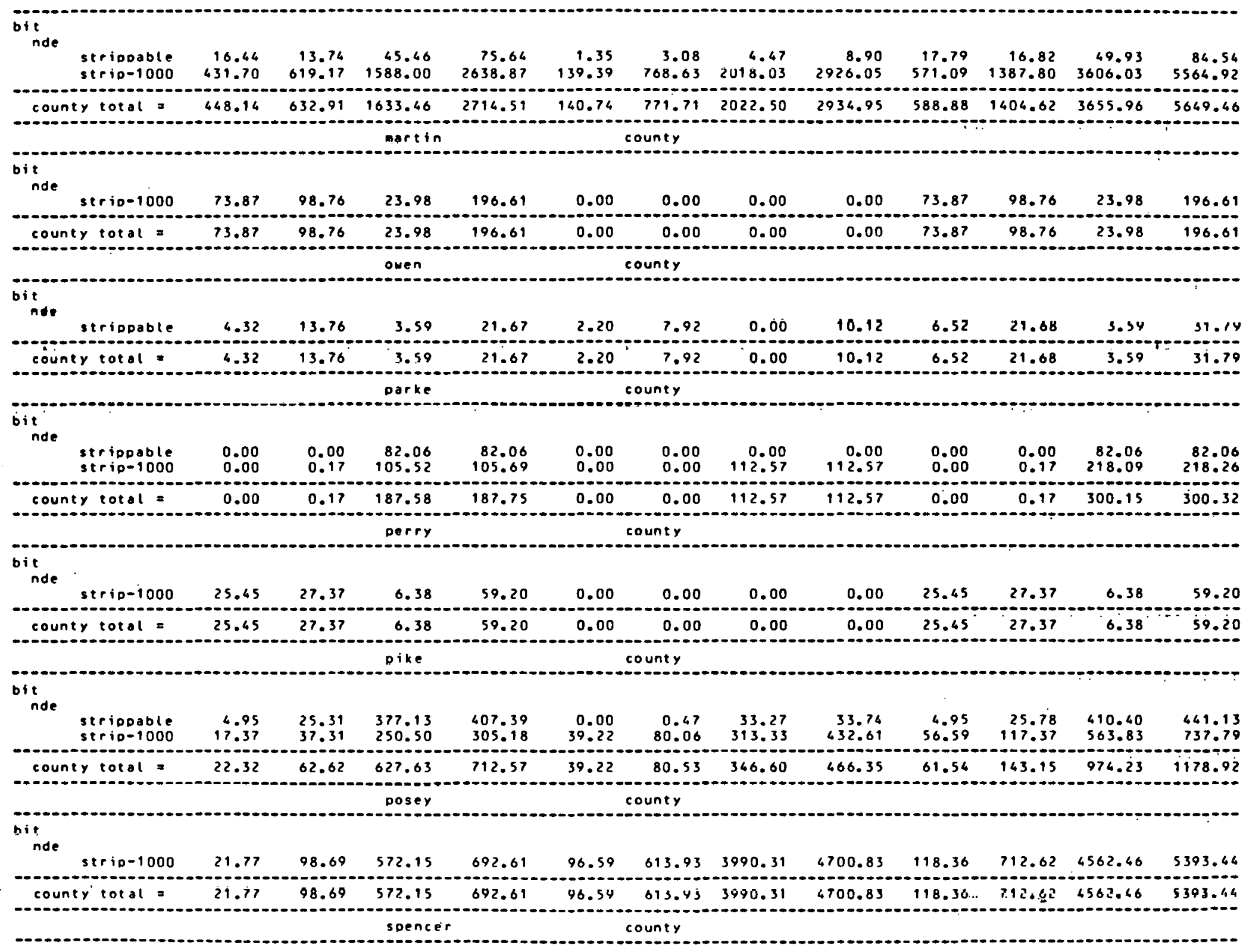




\begin{tabular}{|c|c|c|c|c|c|c|c|c|c|c|c|c|c|}
\hline \multicolumn{14}{|l|}{ it } \\
\hline & $\begin{array}{l}\text { strippable } \\
\text { strip-1000 }\end{array}$ & $\begin{array}{l}63.03 \\
36.63\end{array}$ & $\begin{array}{l}58.35 \\
61.12\end{array}$ & $\begin{array}{l}24.34 \\
26.90\end{array}$ & $\begin{array}{l}125.72 \\
124.65\end{array}$ & $\begin{array}{l}0.00 \\
0.00\end{array}$ & $\begin{array}{l}0.00 \\
0.00\end{array}$ & $\begin{array}{l}0.00 \\
0.00\end{array}$ & $\begin{array}{l}0.00 \\
0.00\end{array}$ & $\begin{array}{l}43.03 \\
36.63\end{array}$ & $\begin{array}{l}58.35 \\
61.12\end{array}$ & $\begin{array}{l}24.34 \\
26.90\end{array}$ & $\begin{array}{l}125.72 \\
124.65\end{array}$ \\
\hline count & $y \operatorname{total}=$ & 79.66 & 199.47 & 51.24 & 250.37 & 0.00 & 0.00 & 0.00 & 0.00 & 79.66 & 119.47 & 51.24 & 250.37 \\
\hline & & & & sultis & & & ity & & & & & & \\
\hline \multicolumn{14}{|l|}{ bit } \\
\hline & $\begin{array}{l}\text { strippable } \\
\text { strip-1000 }\end{array}$ & $\begin{array}{r}3.25 \\
98.00 \\
\end{array}$ & $\begin{array}{l}170.36 \\
286.50\end{array}$ & $\begin{array}{r}276.39 \\
1812.27\end{array}$ & $\begin{array}{r}450.00 \\
2196.77\end{array}$ & $\begin{array}{r}0.00 \\
339.36\end{array}$ & $\begin{array}{r}29.20 \\
2022.80\end{array}$ & $\begin{array}{r}32.15 \\
2563.51\end{array}$ & $\begin{array}{r}61.35 \\
4917.67\end{array}$ & $\begin{array}{r}3.25 \\
429.36\end{array}$ & $\begin{array}{r}199.56 \\
2309.30\end{array}$ & $\begin{array}{r}308.56 \\
4375.78\end{array}$ & $\begin{array}{r}511.35 \\
7114.64\end{array}$ \\
\hline count & $y \operatorname{total}=$ & 101.25 & 456.86 & 2088.66 & 2646.77 & 331.36 & 2052.00 & 2595.66 & 4979.02 & 432.61 & 2508.86 & 6684.32 & 7625.79 \\
\hline & & & & vander & burgh & & county & & & & & & \\
\hline \multicolumn{14}{|l|}{ bit } \\
\hline & SEFip-1000 & 20.58 & 75,56 & 413.18 & 518.33 & 180.03 & 421.12 & 1066.45 & 1667.60 & 209.62 & 496.68 & 1679.63 & 2185.93 \\
\hline counts & $y \operatorname{total}=$ & 29.59 & 75.56 & 413.18 & 518.33 & 180.03 & 421.12 & 1066.45 & 1667.60 & 209.62 & 496.68 & 1479.63 & 2785.93 \\
\hline & & & & vermil & lion & & county & & & & & & \\
\hline \multicolumn{14}{|l|}{ bit } \\
\hline & $\begin{array}{l}\text { strippable } \\
\text { strip-to00 }\end{array}$ & $\begin{array}{l}0.04 \\
7.37\end{array}$ & $\begin{array}{r}0.88 \\
10.00 \\
\end{array}$ & $\begin{array}{r}51.74 \\
478.88 \\
\end{array}$ & $\begin{array}{r}52.66 \\
496.25\end{array}$ & $\begin{array}{l}0.00 \\
2.59\end{array}$ & $\begin{array}{l}0.00 \\
5.04\end{array}$ & $\begin{array}{r}8.58 \\
657.52 \\
\end{array}$ & $\begin{array}{r}8.58 \\
605.15 \\
\end{array}$ & $\begin{array}{l}0.04 \\
9.96\end{array}$ & $\begin{array}{r}0.88 \\
15.04\end{array}$ & $\begin{array}{r}60.32 \\
1136.40\end{array}$ & $\begin{array}{r}61.24 \\
1161.40\end{array}$ \\
\hline couner & $y \operatorname{total}=$ & 7.41 & 10.88 & 530.62 & 548.91 & 2.59 & 5.04 & 666.10 & 673.73 & 10.00 & 15.92 & 1196.72 & 1222.64 \\
\hline & & & & vigo & & & county & & & & & & \\
\hline \multicolumn{14}{|l|}{$\begin{array}{l}\text { bit } \\
\text { nde }\end{array}$} \\
\hline & $\begin{array}{l}\text { stripdable } \\
\text { strip-1000 }\end{array}$ & $\begin{array}{r}2.70 \\
25.68\end{array}$ & $\begin{array}{r}91.93 \\
131.36\end{array}$ & $\begin{array}{r}319.55 \\
1521.17\end{array}$ & $\begin{array}{r}496.18 \\
1678.21\end{array}$ & $\begin{array}{r}0.00 \\
90.26\end{array}$ & $\begin{array}{r}19.40 \\
358.36\end{array}$ & $\begin{array}{r}28.39 \\
1902.71 \\
\end{array}$ & $\begin{array}{r}47.79 \\
2351.33 \\
\end{array}$ & $\begin{array}{r}2.70 \\
115.94 \\
\end{array}$ & $\begin{array}{l}111.33 \\
489.72\end{array}$ & $\begin{array}{r}347.94 \\
3423.88\end{array}$ & $\begin{array}{r}461.97 \\
4029.54\end{array}$ \\
\hline cou & y total $=$ & 28.38 & 223.29 & 1840.72 & 2092.39 & 90.26 & 377.76 & 1931.10 & 2399.12 & 118.64 & 601.05 & 3771.82 & 4491.51 \\
\hline & & & & varric & & & county & & & & & & \\
\hline \multicolumn{14}{|l|}{ bit } \\
\hline & $\begin{array}{l}\text { strippable } \\
\text { strip-1000 }\end{array}$ & $\begin{array}{l}28.86 \\
23.43\end{array}$ & $\begin{array}{r}72.11 \\
115.26\end{array}$ & $\begin{array}{l}443.03 \\
476.45\end{array}$ & $\begin{array}{l}544.00 \\
615.14\end{array}$ & $\begin{array}{r}1.21 \\
26.25\end{array}$ & $\begin{array}{r}3.22 \\
50.20\end{array}$ & $\begin{array}{r}19.97 \\
425.41\end{array}$ & $\begin{array}{r}24.40 \\
501.86\end{array}$ & $\begin{array}{l}30.07 \\
49.68\end{array}$ & $\begin{array}{r}75.33 \\
165.46\end{array}$ & $\begin{array}{l}463.00 \\
901.86\end{array}$ & $\begin{array}{r}568.40 \\
1117.00\end{array}$ \\
\hline count, & totel $=$ & 52.29 & 187.37 & 919.48 & 1159.14 & 27.46 & 53.42 & 445.38 & 526.26 & 79.75 & 240.79 & 1364.86 & 1685.40 \\
\hline \multicolumn{14}{|c|}{ 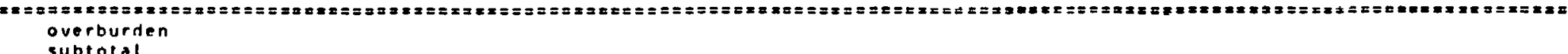 } \\
\hline & $\begin{array}{l}\text { Strippable } \\
\text { strip-1000 }\end{array}$ & $\begin{array}{r}183.31 \\
1031.40\end{array}$ & $\begin{array}{r}613.83 \\
2029.52 \\
-\end{array}$ & $\begin{array}{l}2244.34 \\
8909.99\end{array}$ & $\begin{array}{r}3061.48 \\
11970.91\end{array}$ & $\begin{array}{r}61.03 \\
1084.86\end{array}$ & $\begin{array}{r}111.64 \\
4877.40\end{array}$ & $\begin{array}{r}330.02 \\
15835.74\end{array}$ & $\begin{array}{r}687.69 \\
21798.00\end{array}$ & $\begin{array}{r}224.34 \\
2116.26\end{array}$ & $\begin{array}{r}725.47 \\
6906.92\end{array}$ & $\begin{array}{r}2574.36 \\
24745.73\end{array}$ & $\begin{array}{r}3524.17 \\
33768.91\end{array}$ \\
\hline $\begin{array}{l}t 0 t \\
0=x=\end{array}$ & $a l$ & $\begin{aligned} & 1214.71 \\
= & =2=302=\end{aligned}$ & 2643.35 & 11154.33 & 15012.39 & 1125.89 & 4989.04 & 16165.76 & 22280.69 & 2340.60 & 7632.39 & 27320.09 & 37293.08 \\
\hline
\end{tabular}




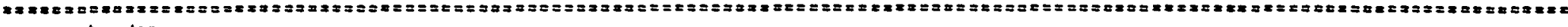
overburden

total

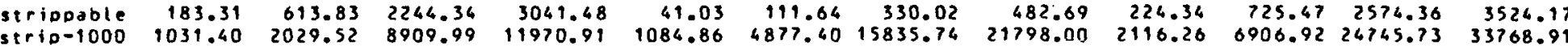

grand total $=1214.71 \quad 2643.3511954 .33 \quad 15012.39 \quad 1125.89 \quad 4989.0416165 .76 \quad 22280.69 \quad 2340.00 \quad 7632.3927320 .09 \quad 37293.08$ ende

total tonnage of identified coal resources in indiana

resource tigures taken from:

$\begin{array}{lll}\text { source } & \text { year base yes of resource } \\ \text { usgs circ } 266 & 1953 & \text { original }\end{array}$

usgs circ 266

1953

original
37293.08 
average analyses of coal in indiand

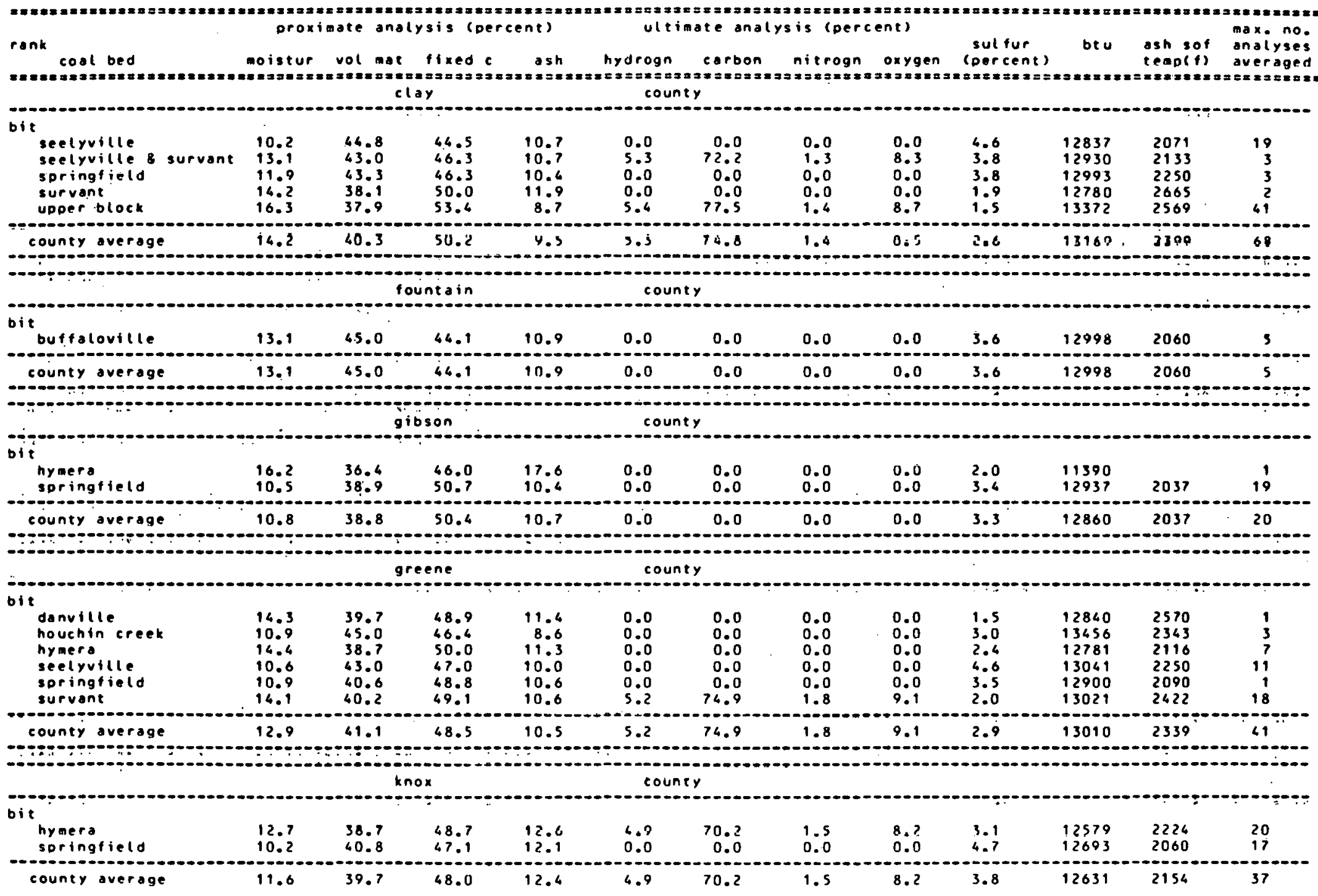




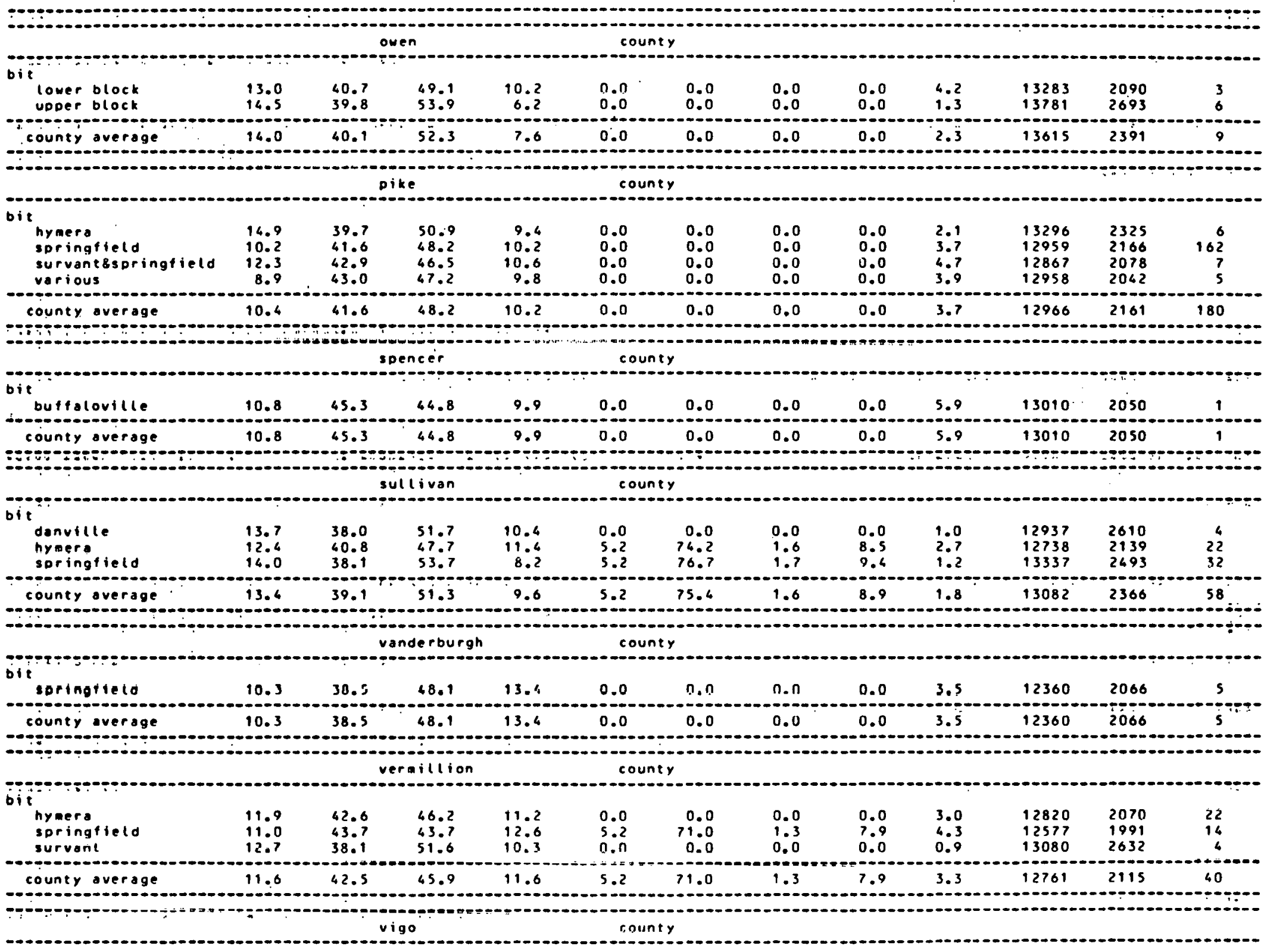




\begin{tabular}{|c|c|c|c|c|c|c|c|c|c|c|c|c|}
\hline $\begin{array}{l}\text { danville } \\
\text { hymera } \\
\text { seelyville } \\
\text { springfield } \\
\text { survant }\end{array}$ & $\begin{array}{l}14.1 \\
13.1 \\
10.9 \\
11.3 \\
12.1\end{array}$ & $\begin{array}{l}42.0 \\
43.8 \\
41.1 \\
42.1 \\
39.3\end{array}$ & $\begin{array}{l}48.6 \\
46.0 \\
45.9 \\
43.9 \\
50.2\end{array}$ & $\begin{array}{r}9.4 \\
10.2 \\
13.1 \\
13.9 \\
10.5\end{array}$ & $\begin{array}{l}0.0 \\
5.2 \\
0.0 \\
4.9 \\
5.3\end{array}$ & $\begin{array}{r}0.0 \\
72.1 \\
0.0 \\
67.5 \\
72.8\end{array}$ & $\begin{array}{l}0.0 \\
1.4 \\
0.0 \\
1.3 \\
1.6\end{array}$ & $\begin{array}{l}0.0 \\
8.7 \\
0.0 \\
7.1 \\
9.0\end{array}$ & $\begin{array}{l}1.3 \\
3.7 \\
4.0 \\
4.2 \\
1.4\end{array}$ & $\begin{array}{l}13030 \\
12966 \\
12522 \\
12335 \\
12956\end{array}$ & $\begin{array}{l}2370 \\
1996 \\
2131 \\
2052 \\
2671\end{array}$ & $\begin{array}{r}2 \\
3 \\
12 \\
30 \\
45\end{array}$ \\
\hline county average & 11.8 & 40. & 47.4 & 11.9 & 5.1 & 70.0 & $i .4$ & 8.0 & 2.7 & $\begin{array}{r}12698 \\
-\end{array}$ & 2218 & 92 \\
\hline & & & $c k$ & & & & & & & & & \\
\hline $\begin{array}{l}\text { danuille } \\
\text { hymera } \\
\text { springfield }\end{array}$ & $\begin{array}{l}14.9 \\
11.3 \\
10.4\end{array}$ & $\begin{array}{l}36.0 \\
39.8 \\
41.5\end{array}$ & $\begin{array}{l}55.4 \\
50.6 \\
47.4\end{array}$ & $\begin{array}{r}8.6 \\
9.6 \\
11.1\end{array}$ & $\begin{array}{l}0.0 \\
5.1 \\
5.1\end{array}$ & $\begin{array}{r}0.0 \\
75.4 \\
71.5\end{array}$ & $\begin{array}{l}0.0 \\
1.5 \\
1.3\end{array}$ & $\begin{array}{r}0.0 \\
10.1 \\
8.3\end{array}$ & $\begin{array}{l}0.9 \\
3.1 \\
4.4\end{array}$ & $\begin{array}{l}12982 \\
12792 \\
12703\end{array}$ & $\begin{array}{l}2677 \\
2317 \\
2066\end{array}$ & $\begin{array}{r}4 \\
5 \\
64\end{array}$ \\
\hline unty average & 10.7 & 41.1 & 48.0 & 10.8 & 5.1 & 72.8 & 1.4 & 8.9 & 4.1 & 12725 & 2124 & 73 \\
\hline
\end{tabular}

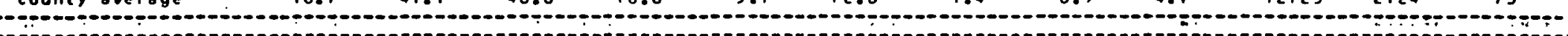

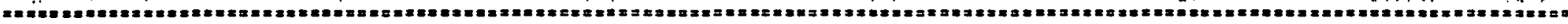


Indiana

\begin{tabular}{|c|c|}
\hline 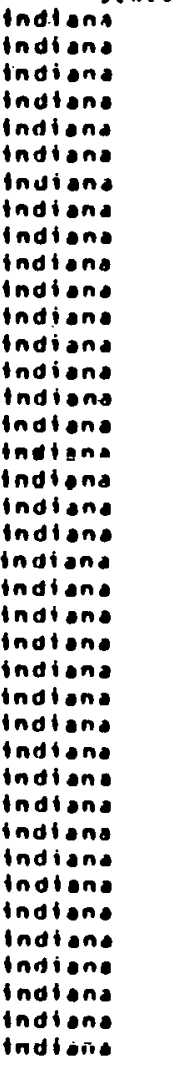 & 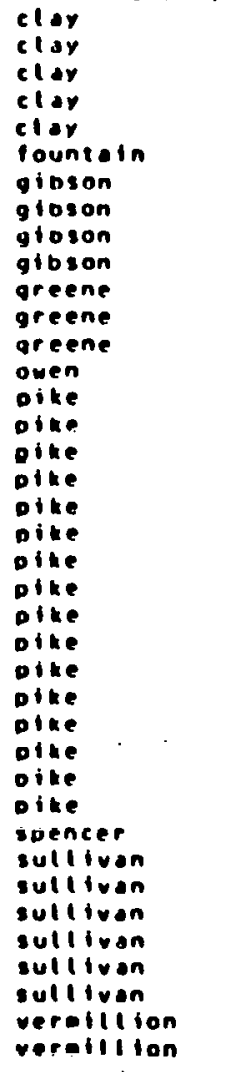 \\
\hline
\end{tabular}

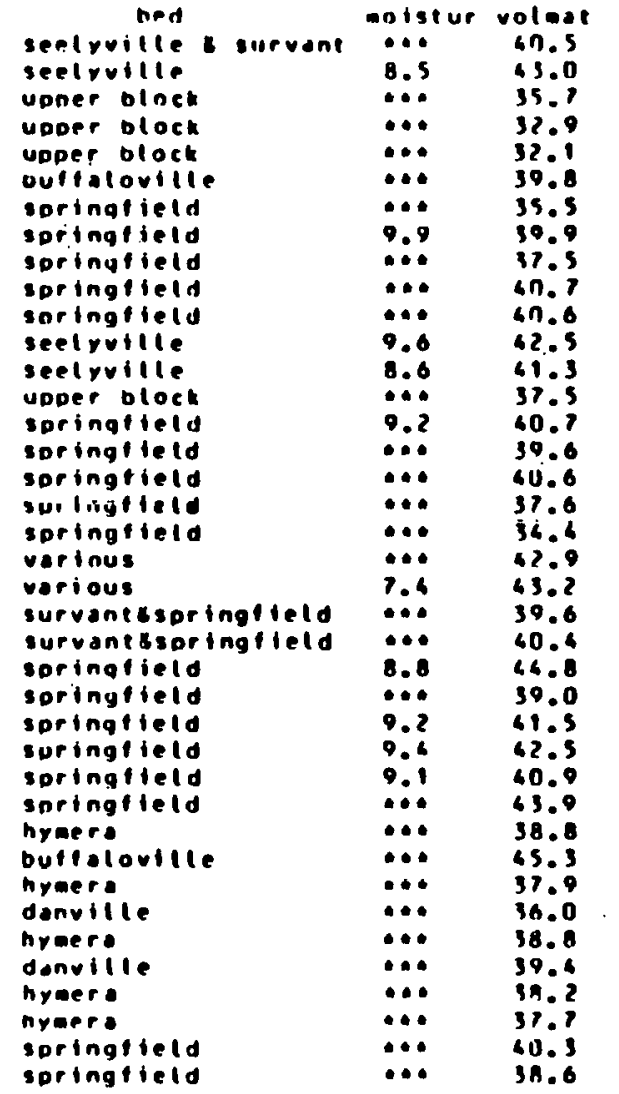

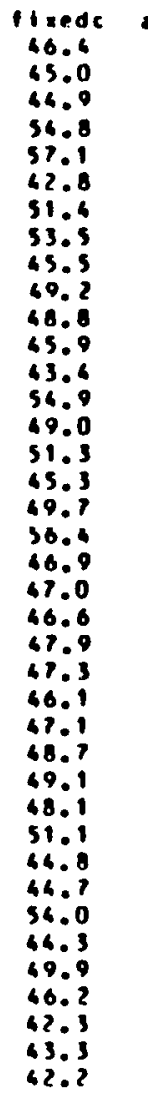

$\begin{array}{lll}\text { sh } & \text { sulfur neu } \\ 13.1 & 6.0 & 12510 \\ 12.0 & 6.4 & 12390\end{array}$

$\begin{array}{lll}10.0 & 5.3 & 12390 \\ 11570\end{array}$

$12.31 .7 \quad 12820$

$10.8 \quad 13020$

$13.12 .4 \quad 12520$

$17.0 \quad 2.1 \quad 13500$

10.1 i.3 13100

119.6 $3.5 \quad 12900$

$11.80 .4 \quad 12580$

15.350 .911980

$10.62 .6 \quad 13680$

$9.1 \quad 3.1 \quad 13230$

$14.13 .9 \quad 12270$

14.9 3.9 12270

$10.2 \quad 4.2 \quad 12900$

$9.84 .2 \quad 1297$

$11.75 .2 \quad 12710$

$1.93 .1 \quad 13260$

16.9 6.7 12100

11.64 .612590

10.8 3050

$\begin{array}{lll}8.0 & 3.4 & 13400\end{array}$

$10.1 \quad 2.2 \quad 12930$

$9.9 \quad 5.9 \quad 13010$

$17.63 .0 \quad 11810$

$17.00 .7 \quad 12950$

$16.93 .3 \quad 11890$

in.? $1.9 \quad 12850$

$15.62 .8 \quad 11870$

$\begin{array}{lll}15.0 & 3.1 \quad 11320\end{array}$

$19.28 .7 \quad 11500$

\begin{tabular}{|c|c|}
\hline $\begin{array}{c}n 9110 \\
60 \\
0 \\
0 \\
0 \\
0 \\
0 \\
0 \\
0 \\
0 \\
0 \\
51 \\
51 \\
55 \\
59 \\
53 \\
66 \\
0 \\
0 \\
0 \\
0 \\
0 \\
0 \\
0 \\
0 \\
0 \\
0 \\
0 \\
56 \\
60 \\
00 \\
0 \\
00 \\
57 \\
52 \\
52 \\
00 \\
0 \\
00 \\
69 \\
07\end{array}$ & 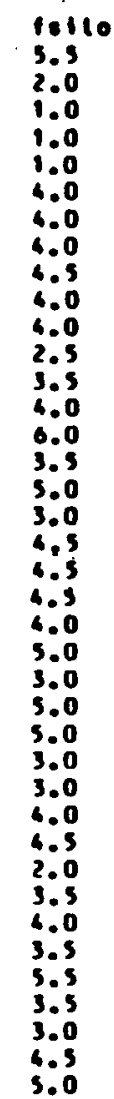 \\
\hline
\end{tabular}

continued..... 


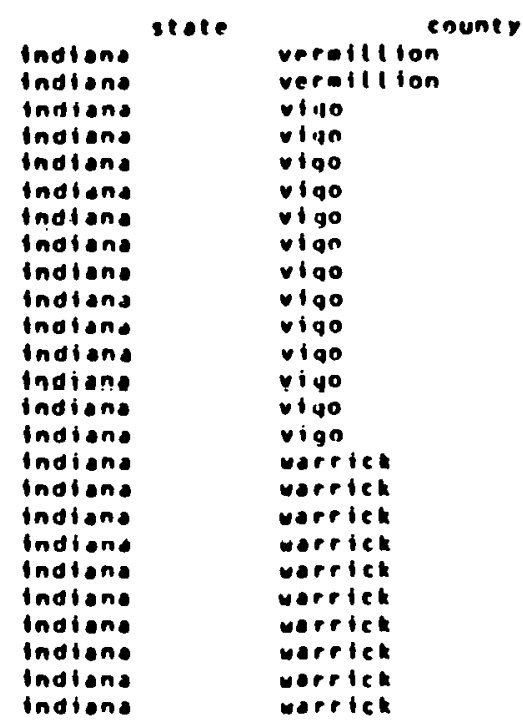

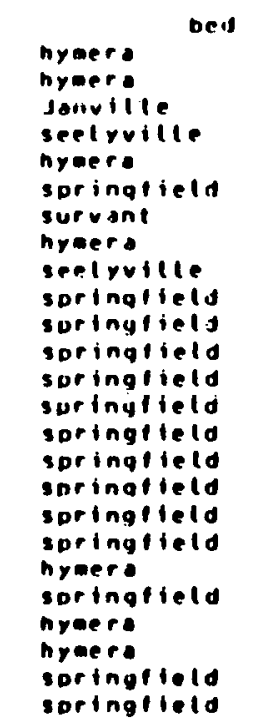

\begin{tabular}{|c|c|c|c|c|c|c|c|}
\hline an & vnle & Il aeds & $\cos$ & 80 & ort & nallo & 181 \\
\hline$\cdots$ & 10.6 & 45.8 & 16.8 & 3.6 & 12170 & 63 & 3.5 \\
\hline$\ldots$ & 29.1 & $4 . n$ & 17.9 & 3.5 & 11560 & 52 & 3.5 \\
\hline$\cdots$ & 30.6 & 47.1 & 12.7 & 1.6 & 12660 & 0 & 4.0 \\
\hline$\cdots$ & 14.8 & 69.3 & 23.9 & 5.6 & 10140 & o & 3.5 \\
\hline$\cdots$ & 49.5 & 66.5 & 110.0 & 4.4 & 12490 & 61 & 5 \\
\hline$\cdots$ & 41.3 & 43.1 & 15.6 & 5.0 & 11980 & 0 & 5.0 \\
\hline$\cdots$ & 17.3 & 51.8 & 10.9 & 1.0 & 12840 & 66 & \\
\hline$\cdots$ & 699 & 66.4 & 0.1 & 2.9 & 13001 & 59 & 5.0 \\
\hline$\ldots$ & $1 x .3$ & 46.5 & 16.7 & 3.5 & 11850 & 66 & 5.0 \\
\hline$\ddot{*}$ & 38.8 & $4 n .2$ & 21.0 & 4.7 & 11080 & n & \\
\hline$\cdots$ & $4 ? .1$ & 41.8 & 16.1 & 4.5 & 11890 & 0 & 3.0 \\
\hline$\cdots$ & un. & 62.6 & 16.6 & 5.2 & 11820 & n & 3.5 \\
\hline$\because$ & 39.8 & 42.1 & 19.1 & 4.7 & 11750 & v & \\
\hline 0.8 & 4.5 & 42.1 & 16.4 & 3.3 & 12420 & d & \\
\hline 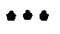 & 6?.9 & 63.1 & 14.0 & 8 & 12480 & .57 & 3.3 \\
\hline$\cdots$ & 38.2 & sn. 4 & 11.4 & 3.3 & 12600 & 66 & 1.0 \\
\hline $\begin{array}{l}9.6 \\
9.9\end{array}$ & 63.1 & 14.6 & 12.5 & 6,7 & 12420 & 61 & \\
\hline 9.9 & $\$ 1.6$ & 45.2 & 13.2 & 5.2 & 12240 & 0 & 4.0 \\
\hline$\ldots$ & in. & $\begin{array}{l}67.6 \\
67.7\end{array}$ & $11 \%$ & 3.8 & 12350 & ?) & \\
\hline 0.5 & 41.5 & 46.0 & 13.9 & 4.3 & 12190 & 0 & \\
\hline 9.0 & 39.9 & 67.9 & 12.2 & 3.9 & 12270 & 31 & 3.0 \\
\hline 9.6 & 41.9 & 45.1 & 13.0 & 4.3 & 12020 & 30 & \\
\hline 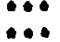 & $\begin{array}{l}39.1 \\
39.1\end{array}$ & $\begin{array}{l}48.5 \\
48.6\end{array}$ & $\begin{array}{l}12.4 \\
12.3\end{array}$ & 4. & $\begin{array}{l}12650 \\
12500\end{array}$ & $\begin{array}{l}62 \\
36\end{array}$ & 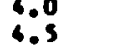 \\
\hline
\end{tabular}



National coal Resources oata systen

U.S. Geological survey

identified coal resources in iowa

(In millions of short tons) $\cos 20$ measured and indicated (inches)
meses interred (inches)

total (inches)

coal bed

$14-2828-42$ over 42 total $14-2828-42$ over 4

total $14-2828-42$ over 42 total

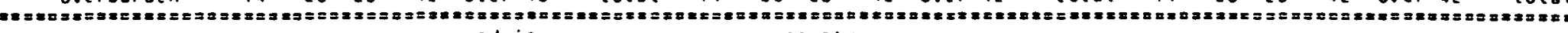
County
cound

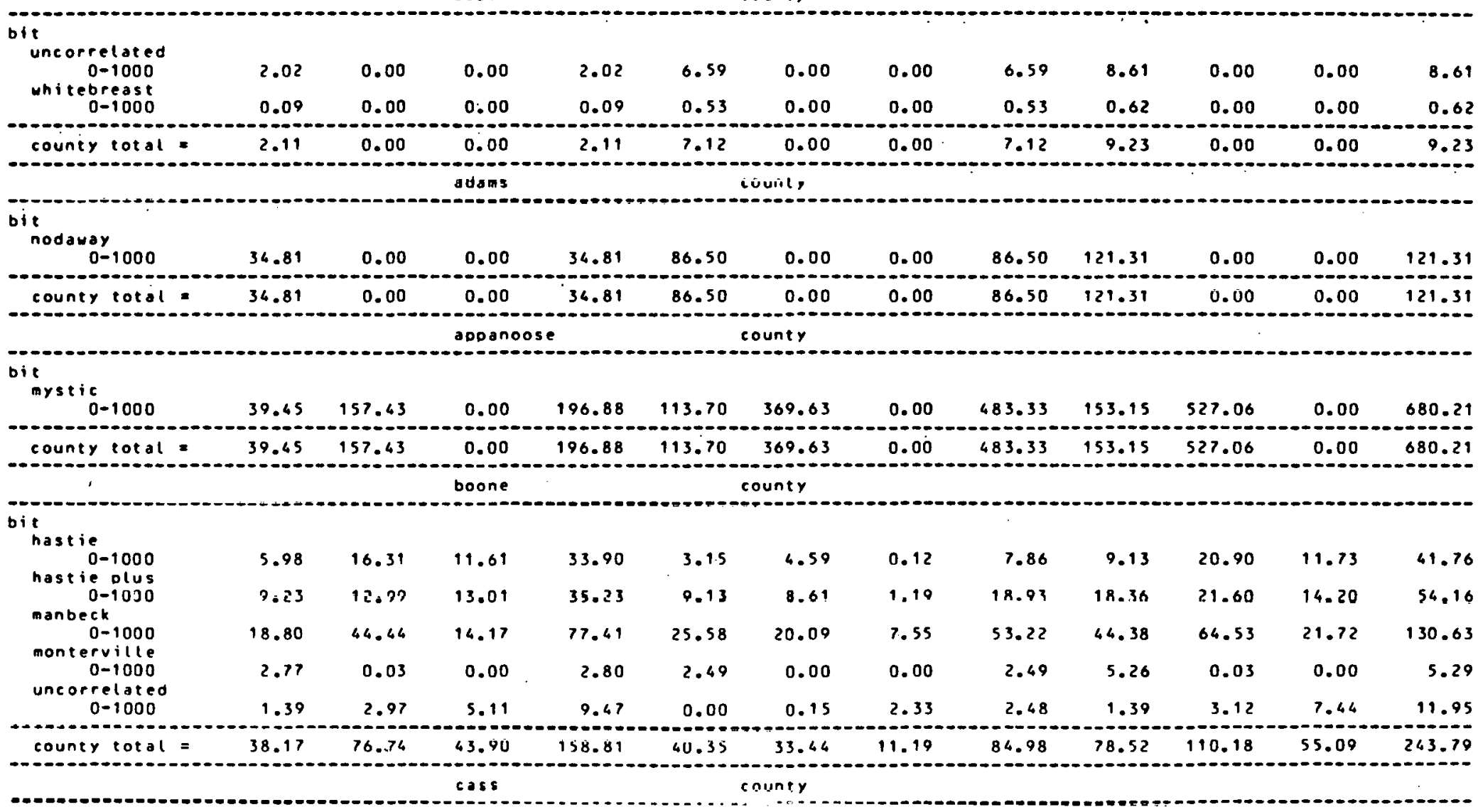

bit 


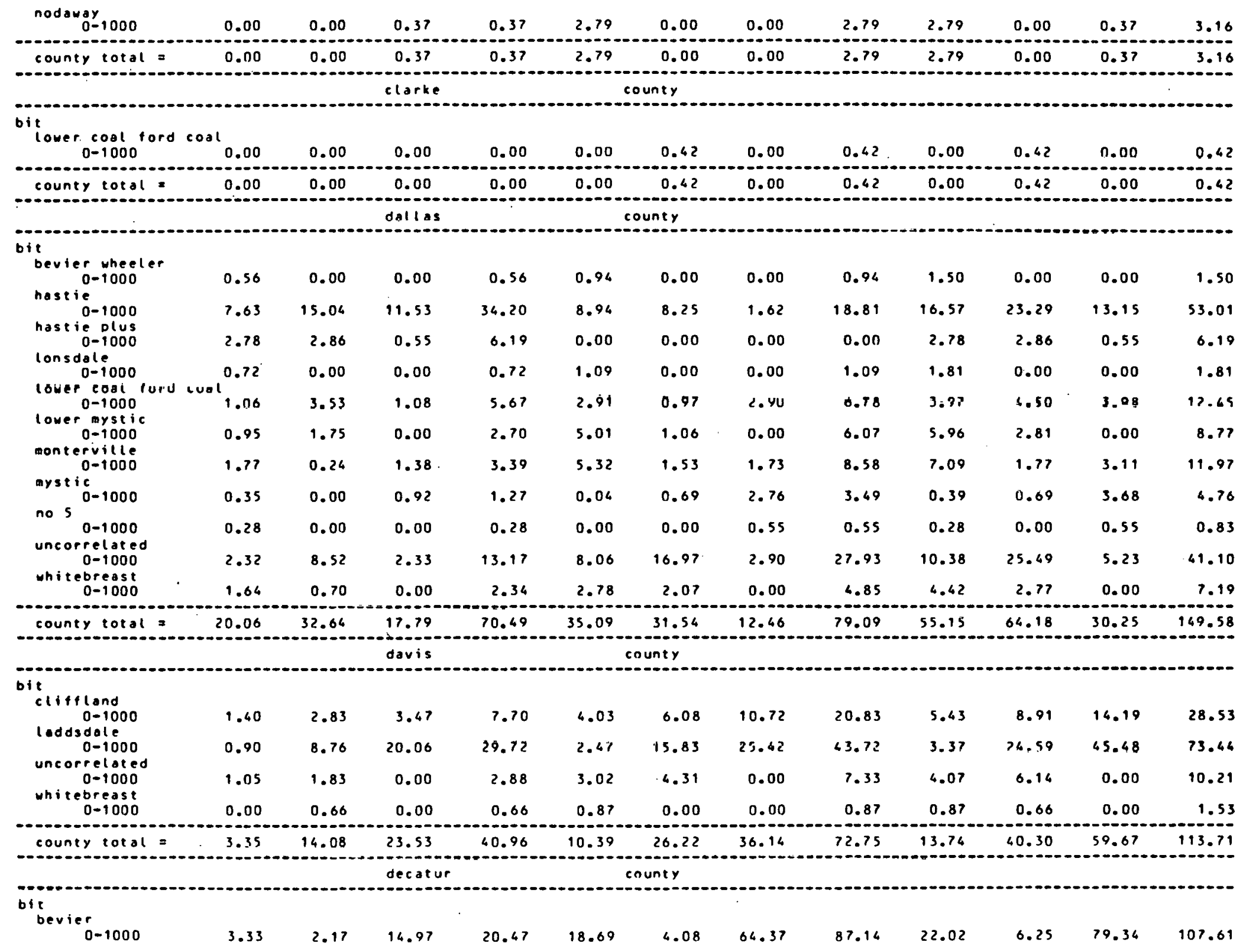




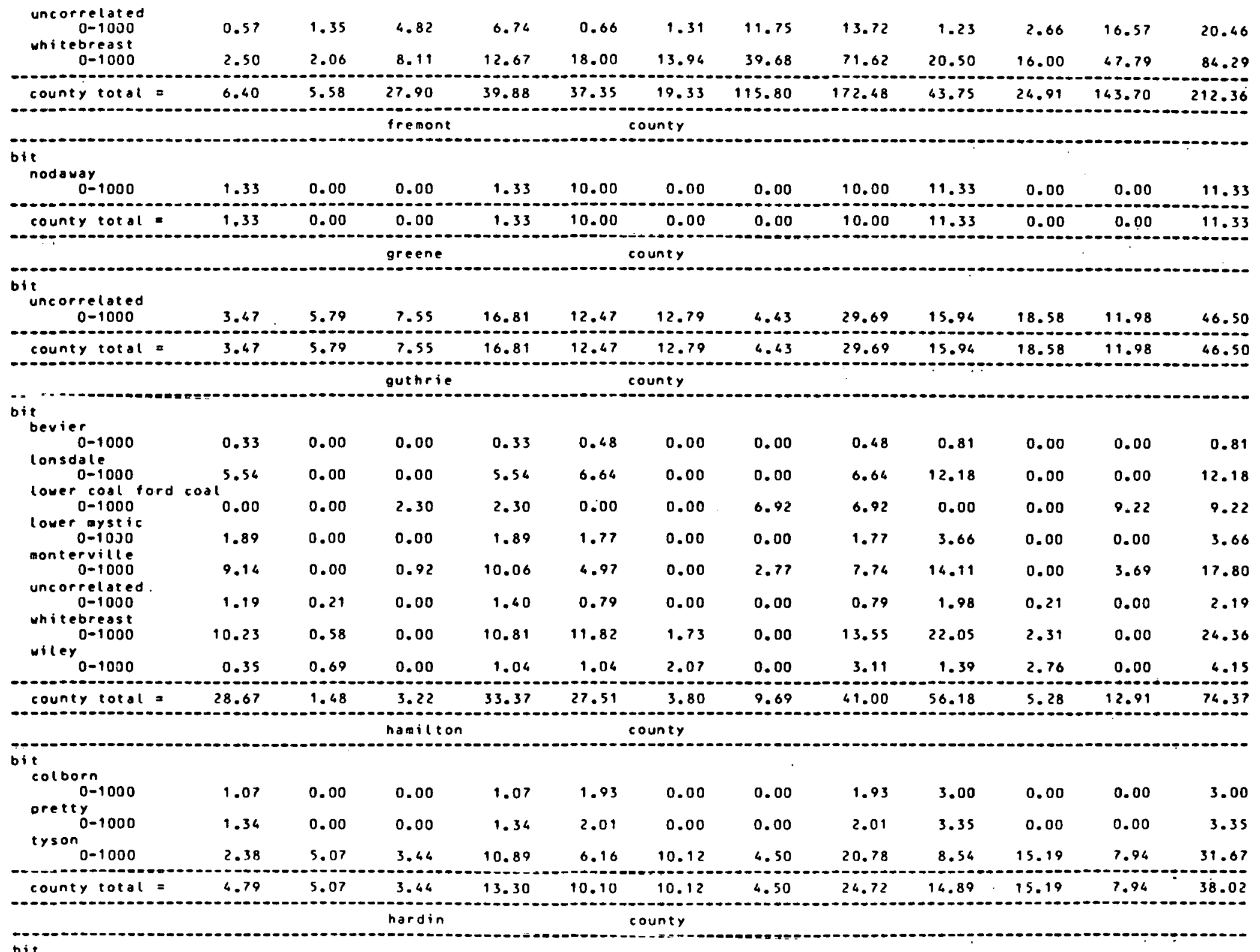




\begin{tabular}{|c|c|c|c|c|c|c|c|c|c|c|c|c|}
\hline $\begin{array}{l}\text { rrelated } \\
0-1000\end{array}$ & 0.40 & 1.38 & 3.22 & 5.00 & 0.04 & 4.68 & 2.03 & 6.75 & 0.44 & 6.06 & 5.25 & 11.75 \\
\hline county total & 0.40 & 1.38 & 3.22 & 5.00 & 0.04 & 4.68 & 2.03 & 6.75 & 0.64 & 6.06 & 5.25 & 11.75 \\
\hline
\end{tabular}

bit

\begin{tabular}{|c|c|c|c|c|c|c|c|c|c|c|c|c|}
\hline $\begin{array}{l}0-1000 \\
\text { ctifftand }\end{array}$ & 0.00 & 0.00 & 0.23 & 0.23 & 0.00 & 0.00 & 0.27 & 0.27 & 0.00 & 0.00 & $0.5 n$ & 0.50 \\
\hline $0-1000$ & 0.25 & 0.52 & 0.48 & 1.25 & 0.46 & 1.42 & 0.00 & 1.88 & 0.71 & 1.96 & 0.48 & 3.13 \\
\hline county & 0.25 & 0.52 & 0.71 & 1.48 & 0.46 & 1.42 & 0.27 & 2.15 & 0.71 & 1.94 & 0.98 & 3.63 \\
\hline
\end{tabular}

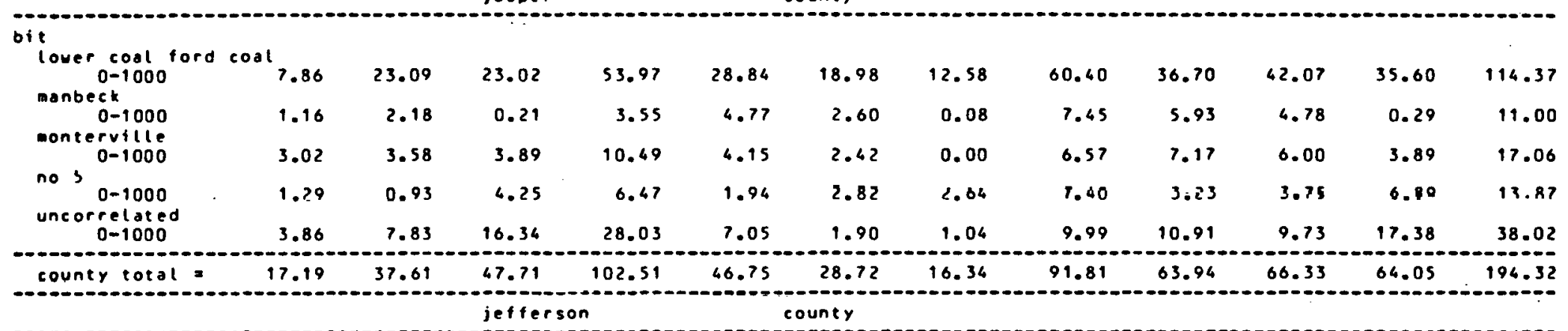

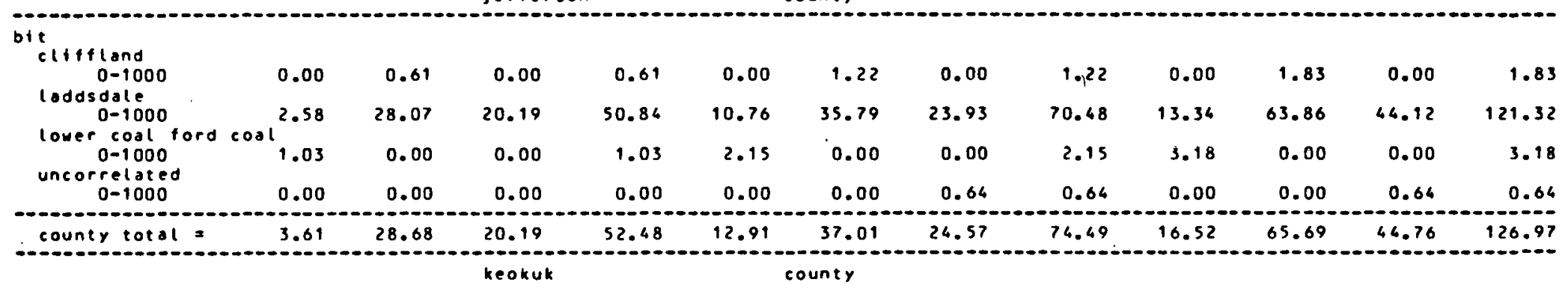

big
bit

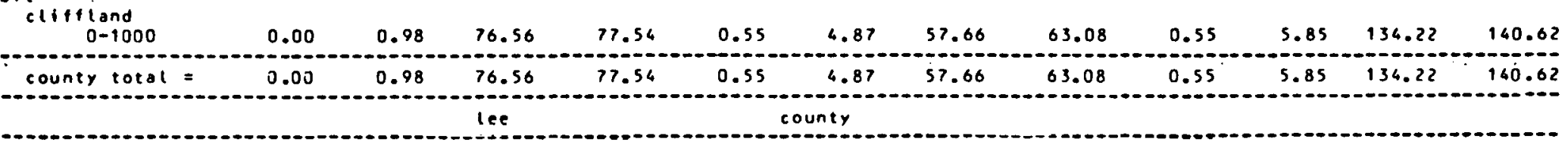
bit

\begin{tabular}{|c|c|c|c|c|c|c|c|c|c|c|c|c|}
\hline $\begin{array}{c}\text { Prelated } \\
0-1000\end{array}$ & 2.17 & 8.00 & 0.71. & 4.88 & 5.05 & 4.71 & 1.62 & 11.38 & 7.22 & 6.71 & 2.33 & 16.26 \\
\hline at & 2.17 & 2.00 & 0.71 & 4.88 & 5.05 & 4.71 & 1.02 & 11.38 & 7.22 & 6.31 & 2.33 & 16.26 \\
\hline
\end{tabular}


lucas

county

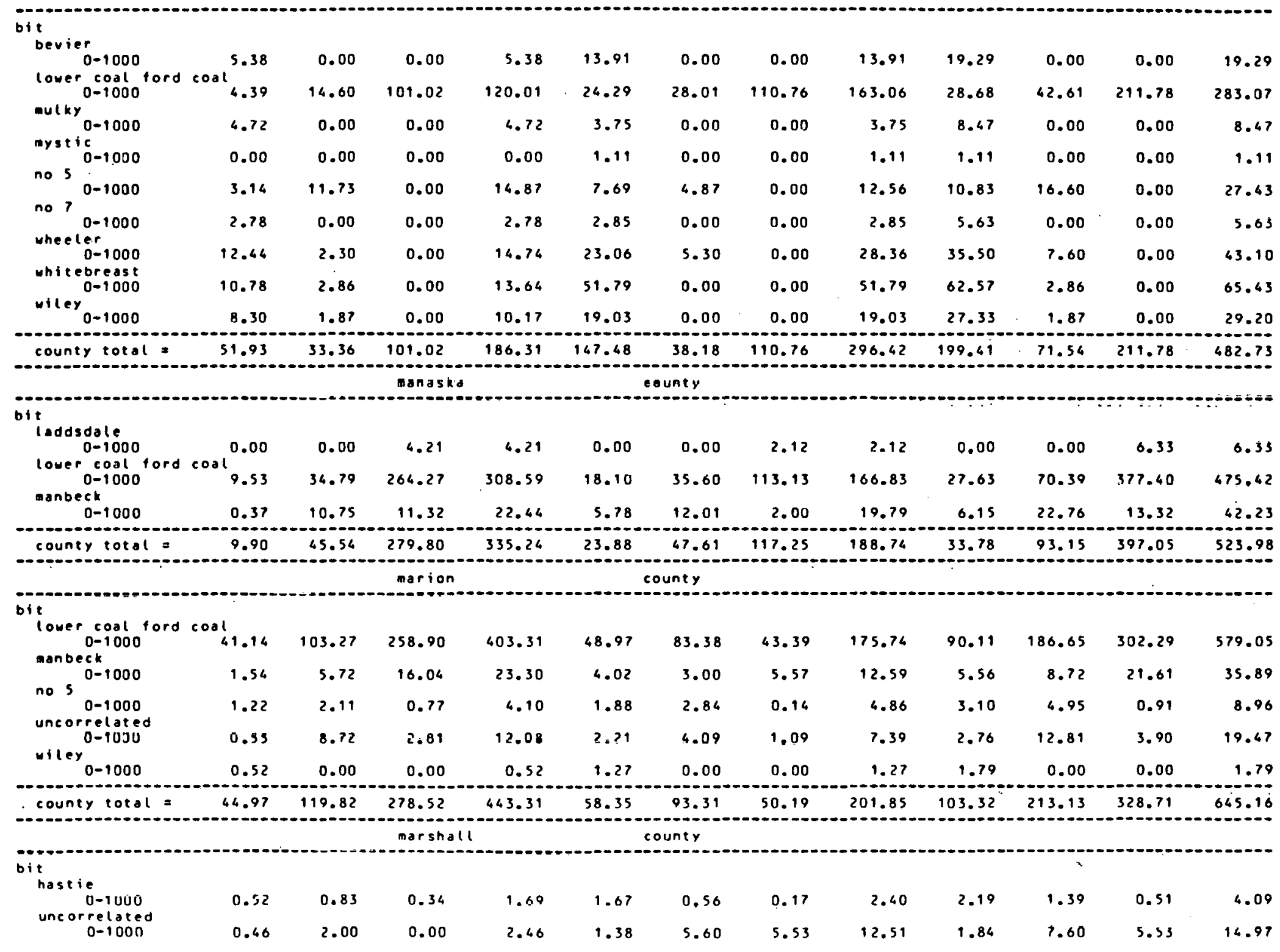




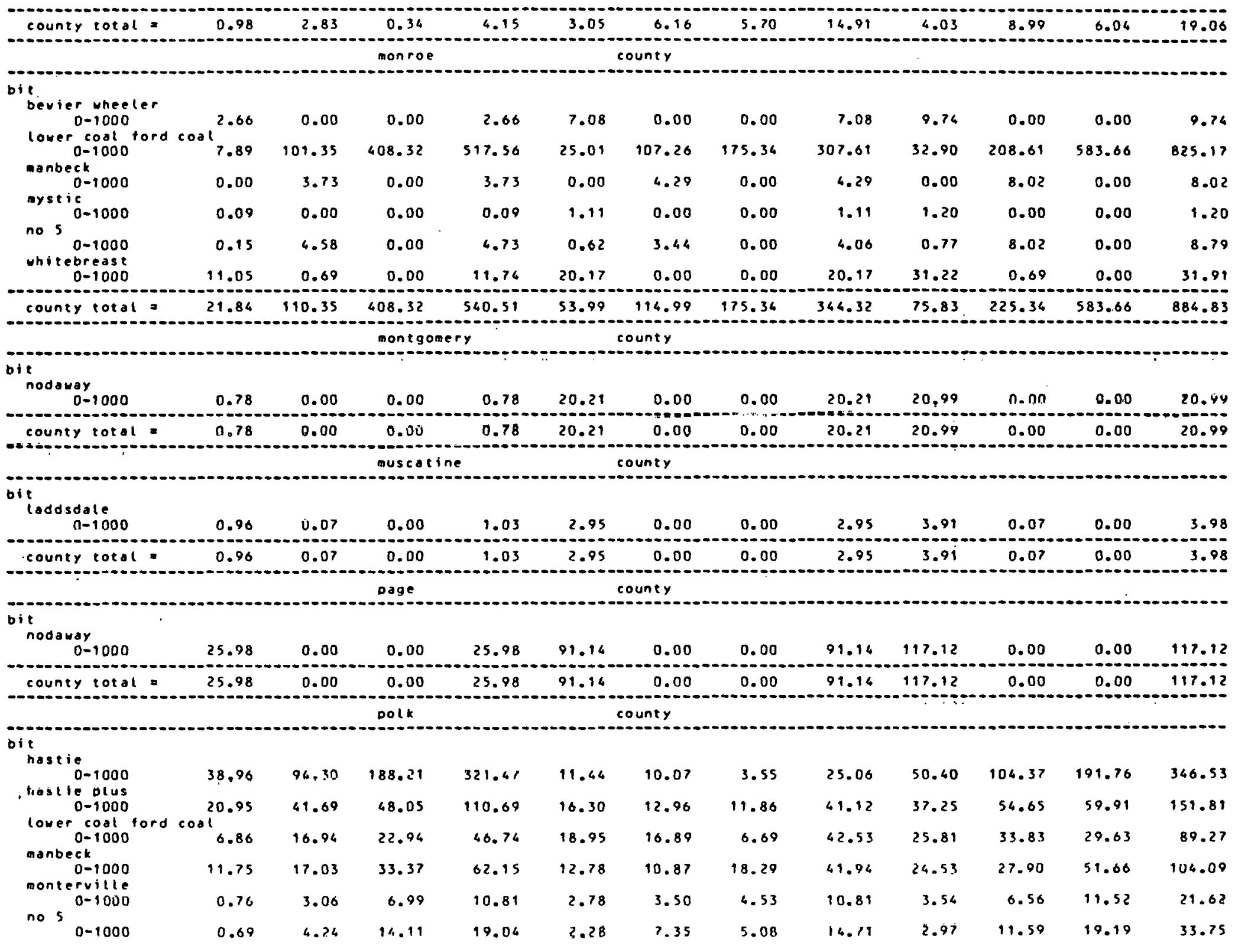




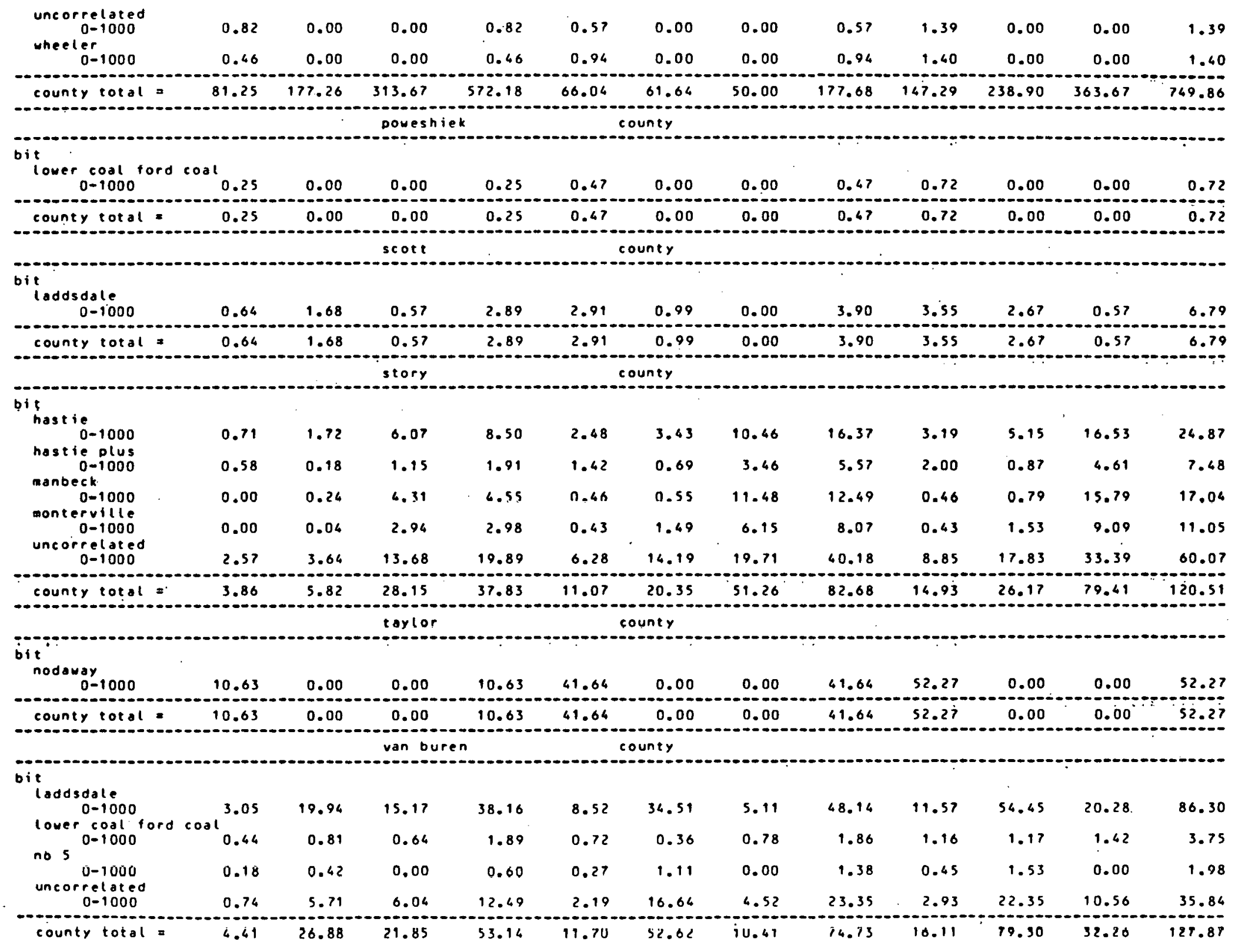




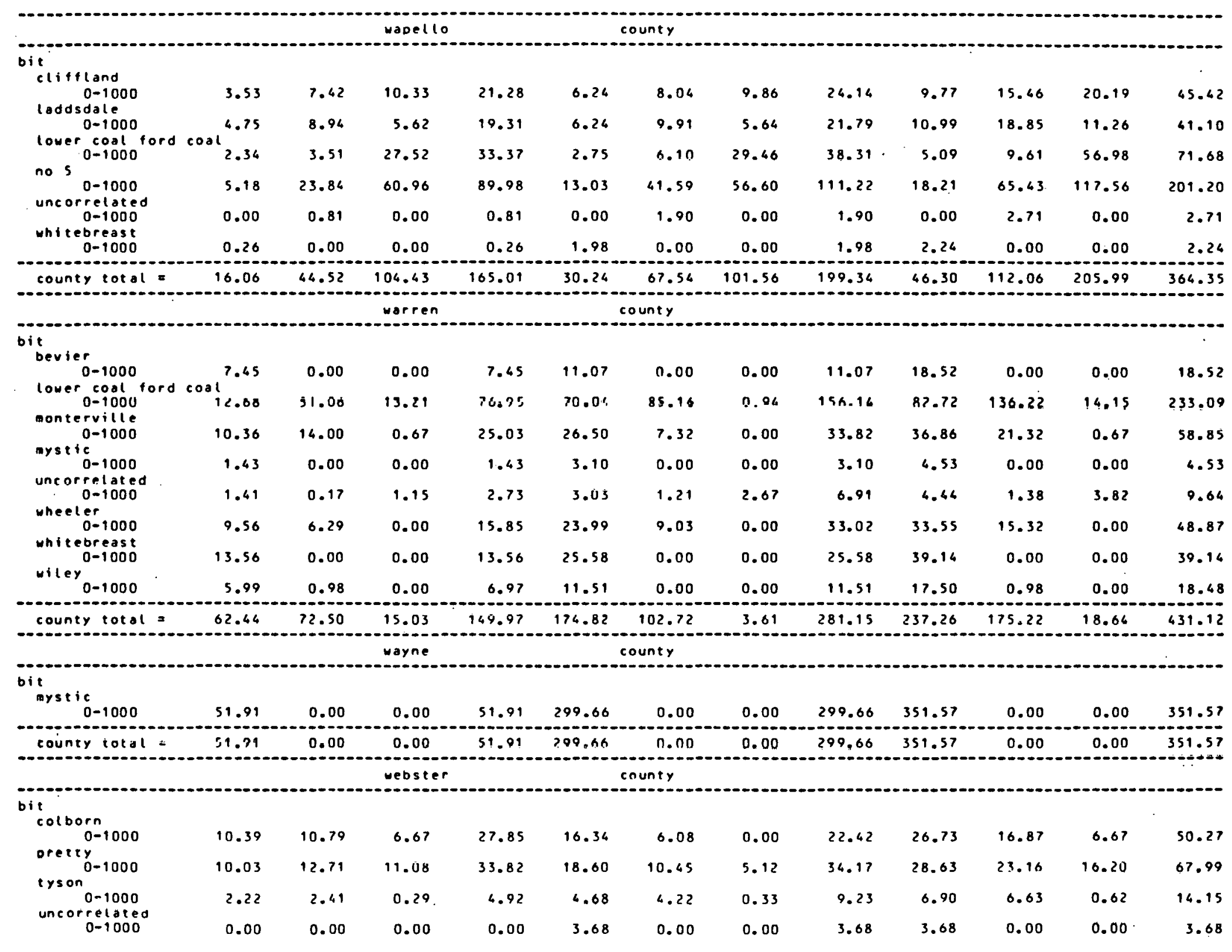




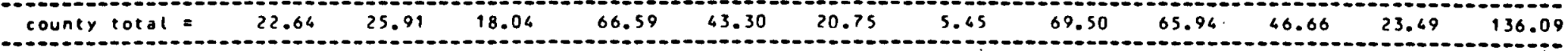
0 overburden

$\begin{array}{lllllllllll}0-1000 & 617.66 & 1036.52 & 1846.54 & 3500.72 & 1542.03 & 1215.56 & 978.23 & 3735.82 & 2159.69 \quad 2252.08 & 2824.77 \quad 7236.54\end{array}$

total = $617.66 \quad 1036.52 \quad 1846.54 \quad 3500.72 \quad 1542.03 \quad 1215.56 \quad 978.23 \quad 3735.82 \quad 2159.69 \quad 2252.08 \quad 2824.77 \quad 3236.54$

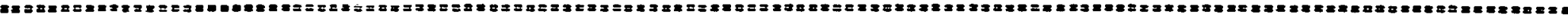
overburden

total

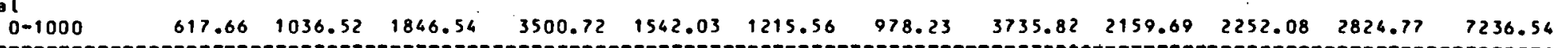
grand total $617.66 \quad 1036.52 \quad 1846.54 \quad 3500.72 \quad 1542.03 \quad 1215.56 \quad 978.23 \quad 3735.82 \quad 2159.69 \quad 2252.08 \quad 2824.77 \quad 7236.56$

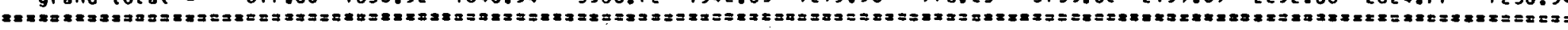

resource figures taken from:
source
year
base year of resource
ioua gs tech paper 4
1965
original 
average analyses of coal in iowa

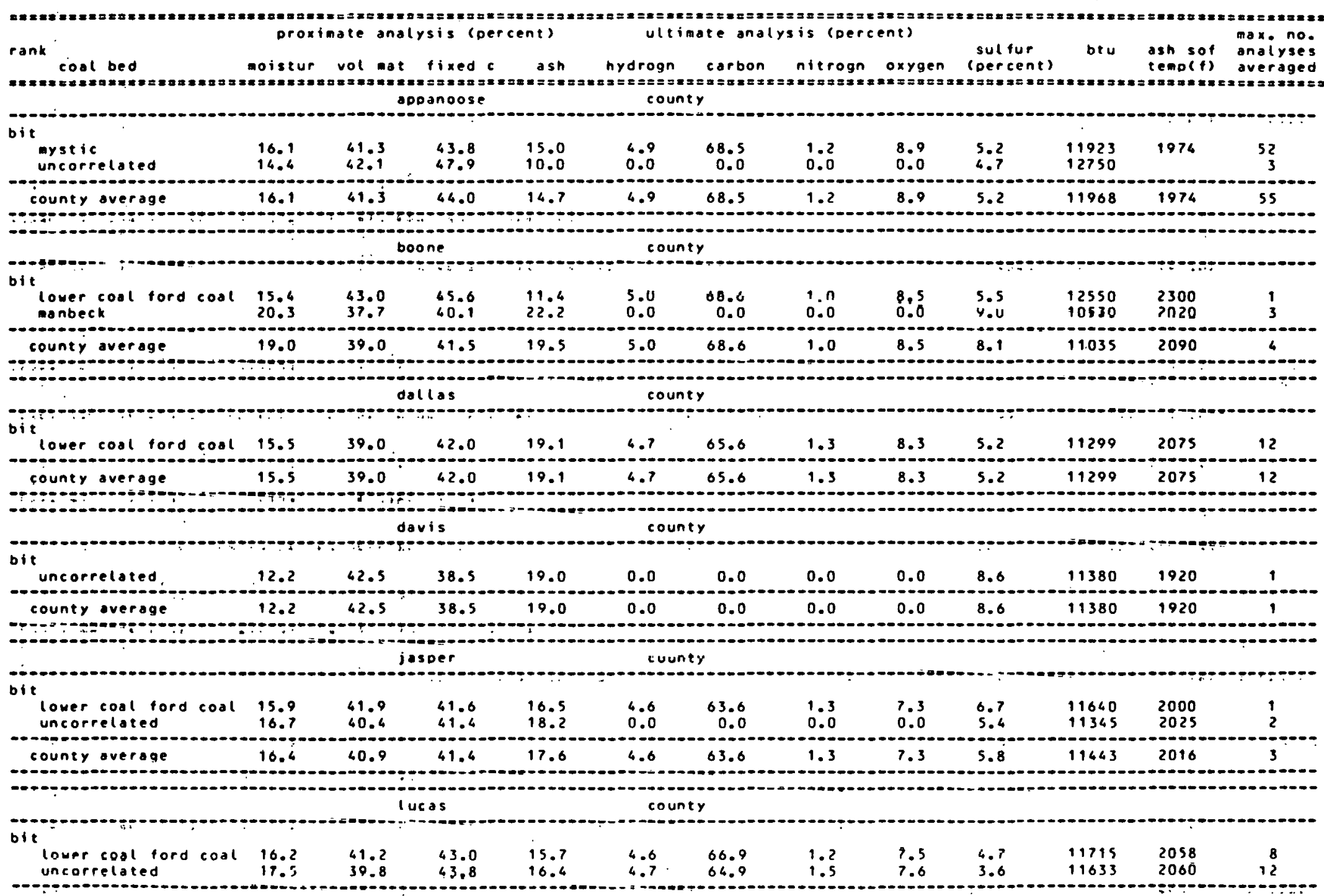




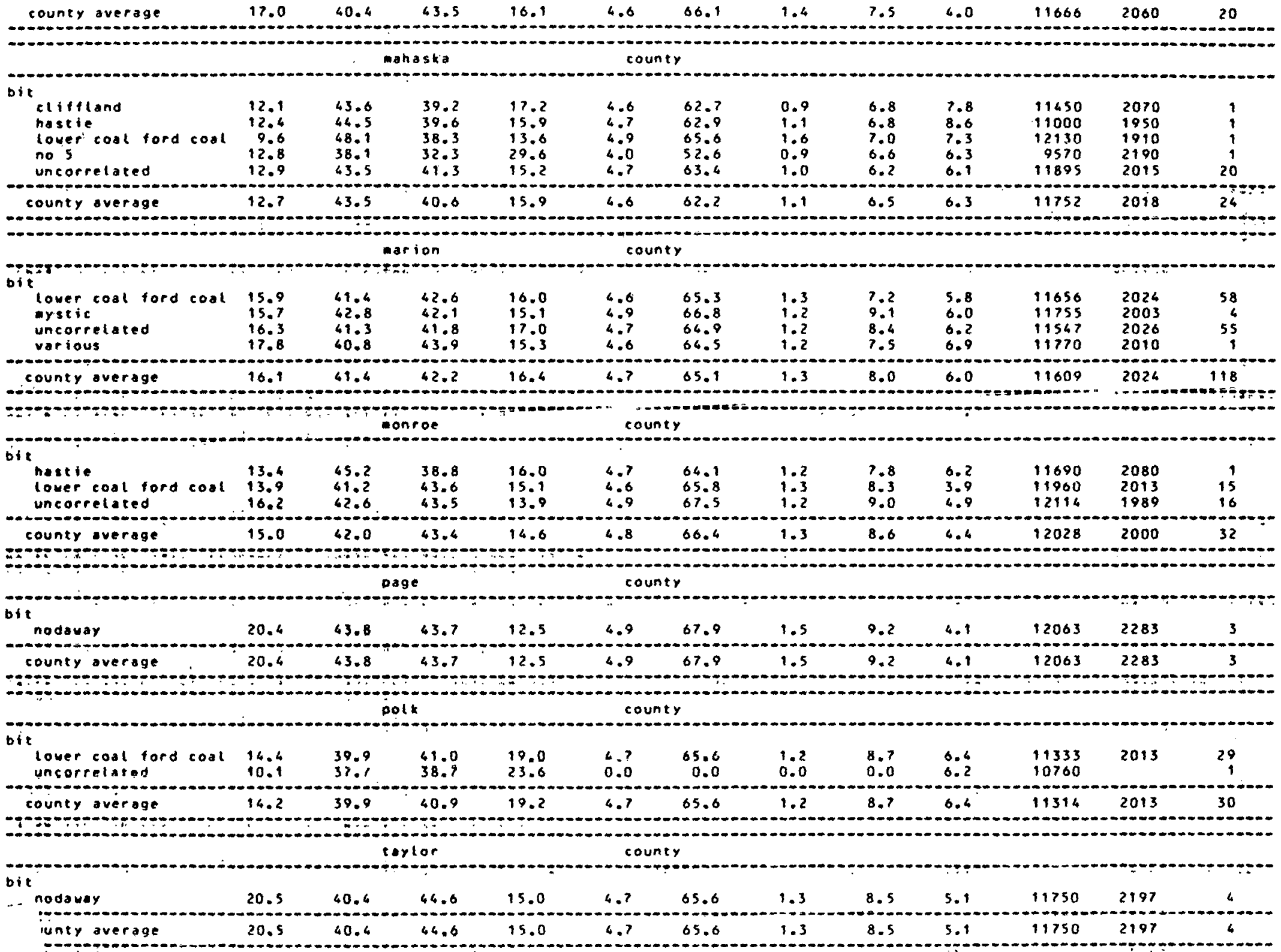


van buren county

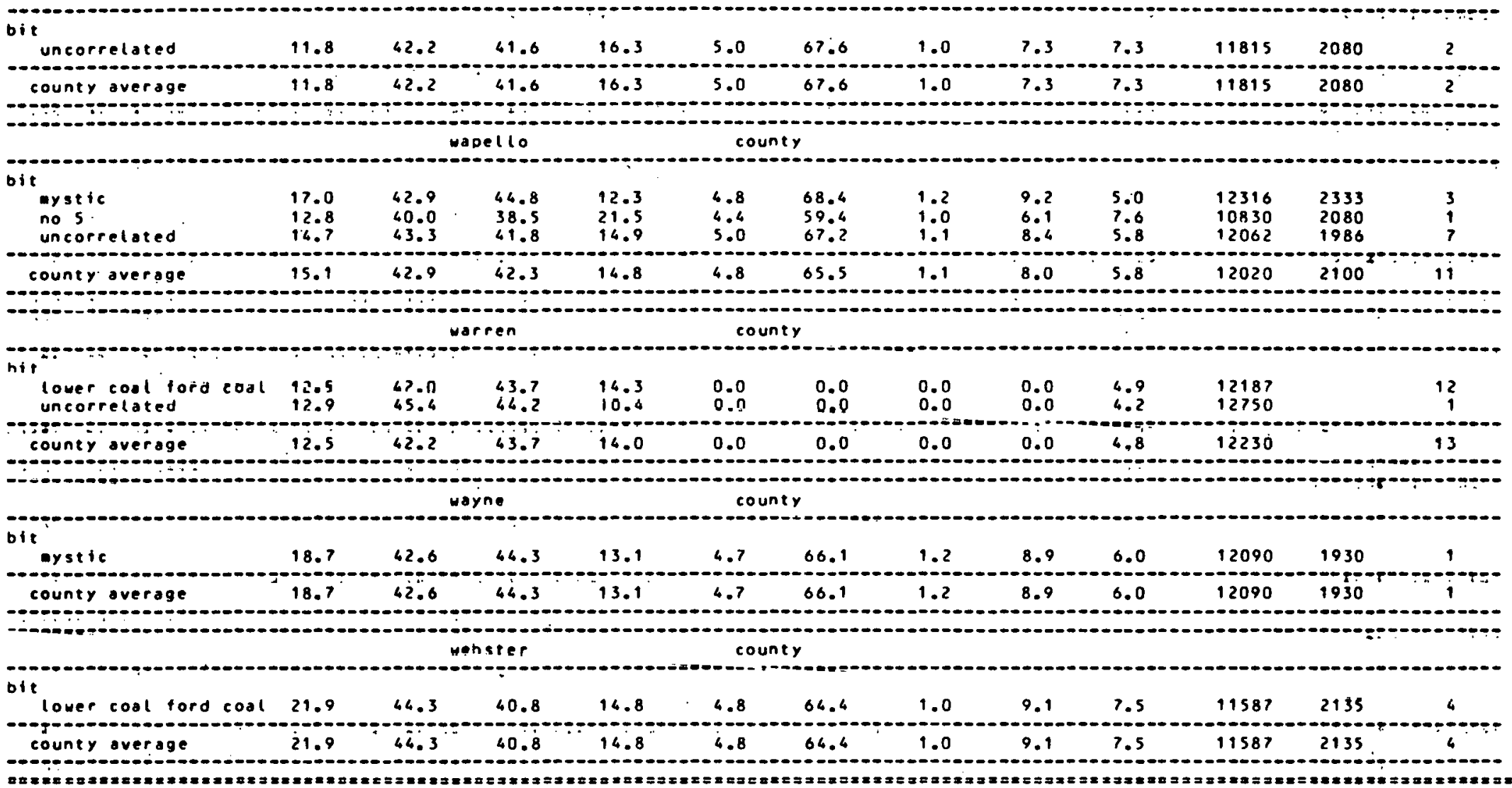


Iowa

\begin{tabular}{|c|c|}
\hline 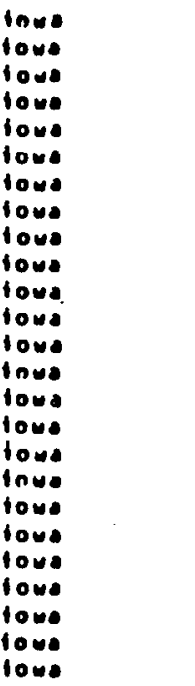 & 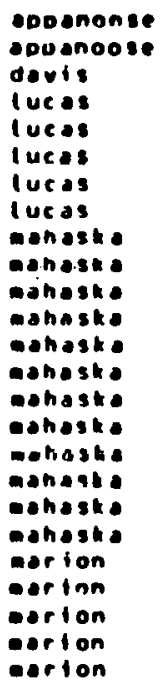 \\
\hline
\end{tabular}

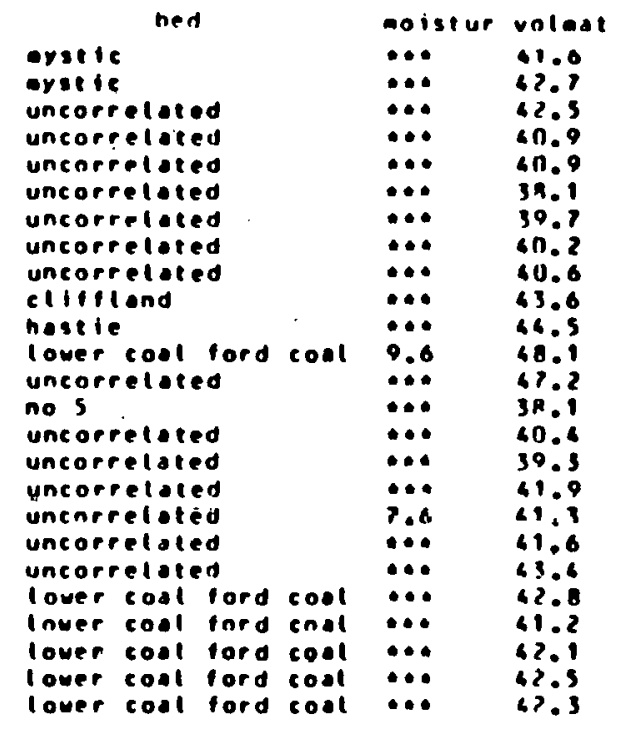

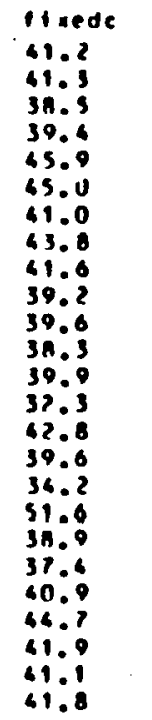

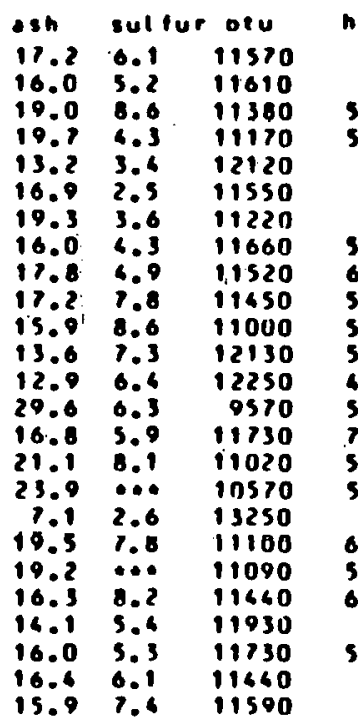

$n g 110$
0
0
59
58
0
0
0
55
66
52
51
50
66
59
70
58
59
0
63
53
69
0
59
0
0

18110
2.5
3.5
3.0
3.0
3.5
2.5
3.0
2.0
2.3
5.0
3.0
3.5
3.0
1.0
4.0
3.0
6.5
2.5
1.0
3.0
3.5
1.0
3.5
2.5
2.0

continued,..... 


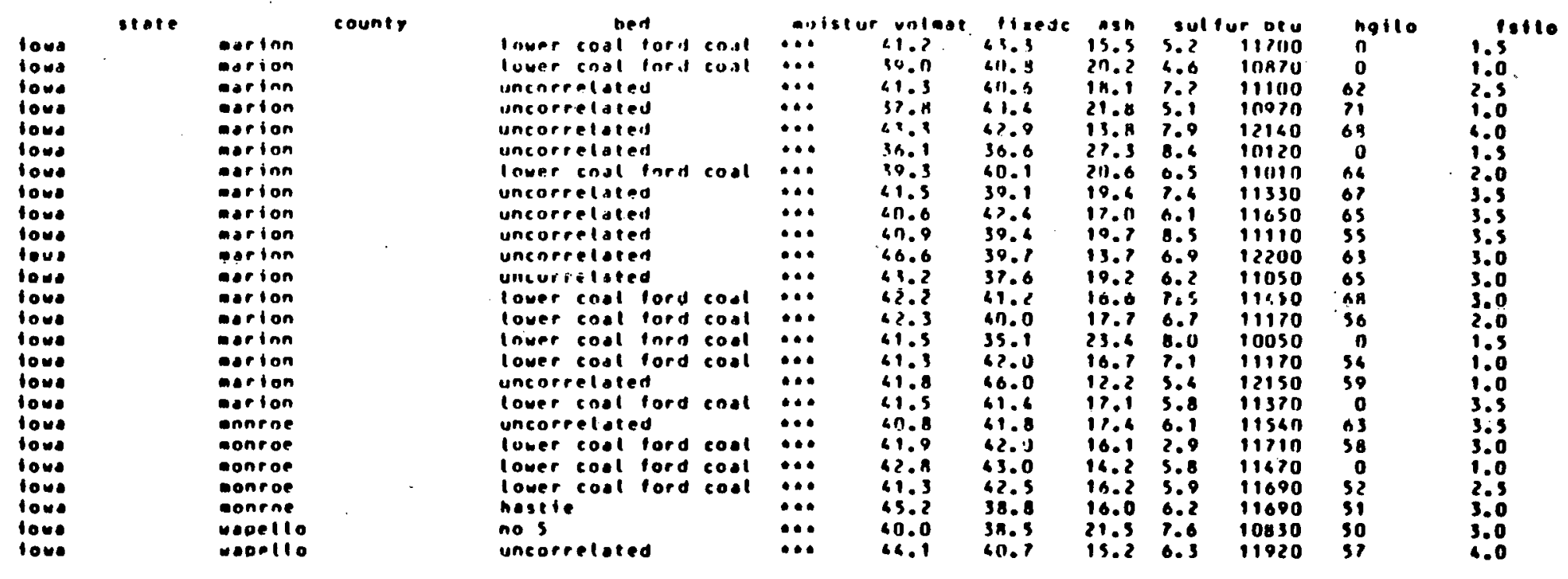




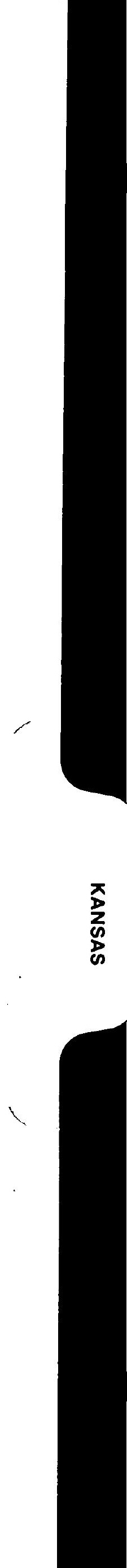


overage onalyses of coal in kansas

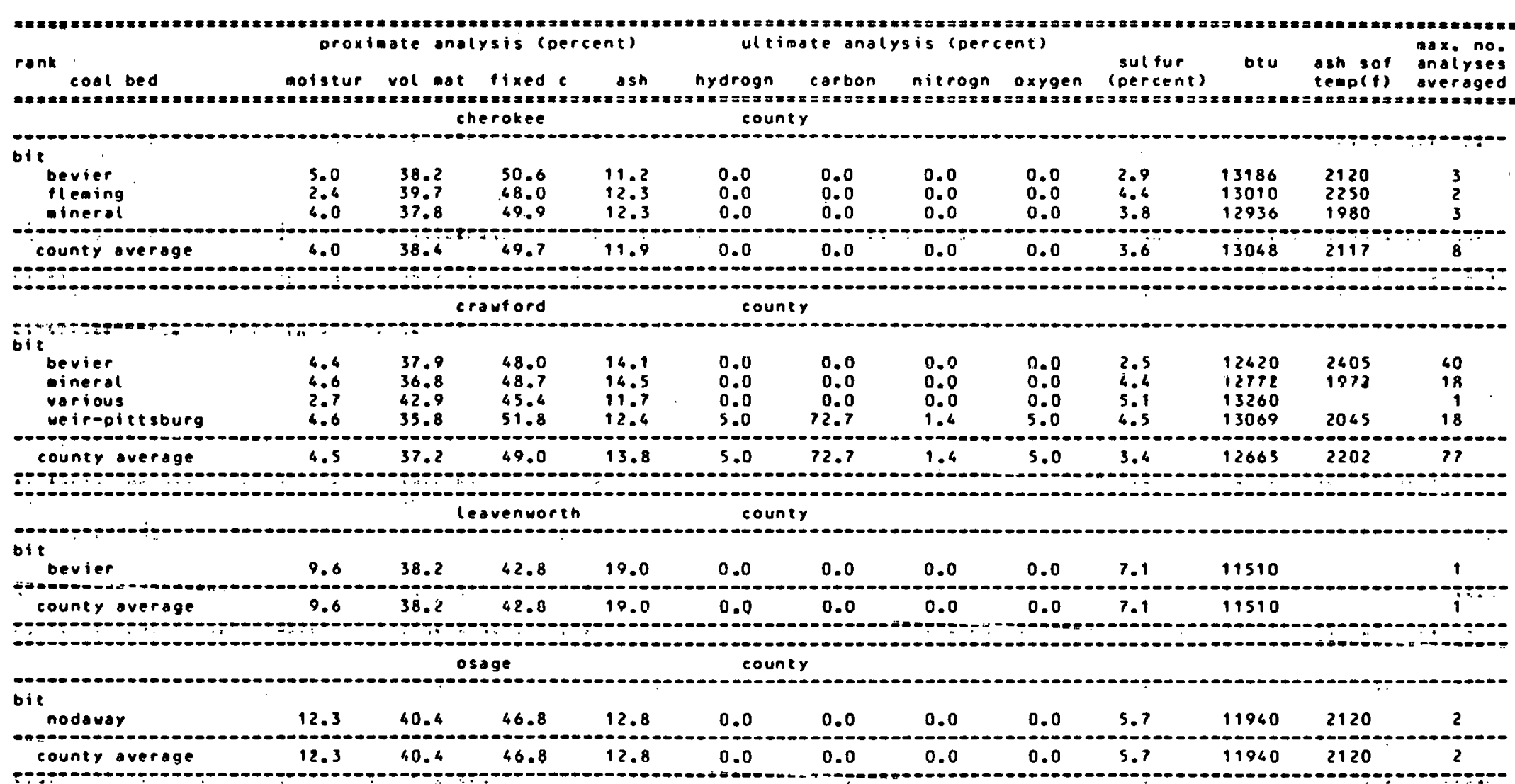

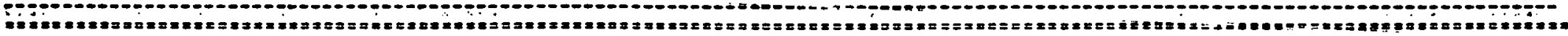


Kansa8

\begin{tabular}{|c|c|c|c|c|c|c|c|c|c|c|}
\hline 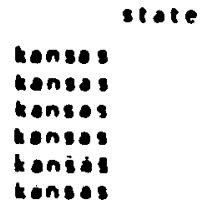 & $\begin{array}{l}\text { couner } \\
\text { chernkes } \\
\text { cherokee } \\
\text { creutord } \\
\text { croutord } \\
\text { irouford } \\
\text { crantort }\end{array}$ & 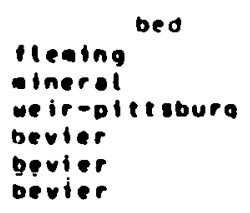 & $\begin{array}{l}\text { mostcur } \\
? .4 \\
3.8 \\
5.8 \\
6.0 \\
8.4 \\
0.1\end{array}$ & $\begin{array}{l}\text { vol } 0.41 \\
39.7 \\
36.2 \\
36.5 \\
37.5 \\
37.9 \\
17.0\end{array}$ & $\begin{array}{l}11 \text { inedc } \\
36.4 \\
50.9 \\
50.9 \\
50.0 \\
30.2 \\
6 n .4\end{array}$ & $\begin{array}{l}03 n \\
15.9 \\
12.9 \\
14: 9 \\
12.9 \\
11: 9 \\
14.8\end{array}$ & $\begin{array}{l}\text { sulfur } \\
5.2 \\
\because: 1 \\
\because: 7 \\
2.3 \\
2.2 \\
2.0\end{array}$ & $\begin{array}{l}010 \\
12780 \\
127170 \\
12800 \\
12870 \\
12890 \\
12430\end{array}$ & $\begin{array}{l}\text { ngillo } \\
0 \\
0 \\
03 \\
60 \\
0.1 \\
0\end{array}$ & $\begin{array}{l}1.110 \\
8.5 \\
8.0 \\
8.0 \\
8.5 \\
7.5 \\
7.5\end{array}$ \\
\hline
\end{tabular}



National Coat Resources Data Systen

U.S. Geological surver

identified coal resources in eastern kentucky

(In millions of short tons)

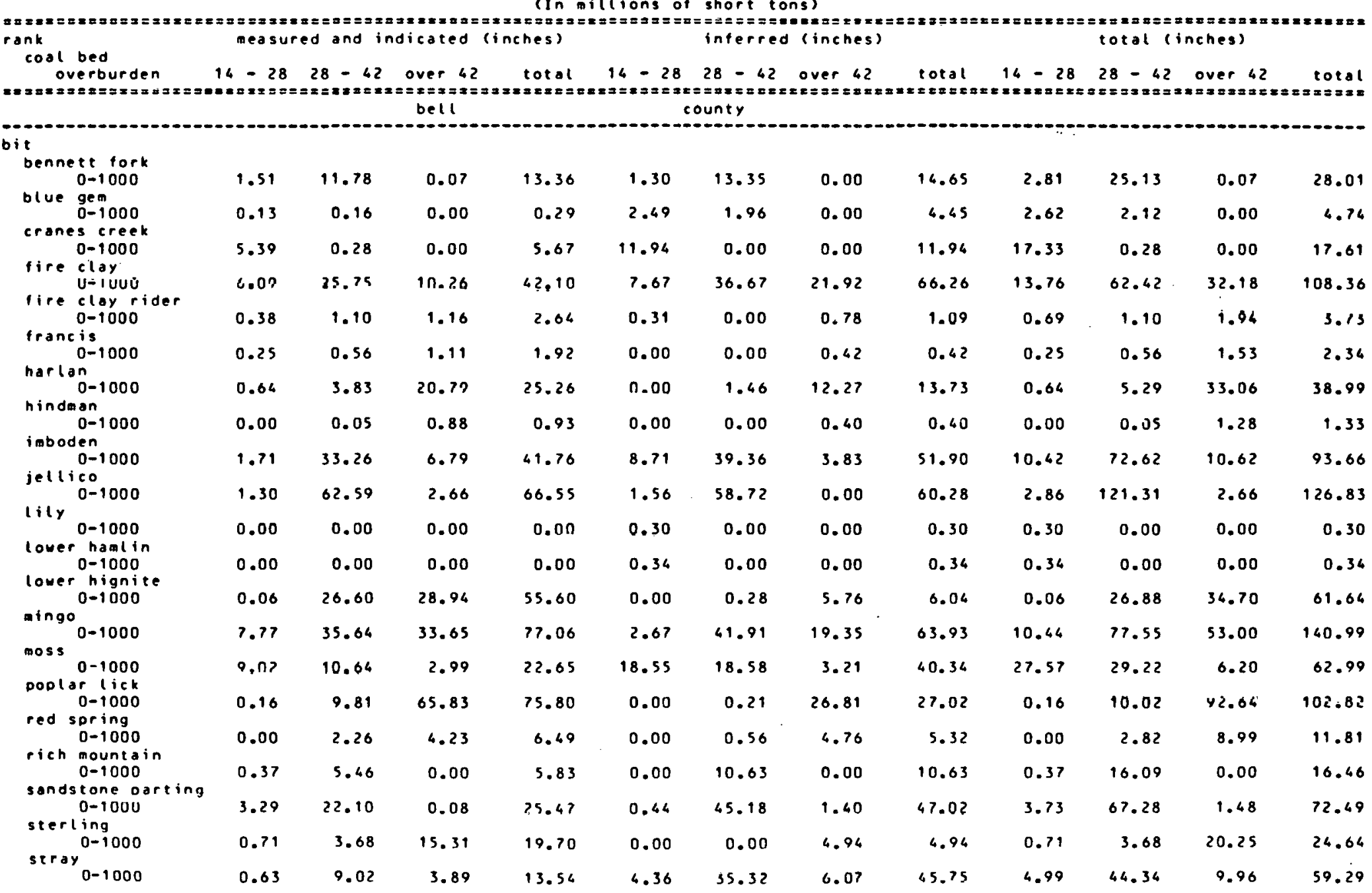




\begin{tabular}{|c|c|c|c|c|c|c|c|c|c|c|c|c|}
\hline county total = & 39.41 & 264.57 & 198.64 & 502.62 & 60.64 & 304.19 & 111.92 & 476.75 & 100.05 & 568.76 & 310.56 & 979.37 \\
\hline & & & boyd & & & inty & & & & & & \\
\hline \multicolumn{13}{|l|}{ t } \\
\hline 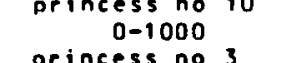 & 0.00 & 0.00 & 0.00 & 0.00 & 3.19 & 0.00 & 0.00 & 3.19 & 3.19 & 0.00 & 0.00 & 3.19 \\
\hline $0-1000$ & 1.90 & 0.00 & 0.00 & 1.90 & 15.71 & 3.56 & 0.00 & 19.27 & 17.61 & 3.56 & 0.00 & 21.17 \\
\hline $\begin{array}{r}\text { Princess no } 4 \\
0-10 n 0^{4}\end{array}$ & 0.45 & 0.00 & 0.00 & 0.45 & 8.06 & 0.00 & 0.00 & 8.06 & 8.51 & 0.00 & 0.00 & 8.51 \\
\hline $\begin{array}{r}\text { princess no } 5 \\
0=1000\end{array}$ & 12.90 & 15.34 & 2.27 & 30.51 & 19.00 & 12.24 & 0.00 & 31.26 & 31.90 & 27.58 & 2.27 & 61.75 \\
\hline $\begin{array}{r}\text { Princess no } 5 \text { a } \\
0-1000\end{array}$ & 11.59 & 6.04 & 0.00 & 17.63 & 25.81 & 3.07 & 0.00 & 28.88 & 37.60 & 9.11 & 0.00 & 46.51 \\
\hline $\begin{array}{c}\text { princess no } 50 \\
0-1000\end{array}$ & 7.75 & 0.00 & 0.00 & 7.75 & 7.04 & 0.00 & 0.00 & 7.04 & 14.79 & 0.00 & 0.00 & 14.79 \\
\hline $\begin{array}{r}\text { orincess no } 6 \\
0-1000\end{array}$ & 7.51 & 17.51 & 7.28 & 32.30 & 20.00 & 13.10 & 3.34 & 36.44 & 27.51 & 30.61 & 10.62 & 68.74 \\
\hline $\begin{array}{r}\text { orincess no } 7 \\
0-1000 \\
\text { princoses nn }\end{array}$ & 19.13 & 53.45 & 33.28 & 105.86 & 12.67 & 40.34 & 7.22 & 60.23 & 31.80 & 93.79 & 40.50 & 166.09 \\
\hline $\begin{array}{c}0-1000 \\
\text { orincess no }\end{array}$ & 9.76 & 3.45 & $3: 43$ & 16.64 & 78.54 & 1.13 & 0.00 & 29.67 & 38.30 & 4.58 & 3.43 & 46.31 \\
\hline $0-1000$ & 2.23 & 0.00 & 0.00 & 2.23 & 13.04 & 4.17 & 0.00 & 17.21 & 15.27 & 4.17 & 0.00 & 19.64 \\
\hline county total = & 73.22 & 95.79 & 46.26 & 215.27 & 153.06 & 77.61 & 10.56 & 241.23 & 226.28 & 173.40 & 56.82 & 456.50 \\
\hline & & & brea & & & it & & & & & & \\
\hline \multicolumn{13}{|l|}{ bit } \\
\hline colvin & 15.44 & 9.83 & 0.00 & 25.27 & 30.00 & 40.79 & 0.00 & 70.79 & 45.44 & 50.62 & 0.00 & 96.06 \\
\hline $\begin{array}{c}0-1000 \\
\text { copland }\end{array}$ & 11.80 & 0.20 & 0.00 & 12.00 & 0.00 & 0.00 & 0.00 & 0.00 & 11.80 & 0.20 & 0.00 & 12.00 \\
\hline $\begin{array}{l}0-1.000 \\
\text { tire elay }\end{array}$ & 0.00 & 6.19 & 0.82 & 7.01 & 10.84 & 0.00 & 0.00 & 10.84 & 10.84 & 6.19 & 0.82 & 17.85 \\
\hline $\begin{array}{l}0-1000 \\
\text { fire clay rider }\end{array}$ & 36.55 & 1.45 & 0.00 & 38.00 & 36.42 & 1.21 & 0.00 & 37.63 & 72.97 & 2.66 & 0.00 & 75.63 \\
\hline $\begin{array}{l}0-1000 \\
\text { francis }\end{array}$ & 112.41 & 102.52 & 3.79 & 218.72 & 17.26 & 4.36 & 0.00 & 21.62 & 129.67 & 106.88 & 3.79 & 240.34 \\
\hline haddix $0-1000$ & 15.44 & 59.79 & 95.92 & 171.15 & 22.53 & 9.08 & 5.73 & 37.34 & 37.97 & 68.87 & 101.65 & 208.69 \\
\hline hazard $0-1000$ & 84.52 & 74.31 & 15.44 & 176.2 .7 & 67.80 & 7.82 & 0.03 & 75.65 & 152.32 & 82.13 & 15.47 & 249.92 \\
\hline $\begin{array}{r}0-1000 \\
\text { hazard no? }\end{array}$ & 81.37 & 66.66 & 14.08 & 162.11 & 45.94 & 7.46 & 0.00 & 53.40 & 127.31 & 74.12 & 14.08 & 215.51 \\
\hline $\begin{array}{l}0-1000 \\
\text { nindman }\end{array}$ & 54.10 & 67.06 & 165.41 & 286.57 & 50.17 & 17.54 & 0.12 & 73.83 & 104.27 & 84.60 & 171.53 & 360.40 \\
\hline $\begin{array}{l}0-1000 \\
\text { lower whitesburg }\end{array}$ & 4.11 & 0.00 & 1.00 & 5.11 & 2.62 & 0.00 & 0.00 & 2.62 & 6.73 & 0.00 & 1.00 & 7.73 \\
\hline skyline & 28.42 & 1.96 & 0.00 & 30.38 & 00.58 & 19.96 & 0.00 & 80.54 & 89.00 & 29.92 & 0.00 & 110.92 \\
\hline $\begin{array}{c}0-1000 \\
\text { uncorrelated }\end{array}$ & 0.18 & 3.07 & 18.91 & 22.16 & 0.12 & 1.98 & 0.00 & 2.10 & 0.30 & 5.05 & 18.91 & 26.26 \\
\hline $\begin{array}{l}0-1000 \\
\text { upder elkhorn no }\end{array}$ & 14.11 & 3.06 & 0.00 & 7.17 & 15.41 & 0.00 & 0.00 & 15.41 & 19.52 & 3.06 & 0.00 & 22.58 \\
\hline $0-1030$ & 3.70 & 1.70 & $0.0 n$ & $? .40$ & 12.55 & 0.37 & 0.00 & 12.92 & 18.25 & 2.07 & 0.00 & 20.32 \\
\hline
\end{tabular}




\begin{tabular}{|c|c|c|c|c|c|c|c|c|c|c|c|c|}
\hline $\begin{array}{c}\text { upder elkhorn no } 3 \\
0-1000 \\
\text { zochariah } \\
0-1000\end{array}$ & $\begin{array}{l}60.38 \\
14.70\end{array}$ & $\begin{array}{r}31.73 \\
0.00\end{array}$ & $\begin{array}{l}4.80 \\
0.00\end{array}$ & $\begin{array}{l}96.91 \\
14.70\end{array}$ & $\begin{array}{l}70.16 \\
38.50\end{array}$ & $\begin{array}{l}4.79 \\
0.00\end{array}$ & $\begin{array}{l}0.00 \\
0.00\end{array}$ & $\begin{array}{l}74.95 \\
38.50\end{array}$ & $\begin{array}{r}130.54 \\
53.20\end{array}$ & $\begin{array}{r}36.52 \\
0.00\end{array}$ & $\begin{array}{l}4.80 \\
0.00\end{array}$ & $\begin{array}{r}171.86 \\
53.20\end{array}$ \\
\hline county total = & 529.23 & 429.53 & 320.17 & 1278.93 & 480.90 & 115.36 & 11.88 & 608.14 & 1010.13 & 544.89 & 332.05 & 1887.07 \\
\hline & & & $\operatorname{car} t$ & & & unty & & & & & & \\
\hline tire ol & & & & & & & & & & & & \\
\hline $0-1000$ & 4.53 & 6.55 & 2.83 & 13.99 & 7.37 & 8.49 & 0.00 & 15.86 & 11.90 & 15.04 & 2.83 & 29.77 \\
\hline $\begin{array}{c}\text { grassy } \\
0-1000\end{array}$ & 1.41 & 0.00 & 0.00 & 1.41 & 14.08 & 0.00 & 0.00 & 14.08 & 15.49 & 0.00 & 0.00 & 15.69 \\
\hline $\begin{array}{l}\text { gun creek } \\
0-1000\end{array}$ & 2.57 & 0.00 & 0.00 & 2.57 & 10.28 & 0.00 & 0.00 & 10.28 & 12.85 & 0.00 & 0.00 & 12.85 \\
\hline $\begin{array}{c}\text { lee coal beds } \\
0-1000\end{array}$ & 0.00 & 0.00 & 0.00 & 0.00 & 5.28 & 0.00 & 0.00 & 5.28 & 5.28 & 0.00 & 0.00 & 5.28 \\
\hline $\begin{array}{c}\text { orincess no } 3 \\
0-1000\end{array}$ & 17.43 & 8.70 & 0.00 & 26.13 & 36.51 & 0.49 & 0.00 & 36.92 & 53.94 & 9.11 & 0.00 & 63.05 \\
\hline $\begin{array}{c}\text { Princess no } \\
0-1000\end{array}$ & 3.78 & 4.49 & 0.00 & 8.27 & 15.95 & 1.87 & 0.00 & 17.82 & 19.73 & 6.36 & 0.00 & 26.09 \\
\hline $\begin{array}{r}\text { orincess no } 1000 \\
\text { und }\end{array}$ & 15.84 & 12.97 & 8.09 & 36.50 & 36.19 & 24.47 & 6.08 & 66.74 & 51.63 & 37.44 & 14.17 & 103.26 \\
\hline orinces $0-1000$ & 2.95 & 0.00 & 0.00 & 2.95 & 10.11 & 0.00 & 0.00 & 10.11 & 13.06 & 0.00 & 0.00 & 13.06 \\
\hline $0-1000$ & 5.46 & 4.49 & 0.00 & 9.95 & 23.14 & 17.44 & 2.54 & 43.12 & 28.60 & 21.93 & 2.54 & 53.07 \\
\hline $0-1000$ & 8.63 & 38.78 & 36.53 & 83.94 & 7.28 & 22.48 & 7.08 & 36.84 & 15.91 & 61.26 & 43.61 & 120.78 \\
\hline $\begin{array}{l}0-1000 \\
\text { tom cooper }\end{array}$ & 2.18 & 2.74 & 0.00 & 4.92 & 5.58 & 7.94 & 0.00 & 13.52 & 7.76 & 10.68 & 0.00 & $18.44^{\circ}$ \\
\hline $0-1000$ & 35.69 & 34.66 & 8.03 & 78.38 & 35.92 & 13.75 & 0.00 & 49.67 & 71.61 & 48.41 & 8.03 & 128.05 \\
\hline county total $=$ & 100.07 & 113.38 & 55.68 & 268.93 & 207.69 & 96.85 & 15.70 & 320.24 & 307.76 & 210.23 & 71.18 & 589.17 \\
\hline - - & & & cloy & & & unty & & & & & & \\
\hline $\begin{array}{l}\text { it } \\
\text { bacon creek }\end{array}$ & & & & & & & & & & & & \\
\hline blue gem & 0.00 & 0.00 & 0.00 & 0.00 & 1.88 & 0.00 & 0.00 & 1.88 & 9.88 & 0.00 & 0.00 & 1.88 \\
\hline $0-1000$ & 0.00 & 0.00 & 0.00 & 0.00 & 0.00 & 3.77 & 0.00 & 3.77 & 0.00 & 3.77 & 0.00 & 3.77 \\
\hline $\begin{array}{c}\text { Hre clay } \\
0=1000 \\
\text { fire clay rider }\end{array}$ & 64.36 & 49.61 & 10.89 & 104.84 & 80.46 & 30.05 & 0.00 & 110.51 & 124.80 & 79.66 & 10.89 & 215.35 \\
\hline $\begin{array}{l}0-1000 \\
\text { francis }\end{array}$ & 7.32 & 9.33 & 3.39 & 20.06 & 25.13 & 6.46 & 0.00 & 31.59 & 32.45 & 15.74 & 3.39 & 59.63 \\
\hline haddix $0-1000$ & 0.00 & 0.00 & 0.00 & 0.00 & 0.11 & 1.02 & 4.40 & 5.53 & 0.11 & 1.02 & 4.40 & 5.53 \\
\hline hozard $0-1000$ & 2.93 & 2.85 & 6.99 & 12.77 & 17.10 & 10.16 & 14.41 & 41.67 & 20.03 & 13.01 & 21.40 & 54.64 \\
\hline jeltico & 0.00 & 0.00 & 0.00 & 0.00 & 3.01 & 0.00 & 0.00 & 3.01 & 3.01 & 0.00 & 0.00 & 3.01 \\
\hline $0=1000$ & 17.32 & 17.54 & 0.00 & 34.86 & 60.45 & 47.52 & 0.00 & 107.97 & 77.77 & 65.06 & 0.00 & 142.83 \\
\hline $\begin{array}{c}0-1000 \\
\text { lower haml in }\end{array}$ & 67.70 & 109.17 & 0.32 & 177.19 & TUU. 81 & 38.05 & 0.00 & 139,64 & 168.51 & 147.82 & 0.32 & 316.65 \\
\hline$n=1 n n n$ & 7.02 & 16.25 & 3.31 & 26.58 & 27.70 & 37.22 & 26.68 & 91.60 & 34.72 & 53.47 & 29.99 & 118.18 \\
\hline
\end{tabular}




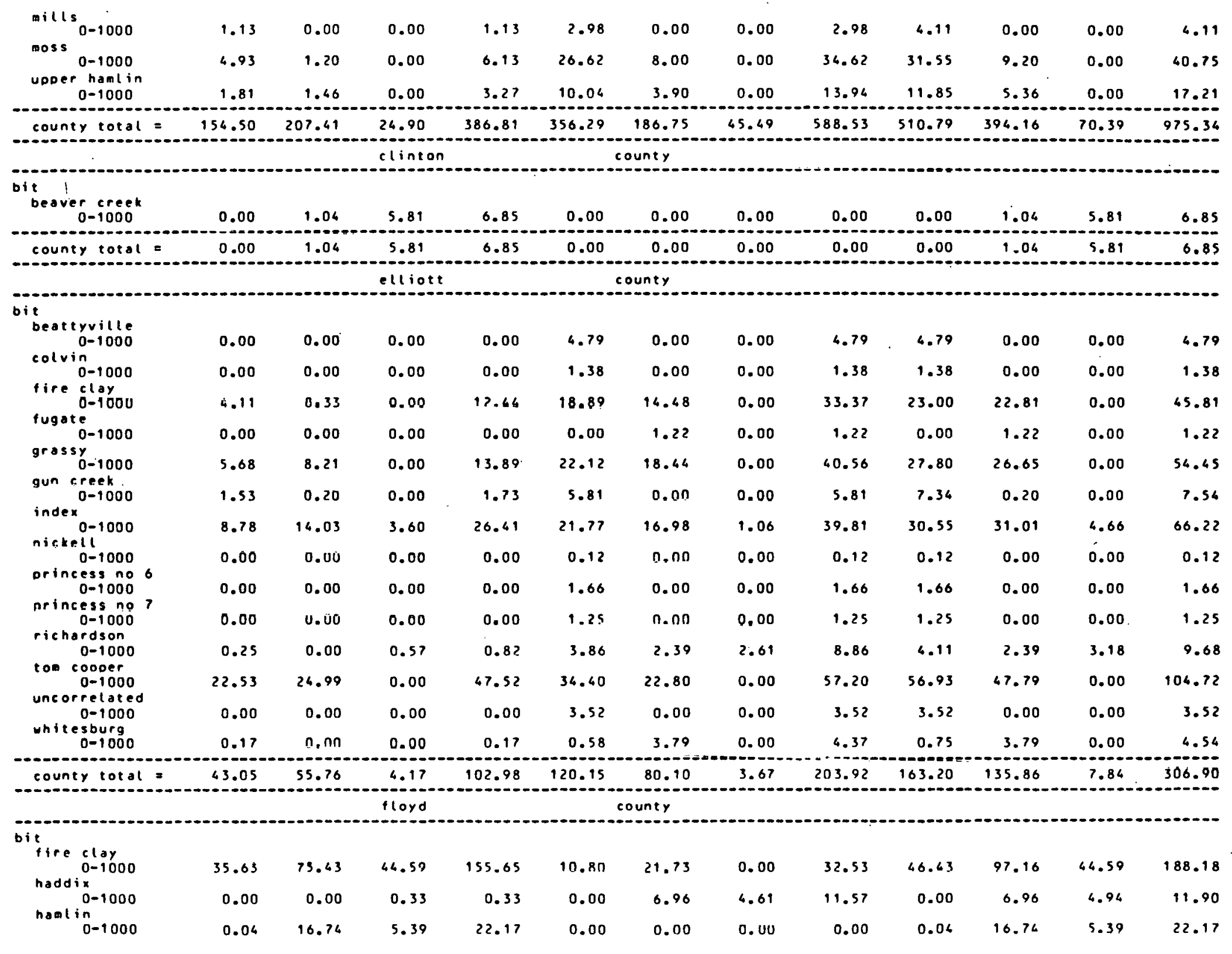




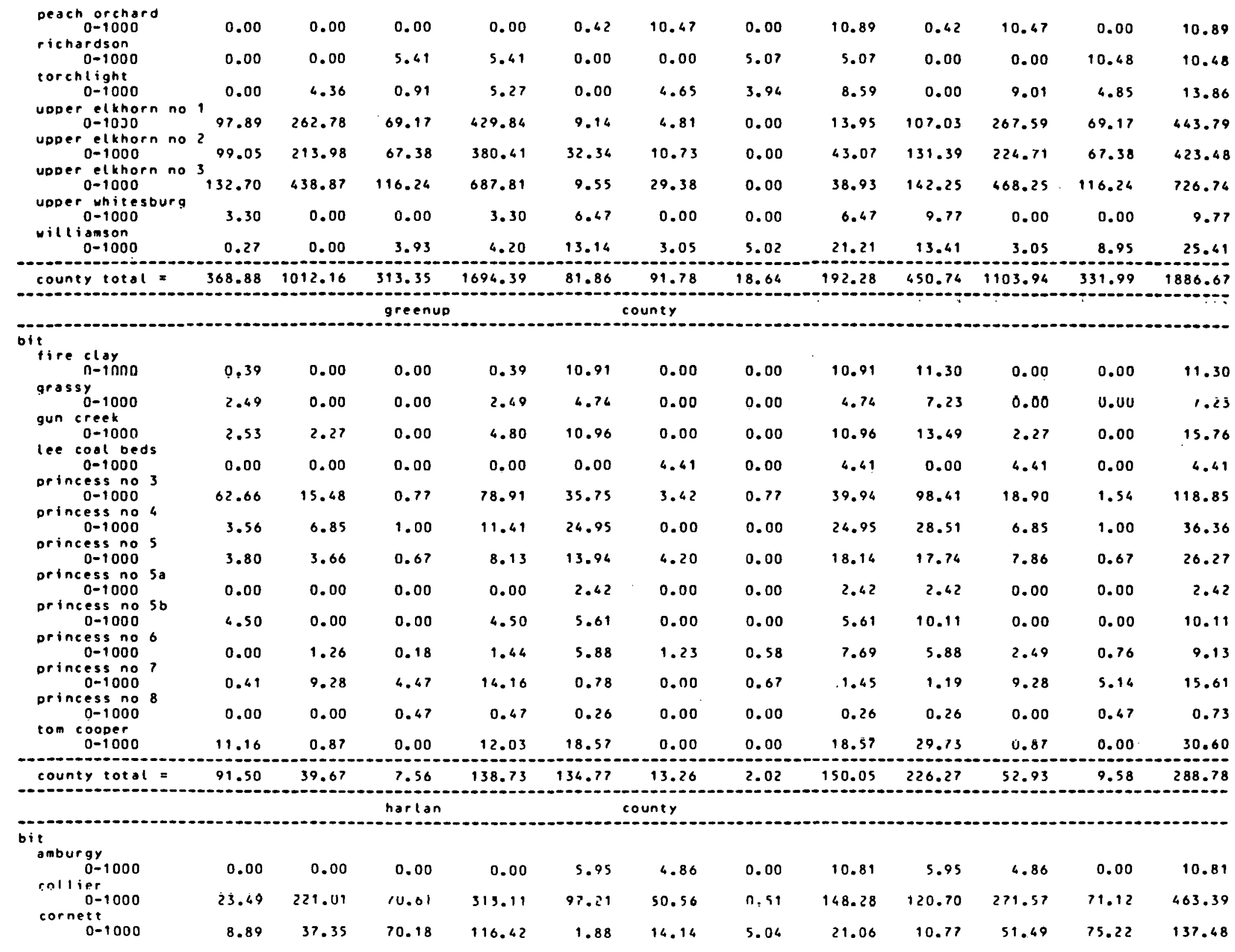




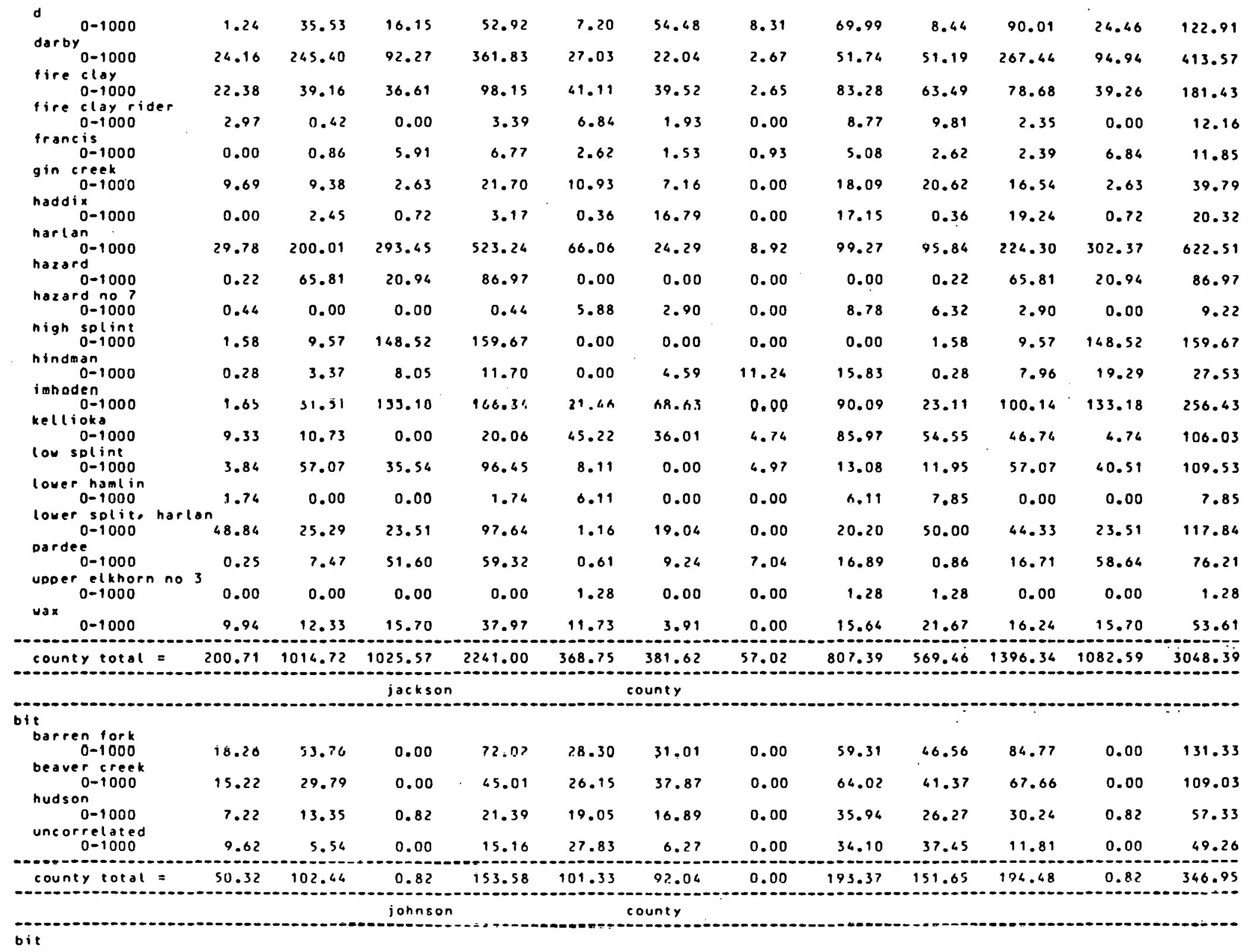




\begin{tabular}{|c|c|c|c|c|c|c|c|c|c|c|c|c|}
\hline 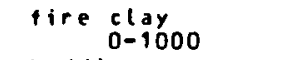 & 20.89 & 21.33 & 1.49 & 63.71 & 53.07 & 30.54 & 0.00 & 83.61 & 73.96 & 51.87 & 1.49 & 127.32 \\
\hline $\begin{array}{l}\text { haddix } \\
\qquad 0-1000\end{array}$ & 0.00 & 8.17 & 22.91 & 31.08 & 4.92 & 38.33 & 12.87 & 56.12 & 4.92 & 46.50 & 35.78 & 87.20 \\
\hline $\begin{array}{l}\text { lower whitesburg } \\
0-1000\end{array}$ & 15.34 & 4.04 & 0.00 & 19.38 & 32.27 & 1.27 & 0.00 & 33.54 & 67.61 & 5.31 & 0.00 & 52.92 \\
\hline mine $0-1000$ & 1.14 & 0.00 & 0.00 & 1.14 & 5.18 & 0.00 & 0.00 & 5.18 & 6.32 & 0.00 & 0.00 & 6.32 \\
\hline $\begin{array}{c}0-1000 \\
\text { richardson }\end{array}$ & 0.96 & 0.00 & 6.72 & 7.68 & 1.38 & 5.30 & 21.00 & 27.68 & 2.34 & 5.30 & 27.72 & 35.36 \\
\hline $0-1000$ & 0.00 & 0.00 & 2.46 & 2.46 & 0.00 & 0.00 & 7.62 & 7.62 & 0.00 & 0.00 & 10.08 & 10.08 \\
\hline $\begin{array}{l}0-1000 \\
\text { upoer elkhorn no }\end{array}$ & 0.00 & 0.94 & 12.35 & 13.29 & 0.00 & 5.60 & 3.12 & 8.52 & 0.00 & 6.34 & 95.47 & 21.81 \\
\hline $0-1000$ & 0.00 & 6.47 & 0.00 & 6.47 & 22.26 & 0.00 & 0.00 & 22.26 & 22.26 & 6.47 & 0.00 & 28.73 \\
\hline $\begin{array}{l}0-1000 \\
\text { upoer uhitesburg }\end{array}$ & 74.60 & 177.33 & 8.41 & 260.34 & 52.49 & 75.54 & 0.00 & 128.03 & 127.09 & 252.87 & 8.41 & 388.37 \\
\hline $0-1000$ & 20.15 & 0.00 & 0.00 & 20.15 & 32.77 & 0.00 & 0.00 & 32.77 & 52.92 & 0.00 & 0.00 & 52.92 \\
\hline $\begin{array}{r}r \text { anson } \\
0-1000\end{array}$ & 5.21 & 0.00 & 0.00 & 5.21 & 3.43 & 0.00 & 0.00 & 3.43 & 8.64 & 0.00 & 0.00 & 8.64 \\
\hline county total = & 138.29 & 218.28 & 54.34 & 410.91 & 207.77 & 156.38 & 44.61 & 408.76 & 346.06 & 374.66 & 98.95 & 819.67 \\
\hline 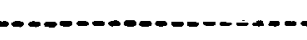 & $\cdots$ & & $=-$ & & & jom & & & & & & \\
\hline bit & & & & & & & & & & & & \\
\hline colvin & 83.61 & 57.53 & 0.00 & 141.14 & 27.55 & 49.51 & 0.00 & 77.06 & 111.16 & 107.04 & 0.00 & 218.20 \\
\hline $0-1000$ & 4.95 & 0.00 & 0.00 & 4.15 & 0.00 & 0.00 & 0.00 & 0.00 & 4.15 & 0.00 & 0.00 & 4.15 \\
\hline $0-1000$ & 211.12 & 222.18 & 98.05 & 531.35 & 40.46 & 0.50 & 0.00 & 40.96 & 251.58 & 222.68 & 98.05 & 572.31 \\
\hline francis & 1.75 & 0.00 & 0.00 & 1.75 & 1.56 & 0.00 & 0.00 & 1.56 & 3.31 & 0.00 & 0.00 & 3.31 \\
\hline hoddix $0-1000$ & 2.45 & 6.72 & 5.64 & 14.61 & 0.00 & 4.34 & 2.08 & 6.42 & 2.65 & 11.06 & 7.52 & 21.03 \\
\hline hazard $0-1000$ & 28.44 & 36.58 & 3.42 & 68.44 & 1.17 & 0.00 & 0,00 & 1.17 & 29.61 & 36.58 & 3.42 & 69.61 \\
\hline $\begin{array}{r}0-1000 \\
\text { hozard no } 7\end{array}$ & 15.26 & 29.73 & 12.22 & 57.21 & 8.69 & 8.99 & 0.00 & 17.68 & 23.95 & 38.72 & 12.22 & 74.89 \\
\hline $0-1000$ & 11.32 & 113.95 & 247.27 & 372.54 & 9.81 & 14.71 & 4.82 & 29.34 & 21.13 & 128.66 & 252.09 & 401.88 \\
\hline $\begin{array}{c}0-1000 \\
\text { skyline }\end{array}$ & 6.78 & 10.18 & 63.46 & 80.42 & 0.00 & 2.39 & 7.22 & 9.61 & 6.78 & 12.57 & 70.68 & 90.03 \\
\hline $\begin{array}{c}0-1000 \\
\text { upper elkhorn no }\end{array}$ & 0.00 & 0.21 & 0.52 & 0,73 & 0.00 & 0.07 & 0.04 & 0.11 & 0.00 & 0.28 & 0.56 & 0.84 \\
\hline $\begin{array}{c}0-1000 \\
\text { upder elkhorn no }\end{array}$ & $2^{80.33}$ & 42.91 & 18.67 & 149.91 & 16.61 & 0.00 & 0.00 & 16.61 & 96.94 & 42.91 & 18.67 & 158.52 \\
\hline $\begin{array}{c}0-1000 \\
\text { upper elkhorn no }\end{array}$ & $3^{123.09}$ & 91.04 & 2.37 & 216.50 & 14.60 & 3.29 & 0.00 & 17.89 & 137.69 & 94.33 & 2.37 & 234.39 \\
\hline $\begin{array}{c}0-1000 \\
\text { upper whitestourg }\end{array}$ & 41.43 & 334.88 & 115.93 & 492.24 & 48.65 & 22.26 & 0.00 & 70.91 & 90.08 & 357.14 & 195.93 & 563.15 \\
\hline $0-1000$ & 59.95 & 28.80 & 0.00 & 88.75 & 16.03 & 5.06 & 0.00 & 19.09 & 73.98 & 33.86 & 0.00 & 107.84 \\
\hline county total $=$ & 669.68 & 974.71 & 567.35 & 2211.74 & 183.13 & 111.12 & 14.16 & 308.41 & 852.81 & 1085.83 & 581.51 & 2520.15 \\
\hline
\end{tabular}




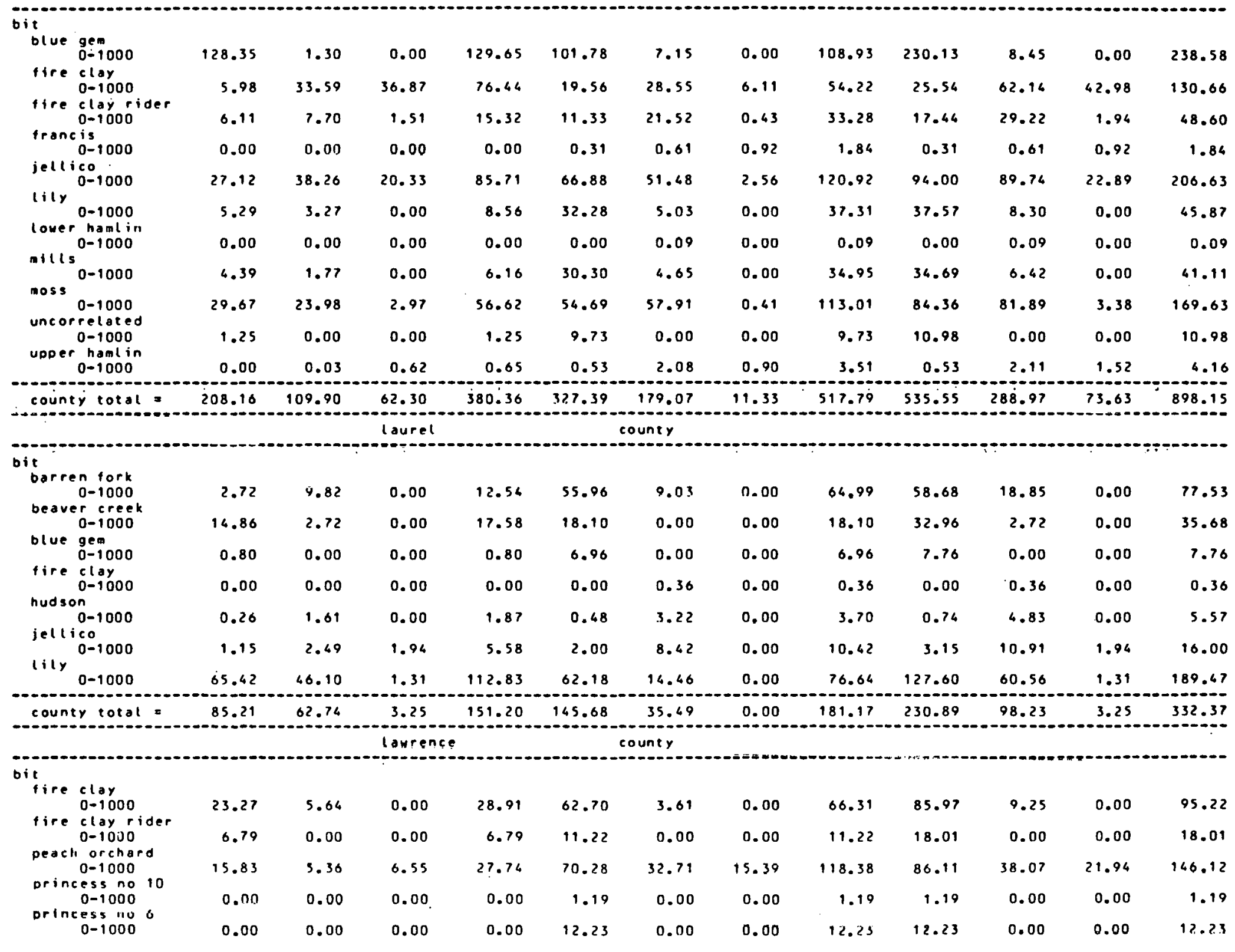




\begin{tabular}{|c|c|c|c|c|c|c|c|c|c|c|c|c|}
\hline $\begin{array}{c}\text { princess no } \\
0-1000\end{array}$ & 2.34 & 3.80 & 4.61 & 10.75 & 9.32 & 4.72 & 6.44 & 20.48 & 19.66 & 8.52 & 11.05 & 31.23 \\
\hline $\begin{array}{c}\text { princess no } 8 \\
0-1000\end{array}$ & 7.77 & 17.69 & 1.38 & 26.84 & 48.91 & 0.00 & 0.00 & 48.91 & 56.68 & 17.69 & 1.38 & 75.75 \\
\hline $\begin{array}{r}\text { richardson } \\
0-1000\end{array}$ & 3.93 & 4.98 & 30.15 & 39.06 & 29.83 & 53.57 & 0.44 & 83.84 & 33.70 & 58.55 & 30.59 & 122.90 \\
\hline $\begin{array}{r}0-1000 \\
\text { topchlight }\end{array}$ & 0.00 & 0.00 & 0.00 & 0.00 & 10.68 & 2.02 & 0.00 & 12.70 & 10.68 & 2.02 & 0.00 & 12.70 \\
\hline $0-1000$ & 4.49 & 12.67 & 6.03 & 23.19 & 23.62 & 5.70 & 0.00 & 29.32 & 28.11 & 18.37 & 6.03 & 52.51 \\
\hline $\begin{array}{r}\text { Whitesburg } \\
0-1000\end{array}$ & 4.60 & 0.00 & 0.00 & 4.60 & 9.01 & 0.00 & 0.00 & 9.01 & 13.61 & 0.00 & 0.00 & 13.61 \\
\hline county total = & 69.02 & 50.14 & 48.72 & 167.88 & 288.99 & 102.33 & 22.27 & 413.59 & 358.01 & 152.47 & 70.99 & 581.47 \\
\hline & & & lee & & & unty & & & & & & \\
\hline \multicolumn{13}{|l|}{ bit } \\
\hline $\begin{array}{c}0-1000 \\
0\end{array}$ & 3.33 & 0.00 & 0.00 & 3.33 & 11.65 & 0.00 & 0.00 & 11.65 & 14.78 & 0.00 & 0.00 & 14.78 \\
\hline $0-1000$ & 22.07 & 23.46 & 7.55 & 53.08 & 49.46 & 32.20 & 10.41 & 84.05 & 63.51 & 55.66 & 17.96 & 137.13 \\
\hline $0-1000$ & 0.00 & 0.00 & 0.00 & 0.00 & 0.08 & 1.49 & 0.00 & 1.57 & 0.08 & 1.49 & 0.00 & 1.57 \\
\hline $0-1000$ & 2.92 & 2.48 & 0.00 & 5.60 & 4.36 & 0.88 & 0.00 & 5.22 & 7.26 & 3.36 & 0.00 & 10.62 \\
\hline $0-1000$ & 10.68 & 7.56 & 0.50 & 18.72 & 11.66 & 6.09 & 0.00 & 17.65 & 22.32 & 13.55 & 0.50 & 36.37 \\
\hline county total $=$ & 39.00 & 33.48 & 8.05 & 80.53 & 68.95 & 40.58 & 10.41 & 119.94 & 107.95 & 74.06 & 18.46 & 200.47 \\
\hline & & & & & & & & & & & & \\
\hline \multicolumn{13}{|l|}{ bit } \\
\hline $\begin{array}{l}\text { anourgy } \\
\text { o- } 1000 \\
\text { fire clay }\end{array}$ & 27.97 & 39.62 & 0.00 & 67.59 & 59.48 & 65.55 & 0.00 & 125.03 & 87.45 & 105.17 & 0.00 & 192.62 \\
\hline $\begin{array}{r}\text { fire clay } \\
0-1000\end{array}$ & 164.23 & 239.05 & 101.70 & 504.98 & 130.23 & 78.14 & 23.46 & 231.83 & 294.46 & 317.19 & 125.16 & 736.81 \\
\hline $\begin{array}{l}\text { fire clay rider } \\
0-1000\end{array}$ & 152.93 & 115,74 & 28.36 & 297.03 & 119.64 & 11.50 & 4.33 & 135.47 & 272.57 & 127.24 & 32.69 & 432.50 \\
\hline $\begin{array}{c}\text { Francis } \\
0-1000\end{array}$ & 5.59 & 11.96 & 15.71 & 33.26 & 14.08 & 17.63 & 12.52 & 44.03 & 19.67 & 29.39 & 28.23 & 77.29 \\
\hline $0-1000$ & 1.51 & 5.19 & 1.08 & 7.78 & 19.25 & 6.07 & 0.00 & 25.32 & 20.76 & 11.26 & 1.08 & 33.10 \\
\hline $0-1000$ & 65.05 & 165.69 & 69.16 & 299.90 & 32.72 & 26.62 & 6.12 & 65.46 & 97.77 & 192.31 & 75.28 & 365.36 \\
\hline $0-1000$ & 39.87 & 45.51 & 17.53 & 102.91 & 18.93 & 18.65 & 0.35 & 37.93 & 58.80 & 64.16 & 17.88 & 140.84 \\
\hline $0-1000$ & 1.60 & 3.00 & 16.82 & 29.42 & 19.74 & 16.33 & 48.00 & 84.07 & 21.34 & 19.33 & 64.82 & 105.49 \\
\hline $0-1000$ & 0.00 & 0.00 & 0.00 & 0.00 & 27.82 & 0.00 & 0.00 & 27.82 & 27.82 & 0.00 & 0.00 & 27.82 \\
\hline $\begin{array}{c}0-1000 \\
\text { upoer elkhorn no }\end{array}$ & $3^{31.55}$ & 3.31 & 1.17 & 36.03 & 2.52 & 0.00 & 0.00 & 2.52 & 34.07 & 3.31 & 1.17 & 38.55 \\
\hline $0-1000$ & 2.14 & 4.80 & 0.00 & 6.911 & 22.41 & 25.00 & n. no & 47.41 & 24.55 & 29.80 & 0.00 & 54.35 \\
\hline $0-1000$ & 3.03 & 0.50 & 0.00 & 3.53 & 18.82 & 5.30 & 0.20 & 24.32 & 21.85 & 5.80 & 0.20 & 27.85 \\
\hline $0-1000$ & 9.53 & 0.00 & 0.00 & 1.53 & 9.37 & 0.00 & 0.00 & 9.37 & 10,90 & 0.00 & 0.00 & 10.90 \\
\hline
\end{tabular}




\begin{tabular}{|c|c|c|c|c|c|c|c|c|c|c|c|c|}
\hline county total $=$ & 497.00 & 634.37 & 251.53 & 1382.90 & 495.01 & 270.59 & 94.98 & 860.58 & 992.01 & 904.96 & 346.51 & 2243.48 \\
\hline & & & letch & & & nty & & & & & & \\
\hline \multicolumn{13}{|l|}{ it } \\
\hline $\begin{array}{l}\text { amburgy } \\
\qquad 0-1000\end{array}$ & 58.22 & 65.80 & 25.49 & 149.51 & 107.90 & 34.29 & 1.77 & 143.96 & 166.12 & 100.09 & 27.26 & 293.67 \\
\hline collier & 0.00 & 0.00 & 0.00 & 0.00 & 6.25 & 0.00 & 0.00 & 6.25 & 6.25 & 0.00 & 0.00 & 6.25 \\
\hline $\begin{array}{l}\text { cornett } \\
0-1000\end{array}$ & 0.00 & 0.00 & 0.64 & 0.64 & 0.00 & 2.40 & 0.67 & 3.07 & 0.00 & 2.40 & 1.31 & 3.71 \\
\hline darby $0-1000$ & 0.00 & 0.00 & 0.00 & 0.00 & 1.22 & 0.37 & 0.00 & 1.59 & 1.22 & 0.37 & 0.00 & 1.59 \\
\hline $\begin{array}{c}\text { elkhorn leader } \\
0-1000\end{array}$ & 55.17 & 16.29 & 1.30 & 12.76 & 32.06 & 0.00 & 0.00 & 32.06 & 87.23 & 16.29 & 1.30 & 106.82 \\
\hline $\begin{array}{l}\text { tire elay } \\
0-1000\end{array}$ & 21.95 & 78.57 & 180.32 & 280.84 & 8.29 & 54.75 & 1.35 & 64.39 & 30.24 & 133.32 & 181.67 & 345.23 \\
\hline $\begin{array}{c}\text { Francis } \\
0-1000\end{array}$ & 0.00 & 0.00 & 0.00 & 0.00 & 0.00 & 1.56 & 0.00 & 1.56 & 0.00 & 1.56 & 0.00 & 1.56 \\
\hline haddix $0-1000$ & 0.00 & 2.53 & 18.18 & 20.71 & 0.00 & 4.62 & 0.00 & 4.62 & 0.00 & 7.15 & 18.18 & 25.33 \\
\hline $\begin{array}{l}\text { harlan } \\
\text { hasard } 0-1000\end{array}$ & 3.02 & 8.83 & 0.00 & 11.85 & 3.41 & 0.00 & 0.00 & 3.41 & 6.43 & 8.83 & 0.00 & 15.26 \\
\hline n-1 nno & 1.76 & 11.06 & 59.27 & 64.09 & 0.04 & 5.18 & 1.49 & 6.71 & 1.80 & 16.24 & 52.76 & 70.80 \\
\hline $0-1000$ & 0.71 & 2.71 & 12.40 & 15.88 & 2.16 & 2.44 & 2.57 & 7.17 & 3.87 & 5.15 & 15.03 & 23.05 \\
\hline $\begin{array}{l}\text { high splint } \\
0-1000 \\
\text { ingon }\end{array}$ & 0.00 & 1.60 & 3.03 & 4.63 & 1.18 & 0.00 & 0.00 & 1.18 & 1.18 & 1.60 & 3.03 & 5.81 \\
\hline $\begin{array}{l}\text { Imogoen } \\
0-1000\end{array}$ & 0.96 & 11.39 & 97.2 .7 & 109.62 & 14.52 & 0.00 & 0.00 & 14.52 & 15.48 & 11.39 & 97.27 & 124.14 \\
\hline $\begin{array}{c}\text { kellioka } \\
0-1000\end{array}$ & 0.00 & 8.11 & 0.00 & 8.11 & 17.60 & 5.87 & 0.00 & 23.47 & 17.60 & 13.98 & 0.00 & 31.58 \\
\hline $\begin{array}{c}\text { Low splint } \\
0-1000\end{array}$ & 0.00 & 0.00 & 0.00 & 0.00 & 0.73 & 8.99 & 11.17 & 20.89 & 0.73 & 8.99 & 11.17 & 20.89 \\
\hline $\begin{array}{c}\text { lower elkhorn } \\
0-1000\end{array}$ & 5.58 & 8.37 & 0.00 & 13.95 & 16.39 & 0.00 & 0.00 & 16.39 & 21.97 & 8.37 & 0.00 & 30.36 \\
\hline $\begin{array}{c}\text { upper elkhorn no } \\
0-1000 \\
\text { upper elkhorn no } 2\end{array}$ & 51.62 & 0.65 & 0.45 & 52.72 & 15.17 & 0.00 & 0.00 & 15.17 & 66.79 & 0.65 & 0.45 & 67.89 \\
\hline $0-1000$ & 55.03 & 2.75 & 0.00 & 57.78 & 35.41 & 9.09 & 0.00 & 44.50 & 90.44 & 11.84 & 0.00 & 102.28 \\
\hline $\begin{array}{c}\text { upder elkhorn no } 3 \\
0-1000\end{array}$ & 35.29 & 111.66 & 289.11 & 436.06 & 25.36 & 8.09 & 24.59 & 58.04 & 60.65 & 119.75 & 313.70 & 494.10 \\
\hline $\begin{array}{l}\text { UDDer hamlin } \\
0-1000\end{array}$ & 0.59 & 1.18 & 25.95 & 27.72 & 3.06 & 6.78 & 0.00 & 9.84 & 3.65 & 7.96 & 25.95 & 37.56 \\
\hline $0-1000$ & 25.16 & 45.70 & 14.85 & 85.71 & 55.79 & 3.77 & 0.00 & 59.56 & 80.95 & 49.47 & 14.85 & 145.27 \\
\hline \multirow{2}{*}{ cou } & 315.06 & 377.20 & 720.32 & 1412.58 & 346.54 & 148.20 & 43.61 & 538.35 & 661.60 & 525.40 & 763.93 & 1950.93 \\
\hline & & & mag of & & & ty & & & & & & \\
\hline \multicolumn{13}{|l|}{ bit } \\
\hline $\begin{array}{l}\ln \\
0=1000\end{array}$ & 10.12 & 1.38 & 0.00 & 11.50 & 0.00 & 0.00 & 0.00 & 0.00 & 10.12 & 1.38 & 0.00 & 11.50 \\
\hline $\begin{aligned} \text { fire clay } \\
0-1000\end{aligned}$ & 110.70 & 35.65 & 0.00 & 146.35 & 23.83 & 2.25 & 0.00 & 26.08 & 134.53 & 37.90 & 0.00 & 172.43 \\
\hline $0-1000$ & 0.93 & 0.00 & 0.00 & 0.93 & 2.11 & 0.00 & 0.00 & 2.11 & 3.04 & 0.00 & 0.00 & 3.04 \\
\hline
\end{tabular}




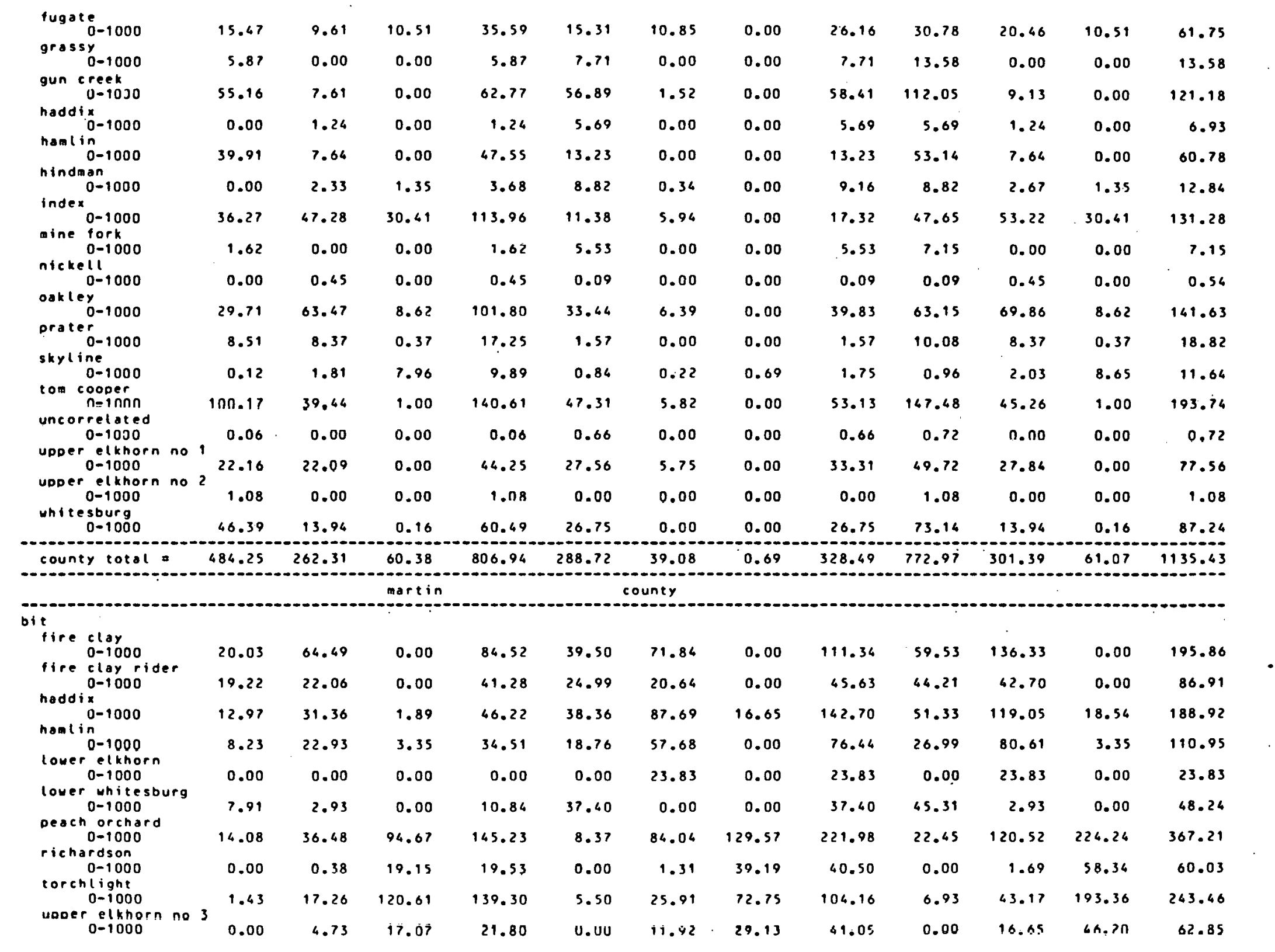




\begin{tabular}{|c|c|c|c|c|c|c|c|c|c|c|c|c|}
\hline $\begin{array}{c}\text { upder whitesburg } \\
0-1000 \\
\text { williamson } \\
0-1000\end{array}$ & $\begin{array}{l}0.00 \\
0.00\end{array}$ & $\begin{array}{l}0.00 \\
1.69\end{array}$ & $\begin{array}{l}0.00 \\
0.00\end{array}$ & $\begin{array}{l}0.00 \\
1.41\end{array}$ & $\begin{array}{l}7.60 \\
5.84\end{array}$ & $\begin{array}{r}0.00 \\
25.36\end{array}$ & $\begin{array}{l}0.00 \\
0.00\end{array}$ & $\begin{array}{r}7.60 \\
31.20\end{array}$ & $\begin{array}{l}7.60 \\
5.84\end{array}$ & $\begin{array}{r}0.00 \\
26.77\end{array}$ & $\begin{array}{l}0.00 \\
0.00\end{array}$ & $\begin{array}{r}7.60 \\
32.61\end{array}$ \\
\hline county total $=$ & 83.87 & 204.03 & 256.74 & 544.64 & 186.32 & 410.22 & 287.29 & 883.83 & 270.19 & 614.25 & 544.03 & 1428.47 \\
\hline & & & mccrea & & & unty & & & & & & \\
\hline \multicolumn{13}{|l|}{ it } \\
\hline $\begin{array}{r}\text { bacon creek } \\
0-1000 \\
\text { barren fork }\end{array}$ & 1.87 & 0.00 & 0.00 & 1.87 & 0.00 & 0.00 & 0.00 & 0.00 & 1.87 & 0.00 & 0.00 & 1.87 \\
\hline $\begin{array}{c}0-1000 \\
\text { beaver creek }\end{array}$ & 30.77 & 26.21 & 0.00 & 56.98 & 37.50 & 59.74 & 0.00 & 97.24 & 68.27 & 85.95 & 0.00 & 156.22 \\
\hline blue gem $0-1000$ & 28.14 & 73.50 & 68.56 & 170.18 & 6.61 & 0.92 & 14.60 & 22.13 & 34.75 & 74.42 & 83.14 & 192.31 \\
\hline huds on & 2.72 & 0.00 & 0.00 & 2.72 & 5.30 & 0.00 & 0.00 & 5.30 & 8.02 & 0.00 & 0.00 & 8.02 \\
\hline jeltico & 20.20 & 39.31 & 34.14 & 93.65 & 0.00 & 0.00 & 0.00 & 0.00 & 20.20 & 39.31 & 34.14 & 93.65 \\
\hline $0-1000$ & 1.46 & 0.32 & 0.00 & 1.78 & 6.54 & 1.28 & 0.00 & 7.82 & 8.00 & 1.60 & 0.00 & 9.60 \\
\hline $\begin{array}{c}0-1000 \\
\text { stearns.no } 11 / 2\end{array}$ & 25.59 & 2.28 & 0.00 & 27.87 & 33.33 & 1.84 & 0.00 & 35.17 & 58.92 & 4.12 & 0.00 & 63.04 \\
\hline $\begin{array}{c}0-1000 \\
\text { uncorrelated }\end{array}$ & 7.62 & 1.32 & $\dot{y} .12$ & 20.20 & $1: 03$ & 0.00 & 0.00 & 1.83 & 9.69 & $7.5 ?$ & 9.12 & 36.09 \\
\hline $0-1030$ & 3.34 & 0.00 & 0.00 & 3.34 & 14.11 & 0.00 & 0.00 & $\begin{array}{l}14.11 \\
.0 .-\ldots\end{array}$ & 17.45 & 0.00 & 0.00 & 17.45 \\
\hline county total = & 121.71 & 149.14 & 111.80 & 382.65 & 105.22 & 63.78 & 14.60 & 183.60 & 226.93 & 212.92 & 126.40 & 566.25 \\
\hline \multicolumn{13}{|l|}{ bit } \\
\hline $\begin{array}{r}\text { beattyville } \\
0-1000\end{array}$ & 18.46 & 4.80 & 0.00 & 23.26 & 7.66 & 0.00 & 0.00 & 7.66 & 26.12 & 4.80 & 0.00 & 30.92 \\
\hline county total & 18.46 & 4.80 & 0.00 & 23.26 & 1.66 & 0.00 & 0.00 & 7.66 & 26.12 & 4.80 & 0.00 & 30.92 \\
\hline \multirow{2}{*}{\multicolumn{13}{|c|}{$\begin{array}{l}\text { bit } \\
\text { adele }\end{array}$}} \\
\hline & & & & & & & & & & & & \\
\hline $\begin{array}{l}0-1000 \\
\text { fire clay }\end{array}$ & 0.58 & 0.00 & 0.00 & 0.58 & 0.00 & 0.00 & 0.00 & 0.00 & 0.58 & 0.00 & 0.00 & 0.58 \\
\hline fugate & 19.62 & 5.96 & 1.56 & 27.14 & 6.58 & 1.55 & 0.00 & 8.13 & 26.20 & 7.51 & 1.56 & 35.27 \\
\hline grassy & 0.34 & 0.92 & 0.00 & 7.26 & 0.00 & $n, n n$ & 0.00 & 0.00 & 6.34 & 0.92 & 0.00 & 7.26 \\
\hline $\begin{array}{l}0-1000 \\
\text { gun creek }\end{array}$ & 58.77 & 0.00 & 0.00 & 58.77 & 15.70 & 0.00 & 0.00 & 15.70 & 74.47 & 0.00 & 0.00 & 74.47 \\
\hline haddix $0-1000$ & 17.98 & 20.75 & 0.00 & 38.73 & 13.43 & 0.36 & 0.00 & 13.79 & 31.41 & 21.11 & 0.00 & 52.52 \\
\hline haml in & 0.00 & 0.30 & 0.00 & 0.30 & 0.07 & 0.00 & 0.00 & 0.07 & 0.07 & 0.30 & 0.00 & 0.37 \\
\hline index $^{0-1000}$ & 1.59 & 0.00 & .00 & 1.59 & 10.44 & 0.00 & 0.00 & 10.46 & 12.03 & 0.00 & 0.00 & 12.03 \\
\hline mine fork & 19.52 & 3.91 & 0.00 & 23.43 & 51.30 & 37.58 & 0.00 & 88.88 & 70.82 & 41.49 & 0.00 & 112.31 \\
\hline $0-1000$ & 1.93 & 0.00 & 0.00 & 1.93 & 3.44 & 0.00 & 0.00 & 3.64 & 5.37 & 0.00 & 0.00 & 5.37 \\
\hline
\end{tabular}




\begin{tabular}{|c|c|c|c|c|c|c|c|c|c|c|c|c|}
\hline $\begin{array}{l}\text { nickell } \\
0-1000 \\
\text { oakley }\end{array}$ & 1.18 & 0.00 & 0.00 & 1.18 & 0.69 & 0.00 & 0.00 & 0.69 & 1.87 & 0.00 & 0.00 & 1.87 \\
\hline $\begin{array}{r}0-1000 \\
\text { richardson }\end{array}$ & 8.43 & 0.00 & 0.00 & 8.43 & 2.46 & 4.75 & 0.00 & 7.29 & 10.89 & 4.75 & 0.00 & 15.64 \\
\hline $0-1000$ & 0.00 & 0.00 & 4.93 & 4.93 & 0.00 & 0.00 & 3.99 & 3.99 & 0.00 & 0.00 & 8.92 & 8.92 \\
\hline $\begin{array}{c}\text { tom cooper } \\
0-1000 \\
\text { unc orrelated }\end{array}$ & 62.57 & 17.31 & 0.00 & 79.88 & 39.67 & 39.61 & 0.00 & 79.28 & 102.24 & 56.92 & 0.00 & 159.16 \\
\hline $0-1000$ & 0.00 & 0.00 & 0.00 & 0.00 & 0.42 & 0.00 & 0.00 & 0.42 & 0.42 & 0.00 & 0.00 & 0.42 \\
\hline county total = & 198.51 & 49.15 & 6.49 & 254.15 & 144.20 & 83.85 & 3.99 & 232.04 & 342.71 & 133.00 & 10.48 & 486.19 \\
\hline & & & ousley & & & ounty & & & & & & \\
\hline \multicolumn{13}{|c|}{ bit } \\
\hline $\begin{array}{r}0-1000 \\
\text { beaver creek }\end{array}$ & 2.68 & 0.00 & 0.00 & 2.68 & 2.23 & 0.00 & 0.00 & 2.23 & 4.91 & 0.00 & 0.00 & 4.91 \\
\hline jellico & 0.00 & 0.00 & 0.00 & 0.00 & 5.13 & 3.37 & 0.00 & 8.50 & 5.13 & 3.37 & 0.00 & 8.50 \\
\hline$x^{0-1000}$ & 0.00 & 0.00 & 0.00 & 0.00 & 0.00 & 1.43 & 0.00 & 1.43 & 0.00 & 1.43 & 0.00 & 1.43 \\
\hline $\begin{array}{c}0-1000 \\
\text { uncorrelated }\end{array}$ & 0.64 & 2.12 & 0.66 & 3.42 & 2.16 & 3.71 & 0.00 & 5.87 & 2.80 & 5.83 & 0.66 & 0.29 \\
\hline $0-1000$ & 0.00 & 0.00 & 0.00 & 0.00 & 0.19 & 0.44 & 0.00 & 0.63 & 0.19 & 0.44 & 0.00 & 0.63 \\
\hline county total= & 3.32 & 2.12 & 0.66 & 6.10 & 9.71 & 8.95 & 0.00 & 18.66 & 13.03 & 11.07 & 0.66 & 24.76 \\
\hline & & & Derry & & & ounty & & & & & & \\
\hline \multicolumn{13}{|l|}{ it } \\
\hline $0-1000$ & 46.24 & 64.81 & 0.00 & 111.05 & 50.85 & 199.23 & 0.00 & 250.08 & 97.09 & 264.04 & 0.00 & 361.13 \\
\hline $\begin{array}{l}\text { fire clay } \\
0-1000 \\
\text { fire clay rider }\end{array}$ & 167.81 & 234.19 & 118.38 & 520.38 & 30.98 & 10.22 & 23.86 & 65.06 & 198.79 & 244.49 & 162.24 & 585.44 \\
\hline $\begin{array}{l}0-1000 \\
\text { francis }\end{array}$ & 65.17 & 27.78 & 0.59 & 93.54 & 1.73 & 0.00 & 0.00 & 1.73 & 66.90 & 27.78 & 0.59 & 95.27 \\
\hline $\begin{array}{l}0-1000 \\
\text { haddix }\end{array}$ & 15.55 & 30.95 & 28.96 & 75.46 & 3.16 & 8.31 & 8.29 & 19.76 & 18.71 & 39.26 & 37.25 & 95.22 \\
\hline hazard $0-1000$ & 38.58 & 26.78 & 4.62 & 69.78 & 3.75 & 0.00 & 0.00 & 3.75 & 62.33 & 26.78 & 4.42 & 73.53 \\
\hline $\begin{array}{r}0-1000 \\
\text { hazard no? }\end{array}$ & 79.86 & 229.26 & 171.40 & 480.52 & 9.19 & 5.30 & 1.89 & 16.38 & 89.05 & 234.56 & 173.29 & 496.90 \\
\hline $\begin{array}{l}0-1000 \\
\text { hindman }\end{array}$ & 26.28 & 64.51 & 421.67 & 510.46 & 12.03 & 19.72 & 14.31 & 46.06 & 36.31 & 84.23 & 435.98 & 556.52 \\
\hline $\begin{array}{c}0-1000 \\
\text { upper elkhorn no }\end{array}$ & 1.55 & $? 2.69$ & 67.82 & 92.06 & 0.00 & 3.52 & 14.30 & 17.82 & 1.55 & 26.21 & 82.12 & 109.88 \\
\hline $0-1000$ & 8.62 & 21.41 & 0.00 & 30.03 & 30.64 & 20.84 & 0.00 & 59.48 & 39.26 & 42.25 & 0.00 & B1. 51 \\
\hline county total = & 447.66 & 722.38 & 813.24 & 1983.28 & 142.33 & 267.14 & 62.65 & 472.12 & 589.99 & 989.52 & 875.89 & 2455.40 \\
\hline & & & Dike & & & ney & & & & & & \\
\hline \multicolumn{13}{|l|}{ bit } \\
\hline $\begin{array}{l}0-1000 \\
\text { bingham }\end{array}$ & & 37.48 & & 118.56 & 79.07 & $3.56^{\circ}$ & 2.77 & 85.40 & 153.50 & 04 & 9.42 & 203.96 \\
\hline $0-1000$ & 74.64 & 96.16 & 70.64 & 241.24 & 137.49 & 21. & 0.00 & 158.75 & 211.93 & 117.42 & 70.64 & 399.99 \\
\hline
\end{tabular}




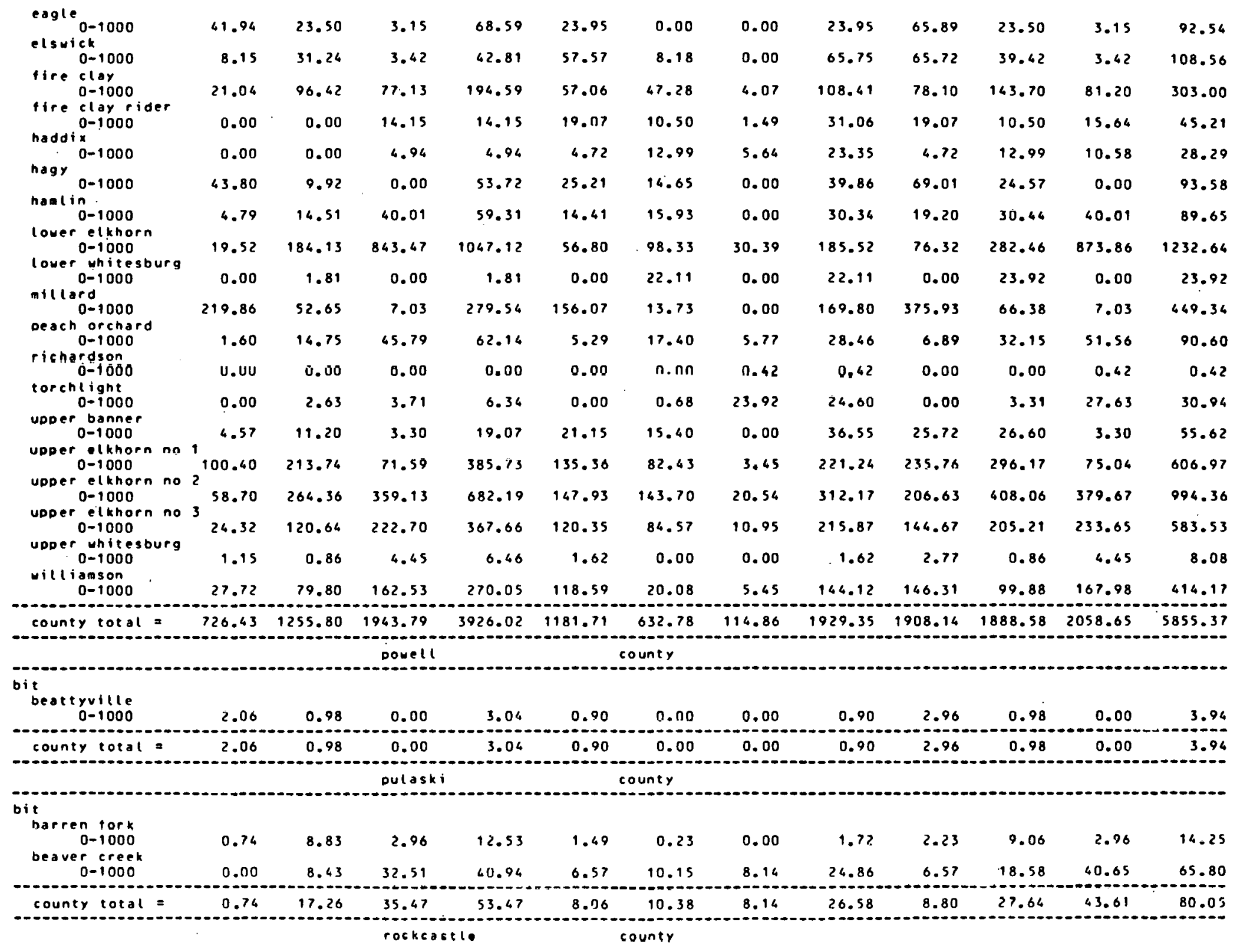




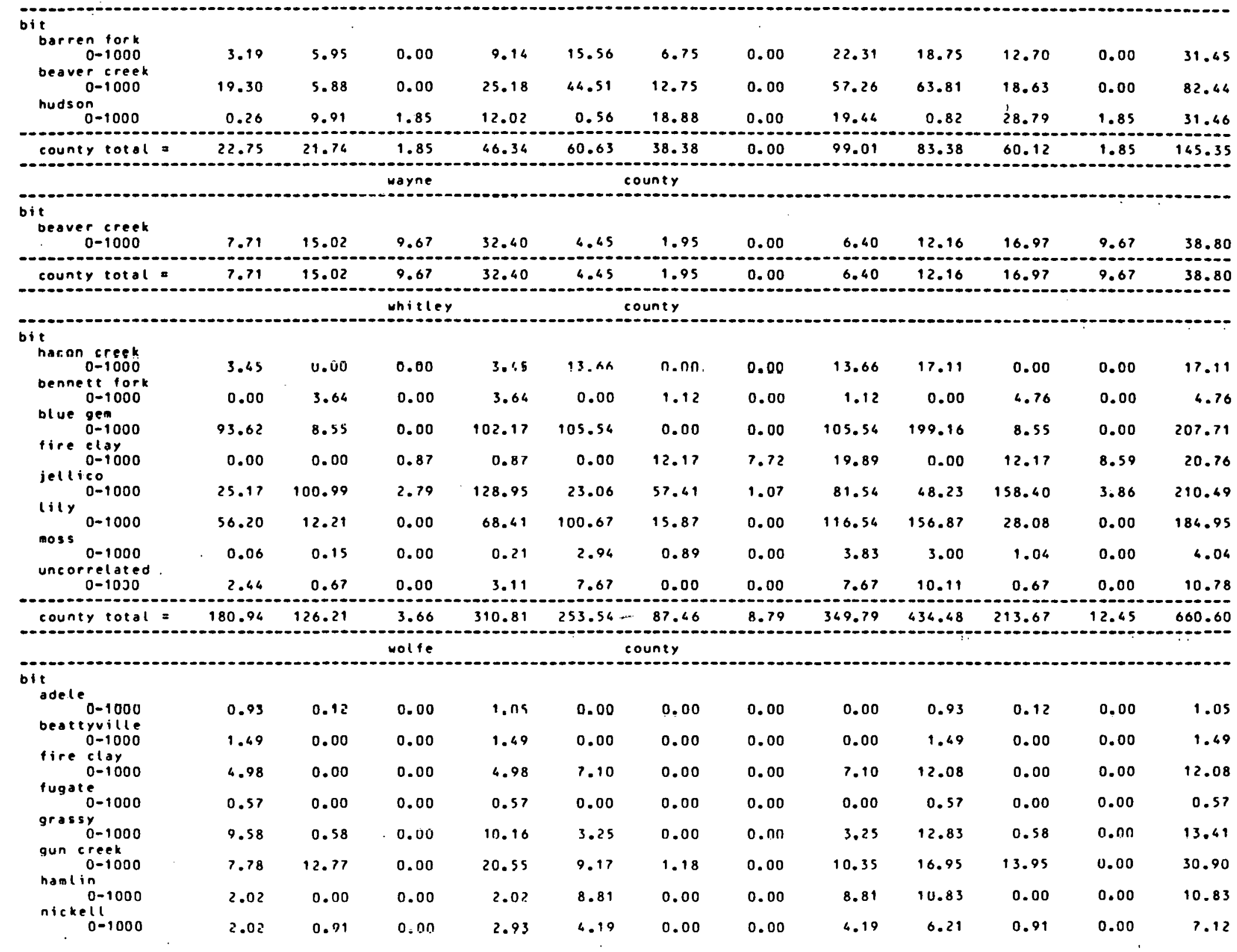




\begin{tabular}{|c|c|c|c|c|c|c|c|c|c|c|c|c|}
\hline $\begin{array}{c}\text { tom cooper } \\
0-10 j 0 \\
\text { uncorrelated } \\
0-1000\end{array}$ & $\begin{array}{l}15.21 \\
14.23\end{array}$ & $\begin{array}{l}5.73 \\
0.00\end{array}$ & $\begin{array}{l}0.00 \\
0.00\end{array}$ & $\begin{array}{l}20.94 \\
14.23\end{array}$ & $\begin{array}{l}14.56 \\
20.21\end{array}$ & $\begin{array}{l}7.97 \\
0.00\end{array}$ & $\begin{array}{l}0.00 \\
0.00\end{array}$ & $\begin{array}{l}22.53 \\
20.21\end{array}$ & $\begin{array}{l}29.77 \\
34.64\end{array}$ & $\begin{array}{r}13.70 \\
0.00\end{array}$ & $\begin{array}{l}0.00 \\
0.00\end{array}$ & $\begin{array}{l}43.47 \\
34.44\end{array}$ \\
\hline $\begin{array}{c}\text { zochariah } \\
0-1000 \\
\text { zachariah rider } \\
0-1000\end{array}$ & $\begin{array}{l}9.07 \\
1.06\end{array}$ & $\begin{array}{r}13.59 \\
0.00\end{array}$ & $\begin{array}{l}0.99 \\
0.00\end{array}$ & $\begin{array}{r}23.65 \\
1.06\end{array}$ & $\begin{array}{r}26.48 \\
0.00\end{array}$ & $\begin{array}{l}3.41 \\
0.00\end{array}$ & $\begin{array}{l}0.00 \\
0.00\end{array}$ & $\begin{array}{r}29.89 \\
0.00\end{array}$ & $\begin{array}{r}35.55 \\
1.06\end{array}$ & $\begin{array}{r}17.00 \\
0.00\end{array}$ & $\begin{array}{l}0.99 \\
0.00\end{array}$ & $\begin{array}{r}53.56 \\
1.06\end{array}$ \\
\hline county total & 68.94 & 33.70 & 0.99 & 103.63 & 93.77 & 12.56 & 0.00 & 106.33 & 162.71 & 66.26 & 0.98 & 209.96 \\
\hline
\end{tabular}

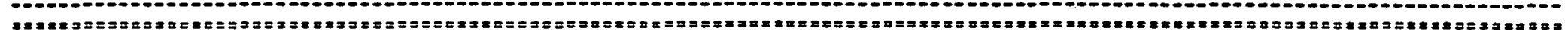

overburden
subtotal

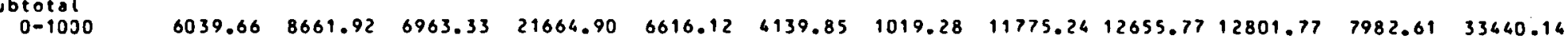

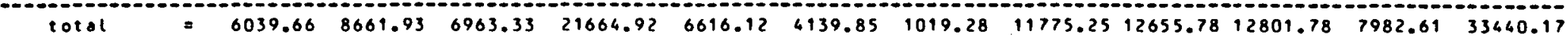
(a)

Ex:

overburden

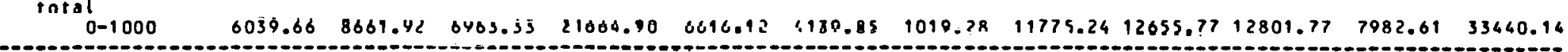

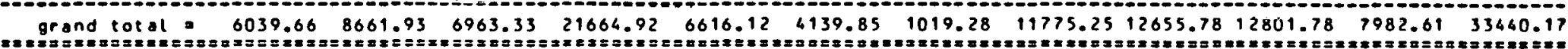

total tonnage of identified coal resources in eastern kentueky

33440.17

resource figures taken from:

$\begin{array}{lll}\text { source } & \text { year } & \text { base year of resource } \\ \text { usgs bull } 1120 & 1963 & \text { original }\end{array}$


National Coal Resources Data Systen

U.S. Geological Surver

identified coal resources in vestern kentucky

(In nillions of short tons)

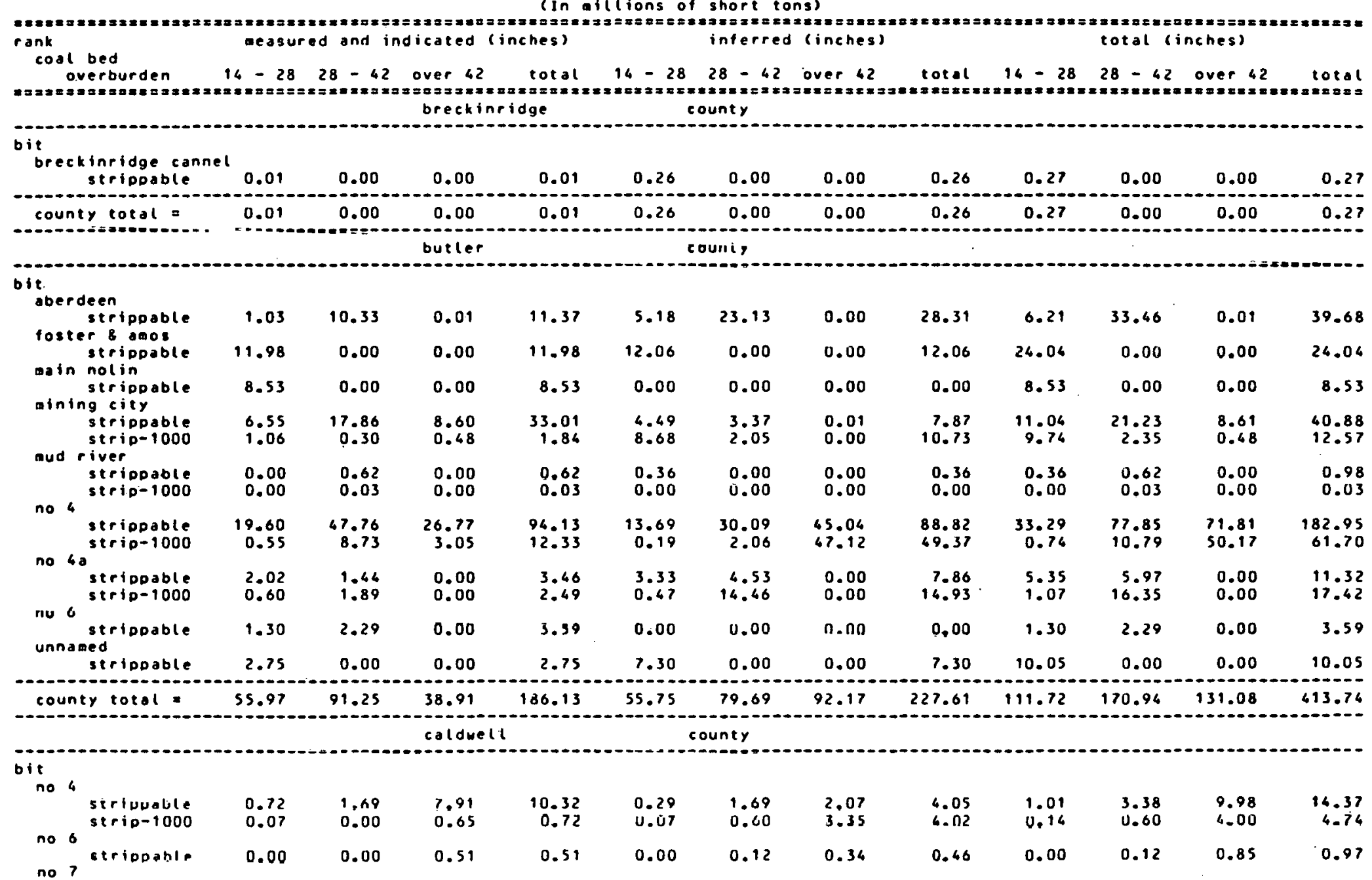




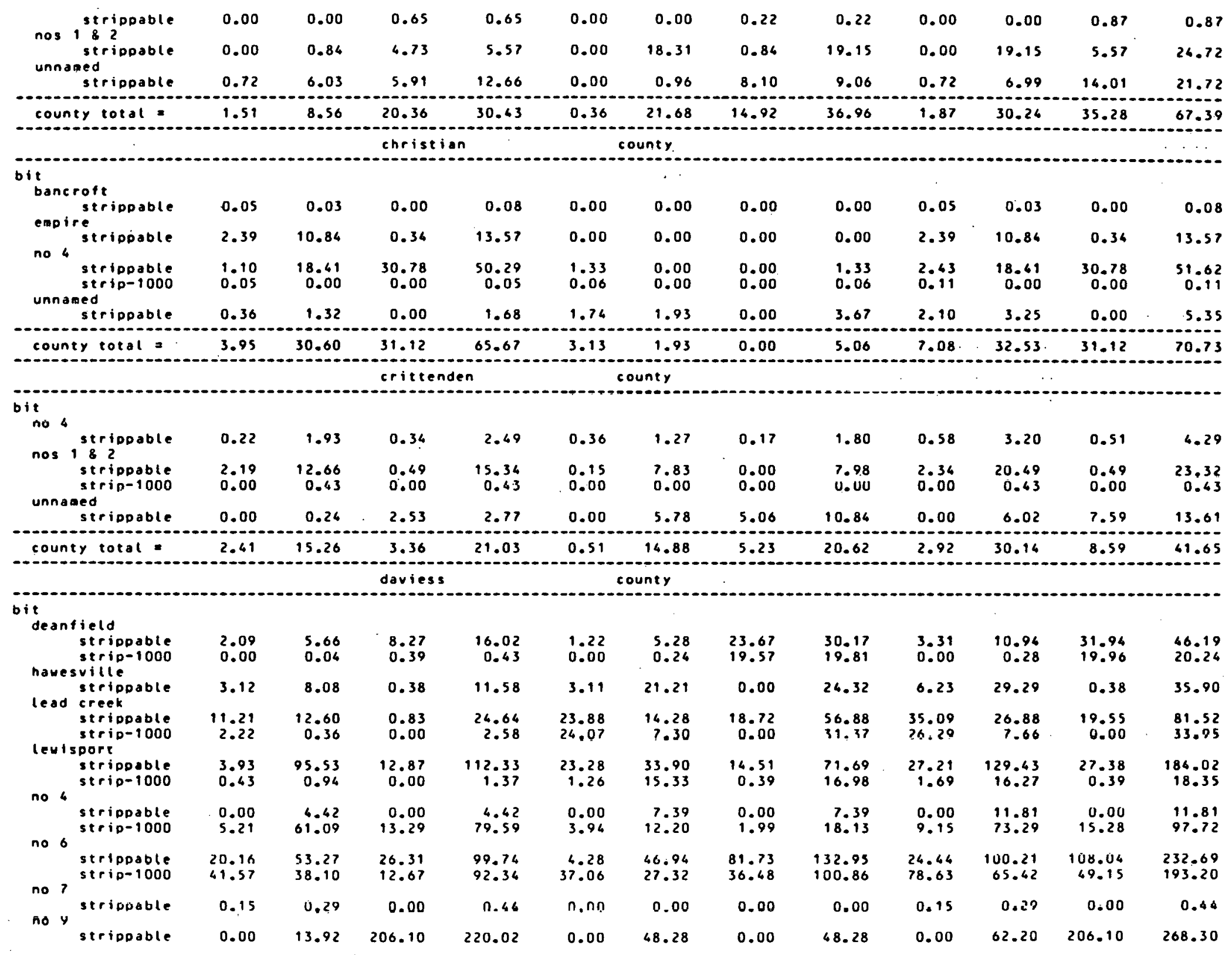




\begin{tabular}{|c|c|c|c|c|c|c|c|c|c|c|c|c|}
\hline $\begin{array}{l}\text { strip-1000 } \\
\text { white ash } \\
\text { strippable }\end{array}$ & $\begin{array}{l}0.00 \\
0.58\end{array}$ & $\begin{array}{l}1.70 \\
2.54\end{array}$ & $\begin{array}{r}91.66 \\
0.00\end{array}$ & $\begin{array}{r}93.36 \\
3.12\end{array}$ & $\begin{array}{l}0.00 \\
6.03\end{array}$ & $\begin{array}{l}2.96 \\
0.60\end{array}$ & $\begin{array}{l}0.00 \\
0.00\end{array}$ & $\begin{array}{l}2.96 \\
6.63\end{array}$ & $\begin{array}{l}0.00 \\
6.61\end{array}$ & $\begin{array}{l}4.66 \\
3.14\end{array}$ & $\begin{array}{r}91.66 \\
0.00\end{array}$ & $\begin{array}{r}96.32 \\
9.75\end{array}$ \\
\hline county total = & 90.67 & 298.54 & 372.77 & 761.98 & 128.13 & 243.23 & 197.06 & 568.42 & 218.80 & 541.77 & 569.83 & 1330.40 \\
\hline & & & edno & & & ney & & & & & & \\
\hline it & & & & & & & & & & & & \\
\hline $\begin{array}{l}\text { Stripoable } \\
\text { unnamed }\end{array}$ & $\begin{array}{r}17.49 \\
2.84\end{array}$ & $\begin{array}{r}100.76 \\
0.27\end{array}$ & 0.00 & $\begin{array}{r}118.17 \\
3.11\end{array}$ & $\begin{array}{r}11.49 \\
7.45\end{array}$ & $\begin{array}{r}0.12 \\
19.28\end{array}$ & $\begin{array}{l}0.00 \\
0.00\end{array}$ & $\begin{array}{l}11.61 \\
26.73\end{array}$ & 28.90 & $\begin{array}{r}100.88 \\
19.55\end{array}$ & $\begin{array}{l}0.00 \\
0.00\end{array}$ & $\begin{array}{r}129.78 \\
29.84\end{array}$ \\
\hline county totol = & 20.25 & 101.03 & 0.00 & 121.28 & 18.96 & 19.40 & 0.00 & 38.34 & 39.19 & 120.63 & 0.00 & 159.62 \\
\hline & & & grays & & & unty & & & & & & \\
\hline it & & & & & & & & & & & & \\
\hline $\begin{array}{l}\text { main nollin } \\
\text { stripoble } \\
\text { unnaged }\end{array}$ & 0.00 & 90.64 & 0.00 & 90.64 & 0.00 & 0.00 & 0.00 & 0.00. & 0.00 & 90.64 & 0.00 & 90.64 \\
\hline sipipoable & 11.37 & 0,00 & 0.00 & 11.37 & 3.03 & 0.00 & 0.00 & 3.03 & 14.40 & 0.00 & 0.00 & 14.60 \\
\hline county total = & 11.37 & 90.64 & 0.00 & 102.01 & 3.03 & 0.00 & 0.00 & 3.03 & 16.60 & 90.66 & U. Ũú & 105.04 \\
\hline & & & hancoc & & & unty & & & & & & \\
\hline & & & & & & & & & & & & \\
\hline $\begin{array}{c}\text { deantield } \\
\text { stripoable } \\
\text { hawesville }\end{array}$ & 3.91 & 0.00 & 0.00 & 3.91 & 2.02 & 0.00 & 0.00 & 2.02 & 5.93 & 0.00 & 0.00 & 5.93 \\
\hline $\begin{array}{l}\text { strippable } \\
\text { strip-1000 } \\
\text { lead creek }\end{array}$ & $\begin{array}{l}0.00 \\
0.00\end{array}$ & $\begin{array}{l}3.47 \\
0.00\end{array}$ & $\begin{array}{l}0.00 \\
0.00\end{array}$ & $\begin{array}{l}3.47 \\
0.00\end{array}$ & $\begin{array}{l}2.91 \\
0.00\end{array}$ & $\begin{array}{r}25.64 \\
3.95\end{array}$ & $\begin{array}{l}0.00 \\
0.00\end{array}$ & $\begin{array}{r}28.55 \\
3.95\end{array}$ & $\begin{array}{l}2.99 \\
0.00\end{array}$ & $\begin{array}{r}29.11 \\
3.95\end{array}$ & $\begin{array}{l}0.00 \\
0.00\end{array}$ & $\begin{array}{r}32.02 \\
3.95\end{array}$ \\
\hline $\begin{array}{l}\text { strippable } \\
\text { strip-1000 }\end{array}$ & $\begin{array}{r}12.82 \\
0.05\end{array}$ & $\begin{array}{r}10.28 \\
0.00\end{array}$ & $\begin{array}{l}0.00 \\
0.00\end{array}$ & $\begin{array}{r}23.10 \\
0.05\end{array}$ & $\begin{array}{r}13.23 \\
0.06\end{array}$ & $\begin{array}{r}10.24 \\
0.00\end{array}$ & $\begin{array}{l}7.16 \\
0.00\end{array}$ & $\begin{array}{r}30.63 \\
0.06\end{array}$ & $\begin{array}{r}26.05 \\
0.11\end{array}$ & $\begin{array}{r}20.52 \\
0.00\end{array}$ & $\begin{array}{l}7.16 \\
0.00\end{array}$ & $\begin{array}{r}53.73 \\
0.11\end{array}$ \\
\hline $\begin{array}{l}\text { lewisport } \\
\text { strippable } \\
\text { unnaped }\end{array}$ & 0.67 & 3.12 & 3.66 & 7.65 & 0.02 & 0.08 & 1.64 & 1.76 & 0.69 & 3.20 & 5.30 & 9.19 \\
\hline $\begin{array}{r}\text { stripoable } \\
\text { strip-to00 }\end{array}$ & $\begin{array}{l}1.20 \\
0.01\end{array}$ & $\begin{array}{l}5.08 \\
0.00\end{array}$ & $\begin{array}{l}0.00 \\
0.00\end{array}$ & $\begin{array}{l}6.28 \\
0.01\end{array}$ & $\begin{array}{l}1.70 \\
0.10\end{array}$ & $\begin{array}{r}57.04 \\
0.00\end{array}$ & $\begin{array}{l}0.00 \\
0.00\end{array}$ & $\begin{array}{r}58.74 \\
0.10\end{array}$ & $\begin{array}{l}2.90 \\
0.11\end{array}$ & $\begin{array}{r}62.12 \\
0.00\end{array}$ & $\begin{array}{l}0.00 \\
0.00\end{array}$ & $\begin{array}{r}65.02 \\
0.11\end{array}$ \\
\hline $\begin{array}{l}\text { White ash } \\
\text { stripoable }\end{array}$ & 0.12 & 0.00 & 0.00 & 0.12 & 0.09 & 0.00 & 0.00 & 0.09 & 0.21 & 0.00 & 0.00 & 0.21 \\
\hline county total $=$ & 18.78 & 21.93 & 3.00 & $\$ 4.37$ & 20.13 & 94.95 & 8.80 & 125.88 & 38.91 & 118.90 & 12.46 & 170.27 \\
\hline & & & hender & & & unty & & & & & & \\
\hline it & & & & & & & & & & & & \\
\hline $\begin{array}{r}\text { strippable } \\
\text { strip-1000 }\end{array}$ & $\begin{array}{l}0.23 \\
2.42\end{array}$ & $\begin{array}{r}0.00 \\
135.84\end{array}$ & $\begin{array}{r}0.00 \\
217.86\end{array}$ & $\begin{array}{r}0.23 \\
356.12\end{array}$ & $\begin{array}{r}0.00 \\
12.23\end{array}$ & $\begin{array}{r}0.40 \\
38.40\end{array}$ & $\begin{array}{r}0.00 \\
84.92\end{array}$ & $\begin{array}{r}0.40 \\
135.55\end{array}$ & $\begin{array}{r}0.23 \\
14.65\end{array}$ & $\begin{array}{r}0.40 \\
174.24\end{array}$ & $\begin{array}{r}0.00 \\
302.78\end{array}$ & $\begin{array}{r}0.63 \\
491.67\end{array}$ \\
\hline strip-1000 & 0.00 & 0.00 & 0.00 & 0.00 & 0.00 & 24.58 & 4.05 & 28.63 & 0.00 & 24.58 & 4.05 & 28.63 \\
\hline no $\sigma^{\text {strip-1000 }}$ & 19.78 & 71.82 & 18.56 & iบ่2.10 & 115.53 & 782.49 & 330.91 & 1237.85 & 127.31 & 854.23 & 358.47 & 1340.01 \\
\hline $\begin{array}{l}\text { strippable } \\
\text { strip-1000 }\end{array}$ & $\begin{array}{l}0.00 \\
4.25\end{array}$ & $\begin{array}{r}4.22 \\
507.68\end{array}$ & $\begin{array}{r}1.00 \\
978.95\end{array}$ & $\begin{array}{r}5.22 \\
1690.88\end{array}$ & $\begin{array}{l}0.00 \\
3.47\end{array}$ & $\begin{array}{r}0.48 \\
132.18\end{array}$ & $\begin{array}{r}16.87 \\
140.42\end{array}$ & $\begin{array}{r}17.35 \\
276.07\end{array}$ & $\begin{array}{l}0.00 \\
7.72\end{array}$ & $\begin{array}{r}6.70 \\
639.86\end{array}$ & $\begin{array}{r}17.87 \\
1119.37\end{array}$ & $\begin{array}{r}22.57 \\
1766.95\end{array}$ \\
\hline
\end{tabular}




\begin{tabular}{|c|c|c|c|c|c|c|c|c|c|c|c|c|c|}
\hline 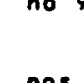 & $\begin{array}{l}\text { strippable } \\
\text { strip-1000 }\end{array}$ & $\begin{array}{l}0.00 \\
0.22\end{array}$ & $\begin{array}{l}10.36 \\
22.71\end{array}$ & $\begin{array}{r}123.46 \\
1935.11\end{array}$ & $\begin{array}{r}133.82 \\
1958.04\end{array}$ & $\begin{array}{l}0.00 \\
0.00\end{array}$ & $\begin{array}{l}0.00 \\
0.00\end{array}$ & $\begin{array}{l}0.00 \\
0.00\end{array}$ & $\begin{array}{l}0.00 \\
0.00\end{array}$ & $\begin{array}{l}0.00 \\
0.22\end{array}$ & $\begin{array}{l}10.36 \\
22.71\end{array}$ & $\begin{array}{r}123.46 \\
1935.11\end{array}$ & $\begin{array}{r}133.82 \\
1958.04\end{array}$ \\
\hline & $\begin{array}{l}\text { stripdable } \\
\text { strip-1000 }\end{array}$ & $\begin{array}{l}0.00 \\
9.62\end{array}$ & $\begin{array}{r}0.00 \\
91.04\end{array}$ & $\begin{array}{r}5.58 \\
544.04\end{array}$ & $\begin{array}{r}5.58 \\
644.70 \\
\end{array}$ & $\begin{array}{r}0.00 \\
17.35\end{array}$ & $\begin{array}{r}0.00 \\
111.96\end{array}$ & $\begin{array}{r}29.74 \\
301.13\end{array}$ & $\begin{array}{r}29.76 \\
430.44\end{array}$ & $\begin{array}{r}0.00 \\
26.97\end{array}$ & $\begin{array}{r}0.00 \\
203.00\end{array}$ & $\begin{array}{r}35.32 \\
845.17\end{array}$ & $\begin{array}{r}35.32 \\
1075.16\end{array}$ \\
\hline coun & nty totat = & 28.52 & 843.67 & 3824.56 & 4696.75 & 148.58 & 1090.41 & 917.04 & 2156.03 & 177.10 & 1934.08 & 4741.60 & 6852.78 \\
\hline & & & & nopkin & & & county & & & & 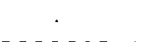 & & \\
\hline \multicolumn{14}{|l|}{ it } \\
\hline & $\begin{array}{l}\text { strippable } \\
\text { strip-1000 }\end{array}$ & $\begin{array}{l}1.62 \\
0.03\end{array}$ & $\begin{array}{l}0.00 \\
0.00\end{array}$ & $\begin{array}{l}0.00 \\
0.00\end{array}$ & $\begin{array}{l}1.62 \\
0.03\end{array}$ & $\begin{array}{l}0.84 \\
0.01\end{array}$ & $\begin{array}{l}0.70 \\
0.06\end{array}$ & $\begin{array}{l}0.00 \\
0.00\end{array}$ & $\begin{array}{l}1.54 \\
0.07\end{array}$ & $\begin{array}{l}2.46 \\
0.04\end{array}$ & $\begin{array}{l}0.70 \\
0.06\end{array}$ & $\begin{array}{l}0.00 \\
0.00\end{array}$ & $\begin{array}{l}3.16 \\
0.10\end{array}$ \\
\hline $\begin{array}{l}\text { enpi } \\
\text { no } 1\end{array}$ & 13 stripoable & 3.7 & 3.13 & .00 & 6 & .00 & 0.00 & 0.00 & 0.00 & 3.76 & 3.13 & 0.00 & 6.89 \\
\hline & $\begin{array}{l}\text { strippable } \\
\text { strip-1000 }\end{array}$ & $\begin{array}{r}6.56 \\
10.18\end{array}$ & $\begin{array}{r}3.86 \\
31.91\end{array}$ & $\begin{array}{l}36.23 \\
57.36\end{array}$ & $\begin{array}{l}66.65 \\
99.45\end{array}$ & $\begin{array}{r}0.42 \\
39.98\end{array}$ & $\begin{array}{r}0.63 \\
191.81\end{array}$ & $\begin{array}{r}58.26 \\
173.46\end{array}$ & $\begin{array}{r}59.11 \\
405.25\end{array}$ & $\begin{array}{r}6.98 \\
50.16\end{array}$ & $\begin{array}{r}4.29 \\
223.72\end{array}$ & $\begin{array}{r}94.49 \\
230.82\end{array}$ & $\begin{array}{l}105.76 \\
504.70\end{array}$ \\
\hline & $\begin{array}{l}\text { strippable } \\
\text { strip-1000 }\end{array}$ & $\begin{array}{l}3.45 \\
0.00\end{array}$ & $\begin{array}{r}0.00 \\
13.60\end{array}$ & $\begin{array}{l}59.75 \\
68.92\end{array}$ & $\begin{array}{l}63.20 \\
82,32\end{array}$ & $\begin{array}{l}0.92 \\
0.00\end{array}$ & $\begin{array}{r}0.00 \\
179.89\end{array}$ & $\begin{array}{r}62.20 \\
341.99\end{array}$ & $\begin{array}{r}63.12 \\
521.88\end{array}$ & $\begin{array}{l}4.37 \\
0.00\end{array}$ & $\begin{array}{r}0.00 \\
193.29\end{array}$ & $\begin{array}{l}121.95 \\
410.91\end{array}$ & $\begin{array}{l}126.32 \\
606.20\end{array}$ \\
\hline & $\begin{array}{l}\text { strippoble } \\
\text { strip-1000 }\end{array}$ & $\begin{array}{l}0.00 \\
0.00\end{array}$ & $\begin{array}{l}3.25 \\
0.36\end{array}$ & $\begin{array}{l}0.00 \\
0.00\end{array}$ & $\begin{array}{l}3.25 \\
0.36\end{array}$ & $\begin{array}{l}0.00 \\
0.00\end{array}$ & $\begin{array}{l}0.60 \\
0.72\end{array}$ & $\begin{array}{l}0.00 \\
0.00\end{array}$ & $\begin{array}{l}0.60 \\
0.72\end{array}$ & $\begin{array}{l}0.00 \\
0.00\end{array}$ & $\begin{array}{l}3.85 \\
1.08\end{array}$ & $\begin{array}{l}0.00 \\
0.00\end{array}$ & $\begin{array}{l}3.85 \\
1.08\end{array}$ \\
\hline na & $\begin{array}{l}\text { strippable } \\
\text { strip-1000 }\end{array}$ & $\begin{array}{l}18.44 \\
47.23\end{array}$ & $\begin{array}{r}41.68 \\
188.37\end{array}$ & $\begin{array}{l}130.91 \\
250.55\end{array}$ & $\begin{array}{l}191.03 \\
486.15\end{array}$ & $\begin{array}{l}14.03 \\
60.48\end{array}$ & $\begin{array}{r}34.06 \\
621.68\end{array}$ & $\begin{array}{r}22.73 \\
311.75\end{array}$ & $\begin{array}{r}70.82 \\
993.71\end{array}$ & $\begin{array}{r}32.47 \\
107.71\end{array}$ & $\begin{array}{r}75.74 \\
809.85\end{array}$ & $\begin{array}{l}153.64 \\
562.30\end{array}$ & $\begin{array}{r}261.85 \\
1679.86\end{array}$ \\
\hline no 6 & $\begin{array}{l}6 \text { stippable } \\
\text { strip-1000 }\end{array}$ & $\begin{array}{l}16.39 \\
35.82\end{array}$ & $\begin{array}{r}26.43 \\
269.78\end{array}$ & $\begin{array}{r}9.80 \\
179.57\end{array}$ & $\begin{array}{r}50.62 \\
485.47\end{array}$ & $\begin{array}{l}26.22 \\
47.55\end{array}$ & $\begin{array}{r}15.29 \\
324.31\end{array}$ & $\begin{array}{r}15.28 \\
366.49\end{array}$ & $\begin{array}{r}56.79 \\
738.35\end{array}$ & $\begin{array}{l}40.61 \\
83.37\end{array}$ & $\begin{array}{r}41.72 \\
594.09\end{array}$ & $\begin{array}{r}25.08 \\
546.06\end{array}$ & $\begin{array}{r}107.43 \\
1223.52\end{array}$ \\
\hline 008 & $\begin{array}{l}\text { strippable } \\
\text { strip-1000 } \\
8\end{array}$ & $\begin{array}{l}9.87 \\
3.70\end{array}$ & $\begin{array}{r}19.13 \\
7.50\end{array}$ & $\begin{array}{l}3.86 \\
0.17\end{array}$ & $\begin{array}{l}24.86 \\
11.37\end{array}$ & $\begin{array}{l}16.80 \\
18.88\end{array}$ & $\begin{array}{l}33.24 \\
55.22\end{array}$ & $\begin{array}{l}0.97 \\
4.05\end{array}$ & $\begin{array}{l}50.21 \\
78.15\end{array}$ & $\begin{array}{l}26.67 \\
22.58\end{array}$ & $\begin{array}{l}66.37 \\
62.72\end{array}$ & $\begin{array}{l}4.03 \\
4.22\end{array}$ & $\begin{array}{l}75.07 \\
89.52\end{array}$ \\
\hline & stripoable & 0.15 & 1.57 & 0.00 & 1.72 & 0.07 & 17.23 & 0.00 & 17.30 & 0.22 & 18.80 & 0.00 & 19.02 \\
\hline nos & $\begin{array}{l}\text { serippable } \\
\text { strip-1000 } \\
11812\end{array}$ & $\begin{array}{l}0.00 \\
0.00\end{array}$ & $\begin{array}{r}10.24 \\
1.39\end{array}$ & $\begin{array}{r}365.50 \\
1313.94\end{array}$ & $\begin{array}{r}375.74 \\
1315.33\end{array}$ & $\begin{array}{l}0.00 \\
0.00\end{array}$ & $\begin{array}{l}0.00 \\
0.00\end{array}$ & $\begin{array}{l}0.00 \\
0.00\end{array}$ & $\begin{array}{l}0.00 \\
0.00\end{array}$ & $\begin{array}{l}0.00 \\
0.00\end{array}$ & $\begin{array}{r}10.24 \\
1.39\end{array}$ & $\begin{array}{r}365.50 \\
1313.96\end{array}$ & $\begin{array}{r}375.74 \\
1315.33\end{array}$ \\
\hline unna & $\begin{array}{l}\text { stripoable } \\
\text { strip-1000 } \\
\text { aned } \\
\text { etrippable }\end{array}$ & $\begin{array}{l}0.00 \\
5.01 \\
0.00\end{array}$ & $\begin{array}{r}0.24 \\
25.00 \\
12.90\end{array}$ & $\begin{array}{r}487.22 \\
1103.53 \\
0.00\end{array}$ & $\begin{array}{r}487.46 \\
1133.54 \\
12.90\end{array}$ & $\begin{array}{l}0.00 \\
1.71 \\
0.00\end{array}$ & $\begin{array}{r}0.00 \\
0.31 \\
20.59\end{array}$ & $\begin{array}{r}79.54 \\
775.43 \\
0.00\end{array}$ & $\begin{array}{r}79.54 \\
777.45 \\
20.59\end{array}$ & $\begin{array}{l}0.00 \\
6.72 \\
0.00\end{array}$ & $\begin{array}{r}0.26 \\
25.31 \\
33.49\end{array}$ & $\begin{array}{r}566.76 \\
1878.96 \\
0.00\end{array}$ & $\begin{array}{r}567.00 \\
1910.99 \\
33.49\end{array}$ \\
\hline coun & ney total $=$ & 160.21 & 652.16 & $\begin{array}{r}4067.31 \\
\text { mclean }\end{array}$ & 4879.66 & 227.91 & 1695.94 & 2211.35 & 3935.20 & 388.12 & 2148.08 & 6278.60 & 8814.86 \\
\hline it & 10 & & & & & & & & & & & & \\
\hline no 1 & $13^{\operatorname{ser} 10-1000}$ & 2.17 & 19.94 & 0.00 & 22.11 & 0.62 & 33.59 & 0.00 & 34.21 & 2.79 & 53.53 & 0.00 & 56.32 \\
\hline & $\begin{array}{r}\text { strippable } \\
\text { serip-1000 }\end{array}$ & $\begin{array}{r}0.00 \\
28.68\end{array}$ & $\begin{array}{r}0.62 \\
25.74\end{array}$ & $\begin{array}{r}1.36 \\
25.75\end{array}$ & $\begin{array}{r}9.98 \\
79.97\end{array}$ & $\begin{array}{r}0.00 \\
16.52\end{array}$ & $\begin{array}{r}0.00 \\
72.78\end{array}$ & $\begin{array}{r}0.00 \\
160.13\end{array}$ & $\begin{array}{r}0.00 \\
249.43\end{array}$ & $\begin{array}{r}0.00 \\
45.00\end{array}$ & $\begin{array}{r}0.62 \\
98.52\end{array}$ & $\begin{array}{r}1.36 \\
185.88\end{array}$ & $\begin{array}{r}1.98 \\
329.60\end{array}$ \\
\hline no 4 & 4 strip-1000 & 0.00 & 4.83 & 0.00 & 4.83 & 0.00 & 59.55 & 0.00 & 59.55 & 0.00 & 64.38 & 0.00 & 64.38 \\
\hline & strippable & 0.00 & 2.90 & 0.00 & 2.90 & 0.00 & 2.05 & 0.00 & 2.05 & 0.00 & 4.95 & 0.00 & 4.95 \\
\hline
\end{tabular}




\begin{tabular}{|c|c|c|c|c|c|c|c|c|c|c|c|c|c|}
\hline & strip-1000 & 0.00 & 34.53 & 65.21 & 99.74 & 20.06 & 272.99 & 169.08 & 462.13 & 20.08 & 307.52 & 214.29 & 561.87 \\
\hline & $\begin{array}{l}\text { strippable } \\
\text { strip-1000 }\end{array}$ & $\begin{array}{r}0.00 \\
12.57\end{array}$ & $\begin{array}{r}6.69 \\
339.70\end{array}$ & $\begin{array}{r}0.00 \\
80.84\end{array}$ & $\begin{array}{r}6.49 \\
433.11\end{array}$ & $\begin{array}{l}0.72 \\
7.10\end{array}$ & $\begin{array}{r}5.48 \\
145.12\end{array}$ & $\begin{array}{r}4.63 \\
187.57\end{array}$ & $\begin{array}{r}10.83 \\
339.79\end{array}$ & $\begin{array}{r}0.72 \\
19.67\end{array}$ & $\begin{array}{r}11.89 \\
484.82\end{array}$ & $\begin{array}{r}4.63 \\
268.41\end{array}$ & $\begin{array}{r}17.24 \\
772.90\end{array}$ \\
\hline no & $\begin{array}{l}\text { strippable } \\
\text { strip-1000 }\end{array}$ & $\begin{array}{l}0.00 \\
0.00\end{array}$ & $\begin{array}{l}7.78 \\
5.50\end{array}$ & $\begin{array}{l}106.38 \\
773.17\end{array}$ & $\begin{array}{l}116.16 \\
778.67\end{array}$ & $\begin{array}{l}0.00 \\
0.00\end{array}$ & $\begin{array}{l}0.00 \\
0.00\end{array}$ & $\begin{array}{r}0.00 \\
204.75\end{array}$ & $\begin{array}{r}0.00 \\
204.75\end{array}$ & $\begin{array}{l}0.00 \\
0.00\end{array}$ & $\begin{array}{l}7.78 \\
5.50\end{array}$ & $\begin{array}{l}106.38 \\
977.92\end{array}$ & $\begin{array}{l}114.16 \\
983.42\end{array}$ \\
\hline nos & $\begin{array}{l}\text { strippable } \\
\text { strip-1000 }\end{array}$ & $\begin{array}{r}0.00 \\
41.23\end{array}$ & $\begin{array}{r}0.00 \\
20.42\end{array}$ & $\begin{array}{r}1.52 \\
133.14\end{array}$ & $\begin{array}{r}1.52 \\
194.79\end{array}$ & $\begin{array}{r}0.00 \\
32.83\end{array}$ & $\begin{array}{r}0.00 \\
17.69\end{array}$ & $\begin{array}{r}14.75 \\
428.23\end{array}$ & $\begin{array}{r}14.75 \\
478.75\end{array}$ & $\begin{array}{r}0.00 \\
74.06\end{array}$ & $\begin{array}{r}0.00 \\
38.11\end{array}$ & $\begin{array}{r}10.27 \\
561.37\end{array}$ & $\begin{array}{r}16.27 \\
673.54\end{array}$ \\
\hline count & is total & 84.45 & 668.37 & 1187.37 & 1740.19 & 77.85 & 609.25 & 1149.14 & 1836.26 & 162.30 & 1077.62 & 2336.51 & 3576.43 \\
\hline & muhle & & & unty & & & & & & \\
\hline \multicolumn{14}{|l|}{ it } \\
\hline \multirow{2}{*}{ mane } & $\begin{array}{l}\text { strippable } \\
\text { strip-1000 }\end{array}$ & $\begin{array}{r}25.11 \\
0.46\end{array}$ & $\begin{array}{l}5.61 \\
1.64\end{array}$ & $\begin{array}{l}3.14 \\
0.20\end{array}$ & $\begin{array}{r}33.66 \\
2.08\end{array}$ & $\begin{array}{r}27.64 \\
0.69\end{array}$ & $\begin{array}{l}13.48 \\
32.01\end{array}$ & $\begin{array}{l}0.00 \\
0.00\end{array}$ & $\begin{array}{l}41.12 \\
32.50\end{array}$ & $\begin{array}{r}52.75 \\
0.93\end{array}$ & $\begin{array}{l}18.89 \\
33.45\end{array}$ & $\begin{array}{l}3.14 \\
0.20\end{array}$ & $\begin{array}{l}74.78 \\
34.58\end{array}$ \\
\hline & $\begin{array}{l}\text { Pver } \\
\text { strippable } \\
\text { strip-1000 }\end{array}$ & $\begin{array}{l}1.52 \\
0.15\end{array}$ & $\begin{array}{l}7.59 \\
0.28\end{array}$ & $\begin{array}{l}4.92 \\
0.37\end{array}$ & $\begin{array}{r}14.03 \\
0.80\end{array}$ & $\begin{array}{r}2.59 \\
18.80\end{array}$ & $\begin{array}{l}0.60 \\
0.00\end{array}$ & $\begin{array}{l}0.00 \\
0.00\end{array}$ & $\begin{array}{r}3.19 \\
18.80\end{array}$ & $\begin{array}{r}4.03 \\
18.95\end{array}$ & $\begin{array}{l}8.19 \\
0.28\end{array}$ & $\begin{array}{l}4.92 \\
0.37\end{array}$ & $\begin{array}{l}17.16 \\
19.60\end{array}$ \\
\hline no 1 & $\begin{array}{l}0 \\
\text { strippable } \\
\text { strid }-1000\end{array}$ & $\begin{array}{l}0.22 \\
4.37\end{array}$ & $\begin{array}{r}2.89 \\
13.59\end{array}$ & $\begin{array}{l}0.04 \\
3.16\end{array}$ & $\begin{array}{r}3.15 \\
21.12\end{array}$ & $\begin{array}{l}0.00 \\
1.73\end{array}$ & $\begin{array}{r}4.22 \\
21.02\end{array}$ & $\begin{array}{l}0.00 \\
0.00\end{array}$ & $\begin{array}{r}4.22 \\
22.75\end{array}$ & $\begin{array}{l}0.22 \\
6.10\end{array}$ & $\begin{array}{r}7.11 \\
34.61\end{array}$ & $\begin{array}{l}0.04 \\
3.18\end{array}$ & $\begin{array}{r}7.37 \\
43.87\end{array}$ \\
\hline no & $\begin{array}{l}3 \\
\text { strippable } \\
\text { strip-1000 }\end{array}$ & $\begin{array}{l}61.12 \\
43.75\end{array}$ & $\begin{array}{r}22.75 \\
9.11\end{array}$ & $\begin{array}{l}10.40 \\
23.14\end{array}$ & $\begin{array}{l}94.27 \\
76.00\end{array}$ & $\begin{array}{r}49.48 \\
5.98\end{array}$ & $\begin{array}{l}0.00 \\
1.46\end{array}$ & $\begin{array}{r}0.00 \\
38.54\end{array}$ & $\begin{array}{l}49.48 \\
45.98\end{array}$ & $\begin{array}{r}110.60 \\
49.73\end{array}$ & $\begin{array}{l}22.75 \\
10.57\end{array}$ & $\begin{array}{l}10.60 \\
61.68\end{array}$ & $\begin{array}{l}143.75 \\
121.98\end{array}$ \\
\hline & $\begin{array}{l}4 \\
\text { strippable } \\
\text { strip- } 1000\end{array}$ & $\begin{array}{l}5.65 \\
0.93\end{array}$ & $\begin{array}{r}3.28 \\
11.75\end{array}$ & $\begin{array}{l}63.85 \\
25.31\end{array}$ & $\begin{array}{l}72.78 \\
37.99\end{array}$ & $\begin{array}{r}14.19 \\
3.66\end{array}$ & $\begin{array}{r}8.17 \\
18.93\end{array}$ & $\begin{array}{l}0.00 \\
0.72\end{array}$ & $\begin{array}{l}22.36 \\
23.31\end{array}$ & $\begin{array}{r}19.86 \\
4.59\end{array}$ & $\begin{array}{l}11.45 \\
30.68\end{array}$ & $\begin{array}{l}63.85 \\
26.03\end{array}$ & $\begin{array}{l}95.14 \\
61.30\end{array}$ \\
\hline no 15 & 5 stripoable & 4.57 & 0.00 & 0.00 & 4.57 & 0.00 & 0.00 & 0.00 & 0.00 & 4.57 & 0.00 & 0.00 & 4.57 \\
\hline no 4 & $\begin{array}{l}\text { strippable } \\
\text { strip-1000 }\end{array}$ & $\begin{array}{l}5.56 \\
9.71\end{array}$ & $\begin{array}{r}14.60 \\
119.75\end{array}$ & $\begin{array}{l}17.27 \\
36.01\end{array}$ & $\begin{array}{r}37.43 \\
165.47\end{array}$ & $\begin{array}{r}1.91 \\
22.33\end{array}$ & $\begin{array}{r}64.18 \\
557.28\end{array}$ & $\begin{array}{r}4.59 \\
56.16\end{array}$ & $\begin{array}{r}50.60 \\
635.77\end{array}$ & $\begin{array}{r}7.47 \\
32.04\end{array}$ & $\begin{array}{r}58.78 \\
677.03\end{array}$ & $\begin{array}{l}21.78 \\
92.17\end{array}$ & $\begin{array}{r}88.03 \\
801.24\end{array}$ \\
\hline 0 & $\begin{array}{l}\text { strippable } \\
\text { strip-1000 }\end{array}$ & $\begin{array}{l}8.73 \\
6.45\end{array}$ & $\begin{array}{l}27.42 \\
64.54\end{array}$ & $\begin{array}{r}2.50 \\
91.07\end{array}$ & $\begin{array}{r}38.65 \\
142.06\end{array}$ & $\begin{array}{l}17.15 \\
69.24\end{array}$ & $\begin{array}{r}36.20 \\
131.79\end{array}$ & $\begin{array}{r}0.68 \\
642.63\end{array}$ & $\begin{array}{r}54.03 \\
643.66\end{array}$ & $\begin{array}{l}25.88 \\
75.69\end{array}$ & $\begin{array}{r}63.62 \\
176.33\end{array}$ & $\begin{array}{r}3.18 \\
533.70\end{array}$ & $\begin{array}{r}92.68 \\
785.72\end{array}$ \\
\hline no 9 & $\begin{array}{l}\text { strippable } \\
\text { strip-1000 } \\
11812\end{array}$ & $\begin{array}{l}0.00 \\
0.00\end{array}$ & $\begin{array}{r}59.13 \\
163.97\end{array}$ & $\begin{array}{l}370.95 \\
512.13\end{array}$ & $\begin{array}{l}430.08 \\
676.10\end{array}$ & $\begin{array}{l}0.00 \\
0.00\end{array}$ & $\begin{array}{l}0.00 \\
0.00\end{array}$ & $\begin{array}{l}0.00 \\
0.00\end{array}$ & $\begin{array}{l}0.00 \\
0.00\end{array}$ & $\begin{array}{l}0.00 \\
0.00\end{array}$ & $\begin{array}{r}59.13 \\
163.97\end{array}$ & $\begin{array}{l}370.95 \\
512.13\end{array}$ & $\begin{array}{l}630.08 \\
676.10\end{array}$ \\
\hline nos & $\begin{array}{l}\text { strippable } \\
\text { strip-1000 }\end{array}$ & $\begin{array}{l}0.00 \\
0.00\end{array}$ & $\begin{array}{r}72.76 \\
9.92\end{array}$ & $\begin{array}{l}688.94 \\
314.77\end{array}$ & $\begin{array}{l}761.68 \\
324.69\end{array}$ & $\begin{array}{l}0.00 \\
0.00\end{array}$ & $\begin{array}{l}0.00 \\
5.92\end{array}$ & $\begin{array}{l}82.26 \\
51.44\end{array}$ & $\begin{array}{l}82.26 \\
57.36\end{array}$ & $\begin{array}{l}0.00 \\
0.00\end{array}$ & $\begin{array}{l}72.74 \\
15.84\end{array}$ & $\begin{array}{l}771.20 \\
366.21\end{array}$ & $\begin{array}{l}843.94 \\
382.05\end{array}$ \\
\hline \multirow[t]{2}{*}{ coun } & ty total = & 178.28 & 590.16 & 2168.17 & 2936.61 & 235.11 & 875.26 & 676.96 & 1787.31 & 413.39 & 1603.02 & 2065011 & 6733.02 \\
\hline & & & & ohio & & & unty & & & & & & \\
\hline \multicolumn{2}{|l|}{ d } & \\
\hline$e \ln$ & $\begin{array}{l}\text { stripoable } \\
\text { strip-1000 } \\
\text { lick }\end{array}$ & $\begin{array}{r}19.74 \\
1.72\end{array}$ & $\begin{array}{r}33.10 \\
5.44\end{array}$ & $\begin{array}{l}33.77 \\
11.63\end{array}$ & $\begin{array}{l}86.61 \\
18.79\end{array}$ & $\begin{array}{l}2.57 \\
0.38\end{array}$ & $\begin{array}{l}16.95 \\
10.32\end{array}$ & $\begin{array}{l}48.10 \\
34.49\end{array}$ & $\begin{array}{l}67.62 \\
65.19\end{array}$ & $\begin{array}{r}22.31 \\
2.10\end{array}$ & $\begin{array}{l}50.05 \\
15.76\end{array}$ & $\begin{array}{l}81.87 \\
66.12\end{array}$ & $\begin{array}{r}154.23 \\
63.98\end{array}$ \\
\hline lead & $\begin{array}{l}\text { strippable } \\
\text { strip-t000 } \\
\text { creek }\end{array}$ & $\begin{array}{l}6.85 \\
0.27\end{array}$ & $\begin{array}{r}20.70 \\
2.83\end{array}$ & $\begin{array}{r}14.66 \\
0.00\end{array}$ & $\begin{array}{r}40.01 \\
3.10\end{array}$ & $\begin{array}{r}11.86 \\
1.50\end{array}$ & $\begin{array}{r}15.66 \\
7.11\end{array}$ & $\begin{array}{l}1.59 \\
0.00\end{array}$ & $\begin{array}{r}29.11 \\
8.70\end{array}$ & $\begin{array}{r}16.71 \\
1.86\end{array}$ & $\begin{array}{r}36.36 \\
9.94\end{array}$ & $\begin{array}{r}16.05 \\
0.00\end{array}$ & $\begin{array}{l}69.12 \\
11.80\end{array}$ \\
\hline & $\begin{array}{l}\text { strippable } \\
\text { strip-iooo }\end{array}$ & $\begin{array}{l}0.00 \\
0.00\end{array}$ & $\begin{array}{l}9.25 \\
0.03\end{array}$ & $\begin{array}{l}0.00 \\
n, 0 n\end{array}$ & $\begin{array}{l}9.25 \\
0.03\end{array}$ & $\begin{array}{l}0.00 \\
0.00\end{array}$ & $\begin{array}{r}42.96 \\
4.13\end{array}$ & $\begin{array}{l}0.00 \\
0.00\end{array}$ & $\begin{array}{r}42.96 \\
4.13\end{array}$ & $\begin{array}{l}0.00 \\
0.00\end{array}$ & $\begin{array}{r}52.21 \\
4.16\end{array}$ & $\begin{array}{l}0.00 \\
0.00\end{array}$ & $\begin{array}{r}52.21 \\
4.16\end{array}$ \\
\hline
\end{tabular}




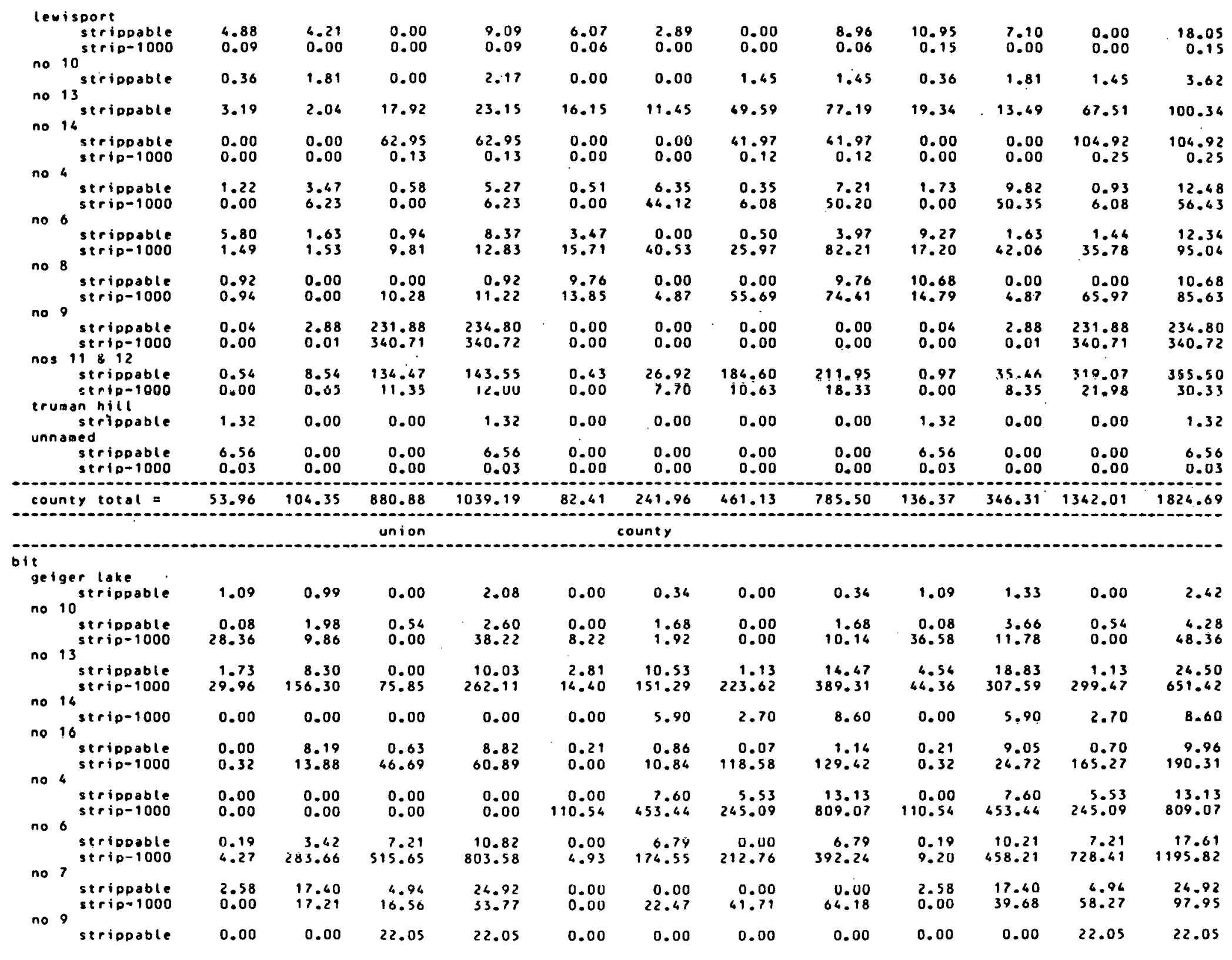




\begin{tabular}{|c|c|c|c|c|c|c|c|c|c|c|c|c|}
\hline \multirow{2}{*}{$\begin{array}{l}\text { strip-1000 } \\
\text { nos } 118 \text { \& } 12 \\
\text { strippable } \\
\text { strip-1000 }\end{array}$} & 0.00 & 0.00 & 1628.54 & 1628.56 & 0.00 & 0.00 & 0.00 & 0.00 & 0.00 & 0.00 & 1628.54 & 1628.56 \\
\hline & $\begin{array}{l}0.72 \\
6.27\end{array}$ & $\begin{array}{l}17.68 \\
57.64\end{array}$ & $\begin{array}{r}37.53 \\
1195.61\end{array}$ & $\begin{array}{r}55.73 \\
1259.52\end{array}$ & $\begin{array}{l}0.00 \\
7.05\end{array}$ & $\begin{array}{r}4.93 \\
22.03\end{array}$ & $\begin{array}{r}15.78 \\
393.11\end{array}$ & $\begin{array}{r}20.71 \\
422.19\end{array}$ & $\begin{array}{r}0.72 \\
13.32\end{array}$ & $\begin{array}{l}22.41 \\
79.67\end{array}$ & $\begin{array}{r}53.31 \\
1588.72\end{array}$ & $\begin{array}{r}76.44 \\
1681.71\end{array}$ \\
\hline county total = & 75.57 & 596.31 & $3551.80^{\circ}$ & 4223.68 & 148.16 & 875.17 & 1260.08 & 2283.61 & 223.73 & 1671.48 & 4811.88 & 6507.09 \\
\hline \multicolumn{2}{|c|}{-... } & & warr & & & ounty & & & & & & \\
\hline \multicolumn{13}{|l|}{ it } \\
\hline \multirow{2}{*}{$\begin{array}{c}\text { main nolin } \\
\text { strippable } \\
\text { unnamed } \\
\text { strippable }\end{array}$} & 2.03 & 3.37 & 0.00 & 5.40 & 0.01 & 0.00 & 0.00 & 0.01 & 2.04 & 3.37 & 0.00 & 5.41 \\
\hline & 1.66 & 0.72 & 0.00 & 2.38 & 0.36 & 0.00 & 0.00 & 0.36 & 2.02 & 0.72 & 0.00 & 2.76 \\
\hline couner total $=$ & 3.69 & 4.09 & 0.00 & 7.78 & 0.37 & 0.00 & 0.00 & 0.37 & 4.06 & 4.09 & 0.00 & 8.15 \\
\hline \multicolumn{13}{|c|}{ webster } \\
\hline \multicolumn{13}{|l|}{ bit } \\
\hline $\begin{array}{r}\text { strippable } \\
\text { no } 13 \\
\text { strip-1000 }\end{array}$ & $\begin{array}{l}0.00 \\
n, 0 n\end{array}$ & $\begin{array}{l}0.00 \\
0.00\end{array}$ & $\begin{array}{l}0.00 \\
0.00\end{array}$ & $\begin{array}{l}0.00 \\
0.00\end{array}$ & $\begin{array}{l}0.00 \\
0.00\end{array}$ & $\begin{array}{l}0.84 \\
8.56\end{array}$ & $\begin{array}{l}0.00 \\
0.00\end{array}$ & $\begin{array}{l}0.84 \\
8.56\end{array}$ & $\begin{array}{l}0.00 \\
0.00\end{array}$ & $\begin{array}{l}0.84 \\
8.56\end{array}$ & $\begin{array}{l}0.00 \\
0.00\end{array}$ & $\begin{array}{l}0.86 \\
8.56\end{array}$ \\
\hline $\begin{array}{l}\text { strippable } \\
\text { strip-.t000 }\end{array}$ & $\begin{array}{l}0.00 \\
1.94\end{array}$ & $\begin{array}{r}0.45 \\
11.85\end{array}$ & $\begin{array}{r}52.60 \\
232.08\end{array}$ & $\begin{array}{r}53.05 \\
245.87\end{array}$ & $\begin{array}{l}0.00 \\
2.50\end{array}$ & $\begin{array}{r}0.00 \\
19.86\end{array}$ & $\begin{array}{r}2.72 \\
606.01\end{array}$ & $\begin{array}{r}2.72 \\
428.37\end{array}$ & $\begin{array}{l}0.00 \\
4.64\end{array}$ & $\begin{array}{r}0.65 \\
31.71\end{array}$ & $\begin{array}{r}55.32 \\
638.09\end{array}$ & $\begin{array}{r}33.18 \\
674.24\end{array}$ \\
\hline no 15 strip-1000 & 0.00 & 13.28 & 15.34 & 28.62 & 0.00 & 27.38 & 131.94 & 159.32 & 0.00 & 40.66 & 147.28 & 187.94 \\
\hline $\begin{array}{l}\text { strippable } \\
\text { strip-1000 }\end{array}$ & $\begin{array}{l}0.00 \\
0.00\end{array}$ & $\begin{array}{l}6.63 \\
0.26\end{array}$ & $\begin{array}{r}0.00 \\
28.07\end{array}$ & $\begin{array}{r}6.63 \\
28.31\end{array}$ & $\begin{array}{l}0.00 \\
0.00\end{array}$ & $\begin{array}{l}7.23 \\
0.00\end{array}$ & $\begin{array}{l}0.00 \\
0.00\end{array}$ & $\begin{array}{l}7.23 \\
0.00\end{array}$ & $\begin{array}{l}0.00 \\
0.00\end{array}$ & $\begin{array}{r}13.86 \\
0.24\end{array}$ & $\begin{array}{r}0.00 \\
28.07\end{array}$ & $\begin{array}{l}13.86 \\
28.31\end{array}$ \\
\hline $\begin{array}{r}\text { strippable } \\
\text { strip-1000 }\end{array}$ & $\begin{array}{l}1.44 \\
1.99\end{array}$ & $\begin{array}{r}2.77 \\
68.93\end{array}$ & $\begin{array}{l}0.00 \\
0.26\end{array}$ & $\begin{array}{r}4.21 \\
71.18\end{array}$ & $\begin{array}{r}0.22 \\
49.33\end{array}$ & $\begin{array}{r}19.16 \\
497.81\end{array}$ & $\begin{array}{r}0.00 \\
105.87\end{array}$ & $\begin{array}{r}19.38 \\
653.01\end{array}$ & $\begin{array}{r}1.66 \\
51.32\end{array}$ & $\begin{array}{r}21.93 \\
566.74\end{array}$ & $\begin{array}{r}0.00 \\
106.13\end{array}$ & $\begin{array}{r}23.59 \\
724.19\end{array}$ \\
\hline $\begin{array}{r}\text { strippable } \\
\text { strip-1000 }\end{array}$ & $\begin{array}{l}0.00 \\
4.89\end{array}$ & $\begin{array}{r}12.77 \\
163.36\end{array}$ & $\begin{array}{r}7.59 \\
137.20\end{array}$ & $\begin{array}{r}20.36 \\
305.45\end{array}$ & $\begin{array}{r}0.00 \\
35.63\end{array}$ & $\begin{array}{r}9.04 \\
235.50\end{array}$ & $\begin{array}{r}7.93 \\
328.93\end{array}$ & $\begin{array}{r}16.97 \\
600.06\end{array}$ & $\begin{array}{r}0.00 \\
60.52\end{array}$ & $\begin{array}{r}21.81 \\
398.80\end{array}$ & $\begin{array}{r}15.52 \\
466.13\end{array}$ & $\begin{array}{r}37.33 \\
905.51\end{array}$ \\
\hline striopable & 0.22 & 1.44 & 0.00 & 1.66 & 0.51 & 0.12 & 0.00 & 0.63 & 0.73 & 1.56 & 0.00 & 2.29 \\
\hline strippable & 0.94 & 0.12 & 0.00 & 1.06 & 0.00 & 0.00 & 0.00 & 0.00 & 0.94 & 0.12 & 0.00 & 1.06 \\
\hline $\begin{array}{l}\text { strippable } \\
\text { strip-1000 } \\
\text { not } 11^{8} 1 ?\end{array}$ & $\begin{array}{l}0.00 \\
0.00\end{array}$ & $\begin{array}{r}0.36 \\
34.58\end{array}$ & $\begin{array}{r}106.51 \\
1567.78\end{array}$ & $\begin{array}{r}104.87 \\
1582.36\end{array}$ & $\begin{array}{l}0.00 \\
0.00\end{array}$ & $\begin{array}{l}0.00 \\
0.00\end{array}$ & $\begin{array}{l}0.00 \\
0.00\end{array}$ & $\begin{array}{l}0.00 \\
0.00\end{array}$ & $\begin{array}{l}0.00 \\
0.00\end{array}$ & $\begin{array}{r}0.36 \\
34.58\end{array}$ & $\begin{array}{r}104.51 \\
1547.78\end{array}$ & $\begin{array}{r}104.87 \\
1582.36\end{array}$ \\
\hline $\begin{array}{l}\text { strippable } \\
\text { strip-1000 }\end{array}$ & $\begin{array}{l}0.00 \\
5.43\end{array}$ & $\begin{array}{r}3.01 \\
54.65\end{array}$ & $\begin{array}{r}34.28 \\
747.15\end{array}$ & $\begin{array}{r}37.29 \\
807.23\end{array}$ & $\begin{array}{r}0.00 \\
10.63\end{array}$ & $\begin{array}{r}1.21 \\
46.43\end{array}$ & $\begin{array}{r}6.53 \\
1062.83\end{array}$ & $\begin{array}{r}7.74 \\
1119.89\end{array}$ & $\begin{array}{r}0.00 \\
16.06\end{array}$ & $\begin{array}{r}4.22 \\
101.08\end{array}$ & $\begin{array}{r}40.81 \\
1804.98\end{array}$ & $\begin{array}{r}45.03 \\
1927.12\end{array}$ \\
\hline county total $=$ & 16.85 & 374.46 & 2906.86 & 3298.15 & 98.82 & 873.14 & 2052.76 & 3024.72 & 115.67 & 1267.58 & 4959.62 & 6322.87 \\
\hline $\begin{array}{l}\text { overburden } \\
\text { subtotal }\end{array}$ & & & & & & & & & & & & \\
\hline $\begin{array}{l}\text { strippable } \\
\text { strip-1000 }\end{array}$ & $\begin{array}{l}386.15 \\
620.30\end{array}$ & $\begin{array}{l}1083.78 \\
3207.58\end{array}$ & $\begin{array}{r}3611.42 \\
15465.71\end{array}$ & $\begin{array}{r}5081.35 \\
19073.59\end{array}$ & $\begin{array}{l}382.12 \\
867.33\end{array}$ & $\begin{array}{r}820.20 \\
5718.69\end{array}$ & $\begin{array}{r}968.74 \\
8077.88\end{array}$ & $\begin{array}{r}2171.06 \\
14663.90\end{array}$ & $\begin{array}{r}768.27 \\
1287.63\end{array}$ & $\begin{array}{l}1903.98 \\
8926.27\end{array}$ & $\begin{array}{r}4580.16 \\
23523.59\end{array}$ & $\begin{array}{r}7252.41 \\
33737.48\end{array}$ \\
\hline $\begin{array}{l}\text { total } \\
=\neq===\end{array}$ & $\begin{array}{l}806.45 \\
x=x=x=2\end{array}$ & $\begin{array}{l}4291.36 \\
=x=3 x=0\end{array}$ & $\begin{array}{l}19057.13 \\
=x=x=x=x=\end{array}$ & $\begin{array}{l}26154.46 \\
=232=30=8\end{array}$ & +249.49 & 6538.09 & 9066.62 & $16834.9 \mathrm{~h}$ & $\begin{array}{l}? 055.90 \\
=x=x==x=\end{array}$ & $\begin{array}{l}10830.25 \\
==3= \pm=x=0\end{array}$ & $\begin{array}{l}28103.75 \\
=3===33 \pm E\end{array}$ & $\begin{array}{l}40989.90 \\
x=x== \pm=x\end{array}$ \\
\hline
\end{tabular}




\begin{tabular}{|c|c|c|c|c|c|c|c|c|c|c|c|c|}
\hline $\begin{array}{l}\text { strippable } \\
\text { strip-1000 }\end{array}$ & $\begin{array}{l}386.15 \\
420.30\end{array}$ & $\begin{array}{l}1083.78 \\
3207.58\end{array}$ & $\begin{array}{r}3611.42 \\
15445.71\end{array}$ & $\begin{array}{r}5081.35 \\
19073.59\end{array}$ & $\begin{array}{l}382.12 \\
867.33\end{array}$ & $\begin{array}{r}820.20 \\
5718.69\end{array}$ & $\begin{array}{r}968.74 \\
8077.88\end{array}$ & $\begin{array}{r}2171.06 \\
14663.90\end{array}$ & $\begin{array}{r}768.27 \\
1287.63\end{array}$ & $\begin{array}{l}1903.98 \\
8926.27\end{array}$ & $\begin{array}{r}45.80 .16 \\
23523.59\end{array}$ & $\begin{array}{r}7252.41 \\
33737.48\end{array}$ \\
\hline total $=$ & 806.45 & $\begin{array}{l}4291.36 \\
321.3==3\end{array}$ & $\begin{array}{l}19057.13 \\
=x=37=02=0\end{array}$ & $\begin{array}{l}26156.94 \\
x=35=8=0\end{array}$ & $\begin{array}{l}1269.45 \\
=3=32 x=30=\end{array}$ & $\begin{array}{l}6538.89 \\
x=8=8=3\end{array}$ & 9066.62 & $\begin{array}{r}16834.96 \\
=16834=0\end{array}$ & 2055.90 & $\begin{array}{l}10830.25 \\
n=8=0.25\end{array}$ & 28103.75 & 4098 \\
\hline
\end{tabular}

total tonnage of dientified coal resources in western kentucky

40989.90

resource tigures taken from:

$\begin{array}{lll}\text { source } & \text { year } & \text { base year of resource } \\ \text { kentucky gs of }-4 k y & 1978 & \text { orfiginal }\end{array}$

kentucky gs of $-4 k y \quad 1978 \quad$ orfiginal 
average analyses of coal in kentucky

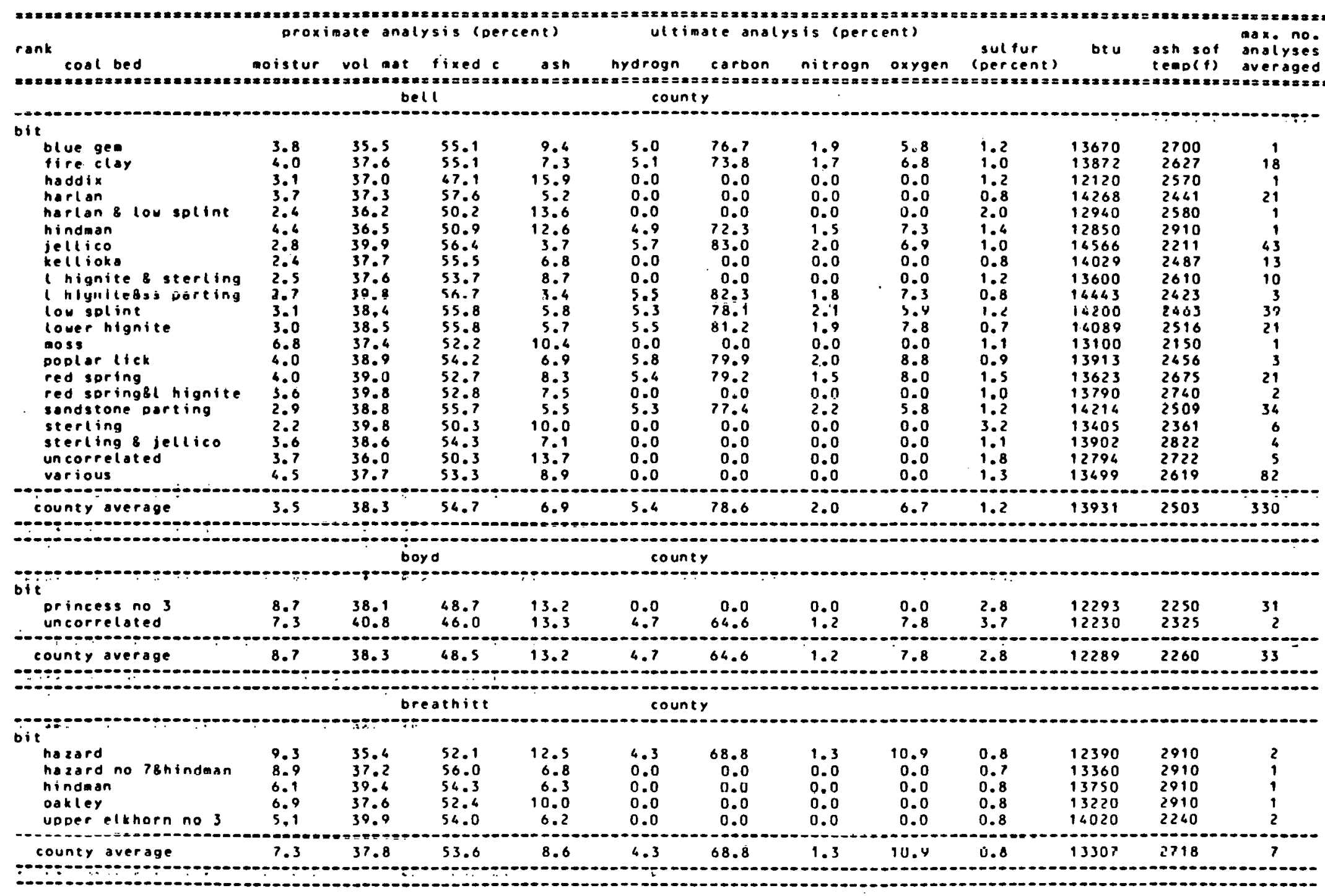


butler

county

\begin{tabular}{|c|c|c|c|c|c|c|c|c|c|c|c|c|}
\hline no 6 & 10.8 & 40.9 & 53.3 & 5.8 & 0.0 & 0.0 & 0.0 & 0.0 & 1.5 & 13800 & 2178 & 10 \\
\hline county average & 10.8 & 40.9 & 53.3 & 5.8 & 0.0 & 0.0 & 0.0 & 0.0 & 1.5 & 13800 & 2178 & 10 \\
\hline & & & ter & & & & & & & & & \\
\hline $\begin{array}{l}\text { princess no } 3 \\
\text { tom cooper }\end{array}$ & $\begin{array}{l}8.7 \\
7.7\end{array}$ & $\begin{array}{l}38.4 \\
39.0\end{array}$ & $\begin{array}{l}51.8 \\
52.6\end{array}$ & $\begin{array}{l}9.7 \\
8.4\end{array}$ & $\begin{array}{l}5.1 \\
5.4\end{array}$ & $\begin{array}{l}76.1 \\
79.6\end{array}$ & $\begin{array}{l}1.5 \\
1.7\end{array}$ & $\begin{array}{r}10.0 \\
9.2\end{array}$ & $\begin{array}{l}1.5 \\
1.2\end{array}$ & $\begin{array}{l}12928 \\
13265\end{array}$ & $\begin{array}{l}2634 \\
2807\end{array}$ & $\begin{array}{r}17 \\
7\end{array}$ \\
\hline county overage & 8.4 & 38.6 & 52.1 & 9.3 & 5.3 & 77.9 & 1.6 & 9.6 & 1.4 & 13027 & 2711 & 26 \\
\hline no 6 & 10.4 & 39.1 & 53.9 & 6.9 & 5.3 & 78.5 & 1.9 & 8.3 & 2.6 & 13550 & 2066 & 6 \\
\hline county average & 10.4 & 39.1 & 53.9 & 6.9 & 5.3 & 18.5 & 1.9 & .8 .3 & 2.6 & 13550 & 2066 & 6 \\
\hline & & & & & & & & & & & & \\
\hline $\begin{array}{l}\text { fire clay } \\
\text { fire clay } 8 \text { lily } \\
\text { hazard no } 7 \\
\text { lily } \\
\text { uncorrelated } \\
\text { various }\end{array}$ & $\begin{array}{l}3.7 \\
3.6 \\
5.6 \\
3.8 \\
3.2 \\
3.8 \\
-.-2 .\end{array}$ & $\begin{array}{l}39.1 \\
41.6 \\
33.6 \\
38.9 \\
39.2 \\
38.3\end{array}$ & $\begin{array}{l}56.8 \\
52.7 \\
52.4 \\
56.2 \\
48.3 \\
55.0\end{array}$ & $\begin{array}{r}6.1 \\
5.8 \\
14.0 \\
6.9 \\
12.5 \\
6.7 \\
-9\end{array}$ & $\begin{array}{l}5.5 \\
0.0 \\
0.0 \\
5.4 \\
0.0 \\
0.0\end{array}$ & $\begin{array}{r}79.8 \\
0.0 \\
0.0 \\
76.4 \\
0.0 \\
0.0 \\
\end{array}$ & $\begin{array}{l}1.8 \\
0.0 \\
0.0 \\
1.3 \\
0.0 \\
0.0\end{array}$ & $\begin{array}{l}7.7 \\
0.0 \\
0.0 \\
6.3 \\
0.0 \\
0.0 \\
-\end{array}$ & $\begin{array}{l}1.0 \\
2.1 \\
0.8 \\
1.7 \\
3.1 \\
1.2\end{array}$ & $\begin{array}{l}14042 \\
14126 \\
12590 \\
14000 \\
13030 \\
13922\end{array}$ & $\begin{array}{l}2677 \\
2166 \\
2910 \\
2426 \\
2250 \\
2468\end{array}$ & $\begin{array}{r}130 \\
3 \\
1 \\
36 \\
1 \\
15\end{array}$ \\
\hline county average & 3.8 & 39.0 & 56.6 & 6.4 & 5.4 & 79.4 & 1.7 & 7.6 & 1.2 & 14012 & 2604 & 186 \\
\hline & & & iess & & & & & & & & & \\
\hline $\begin{array}{l}\text { no } 9 \\
\text { uncorrelated }\end{array}$ & $\begin{array}{l}11.3 \\
12.2\end{array}$ & $\begin{array}{l}39.9 \\
43.0\end{array}$ & $\begin{array}{l}66.6 \\
45.2\end{array}$ & $\begin{array}{l}13.5 \\
11.7\end{array}$ & $\begin{array}{l}0.0 \\
0.0\end{array}$ & $\begin{array}{r}0.0 \\
0.0 \\
0.0-0\end{array}$ & $\begin{array}{r}0.0 \\
0.0\end{array}$ & $\begin{array}{l}0.0 \\
0.0\end{array}$ & $\begin{array}{l}4.0 \\
6.7\end{array}$ & $\begin{array}{r}12151 \\
12595\end{array}$ & $\begin{array}{r}2096 \\
2080\end{array}$ & $\begin{array}{r}12 \\
4\end{array}$ \\
\hline county average & 11.5 & 40.7 & 46.3 & 13.1 & 0.0 & 0.0 & 0.0 & 0.0 & 4.7 & 12262 & 2092 & 16 \\
\hline & & & & & & & & & & & & \\
\hline $\begin{array}{l}\text { haddixepeach orchard } \\
\text { u elkhorn nos } 1882 \\
\text { uelkhorn nos } 1883 \\
\text { uelkhorn nos } 2883 \\
\text { uncorrelated } \\
\text { upper elkhorn no } \\
\text { udoer eliknorn no } \\
\text { upoer elkhorn no } 3 \\
\text { various }\end{array}$ & $\begin{array}{l}5.4 \\
4.6 \\
6.7 \\
5.3 \\
6.9 \\
4.1 \\
4.3 \\
4.5 \\
5.0 \\
-. .9\end{array}$ & $\begin{array}{l}35.1 \\
38.2 \\
35.0 \\
37.9 \\
37.1 \\
38.7 \\
37.9 \\
38.4 \\
37.9\end{array}$ & $\begin{array}{l}50.7 \\
56.0 \\
55.2 \\
54.5 \\
54.6 \\
54.7 \\
56.5 \\
56.6 \\
54.8\end{array}$ & $\begin{array}{r}14.2 \\
5.8 \\
9.8 \\
7.6 \\
8.3 \\
6.6 \\
5.6 \\
5.0 \\
7.3 \\
-\end{array}$ & $\begin{array}{l}0.0 \\
0.0 \\
0.0 \\
0.0 \\
0.0 \\
5.5 \\
5.3 \\
5.4 \\
0.0\end{array}$ & $\begin{array}{r}0.0 \\
0.0 \\
0.0 \\
0.0 \\
0.0 \\
79.1 \\
79.5 \\
80.5 \\
0.0\end{array}$ & $\begin{array}{l}0.0 \\
0.0 \\
0.0 \\
0.0 \\
0.0 \\
1.0 \\
1.0 \\
1.7 \\
0.0\end{array}$ & $\begin{array}{l}0.0 \\
0.0 \\
0.0 \\
0.0 \\
0.0 \\
8.0 \\
8.0 \\
8.4 \\
0.0\end{array}$ & $\begin{array}{l}0.9 \\
0.9 \\
1.2 \\
1.2 \\
0.0 \\
1.0 \\
1.1 \\
0.8 \\
1.1 \\
. .1\end{array}$ & $\begin{array}{l}12440 \\
14057 \\
13420 \\
13733 \\
13240 \\
13970 \\
14077 \\
14205 \\
13829\end{array}$ & $\begin{array}{l}2910 \\
2515 \\
2520 \\
2436 \\
2910 \\
2527 \\
2565 \\
2576 \\
2577\end{array}$ & $\begin{array}{r}1 \\
17 \\
1 \\
3 \\
1 \\
43 \\
21 \\
11 \% \\
20\end{array}$ \\
\hline
\end{tabular}




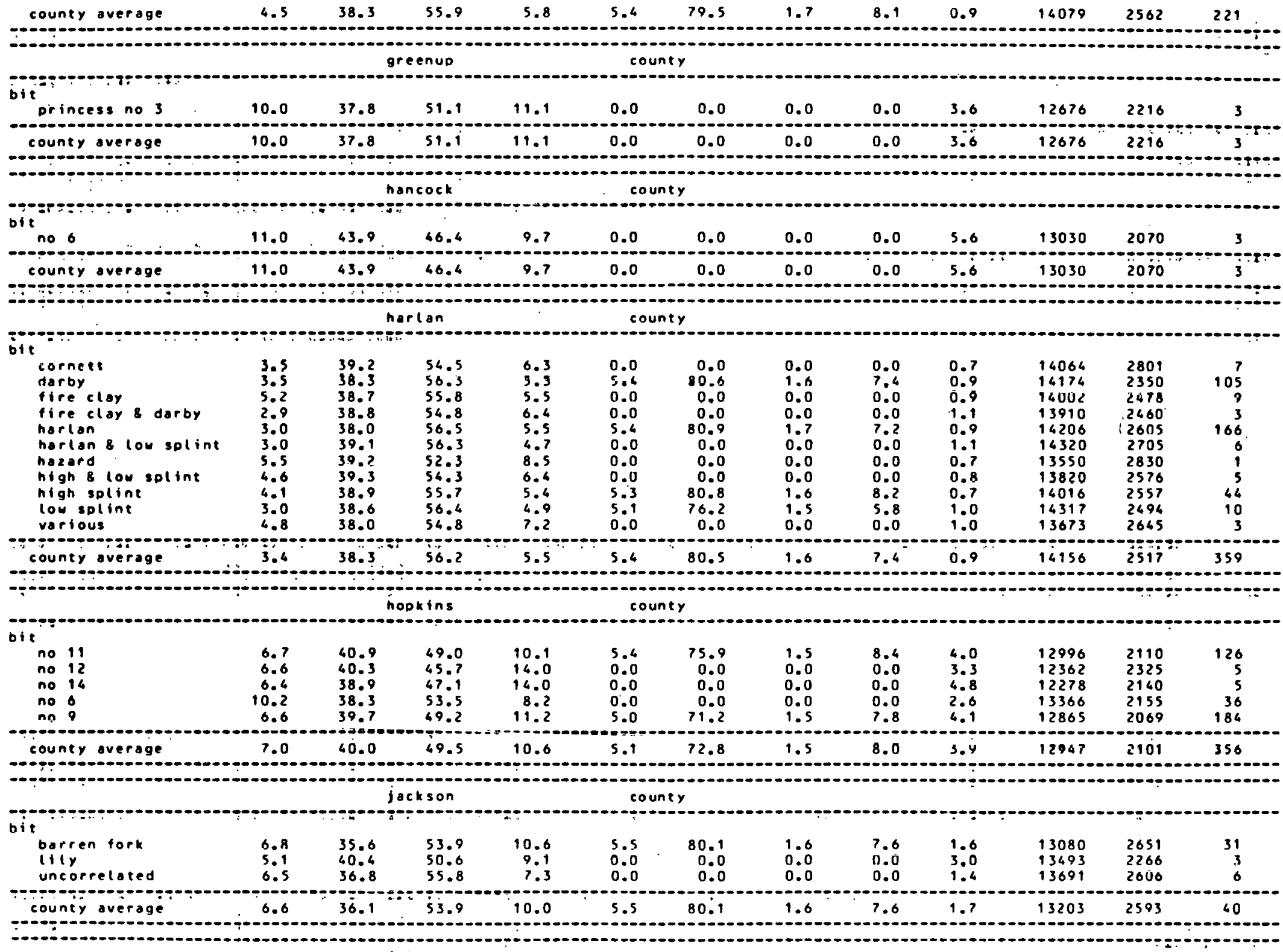




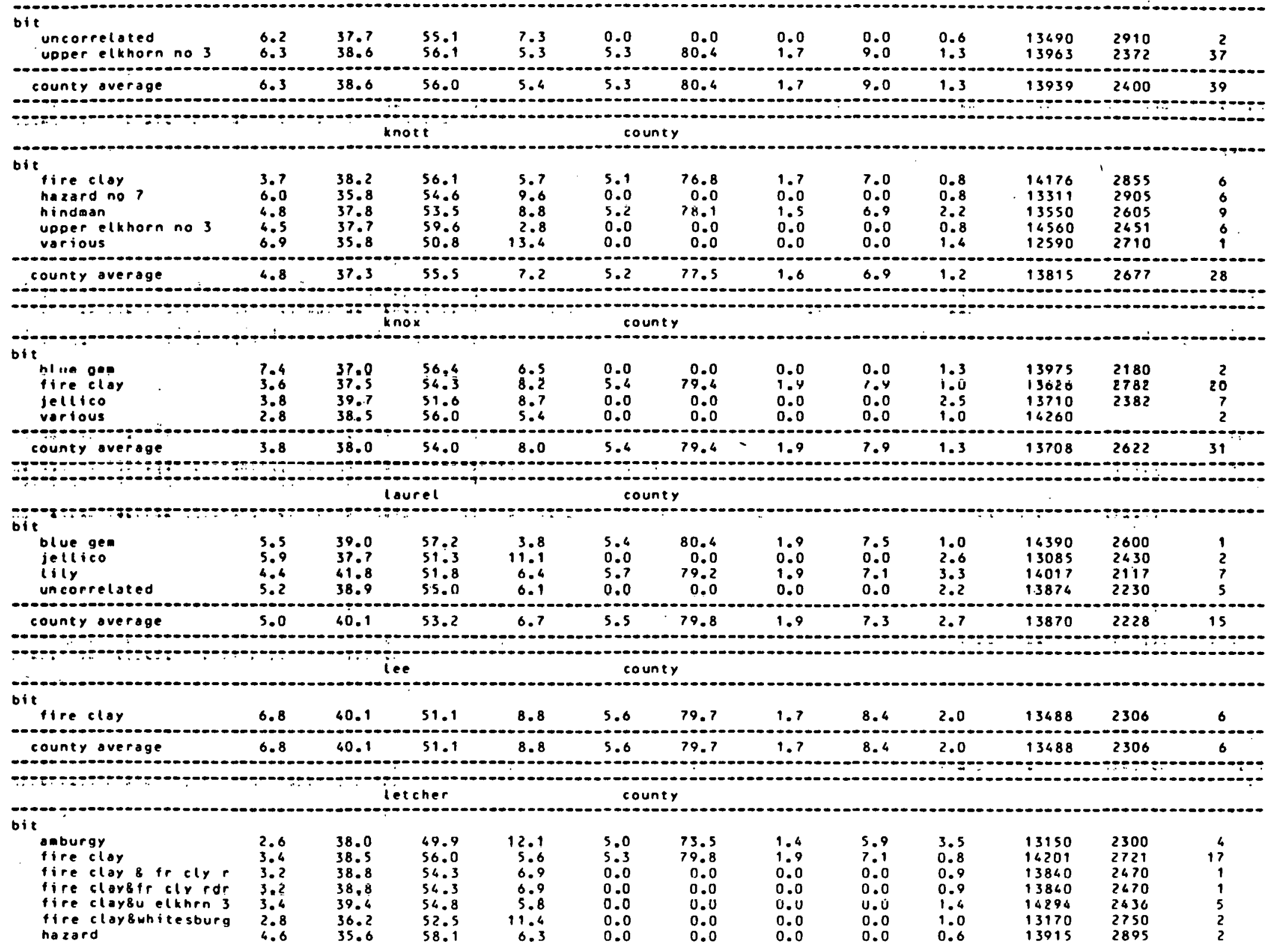




\begin{tabular}{|c|c|c|c|c|c|c|c|c|c|c|c|c|}
\hline $\begin{array}{l}\text { hazard no? } \\
\text { lower whitesburg } \\
\text { uelkhorn } 38 \text { whitsorg } \\
\text { uelkhorn nos } 1828 \\
\text { uelkhrn } 38 \text { elkhn rdr } \\
\text { upper elkhorn no } 3 \\
\text { various }\end{array}$ & $\begin{array}{l}5.4 \\
4.1 \\
5.5 \\
3.2 \\
5.4 \\
3.2 \\
3.8\end{array}$ & $\begin{array}{l}36.4 \\
36.3 \\
37.0 \\
37.0 \\
36.1 \\
38.5 \\
37.0\end{array}$ & $\begin{array}{l}53.6 \\
55.0 \\
54.5 \\
59.2 \\
56.0 \\
56.5 \\
54.2\end{array}$ & $\begin{array}{l}10.0 \\
8.7 \\
8.4 \\
3.2 \\
7.9 \\
5.0 \\
8.7\end{array}$ & $\begin{array}{l}0.0 \\
0.0 \\
0.0 \\
5.5 \\
0.0 \\
5.4 \\
0.0\end{array}$ & $\begin{array}{r}0.0 \\
0.0 \\
0.0 \\
83.2 \\
0.0 \\
81.0 \\
0.0\end{array}$ & $\begin{array}{l}0.0 \\
0.0 \\
0.0 \\
1.6 \\
0.0 \\
1.6 \\
0.0\end{array}$ & $\begin{array}{l}0.0 \\
0.0 \\
0.0 \\
6.5 \\
0.0 \\
6.7 \\
0.0\end{array}$ & $\begin{array}{l}0.7 \\
1.2 \\
1.5 \\
0.7 \\
1.1 \\
1.2 \\
1.2\end{array}$ & $\begin{array}{l}13300 \\
13566 \\
13635 \\
14636 \\
13610 \\
14331 \\
13636\end{array}$ & $\begin{array}{l}2910 \\
2736 \\
2650 \\
2583 \\
2600 \\
2368 \\
2545\end{array}$ & $\begin{array}{r}1 \\
3 \\
2 \\
6 \\
1 \\
60 \\
11\end{array}$ \\
\hline county & 3.4 & 38.1 & 55.9 & 6.0 & 5.4 & 80.9 & 1.6 & 6.7 & 1.2 & 14134 & 2687 & 116 \\
\hline & & & of $\operatorname{tin}$ & & & & & & & & & \\
\hline $\begin{array}{l}\text { Thindnan } \\
\text { oakley }\end{array}$ & $\begin{array}{l}6.8 \\
6.7\end{array}$ & $\begin{array}{l}36.8 \\
37.4\end{array}$ & $\begin{array}{l}55.0 \\
54.8\end{array}$ & $\begin{array}{l}8.2 \\
7.8\end{array}$ & $\begin{array}{l}0.0 \\
0.0\end{array}$ & $\begin{array}{l}0.0 \\
0.0\end{array}$ & $\begin{array}{l}0.0 \\
0.0\end{array}$ & $\begin{array}{l}0.0 \\
0.0\end{array}$ & $\begin{array}{l}0.9 \\
0.9\end{array}$ & $\begin{array}{l}13400 \\
13345\end{array}$ & $\begin{array}{l}2905 \\
2910\end{array}$ & $\begin{array}{l}8 \\
2\end{array}$ \\
\hline county average & 6.8 & 36.9 & 55.0 & 8.1 & 0.0 & 0.0 & 0.0 & 0.0 & 0.9 & 13389 & 2906 & 10 \\
\hline & & & in & & & & & & & & & \\
\hline $\begin{array}{l}\text { haddix } \\
\text { lower elkhorn }\end{array}$ & $\begin{array}{l}4.8 \\
6.8\end{array}$ & $\begin{array}{l}37.7 \\
37.8\end{array}$ & $\begin{array}{l}52.7 \\
53.8\end{array}$ & $\begin{array}{l}9.6 \\
8.4\end{array}$ & $\begin{array}{l}5.4 \\
5.1\end{array}$ & $\begin{array}{l}78.9 \\
76.4\end{array}$ & $\begin{array}{l}1.6 \\
1.6\end{array}$ & $\begin{array}{l}8.8 \\
1.4\end{array}$ & $\begin{array}{l}0.7 \\
i .1\end{array}$ & $\begin{array}{l}13363 \\
13670\end{array}$ & $\begin{array}{l}2899 \\
2680\end{array}$ & 12 \\
\hline county average & 4.7 & 37.7 & 52.8 & 9.5 & 5.3 & 77.6 & 1.6 & 8.1 & 0.7 & 13386 & 2879 & 13 \\
\hline & & & reary & & & & & & & & & \\
\hline $\begin{array}{l}\text { barren fork } \\
\text { beaver creek } \\
\text { hudson } \\
\text { hudsonsstearns } 11 / 2 \\
\text { jellico } \\
\text { lily } \\
\text { stearns no } 11 / 2 \\
\text { verious }\end{array}$ & $\begin{array}{l}3.2 \\
2.7 \\
3.5 \\
3.6 \\
6.0 \\
3.2 \\
4.4 \\
3.1\end{array}$ & $\begin{array}{l}49.3 \\
41.6 \\
40.4 \\
38.1 \\
35.3 \\
41.5 \\
39.0 \\
39.8\end{array}$ & $\begin{array}{l}47.6 \\
47.2 \\
51.2 \\
54.1 \\
52.9 \\
48.6 \\
55.6 \\
52.4\end{array}$ & $\begin{array}{r}11.2 \\
11.2 \\
8.4 \\
7.7 \\
11.8 \\
9.9 \\
5.5 \\
7.8\end{array}$ & $\begin{array}{l}0.0 \\
4.8 \\
0.0 \\
0.0 \\
4.9 \\
0.0 \\
0.0 \\
0.0\end{array}$ & $\begin{array}{r}0.0 \\
68.1 \\
0.0 \\
0.0 \\
73.3 \\
0.0 \\
0.0 \\
0.0\end{array}$ & $\begin{array}{l}0.0 \\
1.1 \\
0.0 \\
0.0 \\
1.7 \\
0.0 \\
0.0 \\
0.0\end{array}$ & $\begin{array}{l}0.0 \\
6.2 \\
0.0 \\
0.0 \\
7.1 \\
0.0 \\
0.0 \\
0.0\end{array}$ & $\begin{array}{l}4.0 \\
3.8 \\
2.2 \\
1.7 \\
1.2 \\
4.1 \\
0.5 \\
2.5\end{array}$ & $\begin{array}{l}13115 \\
13197 \\
13618 \\
13797 \\
13106 \\
13650 \\
16085 \\
13710\end{array}$ & $\begin{array}{l}2150 \\
2064 \\
2535 \\
2386 \\
2663 \\
2122 \\
2656\end{array}$ & $\begin{array}{r}2 \\
29 \\
19 \\
4 \\
3 \\
4 \\
16 \\
5\end{array}$ \\
\hline county average & 3.5 & 40.3 & 50.7 & 9.0 & 4.9 & 70.7 & 1.4 & 6.6 & 2.5 & 13535 & 2603 & 82 \\
\hline & & & lenber & & & - & & & & & & 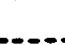 \\
\hline $\begin{array}{lll}\text { t no } & 11 \\
\text { no } & 12 \\
\text { no } & 14 \\
\text { no } & 6 \\
\text { no } & 9 \\
\text { nos } & 118 & \\
\text { various } & 12\end{array}$ & $\begin{array}{r}7.4 \\
7.4 \\
10.3 \\
8.7 \\
7.7 \\
7.0 \\
7.6\end{array}$ & $\begin{array}{l}42.1 \\
36.7 \\
39.7 \\
41.0 \\
40.0 \\
43.1 \\
40.7\end{array}$ & $\begin{array}{l}47.8 \\
46.1 \\
54.4 \\
50.1 \\
49.0 \\
48.0 \\
49.0\end{array}$ & $\begin{array}{r}10.1 \\
17.3 \\
6.0 \\
8.9 \\
11.0 \\
8.9 \\
10.3\end{array}$ & $\begin{array}{l}0.0 \\
0.0 \\
0.0 \\
5.1 \\
5.9 \\
0.0 \\
0.0\end{array}$ & $\begin{array}{r}0.0 \\
0.0 \\
0.0 \\
76.5 \\
72.6 \\
0.0 \\
0.0\end{array}$ & $\begin{array}{l}0.0 \\
0.0 \\
0.0 \\
1.7 \\
1.0 \\
0.0 \\
0.0\end{array}$ & $\begin{array}{l}0.0 \\
0.0 \\
0.0 \\
8.8 \\
8.5 \\
0.0 \\
0.0\end{array}$ & $\begin{array}{l}4.1 \\
3.9 \\
1.9 \\
3.4 \\
4.0 \\
3.5 \\
4.0\end{array}$ & $\begin{array}{l}12922 \\
19705 \\
13585 \\
13228 \\
1285 \\
13170 \\
12870\end{array}$ & $\begin{array}{l}2103 \\
2130 \\
2300 \\
2148 \\
2069 \\
2170 \\
2102\end{array}$ & $\begin{array}{r}34 \\
4 \\
2 \\
9 \\
319 \\
1 \\
15\end{array}$ \\
\hline county average & 7.7 & 40.2 & 48.9 & 10.8 & 5.1 & 73.6 & 1.6 & 8.6 & 4.0 & 12861 & 2079 & 384 \\
\hline
\end{tabular}




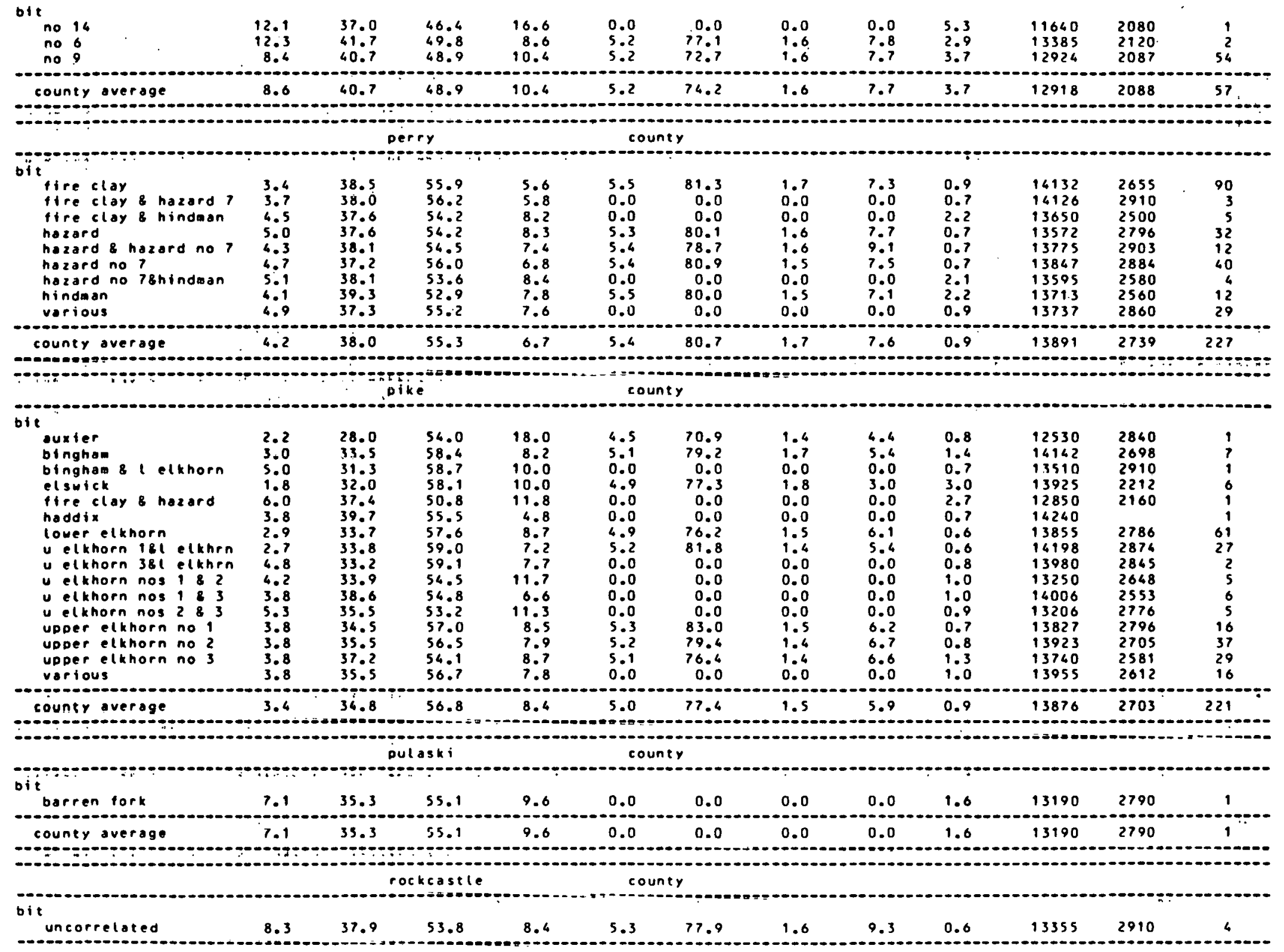




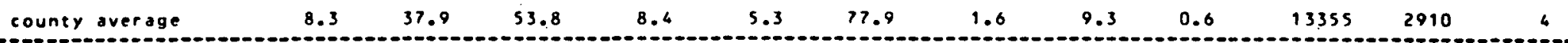

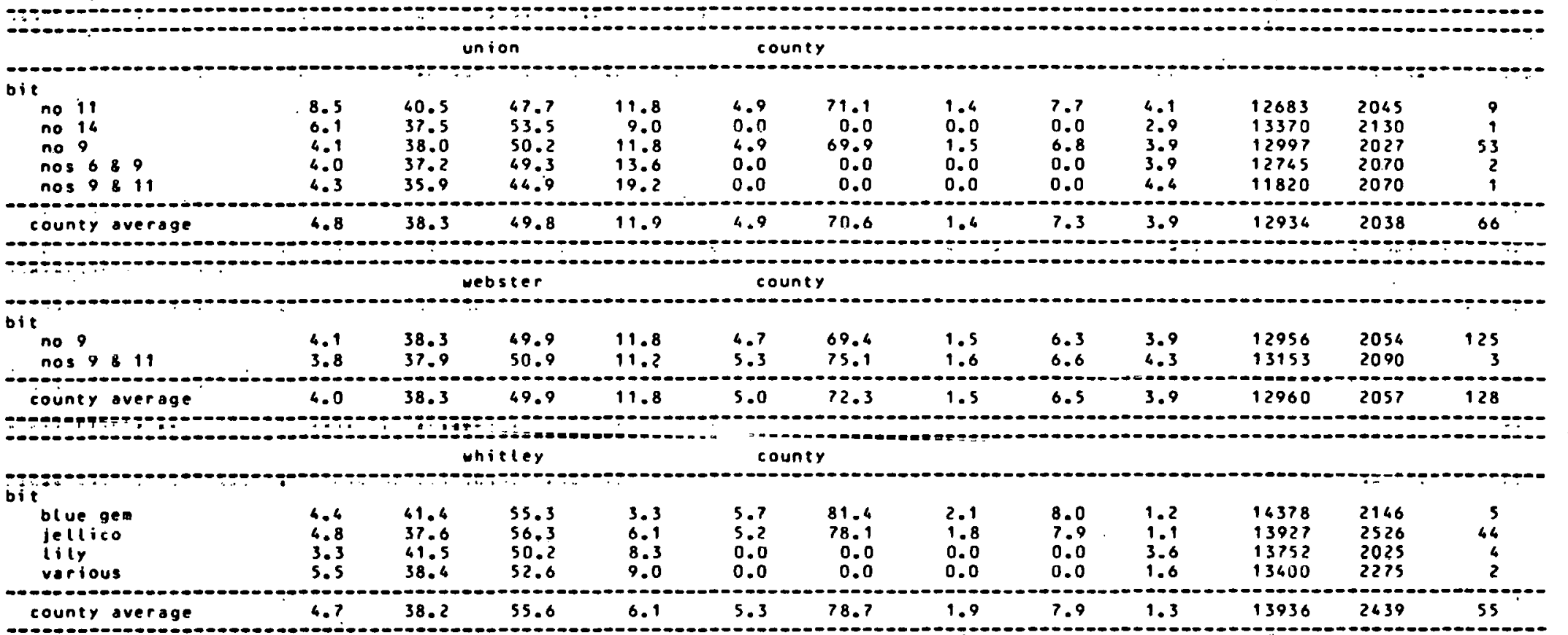

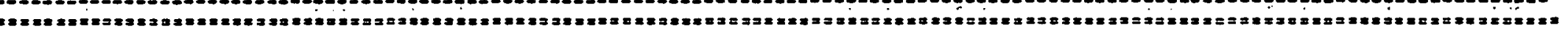


Rentuc ky

\begin{tabular}{|c|c|c|c|c|c|c|c|c|c|c|c|}
\hline 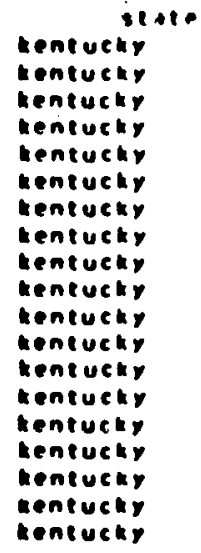 & $\begin{array}{l}\text { Dell } \\
\text { bell } \\
\text { bell } \\
\text { bell } \\
\text { bell } \\
\text { bell } \\
\text { bell } \\
\text { bell } \\
\text { bell } \\
\text { bell } \\
\text { bell } \\
\text { bell } \\
\text { bell } \\
\text { bell } \\
\text { bell } \\
\text { bell } \\
\text { bell } \\
\text { bell } \\
\text { bell }\end{array}$ & county & 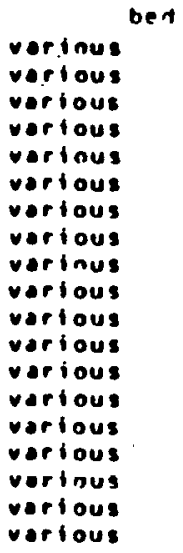 & $\begin{array}{l}00 \text { istur } \\
4.6 \\
4.6 \\
3.2 \\
3.9 \\
7.1 \\
6.7 \\
7.4 \\
6.5 \\
6.1 \\
8.2 \\
7.0 \\
3.0 \\
3.2 \\
3.3 \\
3.5 \\
4.2 \\
3.7 \\
4.5 \\
3.9\end{array}$ & $\begin{array}{l}\text { rol } \\
31.21 \\
37.2 \\
37.4 \\
35.3 \\
37.9 \\
36.8 \\
36.5 \\
36.0 \\
37.2 \\
32.4 \\
36.1 \\
39.2 \\
37.5 \\
37.2 \\
31.9 \\
31.9 \\
314.4 \\
38.8 \\
38.0\end{array}$ & $\begin{array}{l}11 \times e d c \\
53.9 \\
53.9 \\
53.8 \\
52.7 \\
52.2 \\
52.5 \\
51.7 \\
51.2 \\
54.2 \\
65.9 \\
69.1 \\
52.8 \\
54.1 \\
52.7 \\
52.8 \\
53.8 \\
52.9 \\
52.9 \\
52.9\end{array}$ & $\begin{array}{r}98 n \\
8.9 \\
8.9 \\
8.9 \\
11.8 \\
10.6 \\
10.8 \\
11.8 \\
12.8 \\
8.6 \\
21.8 \\
16.8 \\
8.0 \\
8.4 \\
10.1 \\
8.4 \\
1.1 \\
1.7 \\
1.3 \\
3.5\end{array}$ & $\begin{array}{l}\text { sulfur } \\
1.6 \\
1.94 \\
1.2 \\
1.0 \\
1.2 \\
1.2 \\
1.1 \\
1.0 \\
1.2 \\
1.7 \\
1.2 \\
1.3 \\
0.7 \\
0.9 \\
1.8 \\
1.3 \\
1.9 \\
1.9 \\
1.5\end{array}$ & $\begin{array}{l}760 \\
13680 \\
13410 \\
13610 \\
12960 \\
13130 \\
13110 \\
12870 \\
12660 \\
13580 \\
11170 \\
12010 \\
13760 \\
13660 \\
13370 \\
13580 \\
13660 \\
13560 \\
13620 \\
13590\end{array}$ & $\begin{array}{l}\text { Majlo } \\
0 \\
0 \\
0 \\
0 \\
0 \\
0 \\
0 \\
0 \\
0 \\
0 \\
0 \\
0 \\
0 \\
0 \\
0 \\
0 \\
0 \\
0 \\
0\end{array}$ & $\begin{array}{l}1.110 \\
6.0 \\
1.0 \\
1.5 \\
3.0 \\
3.0 \\
1.5 \\
1.5 \\
1.0 \\
6.5 \\
1.0 \\
1.0 \\
5.5 \\
3.0 \\
5.0 \\
4.0 \\
2.5 \\
4.5 \\
1.0 \\
4.5\end{array}$ \\
\hline
\end{tabular}

sontinued...... 


\begin{tabular}{|c|c|c|c|c|c|c|c|c|c|c|c|}
\hline \multicolumn{2}{|c|}{ state } & \multirow[t]{2}{*}{ enuney } & \multirow{2}{*}{ various bed } & \multicolumn{2}{|c|}{ moistur volma } & \multicolumn{2}{|c|}{ fixedc ash } & \multicolumn{2}{|c|}{ sulfur otu } & hạilo & fsilo \\
\hline kentucky & sell & & & 4.3 & 38.6 & 53.3 & 8.1 & 1.1 & 13680 & 0 & 4.0 \\
\hline kentucky & bell & & various & 4.0 & 38.2 & 54.4 & 7.6 & 0.9 & 13770 & 0 & $4: 0$ \\
\hline kentucky & belt & & various & 3.7 & 38.0 & 52.6 & 9.4 & 0.9 & 13600 & 0 & 3.0 \\
\hline kentucky & beil & & various & 6.3 & 39.1 & 53.9 & 8. 8 & 2.0 & 13480 & 0 & 1.5 \\
\hline kentucky & oell & & various & 5.? & 37.3 & 53.9 & 3.8 & 1.3 & 13660 & 0 & 5.0 \\
\hline kentucky & bell & & various & 4.5 & 37.8 & 53.? & 9.0 & 1.6 & 13470 & 0 & 1.0 \\
\hline kentucky & bell & & various & 3.0 & 37.3 & 53.2 & 9.5 & 1.4 & 13470 & 0 & 5.0 \\
\hline kentucky & bell & & varinus & 6.0 & 36.6 & 59.8 & 11.6 & 1.3 & 12960 & 0 & 1.5 \\
\hline kentucky & sell & & various & 5.5 & 37.9 & 53.3 & 3.8 & 1.3 & 13550 & 0 & 1.0 \\
\hline kentucky & bell & & various & 5.0 & 37.1 & 53.4 & 9.5 & 1.7 & 13380 & 0 & 5.5 \\
\hline kentucky & beli & $\$$ & various & 5.5 & 36.4 & $5 \times .1$ & 10.5 & 1.5 & 13200 & 0 & 1.0 \\
\hline kentucky & beli & & varinus & 0.5 & 35.0 & $5 \geqslant .6$ & 12.4 & 1.3 & 12830 & 0 & 2.5 \\
\hline kentucky & bell & & various & 4.5 & 36.8 & 54.9 & 9.3 & 1.0 & 13530 & 0 & 4.0 \\
\hline kentucky & bell & & various & 5.8 & 37.5 & 52.3 & 10.2 & 1.8 & 13240 & 0 & 5.0 \\
\hline kentucky & bell & & various & 4.7 & 37.8 & 53.4 & 8.8 & 1.6 & 13530 & 0 & 4.0 \\
\hline kentucky & beil & & varinus & o.? & 37.4 & 53.9 & 8.7 & $1 . ?$ & 13470 & 0 & 1.5 \\
\hline kentucky & nell & & various & 5.4 & 37.1 & 54.8 & 8.1 & 1.0 & 13560 & 0 & 1.5 \\
\hline Reriuck, & boil & & various & 5.5 & 37.2 & 53.1 & 9.7 & 1.1 & 13360 & 0 & 3.0 \\
\hline kentucky & bell & & various & $\cdots$ & $35=3$ & 56.1 & $8 n$ & 1.1 & 13330 & $n$ & 1.5 \\
\hline kentucky & Dell & & various & 6.8 & 35.1 & 54.4 & 10.5 & 1.4 & 13010 & 0 & 1.0 \\
\hline kentucky & bell & & various & 7.7 & $3 ? .9$ & 54.4 & 11.7 & 1.2 & 12850 & 0 & 1.5 \\
\hline kentiscky & Deil & & red soring & 4.6 & 39.0 & 52.7 & 8.3 & 1.1 & 13680 & 48 & 5.0 \\
\hline kentucky & bell & & red soring & 3.9 & $4 n .1$ & 47.4 & 12.5 & 4.0 & 13000 & 43 & 5.0 \\
\hline kentucky & beli & & red snring & 5.4 & $3 \% .3$ & 52.0 & 11.7 & 2.6 & 13080 & 45 & 5.5 \\
\hline kentucky & bell & & red soring & 5.2 & 36.1 & $4 \% .6$ & 16.3 & 2.9 & 12310 & 4.8 & 4.5 \\
\hline kentucky & beil & & fire clay & 7.1 & 37.3 & 56.9 & 5.8 & 0.9 & 13890 & $\ll 4$ & 2.0 \\
\hline kentucky & bell & & low solint & 5.8 & 36.4 & 56.6 & 7.0 & 0.8 & 13630 & 40 & 1.5 \\
\hline kentucky & bell & & low sntint & 3.6 & 35.4 & 54.8 & 9.8 & 0.8 & 13420 & 40 & 4.5 \\
\hline kentucky & beil & & low solint & 5.7 & 39.5 & 56.5 & 4.9 & 1.9 & 14210 & 43 & 5.5 \\
\hline kentucky & beli & & low solint & 5.0 & 39.2 & 56.5 & 5.2 & 1.6 & 14130 & 43 & 0.0 \\
\hline kentucky & bell & & various & 7.8 & 37.2 & 54.7 & 8.1 & 0.9 & 13520 & 0 & $? .5$ \\
\hline kentucky & bell & & varinus & 6.3 & 34.9 & 50.8 & 14.3 & 1.1 & 12590 & 0 & 1.0 \\
\hline kentucky & beli & & sandstone parting & 2.7 & 30.0 & 55.6 & 4.8 & 1.5 & 14360 & 0 & 6.0 \\
\hline kentucky & beli & & sandstone parting & 3.6 & 36.6 & 54.8 & 8.6 & 1.6 & 13760 & 0 & 6.5 \\
\hline kentucky & bell & & harlan & 5.1 & 36.4 & 58.9 & 4.7 & 0.7 & 14220 & $n$ & 1.0 \\
\hline kentuckv & Dell & & narlan & 4.1 & 37.1 & 56.8 & 0.1 & 0.8 & 14070 & 0 & 5.5 \\
\hline kentucky & bell & & narlan & 5.4 & 36.0 & 60.0 & 4.0 & 0.9 & 14360 & 0 & 5.0 \\
\hline kentucky & beii & & harlan & 2.9 & 39.8 & 57.8 & 3.4 & 0.9 & 14580 & 47 & 5.5 \\
\hline kentucky & Dell & & hartan & 3.4 & 34.7 & 51.2 & $1 \% .1$ & 0.9 & 12760 & 48 & 5.5 \\
\hline kentucky & heli & & harlan & 4.6 & 35.1 & 54.6 & 10.3 & 1.1 & 13430 & 49 & 7.5 \\
\hline kentucky & beli & & haddix & $3=1$ & 37.0 & 67.1 & 15.9 & 1.2 & 12120 & 43 & 1.0 \\
\hline kentucky & $b+i i$ & & fire clay & 3.? & $36 . ?$ & 46.9 & 18.8 & 9.2 & 11990 & 43 & 4.3 \\
\hline kentucky & beii & & sandstone oarting & 2.6 & ? ? ? & $; 1.1$ & 9.7 & 2.6 & 13620 & 0 & 7.0 \\
\hline kentucky & beli & & sandsinne oarting & 2.3 & 51.2 & 30.7 & 0.1 & 2.2 & 13690 & 59 & 7.5 \\
\hline kentucky & bell & & blue gem & 3.3 & 35.5 & 55.1 & 9.4 & 1.2 & 13670 & 4.3 & 6.5 \\
\hline kentucky & beli & & harlan 8 low splint & 2.4 & $36 . ?$ & 50.2 & 13.6 & $? 0$ & 12940 & 55 & 6.5 \\
\hline kentucky & bell & & jellico & $? .9$ & 30.4 & 50.5 & 4.0 & 0.9 & 14500 & 0 & 4.5 \\
\hline kentuckv & bell & & jellicn & 2.0 & 37.0 & 56.2 & 6.9 & 0.8 & 14020 & 0 & 0.0 \\
\hline kentucky & $0 \div 11$ & & jellico & 3.5 & 37.0 & 57.0 & 5.1 & 0.9 & 16351 & j & 6.5 \\
\hline Eentucky & $0: 11$ & & fire clay & 5.9 & 3.9 .1 & 54.3 & 7.6 & 0.7 & 13780 & 49 & 3.5 \\
\hline
\end{tabular}




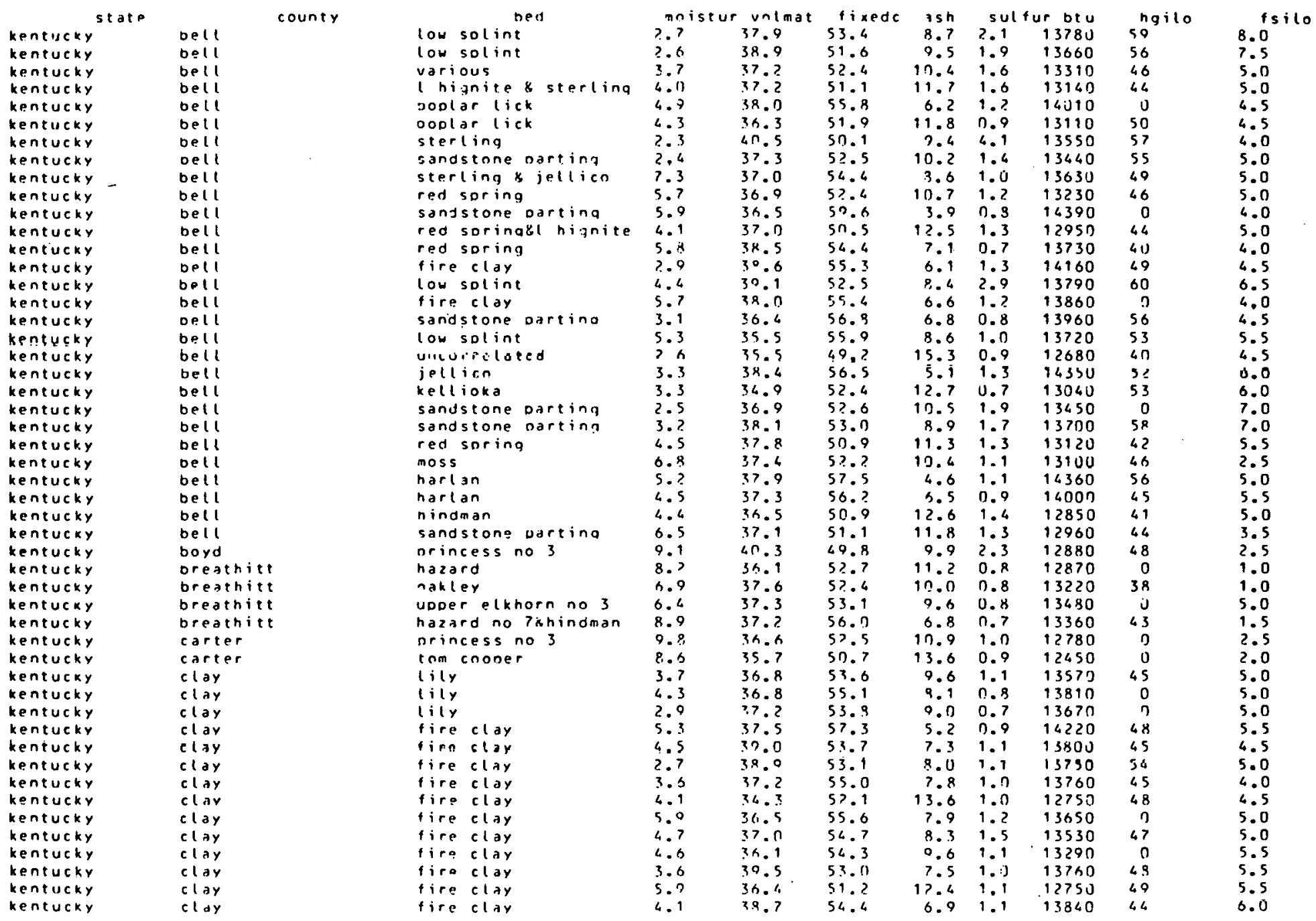




\begin{tabular}{|c|c|c|c|c|c|c|c|c|c|c|c|}
\hline . & state & county & bed & $m \cap i$ & if volmat & tixedc & As $n$ & sul & Ir btu & nailo & fsilo \\
\hline kentucky & $c \cos$ & lily & & 3.7 & 41.1 & 50.0 & 8.9 & $3 . ?$ & 13580 & 47 & 4.5 \\
\hline kentucky & $c l a y$ & fire & $c \operatorname{tay}$ & 5.5 & $3 \wedge .8$ & $5<.1$ & 9.1 & 1.0 & 13430 & $5 n$ & 5.0 \\
\hline kentucky & $c \operatorname{coy}$ & firr & clay & 3.5 & $4 n \cdot 0$ & 54.1 & 5.9 & 1.7 & 16070 & 46 & 4.5 \\
\hline kentucky & $c l a y$ & fire & ctay & 4.8 & 37.3 & 56.2 & 8.4 & 1.0 & 13030 & 47 & 5.0 \\
\hline kentucky & clay & fire & clay & 5.1 & 34.7 & 56.0 & 7.3 & 1.2 & 13730 & 48 & 5.0 \\
\hline kentucky & $c l a y$ & hazarr & d no 7 & 5.6 & 33.6 & $5 ? .4$ & 14.0 & 0.8 & 12590 & 46 & $? 0$ \\
\hline kentucky & $c l a y$ & uncor & related & $3 . ?$ & 30.2 & 48.3 & 12.5 & 3.1 & 13030 & 45 & 7.0 \\
\hline kentucky & clay & tire & $c t a y 81 i l y$ & 4.0 & 47.4 & $5 ? .4$ & 7.2 & 2.2 & 13900 & 0 & 4.5 \\
\hline kentucky & ctay & tire & clay & 4.? & 39.4 & 53.5 & 3.1 & 1.1 & 13760 & 48 & 5.0 \\
\hline kentucky & clay & fire & clay & 3.0 & $34 . ?$ & 53.9 & 7.9 & 1.0 & 13700 & 49 & 4.0 \\
\hline kentucky & clay & fire & clay & 4.1 & 37.7 & $5 ? .6$ & 9.7 & 1.9 & 13390 & 49 & 5.0 \\
\hline kentucky & clay & fire & clay & 4.5 & $3^{2} .2$ & 54.4 & 7.4 & 1.3 & 13740 & 0 & 4.0 \\
\hline kentucky & ctay & fire & clay & 4.5 & 37.0 & 52.0 & 11.0 & 2.9 & 13240 & 51 & 5.0 \\
\hline kentucky & clay & fire & clay & 5.0 & 38.7 & 53.9 & 7.6 & 1.1 & 13740 & $5 ?$ & 4.5 \\
\hline kentucky & ctay & fire & clay & 4.6 & 32.7 & 53.9 & 7.4 & 1.1 & 13740 & 51 & 4.0 \\
\hline kentucky & clay & $\operatorname{lily}$ & & 3.1 & 41.5 & $5 \pi . ?$ & 8.3 & 3.8 & 13720 & 45 & 5.0 \\
\hline kentucky & clay & tire & clay & 4.0 & 30.1 & 52.8 & 8.1 & 1.2 & 13710 & 68 & 4.0 \\
\hline bentucky & clay & fire & clay & 6.3 & 39.7 & 55.3 & 6.0 & 1.0 & 14100 & 47 & 5.0 \\
\hline kentucky & clay & ilne & (1) & 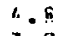 & 37.9 & $54 . ?$ & 7.9 & 0.8 & 13540 & 9 & 5.0 \\
\hline kentucky & clay & lily & & 3.7 & 41.3 & 51.2 & 7.5 & 3.8 & 13190 & $4 b$ & 2.0 \\
\hline kentucky & clay & lity & & 5.7 & 30.4 & 53.1 & 7.5 & 1.1 & 13860 & 50 & 5.0 \\
\hline kentucky & clay & itire & clay & 5.0 & 36.4 & 56.7 & 6.9 & 0.9 & 93840 & 0 & 5.0 \\
\hline kentucky & clay & tire & clay & 5.0 & 37.8 & 54.1 & 8.1 & 1.0 & 13630 & 0 & 4.5 \\
\hline kentueky & clay & fire & clay & 5.1. & 36.6 & 55.1 & 8.3 & 1.3 & 13600 & 50 & 0.0 \\
\hline kentucky & clay & tire & $c l a y$ & 5.0 & $3 n .9$ & 57.4 & 5.7 & 0.7 & 16200 & 0 & 5.0 \\
\hline kentucky & clay & fire & clay & 5.7 & 37.3 & 54.8 & 7.9 & 1.3 & 13870 & 49 & 3.5 \\
\hline kentucky & clay & fire & clay & 3.9 & 25.8 & 55.4 & 7.8 & 1.2 & 13940 & 48 & 5.0 \\
\hline kentucky & clay & fire & clay & 3.0 & 38.0 & 55.2 & 6.8 & 0.9 & $1<050$ & 0 & 4.5 \\
\hline kentucky & clay & fire & clay & $n .0$ & $3 \% .6$ & 55.0 & 10.4 & 0.9 & 13240 & 47 & 3.0 \\
\hline kentucky & $c l a y$ & fire & clay & 4.8 & 37.3 & 53.3 & 9.4 & 1.2 & 13510 & 0 & 3.5 \\
\hline kentucky & floyd & uncort & related & 6.9 & 27.1 & 54.6 & 8.3 & 0.6 & 1.3240 & 40 & 1.0 \\
\hline kentucky & floyd & voder & Plkhorn no 3 & 7.1 & 33.4 & 5 s. 0 & 8.6 & 0.8 & 13680 & 0 & 5.0 \\
\hline kentucky & floyd & upDer & elkhorn no 3 & 6.3 & 34.1 & 57.6 & 8.3 & 0.7 & 13790 & 48 & 5.0 \\
\hline kentucky & floyt & variou & sus & 4.9 &.$n .2$ & 59.2 & $1 ? .6$ & 1.5 & $130) 30$ & 0 & 5.5 \\
\hline kentucky & $410 Y 0$ & $u e(k)$ & khorn nos 14 ? & 3.6 & 39.3 & 49.6 & 11.1 & 1.9 & 13210 & 44 & 5.5 \\
\hline kentucky & floyd & voner & elkhorn no 1 & $4 \cdot 1$ & 39.4 & 53.0 & 8.6 & 0.8 & 13530 & 38 & 4.5 \\
\hline kentucky & + loyd & upoer & olkhorn no 1 & 4.1 & $\ln .0$ & 55.3 & 4.7 & 0.9 & 14370 & 36 & 5.5 \\
\hline kentucky & floyd & unver & - olkhorn no 1 & 4.9 & 36.3 & 55.5 & $8 . ?$ & 9.2 & 13650 & 0 & 5.5 \\
\hline kentucky & $f$ loyd & hadd $\mathbf{i}$ & ixkneach orchard & 5.4 & 35.1 & 50.7 & $14 . ?$ & 0.9 & 12440 & $4 ?$ & 1.5 \\
\hline kentucky & flord & varic & ous & 6.3 & 35.0 & 54.7 & 10.3 & 1.9 & 13240 & 47 & 3.0 \\
\hline kentucky & pioynt & uoper & eltharn nn? & 5.9 & 35,6 & 57.3 & 7.1 & 1.1 & 13810 & : & 3.5 \\
\hline kentucky & floyd & II $\rho \mid k$ & khorn nos? 43 & 8.3 & 35.0 & 53.1 & +1.0 & 1.3 & $i \geqslant 970$ & 0 & $4: 0$ \\
\hline kentucky & floyd & rer & elkhorn no? & ?.? & 43.8 & 59.3 & 4.9 & 3.0 & 14190 & 44 & 4.0 \\
\hline kentucky & floyd & varin & ous & 6.4 & 33.2 & 52.8 & 10.0 & 1.2 & 13390 & 65 & 4.5 \\
\hline kentucky & floya & uoner & elkhorn no 2 & 7.1 & 36.7 & 57.2 & 2.1 & 1.6 & 13610 & 0 & 3.5 \\
\hline kentucky & floyd & מoper & elkhorn no 1 & 7.5 & 34.4 & 52.0 & 12.6 & $1 . ?$ & 12860 & 0 & 4.0 \\
\hline kentucky & finyat & variou & us & 5.6 & 35.8 & 54.3 & 9.9 & 1.1 & $1336 n$ & 47 & 4.5 \\
\hline kentucky & floves & $u=(k)$ & knorn nns $1 \times 2$ & $? .1$ & 35.3 & 57.5 & $7 . ?$ & $1.1)$ & 13850 & 0 & 4.0 \\
\hline kentucky & floyd & $u e|k|$ & horn nos $1 \mathrm{k}$ ? & 0.3 & 34.3 & 54.1 & 11.6 & 1.2 & 13140 & 0 & 4.5 \\
\hline kentucky & floyd & inoer & elkhorn no ? & 6.4 & 35.5 & 56.6 & 7.9 & $1 . n$ & 13600 & ? & 3.5 \\
\hline
\end{tabular}




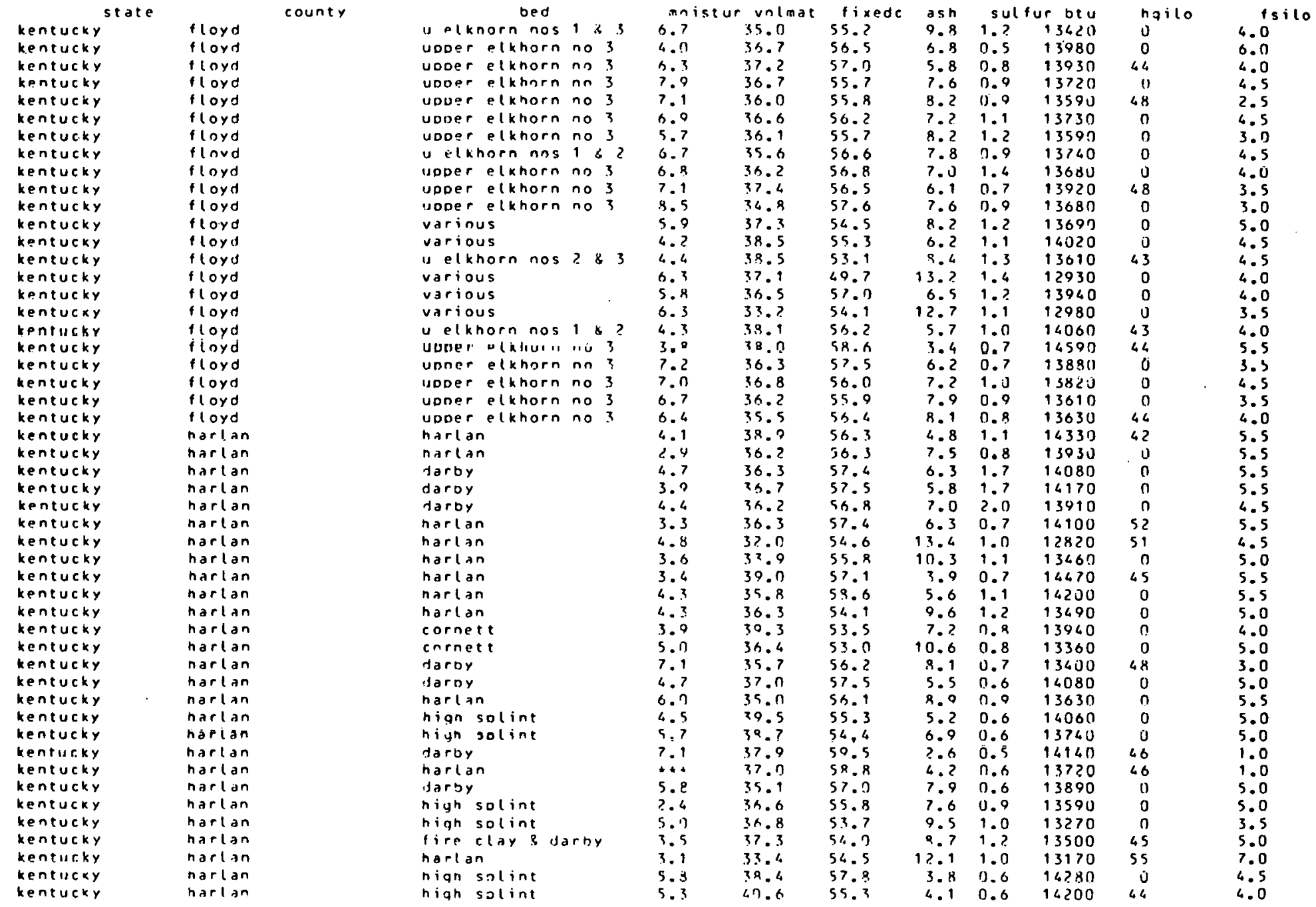




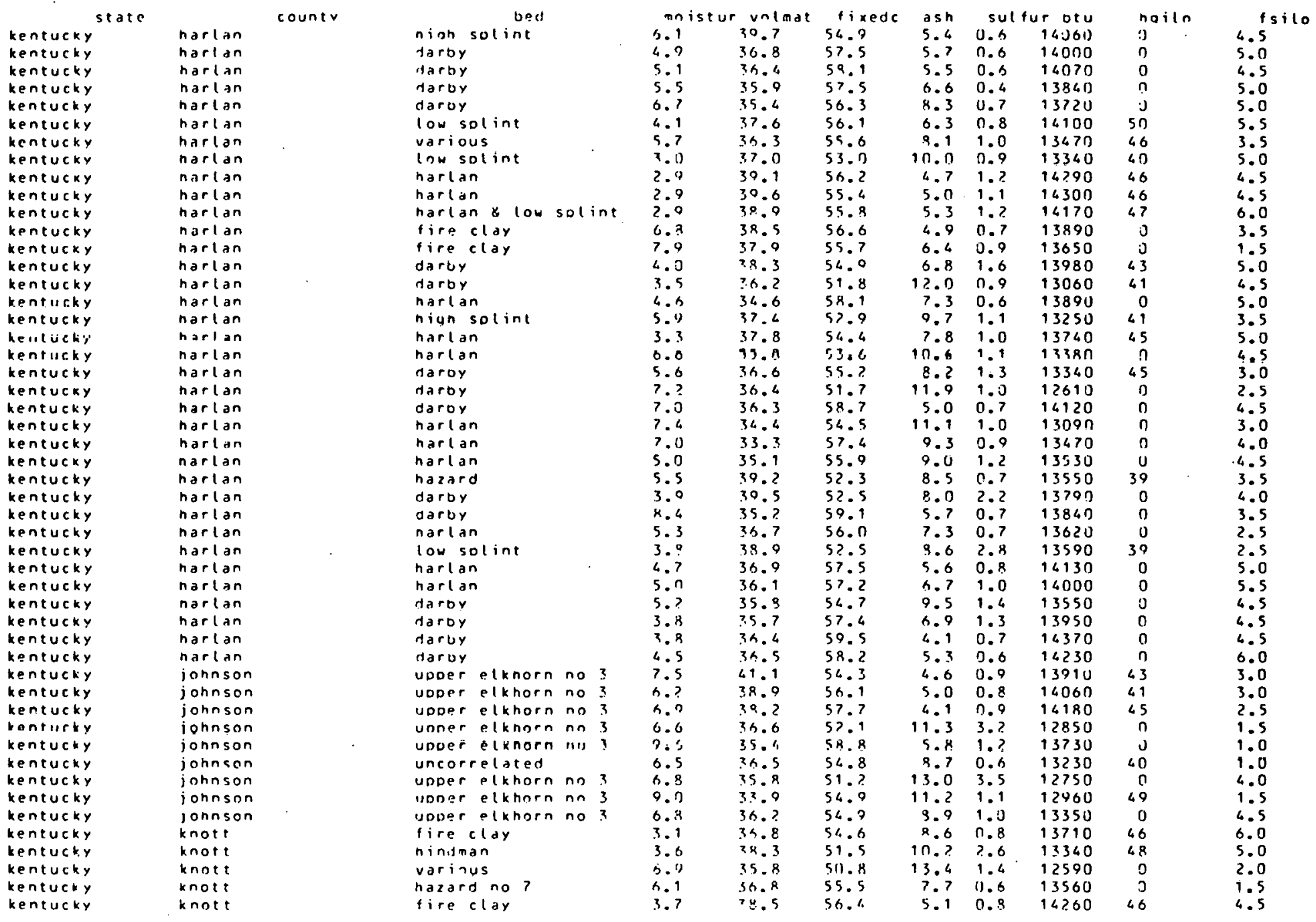




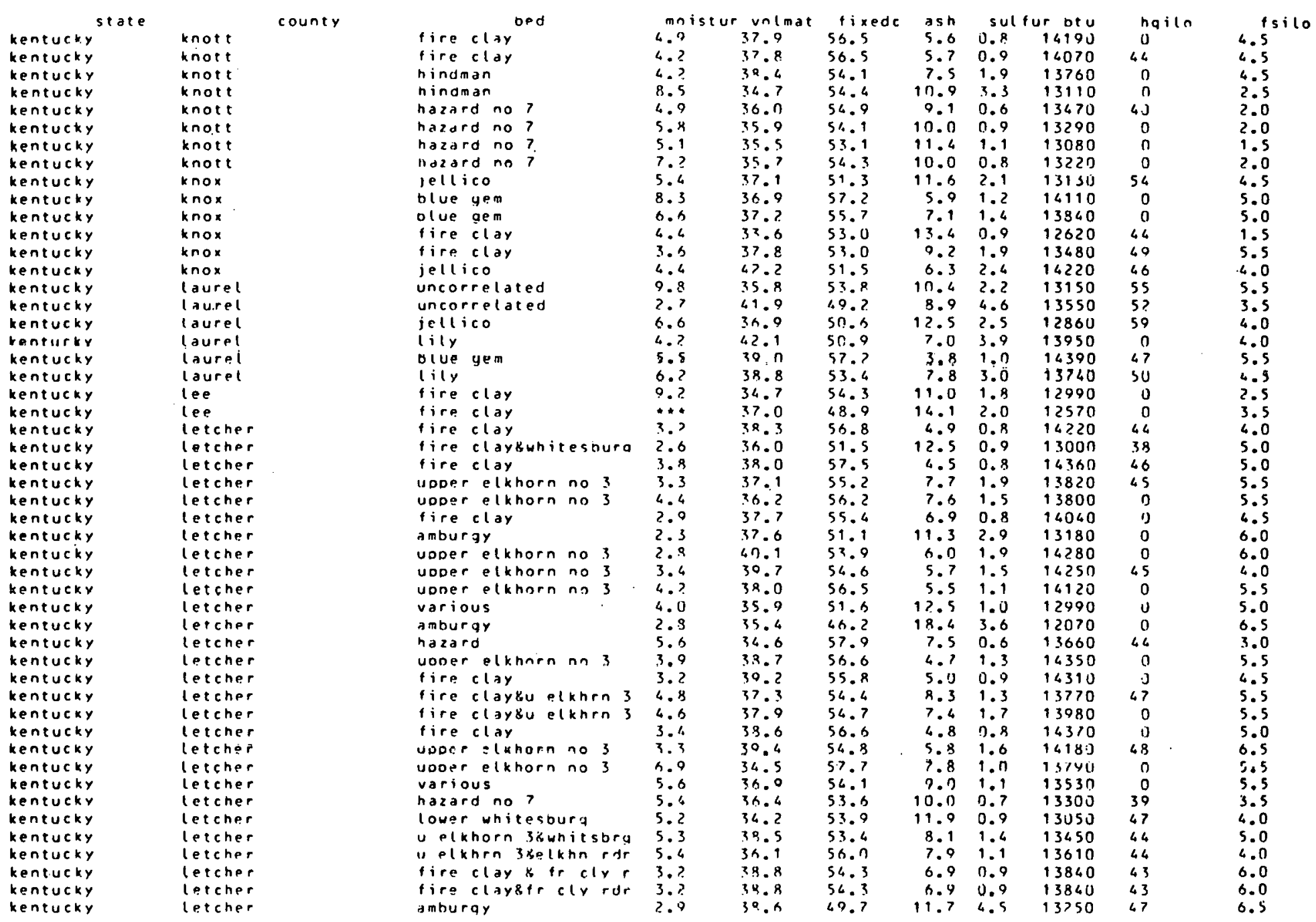




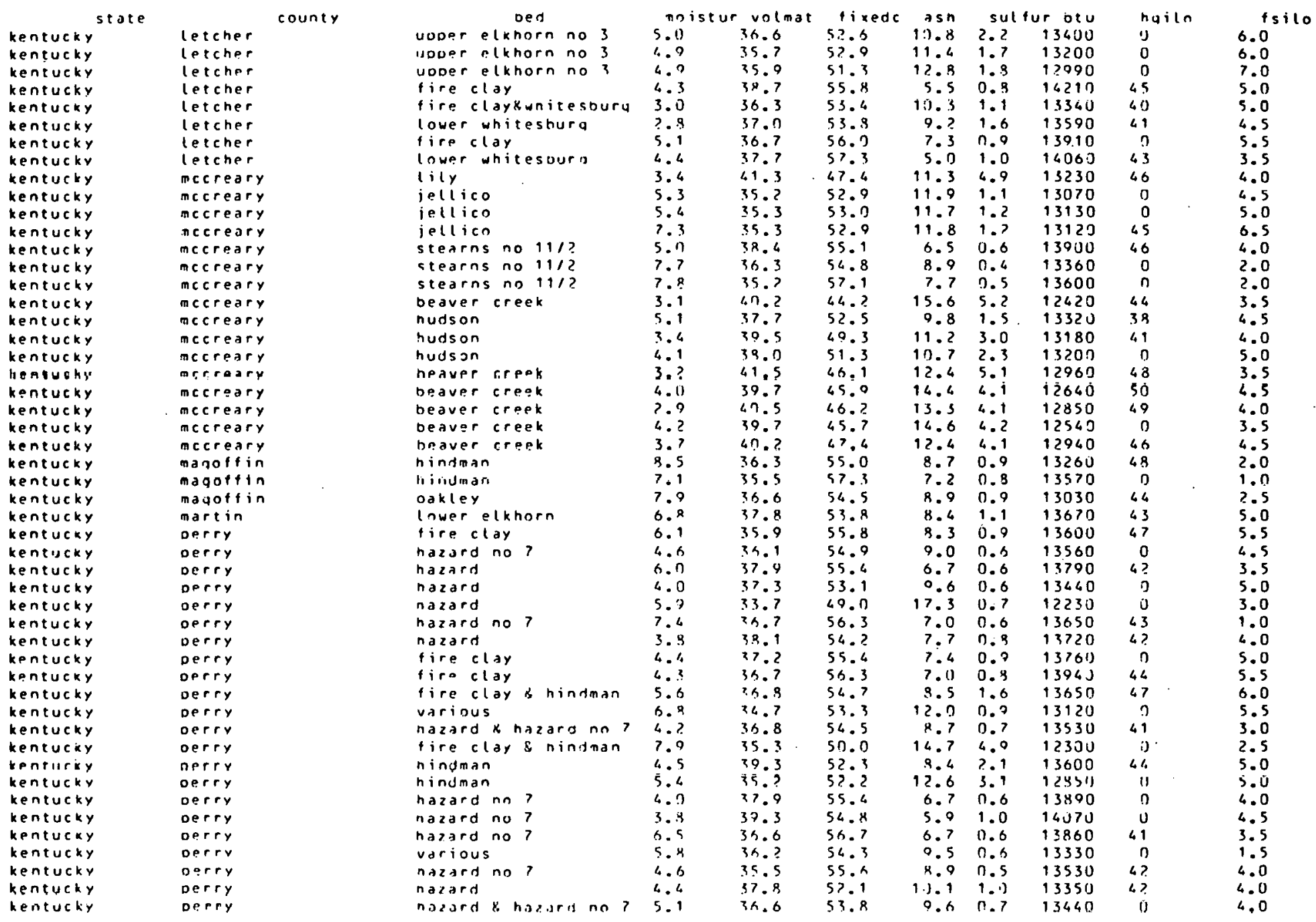




\begin{tabular}{|c|c|c|c|c|c|c|c|c|c|c|c|c|c|}
\hline & & county & & & bed & $\operatorname{mni}$ & r volmat & fixed & ash & sul & ur btu & hails & isilo \\
\hline kentucky & Derry & & nazard & no & & 4.5 & 36.3 & 55.3 & 8.6 & 0.5 & 13020 & 0 & 5.0 \\
\hline kentucky & Derry & & firec & $=1.3 y$ & & 3.1 & 37.8 & 55.4 & 0.8 & 0.8 & 13990 & 0 & 5.0 \\
\hline kentucky & Derry & & tirec & lay & & 4.7 & 37.7 & 54.7 & 7.6 & 0.9 & 13760 & 0 & 5.5 \\
\hline kentucky & perry & & tire $e$ & elay & & 3.8 & 36.0 & $5 ? .8$ & 10.2 & $n .9$ & 13330 & $n$ & 5.5 \\
\hline tentucky & perry & & nindma & & & 3.1 & 33.2 & 52.3 & 0.5 & 2.7 & $13441)$ & 40 & 5.0 \\
\hline kentucky & Derry & & hazard & no & $7 \mathrm{ghindman}$ & 4.5 & 36.9 & 54.0 & 9.1 & 2.2 & 13470 & $4 ?$ & 4.5 \\
\hline kentucky & oerry & & hazard & & & 6.3 & 32.2 & 54.4 & 7.4 & 0.7 & 13670 & D & 1.0 \\
\hline kentucky & Derry & & nazard & & & 7.7 & 37.7 & 54.9 & 7.4 & 0.6 & 13540 & 0 & 1.0 \\
\hline kentucky & oerry & & nazard & & & 6.5 & 36.8 & 54.1 & 9.1 & 0.7 & 13360 & 0 & 1.0 \\
\hline kentucky & Derry & & hazdrd & & & 5.8 & 37.9 & 53.8 & 9.1 & 0.6 & 13350 & 0 & 1.5 \\
\hline kentucky & Derry & & hazard & & & 5.6 & 38.0 & 53.1 & 8.9 & 0.7 & 13690 & 49 & 1.0 \\
\hline kentucky & Derry & & hazard & & & 7.0 & 37.5 & 53.5 & 9.0 & 0.6 & 13350 & 0 & 1.5 \\
\hline kentucky & Derry & & hazard & & & 4.3 & 37.1 & 54.0 & 8.9 & 0.6 & 13420 & 0 & 1.5 \\
\hline kentucky & Derry & & firec & lay & & 1.8 & 37.3 & 55.6 & 7.1 & 0.7 & 13950 & 48 & 6.0 \\
\hline kentucky & Derry & & hazard & no & 7 & 7.8 & 36.5 & 57.7 & 5.8 & 0.5 & 13810 & 42 & 1.5 \\
\hline kentucty & nerry & & nazard & & & 5.1 & 30.8 & 51.5 & 11.7 & 0.9 & 12820 & 47 & 4.5 \\
\hline kentucky & Derry & & hazard & & & 3.4 & 30.3 & 54.9 & 5.8 & 0.5 & 14110 & $3 x$ & 4.0 \\
\hline kentucky & Derry & & hindma & & & 4.4 & 38.6 & 57.1 & 9.3 & 2.5 & 13410 & 0 & 5.5 \\
\hline kentucky & Derry & & hindma & & & 5.1 & 38.3 & 51.8 & 90 & 1.9 & 13250 & 41 & 3.0 \\
\hline kentucky & perry & & varinu & & & 3.5 & 30.2 & 53.6 & 7.2 & 0.9 & 13880 & 0 & 4.5 \\
\hline kentucky & perry & & hazard & 48 & hazard no 7 & 4.5 & 36.7 & $5 ? .5$ & 10.8 & 1.0 & 13190 & 0 & 4.0 \\
\hline kentucky & werty & & hazord & 8 & hozord no? & 4.3 & 37.2 & 50,7 & 17.1 & 0.9 & 12910 & 40 & 4.0 \\
\hline kentucky & Derry & & nindma & $n$ & & 5.0 & 34.9 & 53.2 & 11.9 & 3.6 & 13070 & 0 & 5.0 \\
\hline kentucky & Derry & & nazard & no & 78 hindman & 8.5 & 34.3 & 51.1 & 14.6 & 3.3 & 12550 & 0 & 4.5 \\
\hline kentucky & Derry & & hazard & no & 7 & 6.7 & 36.6 & 54.1 & 9.3 & 0.7 & 131213 & 49 & 1.0 \\
\hline kentucky & Derry & & firec & $c l a y$ & & 1.9 & 37.0 & $57 . ?$ & 5.8 & 0.8 & 141110 & 53 & 6.0 \\
\hline kentucky & perry & & firec & clay & & 3.3 & 37.9 & 55.3 & 0.8 & 0.9 & 13870 & so & 5.0 \\
\hline kentucky & Derry & & fire c & clay & & 4.1 & 38.0 & 55.1 & 6.9 & 0.9 & 13980 & 44 & 4.5 \\
\hline kentucky & Derry & & firec & Clay & $x$ hazard 7 & 4.4 & 35.0 & 55.2 & 8.8 & 0.8 & 13640 & 0 & 5.5 \\
\hline kentucky & perry & & firec & Clay & & 3.1 & ห?.? & 57.0 & 4.8 & 0.8 & 14320 & 0 & 5.0 \\
\hline kentucky & Derry & & firec & clay & & 4.6 & 36.9 & 55.5 & 7.6 & 0.8 & 13880 & $n$ & 6.0 \\
\hline kentucky & Derry & & firee & clay & & $3 . ?$ & 30.6 & 54.4 & 6.0 & 0.9 & 14030 & 3 & 5.0 \\
\hline kentucky & dike & & lower & elk & knorn & 4.0 & 34.0 & 56.1 & 9.9 & 0.7 & 13570 & 48 & 7.0 \\
\hline kentucky & oike & & upper & elk & ehorn no 1 & 4.0 & 35.8 & 50.5 & 7.7 & 0.7 & 14090 & 0 & 6.5 \\
\hline kentucky & Dike & & under & elk & horn no 3 & 2.9 & 37.7 & 55.1 & $7 ?$ & 9.6 & 14130 & 0 & 6.0 \\
\hline kentucky & pike & & unner & $e l k$ & ghorn nn i & 0.9 & 34.9 & 57.9 & 7.2 & 0.0 & 13700 & 0 & 1.0 \\
\hline kentucky & pike & & $\begin{array}{l}\text { uoper } \\
\text { uop }\end{array}$ & elk & khorn no: & 7.0 & 34.0 & 50.8 & 5.3 & 11.5 & $1<270$ & 50 & 3.5 \\
\hline kentucky & pike & & lower & elk & shorn & 2.5 & 32.6 & 57.7 & 9.7 & 0.6 & 13940 & so & 8.0 \\
\hline kentucky & Dike & & under & elk & khorn no? & 6.5 & 35.3 & 311.1 & 14.6 & 1.1 & 123010 & 49 & 1.5 \\
\hline kentucky & Dike & & Inwer & $e l k$ & horn & 3.6 & 31.3 & 55.0 & 13.7 & 0.6 & 13130 & 53 & 9.0 \\
\hline kentucky & Dike & & lower & olk & ghorn & 2.5 & 37.4 & 55.6 & 12.0 & 0.8 & 13220 & 52 & 8.0 \\
\hline kentucky & nike & & upper & eik & hoorn no? & 6.4 & 34.8 & 56.7 & 8.5 & 0.7 & 13850 & 49 & 6.5 \\
\hline kentucky & nike & & binuna & & 10 & 2.5 & $2 ? .5$ & 56.9 & 9.6 & 1.2 & 13930 & 58 & 7.5 \\
\hline kentucky & oike & & unner & $e l k$ & xharn no 3 & 3.1 & $? 8.9$ & 53.7 & 7.5 & 1.5 & 14003 & 64 & 6.0 \\
\hline kentucky & wike & & variou & & & 4.0 & 31.7 & $50 . ?$ & 9.1 & 1.0 & 14010 & 0 & 8.0 \\
\hline kentucky & pike & & bingha & & & $? .1$ & 33.6 & 58.6 & 7.8 & 1.6 & 14250 & 64 & 7.0 \\
\hline kentucky & pike & & under & $e l k$ & shorn no? & 4.1 & 38.0 & 54.3 & 7.7 & 1.0 & 13800 & 46 & 4.5 \\
\hline kentucky & oike & & unner & elk & khorn an? & 3.7 & 38.2 & 55.01 & 6.8 & 1.0 & 14040 & 42 & 5.5 \\
\hline kentuery & nikp & & uuper & plk & horn no 3 & 2.11 & 3 sh. 3 & 53.6 & 10.1 & 2.4 & 13610 & 49 & 6.0 \\
\hline kentucky & Dike & & udoer & $e i k$ & chorn no 1 & 3.7 & 37.7 & 56.5 & 12.8 & 0.9 & 13160 & 51 & 0.5 \\
\hline
\end{tabular}




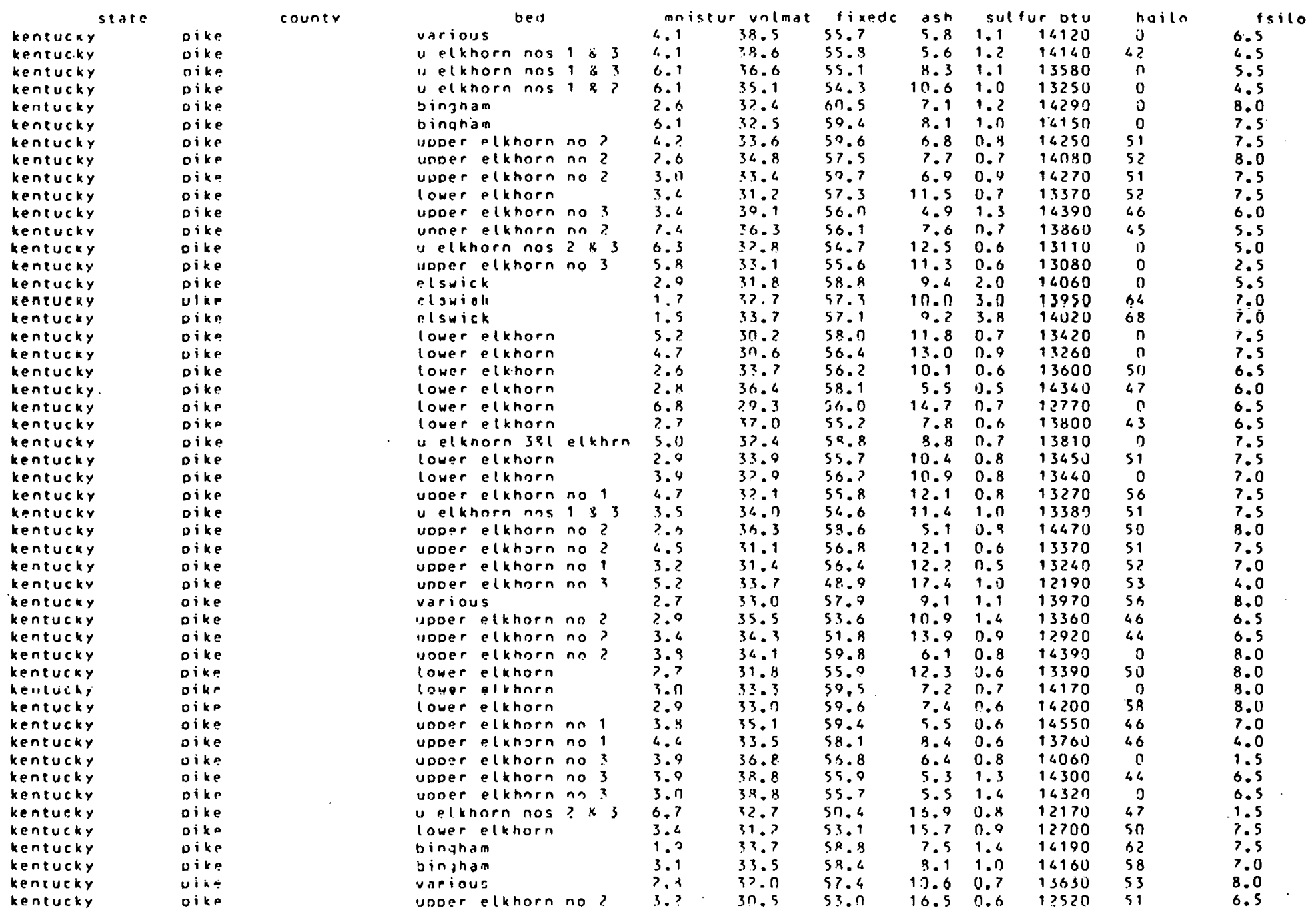




\begin{tabular}{|c|c|c|c|c|c|c|c|c|c|c|c|c|}
\hline & county & \multicolumn{3}{|c|}{ bed } & \multicolumn{2}{|c|}{ moistur volmat } & \multicolumn{2}{|c|}{ fixede ash } & \multicolumn{2}{|c|}{ sulfur btu } & hailn & tsilo \\
\hline kentucky & oike & lower & elknorn & & 2.5 & 31.9 & 34.9 & 13.2 & 0.8 & 13150 & 52 & 8.0 \\
\hline kentucky & oike & lower & elkhorn & & 4.11 & 33.4 & $5 \times .6$ & 13.0 & $n .9$ & 12860 & 48 & 5.5 \\
\hline kentucky & oike & u elkn & orn $1 \& 1$ & elkhrn & 3.6 & $3 \because 0$ & 50.3 & 7.7 & 0.7 & 14120 & 0 & 7.0 \\
\hline kentucky & pike & u elkn & inrn isi & elkhen & $5 . ?$ & 33.1 & 60.2 & 6.7 & 0.6 & 14230 & 0 & 7.5 \\
\hline kentucky & vike & inger & elknorn & & 3.6 & 36.1 & 58.6 & 7.3 & 0.9 & 16160 & 54 & 7.5 \\
\hline kentucky. & Dike & wDDer & elkhorn & no $>$ & 4.9 & 35.2 & on. 3 & 4.5 & 0.7 & 14450 & 0 & 0.0 \\
\hline kentucky & oike & uoder & elkhorn & no 2 & 4.3 & 36.0 & 56.5 & 7.5 & 1.4 & 14030 & 48 & 5.5 \\
\hline kentucky & pike & Wooer & olkhorn & nn? & 5.? & 34.4 & 57.3 & 8.3 & 0.7 & 13680 & 0 & 1.5 \\
\hline kentucky & pikn & uDDet & elknorn & no? & 3.0 & $3 R .4$ & S3.? & 5.4 & 1.0 & 14350 & 45 & 7.0 \\
\hline kentucky & pike & upper & elkhorn & no? & 3.1 & 35.6 & 59.6 & 4.8 & 0.7 & 14530 & 52 & 7.5 \\
\hline kentucky & oike & upper & elkhorn. & no 3 & 2.7 & $36 . n$ & 55.1 & 8.9 & 1.6 & 13840 & 46 & 7.5 \\
\hline kentucky & pike & firec & lay \& ha & azard & 0.0 & 37.4 & SO.8 & 11.8 & 2.7 & 12850 & 51 & 5.5 \\
\hline kentucky & oike & $u$ elkn & orn nos & $2 \times 3$ & 4.6 & 37.0 & 54.3 & 8.7 & 0.9 & 13710 & 45 & 5.5 \\
\hline kentucky & oike & upoer & elkhorn & no" 1 & 2.0 & 36.9 & 59.7 & 3.4 & 0.6 & 14720 & 44 & 7.0 \\
\hline kentucky & Dike & UDDer & elknorn & no 3 & 6.? & 35.0 & 47.3 & 17.7 & 2.6 & 12220 & 0 & 5.5 \\
\hline kentucky & ulke & Uno? & olthnorn & $\mathrm{nn}\}$ & 4.? & 35.5 & $4>.3$ & 17.2 & 2.1 & 12310 & 47 & 6.0 \\
\hline kentucky & oike & uoder & plkhorn & no 3 & 5.3 & 34.2 & 46.6 & 19.2 & 1.4 & I UIU & u & 3.9 \\
\hline kentucky & Dike & UDDer & elkhorn & no 3 & 6.7 & 37.8 & $4 \% .1$ & 20.1 & 1.8 & 11800 & 0 & 5.0 \\
\hline kentucky & Dike & lower & elkhorn & & 5.9 & 32.7 & 55.9 & 11.4 & 0.5 & 13270 & 0 & 4.0 \\
\hline kentucky & oike & lower & elkhorn & & 2.5 & $3 n .3$ & 53.3 & 16.4 & 0.5 & 12690 & 0 & 7.5 \\
\hline kentucky & pike & lover & elkhorn & & 2.6 & 37.6 & on. 1 & 7.3 & 0.6 & 14200 & 57 & 8.5 \\
\hline kentucky & Dike & bingtha & on & & 2.9 & 35.1 & 56.0 & 8.9 & 2.4 & 14030 & 63 & 7.0 \\
\hline kentucky & Dike & oingha & $m \& 1$ el & lkhorn & 5.0 & 31.3 & 58.7 & 10.0 & 0.7 & 13510 & 60 & 6.5 \\
\hline kentucky & pike & varinu & & & 3.4 & 30.0 & 56.3 & 4.7 & 9.0 & 14300 & 0 & 5.0 \\
\hline kentucky & Dike & variou & & & 3.3 & 37.8 & 56.4 & 5.8 & 0.8 & 14230 & i) & 5.0 \\
\hline kentucky & oike & udoer & elkhorn & no 3 & 3.5 & 39.4 & 56.0 & 4.6 & 1.2 & 16360 & 40 & 0.0 \\
\hline kentucky & oike & uoper & etkhorn & no 3 & 4.0 & 30.4 & 55.1 & 5.5 & 9.1 & $14 \$ 10$ & $4 ?$ & 4.0 \\
\hline kentucky & pike & uooer & plkhorn & no? & 3.3 & 34.07 & 55.1 & 10.2 & 0.8 & 13520 & 45 & 6.5 \\
\hline kentucky & Dike & upper & elkhorn & no 1 & 3.5 & 34.7 & 57.0 & 8.3 & 0.8 & 13830 & 48 & 6.0 \\
\hline kentucky & oike & udoer & elkhorn & no 1 & 3.4 & 34.5 & $57 . ?$ & 8.3 & 1.0 & 13720 & so & 0.5 \\
\hline kentucky & pike & variou & & & 4.4 & 36.5 & 51.9 & 11.6 & 1.6 & 13190 & 46 & 5.5 \\
\hline kentucky & pike & $u$ el kh & inrn nos & $? \& 3$ & 5.6 & 36.2 & 54.6 & 0.2 & 1.2 & 13480 & 0 & 6.0 \\
\hline kentueky & Dike & uelkh & forn nos & 182 & 3.7 & $3 ? .9$ & 56.3 & 10.8 & 0.8 & 13650 & u & 6.5 \\
\hline kentucky & Dike & uoper & elkhorn & no 3 & 5.0 & 32.6 & 56.6 & 10.8 & 1.0 & 13440 & so & 7.0 \\
\hline kentucky & oike & lower & elkhorn & & 3.5 & $3 ? .6$ & 57.6 & 9.8 & ก. 8 & 13710 & 59. & 7.0 \\
\hline kentucky & Dike & Inwer & elkhorn & & 2.1 & 33.6 & 60.7 & 5.7 & 0.9 & 14580 & 39 & 8.5 \\
\hline kentucky & bike & isooer & elkhorn & no 1 & 3.5 & 30.5 & 55.9. & 13.6 & 0.7 & 13110 & 58 & 8.0 \\
\hline kenlucky & Dike & $\| P / k h$ & nen nos & $1 \& ?$ & 2.9 & 31.9 & 55.4 & $1 ? .7$ & 0.6 & 13300 & 53 & 3.0 \\
\hline kentucky & oike & 1) elkh & iorn nos & 182 & 4.3 & $3 \geqslant .1$ & 59.6 & 16.5 & 1.3 & 12500 & 57 & 8.0 \\
\hline kentucky & oike & lower & elkhorn & & ? $=?$ & 31.4 & 58.8 & 9.8 & 0.7 & 13840 & 56 & 8.5 \\
\hline kentucky & Dike & lower & elkhorn & & $\because .7$ & 31.4 & 57.0 & 11.6 & 0.6 & 13550 & So & 9.0 \\
\hline kentucky & pike & uoper & elkhorn & no? & 4.1 & 34.0 & 57.5 & 3.5 & 0.7 & 13910 & 49 & 6.5 \\
\hline kentucky & Dike & variou & 5 & & 3.6 & 37.0 & 54.7 & 8.3 & 1.7 & 13860 & 46 & 7.0 \\
\hline kentucky & Dikp & unner & elkhorn & no? & 4.6 & 34.0 & 57.7 & 7.4 & 0.6 & 13900 & 0 & 6.0 \\
\hline kentucky & o ike & vooer & - lkhorn & กก 3 & 5.3 & 35.0 & 58.5 & 5.6 & 0.0 & 14240 & 53 & 6.0 \\
\hline kentucky & oike & u elktis & orn $3 \times 1$ & nlkhen & 4.7 & 33.9 & 59.4 & 6.7 & 0.9 & 14950 & 57 & 7.0 \\
\hline kentucky & pulaski & barren & fork & & 7.1 & 35.3 & 55.1 & 0.6 & 1.6 & 13100 & 48 & 3.5 \\
\hline kentucky & rorkgastle & uncorr & elated & & 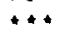 & 35.0 & 53.3 & 11.7 & 0.4 & 12610 & 50 & 1.5 \\
\hline kentucky & whillay & jellic & 0 & & 7.4 & 37.4 & 56.8 & 5.9 & 1.3 & 16010 & 48 & 4.5 \\
\hline kentucky & whitley & jellic & & & 8.8 & 35.4 & 57.6 & 7.0 & 0.9 & 13620 & 57 & 1.0 \\
\hline
\end{tabular}




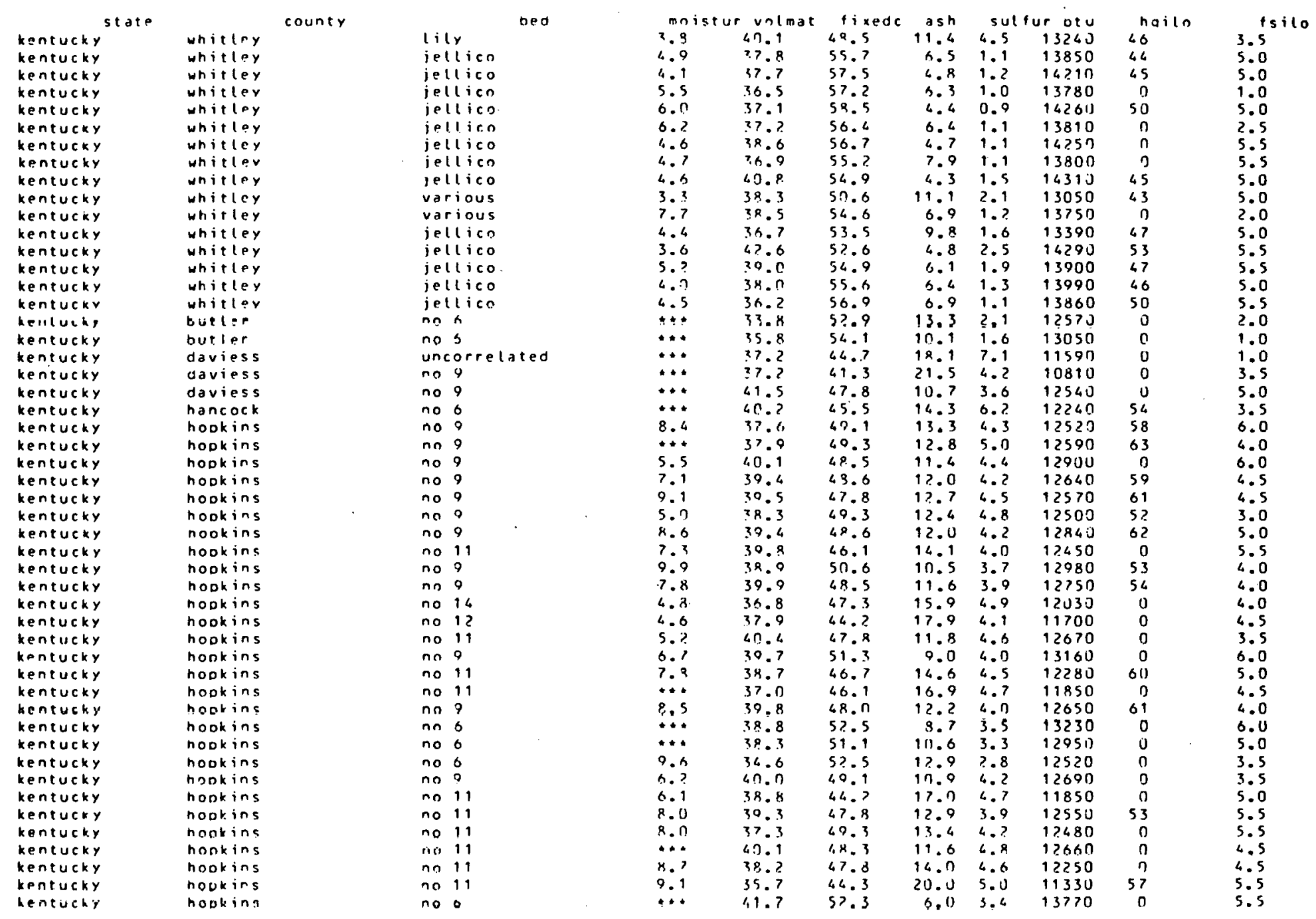




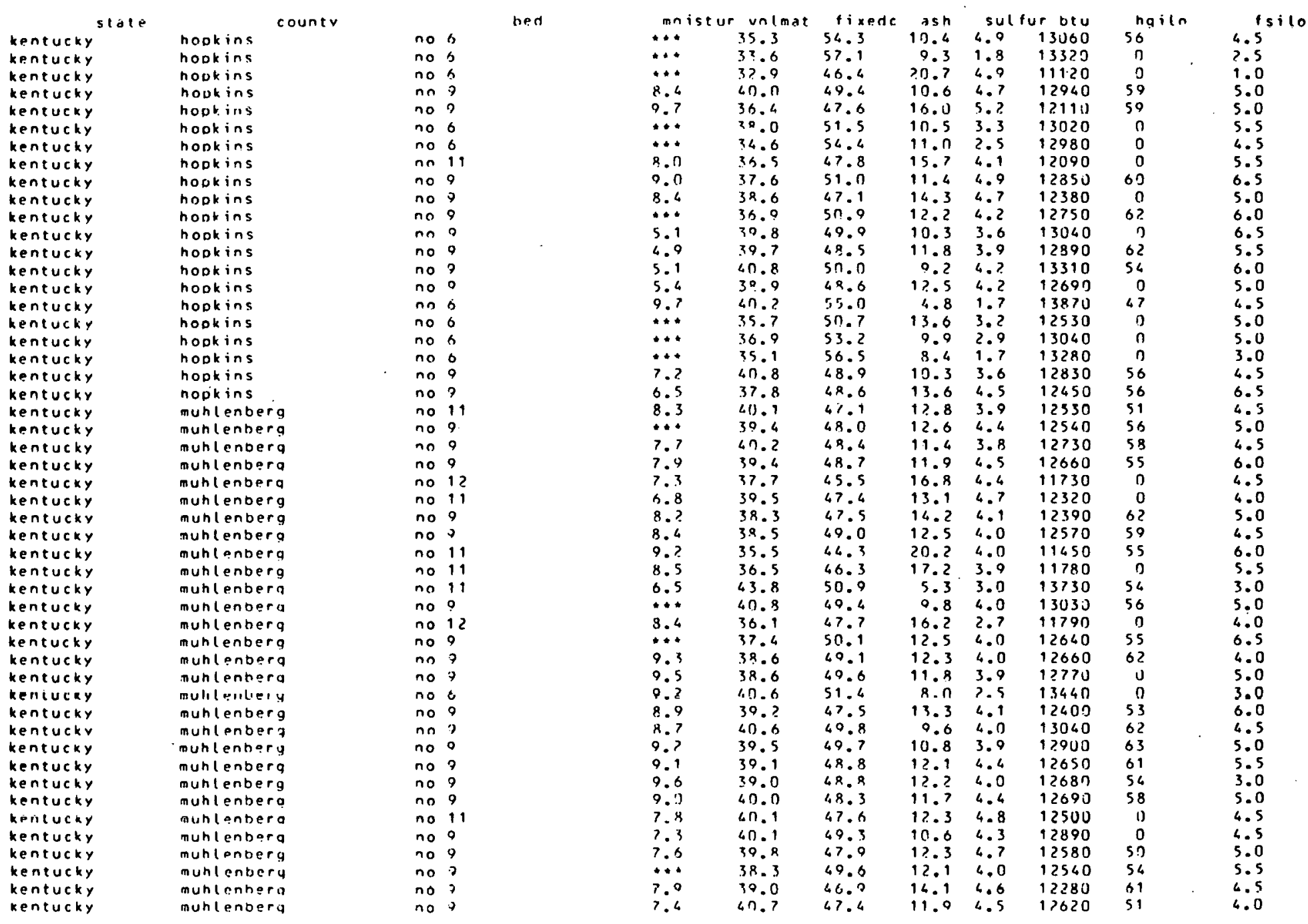




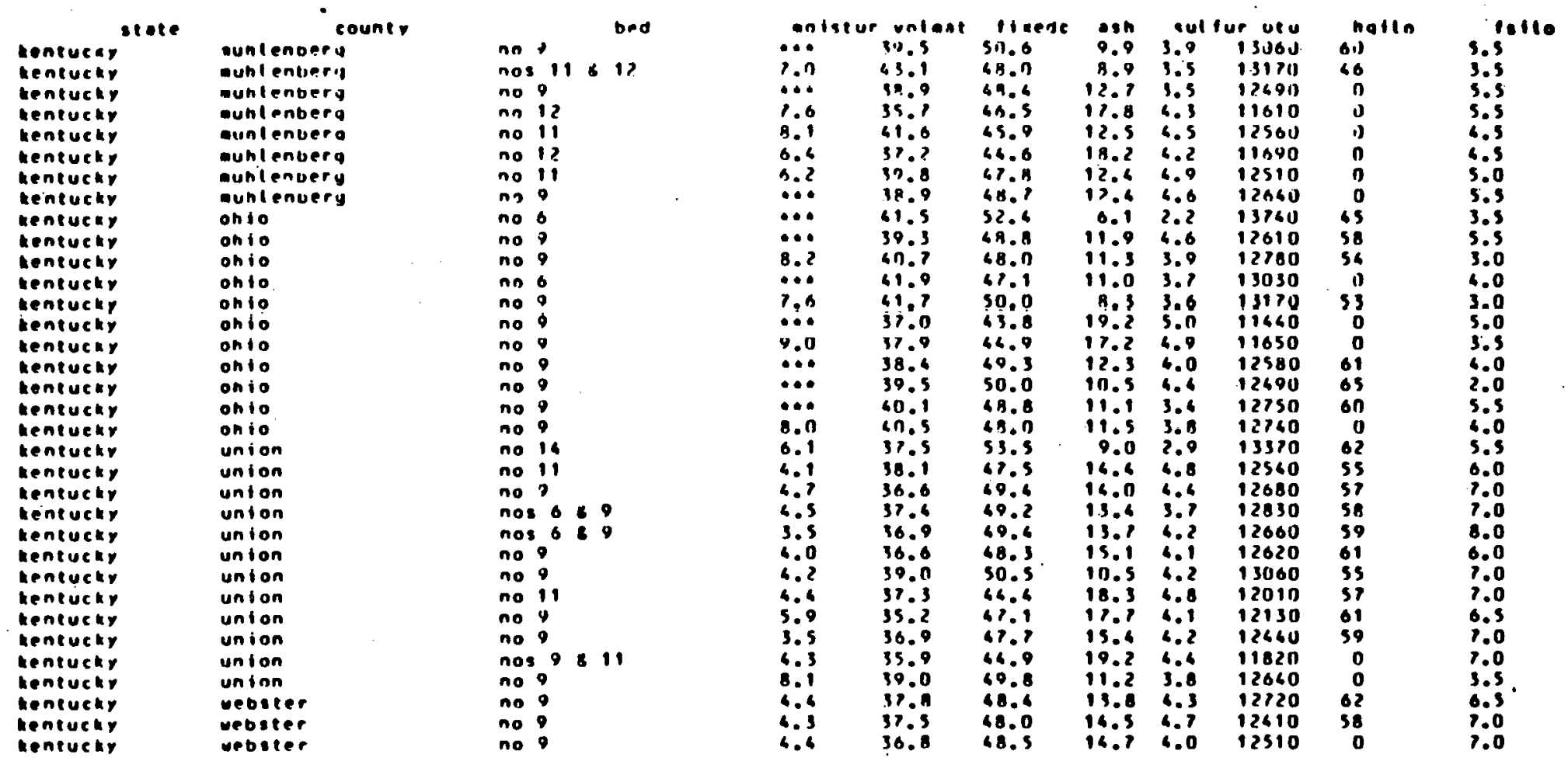



National Coal Resources Data Systea

U.S. Geological survey

identified coal resources in maryland

(In elllions of short tons)

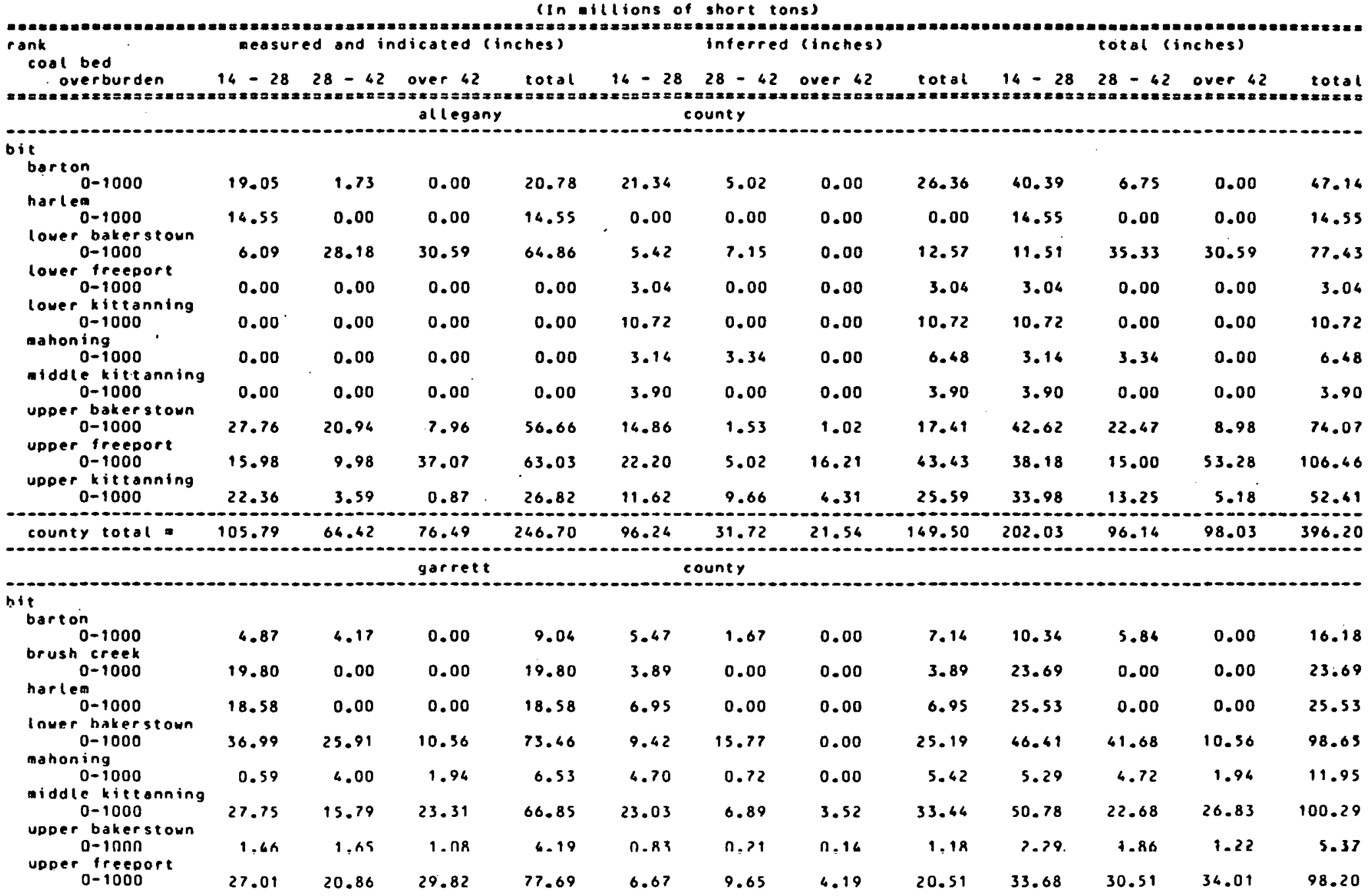




\begin{tabular}{|c|c|c|c|c|c|c|c|c|c|c|c|c|}
\hline $\begin{array}{c}\text { UDDer } \\
0-1000\end{array}$ & 35.70 & 32.09 & 0.00 & 67.79 & 12.52 & 2.63 & 0.00 & 15.15 & 48.22 & 34.72 & 0.00 & 82.94 \\
\hline county total & 172.75 & 104.47 & 66.71 & 343.93 & 73.48 & .37 .56 & 7.85 & 118.87 & 246.23 & 142.01 & 76.56 & 662.80 \\
\hline $\begin{array}{c}\text { overburden } \\
\text { subtotal } \\
0-1000\end{array}$ & 278.54 & 168.89 & 143.20 & 590.63 & 169.72 & 69.26 & 29.39 & 268.37 & 468.26 & 238.15 & 172.59 & 859.00 \\
\hline $\begin{array}{l}t o t a l \\
y=z=02\end{array}$ & $\begin{array}{l}278.54 \\
==2=8=0\end{array}$ & $\begin{array}{l}168.89 \\
=x=x=2\end{array}$ & 143.20 & 590.63 & $\begin{array}{l}169.72 \\
x=x=x=2\end{array}$ & $\begin{array}{l}69.26 \\
:=2 x=3=\end{array}$ & 29.39 & 268.37 & 468.26 & 238.15 & 172.59 & $\begin{aligned} 859.00 \\
=x=20 x\end{aligned}$ \\
\hline
\end{tabular}

\begin{tabular}{|c|c|c|c|c|c|c|c|c|c|c|c|c|}
\hline $0-1000$ & 278.54 & 168.89 & 143.20 & 590.63 & 169.72 & 69.26 & 29.39 & 268.37 & 448.26 & 238.15 & 172.59 & 859.00 \\
\hline & 36 & 168.89 & 143.20 & 590.63 & 169.72 & 69.26 & 29.39 & 268.37 & 448.26 & 238.15 & 172.59 & \\
\hline
\end{tabular}
grand total $=278.56 \quad 168.89 \quad 143.20 \quad 590.63 \quad 169.72 \quad 69.26 \quad 29.39 \quad 268.37 \quad 448.26 \quad 238.15 \quad 172.59 \quad 859.00$

resource figures taken from:

$\begin{array}{lll}\text { source } & \text { year } & \text { base year of resource } \\ \text { usbm bull SO7 } & 1952 & 1950 \\ \text { usbor tech paper } 725 & 1949 & \text { original }\end{array}$


overage analyses of coal in marylano

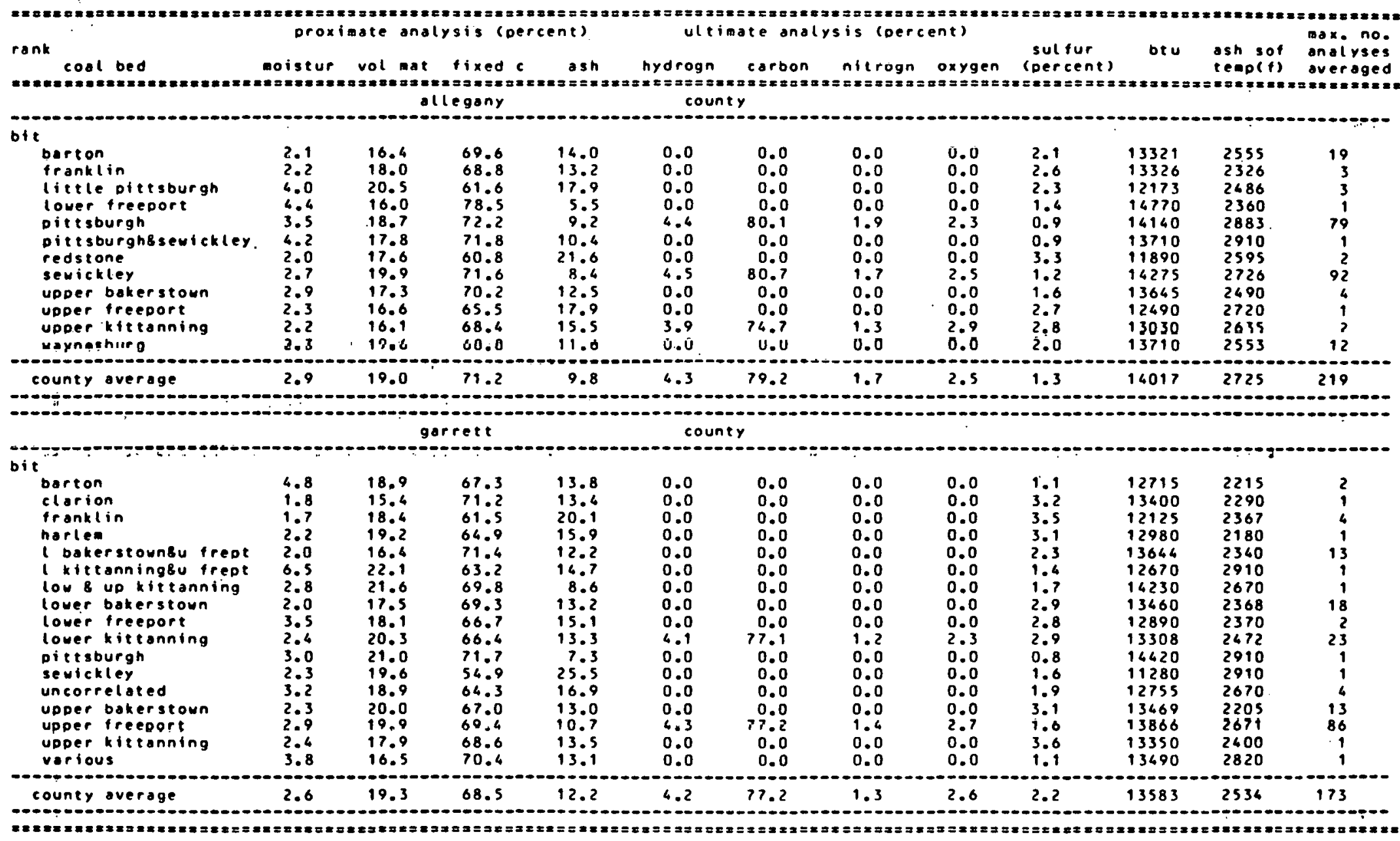


Maryland

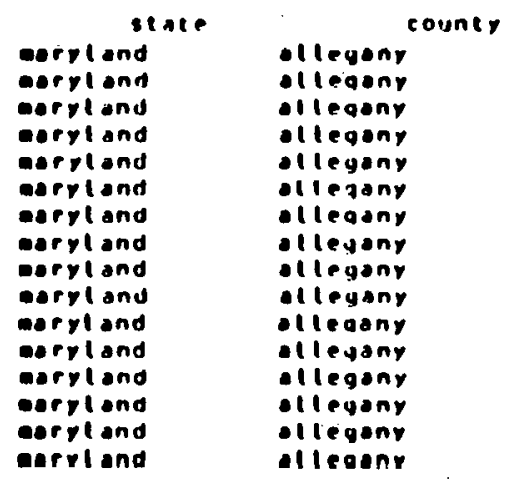

\begin{tabular}{|c|}
\hline 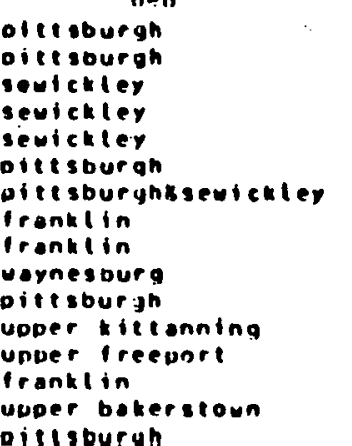 \\
\hline
\end{tabular}

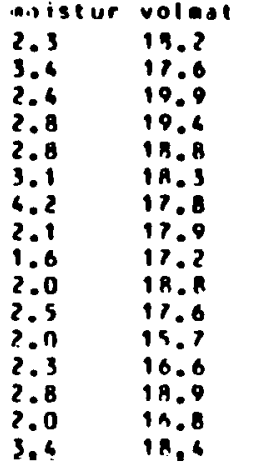

Higede
70.3
68.3
68.8
71.2
69.0
11.1
71.8
70.3
69.2
64.1
72.9
69.3
65.5
61.0
60.8
71.6

asn sultur ptuo

$\begin{array}{lll}11.3 & 0.9 & 13860 \\ 14.1 & 1.2 & 13320 \\ 11.3 & 1.2 & 13880\end{array}$

$0.4 \quad 1.2 \quad 1480$

$12.21 .7 \quad 13660$

$10.60 .9 \quad 13490$

10.40 .913710

$13.6 \quad 3.6 \quad 13270$

9.50 .914110

$17.92 .7 \quad 12490$

$16.13 .1 \quad 13210$

$\begin{array}{lll}22.6 & 7.8 & 11880 \\ 11.0 & 1.0 & 13830\end{array}$

$\begin{array}{cc}\text { ngilo } & 9.8110 \\ 0 & 9.0 \\ 0 & 9.0 \\ 0 & 8.5 \\ 0 & 9.0 \\ 0 & 9.0 \\ 99 & 3.5 \\ 0 & 3.0 \\ 0 & 9.0 \\ 0 & 9.0 \\ 0 & 8.5 \\ 0 & 5.5 \\ 0 & 8.5 \\ 0 & 9.0 \\ 0 & 9.0 \\ 0 & 8.0\end{array}$

continued...... 


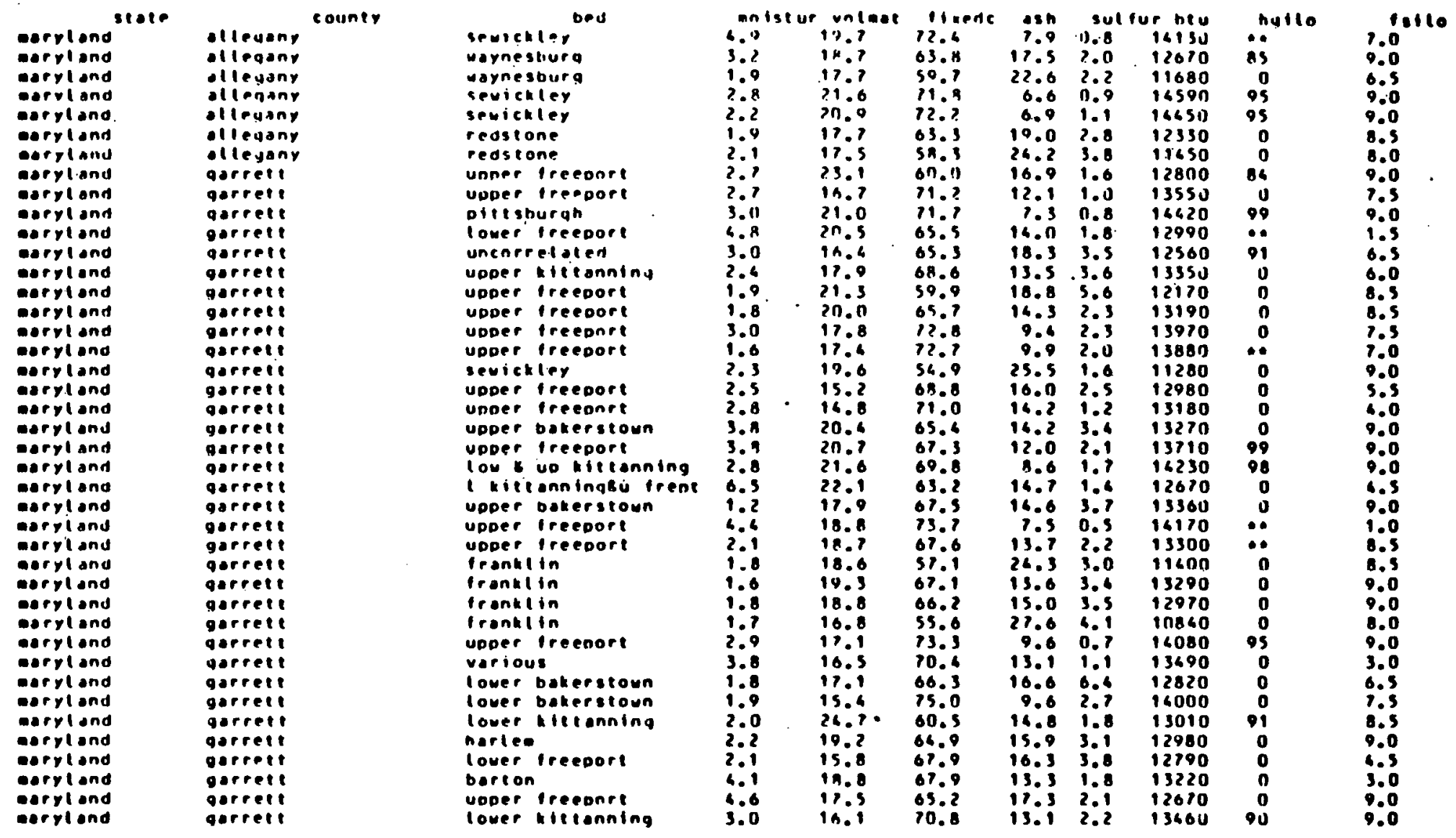



National coal Resources data system

U.S. Geological surver

identified coal resources in atchigan

(In millions of short tons)

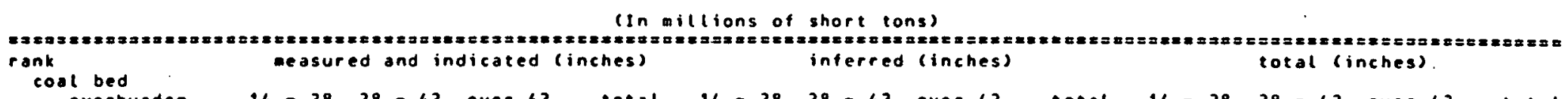

coal bed $14-2828-42$ over 42 averbyrden $28-42$ over 42 lotal $14-28$

(10tal

bit

\begin{tabular}{|c|c|c|c|c|c|c|c|c|c|c|c|c|}
\hline $0-1000$ & 6.20 & 34.82 & 7.64 & 48.66 & 0.00 & 0.00 & 0.00 & 0.00 & 6.20 & 34.82 & .7 .64 & 48.66 \\
\hline county total & 6.20 & 34.82 & 7.64 & 48.66 & 0.00 & 0.00 & 0.00 & 0.00 & 6.20 & 34.82 & 7.64 & 48.66 \\
\hline
\end{tabular}

bit

\begin{tabular}{|c|c|c|c|c|c|c|c|c|c|c|c|c|}
\hline $0-1000$ & 0.00 & 0.94 & 0.00 & 6.94 & 0.00 & 0.00 & 0.00 & 0.00 & 0.00 & 6.96 & 0.00 & 6.94 \\
\hline county tote & 0.00 & 6.94 & 0.00 & 6.94 & 0.00 & 0.00 & 0.00 & 0.00 & 0.00 & 6.96 & 0.00 & 6.94 \\
\hline
\end{tabular}

bit

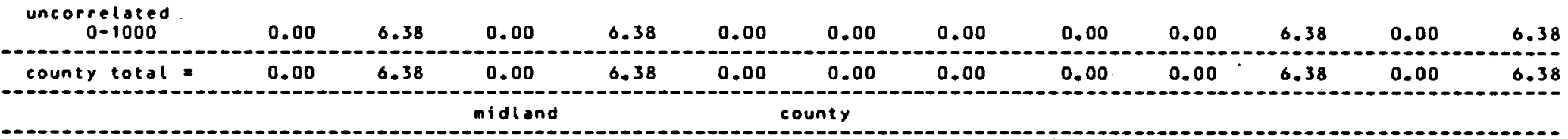

bit
uncorrelated

\begin{tabular}{|c|c|c|c|c|c|c|c|c|c|c|c|c|}
\hline $0-1000$ & 1.32 & 0.00 & 0.00 & 1.32 & 0.00 & 0.00 & 0.00 & 0.00 & 1.32 & 0.00 & 0.00 & 1.32 \\
\hline county & 1.32 & 0.00 & 0.00 & 1.32 & 0.00 & 0.00 & 0.00 & 0.00 & 1.32 & 0.00 & 0.00 & 1.32 \\
\hline
\end{tabular}

bit

uncorrelated
$0-1000$

\begin{tabular}{|c|c|c|c|c|c|c|c|c|c|c|c|c|}
\hline $\begin{array}{c}\text { uncorrelated } \\
0-1000\end{array}$ & 11.27 & 18.46 & 4.15 & 33.88 & 0.00 & 0.00 & 0.00 & 0.00 & 11.27 & 18.46 & 4.15 & 33.88 \\
\hline total & 11.27 & 18.46 & 4.15 & 33.88 & 0.00 & 0.00 & 0.00 & 0.00 & 11.27 & 18.46 & 4.15 & 33.88 \\
\hline
\end{tabular}

bit

shiawassee county

uncorrelated

$\begin{array}{lll}0.99 & 2.02 & 0.00\end{array}$

3.01

0.00

0.00

0.00

0.00

0.99

$2.02 \quad 0.00$

3.01 


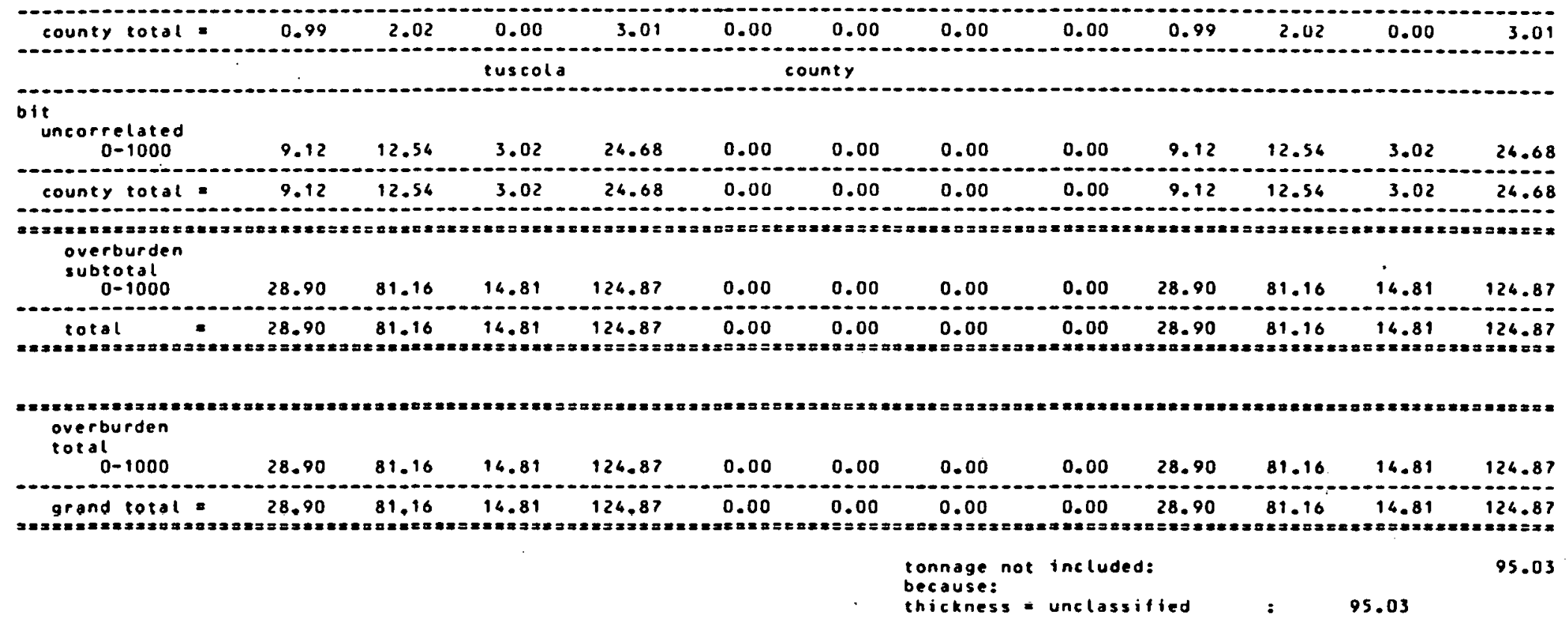

total tonnage of identified coal resources in michigan

219.90

resource figures taken from:

$\begin{array}{lll}\text { source } & \text { year } & \text { base year of resource } \\ \text { usgs circ } 77 & 1950 & 1950\end{array}$

records containing unclassified thickness and/or reliabiltty

\begin{tabular}{|c|c|c|c|c|c|c|c|c|}
\hline state & county & bed & rank & $\begin{array}{l}\text { base } \\
\text { year }\end{array}$ & $\begin{array}{l}\text { thicknes } \\
\text { Cinches or }\end{array}$ & feet) ${ }^{\text {overburden }}$ (feet) & reliabiltty & tonnage \\
\hline $\begin{array}{l}\text { mich } \\
\text { mich } \\
\text { mich } \\
\text { mich } \\
\text { mich } \\
\text { mich } \\
\text { mich } \\
\text { mich } \\
\text { mich } \\
\text { mich }\end{array}$ & $\begin{array}{l}\text { bay } \\
\text { bay } \\
\text { bay } \\
\text { bay } \\
\text { bay } \\
\text { bay } \\
\text { bay } \\
\text { bay } \\
\text { bay } \\
\text { bay }\end{array}$ & $\begin{array}{l}\text { uncorrelated } \\
\text { uncorrelated } \\
\text { uncorrelated } \\
\text { uncorrelated } \\
\text { uncorrelated } \\
\text { uncorrelated } \\
\text { uncorrelated } \\
\text { uncorrelated } \\
\text { uncorrelated } \\
\text { uncorrelated }\end{array}$ & $\begin{array}{l}\text { bit } \\
\text { bit } \\
\text { bit } \\
\text { bit } \\
\text { bit } \\
\text { bit } \\
\text { bit } \\
\text { bit } \\
\text { bit } \\
\text { bit }\end{array}$ & $\begin{array}{l}1950 \\
1950 \\
1950 \\
1950 \\
1950 \\
1950 \\
1950 \\
1950 \\
1950 \\
1950\end{array}$ & $\begin{array}{l}\text { unclass } \\
\text { unclass } \\
\text { unclass } \\
\text { unclass } \\
\text { unclass } \\
\text { unclass } \\
\text { unclass } \\
\text { unclass } \\
\text { unclass } \\
\text { unclass }\end{array}$ & $\begin{array}{l}0-1000 \\
0-1000 \\
0-1000 \\
0-1000 \\
0-1000 \\
0-1000 \\
0-1000 \\
0-1000 \\
0-1000 \\
0-1000\end{array}$ & $\begin{array}{l}\text { indicatd } \\
\text { inferred } \\
\text { indicatd } \\
\text { inferred } \\
\text { indicatd } \\
\text { inferred } \\
\text { indicatd } \\
\text { inferred } \\
\text { indicatd } \\
\text { inferred }\end{array}$ & $\begin{array}{l}0.57 \\
0.98 \\
0.43 \\
1.12 \\
1.62 \\
4.88 \\
0.79 \\
2.15 \\
6.24 \\
12.01\end{array}$ \\
\hline
\end{tabular}




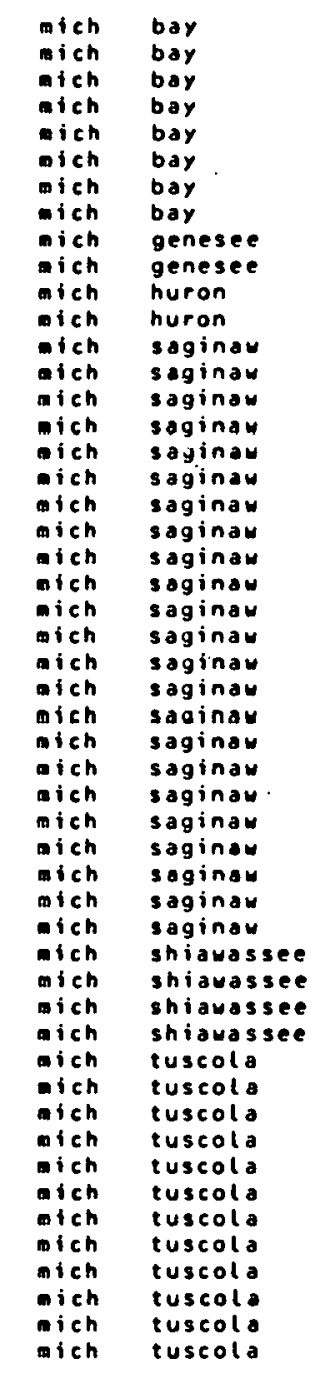

uncorrelated
uncorrelated
uncorrelated
uncorrelated
uncorrelated
uncorrelated
uncorrelated
uncorrelated
uncorrelated
uncorrelated
uncorrelated
uncorrelated
uncorrelated
uncorrelated
uncorrelated
uncorrelated
uncorrelated
uncorrelated
uncorrelated
uncorrelated
uncorrelated
uncorrelated
uncorrelated
uncorrelated
uncorrelated
uncorrelated
uncorrelated
uncorrelated
uncorrelated
uncorrelated
uncorrelated
uncorrelated
uncorrelated
uncorrelated
uncorrelated
uncorrelated
uncorrelated
uncorrelated
uncorrelated
uncorrelated
uncorrelated
uncorrelated
uncorrelated
uncorrelated
uncorrelated
uncorrelated
uncorrelated
uncorrelated
uncorrelated
uncorrelated
uncorrelated

\begin{tabular}{|c|c|c|c|c|}
\hline bit & 1950 & unctass & & $0-1000$ \\
\hline bit & 1950 & unclass & & $0-1000$ \\
\hline bit & 1950 & unctass & & $0-1000$ \\
\hline bit & 1950 & uncloss & & $0-1000$ \\
\hline bit & 1950 & unclass & & $0-1000$ \\
\hline bit & 1950 & uncilass & & $0-1000$ \\
\hline bit & 1950 & unclass & & $0-1000$ \\
\hline bit & 1950 & unctass & & $0-1000$ \\
\hline bit & 1950 & unclass & & $0-1000$ \\
\hline bit & 1950 & unclass & & $0-1000$ \\
\hline bit & 1950 & unclass & & $0-1000$ \\
\hline bit & 1950 & unclass & & $0-1000$ \\
\hline bit & 1950 & unclass & & $0-1000$ \\
\hline bit & 1950 & unclass & & $0-1000$ \\
\hline bit & 1950 & unclass & & $0-1000$ \\
\hline bit & 1950 & unclass & & $0-1000$ \\
\hline bit & 1950 & unclass & & $0-1000$ \\
\hline bit & 1950 & unclass & & $0-1000$ \\
\hline bit & 1950 & unclass & & $0-1000$ \\
\hline bit & 1950 & unctoss & & $0-1000$ \\
\hline bit & 1950 & unclass & & $0-1000$ \\
\hline bit & 1950 & unclass & & $0-1000$ \\
\hline bit & 1950 & unclass & & $0-1000$ \\
\hline bit & 1950 & unctass & & $0-1000$ \\
\hline bit & 1950 & unclass & & $0-1000$ \\
\hline bit & 1950 & unclass & & $0-1000$ \\
\hline bit & 1950 & unctass & & $0-1000$ \\
\hline bit & 1950 & unclass & & $0-1000$ \\
\hline bit & 1950 & unclass & , & $0-1000$ \\
\hline bit & 1950 & unclass & & $0-1000$ \\
\hline bit & 1950 & unclass & & $0-1000$ \\
\hline bit & 1950 & unctass & & $0-1000$ \\
\hline bit & 1050 & unclass & & $0-1000$ \\
\hline bit & 1950 & uncioss & & $0-1000$ \\
\hline bit & 1950 & unclass & & $0-1000$ \\
\hline bit & 1950 & unciass & & $0-1000$ \\
\hline bit & 1950 & uncless & & $0-1000$ \\
\hline bit & 1950 & unclass & & $0-1000$ \\
\hline bit & 1950 & unclass & & $0-1000$ \\
\hline bit & 1950 & uncioss & & $0-1000$ \\
\hline bit & 1950 & unclass & & $0-1000$ \\
\hline bit & 1950 & unclass & & $0-1000$ \\
\hline bit & 1950 & unclass & & $0-1000$ \\
\hline bit & 1950 & unclass & & $0-1000$ \\
\hline bit & 1950 & unclass & & $0-1000$ \\
\hline bit & 1950 & unciass & & $0-1000$ \\
\hline bit & 1950 & unclass & & $0-1000$ \\
\hline bit & 1950 & unctass & & $0-1000$ \\
\hline bit & 1950 & unctass & & $0-1000$ \\
\hline bit & 1950 & unclass & & $0-1000$ \\
\hline bit & 1950 & unclass & & $0-1000$ \\
\hline
\end{tabular}

indicatd

interred

indicatd

interred

indicatd

interred

indicatd

inferred

inferred

inferred

interred

indicatd

indicated

indicaed

inferred

indicatd
interred

indicatd

interred

indicatd

interred

indicatd

inficat

inferred

indiceto

inferred

indicatd

inferred

indicatd

indicatd

indicatd

interred

indicaed

inferred

indicatd

inferred

indicatd

indicatd

inferred

indicato

inferred

indicotd

inferred

indicatd

interred

indicatd

4.34
8.98
0.25
0.62
0.22
0.58
0.56
1.61
0.09
0.20
0.08
0.19
0.16
0.19
0.40
1.25
0.22
0.60
1.35
3.12
0.65
0.95
1.08
2.53
0.16
0.47
1.39
3.88
0.95
1.49
0.07
0.22
0.49
0.20
0.59
0.24
0.31
0.27
0.43
0.50
1.45
1.89
4.98
0.41
1.59
2.07
4.67
0.68
2.00
1.08
1.74 
average analyses of coal in wichigan

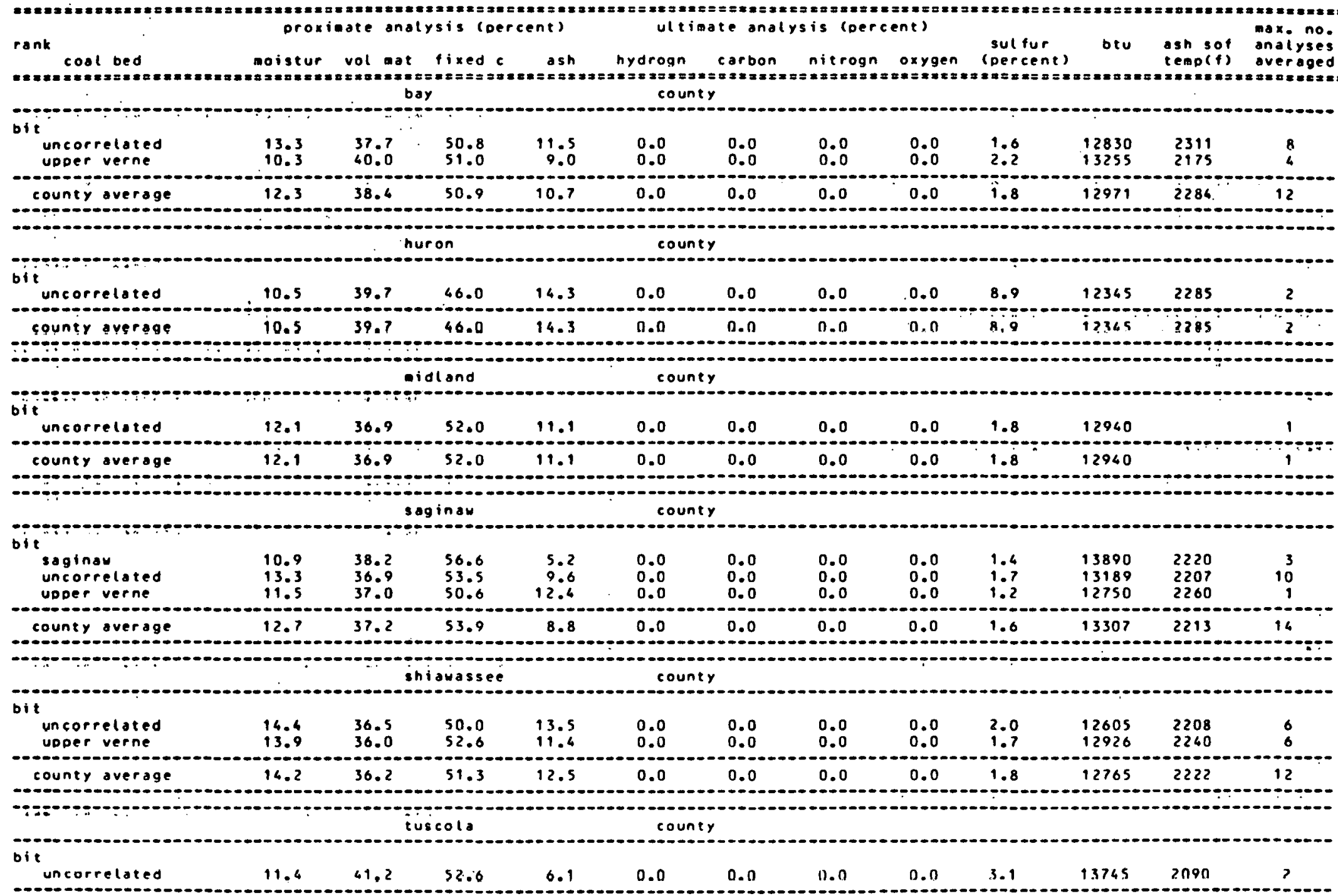


county overage

$11.4 \quad 41.2 \quad 52.6$

6.1

$\begin{array}{lllll}0.0 & 0.0 & 0.0 & 0.0 & 3.1\end{array}$

13745

2090 2

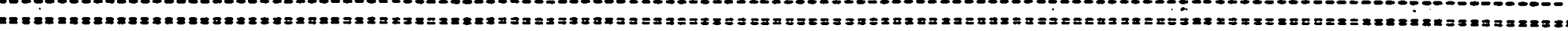




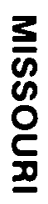


U.S. Geological Survey

identified coal resources in nissouri

(In millions of short tons)

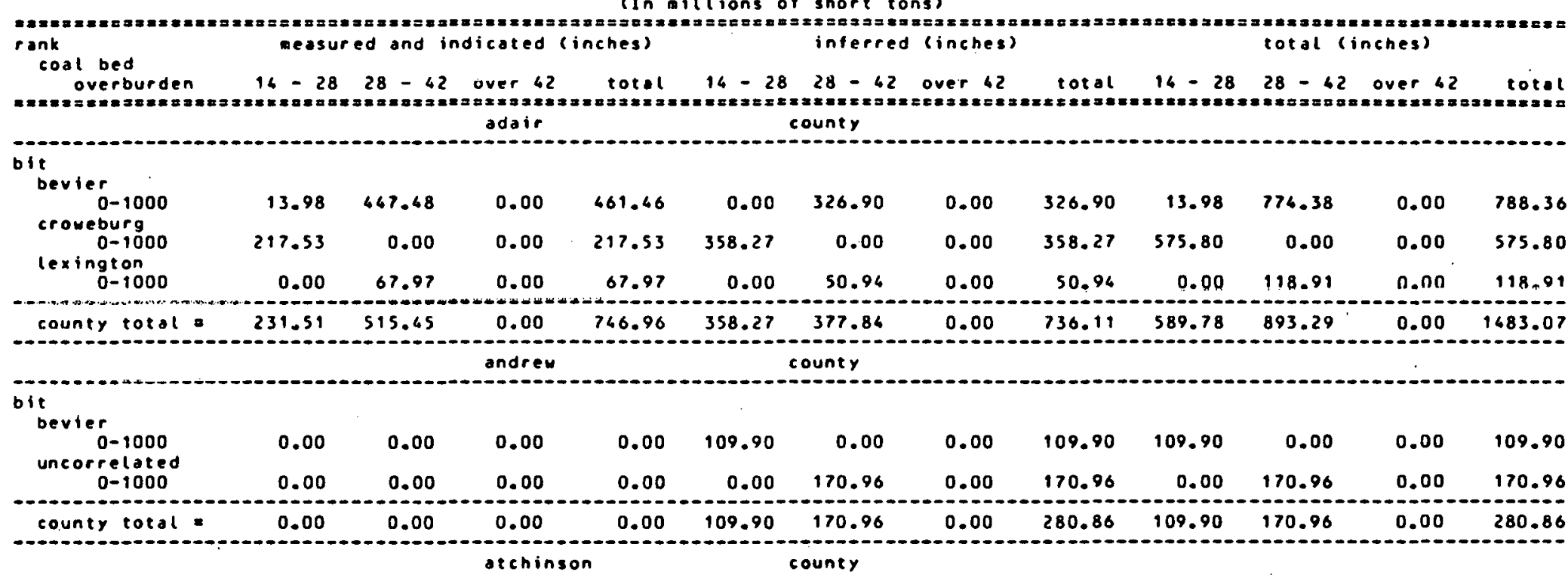
(2)

uncorrelated

\begin{tabular}{|c|c|c|c|c|c|c|c|c|c|c|c|c|}
\hline $0-1000$ & 0.00 & 0.00 & 0.00 & 0.00 & 263.95 & 0.00 & 0.00 & 263.95 & 263.95 & 0.00 & 0.00 & 263.95 \\
\hline count & 0.00 & 0.00 & 0.00 & 0.00 & 263.95 & 0.00 & 0.00 & 263.95 & 263.95 & 0.00 & 0.00 & 263.95 \\
\hline
\end{tabular}

bit

mulky

\begin{tabular}{|c|c|c|c|c|c|c|c|c|c|c|c|c|}
\hline $0-1000$ & 0.00 & 568.86 & 0.00 & 548.86 & 0.00 & 0.00 & 0.00 & 0.00 & 0.00 & 568.86 & 0.00 & 568.86 \\
\hline county total & 0.00 & 548.86 & 0.00 & 548.86 & 0.00 & 0.00 & 0.00 & 0.00 & 0.00 & 548.86 & 0.00 & 548.86 \\
\hline
\end{tabular}
bit

$\begin{array}{crrrrrrrrrrrr}\text { rove } \begin{array}{c}0-1000 \\ \text { veir-pittsburg } \\ 0-1000\end{array} & 23.3 .46 & 0.00 & 0.00 & 233.44 & 24.77 & 0.00 & 0.00 & 24.77 & 258.21 & 0.00 & 0.00 & 258.21 \\ & 0.00 & 109.36 & 0.00 & 109.36 & 0.00 & 0.00 & 0.00 & 0.00 & 0.00 & 109.36 & 0.00 & 109.36\end{array}$




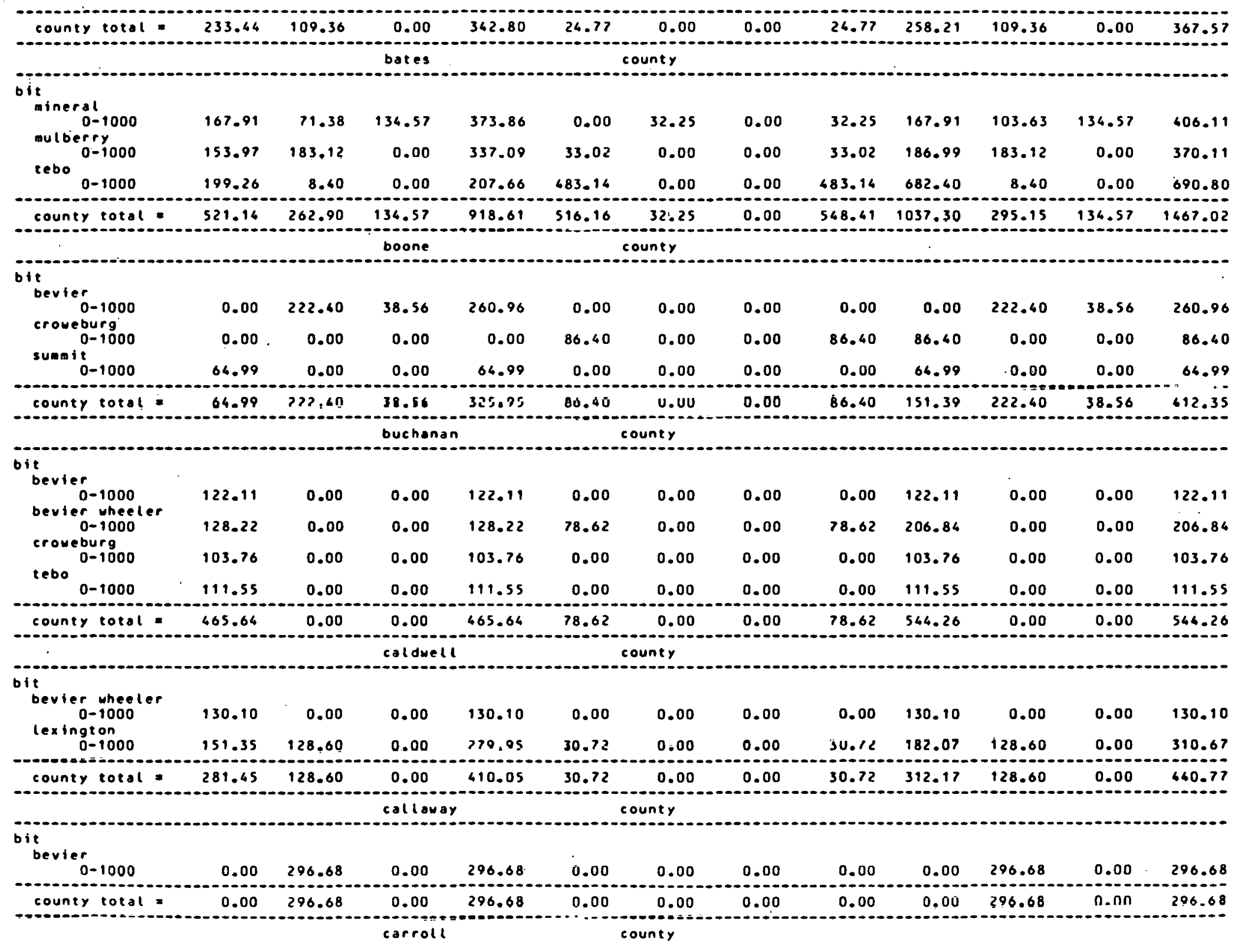




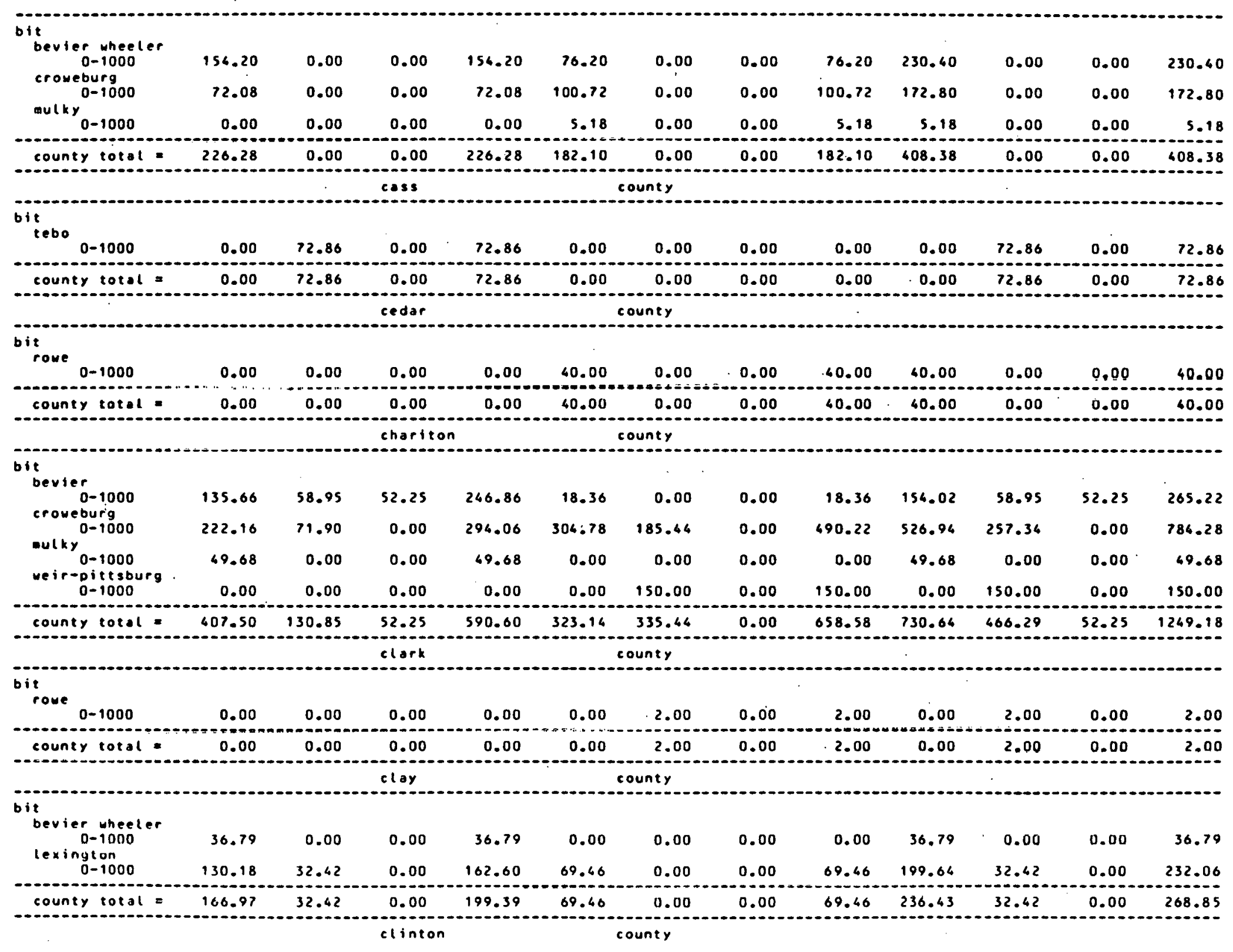




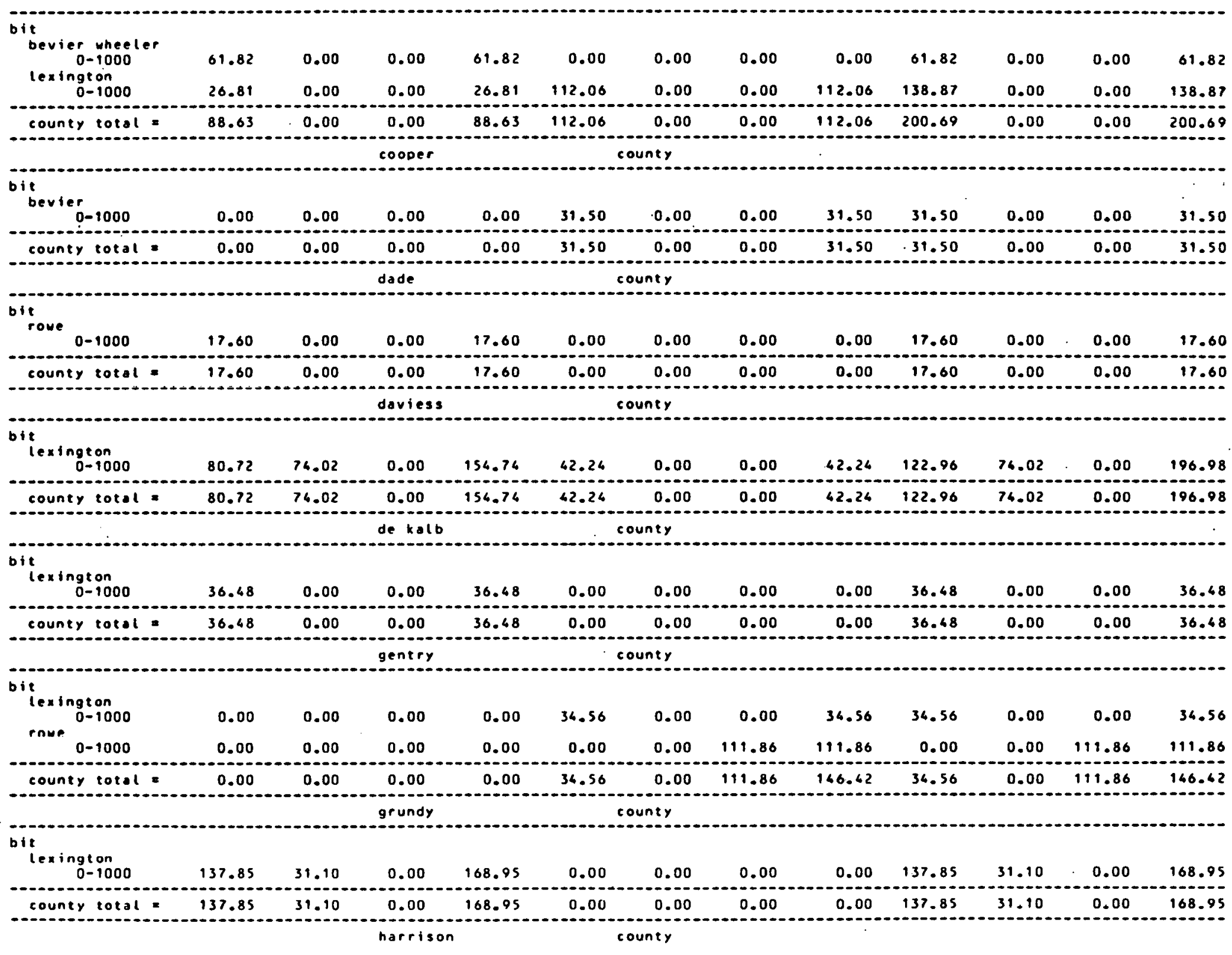




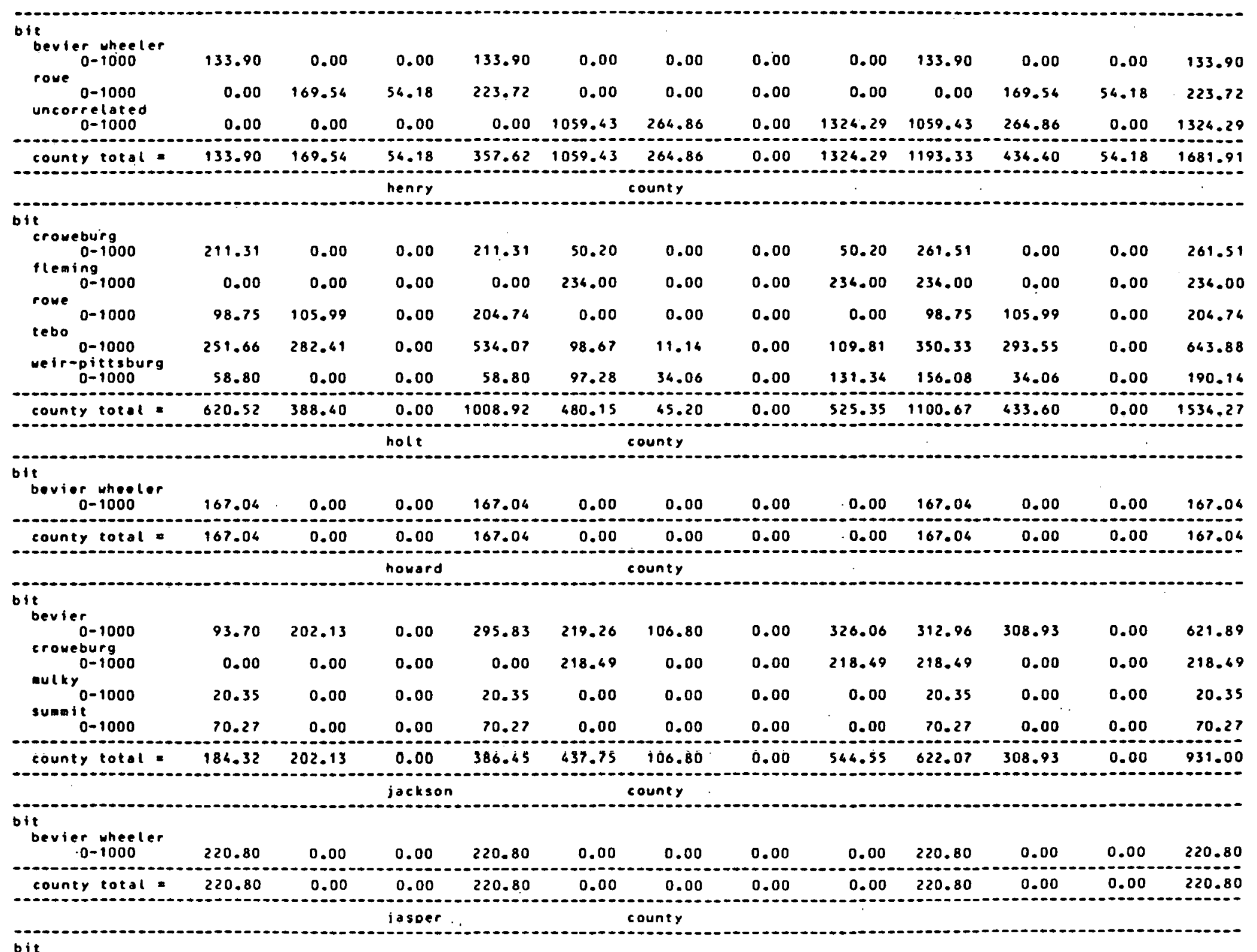




\begin{tabular}{|c|c|c|c|c|c|c|c|c|c|c|c|c|}
\hline $0-1000$ & 6.00 & 0.00 & 0.00 & 6.00 & 87.60 & 0.00 & 0.00 & 87.60 & 93.60 & 0.00 & 0.00 & 93.60 \\
\hline couney totol & 6.00 & $0.00^{\circ}$ & 0.00 & 6.00 & 87.60 & 0.00 & 0.00 & 87.60 & 93.60 & 0.00 & 0.00 & 93.60 \\
\hline & & & johnson & & & nty & & & & & & \\
\hline \multicolumn{13}{|l|}{ bit } \\
\hline $0-1000$ & 25.24 & 0.00 & 0.00 & 25.24 & 607.05 & 0.00 & 0.00 & 607.05 & 632.29 & 0.00 & 0.00 & 632.29 \\
\hline $\mathrm{ky}^{0-1000}$ & 167.64 & 0.00 & 0.00 & 167.64 & 602.52 & 0.00 & 0.00 & 602.52 & 770.16 & 0.00 & 0.00 & 770.16 \\
\hline $0-1000$ & 54.09 & 0.00 & 0.00 & 54.09 & 116.60 & 0.00 & 0.00 & 116.60 & 170.69 & 0.00 & 0.00 & 170.69 \\
\hline $0-1000$ & 115.20 & 0.00 & 67.54 & 182.74 & 0.00 & 0.00 & 0.00 & 0.00 & 115.20 & 0.00 & 67.54 & 182.74 \\
\hline $0-1000$ & 300.81 & 20.04 & 0.00 & 320.85 & 976.12 & 0.00 & 0.00 & 976.12 & 1276.93 & 20.04 & 0.00 & 1296.97 \\
\hline county total = & 662.98 & 20.04 & 67.54 & 750.56 & 2302.29 & 0.00 & 0.00 & 2302.29 & 2965.27 & 20.04 & 67.54 & 3052.85 \\
\hline & & & lafayet & & & nty & & & & & & \\
\hline \multicolumn{13}{|l|}{ bie } \\
\hline nulky. & 272.08 & 0.000 & 0.00 & 272.09 & 74.54 & 0.00 & 0.00 & 76.54 & 346.63 & 0.00 & 0.00 & 346.63 \\
\hline rove $0-1000$ & 133.32 & 0.00 & 0.00 & 133.32 & 66.96 & 0.00 & 0.00 & 66.96 & 200.28 & 0.00 & 0.00 & 200.28 \\
\hline $0-1000$ & 0.00 & 0.00 & $0.00^{\circ}$ & 0.00 & 266.00 & 18.24 & 0.00 & 282.24 & 264.00 & 18.26 & 0.00 & 282.24 \\
\hline county total = & 405.61 & 0.00 & 0.00 & 405.41 & 405.50 & 18.24 & 0.00 & 623.74 & 810.91 & 18.24 & 0.00 & 829.15 \\
\hline & & & levis & & & ney & & & & & & \\
\hline \multicolumn{13}{|l|}{ bit } \\
\hline $0-1000$ & 0.00 & 0.00 & 0.00 & 0.00 & 0.00 & 0.05 & 0.05 & 0.10 & 0.00 & 0.05 & 0.05 & 0.10 \\
\hline county total = & 0.00 & 0.00 & 0.00 & 0.00 & 0.00 & 0.05 & 0.05 & 0.10 & 0.00 & 0.05 & 0.05 & 0.10 \\
\hline 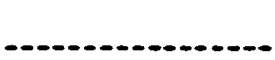 & & & $\operatorname{linn}$ & & & unty & & & & $\therefore$ & $\ldots .-$. & $\therefore$ \\
\hline \multicolumn{13}{|l|}{ bit } \\
\hline & 218.69 & 0.00 & 0.00 & 218.69 & 0.00 & 0.00 & 0.00 & 0.00 & 218.69 & 0.00 & 0.00 & 218.69 \\
\hline $\begin{array}{c}0=1000 \\
\text { wetr-pittsburg }\end{array}$ & 223.01 & 143.13 & 0.00 & 368.16 & 261.41 & 21.50 & $0 . \overline{0} \overline{0}$ & 282.91 & 484.42 & 166.65 & 0.00 & 651.07 \\
\hline $0-1000$ & 0.00 & 419.54 & 0.00 & 419.54 & 0.00 & 0.00 & 0.00 & 0.00 & 0.00 & 419.54 & 0.00 & 619.54 \\
\hline county total : & 641.70 & 566.69 & 0.00 & 1006.39 & 261.41 & 21.50 & 0.00 & 282.91 & 703.91 & 586.19 & 0.00 & 1289.30 \\
\hline$-\infty$ & & & livings & & & unty & & & & & & \\
\hline $\begin{array}{l}\text { bit } \\
\text { bevier wheeler } \\
0-1000\end{array}$ & 319.68 & 0.00 & 0.00 & 319.68 & 0.00 & 0.00 & 0.00 & n. no & 319.68 & $\begin{array}{c}0.00 \\
0.0 .\end{array}$ & n. no & 319.68 \\
\hline county total $=$ & 319.68 & 0.00 & 0.00 & 319.68 & 0.00 & 0.00 & 0.00 & 0.00 & 319.68 & 0.00 & 0.00 & 319.68 \\
\hline
\end{tabular}


macon

county

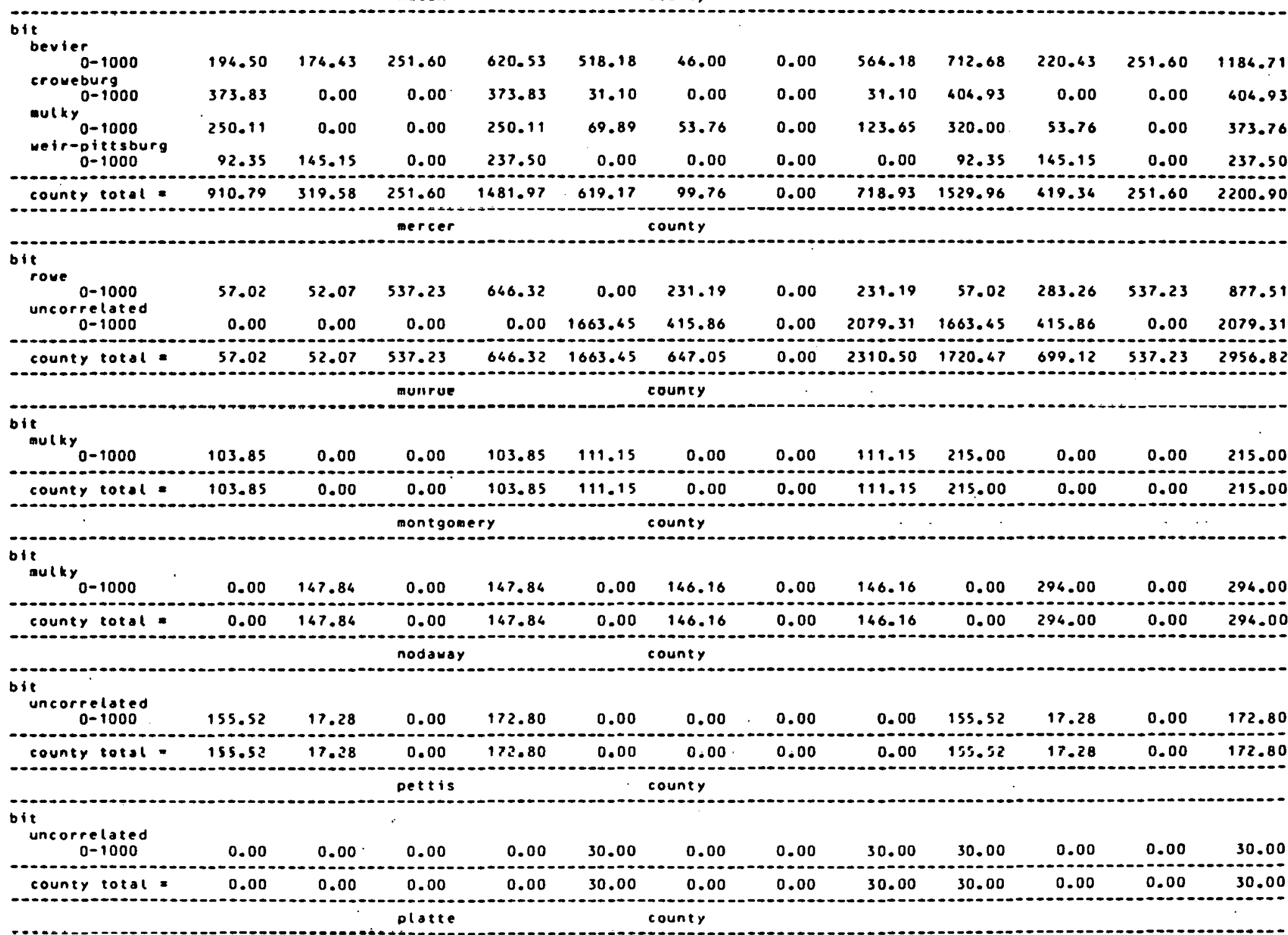




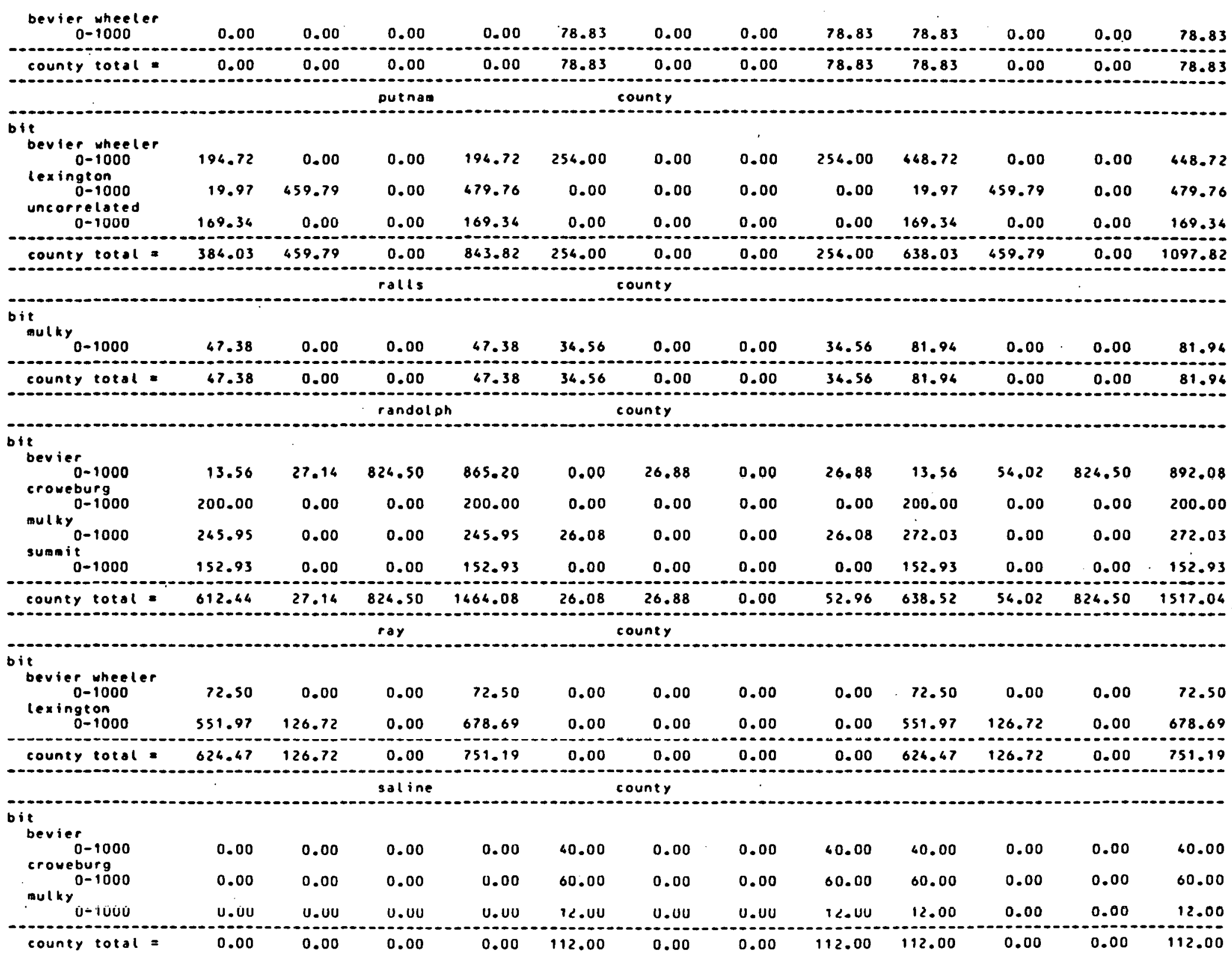




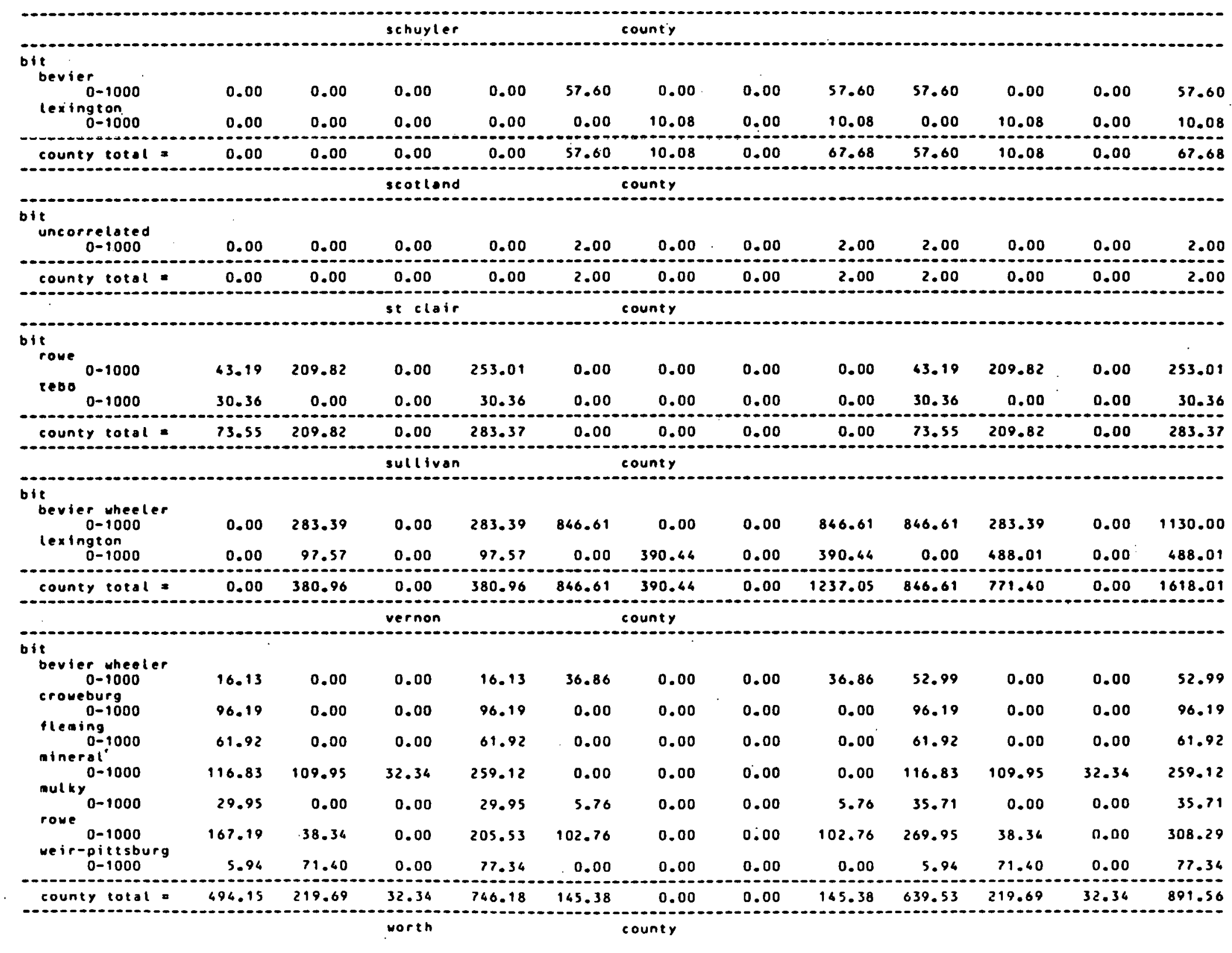




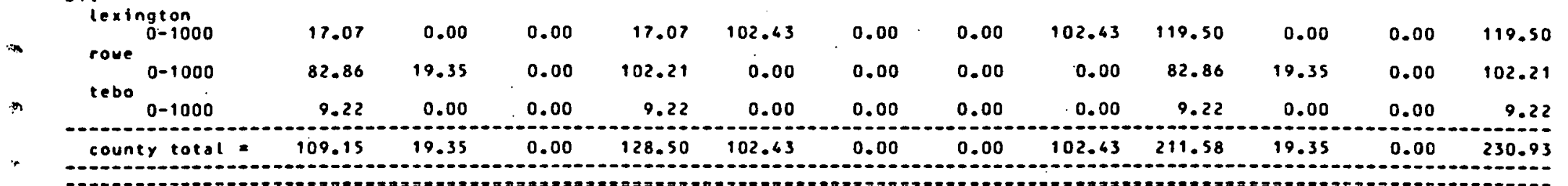

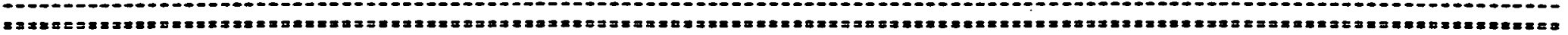

overburden
subtotal

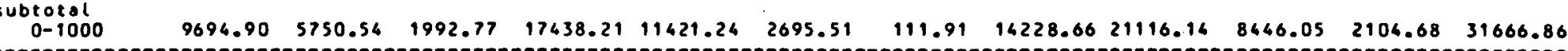

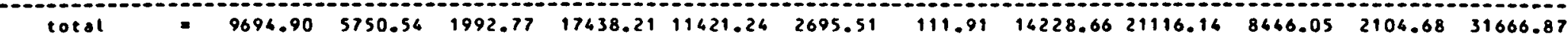

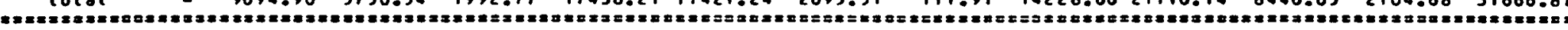

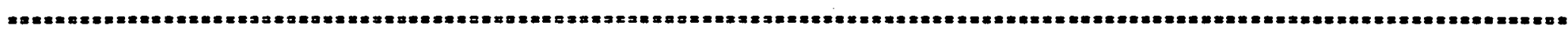
overburden

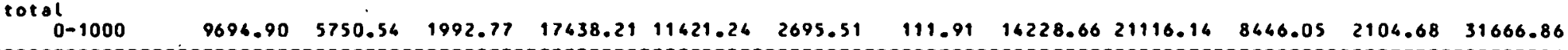
grand total $9694.90 \quad 5750.54 \quad 1992.77 \quad 17438.21 \quad 11421.24 \quad 2695.51 \quad 119.91 \quad 14228.6621916 .14 \quad 8466.05 \quad 2106.68 \quad 31666.87$

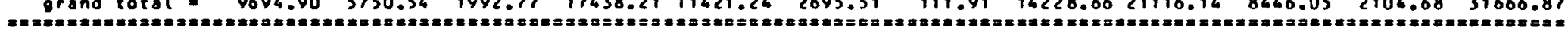

resource figures taken from:

$\begin{array}{lll}\text { source } & \text { year base year of resource } \\ \text { missouri gs ri } 48 & 1971 & \text { original }\end{array}$


average onatyses of coat in issour

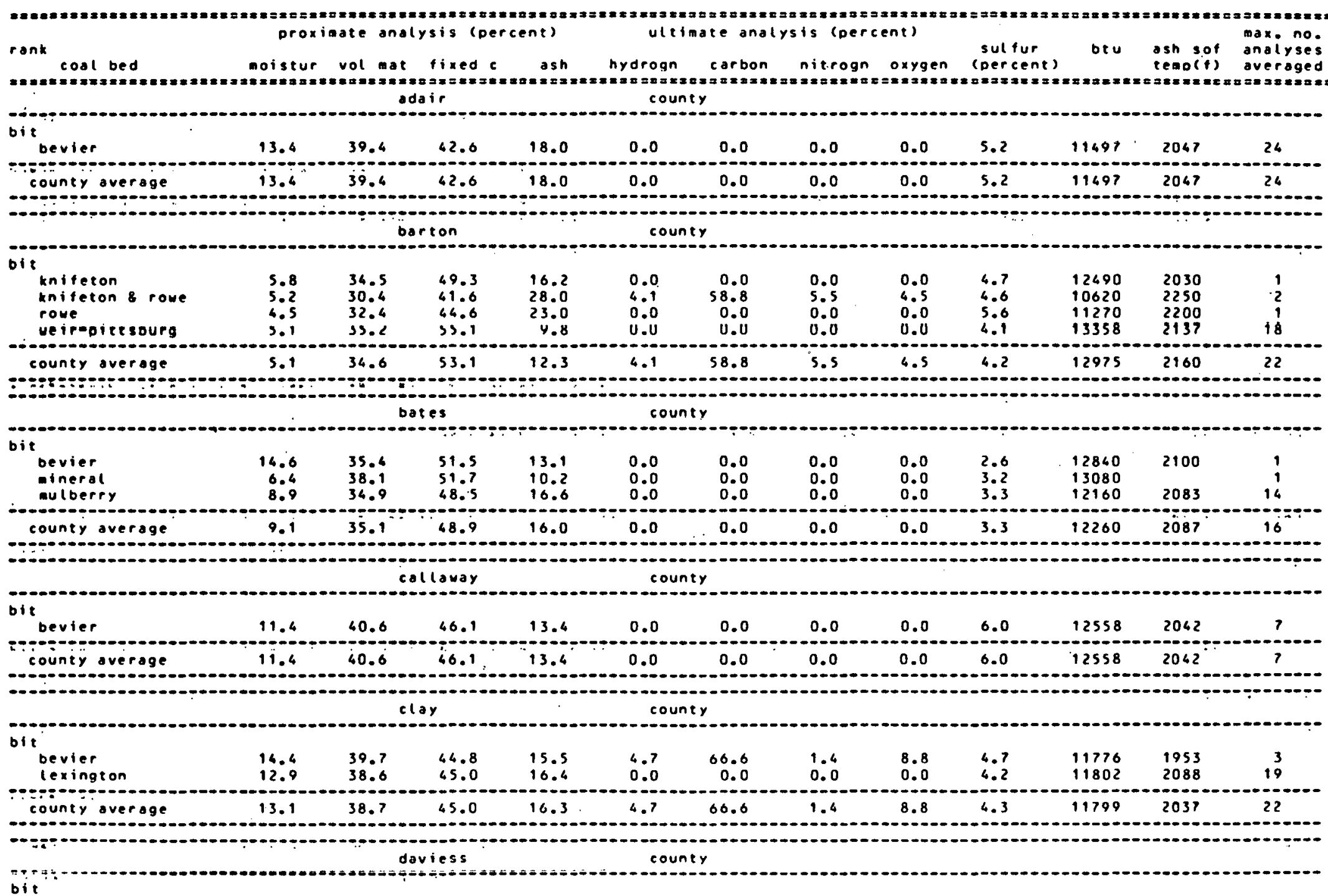




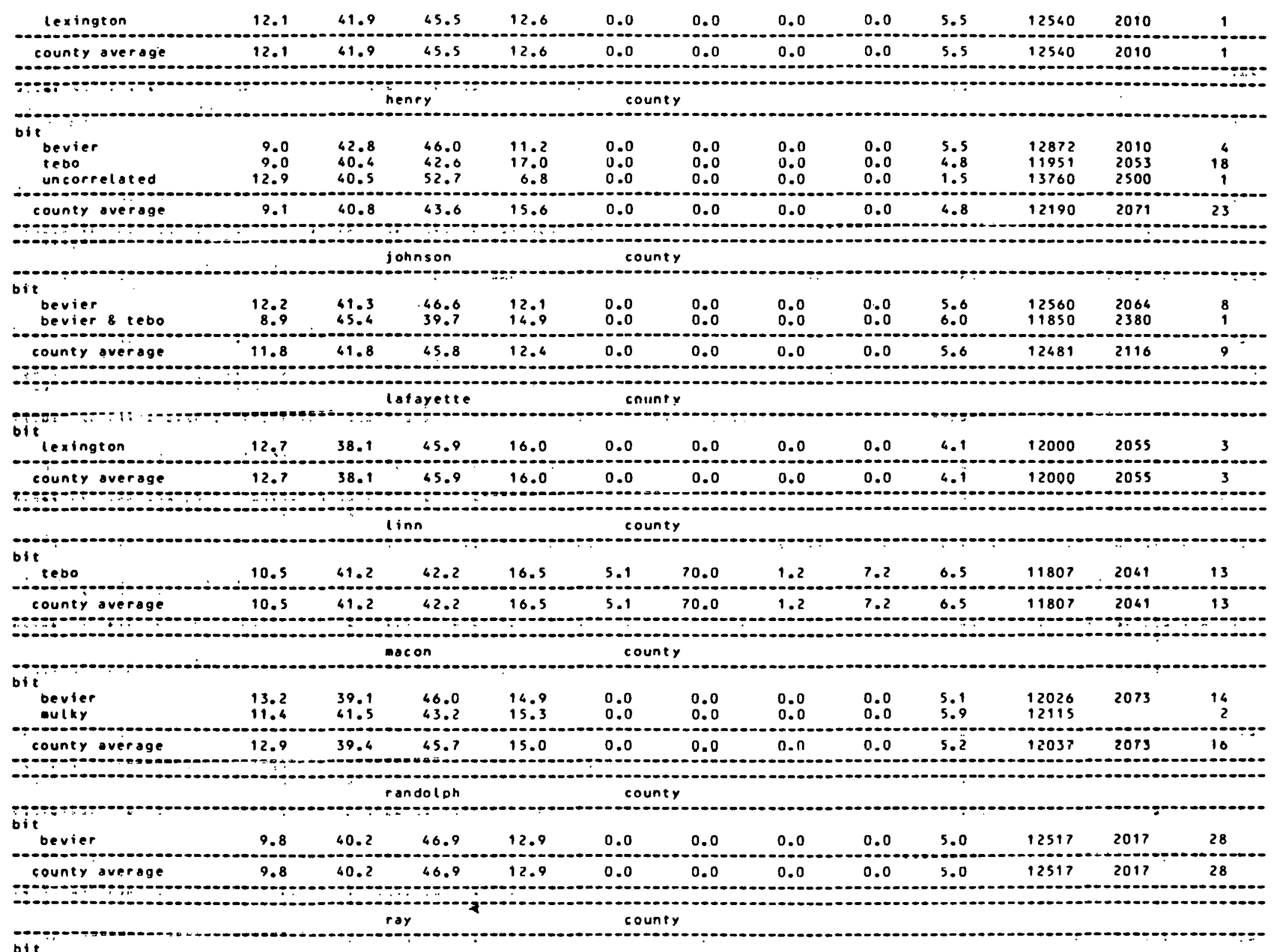




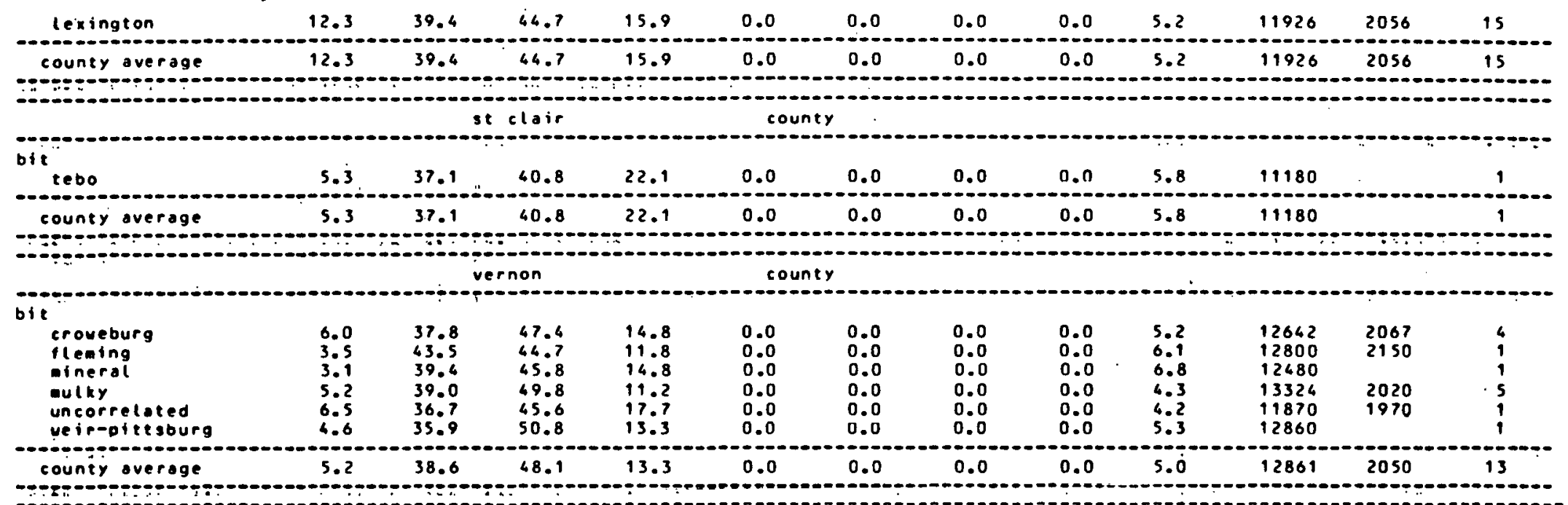


M1880ur1

\begin{tabular}{|c|c|c|}
\hline secte & counir & nees \\
\hline 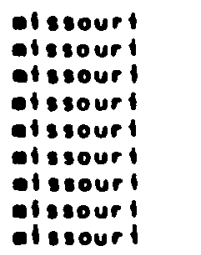 & $\begin{array}{l}\text { berton } \\
\text { berion } \\
\text { barton } \\
\text { berion } \\
\text { bates } \\
\text { cley } \\
\text { henry } \\
\text { rendolon } \\
\text { vernon }\end{array}$ & 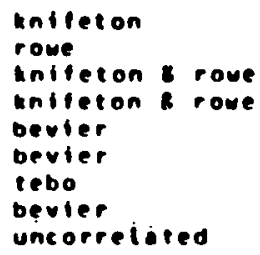 \\
\hline $\begin{array}{l}\text { arsenur } \\
\text { arsour }\end{array}$ & $\begin{array}{l}\text { vernmn } \\
\text { vernosn }\end{array}$ & $\begin{array}{l}\text { ernuevuru } \\
\text { ileminy }\end{array}$ \\
\hline
\end{tabular}

\begin{tabular}{|c|c|c|c|c|c|c|c|}
\hline moistur & votant & Piemdc & $\sin$ & sul in & en & mallo & 12110 \\
\hline $\begin{array}{l}5.8 \\
6.5 \\
5.2 \\
5.2 \\
0.2 \\
0 . .9 \\
0.0 \\
9.1 \\
6.5\end{array}$ & $\begin{array}{l}36.5 \\
37.5 \\
30.4 \\
30.4 \\
35.6 \\
37.9 \\
38.9 \\
32.9 \\
36.9\end{array}$ & $\begin{array}{l}49.3 \\
44.8 \\
41.6 \\
41.6 \\
51.5 \\
43.1 \\
41.4 \\
36.3 \\
45.6\end{array}$ & $\begin{array}{l}10.2 \\
23.0 \\
28.0 \\
28.0 \\
13.1 \\
19.0 \\
20.2 \\
311.8 \\
17.8\end{array}$ & $\begin{array}{l}4.7 \\
3.6 \\
4.6 \\
4.6 \\
2.6 \\
4.6 \\
5.4 \\
3.0 \\
4.2\end{array}$ & $\begin{array}{l}12490 \\
11270 \\
10620 \\
10620 \\
12860 \\
11080 \\
11150 \\
9540 \\
11870\end{array}$ & $\begin{array}{l}37 \\
37 \\
65 \\
65 \\
70 \\
68 \\
58 \\
62 \\
66\end{array}$ & $\begin{array}{l}1.5 \\
8.0 \\
6.0 \\
6.0 \\
5.5 \\
3.0 \\
2.5 \\
3.0 \\
7.0\end{array}$ \\
\hline $\begin{array}{l}4.5 \\
3.5\end{array}$ & $\begin{array}{l}14.2 \\
43.5\end{array}$ & $\begin{array}{l}64.6 \\
64.7\end{array}$ & $\begin{array}{l}15 . ? \\
11.8\end{array}$ & $\begin{array}{l}6.3 \\
6.1\end{array}$ & $\begin{array}{l}12310 \\
12800\end{array}$ & $\begin{array}{l}59 \\
51\end{array}$ & $\begin{array}{l}8.0 \\
7.5\end{array}$ \\
\hline
\end{tabular}



average analyses of coal in montana

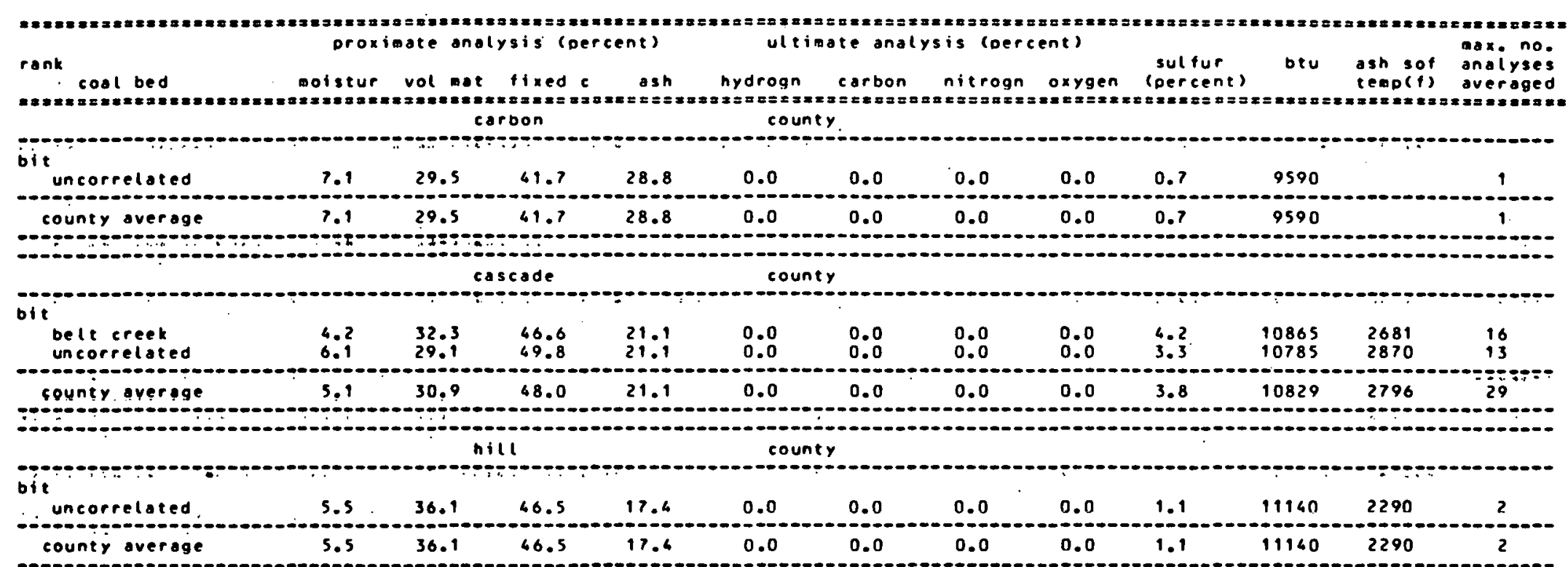

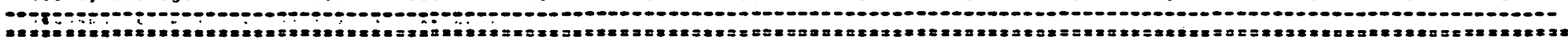



average analyses of coal in nevada

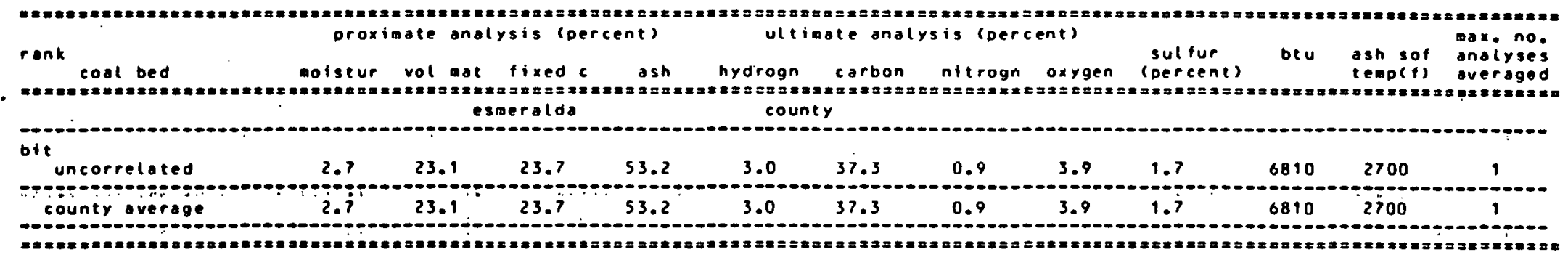



(In millions of short tons)

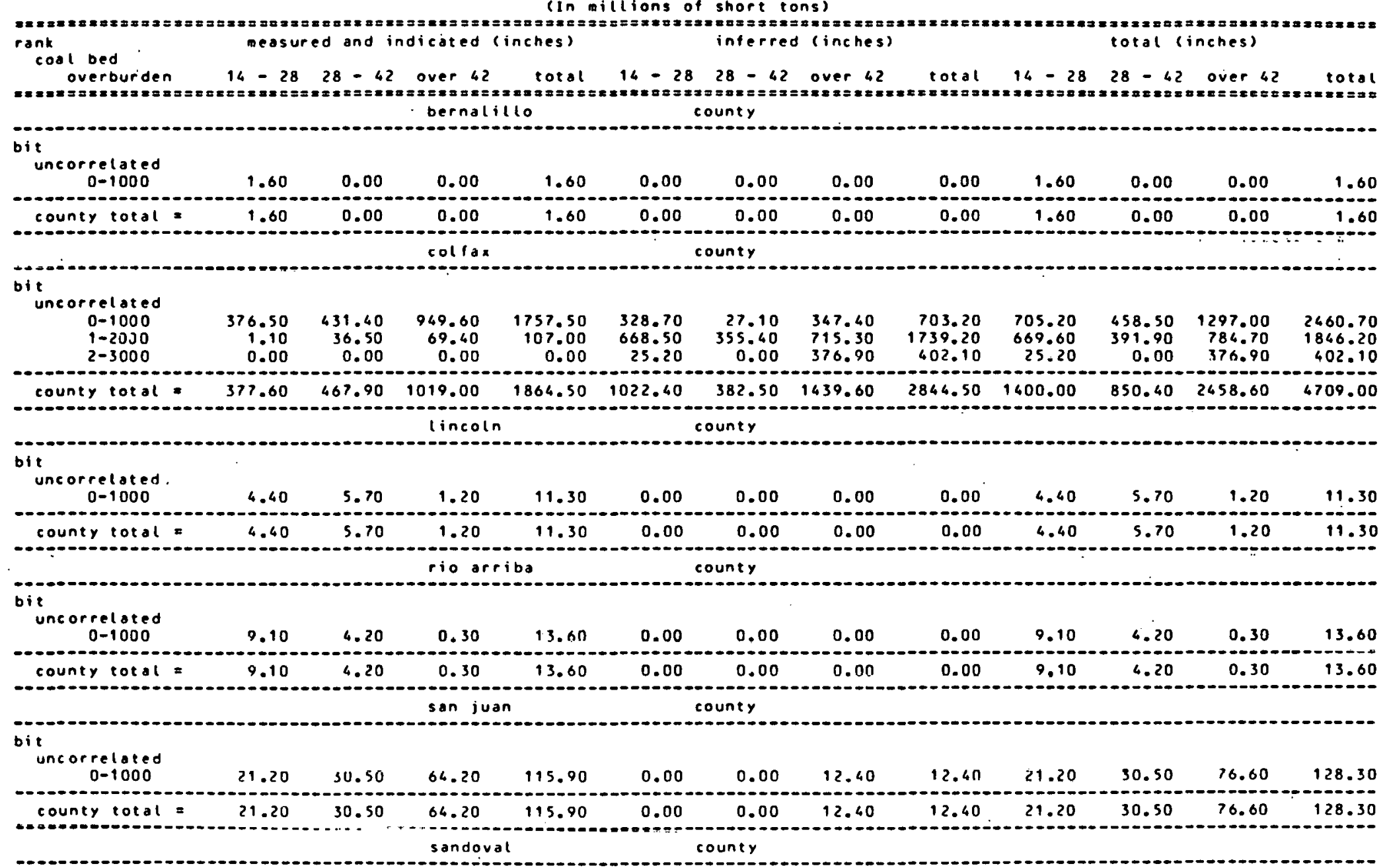




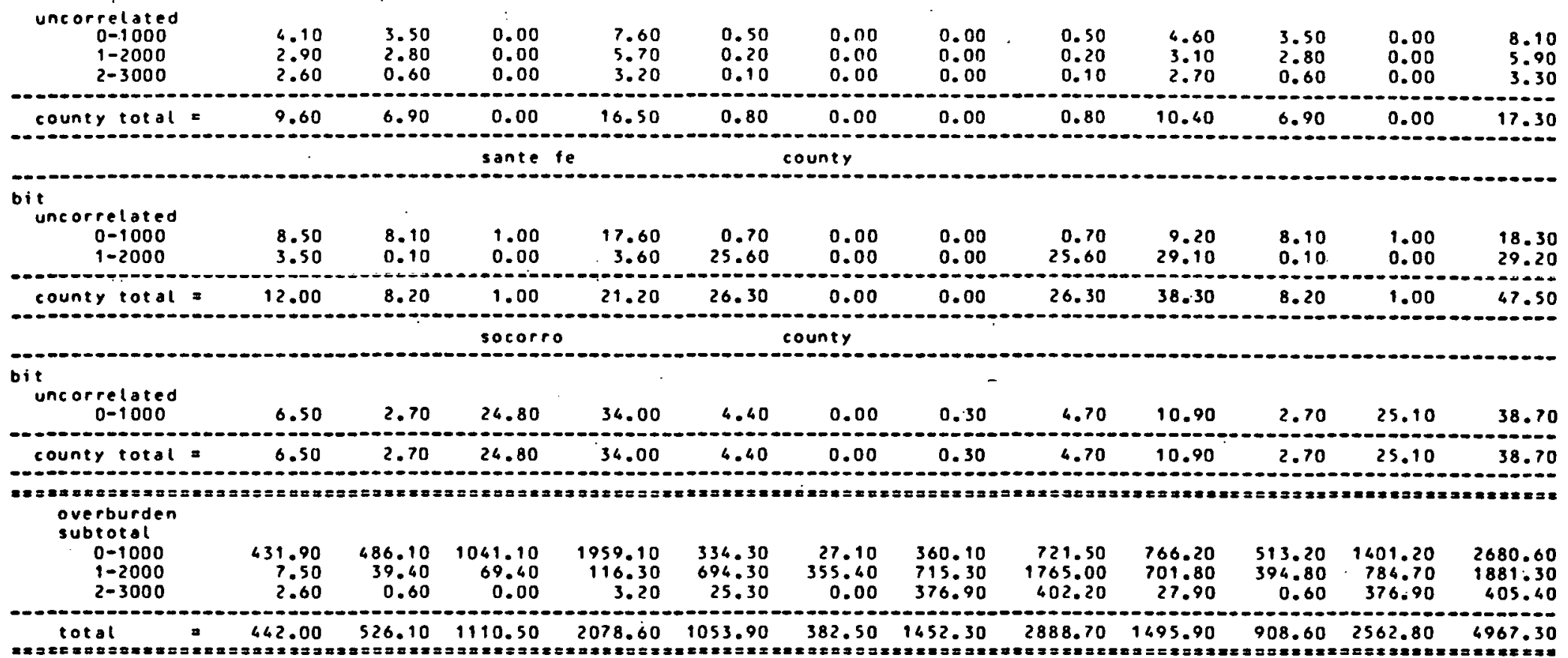

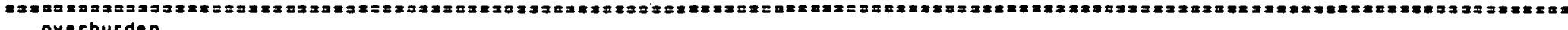 overburden

\begin{tabular}{|c|c|c|c|c|c|c|c|c|c|c|c|c|}
\hline $\begin{array}{l}0-1000 \\
1-2000 \\
2-3000\end{array}$ & $\begin{array}{r}439.90 \\
7.50 \\
2.60\end{array}$ & $\begin{array}{r}486.10 \\
39.40 \\
0.60\end{array}$ & $\begin{array}{r}1049.10 \\
69.40 \\
0.00\end{array}$ & $\begin{array}{r}1959.10 \\
116.30 \\
3.20\end{array}$ & $\begin{array}{r}336.30 \\
694.30 \\
25.30\end{array}$ & $\begin{array}{r}27.10 \\
355.40 \\
0.00\end{array}$ & $\begin{array}{l}360.10 \\
715.30 \\
376.90\end{array}$ & $\begin{array}{r}721.50 \\
1765.00 \\
402.20\end{array}$ & $\begin{array}{r}766.20 \\
701.80 \\
27.90\end{array}$ & $\begin{array}{r}513.20 \\
394.80 \\
0.60\end{array}$ & $\begin{array}{r}1401.20 \\
784.70 \\
376.90\end{array}$ & $\begin{array}{r}2680.60 \\
1881.30 \\
405.40\end{array}$ \\
\hline grand tat & 442.00 & 526.10 & 1110.50 & 2078.60 & 1053.90 & 382.50 & 1452.30 & 2888.70 & 1495.90 & 908.60 & 2562.80 & 4967. \\
\hline
\end{tabular}

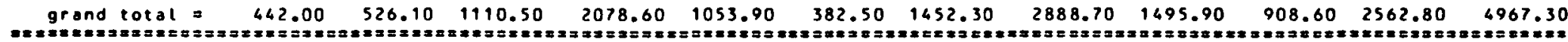

tonnage not included:

5980.40

thickness a classitied by zone: 5980.40

total tonnage of identified coal resources in neu mexico

resource tigures taken froms

\begin{tabular}{|c|c|c|}
\hline source & year & base yeor of resource \\
\hline usys circ 89 & 1950 & Original \\
\hline
\end{tabular}


records containing unclassified tnickness and/or reliability

stote county

$\begin{array}{ll}\text { new } & \text { lincoln } \\ \text { new lincoln } \\ \text { new lincoln } \\ \text { new otero } \\ \text { new otero } \\ \text { new otero } \\ \text { new rioarriba } \\ \text { new rio arriba } \\ \text { new rio arriba } \\ \text { new san juan } \\ \text { new san juan } \\ \text { new son juan }\end{array}$

bed

uncorrelated

uncorrelated

uncorretated

uncorrelated

uncorrelated

uncorrelated

uncorreloted

uncorrelated

uncorrelated rank

bit
bit
bit
bit
bit
bit
bit
bit
bit
bit
bit
bit

base thickness

(inches or teet) (ferburden

(feet)

reliability

tonnage

Orig class by zone
orig class by zone

orig class by zone

orig class by zone

oria class by cone

orig class by zone

orig class by zone

orig class by zone

orig class by zone

orig class by zone

orig class by zone
$0-1000$
$1-2000$

$1-2000$
$2-3000$

$0-1000$

$1-2000$

$2-3000$
$0=1000$

$0=1000$

$1-2000$

$2-3000$
$0-1300$

$0-1300$
$1-2000$

$1-2000$
$2-3000$ inferred $\quad 679.80$

inferred

inferred

interred 101.60

18.30

77.30

126.30

inferred 1432.90

inferred 1127.60 interred 457.10 
average analyses of coal in new mexico

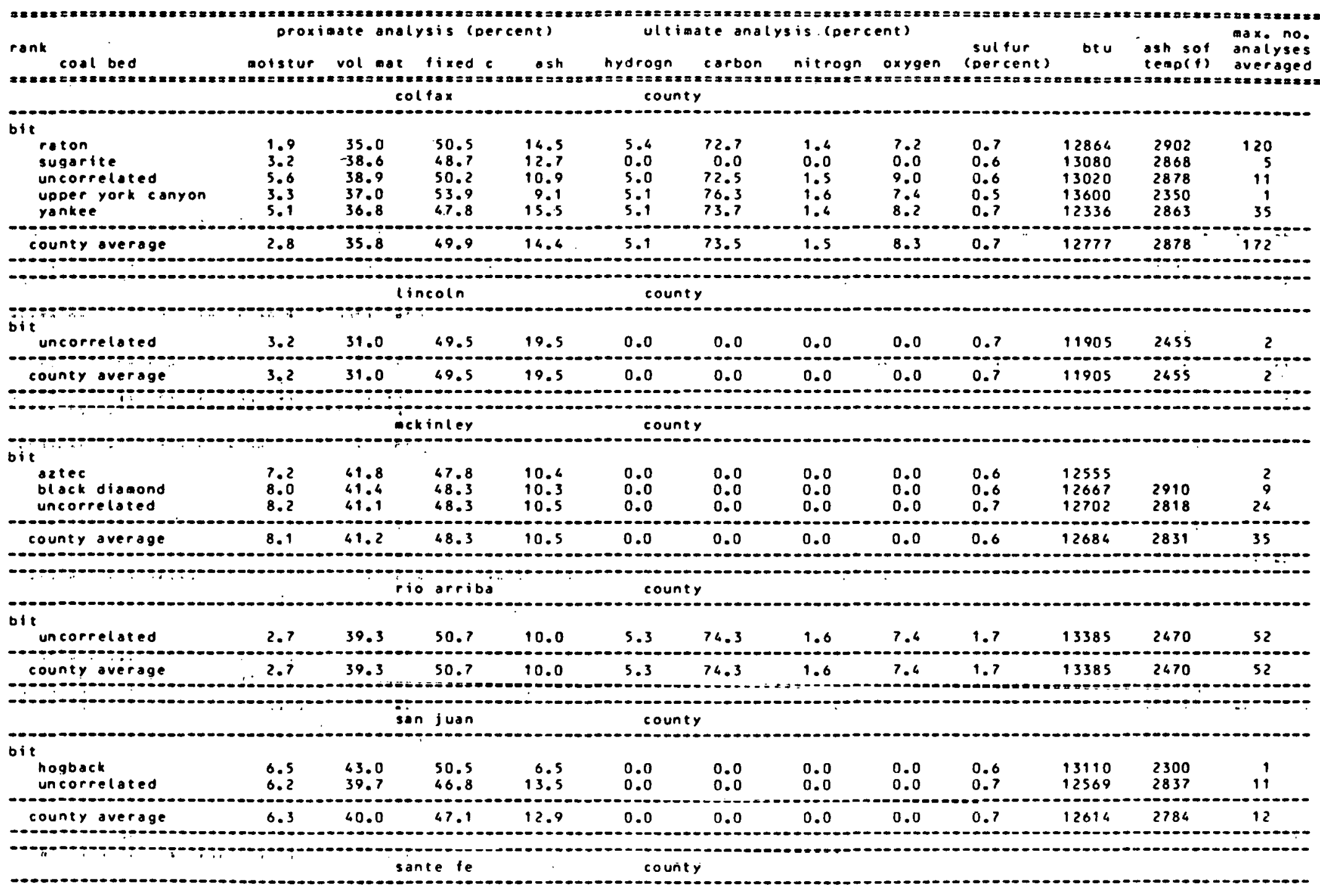


bit

\begin{tabular}{|c|c|c|c|c|c|c|c|c|c|c|c|c|}
\hline $\begin{array}{l}\text { cook } 8 \text { wnite } \\
\text { uncorrelated }\end{array}$ & $\begin{array}{l}2.2 \\
2.5\end{array}$ & $\begin{array}{l}33.5 \\
27.3\end{array}$ & $\begin{array}{l}54.1 \\
63.8\end{array}$ & $\begin{array}{r}12.3 \\
9.0\end{array}$ & $\begin{array}{l}0.0 \\
0.0\end{array}$ & $\begin{array}{l}0.0 \\
0.0\end{array}$ & $\begin{array}{l}0.0 \\
0.0\end{array}$ & $\begin{array}{l}0.0 \\
0.0\end{array}$ & $\begin{array}{l}0.8 \\
1.1\end{array}$ & $\begin{array}{l}12998 \\
12995\end{array}$ & $\begin{array}{l}2322 \\
2760\end{array}$ & $\begin{array}{r}58 \\
2\end{array}$ \\
\hline ounty average & 2.2 & 33.3 & 54.5 & 12.2 & 0.0 & 0.0 & 0.0 & 0.0 & 0.8 & 12998 & 2647 & 60 \\
\hline
\end{tabular}
county

bit carthage
county average

0
0

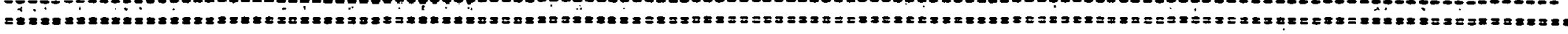


New Mexico

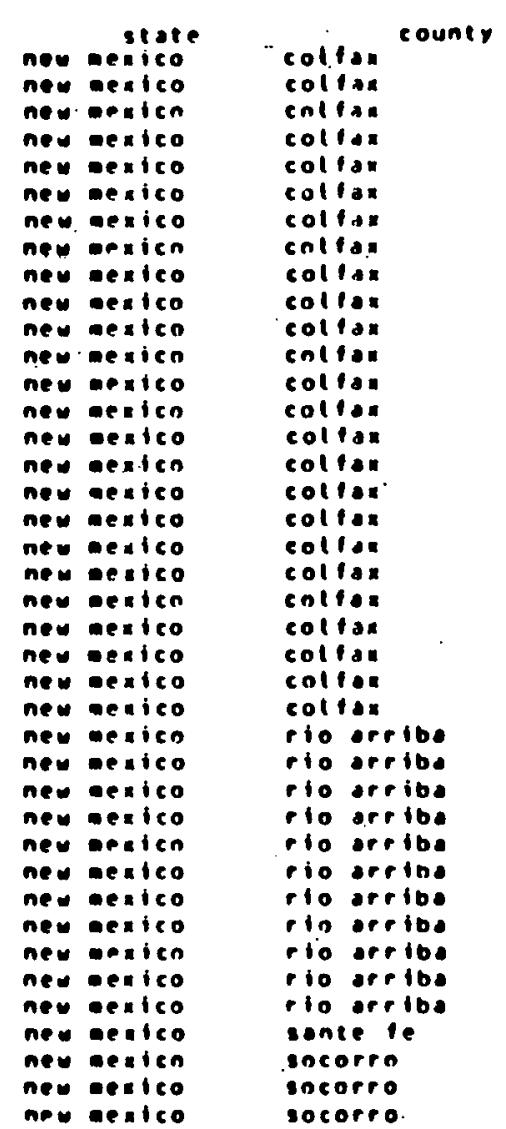

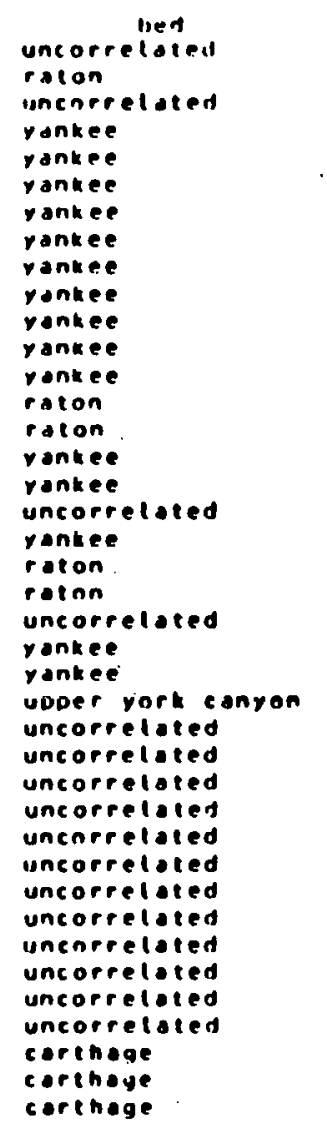

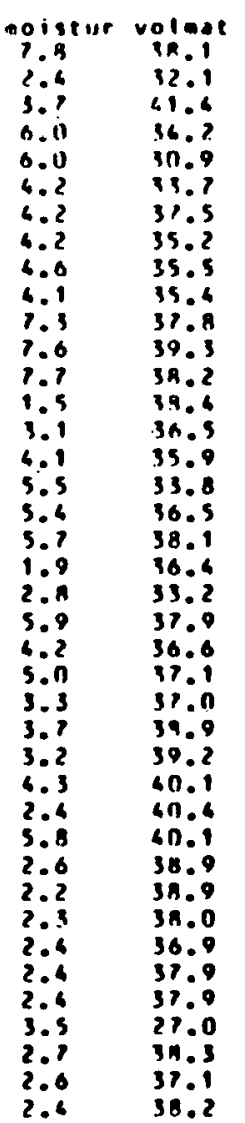

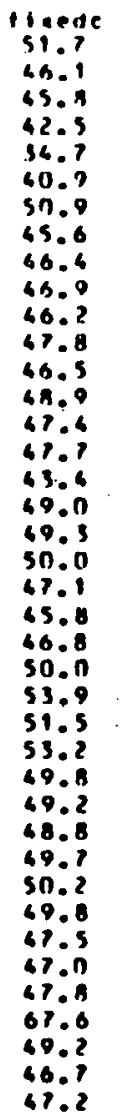

"inn. sulfur oris

21.8 n.8 11660

23.3 0.7 liv611

$36.6 \quad 0.7 \quad 9300$

190.80020

$19.20 .8 \quad 11420$

$18.10 .9 \quad 1199$ n

$11.7 \quad 1.0 \quad 12100$

$16.00 .5 \quad 12150$

$15.30 .7 \quad 12170$

$12.70 .6 \quad 13120$

$16.10 .7 \quad 12560$

$22.80 .7 \quad 11280$

$\begin{array}{lll}14.5 & 0.7 \quad 12380\end{array}$

$\begin{array}{lll}12.6 & 0.6 \quad 12800\end{array}$

19.7 0.9 12830

16.30 .912270

$16.60 .6 \quad 12120$

9.1 n.5 13600

$0.6 \quad 1.0 \quad 13310$

$7.6 \quad 0.6 \quad 13750$

$10.4 \quad 3.2 \quad 13250$

$11.1 \quad 1.6 \quad 12610$

$11.4 \quad 2.9 \quad 13150$

$12.23 .2 \quad 13060$

$15.04 .6 \quad 12660$

$15.13 .8 \quad 12500$

$16.3 \quad 6.2 \quad 12660$

$\begin{array}{lll}5.4 & 1.2 & 12750\end{array}$

$12.50 .6 \quad 13000$

$\begin{array}{lll}16.2 & 1.1 & 12370 \\ 16.6 & 0.8 & 12550\end{array}$

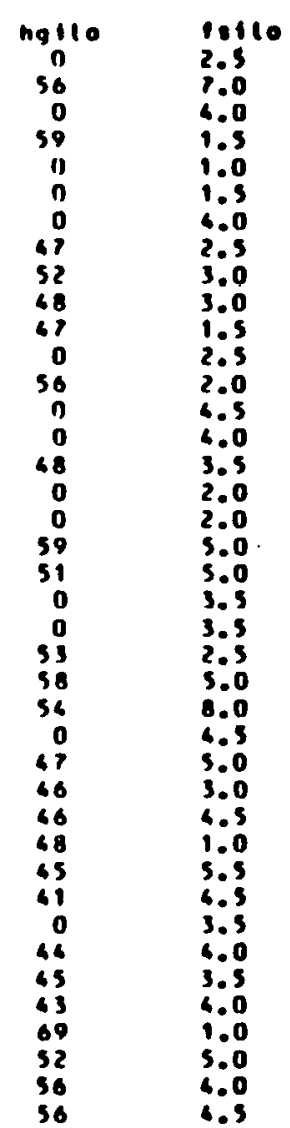



National coal Resources Data System

U.s. Geological survey

identified coal resources in north carolina

(In millions of short tons)

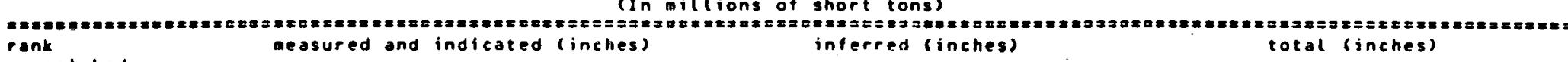

overburden $14-28 \quad 28-42$ over 42 total $14-28 \quad 28-42$ over 42 total $14-2828-42$ over 42 total chatham county

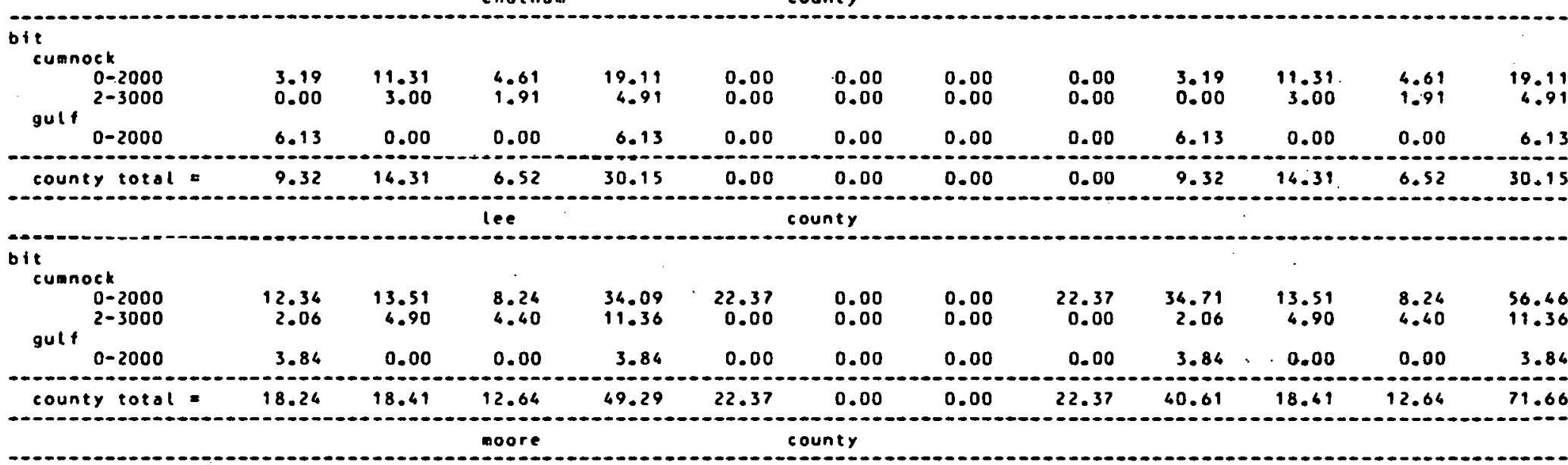

bit

bit cumnoct

\begin{tabular}{l}
$\begin{array}{c}\text { cumnock } \\
0-2000\end{array}$ \\
\hdashline county total
\end{tabular}
overburden

subtotal

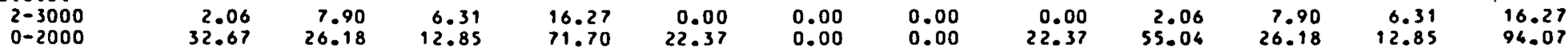

total $34.73 \quad 34.08 \quad 19.16 \quad 87.97 \quad 22.37 \quad 0.00 \quad 0.00 \quad 22.37 \quad 57.10 \quad 34.08 \quad 19.16 \quad 110.36$

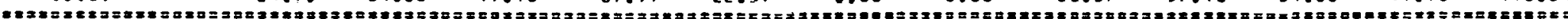

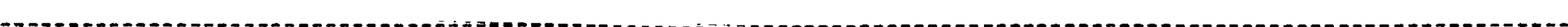

overburden

z-suuu

$2-3000$
$0-2000$

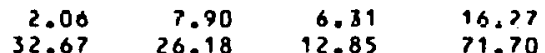

0.00
22.37

0.00

0.00
0.00

0.00
22.37

2.06
33.04

$7.90 \quad 6.31$

$\begin{array}{ll}6.31 & 16.27\end{array}$ 


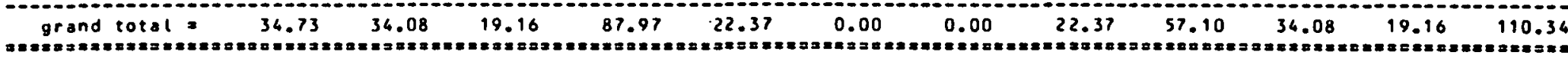

total tonnage of identified coal resources in north carolina

110.34

resource figures taken from:

source

usgs Drof Daper 246 year

1950 base year of resource

1950 

National Coal Resources Data System

U.S. Geological. Survey

identified coal resources in ohio

(In millions of short tons)

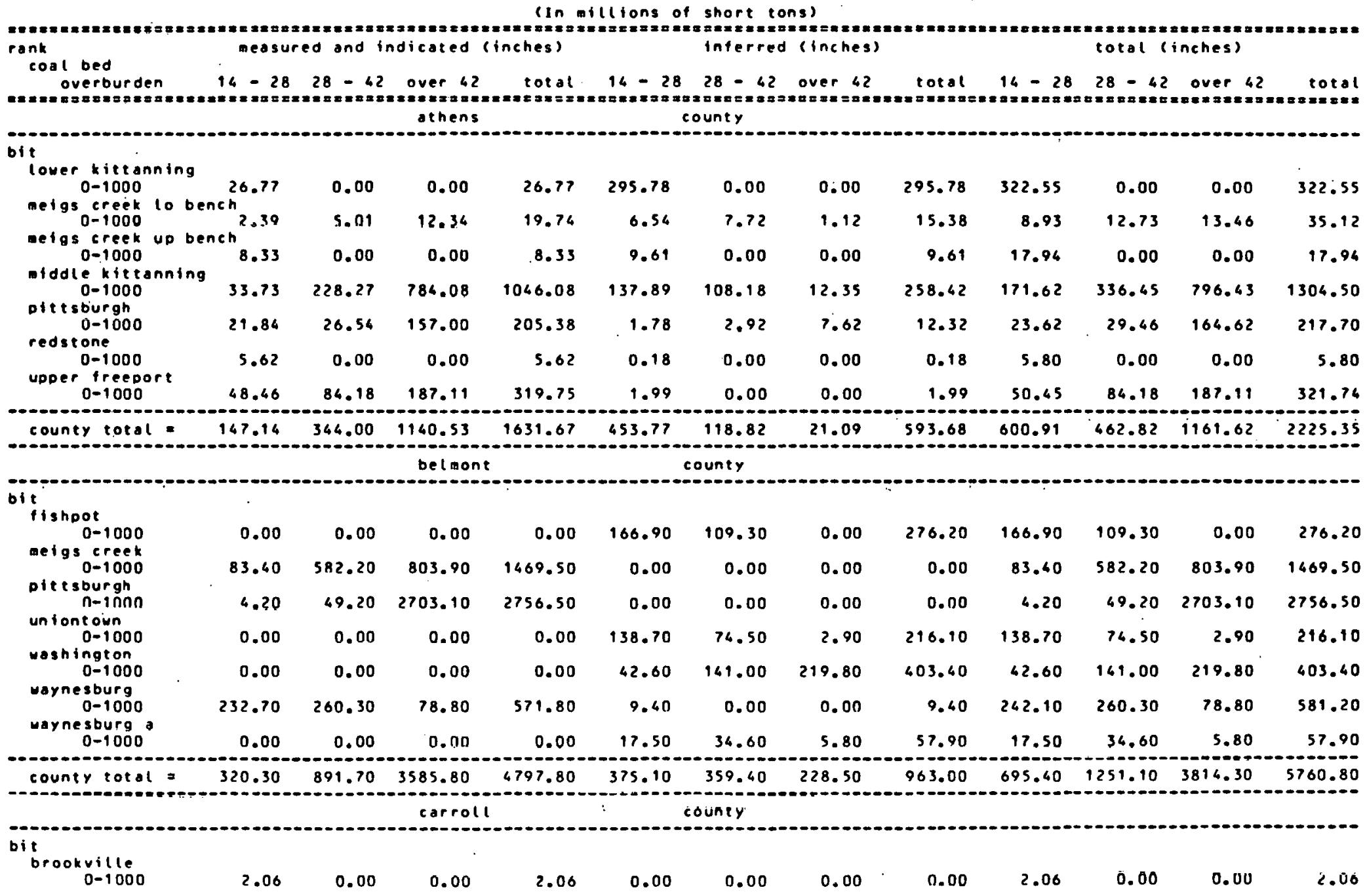




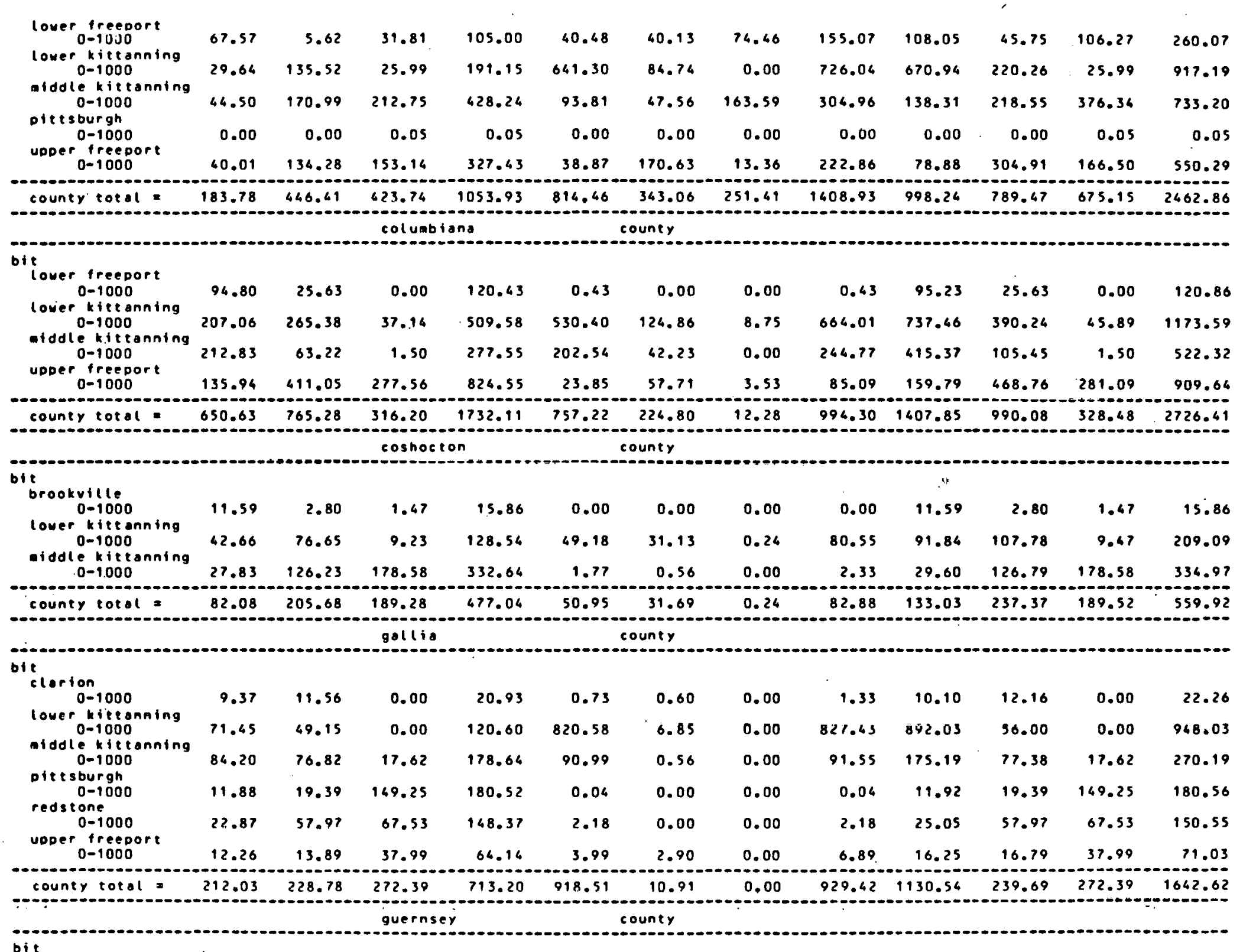




\begin{tabular}{|c|c|c|c|c|c|c|c|c|c|c|c|c|}
\hline $\begin{array}{c}\text { lower kittanning } \\
0-1000\end{array}$ & 28.18 & 53.80 & 43.68 & 125.66 & 692.10 & 219.66 & 7.10 & 918.86 & 120.28 & 273.46 & 50.78 & 1044.52 \\
\hline meigs creek lo ben & en 1.76 & 4.71 & 10.58 & 17.05 & 0.10 & 0.00 & 0.00 & 0.10 & 1.86 & 4.71 & 10.58 & 17.15 \\
\hline $\begin{array}{c}\text { weigs creek up ben } \\
0-1000\end{array}$ & ich 0.88 & 0.33 & 0.00 & 1.21 & 0.00 & 0.00 & 0.00 & 0.00 & 0.88 & 0.33 & 0.00 & 1.21 \\
\hline $\begin{array}{c}\text { - Hdole kitzanning } \\
0-1000\end{array}$ & 84.02 & 601.50 & 46.31 & 529.83 & 174.19 & 338.19 & 0.00 & 512.38 & 258.21 & 739.69 & 46.31 & 1042.21 \\
\hline $\begin{array}{r}\text { Dietsourgh } \\
0-1000\end{array}$ & 1.72 & 12.59 & 49.36 & 63.67 & 0.22 & 0.44 & 0.46 & 1.12 & 1.94 & 13.03 & 49.82 & 64.79 \\
\hline $\begin{array}{l}\text { Udoer Preeport } \\
0-1000\end{array}$ & 58.54 & 146.34 & 677.77 & 682.65 & 7.75 & 6.42 & 8.12 & 22.29 & 66.29 & 152.76 & 485.89 & 704.94 \\
\hline county total & 175.10 & 619.27 & 625.70 & 1420.07 & 874.36 & 564.71 & 15.68 & 1454.75 & 1049.46 & 1183.98 & 641.38 & 2874.82 \\
\hline & & & harri & & & ounty & & & & & & \\
\hline & & & & & & & & & & & & \\
\hline $0-1000$ & 11.85 & 35.35 & 411.36 & 458.56 & 32.59 & 30.09 & 44.84 & 107.52 & 44.44 & 65.44 & 456.20 & 566.08 \\
\hline $0-1000$ & 16.68 & 30.40 & 3.96 & 49.04 & 598.32 & 21.94 & 0.00 & 620.26 & 613.00 & 52.36 & 3.96 & 669.30 \\
\hline $\begin{array}{c}\text { metgs creek lo beñc } \\
0-1000\end{array}$ & 6.08 & 31.32 & 92.20 & 129.60 & 0.00 & 0.00 & 0.00 & 0.00 & 6.08 & 31.32 & 92.20 & 129.60 \\
\hline $0-1000$ & 3.43 & 0.00 & 0.00 & 3.43 & 0.00 & 0.00 & 0.00 & 0.00 & 3.43 & 0.00 & 0.00 & 3.43 \\
\hline $0-1000$ & 67.97 & 254.61 & 278.22 & 600.80 & 42.63 & 70.68 & 9.68 & 122.99 & 110.60 & 325.29 & 287.90 & 723.79 \\
\hline $\begin{array}{l}\text { sburgh } \\
0-1000\end{array}$ & 0.34 & 7.87 & 478.54 & 486.75 & 0.02 & 0.00 & 3.96 & 3.98 & 0.36 & 7.87 & 482.50 & 690.73 \\
\hline $0-1000$ & 50.29 & 217.52 & 73.26 & 341.07 & 13.07 & 31.91 & 0.00 & 44.98 & 63.36 & 249.43 & 73.26 & 386.05 \\
\hline county total $=$ & 154.64 & 577.07 & 1337.54 & 2069.25 & 686.63 & 154.62 & 58.48 & 899.73 & 841.27 & 731.69 & 1396.02 & 2968.98 \\
\hline & & & hock & & & $\ln t$ & & & & & & \\
\hline 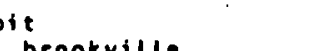 & & & & & & & & & & & & \\
\hline $0-1000$ & 0.00 & 0.20 & 0.00 & 0.20 & 0.00 & 0.00 & 0.00 & 0.00 & 0.00 & 0.20 & 0.00 & 0.20 \\
\hline $\begin{array}{l}0-1000 \\
\text { lower kittanning }\end{array}$ & 27.60 & 0.94 & 0.00 & 28.54 & 0.30 & 0.00 & 0.00 & 0.30 & 27.90 & 0.94 & 0.00 & 28.84 \\
\hline indale ktttanning & 37.73 & 3.16 & 2.62 & 43.51 & 4.76 & 0.00 & 0.00 & 4.76 & 62.69 & 3.16 & 2.62 & 48.27 \\
\hline $0-1000$ & 0.00 & 1.37 & 192.53 & 193.90 & 0.00 & 0.00 & 0.00 & 0.00 & 0.00 & 1.37 & 192.53 & 193.90 \\
\hline $0-1000$ & 3.66 & 8.25 & 13.31 & 25.22 & 0.00 & 0.00 & 0.00 & 0.00 & 3.66 & 8.25 & 13.31 & 25.22 \\
\hline county total & 68.99 & 13.92 & 208.46 & 299.37 & 5.06 & 0.00 & 0.00 & 5.06 & 74.05 & 13.92 & 208.46 & 296.43 \\
\hline & & & nolm & & & $\ln t y$ & & & & & & \\
\hline ott & & & & & & & & & & & & \\
\hline $\begin{array}{l}\text { brookville } \\
\text { u-luuu } \\
\text { lower kittanning }\end{array}$ & 12.48 & 38 & .00 & 13.00 & .00 & 0.00 & 0.00 & $n . n n$ & 12.48 & .38 & 0.00 & 13.86 \\
\hline $\begin{array}{c}0-1000 \\
\text { ardole kittonm }\end{array}$ & 18.72 & 53.27 & 6.92 & 78.91 & 1.87 & 12.29 & 0.00 & 14.16 & 20.59 & 65.56 & 6.92 & 93.07 \\
\hline $0-1000$ & 12.97 & 8.29 & 0.81 & 22.07 & 0.13 & 0.00 & 0.00 & 0.13 & 13.10 & 8.29 & 0.81 & 22.20 \\
\hline
\end{tabular}




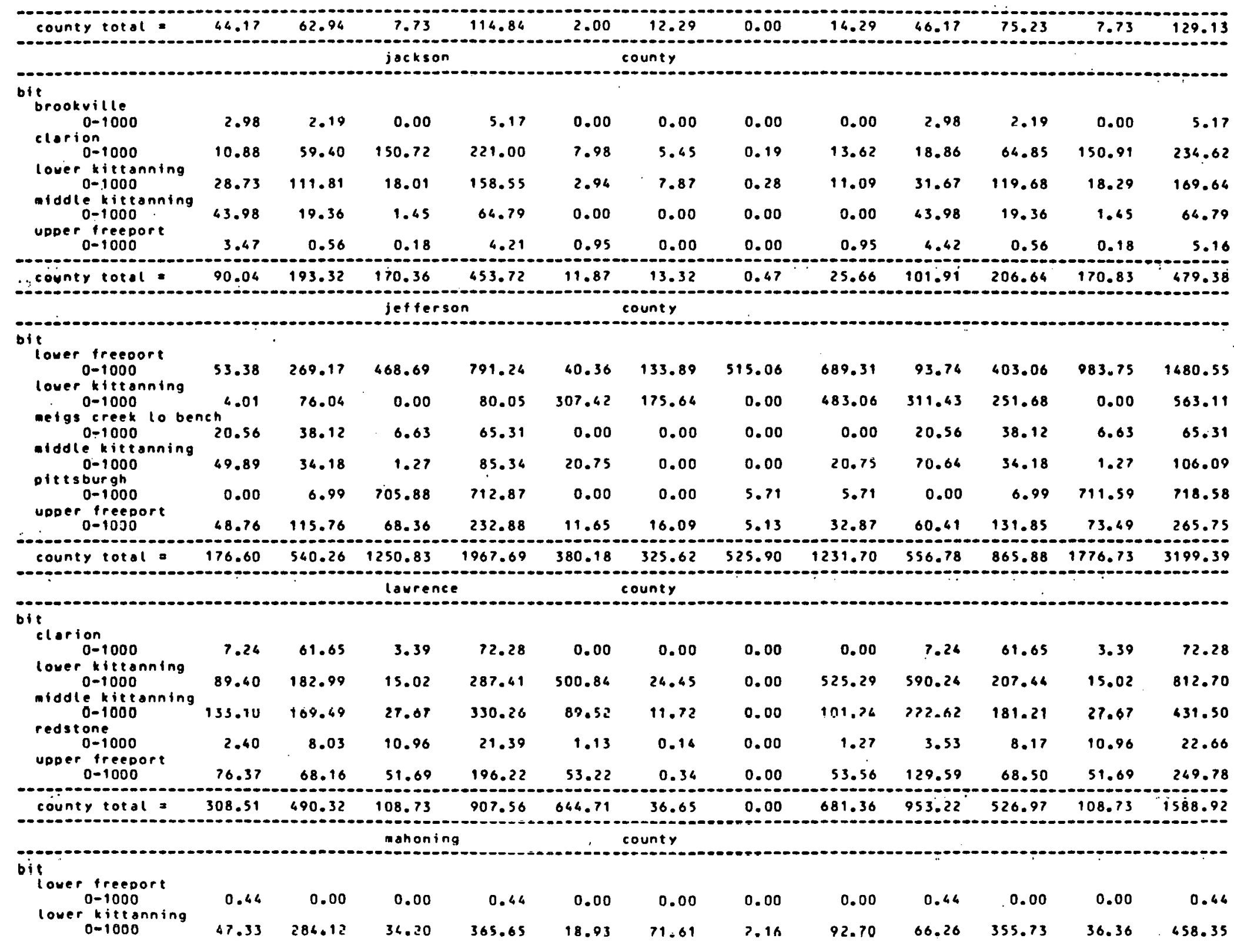




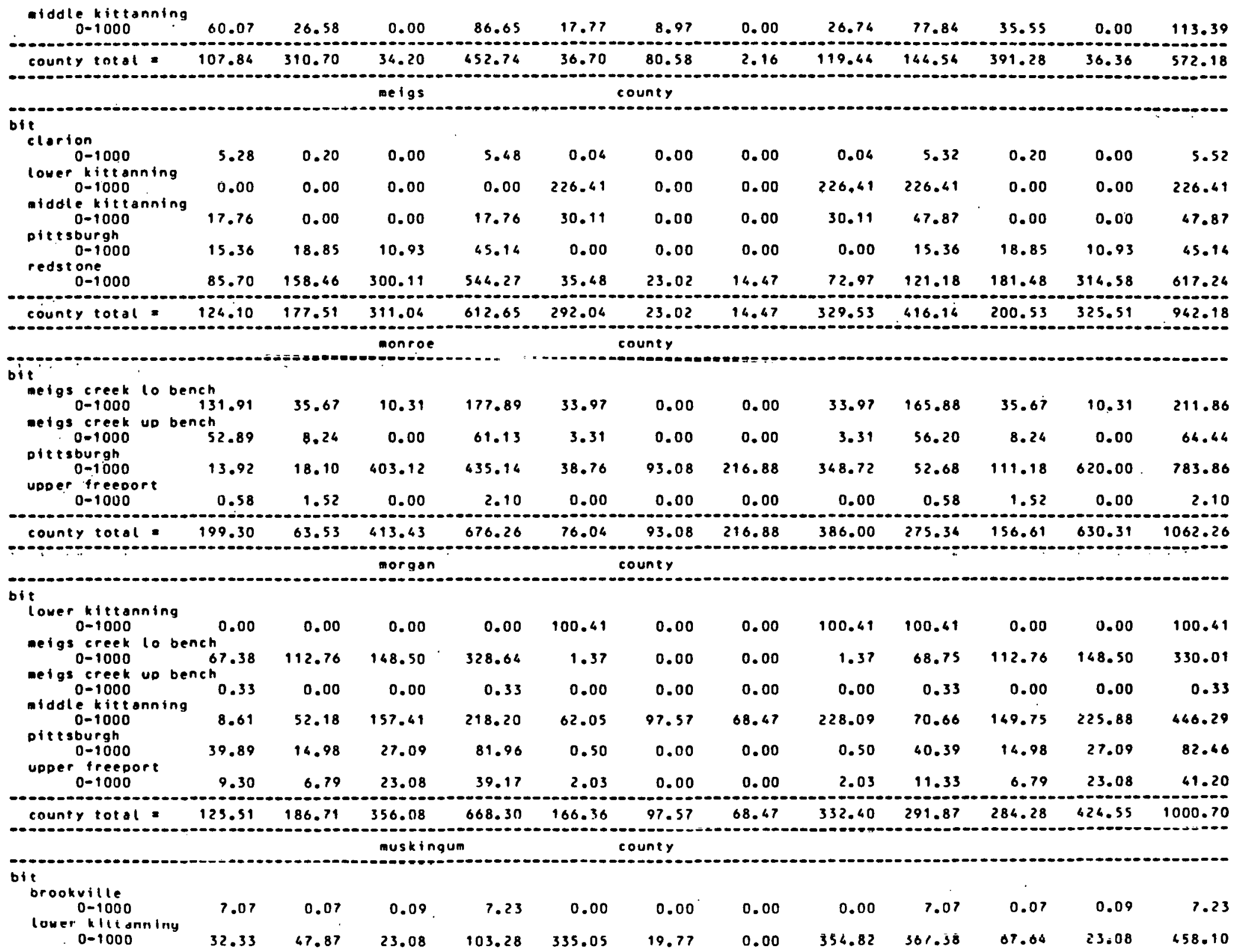




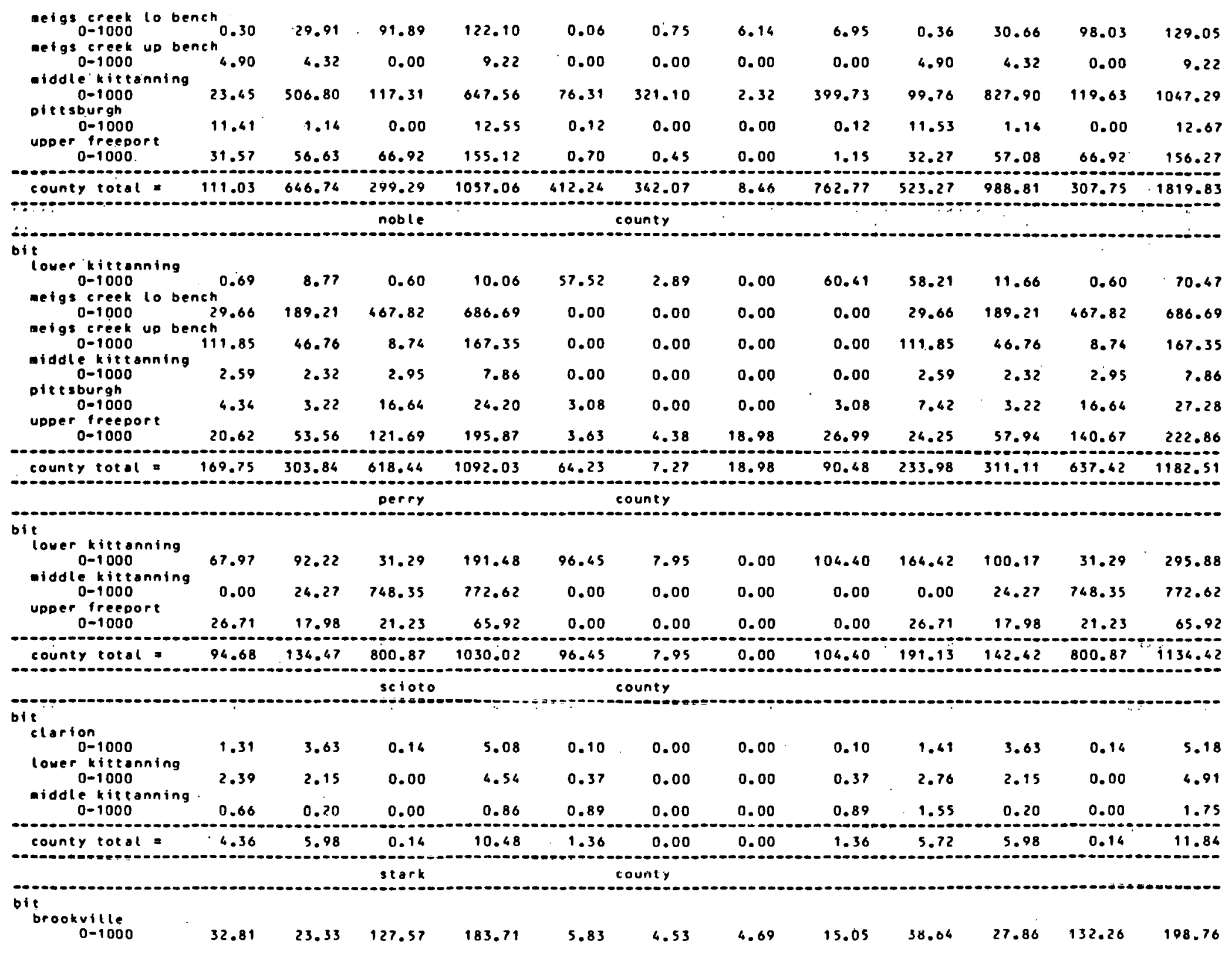




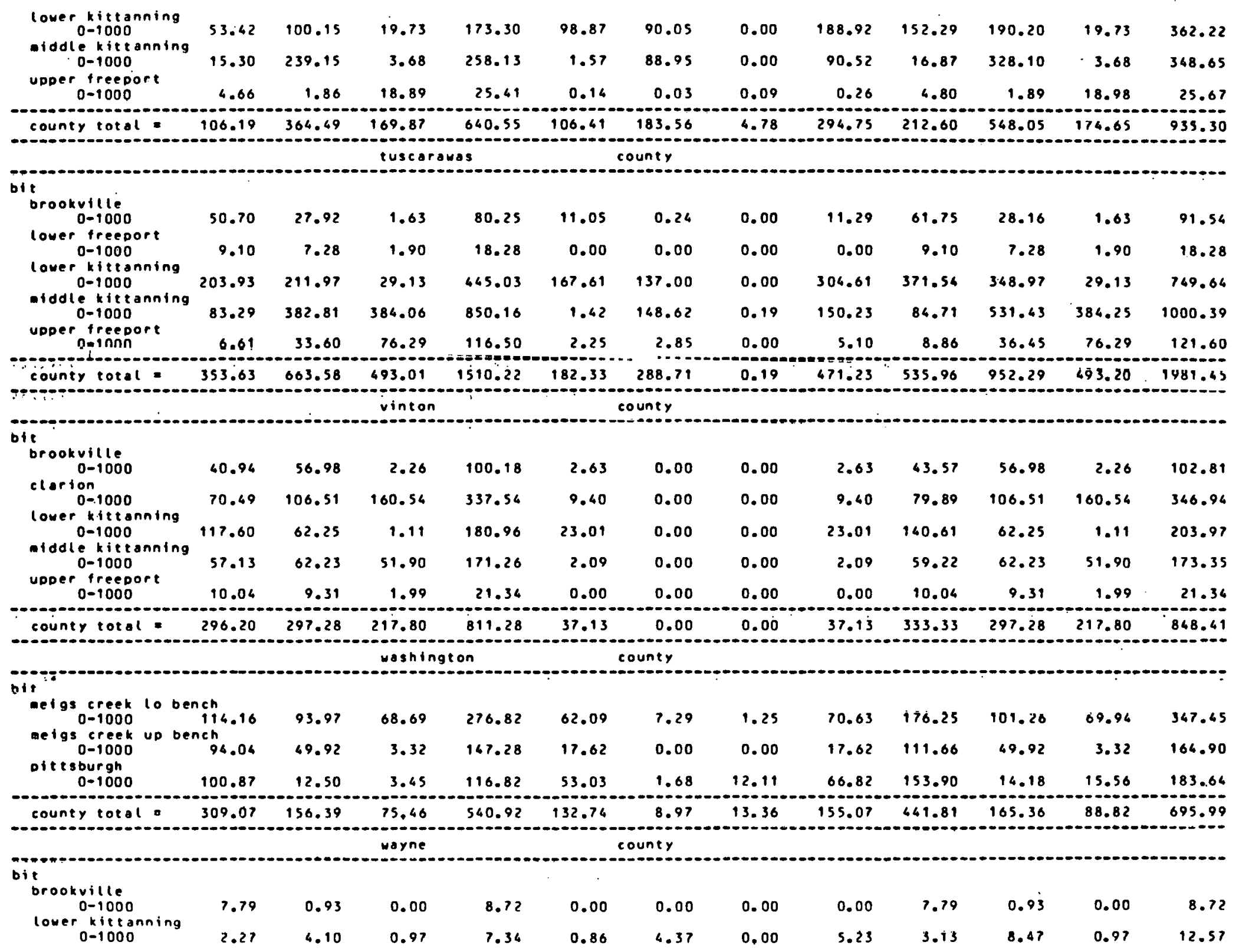




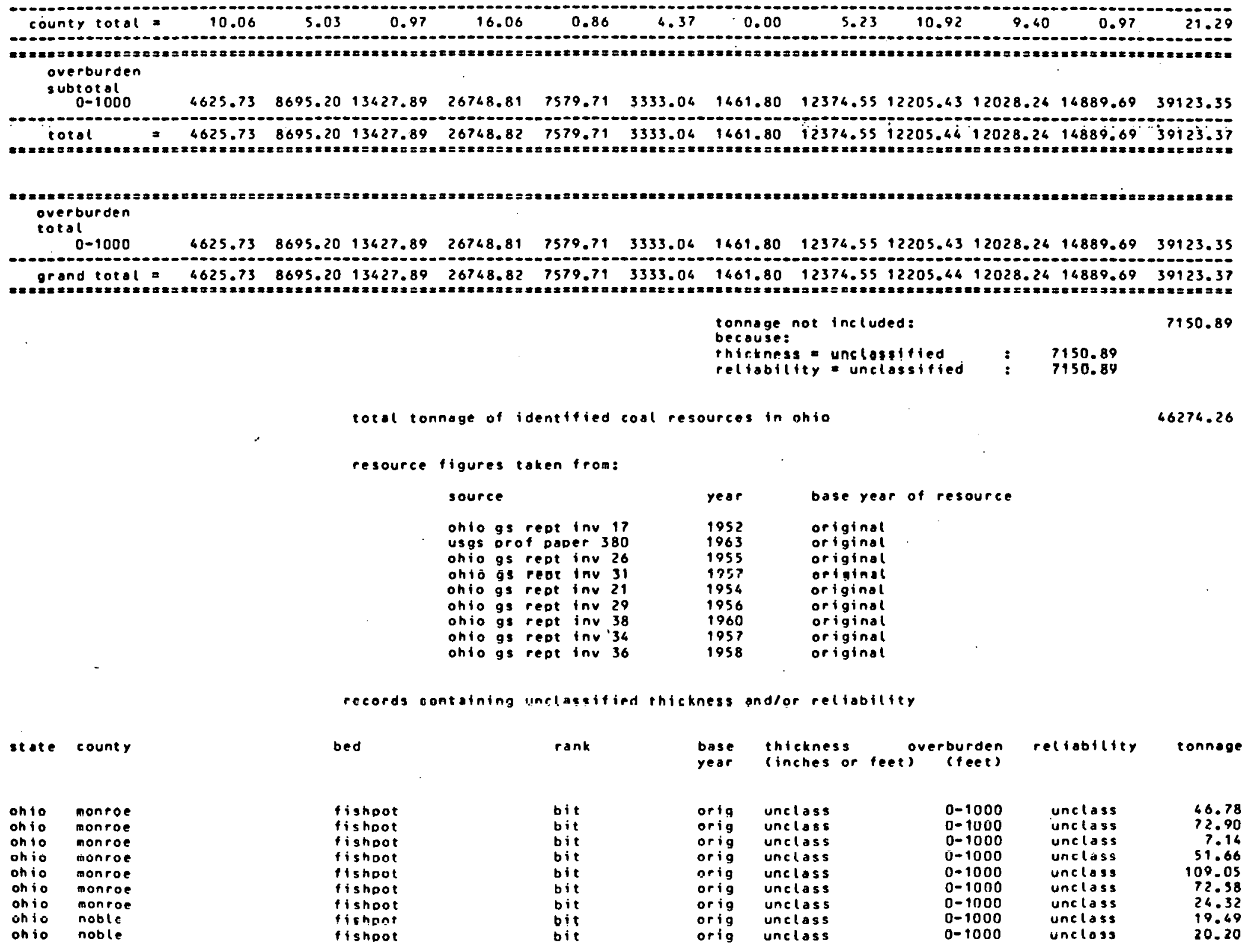




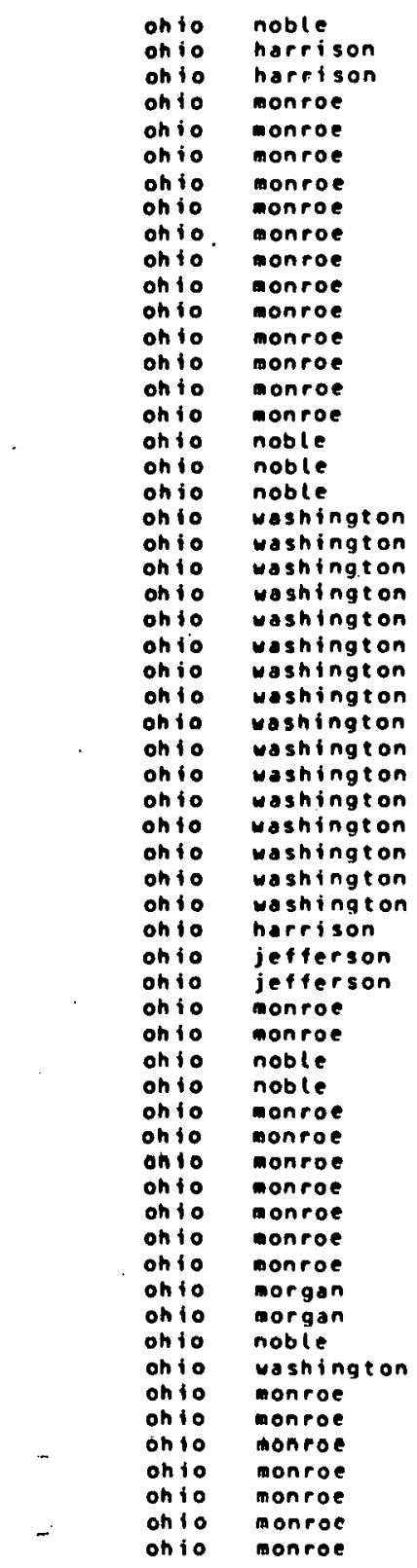

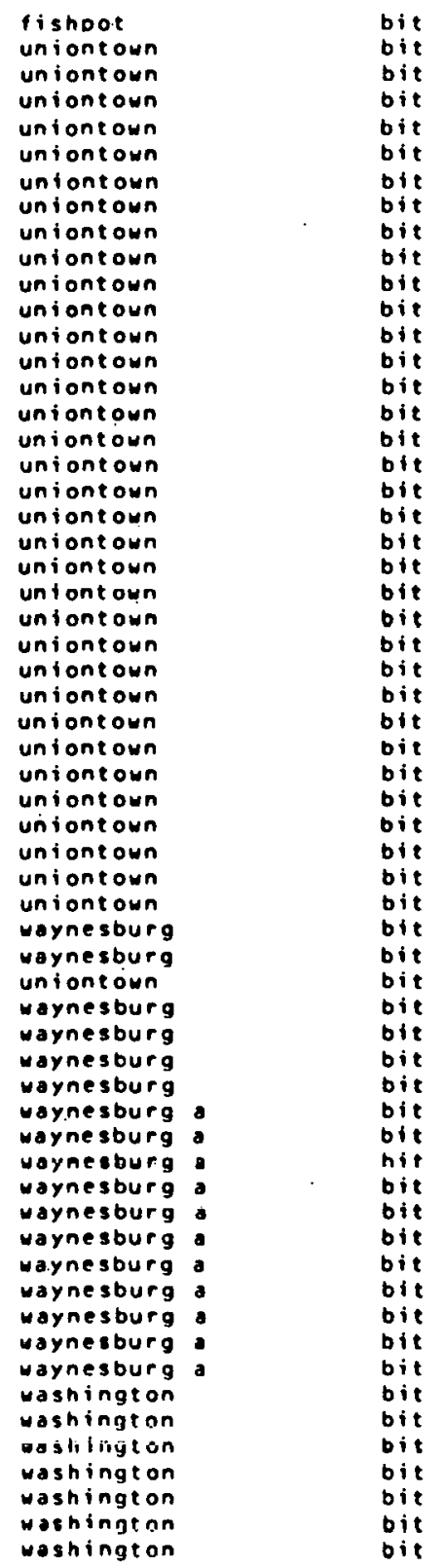

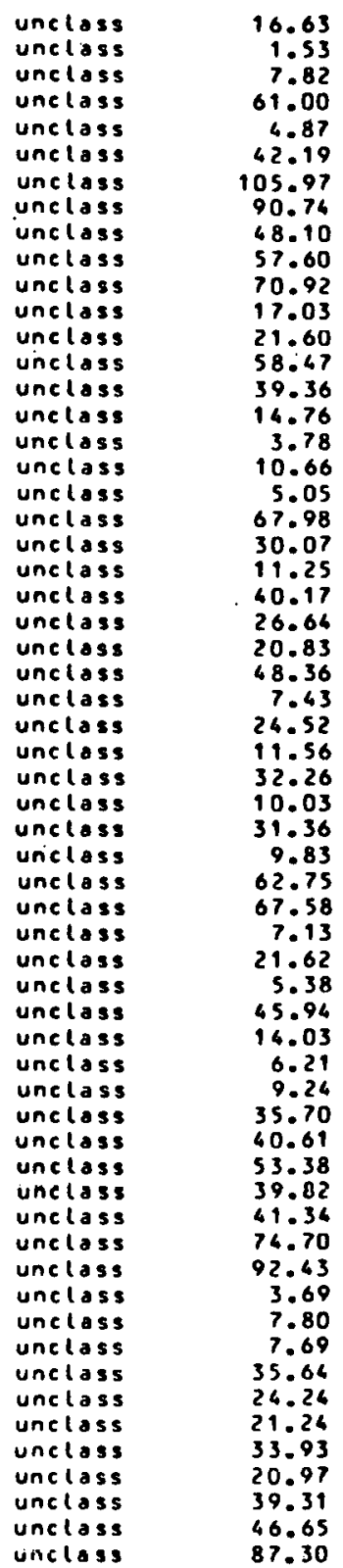

6.63
1.53 7.82
61.00 4.87
42.19 105.97 48.10 58.60
70.92 7.03 21.60
58.47 39.36
14.76 3.78
10.66 5.05 30.07
31.07 19.25 (10.64 48.36
7.43 7.43
24.52 11.56 10.03 1.36 2.75 7.58 21.62 45.94 6.21 9.24
50.70
53.69 3.38 39.82 4.70 7.63
3.69 7.80 35.66 21.24 33.93
20.97
39.31 46.65
87.30 

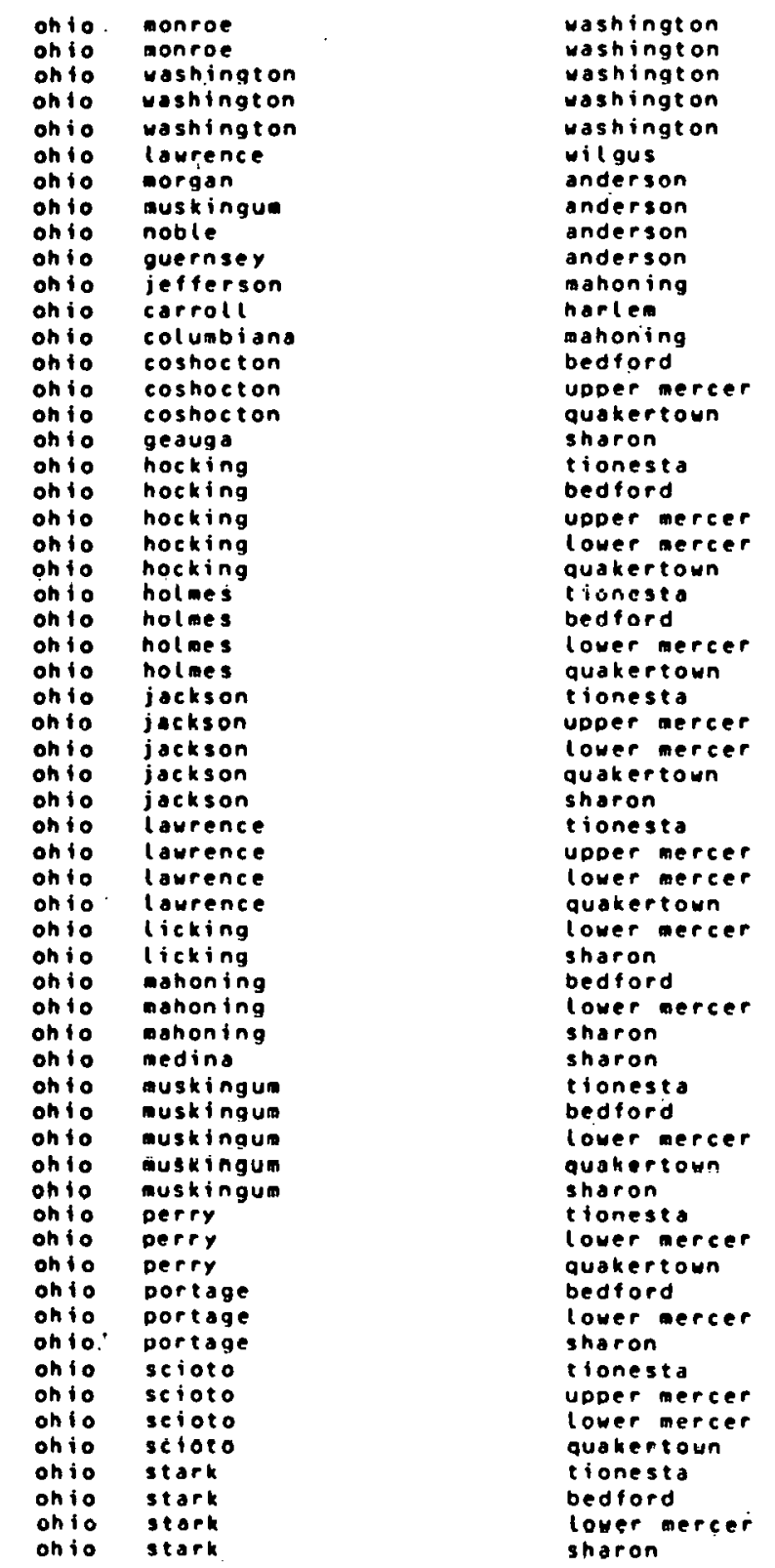

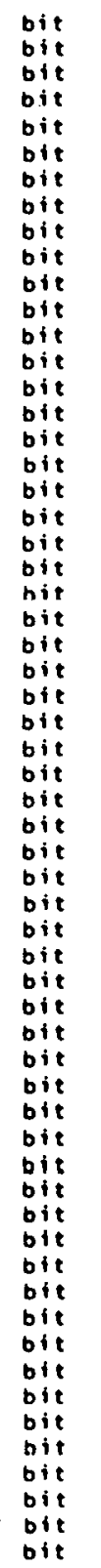

\begin{tabular}{|c|c|c|}
\hline $\begin{array}{l}\text { Orig } \\
\text { orig } \\
\text { orig } \\
\text { orig }\end{array}$ & $\begin{array}{l}\text { unclass } \\
\text { unclass } \\
\text { unclass } \\
\text { unclass }\end{array}$ & $\begin{array}{l}0-1000 \\
0-1000 \\
0=1000 \\
0-1000\end{array}$ \\
\hline orig & unclass & $0-1000$ \\
\hline $\begin{array}{l}\text { Orig } \\
\text { orig }\end{array}$ & unc los 3 & $\begin{array}{l}0-1000 \\
0-1000\end{array}$ \\
\hline orig & unclass & $0-1000$ \\
\hline orig & unclass & $0-1000$ \\
\hline orig & unctass & $0-1000$ \\
\hline orig & uncloss & $0-1000$ \\
\hline orig & uncloss & $0-1000$ \\
\hline orig & unclass & $0-1000$ \\
\hline orig & unclass & $0-1000$ \\
\hline orig & unclass & $0-1000$ \\
\hline orig & unclass & $0-1000$ \\
\hline orig & unclass & $0-1000$ \\
\hline orig & unclass & $0-1000$ \\
\hline orig & unclass & $0 \div 1000$ \\
\hline orig & unclass & $0-1000$ \\
\hline orig & unclass & $\begin{array}{c}0-1000 \\
0-1000\end{array}$ \\
\hline $\begin{array}{l}\text { orig } \\
\text { orig }\end{array}$ & $\begin{array}{l}\text { unclass } \\
\text { unclass }\end{array}$ & $\begin{array}{l}0-1000 \\
0-1000\end{array}$ \\
\hline orig & unclass & $0=1000$ \\
\hline orig & unclass & $0-1000$ \\
\hline orig & unclass & $0-1000$ \\
\hline $\begin{array}{l}\text { orig } \\
\text { orig }\end{array}$ & $\begin{array}{l}\text { uncloss } \\
\text { uncloss }\end{array}$ & $\begin{array}{l}0-1000 \\
0-1000\end{array}$ \\
\hline orig & unclass & $0-1000$ \\
\hline orig & unclass & $0-1000$ \\
\hline orig & uncloss & $\begin{array}{l}0=1000 \\
0=1000\end{array}$ \\
\hline orig & unclass & $0-1000$ \\
\hline orig & uncloss & $0-1000$ \\
\hline orig & uncloss & $0-1000$ \\
\hline orig & unclass & $\begin{array}{l}0-1000 \\
0-1000\end{array}$ \\
\hline orig & unclass & $\begin{array}{l}0-1000 \\
0-1000\end{array}$ \\
\hline orig & unctoss & $\begin{array}{l}0-1000 \\
0-1000\end{array}$ \\
\hline $\begin{array}{l}\text { Orig } \\
\text { orig }\end{array}$ & $\begin{array}{l}\text { unclass } \\
\text { unctass }\end{array}$ & $0-1000$ \\
\hline orig & unclass & $0-1000$ \\
\hline orig & uncloss & $0-1000$ \\
\hline orig & unclass & $0-1000$ \\
\hline orig & uncloss & $0-1000$ \\
\hline orig & unclass & $0-1000$ \\
\hline orig & uncless & $0-1000$ \\
\hline orig & unclass & $0-1000$ \\
\hline orig & uncloss & $0-1000$ \\
\hline orig & uncless & $0-1000$ \\
\hline ortg & unclass & $0-1000$ \\
\hline orig & uncless & $0-1000$ \\
\hline orig & uncloss & $0-1000$ \\
\hline orig & uncloss & $0-1000$ \\
\hline orig & unclass & $0-1000$ \\
\hline orig & uncloss & $0-1000$ \\
\hline orig & unclass & $0-1000$ \\
\hline orts & unclass & $0-1000$ \\
\hline orig & unclass & $0-1000$ \\
\hline orig & unclass & $0-1000$ \\
\hline ortig & unclass & $0-1000$ \\
\hline orig & Unctass & \\
\hline
\end{tabular}

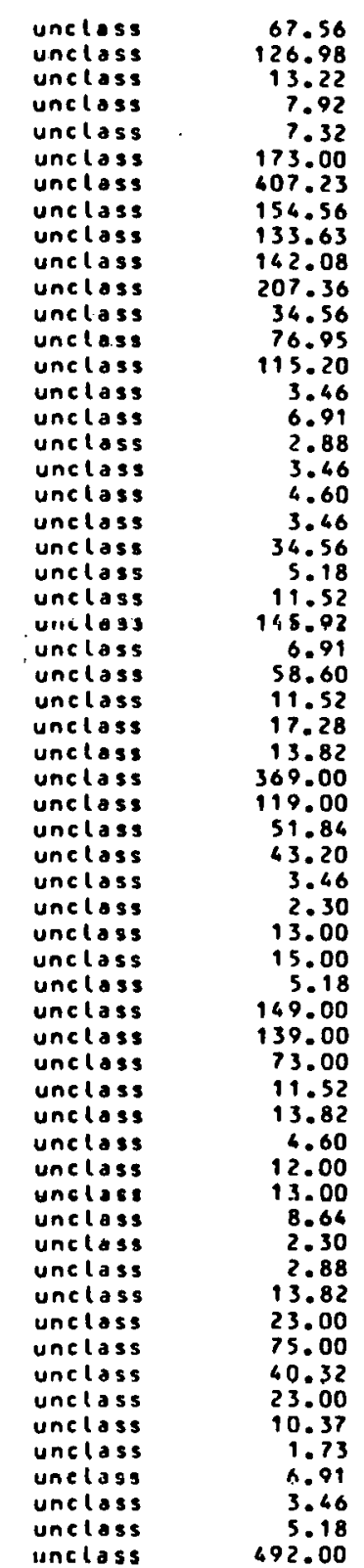




ohio sumnit
ohio trumbull
ohio tuscarawas
ohto tuscarawas
ohio vinton
onio vinton
ohio vinton
ohio wayne
ohio wayne
ohto wayne
ohio vayne
ohio wayne
ohio wayne

sharon
sharon

tionesta

upper mercer

upDer mercer

quakertoun
tionesta

bedford

upper mercer

lower mercer

quakertoun

sharon

bit
bit
bit
bit
bit
bit
bit
bit
bit
bit
bit
bit
bit

orig unclass

unclass
unclass

unclioss
unclioss

unclass

uncloss

unclass

uncloss

uncloss

uncloss

$0-1000$

$0-1000$

$0-1000$

$0-1000$

$0-1000$
$0-1000$

$0=1000$

$0=1000$

$0-1000$

$0-1000$

$0-1000$

$0-1000$
$0-1000$
$0-1000$

0-1000

unclass

unclass $\quad 228.00$

unclass 27.65

uncloss $\quad 17.00$

unclass 25.92

unclass $\quad 269.00$

unclass

unclass 3.91

unclass $\quad 3.46$

$\begin{array}{lr}\text { unclass } & 2.30 \\ \text { unclass } & 5.19\end{array}$

unclass 215.00

orig unclass.
orig uncless 
overage analyses of coal in onto

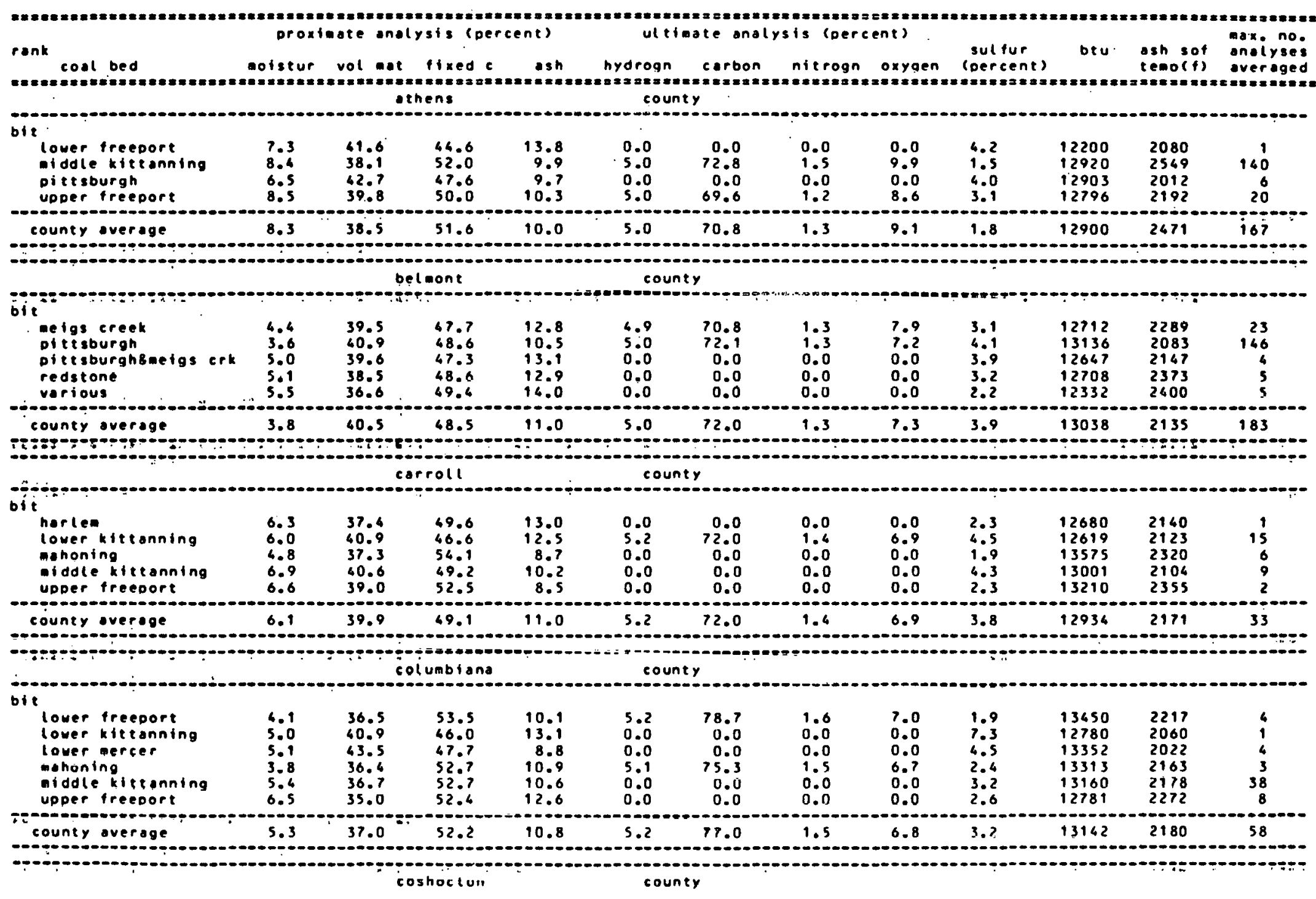




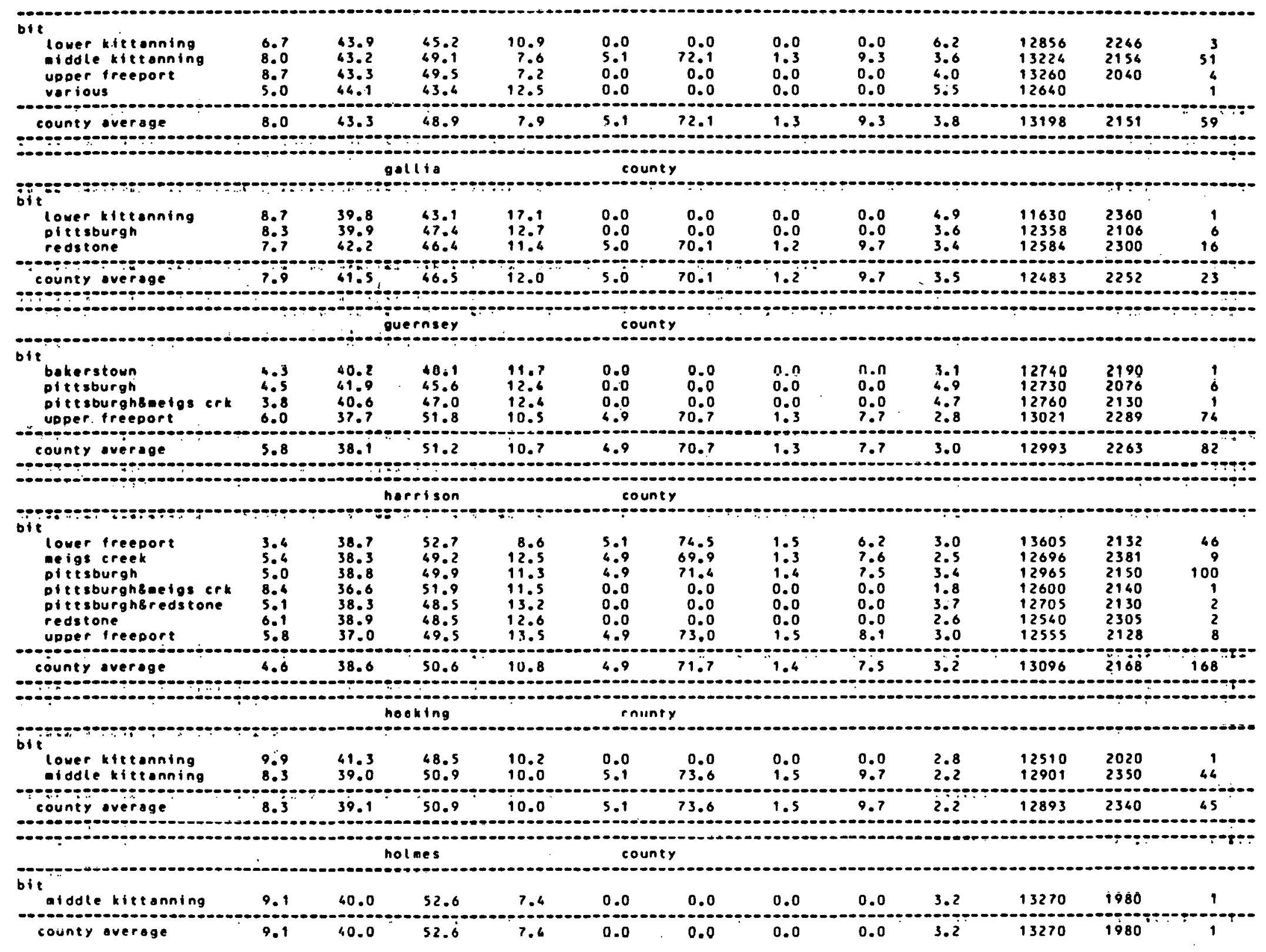




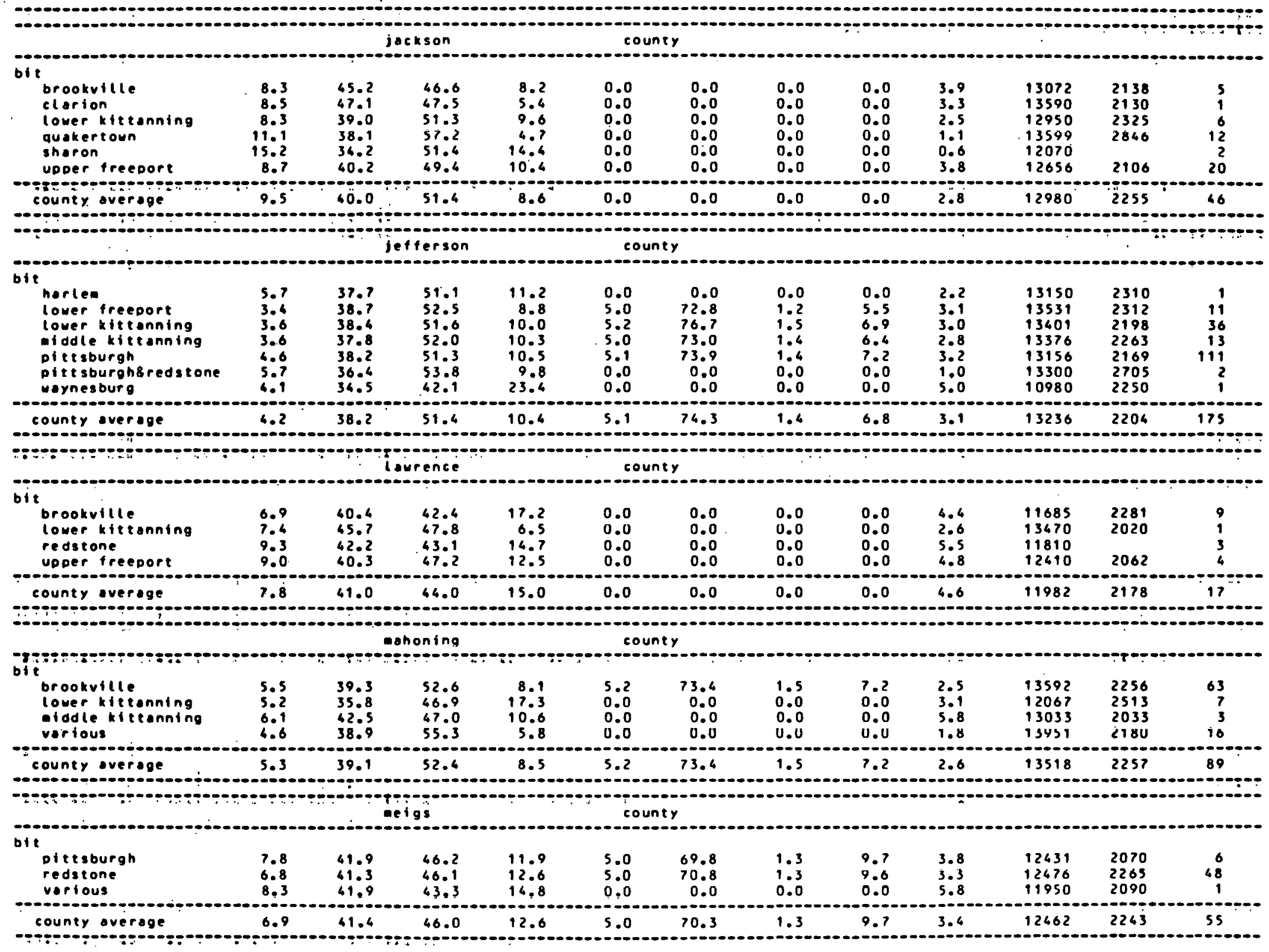


morgan

county

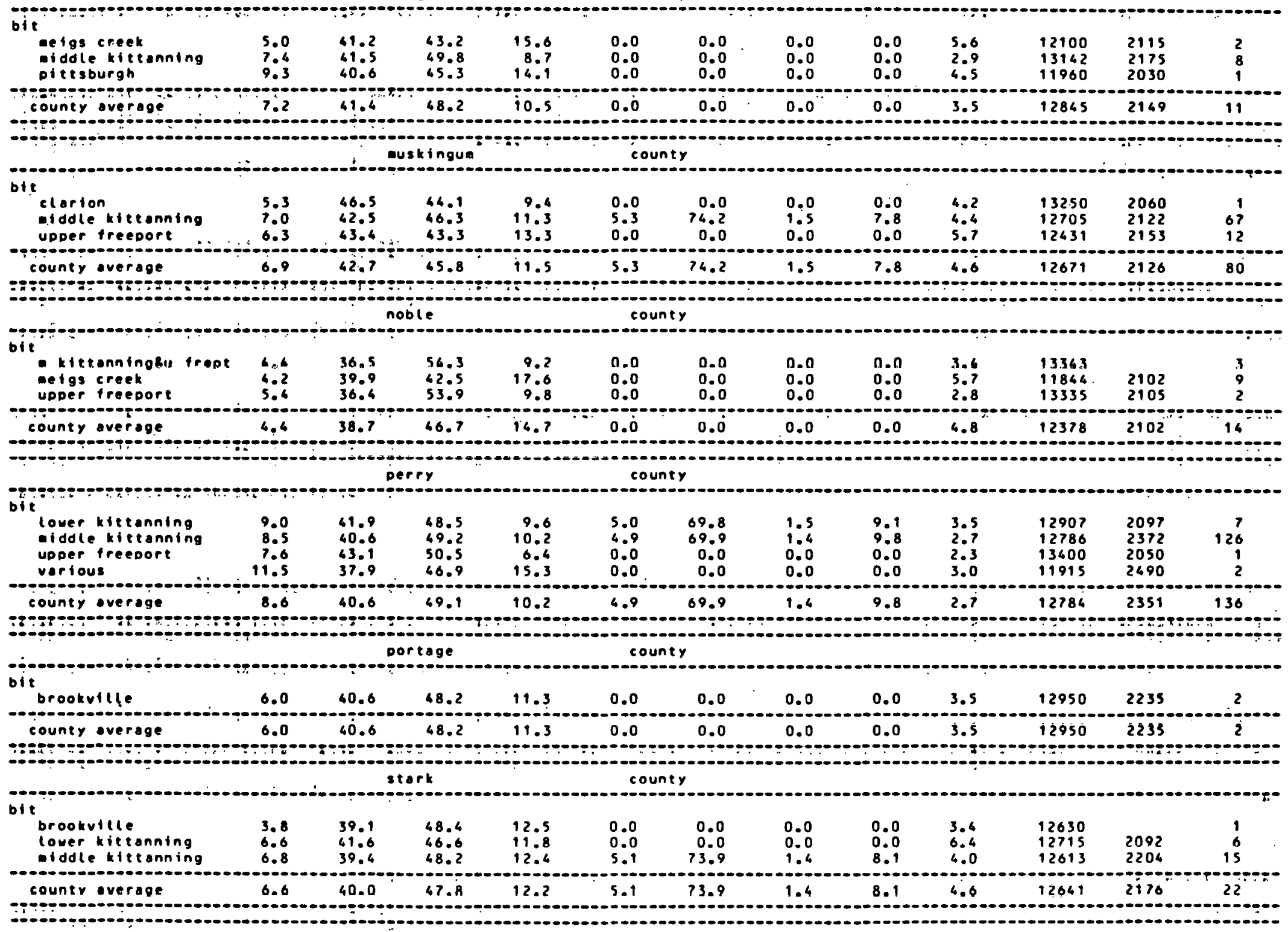


tuscaranas

county

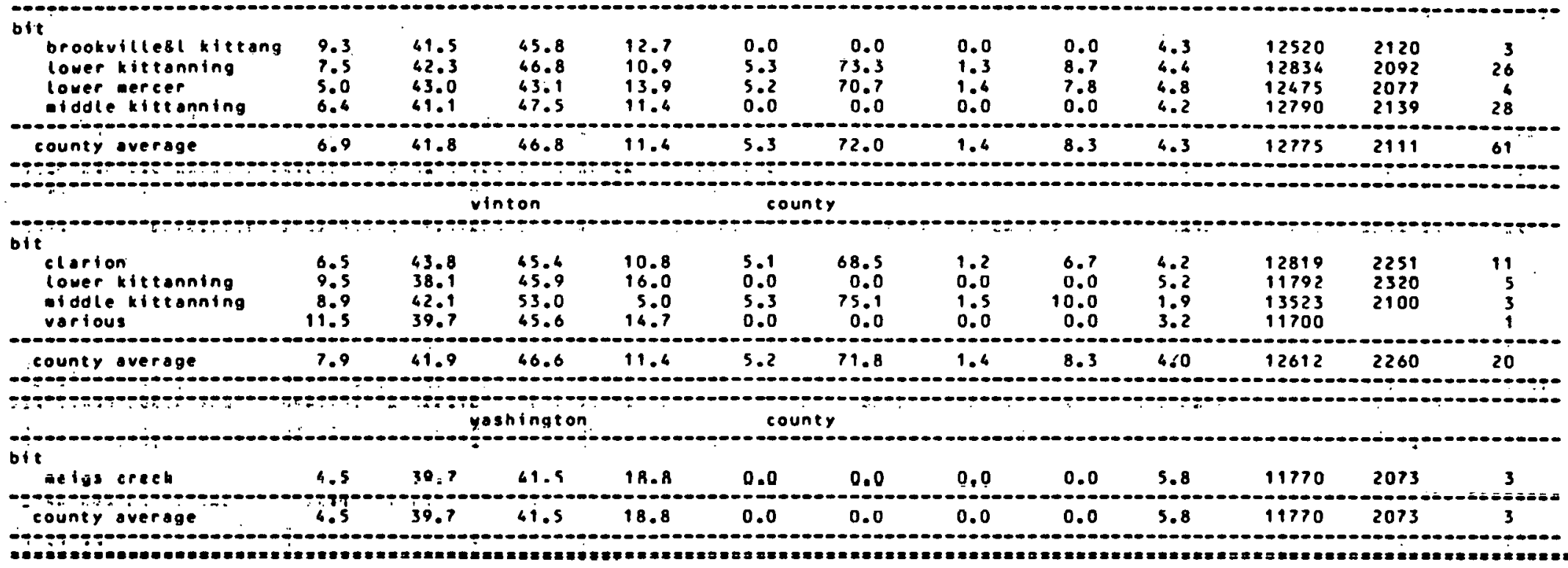


Ohio

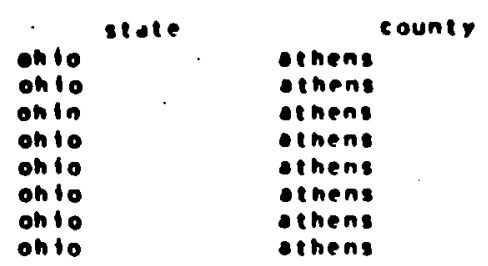

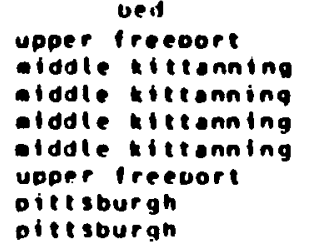

\begin{tabular}{|c|c|c|c|c|c|c|}
\hline $\begin{array}{l}01 \text { istur } \\
7.9 \\
9.9 \\
\because \because 9 \\
\because \cdots 9 \\
9.9 \\
8.1 \\
6.5 \\
9.1\end{array}$ & $\begin{array}{r}010.8 \\
43.6 \\
36.2 \\
35.6 \\
36.1 \\
34.5 \\
46.1 \\
46.9\end{array}$ & $\begin{array}{r}11 \text { redc } \\
67.0 \\
51.5 \\
31.0 \\
31.8 \\
50.9 \\
65.8 \\
44.5 \\
47.1\end{array}$ & $\begin{array}{r}0.15 \\
9.4 \\
12.3 \\
13.4 \\
13.1 \\
16.6 \\
11.1 \\
11.6 \\
9.5\end{array}$ & $\begin{array}{l}301 \text { lur } \\
5.3 \\
1.0 \\
1.8 \\
1.0 \\
1.3 \\
5.2 \\
5.0 \\
4.0\end{array}$ & $\begin{array}{l}080 \\
12940 \\
12520 \\
12320 \\
12390 \\
12070 \\
12810 \\
12670 \\
12700\end{array}$ & $\begin{array}{c}\text { ny jio } \\
0 \\
0 \\
0 \\
0 \\
0 \\
33 \\
53 \\
52\end{array}$ \\
\hline
\end{tabular}

continued...... 


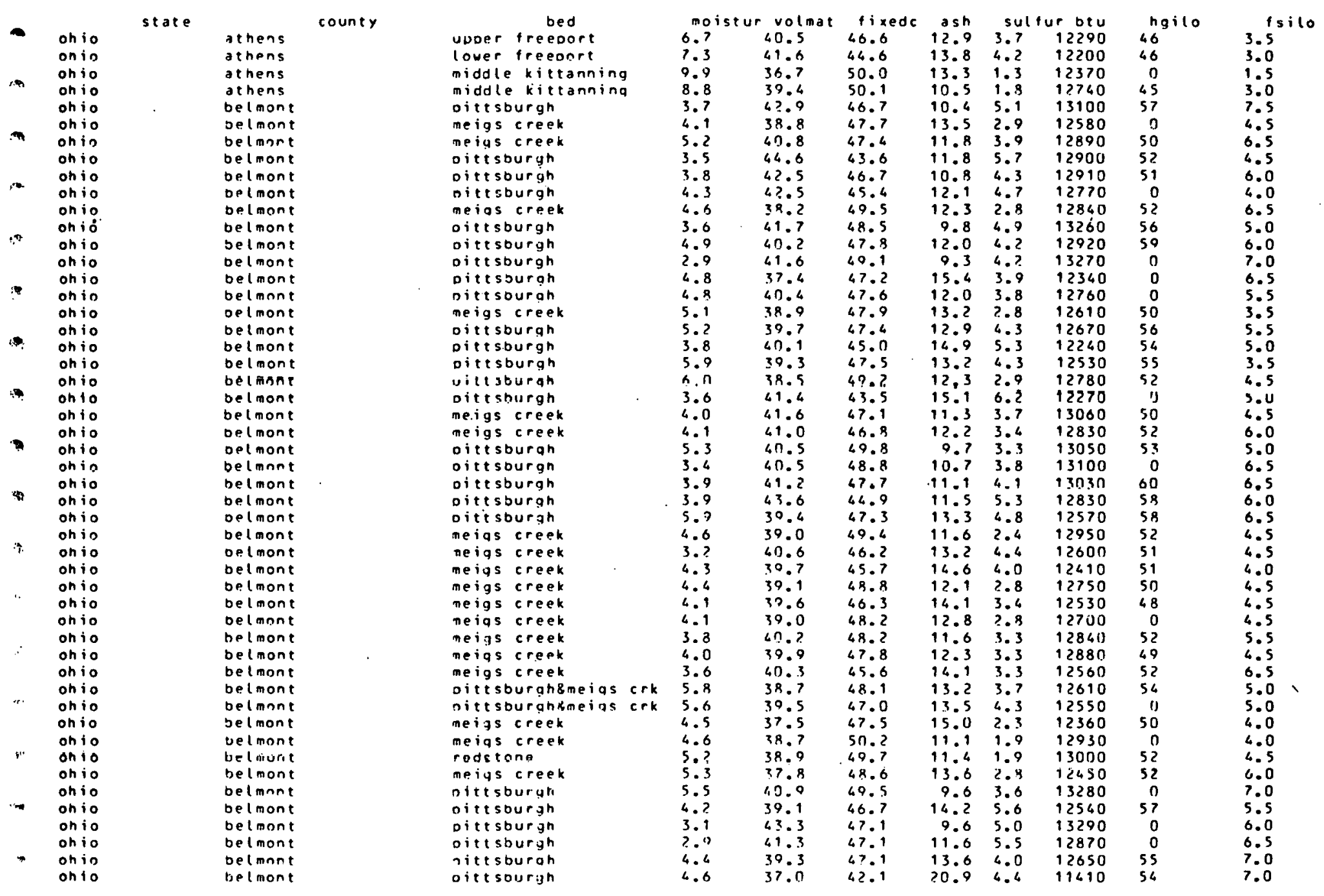




\begin{tabular}{|c|c|c|c|c|c|c|c|c|c|c|c|}
\hline & state & county & bed & moi & volmat & $f i x e$ & $\operatorname{ash}$ & sul & ur otu & hgilo & Isito \\
\hline ohio & & Delmant & meias creek & 5.1 & 38.6 & 49.0 & 12.6 & 2.6 & 12830 & 48 & $5.0^{\circ}$ \\
\hline onio & & belmont & neifs creek & 4.0 & 10.4 & 47.3 & 12.0 & 3.3 & 12860 & 48 & 3.0 \\
\hline onio & & belmont & Dittsburah & 7.9 & 41.3 & 45.8 & 12.9 & 5.1 & 12740 & 59 & 7.0 \\
\hline ohin & & belmont & oitesburgh & 3.6 & 41.4 & $4 R .0$ & 10.6 & 4.3 & 13900 & 0 & 6.0 \\
\hline onio & & bel mont & neigs creek & 4.6 & 41.3 & 67.6 & 11.1 & 3.7 & 12930 & 0 & 6.5 \\
\hline onio & & belmont & Ditisburyh & 4.5 & 39.5 & 49.7 & 10.8 & 3.3 & 12940 & 0 & 7.0 \\
\hline onio & & belmont & meiys creek & 3.9 & 39.9 & 44.8 & 15.3 & 4.1 & 12280 & 59 & 6.5 \\
\hline onio & & carroll & uDDer freeoort & 5.8 & 41.6 & 53.4 & 5.0 & 2.2 & 13700 & 53 & 4.0 \\
\hline on io & & carroli & lower kittanning & 5.8 & 39.6 & 43.7 & 16.7 & 4.9 & 11860 & 54 & 4.0 \\
\hline onio & & carroll & middle kittanning & 5.6 & 40.3 & 48.6 & 11.1 & $4 . ?$ & 12700 & so & 5.0 \\
\hline on io & & carroll & lower kittannirg & 5.8 & 41.4 & 43.4 & 15.2 & 4.1 & 12240 & 52 & 3.5 \\
\hline onto & & carroll & lower kittanning & 5.0 & 42.9 & 46.8 & 9.3 & 5.0 & 13090 & 52 & 4.5 \\
\hline onio & & carroll & upder freedort & 7.3 & 36.4 & 51.6 & 12.0 & 2.4 & 12720 & 59 & 5.0 \\
\hline onio & & carroll & middele kittanning & 5.4 & 41.2 & 49.0 & 9.8 & 4.6 & 13000 & 0 & 3.5 \\
\hline onio & & carroli & lower kittanning & 7.0 & $4 ? .3$ & 48.5 & $9 . ?$ & 3.6 & 13320 & 51 & 4.0 \\
\hline onio & & carroli & Inver kittanning & 4.3 & 44.6 & 45.0 & 10.4 & 4.8 & 13040 & si & 2.0 \\
\hline onio & & carroll & middle kittanning & 5.7 & 40.2 & 50.0 & 9.8 & 4.9 & 12920 & 49 & 5.0 \\
\hline onio & & carroll & lower kittannina & 5.9 & $4 ? .7$ & 47.7 & 9.6 & 4.6 & 12990 & 32 & 4.0 \\
\hline ohio & & carroll & harlem & 6.3 & 37.4 & 40.6 & 13.0 & 2.3 & 12680 & 53 & 2.0 \\
\hline onio & & carroll & nahoning & 4.7 & 36.4 & 53.4 & 10.2 & 2.9 & 13360 & 53 & 5.5 \\
\hline ahio & & carroli & manoning & 5.1 & 36.9 & 53.7 & 9.6 & 1.8 & 13390 & 50 & 5.5 \\
\hline on io & & columbiana & middle kittamina & 3.4 & 37.6 & 53.6 & 8.8 & 3,7 & 13510 & 61 & 7.0 \\
\hline onio & & columbiana & middle kittanning & 4.6 & 36.6 & $\begin{array}{l}53.8 \\
53.8\end{array}$ & 9.6 & 4.0 & 13430 & 56 & 7.0 \\
\hline onio & & columbiana & udper treedort & 4.3 & 35.7 & 54.8 & 9.5 & 1.2 & 13620 & 57 & 5.0 \\
\hline onio & & columbiana & upder freedort & 5.3 & 34.7 & 53.6 & 11.7 & 1.5 & 13100 & 55 & 6.5 \\
\hline onio & & columbiana & uoder frpenart & 4.3 & 36.1 & 56.2 & 7.7 & 1.7 & 13730 & 58 & 6.5 \\
\hline ohio & & columbiana & middle kittonning & 4.7 & 36.6 & 54.5 & 9.1 & 3.1 & 13520 & 56 & 7.0 \\
\hline onio & & columbiana & middle kittanning & 4.7 & 33.7 & 56.5 & 8.8 & 3.3 & 13580 & 57 & 7.0 \\
\hline on io & & columbiana & middle kittanning & 4.4 & 34.2 & 51.0 & 14.8 & $? .9$ & 12600 & 56 & 7.0 \\
\hline ohio & & columbiana & miadle kittannina & 4.4 & 37.1 & 53.5 & 9.4 & 3.2 & 13270 & 58 & 7.5 \\
\hline ohio & & columbiana & middle kittanning & 0.3 & 38.5 & 54.0 & 7.5 & 2.8 & 13600 & 46 & 4.5 \\
\hline onio & & columeiana & middle kittannina & 4.6 & 37.5 & 51.9 & 10.6 & 2.4 & 13230 & 54 & 5.0 \\
\hline onio & & columbiana & middle kittanning & 6.2 & 34.0 & 50.7 & 15.3 & 1.5 & 12630 & 54 & 6.0 \\
\hline ohin & & columbiana & middle kittannina & 4.4 & 36.5 & 51.9 & 11.6 & $? .4$ & 13090 & 53 & 4.0 \\
\hline ohio & & columbiana & middle kittanning & 4.9 & $3 n .2$ & $5 ? .9$ & 10.9 & 2.3 & 13160 & 53 & 3.5 \\
\hline onio & & columbiana & miadle kittanning & 6.3 & 34.4 & 53.4 & $1 ? .2$ & 1.2 & 12850 & 57 & 2.5 \\
\hline onio & & columbiana & lower kittanning & 5.0 & 40.9 & 46.0 & 13.1 & 7.3 & 12780 & 56 & 5.5 \\
\hline onin & & columbiana & worer freeonrt & 2.0 & 37.5 & 42.8 & 19.7 & 8.7 & 11770 & 69 & 5.5 \\
\hline ohio & & columbiana & upper frpeport & $\cdots$ & 34.1 & 50.3 & 15.6 & 2.6 & 12040 & 67 & 1.0 \\
\hline ohio & & columbiona & middle kittanning & 4.2 & 35.0 & 52.0 & 13.0 & $? .5$ & 12950 & 54 & 6.5 \\
\hline onio & & columbiana & udoer freeoort & 8.1 & 34.9 & $53 . ?$ & 11.9 & 2.2 & 12390 & 56 & 4.0 \\
\hline on in & & columbiana & upoer freeonit & 4.7 & 35.8 & 54.8 & 9.4 & 2.6 & 13610 & 57 & 7.5 \\
\hline ohio & & columbiana & middle kiteanninu & 4.9 & 38.2 & 53.0 & 7.9 & 3.8 & 13690 & 61 & 8.0 \\
\hline onio & & columbiana & midule kittannina & 7.0 & $4 ? .1$ & 49.1 & 8.8 & 0.5 & 13300 & $5 ?$ & 3.5 \\
\hline on io & & columbiana & middle kittannina & 5.5 & 36.7 & 53.9 & 9.4 & 3.9 & 13420 & 56 & 6.5 \\
\hline ohin & & columbiana & middle kittannina & 5.1 & 35.7 & 49.2 & 14.9 & 4.4 & 12670 & 53 & 6.5 \\
\hline onio & & columoiana & riddle kittannina & 5.0 & 35.4 & 54.5 & 10.1 & 2.5 & 13360 & 58 & 7.0 \\
\hline onio & & columbiana & lower freeport & 5.3 & 32.0 & 49.8 & 18.2 & 2.6 & 12150 & 55 & 5.0 \\
\hline oh io & & coshnct on & niddle kittanning & 9.5 & $\angle 2.4$ & 48.8 & 8.8 & 4.4 & 13150 & 53 & 3.5 \\
\hline ohio & & conenactonn & middle kittanning & $\ldots$ & 61.0 & 53.6 & 5.4 & 2.2 & 13250 & 0 & 1.0 \\
\hline
\end{tabular}




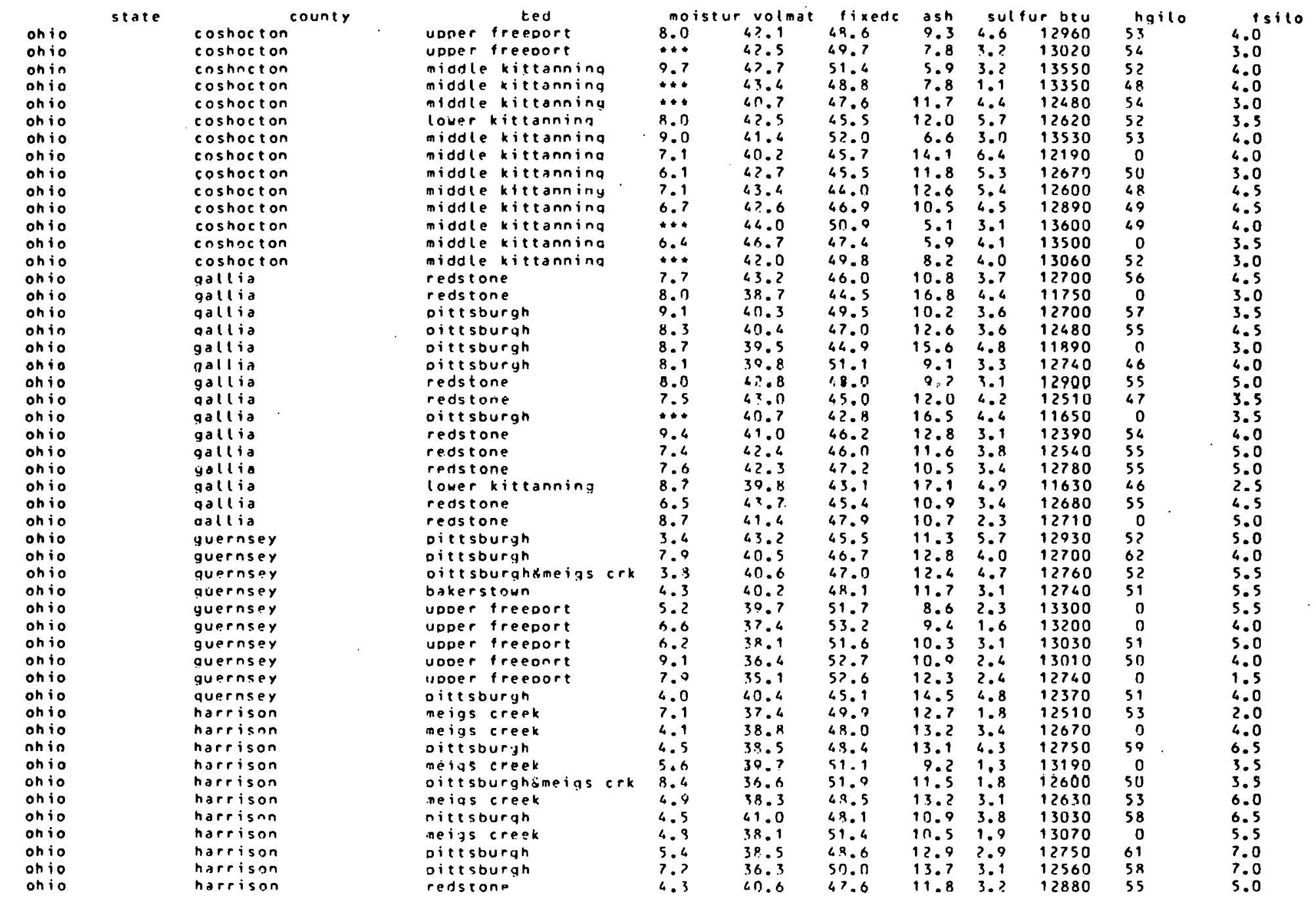




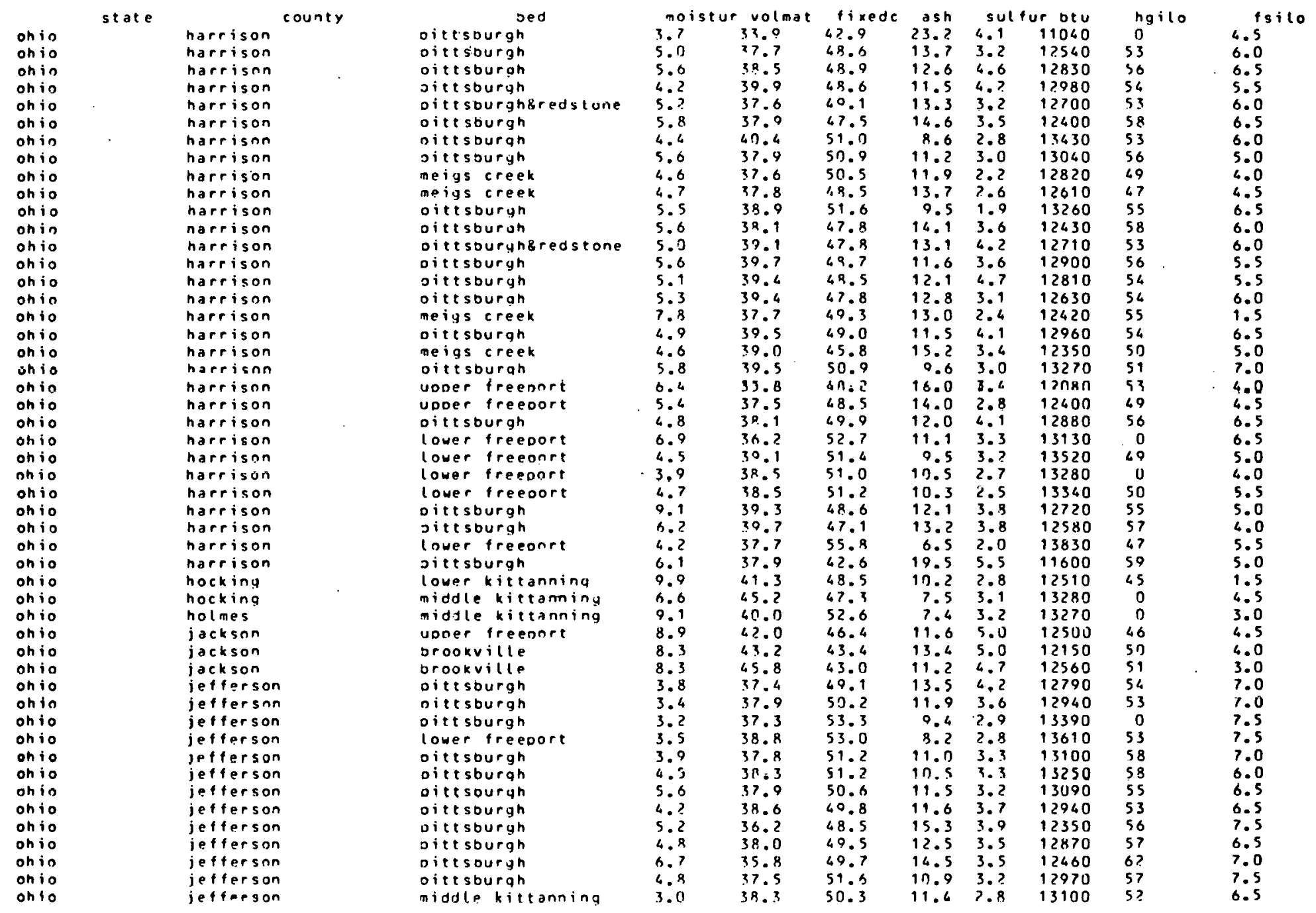




\begin{tabular}{|c|c|c|c|c|c|c|c|c|c|c|c|}
\hline & state & county & bed & $m n$ & volmat & fixedc & ash & sul & ur betu & hailo & isilo \\
\hline onio & & jetterson & oitesburgh & 3.4 & 38.8 & 53.8 & 7.4 & $? .8$ & 13690 & 0 & $\begin{array}{l}7.5 \\
6.5\end{array}$ \\
\hline ohio & & jefferson & lower kittanning & 0.5 & 35.9 & $51 . ?$ & 12.9 & 3.4 & 12830 & 0 & 6.5 \\
\hline ohio & & jefferson & lower kittanning & 4.5 & 30.0 & $5 ? .0$ & 9.0 & $3 . n$ & 13340 & 48 & 5.0 \\
\hline ohio & & jefferson & lower kittanning & 3.6 & 39.1 & 49.6 & 11.3 & 4.1 & 13150 & 52 & 6.5 \\
\hline onio & & jefferson & Lower kittanning & 3.4 & 60.7 & $5 \pi .4$ & 8,9 & $3 . ?$ & 13550 & 55 & 8.0 \\
\hline onio & & jefferson & lower freeoort & 4.9 & 37.1 & 53.8 & 9.1 & 2.7 & 13130 & so & 7.0 \\
\hline ohio & & jefferson & Dittsburan & 7.8 & 36.6 & 51.0 & 12.4 & 2.4 & 12630 & 0 & 2.5 \\
\hline onio & & jefterson & Dittsburgh & 4.3 & 40.3 & $5 n .1$ & 9.6 & 3.8 & 13270 & 52 & 7.0 \\
\hline ohin & & jefferson & oittsourgh & 5.9 & 37.8 & $5 n .7$ & 11.5 & 2.8 & $1309 n$ & 50 & 7.0 \\
\hline ohin & & jefterson & Inwer treeonrt & 5.0 & 35.7 & 55.6 & 7.9 & 9.5 & 13720 & 54 & 7.0 \\
\hline onio & & iefferson & lower kittanning & 5.8 & 35.1 & 51.0 & 13.9 & 3.2 & 12710 & 0 & 6.5 \\
\hline ohio & & jefferson & lower kitianning & 8.1 & 36.0 & $5 n .9$ & 13.1 & 3.4 & 12890 & 0 & 5.5 \\
\hline ohio & & jefferson & lower kittanning & 5.8 & 35.1 & 40.9 & 15.1 & 2.8 & 12690 & 0 & 6.5 \\
\hline ohio & & jefterson & middle kittanning & 6.2 & 37.3 & 54.1 & 8.0 & 2.4 & 13590 & 54 & 6.5 \\
\hline onio & & jefterson & oittsburgh & 4.3 & 37.4 & $52 . ?$ & 10.4 & 2.6 & 13280 & 57 & 7.5 \\
\hline onio & & jefferson & oitesburgh & 4.8 & 37.9 & 50.7 & 11.4 & 3.5 & 12970 & 55 & 6.5 \\
\hline onio & & jefforson & Dittsburgh & 6.4 & 36.2 & 52.5 & 11.3 & 1.1 & 13010 & 0 & 4.5 \\
\hline on io & & jeffersan & Dittsburgh & 5.0 & 38.3 & 51.0 & 10.7 & 3.5 & 13220 & 55 & 5.5 \\
\hline ohio & & jeffersen & nittsburah & 5.7 & 36.9 & 513.4 & $12 . ?$ & 3.6 & 12750 & 0 & 6.5 \\
\hline oh io & & iefferson & oitesburghsredstone & 5.3 & 36.9 & 54.0 & 9.1 & 1.0 & 13420 & 0 & 5.5 \\
\hline ohio & & jefferson & oittsburgh & 3.15 & 39.0 & .9 .1 & 13.1 & 4.9 & 17980 & 55 & 0.0 \\
\hline onio & & jefferson & dittsburyh & 4.7 & $6 n .4$ & 49.9 & 0.7 & 3.7 & 13250 & 54 & 5.5 \\
\hline ohio & & jefferson & nittsburgh & 7.1 & 34.2 & 50.0 & 15.8 & 0.9 & 12310 & 57 & 6.5 \\
\hline ohio & & jefterson & harlem & 5.7 & 37.7 & 51.1 & 11.2 & 2.2 & 13150 & 52 & 6.0 \\
\hline ohio & & jefferson & oittsburgh & 3.8 & 35.8 & 49.1 & 15.1 & 4.1 & 12450 & 55 & 6.5 \\
\hline onio & & jefterson & vaynesburg & 4.1 & 34.5 & $4 ? .1$ & 23.4 & 5.0 & 10980 & 55 & 4.5 \\
\hline ohio & & jefferson & oitesourah & 4.1 & 38.1 & 69.3 & 12.6 & $3 \cdot 3$ & 12680 & 55 & 6.0 \\
\hline on io & & jefferson & jittsburgh & 4.6 & $3.9 . ?$ & 50.3 & 11.5 & 3.1 & 13000 & 54 & 6.5 \\
\hline ohio & & jefterson & oittsourgh & 4.5 & 38.3 & 59.1 & 10.6 & 3.3 & 13170 & 0 & 6.5 \\
\hline ohio & & jefferson & Dittsburgh & 4.4 & 37.3 & 50.1 & 12.6 & 3.2 & 12820 & 56 & 6.5 \\
\hline onio & & jefferson & oittsburgh & 6.11 & 37.2 & 53.1 & 9.7 & 2.4 & 13190 & 54 & 7.0 \\
\hline onio & & jeffersan & Dittsburgh & 6.0 & 38.0 & 47.0 & 15.0 & 4.0 & 12440 & 54 & 5.5 \\
\hline ohio & & jefferson & oittsburgh & 4.5 & 38.1 & 50.7 & 11.9 & 3.4 & 12990 & 54 & 6.5 \\
\hline onio & & jefferson & lower freeport & 2.4 & 30.0 & 50.6 & 10.4 & 5.1 & 13310 & 58 & 6.5 \\
\hline ohio & & jefferson & lower freeport. & 3.6 & 40.9 & 40.4 & 9.7 & 5.3 & 13450 & 58 & 7.0 \\
\hline ohio & & jefterson & oittsburgh & 4.5 & 36.8 & 50.9 & 12.3 & 2.7 & 1.2950 & 55 & 7.0 \\
\hline onio & & jefferson & oitesburah & 3.7 & 39.0 & 50.6 & 10.4 & 3.3 & 13130 & 0 & 7.5 \\
\hline ohio & & lawrence & unper freeonrt & \#* & 38.8 & 45.7 & 15.5 & 4.3 & 11920 & 53 & 3.0 \\
\hline ohio & & mahoning & Drookville & 4.8 & 39.4 & 51.5 & 9.1 & 3.0 & 13530 & 52 & 6.5 \\
\hline ohio & & mahoning & srookville & 5.0 & 30.8 & 52.1 & 9.1 & 3.3 & 13760 & 54 & 5.5 \\
\hline onio & & manoning & Inwer kittannina & 9.3 & $4 n .2$ & 53.1 & 0.7 & 2.4 & 13880 & 54 & 3.5 \\
\hline ohio & & mahoning & lower kittanning & 4.9 & 39.4 & 56.6 & 4.0 & 1.8 & 14230 & 53 & 4.5 \\
\hline ohio & & mahoniny & bronkville & 3.8 & 35.5 & $63, n$ & 16.5 & 3.5 & 12200 & 55 & 4.5 \\
\hline ohio & & mananing & brookville & 5.3 & 46.9 & 48.6 & 7.3 & 3.7 & 13520 & 51 & 4.0 \\
\hline onio & & mahoning & brookville & 5.0 & 43.9 & 48.9 & 7.2 & 4.7 & 13650 & $5 ?$ & 3.5 \\
\hline ohin & & mahnning & Inver kittannind & 3.9 & 30.4 & 38.0 & 31.6 & 4.4 & 9750 & S? & 3.0 \\
\hline onio & & manoning & lower kittanning & 5.8 & 31.7 & $41) .4$ & 27.9 & 3.7 & 10370 & 0 & 5.0 \\
\hline ohio & & mahoning & brookville & 4.5 & 33.4 & $5 ? .5$ & 9.1 & 2.9 & 13550 & 55 & 5.5 \\
\hline ohio & & mahoning & brookville & 4.3 & 30.4 & 51.1 & 9.5 & 2.3 & 13340 & 50 & 5.5 \\
\hline onio & & mahnnina & orookville & 6.5 & 37.4 & 54.8 & 7.8 & 2.6 & 13650 & 52 & .0 \\
\hline
\end{tabular}




\begin{tabular}{|c|c|c|c|c|c|c|c|c|c|c|c|}
\hline & state & courity & bed & moi & r volmat & fixedc & ash & sut & ur otu & ngilo & isilo \\
\hline ohio & scace & mahoning & midale hittanning & 5.1 & $4 ? .0$ & 47.4 & 9.7 & 5.7 & 13220 & 50 & 5.0 \\
\hline onio & & mahoning & niddle kittanning & 8.5 & 40.4 & 46.8 & 12.8 & 6.0 & 12630 & 55 & 2.0 \\
\hline onio & & mahonina & brookville & 0.5 & 34.9 & 47.6 & 17.5 & 4.8 & 12030 & 0 & 6.0 \\
\hline onio & & mahoning & Drookville & 5.1 & 36.7 & $4 ? .3$ & 21.0 & 1.3 & 11600 & 33 & 1.0 \\
\hline ohio & & mahoniny & orookville & 5.5 & $4 n .5$ & 44.8 & 14.7 & 6.3 & 12240 & 51 & 3.5 \\
\hline on io & & manoning & brookville & 7.4 & 37.7 & 45.7 & 15.6 & 3,5 & 12270 & so & 3.0 \\
\hline onio & & mahoning & brookville & 5.6 & 41.9 & 43.0 & 15.1 & 7.7 & 12330 & 49 & 4.5 \\
\hline on io & & mahoning & orookville & 5.4 & 37.5 & 56.2 & 3.3 & 0.6 & 13920 & 58 & 4.5 \\
\hline on io & & mahoniny & orookville & 5.2 & 39.7 & 55.3 & 5.0 & $? .4$ & 14070 & 53 & 4,5 \\
\hline on io & & mahoning & brookville & 5.3 & 40.7 & 53.8 & 5.5 & $? .2$ & 14030 & 55 & 3.5 \\
\hline onio & & gatoning & various. & 5.2 & 40.0 & 55.0 & 5.0 & 9.7 & 14140 & 0 & 5.0 \\
\hline ohin & & mahoning & bronkville & 4.0 & 36.8 & 43.9 & 19.3 & 2.8 & 11960 & 38 & 2.0 \\
\hline on io & & nahoniny & orookville & $6 . ?$ & 36.0 & 49.9 & 16.1 & 2.4 & 12650 & 0 & 4.5 \\
\hline onin & & mahoning & brookville & 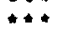 & 34.7 & 55.8 & 9.5 & 2.8 & 13460 & 0 & 5.0 \\
\hline ohio & & mahoning & orookville & 0.0 & 37.7 & 55.8 & 6.5 & 1.5 & 13830 & 0 & 5.0 \\
\hline onio & & manoning & brookville & 9.4 & 35.3 & 53.7 & 11.0 & 2.5 & 13110 & 0 & 4.0 \\
\hline $\begin{array}{l}\text { onio } \\
\text { on }\end{array}$ & & mahoning & brookville & 3.9 & 36.4 & $54 . ?$ & 11.4 & 2.6 & 13020 & 0 & 5.0 \\
\hline ohio & & mahoniny & lower kittanning & 3.5 & 35.6 & 44.7 & 10.7 & 3.4 & 11670 & 55 & 6.0 \\
\hline onio & & mahoning & lower kittanninu & 3.4 & 35.4 & 45.4 & 19.2 & 3.0 & 11690 & 55 & 5.5 \\
\hline onio & & mahoning & brookville & 4.1 & 39.4 & 49.9 & 10.7 & 3.6 & 13260 & 52 & 6.5 \\
\hline ohin & & manoning & hrookville & 3.9 & 61.0 & 53.8 & 0.2 & 3.4 & 13970 & 54 & 7.0 \\
\hline ohio & & neigs & reostone & $0: 2$ & 4.8 & 9.7 .8 & 0.7 & 2.1 & $1>97 n$ & 5? & 3.5 \\
\hline onio & & meigs & redstone & 6.8 & 43.3 & 46.0 & 10.7 & 3.2 & 12820 & 51 & 4.0 \\
\hline onio & & meigs & Dittsburgh & 6.5 & $4 ? .1$ & 46.3 & 11.6 & 3.5 & 12600 & 0 & 4.5 \\
\hline ohio & & meias & Dittsburgh & 5.7 & $4 ? .1$ & 44.0 & 13.9 & 5.3 & 12240 & 51 & 5.5 \\
\hline on io & & meias & redstone & 6.7 & 42.1 & 45.2 & 12.7 & 2.3 & 12540 & $5 ?$ & 4.0 \\
\hline onio & & meiqs & reostone & 7.8 & 39.9 & 66.2 & 13.9 & 3.1 & 12300 & 53 & 4.0 \\
\hline on io & & meigs & redstone & $9 . ?$ & 30.8 & 44.6 & 15.6 & 3.3 & 12030 & 0 & 4.0 \\
\hline on io & & meias & redstone & 7.5 & 43.0 & 46.5 & 10.5 & 5.3 & 12650 & 58 & 5.0 \\
\hline on io & & meias & redstone & 7.3 & 42.9 & 45.7 & 11.4 & 3.6 & 12570 & 49 & 5.0 \\
\hline onio & & meigs & oittsburon & 9.1 & 41.5 & 48.3 & $10 . ?$ & 3.0 & 12650 & $\mathbf{0}$ & 3.5 \\
\hline ohio & & meijs & redstone & 8.1 & $4 \pi .3$ & 45.2 & 14.5 & 5.4 & 12030 & 0 & 1.5 \\
\hline onio & & morgan & meigs creek & 4.7 & 42.6 & 44.0 & 13.4 & 5.7 & 12350 & 0 & 6.0 \\
\hline onio & & morjan & meigs creek & 5.3 & 39.8 & 42.4 & 17.8 & 5.5 & 11850 & 0 & 4.0 \\
\hline onio & & moraan & niddle kittanning & R.? & 43.6 & 47.7 & 8.7 & 3.5 & 13090 & ss & 4.5 \\
\hline onio & & muskinoum & udoer frepoort & 7.7 & 37.5 & 43.7 & 18.8 & 3.3 & 11590 & 53 & 4.5 \\
\hline $\begin{array}{l}\text { on io } \\
\text { on }\end{array}$ & & muskingum & middle kittanning & 7.5 & 1.4 .3 & $46 . ?$ & 9.5 & 4.2 & 12970 & 52 & 5.0 \\
\hline onio & & muskingum & middle kittanning & 9.3 & 40.5 & 69.5 & 10.0 & 3.8 & 12790 & 50 & 3.0 \\
\hline on io & & muskingum & midde kittanning & 8.4 & 40.9 & 49.7 & 0.4 & 2.7 & 13000 & 47 & 2.0 \\
\hline ohin & & muskingum & niddle kittannina & 6.9 & 43.? & 45.2 & 11.6 & 6.0 & 12730 & 0 & 4.5 \\
\hline on io & & muskingum & clarion & 5.3 & 64.5 & 46.1 & 9.6 & 4.2 & 13250 & 44 & 2.5 \\
\hline on io & & muskingum & middle kittanning & 5.5 & 43.5 & 43.8 & 12.7 & 5.1 & 12440 & 0 & 3.5 \\
\hline on io & & muskingum & middle kittannina & 5.8 & 43.8 & 44.6 & 19.6 & 5.4 & 12610 & 0 & 3.5 \\
\hline otition & & musktnulum & "idole hitcannina & 3.3 & 69,3 & 45.4 & 13.3 & 4.4 & 12370 & 0 & 5.5 \\
\hline onio & & muskinuum & middle kiltanning & 0.2 & 63.2 & 45.1 & 11.7 & 5.1 & 12550 & 0 & 4.0 \\
\hline onio & & muskingum & middle kittanning & 8.1 & $4 \cap .5$ & 45.0 & 14.5 & 5.6 & 12160 & 0 & 3.0 \\
\hline ohio & & muskinqum & middle kittanning & 7.2 & 40.4 & 42.8 & 16.8 & 5.2 & 11780 & 53 & 4.5 \\
\hline onio & & muskiraum & middle kitcanning & 8.7 & 37.9 & 46.1 & 18.0 & 5.8 & 11590 & 0 & 3.5 \\
\hline onio & & muskingum & upder treeoort & 7.3 & 43.5 & 41.0 & 15.5 & 7.9 & 12050 & 53 & 2.5 \\
\hline onio & & muskinoum & uoper freeoort & 9.5 & 44.5 & 40.1 & 15.4 & 0.7 & 12000 & 57 & 3.5 \\
\hline
\end{tabular}




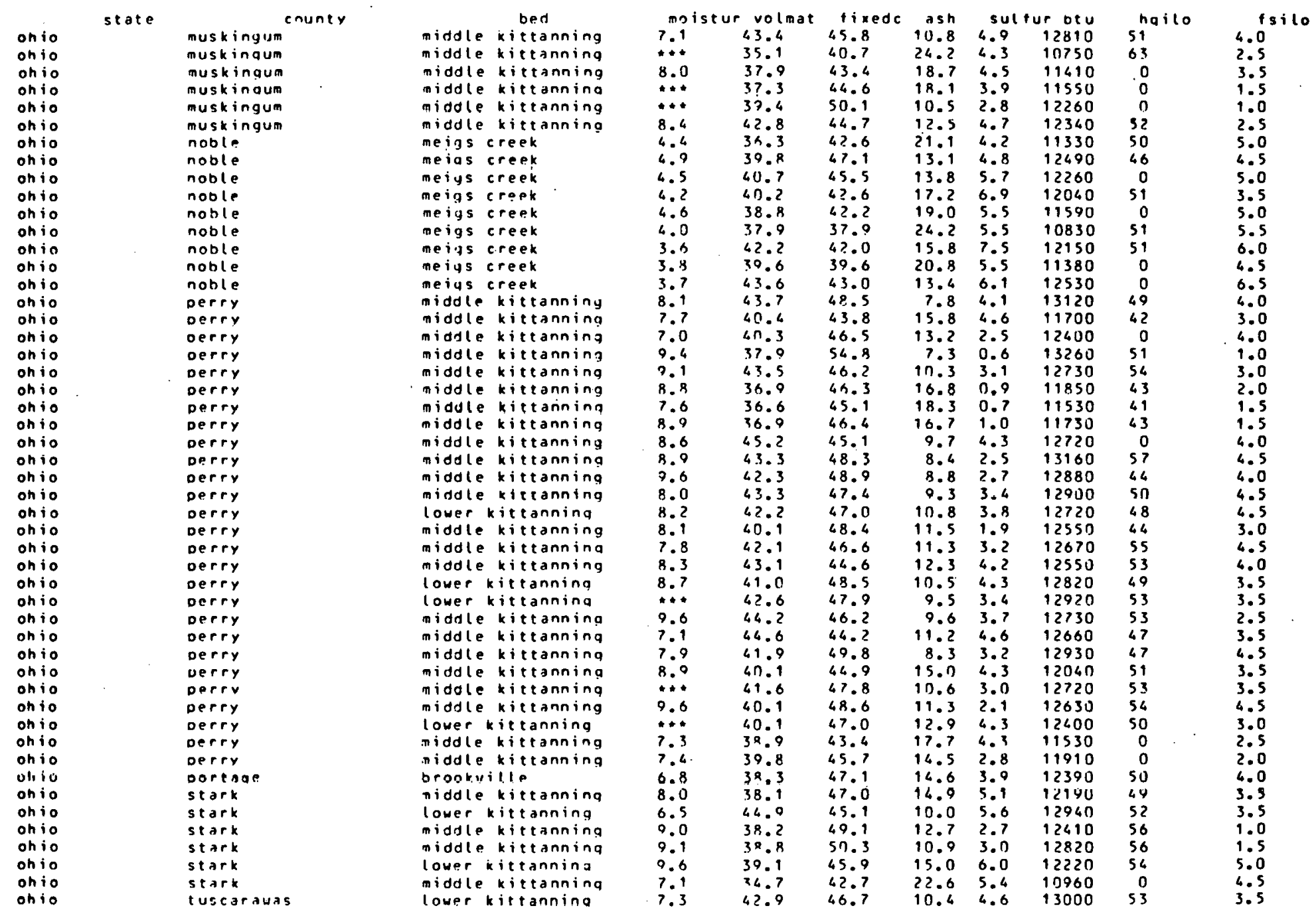




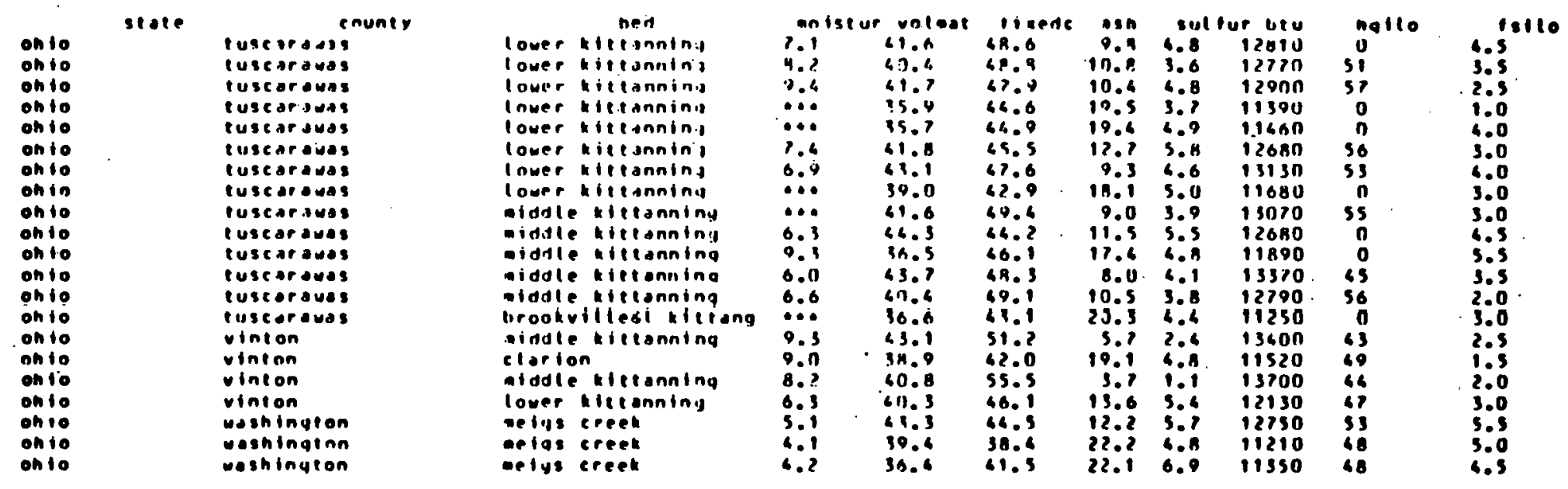


-

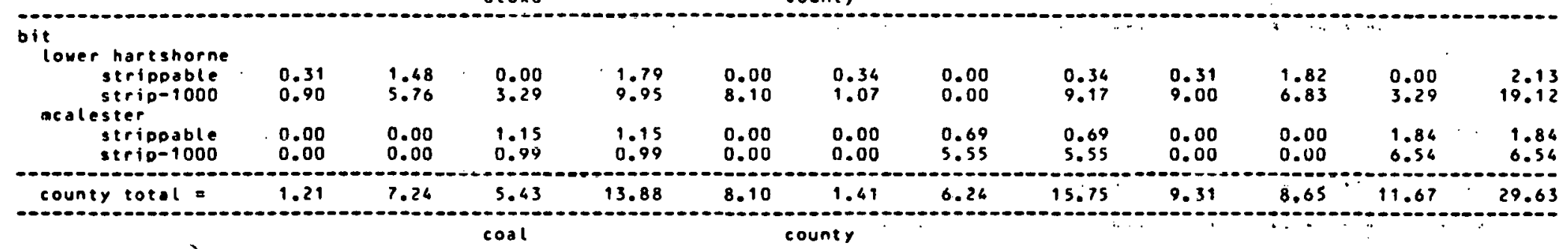

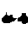
bit

- lower hartshorne

$-1$$$
-
$$$$
\text { - bi }
$$

\begin{tabular}{|c|c|c|c|c|c|c|c|c|c|c|c|c|}
\hline $\begin{array}{l}1-2000 \\
\text { strippoble } \\
\text { strip-1000 }\end{array}$ & $\begin{array}{l}0.00 \\
0.75 \\
0.61\end{array}$ & $\begin{array}{l}0.00 \\
0.52 \\
6.81\end{array}$ & $\begin{array}{l}0.00 \\
0.00 \\
0.00\end{array}$ & $\begin{array}{l}0.00 \\
1.27 \\
7.42\end{array}$ & $\begin{array}{l}0.10 \\
0.00 \\
2.43\end{array}$ & $\begin{array}{r}2.60 \\
0.76 \\
13.74\end{array}$ & $\begin{array}{l}0.00 \\
0.00 \\
0.00\end{array}$ & $\begin{array}{r}2.70 \\
0.76 \\
16.17\end{array}$ & $\begin{array}{l}0.10 \\
0.75 \\
3.04\end{array}$ & $\begin{array}{r}2.60 \\
1.26 \\
20.55\end{array}$ & $\begin{array}{l}0.00 \\
0.00 \\
0.00\end{array}$ & $\begin{array}{r}2.70 \\
2.03 \\
23.59\end{array}$ \\
\hline $\begin{array}{l}1-2000 \\
2-3000 \\
>3000 \\
\text { sirippable } \\
\text { sirip-1000 }\end{array}$ & $\begin{array}{l}0.00 \\
0.00 \\
0.00 \\
0.00 \\
0.00\end{array}$ & $\begin{array}{l}0.00 \\
0.00 \\
0.00 \\
0.36 \\
0.00\end{array}$ & $\begin{array}{r}36.41 \\
1.23 \\
0.00 \\
2.88 \\
55.13\end{array}$ & $\begin{array}{r}36.41 \\
1.23 \\
0.00 \\
3.24 \\
55.13\end{array}$ & $\begin{array}{l}0.00 \\
0.00 \\
0.00 \\
0.00 \\
0.00\end{array}$ & $\begin{array}{l}0.00 \\
0.00 \\
0.00 \\
0.00 \\
0.00\end{array}$ & $\begin{array}{r}84.92 \\
42.44 \\
1.15 \\
0.00 \\
40.05\end{array}$ & $\begin{array}{r}84.92 \\
42.44 \\
1.15 \\
0.00 \\
40.05\end{array}$ & $\begin{array}{l}0.00 \\
0.00 \\
0.00 \\
0.00 \\
0.00\end{array}$ & $\begin{array}{l}0.00 \\
0.00 \\
0.00 \\
0.36 \\
0.00\end{array}$ & $\begin{array}{r}121.33 \\
43.67 \\
1.15 \\
2.88 \\
95.18\end{array}$ & $\begin{array}{r}121.33 \\
43.67 \\
1.15 \\
3.24 \\
95.18\end{array}$ \\
\hline ouney total & 1.36 & 7.69 & 95.65 & 104.70 & 2.53 & 17.10 & 168.56 & 188.10 & 3.89 & $24: 79$ & 264.21 & 292.89 \\
\hline
\end{tabular}
county

- bit cranebura

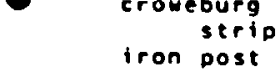
$\begin{array}{ccc}\text { iron post } & \\ \text { strippable } & 27.93 & 0.00 \\ & 24.42 & 0.00\end{array}$ aineral strippable

\begin{tabular}{c} 
strippadoe \\
strip-1000 \\
\hline county total $=$
\end{tabular}

$\rightarrow$

- bit dows on 


\begin{tabular}{|c|c|c|c|c|c|c|c|c|c|c|c|c|c|}
\hline$m$ & $\begin{array}{l}1-2000 \\
2-3000 \\
\text { strippable } \\
\text { strip-1000 }\end{array}$ & $\begin{array}{r}0.15 \\
0.00 \\
2.16 \\
13.03\end{array}$ & $\begin{array}{l}0.40 \\
0.00 \\
0.66 \\
9.62\end{array}$ & $\begin{array}{l}0.00 \\
0.00 \\
0.00 \\
0.00\end{array}$ & $\begin{array}{r}0.55 \\
0.00 \\
2.82 \\
22.65\end{array}$ & $\begin{array}{r}27.90 \\
9.80 \\
1.42 \\
19.78\end{array}$ & $\begin{array}{r}8.05 \\
0.00 \\
1.15 \\
18.40\end{array}$ & $\begin{array}{l}0.00 \\
0.00 \\
0.00 \\
0.00\end{array}$ & $\begin{array}{r}35.95 \\
9.80 \\
2.57 \\
38.18\end{array}$ & $\begin{array}{r}28.05 \\
9.80 \\
3.58 \\
32.81\end{array}$ & $\begin{array}{r}8.45 \\
0.00 \\
1.81 \\
28.02\end{array}$ & $\begin{array}{l}0.00 \\
0.00 \\
0.00 \\
0.00\end{array}$ & $\begin{array}{r}36.50 \\
9.80 \\
5.39 \\
60.83\end{array}$ \\
\hline 9 & $\begin{array}{c}\text { coals above cavanal } \\
1-2000 \\
2-3000 \\
\text { strippable } \\
\text { strip-1000 }\end{array}$ & $\begin{array}{l}0.11 \\
0.00 \\
1.19 \\
6.86\end{array}$ & $\begin{array}{l}0.00 \\
0.00 \\
0.24 \\
0.17\end{array}$ & $\begin{array}{l}0.00 \\
0.00 \\
0.00 \\
0.00\end{array}$ & $\begin{array}{l}0.11 \\
0.00 \\
1.43 \\
7.03\end{array}$ & $\begin{array}{r}15.48 \\
4.09 \\
0.54 \\
11.18\end{array}$ & $\begin{array}{l}0.00 \\
0.00 \\
0.04 \\
0.00\end{array}$ & $\begin{array}{l}0.00 \\
0.00 \\
0.00 \\
0.00\end{array}$ & $\begin{array}{r}15.48 \\
4.09 \\
0.55 \\
11.18\end{array}$ & $\begin{array}{r}15.59 \\
4.09 \\
1.73 \\
18.04\end{array}$ & $\begin{array}{l}0.00 \\
0.00 \\
0.25 \\
0.17\end{array}$ & $\begin{array}{l}0.00 \\
0.00 \\
0.00 \\
0.00\end{array}$ & $\begin{array}{r}15.59 \\
4.09 \\
1.98 \\
18.21\end{array}$ \\
\hline 0 & $\begin{array}{c}\text { cods above stigler } \\
1-2000 \\
\text { strippable } \\
\text { strip- } 1000\end{array}$ & $\begin{array}{l}0.00 \\
4.77 \\
1.43\end{array}$ & $\begin{array}{l}0.00 \\
0.00 \\
0.00\end{array}$ & $\begin{array}{l}0.00 \\
0.00 \\
0.00\end{array}$ & $\begin{array}{l}0.00 \\
4.77 \\
1.43\end{array}$ & $\begin{array}{l}1.96 \\
4.17 \\
9.28\end{array}$ & $\begin{array}{l}0.00 \\
0.00 \\
0.00\end{array}$ & $\begin{array}{l}0.00 \\
0.00 \\
0.00\end{array}$ & $\begin{array}{l}1.96 \\
4.17 \\
9.28\end{array}$ & $\begin{array}{r}1.96 \\
8.94 \\
10.71\end{array}$ & $\begin{array}{l}0.00 \\
0.00 \\
0.00\end{array}$ & $\begin{array}{l}0.00 \\
0.00 \\
0.00\end{array}$ & $\begin{array}{r}1.96 \\
8.94 \\
10.71\end{array}$ \\
\hline 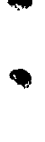 & $\begin{array}{c}\text { hartshorne } \\
1-2000 \\
2-3000 \\
\text { strippable } \\
\text { strip-1000 }\end{array}$ & $\begin{array}{r}0.00 \\
0.00 \\
1.73 \\
10.37\end{array}$ & $\begin{array}{r}61.03 \\
0.00 \\
2.83 \\
61.10\end{array}$ & $\begin{array}{r}12.57 \\
0.35 \\
15.02 \\
113.64\end{array}$ & $\begin{array}{r}53.60 \\
0.35 \\
19.58 \\
184.91\end{array}$ & $\begin{array}{r}23.58 \\
0.00 \\
0.00 \\
20.38\end{array}$ & $\begin{array}{r}34.19 \\
0.00 \\
0.00 \\
44.68\end{array}$ & $\begin{array}{r}67.62 \\
13.96 \\
2.70 \\
17.24\end{array}$ & $\begin{array}{r}125.39 \\
13.96 \\
2.70 \\
82.30\end{array}$ & $\begin{array}{r}23.58 \\
0.00 \\
1.73 \\
30.75\end{array}$ & $\begin{array}{r}75.22 \\
0.00 \\
2.83 \\
105.78\end{array}$ & $\begin{array}{r}80.19 \\
14.31 \\
17.72 \\
130.68\end{array}$ & $\begin{array}{r}178.99 \\
14.31 \\
22.28 \\
267.21\end{array}$ \\
\hline 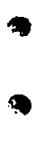 & $\begin{array}{c}\text { lower hartshorne } \\
1-2000 \\
2-3000 \\
23000 \\
\text { strippable } \\
\text { strip-1000 }\end{array}$ & $\begin{array}{l}0.00 \\
0.00 \\
0.00 \\
0.00 \\
0.29\end{array}$ & $\begin{array}{r}8.25 \\
0.00 \\
0.00 \\
7.95 \\
49.19\end{array}$ & $\begin{array}{r}75.58 \\
6.54 \\
0.00 \\
5.79 \\
151.46\end{array}$ & $\begin{array}{r}83.83 \\
6.54 \\
0.00 \\
13.66 \\
200.84\end{array}$ & $\begin{array}{l}0.00 \\
0.00 \\
0.00 \\
0.00 \\
0.00\end{array}$ & $\begin{array}{r}32.84 \\
12.16 \\
0.00 \\
2.68 \\
44.18\end{array}$ & $\begin{array}{r}178.42 \\
111.62 \\
22.07 \\
0.57 \\
48.17\end{array}$ & $\begin{array}{r}211.26 \\
123.78 \\
22.07 \\
3.25 \\
92.35\end{array}$ & $\begin{array}{l}0.00 \\
0.00 \\
0.00 \\
0.00 \\
0.29\end{array}$ & $\begin{array}{r}41.09 \\
12.16 \\
0.00 \\
10.63 \\
93.29\end{array}$ & $\begin{array}{r}254.00 \\
118.16 \\
22.07 \\
6.28 \\
199.61\end{array}$ & $\begin{array}{r}295.09 \\
130.32 \\
22.07 \\
16.91 \\
293.19\end{array}$ \\
\hline 9 & $\begin{array}{c}\text { lower mcalester } \\
1-2000 \\
2-3000 \\
3 t r i p D a b l e \\
\text { strip-1000 }\end{array}$ & $\begin{array}{l}0.25 \\
0.00 \\
0.00 \\
1.55\end{array}$ & $\begin{array}{l}0.00 \\
0.00 \\
0.00 \\
0.00\end{array}$ & $\begin{array}{l}0.00 \\
0.00 \\
0.00 \\
0.00\end{array}$ & $\begin{array}{l}0.25 \\
0.00 \\
0.00 \\
1.55\end{array}$ & $\begin{array}{l}0.12 \\
2.16 \\
0.07 \\
2.70\end{array}$ & $\begin{array}{l}0.45 \\
0.00 \\
0.00 \\
4.11\end{array}$ & $\begin{array}{l}0.00 \\
0.00 \\
0.00 \\
0.00\end{array}$ & $\begin{array}{l}6.57 \\
2.16 \\
0.07 \\
6.81\end{array}$ & $\begin{array}{l}6.37 \\
2.16 \\
0.07 \\
4.25\end{array}$ & $\begin{array}{l}0.45 \\
0.00 \\
0.00 \\
4.11\end{array}$ & $\begin{array}{l}0.00 \\
0.00 \\
0.00 \\
0.00\end{array}$ & $\begin{array}{l}6.82 \\
2.16 \\
0.07 \\
8.36\end{array}$ \\
\hline$\pi$ & $\begin{array}{l}\text { er witteville } \\
\text { s-2000 } \\
\text { strippable } \\
\text { strip-1000 }\end{array}$ & $\begin{array}{l}0.00 \\
0.05 \\
0.00\end{array}$ & $\begin{array}{r}6.53 \\
14.60 \\
2.21\end{array}$ & $\begin{array}{l}0.00 \\
0.00 \\
0.00\end{array}$ & $\begin{array}{r}6.53 \\
14.65 \\
2.21\end{array}$ & $\begin{array}{l}2.37 \\
3.25 \\
0.00\end{array}$ & $\begin{array}{r}11.24 \\
11.69 \\
0.00\end{array}$ & $\begin{array}{l}0.00 \\
0.00 \\
0.00\end{array}$ & $\begin{array}{r}13.61 \\
14.96 \\
0.00\end{array}$ & $\begin{array}{l}2.37 \\
3.30 \\
0.00\end{array}$ & $\begin{array}{r}17.77 \\
26.29 \\
2.21\end{array}$ & $\begin{array}{l}0.00 \\
0.00 \\
0.00\end{array}$ & $\begin{array}{r}20.14 \\
29.59 \\
2.21\end{array}$ \\
\hline 3 & $\begin{array}{l}1-2000 \\
2-3000 \\
\text { strippable } \\
\text { strip- } 1000\end{array}$ & $\begin{array}{l}0.02 \\
0.00 \\
1.50 \\
2.91\end{array}$ & $\begin{array}{r}4.67 \\
0.29 \\
1.95 \\
12.66\end{array}$ & $\begin{array}{r}6.39 \\
0.04 \\
0.04 \\
12.34\end{array}$ & $\begin{array}{r}11.08 \\
0.33 \\
3.49 \\
27.91\end{array}$ & $\begin{array}{l}5.33 \\
0.26 \\
0.00 \\
4.45\end{array}$ & $\begin{array}{r}18.30 \\
19.95 \\
1.92 \\
19.99\end{array}$ & $\begin{array}{r}11.95 \\
16.29 \\
0.00 \\
7.79\end{array}$ & $\begin{array}{l}35.58 \\
36.50 \\
1.12 \\
32.23\end{array}$ & $\begin{array}{l}5.35 \\
0.26 \\
1.50 \\
7.36\end{array}$ & $\begin{array}{l}22.97 \\
20.26 \\
3.07 \\
32.65\end{array}$ & $\begin{array}{r}18.34 \\
16.33 \\
0.04 \\
20.13\end{array}$ & $\begin{array}{r}46.66 \\
36.83 \\
4.61 \\
60.14\end{array}$ \\
\hline 3 & $\begin{array}{l}\text { stigler } \\
1-2000 \\
\text { strippable } \\
\text { strip-1000 }\end{array}$ & $\begin{array}{r}0.00 \\
3.23 \\
11.19\end{array}$ & $\begin{array}{l}0.00 \\
0.00 \\
0.00\end{array}$ & $\begin{array}{l}0.00 \\
0.00 \\
0.00\end{array}$ & $\begin{array}{r}0.00 \\
3.23 \\
11.11\end{array}$ & $\begin{array}{r}19.12 \\
2.55 \\
31.31\end{array}$ & $\begin{array}{l}0.00 \\
0.00 \\
0.00\end{array}$ & $\begin{array}{l}0.00 \\
0.00 \\
0.00\end{array}$ & $\begin{array}{r}19.12 \\
2.55 \\
31.31\end{array}$ & $\begin{array}{r}19.12 \\
5.78 \\
42.62\end{array}$ & $\begin{array}{l}0.00 \\
0.00 \\
0.00\end{array}$ & $\begin{array}{l}0.00 \\
0.00 \\
0.00\end{array}$ & $\begin{array}{r}19.12 \\
5.78 \\
42.42\end{array}$ \\
\hline 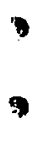 & $\begin{array}{l}\text { hareshorne } \\
1-2000 \\
2-3000 \\
>3000 \\
\text { striposole } \\
\text { stripo-1000 }\end{array}$ & $\begin{array}{r}6.14 \\
0.14 \\
0.00 \\
0.43 \\
11.79\end{array}$ & $\begin{array}{r}18.11 \\
8.10 \\
0.00 \\
6.88 \\
63.35\end{array}$ & $\begin{array}{l}0.00 \\
0.00 \\
0.00 \\
2.53 \\
9.16\end{array}$ & $\begin{array}{r}24.25 \\
8.74 \\
0.00 \\
9.84 \\
84.30\end{array}$ & $\begin{array}{l}20.92 \\
10.27 \\
0.00 \\
0.02 \\
8.63\end{array}$ & $\begin{array}{r}42.96 \\
.8 .24 \\
8.65 \\
0.93 \\
33.30\end{array}$ & $\begin{array}{l}0.00 \\
0.00 \\
0.00 \\
0.42 \\
1.40\end{array}$ & $\begin{array}{r}63.88 \\
18.51 \\
8.65 \\
1.37 \\
43.33\end{array}$ & $\begin{array}{r}27.06 \\
10.41 \\
0.00 \\
0.45 \\
20.42\end{array}$ & $\begin{array}{r}61.07 \\
16.34 \\
8.65 \\
7.81 \\
96.65\end{array}$ & $\begin{array}{r}0.00 \\
0.00 \\
0.00 \\
2.05 \\
10.56\end{array}$ & $\begin{array}{r}88.13 \\
20.75 \\
8.65 \\
11.21 \\
127.63\end{array}$ \\
\hline 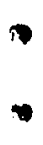 & $\begin{array}{l}\text { mcalester } \\
1-2000 \\
2-3000 \\
\text { striogable } \\
\text { strip-1000 }\end{array}$ & $\begin{array}{l}0.00 \\
0.00 \\
0.09 \\
1.33\end{array}$ & $\begin{array}{l}0.00 \\
0.00 \\
0.00 \\
0.00\end{array}$ & $\begin{array}{l}0.00 \\
0.00 \\
0.00 \\
0.00\end{array}$ & $\begin{array}{l}0.00 \\
0.00 \\
0.09 \\
1.33\end{array}$ & $\begin{array}{l}4.62 \\
0.72 \\
0.00 \\
4.94\end{array}$ & $\begin{array}{l}0.00 \\
0.00 \\
0.00 \\
0.00\end{array}$ & $\begin{array}{l}0.00 \\
0.00 \\
0.00 \\
0.00\end{array}$ & $\begin{array}{l}4.62 \\
0.72 \\
0.00 \\
4.94\end{array}$ & $\begin{array}{l}4.62 \\
0.72 \\
0.09 \\
6.27\end{array}$ & $\begin{array}{l}0.00 \\
0.00 \\
0.00 \\
0.00\end{array}$ & $\begin{array}{l}0.00 \\
0.00 \\
0.00 \\
0.00\end{array}$ & $\begin{array}{l}4.62 \\
0.72 \\
0.09 \\
6.27\end{array}$ \\
\hline & county total $=$ & 82.63 & 320.71 & 411.15 & 814.49 & 279.37 & 379.27 & 500.22 & 1158.86 & 362.00 & 699.98 & 911.37 & 1973.35 \\
\hline
\end{tabular}

te

veir-pitisburg 


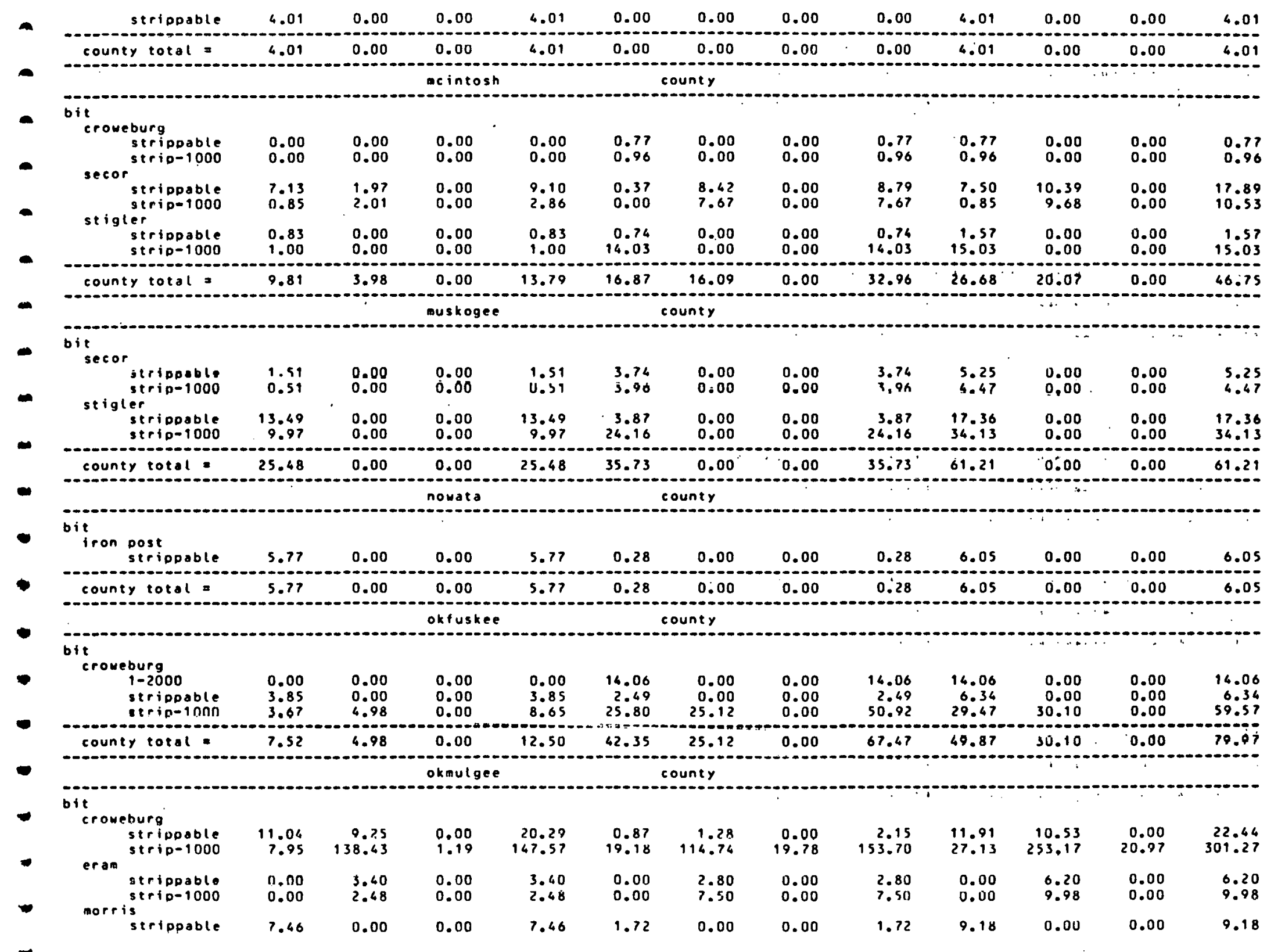




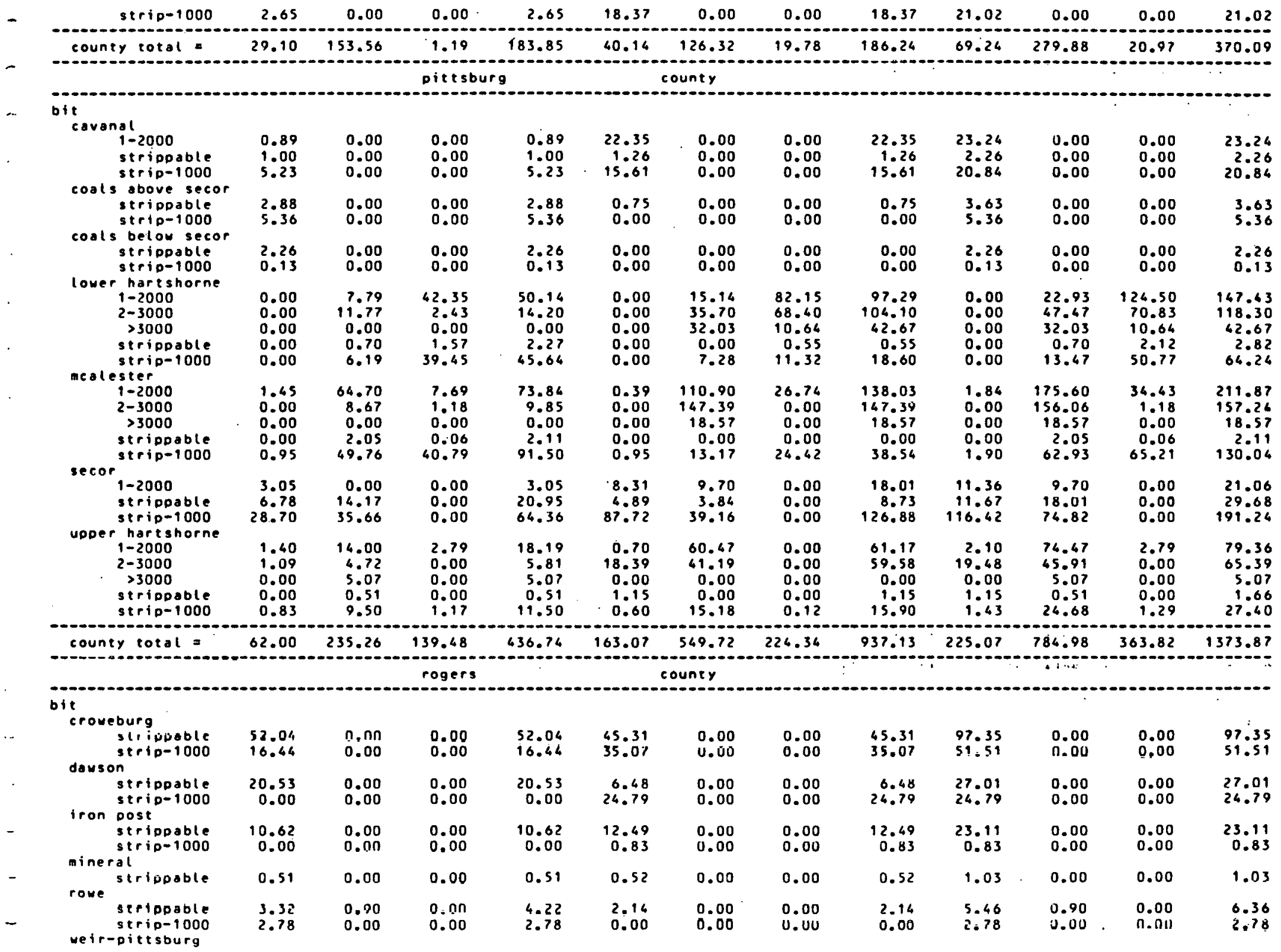




\begin{tabular}{|c|c|c|c|c|c|c|c|c|c|c|c|c|}
\hline strippable & 9.17 & 0.00 & 0.00 & 9.17 & 0.00 & 0.00 & 0.00 & 0.00 & 9.17 & 0.00 & 0.00 & 9.17 \\
\hline county total $=$ & 115.41 & 0.90 & 0.00 & 116.31 & 127.63 & 0.00 & 0.00 & 127.63 & 263.04 & 0.90 & 0.00 & 243.94 \\
\hline & & & sequo & & & ney & & & & & & \\
\hline \multicolumn{13}{|l|}{ bit } \\
\hline $\begin{array}{l}\text { stripoable } \\
\text { strip- } 1000\end{array}$ & $\begin{array}{l}2.69 \\
9.94\end{array}$ & $\begin{array}{l}0.00 \\
0.00\end{array}$ & $\begin{array}{l}0.00 \\
0.00\end{array}$ & $\begin{array}{l}2.69 \\
9.94\end{array}$ & $\begin{array}{r}0.01 \\
14.50\end{array}$ & $\begin{array}{r}0.00 \\
0.00\end{array}$ & $\begin{array}{l}0.00 \\
0.00\end{array}$ & $\begin{array}{r}0.01 \\
14.50\end{array}$ & $\begin{array}{r}2.70 \\
24.64\end{array}$ & $\begin{array}{l}0.00 \\
0.00\end{array}$ & $\begin{array}{r}0.00 \\
0.00\end{array}$ & $\begin{array}{r}2.70 \\
24.44\end{array}$ \\
\hline county total = & 12.63 & 0.00 & 0.00 & 12.63 & 14.51 & 0.00 & 0.00 & 14.51 & 27.14 & 0.00 & 0.00 & 27.14 \\
\hline & & & tulso & & & & & & & & & \\
\hline \multicolumn{13}{|l|}{ bit } \\
\hline $\begin{array}{l}\text { stripdable } \\
\text { strip-1000 }\end{array}$ & $\begin{array}{l}.5 .54 \\
0.42\end{array}$ & $\begin{array}{l}0.00 \\
0.00\end{array}$ & $\begin{array}{l}0.00 \\
0.00\end{array}$ & $\begin{array}{l}5.54 \\
0.42\end{array}$ & $\begin{array}{r}6.12 \\
14.96\end{array}$ & $\begin{array}{l}0.00 \\
0.00\end{array}$ & $\begin{array}{l}0.00 \\
0.00\end{array}$ & $\begin{array}{r}6.12 \\
14.96\end{array}$ & $\begin{array}{l}11.66 \\
15.38\end{array}$ & $\begin{array}{l}0.00 \\
0.00\end{array}$ & $\begin{array}{l}0.00 \\
0.00\end{array}$ & $\begin{array}{l}11.66 \\
15.38\end{array}$ \\
\hline $\begin{array}{l}\text { strippable } \\
\text { strip-1000 }\end{array}$ & $\begin{array}{l}18.38 \\
13.64\end{array}$ & $\begin{array}{l}2.03 \\
0.20\end{array}$ & $\begin{array}{l}0.00 \\
0.00\end{array}$ & $\begin{array}{l}20.61 \\
13.84\end{array}$ & $\begin{array}{l}16.60 \\
55.35\end{array}$ & $\begin{array}{l}0.46 \\
7.40\end{array}$ & $\begin{array}{l}0.00 \\
0.00\end{array}$ & $\begin{array}{l}15.06 \\
62.75\end{array}$ & $\begin{array}{l}32.98 \\
68.99\end{array}$ & $\begin{array}{l}2.49 \\
7.60\end{array}$ & $\begin{array}{l}0.00 \\
0.00\end{array}$ & $\begin{array}{l}35.47 \\
76.59\end{array}$ \\
\hline county total = & 37.98 & 2.23 & 0.00 & 40.21 & 01.03 & 7.86 & 0.00 & 98.89 & 129.01 & 10.09 & 0.00 & 139.10 \\
\hline & & & wagon & & & & & & & & & \\
\hline \multicolumn{13}{|l|}{$\begin{array}{l}\text { bit } \\
\text { croveburg }\end{array}$} \\
\hline $\begin{array}{l}\text { strippable } \\
\text { strip-1000 }\end{array}$ & $\begin{array}{r}15.73 \\
2.18\end{array}$ & $\begin{array}{l}0.00 \\
0.00\end{array}$ & $\begin{array}{l}0.00 \\
0.00\end{array}$ & $\begin{array}{r}15.73 \\
2.18\end{array}$ & $\begin{array}{r}6.85 \\
13.85\end{array}$ & $\begin{array}{l}0.00 \\
0.00\end{array}$ & $\begin{array}{l}0.00 \\
0.00\end{array}$ & $\begin{array}{r}6.85 \\
13.85\end{array}$ & $\begin{array}{l}22.58 \\
16.03\end{array}$ & $\begin{array}{l}0.00 \\
0.00\end{array}$ & $\begin{array}{l}0.00 \\
0.00\end{array}$ & $\begin{array}{l}22.58 \\
16.03\end{array}$ \\
\hline $\begin{array}{l}\text { strippable } \\
\text { strip-1000 }\end{array}$ & $\begin{array}{l}4.42 \\
4.78\end{array}$ & $\begin{array}{l}0.00 \\
0.00\end{array}$ & $\begin{array}{l}0.00 \\
0.00\end{array}$ & $\begin{array}{l}4.42 \\
4.78\end{array}$ & $\begin{array}{r}0.69 \\
15.05\end{array}$ & $\begin{array}{l}0.00 \\
0.00\end{array}$ & $\begin{array}{l}0.00 \\
0.00\end{array}$ & $\begin{array}{r}0.69 \\
15.05\end{array}$ & $\begin{array}{r}5.11 \\
19.83\end{array}$ & $\begin{array}{l}0.00 \\
0.00\end{array}$ & $\begin{array}{l}0.00 \\
0.00\end{array}$ & $\begin{array}{r}5.11 \\
19.83\end{array}$ \\
\hline county totel $=$ & 27.11 & 0.00 & 0.00 & 27.11 & 36.44 & 0.00 & 0.00 & 36.44 & 63.55 & 0.00 & 0.00 & 63.55 \\
\hline
\end{tabular}

\section{bit}

\begin{tabular}{|c|c|c|c|c|c|c|c|c|c|c|c|}
\hline setip-1000 & 0.00 & 0.00 & 0.00 & 0.00 & 4.66 & 0.00 & 0.00 & 4.66 & 4.66 & 0.00 & 0.00 \\
\hline
\end{tabular}

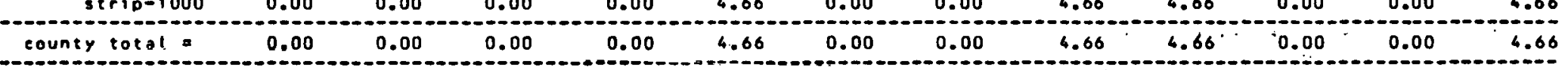

overburden

overburden

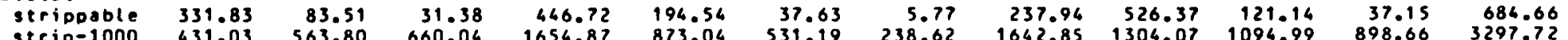

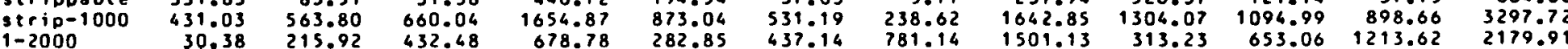
$\begin{array}{llllllll}2-3000 & 30.38 & 215.92 & 432.48 & 678.78 & 283.04 & 531.19 & 238.62 \\ 23000 & 1.23 & 43.04 & 28.01 & 72.28 & 50.30 & 340.65 & 483.14\end{array}$

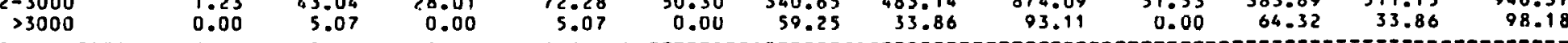

tulal $\quad 704.47 \quad 911.34 \quad 1151.99 \quad 2857.72 \quad 1400.73 \quad 1405.86 \quad 1542.53 \quad 4349.12 \quad 2195.20 \quad 2347.20 \quad 2694.44 \quad 7206.84$

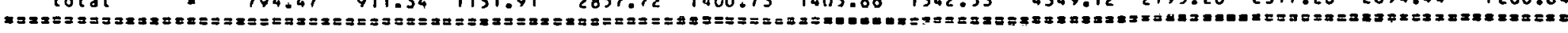




\begin{tabular}{|c|c|c|c|c|c|c|c|c|c|c|c|c|}
\hline $\begin{array}{l}\text { strippable } \\
\text { strip-1000 } \\
1-2000 \\
2-3000 \\
>3000\end{array}$ & $\begin{array}{r}331.83 \\
431.03 \\
30.38 \\
1.23 \\
0.00\end{array}$ & $\begin{array}{r}83.51 \\
563.80 \\
215.92 \\
43.04 \\
5.07\end{array}$ & $\begin{array}{r}31.38 \\
660.04 \\
432.48 \\
28.01 \\
0.00\end{array}$ & $\begin{array}{r}646.72 \\
1654.87 \\
678.78 \\
72.28 \\
5.07\end{array}$ & $\begin{array}{r}194.54 \\
873.04 \\
282.85 \\
50.30 \\
0.00\end{array}$ & $\begin{array}{r}37.63 \\
531.19 \\
437.14 \\
340.65 \\
59.25\end{array}$ & $\begin{array}{r}5.77 \\
238.62 \\
781.14 \\
483.14 \\
33.86\end{array}$ & $\begin{array}{r}237.94 \\
1642.85 \\
1501.13 \\
876.09 \\
93.11\end{array}$ & $\begin{array}{r}526.37 \\
1304.07 \\
313.23 \\
51.53 \\
0.00\end{array}$ & $\begin{array}{r}121.14 \\
1094.99 \\
653.06 \\
383.69 \\
64.32\end{array}$ & $\begin{array}{r}37.15 \\
898.66 \\
1213.62 \\
511.15 \\
33.86\end{array}$ & $\begin{array}{r}684 . \\
3297 . \\
2179 . \\
946 . \\
98\end{array}$ \\
\hline $\begin{array}{l}\text { and total } \\
=\leq x=x=x=0\end{array}$ & $\begin{array}{r}794.47 \\
=x=x=3=0\end{array}$ & $\begin{array}{r}911: 36 \\
x=2=3 x=0\end{array}$ & $\begin{array}{l}1151.99 \\
x=x=x=x=0\end{array}$ & $\begin{array}{r}2857.72 \\
=x=2=3=0\end{array}$ & $\begin{array}{l}1400.73 \\
=x==x=x\end{array}$ & 1405.86 & 1562.53 & $\begin{array}{r}4349.12 \\
=2=0=0=0\end{array}$ & 2195.20 & 2317.20 & 2694.44 & \\
\hline
\end{tabular}

total tonnage of identified coal resources in oklahoada

7206.84

resource tigures taken from:

$\begin{array}{lll}\text { source } & \text { year base year of resource } \\ \text { okgs-ozarks reg comm } & 1974 & 1976\end{array}$


everege enalyses of coal in oklohoma

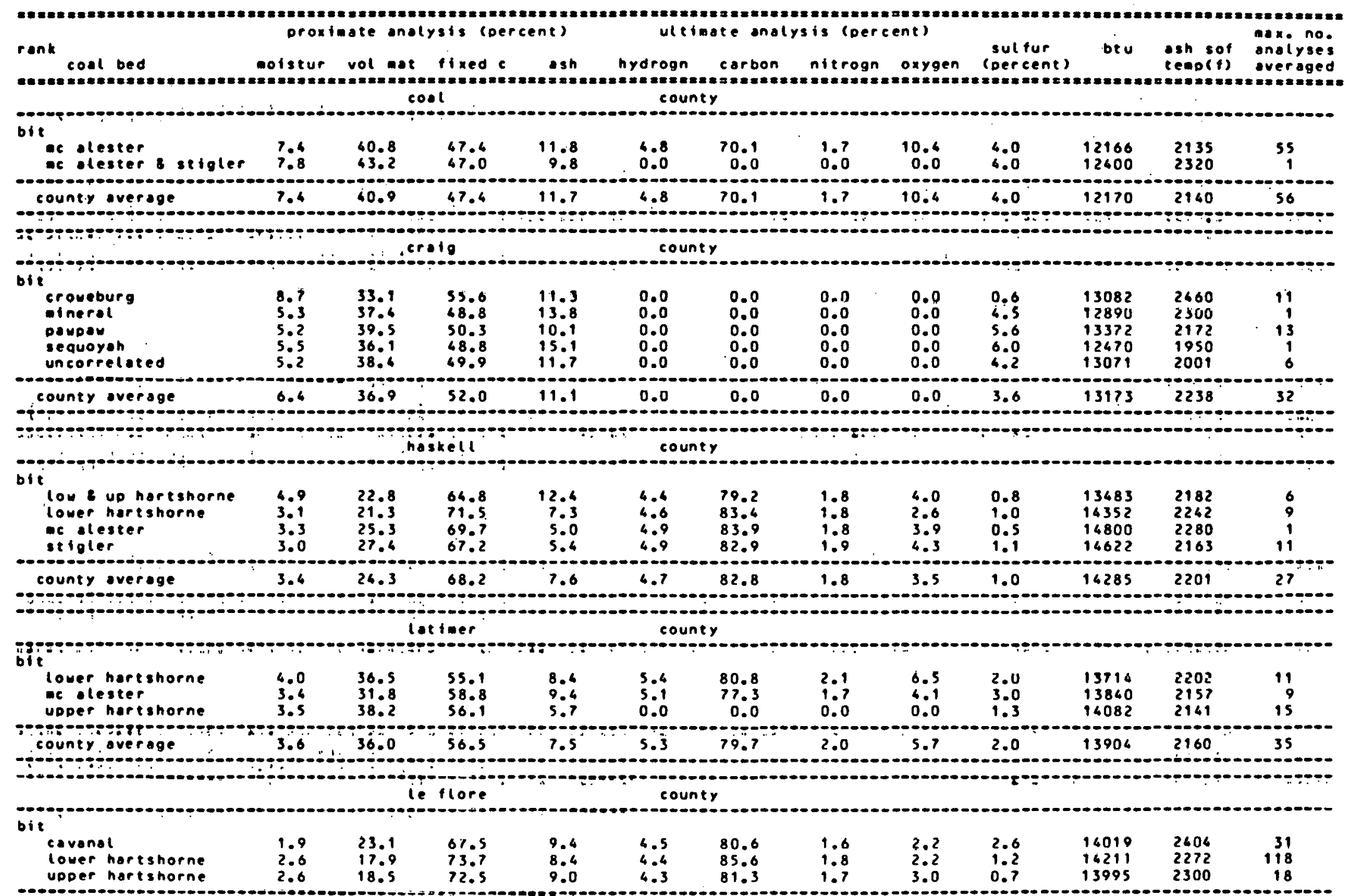




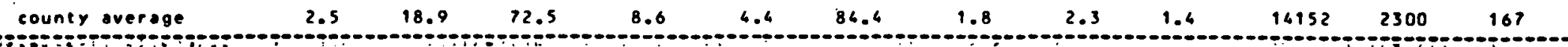

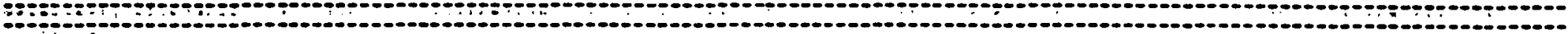
muskogee county

bie

bit Itigler
sounty average
okaulgee county

bit
croweburg

\begin{tabular}{|c|c|c|c|c|c|c|c|c|c|c|c|c|}
\hline $\begin{array}{l}\text { lower nortshorne } \\
\text { oc olester }\end{array}$ & $\begin{array}{l}4.2 \\
3.6\end{array}$ & $\begin{array}{r}38.2 \\
36.1\end{array}$ & $\begin{array}{l}52.2 \\
55.8\end{array}$ & $\begin{array}{l}9.5 \\
8.1\end{array}$ & $\begin{array}{r}5.3 \\
5.2 \\
\end{array}$ & $\begin{array}{l}77.6 \\
79.5\end{array}$ & $\begin{array}{l}1.9 \\
2.0\end{array}$ & $\begin{array}{l}6.1 \\
7.8\end{array}$ & $\begin{array}{l}2.7 \\
0.8\end{array}$ & $\begin{array}{r}13426 \\
13584 \\
-1358\end{array}$ & $\begin{array}{r}2206 \\
2228 \\
\end{array}$ & $\begin{array}{l}20 \\
35\end{array}$ \\
\hline county overoge & 3.8 & 36.9 & 34.5 & 8.6 & 5.2 & 78.9 & 1.9 & 7.2 & 1.5 & 13526 & 2221 & 55 \\
\hline
\end{tabular}
Trger: rogers county

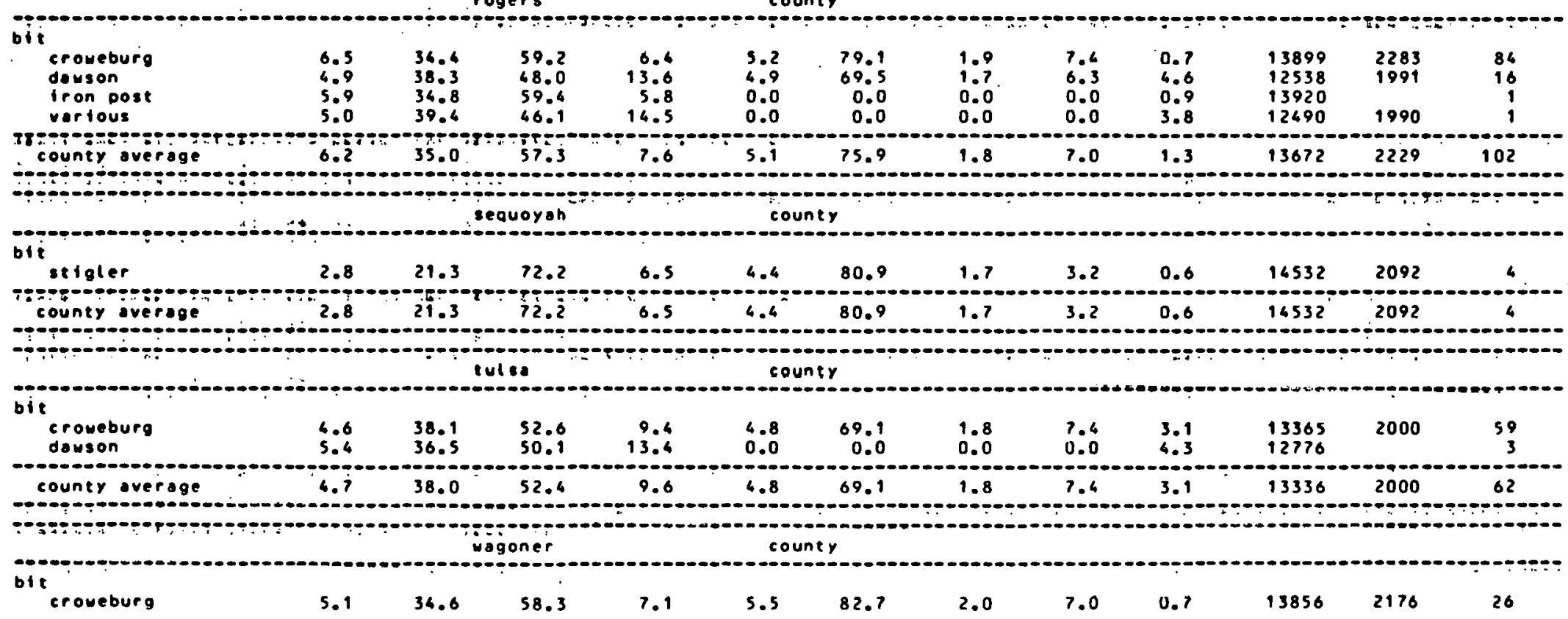




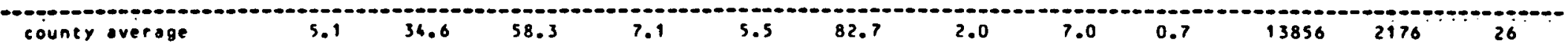

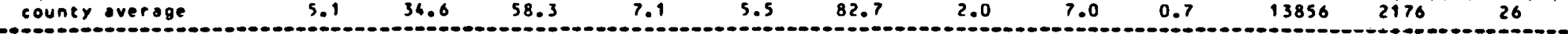

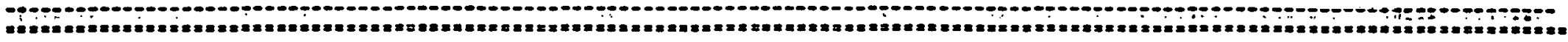


Oklahoma

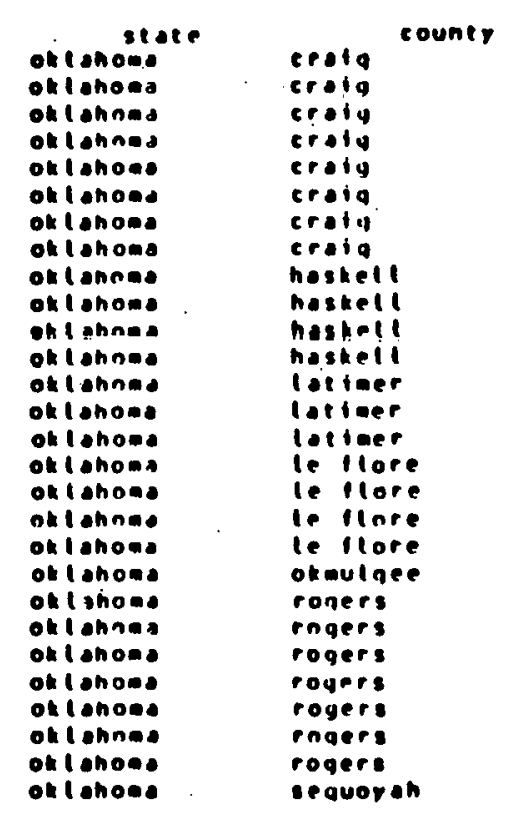

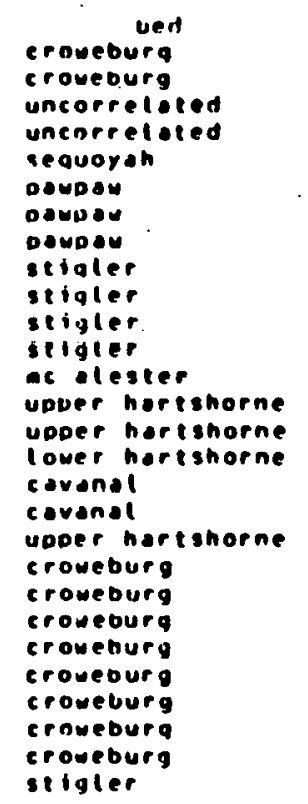

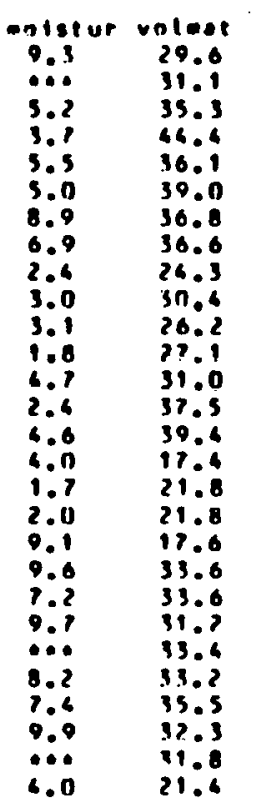

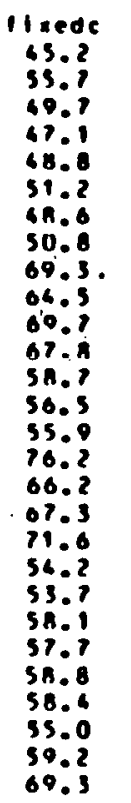

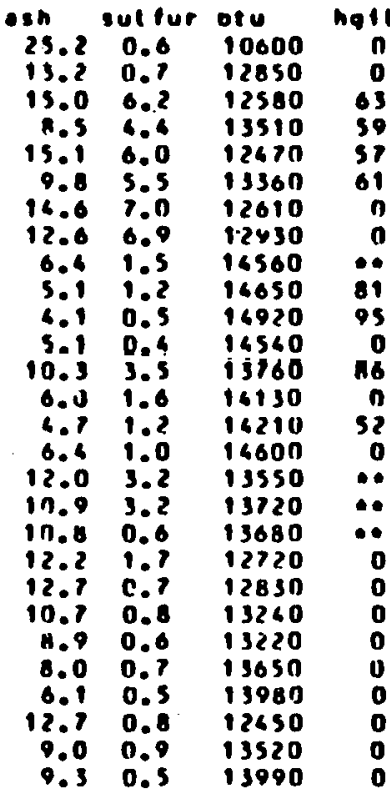

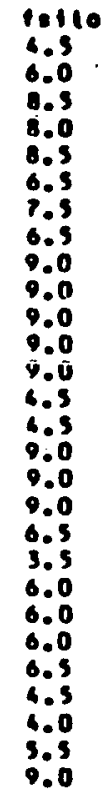



-verage onalyses of coal in oregon

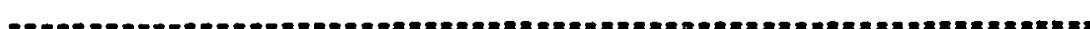
proxinate onolysis (percent) ultiate anolysis (percent)

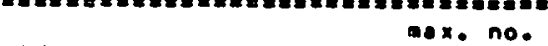
rank coal bed cotstur vol mat tixed c ash hydrogn corbon nitrogn oxyen (pulfur bent) betu ash sof onaiyses w

$$
\text { beaver hill }
$$
$17.4 \quad 49.7 \quad 48.9$

$\begin{array}{llll}68.9 & 9.5 & 5.0 & 68.3 \\ 59.5 & 6.6 & 4.9 & 68.2\end{array}$

$1: 0$

$\begin{array}{ll}1.6 & 16.8 \\ 1.8 & 17\end{array}$

16.4
17.9
17.2

0.4
0.6
0.5

11680
11910

2095
2320

county average $17.641 .7 \quad 49.7$

8.5

$6.9 \quad 68.3$
11756 $21>0$ 3

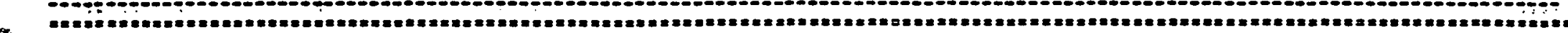




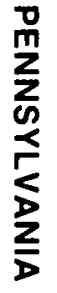


(In willions of short tons)

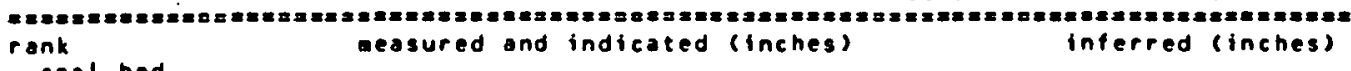

inferred (inches)
total (inches)

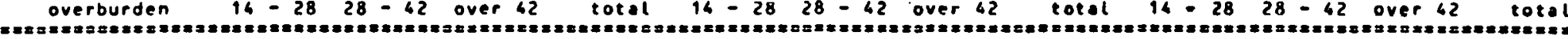

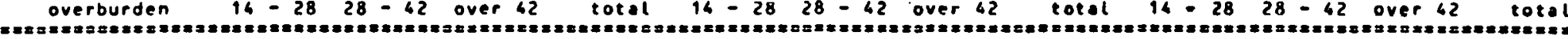

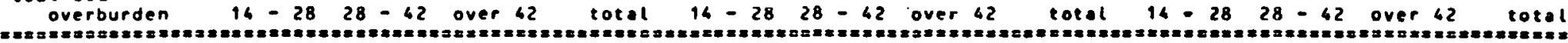
allegheny county

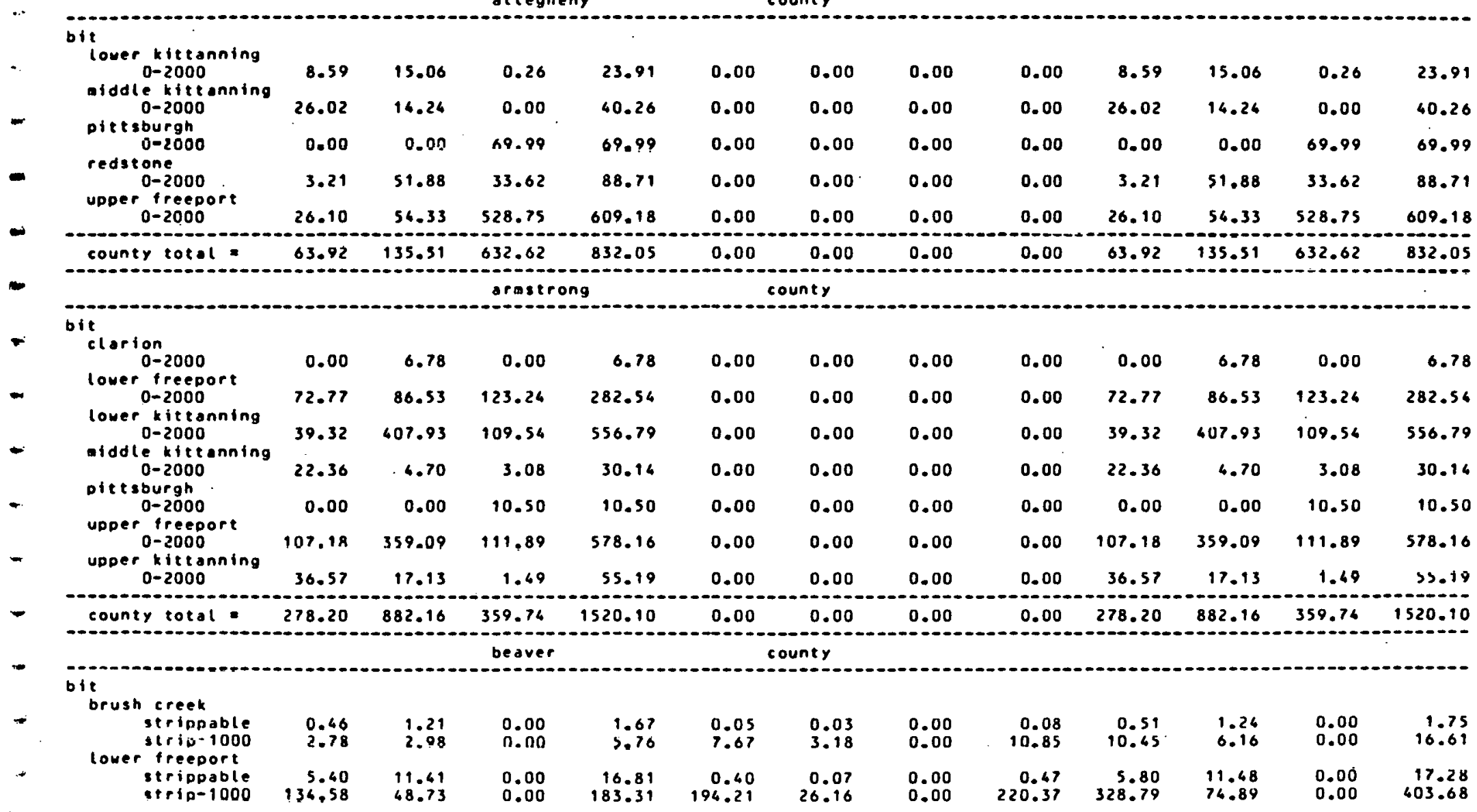




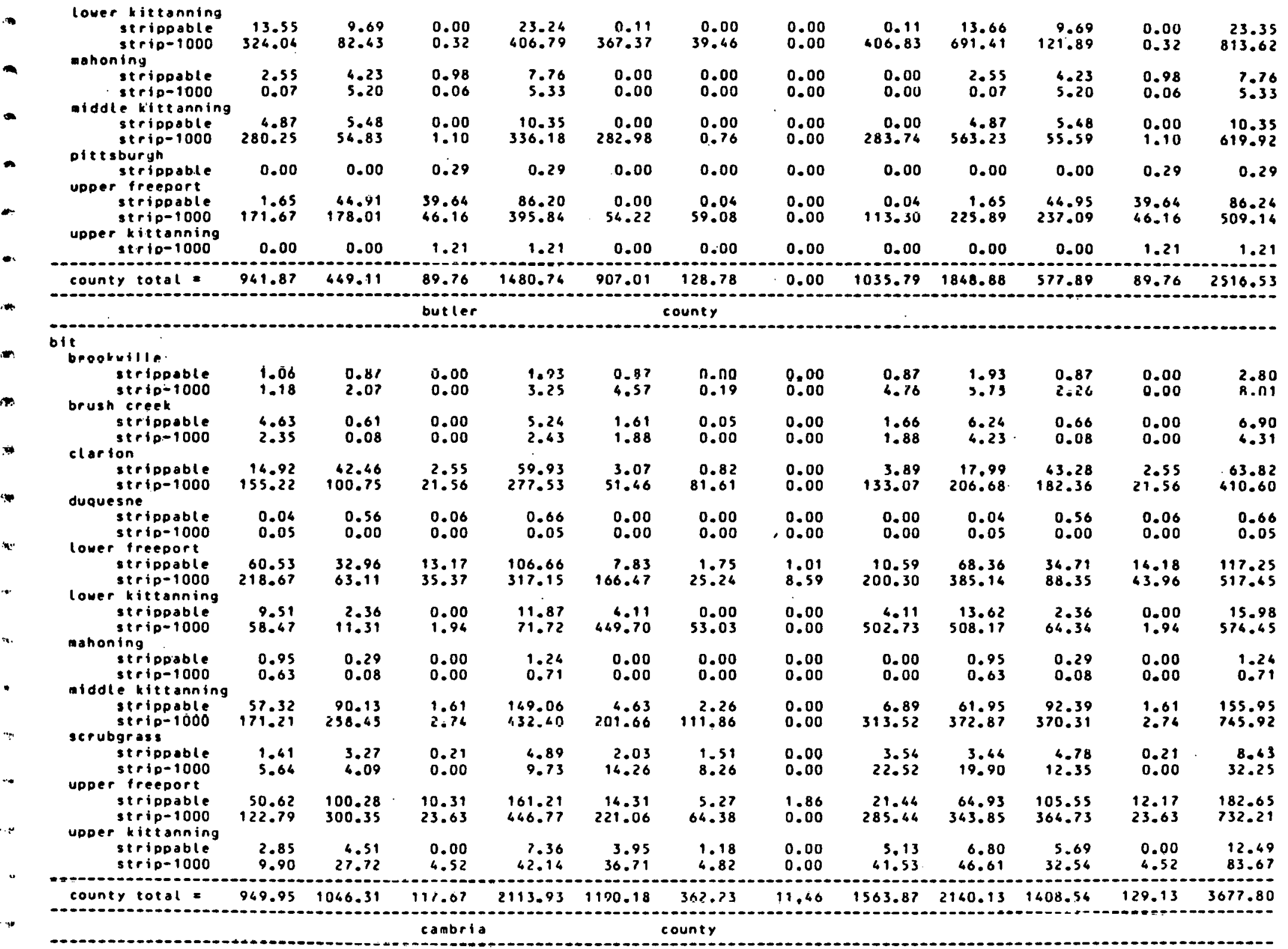


bit

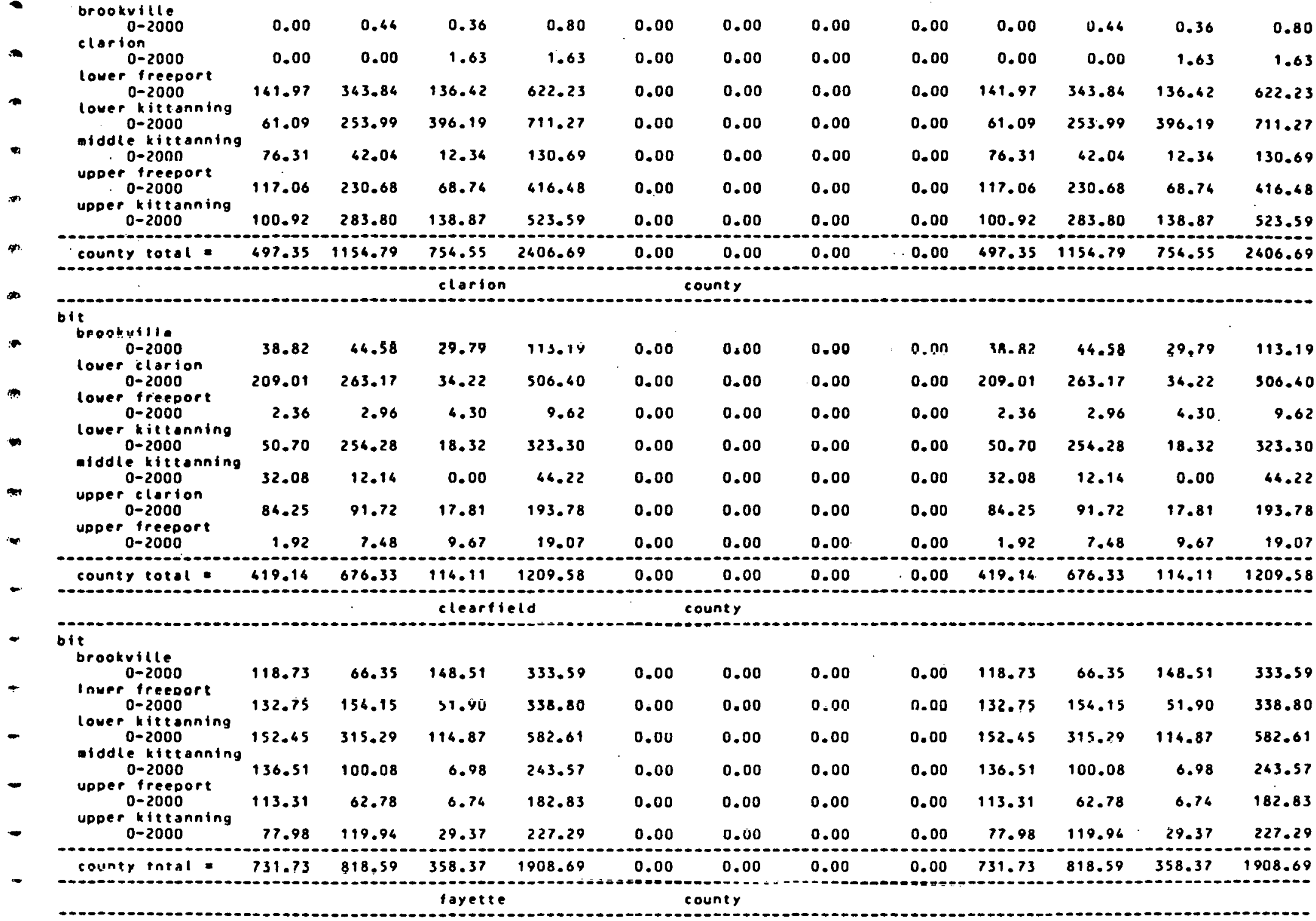

- bit brookville-clarion 


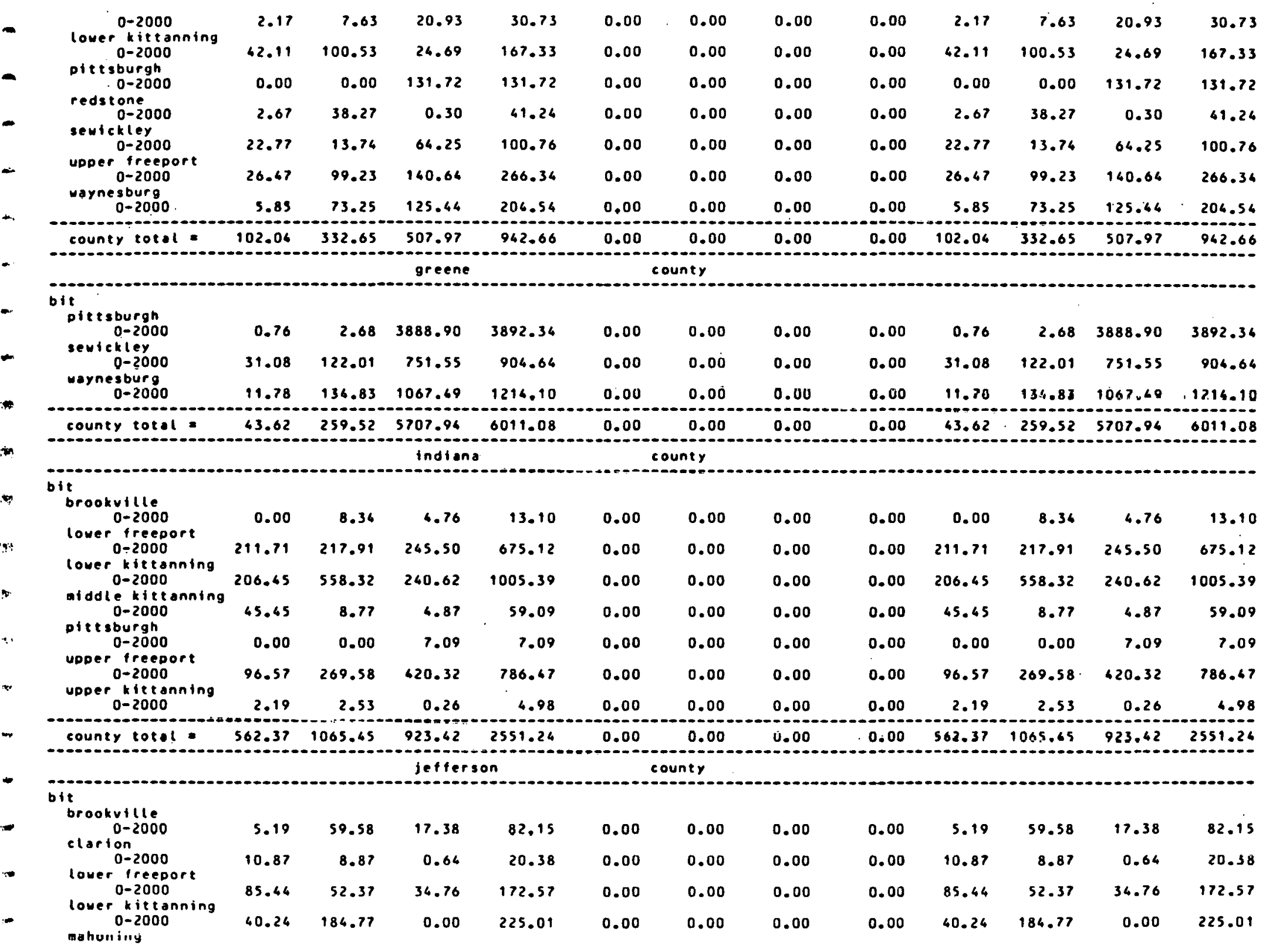




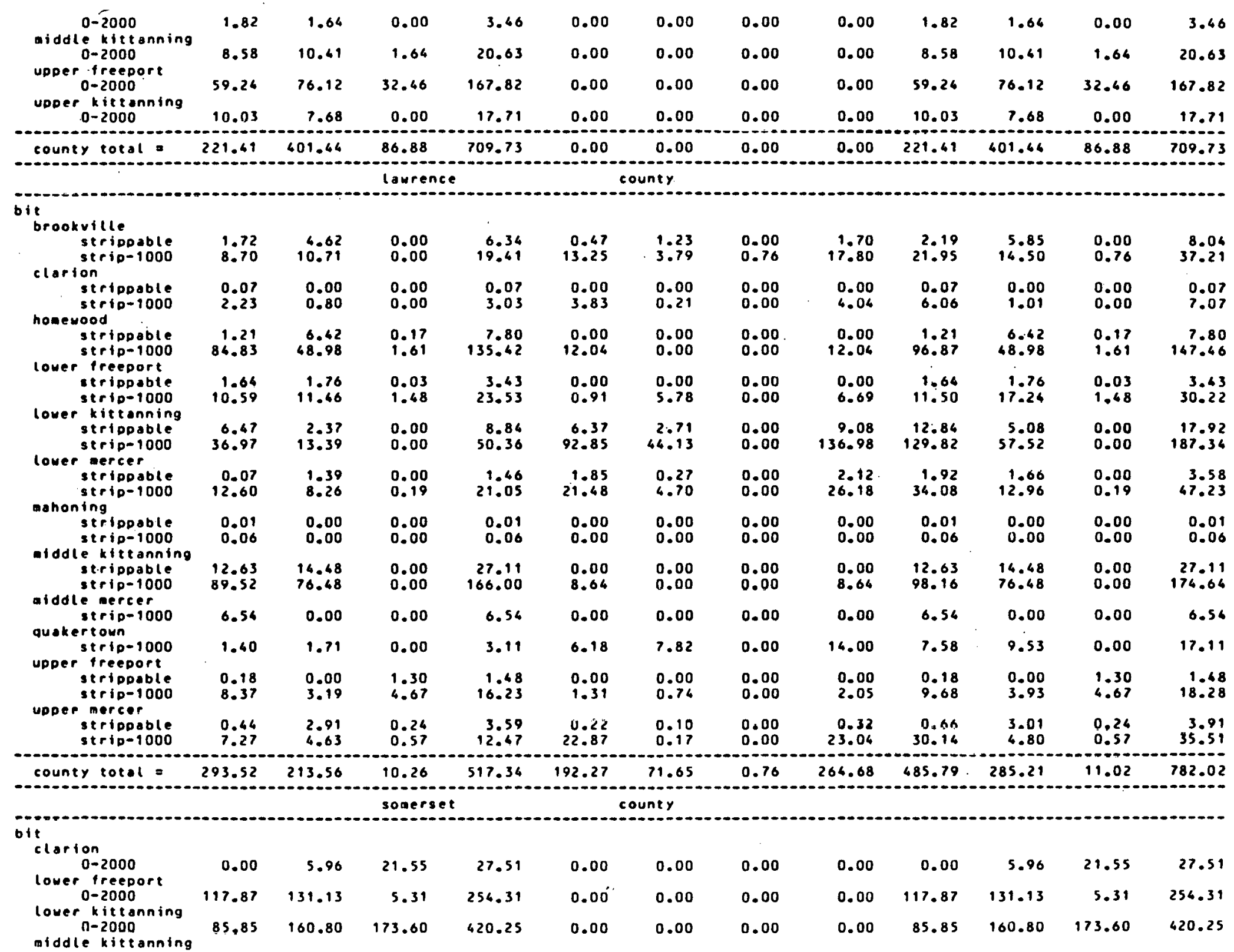




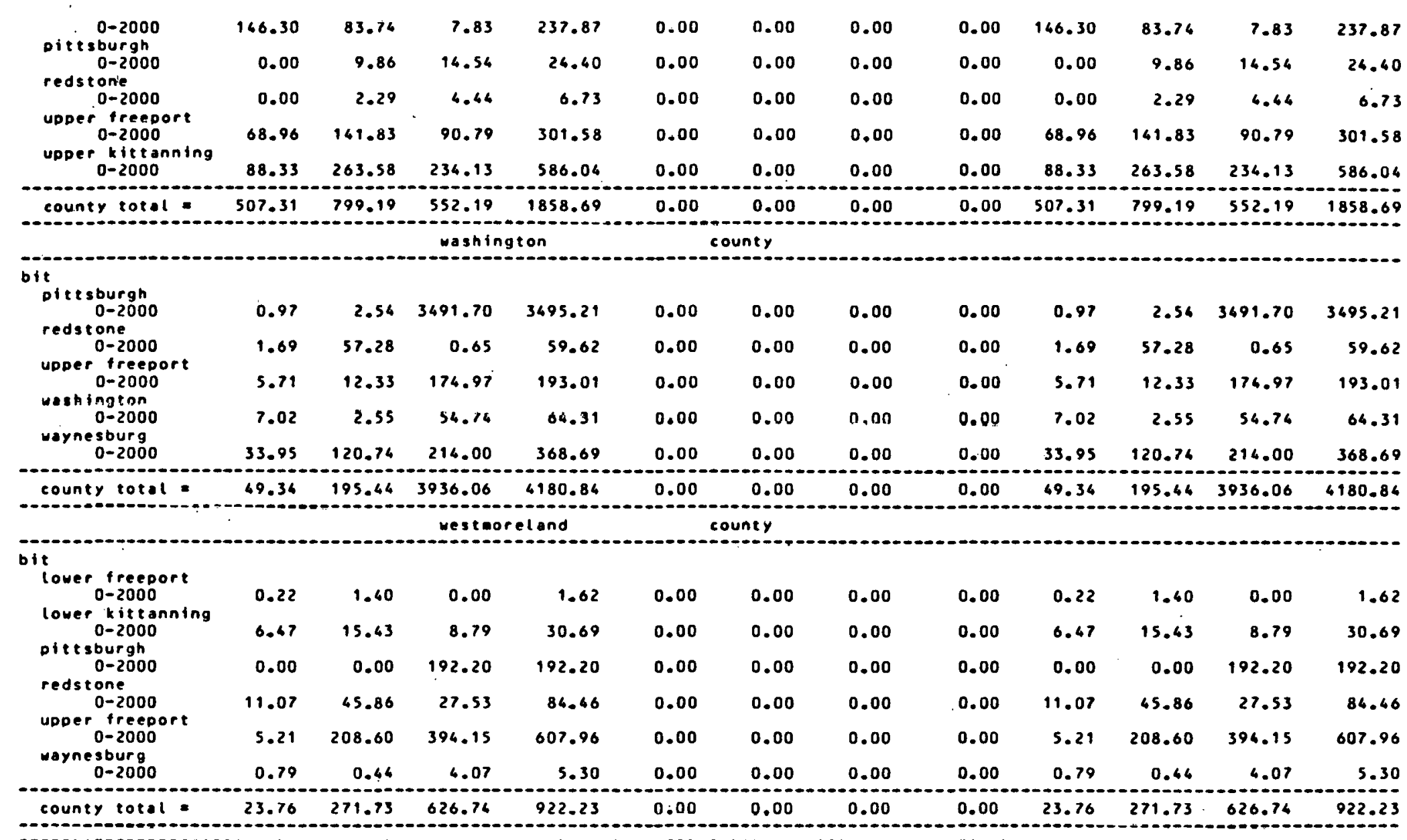

overburden

$\begin{array}{llllllllllllll}\text { strippable } & 256.76 & 389.18 & 70.56 & 716.50 & 51.88 & 17.29 & 2.87 & 72.04 & 308.64 & 406.47 & 73.43 & 788.56\end{array}$

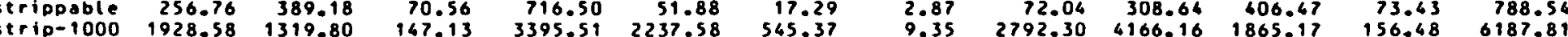

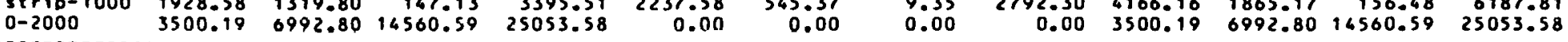

totol $=5685.53 \quad 8701.7814778 .28 \quad 29165.592289 .46 \quad 562.66 \quad 12.22 \quad 2864.34 \quad 7974.99 \quad 9264.4616790 .50 \quad 32029.93$

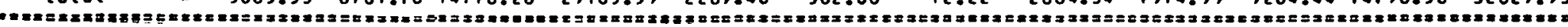




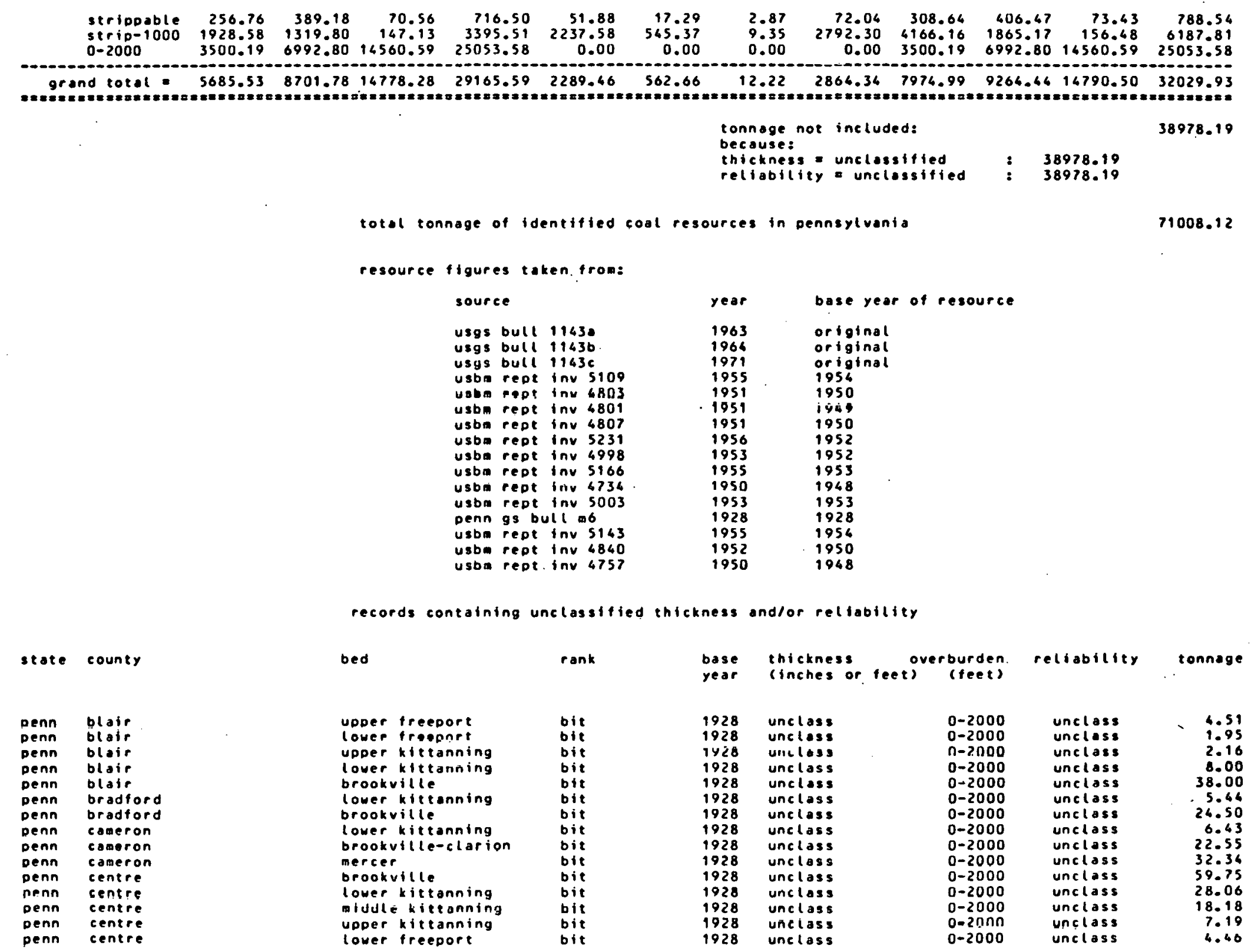




\begin{tabular}{|c|c|}
\hline 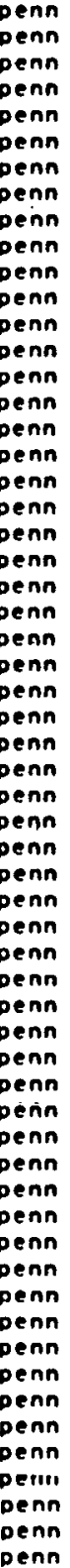 & $\begin{array}{l}\text { centre } \\
\text { centre } \\
\text { centre } \\
\text { centre } \\
\text { centre } \\
\text { centre } \\
\text { centre } \\
\text { centre } \\
\text { centre } \\
\text { cenere } \\
\text { centre } \\
\text { centre } \\
\text { centre } \\
\text { centre } \\
\text { centre } \\
\text { centre } \\
\text { clinton } \\
\text { clinton } \\
\text { clinton } \\
\text { clinton } \\
\text { elk } \\
\text { elk } \\
\text { elk } \\
\text { elk } \\
\text { elk } \\
\text { elk } \\
\text { elk } \\
\text { elk } \\
\text { elk } \\
\text { elk } \\
\text { elk } \\
\text { elk } \\
\text { elk } \\
\text { elk } \\
\text { elk } \\
\text { elk } \\
\text { elk } \\
\text { elk } \\
\text { elk } \\
\text { elk } \\
\text { elk } \\
\text { elk } \\
\text { elk } \\
\text { elk } \\
\text { elk } \\
\text { elk } \\
\text { elk } \\
\text { lycouing } \\
\text { lycoming } \\
\text { lycoijng } \\
\text { mercer } \\
\text { mercer } \\
\text { nercer } \\
\text { mercer } \\
\text { nercer } \\
\text { mckean } \\
\text { mckean } \\
\text { mckean } \\
\text { mckean } \\
\text { mckean }\end{array}$ \\
\hline
\end{tabular}

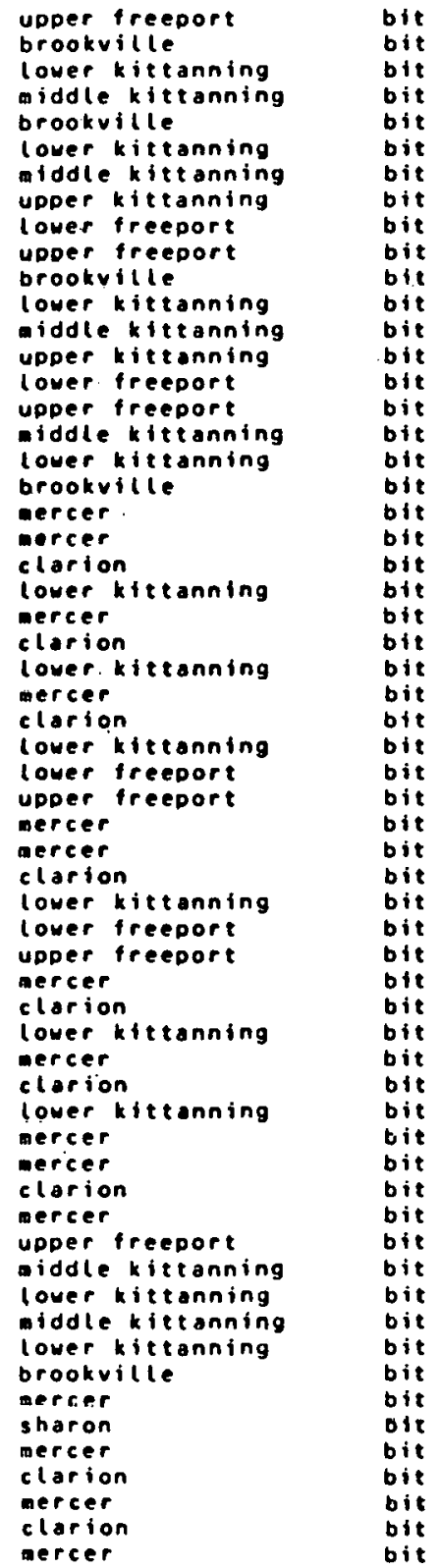

$\begin{array}{ll}1928 & \text { unclass } \\ 1928 & \text { unclass } \\ 1928 & \text { unclass } \\ 1928 & \text { unclass } \\ 1928 & \text { unclass } \\ 1928 & \text { unclass } \\ 1928 & \text { unclass } \\ 1928 & \text { unclass } \\ 1928 & \text { unclass } \\ 1928 & \text { unclass } \\ 1928 & \text { unclass } \\ 1928 & \text { unclass } \\ 1928 & \text { unclass } \\ 1928 & \text { unclass } \\ 1928 & \text { unclass } \\ 1928 & \text { unclass } \\ 1928 & \text { unclass } \\ 1928 & \text { unclass } \\ 1928 & \text { unclass } \\ 1928 & \text { unclass } \\ 1928 & \text { unclass } \\ 1928 & \text { unclass } \\ 1928 & \text { unclass } \\ 1928 & \text { unclass } \\ 1928 & \text { unclass } \\ 1928 & \text { unclass } \\ 1928 & \text { unclass } \\ 1928 & \text { unclass } \\ 1928 & \text { unclass } \\ 1928 & \text { unclass } \\ 1928 & \text { unclass } \\ 1928 & \text { unclass } \\ 1928 & \text { unclass } \\ 1928 & \text { unclass } \\ 1928 & \text { unclass } \\ 1928 & \text { unclass } \\ 1928 & \text { unclass } \\ 1928 & \text { unclass } \\ 1928 & \text { unclass } \\ 1928 & \text { unclass } \\ 1928 & \text { unclass } \\ 1928 & \text { unclass } \\ 1928 & \text { unclass } \\ 1928 & \text { unclass } \\ 1928 & \text { unclass } \\ 1928 & \text { unclass } \\ 1928 & \text { unclass } \\ 1928 & \text { unclass } \\ 1928 & \text { unclass } \\ 1928 & \text { unclass } \\ 1928 & \text { unclass } \\ 1928 & \text { unclass } \\ 1928 & \text { unclass } \\ 1928 & \text { unclass } \\ 1928 & \text { unclass } \\ 1928 & \text { unclass } \\ 1928 & \text { unclass } \\ 1928 & \text { unclass } \\ 1928 & \text { unclass } \\ 1928 & \text { unclass } \\ 1928 & \\ 19\end{array}$

$0-2000$
$0-2000$
$0-2000$
$0-2000$
$0-2000$
$0-2000$
$0-2000$
$0-2000$
$0-2000$
$0-2000$
$0-2000$
$0-2000$
$0-2000$
$0-2000$
$0-2000$
$0-2000$
$0-2000$
$0-2000$
$0-2000$
$0-2000$
$0-2000$
$0-2000$
$0-2000$
$0-2000$
$0-2000$
$0-2000$
$0-2000$
$0-2000$
$0-2000$
$0-2000$
$0-2000$
$0-2000$
$0-2000$
$0-2000$
$0-2000$
$0-2000$
$0-2000$
$0-2000$
$0-2000$
$0-2000$
$0-2000$
$0-2000$
$0-2000$
$0-2000$
$0-2000$
$0-2000$
$0-2000$
$0-2000$
$0-2000$
$0-2000$
$0-2000$
$0-2000$
$0-2000$
$0-2000$
$0-2000$
$0=2000$
$0-2000$
$0-2000$
$0-2000$
$0-2000$

0

\begin{tabular}{|c|c|}
\hline $\begin{array}{l}\text { unclass } \\
\text { unclass } \\
\text { unclass } \\
\text { unclass } \\
\text { unclass } \\
\text { unclass } \\
\text { unclass } \\
\text { unclass } \\
\text { unclass } \\
\text { unclass } \\
\text { unclass } \\
\text { unclass } \\
\text { unclass } \\
\text { unclass } \\
\text { unclass } \\
\text { unclass } \\
\text { unclass } \\
\text { unclass } \\
\text { unclass } \\
\text { unclass } \\
\text { unclass } \\
\text { unclass } \\
\text { unclass } \\
\text { unclass } \\
\text { unclass } \\
\text { unclass } \\
\text { unclass } \\
\text { unclass } \\
\text { unclass } \\
\text { unclass } \\
\text { unclass } \\
\text { unclass } \\
\text { unclass } \\
\text { unclass } \\
\text { unclass } \\
\text { unclass } \\
\text { unclass } \\
\text { unclass } \\
\text { unclass } \\
\text { unclass } \\
\text { unclass } \\
\text { unclass } \\
\text { unclass } \\
\text { unclass } \\
\text { unclass } \\
\text { unclass } \\
\text { unclass } \\
\text { unclass } \\
\text { unclass } \\
\text { unclass } \\
\text { unclass } \\
\text { unclass } \\
\text { unclass } \\
\text { unclass } \\
\text { unclass } \\
\text { unclacs } \\
\text { unclass } \\
\text { unclass } \\
\text { unclass } \\
\text { unclass }\end{array}$ & $\begin{array}{r}0.92 \\
3.08 \\
2.69 \\
0.57 \\
106.86 \\
56.60 \\
2.90 \\
1.66 \\
3.26 \\
0.62 \\
69.50 \\
38.56 \\
21.96 \\
8.53 \\
1.99 \\
0.30 \\
6.38 \\
30.84 \\
34.70 \\
25.24 \\
1.66 \\
0.55 \\
0.30 \\
40.82 \\
16.16 \\
1.57 \\
36.08 \\
50.98 \\
9.65 \\
2.00 \\
0.93 \\
38.72 \\
28.98 \\
55.63 \\
15.07 \\
4.50 \\
1.84 \\
29.70 \\
8.24 \\
0.67 \\
55.55 \\
25.51 \\
6.14 \\
0.36 \\
42.66 \\
2.28 \\
27.72 \\
7.00 \\
19.00 \\
39.00 \\
4.32 \\
12.96 \\
155.00 \\
189.40 \\
58.00 \\
7.73 \\
2.92 \\
12.61 \\
6.67 \\
21.87\end{array}$ \\
\hline
\end{tabular}




\begin{tabular}{|c|c|c|c|c|c|c|c|c|}
\hline & wekean & charion & tre & 1700 & uncess & $0-2000$ & uncloss & 13.61 \\
\hline penn & wckean & mercer & bit & 1928 & unclass & $0-2000$ & uncliass & 14.22 \\
\hline Denn & mckesn & clarion & b i.t & 1928 & unclass & $0-2000$ & uncliass & 12.82 \\
\hline Denn & ackean & mercer & bit & 1928 & unclass & $0-2000$ & unclass & 13.73 \\
\hline Denn & mckean & clarion & bit & 1928 & unclass & $0-2000$ & unclass & 9.50 \\
\hline Denn & mokean & aercer. & bit & 1928 & unctas: & $0-2000$ & unclass & 45.46 \\
\hline Denn & mckean & clarion & bit & 1928 & unclass & $0-2000$ & unclass & 36.37 \\
\hline Denn & mokean & mercer & bit & 1928 & unctass & $0-2000$ & unclass & 10.01 \\
\hline Denn & mckean & clarton & bit & 1928 & uniclass & $0-2000$ & unclass & 9.49 \\
\hline Denn & $\begin{array}{l}\text { mekean } \\
\text { nekean }\end{array}$ & $\begin{array}{l}\text { aercer } \\
\text { clarion }\end{array}$ & bit & 1928 & unclass & $0-2000$ & unclass & $\begin{array}{l}31.43 \\
23.81\end{array}$ \\
\hline $\begin{array}{l}\text { Denn } \\
\text { Denn }\end{array}$ & $\begin{array}{l}\text { mckean } \\
\text { mckean }\end{array}$ & $\begin{array}{l}\text { clarion } \\
\text { mercer }\end{array}$ & bit & $\begin{array}{l}1928 \\
1928\end{array}$ & unclass & $\begin{array}{l}0-2000 \\
0-2000\end{array}$ & unclass & $\begin{array}{l}23.81 \\
53.50\end{array}$ \\
\hline penn & mckean & clarion & bit & .1928 & unclass & $0-2000$ & $\begin{array}{l}\text { unclass } \\
\text { unclass }\end{array}$ & $\begin{array}{l}33.50 \\
33.71\end{array}$ \\
\hline Denn & mokean & mercer & bit & 1928 & unclass & $0-2000$ & unclass & 33.36 \\
\hline Denn & ackean & clarion & bit & 1928 & unclass & $0-2000$ & unclass & 28.71 \\
\hline Denn & tioga & upper kittonning & bit & 1928 & unclass & $0-2000$ & unclass & 33.95 \\
\hline Denn & tioga & widdle kittonning & bit & 1928 & unclass & $0-2000$ & unctass & 30.42 \\
\hline Denn & t toga & lover kittanning & bit & 1928 & unclass & $0-2000$ & uncless & 9.72 \\
\hline Denn & tioga & brookville & bit & 1928 & unclass & $0-2000$ & unclass & $33: 34$ \\
\hline Denn & allegheny & Dittsburgh & bit & 1928 & unc lass & $0-2000$ & unclass & 297.43 \\
\hline penn & allegheny & redstone & bit & 1928 & unclass & $0-2000$ & unclass & 1603.59 \\
\hline Denn & armstrong & brookville & bit & 1928 & unclass & $0-2000$ & unclass & 165.17 \\
\hline Denn & arinstrong & lover freeport & bit & 1928 & unctass & $0-2000$ & unclass & 319.46 \\
\hline Denn & armstrong & lower kittonning & bit & 1928 & unclass & $0-2000$ & unclass & 960.90 \\
\hline Denn & arostrong & upper freeport & bit & 1928 & unclass & $0-2000$ & unclass & 669.79 \\
\hline Denn & arnstrong & upper kittanning & bit & 1928 & uncloss & $0-2000$ & unclass & 71.81 \\
\hline Denn & bedford & clorion. & bit & 1928 & unclass & $0-2000$ & unclass & 112.10 \\
\hline penn & bedford & lover kittanning & bit & 1928 & unclass & $0-2000$ & unclass & 74.12 \\
\hline penn. & bedford & upper freeport & bit & 1928 & uncless & $0-2000$ & unclass & 48.41 \\
\hline Denn & conbrite & brookville & bit & 1928 & unclass & $0-2000$ & unclass & 63.05 \\
\hline Denn & casbria & clarion & bit & 1928 & unclass & $0-2000$ & unclass & 29.77 \\
\hline penn & canbris & lower Ireeport & bit & 1928 & unclass & $0-2000$ & uncless & 630.37 \\
\hline Denn & cambria & lower kittanning & bit & 1928 & unclass & $0-2000$ & unclass & 1082.23 \\
\hline Denn & canbria & upder freedort & bit & 1928 & unclosis & $0-2000$ & unclass & 525.67 \\
\hline Denn & cambria & upper kittanning & bit & 1928 & unctass & $0-2000$ & unclass & 309.21 \\
\hline penn & clorion & brookville & bit & 1928 & unc lass & $0-2000$ & unclass & 237.81 \\
\hline Denn & clarion & lover clarlon & bit & 1928 & unclass & $0-2000$ & unelass & 251.90 \\
\hline Denn & clerton & lover freeport & bit & 1928 & uncloss & $0-2000$ & uncless & 36.58 \\
\hline Denn & clarion & lover kittanning & bit & 1928 & uncless & $0-2000$ & unclass & 95.00 \\
\hline penn & clartion & mercer & bit & 1928 & unclass & $0-2000$ & uncless & 40.90 \\
\hline Denn & clarion & middle kittanning & bit & 1928 & unclass & $0-2000$ & unclass & 14.58 \\
\hline Denn & clartion & upper clarion & bit & 1928 & unctass & $0-2000$ & unclass & 32.42 \\
\hline penn & clarion & upper freeport & bit & 1928 & unclass & $0-2000$ & unclass & 29.83 \\
\hline Denn & clarion & upper kittanning & bit & 1928 & unclass & $0-2000$ & unclass & 34.20 \\
\hline Denn & clearfield & brookville & bit & 1928 & unclass & $0-2000$ & unclass & 53.68 \\
\hline penn & cleartleld & lower freeport & bit & 1928 & unclass & $0-2000$ & unclass & 539.10 \\
\hline penn & clearfield & luwer kiteanning & bit & 1928 & unclass & $0-2000$ & unclass & 828.20 \\
\hline Denn & cleartield & upper freeport & bit & $14<8$ & unclasis & $n \rightarrow>000$ & unclass & 258.68 \\
\hline Denn & clearfield & upper kittanning & bit & 1928 & unclass & $0-2000$ & unclass & 196.05 \\
\hline Denn & fayette & lover kittanning & bit & 1928 & unclass & $0-2000$ & unclass & 130.29 \\
\hline Denn & fayetie & pittsburgh & bit & 1928 & unclass & $0-2000$ & unclass & 1078.02 \\
\hline penn & tarette & redstone & bit & 1928 & unclass & $0-2000$ & unclass & 110.14 \\
\hline Denn & fayette & serickley & bit & 1928 & unclass & $0-2000$ & unclass & 83.29 \\
\hline Denn & forette & upder tree & bit & 1928 & unclass & $0-2000$ & unclass & 1820.16 \\
\hline penn & forette & upper kittanning & bit & 1928 & unclass & $0-2000$ & uncloss & 87.27 \\
\hline Denn & fayette & waynesburg & bit & 1928 & unclass & $0-2000$ & unclass & 109.09 \\
\hline penn & fultan & clarion & bit & 1928 & unclass & $0-2000$ & unclass & 17.90 \\
\hline Denn & fulton & lover kicitambing & bit & 1928 & unclass & $0-2000$ & unclass & 11.88 \\
\hline Denn & greene & upper freeport & bit & 1928 & unclass & $0-200 n$ & mnclass & 1594.08 \\
\hline penn & greene & sevickley & bit & 1928 & unclass & $0-2000$ & unclass & 480.037 \\
\hline
\end{tabular}




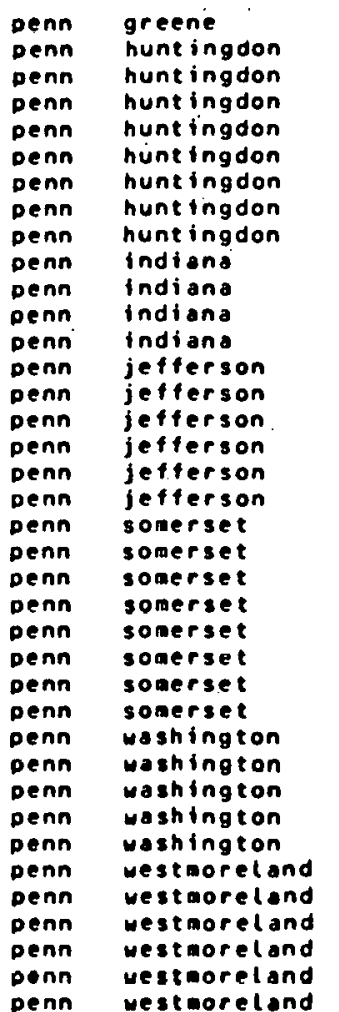

naynesburg

upper treeporing

clarion

lower kittanning

clarion

lover kittanning

upper freepor

lover freedort

lower kittanning

pittsburgh

upper freeport

brookville

lower freeport

iddle kittanning

upper treeport

upper kittanning

brookville

clorion

lover freeport

lower klttanning

Ditteburgh rider

sewickley

upper treeport

upper kittanning

pitsburgh

upper freeport

upper ington

washington
waynesburg

lower ktt tanning

middle kittanning

pittsburgh

redstone

upper freeport

waynesburg bit
bit
bit
bit
bit
bit
bit
bit
bit
bit
bit
bit
bit
bit
bit
bit
bit
bit
bit
bit
bit
bit
bit
bit
bit
bit
bit
bit
bit
bit
bit
bit
bit
bit
bit
bit
bit
bit

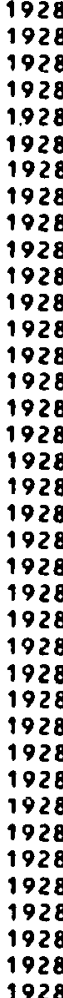

uncloss

unclass

unclass

unclass

unclass

unclass

unclass

unclass

unclass

unclass

unchas

uncloss

uncleass

unclass

unclass

unclass

uncless

unclass

uncloss

unclass

unclass

unclass

unclass

unclas

unclass

unc lass

unclass
$0-2000$

$0-2000$

$0-2000$

$0-2000$

$0-2000$

$0-2000$

$0-2000$

$0-2000$

$0-2000$

0-2000

$0-2000$

$0-2000$

$0-2000$

$0-2000$

$0-2000$

$0-2000$

$0-2000$

$0-2000$

$0-2000$

$0-200$

$0-2000$

0-2000

0-2000

$0-2000$

$0-2000$

$0-2000$

$0-2000$

$0-2000$
$0-2000$

$0-2000$

$0-2000$
$0-2000$

$0-2000$

$0-2000$

$0-2000$

$0-2000$
$0-2000$

$0-2000$ unclass

unclass

unclass

uncless

unclass

unclass

unclass

unclass

unclass

uncloss

unclass

uncloss

unclass

unclass

unclass

unclass

uncloss

uncloss

unciass

unclass

unclass

unclas

unclass

unclass

uncless

unclass
1362.87

69.25
25.96

25.96
5.14

1.65
0.59

15.58
15.85

9.19

1339.08

749.81

20.71

1656.53

244.23

455.69

136.48

136.48
255.79

102.74

61.54

1027.55

8.00

5.10

557.66

2616.61
1039.94

98.54

2196.56

1546.69

907.61
697.34

697.36
706.32

512.65

2079.26

9.71

$\sqrt{2}-2$

$$
\text { thes }
$$

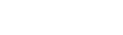


average analyses of coal in pennsylvania

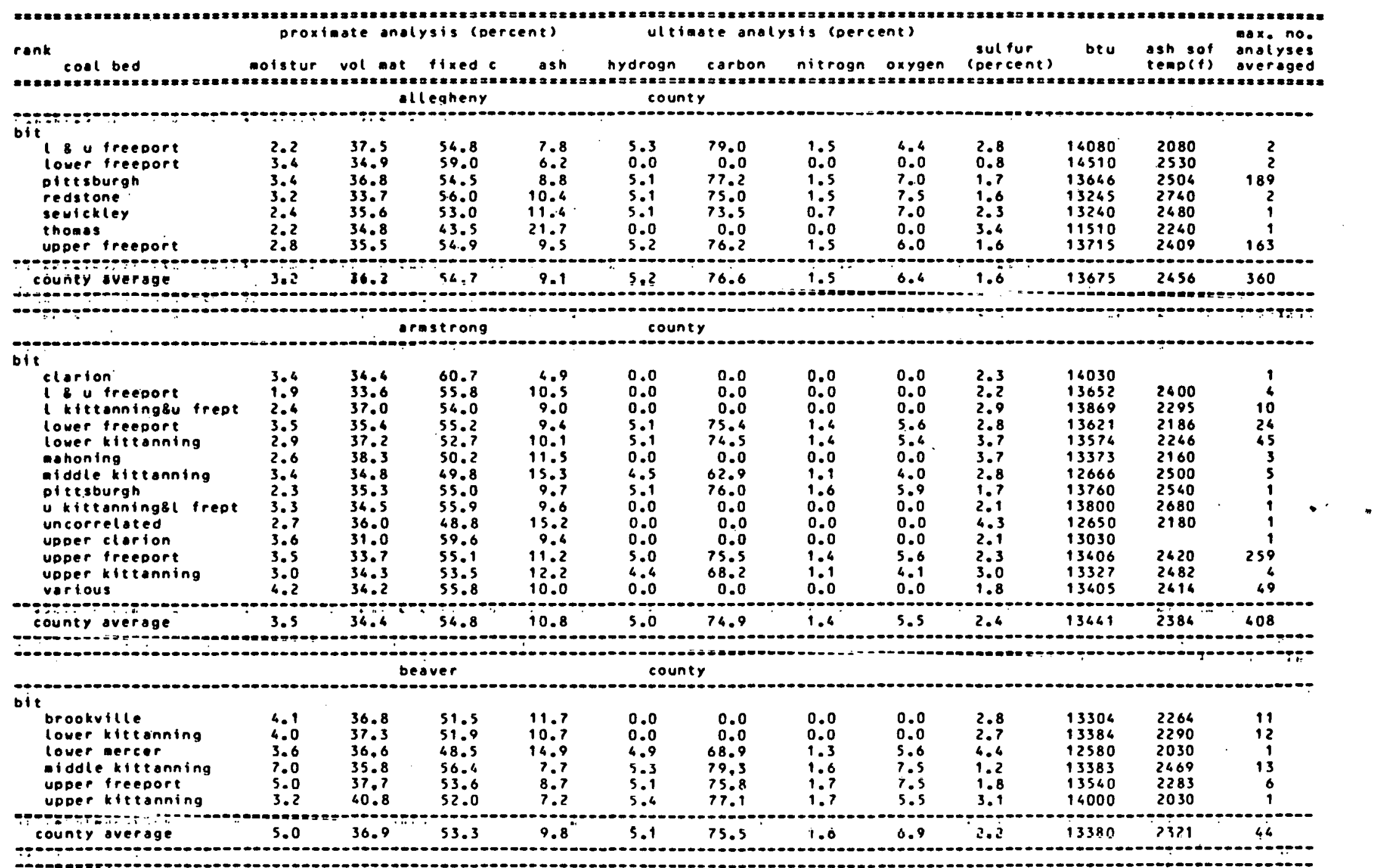




\begin{tabular}{|c|c|c|c|c|c|c|c|c|c|c|c|c|}
\hline $\begin{array}{l}\text { clorion } \\
\text { l kittanninglu frept } \\
\text { lower kittonning } \\
\text { oohoning } \\
\text { pittsburgh } \\
\text { upper treeport } \\
\text { vorious }\end{array}$ & $\begin{array}{l}1.2 \\
1.5 \\
1.5 \\
1.5 \\
1.8 \\
1.5 \\
1.2\end{array}$ & $\begin{array}{l}18.8 \\
18.6 \\
16.6 \\
18.2 \\
15.9 \\
16.9 \\
16.9\end{array}$ & $\begin{array}{l}67.8 \\
69.1 \\
73.0 \\
75.9 \\
66.2 \\
68.5 \\
65.0\end{array}$ & $\begin{array}{l}13.4 \\
12.4 \\
10.6 \\
6.0 \\
18.1 \\
14.5 \\
18.1\end{array}$ & $\begin{array}{l}0.0 \\
0.0 \\
4.3 \\
0.0 \\
0.0 \\
4.0 \\
0.0\end{array}$ & $\begin{array}{r}0.0 \\
0.0 \\
84.3 \\
0.0 \\
0.0 \\
73.0 \\
.0 .0\end{array}$ & $\begin{array}{l}0.0 \\
0.0 \\
1.4 \\
0.0 \\
0.0 \\
1.3 \\
0.0\end{array}$ & $\begin{array}{l}0.0 \\
0.0 \\
2.2 \\
0.0 \\
0.0 \\
1.5 \\
0.0\end{array}$ & $\begin{array}{l}1.8 \\
2.1 \\
1.5 \\
0.7 \\
2.4 \\
2.4 \\
2.6\end{array}$ & $\begin{array}{l}13480 \\
13611 \\
13969 \\
14812 \\
12340 \\
13248 \\
12618\end{array}$ & $\begin{array}{l}2795 \\
2693 \\
2769 \\
2620 \\
2613 \\
2595\end{array}$ & $\begin{array}{r}3 \\
7 \\
33 \\
4 \\
1 \\
23 \\
6\end{array}$ \\
\hline unty overage & 1.5 & 171 & 70.5 & 12.4 & 4.1 & 77.7 & 1.3 & 1.8 & 1.9 & 13619 & 2676 & 77 \\
\hline & & & & & & & & & & & & \\
\hline $\begin{array}{l}\text { brookville } \\
\text { lower kittenning } \\
\text { loddle kittonning } \\
\text { upper freedort } \\
\text { various }\end{array}$ & $\begin{array}{l}4.1 \\
3.8 \\
6.2 \\
2.7 \\
7.5\end{array}$ & $\begin{array}{l}28.3 \\
26.8 \\
29.0 \\
29.9 \\
27.4\end{array}$ & $\begin{array}{l}59.9 \\
65.1 \\
62.6 \\
61.2 \\
60.8\end{array}$ & $\begin{array}{r}11.7 \\
8.1 \\
8.5 \\
8.9 \\
11.8\end{array}$ & $\begin{array}{l}4.6 \\
5.0 \\
0.0 \\
4.9 \\
0.0\end{array}$ & $\begin{array}{r}76.7 \\
81.4 \\
0.0 \\
81.0 \\
0.0 \\
\end{array}$ & $\begin{array}{l}1.2 \\
1.5 \\
0.0 \\
1.5 \\
0.0\end{array}$ & $\begin{array}{l}4.1 \\
4.0 \\
0.0 \\
3.8 \\
0.0 \\
0.0\end{array}$ & $\begin{array}{l}3.3 \\
0.8 \\
2.7 \\
2.3 \\
1.0\end{array}$ & $\begin{array}{l}13457 \\
14110 \\
14010 \\
14103 \\
13140\end{array}$ & $\begin{array}{l}2305 \\
2910 \\
2140 \\
2293 \\
2536\end{array}$ & $\begin{array}{l}4 \\
7 \\
2 \\
3 \\
3\end{array}$ \\
\hline county overage & 4.5 & 27.9 & 62.5 & 9.6 & 4.9 & 80.1 & 1.6 & 4.0 & 1.9 & 13807 & 2502 & 19 \\
\hline$\because$ & & & ord & & & & & & & $-\infty$ & & \\
\hline orion & 2.1 & 18.4 & 69.5 & 12.1 & 4.2 & 78.1 & 1.1 & 3.4 & 1.1 & 13650 & 3910 & 1 \\
\hline county average, & $i$ & is & 69.5 & 12.1 & 4.2 & 78.1 & 1.1 & 3.4 & $i .1$ & 13650 & $2910^{\circ}$ & 1 \\
\hline$-\infty-\infty-\infty$ & $=$ & & & & & & & & 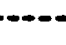 & 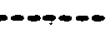 & $\cdots$ & $\cdots$ \\
\hline 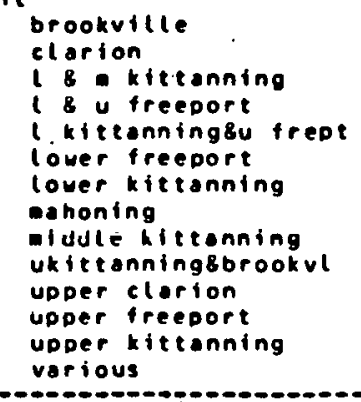 & $\begin{array}{r}4.5 \\
3.8 \\
8.4 \\
5.4 \\
11.1 \\
4.0 \\
6.3 \\
3.2 \\
4.4 \\
4.3 \\
3.9 \\
3.5 \\
3.8 \\
4.2 \\
.2 .\end{array}$ & $\begin{array}{l}36.7 \\
34.8 \\
34.6 \\
32.9 \\
35.6 \\
35.0 \\
38.0 \\
41.0 \\
37.0 \\
37.5 \\
35.9 \\
35.3 \\
35.2 \\
37.3 \\
. . .3\end{array}$ & $\begin{array}{l}52.0 \\
48.2 \\
52.8 \\
57.3 \\
51.7 \\
55.7 \\
49.4 \\
50.7 \\
53.8 \\
54.5 \\
55.7 \\
53.6 \\
56.9 \\
52.6\end{array}$ & $\begin{array}{r}11.4 \\
17.0 \\
12.8 \\
10.0 \\
12.7 \\
9.3 \\
12.7 \\
8.3 \\
10.2 \\
8.0 \\
8.5 \\
11.1 \\
7.9 \\
10.0\end{array}$ & $\begin{array}{l}4.8 \\
0.0 \\
0.0 \\
4.9 \\
0.0 \\
0.0 \\
0.0 \\
0.0 \\
5.1 \\
0.0 \\
0.0 \\
5.1 \\
5.2 \\
0.0\end{array}$ & $\begin{array}{r}71.3 \\
0.0 \\
0.0 \\
76.3 \\
0.0 \\
0.0 \\
0.0 \\
0.0 \\
74.0 \\
0.0 \\
0.0 \\
76.4 \\
79.9 \\
0.0\end{array}$ & $\begin{array}{l}1.3 \\
0.0 \\
0.0 \\
1.4 \\
0.0 \\
0.0 \\
0.0 \\
0.0 \\
1.4 \\
0.0 \\
0.0 \\
1.5 \\
1.5 \\
0.0\end{array}$ & $\begin{array}{l}6.7 \\
0.0 \\
0.0 \\
7.0 \\
0.0 \\
0.0 \\
0.0 \\
0.0 \\
5.5 \\
0.0 \\
0.0 \\
0.2 \\
6.8 \\
0.0 \\
.0\end{array}$ & $\begin{array}{l}2.7 \\
3.8 \\
1.1 \\
1.0 \\
1.4 \\
1.8 \\
3.6 \\
4.0 \\
3.0 \\
1.7 \\
3.1 \\
2.3 \\
2.2 \\
2.7\end{array}$ & $\begin{array}{l}13116 \\
12107 \\
12600 \\
13383 \\
12330 \\
13550 \\
13031 \\
13620 \\
13346 \\
13730 \\
13545 \\
13315 \\
13678 \\
13335 \\
-19.9\end{array}$ & $\begin{array}{l}2344 \\
2505 \\
2680 \\
2666 \\
2270 \\
2382 \\
2275 \\
2284 \\
2495 \\
2373 \\
2440 \\
2246\end{array}$ & $\begin{array}{r}23 \\
4 \\
1 \\
3 \\
1 \\
6 \\
8 \\
1 \\
34 \\
2 \\
2 \\
51 \\
5 \\
14 \\
0.9\end{array}$ \\
\hline couney average & 4.1 & 36.2 & 53.0 & 10.8 & 5.1 & 75.5 & 1.4 & 6.1 & 2.6 & 13270 & 2346 & 155 \\
\hline . & & & oria & & & & & & & & & \\
\hline $\begin{array}{l}\text { clarion } \\
l \& \text { u trapnort } \\
l \quad 8 \text { u kitionning }\end{array}$ & $\begin{array}{l}3.1 \\
2.9 \\
2.3\end{array}$ & $\begin{array}{l}14.2 \\
24.9 \\
23.3\end{array}$ & $\begin{array}{l}68.8 \\
64.5 \\
64.2\end{array}$ & $\begin{array}{l}17.0 \\
10.6 \\
12.5\end{array}$ & $\begin{array}{l}0.0 \\
0.0 \\
0.0\end{array}$ & $\begin{array}{l}0.0 \\
0.0 \\
0.0\end{array}$ & $\begin{array}{l}0.0 \\
0.0 \\
0.0\end{array}$ & $\begin{array}{l}0.0 \\
0.0 \\
0.0\end{array}$ & $\begin{array}{l}3.2 \\
3.7 \\
2.1\end{array}$ & $\begin{array}{l}12290 \\
13907 \\
13562\end{array}$ & $\begin{array}{l}2563 \\
2556\end{array}$ & $\begin{array}{r}1 \\
20 \\
7\end{array}$ \\
\hline
\end{tabular}




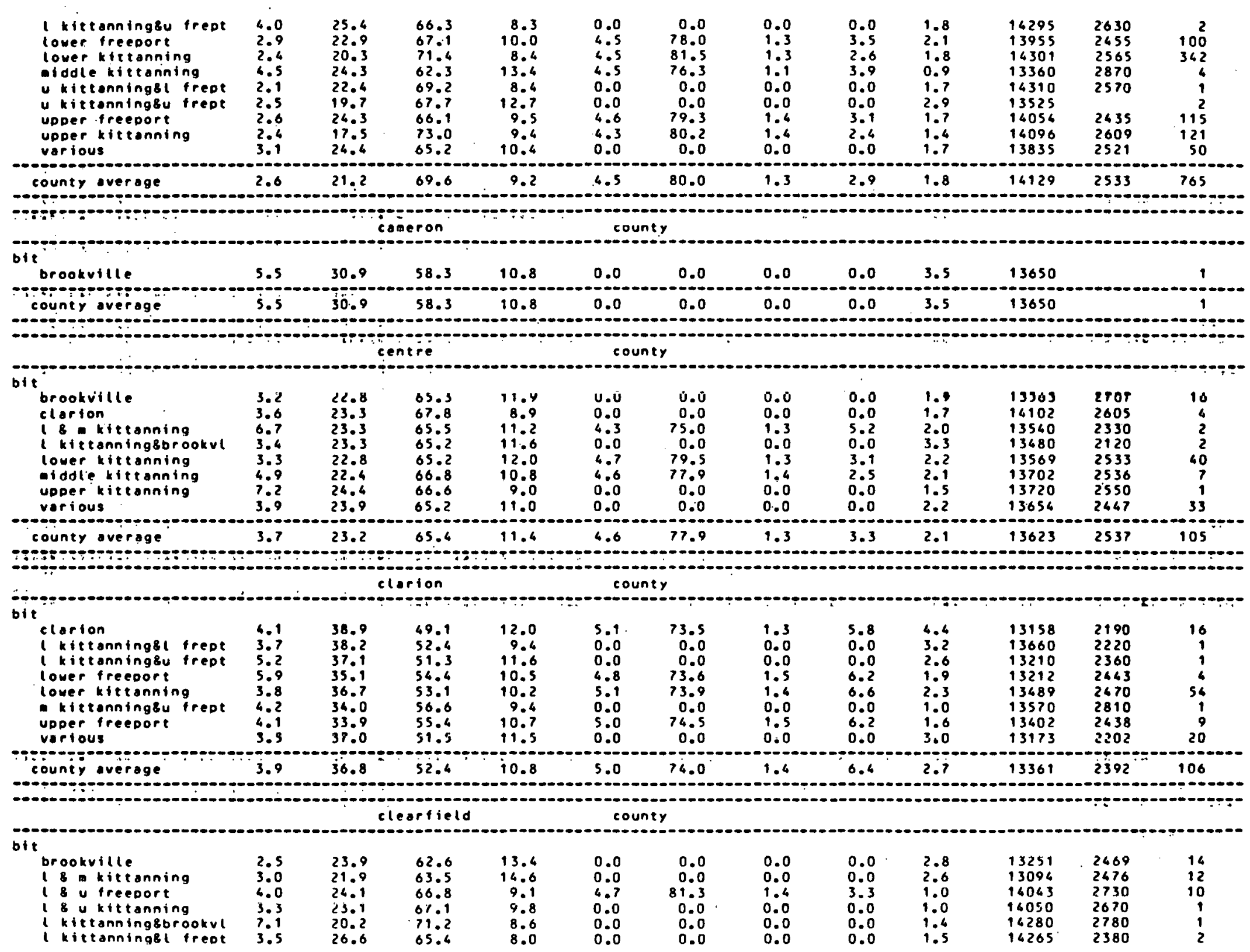




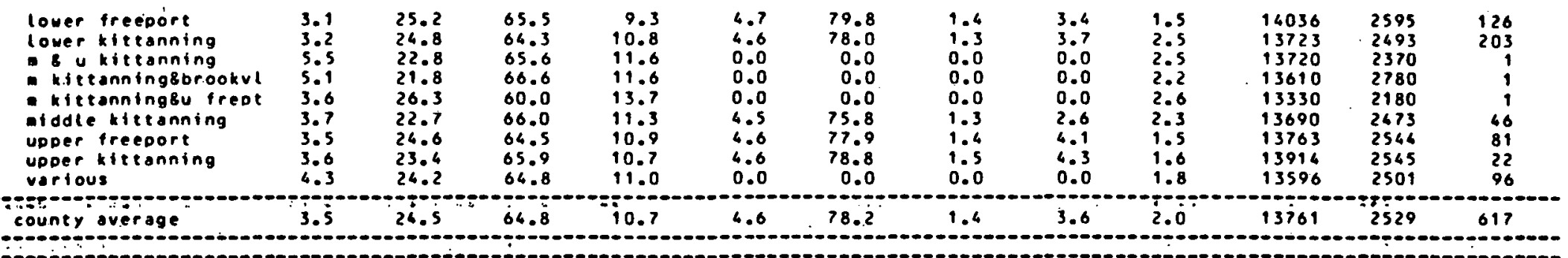

clinton county

\begin{tabular}{|c|c|c|c|c|c|c|c|c|c|c|c|c|}
\hline $\begin{array}{l}\text { l o kittanning } \\
\text { lover freeport } \\
\text { lover kittanning } \\
\text { oiddle kittanning } \\
\text { uoper kittanning }\end{array}$ & $\begin{array}{l}4.1 \\
3.8 \\
7.4 \\
6.7 \\
5.2\end{array}$ & $\begin{array}{l}22.4 \\
24.9 \\
22.5 \\
23.9 \\
24.6\end{array}$ & $\begin{array}{l}66.6 \\
67.4 \\
66.9 \\
65.1 \\
66.1\end{array}$ & $\begin{array}{r}11.0 \\
7.7 \\
10.6 \\
10.9 \\
9.3\end{array}$ & $\begin{array}{l}0.0 \\
0.0 \\
4.4 \\
4.4 \\
0.0\end{array}$ & $\begin{array}{r}0.0 \\
0.0 \\
76.5 \\
75.5 \\
0.0\end{array}$ & $\begin{array}{l}0.0 \\
0.0 \\
1.2 \\
1.2 \\
0.0\end{array}$ & $\begin{array}{l}0.0 \\
0.0 \\
2.3 \\
4.4 \\
0.0\end{array}$ & $\begin{array}{l}2.4 \\
2.1 \\
2.9 \\
2.9 \\
2.4\end{array}$ & $\begin{array}{l}13800 \\
14460 \\
13427 \\
13483 \\
14100\end{array}$ & $\begin{array}{l}2470 \\
2400 \\
2370 \\
2130 \\
2210\end{array}$ & $\begin{array}{l}1 \\
1 \\
6 \\
3 \\
1\end{array}$ \\
\hline Y overoge & 6.3 & 23.4 & 66.3 & 10.3 & 4.4 & 76.0 & 1.2 & 3.3 & $2.7^{\circ}$ & 13652 & 2295 & 10 \\
\hline
\end{tabular}
county

\begin{tabular}{|c|c|c|c|c|c|c|c|c|c|c|c|c|c|}
\hline $\begin{array}{l}\text { les: } \\
\text { lover } \\
\text { lover } \\
\text { aiddle } \\
\text { vortous }\end{array}$ & $\begin{array}{l}\text { kittonning } \\
\text { freeport } \\
\text { kittonning } \\
\text { kittonning } \\
\text { int }\end{array}$ & $\begin{array}{l}4.0 \\
5.7 \\
3.7 \\
3.1 \\
3.9\end{array}$ & $\begin{array}{l}33.6 \\
28.1 \\
32.8 \\
32.7 \\
33.0\end{array}$ & $\begin{array}{l}57.3 \\
63.3 \\
56.7 \\
58.9 \\
58.0\end{array}$ & $\begin{array}{r}9.1 \\
8.6 \\
10.5 \\
8.4 \\
9.0\end{array}$ & $\begin{array}{l}0.0 \\
0.0 \\
0.0 \\
5.1 \\
0.0\end{array}$ & $\begin{array}{r}0.0 \\
0.0 \\
0.0 \\
77.8 \\
0.0\end{array}$ & $\begin{array}{l}0.0 \\
0.0 \\
0.0 \\
1.7 \\
0.0\end{array}$ & $\begin{array}{l}0.0 \\
0.0 \\
0.0 \\
3.0 \\
0.0\end{array}$ & $\begin{array}{l}2.1 \\
1.2 \\
2.7 \\
2.4 \\
1.8\end{array}$ & $\begin{array}{l}13823 \\
13560 \\
13710 \\
14036 \\
13722\end{array}$ & $\begin{array}{l}2614 \\
2394 \\
2460 \\
2388\end{array}$ & $\begin{array}{r}16 \\
1 \\
11 \\
5 \\
11\end{array}$ \\
\hline & & 3.9 & 33.1 & 57.6 & 9.3 & 5.1 & 77.8 & 1.7 & 3.0 & 2.2 & 13788 & 2610 & 44 \\
\hline
\end{tabular}

payet te county

\begin{tabular}{|c|c|c|c|c|c|c|c|c|c|c|c|c|}
\hline $\begin{array}{l}\text { letetanningsorookvl } \\
\text { lower freeport } \\
\text { lower kittanning } \\
\text { oltesburgh } \\
\text { redstone } \\
\text { redstone sewickley } \\
\text { redstoneswoynesourg } \\
\text { sewickley } \\
\text { udoer freeport } \\
\text { upper kit tanning } \\
\text { vorious }\end{array}$ & $\begin{array}{l}7.1 \\
2.8 \\
2.9 \\
2.9 \\
4.3 \\
4.0 \\
3.4 \\
3.8 \\
3.0 \\
2.5 \\
7.5\end{array}$ & $\begin{array}{l}20.2 \\
25.9 \\
25.6 \\
32.6 \\
31.6 \\
33.6 \\
32.6 \\
33.0 \\
30.3 \\
24.4 \\
32.2\end{array}$ & $\begin{array}{l}71.2 \\
58.7 \\
63.9 \\
58.0 \\
55.6 \\
53.8 \\
50.5 \\
53.1 \\
61.1 \\
65.3 \\
56.0\end{array}$ & $\begin{array}{r}8.6 \\
15.4 \\
10.5 \\
9.4 \\
12.8 \\
12.6 \\
16.9 \\
13.9 \\
1.9 \\
9.8 \\
13.8\end{array}$ & $\begin{array}{l}0.0 \\
0.0 \\
5.0 \\
5.1 \\
0.0 \\
0.0 \\
4.6 \\
5.0 \\
9.3 \\
4.6 \\
4.5\end{array}$ & $\begin{array}{r}0.0 \\
0.0 \\
80.1 \\
78.0 \\
0.0 \\
0.0 \\
69.6 \\
72.7 \\
18.7 \\
78.7 \\
71.1\end{array}$ & $\begin{array}{l}0.0 \\
0.0 \\
1.3 \\
1.6 \\
0.0 \\
0.0 \\
1.6 \\
1.4 \\
1.5 \\
1.3 \\
1.4\end{array}$ & $\begin{array}{l}0.0 \\
0.0 \\
3.1 \\
5.1 \\
0.0 \\
0.0 \\
5.5 \\
4.5 \\
3.3 \\
2.8 \\
7.4\end{array}$ & $\begin{array}{l}1.4 \\
2.0 \\
2.6 \\
1.5 \\
1.7 \\
1.8 \\
2.8 \\
3.2 \\
2.6 \\
2.8 \\
2.2\end{array}$ & $\begin{array}{l}14280 \\
12950 \\
13868 \\
13820 \\
13040 \\
13150 \\
12660 \\
12929 \\
14090 \\
14030 \\
12360\end{array}$ & $\begin{array}{l}2780 \\
2570 \\
2385 \\
2637 \\
2605 \\
2610 \\
2650 \\
2355 \\
2250 \\
2666 \\
2230\end{array}$ & $\begin{array}{r}1 \\
2 \\
11 \\
124 \\
4 \\
1 \\
14 \\
2 \\
4 \\
3\end{array}$ \\
\hline county average & 3.1 & 31.8 & 58.1 & 10.1 & 5.0 & 71.0 & 1.5 & 4.8 & 1.8 & 13692 & 2577 & 167 \\
\hline & & & & & & & & & & & & \\
\hline $\begin{array}{l}\text { pittsburgh } \\
\text { ofttsourghsseutickley }\end{array}$ & $\begin{array}{l}2.5 \\
2.6\end{array}$ & $\begin{array}{l}35.8 \\
35.0\end{array}$ & $\begin{array}{l}54.4 \\
54.7\end{array}$ & $\begin{array}{r}9.8 \\
10.3\end{array}$ & $\begin{array}{l}5.1 \\
4.9\end{array}$ & $\begin{array}{l}77.2 \\
74.6\end{array}$ & $\begin{array}{l}1.6 \\
1.3\end{array}$ & $\begin{array}{l}5.4 \\
4.9\end{array}$ & $\begin{array}{l}2.5 \\
2.4\end{array}$ & $\begin{array}{l}13708 \\
13612\end{array}$ & $\begin{array}{l}2224 \\
2296\end{array}$ & $\begin{array}{l}67 \\
12\end{array}$ \\
\hline
\end{tabular}




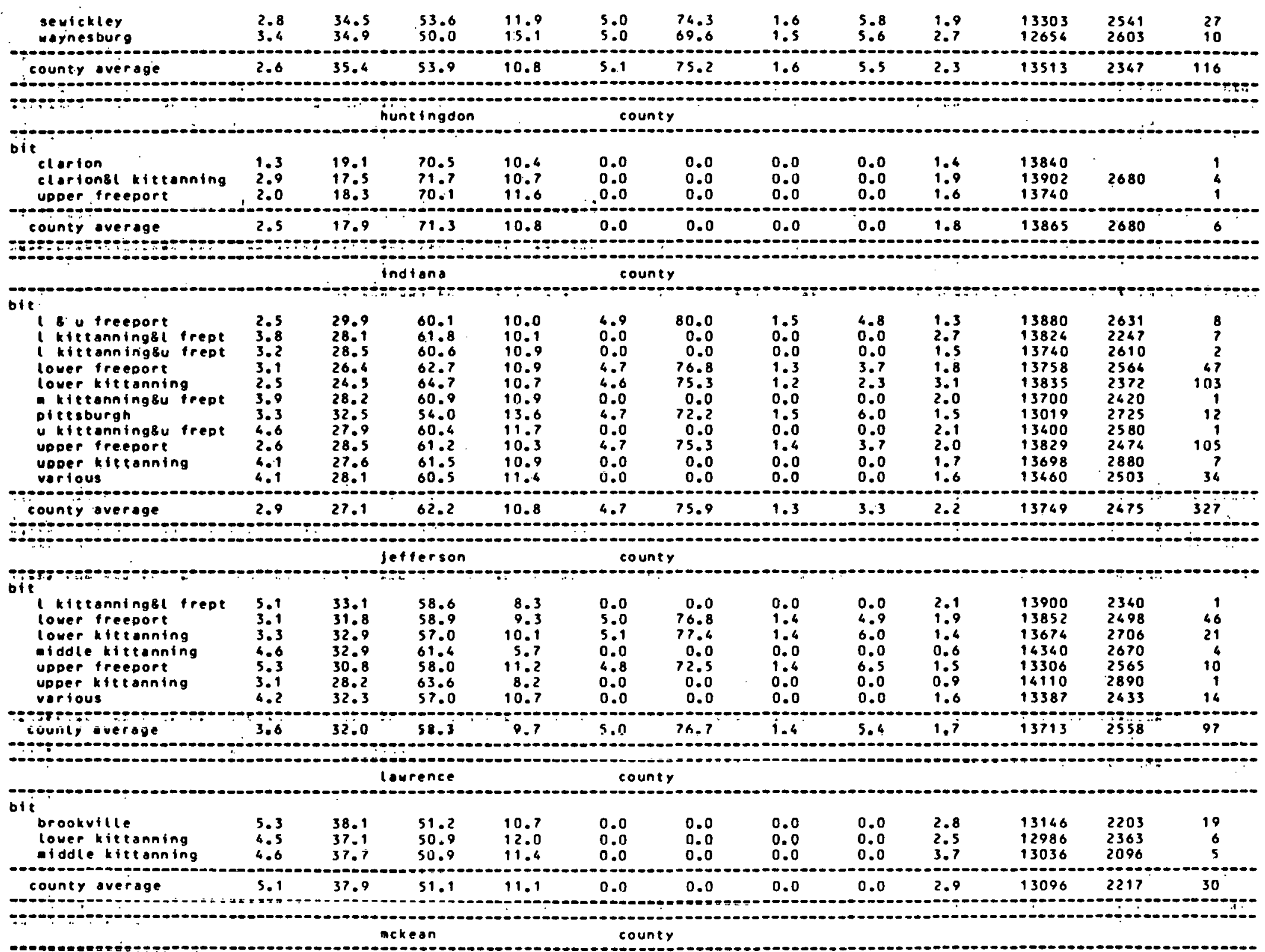




\begin{tabular}{|c|c|c|c|c|c|c|c|c|c|c|c|c|}
\hline lower mercer & 5.7 & 33.0 & 52.5 & 14.5 & 0.0 & 0.0 & 0.0 & 0.0 & 3.2 & 12703 & 2620. & 3 \\
\hline county overage & 5.7 & 33.0 & 52.5 & 14.5 & 0.0 & 0.0 & 0.0 & 0.0 & 3.2 & 12703 & 2420 & 3 \\
\hline$\because$ & & & er & & & & & & & & & \\
\hline $\begin{array}{l}\text { brookville } \\
\text { lover kittonning } \\
\text { middle kltionning } \\
\text { vorious }\end{array}$ & $\begin{array}{l}5.1 \\
5.4 \\
4.7 \\
6.4\end{array}$ & $\begin{array}{l}39.8 \\
37.0 \\
37.2 \\
35.8\end{array}$ & $\begin{array}{l}50.5 \\
50.9 \\
53.2 \\
53.6\end{array}$ & $\begin{array}{r}9.6 \\
12.1 \\
9.6 \\
10.6\end{array}$ & $\begin{array}{l}5.2 \\
0.0 \\
0.0 \\
0.0\end{array}$ & $\begin{array}{r}76.0 \\
0.0 \\
0.0 \\
0.0\end{array}$ & $\begin{array}{l}1.4 \\
0.0 \\
0.0 \\
0.0\end{array}$ & $\begin{array}{l}7.4 \\
0.0 \\
0.0 \\
0.0\end{array}$ & $\begin{array}{l}3.8 \\
2.3 \\
1.6 \\
1.9\end{array}$ & $\begin{array}{l}13314 \\
13002 \\
13360 \\
13130\end{array}$ & $\begin{array}{l}2197 \\
2392 \\
2630 \\
2290\end{array}$ & $\begin{array}{r}45 \\
4 \\
1 \\
2\end{array}$ \\
\hline county overage & $5.2^{\prime}$ & 39.4 & 50.7 & 9.9 & 5.2 & 76.0 & $1.4^{\circ}$ & 7.4 & 3.5 & 13284 & 2224. & 52 \\
\hline י- & & & & & & $\cdots$ & $\cdots$ & & & 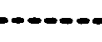 & & ? \\
\hline 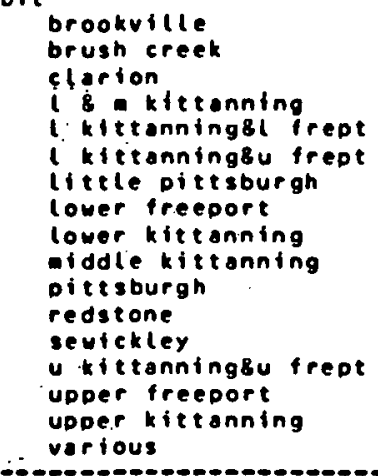 & $\begin{array}{l}3.5 \\
4.0 \\
3.6 \\
2.3 \\
4.0 \\
3.4 \\
3.8 \\
2.9 \\
2.8 \\
3.4 \\
3.9 \\
4.6 \\
2.8 \\
2.6 \\
2.7 \\
2.5 \\
3.2\end{array}$ & $\begin{array}{l}17.8 \\
19.4 \\
19.1 \\
10.1 \\
20.3 \\
18.7 \\
20.9 \\
17.9 \\
17.5 \\
17.5 \\
20.9 \\
20.4 \\
21.4 \\
17.5 \\
18.4 \\
17.2 \\
19.0 \\
19.9\end{array}$ & $\begin{array}{l}79.6 \\
70.3 \\
70.1 \\
73.0 \\
70.8 \\
71.8 \\
69.2 \\
72.3 \\
71.8 \\
68.1 \\
66.1 \\
62.3 \\
67.6 \\
70.1 \\
69.6 \\
71.6 \\
69.8 \\
90.0\end{array}$ & $\begin{array}{r}10.6 \\
10.3 \\
10.8 \\
10.8 \\
8.9 \\
9.6 \\
9.9 \\
9.9 \\
10.7 \\
14.4 \\
13.0 \\
17.4 \\
11.0 \\
12.4 \\
12.0 \\
11.2 \\
11.3\end{array}$ & $\begin{array}{l}4.1 \\
4.0 \\
0.0 \\
4.2 \\
0.0 \\
0.0 \\
0.0 \\
4.3 \\
4.2 \\
4.2 \\
4.3 \\
3.9 \\
4.4 \\
4.3 \\
4.3 \\
4.2 \\
0.0\end{array}$ & $\begin{array}{r}79.2 \\
76.7 \\
0.0 \\
79.0 \\
10.0 \\
0.0 \\
0.0 \\
80.3 \\
79.0 \\
76.6 \\
75.3 \\
70.7 \\
77.8 \\
77.1 \\
78.6 \\
78.6 \\
0.0\end{array}$ & $\begin{array}{l}1.3 \\
1.4 \\
0.0 \\
1.3 \\
0.0 \\
0.0 \\
0.0 \\
1.3 \\
1.3 \\
1.2 \\
1.6 \\
1.6 \\
1.7 \\
1.3 \\
1.4 \\
1.3 \\
0.0 \\
-. .0 .0\end{array}$ & $\begin{array}{l}2.5 \\
3.6 \\
0.0 \\
3.1 \\
0.0 \\
0.0 \\
0.0 \\
2.6 \\
2.2 \\
1.9 \\
2.6 \\
4.5 \\
3.0 \\
1.8 \\
2.7 \\
2.1 \\
0.0 \\
-. .0\end{array}$ & $\begin{array}{l}1.9 \\
2.4 \\
1.6 \\
1.7 \\
2.4 \\
1.3 \\
0.8 \\
1.6 \\
1.9 \\
2.0 \\
1.6 \\
2.0 \\
1.4 \\
1.9 \\
1.7 \\
2.1 \\
1.7 \\
.0 .9\end{array}$ & $\begin{array}{l}13806 \\
13820 \\
13810 \\
13871 \\
14000 \\
14070 \\
14006 \\
14030 \\
13860 \\
13200 \\
13400 \\
12275 \\
13807 \\
13624 \\
13651 \\
13780 \\
13671 \\
1307\end{array}$ & $\begin{array}{l}2830 \\
2293 \\
\\
2680 \\
2660 \\
2805 \\
2876 \\
2607 \\
2579 \\
2662 \\
2619 \\
2560 \\
2749 \\
2535 \\
2645 \\
2526 \\
2619 \\
5\end{array}$ & $\begin{array}{r}3 \\
8 \\
2 \\
1 \\
1 \\
2 \\
3 \\
38 \\
129 \\
4 \\
21 \\
2 \\
10 \\
12 \\
63 \\
162 \\
67 \\
-0.9\end{array}$ \\
\hline county overage & 2.9 & 18.0 & 70.8 & 11.2 & 4.2 & 78.3 & 1.4 & 2.4 & 1.8 & 13764 & 2586 & 528 \\
\hline$-2-0$ & & & & & & 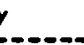 & & & & & & \\
\hline $\begin{array}{l}\text { brookville } \\
\text { idddle klitonning } \\
\text { uncorreloted } \\
\text { upper freeport } \\
\text { upper kittanning } \\
\text { - }\end{array}$ & $\begin{array}{l}2.1 \\
3.3 \\
3.7 \\
3.2 \\
2.8\end{array}$ & $\begin{array}{l}21.3 \\
20.8 \\
17.5 \\
19.8 \\
20.1 \\
0 . . .\end{array}$ & $\begin{array}{l}62.8 \\
65.7 \\
67.9 \\
70.2 \\
61.6\end{array}$ & $\begin{array}{l}15.9 \\
13.7 \\
14.6 \\
10.0 \\
18.4 \\
0 . . .0\end{array}$ & $\begin{array}{l}0.0 \\
0.0 \\
0.0 \\
0.0 \\
0.0 \\
0.0\end{array}$ & $\begin{array}{l}0.0 \\
0.0 \\
0.0 \\
0.0 \\
0.0\end{array}$ & $\begin{array}{l}0.0 \\
0.0 \\
0.0 \\
0.0 \\
0.0\end{array}$ & $\begin{array}{l}0.0 \\
0.0 \\
0.0 \\
0.0 \\
0.0\end{array}$ & $\begin{array}{l}3.4 \\
1.8 \\
1.0 \\
1.6 \\
3.5\end{array}$ & $\begin{array}{l}12930 \\
13250 \\
13150 \\
16010 \\
12503\end{array}$ & $\begin{array}{l}2865 \\
2910 \\
2730 \\
2250\end{array}$ & $\begin{array}{l}1 \\
5 \\
3 \\
1 \\
3\end{array}$ \\
\hline county overage & 3.2 & 19.8 & 65.3 & 16.9 & 0.0 & 0.0 & 0.0 & 0.0 & 2.1 & 13088 & 2697 & 13 \\
\hline & & & 90 & & & & & & & & & \\
\hline $\begin{array}{l}\text { brookville } \\
\text { lower kittenning }\end{array}$ & $\begin{array}{l}4.4 \\
3.0 \\
3.0\end{array}$ & $\begin{array}{l}38.9 \\
38.3\end{array}$ & $\begin{array}{l}49.4 \\
60.7 \\
0.9\end{array}$ & $\begin{array}{l}11.7 \\
15.0 \\
15.0\end{array}$ & $\begin{array}{l}0.0 \\
0.0\end{array}$ & $\begin{array}{l}0.0 \\
0.0\end{array}$ & $\begin{array}{l}0.0 \\
0.0\end{array}$ & $\begin{array}{l}0.0 \\
0.0\end{array}$ & $\begin{array}{l}5.4 \\
5.6\end{array}$ & $\begin{array}{l}13085 \\
12650\end{array}$ & $\begin{array}{l}2100 \\
2050\end{array}$ & 8 \\
\hline county overoge & 6.2 & 38.8 & 49.1 & 12.1 & 0.0 & $n, n$ & 0.0 & 0.0 & 5.5 & 13036 & 2094 & 9 \\
\hline
\end{tabular}




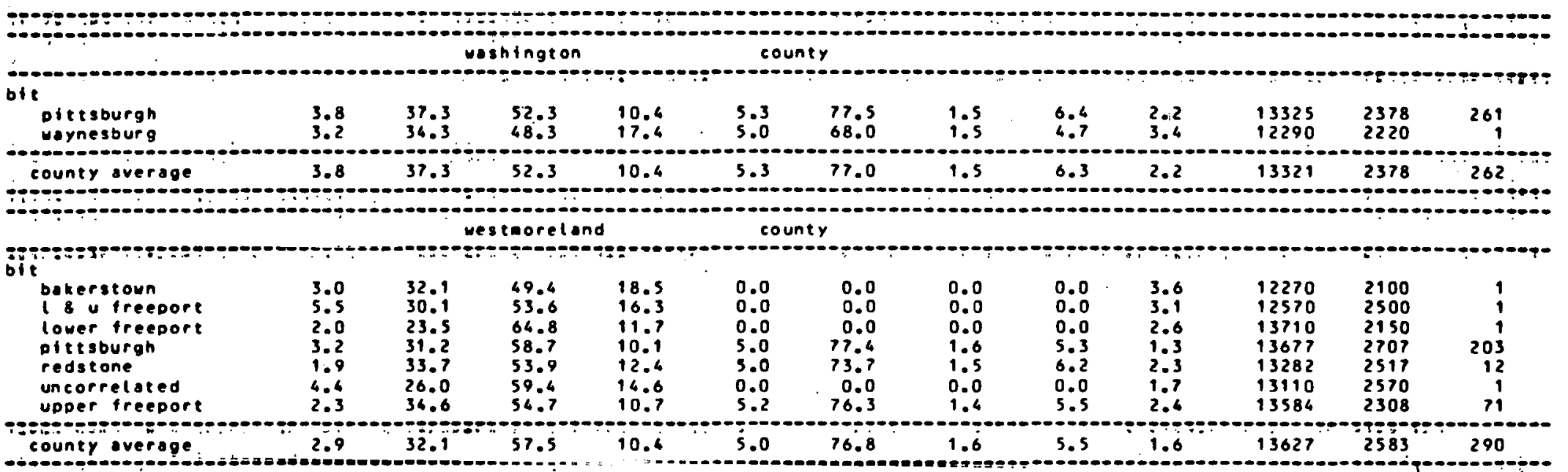




\section{Pennsylvania}

stote county

Dennserluanie. alleghens ued under treedoce moistur

3.4 volest

Pinede
sn. 1
18110

0.5

continued...... 


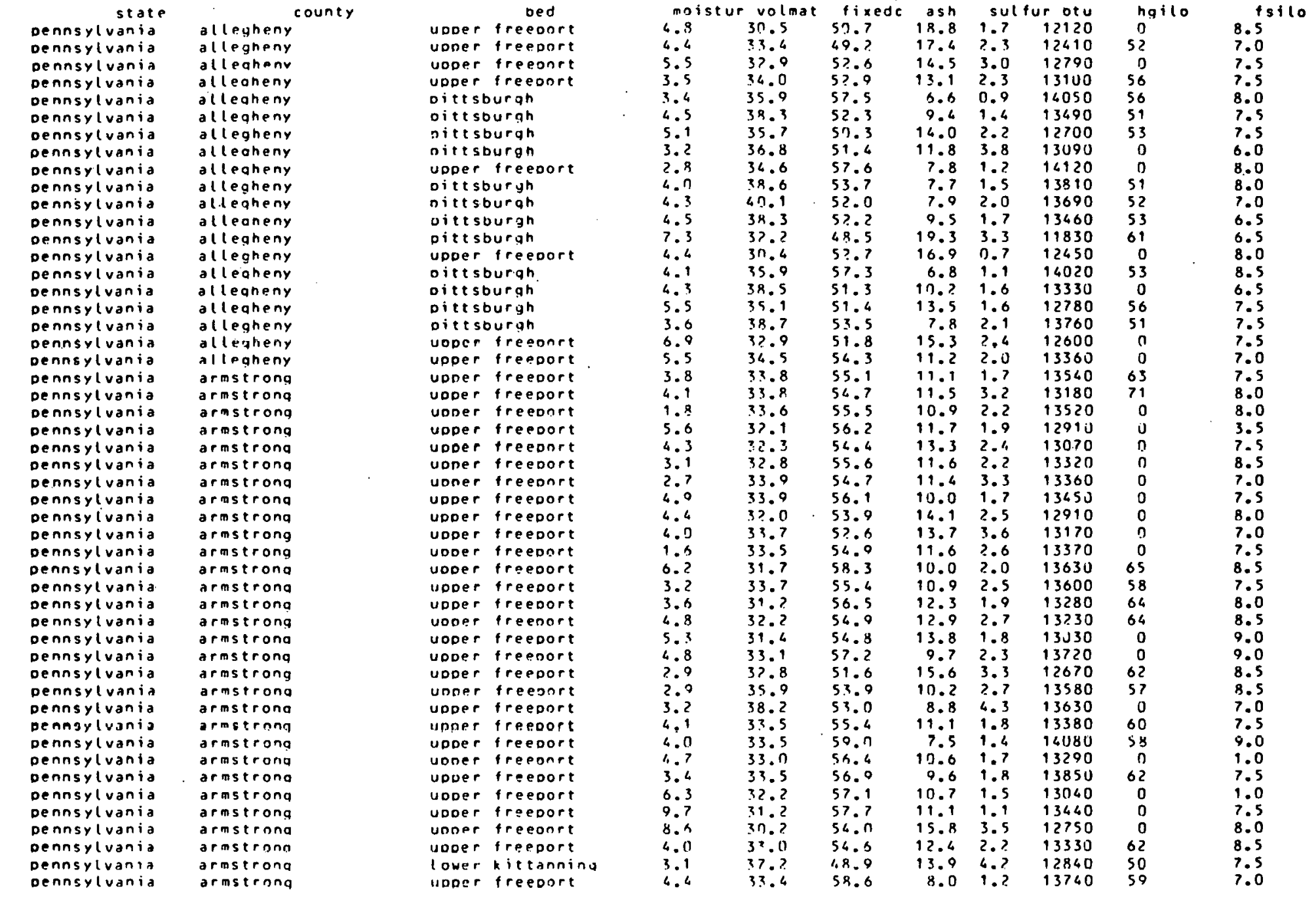




\begin{tabular}{|c|c|c|c|c|c|c|c|c|c|c|c|}
\hline state & county & & bed & moi & $r$ volmat & $f i \times e$ & ash & su & ur btu & hgilo & is 110 \\
\hline Dennsyivania & armstrona & upper & freeport & 0.1 & $>0.3$ & 51.6 & 19.1 & 1.9 & 12210 & 0 & 8.0 \\
\hline dennsylvania & arastrona & upper & freedort & 3.9 & $? 0.8$ & $5 ? .8$ & 17.4 & 1.7 & 12450 & 58 & 7.5 \\
\hline Dennsylvania & armstrona & unoer & treenore & 4.4 & 32.5 & 52.7 & 14.8 & 2.3 & 12900 & 0 & 7.5 \\
\hline pennsyluania & armsirona & uDDer & freeport & 3.0 & 31.8 & 52.0 & 16.2 & 1.6 & 12660 & 0 & 8.0 \\
\hline Dennsyivania & armstrong & upoer & ireeoort & 4.0 & 33.1 & 51.8 & 15.1 & 3.7 & 12760 & 0 & 8.5 \\
\hline Dennisyl vania & armstrong & upper & freeoort & 8.5 & 33.2 & 54.1 & 12.7 & 1.0 & 12760 & 63 & 2.0 \\
\hline pennsyivania & armstrona & under & freenart & 3.? & $3 ? .5$ & 56.6 & 12.9 & 3.0 & 13040 & 57 & 8.0 \\
\hline pennsylvania & armstrona & uoder & freeosrt & 7.3 & 31.1 & 54.7 & 14.2 & 3.7 & 12850 & 0 & 7.0 \\
\hline dennsylvania & armstrong & uDDer & freeoort & 4.8 & 32.0 & 52.6 & 15.4 & 4.6 & 12030 & 0 & 7.0 \\
\hline Dennsylivania & armstrong & upDer & freedort & 4.2 & 29.7 & $5 n .1$ & 20.2 & 2.9 & 11770 & n & 7.0 \\
\hline oennsylvania & armstrono & Iower & treconrt & 2.? & 33.9 & 53.2 & 12.9 & 3.5 & 13150 & 55 & 8.0 \\
\hline Dennsyivania & armstrona & lower & frecoort & 3.2 & 36.2 & 56.9 & 6.9 & 2.3 & 14000 & 59 & 7.5 \\
\hline pennsyivania & armstrong & lower & freedort & 1.6 & 35.5 & 56.8 & 9.7 & 2.8 & 13850 & 62 & 8.0 \\
\hline Denirsyluania & ormstrong & uDDer & freeoort & 3.1 & 35.3 & 51.5 & 13.2 & 3.9 & 13110 & 57 & 9.0 \\
\hline Dennsylvania & armstrona & lover & kittanning & 2.1 & 34.5 & 51.0 & 14.5 & 2.8 & 12900 & 0 & 7.5 \\
\hline Dennsylvania & armstrono & lower & kittanninu & 1.8 & 37.4 & $5 ? .7$ & 9.9 & 4.5 & 13670 & 0 & 8.0 \\
\hline pennsylvania & $\begin{array}{l}\text { armstrong } \\
\text { a }\end{array}$ & udoer & freedort & 3.9 & 34.6 & 55.5 & 0.9 & 1.3 & 13670 & 55 & 8.5 \\
\hline Dennsylvania & armstrong & uoper & freport & 5.0 & 33.2 & 56.5 & 10.3 & 2.1 & 13380 & 0 & 6.5 \\
\hline Dennsyivania & armstrona & unoer & freeonrt & 5.0 & 34.1 & 59.9 & 6.0 & 1.4 & 14210 & 55 & 8.0 \\
\hline pennsylvania & armstrona & lower & treeport & 6.3 & 35.6 & 58.2 & 6.2 & 1.6 & 14100 & 56 & 0.5 \\
\hline pennsyluania & armstrona & variou & & 0.6 & 34.8 & 54.4 & 10.8 & 1.5 & 13110 & 0 & 1.5 \\
\hline Dennsyluania & armstrona & variou & & 2.9 & 35.5 & 56.3 & 8.? & 1.2 & 13750 & 0 & 700 \\
\hline Dennsyluania & armserona & unoer & freeoort & 3.7 & 34.9 & 56.4 & 8.7 & 1.7 & 13790 & 54 & 7.5 \\
\hline Dennsylvania & armstrona & lower & freeport & 3.5 & 35.8 & 57.0 & 7.2 & 1.6 & 14040 & 56 & 7.5 \\
\hline Dennsyluanid & drmsirong & middle & kittanning & 5.6 & 33.7 & 54.0 & 12.3 & 1.0 & 13180 & 56 & $? .0$ \\
\hline Dennsyluania & armstrona & upder & freeoort & 4.6 & 32.0 & 55.4 & $1 \geq .6$ & $? .4$ & 13270 & 67 & 8.5 \\
\hline Dennsylvania & armstrony & lower & kittanning & 8.4 & 36.9 & $3 ? .0$ & 11.1 & 3.9 & 13160 & 65 & 6.5 \\
\hline Dennsylvania. & armstrona & variou & & 3.7 & 32.6 & 55.6 & 11.8 & 2.0 & 13240 & 0 & 6.0 \\
\hline Dennsylvania & armstrong & variou & & 4.0 & 32.1 & 55.8 & 12.1 & 2.1 & 13140 & 0 & 8.0 \\
\hline Dennsyluania & armstrong & udoer & treeport & 4.? & 33.2 & 53.6 & 13.4 & 2.6 & 13140 & 59 & 7.5 \\
\hline Dennsyluania & armstrong & yooer & kittannina & 4.9 & 33.5 & 55.1 & 11.4 & 2.8 & 13460 & 58 & 7.5 \\
\hline Dennsylvania & armstrona & lower & kittanning & 5.0 & 33.1 & 53.9 & 13.0 & 2.4 & 13120 & 60 & 7.5 \\
\hline Dennsylvania & armstrong & ukitt & anningsl fredt & 3.3 & 36.5 & 55.9 & 9.6 & 2.1 & 13800 & 55 & 7.5 \\
\hline Dennsyluania & arinstrong & udoer & ireeoort & 2.7 & 36.1 & 59.0 & 14.9 & 3.8 & 12820 & 58 & 9.0 \\
\hline Dennsylvania & armstrony & wooer & Freeoort & 3.0 & 34.9 & 54.4 & 10.7 & 2.1 & 13480 & 0 & 8.5 \\
\hline Dennsylvania & armstrona & uDDer & frepoort & 4.5 & 33.4 & 56.6 & $11) .0$ & 1.9 & 135.60 & 64 & 6.5 \\
\hline Dennsylvania & armstrona & upper & freeoort & $? .2$ & 34.9 & 56.8 & 8.3 & 2.1 & 13930 & 63 & 8.0 \\
\hline Dennsyluania & armstrong & upoer & treeoort & 2.1 & 34.0 & 57.3 & 8.7 & 1.9 & 13870 & 0 & 8.0 \\
\hline pennsylvania & armstrong & under & freeont & 2.3 & 34.3 & 56.3 & 9.4 & 1.5 & 13790 & 0 & 8.0 \\
\hline Dennsylvania & armstrona & upoer & freesort & 1.8 & 35.4 & 51.6 & 13.2 & 4.7 & 13160 & 64 & 7.5 \\
\hline oennsylvaniag & armstrona & lower & kittanning & 2.3 & 36.3 & 51.6 & 12.1 & 5.4 & 13260 & 6? & 7.5 \\
\hline pennsyluania & armstrong & upper & freedort & 3.8 & 31.1 & 37.8 & 11.1 & 1.5 & 13500 & 69 & 7.0 \\
\hline Dennsylvania & armstrong & uoder & freeonrt & 5.8 & 34.4 & 57.6 & 8.0 & 1.9 & 13780 & 0 & 7.0 \\
\hline Dennsylvania & armstrons & lover. & freeoort & 4.3 & 34.7 & 56.0 & 9.3 & 2.5 & 13620 & 0 & 6.5 \\
\hline Dennsyluania & armstrong & lower & freeonet & 4.2 & 35.2 & 55.9 & 9.9 & 2.8 & 13690 & 0 & 6.5 \\
\hline oennsylvania & armstrong & lomer & freeoort & 4.8 & 35.5 & 54.2 & 10.3 & 3.8 & 13560 & 52 & 6.5 \\
\hline Dennsyluania & armstrong & inwer & freeonrt & 4.7 & 35.0 & 57.6 & 7.4 & 2.5 & 13870 & 0 & 1.5 \\
\hline Dennsyluania & armetrona & lower & freedort & 5.5 & 36.2 & 57.0 & 6.8 & 2.9 & 14020 & 57 & 7.5 \\
\hline Dennsyluania & armstrony & lower & freennet & 4.6 & 36.2 & 55.5 & 8.3 & 3.4 & 13680 & 0 & 6.5 \\
\hline pennsylvania & armstrong & uoper & frecoort & 3.9 & 34.0 & 55.7 & 11.0 & 2.0 & 13370 & 61 & 7.5 \\
\hline
\end{tabular}




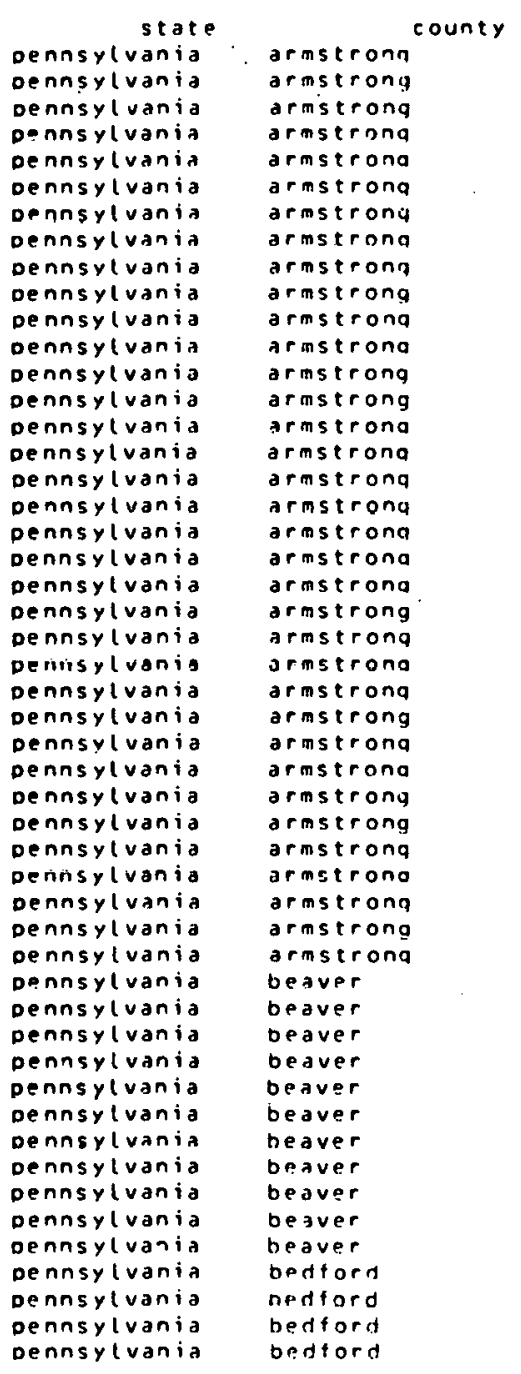

beer freenort

upper freedort

udoer freenart

voper freejort

upder freeport

uoper treedort

unoer treeonrt

lower freevort

uoder freedort

uoder treeport

woper freedort

upder freedort

mahoning

lower kittannina

uoper treeport

udoer freeoort

unoer treeonet

unoer treeoort

uoper freedort

uoder freedort

woner treeonr

upder treeport

middle kittanning

under treeport

udoer treeport

upper freeport

upper treeport

lower kittanning

unner freeonet

nidule kittanning

lower kittannina

miodle kittanning

lower kittannina

middle kittanning

udoer lievurt

udoer freedort

lower kittannina

iddle kittanning

ower kittanning

nuer kittannin.

ower kittannina

lower kittanninu
uover freeoort

\begin{tabular}{|c|c|}
\hline & \\
\hline$?$ & 33.6 \\
\hline $4 . \bar{n}$ & 37.5 \\
\hline 3.9 & 31.0 \\
\hline 7.2 & 30.3 \\
\hline 4.8 & 34.4 \\
\hline 3.6 & 35.1 \\
\hline 4.8 & 33.8 \\
\hline 3.0 & 34.9 \\
\hline 6.9 & $3 ? .3$ \\
\hline 3.0 & $3 ? .8$ \\
\hline 2.7 & 31.3 \\
\hline 2.5 & 31.9 \\
\hline 3.0 & 33.8 \\
\hline 2.4 & 36.8 \\
\hline 1.6 & 28.8 \\
\hline 3.1 & 35.0 \\
\hline 3.1 & 33.4 \\
\hline$? .5$ & 33.6 \\
\hline 2.? & 33.3 \\
\hline 2.3 & 33.7 \\
\hline 2.4 & 34.0 \\
\hline 2.7 & 33.8 \\
\hline 5.8 & 32.1 \\
\hline$? .7$ & 36.0 \\
\hline 4.6 & $34 .$. \\
\hline $3 . n$ & 35.9 \\
\hline 3.1 & 32.7 \\
\hline 3.9 & 34.8 \\
\hline 6.7 & 32.4 \\
\hline 4.0 & $3 ? .2$ \\
\hline 3.6 & 34.8 \\
\hline 3.1 & $3 ? .5$ \\
\hline 4.5 & 31.7 \\
\hline 6.0 & 37.6 \\
\hline 4.2 & 32.9 \\
\hline 5.8 & 33.8 \\
\hline 4.0 & 37.3 \\
\hline 5.? & 14.2 \\
\hline 4.3 & 36.4 \\
\hline 0.3 & 36.3 \\
\hline 4.4 & 34.0 \\
\hline 4.1 & 40.9 \\
\hline 0.2 & 35.6 \\
\hline 6.6 & 34.9 \\
\hline 4.3 & 36.4 \\
\hline 4.8 & 36.5 \\
\hline 1.4 & 15.9 \\
\hline 1.5 & 14.7 \\
\hline$? .9$ & 15.8 \\
\hline & \\
\hline
\end{tabular}

fixedc

$\begin{array}{rl}\text { Ash sulfur btu } \\ 12.9 & 2.0\end{array}$

hgilo

$\begin{array}{llll}53.5 & 12.9 & 2.0 & 13080 \\ 49.5 & ? 1.0 & 1.5 & 11770\end{array}$

$\begin{array}{llll}47.9 & 21.9 & 2.5 & 11720\end{array}$

$\begin{array}{rrrr}48.8 & 20.9 & 3.0 & 11800 \\ 56.8 & 8.8 & 1.6 & 13590\end{array}$

$\begin{array}{llll}55.5 & 9.4 & 2.1 & 13570\end{array}$

$\begin{array}{llll}54.8 & 11.4 & 2.7 & 132.80\end{array}$

$\begin{array}{rrrr}54.7 & 9.9 & 2.8 & 13780 \\ 54.5 & 12.2 & 1.9 & 13360\end{array}$

$\begin{array}{llll}5 ? .2 & 15.0 & 2.4 & 12800\end{array}$

$\begin{array}{llll}47.3 & 19.4 & 2.3 & 12150\end{array}$

$\begin{array}{llll}55.3 & 10.9 & 1.7 & 13570\end{array}$

$48.7 \quad 15.2 \quad 4.4 \quad 12780$

$4 ? .9 \quad 22.1 \quad 5.1 \quad 11540$

$\begin{array}{llll}55.1 & 11.5 & 2.4 & 13500\end{array}$

$\begin{array}{llll}55.1 & 19.3 & 1.8 & 13210\end{array}$

$\begin{array}{llll}55.3 & 11.0 & 2.7 & 13560\end{array}$

$\begin{array}{llll}54.9 & 11.1 & 2.5 & 13560\end{array}$

$\begin{array}{llll}55 . ? & 11.0 & 2.1 & 13580 \\ 54.0 & 13.9 & 1.7 & 12880\end{array}$

$\begin{array}{llll}48.8 & 15.2 & 4.3 & 12650\end{array}$

$\begin{array}{llll}50.5 & 9.2 & 1.5 & 13550\end{array}$

$\begin{array}{llll}43.3 & 15.8 & 0.6 & 12500\end{array}$

$\begin{array}{llll}55.5 & 11.8 & 1.8 & 13460\end{array}$

$\begin{array}{llll}55.9 & 9.3 & 2.1 & 13550\end{array}$

$\begin{array}{llll}50.8 & 17.0 & 2.6 & 12210\end{array}$

$\begin{array}{llll}53.5 & 11.7 & 3.4 & 13280\end{array}$

$\begin{array}{llll}57.0 & 13.5 & 2.1 & 13610\end{array}$

$\begin{array}{llll}59.3 & 9.0 & 1.5 & 13870 \\ 55.6 & 7.0 & 1.6 & 13860\end{array}$

$57.9 \quad 9.2 \quad 2.2 \quad 13880$

$\begin{array}{rrrr}40.5 & 9.7 & 0.9 & 13430\end{array}$

$\begin{array}{llll}55.2 & 10.6 & 0.8 & 13230\end{array}$

$\begin{array}{llll}51.5 & 12.1 & 4.3 & 13020 \\ 53.3 & 13.4 & 1.2 & 12950\end{array}$

$\begin{array}{llll}53.3 & 10.4 & 1.2 & 12950 \\ 50.0 & 12.0 & 3.8 & 13040\end{array}$

$\begin{array}{cccc}57.0 & 12.0 & 2.8 & 13040 \\ 57.7 & 6.4 & 2.1 & 13960\end{array}$

$53.1 \quad 11.3 \quad 2.8 \quad 1332 n$

$53.2 \quad 11.92 .513230$

$\begin{array}{llll}40.8 & 14.4 & .5 & 12000\end{array}$

$67.5 \quad 16.6 \quad 0.8 \quad 12970$

$7 ? .6 \quad 12.72 .1 \quad 13540$

$\begin{array}{llll}79.6 & 12.7 & 2.1 & 13540 \\ 71.9 & 12.4 & 1.8 & 13620 \\ 69.2 & 13.9 & 1.6 & 13480\end{array}$

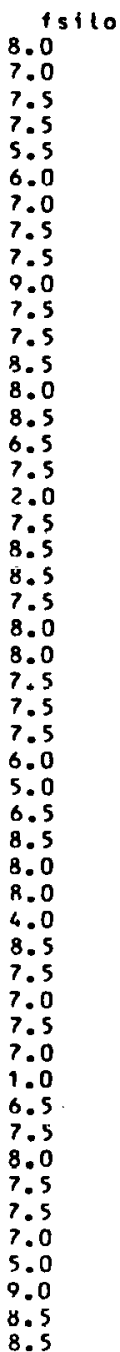




\begin{tabular}{|c|c|c|}
\hline $\begin{array}{r}\text { State } \\
\text { dennsylvanin }\end{array}$ & bedford county & $\begin{array}{l}\text { bed } \\
\text { lower kittanning }\end{array}$ \\
\hline dennsylvania & blair & lower kittanning \\
\hline dennsyivania & blair & lower kittanning \\
\hline Dennsyluanis & blair & various \\
\hline Dennsyluania & blair & middle kittannina \\
\hline Dennsyluanid & blair & midale kittanning \\
\hline pennsylvania & blair & lower kittanning \\
\hline dennsyluania & blair & lover kittanning \\
\hline dennsylivania & Dlair & brookville \\
\hline Dennsylvania & blair & orookville \\
\hline Dennsyivania & butler & niddle kittannina \\
\hline Dennsylvania & butler & broukville \\
\hline Dennsylivania & butler & upder freeoort \\
\hline dénnsplvonis & husler & upder kittanning \\
\hline dennsyluania & butter & middle kittannina \\
\hline Dennsylvanid & butler & niddie kittanntuy \\
\hline dennsylvania & butler & middle kittanning \\
\hline Dennsylvania & butler & middle kittanning \\
\hline Dennsylvania & butler & uoder freenort \\
\hline Dennsylvania & butter & uDper freedort \\
\hline Dennsyluania & butler & Uoder frepoort \\
\hline $\begin{array}{l}\text { Dennsylvania } \\
\text { oennsylvania }\end{array}$ & butler & $\begin{array}{l}\text { middle kittannino } \\
\text { bronkville }\end{array}$ \\
\hline $\begin{array}{l}\text { Dennsylvania } \\
\text { oennsylvania }\end{array}$ & $\begin{array}{l}\text { butler } \\
\text { butler }\end{array}$ & $\begin{array}{l}\text { bronkville } \\
\text { ukitganningsorookve }\end{array}$ \\
\hline dennsylvania & butler & ukit tanningsbrookul \\
\hline dennsyluania & butler & upper treeost \\
\hline Dennsylvania & hucler & I is ufreeoort \\
\hline Dennsyluania & butler & I o kittonning \\
\hline dennsyluania & butter & upder freenort \\
\hline oennsylvania & butler & upper freedort \\
\hline Dennsylvania & butler & uoder freeoort \\
\hline Dennsylvania & butler & uoder treedort \\
\hline dennsyluania & butler & lower treedort \\
\hline Dennsyluania & outler & I kittanningsu fredt \\
\hline dennsylvania & butler & uooer freenort \\
\hline Urnasylyania & butler & udoer fresoort \\
\hline Dennsyluania & butler & brookville \\
\hline dennsylvania & hutler & clarion \\
\hline Dennsylvania & butler & clarion \\
\hline dennsylvania & butter & uover freeoort \\
\hline Dennsylvania & butler & upder freedort \\
\hline oennsyluania & outler & midule kittanning \\
\hline Dennsyluania & butler & middle kittanning \\
\hline dennsylvania & butler & orookville \\
\hline dennsylvania & butler & middle kitetanning \\
\hline oennsylvania & Dutlor & lower freedort \\
\hline denisyluania & butler & Inwer treeroot \\
\hline pennsylvania & outlor & niddle kittanning \\
\hline dennsylvania & notler & lower kittannilly \\
\hline oennsylyạnia & cambria & upoer freeport \\
\hline
\end{tabular}

\begin{tabular}{|c|c|c|c|c|c|c|c|}
\hline \multicolumn{2}{|r|}{ ar volmat } & fixe & ash & \multicolumn{2}{|c|}{ sulfur otu } & noilo & \multirow{2}{*}{ fsilo } \\
\hline 1.4 & 17.6 & 67.8 & 14.6 & 1.0 & 13260 & 0 & \\
\hline 2.9 & 23.8 & 67.5 & 8.7 & 0.9 & 14210 & 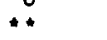 & 9.0 \\
\hline 3.5 & $? 4.8$ & 56.1 & 9.1 & 0.8 & 14140 & 0 & 9.0 \\
\hline 3.4 & 27.2 & 60.0 & 12.8 & 1.4 & 12970 & 0 & 8.5 \\
\hline 5.8 & 28.0 & 65.1 & 6.9 & 1.8 & 14160 & 84 & 8.0 \\
\hline 6.6 & 29.9 & 60.0 & 10.1 & 3.7 & 13860 & 81 & 8.0 \\
\hline 9.5 & 20.4 & 69.3 & 9.3 & 0.6 & 12890 & 90 & 1.0 \\
\hline 4.8 & 28.4 & 64.4 & 7.2 & 0.9 & 14290 & 84 & 8.5 \\
\hline 7.6 & 20.7 & 61.5 & 9.8 & 2.2 & 13750 & 80 & 8.0 \\
\hline 3.6 & 28.9 & 59.0 & 12.9 & 3.6 & 13300 & 87 & 8.0 \\
\hline 3.7 & 35.3 & 55.3 & 8.4 & 0.9 & 13750 & so & 0.5 \\
\hline 4.7 & 38.7 & 50.1 & 11.2 & 4.3 & 13120 & 55 & 5.0 \\
\hline 5.0 & 35.3 & 55.1 & 9.6 & 1.9 & 13500 & so & 7.0 \\
\hline 3.3 & 35.5 & 52.3 & 12.2 & 4.2 & 12940 & 56 & 8.0 \\
\hline 4.4 & 36.9 & 53.3 & 9.8 & 3.4 & 13500 & s5 & 7.0 \\
\hline 5.7 & 37.5 & $5 ? .8$ & 9.7 & 3.3 & 13690 & 55 & 7.0 \\
\hline 4.9 & 38.0 & $90: ?$ & 11.5 & 3.7 & 12970 & 53 & 4.0 \\
\hline 7.5 & 37.3 & 50.6 & 12.3 & 4.2 & 1 suoo & 55 & 6,0 \\
\hline 5.9 & 32.3 & 53.0 & 14.7 & 1.8 & 12780 & 54 & 7.0 \\
\hline 2.8 & 33.9 & 45.4 & 20.7 & 3.7 & 11670 & 0 & 7.5 \\
\hline 4.1 & $31 . ?$ & 46.6 & 22.2 & 3.3 & 11420 & 0 & 0.5 \\
\hline 5.0 & 37,5 & 49.0 & 13.5 & 4.0 & 12820 & so & 5.5 \\
\hline 4.4 & 37.1 & 52.7 & 10.2 & 2.3 & 13630 & 0 & 6.5 \\
\hline 3.4 & 38.7 & 53.9 & 7.4 & 1.4 & 13850 & 0 & 6.5 \\
\hline 5.1 & 36.4 & 55.0 & 9.6 & 1.9 & 13610 & 52 & 5.5 \\
\hline 4.1 & 39.4 & 49.6 & 19.0 & 1.3 & 12030 & 0 & 8.0 \\
\hline 8.4 & 30.4 & 51.5 & 12.1 & 9.6 & 12900 & 64 & 7.0 \\
\hline 8.4 & 34.4 & 52.8 & 12.8 & 1.1 & 12600 & 61 & 1.0 \\
\hline 2.5 & 35.8 & $55 . ?$ & $\begin{array}{r}2.0 \\
9.0\end{array}$ & 1.4 & 13800 & 59 & 7.5 \\
\hline 3.9 & 34.6 & 55.3 & 10.1 & 1.4 & 13550 & 56 & 7.5 \\
\hline 2.2 & 34.6 & 54.7 & 10.7 & 1.2 & 13460 & 0 & 6.5 \\
\hline 5.0 & 33.5 & 51.9 & 14.6 & 1.5 & $1 \geq 820$ & 50 & 7.5 \\
\hline 5.0 & 33.8 & 56.2 & 10.0 & 1.6 & 13380 & 0 & 5.0 \\
\hline 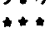 & 35.6 & 51.7 & 12.7 & 1.4 & 12330 & 0 & 3.5 \\
\hline 3.7 & $4 \pi .6$ & 50.2 & 9.2 & 4.5 & 13670 & $6 n$ & 7.0 \\
\hline 3.1 & 39.2 & 48.7 & 12.1 & 4.7 & 13170 & 58 & 7.0 \\
\hline 4.8 & 35.4 & 49.5 & 15.1 & 2.0 & 12490 & so & 5.0 \\
\hline 3.0 & 36.4 & 39.0 & 26.6 & 5.6 & 10690 & So & 4.5 \\
\hline 3.9 & 33.1 & 49.3 & 25.6 & 3.4 & 10680 & 53 & 4.5 \\
\hline 5.3 & 30.7 & 50.9 & 18.4 & 2.0 & $121>0$ & 1) & 7.5 \\
\hline 3.8 & 31.3 & 67.7 & 21.0 & 2.9 & 11800 & 0 & 1.5 \\
\hline 6.1 & 36.9 & $5 ? .7$ & 10.4 & 4.4 & 13300 & 0 & 5.5 \\
\hline 3.7 & 37.9 & $5 \pi .4$ & 11.7 & 4.7 & 13140 & 54 & 6.0 \\
\hline 4.2 & 36.7 & 49.5 & 13.8 & 3.8 & 12880 & 57 & 6.0 \\
\hline 3.5 & 39.2 & $5 n .6$ & 10.4 & 3.7 & 13610 & 48 & 7.5 \\
\hline 4.8 & 34.5 & 53.6 & 11.9 & 1.9 & 13190 & 53 & 7.0 \\
\hline 4.9 & 36.4 & 53.2 & 10.4 & 2.1 & 13320 & 53 & 7.5 \\
\hline 6.9 & 37.5 & 47.3 & 14.7 & 0.2 & 12590 & 52 & 0.5 \\
\hline 6.8 & 34.4 & $55 . ?$ & 10.4 & 0.8 & 13140 & 55 & 1.5 \\
\hline $3 . ?$ & 79.11 & 64.3 & 7.7 & 1.4 & 14270 & $8 ?$ & 8.5 \\
\hline
\end{tabular}




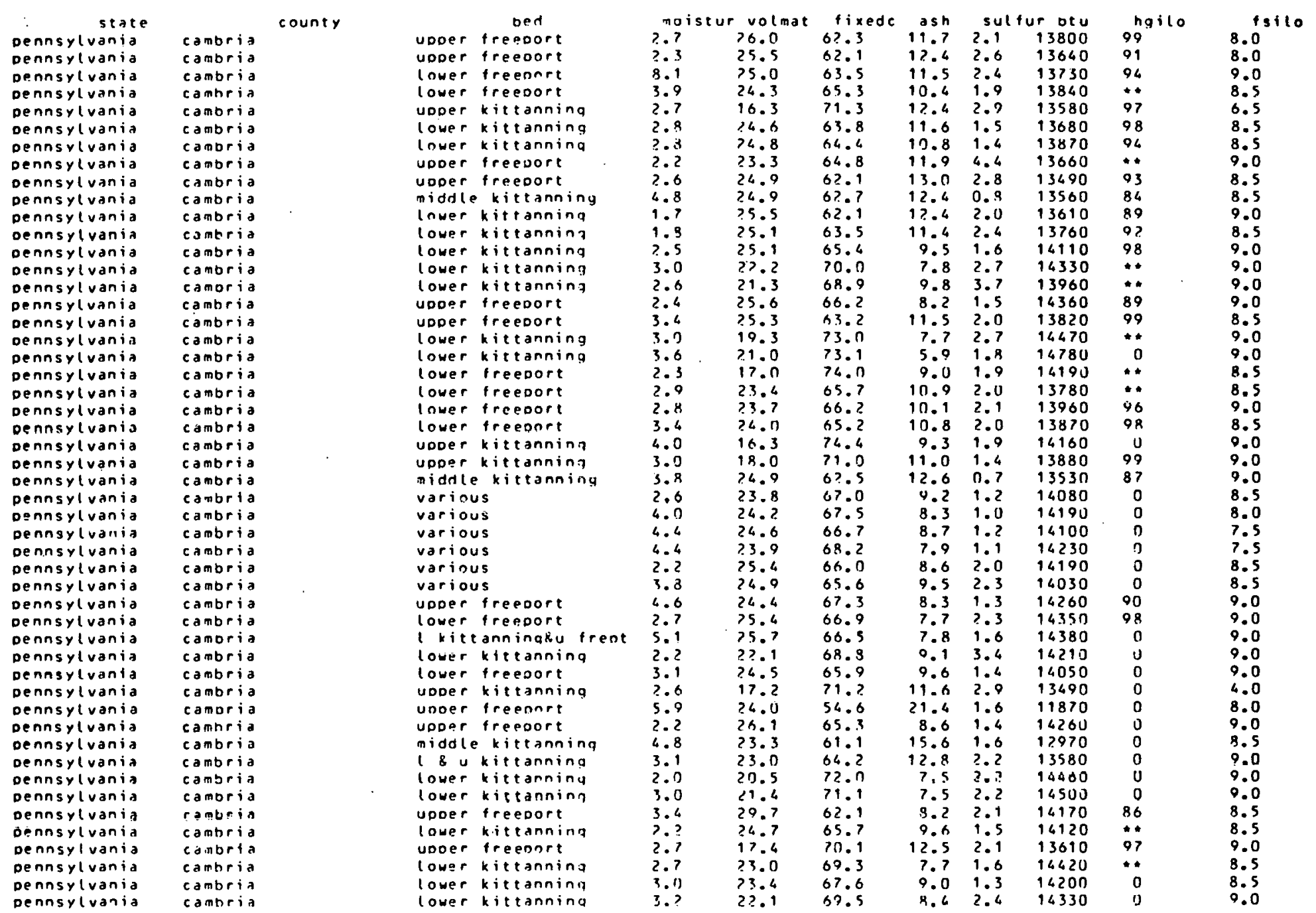




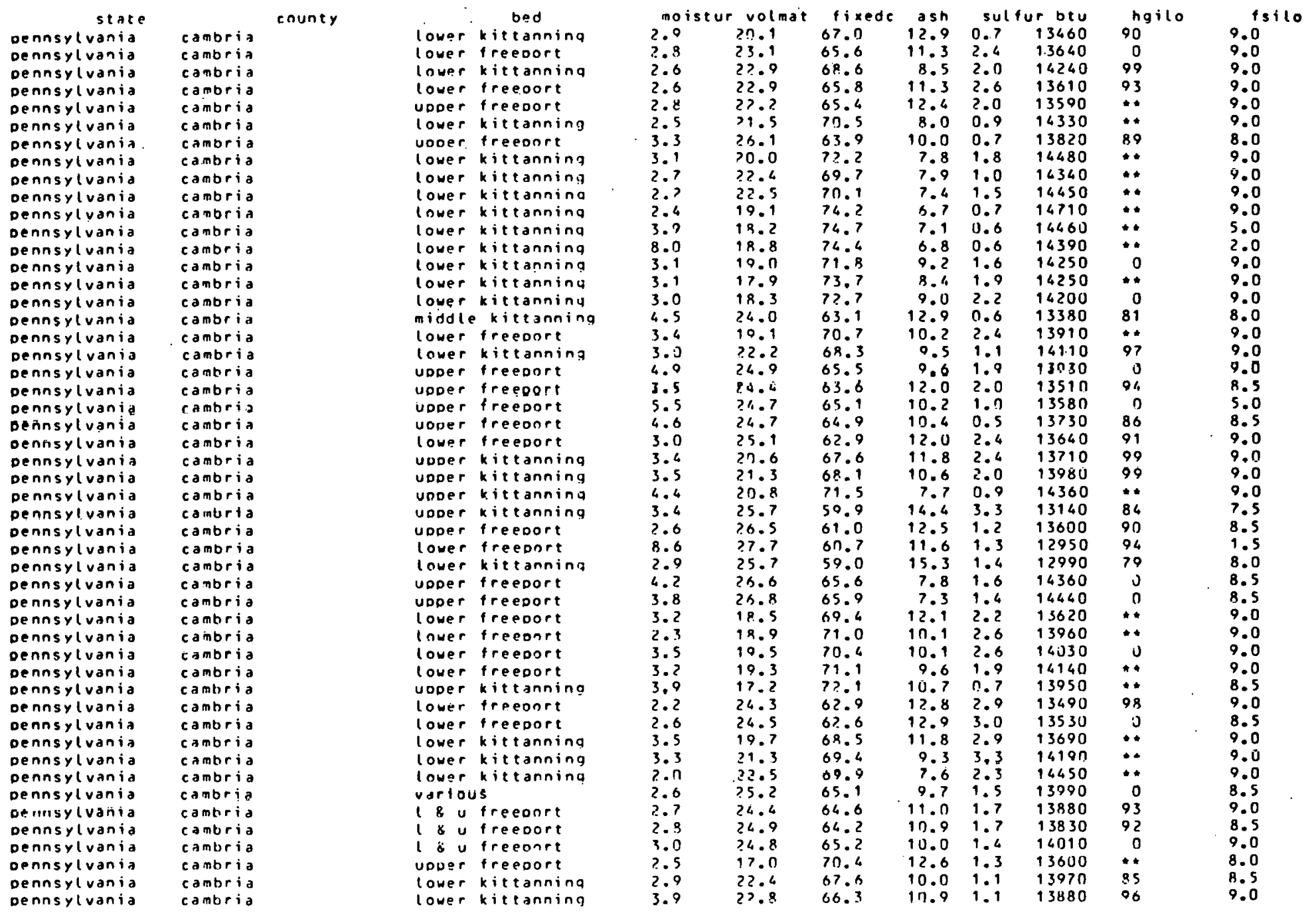




\begin{tabular}{|c|c|}
\hline state & \\
\hline Dennsyluanid & cambria \\
\hline $\begin{array}{l}\text { Dennsylvania } \\
\text { Dennsyluania }\end{array}$ & cambria \\
\hline Dennsyluania & cambria \\
\hline dennsylvania & cambria \\
\hline dennsyluania & cambria \\
\hline Dennsyluaniz & cambria \\
\hline Dennsyluania & cambria \\
\hline Dennsyluania & cambria \\
\hline Dennsyluania & cambria \\
\hline Dennsylvania & cambrid \\
\hline Dennsyluania & cambria \\
\hline dennsyluania & cambria \\
\hline pennsyluania & cambria \\
\hline Dennsyluania & camoria \\
\hline Dennsyluania & cambria \\
\hline Dennsyluania & cambria \\
\hline dennsyluania & cambria \\
\hline Dennsyluania & camoria \\
\hline pennşy lyaniag & sambria \\
\hline Dennsyluania & camoria \\
\hline Dennsyluania & cambria \\
\hline Dennsyluania & cambria \\
\hline Dennsyluania & cambria \\
\hline Dennsyluania & cambria \\
\hline Denneyluania & combria \\
\hline Dennsyivania & cambria \\
\hline Dennsyluania & cambria \\
\hline Dennsylvania & cambria \\
\hline dennsyluania & centre \\
\hline dennsyluania & centre \\
\hline Dennsyluania & centre \\
\hline dennsyluania & centre \\
\hline Dennsyluania & centre \\
\hline Dennsyluania & centre \\
\hline dennsylvanid & centre \\
\hline dennsyluania & centre \\
\hline Dennsyluania & centre \\
\hline Dennsyluania & centre \\
\hline Dennsyluania & centre \\
\hline dennsyluania & centre \\
\hline Dennsyluania & centre \\
\hline Dennsyluania & centre \\
\hline Dennsylvania & centre \\
\hline Dennsyluanid & rentre \\
\hline Dennsyluania & centre \\
\hline Dennsylvania & centre \\
\hline ennsylvania & centre \\
\hline ennsylvania & centre \\
\hline nnsylvania & centre \\
\hline
\end{tabular}

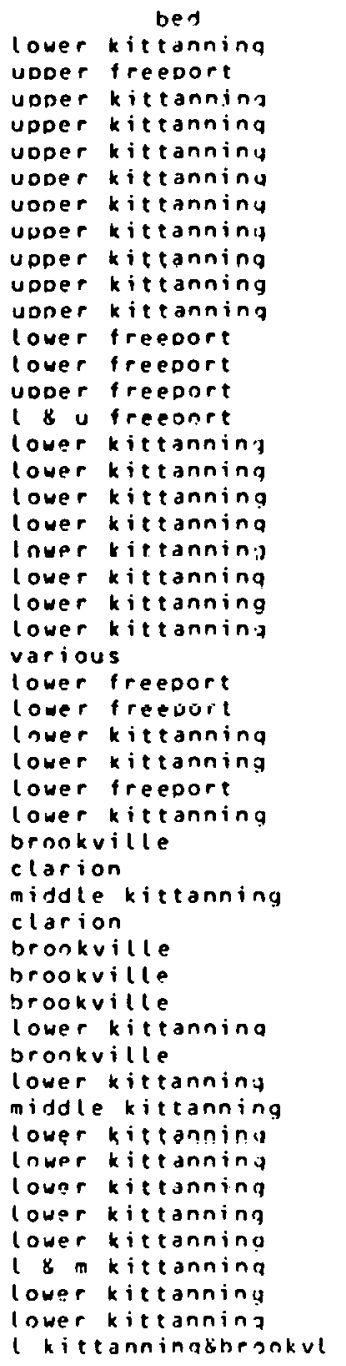

\begin{tabular}{|c|c|}
\hline no & volmat \\
\hline 2.9 & 22.5 \\
\hline 2.5 & 29.0 \\
\hline$? 99$ & 16.3 \\
\hline 1.2 & 16.3 \\
\hline 1.3 & 16.6 \\
\hline 1.8 & $17 . ?$ \\
\hline ?.? & 19.4 \\
\hline 2.6 & 16.4 \\
\hline$? .2$ & 16.5 \\
\hline 3.3 & 17.2 \\
\hline 2.9 & 16.6 \\
\hline$? .5$ & 24.3 \\
\hline 4.3 & 23.7 \\
\hline 3.5 & 74.5 \\
\hline 2.7 & 24.9 \\
\hline$? .4$ & 22.6 \\
\hline 3.6 & 23.7 \\
\hline 4.7 & $? ? .5$ \\
\hline 2.6 & 20.8 \\
\hline$? n$ & ?ח. 5 \\
\hline 2.9 & 21,0 \\
\hline 1.7 & 18.5 \\
\hline 2.1 & 18.1 \\
\hline 4.? & 25.3 \\
\hline 2.5 & 75.5 \\
\hline 2.5 & 24.0 \\
\hline 1.8 & 18.9 \\
\hline 2.3 & 19.0 \\
\hline $3 . n$ & 23.9 \\
\hline$? .6$ & 23.5 \\
\hline 5.3 & 32.4 \\
\hline 3.2 & $? 2.9$ \\
\hline 4.9 & $>1.8$ \\
\hline 2.9 & 72.3 \\
\hline 2.7 & 26.1 \\
\hline 3.0 & 72.7 \\
\hline 2.9 & $? 3.3$ \\
\hline 5.1 & 20.9 \\
\hline 3.5 & $2 ? .0$ \\
\hline 3.3 & 24.0 \\
\hline 3.0 & $? 1.7$ \\
\hline 3.7 & 23.4 \\
\hline 4.9 & 21.2 \\
\hline 2.4 & 2.9 .3 \\
\hline 3.8 & 73.4 \\
\hline 4.6 & $? 3.5$ \\
\hline 3.5 & 22.6 \\
\hline 2.5 & $>1.8$ \\
\hline 5.1 & ??.? \\
\hline 4.4 & 73.5 \\
\hline
\end{tabular}

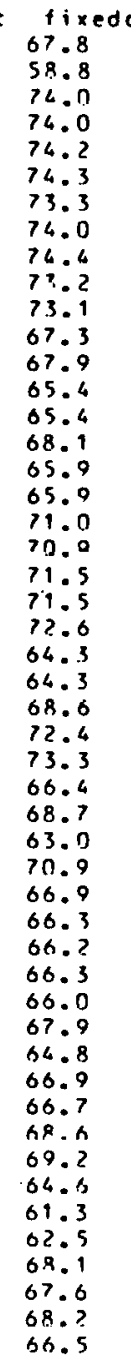

$\begin{array}{rll}\text { ash } & \text { sulfur btu } \\ 9.7 & 1.2 & 14040 \\ 13.2 & 2.0 & 13680 \\ 9.7 & 1.4 & 14060 \\ 9.7 & 0.9 & 14030 \\ 9.2 & 0.9 & 14120 \\ 8.5 & 0.8 & 14280 \\ 10.3 & 1.6 & 13990 \\ 9.6 & 2.1 & 14090 \\ 9.1 & 1.4 & 14220 \\ 9.6 & 1.2 & 14090 \\ 10.3 & 1.3 & 13990 \\ 8.4 & 1.4 & 14300 \\ 8.4 & 1.2 & 14200 \\ 19.1 & 0.8 & 13810 \\ 9.7 & 2.1 & 14010 \\ 9.3 & 1.4 & 14170 \\ 10.4 & 1.8 & 13990 \\ 11.6 & 2.0 & 13850 \\ 8.2 & 2.2 & 14320 \\ 8.6 & 2.3 & 16300 \\ 7.5 & 1.7 & 14670 \\ 10.0 & 2.6 & 14020 \\ 9.3 & 1.8 & 14260 \\ 10.4 & 1.7 & 13910 \\ 10.2 & 2.0 & 13960 \\ 7.4 & 1.7 & 14610 \\ 8.7 & 2.3 & 14250 \\ 8.7 & 2.1 & 14240 \\ 9.7 & 1.4 & 13930 \\ 7.8 & 1.7 & 14320 \\ 14.6 & 4.7 & 12870 \\ 3.2 & 0.8 & 14640 \\ 11.3 & 1.8 & 13710 \\ 11.4 & 1.4 & 13610 \\ 9.7 & 0.9 & 14010 \\ 11.0 & 0.9 & 13810 \\ 10.7 & 1.1 & 13910 \\ 11.2 & 2.9 & 13700 \\ 13.2 & 1.1 & 13610 \\ 9.1 & 1.9 & 14050 \\ 11.6 & 3.9 & 13810 \\ 8.0 & 1.4 & 14350 \\ 9.6 & 1.4 & 14100 \\ 1.9 & 3.9 & 13920 \\ 15.3 & 3.2 & 12970 \\ 14.0 & 3.1 & 13130 \\ 9.3 & 2.9 & 14160 \\ 10.6 & 3.7 & 13900 \\ 9.11 & 2.2 & 13770 \\ 10.0 & 3.0 & 13750 \\ & & \end{array}$

\begin{tabular}{|c|c|}
\hline hgilo & fsilo \\
\hline $\begin{array}{l}96 \\
87\end{array}$ & $\begin{array}{l}9.0 \\
8.0\end{array}$ \\
\hline 0 & 8.0 \\
\hline 0 & 7.5 \\
\hline 0 & 8.5 \\
\hline 0 & 9.0 \\
\hline 0 & 7.5 \\
\hline 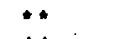 & 8.0 \\
\hline * & 7.0 \\
\hline 0 & 8.0 \\
\hline 96 & 9.0 \\
\hline 92 & 9.0 \\
\hline 96 & 9.0 \\
\hline 9? & 8.0 \\
\hline 91 & 8.5 \\
\hline$\because$ & 9.0 \\
\hline 0 & 9.0 \\
\hline$*$ & 9.0 \\
\hline$\because$ & 9.0 \\
\hline A. & 9.0 \\
\hline$* \star$ & 9.0 \\
\hline$\because *$ & 9.0 \\
\hline ** & 9.0 \\
\hline 0 & 7.5 \\
\hline 94 & 8.5 \\
\hline 96 & 9.0 \\
\hline 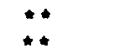 & 9.0 \\
\hline \#* & 9.0 \\
\hline 90 & 8.5 \\
\hline$\because$ & 8.5 \\
\hline * & 6.0 \\
\hline 99 & 7.5 \\
\hline$\ddot{*}$ & 8.0 \\
\hline 98 & 8.0 \\
\hline 0 & 7.5 \\
\hline$*$ & 8.5 \\
\hline 96 & 8.0 \\
\hline * & 7.5 \\
\hline 97 & 7.5 \\
\hline ** & 9.0 \\
\hline ** & 9.0 \\
\hline$\cdots$ & 9.0 \\
\hline ** & 9.0 \\
\hline ** & 9.0 \\
\hline 0 & 9.0 \\
\hline 0 & 9.0 \\
\hline ** & 9.0 \\
\hline & 9.0 \\
\hline & 7.0 \\
\hline & \\
\hline
\end{tabular}




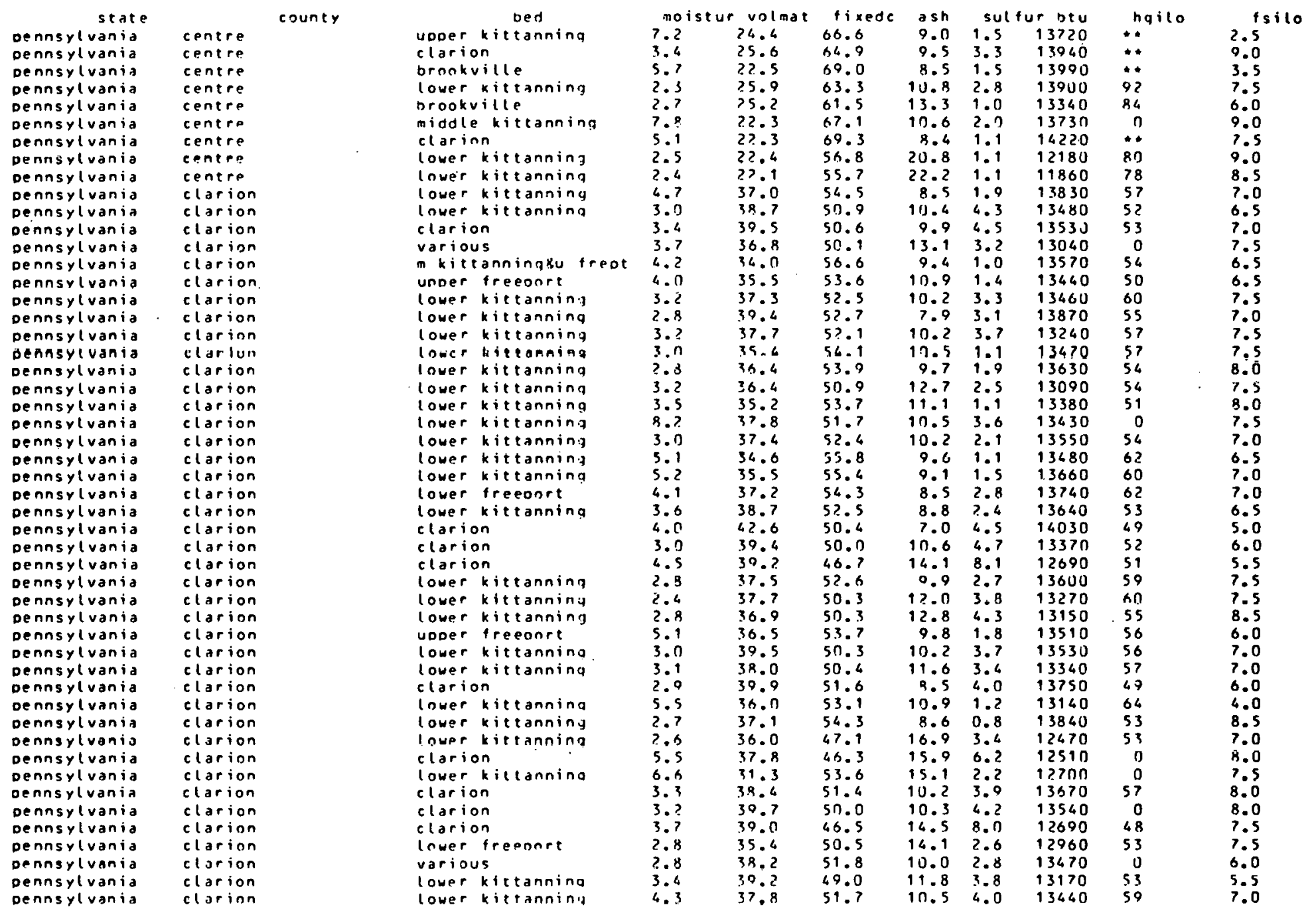




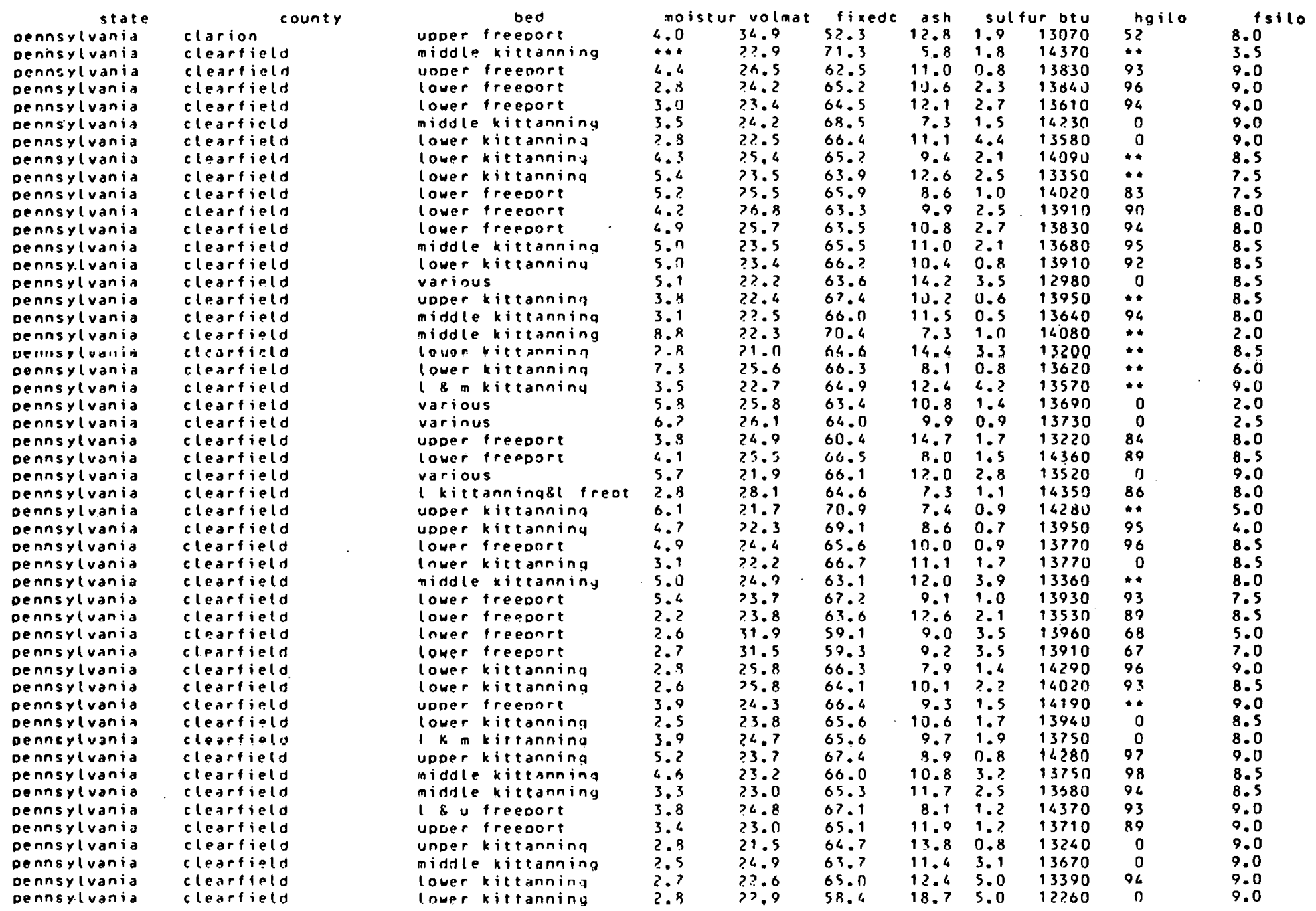




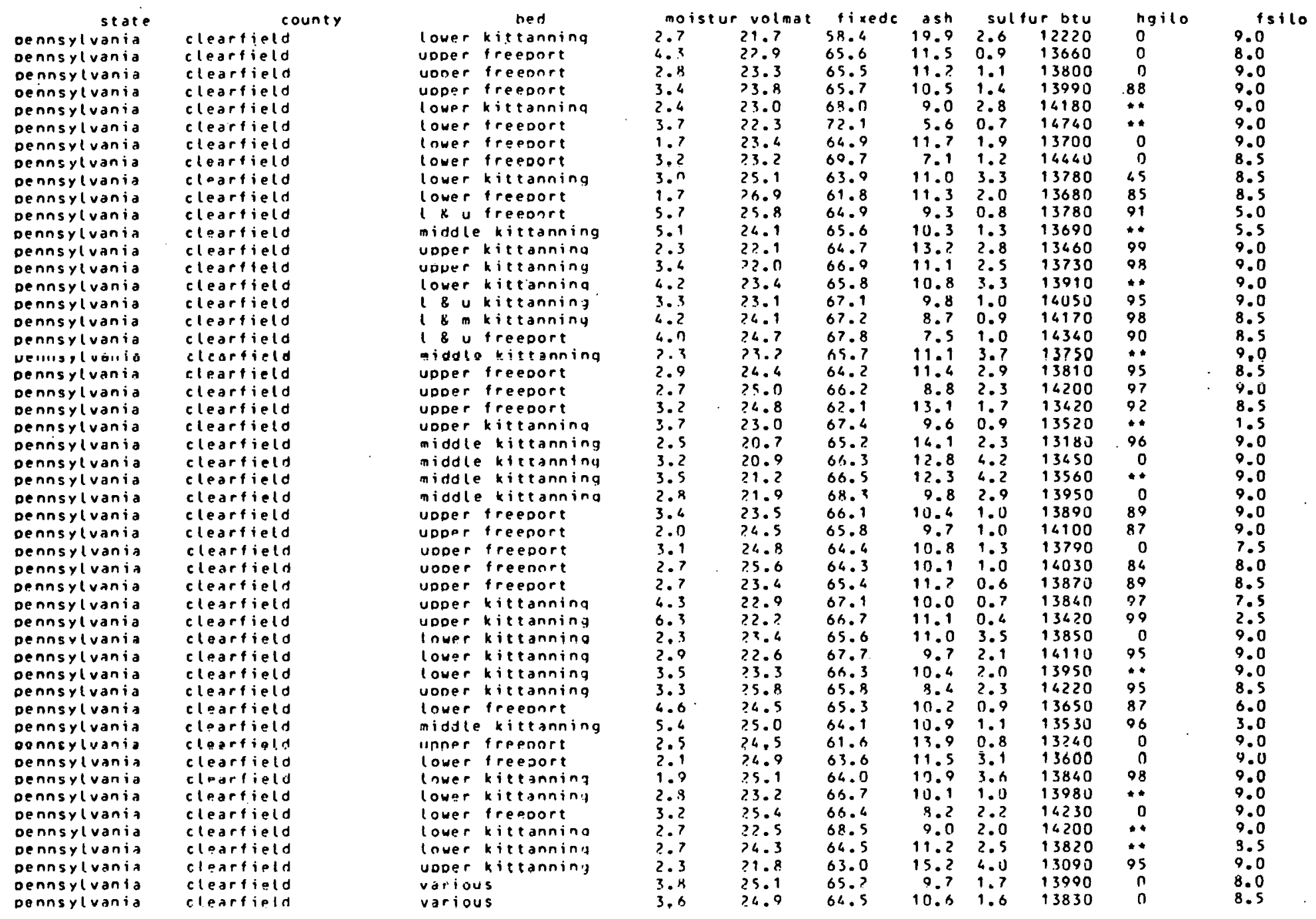




\begin{tabular}{|c|c|}
\hline & \\
\hline Dennsylvania & cleartield \\
\hline ennsyl vania & cleartieto \\
\hline pennsyluania & $\begin{array}{l}\text { clearfield } \\
\text { cleartiela }\end{array}$ \\
\hline Dennsyluania & cleartield \\
\hline pennsyluania & cleartielo \\
\hline Dennsyluania & cleartield \\
\hline Dennsyivania & claparifela \\
\hline Dennsyluania & clearfield \\
\hline Dennsyluania & clearfield \\
\hline Dennsyluania & cleartield \\
\hline Dennsylvania & clearfield \\
\hline pennsyluania & cleartield \\
\hline Dennsyluania & cleartield \\
\hline Dennsyluania & cleartield \\
\hline dennsyluania & cleartield \\
\hline Deninsyluania & cleartield \\
\hline Dennsyluania & clearfield \\
\hline Dennsyivania & clearliela \\
\hline Dennsyivania & cleartield \\
\hline Dennsyluania & clearticlo \\
\hline Dennsyluania & cleartield \\
\hline dennsyivania & cleartiela \\
\hline dennsyluania & cleartielo \\
\hline denngyivania & clearfield \\
\hline Dennsylvania & clearfield \\
\hline Dennsyluania & cleartield \\
\hline Dennsyluania & cleartield \\
\hline Dennsyivania & cleartield \\
\hline Dennsyluania & clearfield \\
\hline pennsylvania & clearfield \\
\hline Dennsylvania & clearfielo \\
\hline denngyivania & clasefielo \\
\hline Dennsylvania & clearfield \\
\hline pennsylvania & cleartield \\
\hline Dennsyivania & cleartield \\
\hline pennsyluania & clearfield \\
\hline Dennsylvania & clearfiela \\
\hline pennsylvania & cleartiela \\
\hline dennsylvania & cleartielo \\
\hline dennsyivania & cleartield \\
\hline Dennsyluania & cleartield \\
\hline dennsylvania & cleartield \\
\hline Dennsylvania & cleartield \\
\hline Dennsyivania & cleartield \\
\hline Dennsyluania & clearfield \\
\hline Dennsyl vania & cleartield \\
\hline Dennsyivania & clearlield \\
\hline inia & clearticla \\
\hline Dennsyluania & clearlield \\
\hline
\end{tabular}

bed
uoper freedor lower freeport lower treeoort lower treecort woper treeport

Uoder reedort lower freeoort lower kittannina various

lower kittanning lower kiteanning lower fre

middle kittannina louer kittannina various

various

midale kittanning uoder freedort

lower treeoort

lower kittanning

voner kittanning

upper kittanning

middle kittanning

lower kittannina

lower treeoort

lower kittonning

lower kittanning

louer kittannina

lower tittanning

lower kittanning

lowar kittanning

lower kittanning

lover kfttanning

l kittanningtorookvl

lower freenot

lower kittanning

$m$ kittanninakbrookv

lover freegnet

lower freesnrt

lower kittanning

lower kitconning

middle kittannina

lower freevort

lower kittannina

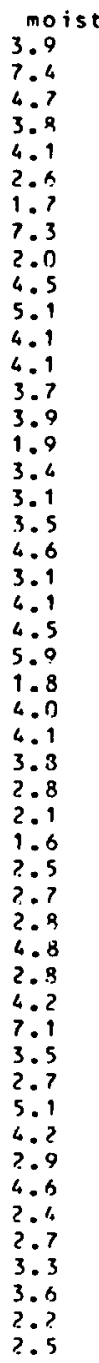

tixedc

$\begin{array}{ll}24.4 & \text { on.4 } \\ 25.3 & 65.1 \\ 25.3 & 64.6 \\ 25.5 & 36.5 \\ 26.3 & 65.7\end{array}$

Ash sulfur bet

$2.0 \quad 13170$

$\begin{array}{lll}0.1 & 1.4 & 13870\end{array}$

$8.01 .5 \quad 14190$

$13.00 .4 \quad 13900$

$13.8 \quad 1.8 \quad 13300$

$\begin{array}{lll}9.9 & 0.6 & 13650\end{array}$

$13.45 .7 \quad 13210$

$\begin{array}{lll}8.4 & 1.1 & 14010\end{array}$

$\begin{array}{lll}7.0 & 0.8 & 14360\end{array}$

$7.7 \quad 2.0 \quad 14220$

$12.6 \quad 1.6 \quad 13370$

$12.61 .6 \quad 13330$

$8.4 \quad 1.214320$

$12.7 \quad 2.8 \quad 13380$

$13.6 \quad 3.6 \quad 13320$

$11.5 \quad 1.9 \quad 13800$

$10.93 .4 \cdot 13790$

$10.7 \quad 3.0 \quad 13850$

$\begin{array}{lll}7.6 & 1.6 & 14330\end{array}$

$2.3 \quad 3.3 \quad 14270$

$13.3 \quad 1.8 \quad 13150$

$\begin{array}{lll}12.6 & 4.3 & 13540\end{array}$

13.8 2.0 13310

$1023.4,13900$

$\begin{array}{lll}9.1 & 3.1 & 13950\end{array}$

$10.0 \quad 4.0 \quad 13850$

$11.2 \quad 3.1 \quad 13750$

$13.7 \quad 3.8 \quad 13180$

$10.0 \quad 3.1 \quad 13840$

$12.7 \quad 3.1 \quad 13310$

$\begin{array}{lll}8.6 & 1.4 & 14280\end{array}$

$10.3 \quad 1.3 \quad 13860$

$11.6 \quad 2.2 \quad 13610$

$10.0 \quad 2.0 \quad 14070$

$13.02 .5 \quad 13360$

$10.23 .4 \quad 14000$

$15.2 \quad 2.7 \quad 13100$

$9.9 \quad 1.3 \quad 13930$

$9.7 \quad 1.2 \quad 141.70$ ngilo istlo

8.

$83 \quad 8.5$

$79 \quad 4.0$

$\begin{array}{ll}82 & 8.5 \\ 95 & 9.0\end{array}$

$88 \quad 5.0$

01
0.0

$91 \quad 5.5$

$67 \quad 8.0$

$0 \quad 8.5$

$\because * \quad 8.5$

$0 \quad 7.5$

8.0
$+\quad 8.0$

$80 \quad 8.5$

8.5
$+4 \quad 9.0$

$94 \quad 9.0$

$88 \quad 7.5$

7.5

8.0

8.0

8.0

8.5
7.0

7.5

8.0

7.5

8.0

8.0

7.5

8.0

8.0

8.0

8.0

8.0 


\begin{tabular}{|c|c|}
\hline state & county \\
\hline ennsyluania & cleartield \\
\hline Dennsyluania & clearfield \\
\hline $\begin{array}{l}\text { Dennsyctuanta } \\
\text { Dennsylvania }\end{array}$ & clearfielo \\
\hline pennsyluania & clearfield \\
\hline Dennsyluania & clearfield \\
\hline Dennsytuania & cleartield \\
\hline Dennsylvania & cleartield \\
\hline pennsyivania & clearfield \\
\hline Dennsyluania & clearfield \\
\hline Dennsylvania & cleartielo \\
\hline Dennsylvania & cleartield \\
\hline Dennsylvania & clearfield \\
\hline Dennsylvania & cleartielo \\
\hline dennsyluania & clearfield \\
\hline veruisyluants & rlpartielo \\
\hline Dennsylvania & clearfield \\
\hline Dennsyluania & clearfielo \\
\hline $\begin{array}{l}\text { Pennsylvaniaa } \\
\text { pennsylvaniad }\end{array}$ & clearfield \\
\hline $\begin{array}{l}\text { Dennsylivania } \\
\text { Dennsyl vania }\end{array}$ & $\begin{array}{l}\text { cleartield } \\
\text { cleartield }\end{array}$ \\
\hline pennsyluania & cleaptield \\
\hline Dennsylvania & cleartield \\
\hline Dennsylvania & cleartield \\
\hline Dennsylvania & clearfield \\
\hline Dennsylvania & clearfield \\
\hline Dennsylvania & clearfield \\
\hline Dennsylvania & cleartield \\
\hline Dennsylvania & cleartielo \\
\hline Dennsyl vania & clearficld \\
\hline $\begin{array}{l}\text { Dennsylvanias } \\
\text { Dennsylvaniag }\end{array}$ & clearfield \\
\hline $\begin{array}{l}\text { Dennsylvania } \\
\text { pennsylvania }\end{array}$ & $\begin{array}{l}\text { clearfield } \\
\text { clearfield }\end{array}$ \\
\hline Dennsylvania & cleartipld \\
\hline Dennsylvania & clearfield \\
\hline Dennsyl vania & cleartield \\
\hline $\begin{array}{l}\text { Dennsyluania } \\
\text { Dennsylvania }\end{array}$ & $\begin{array}{l}\text { clinton } \\
\text { clinton }\end{array}$ \\
\hline $\begin{array}{l}\text { Dennsyl vania } \\
\text { pennsyl vania }\end{array}$ & clinton \\
\hline $\begin{array}{l}\text { Dennsyluania } \\
\text { Dennsylvania }\end{array}$ & $\begin{array}{l}\text { clinton } \\
\text { clinton }\end{array}$ \\
\hline $\begin{array}{l}\text { Dennsylvania } \\
\text { pennsyluanita }\end{array}$ & clinton \\
\hline Dennsyluania & clinton \\
\hline Dennsylvania & clinton \\
\hline Dennsylvania & clinton \\
\hline Dennsylvania & elk \\
\hline Dennsyluania & plk \\
\hline Dennsylvania & elk \\
\hline Dennsylvania & elk \\
\hline Dennsylvania & $e l k$ \\
\hline dennsylvania & $e l k$ \\
\hline
\end{tabular}

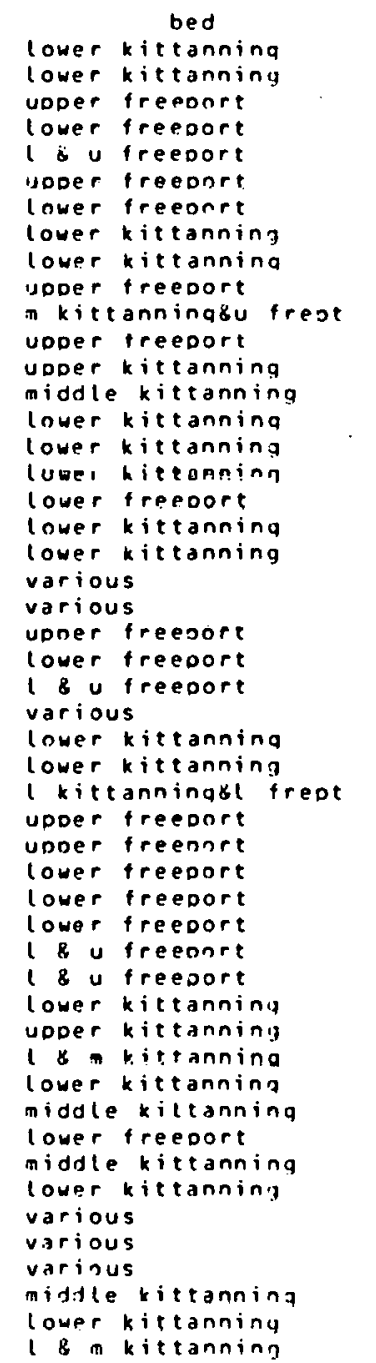

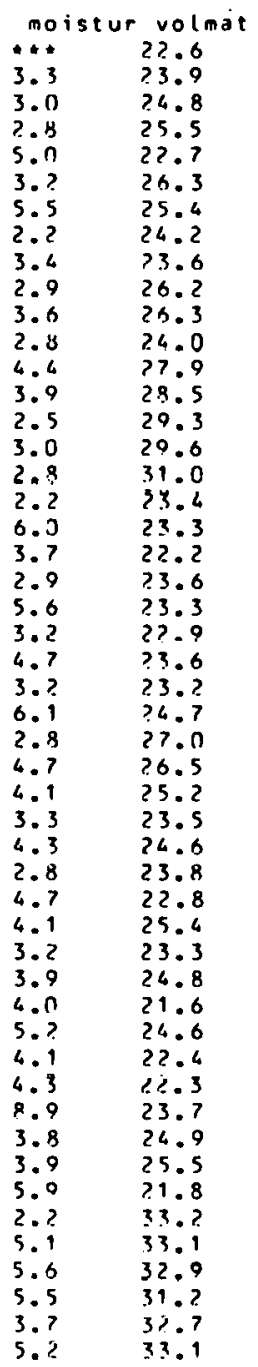

fixedc
65.8
67.5
63.7
67.7
66.2
61.7
65.2
62.9
70.1
62.0
60.7
64.7
56.2
60.8
58.3
60.0
58.1
64.3
66.0
70.1
65.9
67.0
67.2
66.9
68.3
67.5
66.6
65.2
66.2
67.5
67.0
66.9
70.5
65.9
68.2
66.3
66.9
66.1
66.6
63.9
64.5
67.6
66.0
69.3
59.7
59.6
58.7
59.4
56.7
55.5

\begin{tabular}{|c|c|c|}
\hline ds 1 & & \\
\hline $\begin{array}{r}11.6 \\
8.6\end{array}$ & $\begin{array}{l}0.9 \\
1.3\end{array}$ & $\begin{array}{l}12760 \\
14920\end{array}$ \\
\hline 11.5 & 1.4 & 13650 \\
\hline & 1.5 & 16420 \\
\hline 19.1 & 1.0 & 13680 \\
\hline 12.5 & 2.1 & 13560 \\
\hline 9.4 & $1 . ?$ & 1,3990 \\
\hline 12.9 & 3.6 & 13470 \\
\hline 6.3 & 0.8 & 14430 \\
\hline 11.8 & 1.5 & 13670 \\
\hline $93 . ?$ & 2.6 & 13330 \\
\hline 11.3 & 1.1 & 13850 \\
\hline 15.9 & 2.7 & 12870 \\
\hline 10.7 & 1.6 & 13750 \\
\hline 12.6 & 2.6 & 13690 \\
\hline 10.4 & 3.0 & 13800 \\
\hline 10.9 & 3.6 & 13640 \\
\hline 92.9 & 1.6 & I3AKn \\
\hline $10 . ?$ & 2.3 & 13800 \\
\hline 7.7 & 1.0 & 14370 \\
\hline 10.5 & 1.5 & 13860 \\
\hline 9.7 & 1.1 & 13970 \\
\hline 0.9 & 0.7 & 14100 \\
\hline 9.5 & 1.3 & 13720 \\
\hline 8.5 & 0.6 & 14220 \\
\hline 12.8 & 1.9 & $1323 n$ \\
\hline 6.4 & 1.2 & 14690 \\
\hline B. 3 & 2.3 & 16170 \\
\hline 8.6 & 1.9 & 14180 \\
\hline 9.0 & 0.9 & 14160 \\
\hline 3.4 & 0.7 & 13830 \\
\hline 9.3 & 1.5 & 16100 \\
\hline $6 . ?$ & 1.0 & 14470 \\
\hline 8.7 & 1.3 & 14030 \\
\hline 8.5 & 1,2 & 14210 \\
\hline 8.9 & 1.0 & 14160 \\
\hline 11.5 & 4.1 & 13720 \\
\hline & 2.4 & 14100 \\
\hline 1.0 & 2.4 & 13800 \\
\hline 0 & 6.8 & 14170 \\
\hline 11.8 & 2.7 & 13280 \\
\hline 7.7 & 2.1 & 14460 \\
\hline 8.5 & 3.1 & $1<010$ \\
\hline & 2.3 & 13950 \\
\hline 7.1 & 1.6 & 14060 \\
\hline 7.5 & 1.6 & 13840 \\
\hline 8.6 & 1.2 & 13610 \\
\hline 9.4 & 1.9 & 13730 \\
\hline 10.6 & 2.5 & 13620 \\
\hline & & \\
\hline
\end{tabular}

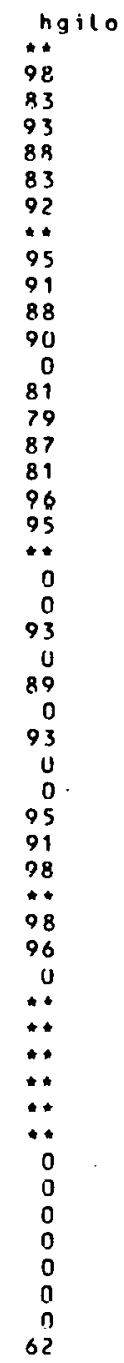

f5ilo
1.0
9.0
9.0
9.0
8.0
8.5
8.5
9.0
8.5
8.5
8.5
8.5
8.0
8.0
8.0
8.0
8.0
9.0
9.0
9.0
6.0
8.5
9.0
7.0
8.5
9.0
8.5
8.5
8.5
8.5
1.5
9.0
8.5
8.0
9.0
9.0
8.5
8.5
9.0
9.0
4.0
9.0
9.00
7.5
8.0
7.5
1.5
8.00
8.5
8.5




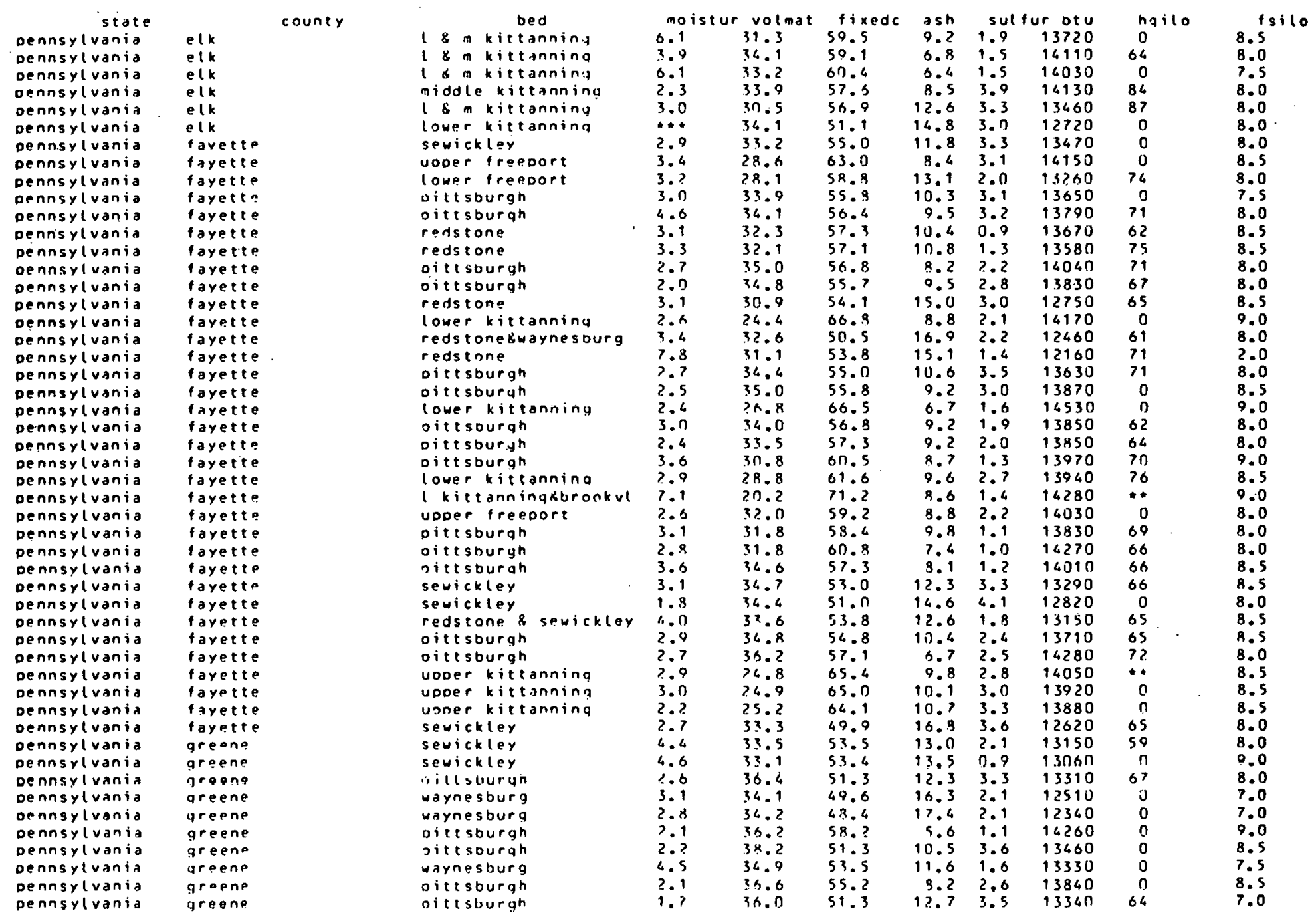




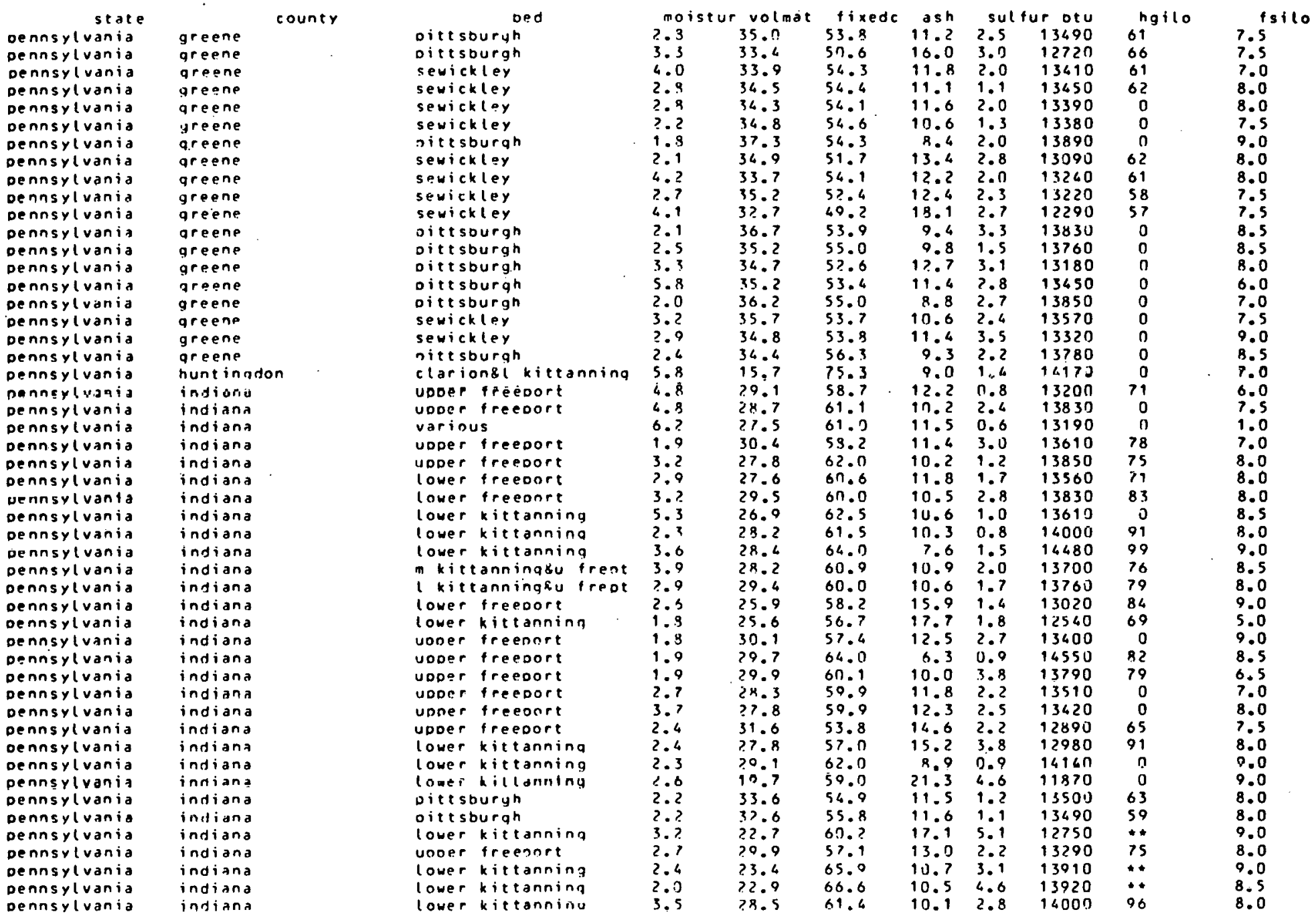




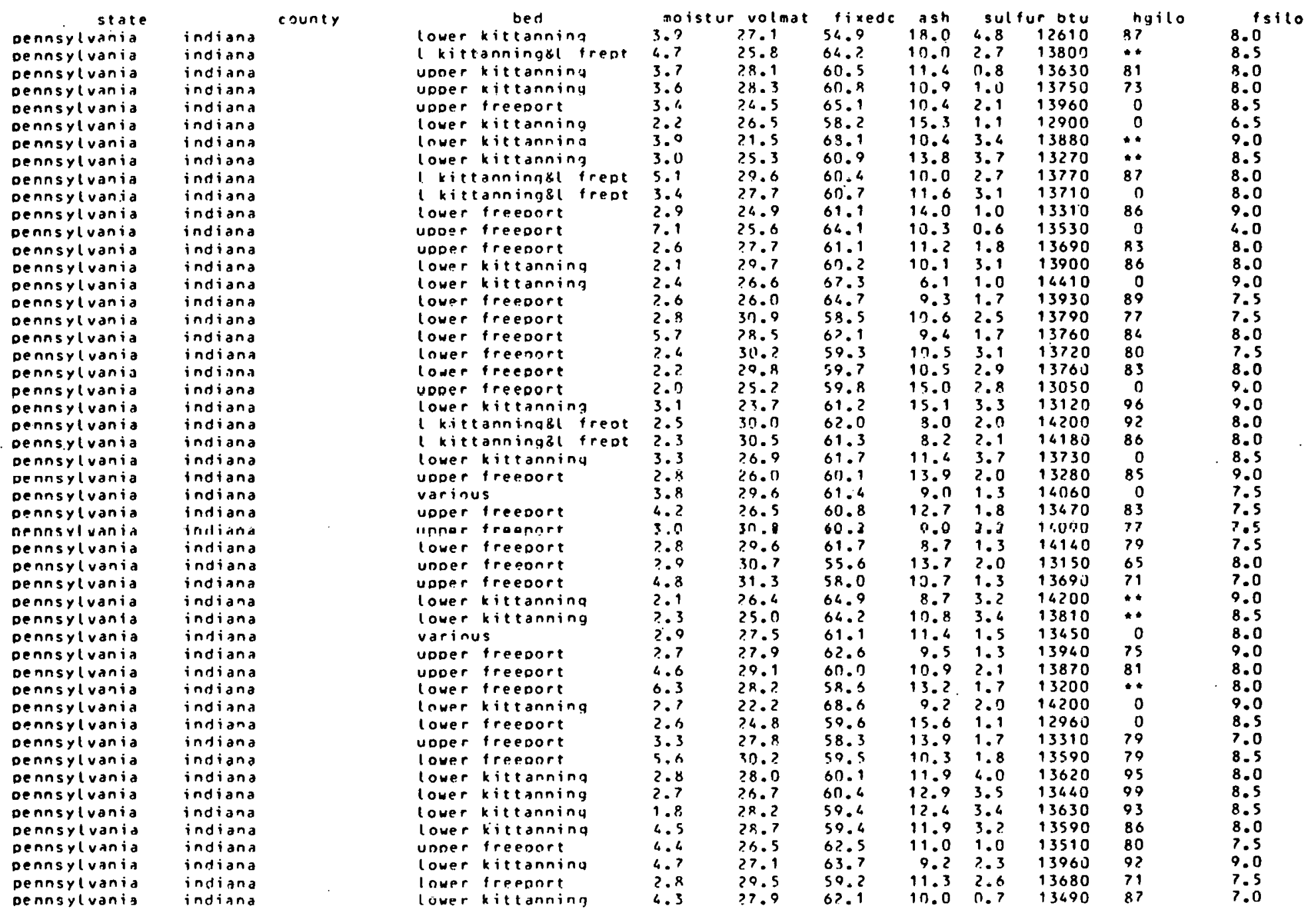




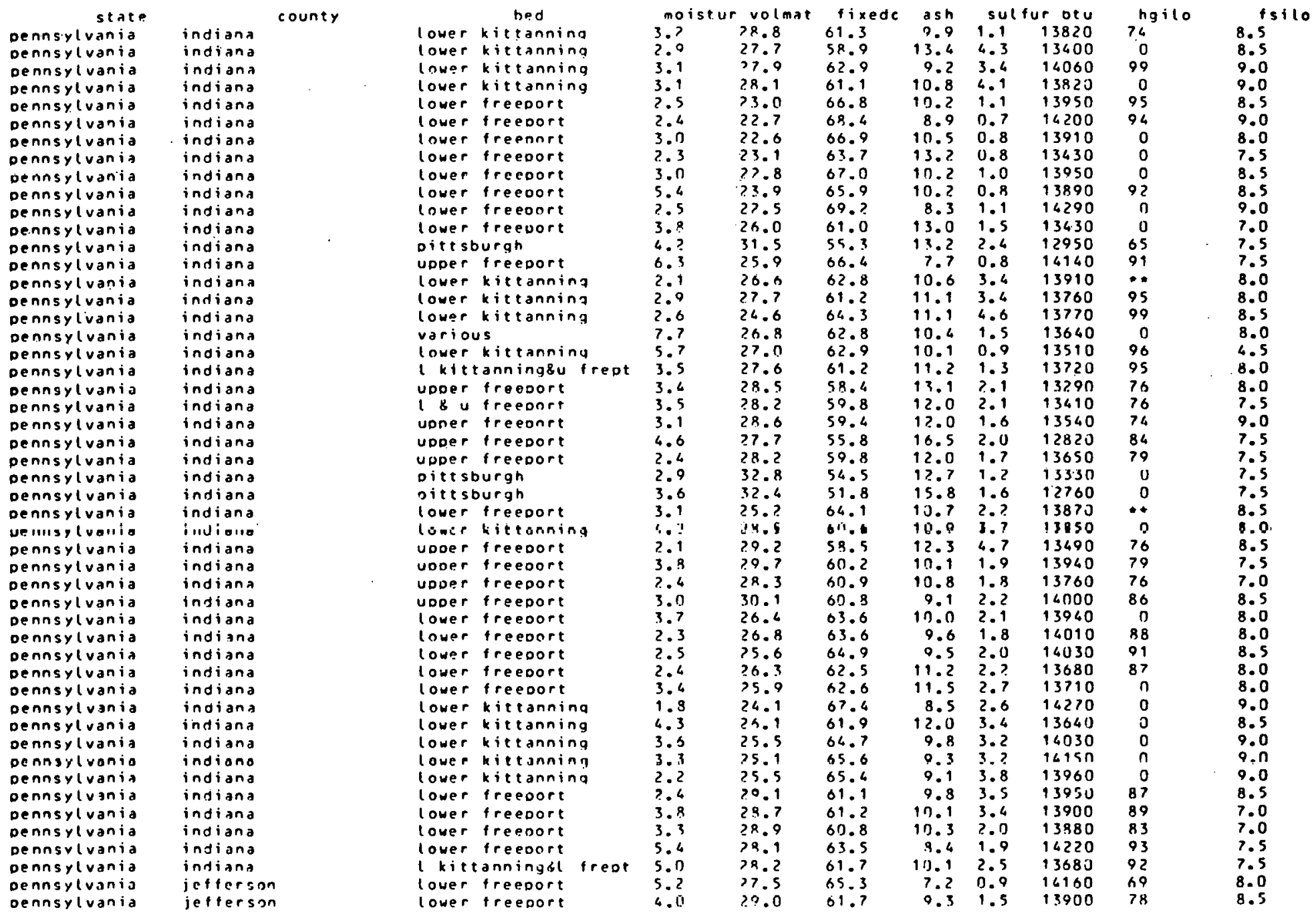




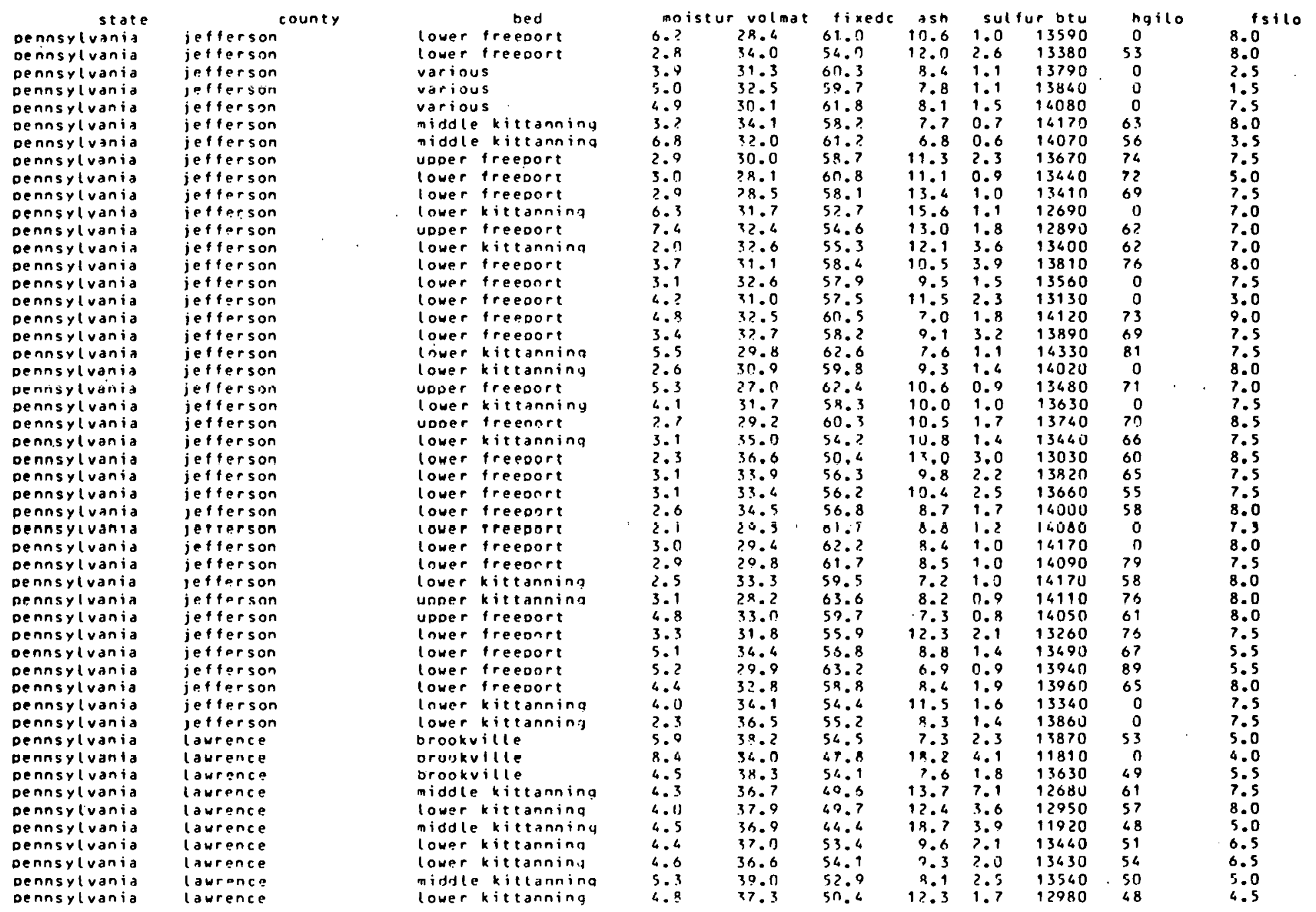




\begin{tabular}{|c|c|c|c|c|c|c|c|c|c|c|}
\hline state & county & bed & mo & r volmat & fixe & ash & sul & & hgilo & tsilo \\
\hline dennsyluania & lawrence & brookville & 4.7 & 40.9 & 46.6 & 12.5 & 5.6 & 12830 & 46 & $5.0^{5}$ \\
\hline oenn'sylvania & laurence & middle kittanning & 4.6 & 38.9 & 55.3 & 5.8 & 2.8 & 13850 & 53 & 5.0 \\
\hline Dennsylvania & lawrance & brookville & 3.9 & $3 R .2$ & 57.1 & 4.7 & 2.5 & 16080 & 0 & 4.5 \\
\hline Dennsyluania & laurence & orookville & 6.9 & 33.8 & 50.7 & 15.5 & 2.2 & 12300 & n & 5.5 \\
\hline Dennsyluania & laurence & brookville & 8.6 & 36.8 & 51.4 & 11.8 & 3.8 & 12960 & 0 & 6.0 \\
\hline Dennsyivania & lawrence & lower kittannina & 4.0 & 37.3 & 48.3 & 14.4 & 3.8 & 12510 & 50 & 5.0 \\
\hline Dennsylvania & mckean & lower nercer & 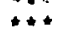 & 31.2 & 5n. 9 & 97.9 & 2.3 & 11800 & 58 & 1.0 \\
\hline Dennsyluania & mercer & brookville & 4.3 & 30.4 & 46.5 & 14.1 & 4.9 & 12560 & 48 & 4.5 \\
\hline Dennsyluania & mercer & brookville & 5.4 & 30.5 & 52.1 & 8.4 & 1.1 & 13460 & 53 & 5.5 \\
\hline dennsyluania & mercer & brookville & 3.9 & 43.4 & 44.7 & 11.9 & 3.3 & 12880 & 43 & 4.0 \\
\hline pennsylvania & mercer & brookvilie & 4.9 & 40.0 & 47.0 & 13.0 & 5.2 & 12650 & 0 & 4.5 \\
\hline Dennsylvania & mercer & middle kittanning & 4.7 & 37.2 & 53.2 & 9.6 & 1.6 & 1.3360 & $5 ?$ & 5.0 \\
\hline Dennsylvania & mercer & $\begin{array}{l}\text { lower kittanning } \\
\text { low }\end{array}$ & 5.3 & 36.5 & $5 \pi .5$ & 13.0 & 2.4 & 12890 & 50 & $\begin{array}{l}3.0 \\
6.0\end{array}$ \\
\hline Dennsyluania & mercer & brookville & 5.9 & 38.5 & 52.6 & 8.9 & 1.7 & 93410 & 55 & 5.0 \\
\hline Dennsyluania & mercer & brookville & 5.7 & $3>.3$ & 55.3 & 7.4 & 9.0 & 13760 & 50 & 5.5 \\
\hline Dennsyluania & somerset & upper kittanning & 2.0 & 17.7 & 70.7 & 11.6 & 1.8 & 13730 & $\star$ & 8.0 \\
\hline dennsyluania & somersat & uoper kittanning & 2.3 & 18.6 & 79.4 & 10.0 & 2.2 & 13970 & 96 & 9.0 \\
\hline pennsyivania & somerset & uoder kittanning & 2.5 & 17.8 & 70.5 & 11.7 & 1.8 & 13750 & 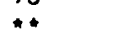 & 8.5 \\
\hline Dennsylvania & somerset & unoer freeont & 3.7 & 17.3 & 67.9 & 14.8 & 2.1 & 13260 & 99 & 8.5 \\
\hline Dennsylvania & somersot & lower freeport & 3.2 & 17.7 & 73.4 & 8.9 & 2.1 & 14240 & $\therefore$ & 9.0 \\
\hline Domagul uania & somerset & midule kittanning & 4.0 & 16.6 & 73.1 & 10.3 & 1.0 & 14010 & 94 & 5.5 \\
\hline pennsyluania & somarset & lower kittanning & 2.6 & $2 \pi .3$ & 64.6 & 15.1 & 1.6 & 13100 & is & $T .3$ \\
\hline Dennsylvania & somerset & lower kittanning & 2.0 & 10.0 & 79.5 & 9.5 & 1.5 & 14040 & $* *$ & 0.0 \\
\hline Dennsylvania & somerset & lower kittanning & 5.2 & $? 0.1$ & 67.7 & 10.2 & 1.2 & 13820 & ** & 4.0 \\
\hline oennsylvania & somerset & lower kittanning & 3.6 & 17.6 & 71.7 & 10.7 & 1.5 & 13800 & n & 9.0 \\
\hline Dennsyluania & somerset & lower kittanning & 3.7 & 17.0 & $7 ?$ & 10.8 & 1.5 & 13860 & $n$ & 6.5 \\
\hline Dennsyivania & somerset & lower freeonrt & 1.9 & 18.8 & 70.2 & 11.0 & 1.2 & 13870 & 97 & 9.0 \\
\hline Dennsylvania & somerset & lower kittanning & 2.5 & 16.7 & 79.9 & 11.6 & 3.2 & 13710 & \# & 9.0 \\
\hline pennsylvania & somerset & uoder kittanning & $2 . R$ & 15.7 & 74.3 & 10.0 & 1.7 & 14070 & $*$ & 8.0 \\
\hline dennsylvania & somerset & upoer kittanning & 3.3 & 16.1 & 73.5 & 10.4 & 1.3 & 14010 & $*$ & 7.5 \\
\hline Dennsyluania & somerset & Inwer kittanning & 2.5 & 19.8 & 69.2 & 12.0 & 2.7 & 13620 & ** & 8.0 \\
\hline dennsyluania & & lower kittanning & 2.7 & 17.3 & $? ?$ & 10.5 & 1.8 & $13880^{\circ}$ & .. & 8.5 \\
\hline pennsyluania & somerset & uoper freeoort & 3.8 & 17.0 & 70.5 & 12.5 & 1.8 & 13510 & 92 & $\begin{array}{l}0.5 \\
8.5\end{array}$ \\
\hline dennsyluania & somerset & $\begin{array}{l}\text { lower kittanniny } \\
\text { low }\end{array}$ & 9.6 & 16.9 & 74.8 & 8.3 & 1.7 & 14310 & 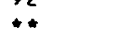 & 9.0 \\
\hline oennsylvania & somerset & vooer kiteannina & 2.7 & 15.9 & 72.2 & 11.9 & 2.8 & 13580 & $\star \star *$ & 7.5 \\
\hline Dennsyivania & somerset & uooer kittanning & 2.0 & 15.4 & 73.8 & 10.8 & 2.9 & 13860 & 99 & 8.0 \\
\hline dennsyluania & somerset & $\begin{array}{l}\text { lower kittanning } \\
\text { lowe }\end{array}$ & 3.2 & 17.9 & 59.7 & 23.4 & 3.3 & 19720 & 82 & 7.5 \\
\hline Dennsylvania & somerset & uoner kittanning & 8.5 & 18.4 & 70.7 & 10.9 & 0.7 & 13450 & ** & 1.5 \\
\hline dennsulvania & somerset & Inwer treonnet & 2.8 & 16.7 & 74.0 & 8.4 & 1.3 & 14290 & ** & 8.5 \\
\hline dennsylvania & somerset & brush creek & $\cdots$ & 21.3 & so.7 & 0.0 & 1.0 & 13510 & \#+ & 1.5 \\
\hline pennsylvania & somerset & brush creek & 3.7 & 17.5 & 69.0 & 14.5 & 4.3 & 13280 & 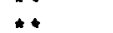 & 8.0 \\
\hline Dennsyluania & somerset & brush creak & 3.1 & 18.6 & 69.9 & 11.5 & 3.0 & 13680 & $\ldots$ & 8.5 \\
\hline dennsylyania & somerset & unoer kittanning & 2.2 & 16.9 & $7 \geqslant .6$ & 10.5 & 0.9 & 13920 & 94 & 5.0 \\
\hline Dennsylvania & somersot & upoer kittanning & 3.7 & 10.8 & $7 \div .8$ & 10.4 & 9.3 & 13000 & 0 & 1.0 \\
\hline dennsyluania & somerset & oittshurah & 6.7 & 21.3 & 64.7 & 94.0 & 1.9 & 12970 & 0 & 9.0 \\
\hline Dennsylvania & somerse? & Dittsourgh & 3.9 & 21.4 & 64.9 & 13.7 & 1.2 & 13230 & g & 9.0 \\
\hline pennsylvania & soperset & oittsburgh & 5.9 & 20.7 & 64.0 & 15.3 & 0.9 & 12680 & 89 & 1.0 \\
\hline pennsyluania & somerset & sittsburuh & 3.4 & 20.9 & 65.8 & 13.3 & 1.0 & 13270 & 88 & 8.0 \\
\hline Dennsylvania & somers?t & uover kittonning & $3 . ?$ & 17.6 & $7 ? .0$ & 10.4 & 0.8 & 13960 & 94 & 0 \\
\hline Dennsyluania & somersot & udoer kittanning & $2 . ?$ & 17.6 & 71.0 & 11.4 & 2.3 & 13750 & 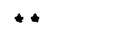 & .0 \\
\hline
\end{tabular}




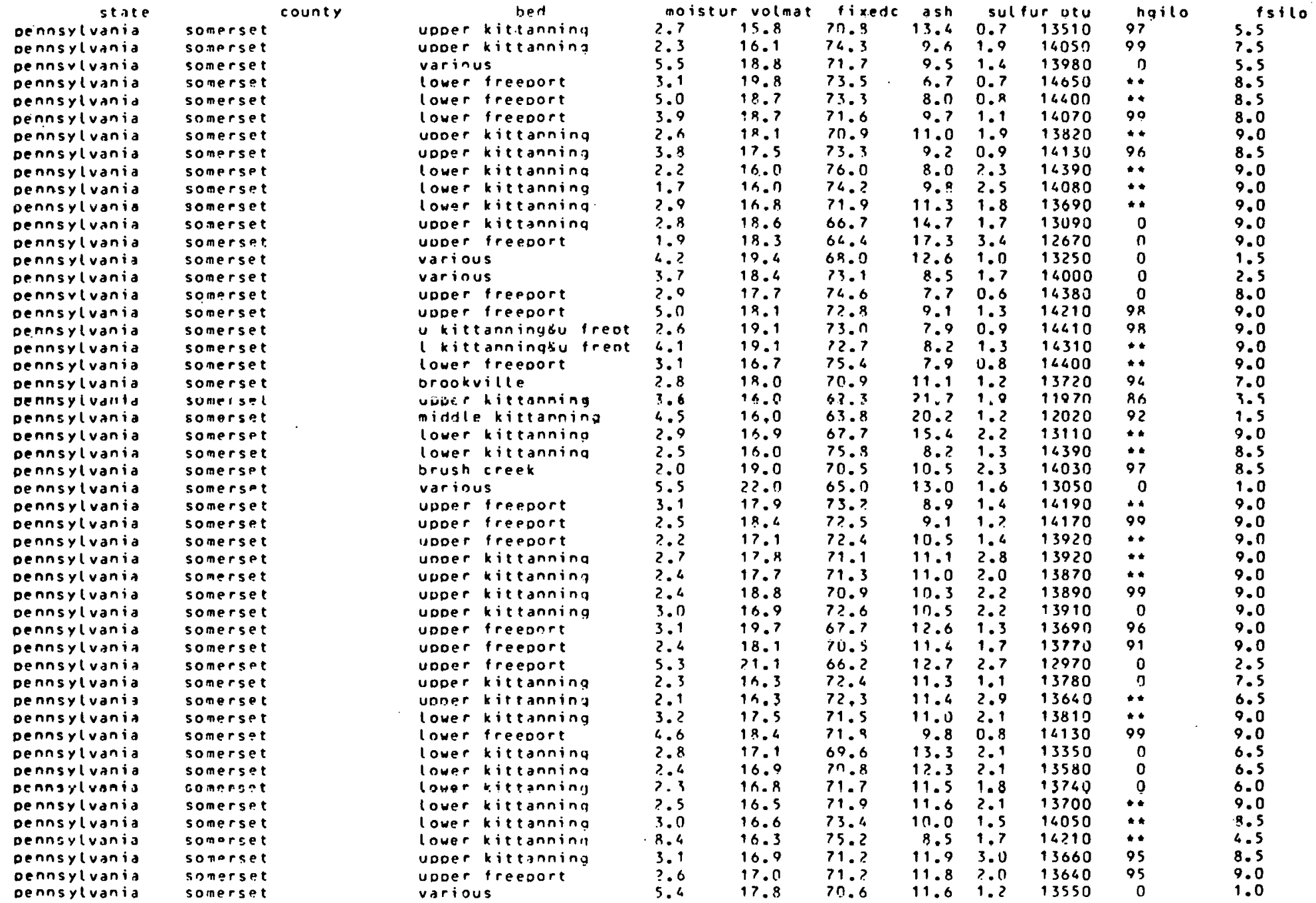




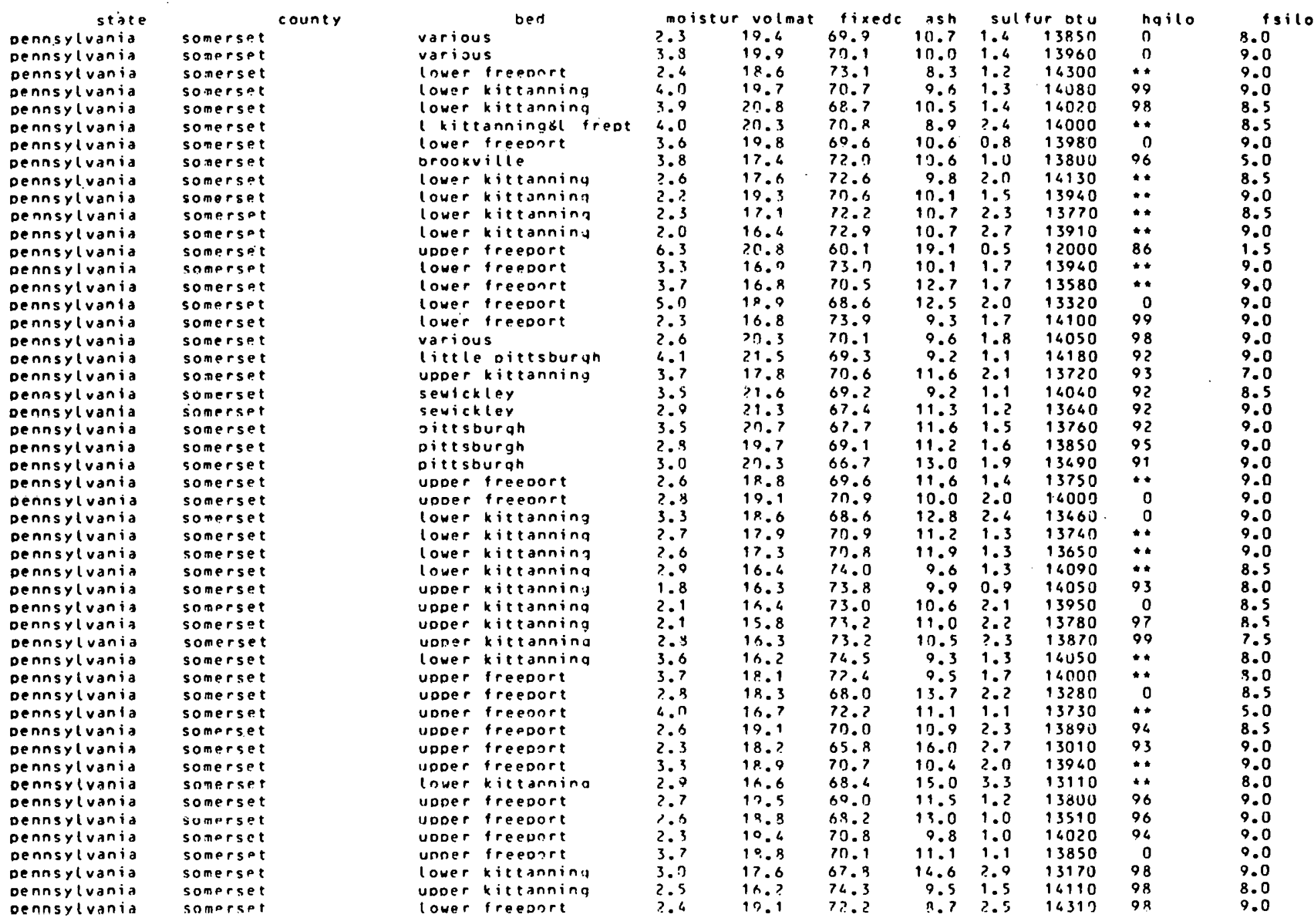




\begin{tabular}{|c|c|c|c|c|c|c|c|c|c|c|c|}
\hline $\begin{array}{c}\text { state } \\
\text { Dennsylvania }\end{array}$ & somerset county & lower & $\begin{array}{l}\text { bed } \\
\text { kittanning }\end{array}$ & $\begin{array}{r}m o \\
4.0\end{array}$ & $\begin{array}{l}\text { volmat } \\
17.7\end{array}$ & $\begin{array}{l}\text { fixedc } \\
>3.5\end{array}$ & $\begin{array}{l}\text { ash } \\
8.8\end{array}$ & $\begin{array}{l}\text { sut } \\
0.9\end{array}$ & $\begin{array}{l}\text { ur btu } \\
14130\end{array}$ & $\begin{array}{l}\text { hgilo } \\
0\end{array}$ & $7.0^{13610}$ \\
\hline Dennsylvania & somerset. & lower & kittanning & 3.1 & 16.7 & 76.6 & 6.7 & 0.9 & 16570 & 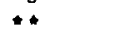 & 8.0 \\
\hline Dennsyluania & somerset & unoer & kittannina & 2.9 & 17.2 & 70.6 & 12.2 & $? .0$ & 13640 & 99 & 8.0 \\
\hline Dennsylvania & somerset & Dittso & urgh & 4.3 & $? 1.5$ & 69.7 & 3.9 & 1.1 & 14270 & 0 & 9.0 \\
\hline Dennsyluania & somerset & upder & freedort & 2.4 & $2 n .0$ & $7 n .0$ & 10.0 & 2.2 & 13900 & 99 & 8.0 \\
\hline Dennsylvania & somerset & lower & kittanning & 3.4 & 17.4 & 71.2 & 11.4 & 1.6 & 13620 & $\because$ & 7.5 \\
\hline Dennsylvania & somerset & lower & kittanning & 1.9 & 18.0 & 70.3 & 11.7 & 2.8 & 13720 & 0 & 5.5 \\
\hline Dennsylvania & somerset & lover & kittannina & 1.3 & 16.8 & 72.9 & 10.4 & 2.0 & 13930 & $\cdots$ & 9.0 \\
\hline pennsylvania & somerset & lower & kittanning & 2.0 & 17.2 & 73.4 & 9.4 & 2.4 & 14100 & 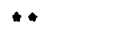 & 8.5 \\
\hline Dennsylvania & somerset & uoper & kittanning & 3.4 & 17.4 & 73.5 & 0.1 & 1.4 & 14180 & 0 & 8.0 \\
\hline Dennsylvania & somerset & lower & kittanning & $? .3$ & 16.4 & $73 . n$ & 10.6 & 3.2 & 13930 & $\star *$ & 9.0 \\
\hline Dennsyluania & somerset & iower & kittanning & 2.2 & 16.2 & 75.4 & 8.4 & 2.7 & 14370 & $*$ & 8.5 \\
\hline Dennsyluania & somerset & lower & kittanniny & 3.0 & 17.6 & 72.4 & 10.0 & 2.1 & 13890 & $\cdots$ & 8.0 \\
\hline Dennsyluania & somerset & lower & freenort & 9.9 & 18.3 & 73.0 & 8.7 & 1.1 & 14200 & $\star \star$ & 9.0 \\
\hline Dennsylvania & somerset & upner & kittannina & 9.3 & $1>.6$ & 68.0 & 14.4 & 4.0 & 13180 & 84 & 7.5 \\
\hline Dennsyivanio & somerset & upDer & kittanning & 2.7 & 17.3 & 66.9 & 15.8 & 4.0 & 12970 & 87 & 8.0 \\
\hline Dennsyluania & somerset & little & oittsburgh & 3.7 & $? 0.8$ & 69.3 & 9.9 & 0.7 & 13930 & 95 & 8.5 \\
\hline Dennsyivania & somerset & little & = oittsburgh & 3.5 & $? \pi .4$ & 69.1 & 10.5 & 0.7 & 13910 & 93 & 9.0 \\
\hline Dennsylivania & somerset & lower & freponet & 2.3 & 19.4 & 69.2 & 11.4 & 2.7 & 13800 & 98 & 9.0 \\
\hline pennsyluania & somerset & lower & kittanning & 3,6 & 16.7 & 72.5 & 10.8 & 2.1 & $138 \times 0$ & $t$ & 0.0 \\
\hline Dennsyluania & somerset & variou & us & 3.8 & 21.5 & 68.3 & 10.2 & 1.0 & 13670 & 0 & 1.5 \\
\hline Dennsyluaria & somerset & lower & treeoort & 3.7 & 16.5 & 74.2 & 9.3 & 2.0 & 14120 & ** & 9.0 \\
\hline Dennsyluania & somerset & Inver & freenort & $3 . ?$ & 16.6 & 73.9 & 9.5 & 2.1 & 96070 & 0 & 8.5 \\
\hline dennsyluania & somerset & lower & freeoort & 1.8 & 17.1 & 71.9 & 11.0 & 0.9 & 13830 & 0 & 8.0 \\
\hline Dennsylvania & somerset & lower & kittanning & $3 . n$ & 18.5 & 69.6 & 11.9 & 2.9 & 13730 & $*$ & 9.0 \\
\hline Dennsyluania & somprset & upDer & freedort & $? .0$ & 18.8 & 68.2 & 13.0 & 1.4 & 13550 & ** & 9.0 \\
\hline pennsyluania & somerset & uDDer & freeonrt & $? .8$ & 13.4 & 71.3 & 10.3 & 2.6 & 13890 & 0 & 2.5 \\
\hline dennsyluania & somerset & upoer & freeport & 3.0 & 17.3 & 70.2 & 12.5 & 2.0 & 13530 & 96 & 8.5 \\
\hline Dennsyluania & tioga & upDer & kittanning & 3.3 & 22.3 & 64.2 & 13.5 & 4.0 & 13240 & 0 & 9.0 \\
\hline pennsylvania & tioga & udoer & freedort & $3 . ?$ & 10.8 & 70.2 & 10.0 & 1.6 & 14010 & 0 & 9.0 \\
\hline pennsyluania & tioaa & uoper & kittannina & 3.5 & 17.6 & 01.4 & 21.0 & 3.8 & 12140 & 0 & 9.0 \\
\hline Dennsyluania & tioad & niddle & Kittannina & 3.3 & 21.4 & 57.8 & 20.8 & 2.1 & 12030 & 0 & 9.0 \\
\hline Dennsyluania & tioar & middle & kittanning & 3.3 & 21.8 & 70.5 & 7.7 & 1.1 & 14360 & 0 & 9.0 \\
\hline pennsyluania & tioga & middle & kittanning & 3.5 & 19.7 & 65.4 & 14.9 & 3.2 & 13000 & 0 & 9.0 \\
\hline Dennsyluania & tioga & midule & kittannina & 3.7 & 20.0 & 60.3 & 10.7 & 1.6 & 13750 & 0 & 9.0 \\
\hline Dennsyluania & tioaa & uncorr & Clated & 3.9 & 17.8 & 73.1 & 9.1 & 0.7 & 14100 & 0 & 9.0 \\
\hline Dennsyluania & I ioga & uncorr & elated & 3.7 & 17.1 & 66.3 & 16.6 & 1.3 & 12770 & 0 & 9.0 \\
\hline Dennsyluania & tioga & uncorr & related & 3.6 & 17.5 & 64.4 & 18.1 & 0.9 & 12580 & 0 & 9.0 \\
\hline pennsyluania & venango & lower & kittanning & 3.0 & 38.3 & 46.7 & 15.0 & 5.6 & 12650 & 0 & 5.5 \\
\hline Dennsyluania & venanoo & brookv & ville & 4.7 & 38.1 & 45.5 & 16.4 & 7.1 & 12330 & 0 & 4.5 \\
\hline Dennsylvania & washinaton & jittsb & burah & 6.8 & 31.4 & 48.5 & 20.1 & 1.3 & 11660 & 59 & 6.5 \\
\hline Dennsylvania & washington & Dittsb & burgh & 3.9 & 37.9 & 45.? & 16.9 & 5.0 & 12070 & 0 & 7.5 \\
\hline dennsylvaficia & washington & nittso & burgh & $\cdots$ & 31.0 & 50.8 & 18.2 & 2.3 & 12080 & 61 & 7.5 \\
\hline Dennsyluania & washinuton & oitesb & burgh & 9.6 & 35.7 & 52.0 & 12.3 & 1.8 & 13000 & 57 & 7.5 \\
\hline Dennsylvania & washington & Dittsb & burah & 2.1 & 35.2 & $57 . ?$ & 7.6 & 1.4 & 14100 & 0 & 7.0 \\
\hline pennsyluania & washington & oittsb & buruh & 5.5 & 75.2 & 50.0 & 14.8 & 2.4 & 12510 & 55 & 6.5 \\
\hline Dennsylvania & washinaton & nittst & burah & 7.5 & 34.4 & 47.9 & 17.7 & 3.? & 11990 & 0 & 5.5 \\
\hline Dennsylvania & washinoton & oittso & burih & 8.5 & 34.1 & 48.6 & 17.3 & 3.7 & 12130 & 0 & 6.5 \\
\hline Dennsylvania & washington & pittsb & burah & 6.6 & 35.3 & 47.2 & 17.5 & 3.7 & 12100 & 59 & 5.5 \\
\hline dennsyluariod & washington & Ditesn & ourgh & 7.6 & $34+1$ & 45.6 & 20.3 & 3.8 & 11660 & 0 & 5.5 \\
\hline
\end{tabular}




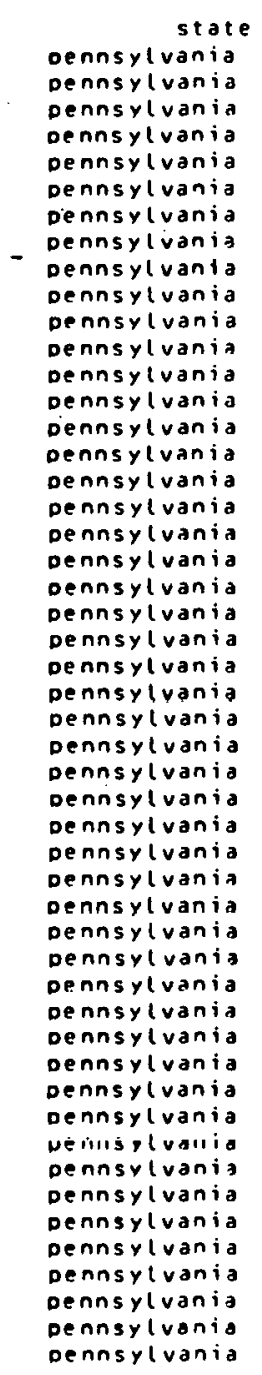

county

washington

washinnton

washinaton

washinaton

washington

washinaton

Washinaton

washington

washinaton

washington

washington

washington

washinaton

washinaton

washington

washington

washinaton

west morel and

westmoreland

westnoreland

vestmoreland

westmoreland

westmoreland

westmoreland
westmoreland

vestmoreland

westmoreland

westmoretand

westmoreland

westmoreland

westmoreland

vestmorel and

west moreland

westmorel and

we stmorel and

westmoreland

westmor eland

uestmureland

westmoreland

westmoreland

westmureland

westmorel and

westmoreland

westmoreland

westmoreland
westmoreland
Ditcsoiurgh

Dittsburgh

Dittsburgh

oittsburyh

Dittsburgh

pittsburgh

Ditesourgh

Dittsburah

oittsburah

oittsburiah

oittsburgh

Dittsburgh

nittsburgh

oittsburah

waynesburg

Dittsourah

Dittsburgh

oittsburah

Dittsburgh

redstone

upoer freenort

dittsburyh

wooer freenner

wover treedor

upper treeport

uoder freedort

uoper freeoor

wooer freeonrt

uoder fraedor

uober freedort

lower freedor

nittsburgh

uoder treeoort

upoer freedor

bakerstown

nitesburgh

upoer treeoor

upoer treeoort

reostone

unoer freedort

unoer treeoort

oitesburgh

nittsburyn

upoer freenor

woder freedort
ude

\begin{tabular}{|c|c|}
\hline mo & \\
\hline 0.9 & $3 ? .9$ \\
\hline $\begin{array}{r}7.4 \\
5.9\end{array}$ & $\begin{array}{r}35.2 \\
34.2\end{array}$ \\
\hline 3.3 & 40.8 \\
\hline 2.9 & 39.4 \\
\hline 6.7 & 39.2 \\
\hline $5 . ?$ & 35.8 \\
\hline 2.0 & 38.1 \\
\hline 2.6 & 35.6 \\
\hline 2.9 & 36.7 \\
\hline 3.7 & 35.6 \\
\hline 4.7 & 33.5 \\
\hline 3.3 & 39.6 \\
\hline 3.8 & 30.3 \\
\hline 4.1 & 37.4 \\
\hline 3.2 & 34.3 \\
\hline 5.2 & 36.4 \\
\hline 5.7 & 36.6 \\
\hline 3.6 & 38.3 \\
\hline 7.7 & 35.3 \\
\hline 2.0 & 34.2 \\
\hline 3.0 & 31.9 \\
\hline 3.1 & $3 ? .7$ \\
\hline 3.3 & 32.9 \\
\hline 1.7 & $3 ? .8$ \\
\hline 3.8 & 31.9 \\
\hline 4.4 & 26.0 \\
\hline 2.0 & 35.2 \\
\hline 2.0 & 35.2 \\
\hline 3.3 & 34.2 \\
\hline 3.2 & 33.0 \\
\hline 3.3 & 34.9 \\
\hline$? .1$ & 34.7 \\
\hline 2.0 & 23.5 \\
\hline 3.1 & 30.6 \\
\hline 2.0 & 35.3 \\
\hline 2.6 & 34.0 \\
\hline 3.0 & $3 ? .1$ \\
\hline 2.2 & $? 9.4$ \\
\hline$? .9$ & $3 \cap .3$ \\
\hline 2.? & 39.6 \\
\hline 4.0 & 33.1 \\
\hline 1.4 & 23.4 \\
\hline 2.4 & 33.6 \\
\hline 2.6 & 32.4 \\
\hline 3.7 & 34.0 \\
\hline 3.9 & 31.5 \\
\hline 2.7 & 34.8 \\
\hline 2,6 & 34.6 \\
\hline 2.5 & 35.5 \\
\hline
\end{tabular}

fixedc
45.3

ash sulfur btu
$20.8 \quad 3.8 \quad 11550$

$\begin{array}{lll}20.8 & 3.8 & 11550 \\ 19.1 & 3.5 & 11810\end{array}$

$17.73 .7 \quad 12080$

$\begin{array}{rrr}7.0 & 3.2 & 13700 \\ 10.6 & 3.5 & 13160\end{array}$

$16.71 .4 \quad 12380$

$13.3 \quad 3.0 \quad 12790$

$\begin{array}{lll}5.6 & 1.5 & 13630\end{array}$

$8.0 \quad 1.6 \quad 13710$

$14.3 \quad 3.3 \quad 12610$

$21.3 \quad 4.1 \quad 11510$

$\begin{array}{rrr}8.4 & 1.4 & 13620 \\ 12.2 & 4.5 & 12910\end{array}$

$\begin{array}{lll}15.1 & 2.7 & 12540\end{array}$

$17.4 \quad 3.4 \quad 12290$

$12.8 \quad 3.1 \quad 12720$

$10.1 ? .7 \quad 13240$

13.73 .512600

$12.3 \quad 2.9 \quad 13390$

$\begin{array}{lll}10.0 & 1.1 & 13680\end{array}$

$11.7 \quad 1.6 \quad 13160$

$0.2 \quad 1.8 \quad 13960$

$14.6 \quad 1.7 \quad 13110$

$\begin{array}{lll}9.5 & 2.4 & 13780\end{array}$

$10.19 .6 \quad 13690$

$\begin{array}{lll}11.9 & 2.6 & 13330 \\ 13.7 & 3.4 & 13500\end{array}$

$\begin{array}{rrr}10.7 & 3.6 & 13500 \\ 8.7 & 2.6 & 13930\end{array}$

$\begin{array}{rrr}8.7 & 2.6 & 13930 \\ 11.7 & 2.6 & 13710\end{array}$

$8.30 .9 \quad 14150$

$1 \mathrm{~J} .1 \quad 1.9 \quad 13680$

$\begin{array}{lll}12.2 & 1.9 & 13370 \\ 18.5 & 3.6 & 12270\end{array}$

$0.6 \quad 2.4 \quad 13910$

$13.2 \quad 0.9 \quad 13190$

110.2 2.5 13780

$13.3 \quad 2.4 \quad 93170$

$8.7 \quad 3.2 \quad 13990$

$\begin{array}{lll}15.9 & 4.9 & 12870 \\ 10.1 & 1.4 & 13640\end{array}$

$\begin{array}{rrr}15.6 & 1.7 & 12280 \\ 0.2 & 2.4 & 13750\end{array}$

$\begin{array}{rrr}8.1 & 1.0 & 13970 \\ 11.9 & 2.8 & 13370\end{array}$

\begin{tabular}{|c|c|}
\hline hailo & isilo \\
\hline $61^{\circ}$ & 6.5 \\
\hline 0 & 5.0 \\
\hline 0 & 7.0 \\
\hline 5? & 7.0 \\
\hline 66 & 7.0 \\
\hline 0 & 8.0 \\
\hline 59 & 7.0 \\
\hline 0 & 8.0 \\
\hline 0 & 8.0 \\
\hline$n$ & 8.0 \\
\hline 0 & 7.5 \\
\hline 0 & 6.5 \\
\hline 51 & 7.0 \\
\hline 50 & 5.5 \\
\hline 53 & 7.0 \\
\hline 0 & 6.0 \\
\hline 57 & 8.0 \\
\hline 0 & 6.0 \\
\hline 0 & 6.0 \\
\hline 0 & 6.0 \\
\hline 0 & 8.0 \\
\hline 69 & 8.5 \\
\hline 66 & 8.5 \\
\hline 60 & 7.5 \\
\hline o & 7.5 \\
\hline 72 & 9.0 \\
\hline 84 & 8.5 \\
\hline 0 & 3.0 \\
\hline 0 & 7.5 \\
\hline 0 & 8.5 \\
\hline 0 & 8.0 \\
\hline 0 & B. 0 \\
\hline 0 & 7.0 \\
\hline ** & 9.0 \\
\hline 78 & 8.5 \\
\hline 60 & 8.0 \\
\hline 61 & 7.5 \\
\hline 63 & 9.0 \\
\hline 0 & 7.0 \\
\hline 0 & 9.0 \\
\hline 69 & 8.5 \\
\hline 5 & 9.0 \\
\hline 32 & $\begin{array}{l}8.5 \\
8.5\end{array}$ \\
\hline 77 & 8.5 \\
\hline 72 & 7.5 \\
\hline 55 & 7.5 \\
\hline 66 & 2.0 \\
\hline 64 & 8.5 \\
\hline $5 B$ & 8.0 \\
\hline $3 ?$ & 8.5 \\
\hline
\end{tabular}




\begin{tabular}{|c|c|}
\hline 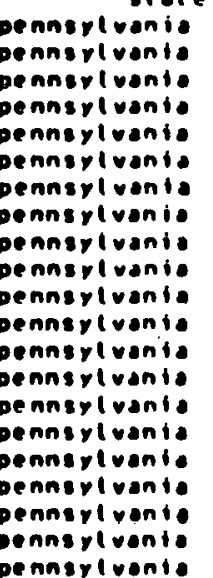 & 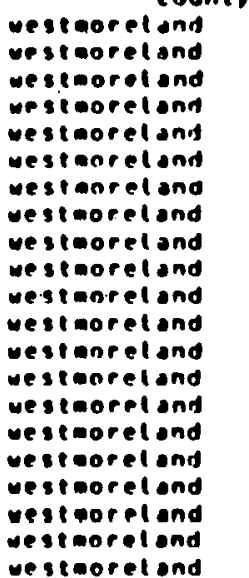 \\
\hline
\end{tabular}
unber ireeoner
under Treegnr
unner freenore
upoer Ireevar
under Ireeg
a
upder Treedort
uoder ireeport
woder Ireedore
uoper treedor
upoer Ireedone
noper Irepdor
upoer treedor
- y Treedor
uoder treedore

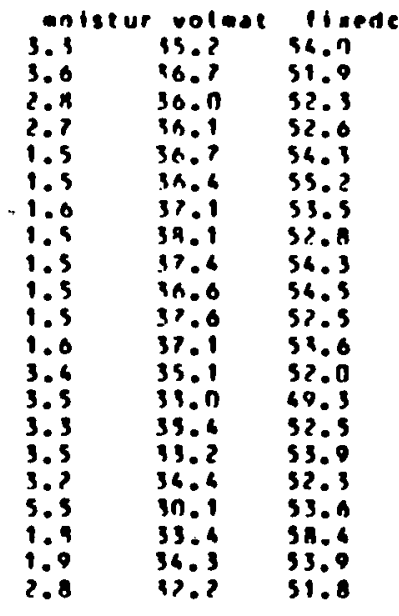

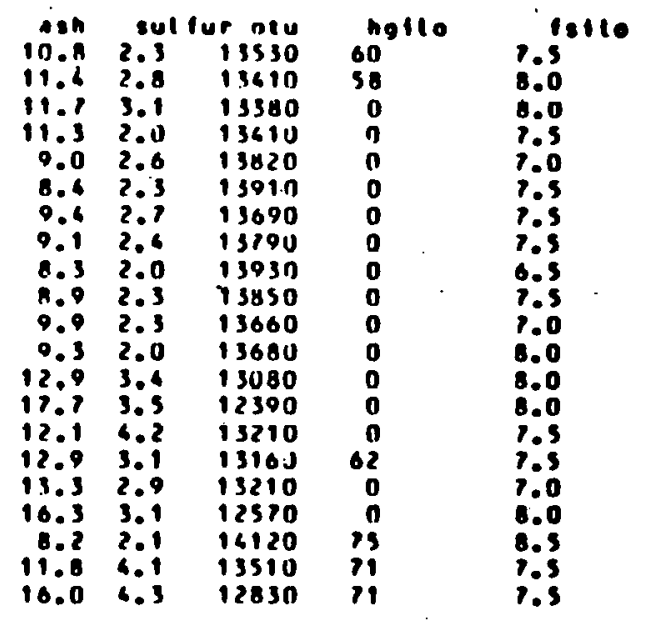



National Coal Resources Data Systen

U.S. Geological survey

identified coal resources in tennessee

(In millions of short tons)

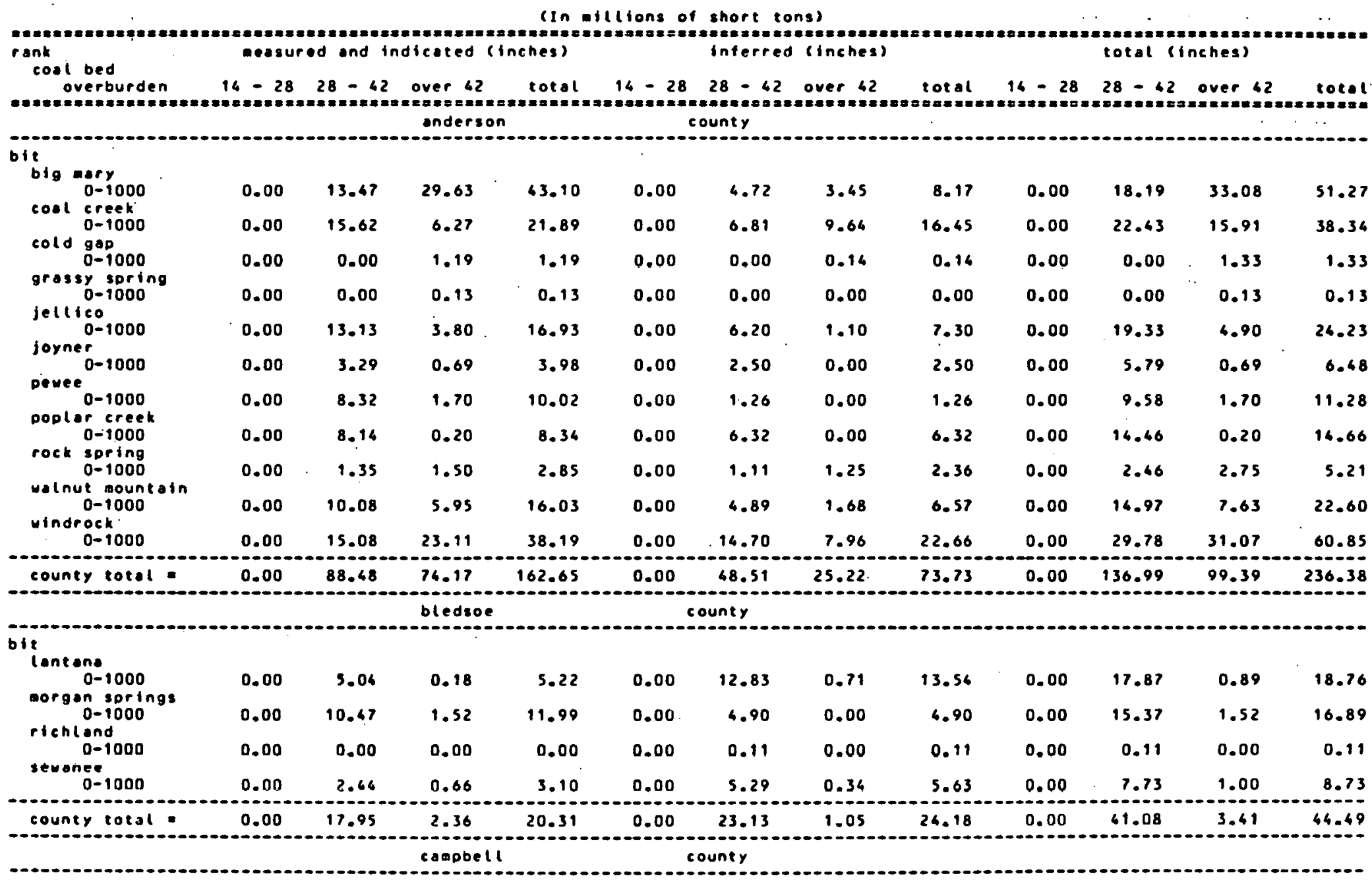




\begin{tabular}{|c|c|c|c|c|c|c|c|c|c|c|c|c|}
\hline $0-1000$ & 0.00 & 8.38 & 14.46 & 22.84 & 0.00 & 17.31 & 6.01 & 23.32 & 0.00 & 25.69 & 20.47 & 46.16 \\
\hline $0-1000$ & 0.00 & 7.95 & 0.28 & 8.23 & 0.00 & 7.85 & 0.00 & 7.85 & 0.00 & 15.80 & 0.28 & 16.08 \\
\hline cold $0-1000$ & 0.00 & 55.99 & 12.61 & 68.60 & 0.00 & 53.59 & 18.59 & 72.18 & 0.00 & 109.58 & 31.00 & 140.58 \\
\hline $\begin{array}{c}0-1000 \\
\text { grassy spring }\end{array}$ & 0.00 & 0.43 & 0,00 & 0.43 & 0.00 & 0.54 & 0.00 & 0.56 & 0.00 & 0.97 & 0.00 & 0.97 \\
\hline $\begin{array}{c}0-1000 \\
\text { hazard no } 5\end{array}$ & 0.00 & 0.62 & 0.47 & 1.09 & $0.00^{\circ}$ & 0.28 & 0.00 & 0.28 & 0.00 & 0.90 & 0.67 & 1.37 \\
\hline jellico & 0.00 & 14.98 & 6.11 & 19.09 & 0.00 & 1.66 & 0.01 & 1.67 & 0.00 & 16.66 & 4.12 & 20.76 \\
\hline jordan & 0.00 & 21.61 & 0.86 & 22.27 & 0.00 & 25.44 & 0.00 & 23.46 & 0.00 & 46.85 & 0.86 & 67.71 \\
\hline $\begin{array}{l}0-1000 \\
\text { joyner }\end{array}$ & 0.00 & 9.32 & 0.00 & 9.32 & 0.00 & 3.43 & 0.00 & 3.63 & 0.00 & 12.75 & 0.00 & 12.75 \\
\hline $\begin{array}{l}0-1000 \\
\text { lower pine bald } \\
0-1000 \\
\text { pewee }\end{array}$ & $\begin{array}{l}0.00 \\
0.00\end{array}$ & $\begin{array}{l}4.13 \\
0.67\end{array}$ & $\begin{array}{l}0.00 \\
0.17\end{array}$ & $\begin{array}{l}4.13 \\
0.84\end{array}$ & $\begin{array}{l}0.00 \\
0.00\end{array}$ & $\begin{array}{r}11.11 \\
0.00\end{array}$ & $\begin{array}{l}0.00 \\
0.00\end{array}$ & $\begin{array}{r}11.11 \\
0.00\end{array}$ & $\begin{array}{l}0.00 \\
0.00\end{array}$ & $\begin{array}{l}15.26 \\
0.67\end{array}$ & $\begin{array}{l}0.00 \\
0.17\end{array}$ & $\begin{array}{l}15.24 \\
0.84\end{array}$ \\
\hline $\begin{array}{l}0-1000 \\
\text { pioneer }\end{array}$ & 0.00 & 18.32 & 2.75 & 21.07 & 0.00 & 5.26 & 0.09 & 5.35 & 0.00 & 23.58 & 2.86 & 26.62 \\
\hline $\begin{array}{c}0-1000 \\
\text { popler creek } \\
0-1000\end{array}$ & $\begin{array}{l}0.00 \\
0.00\end{array}$ & $\begin{array}{l}11.05 \\
19.26\end{array}$ & $\begin{array}{l}0.00 \\
0.00\end{array}$ & $\begin{array}{l}11.05 \\
19.24\end{array}$ & $\begin{array}{l}0.00 \\
0.00\end{array}$ & $\begin{array}{r}5.39 \\
17.66\end{array}$ & $\begin{array}{l}0.00 \\
0.00\end{array}$ & $\begin{array}{r}5.39 \\
17.66\end{array}$ & $\begin{array}{l}0.00 \\
0.00\end{array}$ & $\begin{array}{l}16.44 \\
36.90\end{array}$ & $\begin{array}{l}0.00 \\
0.00\end{array}$ & $\begin{array}{l}16.44 \\
36.90\end{array}$ \\
\hline rex $0-1000$ & 0.00 & 16.90 & 0.34 & 17.26 & 0.00 & 22.75 & 0.00 & 22.75 & 0.00 & .39 .65 & 0.34 & 39.99 \\
\hline $\begin{array}{c}0-1000 \\
\text { wolnut mount ain }\end{array}$ & 0.00 & 1.01 & 9.96 & 10.97 & 0.00 & 3.93 & 0.91 & 4.84 & 0.00 & 4.94 & 10.87 & 15.81 \\
\hline $\begin{array}{l}0-1000 \\
\text { windrock }\end{array}$ & 0.00 & 14.21 & 8.55 & 22.76 & 0.00 & 12.26 & 2.02 & 14.28 & 0.00 & 26.47 & 10.57 & 37.04 \\
\hline $\begin{array}{l}0-1000 \\
\text { county totol }\end{array}$ & 0.00 & 217.50 & $\begin{array}{r}7.13 \\
61.49 \\
-\ldots-0 .\end{array}$ & 278.99 & 0.00 & $\begin{array}{l}20.11 \\
208.57 \\
-20.0 .\end{array}$ & $\begin{array}{l}5.30 \\
33.02 \\
33.02\end{array}$ & 26.59 & $\begin{array}{l}0.00 \\
0.00 \\
0.00\end{array}$ & $\begin{array}{l}33.00 \\
626.07 \\
0.0 .07\end{array}$ & $\begin{array}{l}12.52 \\
0.0 .0 .51 \\
96.51\end{array}$ & $\begin{array}{r}65.52 \\
-.00 .0 .5 \\
520.58\end{array}$ \\
\hline & & & clotibs & & & ounty & &.- & & 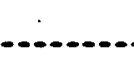 & & \\
\hline big mary & & & & & & & & & & & & \\
\hline olue gen & 0.00 & 1.36 & 1.12 & 2.68 & 0.00 & 1.13 & 0.38 & 1.51 & 0.00 & 2.49 & 1.50 & 3.99 \\
\hline cosl creek & 0.00 & 6.77 & 0.00 & 6.77 & 0.00 & 15.62 & 0.00 & 15.62 & 0.00 & 22.39 & 0.00 & 22.39 \\
\hline jellico $0-1000$ & 0.00 & 19.80 & 6,91 & 26.71 & 0.00 & $28.8 ?$ & 3.59 & 32.61 & 0.00 & 48.62 & 10.50 & 58.12 \\
\hline Dewee & 0.00 & 8.56 & 0.90 & 9.55 & 0.00 & 6.99 & 4.35 & 9.34 & 0.00 & 13.55 & 5.34 & 18.89 \\
\hline $\begin{array}{l}0-1000 \\
\text { pioneer }\end{array}$ & 0.00 & 1.32 & 1.45 & 2.77 & 0.00 & 0.35 & 0.00 & 0.35 & 0.00 & 1.67 & 1.65 & 3.12 \\
\hline rex $0-1000$ & 0.00 & 3.85 & 0.00 & 3.85 & 0.00 & 8.79 & 0.00 & 8.79 & 0.00 & 12.64 & 0.00 & 12.64 \\
\hline $0-1000$ & 0.00 & $\begin{array}{r}1.29 \\
-.2 .-2\end{array}$ & 0.35 & 1.64 & \begin{tabular}{c}
0.00 \\
\hdashline
\end{tabular} & 20.20 & 0.00 & 20.20 & 0.00 & 21.49 & 0.35 & 21.86 \\
\hline county totol = & 0.00 & 62.95 & 10.82 & 53.77 & 0.00 & 79.90 & 8.32 & 88.22 & 0.00 & 122.85 & 19.16 & 161.99 \\
\hline
\end{tabular}




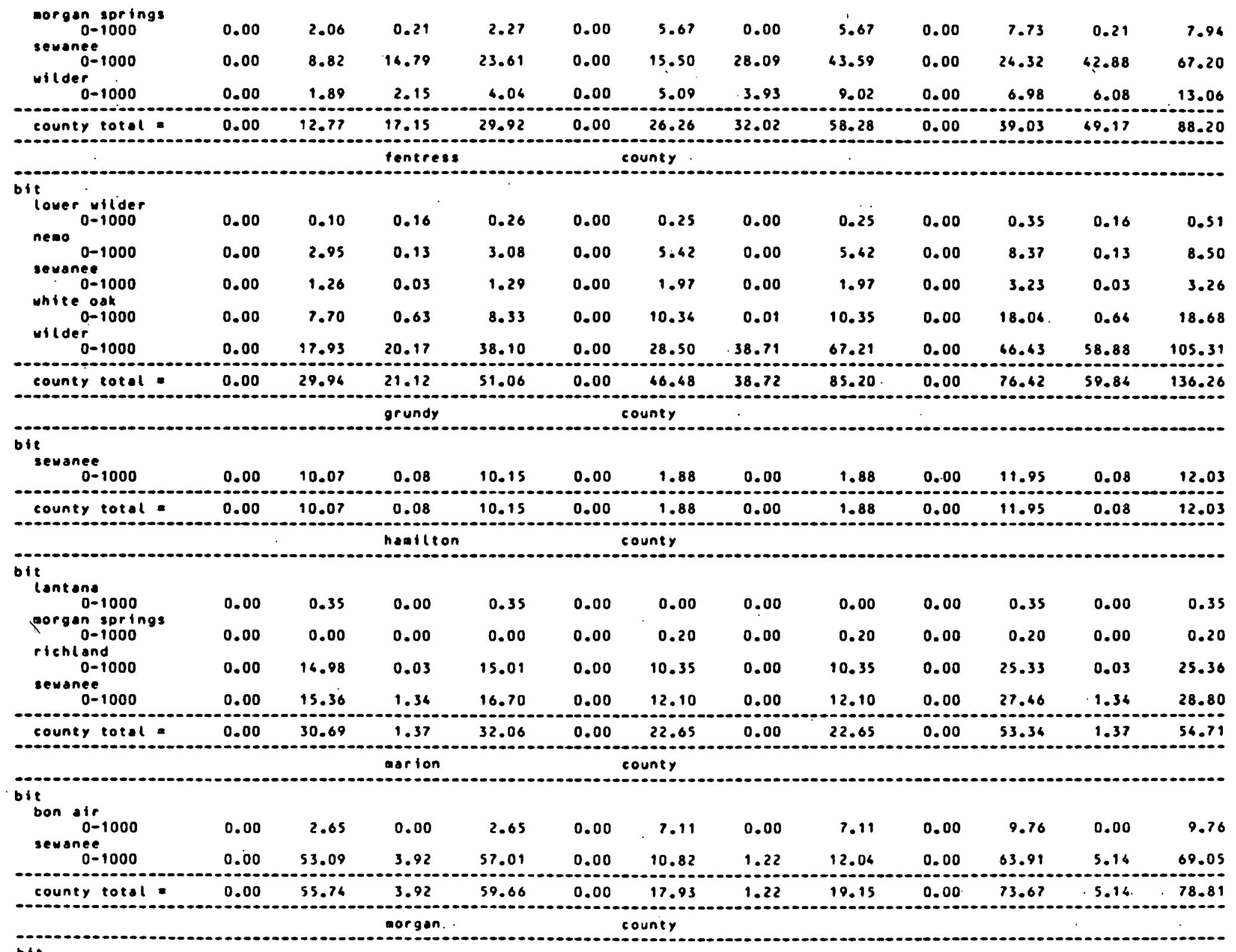




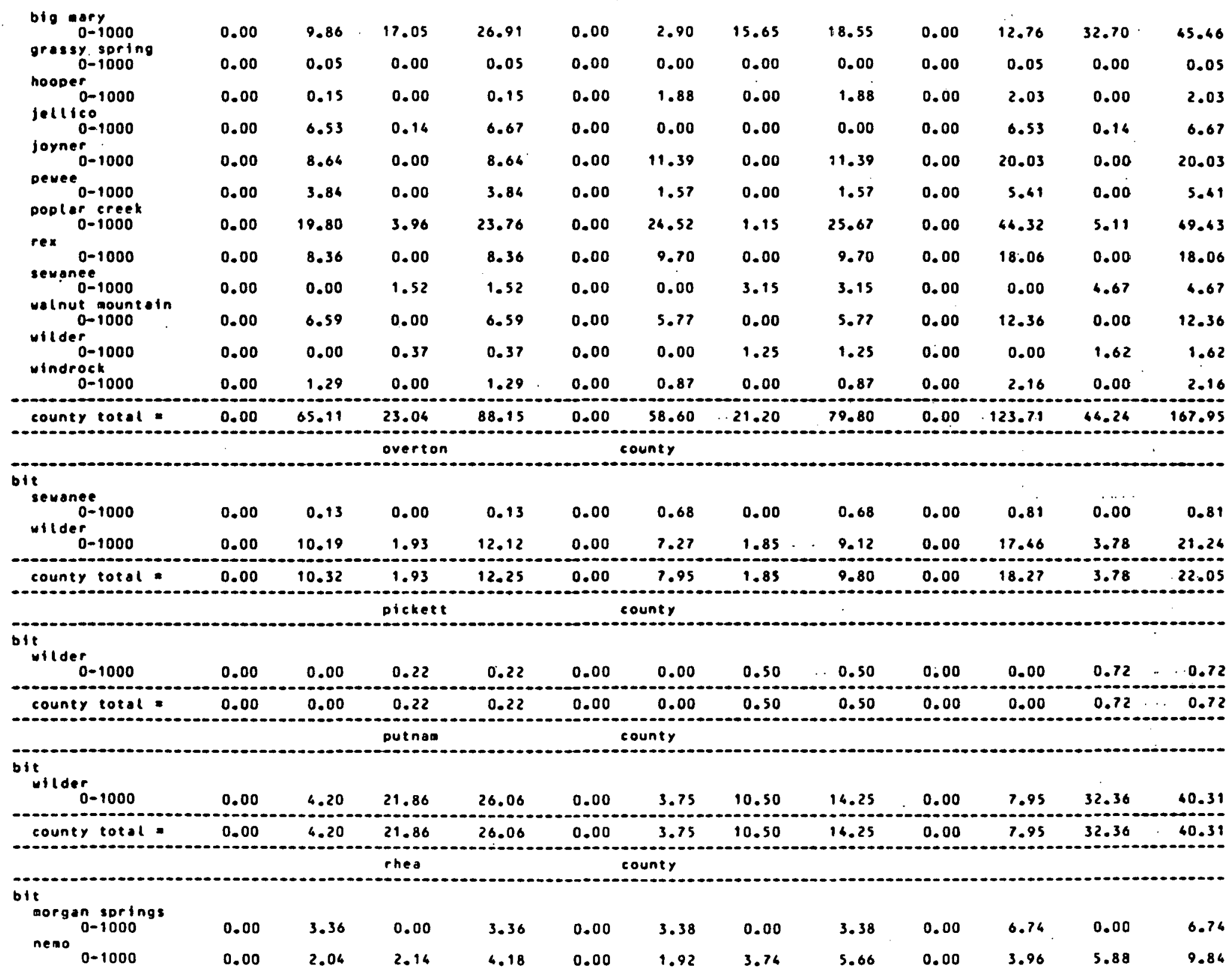




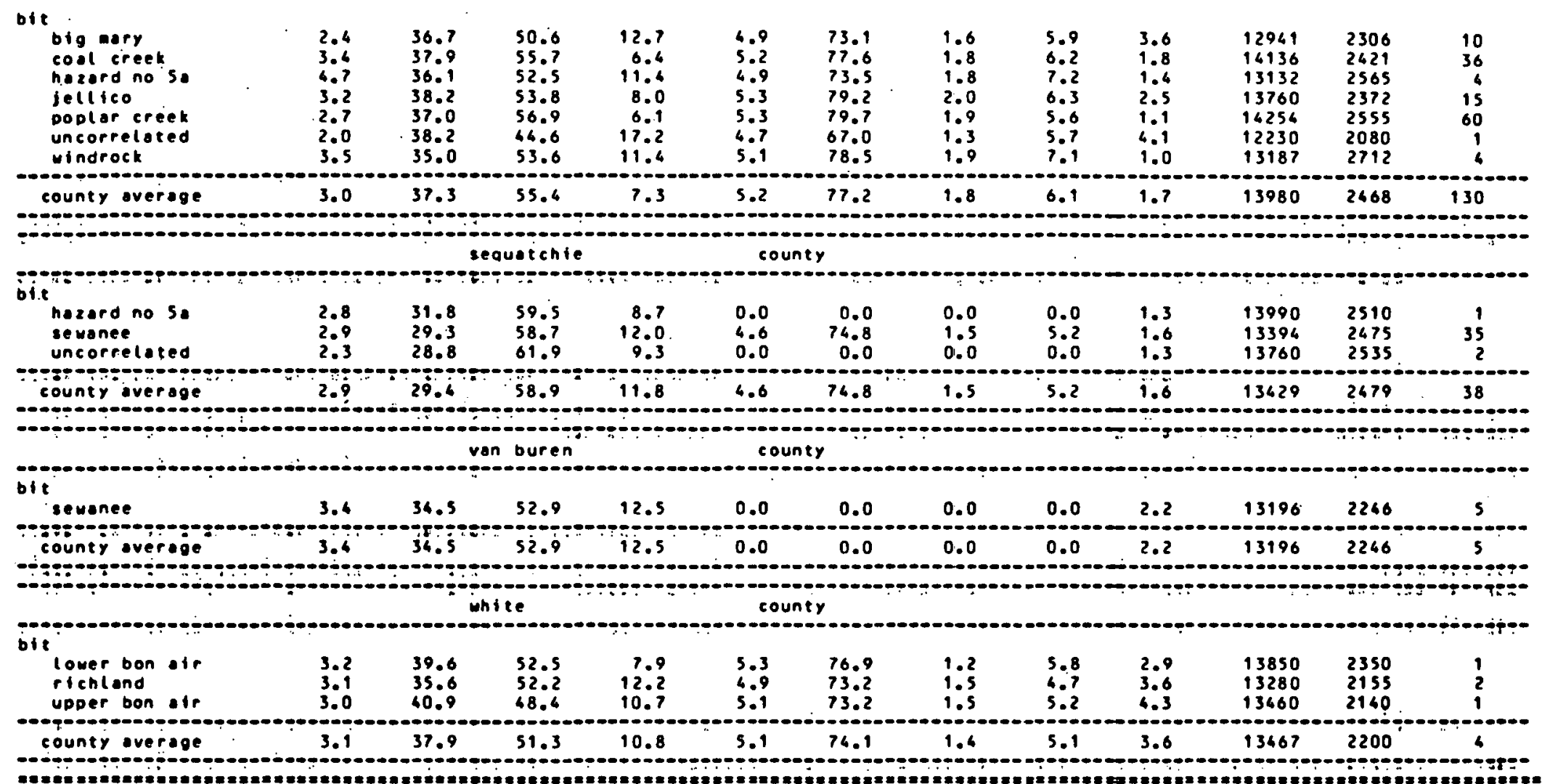




\begin{tabular}{|c|c|c|c|c|c|c|c|c|c|c|c|c|}
\hline $0-1000$ & 0.00 & 5.94 & 0.00 & 5.94 & 0.00 & 9.16 & 0.20 & 9.36 & 0.00 & $\begin{array}{r}15.10 \\
-\ldots \ldots\end{array}$ & 0.20 & 15.30 \\
\hline county totol. & 0.00 & 19.34 & 2.14 & 13.48 & 0.00 & 14.46 & 3.94 & 18.40 & 0.00 & 25.80 & 6.08 & 31.88 \\
\hline & & & roone & & & inty & & & & & & \\
\hline te & & & & & & & & & & & & \\
\hline $0-1000$ & 0.00 & 0.00 & 1.34 & 1.34 & 0.00 & 0.00 & 5.05 & 5.05 & 0.00 & 0.00 & 6.39 & 6.39 \\
\hline county total & 0.00 & 0.00 & 1.34 & 1.34 & 0.00 & 0.00 & 5.05 & 5.05 & 0.00 & 0.00 & 6.39 & 6.39 \\
\hline & & & scott & & & inty & & 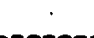 & & & & \\
\hline it & & & & & & & & & & & & \\
\hline $0-1000$ & 0.00 & 15.25 & 17.86 & 33.11 & 0.00 & 5.76 & 6.42 & 12.18 & 0.00 & 21.01 & 24.28 & 45.29 \\
\hline $0-1000$ & 0.00 & 2.16 & 1.94 & 4.10 & 0.00 & 0.27 & 0.34 & 0.61 & 0.00 & 2.43 & 2.28 & 4.71 \\
\hline jellico & 0.00 & 4.43 & 0.00 & 6.63 & 0.00 & 0.80 & 0.00 & 0.80 & 0.00 & 5.23 & $0 .<0$ & 5.23 \\
\hline $0-1000$ & 0.00 & 0.72 & 0.00 & 0.72 & 0.00 & 1.15 & 0.00 & 1.15 & 0.00 & 1.87 & 0.60 & 1.87 \\
\hline $0-1000$ & 0.00 & 3.43 & 0.72 & 4.15 & 0.00 & 2.10 & 0.00 & 2.10 & 0.00 & 5.53 & 0.72 & 6.25 \\
\hline D-1000 & 0.00 & 2.84 & 0.29 & 3.13 & 0.00 & 7.07 & 0.00 & 7.07 & 0.00 & 9.91 & 0.29 & 10.20 \\
\hline $\begin{array}{l}\text { or creek } \\
\text { o-1000 } \\
\text { nut mount oin }\end{array}$ & 0.00 & 30.04 & 1.46 & 31.48 & 0.00 & 24.72 & 0.00 & 24.72 & 0.00 & 56.76 & 1.96 & 56.20 \\
\hline $\begin{array}{l}0-1000 \\
\text { white ook }\end{array}$ & 0.00 & 11.66 & 1.38 & 13.06 & 0.00 & 1.13 & 1.55 & 2.68 & 0.00 & 12.79 & 2.53 & 15.72 \\
\hline $\begin{array}{l}0-1000 \\
\text { windrock }\end{array}$ & 0.00 & 0.56 & 0.10 & 0.66 & 0.00 & 1.95 & 0.01 & 1.96 & 0.00 & 2.51 & 0.11 & 2.62 \\
\hline $0-1000$ & 0.00 & 2.36 & 2.28 & 4.64 & 0.00 & $\begin{array}{r}4.40 \\
.9 .0 .\end{array}$ & 0.00 & 4.40 & 0.00 & 6.76 & 2.28 & 9.04 \\
\hline county totel $=$ & 0.00 & 73.65 & 26.01 & 99.46 & 0.00 & 49.35 & 8.32 & 57.67 & 0.00 & 122.80 & 36.33 & 157.13 \\
\hline & & & $\ldots$ & & & 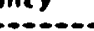 & & & & & & \\
\hline bitehland & & & & & & & & & & & & \\
\hline sewence $0-1000$ & 0.00 & 0.25 & 0.00 & 0.25 & 0.00 & 0.36 & 0.00 & 0.36 & 0.00 & 0.61 & 0.60 & 0.61 \\
\hline $0-1000$ & 0.00 & 59.53 & 5.46 & 66.97 & 0.00 & 21.27 & 2.03 & 23.30 & 0.00 & 80.80 & $\begin{array}{r}7.47 \\
.2 .0 .\end{array}$ & 88.27 \\
\hline county totel & 0.00 & 59.78 & 5.64 & 65.22 & 0.00 & 21.63 & 2.03 & 23.66 & 0.00 & 81.41 & 7.47 & 88.88 \\
\hline & & & & & & 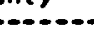 & & & & & 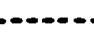 & \\
\hline bon air & & & & & & & & & & & & \\
\hline $\begin{array}{l}0-1000 \\
\text { lontens }\end{array}$ & 0.00 & 0.10 & 0.00 & 0.10 & 0.00 & 0.87 & 0.00 & 0.87 & 0.00 & 0.97 & 0.10 & 0.97 \\
\hline $\begin{array}{l}0-1000 \\
\text { Pfehland }\end{array}$ & 0.00 & 5.93 & 0.47 & 6.40 & 0.00 & 1.89 & 0.26 & 2.15 & 0.00 & 7.82 & 0.73 & 8.55 \\
\hline $\begin{array}{c}0-1000 \\
\text { sevance }\end{array}$ & 0.00 & 4.79 & 0.08 & 4.87 & 0.00 & 5.81 & 0.01 & 5.82 & 0.00 & 10.60 & 0.09 & 10.69 \\
\hline $0-1000$ & 0.00 & 6.08 & 1.97 & 8.05 & 0.00 & 5.37 & 4.76 & 10.13 & 0.00 & 11.45 & 6.73 & 18.18 \\
\hline
\end{tabular}




\begin{tabular}{|c|c|c|c|c|c|c|c|c|c|c|c|c|}
\hline county & 0.00 & 16.90 & 2.52 & 19.42 & 0.00 & 13.96 & 5.03 & 18.97 & 0.00 & 30.86 & 7.55 & 38.39 \\
\hline & & & & & & & & & & & & \\
\hline $0-1000$ & 0.00 & 2.30 & 0.44 & 2.76 & 0.00 & 3.56 & 1.83 & 5.39 & 0.00 & 5.86 & 2.27 & 8.13 \\
\hline $0-1000$ & 0.00 & 5.37 & 1.21 & 6.58 & 0.00 & 1.04 & 0.10 & 1.14 & 0.00 & 6.61 & 1.31 & 7.72 \\
\hline $0-1000$ & 0.00 & 0.28 & 1.09 & 1.37 & 0.00 & 0.00 & 0.11 & 0.11 & 0.00 & 0.28 & 1.20 & 1.48 \\
\hline county cotal & 0.00 & 7.95 & 2.74 & 10.69 & 0.00 & 4.60 & 2.04 & 6.64 & 0.00 & 12.55 & 4.78 & 17.33 \\
\hline
\end{tabular}

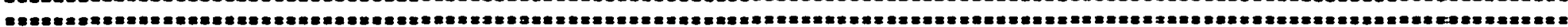
overburden

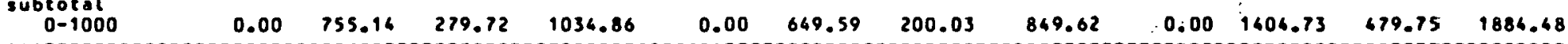

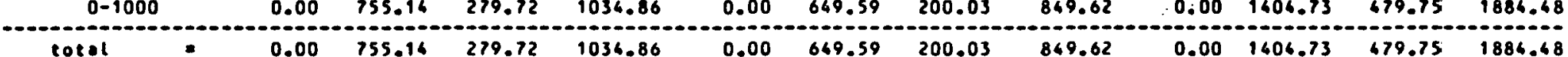

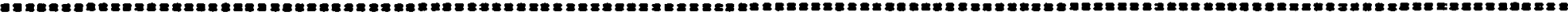
overburden

cotal

$\begin{array}{lllllllllllll}0-1000 & 0.00 & 755.16 & 279.72 & 1034.86 & 0.00 & 669.59 & 200.03 & 869.62 & 0.00 & 1406.73 & 679.75 & 1886.68\end{array}$

grond totel $=0.00 \quad 755.14 \quad 279.72 \quad 1034.86 \quad 0.00 \quad 649.59 \quad 200.03 \quad 869.62 \quad 0.00 \quad 1404.73 \quad 479.75 \quad 1884.48$

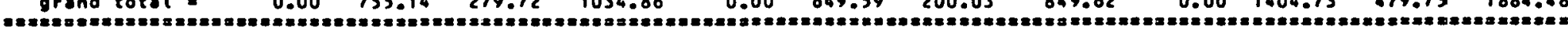

total tonnage of identifted coal resources in tennessee

1884.48

resource figures taken from:

source

tenn dv geol bull 63 rear

1959 base year of resource

1959 
overage onolyses of coal in tennessee

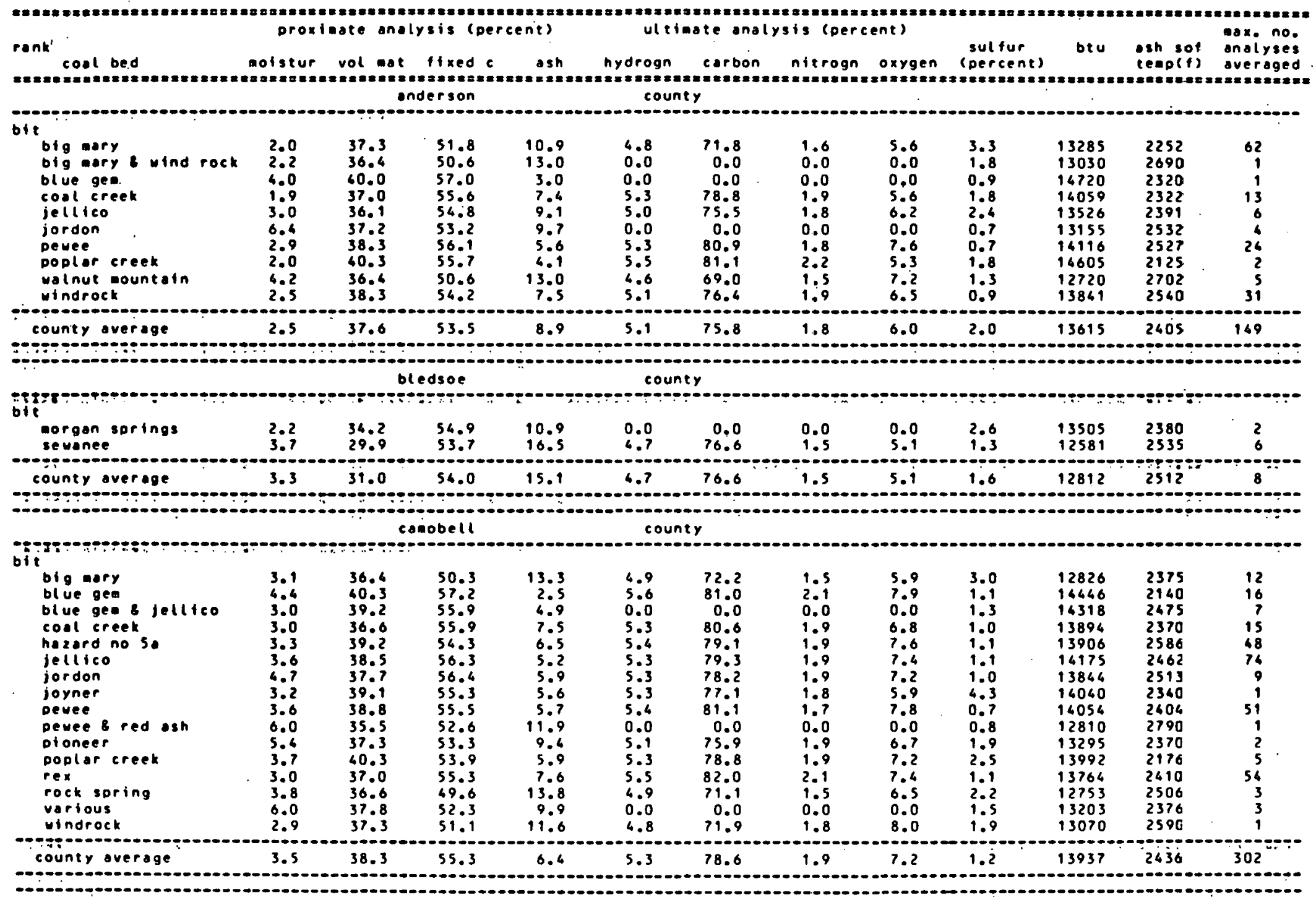


claiborne

county

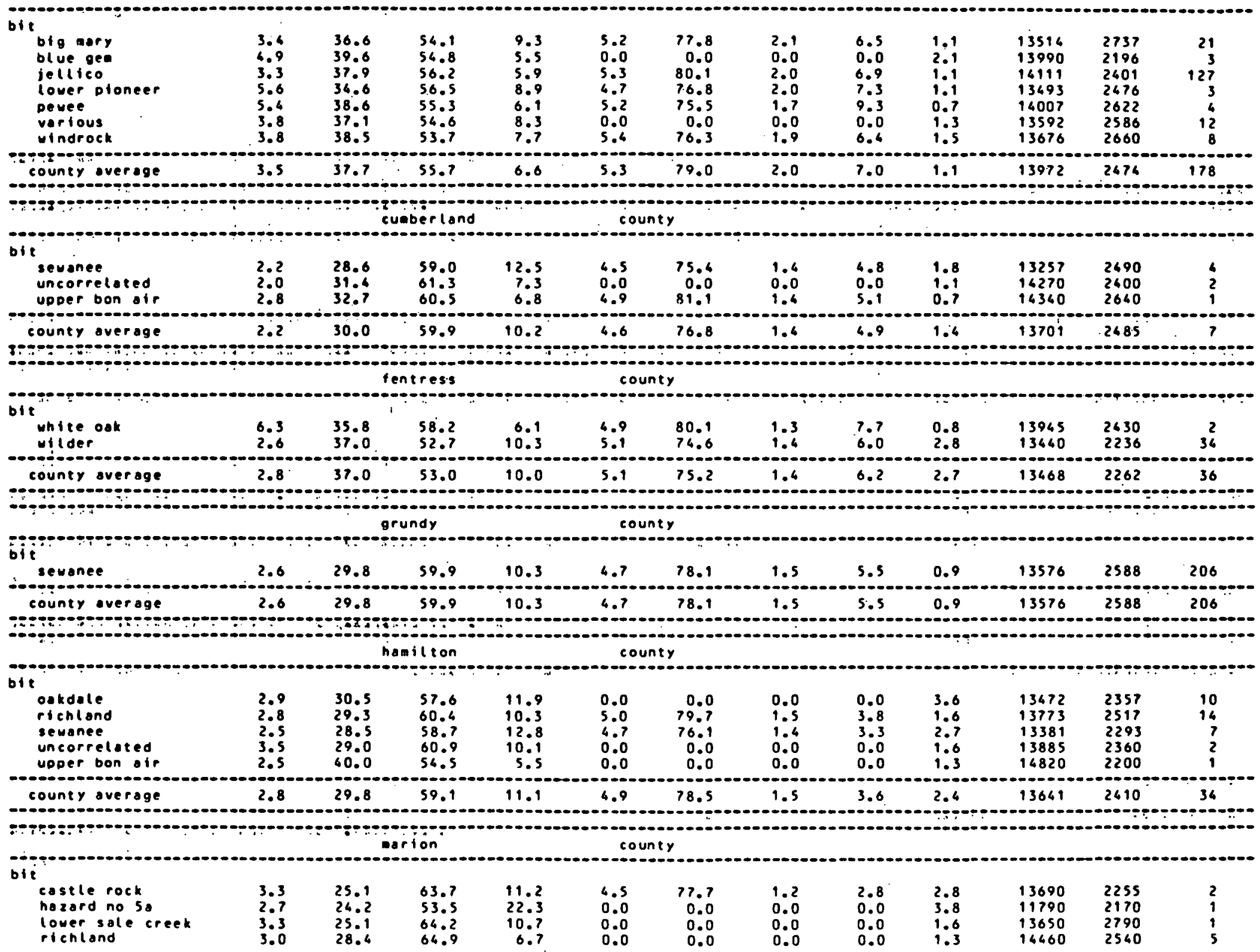




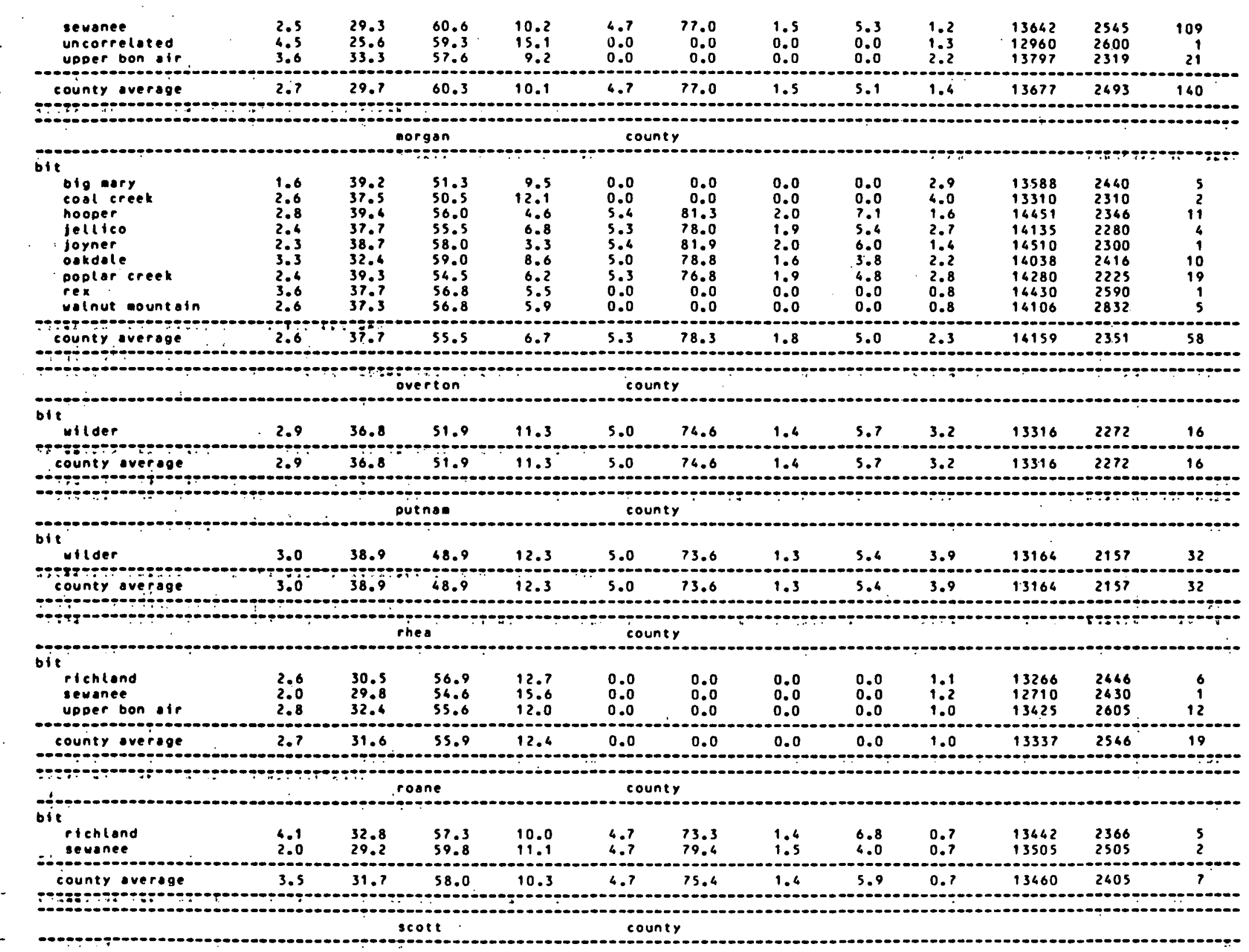


Tennessee

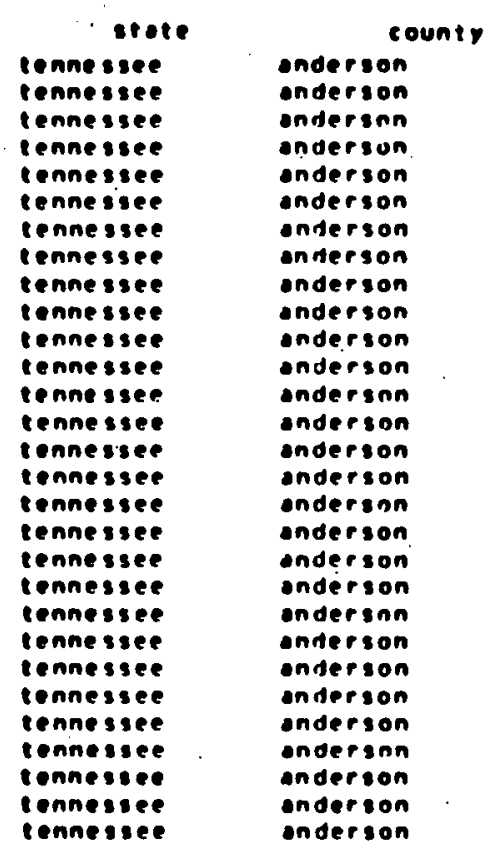

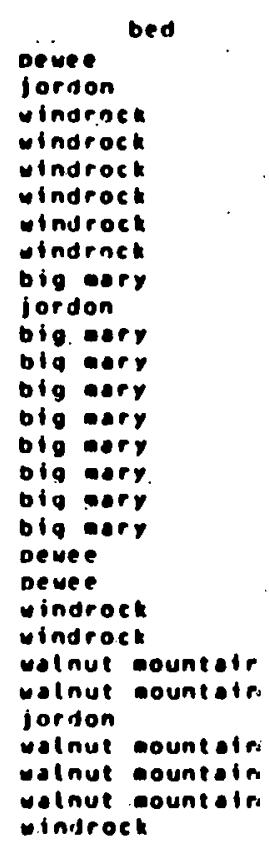

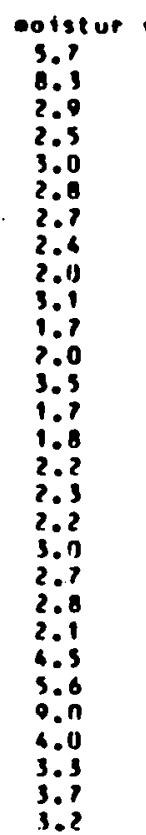

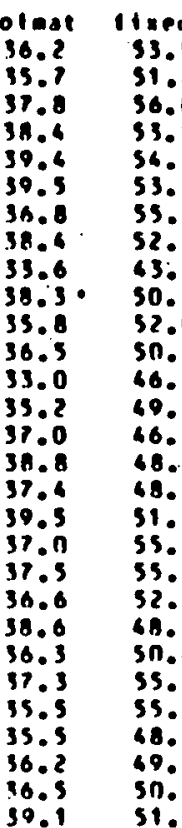

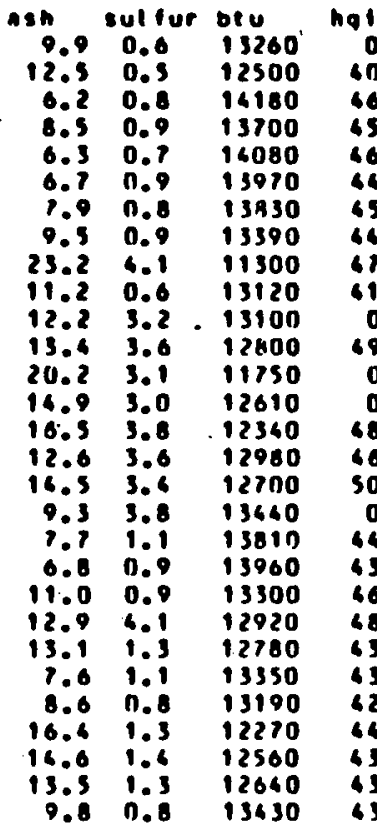

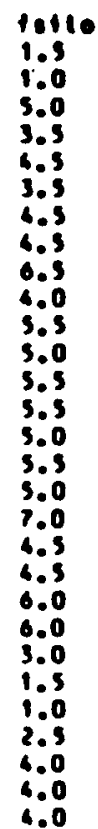

continued...... 


\begin{tabular}{|c|c|c|c|c|c|c|c|c|c|c|c|c|}
\hline \multicolumn{2}{|c|}{ state } & & & ed & noi & volmat & fixe & ash & sut & ur btu & hailo & fs:10 \\
\hline $\begin{array}{l}\text { tennessee } \\
\text { tennessee }\end{array}$ & $\begin{array}{l}\text { anderson } \\
\text { anderson }\end{array}$ & $\begin{array}{l}\text { bian } \\
\text { big? }\end{array}$ & $\begin{array}{l}\text { mary } \\
\text { mary }\end{array}$ & & $\begin{array}{l}2.7 \\
1.9\end{array}$ & $\begin{array}{l}34.9 \\
37.5\end{array}$ & $\begin{array}{l}45.9 \\
47.1\end{array}$ & $\begin{array}{l}17.2 \\
15.4\end{array}$ & $\begin{array}{l}3.5 \\
4.2\end{array}$ & $\begin{array}{l}11910 \\
12520\end{array}$ & $\begin{array}{r}0 \\
45\end{array}$ & $\begin{array}{l}4.5 \\
6.0\end{array}$ \\
\hline tenne ssee. & anderson & bian. & mary 8 & wino ro & $? ?$ & 36.4 & 50.6 & 13.0 & 1.8 & 13030 & 46 & 4.5 \\
\hline tennesseo & bledsne & sewar & inee & & 4.7 & 33.4 & 56.3 & 10.3 & 0.7 & 13570 & 51 & 5.0 \\
\hline tennessee & blensoe & sewar & inee & & 3.5 & 31.5 & 46.5 & 22.0 & 3.1 & 19570 & 58 & 6.5 \\
\hline tennesspe & bledsoe & sevar & inee & & 4.6 & 28.9 & 51.5 & 19.6 & 1.0 & 11960 & 60 & 3.5 \\
\hline tennessee & blensoe & norn & ian sor & ings & 2.3 & 34.9 & 55.6 & 9.5 & 1.7 & 13740 & 54 & 7.0 \\
\hline tennessee & campbell & coal & creek & & $6 . ?$ & 35.6 & 57.5 & 6.9 & 0.7 & 14010 & 47 & 6.0 \\
\hline tennessep & campbell & coal & creek & & 3.3 & 33.5 & 50.2 & 16.3 & 0.7 & 12610 & 48 & 4.5 \\
\hline tennessee & campoe ll & jell & $i<0$ & & 4.6 & 36.8 & 52.5 & 10.7 & 1.8 & 13280 & 52 & 5.0 \\
\hline tennessee & cannbell & jell & $i c o$ & & 4.6 & 38.6 & 56.2 & 5.2 & 0.8 & 14230 & 45 & 4.5 \\
\hline tenne ssee & campoe l l & jell & $i c o$ & & 3.0 & $4 n .1$ & 57.8 & 2.1 & 0.8 & 14610 & 0 & 5.5 \\
\hline tennessee & camobell & jell & ico & & 3.5 & 38.8 & 56.0 & 5.2 & 0.8 & 14270 & 0 & 5.0 \\
\hline tennessee & camndell & jell & $i<0$ & & 5.13 & 35.4 & 54.6 & 10.0 & 1.0 & 13370 & 53 & 5.0 \\
\hline tennessee & campbell & jell & $i c o$ & & 6.7 & 35.4 & 56.2 & 8.6 & 0.9 & 13710 & 49 & 5.5 \\
\hline tennessee & campbell & varic & ous & & 7.3 & 37.2 & 54.3 & 8.5 & 1.0 & 13270 & 46 & 1.5 \\
\hline tennessee & camobell & $j e l l$ & ico & & 4.5 & 36.5 & 56.0 & 7.5 & 1.0 & 13710 & 43 & 4.0 \\
\hline tenne's see & campbell & hazal & ird no & $5 a$ & 4.3 & 38.5 & 51.8 & 9.7 & 0.9 & 13330 & 47 & 5.0 \\
\hline tennessee & camobell & rock & sprin & & 4.0 & 33.4 & 48.5 & 18.1 & 1.0 & 12040 & 42 & 3.0 \\
\hline tennessee & camobell & Dever & & & 4.9 & 35.0 & $5 ? .6$ & 12.4 & 0.6 & 12890 & 0 & 3.0 \\
\hline tenne sspee & campoell & Deres & & & 4.7 & 36.0 & 53.6 & 10.4 & 0.6 & 13200 & 0 & 4.5 \\
\hline tennessee & campbell & Dewe & & & 7.2 & 35.2 & 52.8 & 12.0 & 0.7 & 12970 & 0 & 1.5 \\
\hline tennessee & camobell & oeves & & & 3.2 & 37.9 & 52.7 & 10.0 & 0.8 & 13330 & 0 & 4.5 \\
\hline tennessee & camobell & naza & ro no & $5 a$ & 3.2 & 39.5 & 51.8 & 8.7 & 2.8 & 13490 & 46 & 4.0 \\
\hline tennessee & campoell & bign & mary & & 3.6 & 35.7 & 50.4 & 13.9 & 3.5 & 12770 & 43 & 4.5 \\
\hline tennesspe & campbell & varic & ous & & 6.1 & 36.9 & 53.6 & 9.5 & 1.2 & 13310 & 0 & 2.0 \\
\hline tennessee & campbell & varic & ous & & 4.7 & 30.6 & 48.9 & 11.7 & 2.4 & 13030 & $n$ & 4.5 \\
\hline tennessee & campbell & naza & ro no & $5 a$ & 4.3 & 37.4 & 51.9 & 10.7 & 9.8 & 93960 & 45 & 5.5 \\
\hline tennessee & camnbell & Dinns & eer & & 7.7 & 36.4 & 52.7 & 10.9 & 1.3 & 12930 & 48 & 1.0 \\
\hline tennessee & campbell & newe & e & & 0.0 & 37.3 & 52.5 & 10.2 & 0.7 & 13270 & 44 & 4.5 \\
\hline tennessee & camobell & nazar & ro no & Sa & 5.8 & 38.0 & 54.4 & 7.6 & 1.0 & 13550 & 44 & 2.5 \\
\hline tennessee & camobell & Dewes & e $8 \mathrm{re}$ & d ash & 6.0 & 35.5 & $5 ? .6$ & 19.9 & 0.8 & 12810 & 46 & 2.0 \\
\hline tennessee & campbell & hazai & ird no & 50 & 3.1 & 30.4 & 52.6 & 8.0 & 1.1 & 13140 & 47 & 5.5 \\
\hline tennessee & camnbell & hazar & rd no & $5 \rightarrow$ & 3.4 & 38.1 & 53.4 & 8.5 & 1.1 & 13590 & 47 & 5.5 \\
\hline tennessee & campbell & haza & rd no & $5 a$ & 3.1 & 37.3 & 52.2 & 10.5 & 1.4 & 13280 & 44 & 4.5 \\
\hline tennessee & campbel l & hazar & rd no & Sa & 3.9 & 35.6 & 51.3 & 13.1 & 1.2 & 12800 & 46 & 5.0 \\
\hline tennessee & campoell & oewe & & & 3.3 & 40.0 & 56.1 & 3.9 & 0.6 & 14360 & 0 & 4.5 \\
\hline tennessee & campdell & Dewe & & & 3.4 & $3 R .9$ & 54.3 & 6.8 & 0.6 & 13940 & 39 & 5.0 \\
\hline tennessee & camobell & wind & rock & & $? .9$ & 37.3 & 51.1 & 11.6 & 1.9 & 13070 & $\angle 5$ & 5.0 \\
\hline tennessee & camobell & jords & & & 4.5 & $3 n .5$ & 57.5 & 0.0 & 0.8 & .13810 & 44 & 4.5 \\
\hline tennessep & campbell & jell & ico & & 4.2 & 38.9 & 50.3 & $1 n .8$ & 3.4 & 13200 & 53 & 3.5 \\
\hline tennessee & campoeli & jordc & on & & 4.7 & 37.1 & 58.9 & 4.0 & 0.8 & 14240 & 0 & 4.5 \\
\hline tennessee & camobell & bian & mary & & 2.6 & 35.5 & 47.0 & 17.5 & 4.1 & 12180 & 45 & 0.5 \\
\hline tennessee & camniell & bian & mary & & 2.5 & 35.6 & 46.8 & 17.6 & 4.4 & 12190 & 45 & 6.0 \\
\hline tennessee & camobell & opues & e & & 6.8 & 35.0 & 55.0 & 0.0 & 0.9 & 13530 & 44 & 4.5 \\
\hline tennessee & campbell & hazar & rd no & 53 & 5.0 & 39.0 & 55.4 & 6.6 & 0.8 & 13710 & 46 & 2.5 \\
\hline tennessee & camphell & big. & $\operatorname{mar} y$ & & 3.1 & 34.2 & 50.7 & 15.1 & $? .1$ & 12600 & 67 & 4.5 \\
\hline tennessee & camnell & big & mary. & & $? .9$ & 34.7 & 50.4 & 14.9 & 2.2 & 12610 & 45 & 0.0 \\
\hline tennessee & campbe l l & blue & yem 8 & jellico & 3.7 & 37.1 & 53.6 & 9.3 & 1.6 & 13590 & 0 & 5.0 \\
\hline tennessee & camobell & jell & ico & & 4.1 & 35.5 & 59.3 & 5.2 & 1.1 & 14210 & 0 & 5.5 \\
\hline
\end{tabular}




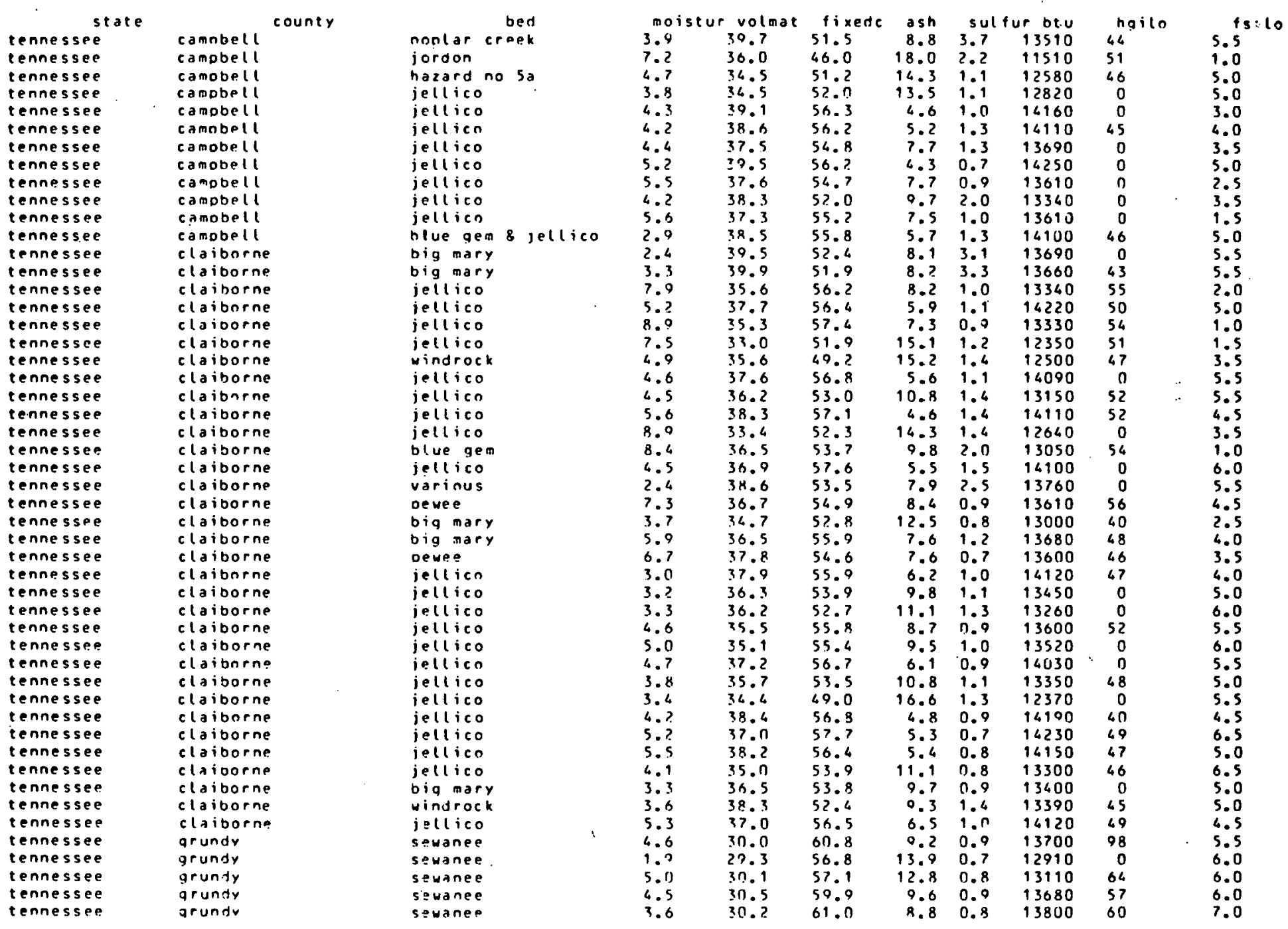




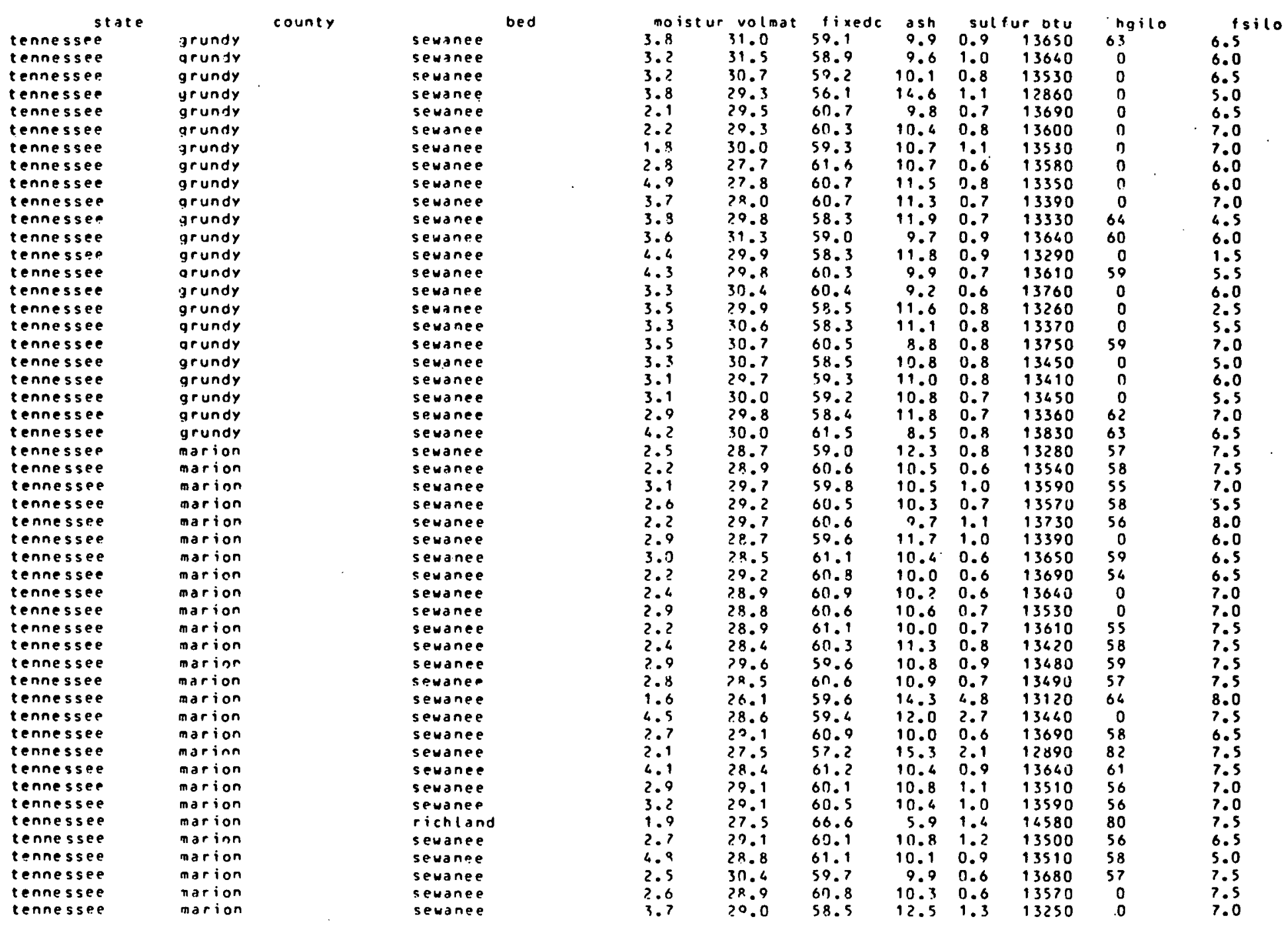




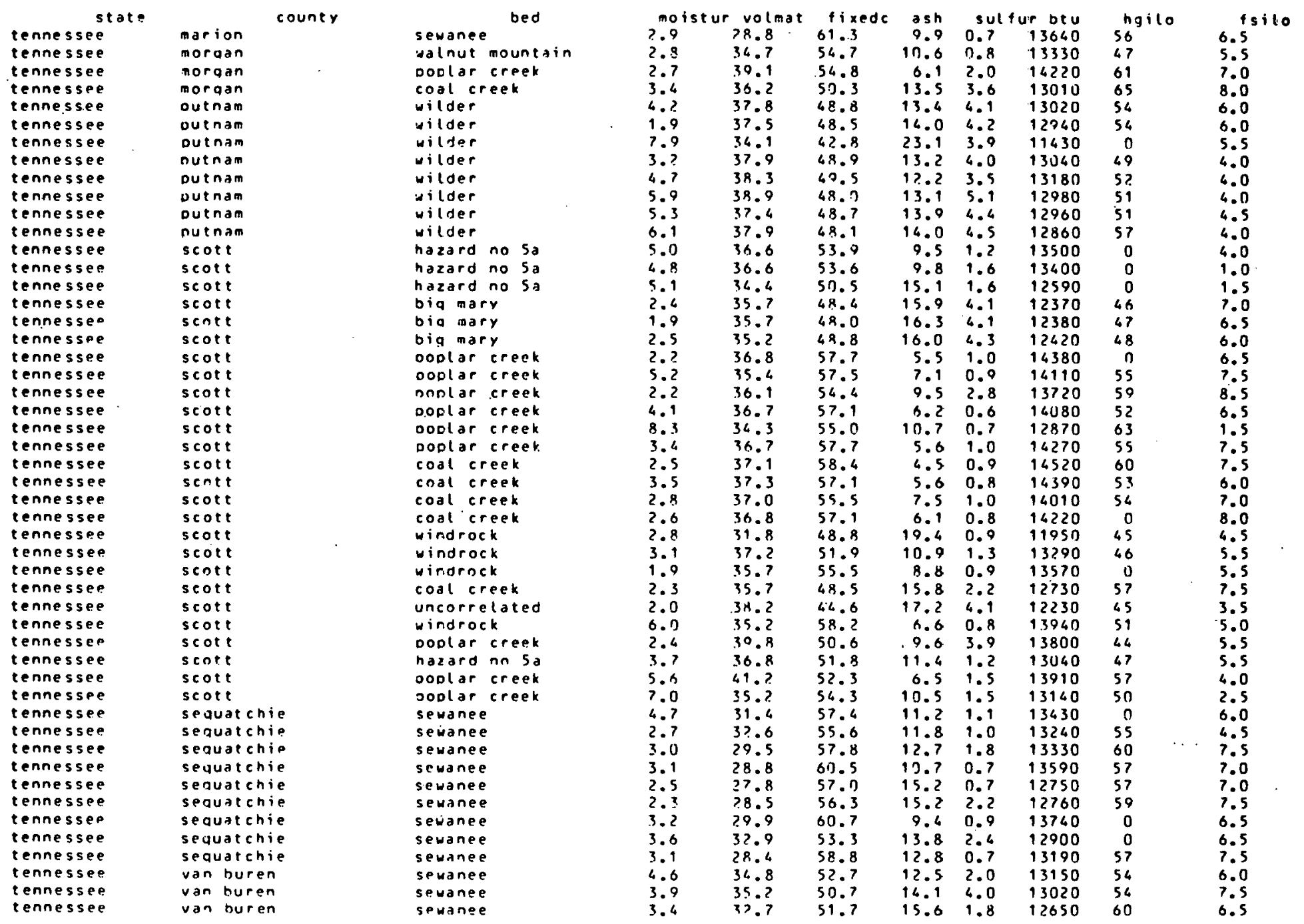



National Coal Resources Data Systen

U.S. Geological survey

identified coal resources in texas

(In willions of short tons)

all records contain unclassified thickness andfor reliabllity

totol tonnages 8976.70

stote county

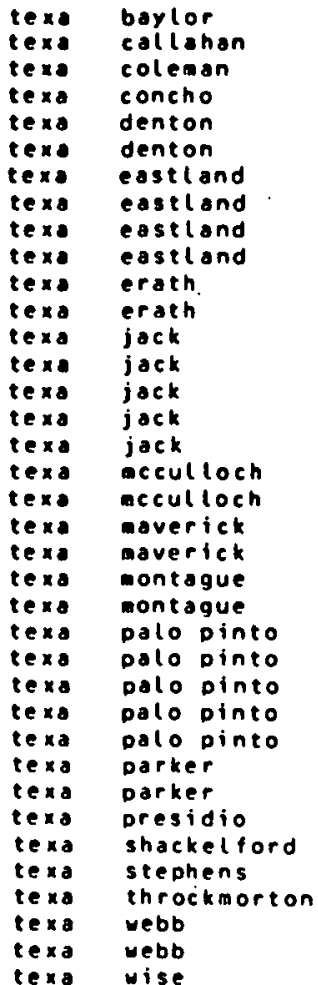

bed

newcastle
newcastle
bull creek
bull creek
bridgeport
bridgeport
thurber
thurber
newcastle
newcastle
inurber
thurber
thurber
thurber
bridgeoort
bridgeport
dalton
bull. creek
chattin
uncorrelated
uncorrelated
bridgeport
bridgeport
thurber
thurber
dalton
abbott
sunday creek
dalton
dalton
uncorrelated
newcastle
newcastle
newcastle
santo tomas
san pedro
thurber

rank

bit
bit
bit
bit
bit
bit
bit
bit
bit
bit
bit
bit
bit
bit
bit
bit
bit
bit
bit
bit
bit
bit
bit
bit
bit
bit
bit
bit
bit
bit
bit
bit
bit
bit
bit
bit
bit

base

base.
year

\begin{tabular}{|c|c|}
\hline $\begin{array}{l}\text { Orig } \\
\text { orig }\end{array}$ & $\begin{array}{l}\text { unclass } \\
\text { unclass }\end{array}$ \\
\hline orig & uncilass \\
\hline rig & unctass \\
\hline ig & unctoss \\
\hline orig & unclass \\
\hline orig & unclass \\
\hline orig & unctoss \\
\hline orig & unclass \\
\hline orig & uncloss \\
\hline orig & uncless \\
\hline orig & unclass \\
\hline orig & unclass \\
\hline orig & unclass \\
\hline orig & unclass \\
\hline orig & unctass \\
\hline orig & unclass \\
\hline orig & uncless \\
\hline orig & unclass \\
\hline orig & unclass \\
\hline$\therefore$ & unclass \\
\hline orig & unclass \\
\hline orig & uncloss \\
\hline & unclass \\
\hline orig & unclass \\
\hline orig & unclass \\
\hline orig & unclass \\
\hline orig & unclass \\
\hline orig & unclass \\
\hline orta & unclass \\
\hline orig & unclass \\
\hline orig & uncloss \\
\hline ori & unclass \\
\hline - & unclass \\
\hline & unctass \\
\hline ori & unctess \\
\hline orig & unclass \\
\hline
\end{tabular}

Gerburden
(feet)

$0-2000$

$0-2000$
$0-2000$

$0-2000$

$0-2000$

$0-3000$

$0-2000$

$0-3000$

$0-2000$

$0=3000$

$0=2000$

$0-3000$

$0-3000$

$0-3000$

$0-3000$

$0-3000$
$0-2000$

$0=2000$

$0-2000$
$0-2000$

$0=2000$

$0=3000$

$0=2000$

$0=3000$

$0-2000$

$0-3000$

$0-2000$

$0-2000$

$0-2000$

$0-2000$

$0-3000$

$0-3000$

$0-2000$

$0-2000$

$0 \div 2000$

$0-3000$

$0-3000$
$0-2000$ reliability tonnage

$\begin{array}{lr}\text { inferred } & 31.20 \\ \text { inferred } & 269.60 \\ \text { inferred } & 128.00 \\ \text { inferred } & 29.10 \\ \text { inferred } & 89.90 \\ \text { inferred } & 116.80 \\ \text { inferred } & 17.10 \\ \text { interred } & 22.20 \\ \text { inferred } & 124.80 \\ \text { inferred } & 141.90 \\ \text { interred } & 27.80 \\ \text { inferred } & 36.10 \\ \text { inferred } & 620.60 \\ \text { inferred } & 806.20 \\ \text { inferred } & 128.20 \\ \text { inferred } & 189.50 \\ \text { inferred } & 1.40 \\ \text { inferred } & 133.90 \\ \text { inferred } & 2.00 \\ \text { inferred } & 125.00 \\ \text { inferred } & 400.00 \\ \text { inferred } & 283.90 \\ \text { inferred } & 308.00 \\ \text { inferred } & 645.00 \\ \text { inferred } & 578.20 \\ \text { inferred } & 75.60 \\ \text { inferred } & 6.00 \\ \text { inferred } & 14.00 \\ \text { inferred } & 175.50 \\ \text { inferred } & 228.00 \\ \text { inferred } & 25.00 \\ \text { inferred } & 32.80 \\ \text { interred } & 97.20 \\ \text { inferred } & 374.90 \\ \text { inferred } & 90.00 \\ \text { inferred } & 24.00 \\ \text { inferred } & 691.20\end{array}$




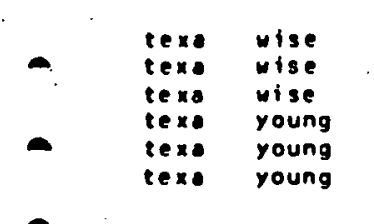

-

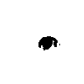

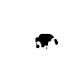

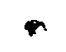

m
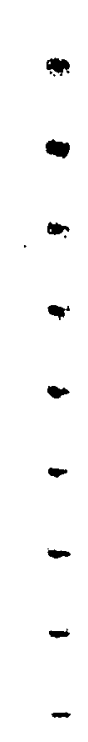

thurber

bridgeport

bridgeport

thurber

newcastle

resource figures taken from:

source

usgs bull 1262d

$\begin{array}{lll}\text { orig unclass } & 0-3000 \\ \text { orig unclass } & 0-2000 \\ \text { orig unclass } & 0-3000 \\ \text { orig unclass } & 0-2000 \\ \text { orig unclass } & 0-3000 \\ \text { orig unclass } & 0-2000\end{array}$

inferred

inferred

interrea

inferred

inferred

897.90

198.00

210.30

70.60

91.70

717.60

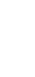

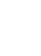

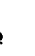

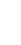

$+$

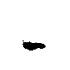

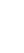


average onalyses of cool in texas

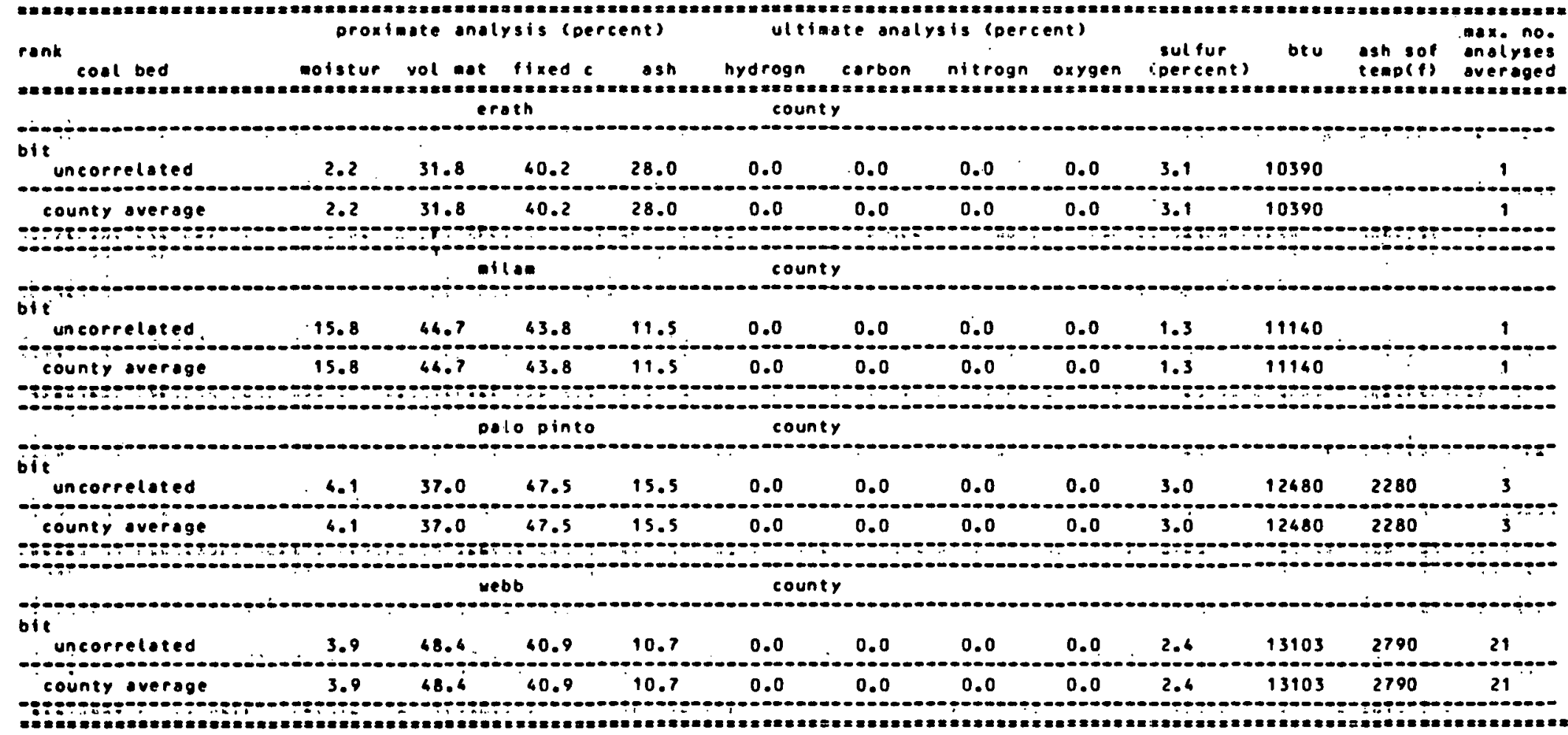


Nattonal Coat Resources Date System

U.S. Geological Survey

identified cool resources in utah

(In alltions of short tons)

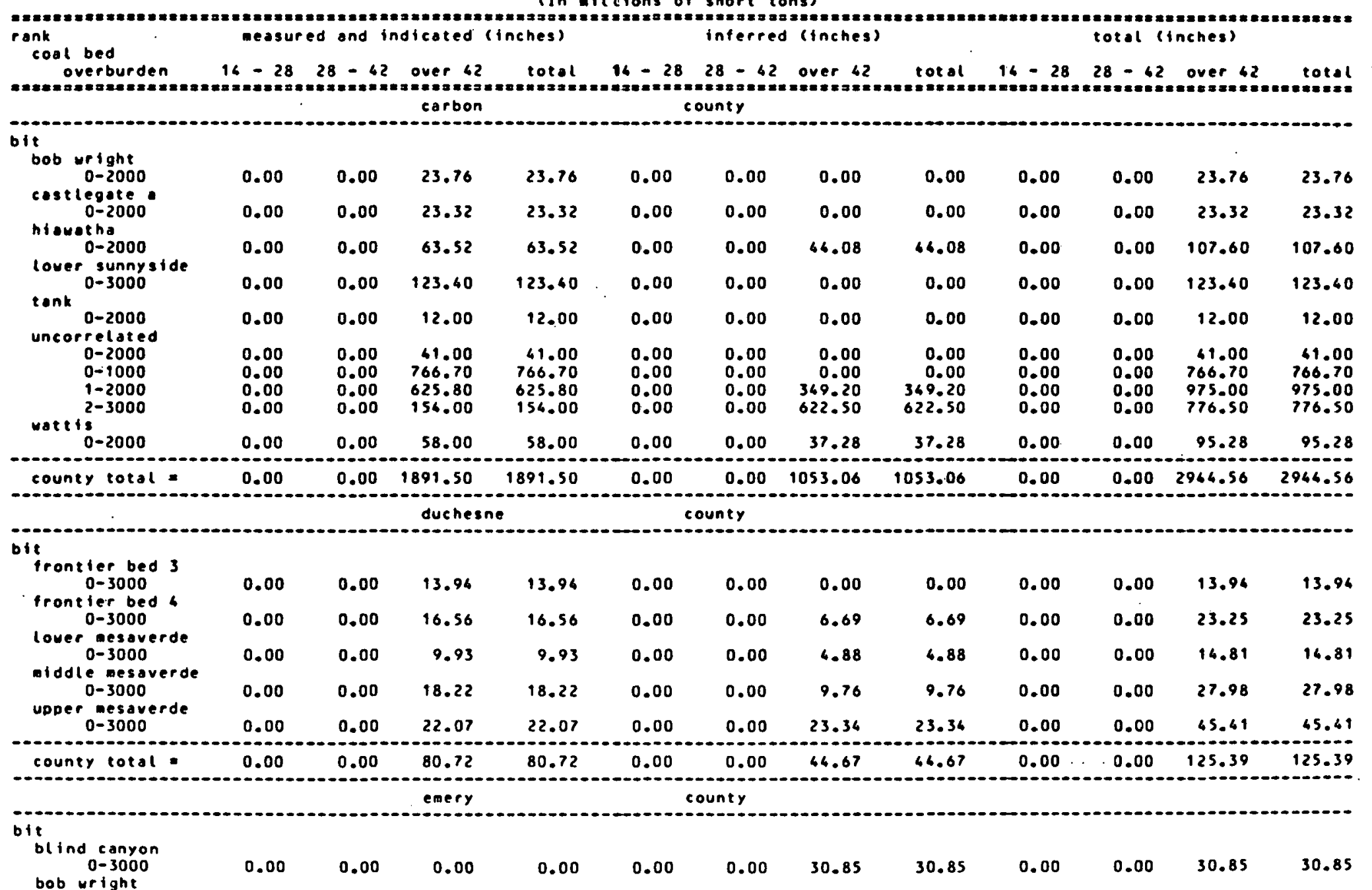




\begin{tabular}{|c|c|c|c|c|c|c|c|c|c|c|c|c|}
\hline $0-2000$ & 0.00 & 0.00 & 23.76 & 23.76 & 0.00 & 0.00 & 0.00 & 0.00 & 0.00 & 0.00 & 23.76 & 23.76 \\
\hline $\begin{array}{l}\text { cand } \text { and } \\
2-3000\end{array}$ & 0.00 & 0.00 & 0.00 & 0.00 & 0.00 & 0.00 & 61.76 & 41.76 & 0.00 & 0.00 & 61.76 & 41.76 \\
\hline $\begin{array}{c}0-3000 \\
0-2000 \\
2-3000\end{array}$ & $\begin{array}{l}0.00 \\
0.00 \\
0.00\end{array}$ & $\begin{array}{l}0.00 \\
0.00 \\
0.00\end{array}$ & $\begin{array}{r}0.00 \\
23.32 \\
0.00\end{array}$ & $\begin{array}{r}0.00 \\
23.32 \\
0.00\end{array}$ & $\begin{array}{l}0.00 \\
0.00 \\
0.00\end{array}$ & $\begin{array}{l}0.00 \\
0.00 \\
0.00\end{array}$ & $\begin{array}{r}19.24 \\
0.00 \\
62.64\end{array}$ & $\begin{array}{r}19.24 \\
0.00 \\
62.64\end{array}$ & $\begin{array}{l}0.00 \\
0.00 \\
0.00\end{array}$ & $\begin{array}{l}0.00 \\
0.00 \\
0.00\end{array}$ & $\begin{array}{l}19.24 \\
23.32 \\
62.64\end{array}$ & $\begin{array}{l}19.26 \\
23.32 \\
62.64\end{array}$ \\
\hline $\begin{array}{r}\text { hrewatha } \\
0-3000 \\
0-2000\end{array}$ & $\begin{array}{l}0.00 \\
0.00\end{array}$ & $\begin{array}{l}0.00 \\
0.00\end{array}$ & $\begin{array}{l}0.00 \\
0.00\end{array}$ & $\begin{array}{l}0.00 \\
0.00\end{array}$ & $\begin{array}{l}0.00 \\
0.00\end{array}$ & $\begin{array}{l}0.00 \\
0.00\end{array}$ & $\begin{array}{l}21.40 \\
44.08\end{array}$ & $\begin{array}{l}21.60 \\
44.08\end{array}$ & $\begin{array}{l}0.00 \\
0.00\end{array}$ & $\begin{array}{l}0.00 \\
0.00\end{array}$ & $\begin{array}{l}21.40 \\
44.08\end{array}$ & $\begin{array}{l}21.40 \\
44.08\end{array}$ \\
\hline $\begin{array}{c}\text { lower coal zone } \\
0-1000 \\
\text { unclass }\end{array}$ & $\begin{array}{l}0.00 \\
0.00\end{array}$ & $\begin{array}{l}0.00 \\
0.00\end{array}$ & $\begin{array}{r}71.73 \\
217.41\end{array}$ & $\begin{array}{r}71.73 \\
217.49\end{array}$ & $\begin{array}{l}0.00 \\
0.00\end{array}$ & $\begin{array}{l}0.00 \\
0.00\end{array}$ & $\begin{array}{l}0.00 \\
0.00\end{array}$ & $\begin{array}{l}0.00 \\
0.00\end{array}$ & $\begin{array}{l}0.00 \\
0.00\end{array}$ & $\begin{array}{l}0.00 \\
0.00\end{array}$ & $\begin{array}{r}71.73 \\
217.41\end{array}$ & $\begin{array}{r}71.73 \\
217.61\end{array}$ \\
\hline $\begin{array}{c}\text { uncorrelated } \\
0-1000 \\
\text { unclass } \\
\text { waters } \\
0-2000\end{array}$ & $\begin{array}{l}0.00 \\
0.00 \\
0.00\end{array}$ & $\begin{array}{l}0.00 \\
0.00 \\
0.00\end{array}$ & $\begin{array}{r}15.54 \\
137.10 \\
0.00\end{array}$ & $\begin{array}{r}15.54 \\
137.10 \\
0.00\end{array}$ & $\begin{array}{l}0.00 \\
0.00 \\
0.00\end{array}$ & $\begin{array}{l}0.00 \\
0.00 \\
0.00\end{array}$ & $\begin{array}{r}0.00 \\
388.78 \\
37.28\end{array}$ & $\begin{array}{r}0.00 \\
388.78 \\
37.28\end{array}$ & $\begin{array}{l}0.00 \\
0.00 \\
0.00\end{array}$ & $\begin{array}{l}0.00 \\
0.00 \\
0.00\end{array}$ & $\begin{array}{r}15.54 \\
525.88 \\
37.28\end{array}$ & $\begin{array}{r}15.54 \\
525.88 \\
37.28\end{array}$ \\
\hline county total & 0.00 & 0.00 & 488.86 & 488.86 & 0.00 & 0.00 & 666.03 & 646.03 & 0.00 & 0.00 & 1136.89 & 1136.89 \\
\hline \multirow{2}{*}{\multicolumn{13}{|c|}{ bit }} \\
\hline & & & & & & & & & & & & \\
\hline $\begin{array}{l}0-3000 \\
0-2000 \\
0-1000\end{array}$ & $\begin{array}{l}0.00 \\
0.00 \\
0.00\end{array}$ & $\begin{array}{l}0.00 \\
0.00 \\
0.00\end{array}$ & $\begin{array}{r}181.25 \\
89.27 \\
154.55\end{array}$ & $\begin{array}{r}181.25 \\
89.27 \\
154.55\end{array}$ & $\begin{array}{l}0.00 \\
0.00 \\
0.00\end{array}$ & $\begin{array}{l}0.00 \\
0.00 \\
0.00\end{array}$ & $\begin{array}{r}316.84 \\
30.18 \\
29.02\end{array}$ & $\begin{array}{r}316.84 \\
30.18 \\
29.02\end{array}$ & $\begin{array}{l}0.00 \\
0.00 \\
0.00\end{array}$ & $\begin{array}{l}0.00 \\
0.00 \\
0.00\end{array}$ & $\begin{array}{l}498.09 \\
119.65 \\
183.57\end{array}$ & $\begin{array}{l}698.09 \\
119.65 \\
183.57\end{array}$ \\
\hline 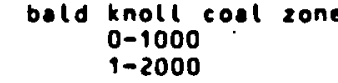 & $\begin{array}{l}0.00 \\
0.00\end{array}$ & $\begin{array}{l}0.00 \\
0.00\end{array}$ & $\begin{array}{l}54.05 \\
2.97\end{array}$ & $\begin{array}{r}54.05 \\
2.97\end{array}$ & $\begin{array}{l}0.00 \\
0.00\end{array}$ & $\begin{array}{l}0.00 \\
0.00\end{array}$ & $\begin{array}{r}0.00 \\
56.72\end{array}$ & $\begin{array}{r}0.00 \\
56.72\end{array}$ & $\begin{array}{l}0.00 \\
0.00\end{array}$ & $\begin{array}{l}0.00 \\
0.00\end{array}$ & $\begin{array}{l}54.05 \\
57.69\end{array}$ & $\begin{array}{l}54.05 \\
57.69\end{array}$ \\
\hline $\begin{array}{c}\text { ehristensen zone } \\
0-3000 \\
0-2000 \\
0-1000\end{array}$ & $\begin{array}{l}0.00 \\
0.00 \\
0.00\end{array}$ & $\begin{array}{l}0.00 \\
0.00 \\
0.00\end{array}$ & $\begin{array}{l}147.20 \\
175.35 \\
271.02\end{array}$ & $\begin{array}{l}147.20 \\
175.35 \\
271.02\end{array}$ & $\begin{array}{l}0.00 \\
0.00 \\
0.00\end{array}$ & $\begin{array}{l}0.00 \\
0.00 \\
0.00\end{array}$ & $\begin{array}{r}486.17 \\
79.55 \\
129.36\end{array}$ & $\begin{array}{r}486.17 \\
79.55 \\
129.36\end{array}$ & $\begin{array}{l}0.00 \\
0.00 \\
0.00\end{array}$ & $\begin{array}{l}0.00 \\
0.00 \\
0.00\end{array}$ & $\begin{array}{l}633.37 \\
25.4 .90 \\
400.38\end{array}$ & $\begin{array}{l}633.37 \\
254.90 \\
400.38\end{array}$ \\
\hline $\begin{array}{c}\text { emery cool zone } \\
0-2000 \\
0-1000\end{array}$ & $\begin{array}{l}0.00 \\
0.00\end{array}$ & $\begin{array}{l}0.00 \\
0.00\end{array}$ & $\begin{array}{r}4.70 \\
119.91\end{array}$ & $\begin{array}{r}4.70 \\
119.91\end{array}$ & $\begin{array}{l}0.00 \\
0.00\end{array}$ & $\begin{array}{l}0.00 \\
0.00\end{array}$ & $\begin{array}{r}19.05 \\
9.48\end{array}$ & $\begin{array}{r}19.05 \\
9.48\end{array}$ & $\begin{array}{l}0.00 \\
0.00\end{array}$ & $\begin{array}{l}0.00 \\
0.00\end{array}$ & $\begin{array}{r}23.75 \\
129.39\end{array}$ & $\begin{array}{r}23.75 \\
129.39\end{array}$ \\
\hline $\begin{array}{c}\text { ferron coal zone } \\
0-2000 \\
0-1000\end{array}$ & $\begin{array}{l}0.00 \\
0.00\end{array}$ & $\begin{array}{l}0.00 \\
0.00\end{array}$ & $\begin{array}{r}22.02 \\
4.18\end{array}$ & $\begin{array}{r}22.02 \\
4.18\end{array}$ & $\begin{array}{l}0.00 \\
0.00\end{array}$ & $\begin{array}{l}0.00 \\
0.00\end{array}$ & $\begin{array}{l}0.00 \\
0.00\end{array}$ & $\begin{array}{l}0.00 \\
0.00\end{array}$ & $\begin{array}{l}0.00 \\
0.00\end{array}$ & $\begin{array}{l}0.00 \\
0.00\end{array}$ & $\begin{array}{r}22.02 \\
4.18\end{array}$ & $\begin{array}{r}22.02 \\
6.18\end{array}$ \\
\hline $\begin{array}{c}\text { henderson coel zone } \\
0-1000 \\
1-2000 \\
2-3000\end{array}$ & $\begin{array}{l}0.00 \\
0.00 \\
0.00\end{array}$ & $\begin{array}{l}0.00 \\
0.00 \\
0.00\end{array}$ & $\begin{array}{r}386.00 \\
75.00 \\
9.00\end{array}$ & $\begin{array}{r}386.00 \\
75.00 \\
9.00\end{array}$ & $\begin{array}{l}0.00 \\
0.00 \\
0.00\end{array}$ & $\begin{array}{l}0.00 \\
0.00 \\
0.00\end{array}$ & $\begin{array}{l}113.00 \\
251.00 \\
128.00\end{array}$ & $\begin{array}{l}113.00 \\
251.00 \\
128.00\end{array}$ & $\begin{array}{l}0.00 \\
0.00 \\
0.00\end{array}$ & $\begin{array}{l}0.00 \\
0.00 \\
0.00\end{array}$ & $\begin{array}{l}499.00 \\
326.00 \\
137.00\end{array}$ & $\begin{array}{l}499.00 \\
326.00 \\
137.00\end{array}$ \\
\hline $\begin{array}{c}\text { rees coal zone } \\
0-3000 \\
0-2000 \\
0-1000\end{array}$ & $\begin{array}{l}0.00 \\
0.00 \\
0.00\end{array}$ & $\begin{array}{l}0.00 \\
0.00 \\
0.00\end{array}$ & $\begin{array}{r}128.63 \\
23.36 \\
4.20\end{array}$ & $\begin{array}{r}128.63 \\
23.36 \\
4.20\end{array}$ & $\begin{array}{l}0.00 \\
0.00 \\
0.00\end{array}$ & $\begin{array}{l}0.00 \\
0.00 \\
0.00\end{array}$ & $\begin{array}{r}62.83 \\
0.00 \\
0.00\end{array}$ & $\begin{array}{r}42.83 \\
0.00 \\
0.00\end{array}$ & $\begin{array}{l}0.00 \\
0.00 \\
0.00\end{array}$ & $\begin{array}{l}0.00 \\
0.00 \\
0.00\end{array}$ & $\begin{array}{r}171.46 \\
23.36 \\
4.20\end{array}$ & $\begin{array}{r}171.46 \\
23.36 \\
4.20\end{array}$ \\
\hline $\begin{array}{c}\text { uncorrelated } \\
0-3000 \\
0-1000\end{array}$ & $\begin{array}{l}0.00 \\
0.00\end{array}$ & $\begin{array}{l}0.00 \\
0.00\end{array}$ & $\begin{array}{r}0.70 \\
29.67\end{array}$ & $\begin{array}{r}0.70 \\
29.67\end{array}$ & $\begin{array}{l}0.00 \\
0.00\end{array}$ & $\begin{array}{l}0.00 \\
0.00\end{array}$ & $\begin{array}{l}0.00 \\
0.00\end{array}$ & $\begin{array}{l}0.00 \\
0.00\end{array}$ & $\begin{array}{l}0.00 \\
0.00\end{array}$ & $\begin{array}{l}0.00 \\
0.00\end{array}$ & $\begin{array}{r}0.70 \\
29.67\end{array}$ & $\begin{array}{r}0.70 \\
29.67\end{array}$ \\
\hline county total = & 0.00 & 0.00 & 1883.03 & 1883.03 & 0.00 & 0.00 & 1689.20 & 1689.20 & 0.00 & 0.00 & 3572.23 & 3572.23 \\
\hline & & & grand & & & $y$ & & & & & & \\
\hline $\begin{array}{c}\text { ballard coal } \\
0-3000\end{array}$ & 0.00 & 0.00 & 85.10 & 85.10 & 0.00 & 0.00 & 5.70 & 5.70 & 0.00 & 0.00 & 90.80 & 90.80 \\
\hline
\end{tabular}




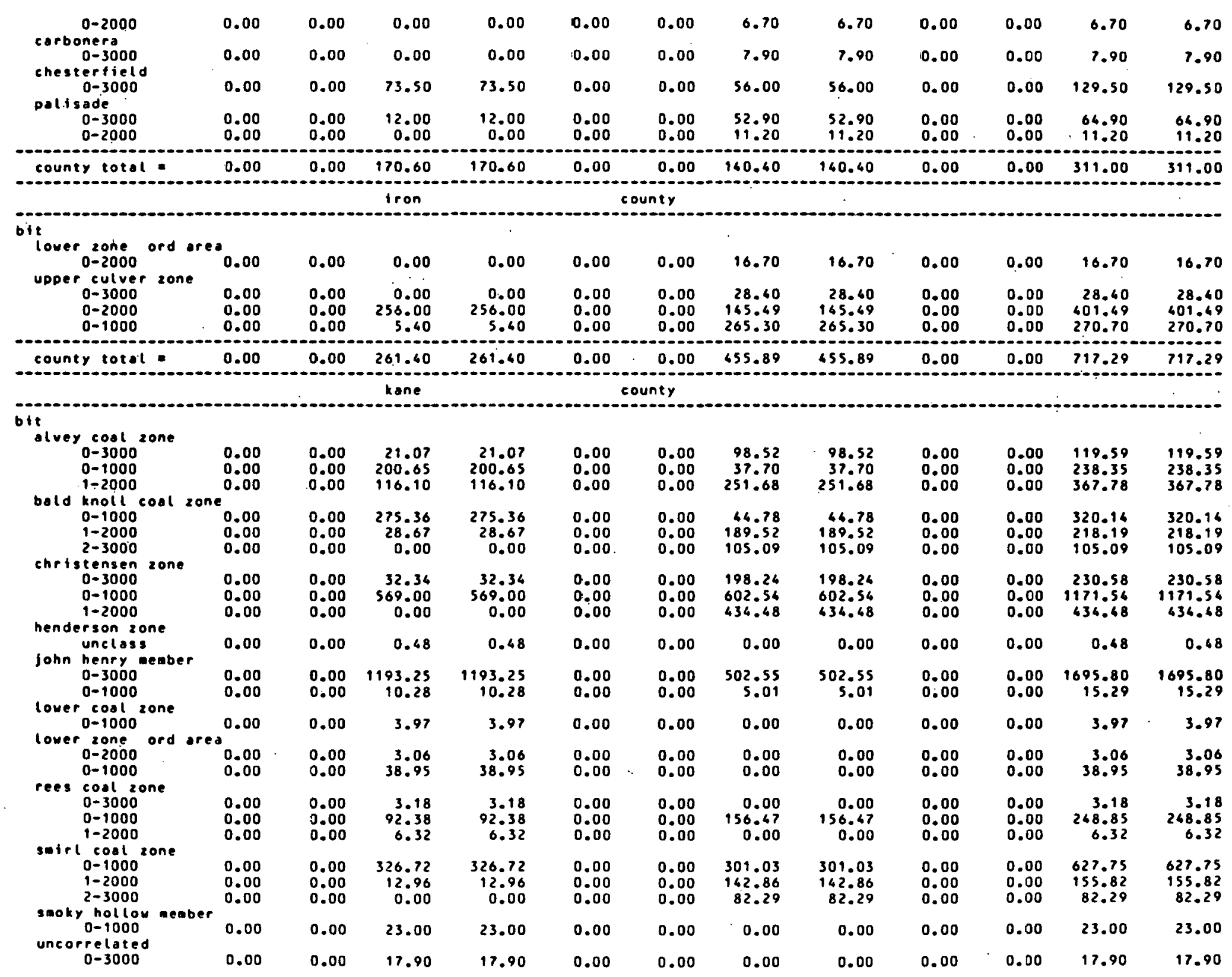




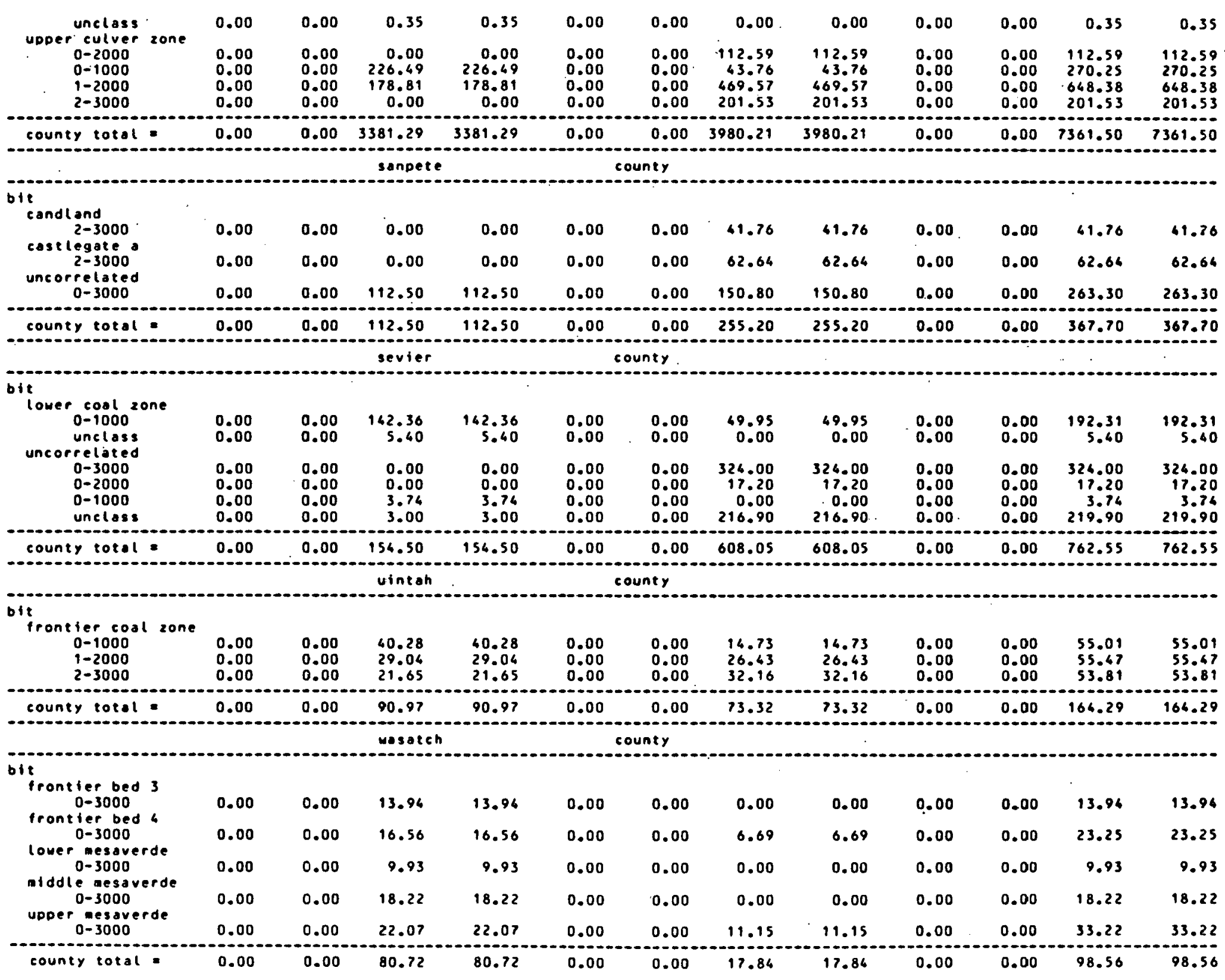




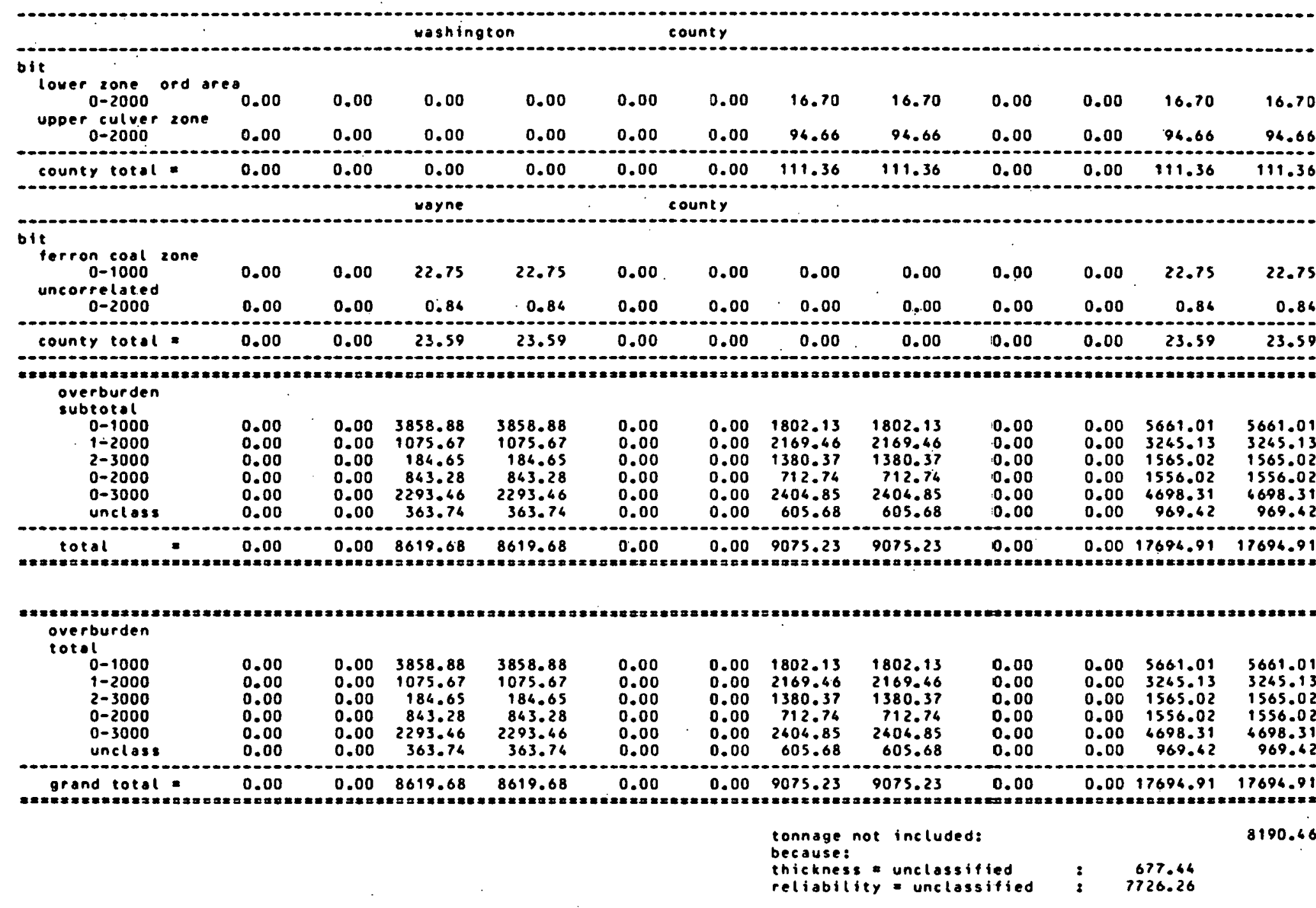




$\begin{array}{lll}\text { utgs of-book cliffs } & 1979 & \text { original } \\ \text { utgs of-wasatch } & 1971 & \text { original } \\ \text { utgs of-coolville } & 1971 & \text { original } \\ \text { utgs monograph } & 1972 & \text { original } \\ \text { utgs of-emery } & 1971 & \text { original } \\ \text { utgs of-koiparowits } & 1970 & \text { original } \\ \text { utgs of-henry atng } & 1971 & \text { original } \\ \text { utgs of-alton } & 1970 & \text { original } \\ \text { utgs monogroph } & 1971 & \text { original } \\ \text { utgs of-kolob-harany } & 1971 & \text { original } \\ \text { utgs of-sevier-sanpt } & 1971 & \text { original } \\ \text { utgs of-vernal } & 1970 & \text { original }\end{array}$

records containing unclassitied thickness andfor reliabllity

state county
s.
utah carbon
utah carbon
utah carbon
utah carbon
utah carbon
utah carbon
utah carbon
utah carbon
utah carbon
utah carbon
utah carbon
utah carbon
utah carbon
utah carbon
utah carbon
utah carbon
utah carbon
utah carbon
utah carbon
utah carbon
utah carbon
utah carbon
utah carbon
utah carbon
utah carbon
utah carbon
utah carbon
utah corbon
utah carbon
utah carbon
utah carbon
utah carbon
utah carbon
utah carbon
utah carbon
utah carbon
utah carbon
utah carbon

bed

upper sunnyside
kenilwarth
upper sunnsside
kenilwarth
sunnyside
hiowatha
uncorrelated
uncorrelated
uncorrelated
castlegate
bob wright
uncorrelated
hiawatha
uncorrelated
castlegate
castlegate
bob wright
castlegate
uncorrelated
costlegate a
uncorrelated
castlegate a
bob uright
castlegate a
bob wright
uncorrelated
uncorrelated
lower gunnyside
rock canyon
gilson sunngide
upper sunnyside
lower sunnyside
rock canyon
gilson
lower sunnyside
upper sunnyside
lower sunnyside
rock canyon

rank

bit
bit
bit
bit
bit
bit
bit
bit
bit
bit
bit
bit
bitt
bit
bit
bit
bit
bit
bit
bit
bit
bit
bit
bit
bit
bit
bit
bit
bit
bit
bit
bit
bit
bit
bit
bit
bit
bit

year

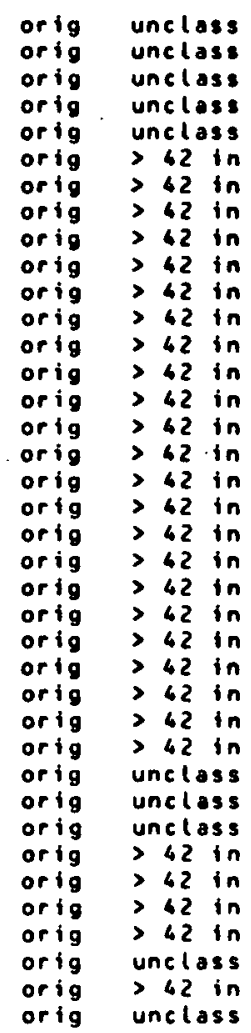

$0-3000$
$0-3000$
$0-3000$
$0-3000$
$0-3000$
$0-3000$
$0-3000$
$0-2000$
$0-2000$
$0-2000$
$0-2000$
$0-2000$
$0-2000$
$0-2000$
$0-2000$
$0-1000$
$0-1000$
$0-1000$
$1-2000$
$0-1000$
$0-1000$
$0-1000$
$0-1000$
$0-1000$
$0-1000$
$0-3000$
$0-3000$
$0-3000$
$0-3000$
$0-3000$
$0-3000$
$0-3000$
$0-3000$
$0-3000$
$0-3000$
$0-3000$
$0-3000$
$0-3000$

religablity tonnage

$\begin{array}{lr}\text { ns } 8 \text { ind } & 10.80 \\ \text { ns } 8 \text { ind } & 2.30 \\ \text { ns } 8 \text { ind } & 18.70 \\ \text { ns } 8 \text { ind } & 5.60 \\ \text { unclass } & 120.90 \\ \text { uncllass } & 46.63 \\ \text { unclass } & 18.00 \\ \text { unclass } & 61.47 \\ \text { unclass } & 245.61 \\ \text { unclass } & 3.28 \\ \text { unclass } & 3.24 \\ \text { unclass } & 102.05 \\ \text { unclass } & 118.00 \\ \text { unclass } & 4.00 \\ \text { unclass } & 73.00 \\ \text { unclass } & 188.00 \\ \text { unclass } & 105.00 \\ \text { unclass } & 31.39 \\ \text { unclass } & 65.95 \\ \text { uncilass } & 24.00 \\ \text { unclass } & 129.75 \\ \text { unclass } & 10.77 \\ \text { unclass } & 8.54 \\ \text { unclass } & 51.67 \\ \text { unclass } & 24.08 \\ \text { unclass } & 94.38 \\ \text { unclass } & 367.97 \\ \text { unclass } & 10.30 \\ \text { unclass } & 3.90 \\ \text { unclass } & 6.10 \\ \text { unclass } & 99.00 \\ \text { unclass } & 98.90 \\ \text { unclass } & 34.10 \\ \text { unclass } & 54.30 \\ \text { unclass } & 2.10 \\ \text { unclass } & 10.80 \\ \text { unclass } & 40.40 \\ \text { unclass } & 7.00\end{array}$




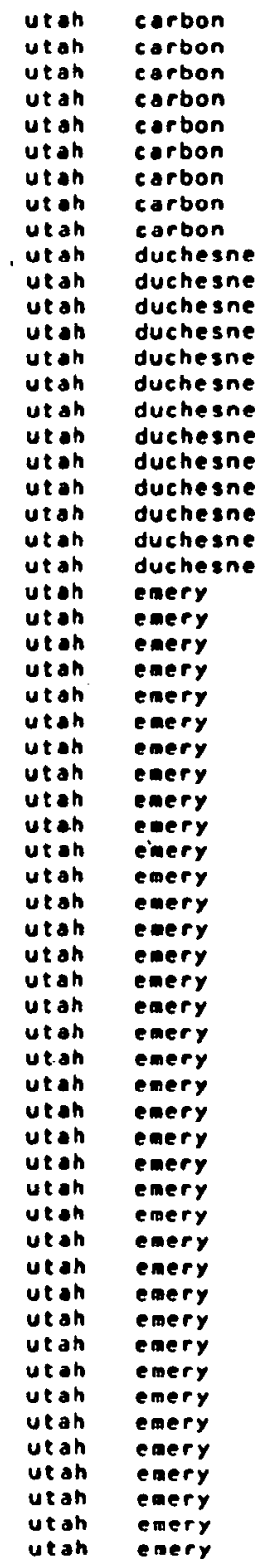

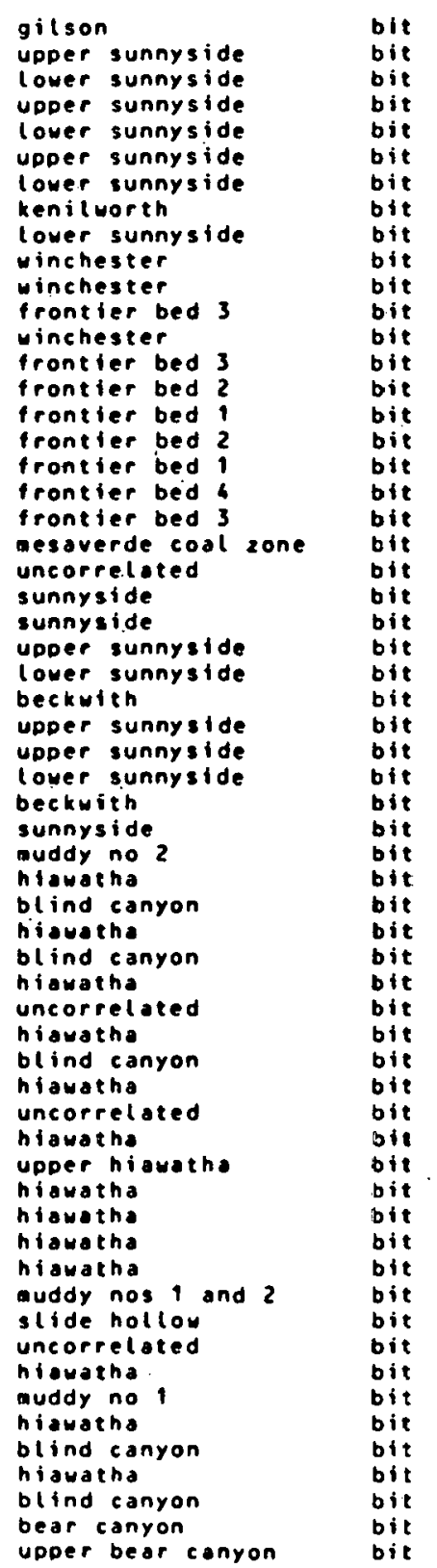

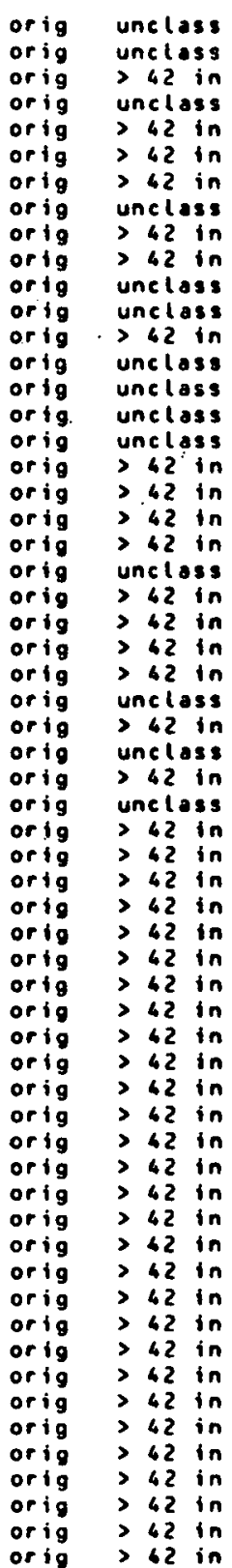
0-3000
$0-3000$
$0-3000$
$0-3000$
$0-3000$
$0-3000$
$0-3000$
$0-3000$
$0-3000$
$0-3000$
$0-3000$
$0-3000$
$0-3000$
$0-3000$
$0-3000$
$0-3000$
$0-3000$
$0-3000$
uncl.oss
$0-300$
$0-300$
$0-3000$
$0-3000$
$0-3000$
$0-3000$
$0-3000$
$0-3000$
$0-3000$
$0-3000$
$0-2000$
$2-3000$
$2-3000$
$2-3000$
$1-2000$
$1-2000$
$1-2000$
$1-2000$
1-2000
$0-1000$
$0-1000$
$0-2000$
$0-2000$
$2-3000$
$2-3000$
$0-2000$
$0-2000$
$0-3000$
$0-3000$
$0-3000$
$0-3000$
$0-3000$
$0-3000$
$0-300$
$0-3000$
$0-3000$
$0-3000$
$0-3000$
$0-3000$
$0-3000$

uncloss

unclass

unclass
unclass

unclass

unclass 


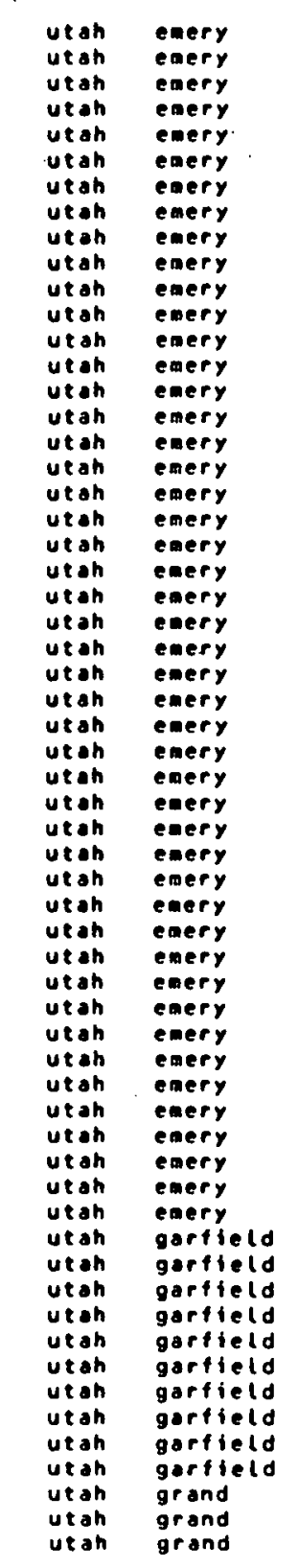

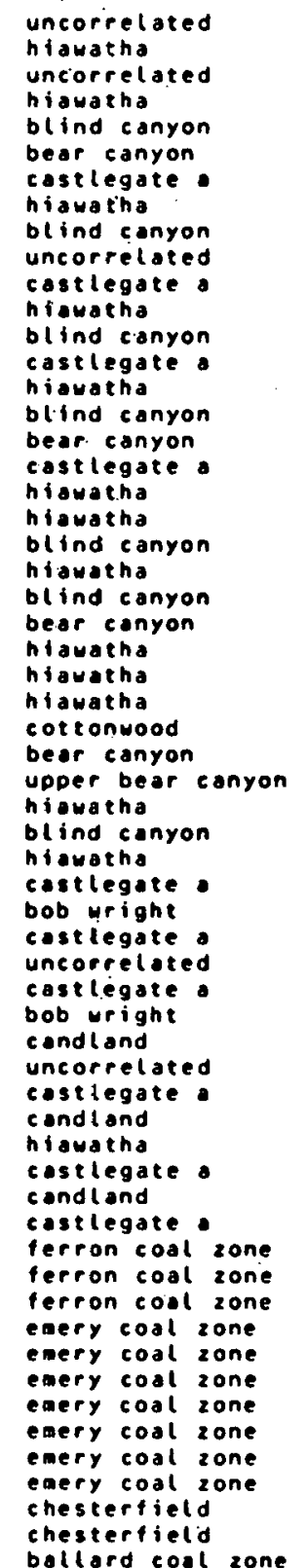

bit
bit
bit
bit
bit
bit
bit
bit
bit
bit
bit
bit
bit
bit
bit
bit
bit
bit
bit
bit
bit
bit
bit
bit
bit
bit
bit
bit
bit
bit
bit
bit
bit
bit
bit
bit
bit
bit
bit
bit
bit
bit
bit
bit
bit
bit
bit
bit
bit
bit
bit
bit
bit
bit
bit
bit
bit
bit
bit
bitt
bit

\begin{tabular}{|c|c|}
\hline $\begin{array}{l}r i g \\
r i g \\
r i g \\
r i g \\
r i g \\
r i g \\
r i g \\
r i g \\
r i g \\
r i g \\
r i g \\
r i g \\
r i g \\
r i g \\
r i g \\
r i g \\
r i g \\
r i g \\
r i g \\
r i g \\
r i g \\
r i g \\
r i g \\
r i g \\
r i g \\
r i g \\
r i g \\
r i g \\
r i g \\
r i g \\
r i g \\
r i g \\
r i g \\
r i g \\
r i g \\
r i g \\
r i g \\
r i g \\
r i g \\
r i g \\
r i g \\
r i g \\
r i g \\
r i g \\
r i g \\
r i g \\
r i g \\
r i g \\
r i g \\
r i g \\
r i g \\
r i g \\
r i g \\
r i g \\
r i g \\
r i g \\
r i g \\
r i g \\
r i g\end{array}$ & 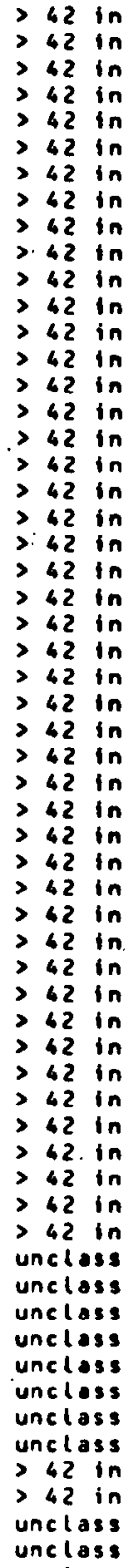 \\
\hline
\end{tabular}

$0-3000$
$0-3000$
$0-3000$
$0-3000$
$0-3000$
$0-3000$
$0-3000$
$0-3000$
$0-3000$
$0-3000$
$0-3000$
$0-3000$
$0-3000$
$0-3000$
$0-3000$
$0-3000$
$0-3000$
$0-3000$
$0-3000$
$0-3000$
$0-3000$
$0-2000$
$0-2000$
$0-2000$
$0-2000$
$0-3000$
$0-3000$
$0-3000$
$0-3000$
$0-3000$
$0-3000$
$0-3000$
$0-3000$
$0-1000$
$0-1000$
$0-1000$
$0-1000$
$0-1000$
$0-1000$
$0-1000$
$0-1000$
$0-1000$
$0-1000$
$0-1000$
$0-1000$
$0-1000$
$0-1000$
$0-1000$
$0-1000$
$0-1000$
$0-1000$
$0-1000$
$0-2000$
$0-1000$
$0-1000$
$0-1000$
$0-1000$
$0-2000$
$0-2000$
$0-2000$
unclioss $\quad 164.50$ uncless $\quad 55.00$ $\begin{array}{ll}\text { unclass } & 3.96 \\ \text { unclass } & 3.35\end{array}$ unclass $\quad 0.90$ unclass $\begin{array}{ll}\text { unclass } & 20.06 \\ \text { unclass } & 27.58\end{array}$ unclass $\quad 18.00$ $\begin{array}{ll}\text { unclass } & 26.24 \\ \text { uncloss } & 87.00\end{array}$ unclass $\quad 126.00$ unclass $\quad 16.00$ $\begin{array}{ll}\text { unclass } & 16.00 \\ \text { unclass } & 112.76\end{array}$ unclass $\quad 49.92$ unclass unclass 8.37 uncloss $\quad 11.30$ unclass $\quad 15.02$ unclass $\quad 78.36$ $\begin{array}{rr}\text { unclass } & 26.69 \\ \text { unclass } & 2.70\end{array}$ unclass 4.61 uncless $\quad 137.58$ $\begin{array}{lr}\text { unclass } & 188.14 \\ \text { uncloss } & 5.00\end{array}$ $\begin{array}{lr}\text { unclass } & 5.00 \\ \text { unclass } & 49.80\end{array}$ unclass $\quad 46.00$ unclass 77.06 unclass $\quad 5.86$ unclass 188.00 unclass $\quad 105.00$ unclass 19.70 unclass
uncloss unclass
unclass $\begin{array}{ll}\text { unclass } & 24.08 \\ \text { unclass } & 52.00\end{array}$ unclass
unclass
unclass unclass $\quad 26.00$ unclass 21.26 unclass $\quad 67.52$ unclass $\quad 30.00$ $\begin{array}{lr}\text { unclass } & 66.30 \\ \text { indicatd } & 0.70\end{array}$ indicatd
indicatd
16.70 $\begin{array}{rr}\text { indicoto } & 26.37 \\ \text { indicatd } & 2.30\end{array}$ $\begin{array}{lr}\text { indicatd } & 2.30 \\ \text { indicatd } & 10.44\end{array}$ $\begin{array}{ll}\text { indicatd } & 10.46 \\ \text { inferred } & 16.72\end{array}$ inferred 21.46 inferred 50.73 uncloss 24.50 unclass 26.50 as 8 ind 4.00 is 8 ind 2.00 


\begin{tabular}{|c|c|}
\hline $\begin{array}{l}\text { utah } \\
\text { utan }\end{array}$ & $\begin{array}{l}\text { grand } \\
\text { grand }\end{array}$ \\
\hline vitah & grand \\
\hline utan & grand \\
\hline vetah & grand \\
\hline utah & grand \\
\hline vetah & grand \\
\hline ut ah & grand \\
\hline utah & grand \\
\hline utah & grand \\
\hline utah & grand \\
\hline utah & grand \\
\hline utan & grand \\
\hline utah & grand \\
\hline ut ah & grand \\
\hline utah & iron \\
\hline utah & iron \\
\hline utan & iron \\
\hline utah & iron \\
\hline utah & iron \\
\hline utah & iron \\
\hline utah & tron \\
\hline utah & iron \\
\hline wetah & iron \\
\hline vetah & iron \\
\hline utan & iron \\
\hline utah & iron \\
\hline vetah & iron \\
\hline utah & kane \\
\hline utsh & sanpete \\
\hline uesen & sanpete \\
\hline vetah & sanpete \\
\hline veah & sanpete \\
\hline utah & sevier \\
\hline utah & seviler \\
\hline utoh & sevier \\
\hline utah & sevier \\
\hline utah & sevier \\
\hline wetah & sevier \\
\hline veah & sevier \\
\hline ue oh & sevier \\
\hline utah & seviler \\
\hline ueteh & seviler \\
\hline utah & sevier \\
\hline utah & sevier \\
\hline utah & sevier \\
\hline uteh & sevier \\
\hline wen & sevier \\
\hline veth & sevier \\
\hline uesh & sevier \\
\hline utah & sevter \\
\hline utah & sevier \\
\hline vean & seviter \\
\hline utah & sevier \\
\hline utsh & sevier \\
\hline utsh & seviter \\
\hline veah & sevier \\
\hline vesh & sevier \\
\hline $\begin{array}{l}\text { utah } \\
\text { utah }\end{array}$ & sevier \\
\hline & \\
\hline
\end{tabular}

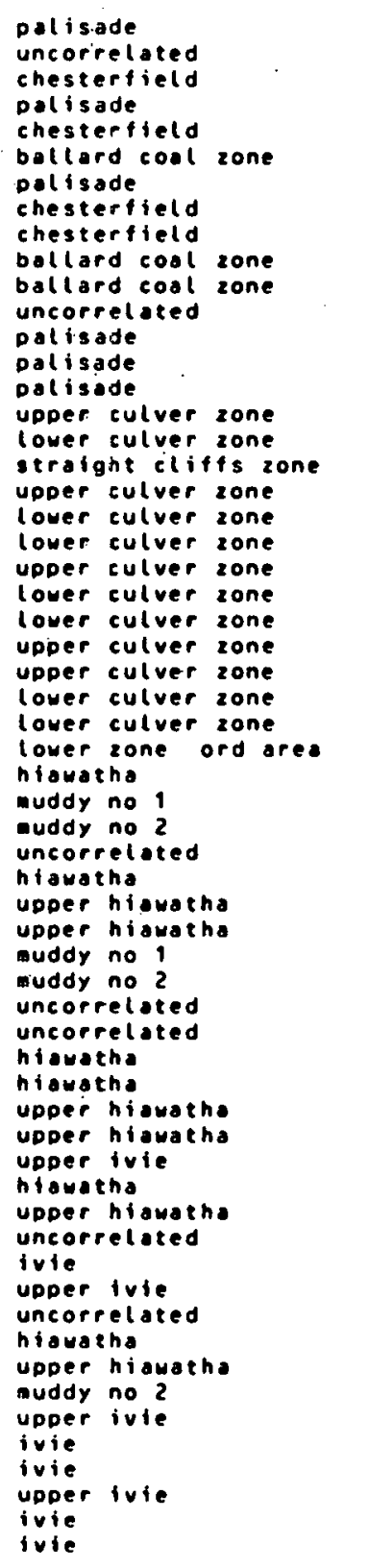

bit
bit
bit
bit
bit
bit
bit
bit
bit
bit
bit
bjt
bit
bit
bit
bit
bit
bit
bit
bit
bit
bit
bit
bit
bit
bit
bit
bit
bit
bit
bit
bit
bit
bit
bit
bit
bit
bit
bit
bit
bit
bit
bit
bit
bit
bit
bit
bit
bit
bit
bit
bit
bit
bit
bit
bit
bit
bit
bit
bit
bit
a

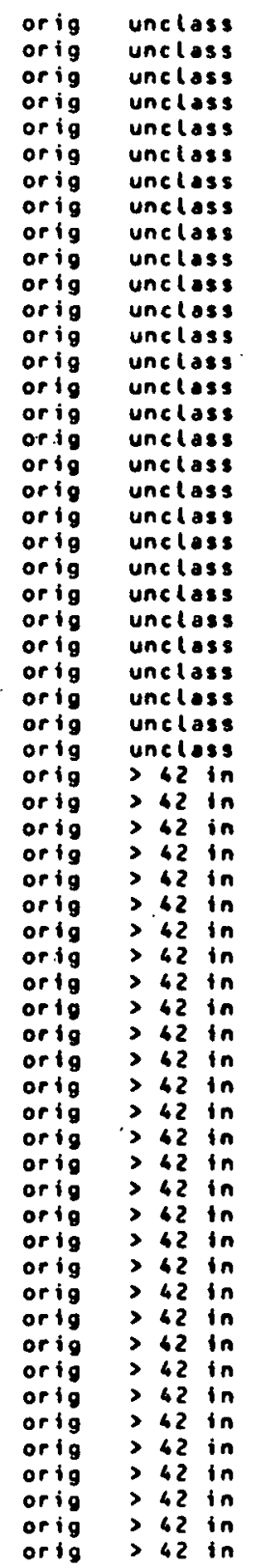

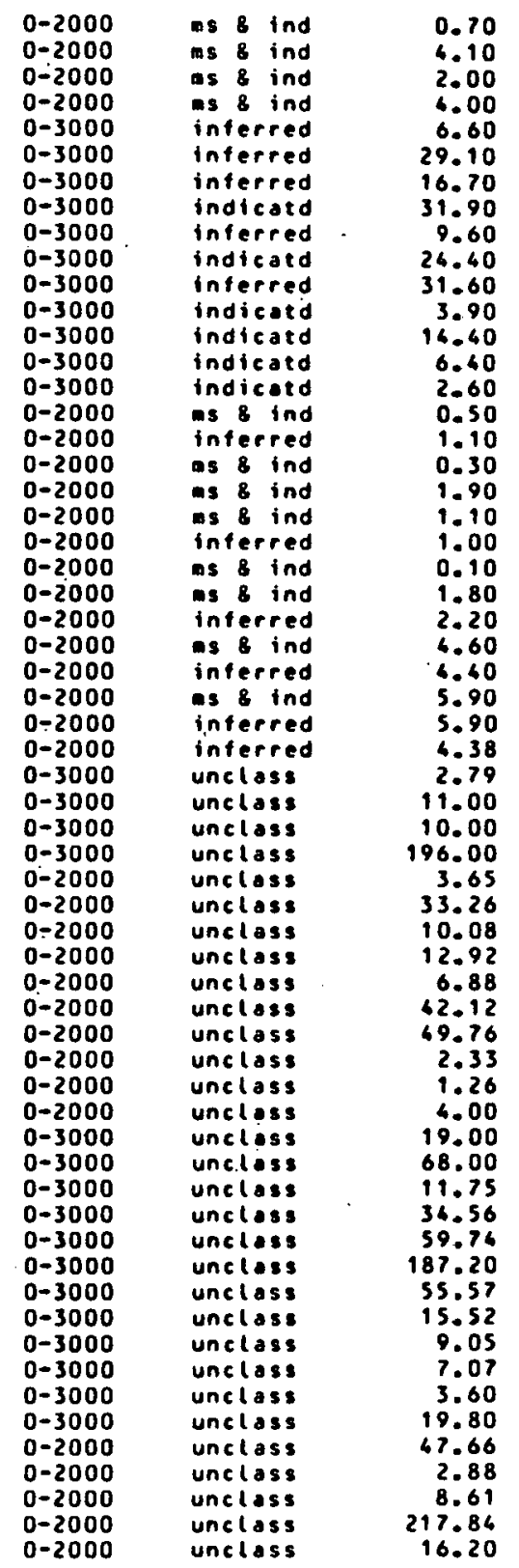




utah sevier
utah sevier
utah sevier
utah sevier
utah sevier
utah sevier
utah sevier
utah sevier
utah sevier
utah sevier
utah sevier
utah sevier
utah sevier
utah sevier
utah sevier.
utah sevier
utah sevier
utah sasatch
utsh uasatch
utah vayne

uncorrelated
ivie .
uncorrelated
hiauatha
muddy. no 1
muddy no
uncorrelated
uncorrelated
uncorrelated
uncorrelated
uncorrelated
lover coal zone
uncorrelated
uncorrelated
lower coal zone
upper ivie
upper ivie
uncorrelated
uncorrelated
enery coal zone

bit
bit
bit
bit
bit
bit
bit
bit
bit
bit
bit
bit
bit
bit
bit
bit
bit
bit
bit
bit

$\begin{array}{llll}\text { orig } & >42 & \text { in } \\ \text { orig } & >42 & \text { in } \\ \text { orig } & >42 & \text { in } \\ \text { orig } & >42 & \text { in } \\ \text { orig } & >42 & \text { in } \\ \text { orig } & >42 & \text { in } \\ \text { orig } & >42 & \text { in } \\ \text { orig } & >42 & \text { in } \\ \text { orig } & >42 & \text { in } \\ \text { orig } & >42 & \text { in } \\ \text { orig } & >42 & \text { in } \\ \text { orig } & >42 & \text { in } \\ \text { orig } & >42 & \text { in } \\ \text { orig } & >42 & \text { in } \\ \text { orig } & >42 & \text { in } \\ \text { orig } & >42 & \text { in } \\ \text { orig } & >42 & \text { in } \\ \text { orig } & >42 & \text { in } \\ \text { orig } & >42 & \text { in } \\ \text { orig } & \text { uncios }\end{array}$

$0-2000$

0-2000

$0-3000$

0-3000

$0-3000$

$0-3000$

$0-2000$

$0-2000$

$0-2000$

$0-2000$

$0-2000$

0

$0-2000$

$0-1000$

$0-3000$

$0-3000$

$0-3000$

$0-1000$

$\begin{array}{lr}\text { unclass } & 7.20 \\ \text { unclass } & 95.60 \\ \text { unclass } & 38.88 \\ \text { unclass } & 29.25 \\ \text { unclass } & 9.00 \\ \text { unclass } & 5.76 \\ \text { unclass } & 99.31 \\ \text { unclass } & 5.20 \\ \text { unclass } & 75.80 \\ \text { unclass } & 5.70 \\ \text { uncloss } & 7.00 \\ \text { unclass } & 2.40 \\ \text { unclass } & 22.60 \\ \text { unclass } & 9.30 \\ \text { unclass } & 25.00 \\ \text { unclass } & 17.25 \\ \text { unclass } & 69.12 \\ \text { unclass } & 50.40 \\ \text { unclass } & 26.25 \\ \text { indicatd } & 4.08\end{array}$


average analyses of coal in utoh

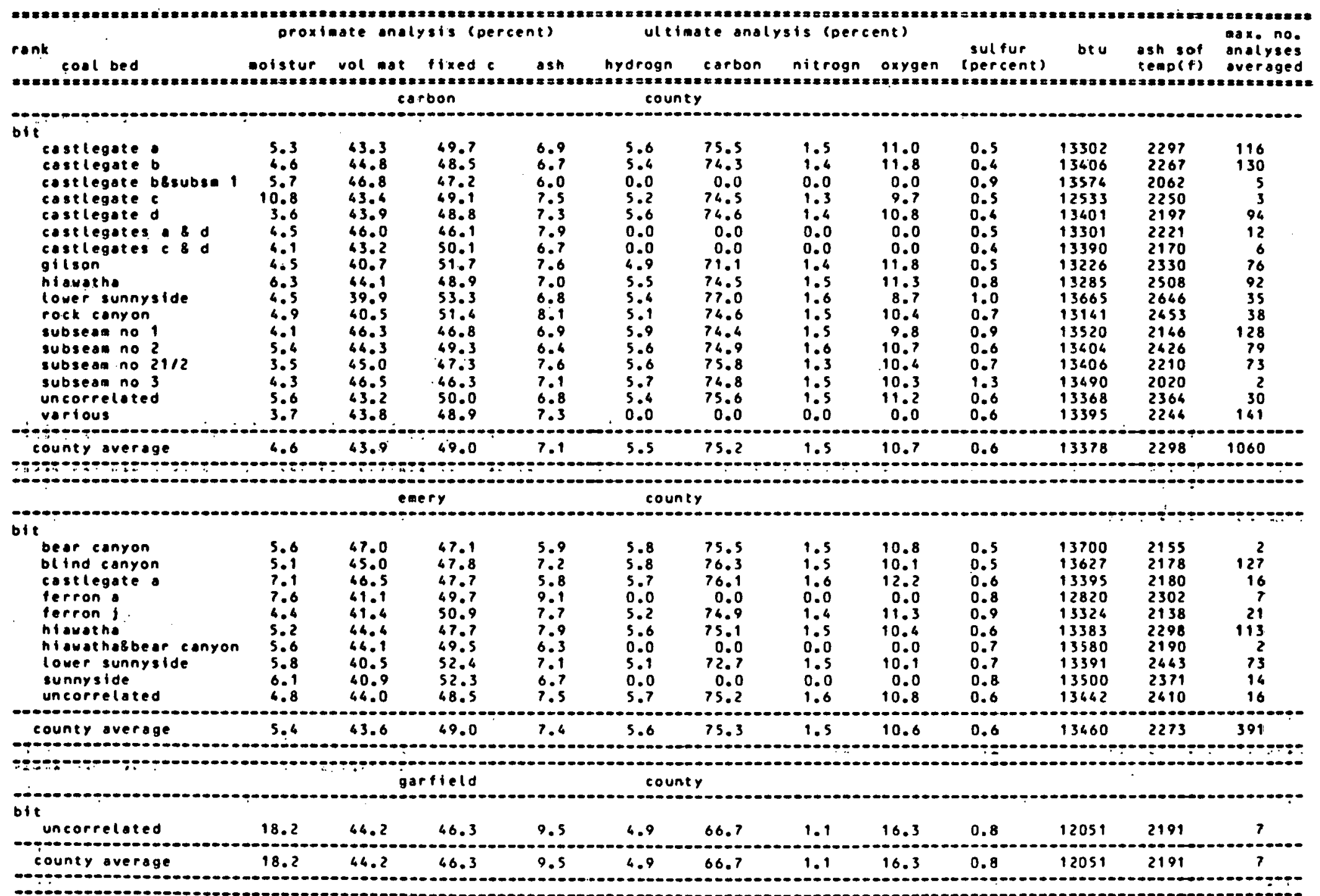


grand

county

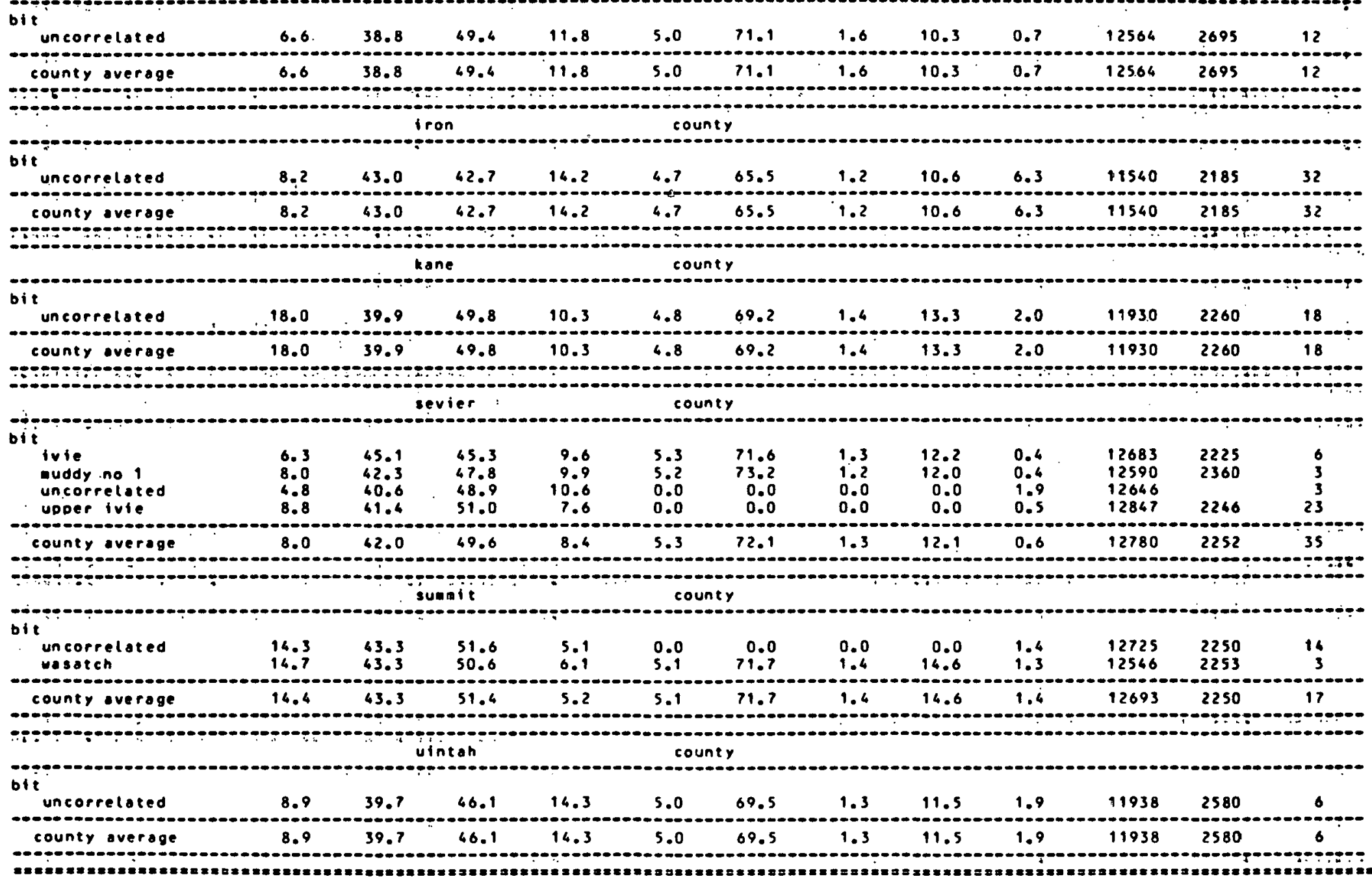




\begin{tabular}{|c|c|c|c|c|c|c|c|c|c|c|c|}
\hline & state & county & bed & mo & r volmat & fixedc & ash & sul & ur otu & hgilo & fsilo \\
\hline $\begin{array}{l}\text { ut an } \\
\text { ut an }\end{array}$ & & $\begin{array}{l}\text { carbonn } \\
\text { carbon }\end{array}$ & $\begin{array}{l}\text { castleate } \\
\text { castleogte }\end{array}$ & $\begin{array}{l}4.6 \\
4.8\end{array}$ & $\begin{array}{l}45.0 \\
44.8\end{array}$ & $\begin{array}{l}48.7 \\
49.1\end{array}$ & $\begin{array}{l}3.3 \\
6.1\end{array}$ & $\begin{array}{l}0.4 \\
0.4\end{array}$ & $\begin{array}{l}13480 \\
13600\end{array}$ & $\begin{array}{l}49 \\
48\end{array}$ & $\begin{array}{l}2.0 \\
1.0\end{array}$ \\
\hline utan & & caroon & castleate b & 4.5 & 45.8 & 47.3 & 6.9 & 0.3 & 13480 & 0 & 2.5 \\
\hline utan & & cartion & castlegate o & 5.0 & 46.3 & 47.1 & 6.6 & 0.3 & 13530 & D & 2.5 \\
\hline utah & & carbon & castlegate & 5.? & 45.6 & 47.6 & 6.8 & 0.3 & 13470 & 0 & 3.0 \\
\hline ut ah & & carbann & castlegate & 5.2 & 45.3 & 47.5 & 7.2 & 0.3 & 13370 & 0 & 1.5 \\
\hline utah & & carbon & castleqate & 6.2 & 42.4 & 45.9 & 10.7 & 0.5 & 12920 & 0 & 5.5 \\
\hline utan & & carbon & castlegate b & 5.8 & 43.0 & 47.1 & 6.9 & 0.4 & 13440 & 0 & 2.5 \\
\hline utah & & carbon & castleate b & 0.2 & 66.3 & 48.5 & 5.2 & 0.4 & 13640 & 0 & 2.0 \\
\hline utan' & & carbon & castleqate b & 0.2 & 48.6 & 46.0 & 5.4 & 0.3 & 13670 & 0 & 2.5 \\
\hline viah & & carbon & castlegate b & 5.7 & 46.1 & 48.? & 5.7 & 0.6 & 13590 & 0 & 1.5 \\
\hline ut an & & carbon & subseamno? & 4.7 & 44.4 & 49.0 & 6.6 & 1.0 & 13690 & 0 & 3.0 \\
\hline ut an & & carbon & castlegate a & 6.0 & 39.4 & 51.2 & 9.4 & 0.5 & 12810 & 48 & 2.0 \\
\hline ut ah & & carbon & castleqate a & 6.4 & 39.6 & 51.3 & 9.1 & 0.5 & 12960 & 0 & 1.5 \\
\hline utah & & carbon & castleqate a & 5.7 & 39.3 & 51.2 & 9.5 & 0.5 & 12830 & 46 & 1.5 \\
\hline ut ah & & carbon & castlegate a & 2.7 & 41.1 & $51 . ?$ & 7.7 & 0.5 & 13080 & 0 & 1.0 \\
\hline utah & & carbon & louer sunnyside & 7.2 & 39.0 & 52.7 & 8.3 & 0.7 & 13470 & 0 & 4.5 \\
\hline utan & & carbonn & castlegate b & $\ldots$ & 39.8 & 47.0 & 13.2 & 0.5 & 11900 & 46 & 1.0 \\
\hline ut $\Rightarrow h$ & & carbon & castlegate o & 0.8 & 45.0 & 49.3 & 5.7 & 0.6 & 13500 & 0 & 1.5 \\
\hline utan & & carbon & castlegate b & 6.5 & 45.5 & 48.2 & 6.3 & 0.5 & 13300 & 0 & 1.5 \\
\hline utah & & carbon & castleqate b & 6.9 & 44.7 & 48.9 & 6.4 & 0.5 & 13270 & 0 & 1.5 \\
\hline utan & & carbon & castleqate 0 & 8.5 & 44.3 & 48.1 & 7.0 & 0.7 & 12850 & 0 & 1.0 \\
\hline utan & & carbon & castlegate & 8.3 & 44.7 & 46.6 & 8.7 & 0.7 & 12590 & 0 & 1.5 \\
\hline ut ah & & carbon & subseam no 1 & 3.4 & 44.9 & 67.4 & 7.7 & 1.7 & 13560 & 46 & 2.0 \\
\hline utah & & carbon & subseam no 1 & 3.7 & $4 R .2$ & 45.9 & 5.9 & 1.3 & 13600 & 0 & 2.5 \\
\hline vitan & & carbon & uncorrelated & 4.5 & 40.4 & 51.8 & 7.8 & 0.8 & $\$ 3360$ & 41 & 1.5 \\
\hline utah & & carbon & lower sunnyside & 3.4 & 40.1 & 51.9 & 8.0 & 0.7 & 13400 & 0 & 4.0 \\
\hline utan & & carbon & castlegate a & 5.7 & 47.7 & 47.2 & 5.1 & 0.4 & 13580 & 48 & 1.5 \\
\hline utah & & carbon & qilson & 4.5 & 40.2 & 50.4 & 9.4 & 0.4 & 12870 & 0 & 1.0 \\
\hline ut ah & & carbon & ailson & 7.0 & 40.5 & 50.7 & 8.8 & 0.4 & 12950 & 0 & 1.5 \\
\hline ut ah & & carbon & gilison & 6.6 & 40.7 & 50.7 & 8.6 & 0.6 & 13120 & 0 & 1.5 \\
\hline utan & & carbon & qilson & 0.1 & 41.1 & 51.? & 7.7 & 0.6 & 13130 & 0 & 1.0 \\
\hline ut an & & carbon & various & $4 . n$ & 45.3 & 46.9 & 7.8 & 0.3 & 13370 & 0 & 2.5 \\
\hline utan & & carban & various & 5.4 & 44.5 & 46.3 & 9.2 & 0.5 & 13040 & 0 & 2.0 \\
\hline viah & . & carbon & uncorrelated & 8.4 & 46.2 & 46.8 & 7.0 & 0.6 & 12970 & 54 & 1.0 \\
\hline ut ah & & carbon & uncorrelated & 8.7 & 44.2 & 49.9 & 5.9 & 0.5 & 13350 & 68 & 1.0 \\
\hline utah & & carbon & castleuate a & 9.3 & 45.8 & 48.6 & 5.6 & 0.5 & 13310 & 50 & 1.0 \\
\hline utan & & carbon & castlegate c & 4.3 & 44.1 & 47.0 & 8.9 & 0.4 & 13220 & 48 & 2.5 \\
\hline utan & & caruon & castleqated & 4.9 & 44.6 & 67.8 & 7.6 & 0.4 & 13380 & 0 & 2.5 \\
\hline ut an & & carbon & castlegate $d$ & 6.5 & 43.5 & 48.9 & 7.6 & 0.4 & 13290 & 46 & 1.5 \\
\hline veah & & carbon & castleqate $A$ & 5.6 & 44.4 & 49.7 & 5.9 & 0.3 & 134.30 & 43 & 1.0 \\
\hline ut ah & & carbon & castlegates a $s 0$ & 8.3 & 44.8 & 4.7 & 6.5 & 0.5 & 13420 & 0 & 2.0 \\
\hline utah & & carbon & gilson & 5.2 & .0 .0 & $5 ? .9$ & 7.1 & 0.4 & 13430 & 0 & 1.5 \\
\hline utah & & carbon & ilson & 6.4 & 49.1 & 51.2 & 7.7 & 0.5 & 13350 & 0 & 2.5 \\
\hline utah & & carbon & qilson & 6.7 & 41.2 & 53.5 & 5.3 & 0.4 & 13730 & 0 & 2.0 \\
\hline utah & & carban & castleqate a & $5 . ?$ & 43.0 & 47.5 & 6.5 & 0.6 & 13620 & 0 & 1.0 \\
\hline utah & & carbon & castleate $u$ & $7 . ?$ & 41.9 & 49.9 & 8.2 & 0.4 & 12900 & 0 & 1.0 \\
\hline ut ah & & cartoon & castlegate b & 7.0 & 43.7 & 50.8 & 5.5 & 0.4 & 13430 & $\angle 0$ & 1.5 \\
\hline ut ah & & carbon & subseam no 1 & 5.4 & $4 x .8$ & 47.8 & 5.4 & 0.9 & 13660 & 0 & 1.0 \\
\hline vetan & & carhan & subseam no 1 & 6.1 & 45.8 & 46.8 & 6.4 & 1.0 & 13580 & 46 & 2.0 \\
\hline
\end{tabular}




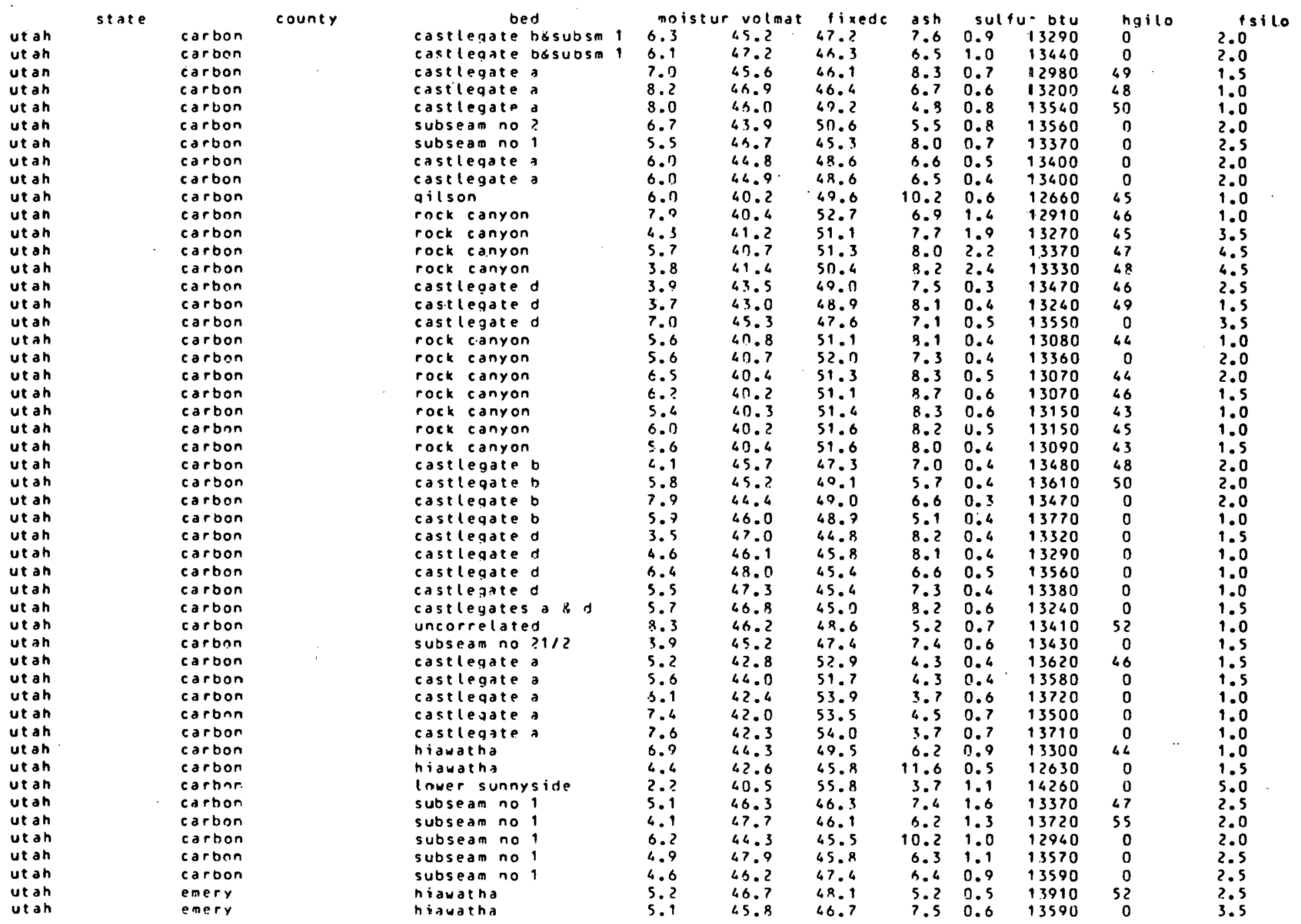




\section{UTAR}

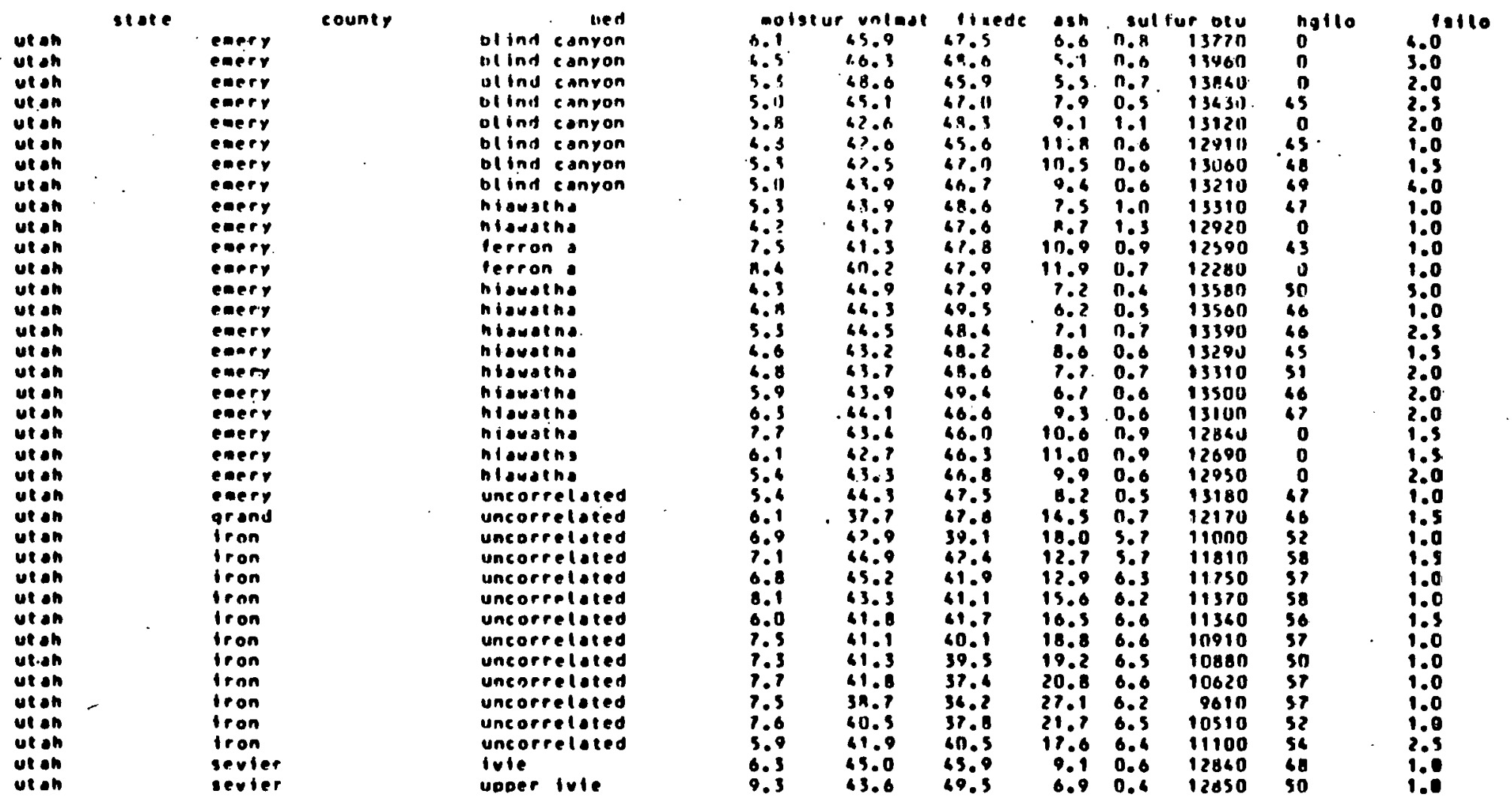




\begin{tabular}{|c|c|c|c|c|c|c|c|c|c|c|c|c|c|}
\hline & state & & county & & bed & moi & $r$ volmat & fixedc & as $n$ & su & ur btu & hailo & isile \\
\hline utah & & enery & & blind & canyon & 7.1 & 46.8 & 43.8 & 9.4 & 0.4 & 13300 & 0 & 3.0 \\
\hline utan & & emery & & blind & canyon & 8.0 & 6.7 .1 & 45.4 & 7.5 & 0.6 & 13630 & 0 & 2.5 \\
\hline ut ah & & emery & & olina & canyon & 7.4 & 1.8 .4 & 64.9 & 0.7 & 0.5 & 13950 & 0 & 3.0 \\
\hline utan & & emery & & olind & canyon & 5.6 & 16.5 & 46.0 & 7.5 & 0.5 & 13560 & 0 & 2.5 \\
\hline utan & & emery & & olind & canyon & 7.6 & 45.6 & 46.7 & 7.7 & 0.6 & 13500 & 0 & 2.5 \\
\hline utah & & emery & & blind & canyon & 6.4 & 49.7 & 43.6 & 7.7 & 0.4 & 13700 & 0 & 2.5 \\
\hline utah & & emery & & niawat & hat & 5.0 & 45.0 & 47.1 & 7.9 & 0.5 & 13540 & 46 & 2.5 \\
\hline ut an & & emery & & niawat & ha & 0.1 & 43.5 & 49.1 & 7.4 & 0.5 & 13240 & 0 & 1.0 \\
\hline utan & & emery & & hiawat & ha & 6.7 & 43.9 & 48.0 & 8.1 & 0.5 & 13170 & 47 & 1.0 \\
\hline ut an & & emery & & hiawat & ha & 3.3 & 63.8 & 40.1 & 7.1 & 0.5 & 13390 & 0 & 1.5 \\
\hline utah & & emery & & lower & sunnyside & 6.4 & 40.4 & 51.5 & 8.1 & 1.0 & 13230 & 43 & 3.0 \\
\hline utah & & emery & & lower & sunnyside & 5.7 & 40.8 & $5 ? .2$ & 7.0 & 0.7 & 13480 & 0 & 2.5 \\
\hline utah & & emery & & lower & sunnyside & 5.9 & $6 n .7$ & 57.3 & 7.0 & 0.7 & 13380 & 0 & 2.0 \\
\hline ut ah & & emery & & lower & sunnyside & 5.5 & 40.6 & 51.9 & 7.5 & 0.7 & 13320 & 0 & 3.0 \\
\hline utah & & emery & & lower & sunnyside & 6.9 & 49.0 & 52.1 & 0.9 & 0.7 & 13520 & 0 & 4.0 \\
\hline utan & & emery & & lower & sunnys ide & 0.7 & 39.8 & 51.1 & 9.1 & 0.8 & 13090 & 47 & 3.5 \\
\hline utan & & emery & & lower & sunnyside & 5.7 & 61.9 & 52.8 & 5.3 & 0.7 & 13490 & 0 & 3.0 \\
\hline ut an & & emery & & lower & sunnys ide & 6.4 & 61.0 & 51.5 & 7.5 & 0.8 & 13240 & 49 & 2.5 \\
\hline utah & & emery & & lower & sunnyside & 5.3 & 40.9 & 52.4 & 6.7 & 0.6 & 13510 & 0 & 3.0 \\
\hline ut an & & emery & - & lower & sunnyside & 6.0 & 49.2 & 52.2 & 6.6 & 0.6 & 13490 & 0 & 2.0 \\
\hline utan & & emery & & lower & sunnyside & 8.1 & 32.5 & 52.7 & 7.8 & 0.9 & 13210 & 0 & 2.5 \\
\hline utah & & emery & & lower & sunnyside & $R .1$ & 40.6 & 52.4 & 7.0 & 0.7 & 13420 & 0 & 3.0 \\
\hline utan & & emery & & lower & sunnyside & 7.6 & 40.8 & 52.7 & 6.5 & 0.9 & 13510 & 0 & 3.5 \\
\hline utah & & emery & & ferron & $j$ & 4.2 & 61.1 & 50.7 & 8.2 & 1.4 & 13380 & 39 & 9.0 \\
\hline utan & & emery & & ferron & $j$ & 4.3 & $4 n .7$ & 52.3 & 7.0 & 0.4 & 13310 & 40 & 1.5 \\
\hline ut an & & emery & & terron & $j$ & 6.4 & 62.4 & 50.1 & 7.5 & 0.8 & 13310 & 43 & 1.5 \\
\hline utan & & emery & & ferron & $j$ & 6.1 & $4 ? .4$ & 49.6 & 8.0 & 0.6 & 13330 & 40 & 1.0 \\
\hline utan & & emery & & ferron & i & 6.1 & $4 ? .1$ & 50.7 & 7.2 & 0.5 & 13360 & 0 & 1.0 \\
\hline utah & & emery & & ferron & $j$ & 4.7 & 41.4 & 52.0 & 6.6 & 0.4 & 13360 & 41 & 2.0 \\
\hline utah & & emery & & hiawat & ina & 6.5 & 45.6 & 47.6 & 6.8 & 0.4 & 13530 & 0 & 2.0 \\
\hline utah & & emery & & hiadat & & 5.2 & 44.8 & 48.3 & 6.9 & 0.4 & 13450 & 0 & 1.5 \\
\hline ut an & & emery & & hiawat & ina & 5.7 & 66.3 & 46.8 & $\begin{array}{l}0.7 \\
6.9\end{array}$ & 0.5 & 13770 & 0 & 3.0 \\
\hline utah & & emery & & hiawat & tha & 8.6 & 46.7 & 46.1 & 7.2 & 0.6 & 13600 & 0 & 3.0 \\
\hline utah & & emery & & hiavat & tha & 5.9 & 47.5 & 43.0 & 9.5 & 0.6 & 13360 & 0 & 3.5 \\
\hline utan & & emery & & $b \operatorname{lin} x$ & canyon & 8.0 & 45.2 & 47.5 & 7.1 & 0.5 & 13530 & 45 & 2.5 \\
\hline utan & & emery & & blind & canyon & 6.4 & 44.8 & 48.8 & 6.4 & 0.6 & 13870 & so & 2.0 \\
\hline utan & & emery & & blind & canyon & 5.5 & 46.7 & 45.1 & 8.2 & 0.4 & 13670 & 0 & 1.0 \\
\hline ut an & & emery & & blind & canyon & 0.6 & 45.0 & 48.3 & 0.7 & 0.6 & 13610 & 0 & 2.5 \\
\hline utah & & emery & & blind & canyon & 0.0 & 44.9 & 49.5 & 5.6 & 0.6 & 13790 & 46 & 3.5 \\
\hline ut an & & emery & & niawat & tha & 5.7 & 43.2 & 47.2 & 9.6 & 0.7 & $130>0$ & 47 & 1.5 \\
\hline utan & & emery & & hiawat & ha & 7.7 & $4 ? .0$ & 46.4 & 11.6 & 0.8 & 12630 & 0 & 1.0 \\
\hline utah & & emery & & hi ayat & tha & 6.8 & 41.7 & 50.7 & 8.2 & 0.8 & 93200 & 0 & 20 \\
\hline vean & & emery & & bear c & canyon & 5.6 & 47.4 & 47.9 & 4.7 & 0.5 & 13850 & so & 1.0 \\
\hline utan & & emery & & bear c & anyon & 5.5 & 46.5 & 46.3 & 7.2 & 0.5 & 13550 & 50 & 1.5 \\
\hline utah & & emery & & hiawat & thatbear canyon & 5.7 & 45.0 & 48.7 & 0.3 & 0.6 & 13620 & 0 & 2.0 \\
\hline utah & & emery & & blind & canyon & 6.3 & 45.4 & 49.6 & 5.0 & 0.6 & 13890 & 48 & 2.5 \\
\hline utan & & emery & & castle & eqate a & 6.8 & 47.0 & 47.5 & 5.5 & 0.7 & 13410 & 53 & 1.5 \\
\hline utah & & emery & & cast le & gate a & 6.9 & 47.5 & 47.7 & 4.8 & 0.4 & 13600 & 49 & 3.0 \\
\hline utah & & emery & & blind & canyon & 4.7 & 47.1 & 47.1 & 5.8 & 0.5 & 13920 & 67 & 3.5 \\
\hline ut ah & & emery & & blind & canyon & 3.2 & 47.2 & 46.8 & 6.0 & 0.5 & 13920 & 0 & 5.0 \\
\hline
\end{tabular}


on

$\mathbf{S}$
$\frac{\mathbf{D}}{\mathbf{2}}$
$\frac{\mathbf{D}}{\mathrm{D}}$ 
National Coal Resources Data Systen

U.S. Grotogical survey

identified coal resources in virginio

(In alllions of short tons)

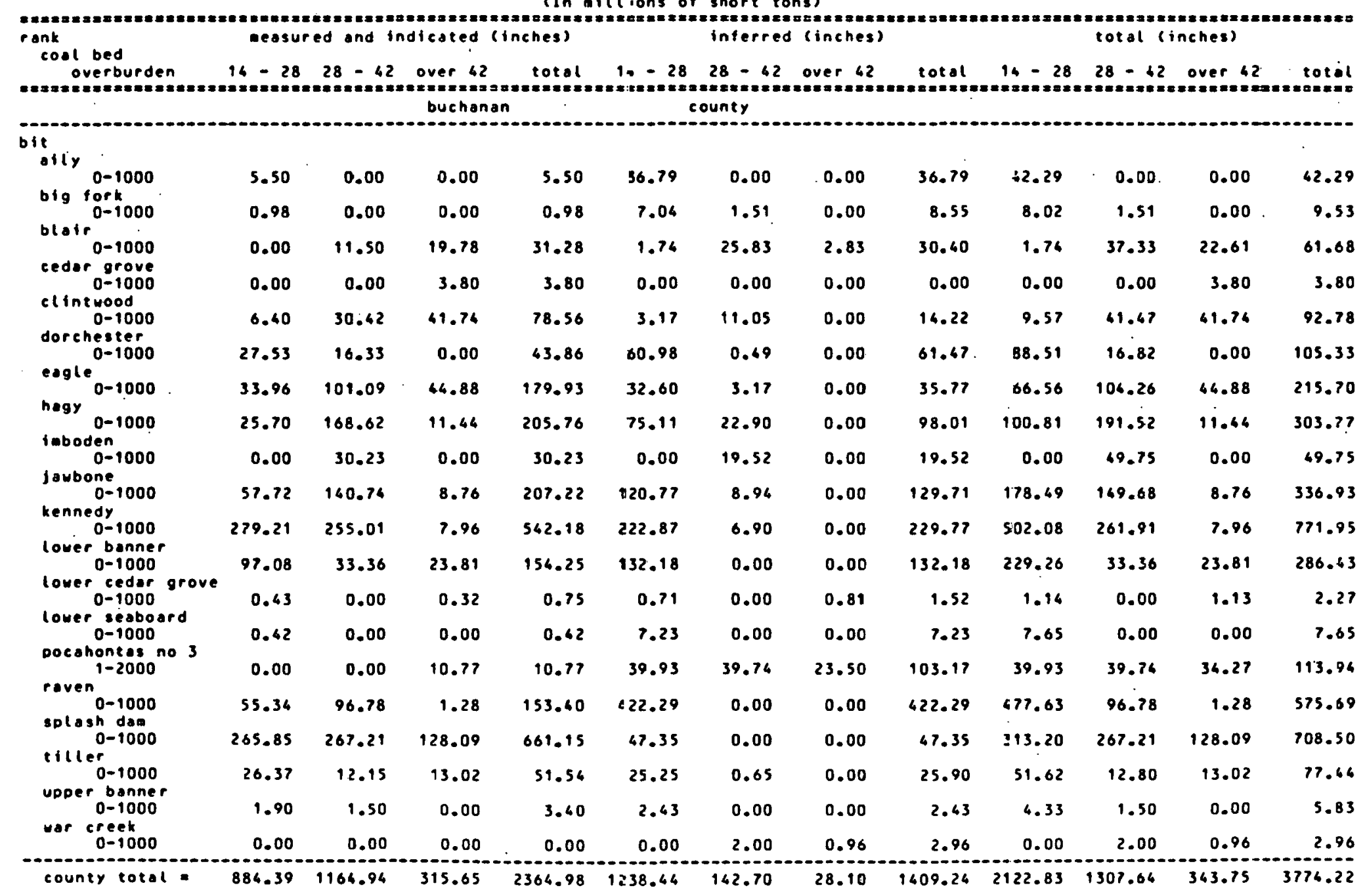




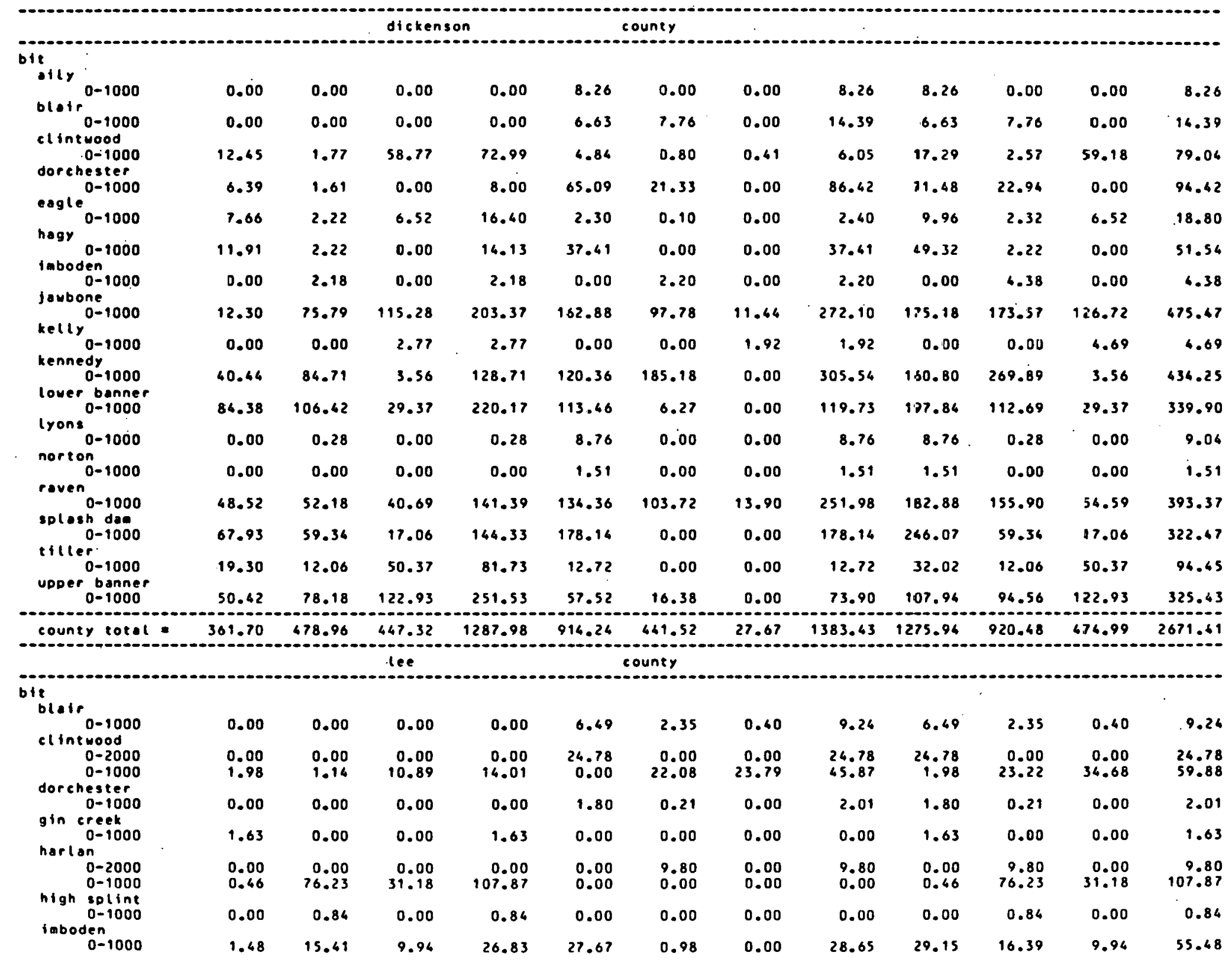




\begin{tabular}{|c|c|c|c|c|c|c|c|c|c|c|c|c|}
\hline kelly $0-1000$ & 0.00 & 9.23 & 0.00 & 9.23 & 2.62 & 4.13 & 0.00 & 6.75 & 2.62 & 13.36 & 0.00 & 15.98 \\
\hline kirk $0-1000$ & 1.76 & 0.00 & 0.00 & 1.76 & 0.00 & 0.00 & 0.00 & 0.00 & 1.76 & 0.00 & 0.00 & 1.76 \\
\hline $\begin{array}{l}\text { lov } 3017 n t \\
0-1000 \\
\text { Lover }\end{array}$ & 0.00 & 2.69 & 16.41 & 19.10 & 2.31 & 0.00 & 0.00 & 2.31 & 2.31 & 2.69 & 16.41 & 21.61 \\
\hline lyons $0=1000$ & 0.00 & 5.70 & 0.00 & 5.70 & 24.50 & 3.43 & 0.00 & 27.93 & 26.50 & 9.13 & 0.00 & 33.63 \\
\hline $\begin{array}{l}0-1000 \\
\text { norris }\end{array}$ & $1^{0.00}$ & 0.00 & 0.00 & 0.00 & 2.55 & 3.22 & 0.00 & 5.77 & 2.55 & 3.22 & 0.00 & 5.77 \\
\hline pardee & 0.00 & 1.63 & 0.06 & 1.67 & 0.00 & 1.10 & 0.00 & 1.10 & 0.00 & 2.73 & 0.04 & 2.77 \\
\hline $\begin{array}{l}0-1000 \\
\text { phillips }\end{array}$ & 0.00 & 0.00 & 1.94 & 1.94 & 0.37 & 0.00 & 0.00 & 0.37 & 0.37 & 0.00 & 1.96 & 2.31 \\
\hline $\begin{array}{l}0-1000 \\
\text { pinhook. }\end{array}$ & 0.00 & 3.06 & 7.98 & 11.04 & 1.65 & 0.48 & 0.00 & 2.13 & 1.65 & 3.54 & 7.98 & 13.17 \\
\hline $\begin{array}{r}0-i 000 \\
\text { stone creek }\end{array}$ & 1.02 & 0.88 & 0.00 & 1.90 & 7.23 & 0.00 & 0.00 & 7.23 & 8.25 & 0.88 & 0.00 & 9.13 \\
\hline $\begin{array}{c}0-1000 \\
\text { toggart }\end{array}$ & 0.63 & 0.00 & 0.00 & 0.63 & 0.00 & 0.00 & 0.00 & 0.00 & 0.63 & 0.00 & 0.00 & 0.63 \\
\hline $\begin{array}{c}0-1000 \\
\text { toggart iarker }\end{array}$ & 0.00 & 35.64 & 11.15 & 46.59 & 0.00 & 0.00 & 0.00 & 0.00 & 0.00 & 35.44 & 11.15 & 66.59 \\
\hline $\begin{array}{c}0-1000 \\
\text { upper st chorles }\end{array}$ & 4.33 & 5.36 & 0.00 & 9.69 & 22.59 & 0.61 & 0.00 & 23.20 & 26.92 & 5.97 & 0.00 & 32.89 \\
\hline $0-1000$ & 0.00 & 3.52 & 0.00 & 3.52 & 3.03 & 0.13 & 0.00 & 3.16 & 3.03 & 3.65 & 0.00 & 6.68 \\
\hline $0-1000$ & 0.00 & 0.00 & 2.46 & 2.46 & 0.00 & 0.00 & 0.00 & 0.00 & 0.00 & 0.00 & 2.46 & 2.46 \\
\hline county total = & 13.29 & 161.13 & 91.99 & 266.41 & 127.59 & 48.52 & 24.19 & 200.30 & 160.88 & 209.65 & 116.18 & 466.71 \\
\hline 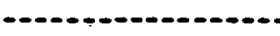 & & & & & & & & & & & & \\
\hline big fork & & & & & & & & & & & & \\
\hline $\begin{array}{c}0-1000 \\
\text { burtons ford }\end{array}$ & 0.00 & 0.00 & 0.00 & 0.00 & 0.00 & 6.80 & 0.00 & 4.80 & 0.00 & 4.80 & 0.00 & 4.80 \\
\hline $\begin{array}{l}0-1000 \\
\text { dorchester }\end{array}$ & 0.00 & 11.82 & 0.77 & 12.59 & 0.42 & 0.00 & 0.00 & 0.62 & 0.42 & 11.82 & 0.77 & 13.01 \\
\hline jaubone & 0.00 & 0.00 & 0.00 & 0.00 & 0.08 & 0.00 & 0.00 & 0.08 & 0.08 & 0.00 & 0.00 & 0.08 \\
\hline $\begin{array}{l}0-1000 \\
\text { kennedy }\end{array}$ & 19.75 & 27.60 & 163.26 & 202.61 & 45.46 & 1.28 & 0.12 & 46.86 & 57.21 & 28.88 & 163.38 & 249.47 \\
\hline $\begin{array}{c}0-9000 \\
\text { lower banner }\end{array}$ & 15.20 & 96.08 & 6.34 & 117.62 & 10.12 & 0.35 & 0.00 & 10.67 & 25.32 & 96.63 & 6.34 & 128.09 \\
\hline roven $0-1000$ & 1.09 & 16.52 & 27.00 & 44.61 & 1.26 & 0.00 & 0.00 & 1.26 & 2.35 & 16.52 & 27.00 & 45.87 \\
\hline $\begin{array}{r}0-1000 \\
\text { splash dan }\end{array}$ & 42.16 & 30.08 & 3.33 & 75.57 & 23.27 & 0.00 & 0.00 & 23.27 & 65.43 & 30.08 & 3.33 & 98.86 \\
\hline tilter ${ }^{0-1000}$ & 0.00 & 0.00 & 0.00 & 0.00 & 8.58 & 0.00 & 0.00 & 8.58 & 8.58 & 0.00 & 0.00 & 8.58 \\
\hline $\begin{array}{c}0-1000 \\
\text { upper banner }\end{array}$ & 18.25 & 24.90 & 103.19 & 146.34 & 8.22 & 0.00 & 0.00 & 8.22 & 26.47 & 24.90 & 103.19 & 154.56 \\
\hline $0-1000$ & 1.58 & 3.67 & 10.75 & 16.00 & 0.00 & 0.00 & 0.00 & 0.00 & 1.58 & 3.67 & 10.75 & 16.00 \\
\hline county total= & 90.03 & 210.67 & 314.64 & 615.34 & 97.41 & 6.43 & 0.12 & 103.96 & 187.44 & 217.10 & 314.76 & 719.30 \\
\hline
\end{tabular}




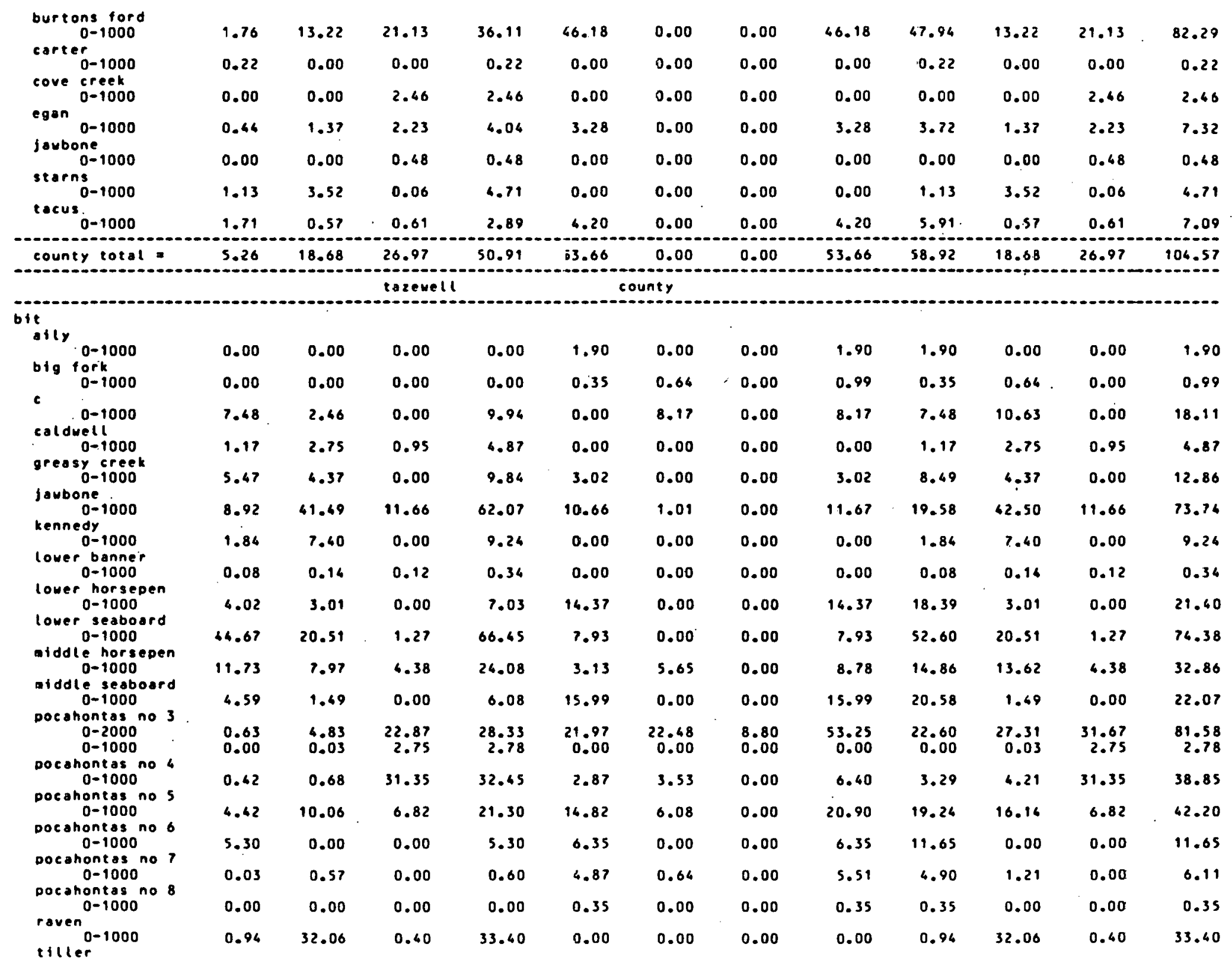




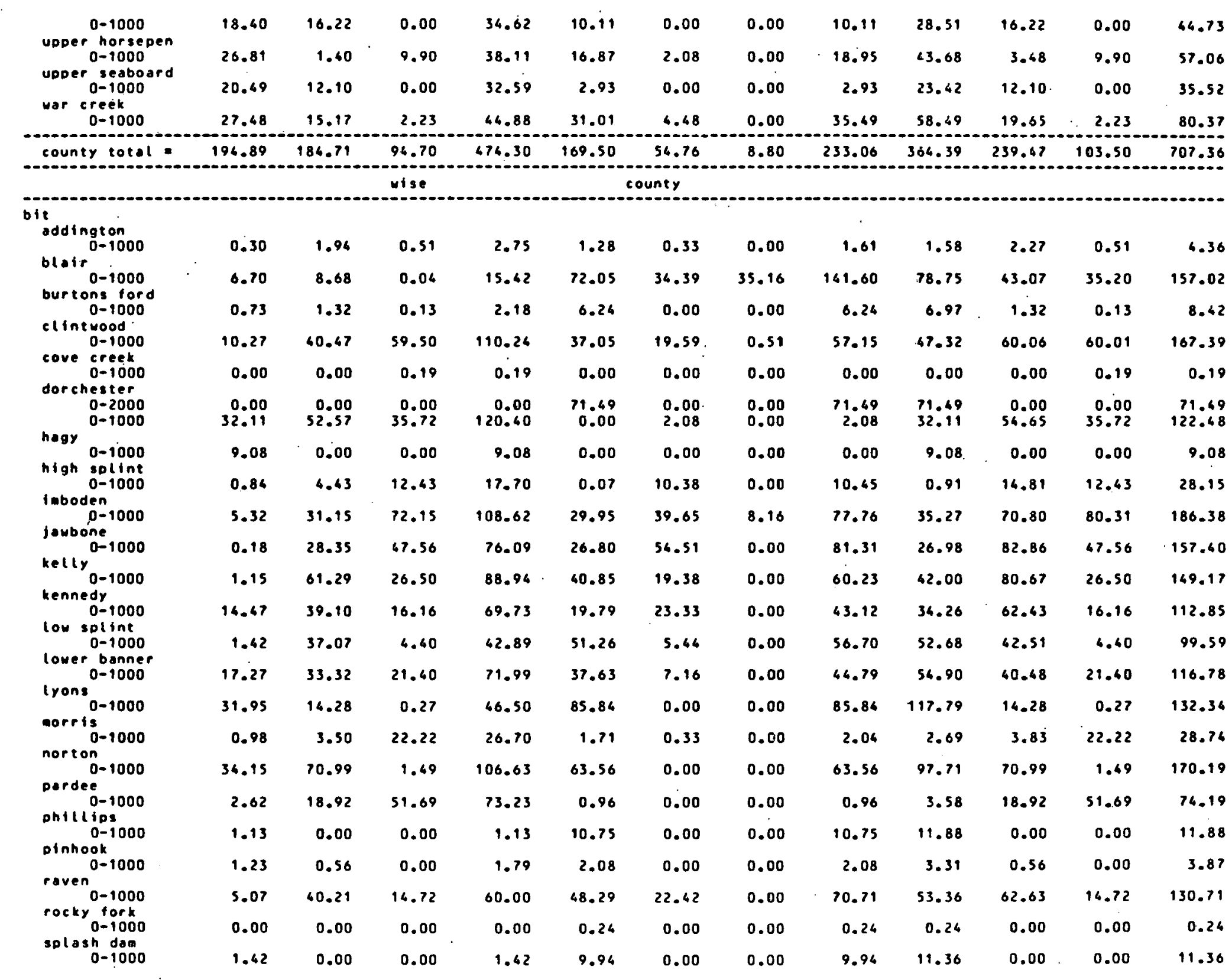




\begin{tabular}{|c|c|c|c|c|c|c|c|c|c|c|c|c|}
\hline $0-1000$ & 2.05 & 34.16 & 79.62 & 115.83 & 9.33 & 4.13 & 0.00 & 5.46 & 3.38 & 38.29 & 79.62 & 121.29 \\
\hline $\begin{array}{c}0-1000 \\
0-90, t\end{array}$ & 12.02 & 71.37 & 0.00 & 83.39 & 2.40 & 0.00 & 0.00 & 2.40 & 14.42 & 71.37 & 0.00 & 85.79 \\
\hline ther & 0.00 & 0.00 & 0.00 & 0.00 & 3.34 & 1.69 & 0.26 & 5.29 & 3.36 & 1.69 & 0.26 & 5.29 \\
\hline $\begin{array}{l}\text { upper Danner } \\
0-1000\end{array}$ & 3.93 & 25.85 & 44.95 & 74.73 & 15.63 & 3.74 & 0.00 & $1^{19.37}$ & 19.56 & 29.50 & 44.95 & 94.10 \\
\hline $0-1000$ & 0.00 & 7.33 & 5.81 & 13.14 & 53.66 & 3.54 & 0.00 & 57.20 & 53.68 & 10.87 & 5.81 & 70.34 \\
\hline inty totol & 6.39 & 6.86 & .46 & 40.71 & 94.19 & 2.09 & 44.09 & 990.37 & $890.5 B$ & 878.95 & 561.55 & 2331.08 \\
\hline
\end{tabular}

overburden

subtotal

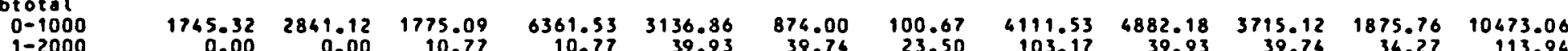

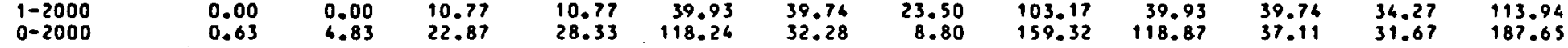

totol $\quad 1745.95 \quad 2845.95 \quad 1808.73 \quad 6400.63 \quad 3295.03 \quad 946.02 \quad 132.97 \quad 4374.02 \quad 5040.98 \quad 3791.97 \quad 1941.70 \quad 10774.65$

\begin{tabular}{|c|c|c|c|c|c|c|c|c|c|c|c|c|}
\hline \multirow{2}{*}{$\begin{array}{l}\text { overburden } \\
\text { total } \\
\qquad-1000 \\
1-2000 \\
0-2000\end{array}$} & \multirow[b]{2}{*}{$\begin{array}{r}1765.32 \\
0.00 \\
0.63\end{array}$} & \multirow[b]{2}{*}{$\begin{array}{r}2841.12 \\
0.00 \\
4.83\end{array}$} & \multirow[b]{2}{*}{$\begin{array}{r}1775.09 \\
10.77 \\
22.87\end{array}$} & \multirow[b]{2}{*}{$\begin{array}{r}6361.53 \\
10.77 \\
28.33\end{array}$} & \multirow[b]{2}{*}{$\begin{array}{r}3136.86 \\
39.93 \\
118.24\end{array}$} & \multirow[b]{2}{*}{$\begin{array}{r}874.00 \\
39.74 \\
32.28\end{array}$} & \multirow[b]{2}{*}{$\begin{array}{r}100.67 \\
23.50 \\
8.80\end{array}$} & \multirow[b]{2}{*}{$\begin{array}{r}4119.53 \\
103.17 \\
159.32\end{array}$} & \multirow[b]{2}{*}{$\begin{array}{r}4882.18 \\
39.93 \\
118.87\end{array}$} & & \multirow[b]{2}{*}{$\begin{array}{r}10473.06 \\
113.94 \\
187.65\end{array}$} \\
\hline & & & & & & & & & & $\begin{array}{r}3715.12 \\
39.74 \\
37.11\end{array}$ & $\begin{array}{r}1875.76 \\
34.27 \\
31.67\end{array}$ & \\
\hline nd to & 1765.95 & 2845.95 & 1808.73 & 6600.63 & 3295.03 & 966.02 & 132.97 & 6374.02 & 5040.98 & 3791.97 & 1961.70 & 10776.65 \\
\hline
\end{tabular}

(1)

total tonnage of identified coal resources in virginia

10774.65

resource tigures token from:

$\begin{array}{lll}\text { source } & \text { year } & \text { base year of resource } \\ \text { usgs circ } 171 & 1952 & 1951\end{array}$


average analyses of coal in virginio

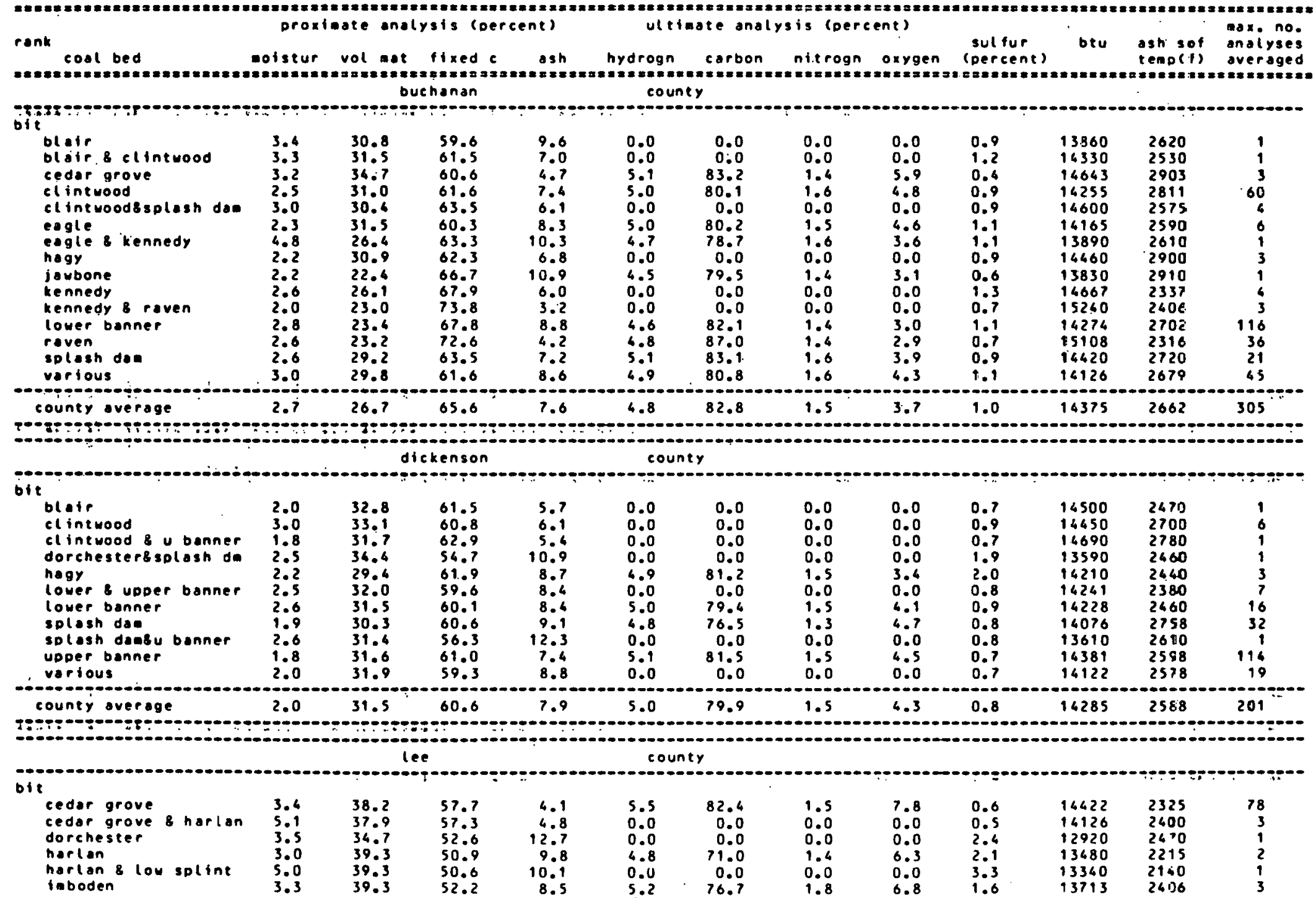




\begin{tabular}{|c|c|c|c|c|c|c|c|c|c|c|c|c|}
\hline $\begin{array}{l}\text { low splint } \\
\text { lou splintsht splint } \\
\text { low splint Bohtllips } \\
\text { pordee } \\
\text { phillips } \\
\text { vortous }\end{array}$ & $\begin{array}{l}3.2 \\
4.1 \\
2.7 \\
3.5 \\
2.9 \\
4.7\end{array}$ & $\begin{array}{l}38.8 \\
39.8 \\
36.5 \\
38.1 \\
37.0 \\
37.5\end{array}$ & $\begin{array}{l}52.3 \\
54.7 \\
54.5 \\
52.5 \\
54.3 \\
52.4\end{array}$ & $\begin{array}{r}8.9 \\
5.5 \\
9.0 \\
9.4 \\
8.8 \\
10.1\end{array}$ & $\begin{array}{l}5.1 \\
5.3 \\
5.1 \\
5.2 \\
5.1 \\
0.0\end{array}$ & $\begin{array}{r}76.5 \\
80.4 \\
75.9 \\
77.2 \\
77.1 \\
0.0\end{array}$ & $\begin{array}{l}1.5 \\
1.6 \\
1.7 \\
1.7 \\
1.7 \\
0.0\end{array}$ & $\begin{array}{l}7.0 \\
8.2 \\
7.6 \\
7.8 \\
7.7 \\
0.0\end{array}$ & $\begin{array}{l}1.8 \\
0.9 \\
0.8 \\
0.9 \\
0.8 \\
1.1\end{array}$ & $\begin{array}{l}13523 \\
14018 \\
13527 \\
13410 \\
13551 \\
13268\end{array}$ & $\begin{array}{l}2437 \\
2340 \\
2836 \\
2630 \\
2890 \\
2462\end{array}$ & $\begin{array}{r}11 \\
5 \\
9 \\
6 \\
10 \\
15\end{array}$ \\
\hline age & 3.5 & 38.1 & 55.7 & 6.3 & 5.3 & 80.0 & 1.6 & 7.6 & $0 . \dot{9}$ & 14008 & $2393^{\circ}$ & 146 \\
\hline & & & gon & & $\|<0$ & & & & & & & \\
\hline merriasac & 1.8 & 12.9 & 60.5 & 26.6 & 0.0 & 0.0 & 0.0 & 0.0 & 0.8 & 11140 & 2910 & 2 \\
\hline county average & 1.8 & 12.9 & 60.5 & 26.6 & 0.0 & 0.0 & 0.0 & 0.0 & 0.8 & 11140 & 2910 & 2 \\
\hline & & & ski $i$ & & & & & & & & & \\
\hline nerrimac & 1.1 & 13.5 & 64.3 & 22.2 & 0.0 & 0.0 & 0.0 & 0.0 & 0.6 & 11590 & 2310 & 3 \\
\hline county average & 1.1 & $i 3.5$ & 64.3 & 22.2 & 0.0 & 0.0 & 0.0 & 0.0 & 0.6 & 11590 & 2310 & $\overline{3}$ \\
\hline & & & sell & & & & & & & & & \\
\hline isubone & 1.0 & 29.1 & 51.4 & 19.5 & 0.0 & 0.0 & 0.0 & 0.0 & 0.5 & 12110 & 2340 & 1 \\
\hline $\begin{array}{l}\text { kennedy } \\
\text { lover } 8 \text { upper banner } \\
\text { lover banner } \\
\text { raven } \\
\text { tiller } \\
\text { upper banner } \\
\text { various }\end{array}$ & $\begin{array}{l}2.2 \\
1.9 \\
2.2 \\
2.6 \\
1.9 \\
2.0 \\
1.9\end{array}$ & $\begin{array}{l}33.3 \\
37.1 \\
35.6 \\
32.8 \\
30.7 \\
36.1 \\
32.4\end{array}$ & $\begin{array}{l}58.9 \\
54.8 \\
56.7 \\
60.1 \\
62.0 \\
57.1 \\
58.9\end{array}$ & $\begin{array}{r}7.8 \\
8.1 \\
7.6 \\
7.1 \\
7.3 \\
6.8 \\
8.7\end{array}$ & $\begin{array}{l}0.0 \\
0.0 \\
5.1 \\
5.2 \\
0.0 \\
5.2 \\
0.0\end{array}$ & $\begin{array}{r}0.0 \\
0.0 \\
79.5 \\
82.8 \\
0.0 \\
81.0 \\
0.0\end{array}$ & $\begin{array}{l}0.0 \\
0.0 \\
1.06 \\
1.5 \\
0.0 \\
1.06 \\
0.0\end{array}$ & $\begin{array}{l}0.0 \\
0.0 \\
5.4 \\
4.5 \\
0.0 \\
5.2 \\
0.0\end{array}$ & $\begin{array}{l}1.0 \\
0.5 \\
0.5 \\
0.8 \\
0.5 \\
0.6 \\
0.8\end{array}$ & $\begin{array}{l}14200 \\
14073 \\
14209 \\
1,6428 \\
14290 \\
14353 \\
14140\end{array}$ & $\begin{array}{l}2200 \\
2293 \\
2330 \\
2297 \\
2300 \\
2285 \\
2320\end{array}$ & $\begin{array}{r}3 \\
3 \\
29 \\
6 \\
1 \\
26 \\
2\end{array}$ \\
\hline county average & 2.1 & 35.3 & 57.2 & 7.5 & 5.2 & 80.7 & 1.6 & 5.1 & 0.7 & 16243 & 2307 & 71 \\
\hline 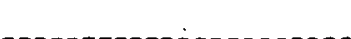 & & & evell & & & & & & & & & \\
\hline $\begin{array}{l}\text { jovbone } \\
\text { lover banner } \\
\text { lower seaboard } \\
\text { middle horsepen } \\
\text { pocahontas no } 3 \\
\text { pocahontas no } 5 \\
\text { roven } \\
\text { upper seaboard } \\
\text { various }\end{array}$ & $\begin{array}{l}2.7 \\
3.6 \\
2.6 \\
3.2 \\
3.2 \\
2.5 \\
2.6 \\
2.5 \\
3.8\end{array}$ & $\begin{array}{l}26.1 \\
28.5 \\
27.4 \\
23.8 \\
21.4 \\
22.3 \\
28.4 \\
29.8 \\
29.4\end{array}$ & $\begin{array}{l}58.2 \\
62.8 \\
66.2 \\
66.6 \\
73.7 \\
73.2 \\
66.3 \\
65.5 \\
59.8\end{array}$ & $\begin{array}{r}15.6 \\
8.7 \\
6.4 \\
9.6 \\
4.9 \\
4.4 \\
5.3 \\
4.7 \\
10.8\end{array}$ & $\begin{array}{l}4.5 \\
4.8 \\
4.9 \\
0.0 \\
4.6 \\
4.7 \\
5.1 \\
5.2 \\
0.0\end{array}$ & $\begin{array}{r}75.6 \\
81.2 \\
82.5 \\
0.0 \\
87.4 \\
87.7 \\
85.4 \\
85.8 \\
0.0\end{array}$ & $\begin{array}{l}1.3 \\
1.5 \\
1.0 \\
0.0 \\
1.2 \\
1.4 \\
1.5 \\
1.4 \\
0.0\end{array}$ & $\begin{array}{l}3.6 \\
3.3 \\
3.4 \\
0.0 \\
3.2 \\
2.8 \\
3.8 \\
3.7 \\
0.0\end{array}$ & $\begin{array}{l}0.6 \\
0.6 \\
1.2 \\
0.7 \\
0.6 \\
0.5 \\
0.7 \\
0.5 \\
0.6\end{array}$ & $\begin{array}{l}13065 \\
14260 \\
14440 \\
14185 \\
14976 \\
15036 \\
14838 \\
16966 \\
13815\end{array}$ & $\begin{array}{l}2845 \\
2720 \\
2260 \\
2770 \\
2512 \\
2268 \\
2301 \\
2338 \\
2433\end{array}$ & $\begin{array}{r}2 \\
3 \\
1 \\
2 \\
20 \\
5 \\
56 \\
5 \\
4\end{array}$ \\
\hline county average & 2.8 & 26.6 & 67.6 & 5.8 & 4.9 & 84.7 & 1.4 & 3.5 & 0.6 & 16768 & 2383 & 98 \\
\hline
\end{tabular}




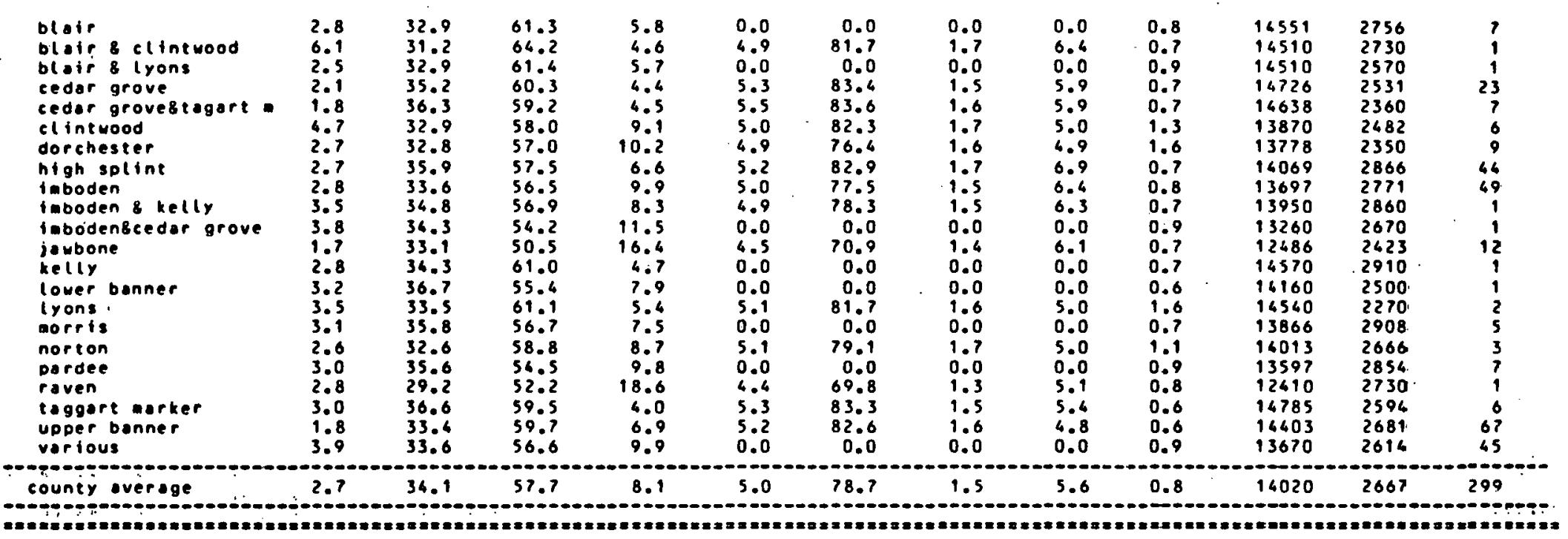




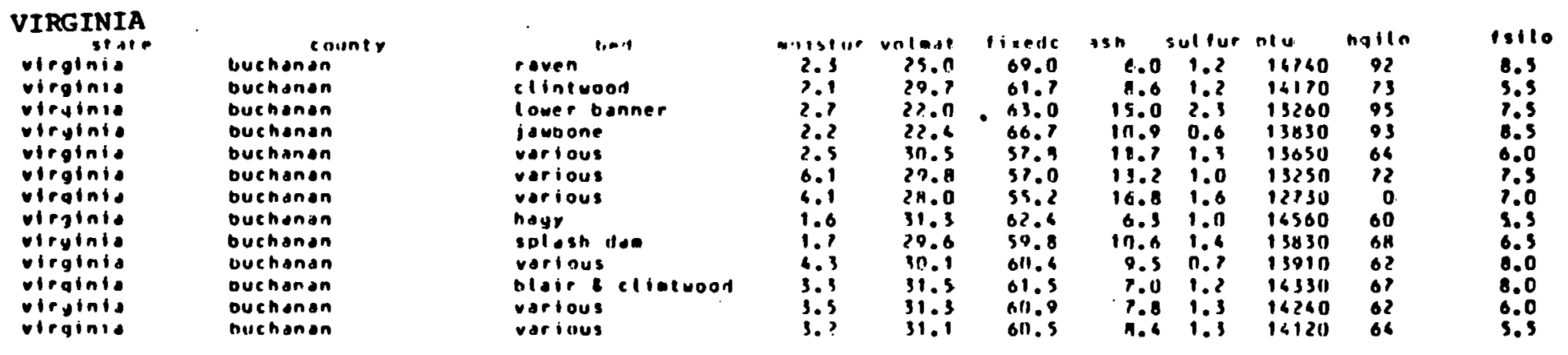




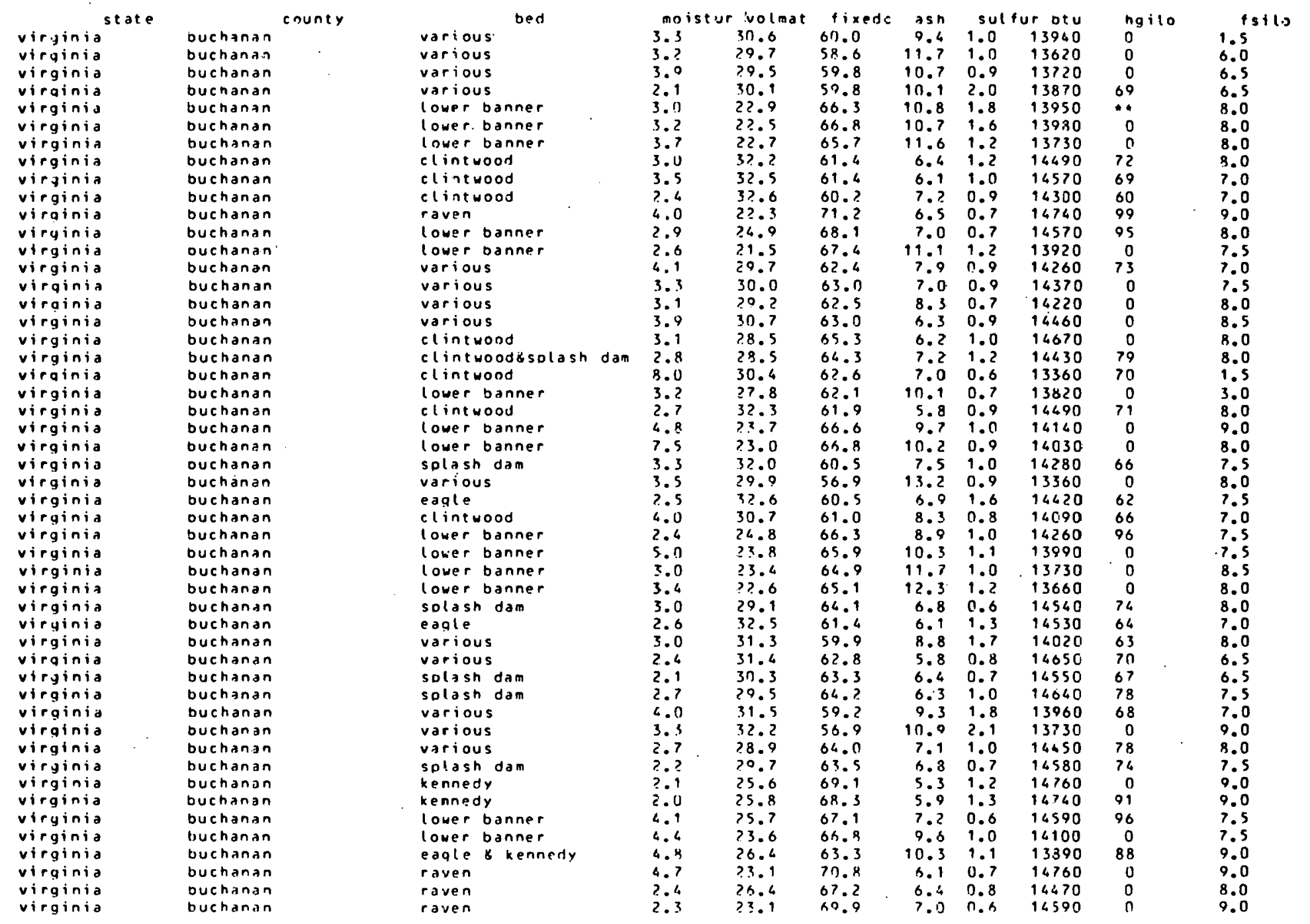




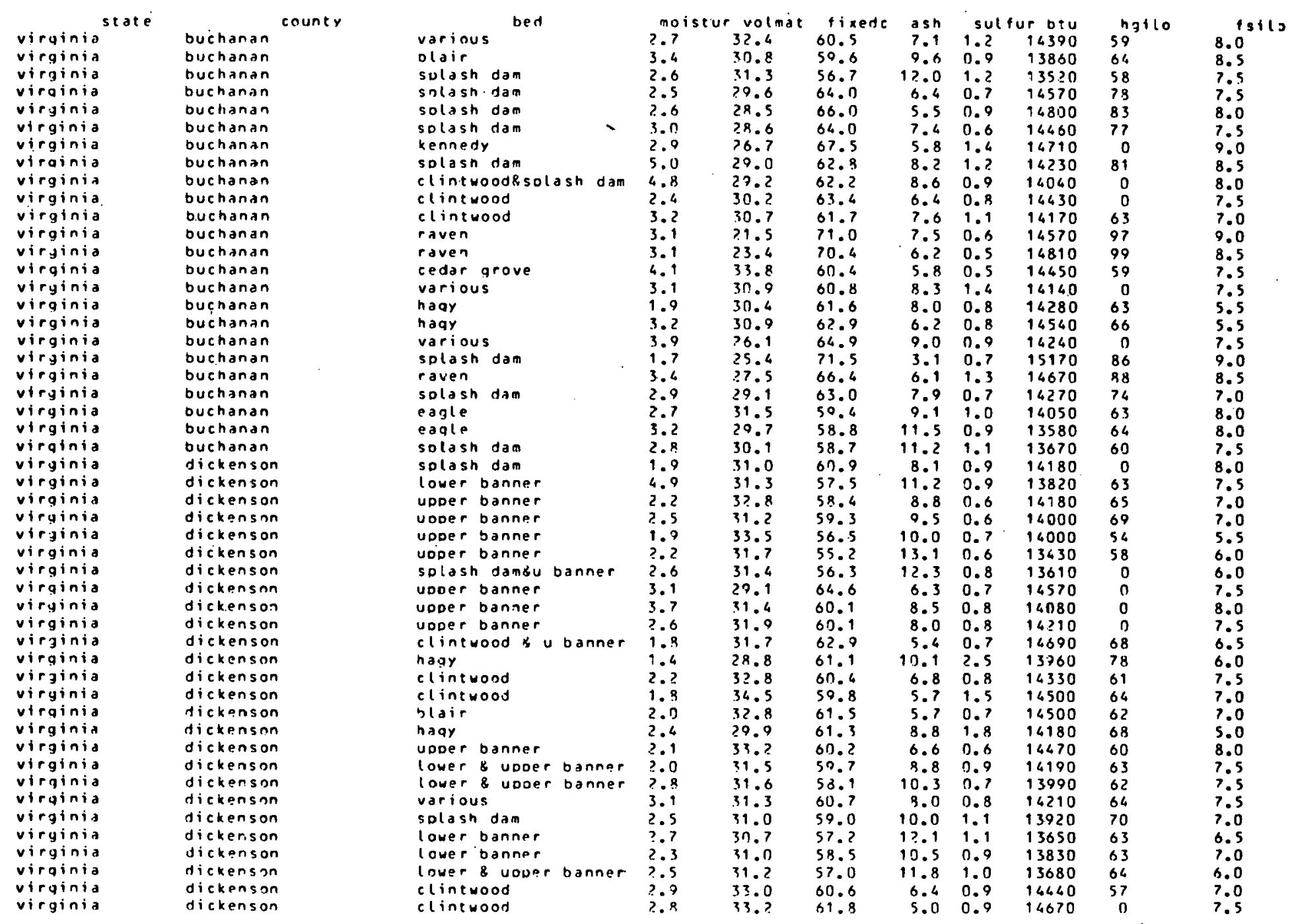




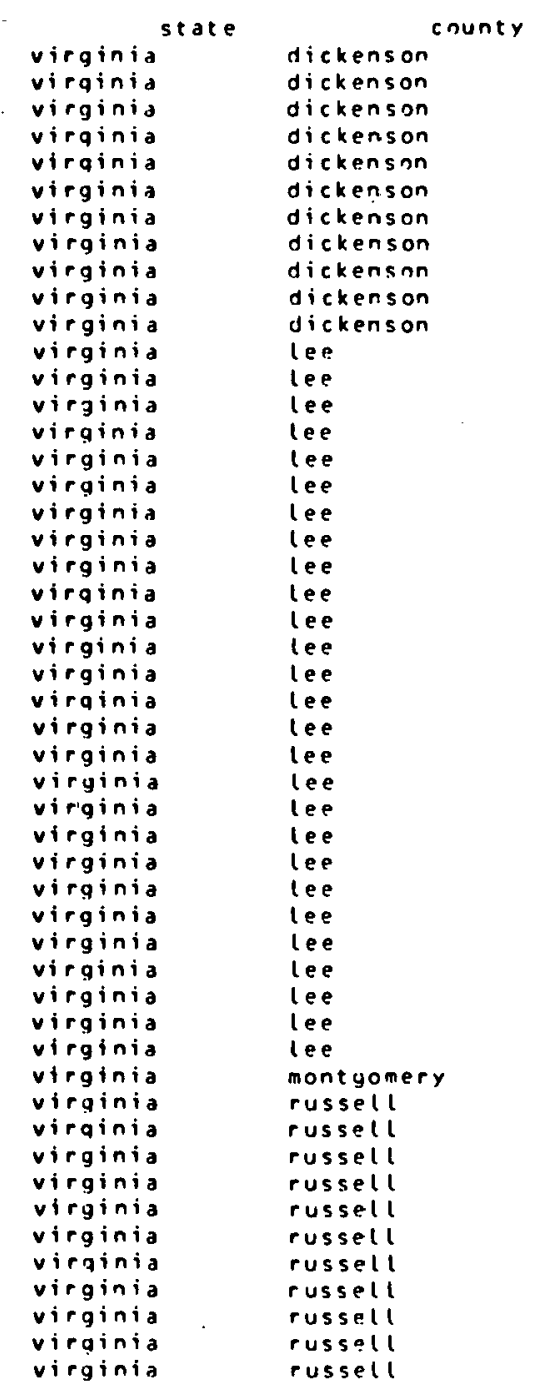

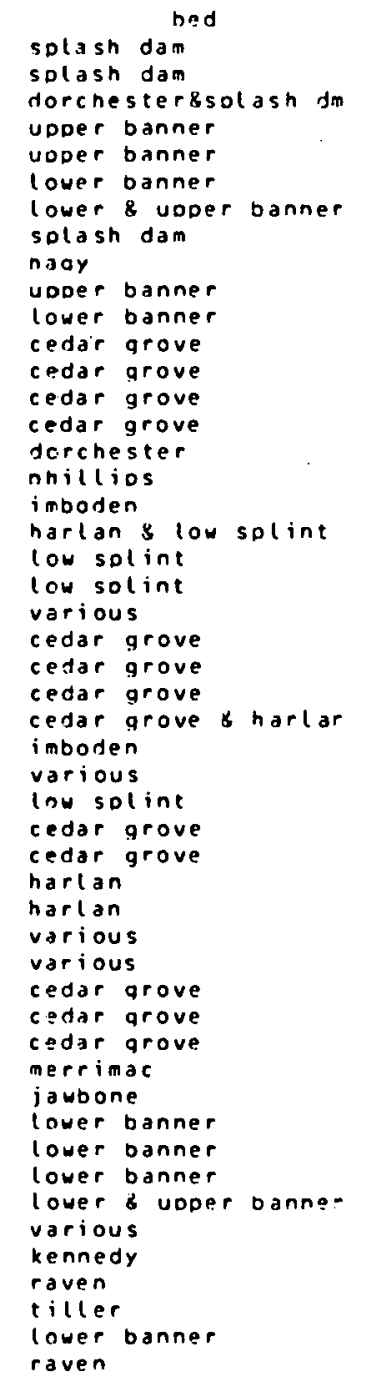

\begin{tabular}{|c|c|}
\hline mo & vol \\
\hline 5.8 & $? 7.2$ \\
\hline 3.4 & $2 \pi .5$ \\
\hline 2.5 & 34.4 \\
\hline$? .2$ & 3.0 \\
\hline 1.9 & $3 ? .9$ \\
\hline 2.3 & 31.8 \\
\hline 3.3 & 31.4 \\
\hline 1.9 & 2.6 .7 \\
\hline 2.7 & 29.6 \\
\hline 1.8 & 35.1 \\
\hline 2.? & 30.4 \\
\hline 4.0 & 38.3 \\
\hline 3.7 & 35.0 \\
\hline 3.0 & 36.6 \\
\hline 3.1 & 36.4 \\
\hline 3.5 & 34.7 \\
\hline 4.2 & 37.2 \\
\hline 4.3 & 36.9 \\
\hline 5.0 & 30.3 \\
\hline 3.6 & 39.7 \\
\hline 5.6 & 37.6 \\
\hline 4.5 & 36.9 \\
\hline 3.? & 36.1 \\
\hline $5 . n$ & 36.8 \\
\hline 5.1 & 36.6 \\
\hline 6.1 & 36.1 \\
\hline 2.9 & 39.7 \\
\hline 3.7 & 37.7 \\
\hline 2.8 & 37.5 \\
\hline 4.4 & 36.4 \\
\hline 6.8 & 36.9 \\
\hline$? .4$ & 37.2 \\
\hline 3.7 & 49.3 \\
\hline 5.8 & 35.4 \\
\hline 6.4 & 35.8 \\
\hline 6.3 & 35.1 \\
\hline 4.7 & 34.0 \\
\hline 4.7 & 33.0 \\
\hline 1.6 & 12.5 \\
\hline 1.0 & 20.1 \\
\hline 1.8 & 35.2 \\
\hline 1.7 & 34.4 \\
\hline 2.4 & 36.9 \\
\hline 2.3 & 35.0 \\
\hline 2.0 & $? 9.9$ \\
\hline$? .8$ & 31.6 \\
\hline 4.3 & 30.4 \\
\hline 1.7 & 30.7 \\
\hline 9.9 & 34.3 \\
\hline 2.8 & 33.6 \\
\hline
\end{tabular}

\begin{tabular}{|c|c|c|c|}
\hline & sh & su & \\
\hline 6.1 & 16.7 & 0.8 & 12630 \\
\hline & 17.3 & 0.8 & 12730 \\
\hline & 10.9 & 1.9 & 13590 \\
\hline & 12.3 & 0.8 & 13500 \\
\hline & 6.9 & 0.6 & 14390 \\
\hline & 8.8 & 1.0 & 14110 \\
\hline & 8.9 & 0.8 & 14130 \\
\hline & $2 ? .5$ & 0.8 & 11900 \\
\hline & 7.2 & 1. & 14490 \\
\hline & 7.7 & 0.7 & 14260 \\
\hline & 10.1 & 0.8 & 13960 \\
\hline & 3.7 & 0. & 16460 \\
\hline & 6.0 & 0.7 & 14180 \\
\hline & 7.9 & 0.9 & 13840 \\
\hline & 6.6 & 0.7 & 16070 \\
\hline & 12.7 & 2.4 & 12920 \\
\hline & 7.8 & 0.7 & 13630 \\
\hline & 11.6 & 1.3 & 13250 \\
\hline & 10.1 & 3.3 & 13340 \\
\hline & 10.8 & 3.7 & 13210 \\
\hline & 11.6 & 2.4 & 12920 \\
\hline & 11.8 & 0.6 & 12990 \\
\hline & 7.3 & 0.5 & 13860 \\
\hline & 6.9 & 0.7 & 13920 \\
\hline & 4.0 & 0.7 & 14650 \\
\hline & 5.8 & 0.6 & 13860 \\
\hline & 9.0 & 1.9 & 13600 \\
\hline .5 & 10.8 & 2.4 & 13250 \\
\hline & 14.9 & 3.4 & 12580 \\
\hline & 9.4 & 0.6 & 13510 \\
\hline & 4.2 & 0.8 & 14400 \\
\hline & 13.3 & 3.2 & 12780 \\
\hline & 6.4 & 1.1 & 14 \\
\hline & 15.6 & 1.3 & 12410 \\
\hline & 14.0 & 0.9 & $1 \geq 460$ \\
\hline & 2.3 & 0.5 & 16 \\
\hline & 6.0 & 0.7 & 14120 \\
\hline & 9.0 & 0.7 & \\
\hline & 39.7 & 0.7 & 10 \\
\hline & 19.5 & 0.5 & 12110 \\
\hline & 7.7 & 0.6 & 14 \\
\hline & 8.4 & 0.7 & .14090 \\
\hline & 8.0 & 0.6 & 142 \\
\hline & 19.1 & 0.6 & 13540 \\
\hline & 9.6 & 0.9 & \\
\hline & 12.5 & 1.2 & 13430 \\
\hline & 13.3 & 0. & 133 \\
\hline & 7.3 & 0.5 & 14290 \\
\hline & 9.9 & 0. & 13970 \\
\hline & 6.5 & 0.7 & 1449 \\
\hline
\end{tabular}

\begin{tabular}{|c|c|}
\hline hgilo & isilo \\
\hline 0 & 2.5 \\
\hline 61 & 8.0 \\
\hline 53 & 7.5 \\
\hline 0 & 7.5 \\
\hline 64 & 7.5 \\
\hline $6 n$ & 7.5 \\
\hline 0 & 7.0 \\
\hline 0 & 7.5 \\
\hline 82 & 0.0 \\
\hline 58 & 6.0 \\
\hline 75 & 7.5 \\
\hline 0 & 5.5 \\
\hline 0 & 5.5 \\
\hline 0 & 5.0 \\
\hline 54 & 6.0 \\
\hline 55 & 5.5 \\
\hline 43 & 5.0 \\
\hline 46 & 5.0 \\
\hline 45 & 5.0 \\
\hline 47 & 5.0 \\
\hline 0 & 2.5 \\
\hline o & 3.5 \\
\hline 0 & 5.5 \\
\hline 0 & 4.5 \\
\hline 0 & 5.5 \\
\hline 0 & 3.0 \\
\hline 49 & 5.0 \\
\hline 45 & 4.5 \\
\hline 44 & 4.5 \\
\hline 0 & 4.5 \\
\hline 0 & 6.5 \\
\hline 49 & 5.0 \\
\hline s1 & 4.5 \\
\hline 0 & 1.0 \\
\hline 0 & 1.5 \\
\hline 43 & 2.0 \\
\hline 0 & 6.0 \\
\hline 0 & 6.5 \\
\hline 69 & 1.0 \\
\hline 0 & 6.5 \\
\hline 51 & 7.0 \\
\hline 52 & 7.0 \\
\hline 43 & 7.0 \\
\hline Si & 7.0 \\
\hline 0 & 8.0 \\
\hline 61 & 7.5 \\
\hline 57 & 8.0 \\
\hline 0 & 8.5 \\
\hline 5? & 7.0 \\
\hline 54 & 7.5 \\
\hline
\end{tabular}




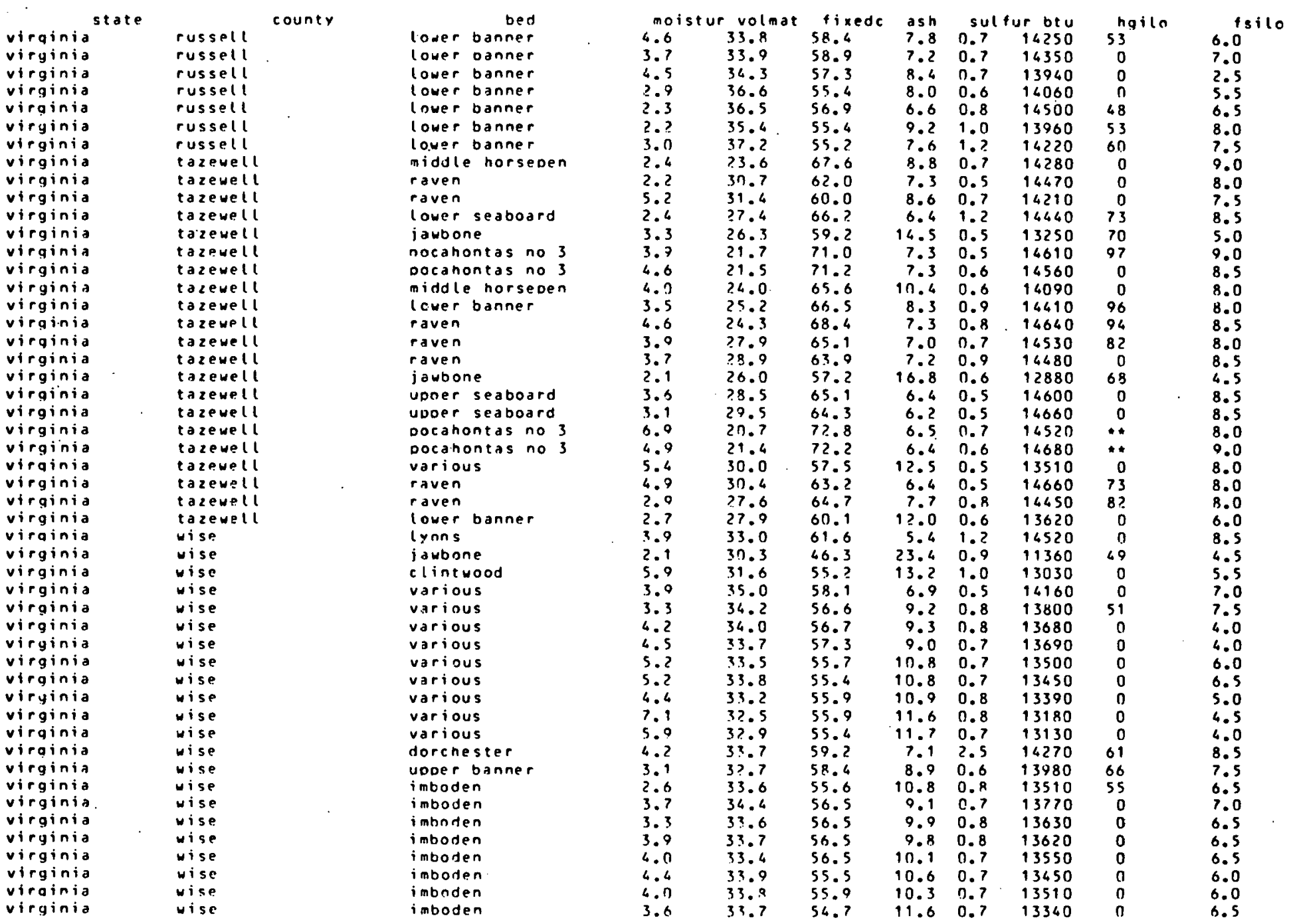




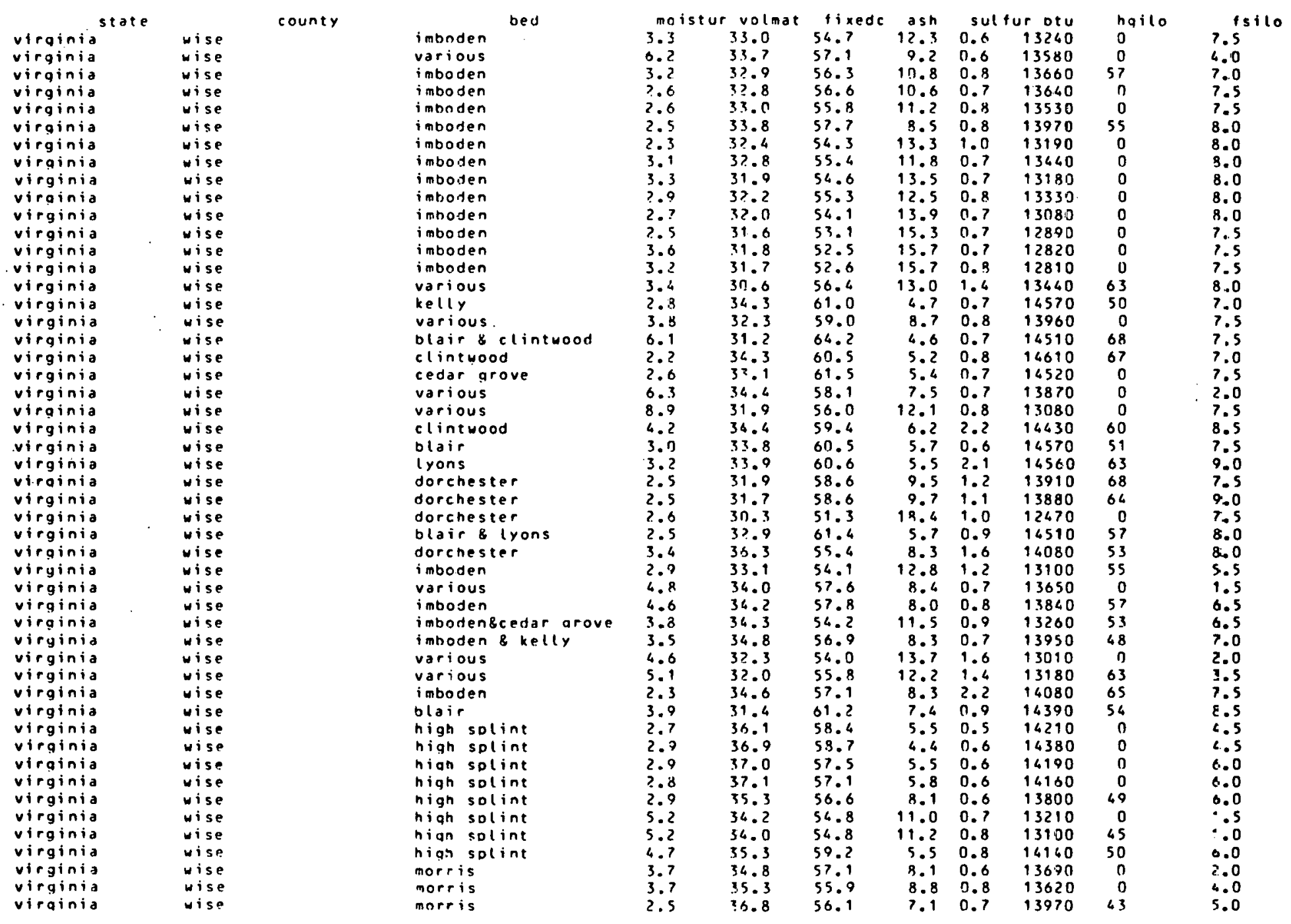




\section{VIRGINIA}

\begin{tabular}{|c|c|c|}
\hline 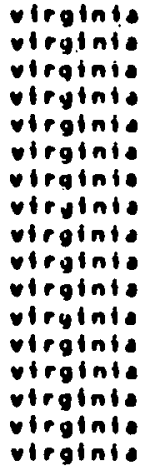 & 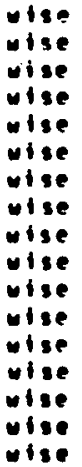 & 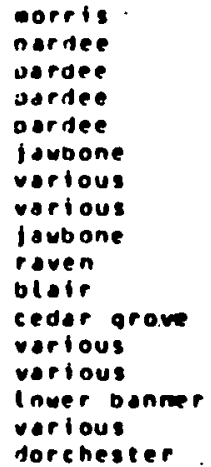 \\
\hline
\end{tabular}

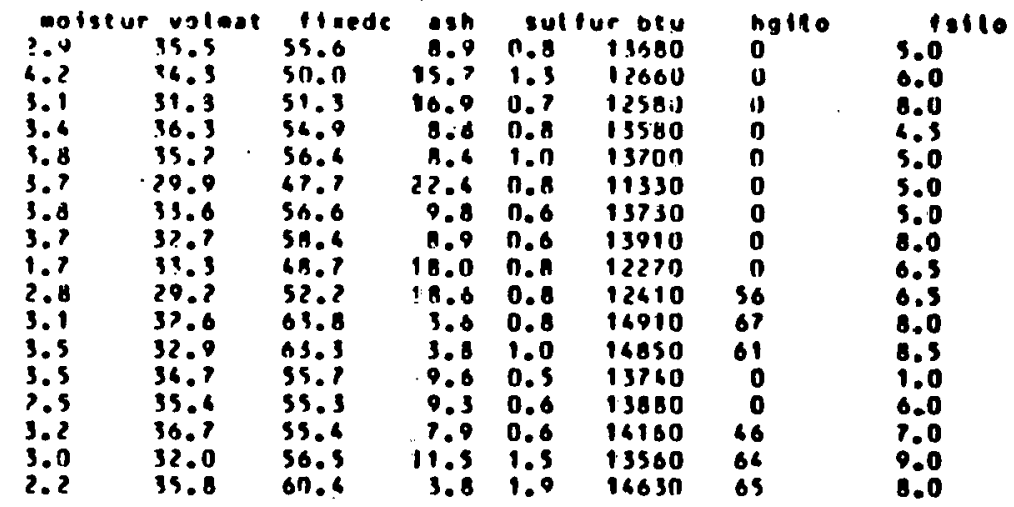



National Coal Resources Data systen

U.S. Geological survey

identified coal resources in wasnington

(In mitllions of short tons)

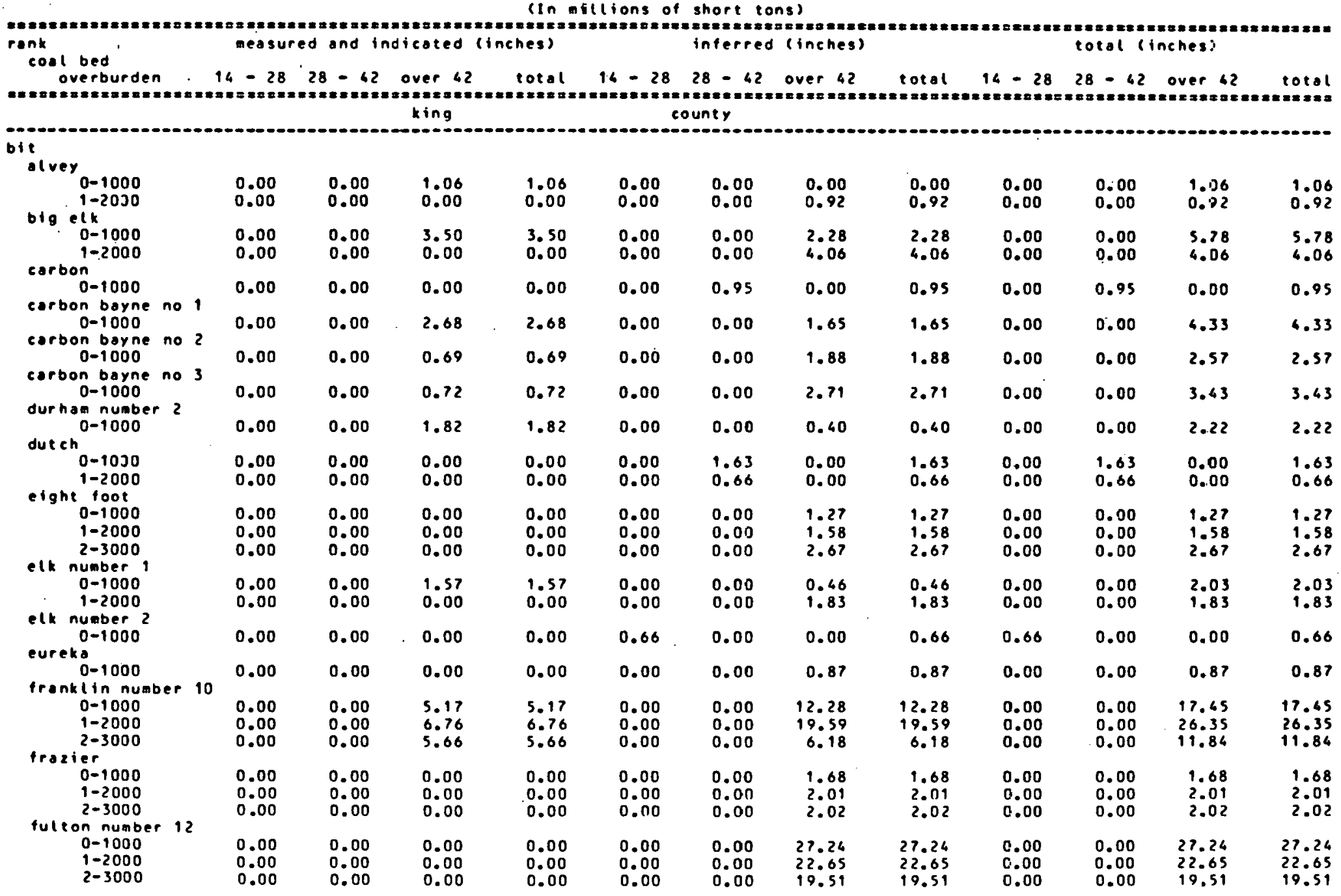




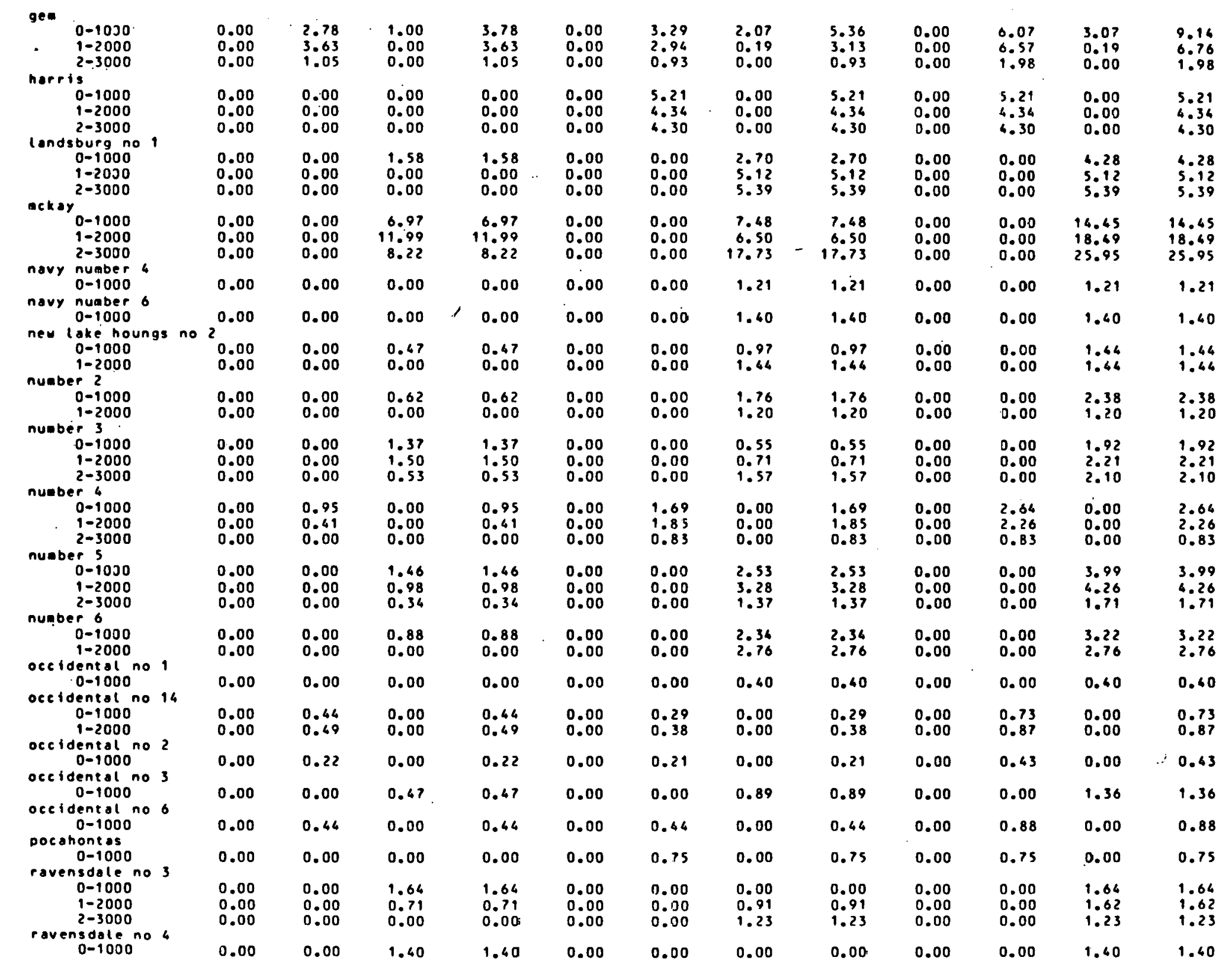




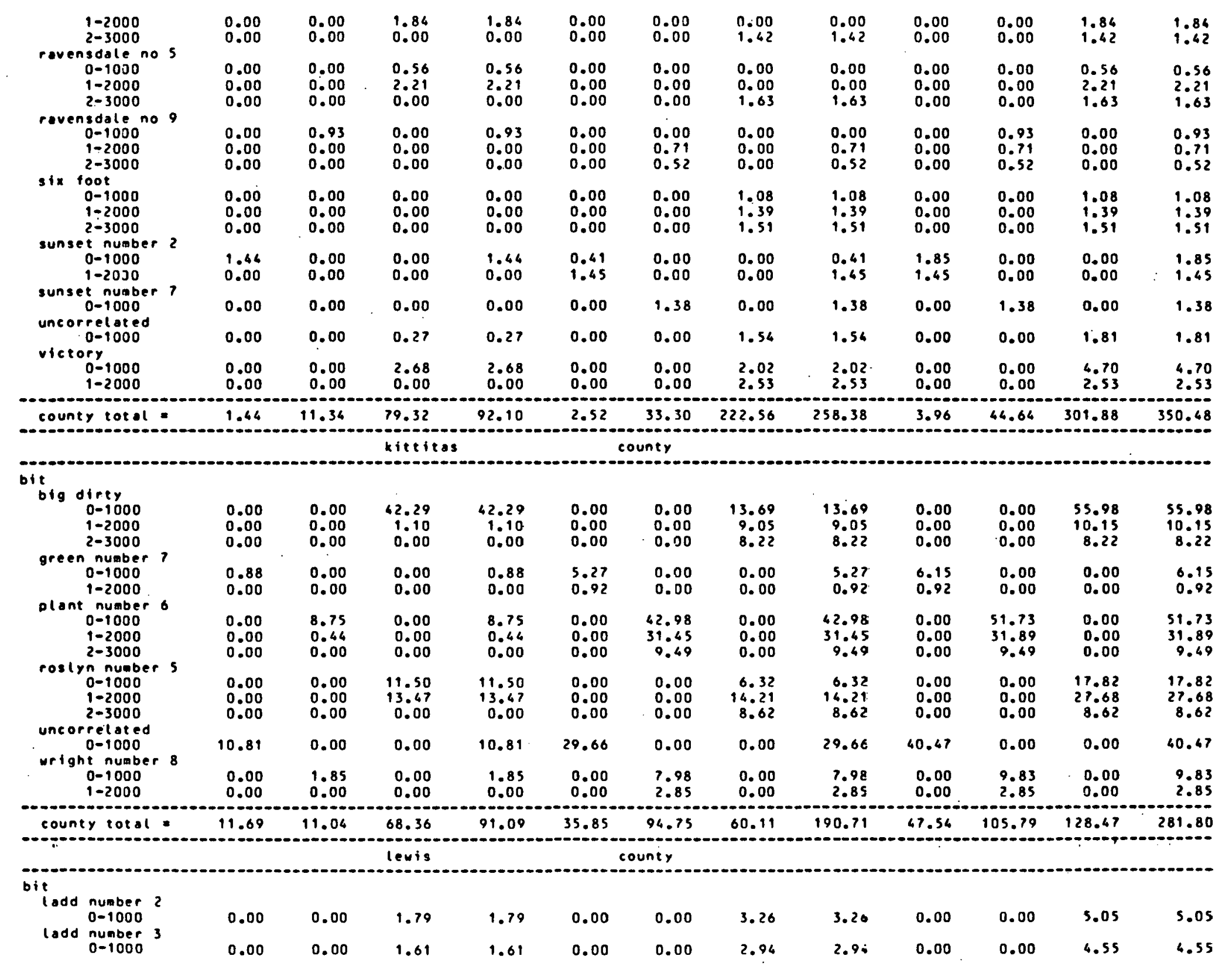




\begin{tabular}{|c|c|c|c|c|c|c|c|c|c|c|c|c|}
\hline $\begin{array}{c}\text { lodd number } 4 \\
0-1000 \\
\text { uncorrelated } \\
0-1000\end{array}$ & $\begin{array}{l}0.00 \\
0.00\end{array}$ & $\begin{array}{l}0.00 \\
0.00\end{array}$ & $\begin{array}{l}1.70 \\
1.92\end{array}$ & $\begin{array}{l}1.70 \\
1.92\end{array}$ & $\begin{array}{l}0.00 \\
1.62\end{array}$ & 0.00 & $\begin{array}{r}3.10 \\
29.38\end{array}$ & $\begin{array}{r}3.10 \\
31.00\end{array}$ & $\begin{array}{l}0.00 \\
1.62\end{array}$ & $\begin{array}{l}0.00 \\
0.00\end{array}$ & $\begin{array}{r}4.80 \\
31.30\end{array}$ & $\begin{array}{r}4.80 \\
32.92\end{array}$ \\
\hline county total = & 0.00 & 0.00 & 7.02 & 7.02 & 1.62 & 0.00 & 38.68 & 60.30 & 1.62 & 0.00 & 45.70 & 47.32 \\
\hline & & & & & & & & & & & & \\
\hline it bio ben & & & & & & & & & & & & \\
\hline $\begin{array}{l}0-1000 \\
\text { black carbon }\end{array}$ & 0.00 & 0.00 & 0.00 & 0.00 & 0.00 & 0.00 & 1.92 & 1.92 & 0.00 & 0.00 & 1.92 & 1.92 \\
\hline $\begin{array}{r}0-1000 \\
1-2000 \\
2-3000 \\
\text { blocksith }\end{array}$ & $\begin{array}{l}0.00 \\
0.00 \\
0.00\end{array}$ & $\begin{array}{l}0.00 \\
0.00 \\
0.00\end{array}$ & $\begin{array}{l}1.18 \\
1.18 \\
1.18\end{array}$ & $\begin{array}{l}1.18 \\
1.18 \\
1.18\end{array}$ & $\begin{array}{l}0.00 \\
0.00 \\
0.00\end{array}$ & $\begin{array}{l}0.00 \\
0.00 \\
0.00\end{array}$ & $\begin{array}{l}1.49 \\
1.93 \\
1.93\end{array}$ & $\begin{array}{l}1.49 \\
1.93 \\
1.93\end{array}$ & $\begin{array}{l}0.00 \\
0.00 \\
0.00\end{array}$ & $\begin{array}{l}0.00 \\
0.00 \\
0.00\end{array}$ & $\begin{array}{l}2.67 \\
3.11 \\
3.11\end{array}$ & $\begin{array}{l}2.67 \\
3.11 \\
3.11\end{array}$ \\
\hline $\begin{array}{r}0-1000 \\
1-2000 \\
2-3000\end{array}$ & $\begin{array}{l}0.37 \\
0.37 \\
0.37\end{array}$ & $\begin{array}{l}0.00 \\
0.00 \\
0.00\end{array}$ & $\begin{array}{l}0.00 \\
0.00 \\
0.00\end{array}$ & $\begin{array}{l}0.37 \\
0.37 \\
0.37\end{array}$ & $\begin{array}{l}0.00 \\
0.00 \\
0.00\end{array}$ & $\begin{array}{l}0.00 \\
0.00 \\
0.00\end{array}$ & $\begin{array}{l}0.00 \\
0.00 \\
0.00\end{array}$ & $\begin{array}{l}0.00 \\
0.00 \\
0.00\end{array}$ & $\begin{array}{l}0.37 \\
0.37 \\
0.37\end{array}$ & $\begin{array}{l}0.00 \\
0.00 \\
0.00\end{array}$ & $\begin{array}{l}0.00 \\
0.00 \\
0.00\end{array}$ & $\begin{array}{l}0.37 \\
0.37 \\
0.37\end{array}$ \\
\hline $\begin{array}{c}0-1000 \\
1-2000 \\
2-3000 \\
\text { corbonado number }\end{array}$ & $\begin{array}{l}0.00 \\
0.00 \\
0.00\end{array}$ & $\begin{array}{l}0.76 \\
0.76 \\
0.76\end{array}$ & $\begin{array}{l}0.00 \\
0.00 \\
0.00\end{array}$ & $\begin{array}{l}0.76 \\
0.76 \\
0.76\end{array}$ & $\begin{array}{l}0.00 \\
0.00 \\
0.00\end{array}$ & $\begin{array}{l}1.60 \\
1.60 \\
1.60\end{array}$ & $\begin{array}{l}0.00 \\
0.00 \\
0.00\end{array}$ & $\begin{array}{l}1.60 \\
1.60 \\
1.60\end{array}$ & $\begin{array}{l}0.00 \\
0.00 \\
0.00\end{array}$ & $\begin{array}{l}2.36 \\
2.36 \\
2.36\end{array}$ & $\begin{array}{l}0.00 \\
0.00 \\
0.00\end{array}$ & $\begin{array}{l}2.36 \\
2.36 \\
2.36\end{array}$ \\
\hline $\begin{array}{l}0-1000 \\
1-2000\end{array}$ & $\begin{array}{l}0.00 \\
0.00\end{array}$ & $\begin{array}{l}0.00 \\
0.00\end{array}$ & $\begin{array}{l}3.06 \\
0.00\end{array}$ & $\begin{array}{l}3.06 \\
0.00\end{array}$ & $\begin{array}{l}0.00 \\
0.00\end{array}$ & $\begin{array}{l}0.00 \\
0.00\end{array}$ & $\begin{array}{l}0.00 \\
3.46\end{array}$ & $\begin{array}{l}0.00 \\
3.16\end{array}$ & $\begin{array}{l}0.00 \\
0.00\end{array}$ & $\begin{array}{l}0.00 \\
0.00\end{array}$ & $\begin{array}{l}3.06 \\
3.16\end{array}$ & $\begin{array}{l}3.06 \\
3.16\end{array}$ \\
\hline $\begin{array}{c}0-1000 \\
1-2030 \\
2-3000 \\
\text { crocker }\end{array}$ & $\begin{array}{l}0.00 \\
0.00 \\
0.00\end{array}$ & $\begin{array}{l}0.00 \\
0.00 \\
0.00\end{array}$ & $\begin{array}{l}1.08 \\
0.00 \\
0.00\end{array}$ & $\begin{array}{l}1.08 \\
0.00 \\
0.00\end{array}$ & $\begin{array}{l}0.00 \\
0.00 \\
0.00\end{array}$ & $\begin{array}{l}0.00 \\
0.00 \\
0.00\end{array}$ & $\begin{array}{l}2.60 \\
2.99 \\
1.59\end{array}$ & $\begin{array}{l}2.60 \\
2.99 \\
1.59\end{array}$ & $\begin{array}{l}0.00 \\
0.00 \\
0.00\end{array}$ & $\begin{array}{l}0.00 \\
0.00 \\
0.00\end{array}$ & $\begin{array}{l}3.48 \\
2.99 \\
1.59\end{array}$ & $\begin{array}{l}3.48 \\
2.99 \\
1.59\end{array}$ \\
\hline $\begin{array}{r}0-1000 \\
1-2000 \\
2-3000 \\
\text { acneill }\end{array}$ & $\begin{array}{l}0.58 \\
0.58 \\
0.58\end{array}$ & $\begin{array}{l}0.00 \\
0.00 \\
0.00\end{array}$ & $\begin{array}{l}0.00 \\
0.00 \\
0.00\end{array}$ & $\begin{array}{l}0.58 \\
0.58 \\
0.58\end{array}$ & $\begin{array}{l}1.12 \\
1.12 \\
1.12\end{array}$ & $\begin{array}{l}0.00 \\
0.00 \\
0.00\end{array}$ & $\begin{array}{l}0.00 \\
0.00 \\
0.00\end{array}$ & $\begin{array}{l}1.12 \\
1.1 .2 \\
1.12\end{array}$ & $\begin{array}{l}1.70 \\
1.70 \\
1.70\end{array}$ & $\begin{array}{l}0.00 \\
0.00 \\
0.00\end{array}$ & $\begin{array}{l}0.00 \\
0.00 \\
0.00\end{array}$ & $\begin{array}{l}1.70 \\
1.70 \\
1.70\end{array}$ \\
\hline $\begin{array}{c}0-1000 \\
1-2000 \\
2-3000 \\
\text { morgon number, }\end{array}$ & $\begin{array}{l}0.00 \\
0.00 \\
0.00\end{array}$ & $\begin{array}{l}0.63 \\
0.82 \\
0.82\end{array}$ & $\begin{array}{l}0.00 \\
0.00 \\
0.00\end{array}$ & $\begin{array}{l}0.63 \\
0.82 \\
0.82\end{array}$ & $\begin{array}{l}0.00 \\
0.00 \\
0.00\end{array}$ & $\begin{array}{l}0.00 \\
0.00 \\
0.00\end{array}$ & $\begin{array}{l}0.00 \\
0.00 \\
0.00\end{array}$ & $\begin{array}{l}0.00 \\
0.00 \\
0.00\end{array}$ & $\begin{array}{l}0.00 \\
0.00 \\
0.00\end{array}$ & $\begin{array}{l}0.63 \\
0.82 \\
0.82\end{array}$ & $\begin{array}{l}0.00 \\
0.00 \\
0.00\end{array}$ & $\begin{array}{l}0.63 \\
0.82 \\
0.82\end{array}$ \\
\hline $\begin{array}{c}0-1000 \\
1-2000 \\
2-3000 \\
\text { nisqually }\end{array}$ & $\begin{array}{l}0.00 \\
0.00 \\
0.00\end{array}$ & $\begin{array}{l}0.00 \\
0.00 \\
0.00\end{array}$ & $\begin{array}{l}1.98 \\
0.22 \\
0.00\end{array}$ & $\begin{array}{l}1.98 \\
0.22 \\
0.00\end{array}$ & $\begin{array}{l}0.00 \\
0.00 \\
0.00\end{array}$ & $\begin{array}{l}0.00 \\
0.00 \\
0.00\end{array}$ & $\begin{array}{l}2.23 \\
5.05 \\
2.29\end{array}$ & $\begin{array}{l}2.23 \\
5.05 \\
2.29\end{array}$ & $\begin{array}{l}0.00 \\
0.00 \\
0.00\end{array}$ & $\begin{array}{l}0.00 \\
0.00 \\
0.00\end{array}$ & $\begin{array}{l}4.21 \\
5.27 \\
2.29\end{array}$ & $\begin{array}{l}4.21 \\
5.27 \\
2.29\end{array}$ \\
\hline $\begin{array}{r}0-1000 \\
1-2000 \\
2-3000 \\
\text { number } 1\end{array}$ & $\begin{array}{l}0.00 \\
0.00 \\
0.00\end{array}$ & $\begin{array}{l}0.00 \\
0.00 \\
0.00\end{array}$ & $\begin{array}{l}0.00 \\
0.00 \\
0.00\end{array}$ & $\begin{array}{l}0.00 \\
0.00 \\
0.00\end{array}$ & $\begin{array}{l}0.00 \\
0.00 \\
0.00\end{array}$ & $\begin{array}{l}0.00 \\
0.00 \\
0.00\end{array}$ & $\begin{array}{l}4.47 \\
4.47 \\
4.47\end{array}$ & $\begin{array}{l}4.47 \\
4.47 \\
4.47\end{array}$ & $\begin{array}{l}0.00 \\
0.00 \\
0.00\end{array}$ & $\begin{array}{l}0.00 \\
0.00 \\
0.00\end{array}$ & $\begin{array}{l}4.47 \\
4.67 \\
4.47\end{array}$ & $\begin{array}{l}4.47 \\
4.47 \\
4.47\end{array}$ \\
\hline $\begin{array}{r}0-1000 \\
1-2000 \\
2-3000 \\
\text { number } 10\end{array}$ & $\begin{array}{l}0.23 \\
0.23 \\
0.23\end{array}$ & $\begin{array}{l}0.00 \\
0.00 \\
0.00\end{array}$ & $\begin{array}{l}0.29 \\
0.00 \\
0.00\end{array}$ & $\begin{array}{l}0.52 \\
0.23 \\
0.23\end{array}$ & $\begin{array}{l}0.19 \\
0.19 \\
0.19\end{array}$ & $\begin{array}{l}0.00 \\
0.00 \\
0.00\end{array}$ & $\begin{array}{l}0.47 \\
0.00 \\
0.00\end{array}$ & $\begin{array}{l}0.66 \\
0.19 \\
0.19\end{array}$ & $\begin{array}{l}0.42 \\
0.42 \\
0.42\end{array}$ & $\begin{array}{l}0.00 \\
0.00 \\
0.00\end{array}$ & $\begin{array}{l}0.76 \\
0.00 \\
0.00\end{array}$ & $\begin{array}{l}1.18 \\
0.42 \\
0.42\end{array}$ \\
\hline $\begin{array}{r}0-1000 \\
1-2000 \\
2-3000 \\
\text { number } 11\end{array}$ & $\begin{array}{l}0.00 \\
0.00 \\
0.00\end{array}$ & $\begin{array}{l}0.00 \\
0.00 \\
0.00\end{array}$ & $\begin{array}{l}2.77 \\
3.02 \\
3.02\end{array}$ & $\begin{array}{l}2.77 \\
3.02 \\
3.02\end{array}$ & $\begin{array}{l}0.00 \\
0.00 \\
0.00\end{array}$ & $\begin{array}{l}0.00 \\
0.00 \\
0.00\end{array}$ & $\begin{array}{l}0.74 \\
1.87 \\
1.87\end{array}$ & $\begin{array}{l}0.74 \\
1.87 \\
1.87\end{array}$ & $\begin{array}{l}0.00 \\
0.00 \\
0.00\end{array}$ & $\begin{array}{l}0.00 \\
0.00 \\
0.00\end{array}$ & $\begin{array}{l}3.51 \\
4.89 \\
4.89\end{array}$ & $\begin{array}{l}3.51 \\
4.89 \\
4.89\end{array}$ \\
\hline $\begin{array}{r}0-1000 \\
1-2000 \\
2-3000 \\
\text { number } 12\end{array}$ & $\begin{array}{l}0.59 \\
0.59 \\
0.59\end{array}$ & $\begin{array}{l}0.88 \\
0.88 \\
0.88\end{array}$ & $\begin{array}{l}0.00 \\
0.00 \\
0.00\end{array}$ & $\begin{array}{l}1.47 \\
1.47 \\
1.47\end{array}$ & $\begin{array}{l}0.39 \\
0.39 \\
0.39\end{array}$ & $\begin{array}{l}0.26 \\
0.79 \\
0.79\end{array}$ & $\begin{array}{l}0.00 \\
0.00 \\
0.00\end{array}$ & $\begin{array}{l}0.65 \\
1.18 \\
1.18\end{array}$ & $\begin{array}{l}0.98 \\
0.98 \\
0.98\end{array}$ & $\begin{array}{l}1.14 \\
1.67 \\
1.67\end{array}$ & $\begin{array}{l}0.00 \\
0.00 \\
0.00\end{array}$ & $\begin{array}{l}2.12 \\
2.65 \\
2.65\end{array}$ \\
\hline
\end{tabular}




\begin{tabular}{|c|c|c|c|c|c|c|c|c|c|c|c|c|}
\hline $\begin{array}{r}0-1000 \\
1-2000 \\
2-3000 \\
\text { nunber } 2\end{array}$ & $\begin{array}{l}0.00 \\
0.00 \\
0.00\end{array}$ & $\begin{array}{l}0.93 \\
0.93 \\
0.93\end{array}$ & $\begin{array}{l}0.00 \\
0.00 \\
0.00\end{array}$ & $\begin{array}{l}0.93 \\
0.93 \\
0.93\end{array}$ & $\begin{array}{l}0.00 \\
0.00 \\
0.00\end{array}$ & $\begin{array}{l}1.76 \\
1.76 \\
1.74\end{array}$ & $\begin{array}{l}0.00 \\
0.00 \\
0.00\end{array}$ & $\begin{array}{l}1.76 \\
1.76 \\
1.74\end{array}$ & $\begin{array}{l}0.00 \\
0.00 \\
0.00\end{array}$ & $\begin{array}{l}2.67 \\
2.67 \\
2.67\end{array}$ & $\begin{array}{l}0.00 \\
0.00 \\
0.00\end{array}$ & $\begin{array}{l}2.67 \\
2.67 \\
2.67\end{array}$ \\
\hline $\begin{array}{l}0-1000 \\
1-2000 \\
2-3000\end{array}$ & $\begin{array}{l}0.56 \\
0.56 \\
0.56\end{array}$ & $\begin{array}{l}0.00 \\
0.00 \\
0.00\end{array}$ & $\begin{array}{l}1.25 \\
0.00 \\
0.00\end{array}$ & $\begin{array}{l}1.81 \\
0.56 \\
0.56\end{array}$ & $\begin{array}{l}0.25 \\
0.25 \\
0.25\end{array}$ & $\begin{array}{l}0.00 \\
0.00 \\
0.00\end{array}$ & $\begin{array}{l}2.37 \\
0.25 \\
0.00\end{array}$ & $\begin{array}{l}2.62 \\
0.50 \\
0.25\end{array}$ & $\begin{array}{l}0.81 \\
0.81 \\
0.81\end{array}$ & $\begin{array}{l}0.00 \\
0.00 \\
0.00\end{array}$ & $\begin{array}{l}3.62 \\
0.25 \\
0.00\end{array}$ & $\begin{array}{l}4.63 \\
1.06 \\
0.81\end{array}$ \\
\hline $\begin{array}{c}\text { number } 2 \\
0-1000 \\
\text { number } 3\end{array}$ & 0.00 & 0.00 & 0.00 & 0.00 & 0.00 & 0.00 & 1.28 & 1.28 & 0.00 & 0.00 & 1.28 & 1.28 \\
\hline $\begin{array}{r}0-1000 \\
1-2000 \\
2-3000 \\
\text { number } 4\end{array}$ & $\begin{array}{l}0.31 \\
0.31 \\
0.31\end{array}$ & $\begin{array}{l}0.36 \\
0.36 \\
0.36\end{array}$ & $\begin{array}{l}0.06 \\
0.00 \\
0.00\end{array}$ & $\begin{array}{l}0.71 \\
0.67 \\
0.67\end{array}$ & $\begin{array}{l}0.07 \\
0.07 \\
0.07\end{array}$ & $\begin{array}{l}0.34 \\
0.34 \\
0.34\end{array}$ & $\begin{array}{l}0.29 \\
0.69 \\
0.00\end{array}$ & $\begin{array}{l}0.70 \\
1.10 \\
0.41\end{array}$ & $\begin{array}{l}0.38 \\
0.38 \\
0.38\end{array}$ & $\begin{array}{l}0.70 \\
0.70 \\
0.70\end{array}$ & $\begin{array}{l}0.33 \\
0.69 \\
0.00\end{array}$ & $\begin{array}{l}1.41 \\
1.77 \\
1.08\end{array}$ \\
\hline $\begin{array}{c}0-1000 \\
1-2000 \\
2-3000 \\
\text { nunber } 41 / 2\end{array}$ & $\begin{array}{l}0.09 \\
0.09 \\
0.09\end{array}$ & $\begin{array}{l}0.45 \\
0.45 \\
0.45\end{array}$ & $\begin{array}{l}0.00 \\
0.00 \\
0.00\end{array}$ & $\begin{array}{l}0.56 \\
0.56 \\
0.54\end{array}$ & $\begin{array}{l}0.04 \\
0.04 \\
0.04\end{array}$ & $\begin{array}{l}0.17 \\
0.17 \\
0.17\end{array}$ & $\begin{array}{l}0.00 \\
0.00 \\
0.00\end{array}$ & $\begin{array}{l}0.21 \\
0.21 \\
0.21\end{array}$ & $\begin{array}{l}0.13 \\
0.13 \\
0.13\end{array}$ & $\begin{array}{l}0.62 \\
0.62 \\
0.62\end{array}$ & $\begin{array}{l}0.00 \\
0.00 \\
0.00\end{array}$ & $\begin{array}{l}0.75 \\
0.75 \\
0.75\end{array}$ \\
\hline $\begin{array}{c}0-1000 \\
1-2000\end{array}$ & $\begin{array}{l}0.00 \\
0.00\end{array}$ & $\begin{array}{l}0.00 \\
0.00\end{array}$ & $\begin{array}{l}0.00 \\
0.00\end{array}$ & $\begin{array}{l}0.00 \\
0.00\end{array}$ & $\begin{array}{l}0.00 \\
0.00\end{array}$ & $\begin{array}{l}0.99 \\
0.65\end{array}$ & $\begin{array}{l}0.00 \\
0.00\end{array}$ & $\begin{array}{l}0.99 \\
0.65\end{array}$ & $\begin{array}{l}0.00 \\
0.00\end{array}$ & $\begin{array}{l}0.99 \\
0.65\end{array}$ & $\begin{array}{l}0.00 \\
0.00\end{array}$ & $\begin{array}{l}0.99 \\
0.65\end{array}$ \\
\hline $\begin{array}{l}0-1000 \\
1-2000 \\
2-3000 \\
\text { under } 6\end{array}$ & $\begin{array}{l}0.18 \\
0.18 \\
0.18\end{array}$ & $\begin{array}{l}0.38 \\
0.38 \\
0.38\end{array}$ & $\begin{array}{l}0.00 \\
0.00 \\
0.00\end{array}$ & $\begin{array}{l}0.56 \\
0.56 \\
0.56\end{array}$ & $\begin{array}{l}0.08 \\
0.08 \\
0.08\end{array}$ & $\begin{array}{l}0.29 \\
0.29 \\
0.29\end{array}$ & $\begin{array}{l}2.32 \\
1.69 \\
0.00\end{array}$ & $\begin{array}{l}2.69 \\
2.06 \\
0.37\end{array}$ & $\begin{array}{l}0.26 \\
0.26 \\
0.26\end{array}$ & $\begin{array}{l}0.67 \\
0.67 \\
0.67\end{array}$ & $\begin{array}{l}2.32 \\
1.69 \\
0.00\end{array}$ & $\begin{array}{l}3.25 \\
2.62 \\
0.93\end{array}$ \\
\hline $\begin{array}{r}0-1000 \\
1-2000 \\
2-3000 \\
\text { nusber? }\end{array}$ & $\begin{array}{l}1.03 \\
1.03 \\
1.03\end{array}$ & $\begin{array}{l}0.47 \\
0.47 \\
0.47\end{array}$ & $\begin{array}{l}1.17 \\
1.17 \\
1.17\end{array}$ & $\begin{array}{l}2.67 \\
2.67 \\
2.67\end{array}$ & $\begin{array}{l}0.80 \\
0.80 \\
0.80\end{array}$ & $\begin{array}{l}0.00 \\
0.00 \\
0.00\end{array}$ & $\begin{array}{l}1.96 \\
3.27 \\
1.09\end{array}$ & $\begin{array}{l}2.76 \\
4.07 \\
1.89\end{array}$ & $\begin{array}{l}1.83 \\
1.83 \\
1.83\end{array}$ & $\begin{array}{l}0.47 \\
0.47 \\
0.47\end{array}$ & $\begin{array}{l}3.13 \\
4.44 \\
2.26\end{array}$ & $\begin{array}{l}3.63 \\
6.74 \\
4.56\end{array}$ \\
\hline $\begin{array}{r}0-1000 \\
1-2000 \\
2-3000 \\
\text { number } 8\end{array}$ & $\begin{array}{l}0.00 \\
0.00 \\
0.00\end{array}$ & $\begin{array}{l}0.00 \\
0.00 \\
0.00\end{array}$ & $\begin{array}{l}2.67 \\
2.67 \\
2.67\end{array}$ & $\begin{array}{l}2.67 \\
2.67 \\
2.67\end{array}$ & $\begin{array}{l}0.00 \\
0.00 \\
0.00\end{array}$ & $\begin{array}{l}0.00 \\
0.00 \\
0.00\end{array}$ & $\begin{array}{l}1.22 \\
2.03 \\
2.03\end{array}$ & $\begin{array}{l}1.22 \\
2.03 \\
2.03\end{array}$ & $\begin{array}{l}0.00 \\
0.00 \\
0.00\end{array}$ & $\begin{array}{l}0.00 \\
0.00 \\
0.00\end{array}$ & $\begin{array}{l}3.89 \\
4.70 \\
4.70\end{array}$ & $\begin{array}{l}3.89 \\
4.70 \\
4.70\end{array}$ \\
\hline $\begin{array}{r}0-1000 \\
1-2000 \\
2-3000\end{array}$ & $\begin{array}{l}0.00 \\
0.00 \\
0.00\end{array}$ & $\begin{array}{l}0.00 \\
0.00 \\
0.00\end{array}$ & $\begin{array}{l}2.88 \\
3.07 \\
3.07\end{array}$ & $\begin{array}{l}2.88 \\
3.07 \\
3.07\end{array}$ & $\begin{array}{l}0.00 \\
0.00 \\
0.00\end{array}$ & $\begin{array}{l}0.00 \\
0.00 \\
0.00\end{array}$ & $\begin{array}{l}1.00 \\
2.12 \\
2.12\end{array}$ & $\begin{array}{l}1.00 \\
2.12 \\
2.12\end{array}$ & $\begin{array}{l}0.00 \\
0.00 \\
0.00\end{array}$ & $\begin{array}{l}0.00 \\
0.00 \\
0.00\end{array}$ & $\begin{array}{l}3.88 \\
5.19 \\
5.19\end{array}$ & $\begin{array}{l}3.88 \\
5.19 \\
5.19\end{array}$ \\
\hline $\begin{array}{c}0-1000 \\
1-2000 \\
2-3000 \\
\text { uncorrelated }\end{array}$ & $\begin{array}{l}0.37 \\
0.37 \\
0.37\end{array}$ & $\begin{array}{l}0.00 \\
0.00 \\
0.00\end{array}$ & $\begin{array}{l}0.00 \\
0.00 \\
0.00\end{array}$ & $\begin{array}{l}0.37 \\
0.37 \\
0.37\end{array}$ & $\begin{array}{l}0.24 \\
0.24 \\
0.24\end{array}$ & $\begin{array}{l}0.00 \\
0.00 \\
0.00\end{array}$ & $\begin{array}{l}0.00 \\
0.00 \\
0.00\end{array}$ & $\begin{array}{l}0.24 \\
0.26 \\
0.24\end{array}$ & $\begin{array}{l}0.61 \\
0.61 \\
0.61\end{array}$ & $\begin{array}{l}0.00 \\
0.00 \\
0.00\end{array}$ & $\begin{array}{l}0.00 \\
0.00 \\
0.00\end{array}$ & $\begin{array}{l}0.61 \\
0.61 \\
0.61\end{array}$ \\
\hline $\begin{array}{c}0-1000 \\
1-2000 \\
2-3000 \\
\text { wilkeson number } 1\end{array}$ & $\begin{array}{l}0.00 \\
0.00 \\
0.00\end{array}$ & $\begin{array}{l}0.00 \\
0.00 \\
0.00\end{array}$ & $\begin{array}{l}0.00 \\
0.00 \\
0.00\end{array}$ & $\begin{array}{l}0.00 \\
0.00 \\
0.00\end{array}$ & $\begin{array}{l}0.00 \\
0.00 \\
0.00\end{array}$ & $\begin{array}{l}0.55 \\
0.55 \\
0.55\end{array}$ & $\begin{array}{l}0.33 \\
0.33 \\
0.33\end{array}$ & $\begin{array}{l}0.88 \\
0.88 \\
0.88\end{array}$ & $\begin{array}{l}0.00 \\
0.00 \\
0.00\end{array}$ & $\begin{array}{l}0.55 \\
0.55 \\
0.55\end{array}$ & $\begin{array}{l}0.33 \\
0.33 \\
0.33\end{array}$ & $\begin{array}{l}0.88 \\
0.88 \\
0.88\end{array}$ \\
\hline $\begin{array}{c}0-1000 \\
1-2000 \\
2-3000 \\
\text { uithes }\end{array}$ & $\begin{array}{l}1.73 \\
0.00 \\
0.00\end{array}$ & $\begin{array}{l}1.86 \\
0.98 \\
0.16\end{array}$ & $\begin{array}{l}0.00 \\
0.00 \\
0.00\end{array}$ & $\begin{array}{l}3.57 \\
0.98 \\
0.96\end{array}$ & $\begin{array}{l}2.88 \\
4.19 \\
0.00\end{array}$ & $\begin{array}{l}1.67 \\
0.63 \\
0.91\end{array}$ & $\begin{array}{l}0.00 \\
0.00 \\
0.00\end{array}$ & $\begin{array}{l}4.55 \\
4.82 \\
0.91\end{array}$ & $\begin{array}{l}4.61 \\
4.19 \\
0.00\end{array}$ & $\begin{array}{l}3.51 \\
1.61 \\
1.07\end{array}$ & $\begin{array}{l}0.00 \\
0.00 \\
0.00\end{array}$ & $\begin{array}{l}8.12 \\
3.80 \\
1.07\end{array}$ \\
\hline $\begin{array}{c}0-1000 \\
1-2000 \\
2-3000 \\
\text { wilkeson number } 3\end{array}$ & $\begin{array}{l}0.00 \\
0.00 \\
0.00\end{array}$ & $\begin{array}{l}0.00 \\
0.00 \\
0.00\end{array}$ & $\begin{array}{r}10.63 \\
2.78 \\
0.14\end{array}$ & $\begin{array}{r}10.63 \\
2.78 \\
0.16\end{array}$ & $\begin{array}{l}0.00 \\
0.00 \\
0.00\end{array}$ & $\begin{array}{l}0.00 \\
0.00 \\
0.00\end{array}$ & $\begin{array}{r}4.26 \\
16.79 \\
8.59\end{array}$ & $\begin{array}{r}4.24 \\
16.79 \\
8.59\end{array}$ & $\begin{array}{l}0.00 \\
0.00 \\
0.00\end{array}$ & $\begin{array}{l}0.00 \\
0.00 \\
0.00\end{array}$ & $\begin{array}{r}14.87 \\
17.57 \\
8.73\end{array}$ & $\begin{array}{r}14.87 \\
17.57 \\
8.73\end{array}$ \\
\hline $\begin{array}{c}0-1000 \\
1-2000 \\
2-3000 \\
\text { wilkeson number } 4\end{array}$ & $\begin{array}{l}0.00 \\
0.00 \\
0.00\end{array}$ & $\begin{array}{l}0.00 \\
0.00 \\
0.00\end{array}$ & $\begin{array}{r}12.51 \\
11.94 \\
0.20\end{array}$ & $\begin{array}{r}12.59 \\
11.94 \\
0.20\end{array}$ & $\begin{array}{l}0.00 \\
0.00 \\
0.00\end{array}$ & $\begin{array}{l}0.00 \\
0.00 \\
0.00\end{array}$ & $\begin{array}{r}5.51 \\
11.99 \\
12.78\end{array}$ & $\begin{array}{r}5.51 \\
11.99 \\
12.78\end{array}$ & $\begin{array}{l}0.00 \\
0.00 \\
0.00\end{array}$ & $\begin{array}{l}0.00 \\
0.00 \\
0.00\end{array}$ & $\begin{array}{l}18.02 \\
23.93 \\
12.98\end{array}$ & $\begin{array}{l}18.02 \\
23.93 \\
12.98\end{array}$ \\
\hline $\begin{array}{l}0-1000 \\
1-2000 \\
2-3000\end{array}$ & $\begin{array}{l}0.00 \\
0.00 \\
0.00\end{array}$ & $\begin{array}{l}4.61 \\
0.00 \\
0.00\end{array}$ & $\begin{array}{l}1.27 \\
0.81 \\
0.00\end{array}$ & $\begin{array}{l}5.88 \\
0.81 \\
0.00\end{array}$ & $\begin{array}{l}0.00 \\
0.00 \\
0.00\end{array}$ & $\begin{array}{l}4.50 \\
7.75 \\
0.72\end{array}$ & $\begin{array}{l}2.20 \\
1.84 \\
0.00\end{array}$ & $\begin{array}{l}6.70 \\
9.59 \\
0.72\end{array}$ & $\begin{array}{l}0.00 \\
0.00 \\
0.00\end{array}$ & $\begin{array}{l}9.11 \\
7.75 \\
0.72\end{array}$ & $\begin{array}{l}3.47 \\
2.65 \\
0.00\end{array}$ & $\begin{array}{r}12.58 \\
10.40 \\
0.72\end{array}$ \\
\hline
\end{tabular}




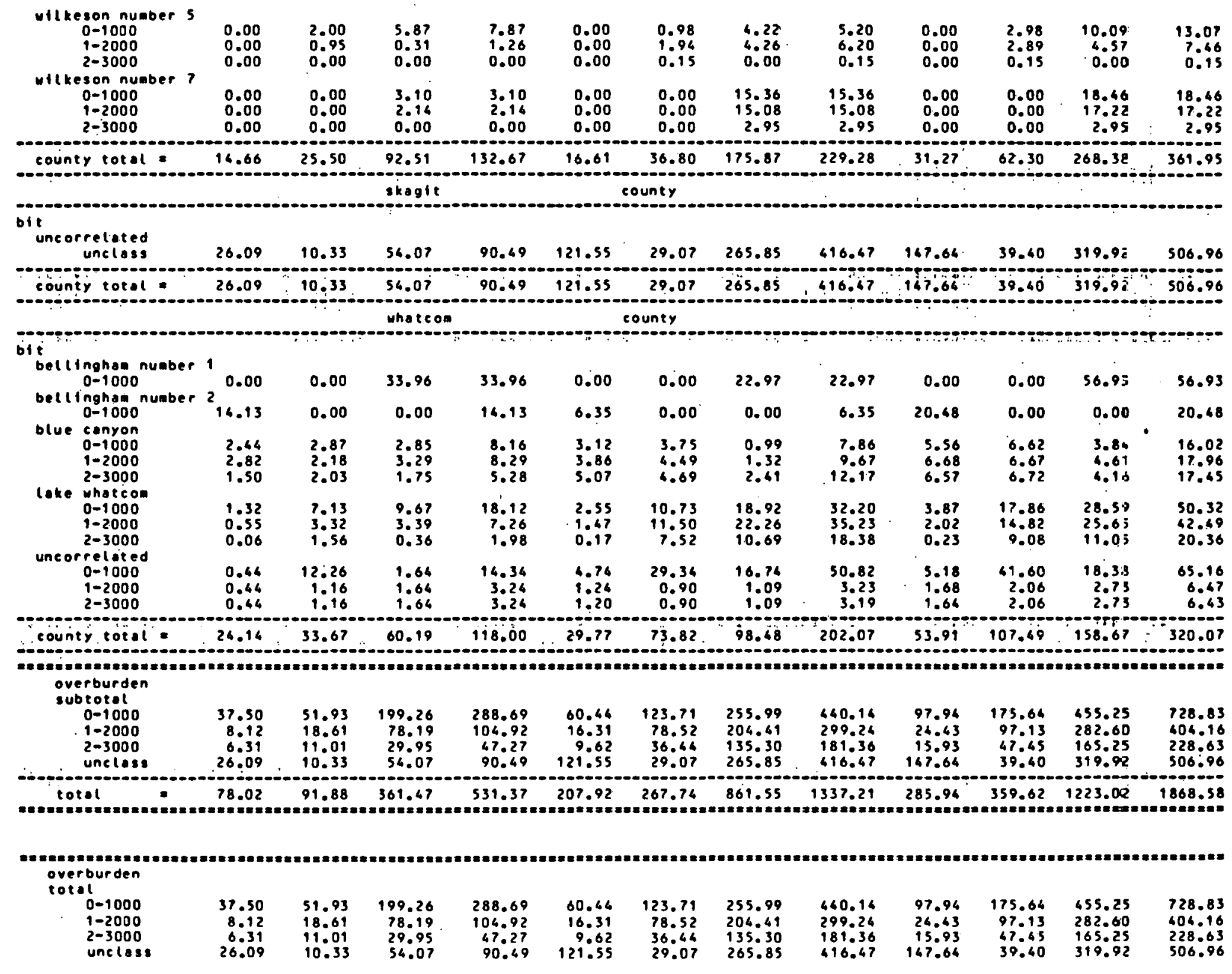




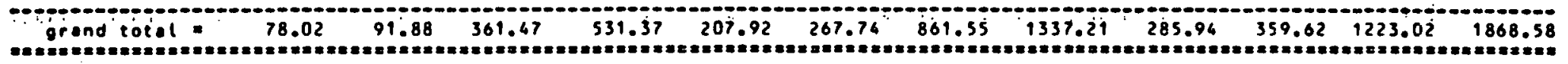

total tonnage of identifted coal resources in washington

186.8 .58

resource flgures taken froms

$\begin{array}{lll}\text { source } & \text { year } & \text { base yeaf of resource } \\ \text { wash dag bull } 67 & 1961 & 1960\end{array}$


average onalyses of coal in washington

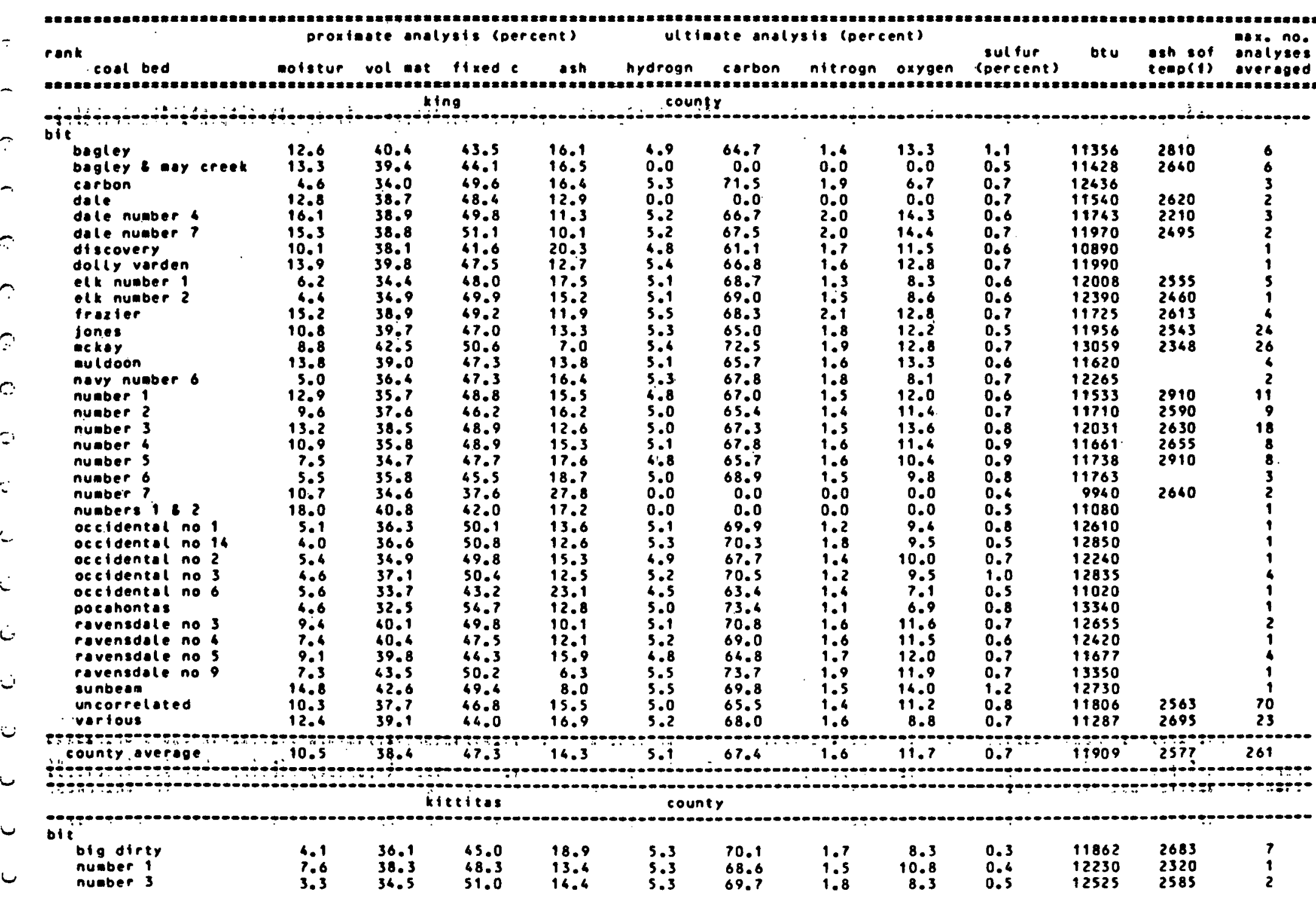




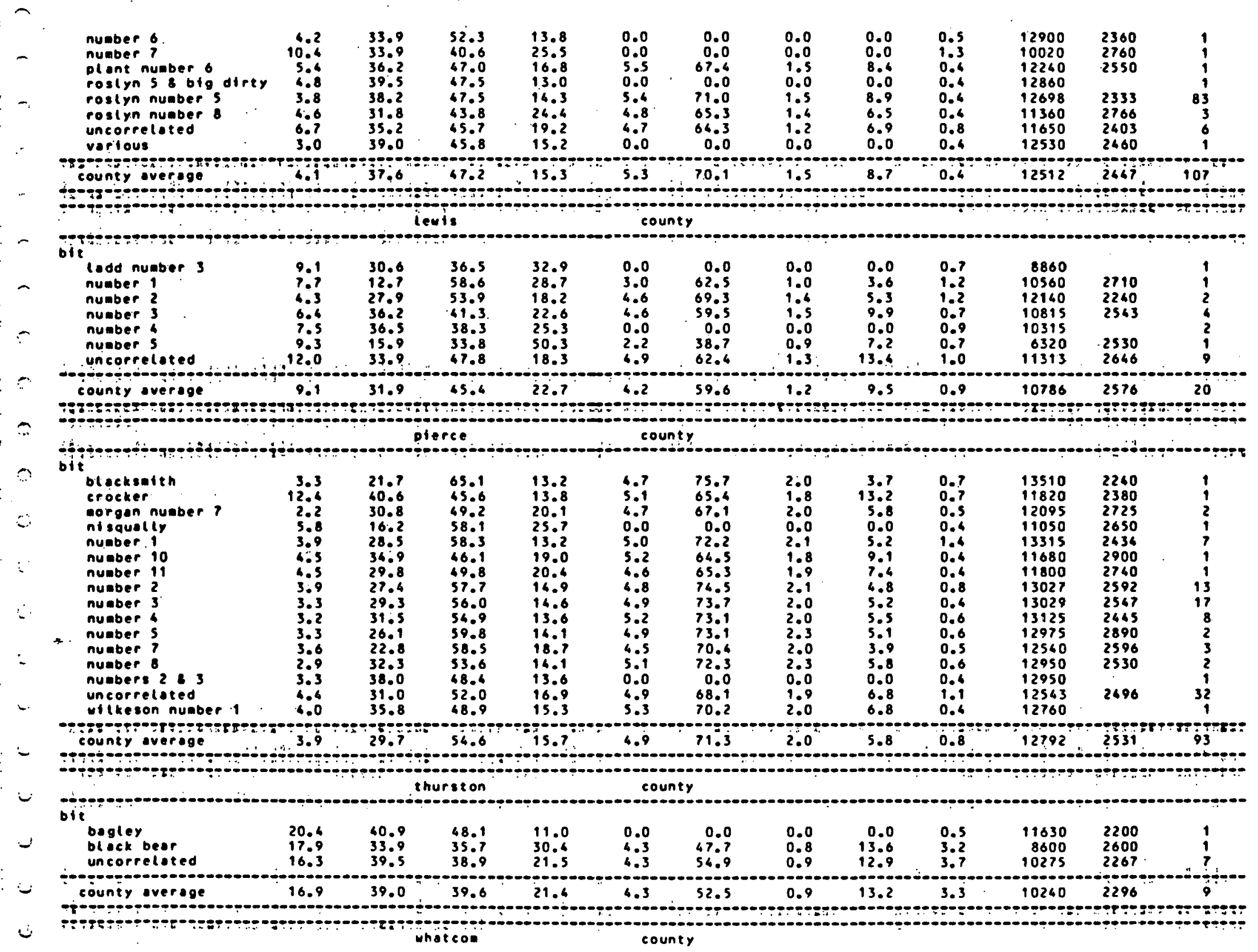


- bi

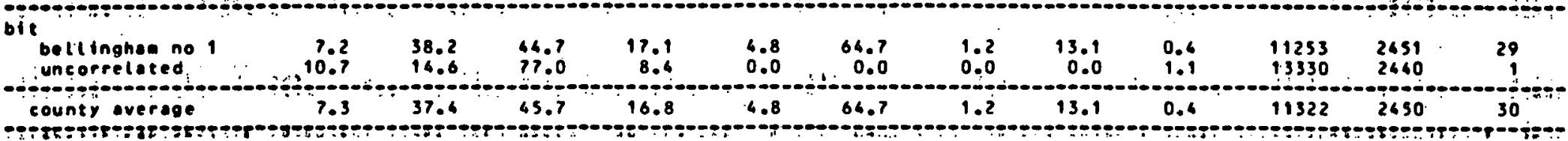




\section{WASHIRIGTON}

side.

uesington ing

uosinington ing

couner

ben
uncerreloted

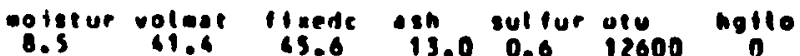

10110 
National Coal Resources Data System

U.S. Geological Survey

identified coal resources in west virginia

- (In aillions of short tons)

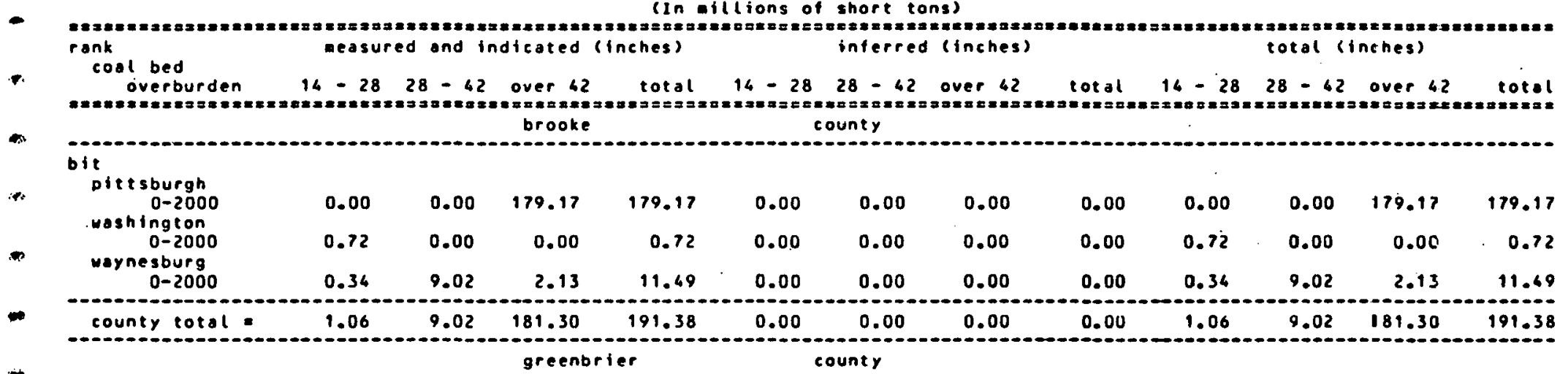

$\rightarrow$ bit

beckley

$\cdots$

$\begin{array}{ccccc}0-1000 & 0.00 & 27.24 & 2.10 & 29.34\end{array}$

$\begin{array}{cccccccccccccc}0-1000 & 0.00 & 27.24 & 2.10 & 29.34 & 0.00 & 7.81 & 0.00 & 7.81 & 0.00 & 35.05 & 2.10 & 37.15\end{array}$ $0-1000$

$0.00 \quad 13.72 \quad 25.97$

39.69

0.00

30.160 .00

47.82

0-1000

0.00

26.61

0.00

30.16

17.66

47.82

0.00

43.88

43.63

87.51

pocahontas no 6

sevell $0-1000$

$0.00 \quad 56.29 \quad 35.62$

91.91

0.00

7.26

0.00

$49.23 \quad 17.22$

58.45

county total

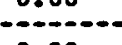

$$
\text { bit }
$$

165.20

\begin{tabular}{ll}
\hline .04 & 134.68 \\
\hline $1094 n$ \\
\hline 5.51 & 352.95 \\
&
\end{tabular}

$0-2000$

$\begin{array}{llll}85.72 & 53.75 \quad 25.10 & 164.57\end{array}$

0.00

0.00

0.00

0.00

85.72

385.51

352.95

888.15

0 campbell cr
$0-2000$

$\begin{array}{llll}100.50 & 574.92 & 264.88 & 940.30\end{array}$

0.00

0.00

0.00

0.00100 .50

53.75

25.10

166.57

0-2000

$\begin{array}{lllll}77.57 & 180.39 & 477.14 & 735.10 & 0.00\end{array}$

0.00

0.00

77.57

574.92

264.88

960.30

$\begin{array}{lll}125.33 & 66.47 & 33.94\end{array}$

225.74

0.00

0.00

0.00

0.00

125.33

180.39

7.14

735.10

$0-2000$

$86.32 \quad 147.60 \quad 171.32$

405.24

0.00

.0 .00

0.00

0.00

86.32

33.9

225.74

ton 2000

142.85

0.05

$0.00 \quad 142.90$

$0.00 \quad 0.00 \cdot 0.00$

0.00

142.85

0.05

0.00

405.24 


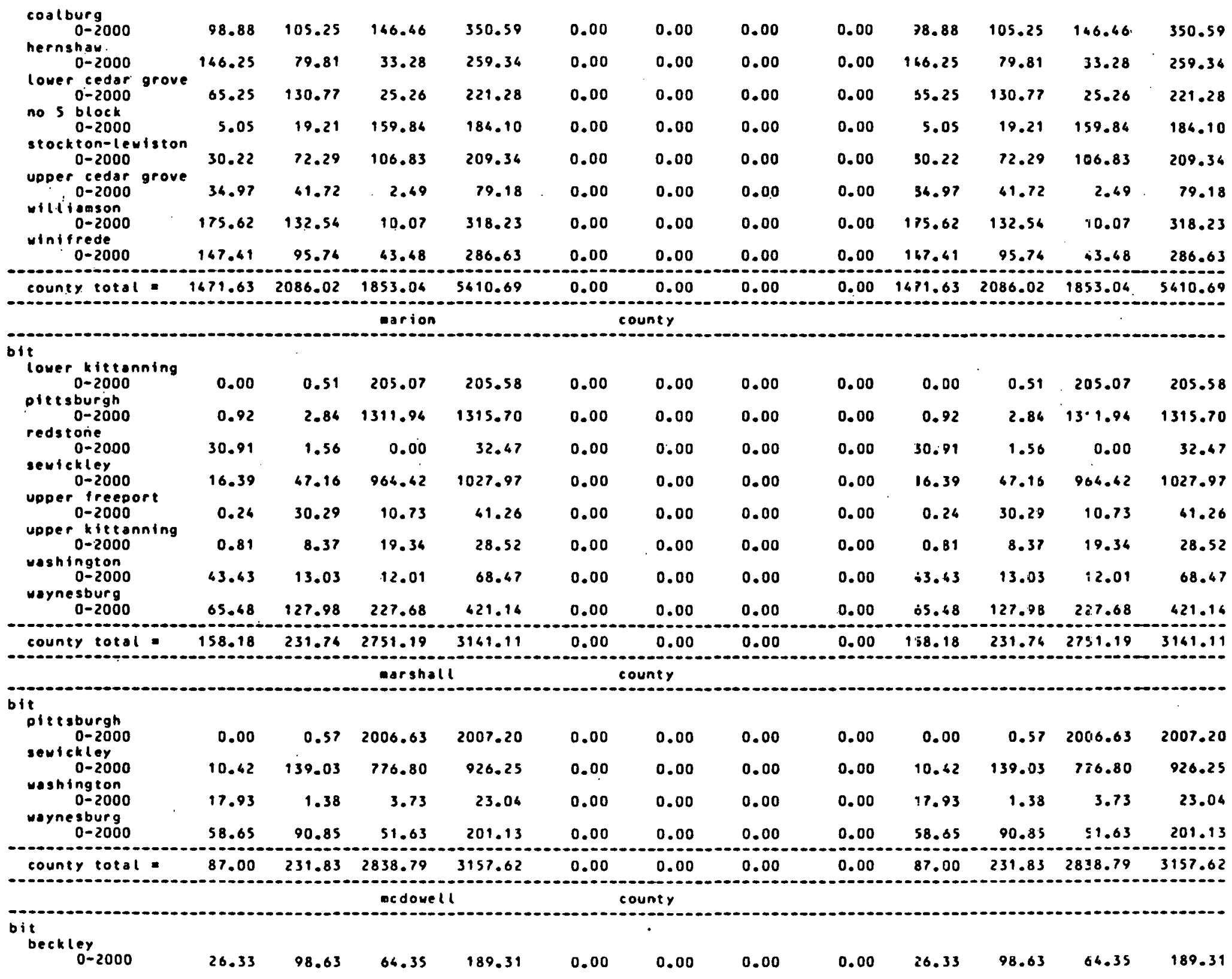




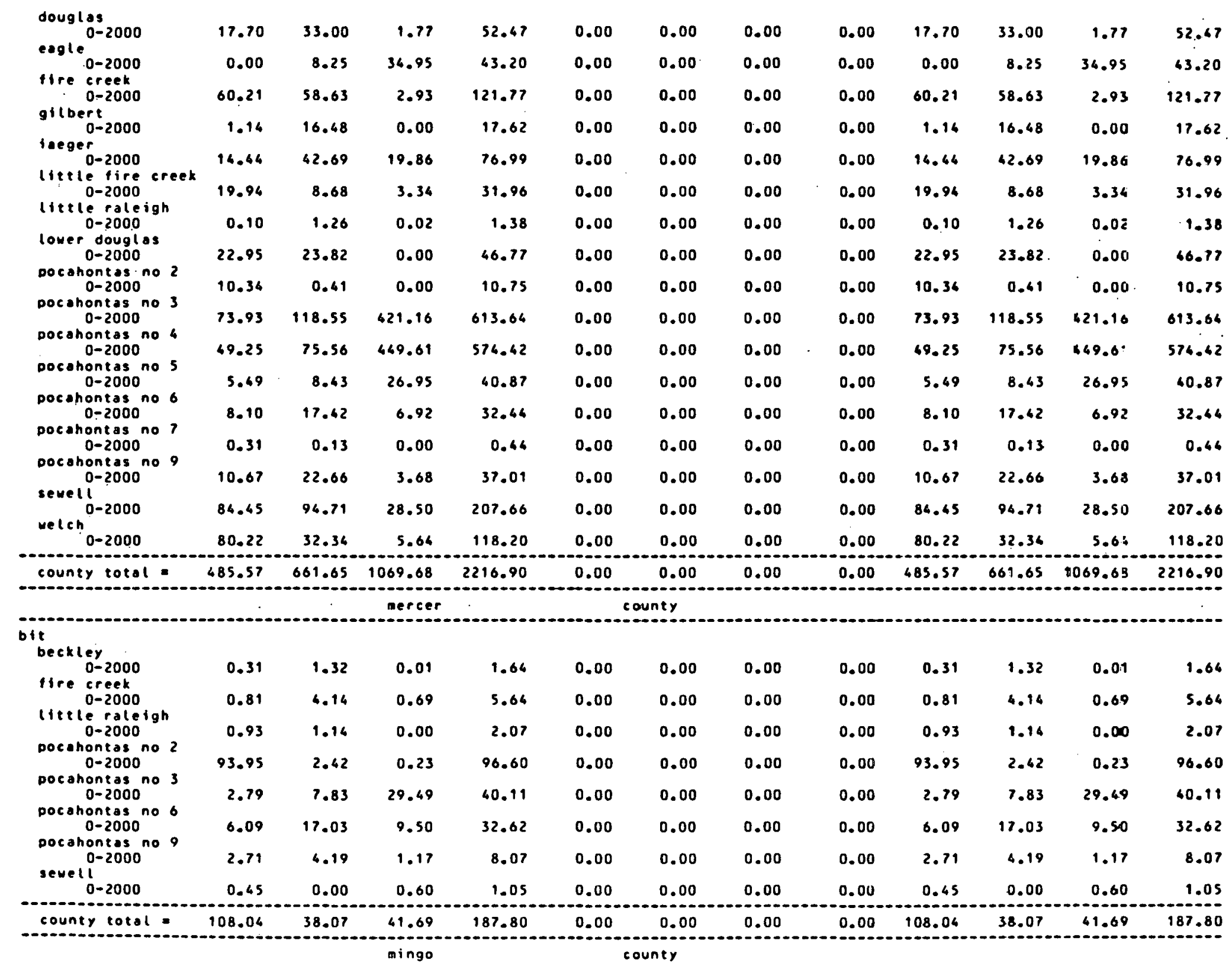




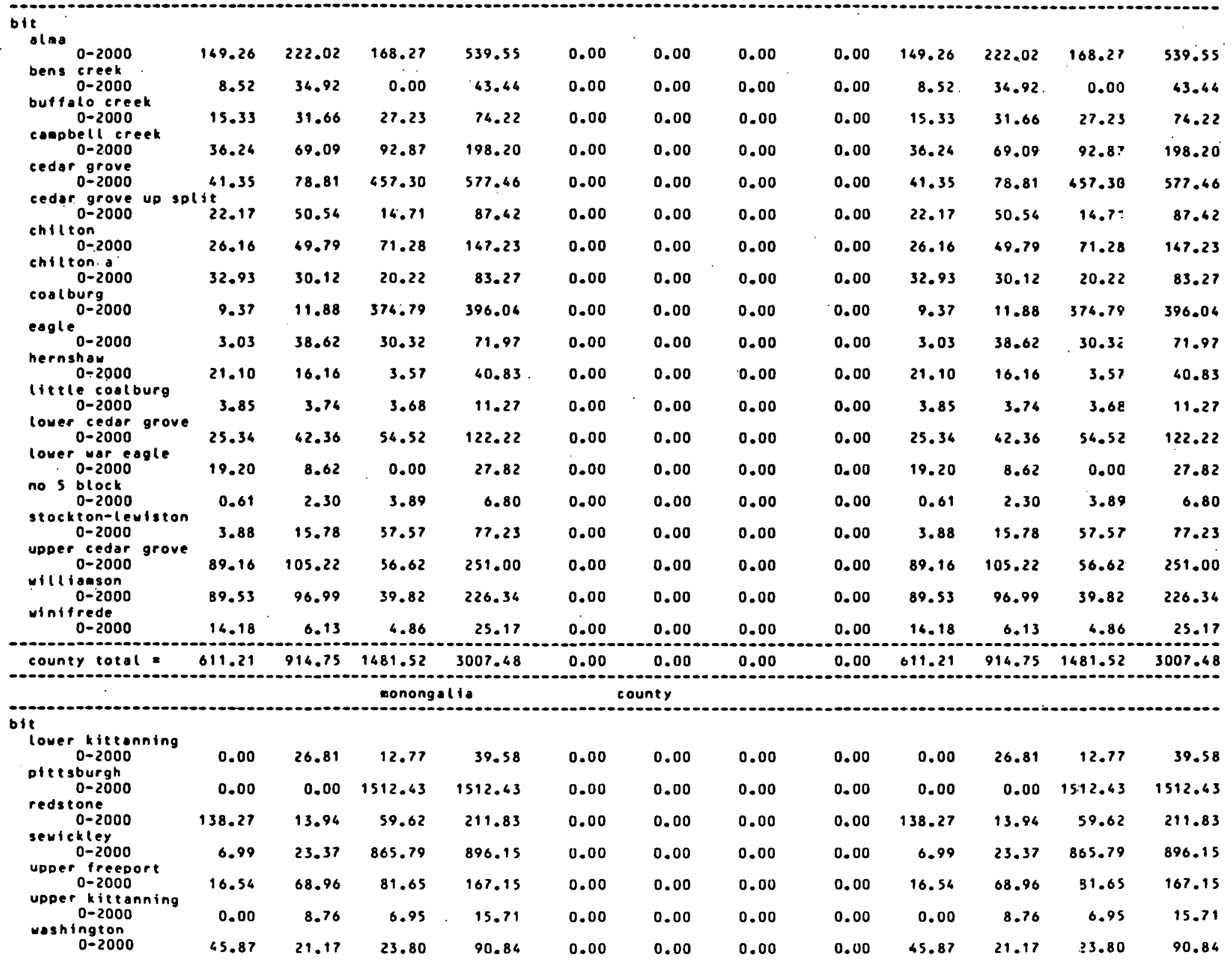




\begin{tabular}{|c|c|c|c|c|c|c|c|c|c|c|c|c|}
\hline $\begin{array}{r}\text { wornesburg } \\
0-2000\end{array}$ & 24.34 & 69.93 & 517.76 & 592.03 & 0.00 & 0.00 & 0.00 & 0.00 & .24 .34 & 69.93 & 517.76 & 592.03 \\
\hline county total = & 232.01 & 212.94 & 3080.77 & 3525.72 & 0.00 & 0.00 & 0.00 & 0.00 & 232.01 & 212.94 & 3080.77 & 3525.72 \\
\hline & & & ohio & & & nty & & & & & & \\
\hline bit & & & & & & & & & & & & \\
\hline $0-2000$ & 0.00 & 0.00 & 343.29 & 363.29 & 0.00 & 0.00 & 0.00 & 0.00 & 0.00 & 0.00 & 343.29 & 363.29 \\
\hline $0-2000$ & 8.79 & 12.42 & 0.75 & 21.96 & 0.00 & 0.00 & 0.00 & 0.00 & 8.79 & 12.42 & 0.75 & 21.96 \\
\hline county totel & 8.79 & 12.42 & 344.04 & 365.25 & 0.00 & 0.00 & 0.00 & 0.00 & 8.79 & 12.42 & 344.04 & 365.25 \\
\hline & & & raleig & & & ty & & & & & & \\
\hline bit & & & & & & & & & & & & \\
\hline $0-2000$ & 0.00 & 4.67 & 36.68 & 41.35 & 0.00 & 0.00 & 0.00 & 0.00 & 0.00 & 4.67. & 36.68 & 41.35 \\
\hline $0-2000$ & 65.56 & 148.59 & 306.58 & 520.71 & 0.00 & 0.00 & 0.00 & 0.00 & 65.54 & 148.59 & 306.58 & 520.71 \\
\hline $0-2000$ & 3.97 & 50.53 & 267.87 & 322.37 & 0.00 & 0.00 & 0.00 & 0.00 & 3.97 & 50.53 & 267.87 & 322.37 \\
\hline $0-2000$ & 0.00 & 4.08 & 3.23 & 7.31 & 0.00 & 0.00 & 0.00 & 0.00 & 0.00 & 4.08 & 3.23 & 7.31 \\
\hline $\begin{array}{l}0-2000 \\
\text { fire creek }\end{array}$ & 4.37 & 15.60 & 268.80 & 268.77 & 0.00 & 0.00 & 0.00 & 0.00 & 6.37 & 15.60 & 248.80 & 268.77 \\
\hline $0-2000$ & 57.44 & 42.33 & 62.79 & 142.56 & 0.00 & 0.00 & 0.00 & 0.00 & 57.64 & 42.33 & 62.79 & 142.56 \\
\hline $\begin{array}{c}0-2000 \\
0\end{array}$ & 7.79 & 17.74 & 0.00 & 25.53 & 0.00 & 0.00 & 0.00 & 0.00 & 7.79 & 17.84 & 0.00 & 25.53 \\
\hline $0-2000$ & 7.31 & 16.23 & 18.57 & 42.11 & 0.00 & 0.00 & 0.00 & 0.00 & 7.31 & 16.23 & 18.57 & 42.11 \\
\hline $\begin{array}{c}0-2000 \\
\text { lower hernshaw }\end{array}$ & 0.79 & 1.90 & 57.31 & 60.00 & 0.00 & 0.00 & 0.00 & 0.00 & 0.79 & 1.90 & 57.31 & 60.00 \\
\hline $0-2000$ & 4.69 & 9.06 & 5.75 & 19.50 & 0.00 & 0.00 & 0.00 & 0.00 & 4.69 & 9.06 & 5.75 & 19.50 \\
\hline $\begin{array}{l}0-2000 \\
\text { lover vinifrede }\end{array}$ & 3.79 & 9.70 & 31.99 & 45.48 & 0.00 & 0.00 & 0.00 & 0.00 & 3.79 & 9.70 & 31.99 & 45.48 \\
\hline $0-2000$ & 0.00 & 0.00 & 9.63 & 9.63 & 0.00 & 0.00 & 0.00 & 0.00 & 0.00 & 0.00 & 9.63 & 9.63 \\
\hline $\begin{array}{c}0-2000 \\
\text { pocahontas no } 2\end{array}$ & 0.00 & 0.00 & 13.51 & 13.51 & 0.00 & 0.00 & 0.00 & .0 .00 & 0.00 & 0.00 & 13.51 & 13.51 \\
\hline $\begin{array}{c}0-2000 \\
\text { pocehontas no } 3\end{array}$ & 30.30 & 7.26 & 0.00 & 37.56 & 0.00 & 0.00 & 0.00 & 0.00 & 30.30 & 7.26 & 0.00 & 37.56 \\
\hline $\begin{array}{c}0-2000 \\
\text { pocahontas no } 4\end{array}$ & 19.22 & 203.20 & 118.52 & 340.94 & 0.00 & 0.00 & 0.00 & 0.00 & 19.22 & 203.20 & 118.52 & 360.94 \\
\hline $\begin{array}{c}0-2000 \\
\text { pocahontas no } 6\end{array}$ & 27.24 & 95.50 & 70.19 & 192.93 & 0.00 & 0.00 & 0.00 & 0.00 & 27.24 & 95.50 & 70.19 & 192.93 \\
\hline $\begin{array}{l}0-2000 \\
\text { poweltiton }\end{array}$ & 15.52 & 17.31 & 4.38 & 37.21 & 0.00 & 0.00 & 0.00 & 0.00 & 15.52 & 17.31 & 4.38 & 37.21 \\
\hline sewell $0-2000$ & 30.72 & 51.61 & 19.75 & 102.08 & 0.00 & 0.00 & 0.00 & 0.00 & 30.72 & 51.61 & 19.75 & 102.08 \\
\hline $\begin{array}{l}0-2000 \\
k \text { ton-levis }\end{array}$ & 11.26 & 28.97 & 75.88 & 116.09 & 0.00 & 0.00 & 0.00 & 0.00 & 19.24 & 28.97 & 75.88 & 116.09 \\
\hline $0-2000$ & 0.16 & 0.00 & 7.15 & 7.31 & 0.00 & 0.00 & 0.00 & 0.00 & 0.16 & 0.00 & 7.15 & 7.31 \\
\hline $0-2000$ & 0.00 & 3.24 & 1.39 & 4.63 & 0.00 & 0.00 & 0.00 & 0.00 & 0.00 & 3.26 & 1.39 & 4.63 \\
\hline
\end{tabular}




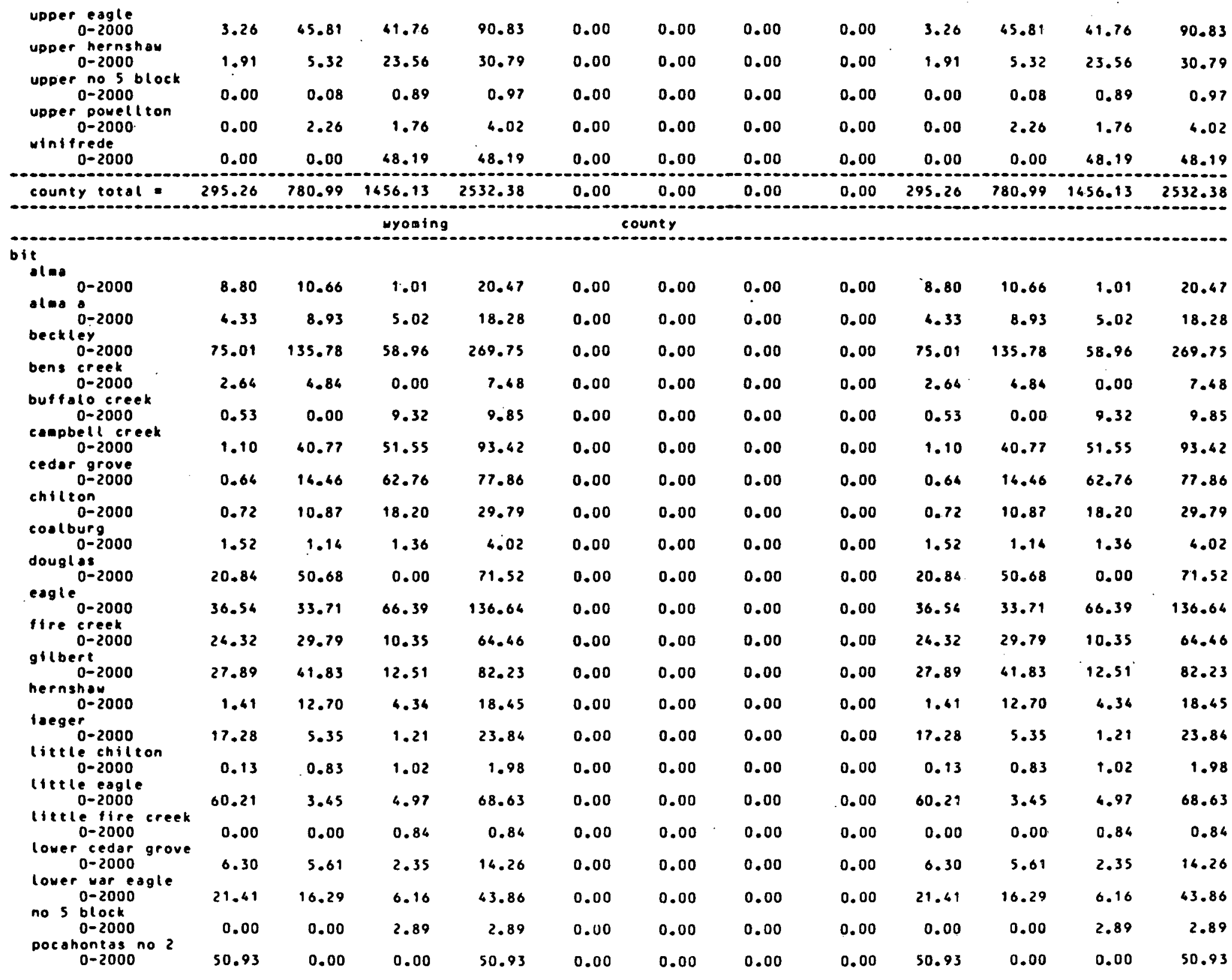




\begin{tabular}{|c|c|c|c|c|c|c|c|c|c|c|c|c|}
\hline $\begin{array}{c}\text { pocahoneas no } 3 \\
0-2000\end{array}$ & 22.62 & 197.74 & 626.14 & 846.50 & 0.00 & 0.00 & 0.00 & 0.00 & 22.62 & 197.74 & 626.14 & 846.50 \\
\hline $\begin{array}{c}0-2000 \\
\text { pocariontas no } 5\end{array}$ & 50.38 & 90.07 & 76.23 & 216.68 & 0.00 & 0.00 & 0.00 & 0.00 & 50.38 & 90.07 & 76.23 & 216.68 \\
\hline $\begin{array}{c}0-2000 \\
\text { pocahontas no } 6\end{array}$ & 0.00 & 0.60 & 0.00 & 0.60 & 0.00 & 0.00 & 0.00 & 0.00 & 0.00 & 0.60 & 0.00 & 0.60 \\
\hline $\begin{array}{c}0-2000 \\
\text { pocahontas no? }\end{array}$ & 34.90 & 89.31 & 84.43 & 198.64 & 0.00 & 0.00 & 0.00 & 0.00 & 36.90 & 89.31 & 74.43 & 198.64 \\
\hline $\begin{array}{c}0-2000 \\
\text { pocahontas no } 9\end{array}$ & 0.00 & 0.10 & 0.00 & 0.10 & 0.00 & 0.00 & 0.00 & 0.00 & 0.00 & 0.10 & 0.00 & 0.10 \\
\hline sevell & 0.00 & 5.36 & 0.28 & 5.66 & 0.00 & 0.00 & 0.00 & 0.00 & 0.00 & 5.36 & 0.28 & 5.64 \\
\hline sevelí & 70.05 & 149.75 & 22.26 & 242.06 & 0.00 & 0.00 & 0.00 & 0.00 & 70.05 & 149.75 & 22.26 & 262.06 \\
\hline $\begin{array}{c}0-2000 \\
\text { stockton-lewiston }\end{array}$ & 0.00 & 0.38 & 0.23 & 0.61 & 0.00 & 0.00 & 0.00 & 0.00 & 0.00 & 0.38 & 0.23 & 0.61 \\
\hline $\begin{array}{r}0-2000 \\
\text { roper }\end{array}$ & 0.22 & 1.03 & 0.21 & 1.46 & 0.00 & 0.00 & 0.00 & 0.00 & 0.22 & 1.03 & 0.21 & 1.46 \\
\hline velch $0-2000$ & 0.00 & 0.04 & 3.28 & 3.32 & 0.00 & 0.00 & 0.00 & 0.00 & 0.00 & 0.04 & 3.28 & 3.32 \\
\hline $\begin{array}{r}0-2000 \\
-141 \text { tason }\end{array}$ & 0.40 & 0.00 & 0.00 & 0.40 & 0.00 & 0.00 & 0.00 & 0.00 & 0.60 & 0.00 & 0.00 & 0.40 \\
\hline $\begin{array}{l}0-2000 \\
\text { winifrede }\end{array}$ & 2.00 & 7.47 & 1.36 & 10.83 & 0.00 & 0.00 & 0.00 & 0.00 & 2.00 & 7.67 & 1.36 & 10.83 \\
\hline $0-2000$ & 1.36 & 3.63 & 22.15 & 27.14 & 0.00 & 0.00 & 0.00 & 0.00 & 1.36 & 3.63 & 22.15 & 27.14 \\
\hline county total & 64.48 & 973.17 & 1147.78 & 2665.63 & 0.00 & 0.00 & 0.00 & 0.00 & $\subseteq 44.48$ & 973.17 & 1747.78 & 2665.63 \\
\hline
\end{tabular}

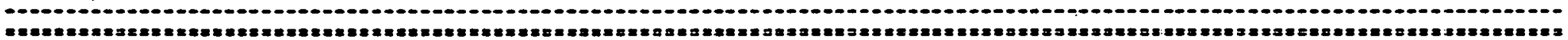
overburden

overburden

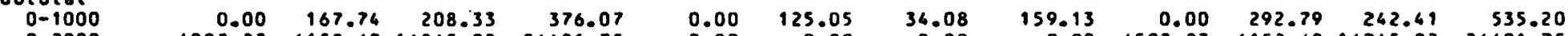

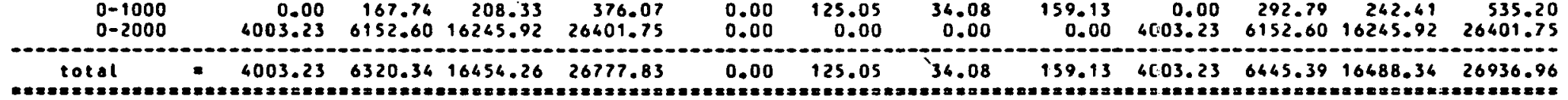

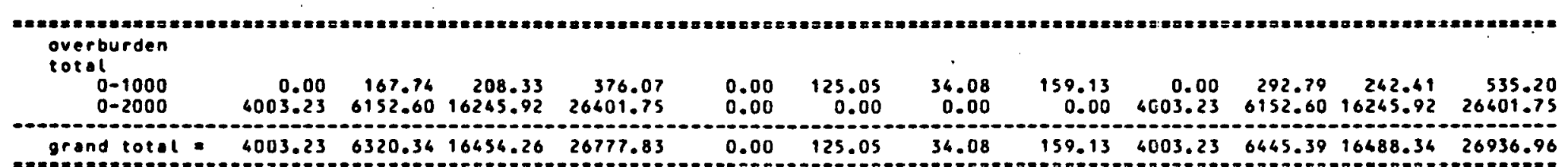

encrand totel = $4003.23 \quad 6320.3416454 .26 \quad 26777.83 \quad 0.00 \quad 125.05 \quad 34.08 \quad 159.13 \quad 4003.23 \quad 6445.3916488 .34 \quad 26936.96$

tonnage not includeds

73362.28

thickness: unclassilied $\quad 73362.28$
reliability = unclassifled $\quad 73362.28$

total tonnage of identified coal resources in west virginia

100299.24

resource figures taken from:

source year base year cf resource 


\begin{tabular}{|c|c|c|c|c|}
\hline $\begin{array}{l}\text { usgs } \\
\text { usbm } \\
\text { usbn } \\
\text { usbm } \\
\text { usbin } \\
\text { usbm } \\
\text { usbm } \\
\text { usbm } \\
\text { usbn } \\
\text { usbm } \\
\text { usbm } \\
\text { usbor } \\
\text { ungs }\end{array}$ & $\begin{array}{l}0 f- \\
\text { rep } \\
\text { rep } \\
r e p \\
r e p \\
r e p \\
r e p \\
r e p \\
r e p \\
r e p \\
r e p \\
\text { rep } \\
\text { vol. }\end{array}$ & $\begin{array}{l}\text { irnbrier wvo } \\
\text { inv } 5267 \\
\text { inv } 5207 \\
\text { inv } 5077 \\
\text { inv } 5068 \\
\text { inv } 5233 \\
\text { inv } 4893 \\
\text { inv } 4966 \\
\text { inv } 5160 \\
\text { inv } 5259 \\
\text { inv } 4924 \\
\text { inv } 5971 \\
\text { ine is }\end{array}$ & $\begin{array}{l}1972 \\
1956 \\
1956 \\
1954 \\
1954 \\
1956 \\
1952 \\
1953 \\
1955 \\
1956 \\
1952 \\
1955 \\
1940\end{array}$ & $\begin{array}{l}\text { original } \\
1955 \\
1954 \\
1953 \\
1953 \\
1954 \\
1949 \\
1951 \\
1956 \\
1954 \\
1951 \\
1956 \\
\text { original }\end{array}$ \\
\hline
\end{tabular}

stote county

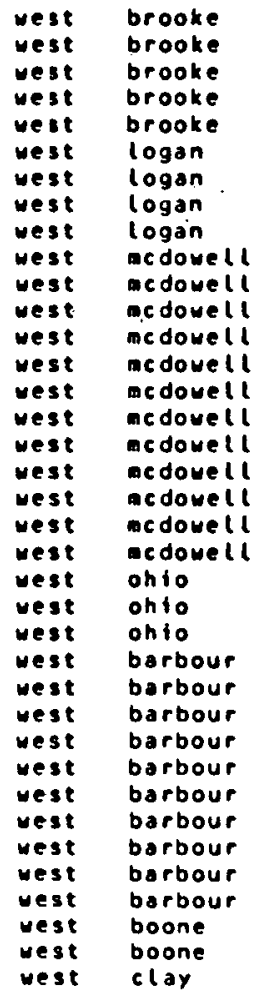

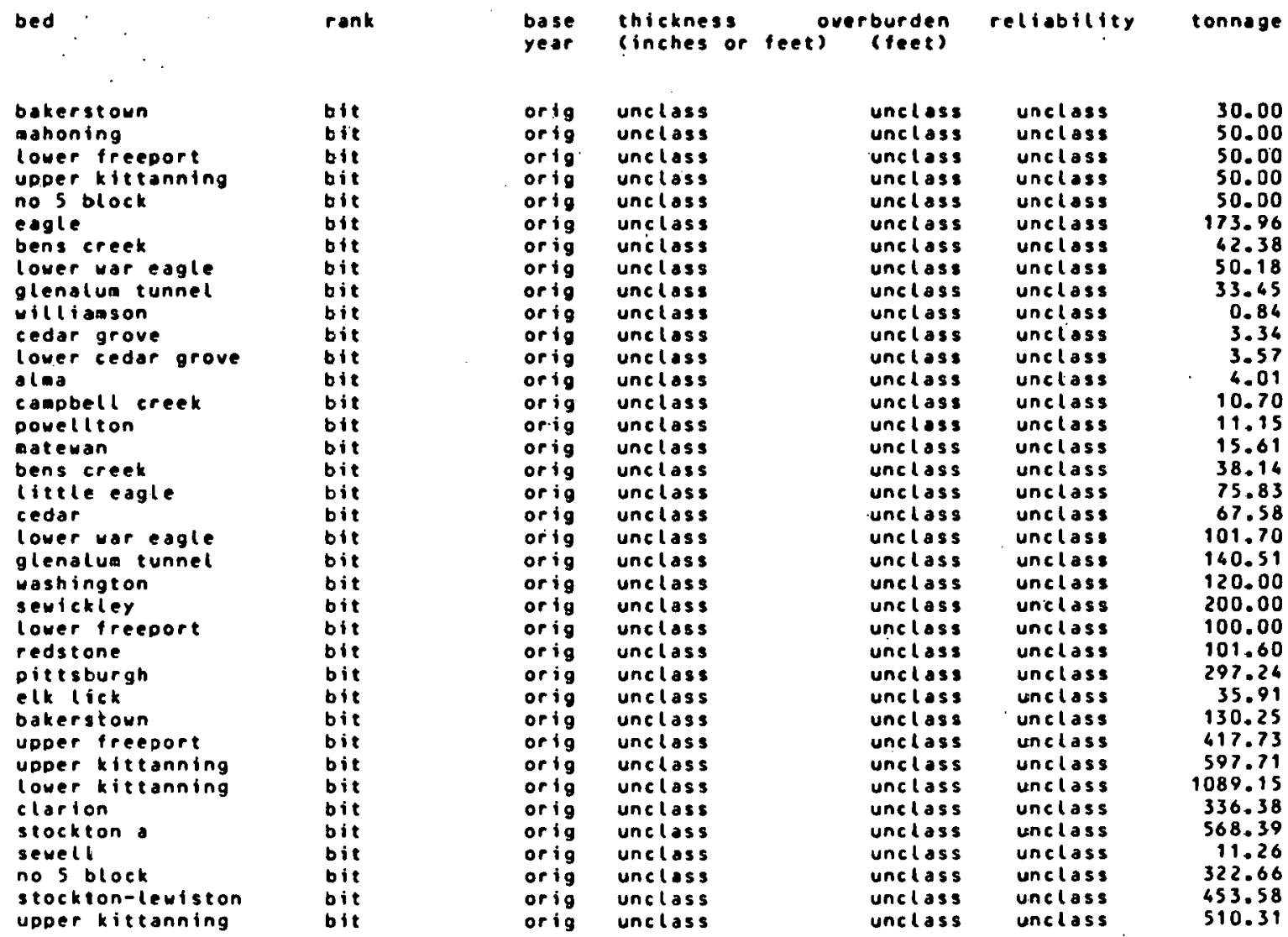




\begin{tabular}{|c|c|}
\hline & $\begin{array}{l}\text { clay } \\
\text { clay }\end{array}$ \\
\hline $\begin{array}{l}\text { vest } \\
\text { west }\end{array}$ & $\begin{array}{l}\text { clay } \\
\text { clay }\end{array}$ \\
\hline & clay \\
\hline west & clay \\
\hline west. & ctay \\
\hline west & clay \\
\hline vest & clay \\
\hline vest & clay \\
\hline vese & doddridge \\
\hline west & doddridge \\
\hline west & doddridge \\
\hline vest & tayette \\
\hline west & tayette \\
\hline vest & fayette \\
\hline west & fayette \\
\hline west & fayette \\
\hline west & fayetie \\
\hline west & fayetie \\
\hline west & fayette \\
\hline west & fayette \\
\hline west & fayette \\
\hline west & fayette \\
\hline west & fayette \\
\hline vest & fayet te \\
\hline west & tayette \\
\hline west & fayetie \\
\hline west & fayette \\
\hline west & fayette \\
\hline west & tayetze \\
\hline west & fayette \\
\hline vest & giliner \\
\hline west & gitwer \\
\hline vest & gilmer \\
\hline west & grant \\
\hline west & grant \\
\hline west & grant \\
\hline west & grant \\
\hline west & grant \\
\hline west & grant \\
\hline west & hancock \\
\hline west & honcock \\
\hline rest & hancock \\
\hline west & hancock \\
\hline vest & hancock \\
\hline vest & hancock \\
\hline $\begin{array}{l}\text { west } \\
\text { west }\end{array}$ & $\begin{array}{l}\text { harrison } \\
\text { harrison }\end{array}$ \\
\hline west & harrison \\
\hline west & harrison \\
\hline vest & harrison \\
\hline west & kanauha \\
\hline west & kanawha \\
\hline west & kanawho \\
\hline west & kanawha \\
\hline west & kanawta \\
\hline vest & kanawha \\
\hline vest & konauha \\
\hline west & Manawha \\
\hline west & kanowha \\
\hline
\end{tabular}

\begin{tabular}{|c|c|}
\hline \multirow[t]{2}{*}{$\begin{array}{l}\text { Diddle } \\
\text { no } 5 \text { blo } \\
\text { clarion }\end{array}$} & $\begin{array}{l}\text { bit } \\
\text { bit } \\
\text { bit } \\
\text { bit } \\
\text { bit }\end{array}$ \\
\hline & bit \\
\hline $\begin{array}{l}\text { cedar grove } \\
\text { caupbell creek }\end{array}$ & $\begin{array}{l}\text { bit } \\
\text { bit }\end{array}$ \\
\hline eagle & bit \\
\hline $\begin{array}{l}\text { washington } \\
\text { uniontown }\end{array}$ & $\begin{array}{l}\text { bit } \\
\text { b.it }\end{array}$ \\
\hline $\begin{array}{l}\text { uniontown } \\
\text { pitts burgh }\end{array}$ & bit \\
\hline $\begin{array}{l}\text { middle kittanning } \\
\text { no } 5 \text { block }\end{array}$ & $\begin{array}{l}\text { bit } \\
\text { bit }\end{array}$ \\
\hline stockton-leutston & bit \\
\hline coalburg & bit \\
\hline $\begin{array}{l}\text { winifrede } \\
\text { chilton }\end{array}$ & $\begin{array}{l}\text { bit } \\
\text { bit }\end{array}$ \\
\hline cedar grove & bit \\
\hline$a \operatorname{lma}$ & bit \\
\hline canpbell creek & bit \\
\hline Dowelliton & bit \\
\hline $\begin{array}{l}\text { eagle } \\
\text { litile eagle }\end{array}$ & $\begin{array}{l}\text { bit } \\
\text { bit }\end{array}$ \\
\hline glenalum tunnel & bit \\
\hline $\begin{array}{l}\text { gilbert } \\
\text { douglas }\end{array}$ & $\begin{array}{l}\text { bit } \\
\text { bit }\end{array}$ \\
\hline sevell & bit \\
\hline tire creek & bit \\
\hline Docahontas no 6 & bit \\
\hline pocahontas no 3 & bit \\
\hline washington & bit \\
\hline Dittsburgh & bit \\
\hline bakerstoun & bit \\
\hline Ditesburgh & bit \\
\hline little pittşburgh & bit \\
\hline elk lick & bit \\
\hline harlem & bit \\
\hline bakerstown & bit \\
\hline upper freeport & bit \\
\hline Dittsburgh & bit \\
\hline brush creek & bit \\
\hline matoning & bit \\
\hline lower freeport & bit \\
\hline niddle kittanning & bit \\
\hline lower kittanning & bit \\
\hline $\begin{array}{l}\text { washington } \\
\text { redstone }\end{array}$ & $\begin{array}{l}\text { bit } \\
\text { bit }\end{array}$ \\
\hline Ditesburgh & bit \\
\hline hiarlem & bit \\
\hline upper kittanning & bit \\
\hline pittsburgh & bit \\
\hline lover kittsnning & bit \\
\hline stocktan-lewiston & bit \\
\hline coalburg & bit \\
\hline winifrede & bit \\
\hline hernshaw & bit \\
\hline cedar gr & bit \\
\hline $\begin{array}{l}\text { campbelt creek } \\
\text { pouelition }\end{array}$ & bit \\
\hline
\end{tabular}

\begin{tabular}{|c|c|}
\hline orig & unclass \\
\hline $\begin{array}{l}\text { orig } \\
\text { origg }\end{array}$ & $\begin{array}{l}\text { unclass } \\
\text { unclass }\end{array}$ \\
\hline & unclass \\
\hline orig & unclass \\
\hline orig & unclass \\
\hline orig & unclass \\
\hline orig & unclass \\
\hline orig & unclass \\
\hline orig & uncloss \\
\hline orig & unclas's \\
\hline orig & unclass \\
\hline orig & uncl ass.s \\
\hline or ig & unclass \\
\hline orig & unctass \\
\hline origg & unclass \\
\hline orig & unclass \\
\hline $\begin{array}{l}\text { orig } \\
\text { ortig }\end{array}$ & uncloss \\
\hline $\begin{array}{l}\text { origg } \\
\text { origg }\end{array}$ & unclass \\
\hline orig & uncloss \\
\hline orig & unclass \\
\hline orig & uncloss \\
\hline orig & unclass \\
\hline or 19 & unclass \\
\hline orig & unclass \\
\hline orig & unclass \\
\hline $\begin{array}{l}\text { orig } \\
\text { ortg }\end{array}$ & unclass \\
\hline 6 & unclass \\
\hline orig & uncloss \\
\hline orig & unclass \\
\hline orig & unctoss \\
\hline orig & unclass \\
\hline or ig & unclas.s \\
\hline orig & uncloss \\
\hline orig & unclass \\
\hline orig & class \\
\hline orig & unclass \\
\hline or $i g$ & unclass \\
\hline orig & unclass \\
\hline orig & unclass \\
\hline orig & unclass \\
\hline orig & unclass \\
\hline orig & class \\
\hline orig & unclass \\
\hline & unclass \\
\hline orig & unclass \\
\hline orig & unclass \\
\hline orig & unclass \\
\hline orig & nclass \\
\hline orig & unclass \\
\hline orig & unclass \\
\hline orig & uncloss \\
\hline orig & unctoss \\
\hline orig & unclass \\
\hline oris & unclass \\
\hline orig & loss \\
\hline orig & unclass \\
\hline or & unctass \\
\hline & \\
\hline
\end{tabular}

\begin{tabular}{|c|c|c|}
\hline $\begin{array}{l}\text { unclass } \\
\text { unclass }\end{array}$ & $\begin{array}{l}\text { unclass } \\
\text { unctass }\end{array}$ & $\begin{array}{l}275.88 \\
691.83\end{array}$ \\
\hline unclass & unclass & 96.01 \\
\hline unclass & unclass & 621.61 \\
\hline unclass & unclass & 605.43 \\
\hline unclass & unclass & 185.00 \\
\hline unclass & unclass & 125.29 \\
\hline unclass & uncloss & 116.23 \\
\hline uncless & unclass & 131.70 \\
\hline unclass & unclass & 396.27 \\
\hline unclass & unclass & 111.51 \\
\hline unclass & unclass & 611.54 \\
\hline unclass & unclass & 10.26 \\
\hline unclass & unclass & 61.95 \\
\hline unctiass & uncioss & 71.74 \\
\hline unc lass & unclass & 115.49 \\
\hline unctass & unctass & 100.63 \\
\hline unclass & unclass & 113.38 \\
\hline unclass & unclass & 203.27 \\
\hline uncioss & unctass & 196.70 \\
\hline unclass & unclass & 767.22 \\
\hline unclass & unclass & 302.69 \\
\hline unctass & unclass & 511.41 \\
\hline unclass & unclass & 267.27 \\
\hline unclass & unclass. & 37.94 \\
\hline unclass & unctass & 34.51 \\
\hline unclass & unclass & 33.59 \\
\hline uncloss & unclass & 967.19 \\
\hline unclass & unclass & 442.85 \\
\hline unctass & unclass & 126.70 \\
\hline unclass & unclass & 77.92 \\
\hline unclass & unctass & 26.76 \\
\hline unctass & unclass & 652.19 \\
\hline unclass & unclass & 540.30 \\
\hline uncloss & unclass & 0.61 \\
\hline unclass & unclass & 0.34 \\
\hline uncless & unclass & 51.52 \\
\hline unclass & uncitass & 45.58 \\
\hline unclass & unclass & 269.97 \\
\hline unclass & unclass & 600.99 \\
\hline unclass & unclass & 5.84 \\
\hline unctass & unclass & 75.00 \\
\hline unclass & unclass & 75.00 \\
\hline unclass & unclass & 194.16 \\
\hline unclass & unclass & 75.00 \\
\hline unclass & unctass & 75.00 \\
\hline unclass & unctoss & 133.48 \\
\hline unclass & unclass & 70.81 \\
\hline unclass & unclass & 1667.35 \\
\hline unclass & unclass & 133.82 \\
\hline unclass & unctass & 167.27 \\
\hline uncl \&s s & unclass & 252.24 \\
\hline unclass & unclass & 1217.73 \\
\hline uncloss & unctass & 1642.71 \\
\hline unclass & unclass & 439.36 \\
\hline unclass & unclass & 263.73 \\
\hline unclass & unclass & 17.66 \\
\hline unctass & unclass & 408.16 \\
\hline unclass & unclass & 1460.55 \\
\hline unclass & unctass & 111.51 \\
\hline
\end{tabular}




\begin{tabular}{|c|c|}
\hline $\begin{array}{l}\text { West } \\
\text { west }\end{array}$ & $\begin{array}{l}\text { kanauh } \\
\text { lewis }\end{array}$ \\
\hline vest & levis \\
\hline west & levis \\
\hline vest & leuts \\
\hline west & levis \\
\hline $\begin{array}{l}\text { vest } \\
\text { vest }\end{array}$ & $\begin{array}{l}\text { lewis } \\
\text { lewis. }\end{array}$ \\
\hline west & lewis \\
\hline west & lincoln \\
\hline vest & lincoln \\
\hline west & lincoln \\
\hline vest & lineoln \\
\hline $\begin{array}{l}\text { vest } \\
\text { west }\end{array}$ & $\begin{array}{l}\text { meson } \\
\text { meson }\end{array}$ \\
\hline west & aineral \\
\hline vest & -ineral \\
\hline $\begin{array}{l}\text { west } \\
\text { west. }\end{array}$ & $\begin{array}{l}\text { mineral } \\
\text { mineral }\end{array}$ \\
\hline west & minerat \\
\hline vest & mineral \\
\hline & minẹral \\
\hline vest & nineral \\
\hline west & - Ineral \\
\hline west & - inerol \\
\hline $\begin{array}{l}\text { west } \\
\text { west }\end{array}$ & $\begin{array}{l}\text { mineral } \\
\text { ineral }\end{array}$ \\
\hline vest & nicholes \\
\hline vest & nficholies \\
\hline vest & nicholes \\
\hline west & nicholas \\
\hline vest & nichol as \\
\hline west & nicholas \\
\hline $\begin{array}{l}\text { west } \\
\text { west }\end{array}$ & nicholas \\
\hline $\begin{array}{l}\text { west } \\
\text { west }\end{array}$ & $\begin{array}{l}\text { nicholars } \\
\text { nicholas }\end{array}$ \\
\hline vest & nicholes \\
\hline west & ntetiolas \\
\hline vest & nicholes \\
\hline west & nicholas \\
\hline $\begin{array}{l}\text { west } \\
\text { west }\end{array}$ & nichol as \\
\hline $\begin{array}{l}\text { west } \\
\text { west }\end{array}$ & $\begin{array}{l}\text { nicholas } \\
\text { nicholas }\end{array}$ \\
\hline west & Docahontas \\
\hline west & Docehontes \\
\hline vest & Docahontas \\
\hline west & preston \\
\hline west & preston \\
\hline west & preston \\
\hline west & Dreston \\
\hline west & preston \\
\hline west & preston \\
\hline west & Dreston \\
\hline west & preston \\
\hline wes & preston \\
\hline west & preston \\
\hline iest & Dutnam \\
\hline west & Dutnaw \\
\hline $\begin{array}{l}\text { west } \\
\text { west }\end{array}$ & $\begin{array}{l}\text { randolph } \\
\text { randolph }\end{array}$ \\
\hline
\end{tabular}

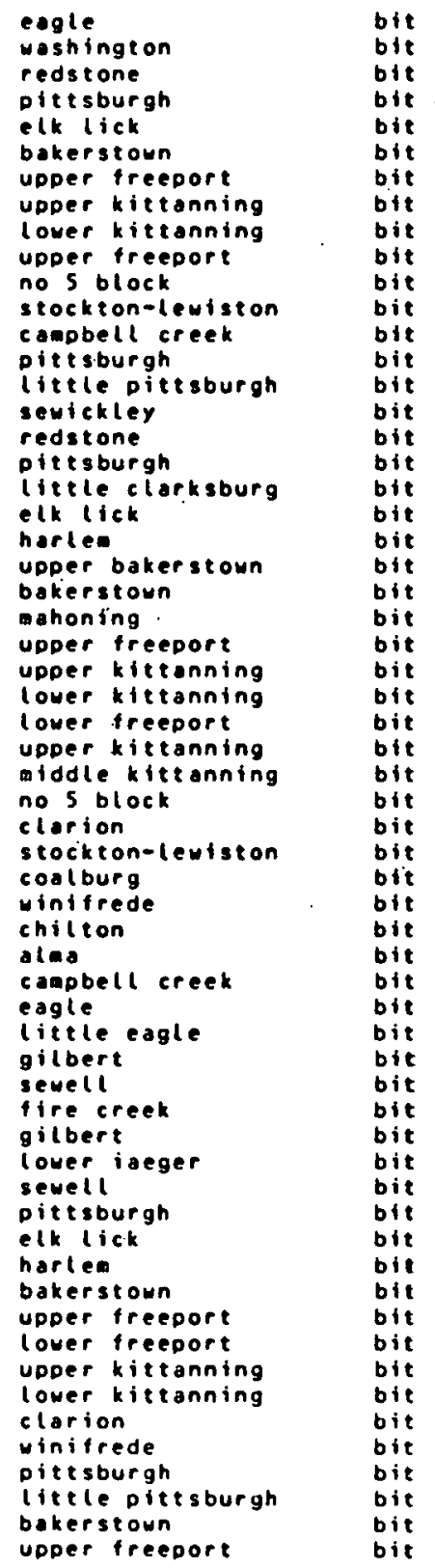

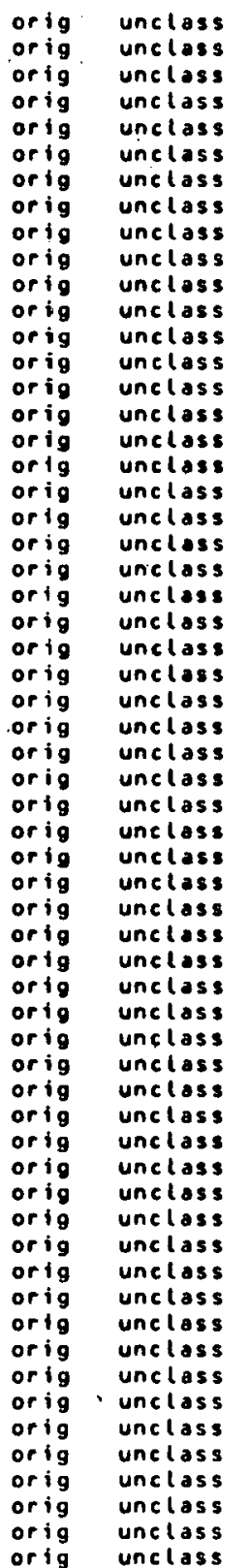

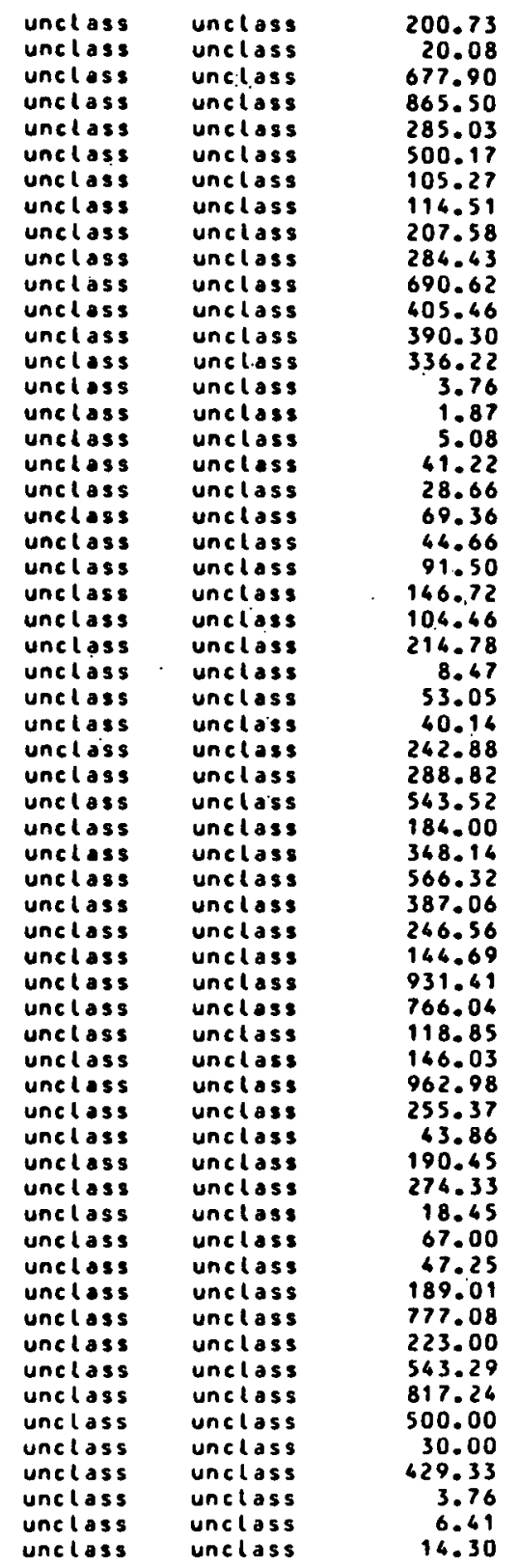




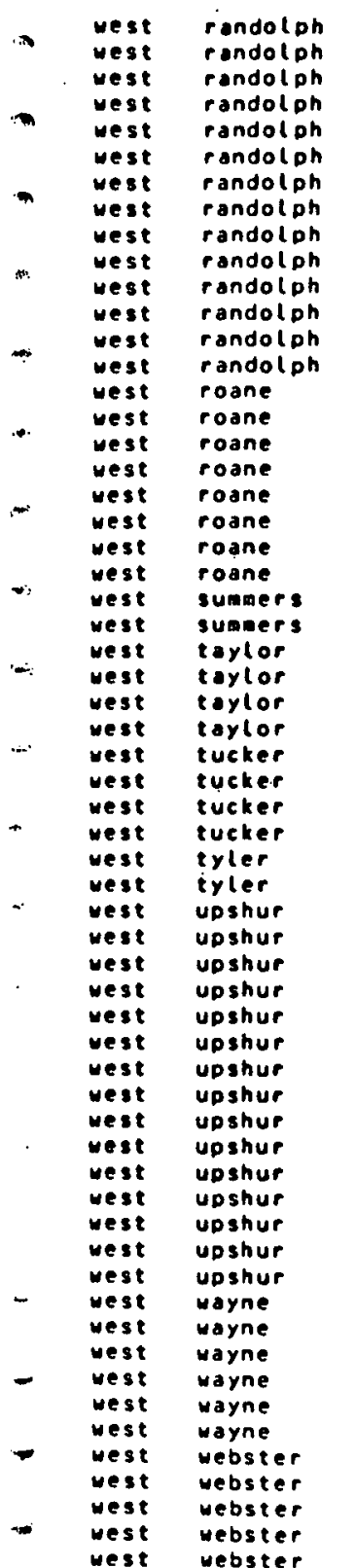

\begin{tabular}{|c|c|}
\hline 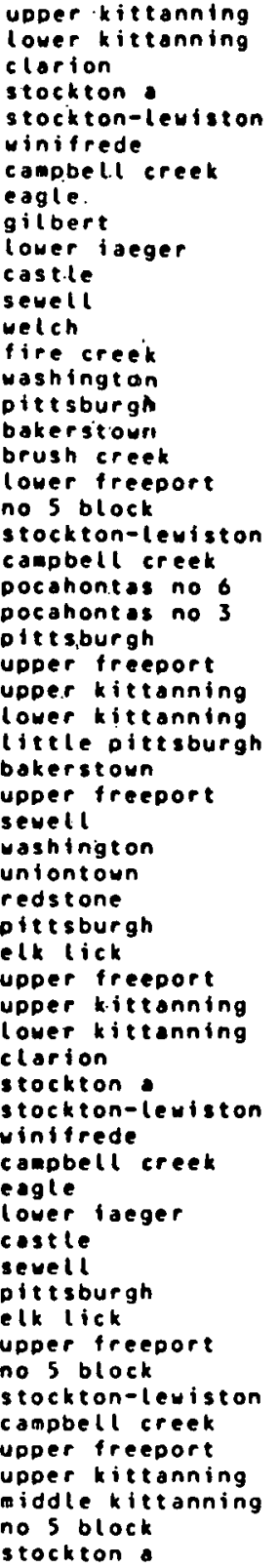 & 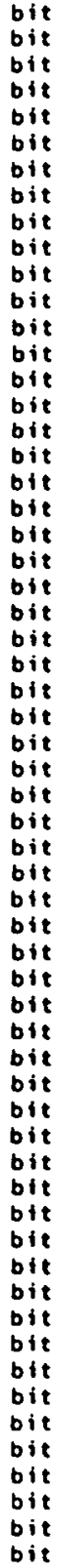 \\
\hline
\end{tabular}

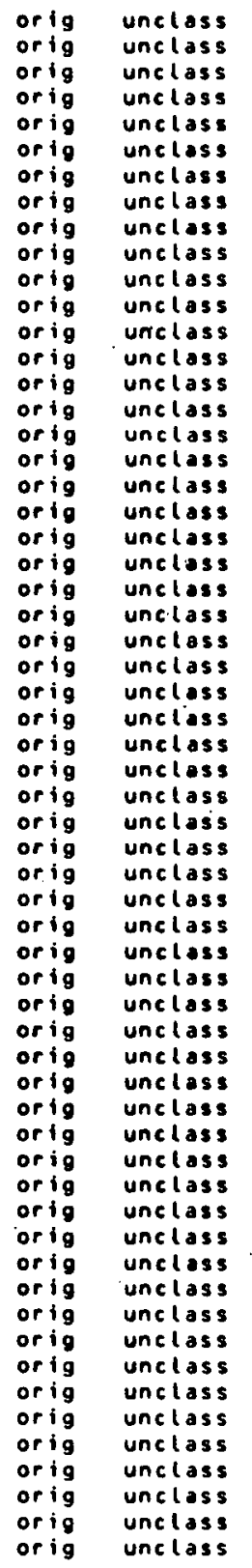

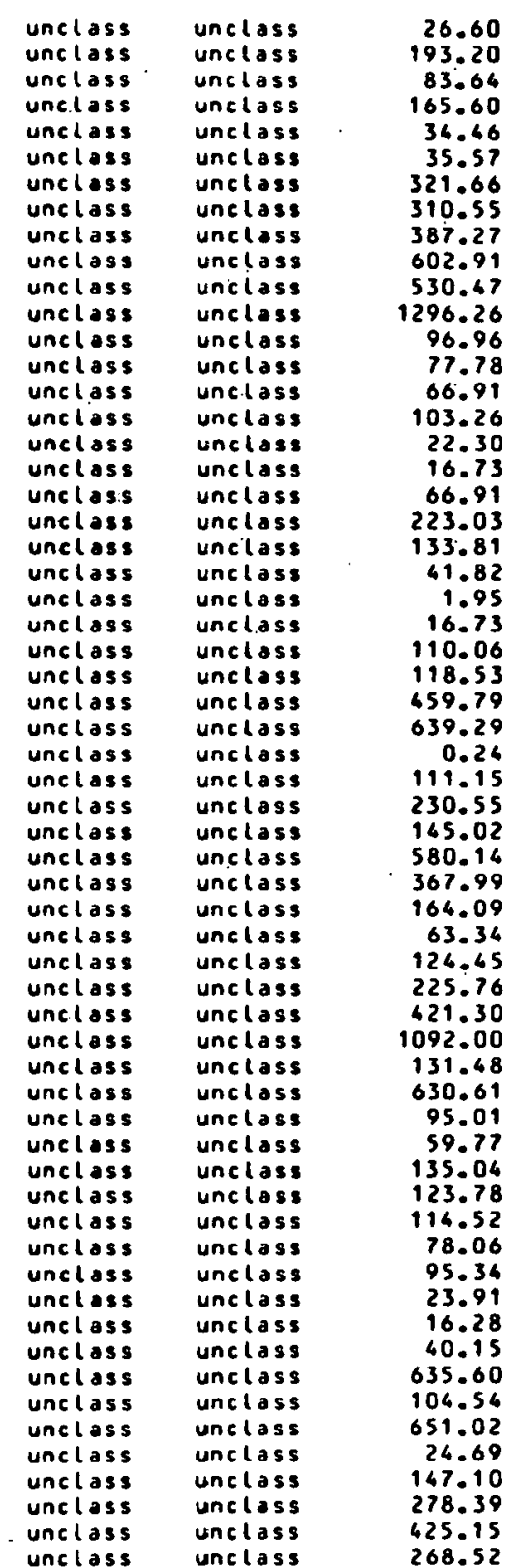




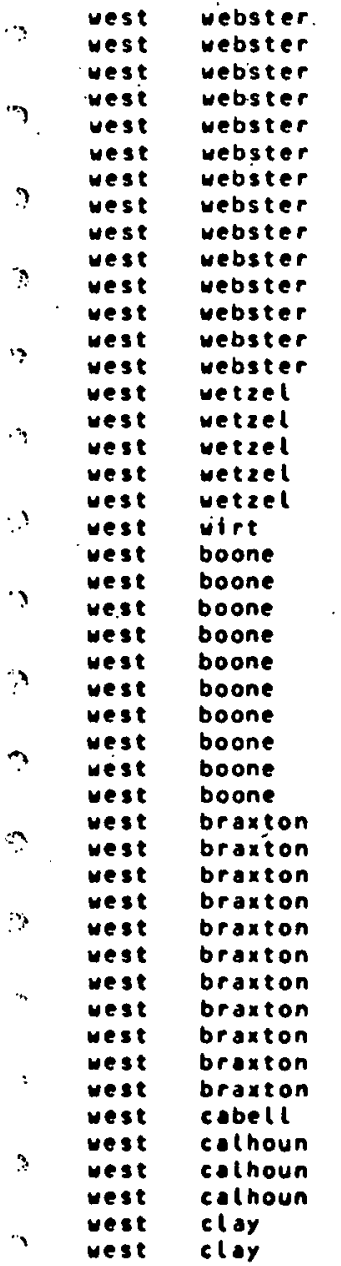

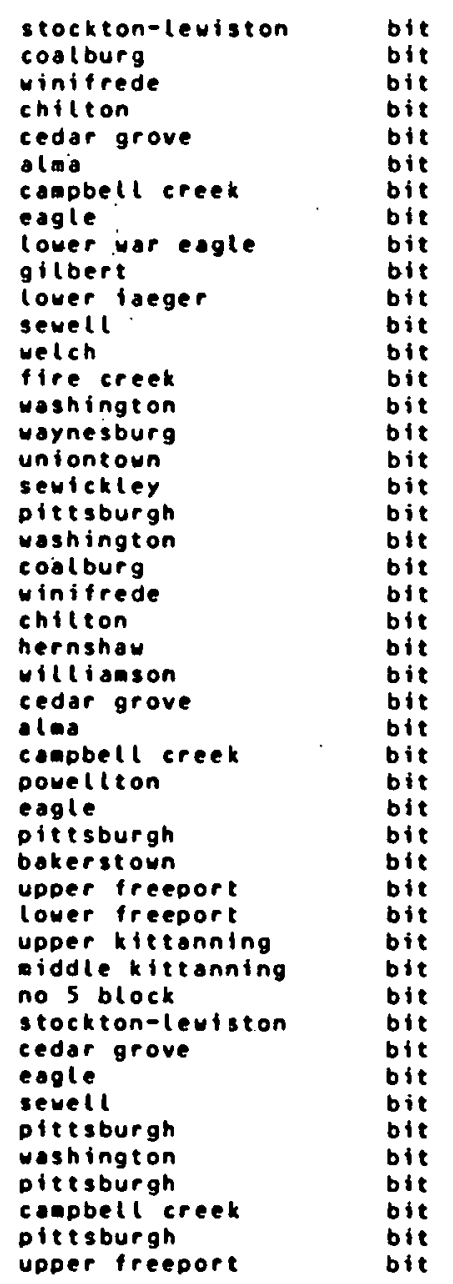

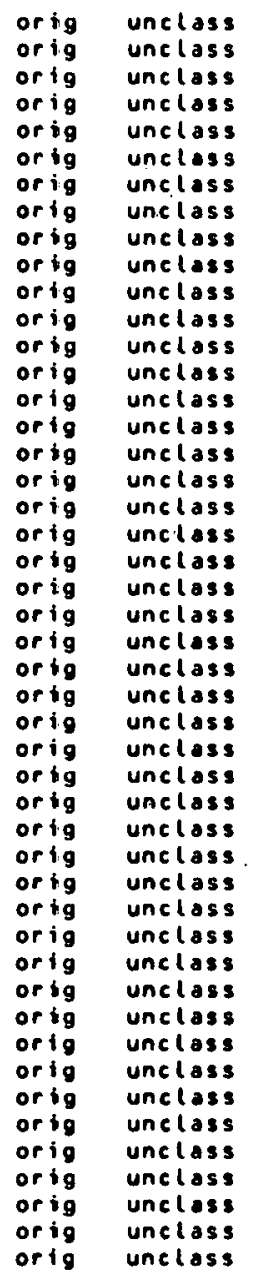

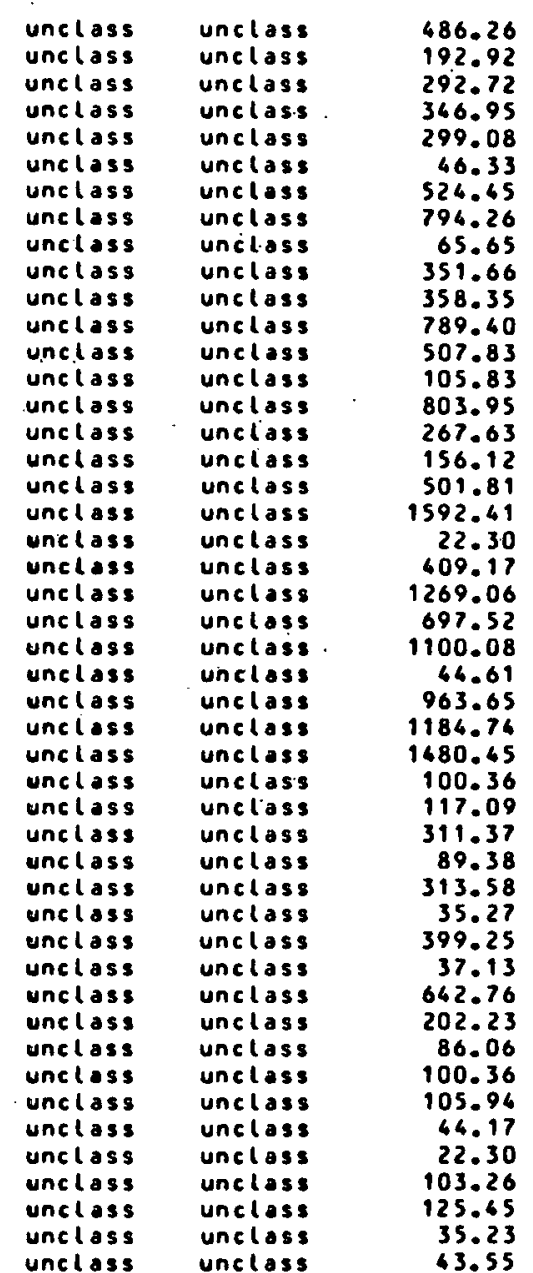


overage analyses of coal in vest virginia

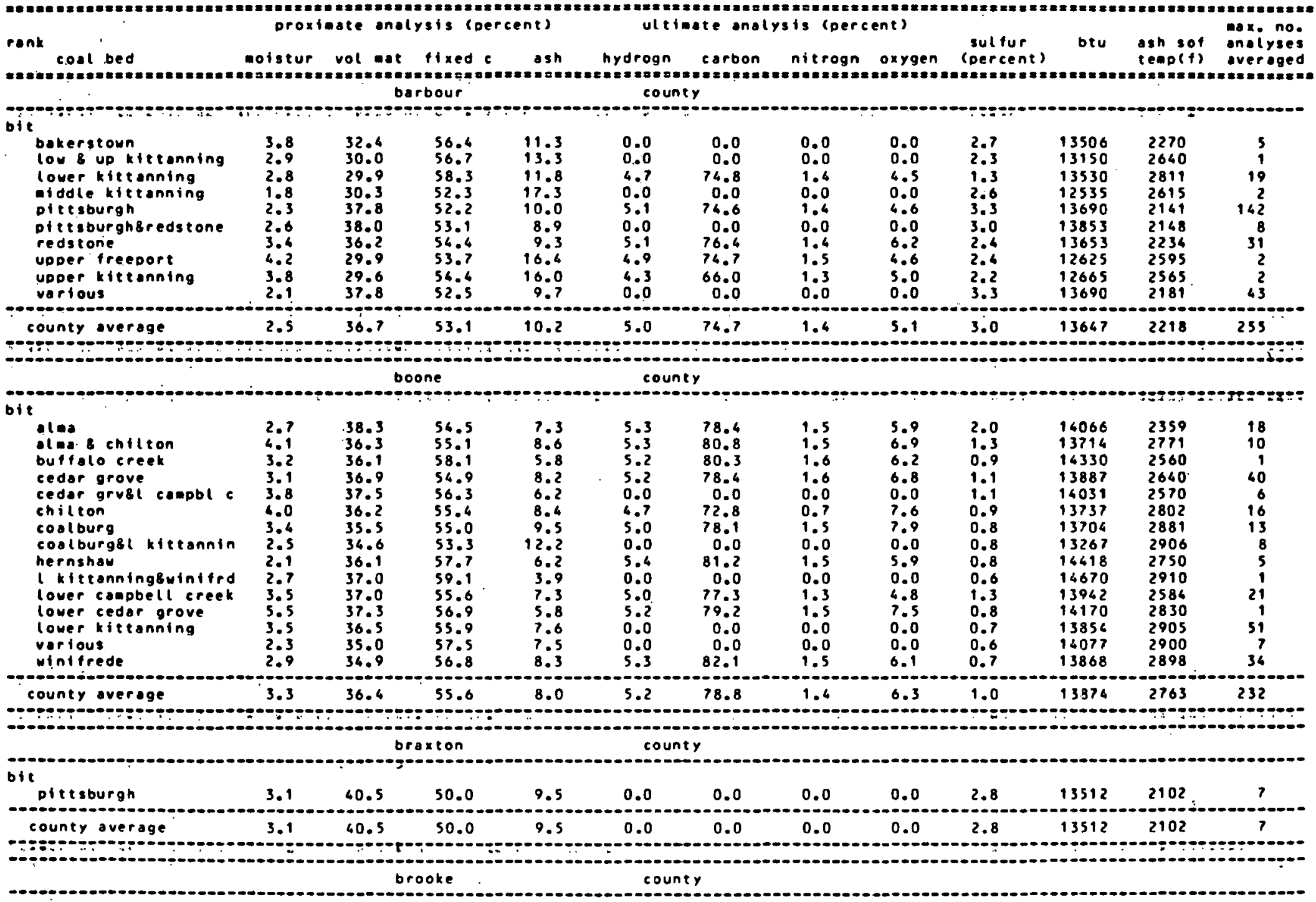




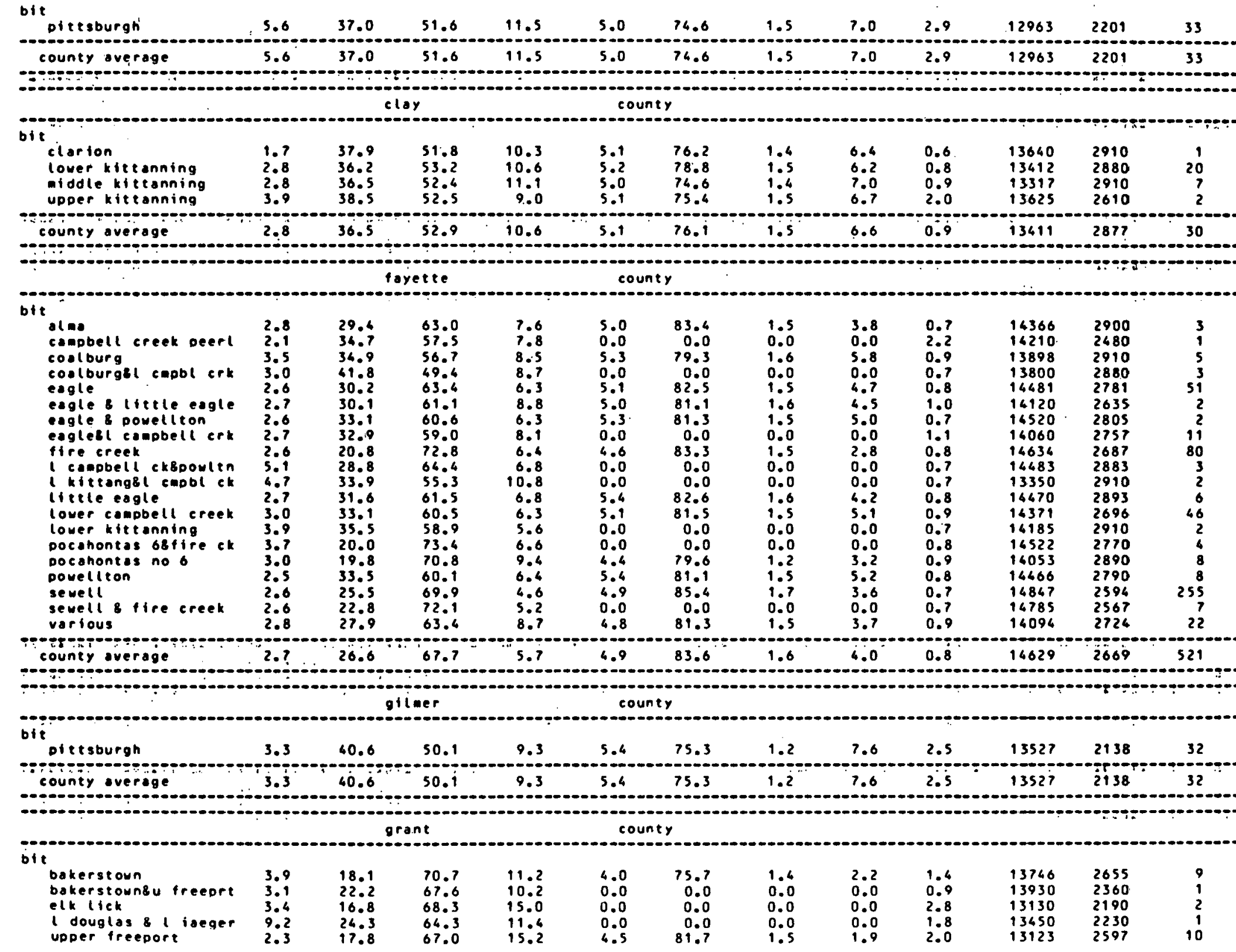




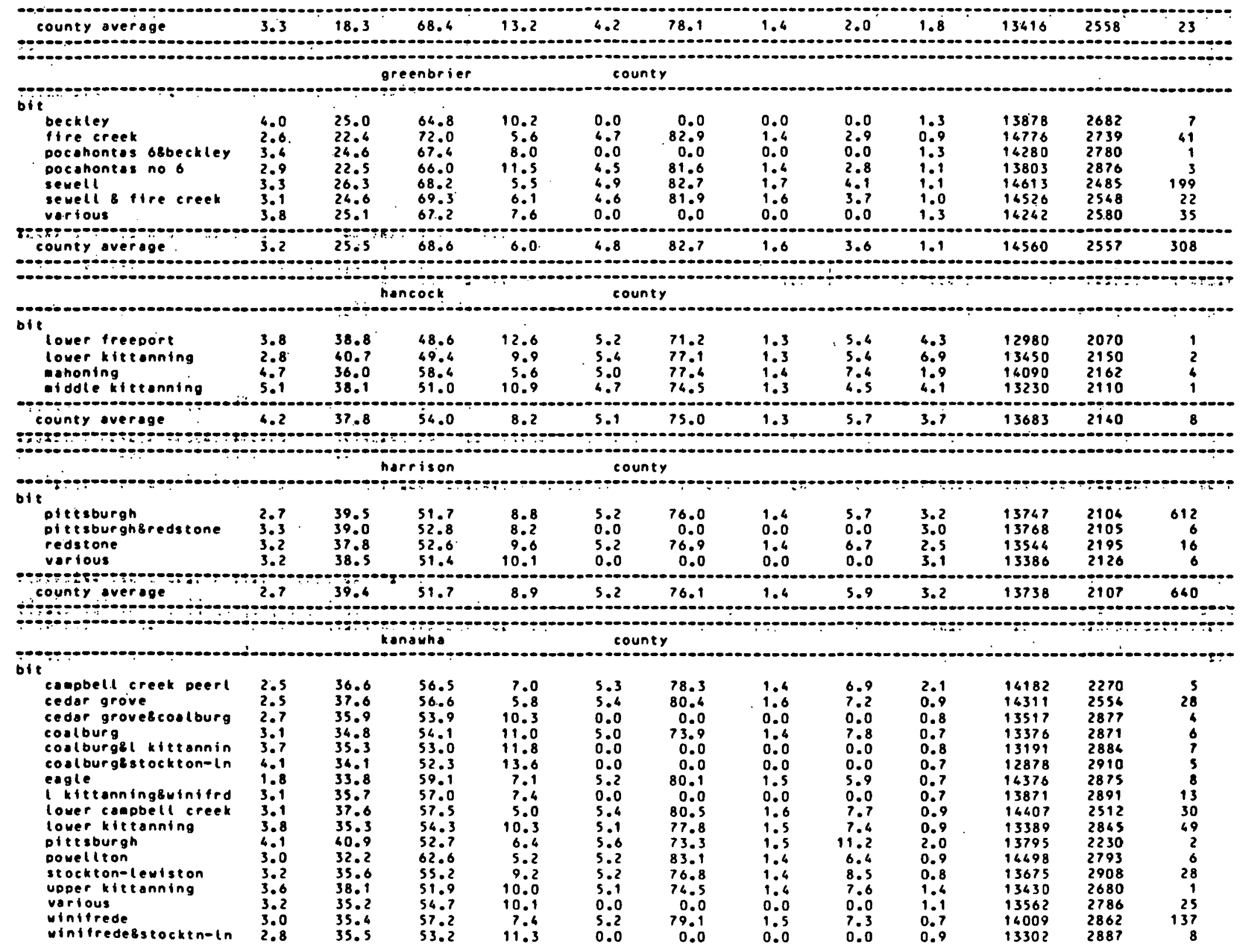


as on

county

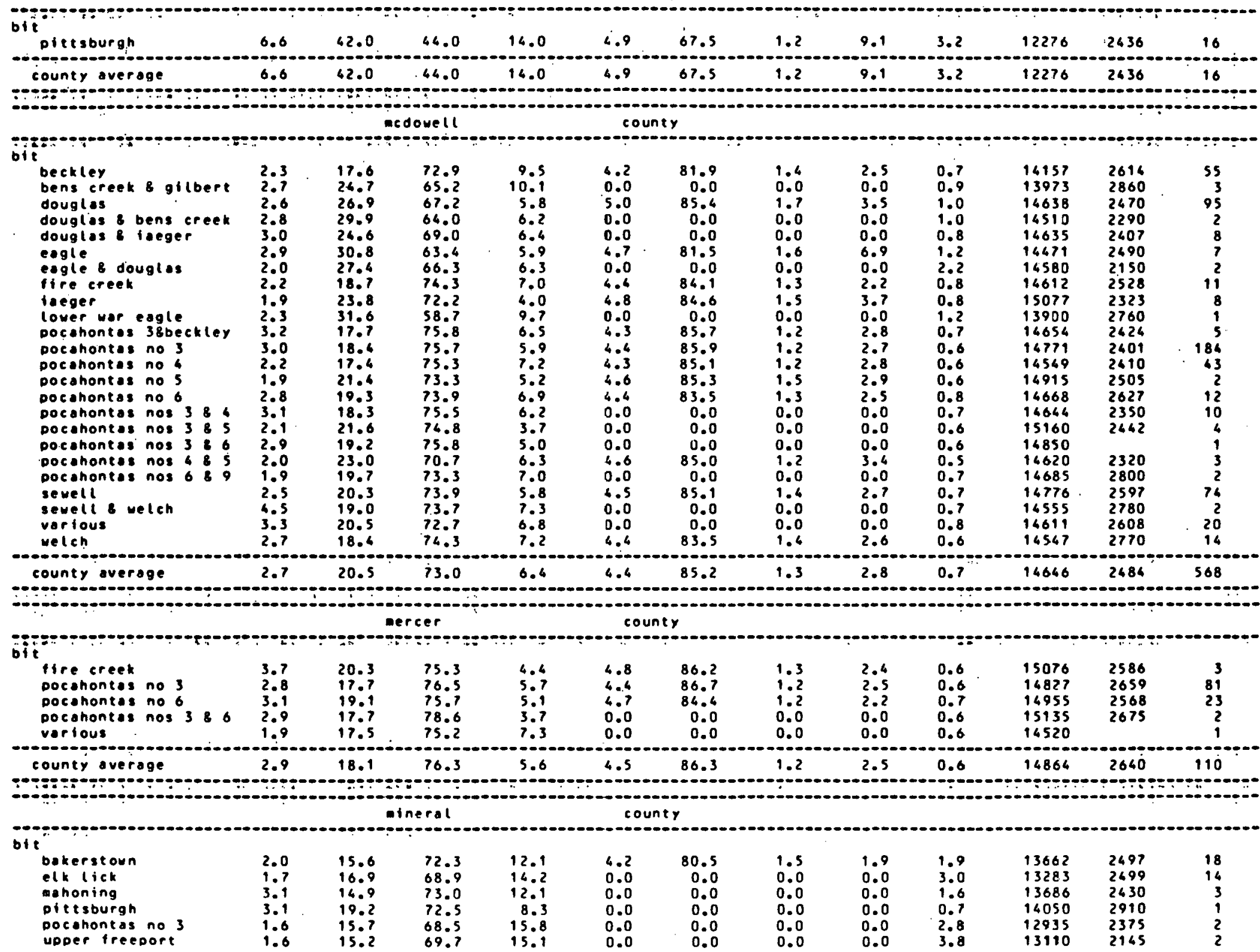




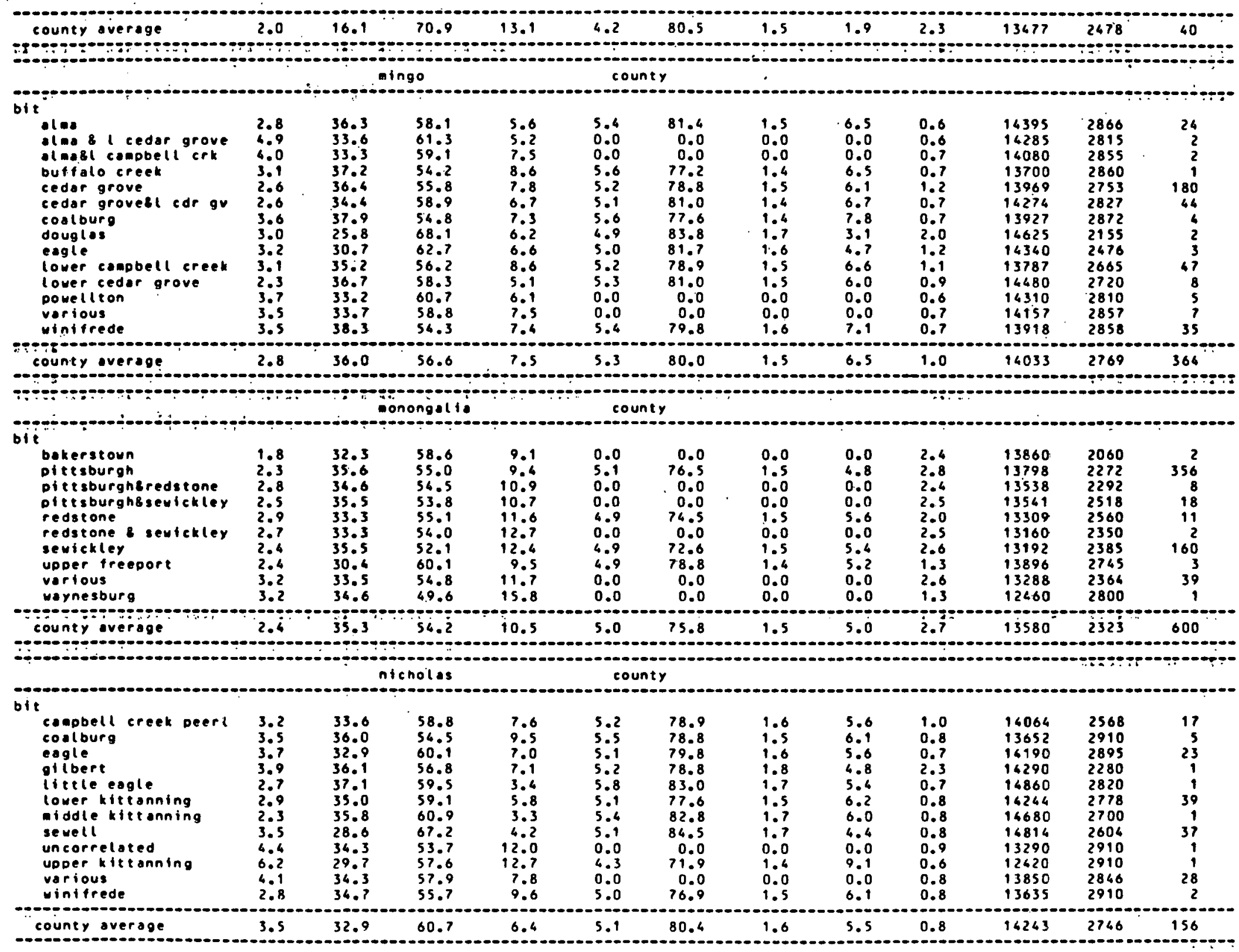


ohio

county

\begin{tabular}{|c|c|c|c|c|c|c|c|c|c|c|c|c|}
\hline pittsburgh & 3.3 & 40.1 & 50.4 & 9.4 & 5.1 & 73.9 & 1.3 & 7.1 & 3.9 & 13389 & 2059 & 35 \\
\hline county average & 3.3 & 40.1 & 50.4 & 9.4 & 5.1 & 73.9 & 1.3 & 7.1 & 3.9 & 13389 & 2059 & 35 \\
\hline & & & & & & & & & & & & \\
\hline sevell & 2.8 & 30.3 & 62.9 & 6.8 & 0.0 & 0.0 & 0.0 & 0.0 & 0.7 & 16496 & 2890 & 37 \\
\hline county overage. & 2.8 & 30.3 & 62.9 & 6.8 & 0.0 & 0.0 & 0.0 & 0.0 & 0.7 & 16696 & 2890 & 37 \\
\hline & & & on & & & & & & & & & \\
\hline $\begin{array}{l}\text { bokerstoun } \\
\text { bokerstownsu freeprt } \\
\text { laver kittonning } \\
\text { mohon ing } \\
\text { middle ktt onning } \\
\text { pittsburgh } \\
\text { upper treeport } \\
\text { upper kittonning } \\
\text { various }\end{array}$ & $\begin{array}{l}2.7 \\
2.8 \\
3.0 \\
2.9 \\
3.3 \\
5.7 \\
2.9 \\
3.8 \\
3.3\end{array}$ & $\begin{array}{l}29.8 \\
29.5 \\
23.6 \\
28.2 \\
24.9 \\
32.4 \\
29.0 \\
29.9 \\
27.7\end{array}$ & $\begin{array}{l}60.4 \\
59.3 \\
65.9 \\
62.1 \\
65.0 \\
58.9 \\
59.2 \\
53.2 \\
59.5\end{array}$ & $\begin{array}{r}9.8 \\
11.2 \\
10.5 \\
9.7 \\
10.1 \\
8.7 \\
11.8 \\
16.8 \\
12.8\end{array}$ & $\begin{array}{l}4.9 \\
0.0 \\
4.5 \\
0.0 \\
4.06 \\
5.0 \\
4.7 \\
4.5 \\
0.0\end{array}$ & $\begin{array}{r}80.0 \\
0.0 \\
76.0 \\
0.0 \\
83.9 \\
77.1 \\
74.8 \\
71.6 \\
0.0\end{array}$ & $\begin{array}{l}1.7 \\
0.0 \\
1.5 \\
0.0 \\
1.4 \\
1.6 \\
1.4 \\
1.3 \\
0.0\end{array}$ & $\begin{array}{l}4.0 \\
0.0 \\
2.7 \\
0.0 \\
3.7 \\
6.2 \\
3.9 \\
4.5 \\
0.0\end{array}$ & $\begin{array}{l}2.0 \\
2.4 \\
2.0 \\
2.7 \\
2.8 \\
1.6 \\
2.1 \\
2.4 \\
2.0\end{array}$ & $\begin{array}{l}13849 \\
13650 \\
13878 \\
13943 \\
13976 \\
13720 \\
13536 \\
12610 \\
13373\end{array}$ & $\begin{array}{l}2295 \\
2273 \\
2579 \\
2160 \\
2330 \\
2392 \\
2548 \\
2803 \\
2538\end{array}$ & $\begin{array}{r}107 \\
7 \\
76 \\
3 \\
3 \\
8 \\
266 \\
3 \\
34\end{array}$ \\
\hline county average & 3.0 & 28.3 & 60.5 & 11.2 & 4.7 & 75.8 & 1.4 & $3.8^{\circ}$ & 2.1 & 13650 & 2485 & 485 \\
\hline Dittsburgh & 3.5 & 41.1 & 50.3 & 8.6 & 5.3 & 76.2 & 1.4 & 7.8 & 2.6 & 13421 & 2160 & 16 \\
\hline county average & 3.5 & $6 i . i$ & 50.3 & 8.6 & 5.3 & 76.2 & 1.4 & 7.8 & 2.6 & 13621 & 2160 & 16 \\
\hline & & & igh & & & & & & & & & \\
\hline 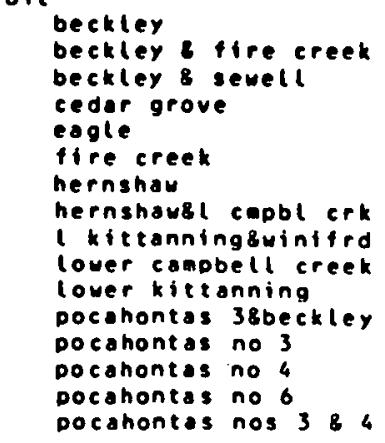 & $\begin{array}{l}2.6 \\
2.7 \\
3.9 \\
2.0 \\
2.6 \\
2.4 \\
1.6 \\
2.4 \\
2.9 \\
3.1 \\
2.3 \\
2.3 \\
2.4 \\
2.9 \\
2.4 \\
2.2\end{array}$ & $\begin{array}{l}18.3 \\
18.5 \\
20.5 \\
34.8 \\
28.7 \\
18.1 \\
34.4 \\
31.2 \\
35.3 \\
30.9 \\
34.0 \\
15.9 \\
16.4 \\
16.8 \\
16.0 \\
15.5\end{array}$ & $\begin{array}{l}76.3 \\
75.5 \\
72.7 \\
58.6 \\
64.1 \\
76.5 \\
58.7 \\
61.4 \\
58.8 \\
61.5 \\
56.9 \\
74.8 \\
76.1 \\
77.5 \\
78.4 \\
74.0\end{array}$ & $\begin{array}{l}5.4 \\
5.9 \\
6.9 \\
6.5 \\
7.2 \\
5.4 \\
6.9 \\
7.4 \\
5.8 \\
7.6 \\
9.2 \\
9.3 \\
7.5 \\
5.7 \\
5.0 \\
10.4\end{array}$ & $\begin{array}{l}4.5 \\
0.0 \\
0.0 \\
0.0 \\
4.9 \\
4.6 \\
5.3 \\
0.0 \\
0.0 \\
0.0 \\
0.0 \\
0.0 \\
4.3 \\
4.3 \\
4.3 \\
0.0\end{array}$ & $\begin{array}{r}86.5 \\
0.0 \\
0.0 \\
0.0 \\
81.5 \\
86.7 \\
81.5 \\
0.0 \\
0.0 \\
0.0 \\
0.0 \\
0.0 \\
85.0 \\
84.1 \\
86.4 \\
0.0\end{array}$ & $\begin{array}{l}1.5 \\
0.0 \\
0.0 \\
0.00 \\
1.5 \\
1.5 \\
1.0 \\
0.0 \\
0.0 \\
0.0 \\
0.0 \\
0.0 \\
1.2 \\
1.3 \\
1.3 \\
0.0\end{array}$ & $\begin{array}{l}2.4 \\
0.0 \\
0.0 \\
0.0 \\
3.9 \\
2.5 \\
4.7 \\
0.0 \\
0.0 \\
0.0 \\
0.0 \\
0.0 \\
2.3 \\
2.5 \\
2.1 \\
0.0\end{array}$ & $\begin{array}{l}0.7 \\
0.8 \\
0.8 \\
1.0 \\
1.0 \\
0.8 \\
1.2 \\
0.9 \\
0.6 \\
0.9 \\
0.7 \\
0.8 \\
0.8 \\
0.8 \\
0.9 \\
0.7\end{array}$ & $\begin{array}{l}14800 \\
14672 \\
14560 \\
14395 \\
14419 \\
14840 \\
14405 \\
14310 \\
14298 \\
14280 \\
13806 \\
14183 \\
14460 \\
14803 \\
14875 \\
13957\end{array}$ & $\begin{array}{l}2774 \\
2766 \\
2630 \\
2606 \\
2776 \\
2666 \\
2375 \\
2733 \\
2908 \\
2640 \\
2910 \\
2874 \\
2841 \\
2750 \\
2700 \\
2890\end{array}$ & $\begin{array}{r}133 \\
13 \\
2 \\
12 \\
10 \\
80 \\
4 \\
3 \\
23 \\
3 \\
3 \\
8 \\
42 \\
33 \\
2 \\
8\end{array}$ \\
\hline
\end{tabular}




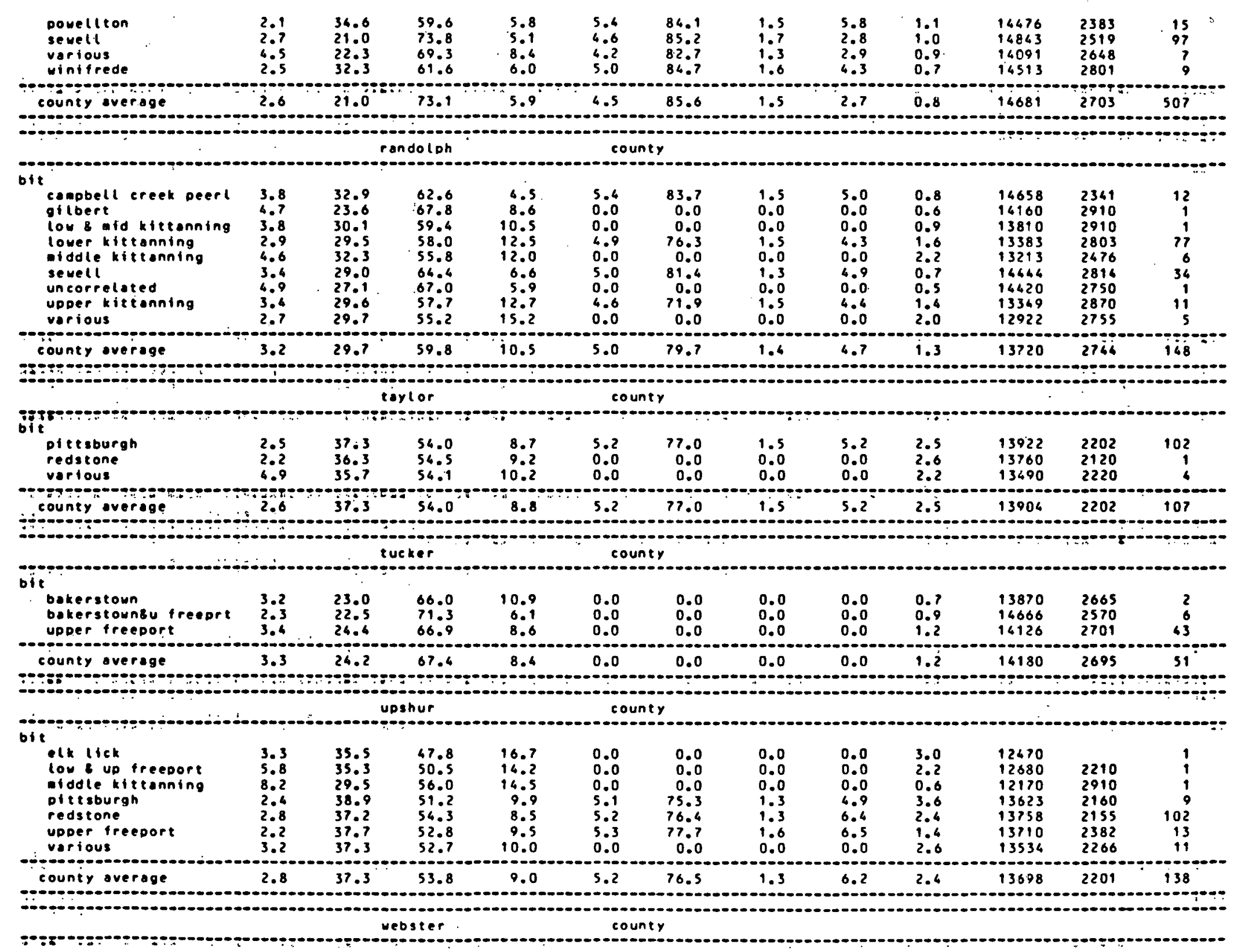




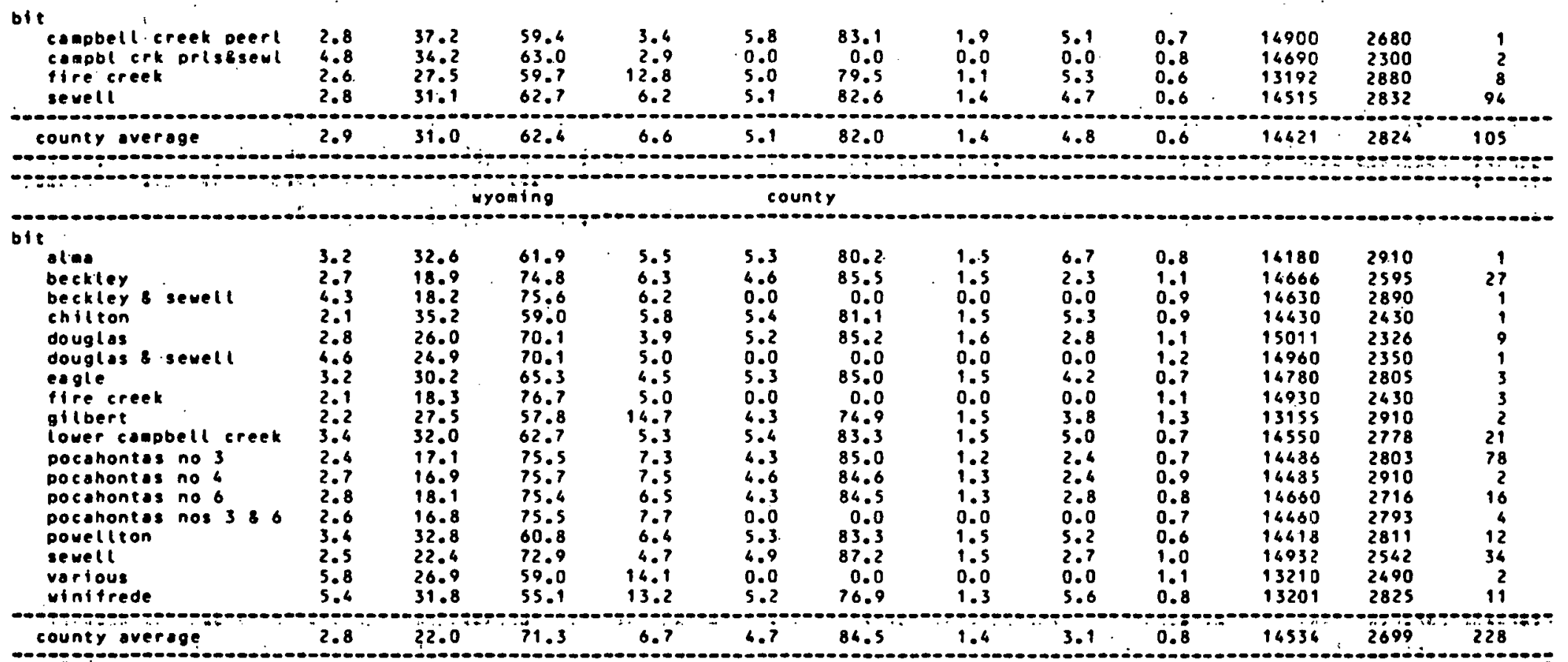

eque 
WEST VIRGINIA

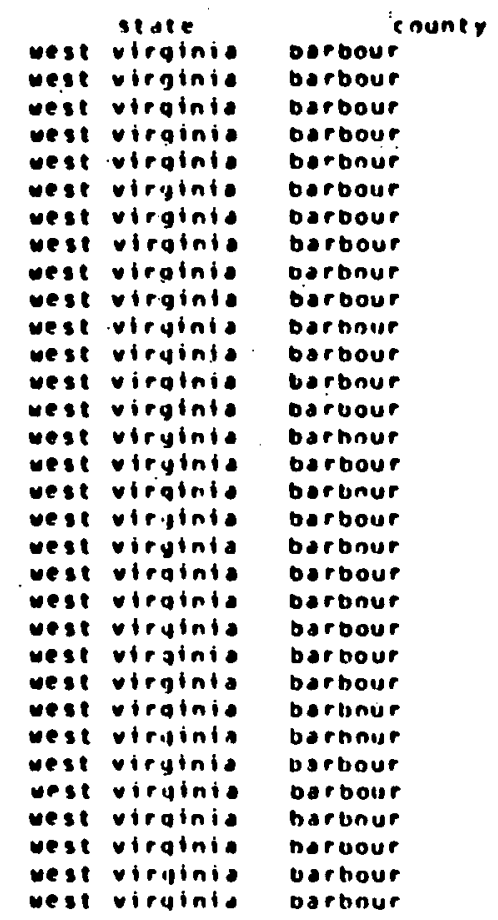

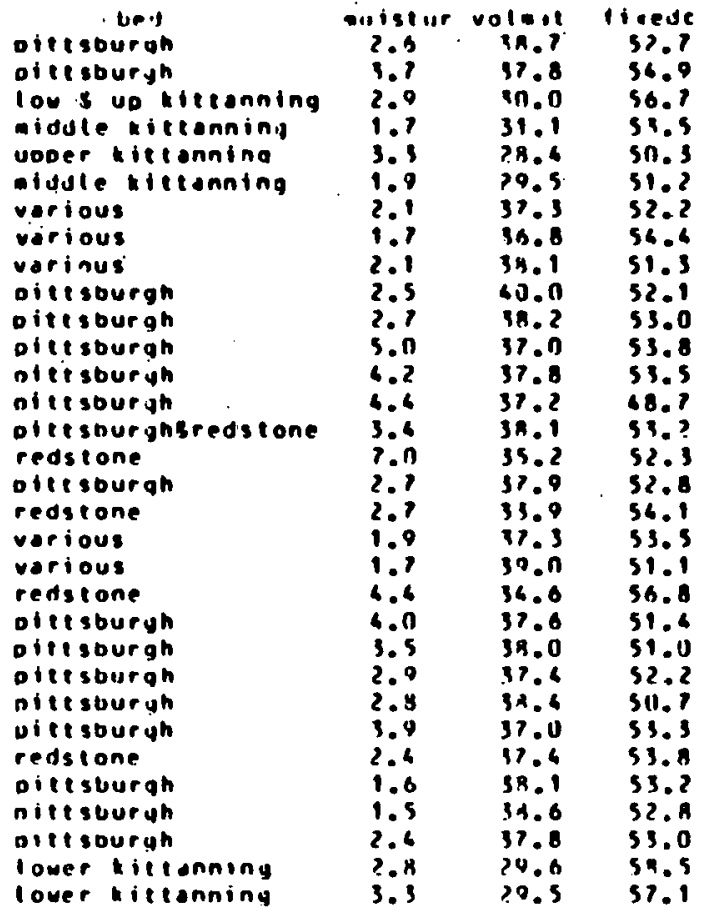

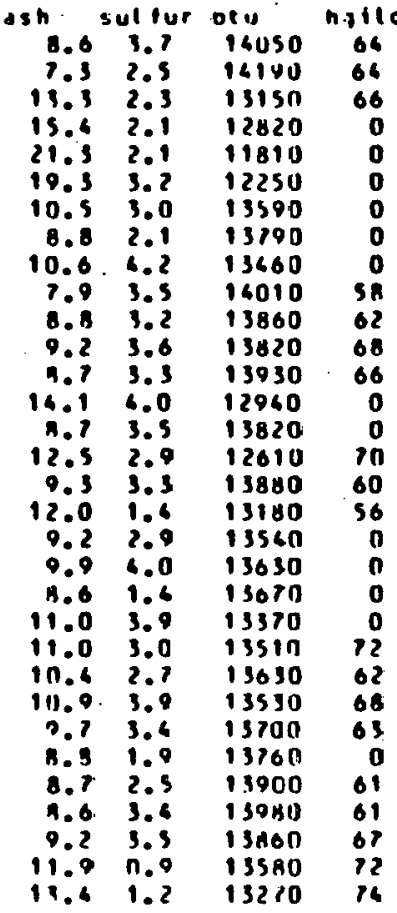

18110
7.5
8.0
8.0
8.0
7.0
7.5
7.5
8.0
7.0
8.0
7.5
7.5
8.0
7.0
8.0
7.5
7.5
8.5
7.5
7.5
7.5
7.0
8.5
8.0
8.0
7.5
8.0
7.5
6.0
7.5
8.0
7.5


- west virainis west virainia

west virainia west virainia

- vese virainia vest virainia

- west virainia west virainia

west virainia west virginia

west virainia

- vest virginia west virginia

- west virginia

west virainia

west virginia

vest virainis

s west virginia west virginia

West virginia vest virginia

west virainia

west virginia

a virginia

west virginia

west virginia

r west virainia west virginia

west virginia

west virginia

- west virqinia

west virainia

west virgini

west virainia county

arbour

barbour

barbour

harhour

harbour

barbcur
barbour

barbour

barbnur

barbour

barbour

barbnur

barbour

barbour

barbour

barbour

barbour

barbour

barbour

barbour

barbour

barbour

barhour

barbour

barbour

barbnisr

barbour

barbour

barbnue

barsour

barbour

barbour

barbor

boone

boon

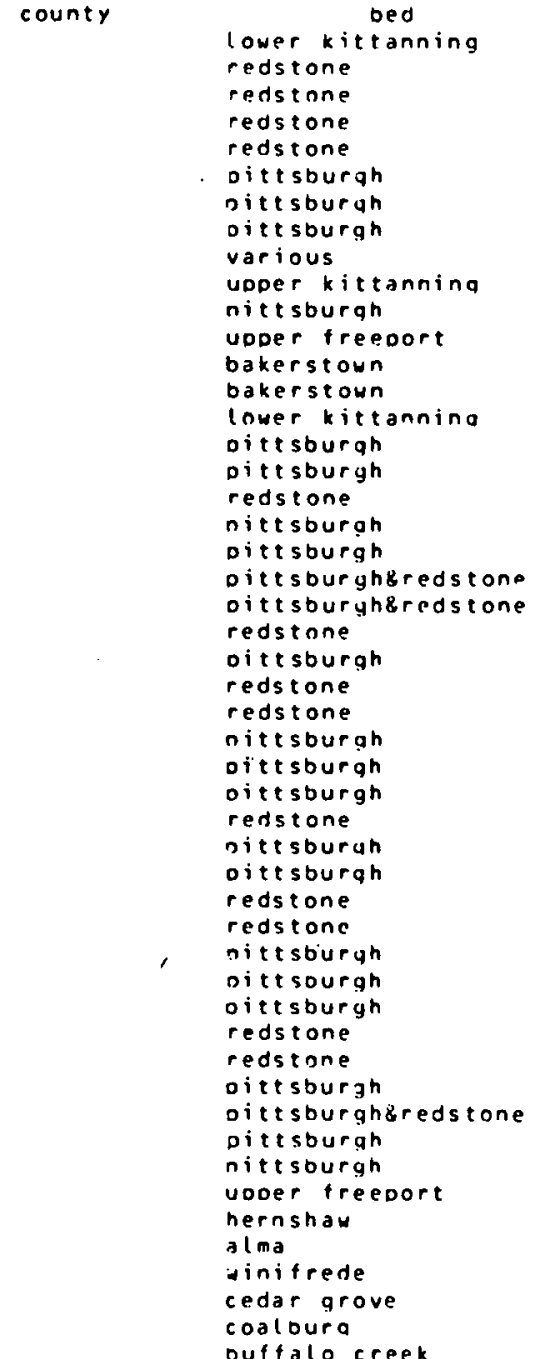

ouftalo creek

\begin{tabular}{|c|c|c|c|c|c|c|c|}
\hline סח & - volmat & fixedc & ash & & ur btu & hailo & \\
\hline .3 & 78.4 & 58.6 & 13.0 & 0.8 & 13370 & 74 & 7.5 \\
\hline 5.5 & 35.1 & 55.2 & 9.7 & 2.6 & 13670 & 64 & 8.0 \\
\hline 4.3 & 36.2 & 53.4 & 10.4 & 3.0 & 13470 & 0 & 7.5 \\
\hline 5.0 & 36.4 & 51.? & 12.4 & 3.4 & 13220 & 0 & 6.0 \\
\hline 2.2 & 36.2 & 55.4 & 8.4 & 1.7 & 13880 & 57 & 8.0 \\
\hline 2.5 & 38.3 & 51.3 & 10.4 & 3.5 & 13670 & 61 & 7.5 \\
\hline 2.9 & 30.6 & 51.3 & 9.1 & 3.4 & 13820 & 60 & 7.5 \\
\hline 1.? & 40.8 & 49.1 & 10.1 & 3.7 & 13750 & 0 & 7.5 \\
\hline 3.? & 36.2 & 51.6 & 12.2 & 3.6 & 13200 & 0 & 7.0 \\
\hline 4.2 & 30.8 & 58.5 & 10.7 & 2.3 & 13520 & 73 & 8.0 \\
\hline 2.0 & 38.1 & 52.8 & 9.1 & 3.? & 13840 & 59 & 8.0 \\
\hline 4.2 & 30.0 & 57.9 & 12.1 & 2.2 & 13370 & 72 & 7.0 \\
\hline 3.2 & 32.1 & 58.5 & 0.4 & 1.7 & 13950 & 68 & 8.0 \\
\hline 4.6 & 31.8 & 55.6 & 12.8 & 3.0 & 13450 & 0 & 6.5 \\
\hline 2.2 & 29.5 & 56.1 & 14.4 & 0.8 & 13170 & 65 & 7.5 \\
\hline 2.9 & 38.0 & 51.0 & 11.0 & 4.0 & 13500 & 64 & 9.0 \\
\hline 5.0 & 37.6 & 50.8 & 11.6 & 4.2 & 13370 & 60 & 7.0 \\
\hline 2.8 & 36.5 & 55.6 & 7.9 & 2.5 & 13870 & 0 & 8.0 \\
\hline 1.9 & 39.7 & 50.3 & 10.0 & 4.2 & 13650 & 0 & 7.0 \\
\hline 3.5 & 37.6 & 54.7 & 7.7 & 2.2 & 14000 & 0 & 8.0 \\
\hline 2.7 & 39.8 & $5 ? .9$ & 7.3 & 3.1 & 14130 & 61 & 7.5 \\
\hline 3.1 & 36.8 & 53.1 & 10.1 & 2.4 & 13620 & 60 & 7.5 \\
\hline 3.1 & 36.0 & 57.0 & 7.0 & 1.7 & 14060 & 60 & 8.0 \\
\hline 3.5 & 40.3 & 50.4 & 9.3 & 4.2 & 13750 & 59 & 6.5 \\
\hline 3.5 & 37.0 & 55.1 & 7.9 & 2.9 & 13890 & 59 & 8.5 \\
\hline 3.7 & 36.8 & 54.4 & 8.8 & 2.6 & 13720 & 62 & 8.5 \\
\hline 2.9 & 38.5 & 53.5 & 9.0 & 3.1 & 13980 & 63 & 8.5 \\
\hline 1.9 & 40.5 & 50.5 & 9.0 & 3.9 & 13810 & 63 & 7.0 \\
\hline 7.4 & 38.0 & 51.3 & 10.7 & 4.1 & 13440 & 64 & 7.5 \\
\hline 3.1 & 39.0 & 51.9 & 9.1 & 2.9 & 13690 & 0 & 7.5 \\
\hline 2.4 & 37.8 & 48.2 & 14.0 & 4.5 & 12970 & 56 & 8.0 \\
\hline 2.9 & 39.2 & 51.1 & 9.7 & 3.9 & 13730 & 64 & 8.5 \\
\hline 4.9 & 36.8 & $54 . ?$ & 9.0 & 2.8 & 13730 & 63 & 7.5 \\
\hline 6.6 & 36.2 & 56.8 & 9.0 & 2.2 & 13700 & 0 & 8.0 \\
\hline$? .1$ & 38.1 & 50.0 & 11.9 & 3.5 & 13360 & 69 & 7.5 \\
\hline 5.7 & 38.1 & 49.0 & 12.9 & 5.2 & 131110 & 0 & 8.0 \\
\hline 2.2 & 49.2 & 40.6 & 9.2 & 3.9 & 13790 & 0 & 7.0 \\
\hline 2.5 & 36.6 & 53.5 & 9.9 & 2.6 & 13600 & 60 & 8.0 \\
\hline 2.4 & 38.2 & 53.3 & 8.5 & 2.8 & 13770 & 58 & 8.0 \\
\hline 2.8 & 39.2 & 51.3 & 9.5 & 3.7 & 13640 & 58 & \\
\hline 2.9 & 36.7 & 54.3 & 0.0 & 2.8 & 13840 & 61 & \\
\hline 3.0 & 36.8 & 50.7 & 12.3 & 5.2 & 13370 & 66 & \\
\hline 2.8 & 39.5 & 54.7 & 9.3 & 4.5 & 13070 & 62 & 7.0 \\
\hline 4.1 & $? 9.8$ & 40.5 & 20.7 & 2.6 & 11880 & 69 & 7.5 \\
\hline 2.7 & $3 t .6$ & $58 . ?$ & $5 . ?$ & 0.8 & 14530 & 53 & 6.5 \\
\hline 3.4 & 36.8 & 55.0 & 8.2 & 1.8 & 13880 & 40 & 7.5 \\
\hline 6.? & 36.0 & 55.8 & 8.2 & 0.7 & 13370 & 40 & 1.5 \\
\hline 2.6 & 37.3 & 55.3 & 7.4 & 1.1 & 16180 & 55 & 7.5 \\
\hline 6.5 & 37.6 & 55.6 & 5.8 & n.? & 13770 & 0 & 2.0 \\
\hline 3.7 & 36.1 & 58.1 & 5.8 & 0.9 & 14330 & 54 & \\
\hline
\end{tabular}




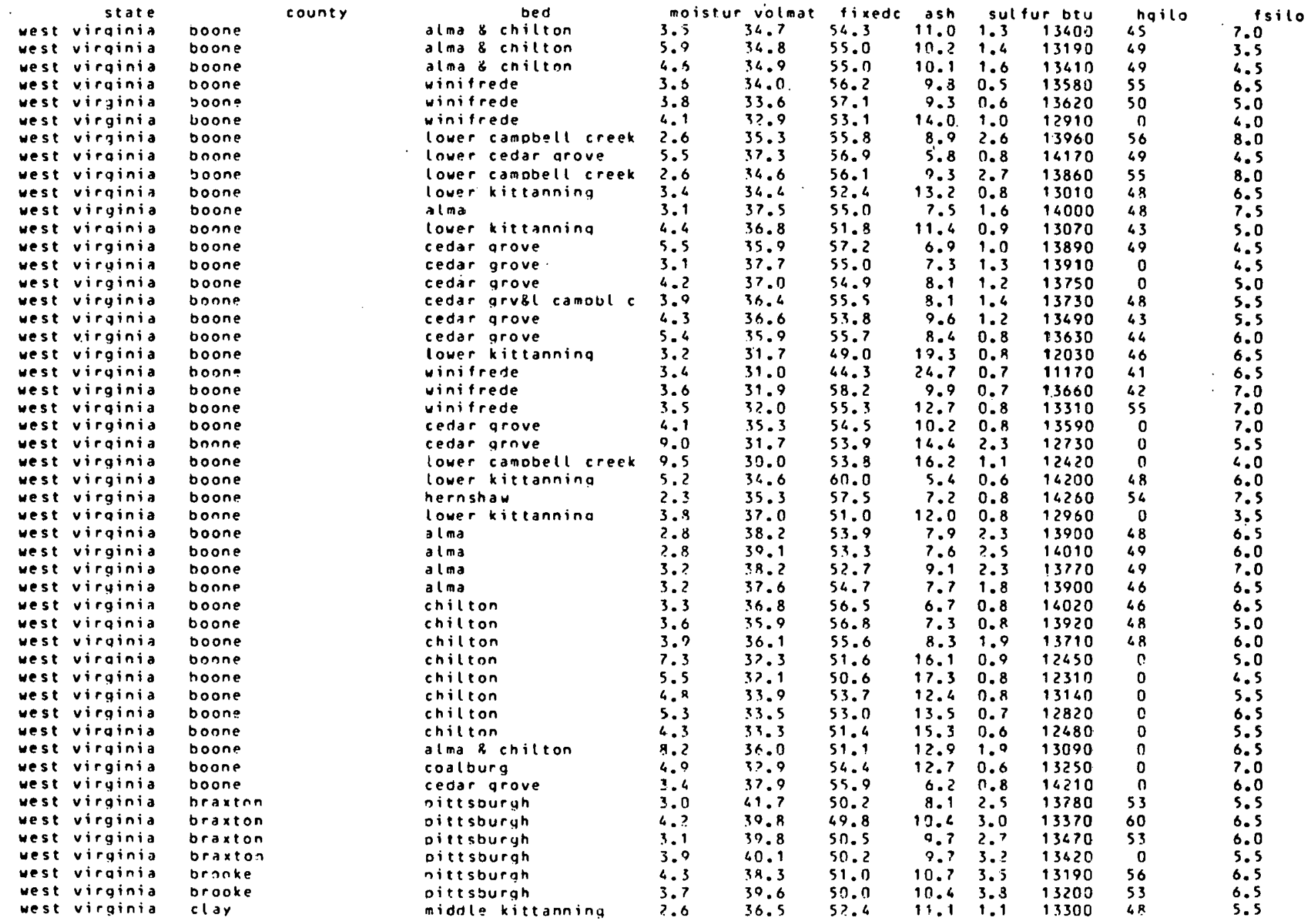




\begin{tabular}{|c|c|c|c|c|c|c|c|c|c|c|c|c|c|}
\hline & state & & county & & bed & moi & volmat & fixedc & ash & sul & ar otu & hailo & is silo \\
\hline west & virginit & clay & & middle & kittanning & 3.1 & 38.8 & 52.1 & 9.1 & 0.8 & 13730 & 43 & 6.0 \\
\hline west & virginia & clay & & clarion & & 1.7 & 37.9 & 51.8 & 10.3 & 0.6 & 13640 & 38 & 3.5 \\
\hline west & virginio & $c$ lay & & uDDer & kittanning & 3.6 & 32.1 & 52.6 & 9.3 & 2.0 & 13630 & 52 & 6.5 \\
\hline vest & virainia & clay & & lower & kittanning & 5.2 & 37.6 & 53.7 & 8.7 & 0.7 & 13800 & 0 & 0.5 \\
\hline west & viryinia & clay & & lower & kittannina & 3.6 & 37.0 & 55.9 & 7.1 & 0.7 & 14060 & 45 & 6.5 \\
\hline west & virainia & clay & & lower & kittannina & 4.8 & 35.2 & 55.7 & 9.1 & 0.7 & 13590 & 0 & 3.0 \\
\hline west & virginia & fayette & & fire or & reek & 3.4 & 19.8 & 74.9 & 5.3 & 0.8 & 14860 & $\approx$ & 8.0 \\
\hline west & virainia & fayette & & Docahor & ntas. ne 6 & 4.6 & 18.8 & $? \geq .7$ & 8.5 & 0.7 & 14270 & 98 & 7.5 \\
\hline west & virainia & fayette & & vocanon & ntas no 6 & 4.4 & 10.9 & 73.8 & 6.3 & 0.7 & 14510 & 0 & 7.0 \\
\hline west & virginia & fayette & 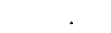 & Docahor & ntas no 6 & 2.1 & 10.2 & 75.1 & 5.7 & 0.9 & 14680 & 0 & 8.5 \\
\hline west & virginia & fayette & & lower & camobell creek & 2.3 & 34.3 & 60.0 & 5.7 & 0.8 & 16350 & 0 & 8.0 \\
\hline west & virainit & fayette & & Docanon & ntas o8fire ck & 2.9 & 19.3 & 75.1 & 5.6 & 0.8 & 14810 & $\ldots$ & $8: 5$ \\
\hline vest & virainia & fayetie & & Docanon & ntas osfireck & 7.2 & 20.6 & 72.4 & 7.0 & 0.7 & 14180 & 0 & 3.0 \\
\hline west & virginia & fayette & & Docahor & ntas no 6 & 3.1 & 13.3 & 72.4 & 9.3 & 0.8 & 14100 & 0 & 7.5 \\
\hline west & virginia & fayette & & sewell & & 2.9 & 27.3 & 67.1 & 5.6 & 0.6 & 14640 & 82 & 8.5 \\
\hline west & virginia & fayette & & seweli & & $? .5$ & 26.3 & 65.3 & 1.9 & 0.8 & 14210 & 0 & 8.0 \\
\hline vest & virginia & fayotie & & eaole & & 5.4 & 31.5 & 57.6 & 10.9 & 0.9 & 13600 & 0 & 8.0 \\
\hline vest & virginia & fayette & & eagle & & $? .1$ & 33.5 & 58.7 & 7.8 & 0.6 & 14140 & so & 5.5 \\
\hline west & virginia & fayette & & eagle & & 2.7 & 33.3 & 60.9 & 5.8 & 0.8 & 94480 & 59 & 8.5 \\
\hline west & virainia & farette & & $i \operatorname{comot}$ & bell ckbonulth & 5.1 & 27.3 & 66.0 & 6.7 & 0.7 & 16640 & 0 & 8.0 \\
\hline west & virainia & fayette & & I campl & bell ck80oulten & 4.7 & 30.0 & 62.9 & 7.1 & 0.6 & 14410 & 0 & 4.5 \\
\hline vest & virginia & fayette & & icamob & beli ckBoowlen & 5.6 & 29.2 & 64.2 & 6.6 & 0.8 & 16600 & 80 & 7.0 \\
\hline west & virginia & fayette & & eagle & & 2.2 & 31.5 & 62.5 & 6.0 & 0.9 & 14700 & 71 & 7.0 \\
\hline west & virainia & fayette & & eaqle & & 3.3 & $3 n .6$ & 61.5 & 7.9 & 0.9 & 94260 & 0 & 7.0 \\
\hline vest & virainia & fayetie & & eagle & & 4.9 & 30.5 & 61,2 & 8.3 & 0.8 & 14090 & 0 & 3.5 \\
\hline vest & virginia & fayette & & litele & eagle & 2.0 & 30.6 & 64.9 & 4.5 & 0.8 & 94780 & 0 & 8.5 \\
\hline west & virginia & fayette & & sewell & & 3.0 & 22.3 & 74.2 & 3.5 & 0.7 & 15060 & 95 & 9.0 \\
\hline west & virginia & fayet te & & Docahor & ntas nn 6 & 2.2 & 20.4 & 66.1 & 13.5 & 1.0 & 13300 & 87 & 8.0 \\
\hline west & virainia & fayette & & Docanor & neas no 6 & 2.1 & $? 1.5$ & 68.4 & 10.1 & 0.9 & 13960 & 0 & 8.5 \\
\hline west & virginia & fayette & & lower & camobell creek & 3.7 & 33.4 & 60.3 & 6.3 & 0.8 & 14380 & 55 & 7.5 \\
\hline west. & virginia & fayette & & lower & campbell creek & 3.0 & 34.1 & 59.0 & 6.9 & 0.9 & 16280 & 53 & 7.5 \\
\hline west & virginia & fayette & & eaqle & & $? .1$ & 20.1 & 66.3 & 4.6 & 0.8 & 14890 & 0 & 8.0 \\
\hline yest & virginia & fayetie & & fire cr & reek & 3.3 & 18.5 & 74.9 & 6.6 & 1.4 & 14550 & $\therefore$ & 8.5 \\
\hline west & virginia & fayetite & & fire es & reek & 3.8 & 19.9 & 73.3 & 7.8 & 0.9 & 14420 & 0 & 8.5 \\
\hline vest & virginia & fayette & & sewell & & 2.9 & 24.1 & 68.0 & 7.9 & 0.7 & 14380 & 0 & 8.0 \\
\hline vest & virginia & fayette & & coalbur & $r a$ & 3.0 & 35.4 & 57.2 & 7.4 & 0.9 & 14060 & 0 & 7.0 \\
\hline west & virainia & fayette & & sewell & & 2.2 & 25.5 & 69.5 & 4.0 & 0.7 & 16850 & 0 & \\
\hline west & virqinia & fayette & & seweil & & 8.2 & $? 3.3$ & 72.0 & 4.7 & 0.7 & 14790 & $\ldots$ & 8.5 \\
\hline west & virginia & tayette & & sewell & & 2.6 & 24.7 & 71.7 & 3.6 & 0.5 & 14860 & $n$ & 8.0 \\
\hline west & virainia & fayette & & seveli & & $4 . ?$ & 73.9 & 71.7 & 4.4 & 0.8 & 16850 & 94 & 8.0 \\
\hline vest & virainia & fayette & & sewell & & 4.8 & 24.6 & 70.0 & 5.4 & 0.8 & 14790 & 0 & 8.5 \\
\hline west & virainia & farette & & sewell & 8 fire creek & 3.5 & 22.1 & 71.9 & 6.0 & 0.6 & 14720 & 0 & 9.0 \\
\hline vest & virginia & fayetie & & fire or & reek & 1.9 & 25.0 & $68 . n$ & 7.0 & 0.8 & 14240 & 88 & 7.5 \\
\hline west & virginia & fayette & & lower & camooell creek & 5.5 & 33.6 & $6 ? .6$ & 3.8 & 0.6 & 14370 & 65 & 7.0 \\
\hline vest & virainia & fayetto & & sewell & & 3.4 & $? 6.2$ & 67.8 & 6.0 & 0.6 & 14540 & 0 & 7.5 \\
\hline west & virainia & fayette & & seweli & & 3.6 & 36.5 & 67.8 & 5.7 & 0.5 & 14590 & 0 & 7.5 \\
\hline vest & virginia & faypte & & lower & kittannina & 4.7 & 35.6 & 58.5 & 5.9 & $n .7$ & 14120 & 45 & 5.5 \\
\hline west & virginia & fayette & & lower & kittannina & 3.1 & 35.4 & 50.3 & 5.3 & $n .7$ & 14250 & 46 & 6.0 \\
\hline west & virainia & fayetto & & sewell & & 4.1 & 24.4 & 79.5 & 4.1 & 0.7 & 15000 & 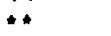 & 9.0 \\
\hline west & virginia & faypte & & sewell & & 4.9 & 23.5 & 70.3 & 6.7 & 0.8 & 14550 & 0 & 9.0 \\
\hline
\end{tabular}




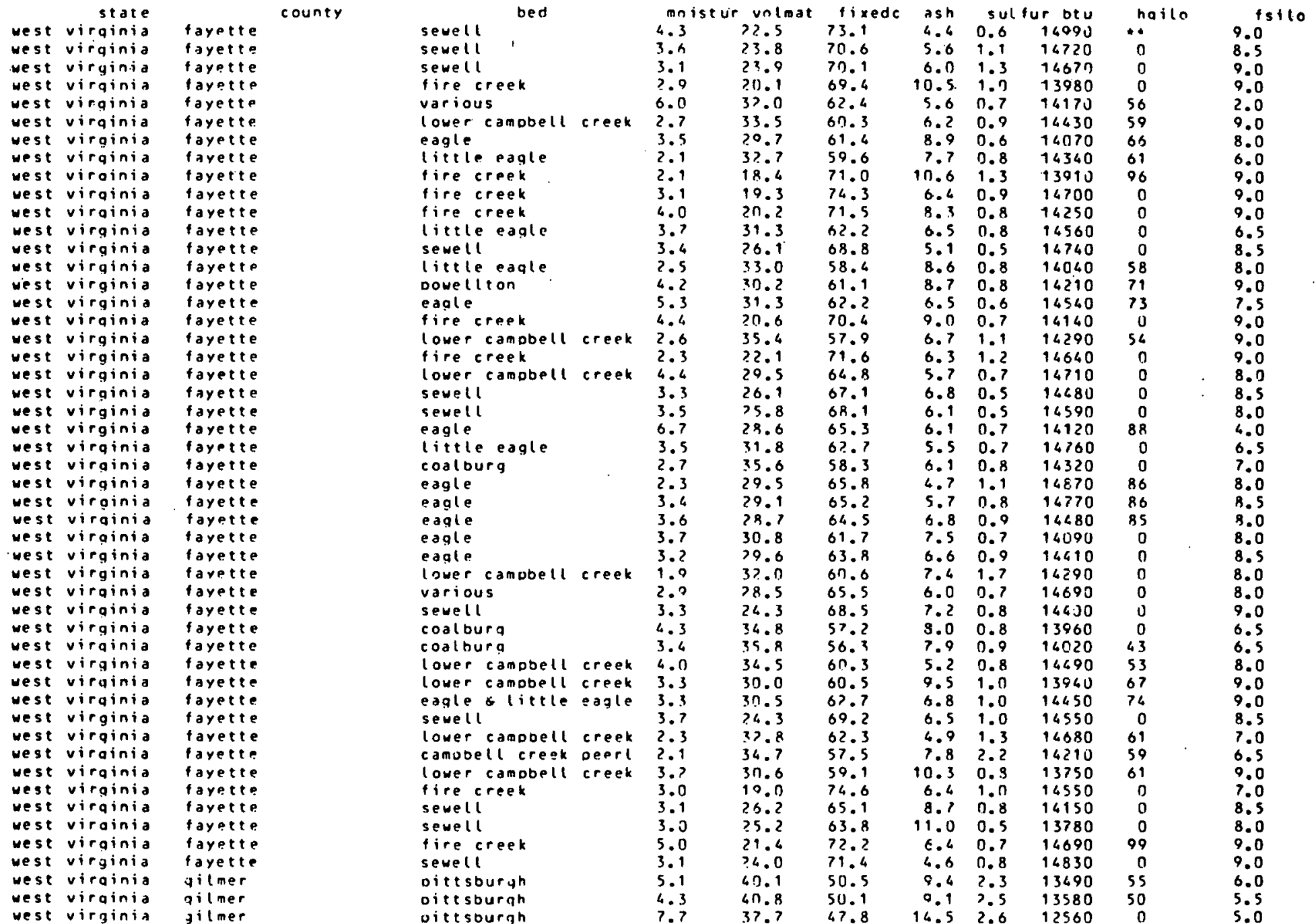




\begin{tabular}{|c|c|c|c|c|c|c|c|c|c|c|c|}
\hline west & $\begin{array}{r}\text { state } \\
\text { virainia }\end{array}$ & county & $\begin{array}{l}\text { bed } \\
\text { gittsourah }\end{array}$ & $\begin{array}{l}\pi 0 i \\
4.2\end{array}$ & $\begin{array}{l}\text { volmat } \\
4 \pi .6\end{array}$ & $\begin{array}{l}\text { fixedc } \\
49.7\end{array}$ & $\begin{array}{l}\text { ash } \\
9.7\end{array}$ & $\begin{array}{l}5.7 \\
2.7\end{array}$ & $\begin{array}{l}\text { ur b.tu } \\
13470\end{array}$ & hailo & $5.5^{15110}$ \\
\hline west & virainia & gilmer & oittsburgh & 3.8 & 39.6 & 49.2 & 19.2 & 2.8 & 13.140 & 53 & $\begin{array}{l}5.5 \\
7.0\end{array}$ \\
\hline west & virainia & yilner & pittsburgh & 3.6 & 39.0 & 51.3 & 9.7 & 2.6 & 13440 & 55 & 7.0 \\
\hline west & virginia & gilmer & ditesburan & 5.3 & 40.3 & 50.8 & 8.9 & 2.8 & 13570 & 56 & 6.5 \\
\hline west & virainia & qilmer & nittsburuh & 3.1 & 39.8 & 52.2 & 8.0 & 9.7 & 13780 & 54 & 6.5 \\
\hline west & virginia & grant & bakerstown & 2.5 & 15.4 & 56.8 & 27.8 & 1.9 & 19050 & 83 & 5.5 \\
\hline west & virginia & grant & elk lick & 2.9 & 17.3 & 61.0 & 21.7 & 3.9 & 12060 & 86 & 8.5 \\
\hline west & virginia & grant & bakerstown & 3.0 & 96.3 & 72.6 & 19.1 & 2.9 & 13870 & $\star *$ & 7.5 \\
\hline west & virginia & arant & bakerstown & 8.8 & 22.9 & 61.6 & 15.5 & 1.3 & 12730 & 0 & 3.5 \\
\hline west & virainia & urant & bakerstown & 6.1 & 26.2 & 64.8 & 9.0 & 2.3 & 13840 & 0 & 7.0 \\
\hline west & virginid & grant & 1 douglas 8 l iaeqer & 9.2 & 76.3 & 64.3 & 11.4 & 1.8 & 13450 & 0 & 5.5 \\
\hline west & virginia & grant & bakerstownsu freeprt & 3.1 & $2 ? . ?$ & 67.6 & 10.2 & 0.9 & 13930 & 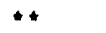 & 9.0 \\
\hline west & virginia & grant & bakerstoun & 3.3 & 16.4 & 76.8 & 6.8 & 0.6 & 14650 & 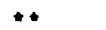 & 9.0 \\
\hline west & virainia & yreentorier & sevell & 3.5 & 27.3 & 68.0 & 4.7 & 1.2 & 16770 & $8 ?$ & 9.0 \\
\hline west & virginia & greenbrier & fire creek & 3.6 & $? 9.8$ & 63.6 & 14.6 & 1.1 & 13130 & 84 & 8.5 \\
\hline west & virginia & greenbrier & sewell & 7.5 & $? 7.7$ & 68.7 & 3.6 & 0.8 & 96950 & 0 & 8.5 \\
\hline wese & virginia & greenbrier & sewali & 3.4 & 26.2 & 63.1 & 10.7 & 0.9 & 13720 & 70 & 8.0 \\
\hline west & virainia & greenorier & sevell & 3.5 & 77.2 & 68.7 & 4.1 & 1.0 & 14930 & 0 & 9.0 \\
\hline west & viruinia & qreenbrier & sewell & 3.8 & 27.0 & 63.4 & 4.6 & 1.0 & 16760 & 82 & 8.5 \\
\hline vest & virginia & yreenorier & sewell & 2.9 & 76.8 & 65.7 & 6.5 & 1.? & 14540 & 80 & 9.0 \\
\hline west & virginia & greenbrier & sevell & 6.8 & 26.9 & 68.3 & 4.8 & 1.1 & 14500 & 86 & 7.0 \\
\hline west & virainia & areenbrier & sewell & $5 . ?$ & 27.6 & 66.9 & 5.5 & 0.8 & 16690 & 80 & 7.5 \\
\hline yest & virginia & greenorier & seckley & 7.2 & 2.4 .9 & 64.5 & 10.6 & 1.3 & 13810 & 81 & 8.0 \\
\hline west & virginia & greenbrier & sevell & $3 . ?$ & 27.7 & 67.9 & 4.4 & 0.8 & 14820 & 85 & 9.0 \\
\hline west & virginia & greenbrier & seweli & 3.6 & 27.0 & 63.8 & 3.2 & 1.1 & 13930 & 0 & 8.0 \\
\hline west & virainia & greenorier & seveli & 3.8 & 27.4 & 67.0 & 5.6 & 1.1 & 14450 & 82 & 8.5 \\
\hline west & virginia & greenbrier & sewell & 3.7 & 23.7 & 36.4 & 6.9 & 1.1 & 16400 & 80 & 9.0 \\
\hline west & virginia & greenbrier & sewell & 4.2 & 29.8 & 36.7 & 6.5 & .9 .2 & 14490 & 0 & 3.5 \\
\hline west & virginia & greenorier & firecreek & 2.4 & 74.0 & 72.5 & 3.5 & 0.8 & 15110 & 0 & 9.0 \\
\hline west & virginia & qreenbrier & fire creek & 2.6 & 20.1 & 60.3 & 10.6 & 1.1 & 13910 & 93 & 8.5 \\
\hline west & virainia & greenorier & sevell & 2.6 & $? 7.5$ & 35.6 & 6.9 & 1.0 & 14390 & 0 & 8.5 \\
\hline west & virginia & greenbrier & sewell & 2.9 & 27.3 & 58.1 & 4.6 & 1.4 & 14720 & 0 & 8.5 \\
\hline wese & virginia & greenbrier & seweli & 3.6 & 26.1 & 38.2 & 5.7 & 1.3 & 14530 & 86 & 9.0 \\
\hline west & virainia & areenorier & sevell & 2.8 & 27.0 & $\$ 9.8$ & 3.2 & 1.1 & 15000 & 0 & 8.5 \\
\hline west & virginia & greenbrier & fire creek & 2.8 & 18.7 & 70.9 & 10.4 & 1.? & 13960 & 97 & 8.0 \\
\hline west & virginia & greenbrier & semell & 2.7 & 23.1 & 72.8 & 4.1 & 0.8 & 15010 & 97 & 9.0 \\
\hline west & virginia & greenbrier & sevell \& fire creek & 4.0 & 23.1 & 89.9 & 7.0 & 1.2 & 14420 & 89 & 9.0 \\
\hline west & virainia & greenbrier & beckley & 4.9 & 25.1 & $66 . ?$ & 8.7 & 1.2 & 14090 & 84 & 7.5 \\
\hline west & virginia & greentrier & fire creek & 2.1 & 20.5 & 72.0 & 7.5 & 1.4 & 14480 & 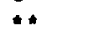 & 9.0 \\
\hline west & virginia & greenbrier & various & 4.5 & 24.9 & 67.5 & 7.6 & 1.4 & 14310 & 0 & 0.5 \\
\hline west & virginia & greenbrior & various & 4.6 & 24.8 & 67.9 & 7.3 & 9.3 & 14380 & 0 & 8.0 \\
\hline west & virainia & greenbrier & various & 4.8 & 25.3 & 68.2 & 6.5 & 1.5 & 16420 & 0 & 8.5 \\
\hline west & virginia & greenbrier & various & 4.5 & 24.7 & 68.2 & 7.8 & 1.0 & 14280 & 0 & \\
\hline west & virginia & greenbrier & various & 4.0 & 24.8 & 68.3 & 6.9 & $1 . ?$ & 14390 & 0 & 8.5 \\
\hline west & virginia & jeenbri=r & various & 4.6 & 26.7 & 66.9 & 8.6 & 1.3 & 13980 & 0 & 3.0 \\
\hline west & virainia & areenbrier & varinus & 3.4 & 25.1 & 68.0 & 6.7 & 1.4 & 14310 & 0 & 0.0 \\
\hline west & virainia & areenhrier & various & 4.3 & 25.4 & 68.1 & 6.5 & 1.6 & 14430 & 0 & 0.0 \\
\hline vese & virginia & greenbrier & various & $? .3$ & 26.9 & 68.? & 5.7 & 9.5 & 14460 & 0 & 8.0 \\
\hline west & virginia & greenbrier & beckley & 3.3 & 35.3 & 60.1 & 5.6 & 1.5 & 14640 & 95 & 8.5 \\
\hline west & virainia & oreenbrier & nocanontas no 6 & $3 . ?$ & 22.5 & 69.9 & 7.5 & 0.8 & 94460 & 97 & 8.5 \\
\hline
\end{tabular}




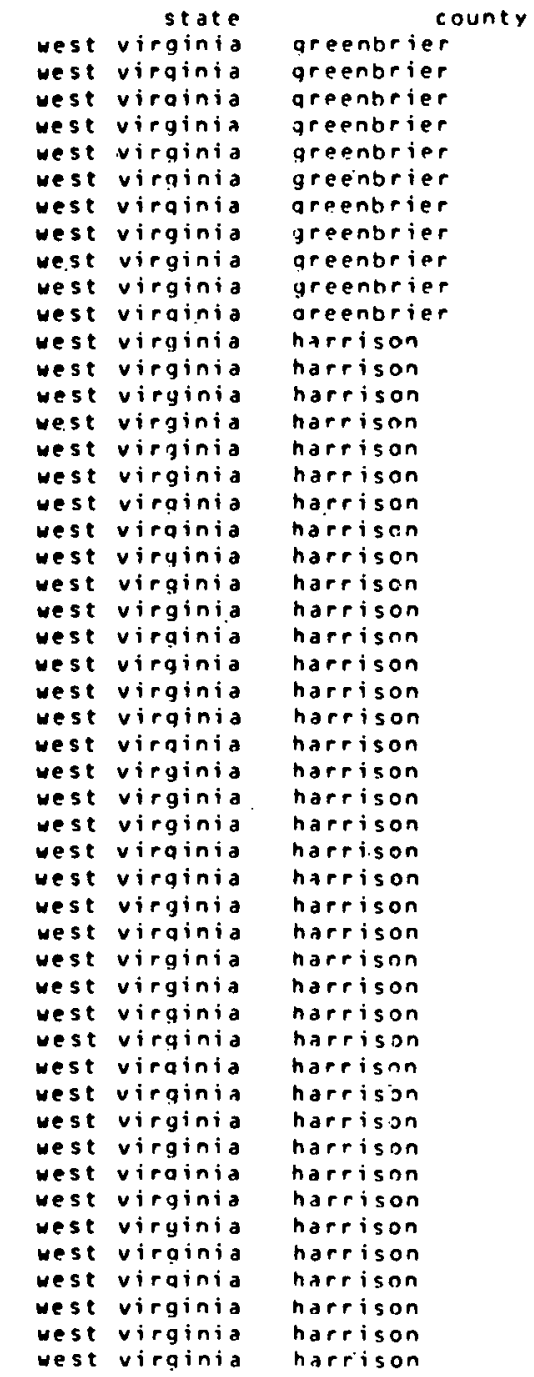

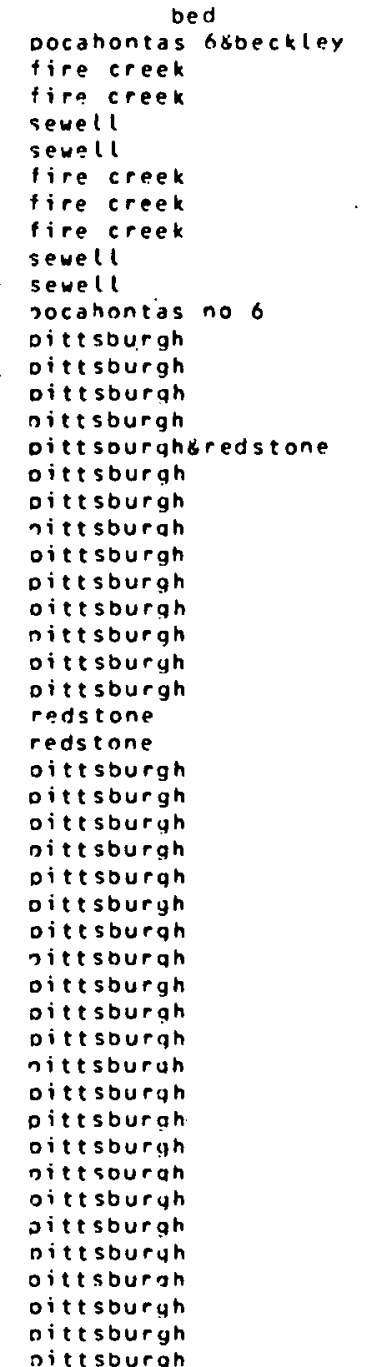

\begin{tabular}{|c|c|c|c|c|c|}
\hline mo & volmat & $f i x \in d$ & as $h$ & sul & \\
\hline 3.4 & 24.6 & 67.6 & 8.0 & 1.3 & 14280 \\
\hline ?.1 & $2 ? .1$ & 72.3 & 5.6 & 0.8 & 14830 \\
\hline 2.8 & 22.3 & 72.4 & 5.3 & 0.8 & 14850 \\
\hline 4.5 & 26.5 & 68.9 & 4.6 & 1.0 & 14750 \\
\hline 4.6 & $2 x .5$ & 68.1 & 5.4 & 0.8 & 14710 \\
\hline 2.1 & 22.0 & 71.3 & 6.7 & 0.8 & 16620 \\
\hline 3.6 & 21.5 & 67.5 & 11.0 & 1.1 & 13890 \\
\hline 3.4 & 23.0 & 69.3 & 7.7 & 0.9 & 14690 \\
\hline 3.7 & 79.2 & 67.3 & 3.5 & 0.7 & 16930 \\
\hline 3.2 & 29.1 & 68.7 & 3.2 & 0.6 & 15090 \\
\hline 2.6 & $? 4.0$ & 67.4 & 8.6 & 1.1 & 14330 \\
\hline 4.2 & 39.7 & 52.0 & 9.3 & 2.8 & 13700 \\
\hline 3.4 & 39.6 & 51.7 & 9.7 & 3.1 & 13530 \\
\hline 4.9 & 36.5 & 53.0 & 10.5 & 2.4 & 13330 \\
\hline 4.2 & 40.3 & 52.1 & 7.6 & 3.9 & 13830 \\
\hline 3.9 & 38.5 & 54.5 & 7.0 & 1.8 & 13820 \\
\hline 2.7 & 39.4 & 50.9 & 9.7 & 3.4 & 13590 \\
\hline 4.1 & 36.8 & 56.8 & 6.4 & 1.4 & 16090 \\
\hline 2.9 & 38.9 & 52.3 & 8.5 & 3.0 & 13910 \\
\hline 2.7 & 40.4 & 50.2 & 9.4 & 4.5 & 13770 \\
\hline 3.? & 40.3 & 50.4 & 9.3 & 3.3 & 13640 \\
\hline 3.5 & 40.2 & 50.3 & 9.5 & 3.6 & 13560 \\
\hline 2.7 & 37.0 & 49.3 & 13.7 & 4.1 & 12870 \\
\hline 2.5 & 42.3 & 50.9 & 6.8 & 2.9 & 14170 \\
\hline$? .1$ & 41.4 & 59.5 & 8.1 & 3.7 & 13870 \\
\hline 3.1 & 37.3 & 51.7 & 11.0 & 2.4 & 13250 \\
\hline 3.4 & 39.8 & $5 ? .0$ & 8.2 & 3.5 & 13710 \\
\hline 3.4 & 38.8 & 50.8 & 10.4 & 2.8 & 13480 \\
\hline 6.7 & 40.3 & 51.1 & 8.6 & 3.8 & 13710 \\
\hline 4.5 & 39.8 & 49.9 & 10.5 & 4.3 & 13440 \\
\hline 2.9 & 41.6 & 49.4 & 9.0 & 4.3 & 13690 \\
\hline 2.7 & 43.8 & 47.1 & 9.1 & 4.8 & 13450 \\
\hline 3.5 & 39.1 & 53.2 & 7.7 & 3.9 & 13890 \\
\hline 5.7 & 38.4 & 53.1 & 8.5 & 2.4 & 13810 \\
\hline 4.0 & 41.5 & 48.8 & 9.7 & 4.9 & 13560 \\
\hline 5.0 & 42.0 & 49.4 & 8.6 & 4.0 & 13660 \\
\hline $7 . ?$ & 41.2 & $5 n .4$ & 8.6 & 3.9 & 13750 \\
\hline 6.4 & $4 ? .1$ & 48.8 & 9.1 & 4.2 & 13650 \\
\hline $5 . ?$ & 40.1 & 50.6 & 9.3 & 3.1 & 13570 \\
\hline 3.5 & 40.5 & 51.6 & 7.9 & 3.6 & 13890 \\
\hline 3.7 & 49.5 & sn. 1 & 8.4 & 3.6 & 13760 \\
\hline 3.9 & 41.6 & 51.0 & 7.4 & 3.4 & 13880 \\
\hline 4.7 & 30.6 & $5 ? .2$ & 8.2 & 3.4 & 13810 \\
\hline 3.4 & 41.4 & 50.0 & 8.6 & 4.1 & 13660 \\
\hline 2.9 & 49.5 & $4^{0} .6$ & 8.9 & 3.9 & 13680 \\
\hline 3.4 & 41.4 & 49.7 & 8.7 & 4.3 & 13720 \\
\hline 2.8 & 47.9 & 48.1 & 9.0 & 4.11 & 13720 \\
\hline 3.3 & 41.0 & 48.7 & 10.1 & 4.0 & 13450 \\
\hline 3.8 & 40.7 & 49. & 9.7 & 3.6 & 13530 \\
\hline 3.3 & 38.7 & 51.5 & 9.8 & 4.0 & 13550 \\
\hline
\end{tabular}

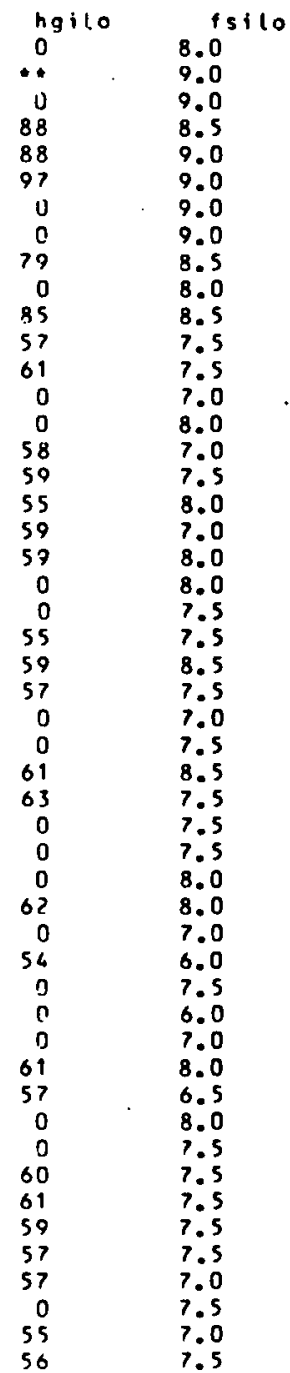




\begin{tabular}{|c|c|c|c|c|c|c|c|c|c|c|c|c|}
\hline & state & & county & bed & moi & r volmat & fixedc & ash & sul & ur btu & ngito & isil.o \\
\hline west & virginia & harrison & & oittsburgh & 2.2 & $4 ? .0$ & $5 n .2$ & 7.8 & 3.2 & 13970 & 56 & 8.0 \\
\hline west & virginia & harrison & & vittsburgh & 1.0 & 40.6 & 51.0 & 8.4 & 3.1 & 13790 & $n$ & 7.0 \\
\hline west & virginia & harrison & & Dittsourụh & 2.1 & 39.1 & 51.3 & 9.6 & 3.3 & 13600 & 0 & 8.0 \\
\hline west & virania & harrison & & oittsourigh & 2.11 & 40.4 & 50.8 & 8.8 & 3.5 & 13730 & 0 & 7.0 \\
\hline west & virainia & harrison & & oittsburgh & 1.5 & 40.7 & 51.5 & 7.8 & 3.1 & 13860 & 0 & 7.5 \\
\hline west & virginia & harrison & & Dittsburgh & 1.7 & $4 n .2$ & 50.2 & 9.6 & 3.9 & 13590 & 0 & 7.0 \\
\hline west & virainia & harrison & & Dittsburan & 1.8 & 40.6 & $50 . \pi$ & 9.4 & 3.7 & 13670 & 0 & 7.0 \\
\hline west & virginia & harrison & & jittsburgh & 1.5 & 30.9 & 49.9 & 10.2 & 6.5 & 13540 & 0 & 7.0 \\
\hline west & virginia & harrison & & oittsburgh & 2.8 & 39.4 & 51.7 & 8.9 & 3.1 & 13730 & 0 & 8.0 \\
\hline west & virginia & harrison & & Dittsburgh & 2.9 & 39.0 & 52.7 & 8.3 & 2.8 & 13780 & 0 & 7.0 \\
\hline west & virainia & harrison & & Ditesourgh & 2.4 & 39.7 & 52.1 & 8.? & 2.7 & 13720 & 0 & 7.5 \\
\hline west & virginia & harrison & & dittsourgh & 3.0 & 40.2 & 51.5 & 8.3 & 3.0 & 13810 & 0 & 7.5 \\
\hline west & virginia & harrison & & Dittsburgh & 2.1 & 60.0 & 51.4 & 8.6 & 3.3 & 13740 & 0 & 8.0 \\
\hline west & virginia & harrison & & Dittsburgh & 2.3 & 30.4 & 59.6 & 9.0 & 3.4 & 13700 & 0 & 7.0 \\
\hline west & virginia & harrison & & oittsburah & $*+$ & 37.8 & 52.2 & 10.0 & 2.9 & 13160 & 69 & 7.0 \\
\hline west & virginia & harrison & & Dittsburgh & 2.6 & 38.4 & 51.9 & 9.7 & 3.3 & 13630 & 0 & 7.5 \\
\hline vest & virginia & harrison & & oitesburgh & 1.7 & 38.0 & 52.6 & 9.4 & 3.4 & 13710 & 0 & 8.5 \\
\hline west & virginia & harrison & & oittsburgh & 9.9 & 37.5 & 53.3 & 9.2 & 2.8 & 13740 & 0 & 7.0 \\
\hline west & viruinia & harrison & & oittsburah & 1.3 & 39.8 & 50.9 & 10.3 & 4.2 & 13570 & 0 & 8.0 \\
\hline west & viruginia & harrison & & oittsburgh & 1.3 & 30.2 & $5 ? .4$ & 8.4 & 2.7 & 13990 & 0 & 7.0 \\
\hline west & virginia & harrison & & oittsburgh & 1.7 & 37.9 & $5 ? .8$ & 9.3 & 2.9 & 13740 & 0 & 7.0 \\
\hline west & virginia & harrison & & oittsburgh & 1.5 & 38.8 & 53.0 & 8.2 & 2.8 & 13980 & 0 & 7.0 \\
\hline west & virqinia & harrison & & Dittsburạh & 2.1 & 39.6 & 53.6 & 7.8 & 2.8 & 13940 & 0 & 8.0 \\
\hline vest & virginia & narrisan & & oittsburgh & 1.9 & 38.6 & 53.0 & 8.4 & 2.8 & 13880 & $\mathbf{0}$ & 8.5 \\
\hline west & virginia & harrison & & oittsburgh & 2.0 & 37.4 & 53.4 & 9.2 & 2.9 & 13750 & 0 & 7.5 \\
\hline west & virginia & harrison & & nittsburgh & 2.4 & 39.1 & 52.3 & 8.6 & $3 . ?$ & 13890 & 0 & 7.5 \\
\hline west & virainia & harrison & & oittsourgh & 2.3 & 38.7 & 53.2 & 8.1 & 2.6 & 13910 & 0 & 7.5 \\
\hline west & virginia & harrison & & pit tsburgh & 2.1 & 38.3 & 53.0 & 8.7 & 3.2 & 13800 & 0 & 7.5 \\
\hline west & virginia & harrison & & oittsburgh & 9.8 & 38.6 & 51.9 & 9.5 & 3.6 & 13720 & 0 & 7.5 \\
\hline west & virainia & harrison & & Dittsburgh & 4.5 & 36.9 & 54.8 & 8.3 & 2.2 & 13820 & 0 & 7.0 \\
\hline west & virainid & harrison & & nittsburan & 2.0 & 39.3 & 51.7 & 10.0 & 3.3 & 13620 & 0 & 8.0 \\
\hline west & virginia & harrison & & oitesburah & 8.? & 35.8 & $57 . ?$ & 7.0 & 2.1 & 13920 & 68 & 7.5 \\
\hline vest & virginia & harrison & & oittsburgh & 3.2 & 37.7 & 51.7 & 10.6 & 3.5 & 13520 & 59 & 7.5 \\
\hline west & virginia & harrison & & oittsburyh & 6.0 & 38.4 & 50.8 & 10.8 & 4.5 & 13510 & 0 & 8.0 \\
\hline vest & viruinia & harrison & & sittsourah & 2.5 & 39.2 & $5 ? .5$ & 8.3 & 3.2 & 13940 & 57 & 7.0 \\
\hline vest & virqinia & harrison & & oittsburgh & 2.4 & 39.8 & 57.5 & 7.7 & 3.0 & 16000 & 60 & 8.0 \\
\hline vest & virainia & harrison & & oitesburgh & 3.0 & 30.3 & 52.7 & 8.0 & 3.3 & 13910 & 59 & 8.0 \\
\hline west & virginia & harrison & & Dittsburgh & 3.6 & 39.5 & 49.6 & 10.9 & 4.2 & 13220 & 62 & 7.0 \\
\hline west & virainia & harrison & & bittsburgh & 4.0 & 40.1 & 50.0 & 9.9 & 3.4 & 13380 & 59 & 7.5 \\
\hline west & virginia & harrison & & oittsourgh & 4.3 & 40.6 & 40.8 & 0.6 & 3.4 & 13430 & 60 & 7.0 \\
\hline west & virainia & hareison & & Dittsburgh & 4.6 & 40.9 & 49.9 & 9.2 & 3.5 & 13570 & 55 & 7.0 \\
\hline west & virainia & harrison & & Dittsburgh & 5.4 & 41.1 & $5 n .1$ & 8.8 & 4.0 & 13680 & 57 & 6.5 \\
\hline west & virainia & harrisnn & & aitesburah & 4.2 & 41.2 & 48.7 & 10.1 & 3.6 & 13450 & 59 & 6.5 \\
\hline west & virginia & harrison & & redstone & 2.0 & 35.6 & $5 ? .4$ & $1 ? .0$ & 3.7 & 13170 & 0 & 7.5 \\
\hline west & virainia & harrison & & oittsburgh & 5.7 & $39 . ?$ & 53.2 & 8.6 & 3.2 & 13820 & 66 & 7.5 \\
\hline west & virginia & harrison & & oittsourgh & 2.6 & 30.1 & 52.5 & 8.4 & 3.1 & 13760 & 60 & 7.0 \\
\hline we st & virginia & harrison & & sittsburgh & 7.6 & 39.2 & 50.4 & 10.4 & 3.8 & 13500 & 61 & 7.5 \\
\hline west & virainia & narrisnn & & sittsourah & 0.2 & 39.6 & $5 ? .3$ & 8.1 & 3.7 & 13830 & 61 & 8.0 \\
\hline west & virginia & harrison & & Jittsburghbredstone & 3.0 & 37.8 & 51.8 & 8.4 & 3.6 & 13770 & 59 & 6.5 \\
\hline west & virainia & harrison & & redstone & 2.4 & 41.4 & $5 n .3$ & 8.3 & 2.6 & 13800 & $\pi$ & 8.0 \\
\hline
\end{tabular}




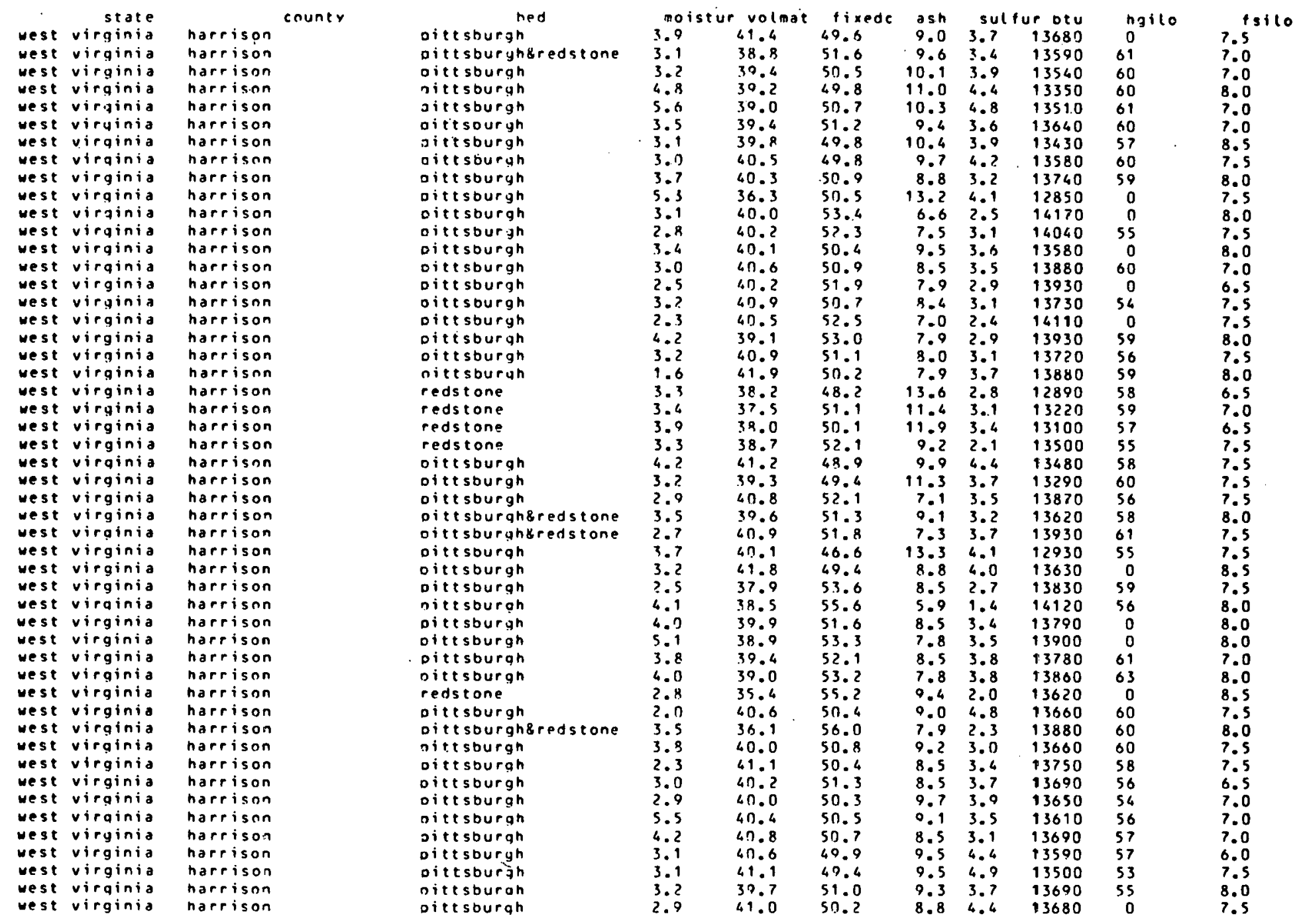




\begin{tabular}{|c|c|c|c|c|c|c|c|c|c|c|c|c|}
\hline & state & & county & bed & mo & r volmat & fixe & Ash & sul & ur beu & hgiln & 13110 \\
\hline west & virginia & harrisnn & & at tesuryn & 2.5 & 40.9 & 49.9 & 9.2 & 3.9 & 13680 & 58 & 7.0 \\
\hline vest & virginia & harrison & & oittsburgh & 3.2 & 39.1 & 49.5 & 11.4 & 4.0 & 13340 & 54 & 7.0 \\
\hline west & virginia & harrison & & Ditesburgh & 4.5 & 40.8 & 69.7 & 9.5 & 3.8 & 13480 & 61 & 8.0 \\
\hline west & virainia & harrison & & oitt sburgh & 2.5 & 39.0 & 53.3 & 7.7 & 2.4 & 13970 & 0 & 7.5 \\
\hline west & virainia & harrison & & oittsburgh & 3.8 & 38.4 & 53.3 & 3.3 & 1.6 & 13820 & 53 & 8.5 \\
\hline west & virginia & narrison & & oittsburgh & 2.7 & 30.3 & 51.7 & 0.0 & 2.9 & 93670 & 54 & 7.5 \\
\hline west & virainia & harrison & & Dittsburgh & 2.7 & 40.3 & 51.7 & 8.0 & 3.5 & 13950 & 0 & 7.0 \\
\hline west & virginia & harrison & & Dittsburgh & 4.0 & 37.9 & 53.7 & 8.4 & 2.5 & 13850 & 0 & 7.5 \\
\hline west & virginia & narrisnn & & Dittsouran & 4.0 & .7 .9 & 53.7 & 8.4 & 2.5 & 13850 & 0 & 7,5 \\
\hline west & virginia & harrison & & dittsburgh & 2.8 & 41.4 & 59.0 & 7.6 & 2.6 & 13870 & 53 & 7.5 \\
\hline west & virqinia & hareison & & Dittsourgh & 3.1 & 40.3 & 51.1 & 8.6 & 4.2 & 13810 & 56 & 7.0 \\
\hline west & virginia & harrison & & dittsburah & 3.0 & 39.8 & 50.7 & 9.5 & 4.4 & 13630 & 58 & 7.5 \\
\hline west & virginia & harrisan & & oittsburgh & 4.0 & 49.0 & 69.0 & 10.0 & 4.0 & 13540 & 58 & 7.0 \\
\hline west & virginia & harrison & & oitesburgh & 5.8 & 41.4 & 49.6 & 10.0 & 3.9 & 13520 & 57 & 7.0 \\
\hline west & virainia & harrison & & Dittsburgh & 2.8 & 30.8 & 50.6 & 9.6 & 3.7 & 13600 & 57 & $7: 0$ \\
\hline vest & virginia & harrison & & oittsburgh & 2.4 & 49.4 & 50.0 & 8.6 & 4.0 & 13750 & 0 & 6.5 \\
\hline west & virginia & harrison & & oittsourgh & 2.5 & 40.4 & 50.1 & 9.5 & 4.0 & 13650 & 59 & 7.5 \\
\hline west & virginia & harrison & & nittsburgh & 3.5 & 39.7 & 50.4 & 9.9 & 4.2 & 13550 & 59 & 7.0 \\
\hline west & virginia & harrison & & Dittsburgh & 2.5 & 40.0 & 50.5 & 9.5 & 4.0 & 13580 & 0 & 6.0 \\
\hline west & virginia & harrison & & Dittsburgh & 6.9 & 30.4 & 49.8 & 10.8 & 4.7 & 13350 & 0 & 8.0 \\
\hline west & virginia & harrison & & redstone & 4.4 & 34.1 & 54.7 & 91.2 & 1.9 & 13270 & 61 & 7.0 \\
\hline west & virninia & narrison & & Dittsburgh & 5.4 & 30.5 & $5 ? .1$ & 8.4 & 3.0 & 13700 & 63 & 8.0 \\
\hline west & virainia & narrison & & Dittsburyh & 2.9 & $6 ? .1$ & 50.0 & 7.9 & 4.1 & 13950 & 60 & 7.5 \\
\hline west & virginia & harrison & & oittsburgh & 3.9 & 38.2 & 54.8 & 7.0 & 2.5 & 13990 & 59 & 8.0 \\
\hline west & virginia & harrison & & oittsburah & 3.1 & $4 ? .9$ & 68.6 & 8.5 & 4.0 & 13710 & 53 & 0.5 \\
\hline west & virainia & harrisan & & oittsourgah & 4.8 & 40.8 & 50.8 & 9.4 & 3.0 & 13610 & 57 & 7.0 \\
\hline west & viryinia & harrison & & oittsourgh & 3.0 & 41.7 & 50.6 & 7.7 & 3.8 & 13820 & 56 & 7.5 \\
\hline west & virginia & harrison & & Ditesburgh & 2.0 & 42.0 & 49.8 & 8.2 & 3.8 & 13790 & 56 & 5.5 \\
\hline west & virginia & harrison & & Dittsourah & 5.0 & 42.1 & 40.6 & 8.3 & 3.1 & 13800 & 57 & 7.5 \\
\hline west & virginia & harrison & & nittsourah & 2.7 & $4 ? .3$ & 48.6 & 0.1 & 4.4 & 13530 & 58 & 7.0 \\
\hline west & virginia & harrison & & Ditesburgh & 1.9 & 40.2 & 51.8 & 8.0 & 2.9 & 13840 & 61 & 7.5 \\
\hline vest & virginia & harrison & & dittsouryh & 2.8 & 38.0 & 53.3 & 8.7 & 1.9 & 13820 & 59 & 7.5 \\
\hline vest & virginia & harrison & & Dittsburah & 2.1 & 40.2 & 50.7 & 0.1 & 3.4 & 13790 & 55 & 0.5 \\
\hline west & virainia & narrison & & redstone & 2.8 & 36.0 & 54.9 & 0.1 & 2.0 & 13690 & 59 & 7.5 \\
\hline west & virginia & kanowha & & Dittsburgh & 4.2 & 39.9 & 53.4 & 6.7 & 2.0 & 13760 & 0 & 5.0 \\
\hline west & virginia & kanawha & & Dittsburgh & 4.0 & 61.8 & 52.0 & 6.2 & 2.0 & 13830 & 0 & 5.0 \\
\hline west & virginia & kanawha & & winifrede & 2.0 & 34.1 & 61.7 & 4.2 & 0.5 & 14100 & 0 & 0.5 \\
\hline west & virginia & kanauha & & lower kittannina & 7.9 & 35.4 & 51.1 & 13.5 & 0.7 & 12920 & 43 & 5.0 \\
\hline vest & virginia & kanauha & & lower kittannina & 3.2 & 34.7 & 51.6 & 13.7 & 0.8 & 12860 & 42 & 5.0 \\
\hline west & virginia & kanawh $\rightarrow$ & & cedar grove & 3.1 & 37.8 & 57.0 & 5.2 & 0.7 & 14410 & 44 & 7.0 \\
\hline west & virginia & kanawha & & lower kittannina & 3.8 & 31.8 & 42.7 & 25.5 & 1.1 & 10790 & 0 & 3.0 \\
\hline west & viroinia & kanawha & & lower kittannina & 5.2 & 33.6 & 50.9 & 15.5 & 0.7 & 12350 & 43 & 1.0 \\
\hline west & virginia & kanauna & & coaldurasl kittannin & 4.8 & 36.8 & 49.1 & 16.1 & 0.8 & 12500 & 0 & 4.0 \\
\hline west & virginia & kanauha & & lower kittanning & 3.9 & 34.3 & 61.8 & 3.9 & 0.7 & 14390 & 0 & 5.5 \\
\hline west & virginia & kanawha & & l kittanninasuinifro & 4.5 & 34.3 & 55.4 & 10.3 & 0.8 & 93340 & 48 & 6.5 \\
\hline west & virginia & kanauha & & i kittanninasuinifro & $5.1)$ & 35.4 & 57.1 & 7.5 & 0.7 & 13850 & 48 & 5.5 \\
\hline west & virginia & kanawha & & eagle & 2.3 & 33.2 & 62.0 & 4.8 & 0.7 & 14680 & 0 & 7.5 \\
\hline west & virginia & tanauha. & & winifrede & 2.5 & 34.5 & 55.5 & 10.0 & 0.7 & 13450 & 0 & 6.0 \\
\hline west & virginia & kanawha & & cedar arove & 2.6 & 37.8 & 55.3 & 6.4 & 0.7 & 14140 & 44 & 0.5 \\
\hline west & virginia & kanauna & & coaluurast kittannin & 4.0 & 33.2 & 49.1 & 17.0 & 0.9 & $123 \geq 0$ & 44 & 2.5 \\
\hline
\end{tabular}




\begin{tabular}{|c|c|c|c|c|c|c|c|c|c|c|c|c|}
\hline west & $\begin{array}{r}\text { state } \\
\text { virginia }\end{array}$ & kanawha & county & $\begin{array}{c}\text { bed } \\
\text { lower kittanning }\end{array}$ & $\begin{array}{l}m 0 i \\
5.0\end{array}$ & $\begin{array}{l}\text { volmat } \\
36.1\end{array}$ & $\begin{array}{l}f i x e d c \\
50.2\end{array}$ & $\begin{array}{l}\text { 25h } \\
4.7\end{array}$ & $\begin{array}{r}50 \\
0.8\end{array}$ & $\begin{array}{l}\Delta r b t u \\
14270\end{array}$ & $4_{40}^{h g i l 0}$ & $4.0^{15 i 10}$ \\
\hline west & virginia & kanauna & & varinus & 3.6 & 39.3 & 56.9 & 11.8 & 1.3 & 13490 & 0 & 7.5 \\
\hline west & virginia & kanauta & & various & 5.9 & 31.9 & 55.8 & 12.3 & 1.3 & 13350 & 0 & 6.5 \\
\hline vest & viruinia & kanawha & & various & 5.3 & $3 ? .1$ & 55.4 & 12.5 & 1.5 & 13250 & 0 & 7.0 \\
\hline west & virginia & kanawha & & various & 3.4 & 32.0 & 57.7 & 10.3 & 1.2 & 13720 & 0 & 8.0 \\
\hline west & virginia & kanawha & & various & 3.8 & 39.5 & 57.2 & 11.3 & 1.3 & 13580 & 0 & 7.0 \\
\hline vest & virqinia & kanawha & & various & 4.0 & 32.5 & 56.4 & 11.1 & 1.5 & 13590 & 0 & 8.5 \\
\hline west & virginia & kanawha & & Dowelltion & 3.3 & 31.8 & 59.0 & 0.2 & 1.7 & 13920 & 64 & 7.5 \\
\hline west & virginia & kanawha & & Dowelition & 4.3 & 31.8 & 59.0 & 0.2 & 0.6 & 13680 & 0 & 4.0 \\
\hline west & virginia & kanauha & & winifrede & 2.4 & 35.6 & 60.2 & 4.2 & 0.7 & 14510 & 0 & 6.0 \\
\hline vest & virainia & kanawha & & camobell creek Deerl & 2.5 & 37.3 & 56.7 & 6.0 & 1.6 & 14300 & 0 & 7.0 \\
\hline west & virginia & kanawha & & lower camobell creek & 2.8 & 37.7 & 57.9 & 4.4 & 1.0 & 14500 & 0 & 6.5 \\
\hline west & virginia & kanawha & & winifrede & 4.5 & 36.5 & 51.3 & 12.2 & 0.7 & 12970 & 45 & 5.0 \\
\hline west & virginia & kanawha & & coatburg & 3.1 & 33.8 & 54.4 & 11.8 & 0.8 & 93220 & 0 & 6.0 \\
\hline west & viroinia & kanawha & & camnbell creek peerl & $? .9$ & 39.3 & 57.1 & 3.6 & 1.2 & 14710 & 0 & 6.5 \\
\hline west & virginia & kanawha & & winifrede & 4.5 & 35.0 & 58.3 & 6.7 & 0.6 & 13900 & 48 & 5.5 \\
\hline west & virginia & kanawha & & lower camodell creek & 4.2 & 32.9 & 57.8 & 9.3 & 1.6 & 13830 & 0 & 8.0 \\
\hline west & virginia & kanawhia & & lower kittanning & 8.4 & 33.3 & 57.0 & 9.7 & 0.5 & 13340 & 0 & 2.5 \\
\hline vest & virainia & kanawha & & winifrede & 5.5 & $3 ? .0$ & 60.0 & 8.0 & 0.5 & 13750 & 55 & 3.5 \\
\hline west & virginia & kanawha & & winifrede & 7.3 & 35.0 & 57.6 & 7.4 & 0.6 & 14030 & 52 & 7.0 \\
\hline west & virginia & Kanawha & & winifrede & 3.9 & 33.7 & 57.5 & 8.8 & 0.7 & 13860 & 54 & 6.5 \\
\hline west & virginia & kanawha & & winifrede & 3.9 & 33.2 & 56.7 & 10.1 & 0.6 & 13600 & 0 & 6.5 \\
\hline west & virginia & kanawha & & Inwer camobell creek & 2.1 & 32.3 & 56.0 & 11.7 & 1.5 & 13550 & $6 ?$ & 8.0 \\
\hline west & virginia & kanawha & & lower kittanning & 2.6 & 35.4 & 58.7 & 5.9 & 1.0 & 14220 & 0 & 6.0 \\
\hline west & virginia & kanawha & & lower campbell creek & 2.0 & 33.0 & $6 n .7$ & 6.3 & 0.5 & 14610 & 0 & 7.5 \\
\hline west & virginia & kanawha & & coalbura & 2.8 & 34.9 & 54.3 & 19.6 & 0.7 & 93250 & 0 & 3.5 \\
\hline west & virainia & kanawha & & eaqle & 2.1 & 36.2 & 58.3 & 7.5 & 0.6 & 16360 & 51 & 6.0 \\
\hline west & virginia & kanawha & & lover kittanning & 3.9 & 36.2 & 51.5 & 14.3 & 0.7 & 12810 & 43 & \\
\hline west & virginia & kanawha & & lower kittanning & 3.7 & 33.9 & 51.4 & 14.7 & 0.8 & 12730 & 0 & 4.5 \\
\hline west & virginia & kanawha & & lower kittannina & 4.9 & 32.1 & 53.2 & 14.7 & 0.6 & 12600 & 0 & 4.0 \\
\hline west & virainia & kanawha & & lover kittannina & 5.9 & 31.3 & 54.1 & 14.6 & 0.7 & 12620 & 0 & \\
\hline west & viryinia & kanawha & & lower kittannina & 5.7 & 32.9 & 50.0 & 17.1 & 0.7 & 12320 & 0 & 4.5 \\
\hline west & virginia & kanawha & & stockton-lewiston & 6.5 & 29.3 & 47.9 & 22.8 & 0.6 & 11350 & 0 & 2.5 \\
\hline west & virainia & kanawha & & stockton-lewiston & 4.5 & 29.3 & 47.1 & 23.6 & 0.6 & 11270 & 0 & 2.0 \\
\hline west & virginia & kanawha & & stocktnn-leviston & 4.5 & 32.0 & 50.1 & 17.9 & 0.0 & 12200 & $\mathbf{0}$ & 4.5 \\
\hline west & virqinia & kanawna & & coalourgostockton-ln & 4.3 & 32.7 & 51.9 & 15.4 & 0.8 & 12580 & 0 & 5.5 \\
\hline west & virginia & kanawha & & coalourgdstockton-ln & 6.6 & 30.5 & 47.5 & 22.0 & 0.6 & 11430 & 0 & 3.0 \\
\hline vest & virginia & kanawha & & lower campbell creek & 3.8 & 35.0 & 56.8 & 8.2 & 1.8 & 13950 & 55 & 8.0 \\
\hline west & virainia & kanawha & & lower camobell creek & 2.9 & 34.8 & 56.3 & 8.9 & 1.8 & 13910 & 0 & 8.5 \\
\hline west & virginia & kanawhd & & stockton-lewistan & 3.0 & 34.9 & 57.3 & 7.8 & 0.6 & 13780 & 0 & 5.5 \\
\hline west & virginia & kanawht & & various & 4.? & 38.3 & 51.6 & 10.1 & 1.6 & 13420 & 0 & 4.5 \\
\hline west & virainia & kanawha & & various & 2.5 & 32.3 & 49.4 & 12.3 & 9.6 & 13050 & 0 & 5.5 \\
\hline west & virginia & kanawha & & wooer kittannina & 3.6 & 39.1 & 51.9 & 10.0 & 1.4 & 13430 & 51 & 5.5 \\
\hline vest & virginia & kanjuha & & lover kittanning & 4.6 & 37.2 & 51.6 & 19.2 & 1.3 & 13230 & 49 & 5.5 \\
\hline west & virginia & kanawna & & winifrede & 2,1 & 35.0 & 56.9 & 7.2 & 0.8 & 14180 & 0 & 7.5 \\
\hline west & virginia & kanawha & & winifrede & 2.6 & 38.4 & 55.5 & 0.1 & 0.6 & 13940 & 0 & 5.5 \\
\hline west & virainia & kanawha & & Winifrede & 8.3 & 37.8 & 53.6 & 8.6 & 0.7 & 13220 & 48 & 9.0 \\
\hline west & virginid & kanawha & & winifrede & 4.4 & 37.7 & 53.6 & 8.7 & 0.7 & 13630 & 41 & 5.0 \\
\hline west & virginia & kanawha & & winifrede & 5.1 & 37.1 & 51.0 & $1: .9$ & 0.7 & 13070 & 43 & 4.5 \\
\hline west & virginia & kangwha & & stockton-leuiston & 2.8 & 36.0 & 58.1 & 5.9 & 0.7 & 14210 & 0 & 6.5 \\
\hline
\end{tabular}




\begin{tabular}{|c|c|c|c|c|c|c|c|c|c|c|c|}
\hline & state & county & bed & mo & rolmar & fixec & osh & sul & btu & ngilo & istlo \\
\hline $\begin{array}{l}\text { west } \\
\text { west }\end{array}$ & $\begin{array}{l}\text { virainia } \\
\text { viruinia }\end{array}$ & $\begin{array}{l}\text { kandwha } \\
\text { kanawha }\end{array}$ & $\begin{array}{l}\text { coalburg } \\
\text { vinifrede }\end{array}$ & $\begin{array}{l}2.5 \\
2.8\end{array}$ & $\begin{array}{l}25.3 \\
37.0\end{array}$ & $\begin{array}{l}51.9 \\
54.6\end{array}$ & $\begin{array}{r}12.8 \\
8.4\end{array}$ & $\begin{array}{l}0.7 \\
1.0\end{array}$ & $\begin{array}{l}13130 \\
13880\end{array}$ & $\begin{array}{l}41 \\
47\end{array}$ & $\begin{array}{l}4.0 \\
5.5\end{array}$ \\
\hline west & viruinia & kanawha & winifrede & 2.9 & 37.2 & 57.0 & 5.8 & 0.8 & 14280 & so & 6.0 \\
\hline west & virainia & kanawna & camonell creek deerl & 1.7 & $34 . ?$ & 53.6 & 9.2 & 3.2 & 13880 & 61 & 8.0 \\
\hline west & virginia & kanawha & camobell creek Deerl & 2.6 & 33.8 & 55.6 & 10.6 & 3.4 & 13670 & 64 & 8.5 \\
\hline west & virg̈inia & kanawha & oovelltion & 4.0 & 31.1 & 64.6 & 4.3 & 0.6 & 14170 & 61 & 2.0 \\
\hline west & virginia & kanauha & lower kittannina & 4.1 & 35.0 & 50.0 & 15.0 & 2.0 & 12470 & 46 & 5.0 \\
\hline west & virainia & kanautia & lower kittannina & 4.2 & 34.4 & 51.4 & 14.2 & 1.9 & 12750 & 44 & 4.0 \\
\hline west & viroinia & kanauha & loder kittanning & 4.0 & 34.0 & 50.3 & 15.7 & 2.2 & 12380 & 49 & 4.0 \\
\hline vest & virginia & kanauha & loxer camobell creek & 3.6 & 35.8 & 55.1 & 9.1 & 0.8 & 13600 & 51 & 5.5 \\
\hline west & virginia & kanautia & lower campbell creok & 5.9 & 36.4 & 57.2 & 6.4 & 0.5 & 14010 & $n$ & 5.5 \\
\hline west & virainia & kanauta & lower kittannina & 5.? & 34.2 & 51.0 & 14.8 & 1.9 & 12590 & 44 & 4.5 \\
\hline west & virginia & kanautid & various & 4.4 & 36.1 & $53: 1$ & 10.8 & 1.7 & 13460 & 43 & 6.0 \\
\hline vest & virginia & kanawtia & various & 4.0 & 31.0 & 47.1 & 29.9 & 0.8 & 11460 & 0 & 4.0 \\
\hline west & virginia & kanawtia & cedar grove & 5.7 & 35.3 & 52.9 & 11.8 & 1.8 & 13260 & 0 & 6.0 \\
\hline west & virainia & kanawha & cedar arove & 2.5 & 38.4 & 57.2 & 4.4 & 1.7 & 14350 & 0 & 6.0 \\
\hline west & virginit & kanawha & coslourgestockton-ln & 2.8 & 32.7 & 49.0 & 18.3 & 0.6 & 12170 & 40 & 4.0 \\
\hline west & virginia & kanauria & winifrede & 3.1 & 35.0 & 51.8 & 13.2 & 1.2 & 12990 & 54 & 6.0 \\
\hline west & virginia & kanawha & lower campoell creek & 3.2 & 37.8 & 57.0 & 5.2 & 0.8 & 14350 & 55 & 7.5 \\
\hline west & virainia & kanawha & lower camodeli creek & 3.3 & 38.6 & 56.9 & 4.5 & 0.7 & 14540 & 53 & 6.5 \\
\hline west & virginia & kanawha & lower camobell creek & 3.7 & 37.5 & 57.5 & 5.0 & 0.6 & 14610 & 53 & 8.0 \\
\hline vest & virginia & kanauha & cedar grovescoaloura & 3.4 & 33.9 & 53.6 & 12.5 & 9.9 & 13100 & 0 & 6.0 \\
\hline west & virginia & kanawha & coalbura & 3.3 & 33.7 & 51.9 & 14.4 & 0.7 & 12780 & 45 & 5.5 \\
\hline west & virainia & kanauta & camobell creek oeerl & 2.7 & 38.3 & 56.3 & 5.4 & 1.2 & 14350 & 46 & 6.5 \\
\hline west & virginia & kanawha & stockton-lewiston & 4.9 & 35.5 & 53.5 & 11.0 & 1.3 & 13340 & 47 & 7.0 \\
\hline west & virginia & lewis & redstone & 3.2 & 37.8 & 47.1 & 15.1 & 3.7 & 12570 & 58 & 8.0 \\
\hline west & virginia & lewis & redstone & 2.9 & 38.7 & $5 ? .2$ & 9.1 & 2.8 & 13620 & 60 & 7.5 \\
\hline west & virainia & lewis & oitesburah & 4.5 & 49.4 & 40.2 & 9.4 & 4.2 & 13450 & 58 & 7.5 \\
\hline west & virginia & lewis & Dittsburgh & 2.5 & 40.3 & 50.6 & 9.1 & 3.2 & 13450 & 0 & 6.0 \\
\hline west & virginia & lewis & Dittsburgh & 5.5 & 39.7 & 50.1 & 11.2 & 3.3 & 13070 & 63 & 0.5 \\
\hline west & virginia & lewis & Dittsburgh & 3.7 & 41.3 & 49.2 & 9.5 & 4.0 & 93500 & 57 & 6.5 \\
\hline west & virainia & lents & jittsourgh & 4.1 & 40.4 & 50.2 & 9.4 & 3.8 & 13500 & 57 & 7.5 \\
\hline west & virginia & lewis & ditisburgh & 3.7 & 49.9 & 50.2 & 7.9 & 3.6 & 13600 & 59 & 6.5 \\
\hline west & virginia & Lewis & oittsourgharedstone & 3.7 & 30.9 & 48.8 & 11.3 & 3.5 & 13160 & 56 & 7.5 \\
\hline west & virginia & lewis & redstone & 4.2 & 38.6 & 49.6 & 11.8 & 4.0 & 13050 & 57 & 6.0 \\
\hline west & virainia & lewis & nittsburan & 3.3 & 41.5 & 47.8 & 10.7 & 4.3 & 13200 & 59 & 7.0 \\
\hline vest & virginia & lewis & various & 3.5 & 41.1 & 50.6 & 8.3 & 3.6 & 13590 & 0 & 6.5 \\
\hline west & virginia & lewis & various & 4.0 & $39 . ?$ & 49.6 & 19.2 & 3.4 & 13950 & 0 & 5.0 \\
\hline vest & virginia & lewis & redstone & 3.7 & 30.7 & 51.9 & 8.4 & 3.5 & 13660 & 62 & 8.0 \\
\hline west & virainia & lewis & nitesburah & 3.7 & 40.1 & 51.3 & 8.6 & 3.3 & 93650 & 57 & 8.0 \\
\hline west & virginia & lewis & ditisburgh & 5.7 & 37.9 & 51.5 & 10.6 & 2.7 & 13150 & 57 & 7.0 \\
\hline west & virginia & Lewis & redstone & 3.6 & 30.3 & 51.? & 9.5 & 3.5 & 13520 & 63 & 7.5 \\
\hline west & virainia & lewis & oit tsburgh & 2.8 & 40.4 & 50.9 & 8.7 & 3.0 & 13540 & 0 & 5.5 \\
\hline west & virginia & lewis & oittsburgh & 3.2 & $40 . n$ & 50.8 & 0.2 & 3.3 & 13470 & 0 & 6.5 \\
\hline west & virainia & lewis & oittsourgh & 4.4 & 40.9 & 51.3 & 7.8 & 3.4 & 13730 & 55 & 6.5 \\
\hline west & virginia & lewis & oitisturgh & 5.4 & 37.7 & 53.9 & 8.4 & $? .8$ & 13570 & 57 & 8.0 \\
\hline west & virainia & lewis & redstone & 2.7 & 37.4 & 46.9 & 14.5 & 4.4 & 12610 & 56 & 7.0 \\
\hline west & virainia & Inoan & crilton & 4.7 & 35.4 & 57.5 & 7.1 & 0.6 & 13860 & 43 & 6.5 \\
\hline west & viruinia & logan & $\rightarrow \operatorname{lma}$ & $? .9$ & 30.4 & 54.1 & 6.5 & 1.7 & 94070 & 46 & 6.5 \\
\hline vest & virginia & $\operatorname{logan}$ & alma & 4.0 & 30.4 & 51.1 & 9.5 & 2.2 & 13430 & 44 & 5.0 \\
\hline
\end{tabular}




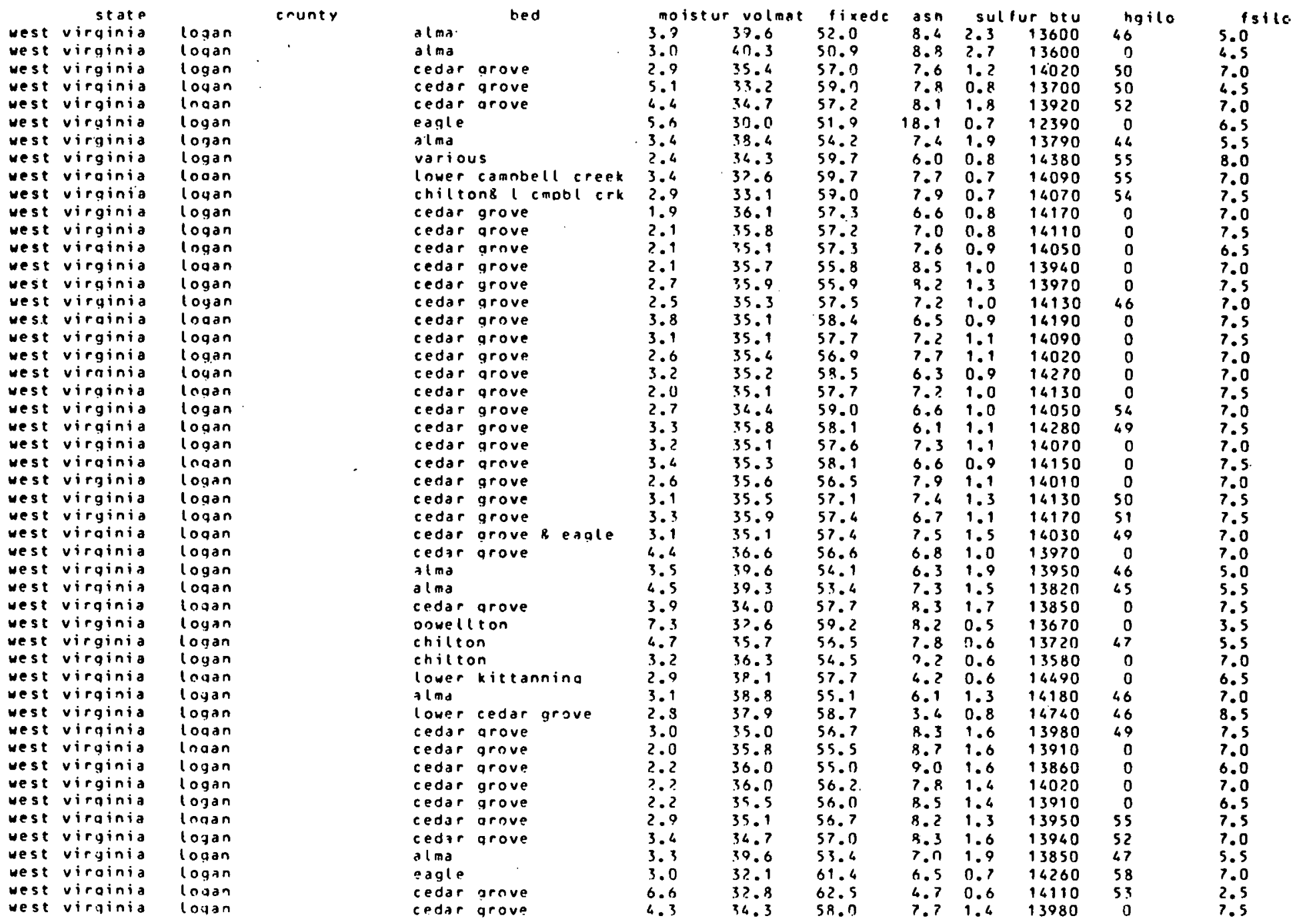




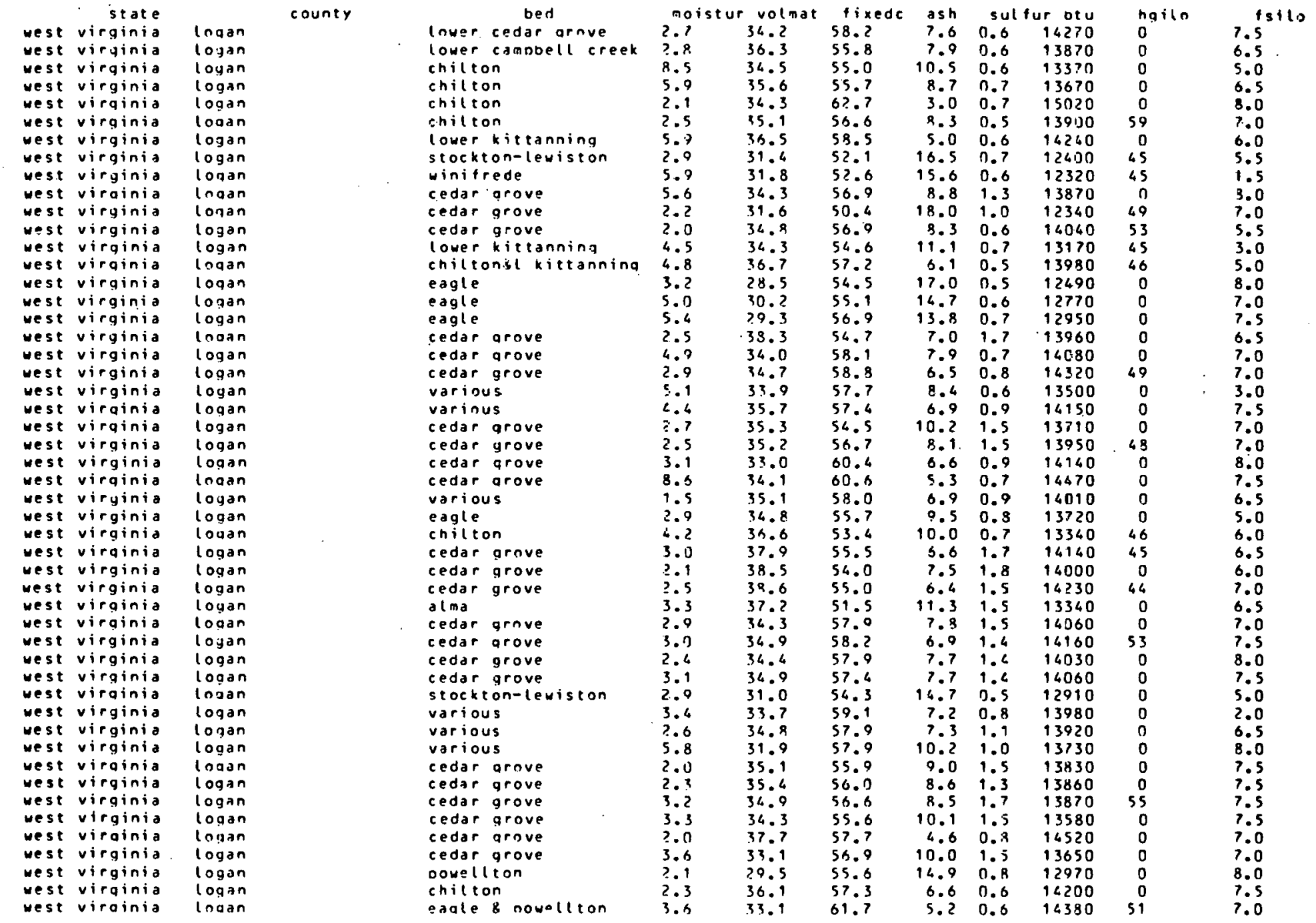




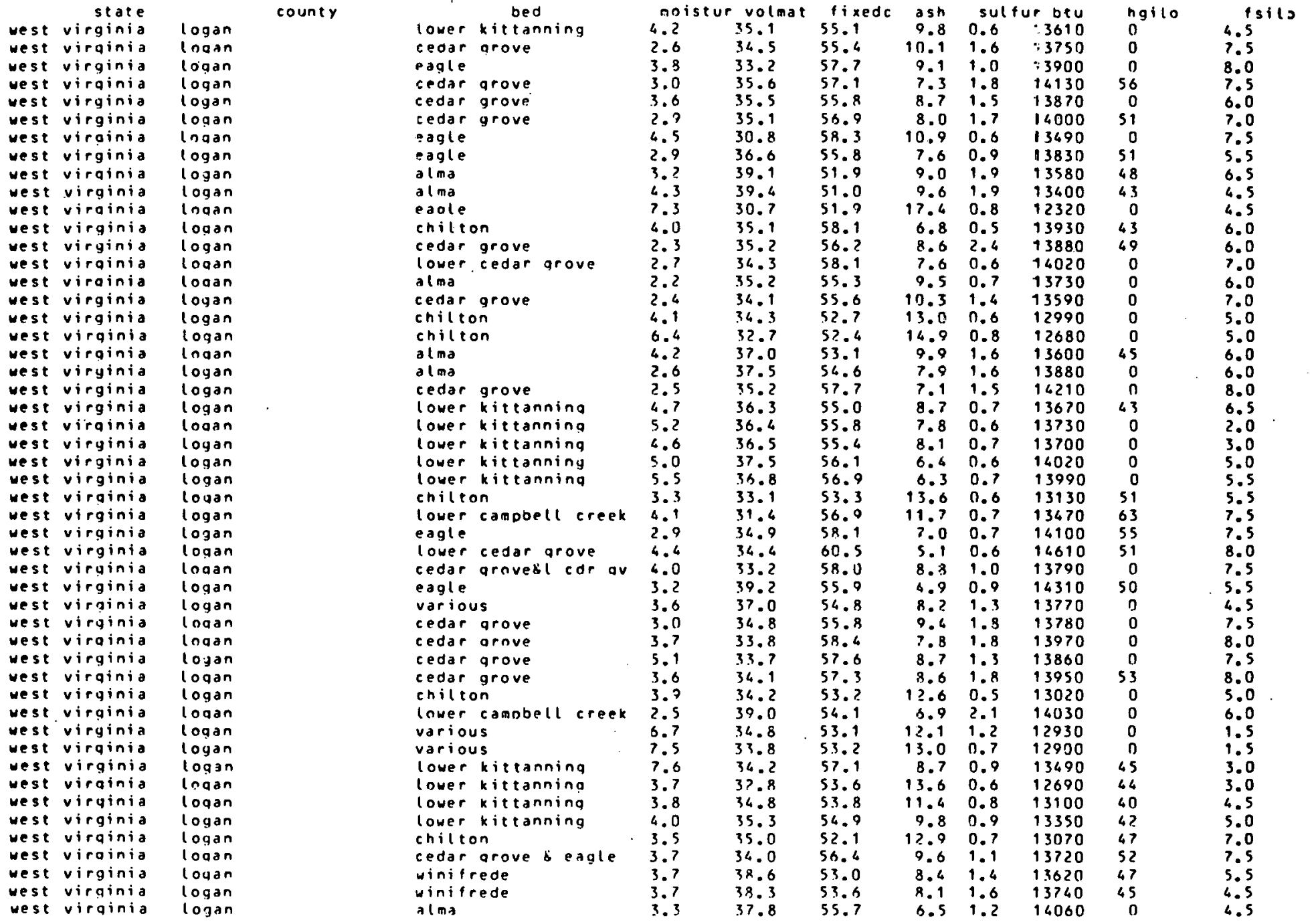




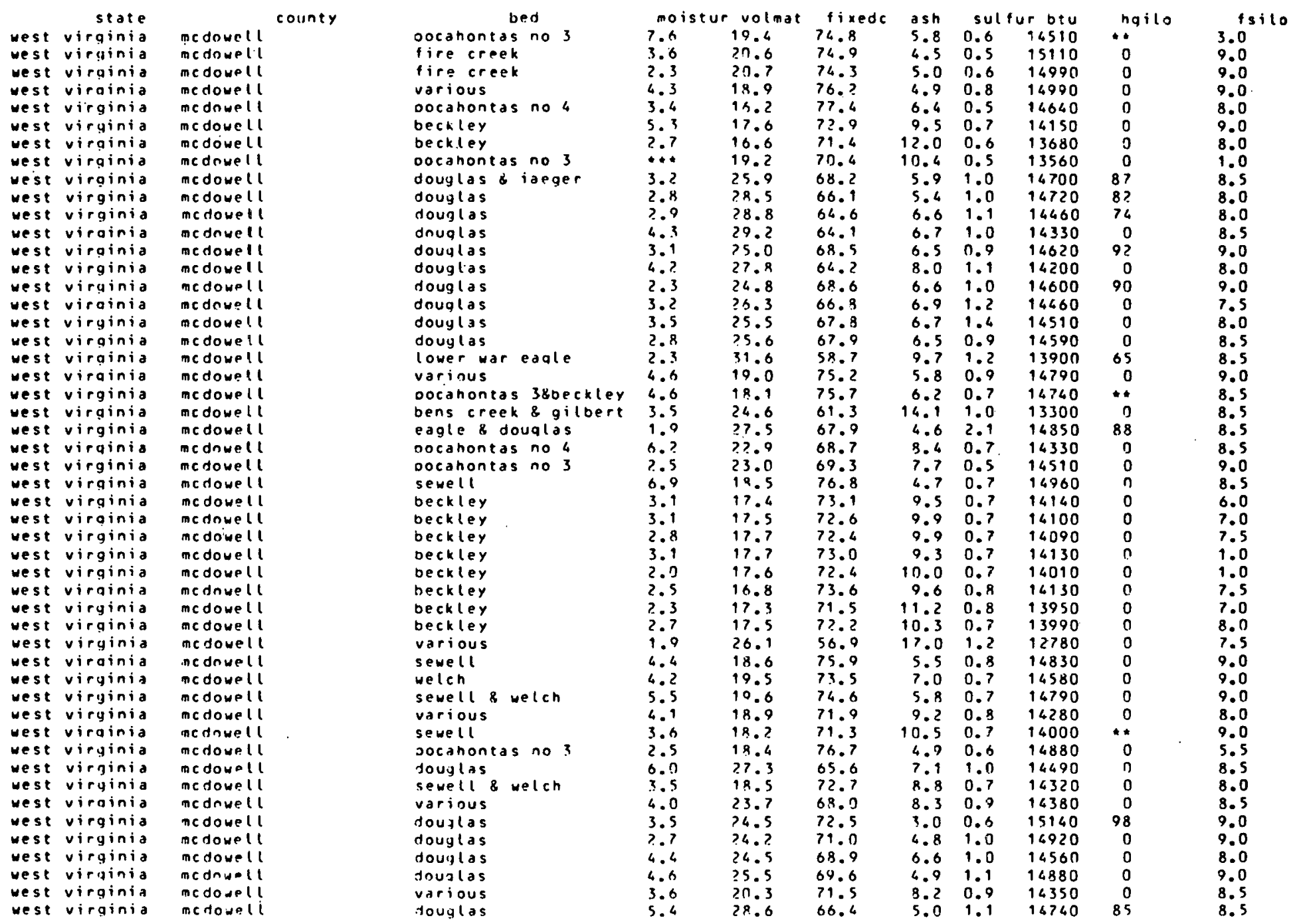




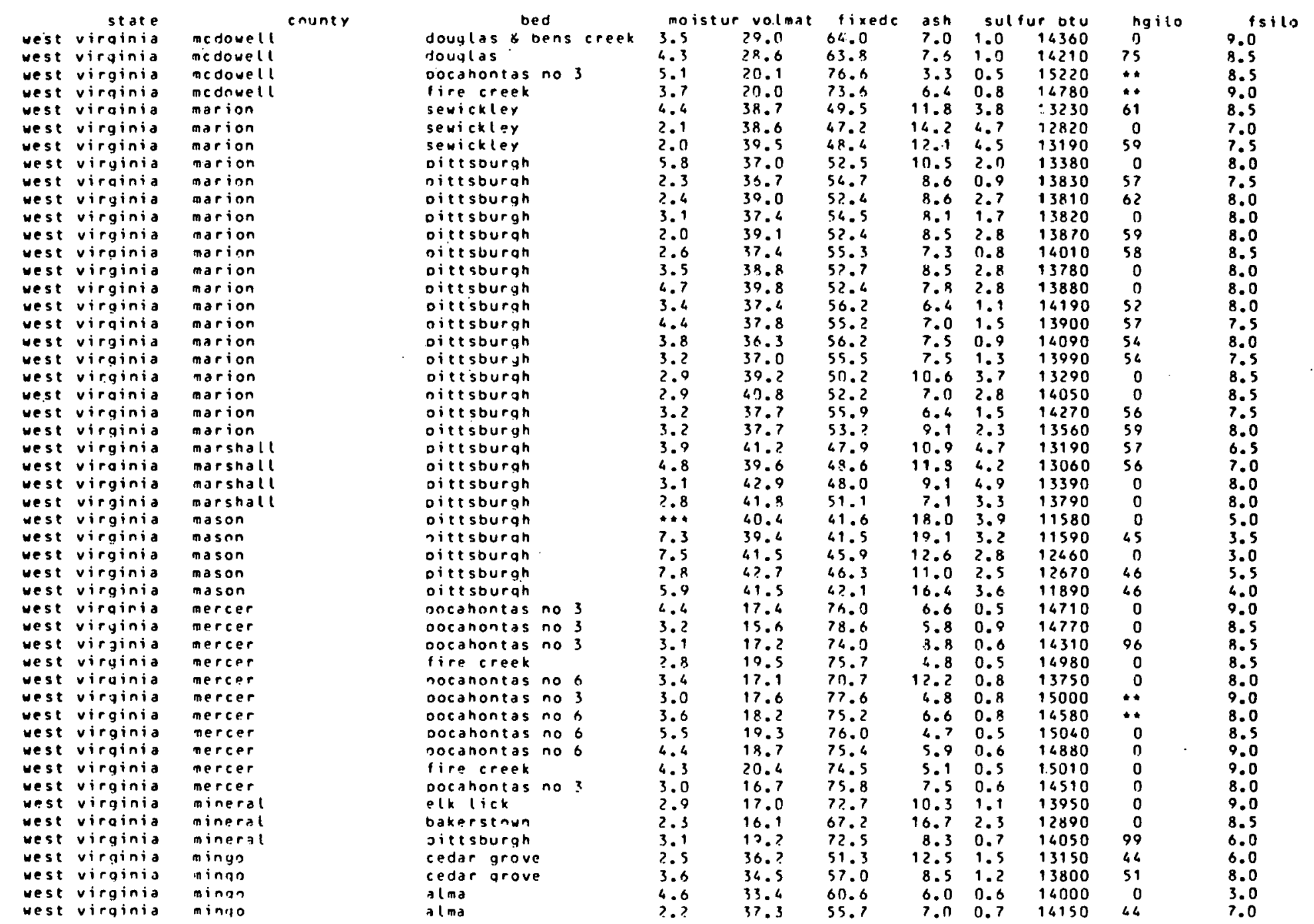




\begin{tabular}{|c|c|c|}
\hline & $\begin{array}{l}\text { state } \\
\text { virginia }\end{array}$ & minan \\
\hline es & virginit & mingn \\
\hline es & virainia & mingo \\
\hline west & virainia & mingo \\
\hline west & virginia & mingo \\
\hline vest & virainia & minan \\
\hline st & virginia & mingo \\
\hline west & virginia & mingo \\
\hline vest & virginia & minao \\
\hline west & virainia & ming̣n \\
\hline west & virginia & mingo \\
\hline west & virqinia & mingo \\
\hline west & virginia & mingo \\
\hline west & virginia & minao \\
\hline west & virginia & mingo \\
\hline west & virginia & mingo \\
\hline west & viryinia & mingo \\
\hline west & virainia & minan \\
\hline we s.t & virginia & mingo \\
\hline west & virginia & $\operatorname{mingo}$ \\
\hline we st & virginia & mingo \\
\hline west & virainia & mingo \\
\hline vest & virginia & mingo \\
\hline est & virginia & mingo \\
\hline st & virginia & mingo \\
\hline vest & virginia & mingn \\
\hline vest & virginia & mingo \\
\hline west & virginia & mingo \\
\hline west & virginia & mingo \\
\hline west & virainia & minqn \\
\hline west & virginia & mingo \\
\hline west & virginia & mingo \\
\hline west & virginia & mingo \\
\hline rest & virainia & minan \\
\hline vest & virginia & mingo \\
\hline west & virginia & minao \\
\hline west & virginia & mingo \\
\hline est & virainia & minan \\
\hline west & virginia & mingo \\
\hline vest & virginia & mingo \\
\hline : 5 & viropinia & mingo \\
\hline west & viroinia & mingn \\
\hline & virginta & mingo \\
\hline 58 & virginid & ingo \\
\hline es & Inda & 0 \\
\hline West & virainia & minon \\
\hline+52 & virginia & $m$ mingo \\
\hline 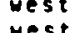 & & \\
\hline st & virainio & minan \\
\hline
\end{tabular}

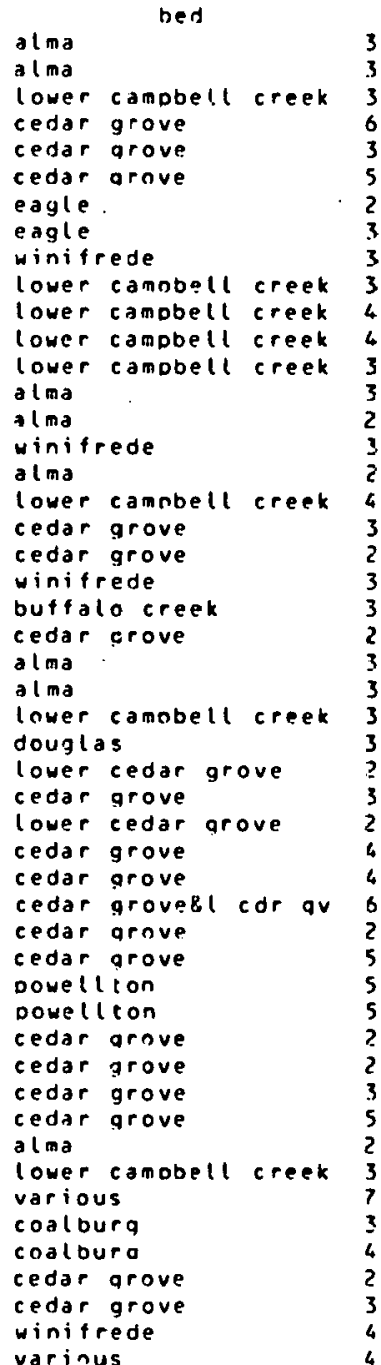

\begin{tabular}{|c|c|c|}
\hline noi & Volma & \\
\hline 3.5 & 35.8 & 54.9 \\
\hline 3.7 & 36.5 & 56.2 \\
\hline 3.2 & 32.1 & 56.8 \\
\hline 6.3 & 34.9 & $56 . ?$ \\
\hline 3.3 & 36.1 & 57.4 \\
\hline 5.9 & 36.6 & 59.0 \\
\hline 2.6 & 30.4 & 65.3 \\
\hline 3.5 & 30.3 & 59.9 \\
\hline 3.2 & 41.4 & 55.2 \\
\hline 3.6 & 38.1 & 55.6 \\
\hline 4.3 & 38.3 & 56.3 \\
\hline .6 & 38.8 & 55.3 \\
\hline 3.2 & 39.2 & 54.7 \\
\hline 3.2 & 36.9 & 50.1 \\
\hline 2.7 & 36.5 & 58.3 \\
\hline 3.6 & 41.4 & 53.3 \\
\hline 2.7 & 36.4 & 54.8 \\
\hline 4.6 & 38.9 & 40.8 \\
\hline 3.1 & 35.3 & 56.0 \\
\hline 2.8 & 36.3 & 55.8 \\
\hline .5 & 38.1 & 55.9 \\
\hline 3.1 & 37.2 & 54.2 \\
\hline 2.4 & 37.2 & 52.8 \\
\hline 3.6 & 32.0 & 57.1 \\
\hline 3.1 & 36.3 & 58.8 \\
\hline 3.0 & 38.1 & 54.6 \\
\hline 3 & 25.6 & 69.5 \\
\hline .0 & 34.1 & 55.5 \\
\hline 3.2 & 34.5 & 58.5 \\
\hline 2.4 & 36.4 & 60.2 \\
\hline 6.8 & 33.9 & 49.7 \\
\hline & 35.0 & 53.7 \\
\hline 6.0 & 31.9 & $5 ? .4$ \\
\hline .1 & 37.0 & 52.7 \\
\hline 5.4 & 32.6 & 57.6 \\
\hline 5.2 & 32.3 & 61.6 \\
\hline & 32.6 & 61.7 \\
\hline & 35.9 & 53.5 \\
\hline 7 & 35.0 & 54.2 \\
\hline & 35.6 & 54.4 \\
\hline 5.2 & 34.7 & 53.1 \\
\hline 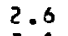 & 37.2 & 56.8 \\
\hline 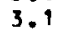 & 39.5 & 54.7 \\
\hline & 33.4 & 55.5 \\
\hline$\bullet$ & 38.1 & 55.5 \\
\hline 1 & 37.7 & $5 ? .8$ \\
\hline & 36.6 & 58.3 \\
\hline. & 36.6 & 56.5 \\
\hline & 36.9 & 49.1 \\
\hline & 32.0 & 58.0 \\
\hline
\end{tabular}

ash sulfu = betu

$\begin{array}{lll}9.3 & 0.7 & 13720 \\ 7.3 & 0.7 & 14060\end{array}$

$11.10 .6 \quad 13430$

$6.5 \quad 1.4 \quad 14250$

$6.4 \quad 1.213960$

$\begin{array}{lll}6.3 & 9.2 & 14750\end{array}$

$\begin{array}{lll}9.8 & 0.9 & 13720 \\ 3.4 & 0.7 & 14647\end{array}$

$6.3 \quad 1.4 \quad 14070$

$5.4 \quad 1.1 \quad 14110$

$\begin{array}{lll}5.9 & 1.5 & 44020\end{array}$

$\begin{array}{lll}4.0 & 0.6 & 14560\end{array}$

$5.2 \quad 0.6 \quad 14390$

$5.3 \quad 1.0 \quad 14220$

$\begin{array}{rll}5.8 & 0.6 & 13910\end{array}$

$12.12 .1 \quad 13080$

7.71 .213790

$6.0 \quad 0.6 \quad 14120$

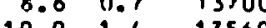

$10.90 .6 \quad 13580$

$1.90 .6 \quad 13580$

.31 .013350

$\begin{array}{rll}5.1 & 1.4 & 14820\end{array}$

$\begin{array}{rll}7.4 & 2.2 & 13630 \\ 3.0 & 0.7 & 14140\end{array}$

$3.4 \quad 0.6 \quad 14780$

$17.2 \quad 2.0 \quad 12670$

$\begin{array}{llll}11.3 & 2.4 & 13630\end{array}$

$15.7 \quad 1.4 \quad 12580$

$11.32 .0 \quad 13600$

$\begin{array}{lll}9.8 & 0.8 & 13570\end{array}$

$5.7 \quad 0.6 \quad 14220$

$10.6 \quad 1.8 \quad 13480$

$10.8 \quad 1.6 \quad 13480$

$\begin{array}{lll}10.0 & 2.1 & 13640 \\ 12.2 & 2.6 & 13270\end{array}$

$6.0 \quad 0.6 \quad 14400$

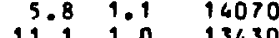

$11.19 .0 \quad 13430$

$\begin{array}{lll}6.4 & 0.7 & 16240\end{array}$

$7.91 .0 \quad 14090$

$6.91 .7 \quad 16080$

$\begin{array}{lll}14.0 & 0.7 & 12760 \\ 10.0 & 0.7 & 13770\end{array}$

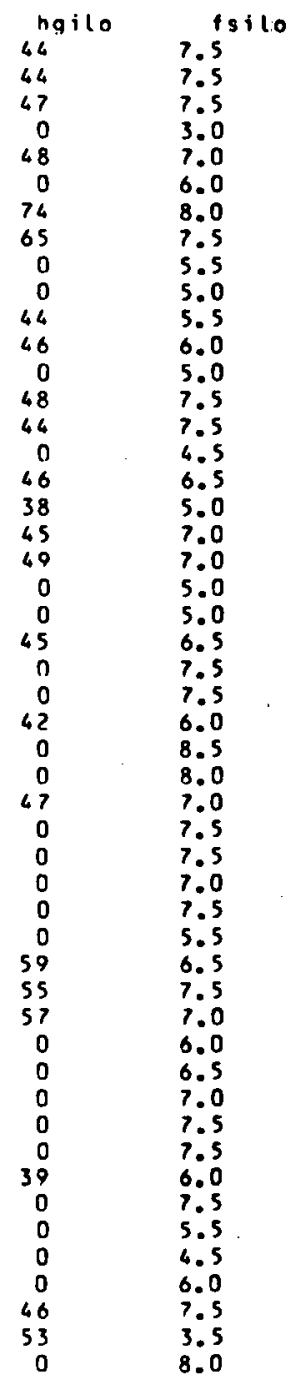




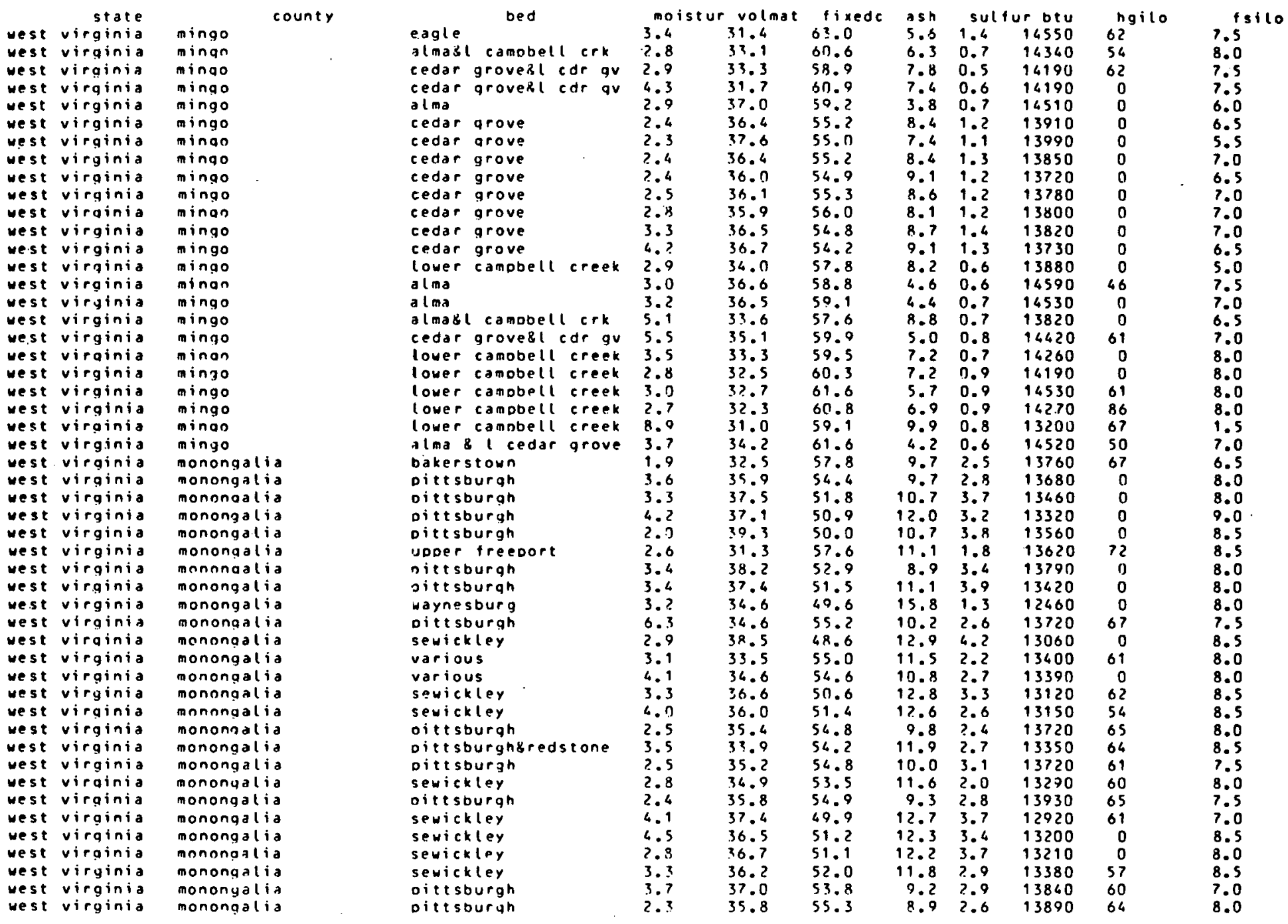


state west virainia west virainia west virginia west viraini West virainia west virainia

west virainia

west virainia

vest virginia

vest virginia

west viruinia

west virginia

west virgini

vest viraini

west viruinia

uest virginia

uest viruinia

vest virainis

vest virginis

vest virginia

west virainia

west virginia

west virginia

west virginio

west virainia

west virainia

vest virginia
uest virainia

west virainia

vest virginia

west virginia

west virginia

west virainia

west virginia

west virginia.

west virainia

west virgini

west virginia

west virainia

west virginia monongalia county monongalia monongalia

monongalia

monoalia

mononali

monongalia

monongalia

monongalia

monongalia

mononqalia

monongalia

mononualia

mongalia

monongalia

mononalia

monongalia

monongalia

mononaalia

mononaalia

monongalia

mononoalia

mononqalia

monongalia

mononaaliz

monongalia

monongalia

nichol as

icholas

nichnitas

nicholas

\begin{tabular}{|c|c|}
\hline pittsburq & \\
\hline oittsourg & \\
\hline nittsbural & \\
\hline bittsburyl & \\
\hline Ditesourg & \\
\hline pitt sburg & \\
\hline oitrsbura & \\
\hline oittsburg & \\
\hline bittsburg & \\
\hline Diteshura & \\
\hline nitesburg & \\
\hline Dittsourg & \\
\hline Dittsourg & \\
\hline Ditesburg & \\
\hline oittsbury & \\
\hline oittsoury & \\
\hline pittsbury & \\
\hline oitesburgl & \\
\hline sewickley & \\
\hline oittsburg & \\
\hline Ditesoura & hyredstone \\
\hline Dittsburg & insredstone \\
\hline Dittsburg & horedstone \\
\hline redstone & 8 sewickley \\
\hline bittsburq & \\
\hline oittsburg & \\
\hline Dittsburg & \\
\hline nittsbury & \\
\hline oittsburg & \\
\hline sewickley & \\
\hline sewickley & \\
\hline redstone & \\
\hline $\begin{array}{l}\text { redstone } \\
\text { redstone }\end{array}$ & \\
\hline redstone & \\
\hline redstone & \\
\hline $\begin{array}{l}\text { Dittsburg } \\
\text { pittsburg }\end{array}$ & \\
\hline Dittsburg & \\
\hline sewickley & \\
\hline sewickley & \\
\hline redstone & \\
\hline $\begin{array}{l}\text { redsione } \\
\text { sewickley }\end{array}$ & \\
\hline pittsbura & \\
\hline $\begin{array}{l}\text { eagle } \\
\text { eagle }\end{array}$ & \\
\hline $\begin{array}{l}\text { eaqle } \\
\text { camoollt }\end{array}$ & creek peerl \\
\hline coalbura & \\
\hline sevell & \\
\hline
\end{tabular}

moist
2.2
2.7
7.7
1.9
1.7
1.6
7.8
2.3
2.7
3.5
3.5
4.7
3.1
4.4
3.5
2.7
2.6
2.5
2.8
3.2
2.3
2.5
2.7
2.8
2.8
3.7
7.5
3.4
2.7
3.5
3.9
2.6
2.6
3.9
2.5
3.2
2.5
1.9
3.7
3.2
3.2
2.0
3.7
2.7
3.1
4.5
3.4
3.4
5.3
3.1

$\begin{array}{ll}\text { volmat } & \$ \text { ixedc } \\ 35.7 & 55.6 \\ 36.8 & 56.5 \\ 35.6 & 55.9 \\ 34.8 & 56.3 \\ 35.6 & 56.1 \\ 34.7 & 55.6 \\ 35.9 & 55.4 \\ 36.4 & 55.7 \\ 34.9 & 53.4 \\ 35.2 & 54.4 \\ 34.8 & 55.7 \\ 34.7 & 53.7 \\ 34.6 & 55.9 \\ 34.6 & 56.0 \\ 35.0 & 55.9 \\ 34.1 & 58.4 \\ 35.1 & 55.3 \\ 35.3 & 55.7 \\ 32.2 & 54.0 \\ 36.5 & 55.0 \\ 35.0 & 53.4 \\ 34.7 & 54.3 \\ 34.4 & 56.4 \\ 33.7 & 54.1 \\ 35.8 & 53.2 \\ 35.9 & 55.0 \\ 37.6 & 53.3 \\ 35.6 & 55.3 \\ 37.3 & 53.9 \\ 35.8 & 53.9 \\ 36.2 & 51.5 \\ 34.0 & 55.0 \\ 37.5 & 55.0 \\ 33.7 & 53.4 \\ 34.0 & 53.9 \\ 31.1 & 57.0 \\ 34.7 & 54.0 \\ 34.7 & 55.5 \\ 33.4 & 54.0 \\ 33.8 & 57.3 \\ 34.0 & 53.0 \\ 37.0 & 55.5 \\ 34.5 & 54.0 \\ 37.9 & 50.6 \\ 35.1 & 53.5 \\ 30.2 & 54.6 \\ 33.3 & 59.5 \\ 33.2 & 55.3 \\ 35.5 & 54.9 \\ 31.0 & 03.7 \\ & \\ 3 & \end{array}$

ash sulfur btu $6.72 .4 \quad 14310$ $8.5 \quad 1.9 \quad 14030$ $8.9 \quad 2.1 \quad 14020$ $8.3 \quad 2.0 \quad 14040$ $9.7 \quad 2.5 \quad 13790$ $8.7 \quad 2.9 \quad 14000$ $11.7 \quad 3.0 \quad 13400$ $10.4 \quad 2.8 \quad 13700$ $9.5 \quad 2.9 \quad 13780$ $\begin{array}{lll}2.1 & 3.0 & 13390\end{array}$ $9.5 \quad 2.9 \quad 13800$ 13013740 $\begin{array}{lll}.5 & 2.5 & 14130\end{array}$ $9.0 \quad 3.313850$ $13.8 \quad 9.2 \quad 13040$ $8.5 \quad 2.9 \quad 13850$ $11.6 \quad ? .5 \quad 13430$ $9.2 \quad 2.1 \quad 13800$ $12.2 \quad 2.8 \quad 13220$ $\begin{array}{lll}1.0 & 2.5 & 13610\end{array}$ $9.1 \quad 2.7 \quad 13910$ $9.111 .9 \quad 13690$ $9.1 \quad 2.6 \quad 13760$ $1.3 \quad 2.6 \quad 13840$ $\begin{array}{lll}12.3 & 3.2 & 13220\end{array}$ $11.0 \quad 1.9 \quad 13440$ $\begin{array}{lll}12.5 & 1.7 & 13200\end{array}$ $12.9 \quad 2.9 \quad 12970$ $\begin{array}{lll}12.1 & 2.2 & 13210 \\ 11.9 & 1.3 & 13260\end{array}$ $\begin{array}{lll}11.9 & 1.3 & 13260 \\ 11.8 & 3.1 & 13360\end{array}$ $08 \quad 3.0 \quad 13740$ $11.7 \quad 3.3 \quad 13340$ $13.9 \quad 1.3 \quad 12830$ $13.061 .9 \quad 13360$ $10.6 \quad 2.9 \quad 13670$ $11.5 \quad 3.5 \quad 13600$ $\begin{array}{llll}11.4 & 2.8 & 13520\end{array}$ $15.20 .6 \quad 12820$ $\begin{array}{llll}7.2 & 0.8 & 14110 \quad 5\end{array}$ $12.51 .0 \quad 13310 \quad 55$ $\begin{array}{llll}9.6 & 0.8 & 13560 & 46\end{array}$

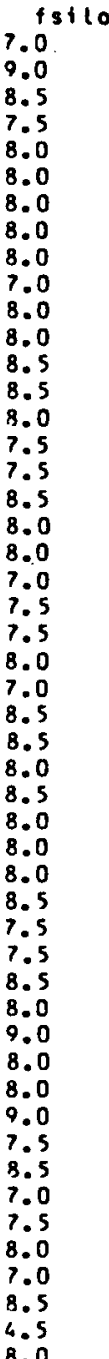




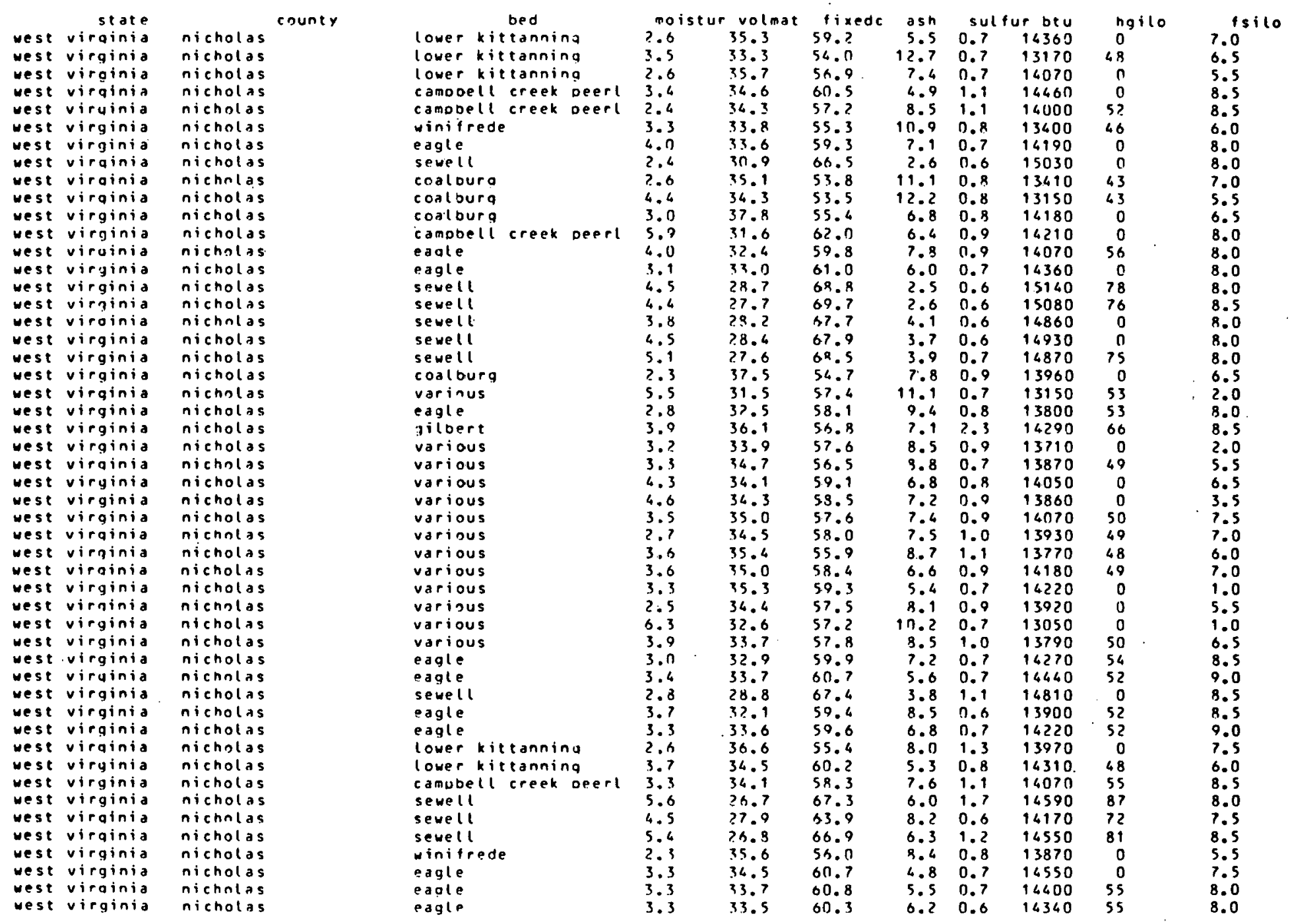




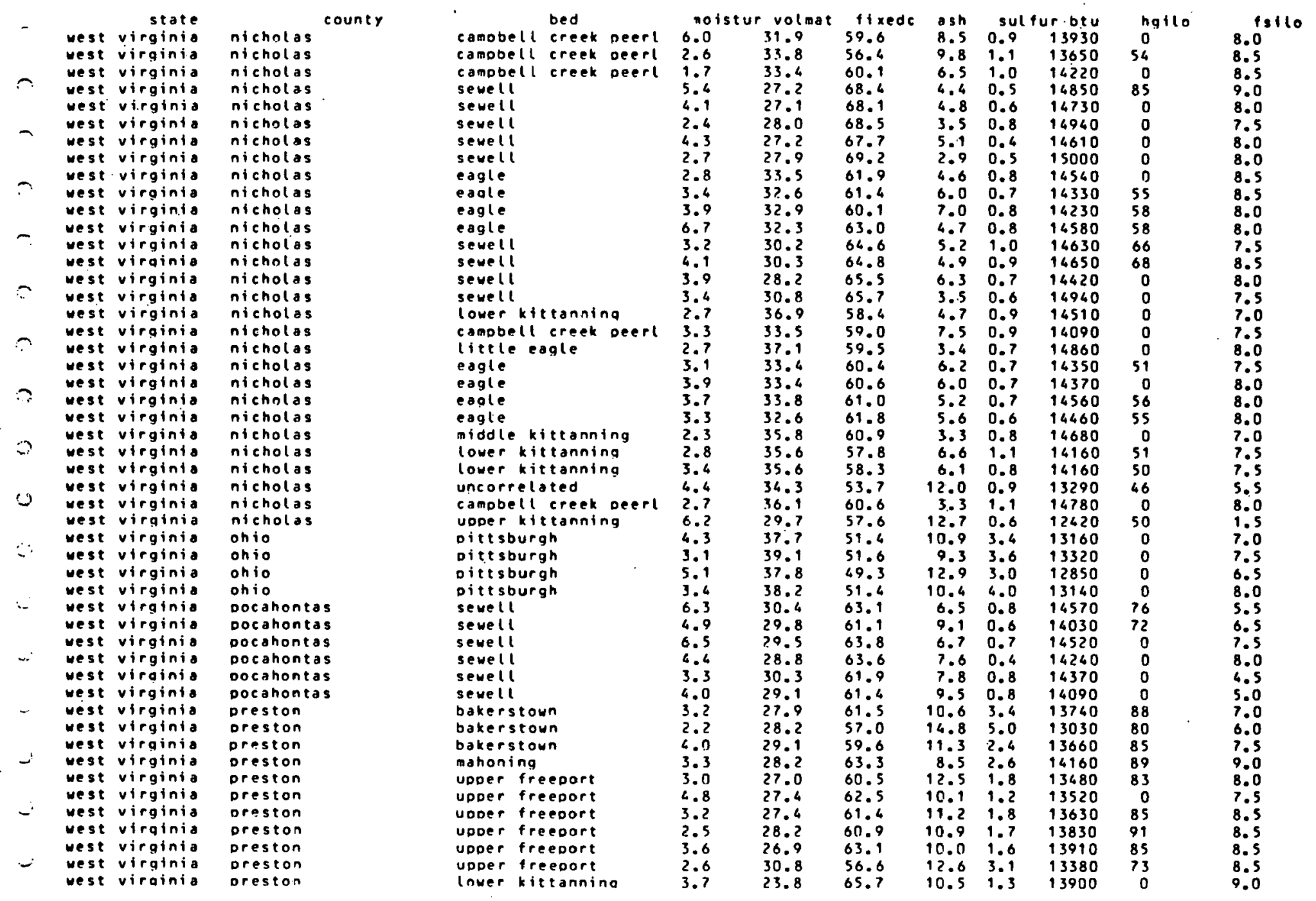




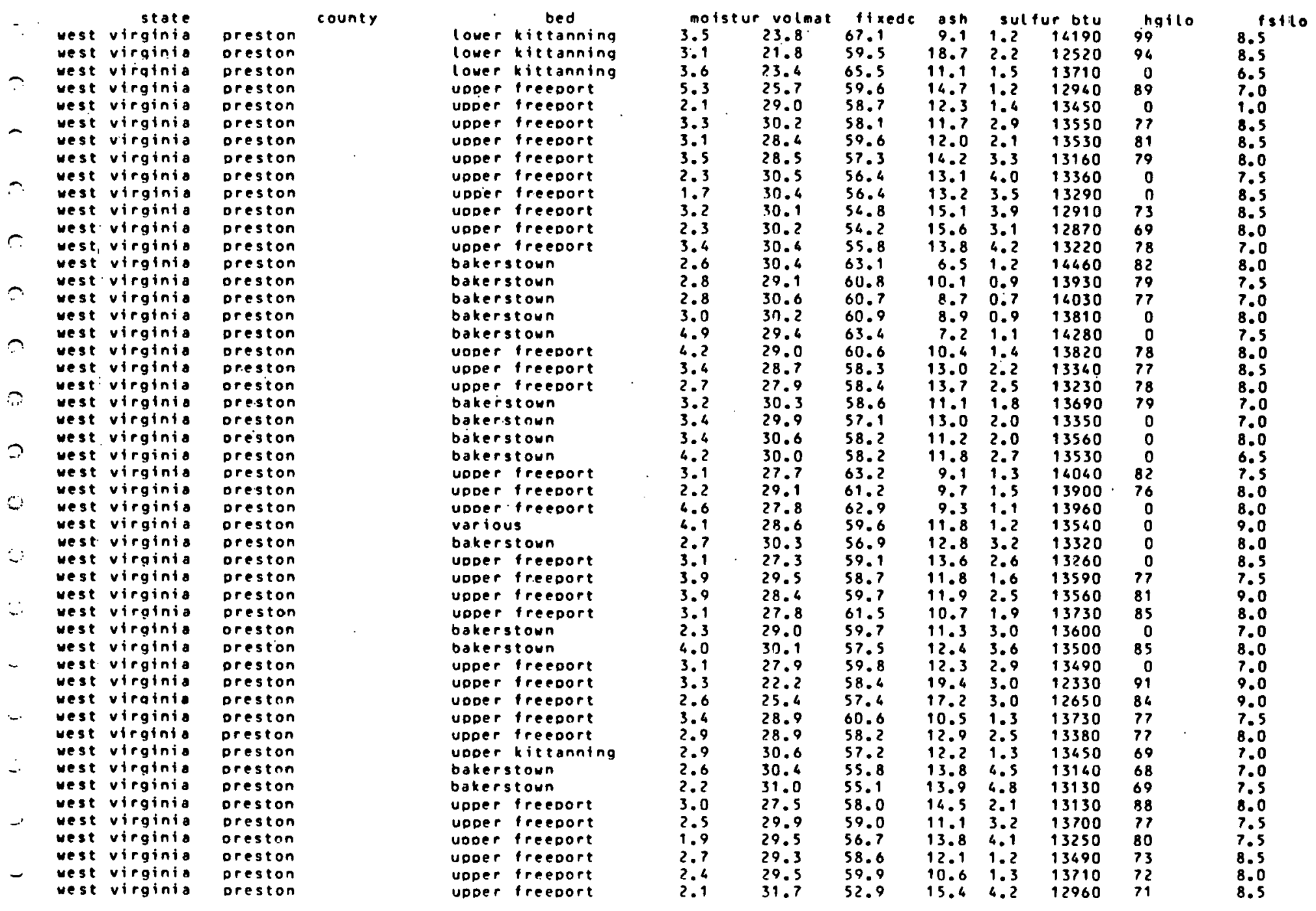


rest virginita

west virginio preston

west virginio preston

west virginia oreston

$C$ west. virginio

Dreston

west virginia Dreston

west virginia preston

west virginia preston

west virginia oreston

west virginta preston

west virginia preston

west virginia preston

west virginia preston

west viratnia oreston

west virginia preston

west virginia preston

- vest virginta oreston

west virginio preston

west virgini preston

west virainis preston

west virginis preston

west virginia oreston

west virginta oreston

west virainis preston

west virginis preston

west virginis preston

west virginia preston

wese virginta oreston

west virginio preston

west virginio preston

vest virginio oreston
vest virginio preston

vest virginia preston

west virginia preston

west virginia oreston

west virgini oreston

west virginia oreston

vese virainio oreston

west virginia preston
- west virginia oreston county

bakerstown

bakerstown

niddle kittanning

upder freedort

bakerstown

bakerstown

bokerstoun

uder freedort

uober ire

uoder freedort

uoder freedort

upper treeport

bakerstoun

bakerstounsu freeprt

uoper freeport

uoder freedort

upper freedort

uoder freeport

upper freeport

upoer freedort

upper freeport

upder freedort

upder freedort

upper freedort

upder freedort

bakerstown

upper treeport

upder ktttanning

lower kftranning

upder freedort

uoper freeport

uoper freeport

Uover treeport

udier treedort

upper freecort

udeer freedort

upper freeport

iddle kittannina

uoder freeoort

uper treedort

udoer frecoort

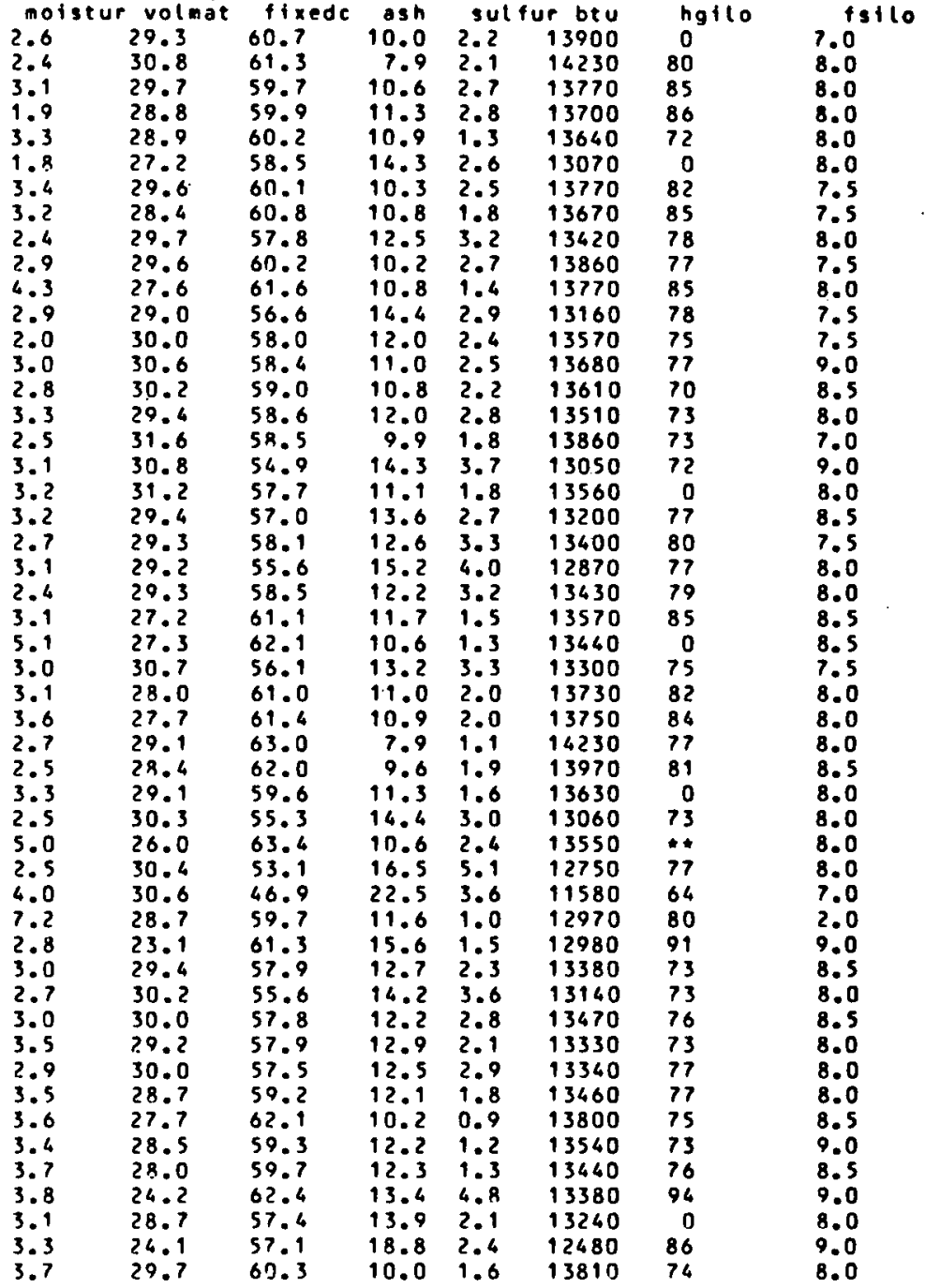


state west virginta vest virainis west virainis west virginia west virainia west virginio vest virginis uest virginia west virginia vest virginio vest virginia west virginis vest virginia west virginia west virginia west virginto vest virgtnia

west virginis west virginis west virginia

west virginta vest virginia west virginia west virginio west virginia vest virgini west virginia

rest virginia west virginia west virainio vest virginia west virgints west virginio vest virghnia west virginia vest virginia west virginia county Dreston Dreston Drestan Dreston oreston preston oreston oreston preston Dreston preston orestan oreston preston preston raleigh raleigh raleigh raleigh raleigh raleigh raleioh raleigh raleigh raleigh raleigh raleigh raleigh raleiah raleigh raleigh raleigh raleigh raleigh raleigh raleigh raleigh raleigh raleigh raleigh raleigh raleigh raleigh raleian
raleigh upper treedors

upoer treepor

uoder treedort

under treedort

upoer treedort

upper freedort

bakerstoun

bakerstown

bakerstoundu ireeprt

bakerstoundu freedrt

bakerst ounbu freeprt

bakerstownsu freeort

Upper kfttanning

Docahontas no 4

various

beckley

beckley

cedar grove

cedar

fire creek

eagle

eagle

eagle

sevel

sevell

sevell

eagle

tire creek

tire creek

lire creek

fire creek

Docanontas no 4

Docahontas no 3

lkittanningerinifrd beckley

sewell

powelltion

Dowellton

Dociltan no

Docahontas no 4

Docahontas no 4

Docahontas no 4

sevell

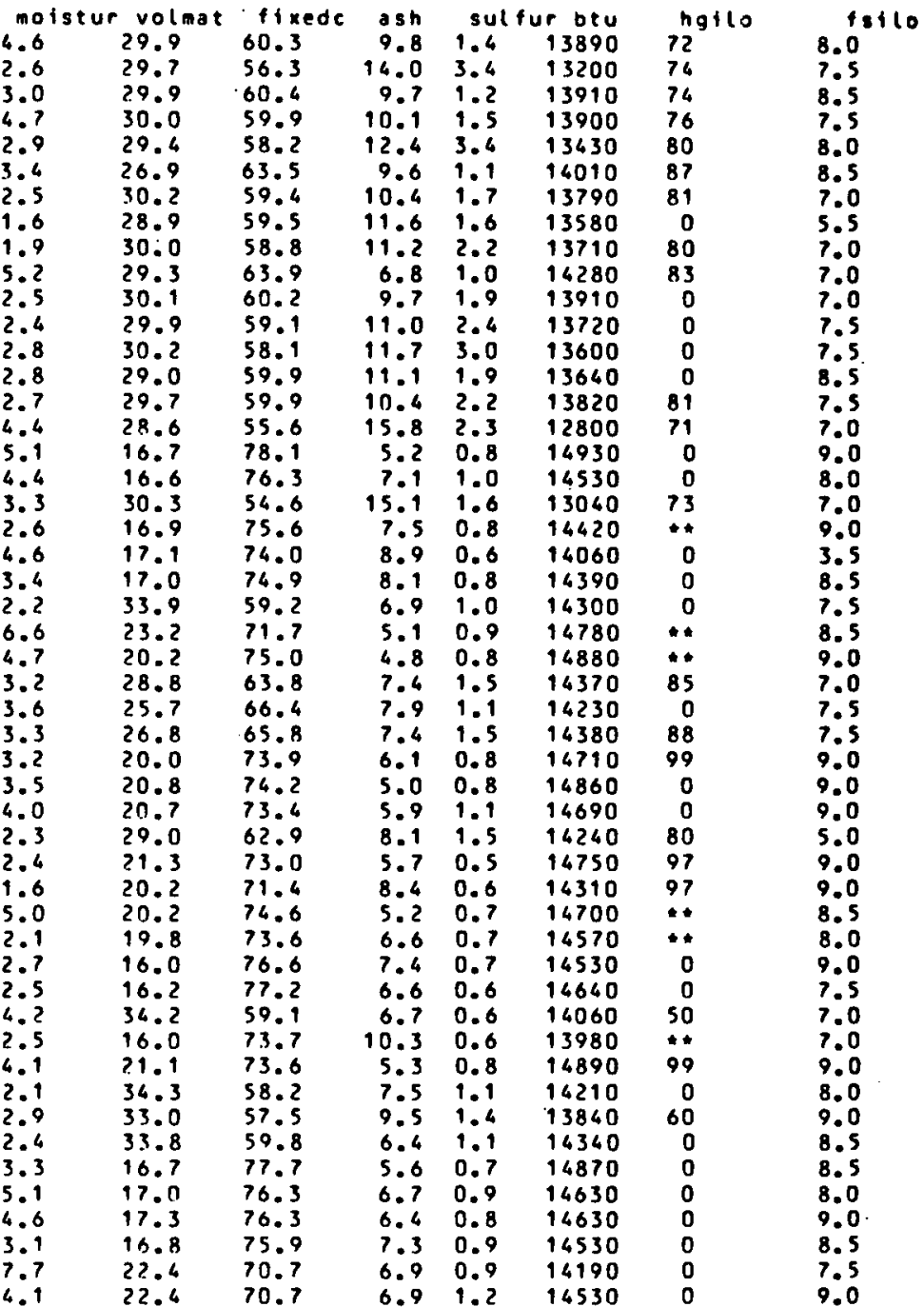

$+3110$

70.7 


\begin{tabular}{|c|c|c|}
\hline & $\begin{array}{l}\text { state } \\
\text { ginia }\end{array}$ & \\
\hline st & virginia & 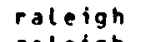 \\
\hline $\begin{array}{l}\text { west } \\
\text { west }\end{array}$ & $\begin{array}{l}\text { virginio } \\
\text { virginia }\end{array}$ & $\begin{array}{l}\text { raleigh } \\
\text { raleight }\end{array}$ \\
\hline 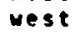 & virginia & raleigh \\
\hline $\begin{array}{l}\text { west } \\
\text { west }\end{array}$ & $\begin{array}{l}\text { virainio } \\
\text { virginia }\end{array}$ & $\begin{array}{l}\text { raleiah } \\
\text { raleigh }\end{array}$ \\
\hline west & virginia & raleigh \\
\hline west & virginia & raleigh \\
\hline $\begin{array}{l}\text { west } \\
\text { west }\end{array}$ & $\begin{array}{l}\text { virginio } \\
\text { virginisa }\end{array}$ & $\begin{array}{l}\text { raleigh } \\
\text { raleigh }\end{array}$ \\
\hline west & virginia & raleigh \\
\hline $\begin{array}{l}\text { west } \\
\text { west }\end{array}$ & $\begin{array}{l}\text { virginis } \\
\text { virginia }\end{array}$ & \\
\hline west & virginia & olo \\
\hline vest & virginio & $r$ \\
\hline west & virginio & randol \\
\hline west & virginio & randolo \\
\hline $\begin{array}{l}\text { vest } \\
\text { rest }\end{array}$ & $\begin{array}{l}\text { virs } \\
\text { virs }\end{array}$ & randolo \\
\hline vest & virginis & randolo \\
\hline west & virs & \\
\hline vest & virginia & $O L$ \\
\hline & virginta & \\
\hline $\begin{array}{l}\text { vest } \\
\text { west }\end{array}$ & $\begin{array}{l}\text { virginta } \\
\text { virginia }\end{array}$ & randoloh \\
\hline west & virginia & randolo \\
\hline vest & virg & \\
\hline west & virginid & 10 \\
\hline vest & viratnia & 10 \\
\hline west & & \\
\hline west & virginia & $\begin{array}{l}\text { randolph } \\
\text { randoloh }\end{array}$ \\
\hline & virginia & $\begin{array}{l}\text { ol on } \\
\text { ol on }\end{array}$ \\
\hline yest & virs & $\begin{array}{l}\text { dolo } \\
\text { dolo }\end{array}$ \\
\hline vest & virginia & rando lo \\
\hline & 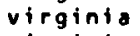 & ranc \\
\hline west & virainta & $\begin{array}{l}\text { randolo } \\
\text { randolo }\end{array}$ \\
\hline west & vtrginta & $\begin{array}{l}\text { randolo } \\
\text { randolp }\end{array}$ \\
\hline west & virginia & randolp \\
\hline vest & virginto & randoln \\
\hline & virginta & \\
\hline $\begin{array}{l}\text { west } \\
\text { vest }\end{array}$ & virgtnia & randolo \\
\hline vest & virginia & randolo \\
\hline west & virginid & randols \\
\hline & dirginia & $r a$ \\
\hline & & ando \\
\hline
\end{tabular}

county

raleion

raleigh

raleigh
aleigh

raleian

andoloh

dol on

toloh

alo

randolon

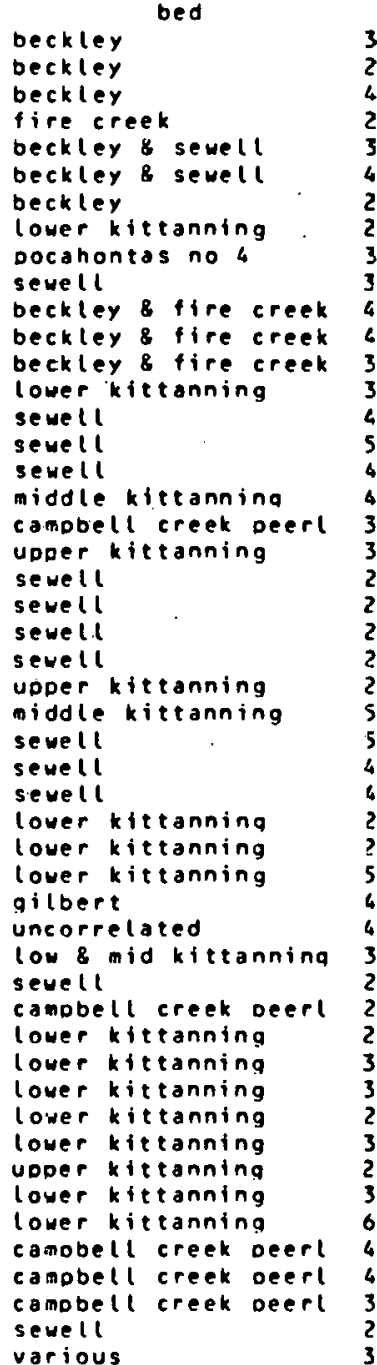

701
3.4
2.7
4.8
2.5
3.7
4.1
2.2
2.9
3.2
3.3
4.5
6.9
3.5
3.0
4.5
5.9
4.0
4.1
3.0
3.1
2.7
2.5
2.9
2.7
2.7
5.8
5.5
4.6
4.3
2.9
2.0
5.3
4.7
4.9
3.8
2.1
2.7
2.5
3.0
3.6
2.9
3.5
2.6
3.2
6.4
4.8
4.1
3.4
2.8
3.2
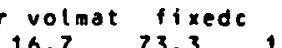

ash sulfur btu

$8.50 .7 \quad 14300$

$9.3 \quad 0.7 \quad 14200$

$\begin{array}{ll}16.5 & 75.0 \\ 16.3 & 74.0\end{array}$

$19.4 \quad 72.8$

$2.0 .9 \quad 73.5$

$21.6 \quad 71.0$

$32.9 \quad 56.2$

16.676 .3

$20.1 \quad 74.1$

$20.0 \quad 73.6$

$\begin{array}{ll}19.6 & 72.7\end{array}$

30.4

2.5 .5

25.1

26.5

34.7

39.459.

$31.1 \quad 64.7$

$30.2 \quad 66.0$

$30.9 \quad 62.6$

$30.6 \quad 58.8$

30.1 65.5

$29.4 \quad 66.1$

$30.7 \quad 63.2$

$30.4 \quad 57.4$

$29.3 \quad 54.8$

$23.6 \quad 67.8$

$27.9 \quad 67.0$

$30.1 \quad 59.4$

$34.6 \quad 63.6$

$29.7 \quad 58.8$

$\begin{array}{ll}29.7 & 58.6 \\ 29.6 & 58.6\end{array}$

$30.1 \quad 57.4$

$29.5 \quad 58.9$

$\begin{array}{ll}28.3 & 53.4 \\ 28.0 & 59.2\end{array}$

28.8 60.7

$32.2 \quad 62.9$

64.0

$30.8 \quad 64.0$ 7.81 .014430 $8.2 \cdot 1.0 \quad 14340$ $\begin{array}{lll}7.3 & 0.9 & 14420\end{array}$ $10.90 .8 \quad 13420$ $\begin{array}{lll}7.1 & 0.8 & 14600\end{array}$ $\begin{array}{lll}3.8 & 0.8 & 14260\end{array}$ $\begin{array}{lll}.8 & 0.8 & 14680\end{array}$ $6.40 .6 \quad 14420$ $9.90 .7 \quad 13900$ $\begin{array}{lll}9.4 & 1.0 & 14440\end{array}$ $6.7 \quad 0.8 \quad 14520$ $1.6 \quad 1.1 \quad 14360$ $\begin{array}{lll}3.5 & 0.8 & 14850\end{array}$ $10.31 .3 \quad 13320$ $4.20 .5 \quad 14940$ $\begin{array}{lll}3.8 & 0.7 & 14880\end{array}$ $10.50 .9 \quad 13800$ $10.3 \quad 0.8 \quad 13300$ $4.4 \quad 0.5 \quad 14190$ $6.10 .5 \quad 14490$ $12.2 \quad 2.1 \quad 13500$ $15.75 .0 \quad 12890$ $8.60 .6 \quad 14160$ $\begin{array}{lll}5.9 & 0.5 & 14420\end{array}$ $10.5 \quad 0.9 \quad 13810$ $\begin{array}{lll}1.7 & 0.7 & 14370\end{array}$ $11.5 \quad 1.2 \quad 13660$ $11.7 \quad 1.0 \quad 13650$ $11.81 .2 \quad 13510$ $\begin{array}{lll}12.5 & 1.8 & 13420\end{array}$ $\begin{array}{lll}11.6 & 1.9 & 13560\end{array}$ $18.32 .3 \quad 12410$ $10.51 .0 \quad 13320$ $4.9 \quad 0.9 \quad 14640$ $\begin{array}{lll}3.3 & 0.8 & 14860\end{array}$ $\begin{array}{lll}5.2 & 0.6 & 14620\end{array}$ $11.4 \quad 1.8 \quad 13560$

\begin{tabular}{|c|c|}
\hline hgilo & \\
\hline 98 & 5.0 \\
\hline 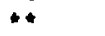 & 7.5 \\
\hline 0 & 7.9 \\
\hline 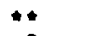 & 9.0 \\
\hline 0 & 9.0 \\
\hline 0 & 8.0 \\
\hline 0 & 9.0 \\
\hline 46 & 7.0 \\
\hline 0 & 7.5 \\
\hline$\because *$ & 9.0 \\
\hline 0 & 8.5 \\
\hline 0 & 8.0 \\
\hline$*$ & 9.0 \\
\hline 0 & 7.5 \\
\hline 85 & 9.0 \\
\hline 82 & 8.0 \\
\hline 0 & 9.0 \\
\hline 72 & 7.5 \\
\hline 0 & 8.5 \\
\hline 0 & 8.0 \\
\hline 57 & 7.5 \\
\hline 0 & 7.0 \\
\hline 0 & 7.5 \\
\hline 63 & 7.5 \\
\hline 77 & 6.0 \\
\hline 75 & 2.5 \\
\hline 0 & 1.5 \\
\hline 60 & 3.5 \\
\hline 62 & 7.5 \\
\hline 0 & 6.5 \\
\hline 69 & 8.0 \\
\hline 78 & 7.0 \\
\hline 70 & 7.5 \\
\hline 71 & 4.5 \\
\hline 79 & 8.0 \\
\hline 80 & 7.5 \\
\hline 0 & 9.0 \\
\hline 0 & 7.0 \\
\hline 75 & 7.5 \\
\hline 77 & 8.0 \\
\hline 0 & 8.0 \\
\hline 76 & 8.0 \\
\hline 71 & $?$ \\
\hline 0 & 7.5 \\
\hline 78 & 5.5 \\
\hline 0 & 8. \\
\hline 61 & 9.0 \\
\hline 0 & 8. \\
\hline 61 & 8 \\
\hline 74 & \\
\hline
\end{tabular}$$
\text { 11. } 1.8 \text { } 13560
$$

74




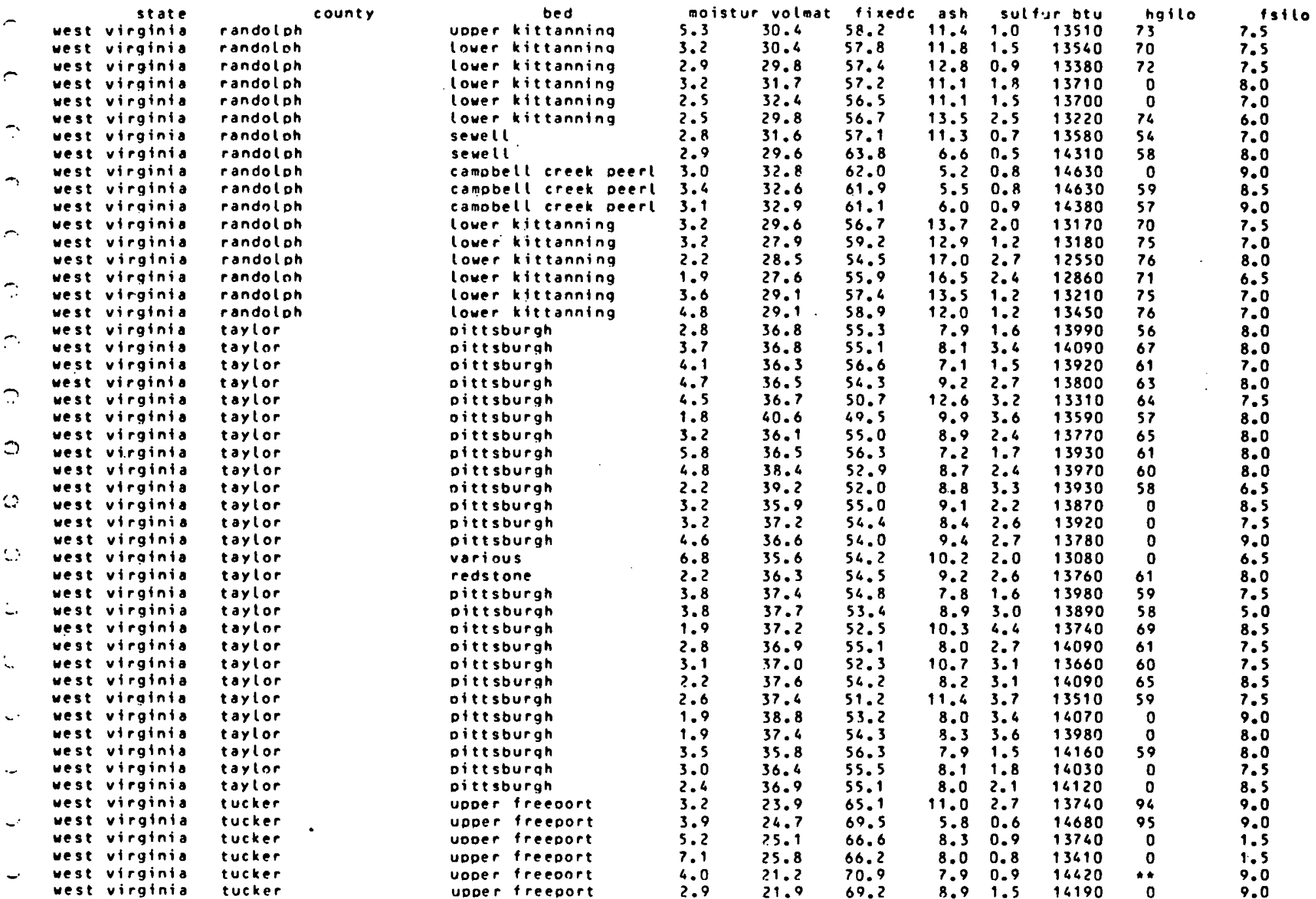




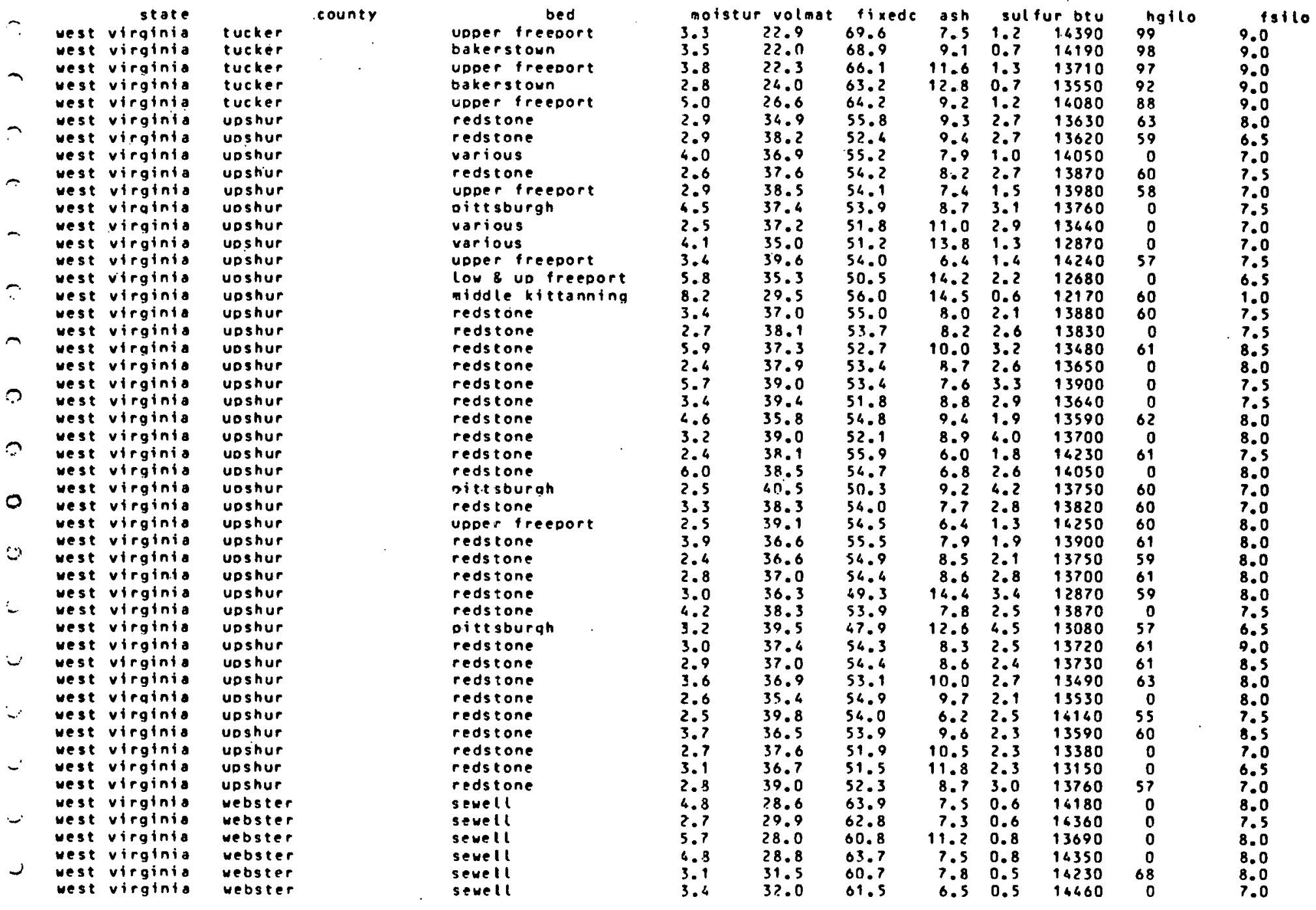




\begin{tabular}{|c|c|c|c|c|c|c|c|c|c|c|c|c|}
\hline rest & $\begin{array}{r}\text { state } \\
\text { virginia }\end{array}$ & wenster & county & bed & 4.4 & $\begin{array}{l}\text { volmat } \\
? 9.1\end{array}$ & $\begin{array}{l}\text { fixedc } \\
64.3\end{array}$ & $\begin{array}{l}\text { ash } \\
6.6\end{array}$ & $\begin{array}{l}\text { sul } \\
0.5\end{array}$ & $\begin{array}{l}\text { ur betu } \\
14480\end{array}$ & hailo & 7.55110 \\
\hline west & viruinia & webster & & fire creek & 2.8 & 28.6 & 62.1 & 9.3 & 0.4 & 13760 & 0 & 7.5 \\
\hline west & virqinia & webster & & fire creek & 3.2 & 78.8 & 63.2 & 8.0 & 0.5 & 14060 & 0 & 7.5 \\
\hline west & virainia & webster & & sevell & 1.9 & 30.9 & 60.9 & 8.2 & 0.9 & 14080 & 0 & 8.5 \\
\hline west & virginia & webster & & sewell & 3.6 & 28.6 & 60.5 & 10.9 & 0.5 & 13790 & 0 & 7.5 \\
\hline west & virginia & webster & & fire creek & 3.0 & 28.2 & 61.3 & 10.5 & 0.5 & 13550 & 57 & 6.5 \\
\hline west & virainia & webster & & sevell & 2.5 & 31.7 & 61.8 & 6.5 & 0.8 & 14460 & 57 & 7.5 \\
\hline west & virọinia & webster & & camobl crk urlsosewl & 5.7 & 33.3 & 62.9 & 3.8 & 0.8 & 14460 & 0 & 6.0 \\
\hline west & virginia & weoster & & sewe ll & 3.5 & 29.7 & 64.7 & 5.6 & 0.5 & 14520 & 0 & 8.0 \\
\hline west & virginia & webster & & sewell & 5.1 & 29.2 & 66.0 & 6.8 & 0.5 & 14080 & 59 & 6.0 \\
\hline west & virạinia & webster & & camobell creek peerl & 2.8 & 37.2 & 59.4 & 3.4 & 0.7 & 16900 & 0 & 8.0 \\
\hline west. & viroinia & webster & & sevell & 6.2 & 29.4 & 65.1 & 5.5 & 0.9 & 14520 & 79 & 8.0 \\
\hline west & virginia & webster & & sevell & 2.4 & 30.7 & 61.6 & 7.7 & 1.0 & 14270 & 67 & 7.5 \\
\hline west & virginia & webster & & sewell & 6.7 & 29.6 & 65.6 & 4.8 & 0.6 & 14190 & 71 & 2.5 \\
\hline west & virainia & webster & & sewell & 5.2 & 29.9 & 64.5 & 5.6 & 0.6 & 14480 & $\gg 1$ & 8.0 \\
\hline west & virainia & webster & & sewell & 3.8 & 28.8 & 63.3 & 7.9 & 1.0 & 14230 & 70 & 8.5 \\
\hline west & virginfa & wyoming & & beckley & 3.1 & 17.6 & 77.8 & 4.6 & 0.6 & 14950 & 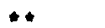 & 7.5 \\
\hline west & virginia & wrominc & & Docahontas no 6 & 3.0 & 17.8 & 75.2 & 7.0 & 0.6 & 14640 & 0 & 9.0 \\
\hline west & virginia & wyoming & & various & 5.8 & 26.9 & 59.0 & 14.1 & 1.1 & 13210 & 70 & 2.5 \\
\hline vest & virainis & Wyomina & & various & 5.8 & 26.9 & 59.0 & 14.1 & 1.1 & 13210 & 70 & 2.5 \\
\hline west & virginia & wroming & & sewell & 2.3 & 2?.0 & 74.5 & 3.5 & 0.7 & 15130 & 0 & 9.0 \\
\hline west & virginia & wyoming & & douglas & 3.9 & 26.3 & 67.9 & 5.8 & 1.0 & 14.700 & 93 & 9.0 \\
\hline west & virginia & wyoming & & douglas & 2.8 & 25.4 & 69.2 & 5.4 & 1.0 & 94730 & 0 & 7.0 \\
\hline vest & virainia & wrom ing & & doualas & 2.9 & 25.4 & 70.0 & 4.6 & 1.1 & 14980 & 0 & 9.0 \\
\hline west & virginia & wyomina & & douglas sewell & 4.6 & $?: 4.9$ & 70.1 & 5.0 & 1.2 & 14960 & 0 & 8.5 \\
\hline west & virginia & wroming & & pocahontas no 3 & 4.4 & 17.0 & 76.4 & 6.6 & 0.6 & 14600 & 0 & 6.0 \\
\hline west & virginia & wyoming & & Docahontas no 4 & 2.9 & 17.4 & 74.0 & 8.6 & 1.0 & 14300 & 0 & 7.5 \\
\hline vest & virainia & Wynming & & Docahonias no 3 & 3.1 & 18.1 & 75.2 & 6.7 & 0.8 & 14550 & 0 & 8.0 \\
\hline west & virginia & wyoming & & Dowelltion & 2.2 & $3 ? .3$ & 62.0 & 5.7 & 0.6 & 14510 & 57 & 8.5 \\
\hline west & virginia & wroming & & oowelltan & 3.1 & $3 ? .9$ & 62.5 & 4.6 & 0.6 & 14730 & 60 & 8.0 \\
\hline west & virginia & wyoming & & oowelltion & 2.9 & 33.4 & 60.9 & 5.7 & 0.7 & $145 ? 0$ & 62 & 8.0 \\
\hline west & virainia & wromina & & ane llt on & 3.6 & 34.1 & 58.6 & 7.3 & 0.6 & 16260 & 0 & 6.0 \\
\hline west & virginia & wyoming & & jowellt on & 2.9 & $3 ? .2$ & 69.3 & 6.5 & 0.7 & 14460 & 58 & 8.0 \\
\hline west & virginia & wroming & & Dowelltan & 2.7 & 37.2 & 61.2 & 6.6 & 0.6 & 14400 & 0 & 8.0 \\
\hline west & virginia & Wyoming & & dinifrede & 3.0 & 35.1 & 58.3 & 6.6 & 0.7 & 14360 & 0 & 6.5 \\
\hline west & virainia & wyomina & & winifrede & 6.2 & 33.1 & 55.4 & 11.5 & 0.8 & 13520 & 60 & 7.5 \\
\hline west & virginia & wroming & & winitrede & 8.0 & 31.3 & 56.4 & 12.3 & 0.7 & 13400 & 0 & 7.5 \\
\hline west & virginia & wromina & & winitrede & 5.9 & 39.4 & 55.5 & 13.1 & 0.8 & 13270 & $n$ & 6.5 \\
\hline west & virainia & wyoming & & winitrede & 2.7 & 31.2 & 54.1 & 14.7 & 0.8 & 12870 & 0 & 7.5 \\
\hline west & virginia & wynmina & & Winifrede & 5.2 & 30.8 & 53.7 & 15.5 & 0.8 & 12750 & 0 & 7.0 \\
\hline west & virainia & wroming & & winitrede & 4.4 & 30.3 & 56.9 & 14.8 & 0.7 & 12890 & 0 & 7.5 \\
\hline west & virginia & wyoming & & vinitrede & 6.1 & 31.0 & 55.4 & 13.6 & 0.7 & 13090 & 0 & 7.5 \\
\hline west & virainia & wyoming & & winitrede & 6.7 & 30.9 & 53.1 & 16.0 & 0.8 & 12710 & 0 & 5.0 \\
\hline west & virainia & wynmina & & winitrente & 7.1 & 30.5 & 53.0 & 16.5 & 0.8 & 12650 & 0 & 7.0 \\
\hline west & virginia & wyoming & & winitrece & 2.1 & 33.8 & 56.1 & 10.1 & 0.9 & 13710 & 0 & 6.5 \\
\hline west & virginia & wyoming & & Docanontas no 4 & 2.5 & 16.4 & 77.3 & 6.3 & 0.8 & 14670 & 0 & 8.5 \\
\hline vest & virginia & wyomina & & Docahontas no 3 & 4.0 & 17.8 & 75.7 & 6.5 & 0.7 & 14640 & 0 & 8.0 \\
\hline west & virainia & Wynmina & & Houglas & 2.4 & $? 7.4$ & 68.1 & 4.5 & 2.4 & 14860 & 0 & 8.5 \\
\hline west & virginia & wroming & & lower camobell & 3.8 & $3 ? .0$ & 64.9 & 3.1 & 0.6 & 14950 & 59 & 8.5 \\
\hline west & virginia & wyoming & & camoocll & 3.4 & 33.2 & 69.6 & 5.2 & 0.7 & 14510 & 55 & 8.0 \\
\hline
\end{tabular}


MEST VIRGINIA

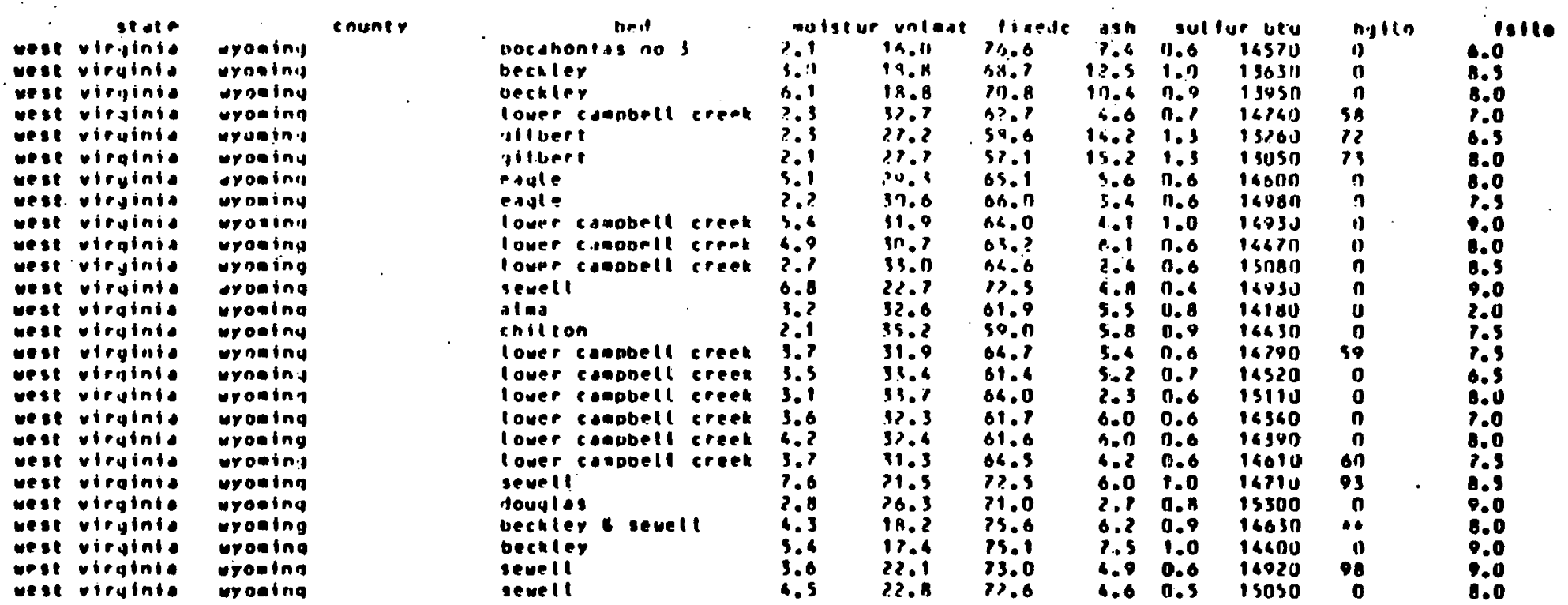



National Coal Resources Dato System

\author{
U.S. Geological survey
}

identified coal resources in wyoming

(In milltions of short tons)

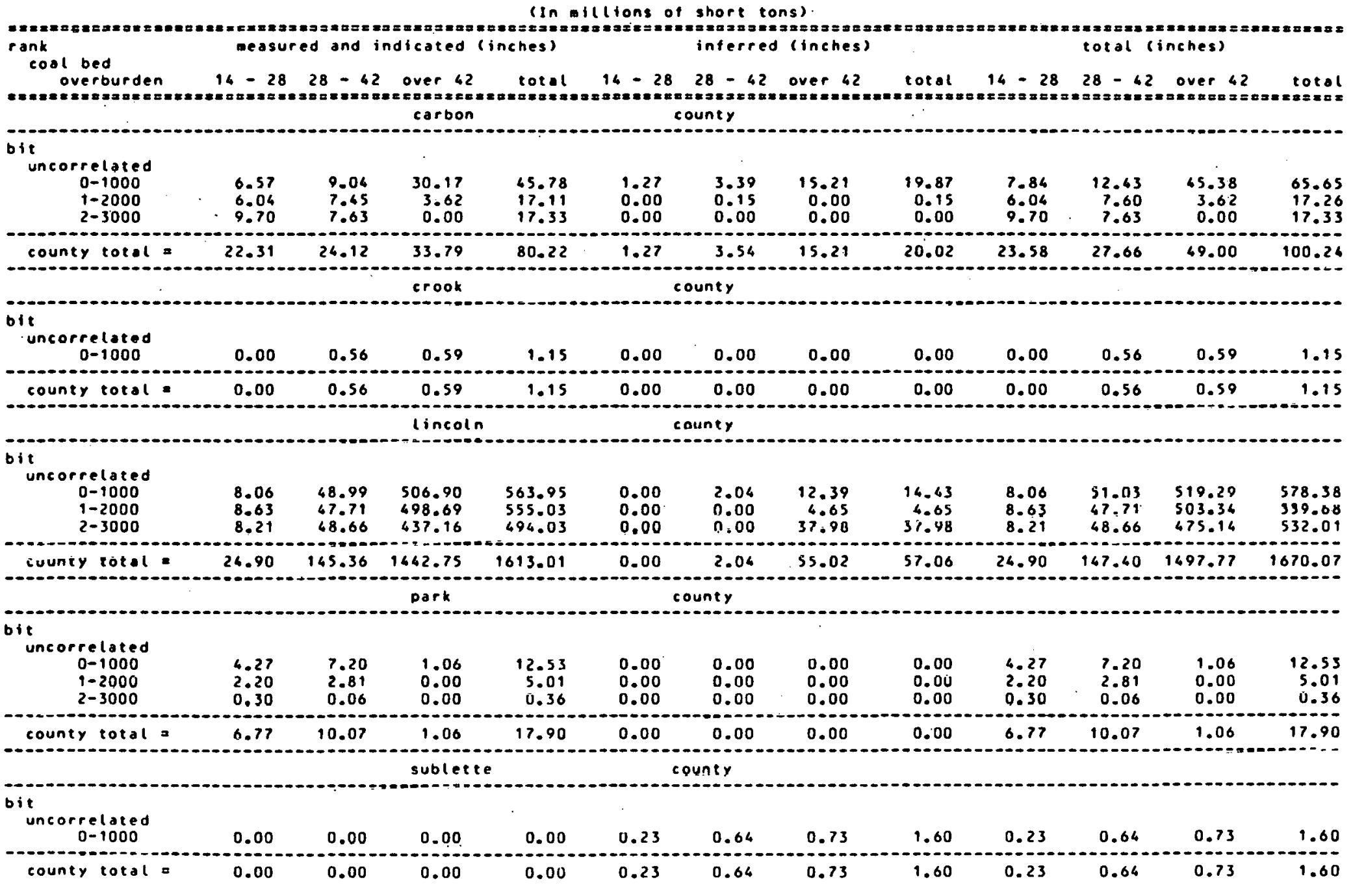




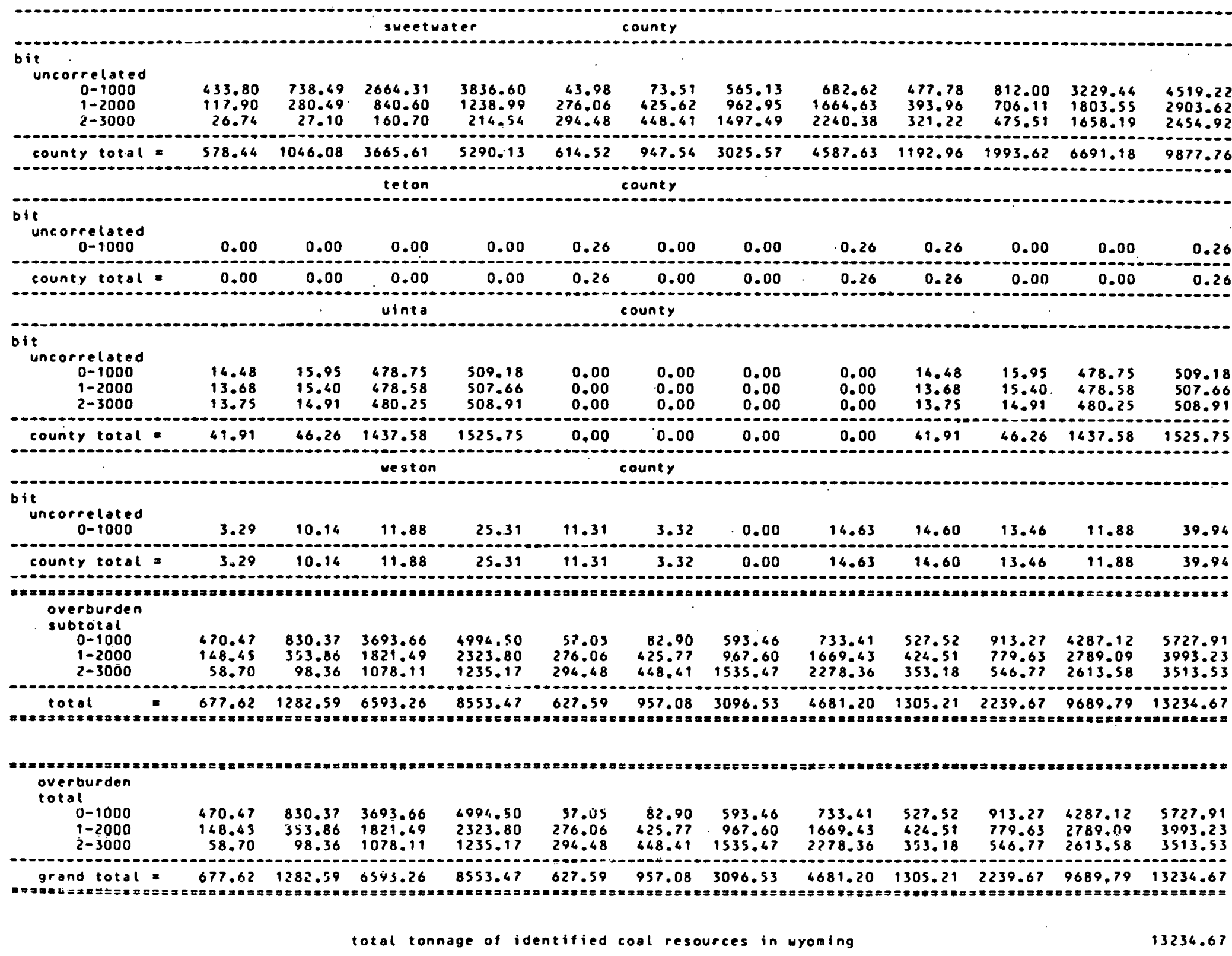


resource figures taken from:

source

year

1950

base year of resource

usgs circ 81

original 
overage onalyses of coal in wyoming

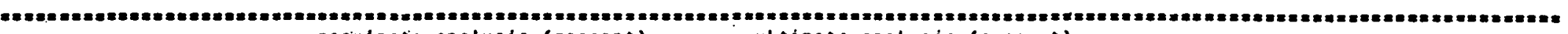

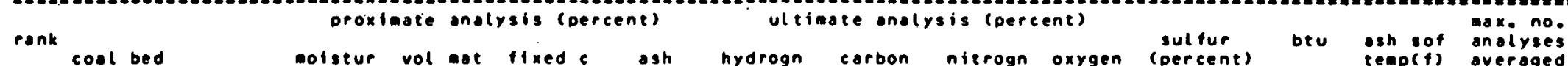

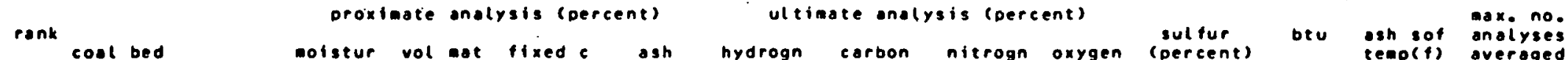

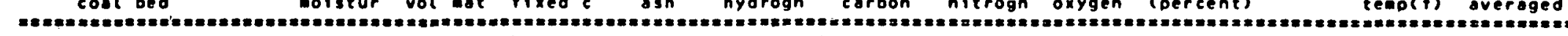

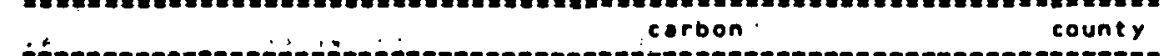

bit uncorreloted
county averoge

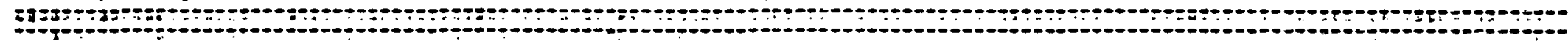

C. 1 county

bit

uncorreloted

willow creek

county average

$\begin{array}{lll}5.6 & 42.7 & 49.8 \\ 6.1 & 40.1 & 51.6\end{array}$

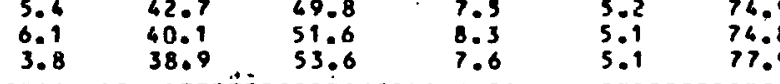

$5.241 .3 \quad 51.0 \quad 7.7$

9

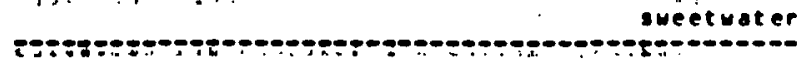

county

\section{rock springs no $1 \quad 7.5 \quad 41.6 \quad 51.2 \quad 7.3 \quad 0.0 \quad 0.0$}

$\begin{array}{lllllll}\text { rock springs no } 3 & 6.5 & 41.6 & 51.2 & 7.3 & 0.0 & 0.0 \\ \text { rock springs } & 41.6 & 53.4 & 5.0 & 5.4 & 76.5\end{array}$

rock springs no?

rock sorings nos 183

vartous

$\begin{array}{lll}6.5 & 41.9 & 54.7 \\ 7.5 & 41.1 & 53.3\end{array}$

$\begin{array}{lll}6.6 & 41.9, & 53.3 \\ 63.7 & 41.7 & 53.7\end{array}$

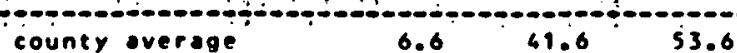

county overage $\quad 6.6 \quad 41.6 \quad 53.6 \quad 6.60$

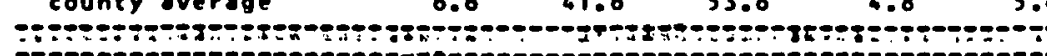

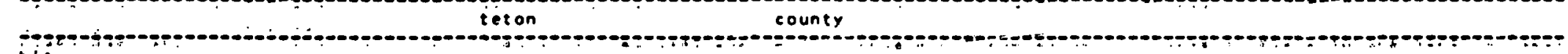

C bis un

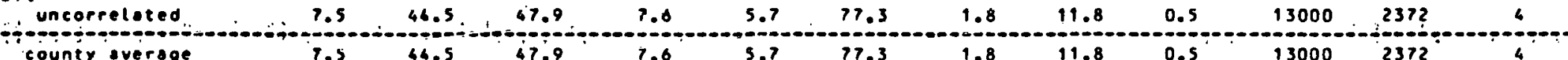

county average

7.546 .5

$47.9 \quad 7.6$

$5.7 \quad 77.3$

county

$\cup \quad$ ind bit

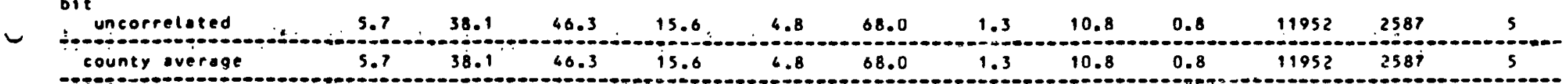

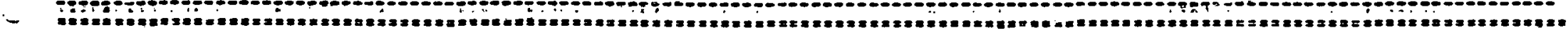




\begin{tabular}{|c|c|c|c|c|c|c|c|c|c|c|}
\hline 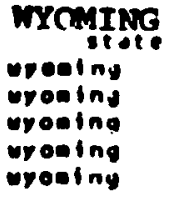 & $\begin{array}{l}\text { lincoleouney } \\
\text { lincoln } \\
\text { lincoln } \\
\text { lincoln } \\
\text { unnteln } \\
\text { ulnte }\end{array}$ & $\begin{array}{l}\text { hed } \\
\text { uncorreleted } \\
\text { uncarreleted } \\
\text { weaberer } \\
\text { uncorreleped } \\
\text { uncorreloted }\end{array}$ & $\begin{array}{c}\text { an ise ur } \\
1.9 \\
0.0 \\
7.1 \\
5.3 \\
5.5\end{array}$ & $\begin{array}{r}010.1 \\
42.1 \\
62.5 \\
66.4 \\
47.3 \\
34.3\end{array}$ & $\begin{array}{r}11 \text { wede } \\
50.5 \\
51.1 \\
69.3 \\
53.2 \\
46.3\end{array}$ & $\begin{array}{r}.9 n \\
1.4 \\
6.4 \\
6.3 \\
6.5 \\
15.4\end{array}$ & $\begin{array}{l}801 \text { tur } \\
0.6 \\
0.7 \\
0.8 \\
0.7 \\
0.8\end{array}$ & $\begin{array}{l}\text { biv } \\
13180 \\
13260 \\
13220 \\
13310 \\
11920\end{array}$ & $\begin{array}{c}1110 \\
55 \\
0 \\
0 \\
0 \\
50\end{array}$ & $\begin{array}{l}18110 \\
1.8 \\
1.5 \\
1.0 \\
1.5 \\
1.5\end{array}$ \\
\hline
\end{tabular}


PROCESS DMISION

WRLLANS BROTHERS ENCENERTANG COMPANY

$$
D O=/ M C / 14584-1193
$$

PREFACE

Appendix A is a catalog of the bituminous coal in 29 states of the contiguous United States which contain identified bituminous coal resources.

PO -401 